\title{
STATISTICAL SUMMARIES OF STREAMFLOW DATA IN NEW MEXICO THROUGH 1985
}

By Scott D. Waltemeyer

\section{U.S. GEOLOGICAL SURVEY}

Water-Resources Investigations Report 88-4228

Prepared in cooperation with the STATE OF NEW MEXICO AND OTHER AGENCIES

Albuquerque, New Mexico 
DEPARTMENT OF THE INTERIOR

MANUEL LUJAN, JR., Secretary

U.S. GEOLOGICAL SURVEY

Dallas L. Peck, Director

For additional information

write to:

District Chief

U.S. Geological Survey

Water Resources Division

Pinetree Office Park

4501 Indian School Rd. NE, Suite 200

Albuquerque, New Mexico 87110
Copies of this report can be purchased from:

U.S. Geological Survey Books and Open-File Reports Federal Center, Building 810 Box 25425

Denver, Colorado 80225 
Abstract........................................... 1

Introduction $\ldots \ldots \ldots \ldots \ldots \ldots \ldots \ldots \ldots \ldots \ldots \ldots \ldots \ldots \ldots \ldots \ldots \ldots \ldots \ldots$

Previous studies....................................... 1

Streamflow records $\ldots \ldots \ldots \ldots \ldots \ldots \ldots \ldots \ldots \ldots \ldots \ldots \ldots \ldots \ldots \ldots \ldots \ldots \ldots \ldots \ldots \ldots$

Statistical summaries............................... 2

Mean monthly and mean annual discharge.................. 4

Low-flow frequency............................. 4

High-flow frequency............................ 4

Flow duration.................................. 6

Effects of regulation $\ldots \ldots \ldots \ldots \ldots \ldots \ldots \ldots \ldots \ldots \ldots \ldots \ldots \ldots \ldots \ldots \ldots$

References cited $\ldots \ldots \ldots \ldots \ldots \ldots \ldots \ldots \ldots \ldots \ldots \ldots \ldots \ldots \ldots \ldots \ldots \ldots \ldots \ldots \ldots$

List of streamflow-gaging stations in downstream order............ 8

Streamflow-gaging-station descriptions and statistical tables......... 13

\section{FIGURES}

Figure 1. Map showing location of streamflow-gaging stations..........

2. Graph showing low-flow frequency curves of Arroyo

Hondo at Arroyo Hondo, New Mexico....................

3. Graph showing high-flow frequency curves of Arroyo

Hondo at Arroyo Hondo, New Mexico....................

4. Graph showing duration curve for daily flows of Arroyo

Hondo at Arroyo Hondo, New Mexico. 


\section{CONVERSION FACTORS}

The inch-pound units in this report can be converted to the metric system of units as follows:

Multiply inch-pound unit

$$
\text { acre }
$$

acre-foot per year

(acre-ft/yr)

cubic foot per second

$$
(\mathrm{ft} / \mathrm{s})
$$

foot ( $f t$ )

inch per year (in/yr)

mile (mi)

square mile $\left(\mathrm{mi}^{2}\right)$
By

$$
0.004047
$$

0.001233

0.02832

0.3048

25.40

1.609

2.590
To obtain metric unit

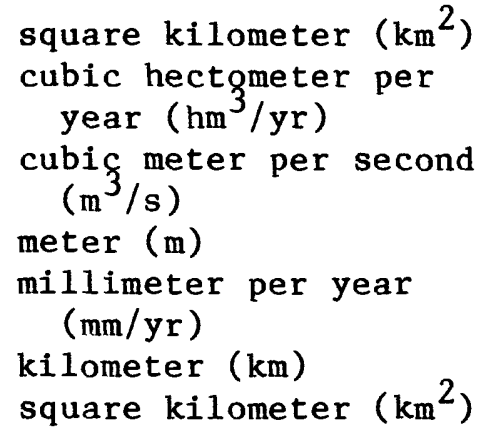

Sea level: In this report "sea level" refers to the National Geodetic Vertical Datum of 1929 (NGVD of 1929)--a geodetic datum derived from a general adjustment of the first-order level nets of both the United States and Canada, formerly called "Sea Level Datum of 1929." 


\title{
STATISTICAL SUMMARIES OF STREAMFLOW DATA IN NEW MEXICO THROUGH 1985
}

\author{
By Scott D. Waltemeyer
}

\begin{abstract}
Statistical summaries of streamflow data collected at selected gaging stations are presented in this report to aid in appraising the hydrology of New Mexico. Streamflow records are presented for 169 gaging stations for their periods of record. Records for 17 stations are separated into periods before, after, and between changes in upstream regulation. For each gaging station, a brief description is given for station location, drainage area, period of record, revisions of previously published records, type and history of gages, regulation and diversions, average discharge, and extremes of discharge. These data are followed by statistical summaries of mean monthly and mean annual flow statistics, low-flow and high-flow frequency, and flowduration information.
\end{abstract}

\section{INTRODUCTION}

As a prerequisite to comprehensive planning for the development and management of the State's surface-water resources, the State of New Mexico is actively appraising the hydrology of the State. The purpose of this report is to present statistical summaries of streamflow data collected at 169 selected streamflow-gaging stations in New Mexico to aid in that appraisal. Mean monthly and mean annual discharge, low-flow and high-flow frequency, and flowduration information were determined for 169 stations. These data will be useful to individuals and agencies concerned with management of surface water in New Mexico. This report was prepared in cooperation with the State of New Mexico and other agencies. Special thanks are extended to two individuals: Gary D. Rogers, U.S. Geological Survey, who wrote the original computer program to merge all statistical data into one table, and Clyde Alexander, U.S. Geological Survey, who modified the program for use on the current Survey computer system.

\section{PREVIOUS STUDIES}

Reiland and Haynes (1963) presented duration of daily mean discharge and selected sequences of low-mean and high-mean discharge for 122 gaging stations. Techniques for determining flow-duration data and low- and highflow frequency data were given. Borland (1970) presented regional relations based upon records of 64 gaging stations. The statewide relations were derived for selected sequences of low- and high-flow frequencies as related to basin and climatic characteristics. Mean monthly and mean annual discharge 
relations also were derived. Reiland (1980) presented flow-duration data for 156 gaging stations in a part I series. A part II series was to include the log-Pearson Type III probability distribution of the low- and high-mean discharges for specified numbers of days. The report herein presents that low- and high-flow frequency data for the gaging stations of New Mexico.

\section{STREAMFLOW RECORDS}

Al1 existing and discontinued streamflow-gaging stations with 10 years or more of record of mean daily discharge were used for this compilation report. Streamflow statistics are presented in this report for the gaging stations shown in figure 1. The station numbering is by the standard downstream order of the U.S. Geological Survey.

Records through September 30, 1985 (or September 30 of the last year of record, if discontinued prior to 1985), were used in computing mean monthly and mean annual discharge, high-flow frequency, and flow-duration information. Low-flow frequency data were computed on the basis of the climatic year, which ends March 31. A station description of the gaging station precedes the statistical summaries for each station. The station description consists of location, drainage area, period of record, type and history of gages, remarks on regulation and diversions, average discharge, and extremes of discharge. The station descriptions are identical to the annual water-data report series such as "Water Resources Data--New Mexico" by Denis and others (1986), except for categories describing revised records, extremes, and remarks for a specific year of record. Detailed definitions and explanations of each category of the station descriptions are given in that report. Some revised records are referenced to "WSP" (Water-Supply Paper), a formal publication series of the Geological Survey.

The natural flow of streams has been altered by regulation and is reflected in the records of 17 stations. The statistical summaries for periods before, after, and between changes of regulation are separated into each respective period.

\section{Statistical Summaries}

Statistical summaries presented for the stations include mean monthly and mean annual discharge, magnitude and probability of annual low flow, magnitude and probability of annual high flow, and duration of flow. The monthly and annual data were processed using the Daily Values Monthly and Annual Statistics (Program W4422) computer program by Price and Meeks (1977). Lowflow, high-flow, and flow-duration data were processed by the Daily Value Statistics (Program A969) computer program by Meeks (1977). Statistics are missing in some cases because of a lack of monthly data, frequency-curve computation failures, or insufficient length of record. These cases are denoted by a dash (--). 


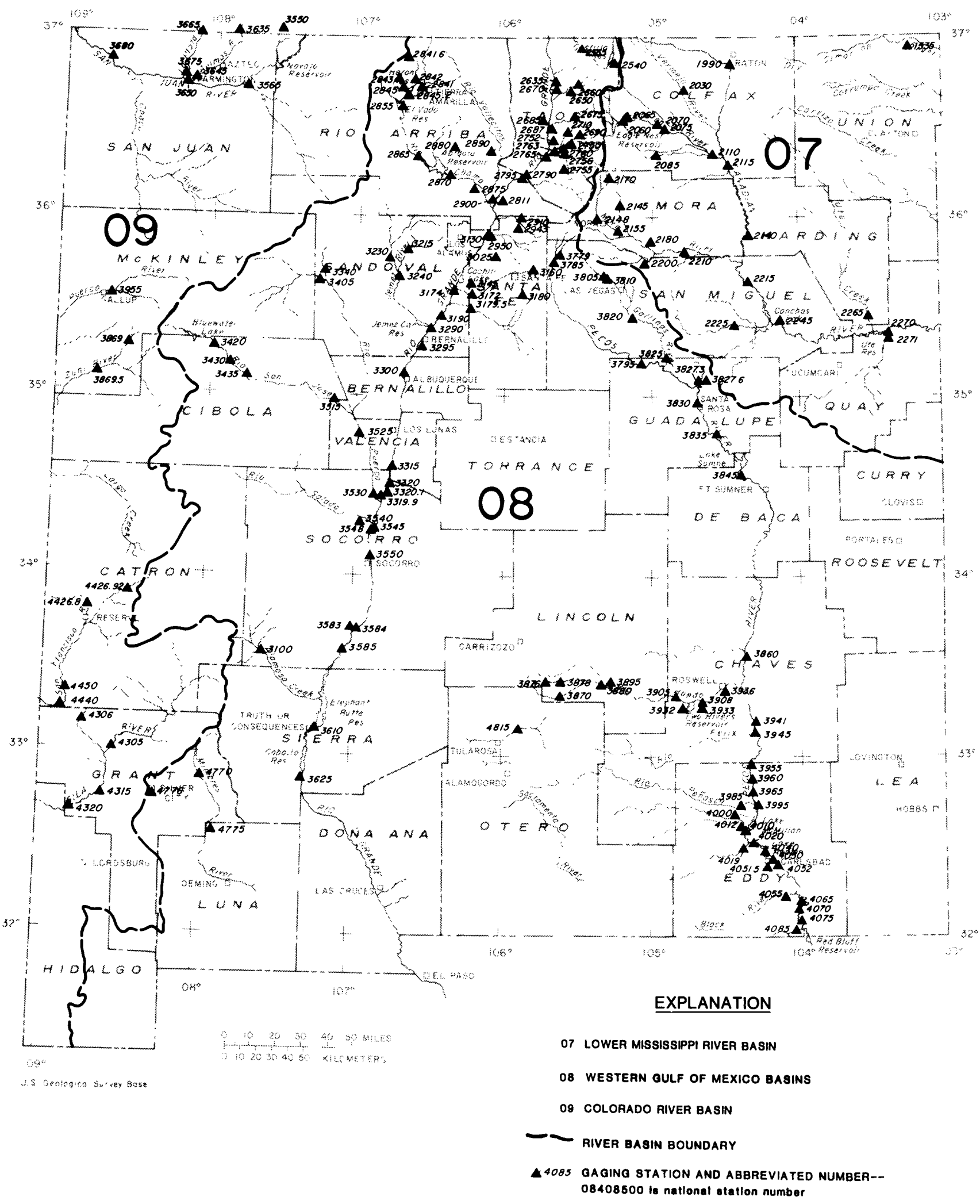

Figure 1.--Location of streamflow-gaging stations. 
Mean Monthly and Mean Annual Discharge

The tabulations for the mean monthly and mean annual discharges for the period of record include the minimum, maximum, mean, standard deviation, coefficient of variation (ratio of the standard deviation to the mean), and the percentage of mean annual runoff for each mean monthly discharge. Mean, standard deviation, and coefficient of variation are defined in standard textbooks on statistics. The general meaning of the mean is a measure of the central tendency of a group of data, standard deviation is a measure of the variability, and coefficient of variation is a dimension-free comparison of the standard deviation in units of the mean.

\section{Low-Flow Frequency}

Low-flow frequency analysis was made by fitting a log-Pearson Type III distribution to each annual series of flows. The log-Pearson Type III distribution is defined in standard textbooks on hydrology. The data necessary to plot standard low-flow frequency curves from the log-Pearson Type III frequency distribution are shown in the low-flow tabulations. Graphs may be desired of the low-flow frequency curves. The resulting plot shows the relation between non-exceedance probability as abscissa and average discharge for specified numbers of days as ordinate. An example low-flow frequency curve is shown in figure 2. The example in figure 2 shows there is a 20-percent chance that the lowest 1-day average flow in a given year will be less than 5.20 cubic feet per second. The smallest mean discharge for consecutive periods of $1,3,7,14,30,60,90,120$, and 183 days for recurrence intervals of $2,5,10,20,50$, and 100 years is shown in the tabulation. The associated non-exceedance probabilities, expressed as percentages, are $50,20,10,5,2$, and 1 percent, respectively. Recurrence intervals generally were extended to only twice the period of record. Records of more than 40 years were extended to the 100-year recurrence interval $(0.01$ non-exceedance probability).

\section{High-Flow Frequency}

High-flow frequency analysis also was made by fitting a log-Pearson Type III distribution to each annual series of flows. The data necessary to plot standard high-flow frequency curves from the log-Pearson Type III frequency distribution are shown in the high-flow tabulations. The resulting plot is a graph showing the relation between exceedance probability as abscissa and high-flow data as ordinate. An example high-flow frequency curve is shown in figure 3. The example shows that a flow of 240 cubic feet per second for 1 day has a 20-percent chance of being equaled or exceeded. The largest mean discharge for consecutive periods of $1,3,7,15,30$, 60, and 90 days for recurrence intervals of $2,5,10,25,50$, and 100 years is shown in the tabulation. The associated exceedance probabilities, expressed as percentages, are $50,20,10,4,2$, and 1 percent, respectively. The criteria for extending records of high-flow data are the same as those for low-flow data. 


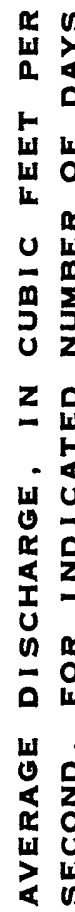

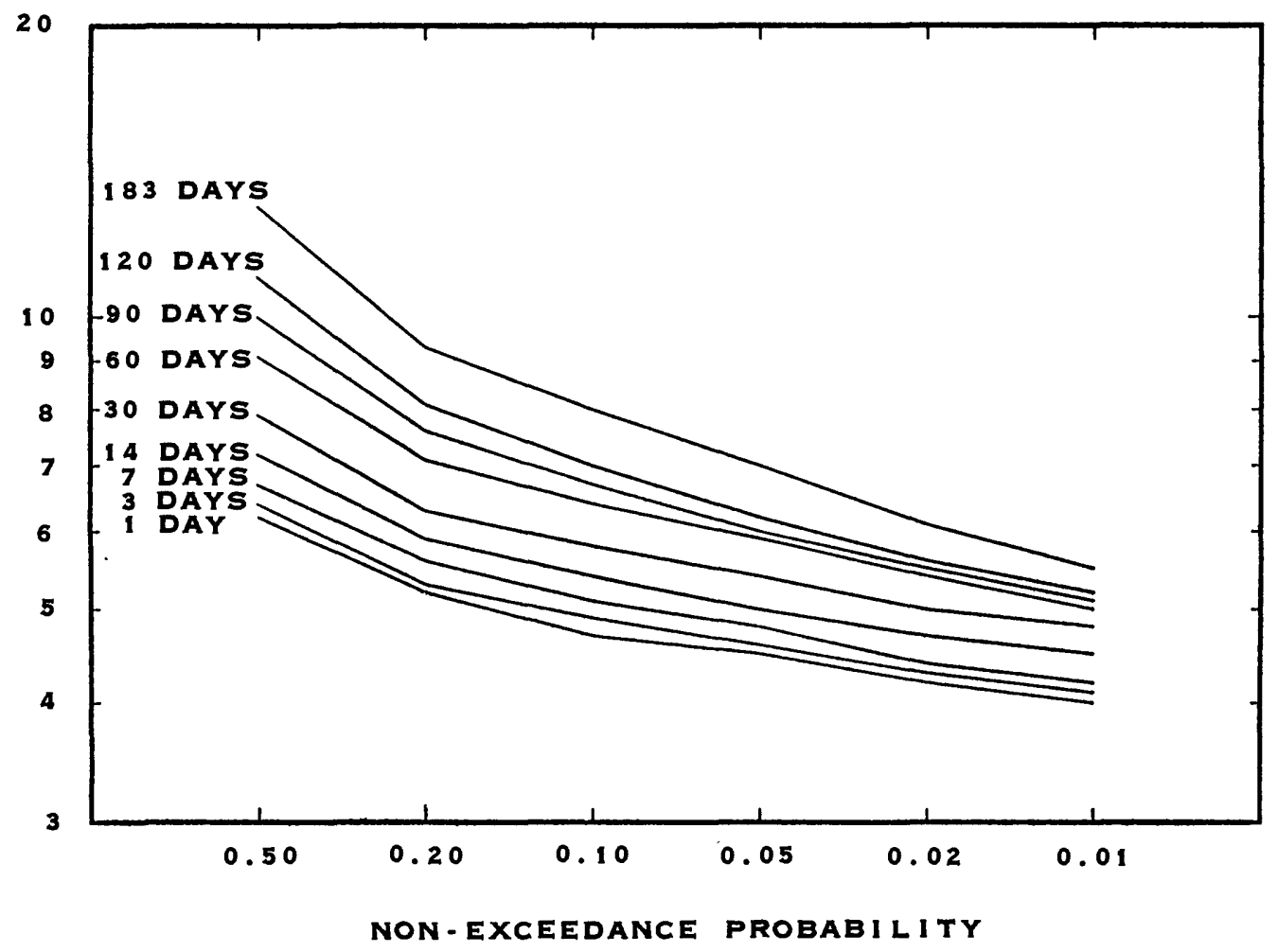

Figure 2.--Low-flow frequency curves of Arroyo Hondo at Arroyo Hondo, New Mexico.

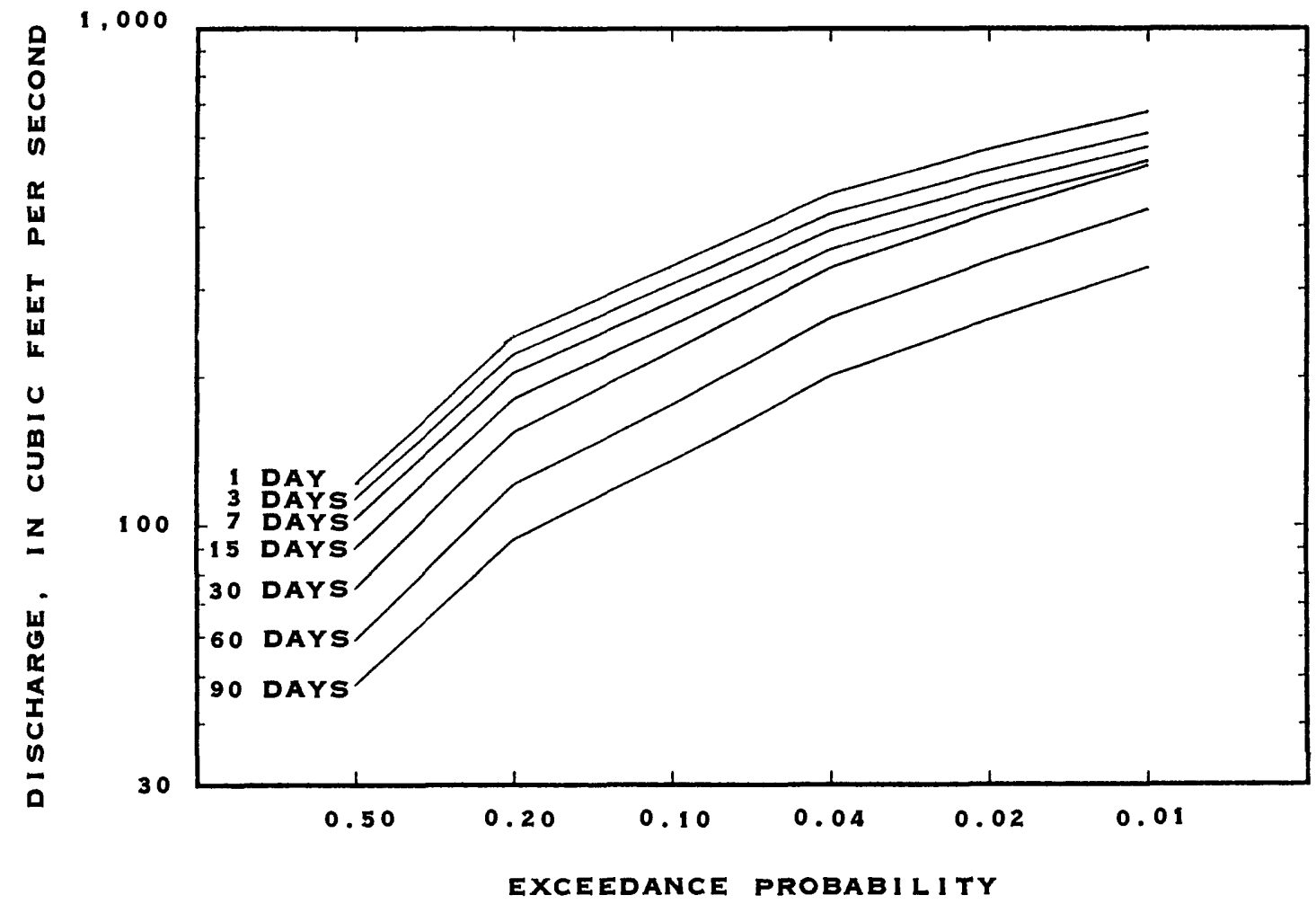

Figure 3.--High-flow frequency curves of Arroyo Hondo at Arroyo Hondo, New Mexico. 
The flow-duration tabulations list the data necessary to plot a standard flow-duration curve. The flow-duration curve is a cumulative frequency curve, also known as cumulative distribution function, that shows the percentage of time that specified discharges were equaled or exceeded. The discharges, in cubic feet per second, that were equaled or exceeded for a given percentage of time are shown in the tabulations. An example flow-duration curve for Arroyo Hondo is shown in figure 4. The example shows that a daily-average discharge of 54 cubic feet per second has been equaled or exceeded 10 percent of the time.

\section{Effects of Regulation}

The natural flow of many streams in New Mexico is altered by regulation by dams or diversions for irrigation. Where these conditions exist, the reported data reflect the pattern of operation of regulation and diversion. The "Remarks" section of the station description indicates known regulations and diversions. Periods of natural flow were segregated from periods of flow affected by regulation by dams or major diversions at 17 stations. If this information is needed for other sites, special requests can be directed to the District Office in Albuquerque, New Mexico. Depending upon the number of sites involved, a cost for computer services may be charged.

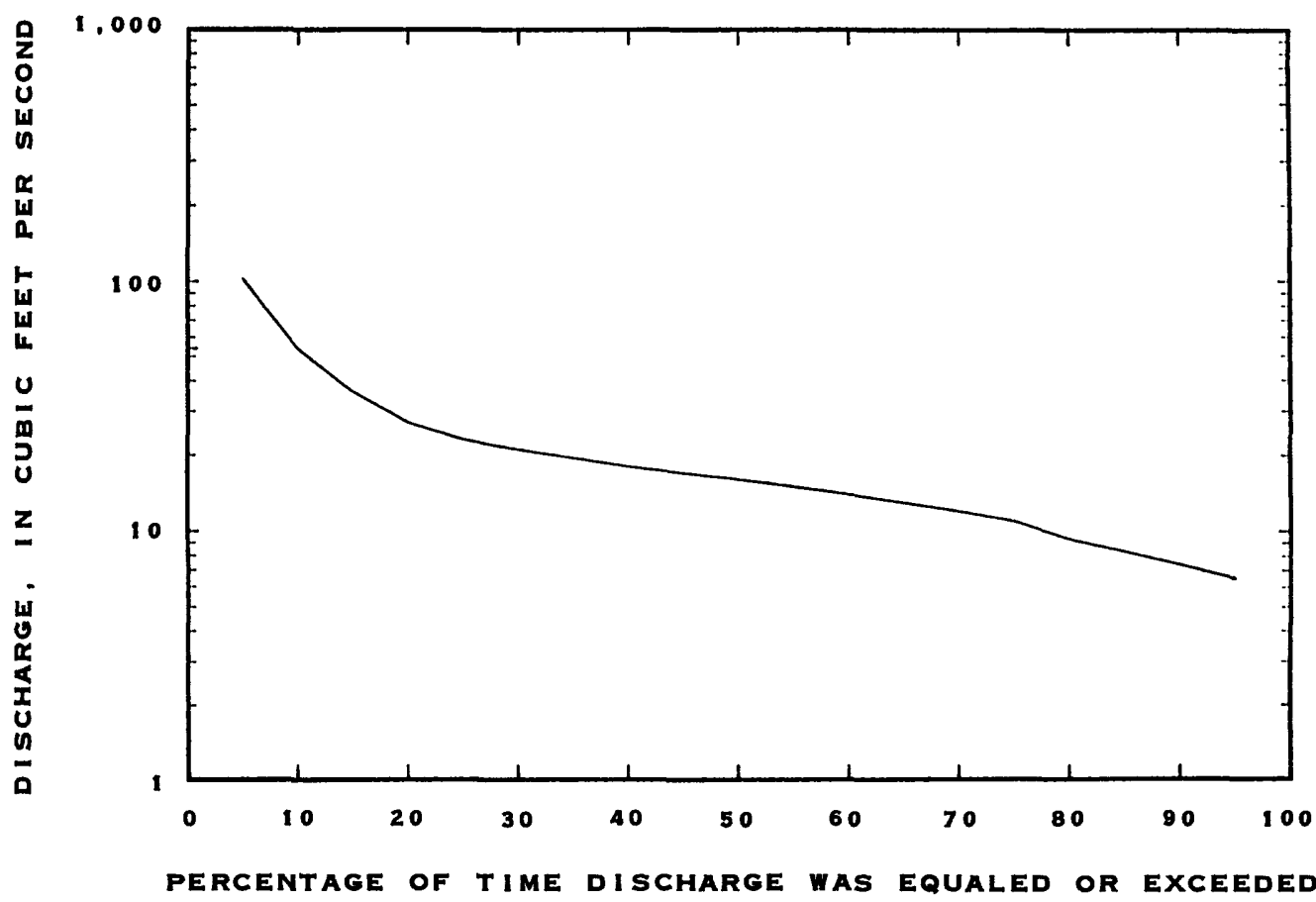

Figure 4.--Duration curve for daily flows of Arroyo Hondo at Arroyo Hondo, New Mexico. 


\section{REPERENCES CITED}

Borland, J.P., 1970, A proposed streamflow-data program for New Mexico: U.S. Geological Survey open-file report, $71 \mathrm{p}$.

Denis, L.D., Beal, L.V., and Allen, H.R., 1986, Water resources data, New Mexico-Water year 1985: U.S. Geological Survey Water-Data Report NM-85-1, 482 p.

Meeks, W.C., 1977, Daily values statistics (Program A969), chapter IV, section G, of WATSTORE User's Guide: U.S. Geological Survey Open-File Report 75-426, v. 1, p. G-1 to G-37.

Price, W.E., Jr., and Meeks, W.C., 1977, Daily values monthly and annual statistics (Program W4422), chapter IV, section F, of WATSTORE User's Guide: U.S. Geological Survey Open-File Report 75-426, v. 1, p. F-1 to F-46.

Reiland, L.J., 1980, Flow characteristics of New Mexico streams: New Mexico State Engineer Special Report, 115 p.

Reiland, L.J., and Haynes, G.L., Jr., 1963, Flow characteristics of New Mexico streams: New Mexico State Engineer Special Report, 342 p. 


\section{LIST OF STREAMIPLOW-GAGING STATIONS IN DOWNSTREAM ORDER}

Station

number

Station name

Page

\section{LOWER MISSISSIPPI RIVER BASIN}

Arkansas River basin

07153500

07199000

07203000

07206000

07206500

07207000

07207500

07208500

07211000

07211500

07214000

07214500

07214800

07215500

07216500

07217000

07218000

07220000

07221000

07221500

07222500

07224500

07226500

07227000

07227100
Dry Cimarron River near Guy, New Mexico...................

Canadian River near Hebron, New Mexico.....................

Vermejo River near Dawson, New Mexico......................

Cimarron River below Eagle Nest Dam, New Mexico..............

Cimarron River at Ute Park, New Mexico.....................

Cimarron River near Cimarron, New Mexico.....................

Ponil Creek near Cimarron, New Mexico.......................

Rayado Creek at Sauble Ranch, near Cimarron, New Mexico.........

Cimarron River at Springer, New Mexico......................

Canadian River near Taylor Springs, New Mexico................

Canadian River near Roy, New Mexico.........................

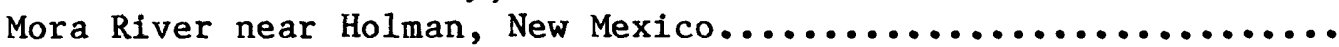

Rio La Casa near Cleveland, New Mexico......................

Mora River at La Cueva, New Mexico.......................

Mora River near Golondrinas, New Mexico.....................

Coyote Creek below Black Lake, New Mexico....................

Coyote Creek near Golondrinas, New Mexico.....................

Sapello River at Sapello, New Mexico........................

Mora River near Shoemaker, New Mexico......................

Canadian River near Sanchez, New Mexico.....................

Conchas River at Variadero, New Mexico......................

Canadian River below Conchas Dam, New Mexico.................

Ute Creek near Logan, New Mexico.........................

Canadian River at Logan, New Mexico.......................

Period after completion of Ute Dam..................... 37

Revuelto Creek near Logan, New Mexico...................... 38

\section{WESTERN GULF OF MEXICO BASINS}

Rio Grande basin

08254000

Costilla Creek below Costilla Dam, New Mexico................

08255500

08263000

08263500

08264000

08265000

08266000

08267000

08267500

08268500

08268700
Costilla Creek near Costilla, New Mexico.....................

Latir Creek near Cerro, New Mexico........................

Rio Grande near Cerro, New Mexico........................

Red River near Red River, New Mexico......................

Red River near Questa, New Mexico.........................

Cabresto Creek near Questa, New Mexico......................

Red River at Mouth, near Questa, New Mexico.................

Rio Hondo near Valdez, New Mexico........................

Arroyo Hondo at Arroyo Hondo, New Mexico...................

Rio Grande near Arroyo Hondo, New Mexico.................... 


\section{LIST OF STREAMFLOW-GAGING STATIONS IN DOWNSTREAM ORDER--Continued}

Station

number

Station name

Page

Rio Grande basin - Continued

08269000

08271000

08275000

08275300

08275500

08275600

08276000

08276300

08276500

08279000

08279500

08281100

08283500

08284100

08284160

08284200

08284300

08284500

08284520

08285500

08286500

08287000

08287500

08288000

08289000

08290000

08291000

08294300

08295000

08302500

08313000

08314500

08316000

08317200

08317400

08317950

08318000
Rio Pueblo de Taos near Taos, New Mexico...................

Rio Lucero near Arroyo Seco, New Mexico.....................

Rio Fernando de Taos near Taos, New Mexico..................

Rio Pueblo de Taos near Ranchito, New Mexico.................

Rio Grande del Rancho near Talpa, New Mexico.................

Rio Chiquito near Talpa, New Mexico......................

Rio Pueblo de Taos at Los Cordovas, New Mexico................

Rio Pueblo de Taos below Los Cordovas, New Mexico..............

Rio Grande below Taos Junction Bridge, near Taos, New Mexico....

Embudo Creek at Dixon, New Mexico.......................

Rio Grande at Embudo, New Mexico.......................

Rio Grande above San Juan Pueblo, New Mexico................

Rio Chama at Park View, New Mexico......................

Rio Chama near La Puente, New Mexico......................

Azotea Tunnel at Outlet, near Chama, New Mexico...............

Willow Creek above Heron Reservoir, near Los Ojos, New Mexico...

Period after completion of Azotea Tunne1................

Horse Lake Creek above Heron Reservoir, near Los 0jos,

New Mexico...................................... 67

Willow Creek near Park View, New Mexico................. 68

Willow Creek below Heron Dam, New Mexico.................. 69

Rio Chama below E1 Vado Dam, New Mexico.................. 70

Period after completion of Heron Dam.................. 71

Rio Chama above Abiquiu Reservoir, New Mexico.............. 72

Rio Chama below Abiquiu Dam, New Mexico.................. 73

Rio Chama near Abiquiu, New Mexico...................... 74

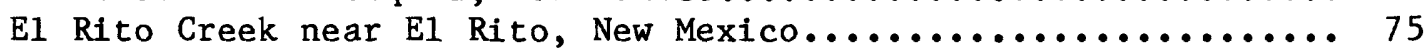

Rio 0jo Caliente at La Madera, New Mexico................. 76

Rio Chama near Chamita, New Mexico..................... 77

Period before regulation of Abiquiu Dam................ 78

Period after completion of Heron Dam.................. 79

Santa Cruz River at Cundiyo, New Mexico................... 80

Rio Nambe at Nambe Falls, near Nambe, New Mexico............ 81

Rio Nambe near Nambe, New Mexico....................... 82

Tesuque Creek above diversions, near Santa Fe, New Mexico...... 83

Rio Grande at Otowi Bridge, near San Ildefonso, New Mexico..... 84

Period after completion of Heron Dam.................. 85

Rio Grande at Cochiti, New Mexico...................... 86

Santa Fe River near Santa Fe, New Mexico.................. 87

Santa Fe River above Cochiti Lake, New Mexico.............. 88

Rio Grande below Cochiti Dam, New Mexico.................. 89

Galisteo Creek below Galisteo Dam, New Mexico............... 90

Galisteo Creek at Domingo, New Mexico.................... 91 


\section{LIST OF STREAMFLOW-GAGING STATIONS IN DOWNSTREAM ORDER--Continued}

Station

number

Station name

Page

\section{Rio Grande basin - Continued}

08319000 Rio Grande at San Felipe, New Mexico......................

Period before regulation of Cochiti Dam and after

completion of Abiquiu Dam......................... 93

Period after completion of Cochiti Dam.................. 94

08321500

Jemez River below East Fork, near Jemez Springs, New Mexico.... 95

08323000

Rio Guadalupe at Box Canyon, near Jemez, New Mexico............

08324000

Jemez River near Jemez, New Mexico.......................

08329000

Jemez River below Jemez Canyon Dam, New Mexico.............. 98

Period after completion of Jemez Canyon Dam............... 99

08329500

Rio Grande near Bernalillo, New Mexico.................... 100

08330000

08331500

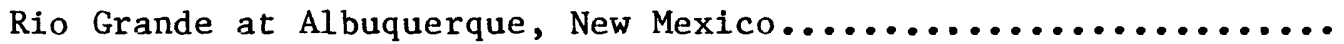

101

Period before regulation of Cochiti Dam and after

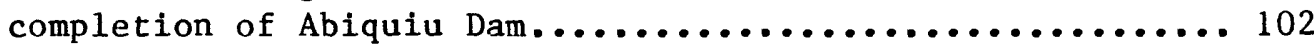

Period after completion of Cochiti Dam.................. 103

08331990

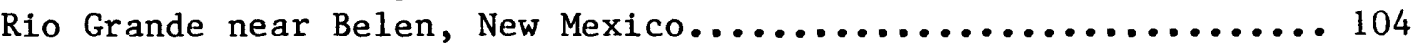

Rio Grande Conveyance Channel near Bernardo, New Mexico........ 105

Period before regulation of Cochiti Dam and after

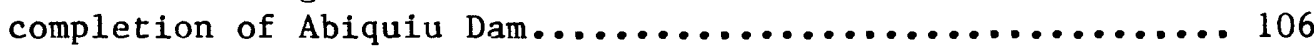

Period after completion of Cochiti Dam................ 107

08332000 Rio Grande near Bernardo, New Mexico...................... 108

08332010 Rio Grande Floodway near Bernardo, New Mexico............... 109

Period after completion of Cochiti Dam................. 110

08334000 Rio Puerco above Arroyo Chico, near Guadalupe, New Mexico....... 111

08340500 Arroyo Chico near Guadalupe, New Mexico.................... 112

08342000 Bluewater Creek near Bluewater, New Mexico.................. 113

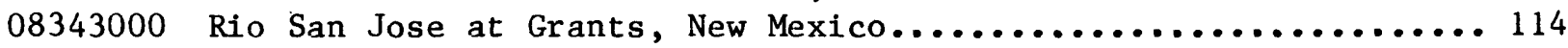

08343500 Rio San Jose near Grants, New Mexico...................... 115

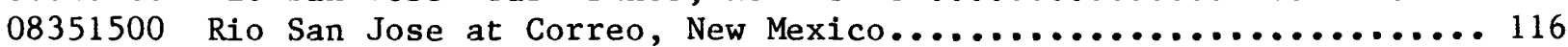

08352500 Rio Puerco at Rio Puerco, New Mexico........................ 117

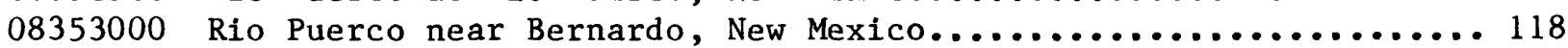

08354000 Rio Salado near San Acacia, New Mexico....................... 119

08354500 Socorro Main Canal North at San Acacia, New Mexico............ 120

08354800 Rio Grande Conveyance Channel at San Acacia, New Mexico........ 121

Period after completion of Cochiti Dam.................. 122

08355000 Rio Grande at San Acacia, New Mexico....................... 123

08358300 Rio Grande Conveyance Channel at San Marcial, New Mexico........ 124

Period before regulation of Cochiti Dam and after

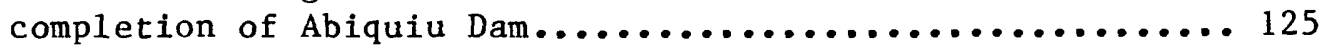

Period after completion of Cochiti Dam................. 126

08358400 Rio Grande Floodway at San Marcial, New Mexico............... 127

Period before regulation of Cochiti Dam and after completion of Abiquiu Dam................................ 128

Period after completion of Cochiti Dam.................. 129

08358500 Rio Grande at San Marcial, New Mexico...................... 130

08360000 Alamosa Creek near Monticello, New Mexico.................. 131 


\section{LIST OF STREAMPLOW-GAGING STATIONS IN DOWNSTREAM ORDER-Continued}

Station

number

Station name

Page

Rio Grande basin - Continued

08361000

08362500

08377900

08378500

08379500

08380500

08381000

08382000

08382500

08382730

08382760

08383000

08383500

08384500

08386000

08387000

08387600

08387800

08388000

08389500

08390500

08390800

08393200

08393300

08393600

08394100

08394500

08395500

08396000

08396500

08398500

08399500

08400000

08401000

08401200

08401900

08402000

08404000

08405000

08405150

Rio Grande below Elephant Butte Dam, New Mexico............. 132

Rio Grande below Caballo Dam, New Mexico.................. 133

Rio Mora near Terrero, New Mexico....................... 134

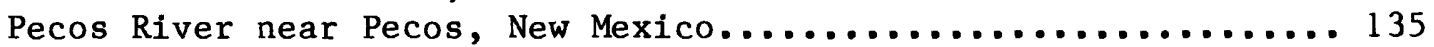

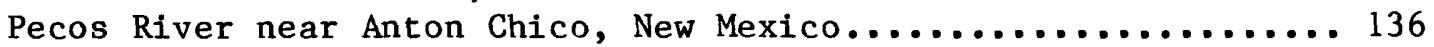

Gallinas Creek near Montezuma, New Mexico.................. 137

Gallinas Creek at Montezuma, New Mexico................... 138

Gallinas River near Lourdes, New Mexico.................... 139

Gallinas River near Colonias, New Mexico................... 140

Los Esteros Creek above Santa Rosa Lake, New Mexico........... 141

Los Esteros Creek tributary above Santa Rosa Lake, New Mexico... 142

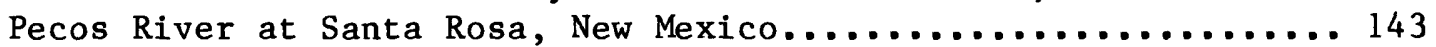

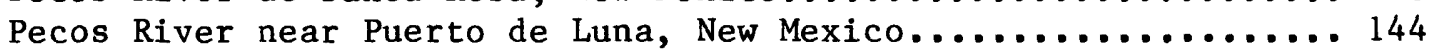

Pecos River below Sumner Dam, New Mexico.................... 145

Period before regulation of Santa Rosa Dam and after

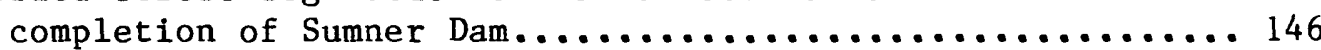

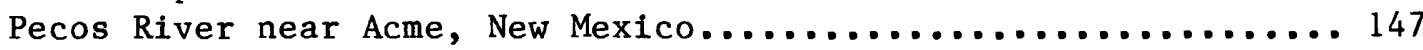

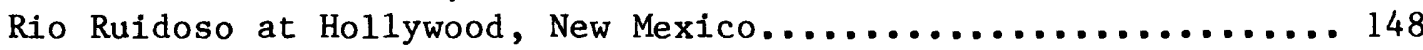

Eagle Creek below South Fork, near Alto, New Mexico........... 149

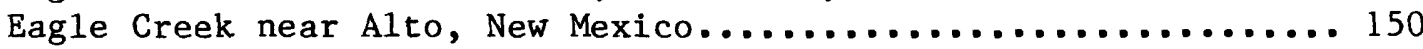

Rio Ruidoso at Hondo, New Mexico........................ 151

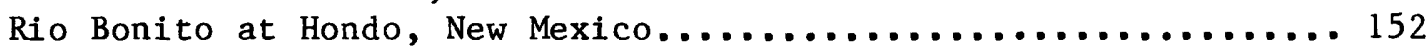

Rio Hondo at Diamond A Ranch, near Roswe11, New Mexico......... 153

Rio Hondo below Diamond A Dam, near Roswel1, New Mexico........ 154

Rocky Arroyo above Two Rivers Reservoir, near Roswe11,

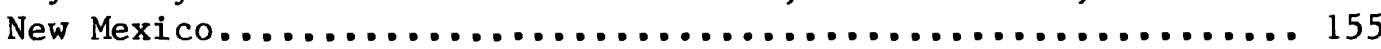

Rocky Arroyo below Rocky Dam, near Roswe11, New Mexico........ 156

North Spring River at Roswel1, New Mexico.................. 157

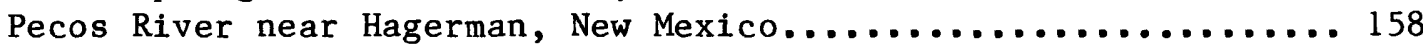

Rio Felix at 0ld Highway Bridge, near Hagerman, New Mexico..... 159

Pecos River near Lake Arthur, New Mexico................... 160

Cottonwood Creek near Lake Arthur, New Mexico................ 161

Pecos River near Artesia, New Mexico..................... 162

Period before regulation of Santa Rosa Dam and after

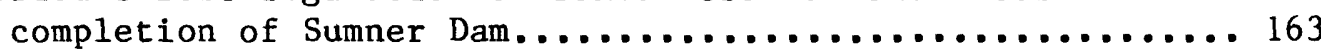

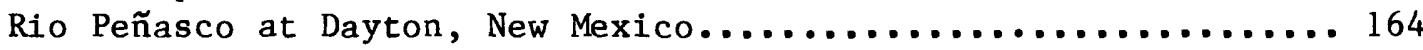

Pecos River (Kaiser Channel) near Lakewood, New Mexico......... 165

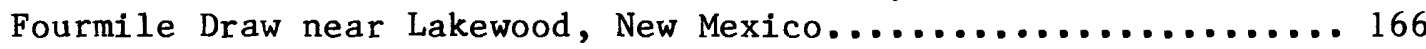

Pecos River below McMillan Dam, New Mexico................. 167

South Seven Rivers near Lakewood, New Mexico................. 168

Rocky Arroyo at Highway Bridge, near Carlsbad, New Mexico...... 169

Pecos River at Damsite 3, near Carlsbad, New Mexico............ 170

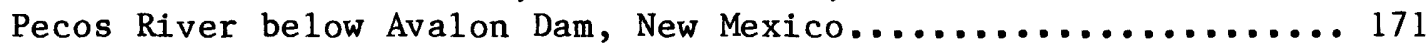

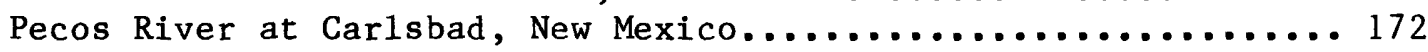

Dark Canyon Draw at Carlsbad, New Mexico.................. 173 


\section{LIST OF STREAMPLOW-GAGING STATIONS IN DOWNSTREAM ORDER--Concluded}

Station

number

Station name

Page

Rio Grande basin - Concluded

08405200

08405500

08406500

08407000

08407500

08408500

08477000

08477500

08477600

08481500
Pecos River below Dark Canyon Draw, at Carlsbad, New Mexico..... Black River above Malaga, New Mexico......................

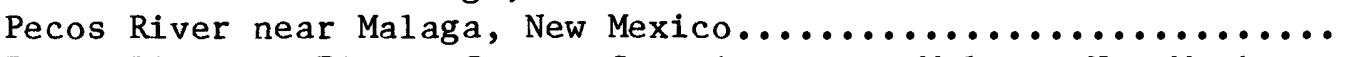
Pecos River at Pierce Canyon Crossing, near Malaga, New Mexico.. Pecos River at Red Bluff, New Mexico...................... Delaware River near Red Bluff, New Mexico..................

Mimbres River basin

Mimbres River near Mimbres, New Mexico.................... 180 Mimbres River near Faywood, New Mexico................... 181 San Vicente Arroyo at Silver City, New Mexico............... 182

Tularosa Valley basin

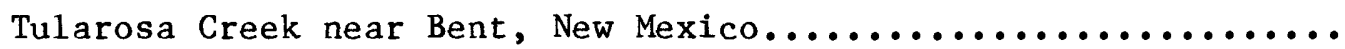
183

\section{COLORADO RIVER BASIN}

San Juan River basin

09355500

09356500

09363500

09364500

09365000

09366500

09367500

09368000

09386900

09386950

09395500

09430500

09430600

09431500

09432000

09442680

09442692

09443000

09444000

San Juan River near Archuleta, New Mexico.................. 184

San Juan River near Blanco, New Mexico................... 185

Animas River near Cedar Hill, New Mexico.................. 186

Animas River at Farmington, New Mexico................... 187

San Juan River at Farmington, New Mexico................. 188

Period after completion of Navajo Dam.................. 189

La Plata River at Colorado-New Mexico State line............. 190

La Plata River near Farmington, New Mexico................. 191

San Juan River at Shiprock, New Mexico.................... 192

Period after completion of Navajo Dam.................. 193

Little Colorado River basin

Rio Nutria near Ramah, New Mexico....................... 194

Zuni River above Black Rock Reservoir, New Mexico............ 195

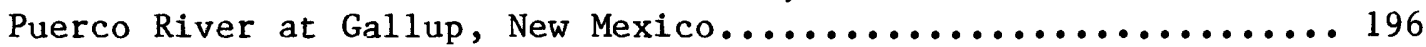

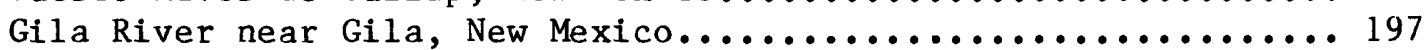

Mogollon Creek near Cliff, New Mexico...................... 198

Gila River near Redrock, New Mexico..................... 199

Gila River below Blue Creek, near Virden, New Mexico.......... 200

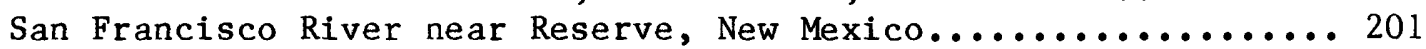

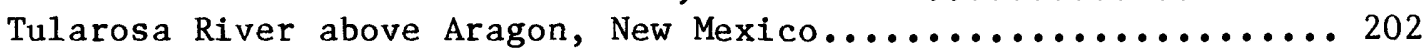

San Francisco River near Alma, New Mexico.................. 203

San Francisco River near Glenwood, New Mexico................ 204 
ARKANSAS RIVER BASIN

07153500 DRY CIMARRON RIVER NEAR GUY, NM

LOCATION.--Lat $36^{\circ} 59^{\prime} 15^{\prime \prime}$, long $10^{\circ} 25^{\prime} 25^{\prime \prime}$, in SEł sec.21, T.32 N., R.33 E., Union County, on right bank 1.5 mi upstream from Baker damsite, $1.7 \mathrm{mi}$ northwest of valley, $3.0 \mathrm{mi}$ upstream from Travesser Creek, $12 \mathrm{mi}$ north of Guy, $26 \mathrm{mi}$ northwest of Kenton, Okla., and at mile 634.5 .

DRAINAGE AREA. $--545 \mathrm{mi}^{2}$.

PERIOD OF RECORD.--April 1942 to December 1973 (discontinued). Prior to October 1965, published as Cimarron River near Guy.

REVISED RECORDS.--WSP 1177: Drainage area.

GAGE.--Water-stage recorder. Altitude of gage is 4,900 ft from topographic map. Prior to Oct. 1 , 1943 , at datum $0.44 \mathrm{ft}$ higher.

REMARKS.--Diversions for irrigation of about 6,500 acres above station.

AVERAGE DISCHARGE. -31 years (water years 1943-73), $10.0 \mathrm{ft}^{3} / \mathrm{s}, 7,240 \mathrm{acre}-\mathrm{ft} / \mathrm{yr}$.

EXTREMES FOR PERIOD OF RECORD.--Maximum discharge, $46,100 \mathrm{ft}^{3} / \mathrm{s}$ Aug, 21,1965 (gage height, $22.00 \mathrm{ft}, \mathrm{from} \mathrm{rating}$ curve extended above $3,000 \mathrm{ft}^{3} / \mathrm{s}$ on basis of slope-area measurements at gage heights $15.7 \mathrm{ft}$ and $22.00 \mathrm{ft}$; no flow at times.

STATISTICAL SUMMARIES

MEAN MONTHLY AND MEAN ANNUAL DISCHARGES 1943-73

\begin{tabular}{|c|c|c|c|c|c|c|}
\hline MONTH & $\begin{array}{l}\text { MI NI MUM } \\
\left(\mathrm{FT}^{3} / \mathrm{S}\right)\end{array}$ & $\begin{array}{c}\text { MAXIMUM } \\
\left(\mathrm{FT}^{3} / \mathrm{S}\right)\end{array}$ & $\begin{array}{l}\text { MEAN } \\
\left(\mathrm{FT}^{3} / \mathrm{S}\right)\end{array}$ & $\begin{array}{l}\text { STAN- } \\
\text { DARD } \\
\text { DEVIA- } \\
\text { TION } \\
\left(\mathrm{FT}^{3} / \mathrm{S}\right)\end{array}$ & $\begin{array}{l}\text { COEFFI - } \\
\text { CIENT OF } \\
\text { VARI- } \\
\text { ATION }\end{array}$ & $\begin{array}{c}\text { PERCENT } \\
\text { OF } \\
\text { ANNUAL } \\
\text { RUNOFF }\end{array}$ \\
\hline \multicolumn{7}{|c|}{ - - - } \\
\hline OCTOBER & .3 & 68 & 6.7 & 16 & 2.37 & 5.5 \\
\hline NOVEMBER & 1.1 & 20 & 3.7 & 3.3 & .91 & 3.0 \\
\hline DECEMBER & 1.2 & 17 & 3.7 & 3.0 & .80 & 3.0 \\
\hline JANUARY & .7 & 18 & 3.8 & 3.1 & .81 & 3.1 \\
\hline FEBRUARY & .8 & 15 & 3.5 & 2.6 & .75 & 2.8 \\
\hline MARCH & .9 & 16 & 3.7 & 3.1 & .84 & 3.0 \\
\hline APRIL & .2 & 52 & 5.2 & 9.5 & 1.83 & 4.2 \\
\hline MAY & .6 & 278 & 18 & 52. & 2.93 & 14.3 \\
\hline JUNE & 0.0 & 97 & 14 & 24 & 1.66 & 11.5 \\
\hline JULY & 1.4 & 61 & 19 & 18 & .95 & 15.1 \\
\hline AUGUST & .8 & 265 & 32 & 50 & 1.55 & 26.0 \\
\hline SEPTEMBER & .7 & 71 & 11 & 17 & 1.62 & 8.5 \\
\hline NNUAL & 3.1 & 39 & 10 & 8.4 & .81 & 100 \\
\hline
\end{tabular}

MAGNITUDE AND PROBABILITY OF ANNUAL LOW FLOW BASED ON PERIOD OF RECORD $1944-73$

\begin{tabular}{|c|c|c|c|c|c|c|}
\hline $\begin{array}{l}\text { PERIOD } \\
\text { (CON- }\end{array}$ & DISCH? & $\begin{array}{l}\text { IN } \\
\text { RVAL } \\
\text { EDAN }\end{array}$ & $\begin{array}{l}\text { S, } \\
\text { YEA } \\
\text { ROBAF }\end{array}$ & $\begin{array}{l}\text { INDI } \\
\text { AND } \\
\text { TY, }\end{array}$ & $\begin{array}{l}D \text { RE } \\
\text { AL N N } \\
\text { ERCE }\end{array}$ & ENCE \\
\hline $\begin{array}{l}\text { TIVE } \\
\text { DAYS) }\end{array}$ & $\begin{array}{c}2 \\
50 \%\end{array}$ & $\begin{array}{c}5 \\
20 \%\end{array}$ & $\begin{array}{l}10 \\
108\end{array}$ & $\begin{array}{l}20 \\
58\end{array}$ & $\begin{array}{l}50 \\
28\end{array}$ & $\begin{array}{r}100 \\
18\end{array}$ \\
\hline $\begin{array}{r}1 \\
3 \\
7 \\
14 \\
30 \\
60 \\
90 \\
120 \\
183\end{array}$ & $\begin{array}{r}.3 \\
.4 \\
.5 \\
.7 \\
1.1 \\
1.7 \\
2.1 \\
2.4 \\
2.7\end{array}$ & $\begin{array}{r}0.0 \\
0.0 \\
.1 \\
.2 \\
.4 \\
.8 \\
1.1 \\
1.6 \\
1.8\end{array}$ & $\begin{array}{r}0.0 \\
0.0 \\
0.0 \\
0.0 \\
.2 \\
.4 \\
.8 \\
1.2 \\
1.4\end{array}$ & $\begin{array}{r}0.0 \\
0.0 \\
0.0 \\
0.0 \\
0.0 \\
.3 \\
.6 \\
1.0 \\
1.2\end{array}$ & $\begin{array}{r}0.0 \\
0.0 \\
0.0 \\
0.0 \\
0.0 \\
.1 \\
.4 \\
.8 \\
1.0\end{array}$ & \\
\hline
\end{tabular}

MAGNITUDE AND PROBABJLITY OF ANNUAL HIGH FLOW BASED ON PERIOD OF RECORD $1943-73$

\begin{tabular}{|c|c|c|c|c|c|c|}
\hline \multirow{2}{*}{$\begin{array}{l}\text { PERIOD } \\
\text { (CON- } \\
\text { SECU- } \\
\text { TIVE } \\
\text { DAYS) }\end{array}$} & \multicolumn{6}{|c|}{$\begin{array}{c}\text { DISCHARGE, IN FT }{ }^{3} / \mathrm{S} \text {, FOR INDICATED RECURRENCE } \\
\text { INTERVAL, IN YEARS, AND ANNUAL } \\
\text { EXCEEDANCE PROBABILITY, IN PERCENT }\end{array}$} \\
\hline & $\begin{array}{c}2 \\
508\end{array}$ & $\begin{array}{c}5 \\
208\end{array}$ & $\begin{array}{l}10 \\
108\end{array}$ & $\begin{array}{l}25 \\
48\end{array}$ & $\begin{array}{l}50 \\
28\end{array}$ & $\begin{array}{r}100 \\
18\end{array}$ \\
\hline 1 & 500 & 1190 & 1960 & 3460 & 5100 & -- \\
\hline 3 & 217 & 546 & 935 & 1730 & 2650 & -- \\
\hline 7 & 114 & 270 & 446 & 797 & 1190 & -- \\
\hline 15 & 64 & 146 & 233 & 392 & 559 & -- \\
\hline 30 & 41 & 89 & 137 & 224 & 310 & - \\
\hline 60 & 26 & 56 & 84 & 132 & 178 & -- \\
\hline 90 & 20 & 42 & 64 & 103 & 141 & -- \\
\hline
\end{tabular}

DURATION TABLE OF DAILY MEAN FLOW FOR PERIOD OF RECORD 1943-73

DISCHARGE, IN FT ${ }^{3} / \mathrm{S}$, THAT WAS FQUALEN OR EXCEEDED FOR INDICATED PERCENT OF TIME

$\begin{array}{cccccccccc}58 & 108 & 158 & 208 & 258 & 308 & 408 & 508 & 608 & 708\end{array}$


ARKANSAS RIVER BASIN

07199000 CANADIAN RIVER NEAR HEBRON, NM

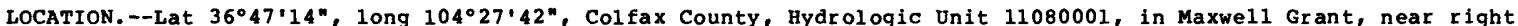
bank at downstream end of bridge pier on U.S. Highways 64 and $85,3.1 \mathrm{mi}$ north of Hebron, $5.0 \mathrm{mi}$ upstream from Chicorica Creek, $8.0 \mathrm{mi}$ south of Raton, and at mile 888.1.

DRAINAGE AREA. $--229 \mathrm{mi}^{2}$.

PERIOD OF RECORD.--June 1946 to current year.

REVISED RECORDS.--WSP 1281: 1946, 1947-48(P), 1949. WSP 1921: 1960 (M).

GAGE.--Water-stage recorder, Elevation of gage is 6,248 ft above National Geodetic Vertical Datum of 1929 , from topographic map. See WSP 1921 for history of changes prior to Aug. 18, 1965.

REMARKS.--Diversions upstream from station for irrigation of a few hundred acres. Part or all of low flow can be diverted to left bank $1.6 \mathrm{mi}$ upstream from station for stock water, off-channel storage and irrigation.

AVERAGE DISCHARGE. - -39 years (water years 1947-85), $8.38 \mathrm{ft} / \mathrm{s}, 6,070$ acre-ft/yr.

EXTREMES FOR PERIOD OF RECORD.--Maximum discharge, $62,400 \mathrm{ft} / \mathrm{s}$, June 17, 1965, gage height, $28.2 \mathrm{ft}$, from floodmarks, present datum, from rating curve extended above $1,300 \mathrm{ft} / \mathrm{s}$ on basis of slope-area measurement of peak flow; no flow for many days most years.

EXTREMES OUTSIDE PERIOD OF RECORD.--F1ood in 1942 reached a stage of about 28 ft, present datum, at site 150 ft upstream, from information by local residents.

STATISTICAL SUMMARIES

MEAN MONTHLY AND MEAN ANNUAL, DISCHARGES 1947-85

\begin{tabular}{|c|c|c|c|c|c|c|}
\hline MONTH & $\begin{array}{l}\text { MINIMUM } \\
\left(\mathrm{FT}^{3} / \mathrm{S}\right)\end{array}$ & $\begin{array}{c}\operatorname{MAXIMUM} \\
\left(\mathrm{FT}^{3} / \mathrm{S}\right)\end{array}$ & $\begin{array}{l}\text { MEAN } \\
\left(\mathrm{FT}^{3} / \mathrm{S}\right)\end{array}$ & $\begin{array}{l}\text { STAN- } \\
\text { DARD } \\
\text { DEVIA- } \\
\text { TION } \\
\left(\text { FT }^{2} / S\right)\end{array}$ & $\begin{array}{l}\text { COEFFI- } \\
\text { CIENT OF } \\
\text { VARI- } \\
\text { ATION }\end{array}$ & $\begin{array}{c}\text { PERCENT } \\
\text { OF } \\
\text { ANNUAL } \\
\text { RUNOFF }\end{array}$ \\
\hline $\begin{array}{l}\text { OCTOBER } \\
\text { NOVEMBER } \\
\text { DECEMBER } \\
\text { JANUARY } \\
\text { FEBRUARY } \\
\text { MARCH } \\
\text { APRIL } \\
\text { MAY } \\
\text { JUNE } \\
\text { JULY } \\
\text { AUGUST } \\
\text { SEPTEMBER }\end{array}$ & $\begin{array}{l}0.0 \\
0.0 \\
0.0 \\
0.0 \\
0.0 \\
0.0 \\
0.0 \\
.1 \\
.1 \\
.2 \\
0.0 \\
0.0\end{array}$ & $\begin{array}{c}19 \\
8.3 \\
4.4 \\
6.2 \\
4.4 \\
18 \\
134 \\
261 \\
461 \\
94 \\
209 \\
83\end{array}$ & $\begin{array}{l}2.4 \\
1.5 \\
1.1 \\
1.0 \\
.9 \\
2.0 \\
10 \\
18 \\
16 \\
16 \\
24 \\
6.8\end{array}$ & $\begin{array}{l}4.4 \\
1.9 \\
1.3 \\
1.3 \\
1.1 \\
4.3 \\
27 \\
47 \\
74 \\
22 \\
41 \\
14\end{array}$ & $\begin{array}{l}1.83 \\
1.32 \\
1.17 \\
1.27 \\
1.15 \\
2.18 \\
2.70 \\
2.58 \\
4.49 \\
1.34 \\
1.73 \\
2.04\end{array}$ & $\begin{array}{r}2.4 \\
1.5 \\
1.1 \\
1.0 \\
.9 \\
2.0 \\
10.0 \\
18.2 \\
16.4 \\
16.0 \\
23.6 \\
6.8\end{array}$ \\
\hline ANNUAL & .4 & 50 & 8.4 & 9.7 & 1.16 & 100 \\
\hline
\end{tabular}

MAGNITUDE AND PROBABILITY OF ANNUAL LOW FLOW BASED ON PERIOD OF RECORD 1948-85

\begin{tabular}{|c|c|c|c|c|c|c|}
\hline \multirow{2}{*}{$\begin{array}{l}\text { PERIOD } \\
\text { (CON- } \\
\text { SECU- } \\
\text { TIVE } \\
\text { DAYS) }\end{array}$} & \multicolumn{6}{|c|}{$\begin{array}{l}\text { DISCHARGE, IN FT } 3 / \text {, FOR INDICATED RECURRENCE } \\
\text { INTERVAL, IN YEARS, AND ANNUAL NON- } \\
\text { EXCEEDANCE PROBABILITY, IN PERCENT }\end{array}$} \\
\hline & $\begin{array}{c}2 \\
508\end{array}$ & $\begin{array}{c}5 \\
208\end{array}$ & $\begin{array}{l}10 \\
108\end{array}$ & $\begin{array}{l}20 \\
58\end{array}$ & $\begin{array}{l}50 \\
28\end{array}$ & \\
\hline $\begin{array}{r}1 \\
3 \\
7 \\
14 \\
30 \\
60 \\
90 \\
120 \\
183\end{array}$ & $\begin{array}{l}0.0 \\
0.0 \\
0.0 \\
0.0 \\
0.0 \\
.1 \\
.2 \\
.3 \\
.5\end{array}$ & $\begin{array}{l}0.0 \\
0.0 \\
0.0 \\
0.0 \\
0.0 \\
0.0 \\
.1 \\
.1 \\
.1\end{array}$ & $\begin{array}{r}0.0 \\
0.0 \\
0.0 \\
0.0 \\
0.0 \\
0.0 \\
0.0 \\
0.0 \\
.1\end{array}$ & $\begin{array}{l}0.0 \\
0.0 \\
0.0 \\
0.0 \\
0.0 \\
0.0 \\
0.0 \\
0.0 \\
0.0\end{array}$ & $\begin{array}{l}0.0 \\
0.0 \\
0.0 \\
0.0 \\
0.0 \\
0.0 \\
0.0 \\
0.0 \\
0.0\end{array}$ & \\
\hline
\end{tabular}

MAGNITUDE AND PROBABILITY OF ANNUAL HIGH FLOW BASED ON PERIOD OF RECORD 1947-85

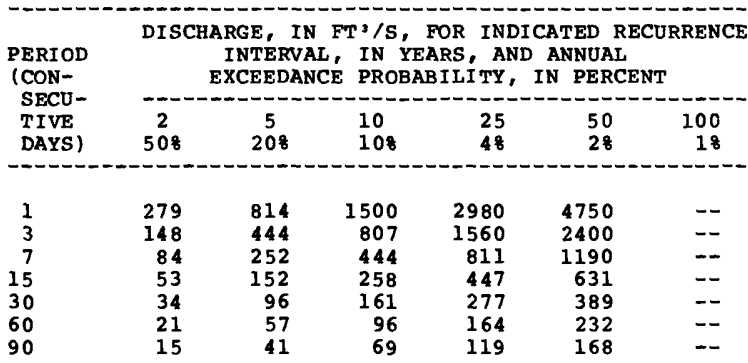

DURATION TABLE OF DAILY MEAN FLOW FOR PERIOD OF RECORD 1947-85

DISCHARGE, IN FT'/S, THAT WAS EQUALED OR EXCEEDED FOR INDICATED PERCENT OF TIM

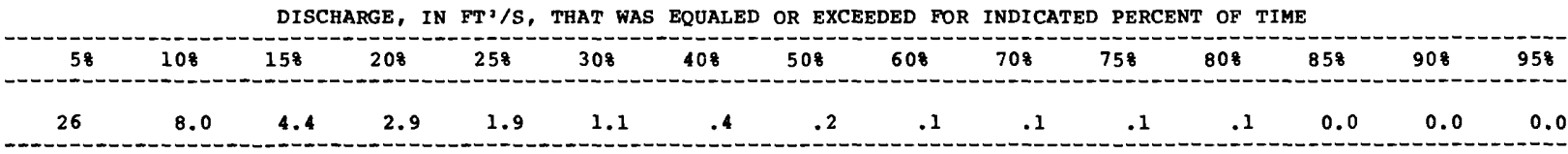




\section{ARKANSAS RIVER BASIN}

07203000 VERMEJO RIVER NEAR DAWSON, NM

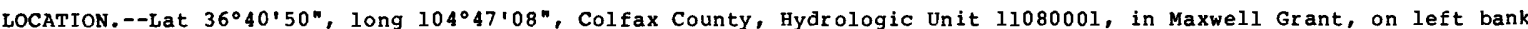
$1.3 \mathrm{mi}$ north of Dawson, $2.3 \mathrm{mi}$ upstream from Rail Canyon, and at mile 28.2.

DRAINAGE AREA. $--301 \mathrm{mi}^{2}$.

PERIOD OF RECORD.--October 1915 to July 1918, April 1919 to May 1921, January 1927 to current year. Monthly discharge only for some periods, published in wSP 1311 .

REVISED RECORDS.--WSP 1117: 1947, drainage area. WSP 1281: 1932(M), 1934(M), 1936-38(M), 1941-42(P), 1944-46(M). GAGE.--Water-stage recorder. Elevation of gage is 6,365 ft above National Geodetic Vertical Datum of 1929 , from topographic map. See wSP 1311 or 1731 for history of changes prior to Sept. $24,1953$.

REMARKS.--Diversions for irrigation of small acreage and mountain meadows upstream from station.

AVERAGE DISCHARGE.--61 years (water years 1916-17, 1920, 1928-85), $18.3 \mathrm{ft}^{3} / \mathrm{s}, 13,260 \mathrm{acre-ft} / \mathrm{yr}$.

EXTREMES FOR PERIOD OF RECORD (SINCE 1926).--Maximum discharge, 12,600 ft 3/s, June 17, 1965, gage height, 15.25 ft, from rating curve extended above $400 \mathrm{ft} / \mathrm{s}$ on basis of slope-area measurement of peak flow; no flow at times.

EXTREMES OUTSIDE PERIOD OF RECORD,--A major flood occurred Aug. 2, 1921, when discharge probably exceeded 10,000 $\mathrm{ft} / \mathrm{s}$.

STATISTICAL SUMMARIES

MEAN MONTHLY AND MEAN ANNUAL DISChARges $\begin{aligned} & 1916-17,1920 \\ & 1928-85\end{aligned}$

\begin{tabular}{|c|c|c|c|c|c|c|}
\hline MONTH & $\begin{array}{c}\text { M I NIMUM } \\
\left(\mathrm{FT}^{3} / \mathrm{S}\right)\end{array}$ & $\begin{array}{c}\text { MAXIMUM } \\
\left(\mathrm{FT} \mathrm{T}^{3 / \mathrm{S})}\right.\end{array}$ & $\begin{array}{l}\text { ME AN } \\
\left(F^{3} T^{3} / S\right)\end{array}$ & $\begin{array}{l}\text { STAN- } \\
\text { DARD } \\
\text { DEVIA- } \\
\text { TION } \\
\left(\text { FT }^{3} / \mathrm{S}\right)\end{array}$ & $\begin{array}{l}\text { COEFFI- } \\
\text { CIENT OF } \\
\text { VARI- } \\
\text { ATION }\end{array}$ & $\begin{array}{c}\text { PERCENT } \\
\text { OF } \\
\text { ANNUAL } \\
\text { RUNOFF }\end{array}$ \\
\hline OCTOBER & .2 & 52 & 8.5 & 8.8 & 1.04 & 3.7 \\
\hline NOVEMBER & 0.0 & 31 & 6.1 & 4.6 & $\begin{array}{r}1.04 \\
.76\end{array}$ & 2.7 \\
\hline DECEMBER & .6 & 16 & 4.8 & 3.5 & .73 & 2.1 \\
\hline JANUARY & .7 & 16 & 4.8 & 3.1 & .64 & 2.1 \\
\hline FEBRUARY & 1.2 & 17 & 6.0 & 3.5 & .59 & 2.6 \\
\hline MARCH & .8 & 18 & 6.0 & 4. & .67 & 2.7 \\
\hline APR IL & 1.2 & 370 & 21 & 49 & 2.34 & 9.3 \\
\hline MAY & 1.0 & 372 & 50 & 64 & 1.28 & 21.9 \\
\hline JUNE & .7 & 179 & 34 & 36 & 1.06 & 15.0 \\
\hline JULY & 1.9 & 138 & 30 & 22 & .74 & 13.0 \\
\hline AUGUST & 4.5 & 147 & 40 & 35 & .87 & 17.6 \\
\hline SEPTEMBER & .4 & 78 & 17 & 17 & 1.02 & 7.3 \\
\hline NNUAL & 2.1 & 89 & 18 & 14 & .77 & 100 \\
\hline
\end{tabular}

MAGNITUDE AND PROBABILITY OF ANNUAL LOW FLOW BASED ON PERIOD OF RECORD 1917, 1920-2I, $1928-85$

\begin{tabular}{|c|c|c|c|c|c|c|}
\hline \multirow{2}{*}{$\begin{array}{l}\text { PERIOD } \\
\text { (CON- } \\
\text { SECU- } \\
\text { TIVE } \\
\text { DAYS) }\end{array}$} & \multicolumn{6}{|c|}{$\begin{array}{l}\text { DISCHARGE, IN FT'3/S, FOR INDICATED RECURRENCE } \\
\text { INTERVAL, IN YEARS, AND ANNUAL NON- } \\
\text { EXCEEDANCE PROBABILITY, IN PERCENT }\end{array}$} \\
\hline & $\begin{array}{c}2 \\
508\end{array}$ & $\begin{array}{c}5 \\
208\end{array}$ & $\begin{array}{l}10 \\
108\end{array}$ & $\begin{array}{l}20 \\
58\end{array}$ & $\begin{array}{l}50 \\
28\end{array}$ & $\begin{array}{r}100 \\
18\end{array}$ \\
\hline $\begin{array}{r}1 \\
3 \\
7 \\
14 \\
30 \\
60 \\
90 \\
120 \\
183\end{array}$ & $\begin{array}{r}.8 \\
.9 \\
1.1 \\
1.6 \\
2.3 \\
3.1 \\
3.8 \\
4.2 \\
4.9\end{array}$ & $\begin{array}{r}.3 \\
.3 \\
.4 \\
.5 \\
.9 \\
1.3 \\
1.7 \\
2.1 \\
2.6\end{array}$ & $\begin{array}{r}.1 \\
.2 \\
.2 \\
.2 \\
.4 \\
.7 \\
1.0 \\
1.3 \\
1.9\end{array}$ & $\begin{array}{r}0.0 \\
0.0 \\
0.0 \\
.1 \\
.2 \\
.4 \\
.6 \\
.9 \\
1.4\end{array}$ & $\begin{array}{r}0.0 \\
0.0 \\
0.0 \\
0.0 \\
.1 \\
.2 \\
.3 \\
.5 \\
.9\end{array}$ & $\begin{array}{r}0.0 \\
0.0 \\
0.0 \\
0.0 \\
.1 \\
.1 \\
.2 \\
.4 \\
.7\end{array}$ \\
\hline
\end{tabular}

MAGNITUDE AND PROBABILITY OF ANNUAL HIGH FLOW BASED ON PERIOD OF RECORD 1916-17, 1920, $1928-85$

\begin{tabular}{|c|c|c|c|c|c|c|}
\hline \multirow{2}{*}{$\begin{array}{l}\text { PERIOD } \\
\text { (CON- } \\
\text { SECU- } \\
\text { TIVE } \\
\text { DAYS) }\end{array}$} & \multicolumn{6}{|c|}{$\begin{array}{l}\text { DISCHARGE, IN FT } 3 / \mathrm{S} \text {, FOR INDICATED RECURRENCE } \\
\text { INTERVAL, IN YEARS, AND ANNUAL } \\
\text { EXCEEDANCE PROBABILITY, IN PERCENT }\end{array}$} \\
\hline & $\begin{array}{c}2 \\
50 \%\end{array}$ & $\begin{array}{c}5 \\
208\end{array}$ & $\begin{array}{l}10 \\
108\end{array}$ & $\begin{array}{l}25 \\
48\end{array}$ & $\begin{array}{l}50 \\
28\end{array}$ & $\begin{array}{r}100 \\
18\end{array}$ \\
\hline $\begin{array}{r}1 \\
3 \\
7 \\
15 \\
30 \\
60 \\
90\end{array}$ & $\begin{array}{r}232 \\
146 \\
103 \\
79 \\
62 \\
46 \\
38\end{array}$ & $\begin{array}{r}502 \\
302 \\
208 \\
155 \\
120 \\
86 \\
69\end{array}$ & $\begin{array}{r}790 \\
460 \\
306 \\
220 \\
166 \\
117 \\
94\end{array}$ & $\begin{array}{r}1340 \\
743 \\
470 \\
318 \\
233 \\
159 \\
127\end{array}$ & $\begin{array}{r}1920 \\
1030 \\
626 \\
404 \\
287 \\
192 \\
153\end{array}$ & $\begin{array}{r}2700 \\
1400 \\
815 \\
500 \\
346 \\
226 \\
181\end{array}$ \\
\hline
\end{tabular}

DURATION TABLE OF DAILY MEAN FLOW FOR PERIOD OF RECORD 1916-17, 1920, 1928-85 DISCHARGE, IN FT $3 / S$, THAT WAS EQUALED OR EXCEEDED FOR INDICATED PERCENT OF TIME

\begin{tabular}{|c|c|c|c|c|c|c|c|c|c|c|c|c|c|c|}
\hline 58 & 108 & 158 & 208 & 258 & 308 & 408 & 508 & 608 & 708 & 758 & 808 & 858 & 908 & 958 \\
\hline 72 & 43 & 31 & 22 & 17 & 14 & 9.1 & 6.8 & 5.0 & 3.8 & 3.3 & 2.7 & 2.1 & 1.5 & .9 \\
\hline
\end{tabular}




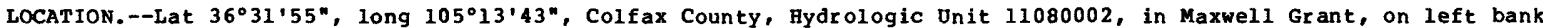
$300 \mathrm{ft}$ downstream from Eagle Nest Dam, $2.5 \mathrm{mi}$ southeast of Eagle Nest, 6.7 mi west of Dte Park, and at mile

48.6 .

DRAINAGE AREA. $--167 \mathrm{mi}^{2}$.

PERIOD OF RECORD.--May 1950 to current year. Published as Cimarron Creek below Eagle Nest Dam October 1952 to September 1965 .

REVISED RECORDS.-WSP 1281: Drainage area.

GAGE.--Water-stage recorder. Parshall flume since May 15, 1951. Elevation of gage is $8,080 \mathrm{ft}$ above National Geodetic Vertical Datum of 1929, from topographic map. Prior to May 15, 1951, at datum $0.81 \mathrm{ft}$ higher.

REMARKS.--Flow regulated by Eagle Nest Lake (station 07205500). Diversions for irrigation of 2,500 acres upstream from station.

AVERAGE DISCHARGE. --35 years (water years 1951-85), $13.6 \mathrm{ft}^{3} / \mathrm{s}, 9,850$ acre-ft/yr.

EXTREMES FOR PERIOD OF RECORD.--Maximum discharge, $205 \mathrm{ft}^{3} / \mathrm{s}$, June 14, 1955; maximum gage height, $3.04 \mathrm{ft}$, April 20 , 1983 ; no flow at times most years.

STATISTICAL SUMMARIES

MEAN MONTHLY AND MEAN ANNUAL DISCHARGES 1951-85

\begin{tabular}{|c|c|c|c|c|c|c|}
\hline MONTH & $\begin{array}{l}\text { MINIMUM } \\
\left(\mathrm{FT}^{3} / \mathrm{S}\right)\end{array}$ & $\begin{array}{c}\text { MAXIKUM } \\
\left(\mathrm{FT}^{3} / \mathrm{S}\right)\end{array}$ & $\begin{array}{l}\text { MEAN } \\
\left(\mathrm{FT}^{3} / \mathrm{S}\right)\end{array}$ & $\begin{array}{l}\text { STAN- } \\
\text { DARD } \\
\text { DEVIA- } \\
\text { TION } \\
\left(\mathrm{FT}^{3} / \mathrm{S}\right)\end{array}$ & $\begin{array}{l}\text { COEFFI- } \\
\text { CIENT OF } \\
\text { VARI- } \\
\text { ATION }\end{array}$ & $\begin{array}{c}\text { PERCENT } \\
\text { OF } \\
\text { ANNUAL } \\
\text { RUNOFF }\end{array}$ \\
\hline $\begin{array}{l}\text { OCTOBER } \\
\text { NOVEMBER } \\
\text { DECEMBER } \\
\text { JANUARY } \\
\text { FEBRUARY } \\
\text { MARCH } \\
\text { APRIL } \\
\text { MAY } \\
\text { JUNE } \\
\text { JULY } \\
\text { AUGUST } \\
\text { SEPTEMBER }\end{array}$ & $\begin{array}{l}.2 \\
0.0 \\
0.0 \\
0.0 \\
0.0 \\
0.0 \\
0.0 \\
.7 \\
5.3 \\
7.2 \\
.7 \\
.1\end{array}$ & $\begin{array}{c}50 \\
26 \\
1.4 \\
3.6 \\
5.2 \\
12 \\
43 \\
102 \\
66 \\
68 \\
48 \\
51\end{array}$ & $\begin{array}{r}16 \\
5.7 \\
.2 \\
.3 \\
.6 \\
3.6 \\
16 \\
28 \\
30 \\
32 \\
17 \\
14\end{array}$ & $\begin{array}{r}9.0 \\
6.0 \\
.4 \\
.8 \\
1.2 \\
3.6 \\
12 \\
22 \\
16 \\
14 \\
12 \\
11\end{array}$ & $\begin{array}{l}.58 \\
1.05 \\
1.58 \\
2.57 \\
2.16 \\
1.00 \\
.80 \\
.79 \\
.54 \\
.44 \\
.69 \\
.76\end{array}$ & $\begin{array}{r}9.6 \\
3.5 \\
.1 \\
.2 \\
.3 \\
2.2 \\
9.5 \\
17.1 \\
18.5 \\
19.5 \\
10.7 \\
8.7\end{array}$ \\
\hline NNUAL & 5.9 & 21 & 14 & 4.3 & .31 & 100 \\
\hline
\end{tabular}

MAGNITUDE AND PROBABILITY OF ANNUAL LOW FLOW BASED ON PERI OD OF RECORD $1952-85$

\begin{tabular}{|c|c|c|c|c|c|c|}
\hline \multirow{2}{*}{$\begin{array}{l}\text { PERIOD } \\
\text { (CON- } \\
\text { SECU- } \\
\text { TIVE } \\
\text { DAYS) }\end{array}$} & \multicolumn{6}{|c|}{$\begin{array}{l}\text { DISCHARGE, IN FT'3/S, FOR INDICATED RECURRENCE } \\
\text { INTERVAL, IN YEARS, AND ANNUAL NON- } \\
\text { EXCEEDANCE PROBABILITY, IN PERCENT }\end{array}$} \\
\hline & $\begin{array}{c}2 \\
508\end{array}$ & $\begin{array}{c}5 \\
208\end{array}$ & $\begin{array}{l}10 \\
108\end{array}$ & $\begin{array}{l}20 \\
58\end{array}$ & $\begin{array}{l}50 \\
28\end{array}$ & \\
\hline $\begin{array}{r}1 \\
3 \\
7 \\
14 \\
30 \\
60 \\
90 \\
120 \\
183\end{array}$ & $\begin{array}{l}0.0 \\
0.0 \\
0.0 \\
0.0 \\
0.0 \\
0.0 \\
0.0 \\
.2 \\
3.7\end{array}$ & $\begin{array}{l}0.0 \\
0.0 \\
0.0 \\
0.0 \\
0.0 \\
0.0 \\
0.0 \\
0.0 \\
2.2\end{array}$ & $\begin{array}{l}0.0 \\
0.0 \\
0.0 \\
0.0 \\
0.0 \\
0.0 \\
0.0 \\
0.0 \\
1.6\end{array}$ & $\begin{array}{l}0.0 \\
0.0 \\
0.0 \\
0.0 \\
0.0 \\
0.0 \\
0.0 \\
0.0 \\
1.3\end{array}$ & $\begin{array}{r}0.0 \\
0.0 \\
0.0 \\
0.0 \\
0.0 \\
0.0 \\
0.0 \\
0.0 \\
.9\end{array}$ & \\
\hline
\end{tabular}

MAGNITUDE AND PROBABILITY OF ANNUAL HIGH FLOW BASED ON PERIOD OF RECORD 1951-85

\begin{tabular}{|c|c|c|c|c|c|c|}
\hline \multirow{2}{*}{$\begin{array}{l}\text { PERIOD } \\
\text { (CON- } \\
\text { SECU- } \\
\text { TIVE } \\
\text { DAYS) }\end{array}$} & \multicolumn{6}{|c|}{$\begin{array}{l}\text { DISCHARGE, IN FT } 3 / S \text {, FOR INDICATED RECURRENCE } \\
\text { INTERVAL, IN YEARS, AND ANNUAL } \\
\text { EXCEEDANCE PROBABILITY, IN PERCENT }\end{array}$} \\
\hline & $\begin{array}{c}2 \\
508\end{array}$ & $\begin{array}{c}5 \\
208\end{array}$ & $\begin{array}{l}10 \\
108\end{array}$ & $\begin{array}{l}25 \\
48\end{array}$ & $\begin{array}{l}50 \\
28\end{array}$ & $\begin{array}{r}100 \\
18\end{array}$ \\
\hline $\begin{array}{r}1 \\
3 \\
7 \\
15 \\
30 \\
60 \\
90\end{array}$ & $\begin{array}{l}89 \\
85 \\
75 \\
61 \\
48 \\
38 \\
34\end{array}$ & $\begin{array}{r}129 \\
124 \\
111 \\
88 \\
65 \\
50 \\
44\end{array}$ & $\begin{array}{r}156 \\
150 \\
135 \\
108 \\
75 \\
58 \\
50\end{array}$ & $\begin{array}{r}188 \\
184 \\
168 \\
134 \\
88 \\
66 \\
55\end{array}$ & $\begin{array}{r}211 \\
208 \\
193 \\
155 \\
98 \\
71 \\
59\end{array}$ & $\begin{array}{l}-- \\
-- \\
-- \\
--\end{array}$ \\
\hline
\end{tabular}

DURATION TABLE OF DAILY MEAN FLOW FOR PERIOD OF RECORD 1951-85

DISCHARGE, IN FT $3 / \mathrm{S}$, THAT WAS EQUALED OR EXCEEDED FOR INDICATED PERCENT OF TIME

\begin{tabular}{|c|c|c|c|c|c|c|c|c|c|c|c|c|c|c|}
\hline 58 & 108 & 158 & 208 & 258 & 308 & 408 & 508 & 608 & 708 & 758 & 808 & 858 & 908 & 958 \\
\hline 51 & 38 & 31 & 26 & 22 & 18 & 11 & 5.3 & .8 & .2 & .1 & 0.0 & 0.0 & 0.0 & \\
\hline
\end{tabular}


ARKANSAS RIVER BASIN

07206500

CIMARRON RIVER AT UTE PARK, NM

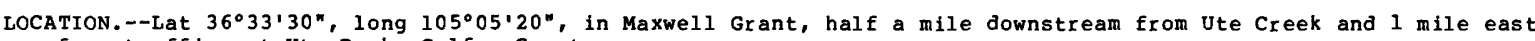
of post office at Ute Park, Colfax County.

DRAINAGE AREA. $--297 \mathrm{mi}^{2}$.

PERIOD OF RECORD.--July 1907 to December 1914, and october 1930 to September 1950 (discontinued) in reports of Geological Survey. July 1907 to December 1931 in reports of state Engineer.

REVISED RECORDS.--WSP 1117: Drainage area.

REMARKS.--Flow regulated by Eagle Nest Reservoir (capacity, 79,120 acre-feet). Diversions above station for irrigation.

AVERAGE DISCHARGE.--30 years (1908-17, 1931-50; since completion of Eagle Nest Dam), 30.0 ft3/s, 21,730 acre-ft/yr.

EXTREMES FOR PERIOD OF RECORD.--Maximum discharge, $404 \mathrm{ft} / \mathrm{s}$ May 12, 1942 (gage height, $3.43 \mathrm{ft}$ ); minimum daily $1.5 \mathrm{ft} / \mathrm{s}$ Jan. $18-20,1936$

STATISTICAL SUMMARIES

MEAN MONTHLY AND MEAN ANNUAL DISCHARGES $\begin{aligned} & 1908-17, \\ & 1931-50\end{aligned}$

\begin{tabular}{|c|c|c|c|c|c|c|}
\hline MONTH & $\begin{array}{l}\text { MINIMUM } \\
\left(\mathrm{FT}^{3} / \mathrm{S}\right)\end{array}$ & $\begin{array}{c}\text { MAXIMUM } \\
\left(\mathrm{FT}^{3} / \mathrm{S}\right)\end{array}$ & $\begin{array}{l}\text { MEAN } \\
\left(\mathrm{FT}^{3} / \mathrm{S}\right)\end{array}$ & $\begin{array}{c}\text { STAN- } \\
\text { DARD } \\
\text { DEVIA- } \\
\text { TION } \\
\left(\mathrm{FT}^{3} / \mathrm{S}\right)\end{array}$ & $\begin{array}{l}\text { COEFFI- } \\
\text { CIENT OF } \\
\text { VARI- } \\
\text { ATION }\end{array}$ & $\begin{array}{c}\text { PERCENT } \\
\text { OF } \\
\text { ANNUAL } \\
\text { RUNOFF }\end{array}$ \\
\hline $\begin{array}{l}\text { OCTOBER } \\
\text { NOVEMBER } \\
\text { DECEMBER } \\
\text { JANUARY } \\
\text { FEBRUARY } \\
\text { MARCH } \\
\text { APRIL } \\
\text { MAY } \\
\text { JUNE } \\
\text { JULY } \\
\text { AUGUST } \\
\text { SEPTEMBER }\end{array}$ & $\begin{array}{r}4.1 \\
4.8 \\
2.4 \\
2.8 \\
3.5 \\
5.5 \\
15 \\
29 \\
15 \\
6.3 \\
8.3 \\
3.1\end{array}$ & $\begin{array}{r}35 \\
31 \\
18 \\
20 \\
35 \\
88 \\
183 \\
388 \\
126 \\
105 \\
76 \\
43\end{array}$ & $\begin{array}{c}18 \\
13 \\
8.3 \\
9.1 \\
11 \\
22 \\
61 \\
110 \\
70 \\
52 \\
34 \\
20\end{array}$ & $\begin{array}{l}8.5 \\
5.7 \\
3.5 \\
4.7 \\
8.1 \\
21 \\
40 \\
77 \\
29 \\
27 \\
16 \\
10\end{array}$ & $\begin{array}{l}.47 \\
.43 \\
.42 \\
.52 \\
.73 \\
.96 \\
.66 \\
.70 \\
.42 \\
.53 \\
.46 \\
.51\end{array}$ & $\begin{array}{r}4.2 \\
3.1 \\
1.9 \\
2.1 \\
2.6 \\
5.1 \\
14.2 \\
25.6 \\
16.3 \\
12.1 \\
8.0 \\
4.7\end{array}$ \\
\hline ANNUAL & 22 & 71 & 36 & 11 & .30 & 100 \\
\hline
\end{tabular}

MAGNITUDE AND PROBABILITY OF ANNUAL LOW FLOW BASED ON PERIOD OF RECORD 1909-17, 1932-50

\begin{tabular}{|c|c|c|c|c|c|c|}
\hline \multirow{2}{*}{$\begin{array}{l}\text { PERIOD } \\
\text { (CON- } \\
\text { SECU- } \\
\text { TIVE } \\
\text { DAYS) }\end{array}$} & \multicolumn{6}{|c|}{$\begin{array}{l}\text { DISCHARGE, IN FT'/S, FOR INDICATED RECURRENCE } \\
\text { INTERVAL, IN YEARS, AND ANNUAL NON- } \\
\text { EXCEEDANCE PROBABILITY, IN PERCENT }\end{array}$} \\
\hline & $\begin{array}{c}2 \\
508\end{array}$ & $\begin{array}{c}5 \\
208\end{array}$ & $\begin{array}{l}10 \\
108\end{array}$ & $\begin{array}{l}20 \\
58\end{array}$ & $\begin{array}{l}50 \\
28\end{array}$ & $\begin{array}{r}100 \\
18\end{array}$ \\
\hline $\begin{array}{r}1 \\
3 \\
7 \\
14 \\
30 \\
60 \\
90 \\
120 \\
183\end{array}$ & $\begin{array}{r}4.6 \\
4.8 \\
5.2 \\
5.6 \\
6.3 \\
7.0 \\
7.5 \\
8.0 \\
11\end{array}$ & $\begin{array}{l}3.0 \\
3.2 \\
3.5 \\
3.8 \\
4.3 \\
4.8 \\
5.1 \\
5.7 \\
8.7\end{array}$ & $\begin{array}{l}2.4 \\
2.6 \\
2.9 \\
3.1 \\
3.5 \\
3.9 \\
4.1 \\
4.8 \\
7.7\end{array}$ & $\begin{array}{l}2.0 \\
2.1 \\
2.4 \\
2.6 \\
2.9 \\
3.2 \\
3.5 \\
4.2 \\
7.1\end{array}$ & $\begin{array}{l}1.6 \\
1.7 \\
2.0 \\
2.2 \\
2.4 \\
2.7 \\
2.9 \\
3.6 \\
6.4\end{array}$ & $\begin{array}{l}-- \\
-- \\
-- \\
-- \\
-- \\
--\end{array}$ \\
\hline
\end{tabular}

MAGNITUDE AND PROBABILITY OF ANNUAL HIGH FLOW BASED ON PERIOD OF RECORD 1908-17, 1931-50

\begin{tabular}{|c|c|c|c|c|c|c|}
\hline \multirow{2}{*}{$\begin{array}{l}\text { PERIOD } \\
\text { (CON- } \\
\text { SECU- } \\
\text { TIVE } \\
\text { DAYS) }\end{array}$} & \multicolumn{6}{|c|}{$\begin{array}{l}\text { DISCHARGE, IN FT'/S, FOR INDICATED RECURRENCE } \\
\text { INTERVAL, IN YEARS, AND ANNUAL } \\
\text { EXCEEDANCE PROBABILI TY, IN PERCENT }\end{array}$} \\
\hline & $\begin{array}{c}2 \\
508\end{array}$ & $\begin{array}{c}5 \\
208\end{array}$ & $\begin{array}{l}10 \\
108\end{array}$ & $\begin{array}{l}25 \\
48\end{array}$ & $\begin{array}{l}50 \\
28\end{array}$ & $\begin{array}{r}100 \\
18\end{array}$ \\
\hline $\begin{array}{r}1 \\
3 \\
7 \\
15 \\
30 \\
60 \\
90\end{array}$ & $\begin{array}{r}172 \\
161 \\
148 \\
131 \\
112 \\
93 \\
81\end{array}$ & $\begin{array}{l}270 \\
249 \\
226 \\
198 \\
167 \\
133 \\
111\end{array}$ & $\begin{array}{l}359 \\
332 \\
299 \\
260 \\
218 \\
167 \\
135\end{array}$ & $\begin{array}{l}509 \\
471 \\
420 \\
365 \\
301 \\
219 \\
170\end{array}$ & $\begin{array}{l}653 \\
606 \\
538 \\
467 \\
381 \\
265 \\
201\end{array}$ & $\begin{array}{l}-- \\
-- \\
-- \\
-- \\
-- \\
--\end{array}$ \\
\hline
\end{tabular}

DURATION TABLE OF DAILY MEAN FLOW FOR PERIOD OF RECORD 1908-17, 1931-50 
ARRANSAS RIVER BASIN

07207000 CIMARRON RIVER NEAR CIMARRON, NM

LOCATION.--Lat 36.31'11", long 104.58'42", Colfax County, Hydrologic Unit 11080002, in Maxwell Grant, on right bank

$1,200 \mathrm{ft}$ downstream from Turkey Creek Canyon, $3.6 \mathrm{mi}$ west of cimarron, and at mile 31.6 .

DRAINAGE AREA. $--294 \mathrm{mi}^{2}$.

PERIOD OF RECORD.--May 1950 to current year. Published as Cimarron Creek near Cimarron, October 1952 to September 1965.

REVISED RECORDS.--WSP 1281: Drainage area.

GAGE.--Water-stage recorder. Concrete control since Nov. 6, 1963 . Datum of gage is $6,599.58 \mathrm{ft}$ above National Geodetic Vertical Datum of 1929.

REMARKS.--Flow regulated by Eagle Nest Lake (station 07205500). Diversions upstream from station for

irrigation of about 3,500 acres, part of which is downstream from station. Philmont ditch (formerly known as

Cimarroncito ditch) diverts from left bank $1.5 \mathrm{mi}$ upstream from station, flumes under river $0.9 \mathrm{mi} u p s t r e a m$ and

bypasses station for of $f$-channel storage and irrigation downstream; Cimarron Diversion pipeline 300 ft upstream

from station for city of Raton water Supply started June, 1983.

AVERAGE DISCHARGE.--35 years (water years 1951-85), 21.0 ft $3 / \mathrm{s}, 15,210$ acre-ft/yr.

EXTREMES FOR PERIOD OF RECORD, --Maximum discharge, 15,500 ft $3 / \mathrm{s}$, June 17, 1965, gage height, $12.42 \mathrm{ft}$, from

floodmark, from rating curve extended above $800 \mathrm{ft} / \mathrm{s}$ on basis of slope-area measurements at gage heights 4.88
$\mathrm{ft}$ and $12.42 \mathrm{ft}$; no flow at times.

STATISTICAL SUMMARIES

MEAN MONTHLY AND MEAN ANNUAL DISCHARGES 1951-85

\begin{tabular}{|c|c|c|c|c|c|c|}
\hline MONTH & $\begin{array}{l}\text { MINIMUM } \\
\left(\mathrm{FT}^{3} / \mathrm{S}\right)\end{array}$ & $\begin{array}{c}\text { MAXI MUM } \\
\left(\mathrm{FT}^{3} / \mathrm{S}\right)\end{array}$ & $\begin{array}{l}\text { MEAN } \\
\left(\mathrm{FT}^{3} / \mathrm{S}\right)\end{array}$ & $\begin{array}{l}\text { STAN- } \\
\text { DARD } \\
\text { DEVIA- } \\
\text { TION } \\
\text { (FT' } 3 \text { /S) }\end{array}$ & $\begin{array}{l}\text { COEFFI- } \\
\text { CIENT OF } \\
\text { VARI- } \\
\text { ATION }\end{array}$ & $\begin{array}{c}\text { PERCENT } \\
\text { OF } \\
\text { ANNUAL } \\
\text { RUNOFF }\end{array}$ \\
\hline OCTOBER & .1 & 45 & 18 & 8.4 & .47 & 7.2 \\
\hline NOVEMBER & 3.7 & 27 & 11 & 5.4 & .51 & 4.2 \\
\hline DECEMBER & 1.3 & 8.3 & 4.2 & 1.6 & .39 & 1.7 \\
\hline JANUARY & 1.1 & 8.5 & 3.5 & 1.6 & .45 & 1.4 \\
\hline FEBRUARY & 1.4 & 8.0 & 3.7 & 1.8 & .48 & 1.5 \\
\hline MARCH & 1.7 & 13 & 6.6 & 3.0 & .4 & 2.6 \\
\hline APRIL & 2.7 & 43 & 27 & 9.5 & .35 & 10.7 \\
\hline MAY & 24 & 115 & 57 & 25 & .43 & 22.5 \\
\hline JUNE & 8.6 & 123 & 47 & 22 & .47 & 18.8 \\
\hline JULY & 6.1 & 71 & 35 & 15 & .44 & 13.8 \\
\hline AUGUST & 2.0 & 44 & 23 & 11 & & 9.3 \\
\hline SEPTEMBER & .1 & 50 & 16 & 9.3 & .58 & 6.4 \\
\hline N NUAL & 9.1 & 32 & 21 & 5.4 & .26 & 100 \\
\hline
\end{tabular}

MAGNI TUDE AND PROBABILITY OF ANNUAL LOW FLOW BASED ON PERIOD OF RECORD $1952-85$

\begin{tabular}{|c|c|c|c|c|c|c|}
\hline \multirow{2}{*}{$\begin{array}{l}\text { PERIOD } \\
\text { (CON- } \\
\text { SECU- } \\
\text { TIVE } \\
\text { DAYS) }\end{array}$} & \multicolumn{6}{|c|}{$\begin{array}{l}\text { DISCHARGE, IN FT3/S, FOR INDICATED RECURRENCE } \\
\text { INTERVAL, IN YEARS, AND ANNUAL NON- } \\
\text { EXCEEDANCE PROBABILITY, IN PERCENT }\end{array}$} \\
\hline & $\begin{array}{c}2 \\
508\end{array}$ & $\begin{array}{c}5 \\
208\end{array}$ & $\begin{array}{l}10 \\
108\end{array}$ & $\begin{array}{l}20 \\
58\end{array}$ & $\begin{array}{l}50 \\
28\end{array}$ & $\begin{array}{r}100 \\
18\end{array}$ \\
\hline $\begin{array}{r}1 \\
3 \\
7 \\
14 \\
30 \\
60 \\
90 \\
120 \\
183\end{array}$ & $\begin{array}{l}1.5 \\
1.7 \\
2.0 \\
2.3 \\
2.6 \\
3.6 \\
3.4 \\
3.9 \\
8.1\end{array}$ & $\begin{array}{r}.7 \\
.9 \\
1.2 \\
1.4 \\
1.8 \\
2.1 \\
2.4 \\
2.8 \\
5.7\end{array}$ & $\begin{array}{r}.4 \\
.6 \\
.8 \\
1.1 \\
1.4 \\
1.3 \\
1.9 \\
2.3 \\
4.3\end{array}$ & $\begin{array}{r}.2 \\
.4 \\
.5 \\
.7 \\
1.1 \\
.7 \\
1.6 \\
1.9 \\
3.2\end{array}$ & $\begin{array}{r}0.0 \\
0.0 \\
0.0 \\
0.0 \\
0.0 \\
.03 \\
1.2 \\
1.5 \\
2.2\end{array}$ & 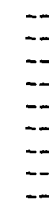 \\
\hline
\end{tabular}

MAGNITUDE AND PROBABILITY OF ANNUAL HIGH FLOW BASED ON PERIOD OF RECORD 1951-85

\begin{tabular}{|c|c|c|c|c|c|c|}
\hline \multirow{2}{*}{$\begin{array}{l}\text { PERIOD } \\
\text { (CON- } \\
\text { SECU- } \\
\text { TIVE } \\
\text { DAYS) }\end{array}$} & \multicolumn{6}{|c|}{$\begin{array}{l}\text { DISCHARGE, IN FT'/S, FOR INDICATED RECURRENC } \\
\text { INTERVAL, IN YEARS, AND ANNUAL } \\
\text { EXCEEDANCE PROBABILITY, IN PERCENT }\end{array}$} \\
\hline & $\begin{array}{c}2 \\
508\end{array}$ & $\begin{array}{c}5 \\
208\end{array}$ & $\begin{array}{l}10 \\
108\end{array}$ & $\begin{array}{l}25 \\
48\end{array}$ & $\begin{array}{l}50 \\
28\end{array}$ & $\begin{array}{r}100 \\
18\end{array}$ \\
\hline $\begin{array}{r}1 \\
3 \\
7 \\
15 \\
30 \\
60 \\
90\end{array}$ & $\begin{array}{l}93 \\
92 \\
89 \\
78 \\
64 \\
52 \\
48\end{array}$ & $\begin{array}{r}157 \\
149 \\
135 \\
111 \\
88 \\
70 \\
62\end{array}$ & $\begin{array}{r}233 \\
207 \\
173 \\
135 \\
105 \\
81 \\
70\end{array}$ & $\begin{array}{r}395 \\
314 \\
229 \\
168 \\
126 \\
95 \\
78\end{array}$ & $\begin{array}{r}588 \\
426 \\
278 \\
193 \\
142 \\
105 \\
83\end{array}$ & $\begin{array}{l}-- \\
-- \\
-- \\
-- \\
-- \\
--\end{array}$ \\
\hline
\end{tabular}

DURATION TABLE OF DAILY MEAN FLOW FOR PERIOD OF RECORD 1951-85 DISCHARGE, IN FT3/S, THAT WAS EQUALED OR EXCEEDED FOR INDICATED PERCENT OF TIME

\begin{tabular}{|c|c|c|c|c|c|c|c|c|c|c|c|c|c|c|}
\hline 58 & 108 & 158 & 208 & 258 & 308 & 408 & 508 & 608 & 708 & 758 & 808 & 858 & 908 & 958 \\
\hline 68 & 52 & 42 & 35 & 30 & 26 & 19 & 13 & 7.7 & 5.2 & 4. & 3.7 & 3.1 & 2.6 & 2.0 \\
\hline
\end{tabular}


ARKANSAS RIVER BASIN

07207500 PONIL CREER NEAR CIMARRON, NM

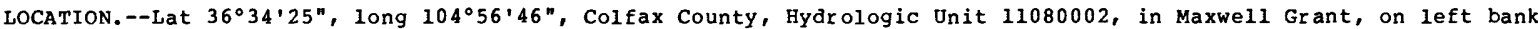
$1.6 \mathrm{mi}$ downstream from confluence of North and South Ponil Creeks, and $4.7 \mathrm{mi}$ northwest of Cimarron.

DRAINAGE AREA. $--171 \mathrm{mi}^{2}$.

PERIOD OF RECORD.--November 1915 to June 1919, August 1919 to July 1925, September 1925, September 1927 to July

1929, May 1950 to current year. Prior to May 1950 monthly discharge only, published in wSP 1311.

REVISED RECORDS.--WSP 1281: Drainage area. WSP 1731: 1920.

GAGE.--Water-stage recorder. Elevation of gage is $6,630 \mathrm{ft}$ above National Geodetic Vertical Datum of 1929 , from topographic map. Prior to May 8, 1922, at site $0.1 \mathrm{mi}$ downstream at different datum. May 8 , 1922 to Aug. 8, 1929, at site $0.4 \mathrm{mi}$ upstream at different datum.

REMARKS.--Diversions for irrigation of about 250 acres upstream from station. Diversions 1,000 ft downstream from station for irrigation of about 300 acres.

AVERAGE DISCHARGE. -43 years (water years 1917-18, 1920-24, 1928, 1951-85), 11.2 ft $3 / \mathrm{s}, 8,110$ acre-ft/yr

EXTREMES FOR PERIOD OF RECORD.--Maximum discharge, $5,630 \mathrm{ft} 3 / \mathrm{s}$, June 17,1965 , gage height, $11.13 \mathrm{ft}$, from rating curve extended above $230 \mathrm{ft} / \mathrm{s}$ on basis of slope-area measurements at gage heights $3.56 \mathrm{ft}$, $5.80 \mathrm{ft}$, $7.15 \mathrm{ft}$, and $11.13 \mathrm{ft}$; no $\mathrm{flow}$ many days most years.

EXTREMES OUTSIDE PERIOD OF RECORD.--Discharge for flood of Aug. 8, 1929, which destroyed gage, was estimated as $5,200 \mathrm{ft}^{3} / \mathrm{s}$ by State Engineer.

STATISTICAL SUMMARIES

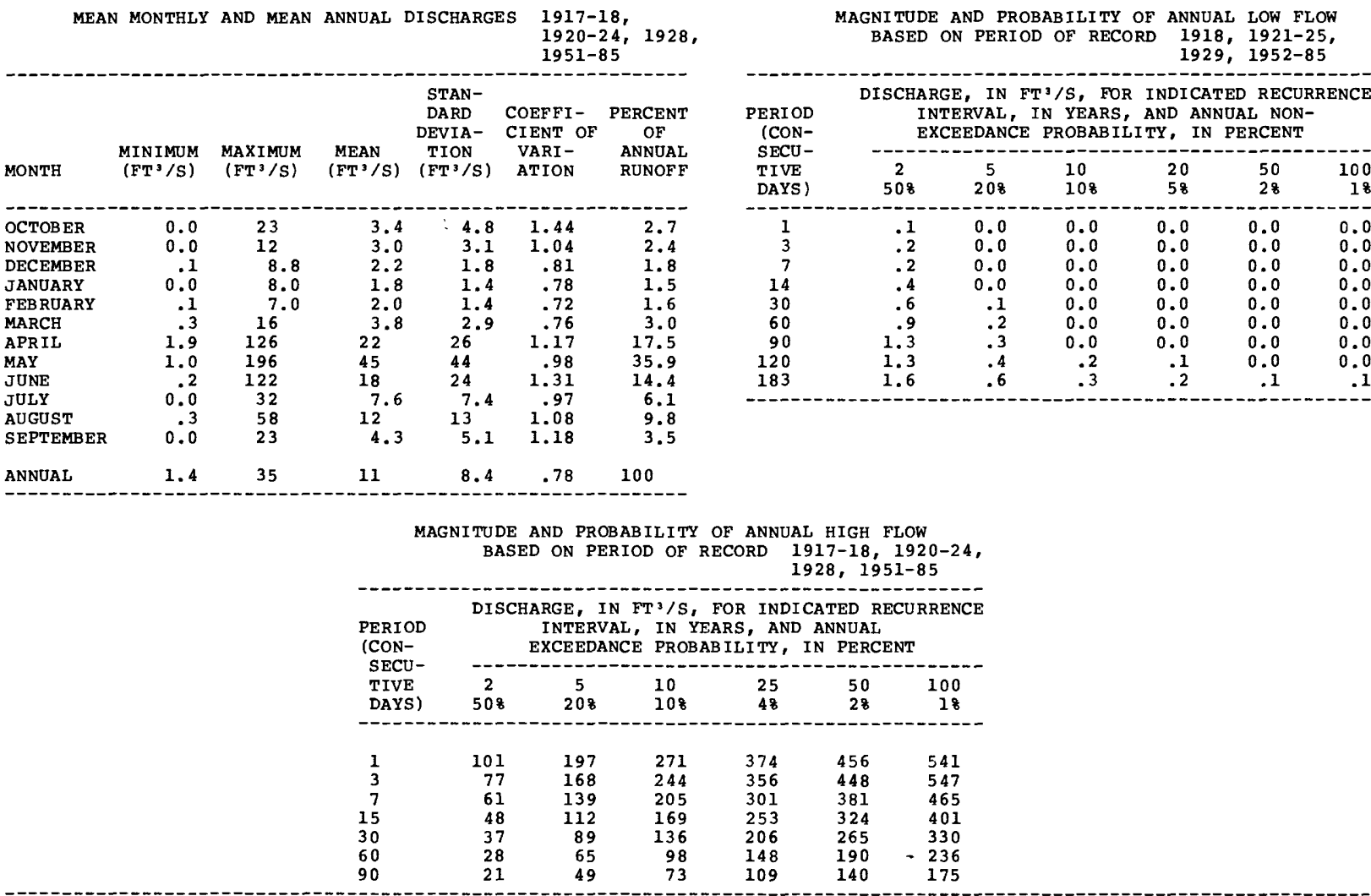

DURATION TABLE OF DAILY MEAN FLOW FOR PERIOD OF RECORD 1917-18, 1920-24, 1928, 1951-85 DISCHARGE, IN FT $3 / \mathrm{S}$, THAT WAS EOUALED OR EXCEEDED FOR INDICATED PERCENT OF TIME

\begin{tabular}{|c|c|c|c|c|c|c|c|c|c|c|c|c|c|c|}
\hline 58 & 108 & 158 & 208 & 258 & 308 & 408 & 508 & 608 & 708 & 758 & 808 & 858 & 908 & 958 \\
\hline 53 & 27 & 16 & 11 & 7.8 & 5.9 & 3.9 & 2.8 & 2.0 & 1.4 & 1.1 & .8 & .6 & .3 & \\
\hline
\end{tabular}


ARKANSAS RIVER BASIN

07208500 RAYADO CREEK AT SAUBLE RANCH, NEAR CIMARRON, NM

LOCATION,--Lat $36^{\circ} 22^{\prime} 20^{*}$, long $104^{\circ} 58^{\prime} 10^{*}$, Colfax County, Hydrologic Unit 11080002, in Maxwell Grant, on right bank at Sauble Ranch (Carson-Maxwell Base Camp of Philmont Scout Ranch), $2.5 \mathrm{mi}$ upstream from State Highway $21,4.0$ mi downstream from Bonito Creek, and $9.8 \mathrm{mi}$ southwest of Cimarron.

DRAINAGE AREA. $--65 \mathrm{mi}^{2}$.

PERIOD OF RECORD.--January 1909 to February 1910, June to August 1910, May 1911 to May 1913 , July 1913 to February 1915, October 1915 to September 1918, March 1919 to September 1920, June 1923 to September 1924 , March to May 1927, August 1927 to current year. Monthly discharge only for some periods, published in wSP 1311 . Records for Apr il and May 1910, published in WSP 287, are unreliable and should not be used. Published as Rayado River "at," "near," or "above" Abreu's Ranch near Cimarron prior to October 1925 and as Rayado River at Sauble Ranch, near Cimarron, October 1925 to September 1952.

REVISED RECORDS.--WSP 1281: 1914, 1934-35(M), 1937(M), 1941(P), 1942(M), 1944(M), drainage area. See also PERIOD OF RECORD.

GAGE.--Water-stage recorder. Concrete control since Oct. 13, 1976. Elevation of gage is $6,720 \mathrm{ft}$ above National Geodetic Vertical Datum of 1929, from topographic map. See WSP 1921 for history of changes prior to Oct. 1 , 1954. Oct. 1, 1954 to June 16, 1965, at site $270 \mathrm{ft}$ downstream at datum $2.79 \mathrm{ft} 10 \mathrm{wer}$.

REMARKS. - No diversion upstream from station.

AVERAGE DISCHARGE.--58 years (water years 1912, 1917-18, 1931-85), 13.0 ft3/s, 9,420 acre-ft/yr.

EXTREMES FOR PERIOD OF RECORD (1909-12, and SINCE 1913).--Maximum discharge, 9,000 ft $3 / \mathrm{s}, \mathrm{June} 17$, 1965, gage height, $11.5 \mathrm{ft}$, from floodmarks, from rating curve extended above $70 \mathrm{ft} / \mathrm{s}$ on basis of $\mathrm{field}$ estimate of peak flow; minimum, $0.03 \mathrm{ft}^{3} / \mathrm{s}$, Dec. 3, 1950, but may have been less during periods of ice effect.

EXTREMES OUTSIDE PERIOD OF RECORD.--The major flood of June 10, 1913, destroyed the gage (stage and discharge not determined). Another major flood probably occurred Sept. 29 or $30,1904$.

STATISTICAL SUMMARIES

MEAN MONTHLY AND MEAN ANNUAL DISChARGES 1912, 1917-18,

MAGNITUDE AND PROBABILITY OF ANNUAL LOW FLOW $1931-85$ BASED ON PERIOD OF RECORD 1917-18, 1931-85

\begin{tabular}{|c|c|c|c|c|c|c|}
\hline MONTH & $\begin{array}{c}\text { MINIMUM } \\
\left(\mathrm{FT}^{3} / \mathrm{S}\right)\end{array}$ & $\begin{array}{c}\text { MAXIMUM } \\
\left(\mathrm{FT}^{3} / \mathrm{S}\right)\end{array}$ & $\begin{array}{l}\text { MEAN } \\
\left(\mathrm{FT}^{3} / \mathrm{S}\right)\end{array}$ & $\begin{array}{c}\text { STAN- } \\
\text { DARD } \\
\text { DEVIA- } \\
\text { TION } \\
\left(\text { FT }^{3} / \mathrm{S}\right)\end{array}$ & $\begin{array}{l}\text { COEFFI- } \\
\text { CIENT OF } \\
\text { VARI - } \\
\text { ATION }\end{array}$ & $\begin{array}{c}\text { PERCENT } \\
\text { OF } \\
\text { ANNUAL } \\
\text { RUNOFF }\end{array}$ \\
\hline $\begin{array}{l}\text { OCTOBER } \\
\text { NOVEMBER } \\
\text { DECEMBER } \\
\text { J ANUARY } \\
\text { FEBRUARY } \\
\text { MARCH } \\
\text { APRIL } \\
\text { MAY } \\
\text { JUNE } \\
\text { JULY } \\
\text { AUGUST } \\
\text { SEPTEMBER }\end{array}$ & $\begin{array}{l}1.2 \\
1.4 \\
1.3 \\
1.6 \\
2.0 \\
3.0 \\
5.2 \\
3.7 \\
1.8 \\
1.4 \\
2.1 \\
.9\end{array}$ & $\begin{array}{c}30 \\
20 \\
8.3 \\
8.0 \\
6.8 \\
24 \\
127 \\
287 \\
231 \\
55 \\
72 \\
23\end{array}$ & $\begin{array}{l}5.7 \\
4.7 \\
3.7 \\
3.4 \\
3.8 \\
6.9 \\
30 \\
52 \\
21 \\
9.7 \\
11 \\
6.5\end{array}$ & $\begin{array}{r}4.7 \\
3.0 \\
1.3 \\
1.0 \\
1.0 \\
3.4 \\
24 \\
49 \\
31 \\
8.7 \\
12 . \\
4.4\end{array}$ & $\begin{array}{r}.82 \\
.63 \\
.35 \\
.29 \\
.27 \\
.48 \\
.81 \\
.95 \\
1.47 \\
.90 \\
1.06 \\
.68\end{array}$ & $\begin{array}{r}3.6 \\
3.0 \\
2.3 \\
2.2 \\
2.4 \\
4.4 \\
18.7 \\
32.7 \\
13.5 \\
6.2 \\
6.9 \\
4.1\end{array}$ \\
\hline NNUAL & 2.8 & 42 & 13 & 8.5 & .65 & 100 \\
\hline
\end{tabular}

\begin{tabular}{|c|c|c|c|c|c|c|}
\hline \multirow{2}{*}{$\begin{array}{l}\text { PERIOD } \\
\text { (CON- } \\
\text { SECU- } \\
\text { TIVE } \\
\text { DAYS) }\end{array}$} & \multicolumn{6}{|c|}{$\begin{array}{l}\text { DISCHARGE, IN FT'/S, FOR INDICATED RECURRENCE } \\
\text { INTERVAL, IN YEARS, AND ANNUAL NON- } \\
\text { EXCEEDANCE PROBABILITY, IN PERCENT }\end{array}$} \\
\hline & $\begin{array}{c}2 \\
508\end{array}$ & $\begin{array}{c}5 \\
208\end{array}$ & $\begin{array}{l}10 \\
108\end{array}$ & $\begin{array}{l}20 \\
58\end{array}$ & $\begin{array}{l}50 \\
28\end{array}$ & $\begin{array}{r}100 \\
18\end{array}$ \\
\hline $\begin{array}{r}1 \\
3 \\
7 \\
14 \\
30 \\
60 \\
90 \\
120 \\
183\end{array}$ & $\begin{array}{l}1.7 \\
2.0 \\
2.3 \\
2.5 \\
2.7 \\
3.1 \\
3.3 \\
3.5 \\
3.8\end{array}$ & $\begin{array}{l}1.0 \\
1.3 \\
1.5 \\
1.7 \\
2.0 \\
2.3 \\
2.5 \\
2.7 \\
2.9\end{array}$ & $\begin{array}{r}.8 \\
1.0 \\
1.2 \\
1.4 \\
1.7 \\
2.0 \\
2.2 \\
2.3 \\
2.6\end{array}$ & $\begin{array}{r}.6 \\
.8 \\
1.0 \\
1.2 \\
1.5 \\
1.8 \\
1.9 \\
2.0 \\
2.3\end{array}$ & $\begin{array}{r}.4 \\
.6 \\
.8 \\
.9 \\
1.2 \\
1.5 \\
1.7 \\
1.8 \\
2.1\end{array}$ & $\begin{array}{r}.5 \\
.6 \\
.8 \\
1.1 \\
1.4 \\
1.5 \\
1.6 \\
2.0\end{array}$ \\
\hline
\end{tabular}

MAGNITUDE AND PROBABILITY OF ANNUAL HIGH FLOW BASED ON PERIOD OF RECORD 1912, 1917-18, $1931-85$

\begin{tabular}{|c|c|c|c|c|c|c|}
\hline \multirow{2}{*}{$\begin{array}{l}\text { PERIOD } \\
\text { (CON- } \\
\text { SECU- } \\
\text { TIVE } \\
\text { DAYS) }\end{array}$} & \multicolumn{6}{|c|}{$\begin{array}{l}\text { DISCHARGE, IN FT' } 3 / S \text {, FOR INDICATED RECURRENCE } \\
\text { INTERVAL, IN YEARS, AND ANNUAL } \\
\text { EXCEEDANCE PROBABILITY, IN PERCENT }\end{array}$} \\
\hline & $\begin{array}{c}2 \\
508\end{array}$ & $\begin{array}{c}5 \\
208\end{array}$ & $\begin{array}{l}10 \\
108\end{array}$ & $\begin{array}{l}25 \\
48\end{array}$ & $\begin{array}{l}50 \\
28\end{array}$ & $\begin{array}{r}100 \\
18\end{array}$ \\
\hline $\begin{array}{r}1 \\
3 \\
7 \\
15 \\
30 \\
60 \\
90\end{array}$ & $\begin{array}{l}80 \\
73 \\
64 \\
53 \\
44 \\
34 \\
27\end{array}$ & $\begin{array}{r}200 \\
176 \\
149 \\
119 \\
96 \\
70 \\
53\end{array}$ & $\begin{array}{r}328 \\
278 \\
227 \\
178 \\
141 \\
100 \\
75\end{array}$ & $\begin{array}{l}558 \\
454 \\
354 \\
270 \\
210 \\
146 \\
108\end{array}$ & $\begin{array}{l}792 \\
623 \\
468 \\
351 \\
270 \\
185 \\
137\end{array}$ & $\begin{array}{r}1090 \\
827 \\
599 \\
442 \\
336 \\
228 \\
167\end{array}$ \\
\hline
\end{tabular}

DURATION TABLE OF DAILY MEAN FLOW FOR PERIOD OF RECORD 1912, 1917-18, 1931-85

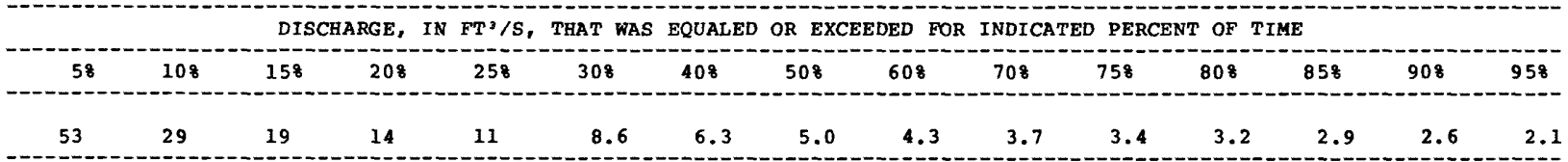


ARKANSAS RIVER BASIN

07211000 CIMARRON RIVER AT SPRINGER, NM

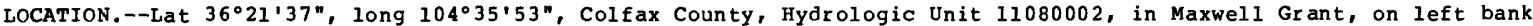
at Springer, $400 \mathrm{ft}$ downstream from bridge on State Highway 199, $0.3 \mathrm{mi}$ upstream from Salado Creek, and at mile 8.2.

DRAINAGE AREA. $--1,032 \mathrm{mi}^{2}$.

PERIOD OF RECORD.--August 1907 to December 1909, January 1921 to February 1922, October 1924 to January 1926 , September 1926 to current year. Monthly discharge only for some periods, published in wSP 1311 . Published as Cimarron Creek at Springer, October 1952 to September 1965.

REVISED RECORDS,--WSP 827: 1934-36(M). WSP 1281: 1942, 1945-46(M).

GAGE.--Water-stage recorder. Concrete control since Nov. 5, 1954 . Elevation of gage is $5,770 \mathrm{ft}$ above National Geodetic Vertical Datum of 1929, from topographic map. See WSP 1311 or 1731 for history of changes prior to July 17, 1942.

REMARKS.--Flow partly regulated by Eagle Nest Lake (station 07205500). Diversions for irrigation of about 23,000 acres upstream from station and a few hundred acres between station and mouth.

AVERAGE DISCHARGE.--60 years (water years 1908-09, 1925, 1927-28, 1931-85), 17.2 ft3/s, 12,460 acre-ft/yr.

EXTREMES FOR PERIOD OF RECORD (SINCE 1930).--Maximum discharge, 29,500 ft3/s, June 18, 1965, gage height, 19.96 ft, from floodmarks, from rating curve extended above $1,800 \mathrm{ft} / \mathrm{s}$ on basis of contracted-opening measurement of peak flow; no flow at times in 1954, 1956-57, 1978, 1983, 1984 .

EXTREMES OUTSIDE PERIOD OF RECORD.--Maximum stage, about $22 \mathrm{ft}$, Sept. 29, 1904 (backwater from debris on railroad bridge). Another major flood occurred June 11, 1913. Maximum discharge of these floods probably extended $10,000 \mathrm{ft}^{3} / \mathrm{s}$, but probably were less than the $1965 \mathrm{flood}$.

STATISTICAL SUMMARIES

\begin{tabular}{|c|c|c|c|c|c|c|}
\hline MEAN & MONTHLY & AND MEAN & ANNUAL & DISCHARG & $\begin{array}{l}1908- \\
1927- \\
1931-\end{array}$ & $\begin{array}{l}9,1925, \\
8, \\
5\end{array}$ \\
\hline ONTH & $\begin{array}{l}\text { MINIMUM } \\
\left(\mathrm{FT}^{3} / \mathrm{S}\right)\end{array}$ & $\begin{array}{c}\text { MAXIMUM } \\
\left(F^{3} / S\right)\end{array}$ & $\begin{array}{l}\text { MEAN } \\
\left(F T^{3} / S\right)\end{array}$ & $\begin{array}{l}\text { STAN- } \\
\text { DARD } \\
\text { DEVIA- } \\
\text { TION } \\
(\text { FT'/S) }\end{array}$ & $\begin{array}{l}\text { COEFFI- } \\
\text { CIENT OF } \\
\text { VARI- } \\
\text { ATION }\end{array}$ & $\begin{array}{c}\text { PERCENT } \\
\text { OF } \\
\text { ANNUAL } \\
\text { RUNOFF }\end{array}$ \\
\hline $\begin{array}{l}\text { OCTOBER } \\
\text { NOVEMBER } \\
\text { DECEMBER } \\
\text { JANUARY } \\
\text { FEBRUARY } \\
\text { MARCH } \\
\text { APRIL } \\
\text { MAY } \\
\text { JUNE } \\
\text { JULY } \\
\text { AUGUST } \\
\text { SEPTEMBER }\end{array}$ & $\begin{array}{r}0.0 \\
.2 \\
.3 \\
.3 \\
.3 \\
.4 \\
.5 \\
.7 \\
1.0 \\
.4 \\
.2 \\
0.0\end{array}$ & $\begin{array}{r}98 \\
68 \\
41 \\
35 \\
45 \\
26 \\
358 \\
928 \\
699 \\
146 \\
116 \\
118\end{array}$ & $\begin{array}{l}8.3 \\
6.6 \\
5.6 \\
5.5 \\
6.0 \\
4.9 \\
20 \\
72 \\
38 \\
11 \\
15 \\
11\end{array}$ & $\begin{array}{c}17 \\
10 \\
6.1 \\
5.3 \\
6.8 \\
4.4 \\
51 \\
159 \\
102 \\
21 \\
22 \\
21\end{array}$ & $\begin{array}{l}2.04 \\
1.57 \\
1.09 \\
.96 \\
1.12 \\
.90 \\
2.55 \\
2.21 \\
2.71 \\
1.93 \\
1.45 \\
1.92\end{array}$ & $\begin{array}{r}4.1 \\
3.3 \\
2.7 \\
2.7 \\
3.0 \\
2.4 \\
9.9 \\
35.3 \\
18.5 \\
5.4 \\
7.5 \\
5.3\end{array}$ \\
\hline & .9 & 121 & 17 & 24 & 1.40 & 100 \\
\hline
\end{tabular}

\begin{tabular}{|c|c|c|c|c|c|c|}
\hline & \multicolumn{4}{|c|}{$\begin{array}{l}\text { MAGNI TUDE AND PROBABILITY OF } \\
\text { BASED ON PERIOD OF RECORD }\end{array}$} & \multicolumn{2}{|c|}{$\begin{array}{l}\text { ANNUAL LOW FLOW } \\
1909,1928 \text {, } \\
1931-85\end{array}$} \\
\hline \multirow{2}{*}{$\begin{array}{l}\text { PERIOD } \\
\text { (CON- } \\
\text { SECU- } \\
\text { TIVE } \\
\text { DAYS) }\end{array}$} & DISCH? & $\begin{array}{l}\text { IN } \\
\text { RVAL } \\
\text { EDANC }\end{array}$ & $\begin{array}{l}\text { /S, } \\
\text { YEAF } \\
\text { ROBAE }\end{array}$ & $\begin{array}{l}\text { INDI } \\
\text { AND } \\
\text { TY, }\end{array}$ & $\begin{array}{l}\text { JAL NO } \\
\text { PERCEN }\end{array}$ & \\
\hline & $\begin{array}{c}2 \\
508\end{array}$ & $\begin{array}{c}5 \\
208\end{array}$ & $\begin{array}{l}10 \\
108\end{array}$ & $\begin{array}{l}20 \\
58\end{array}$ & $\begin{array}{l}50 \\
28\end{array}$ & $\begin{array}{r}100 \\
18\end{array}$ \\
\hline $\begin{array}{r}1 \\
3 \\
7 \\
14 \\
30 \\
60 \\
90 \\
120 \\
183\end{array}$ & $\begin{array}{r}.6 \\
.7 \\
.8 \\
1.0 \\
1.4 \\
2.6 \\
3.0 \\
3.3 \\
3.5\end{array}$ & $\begin{array}{r}.1 \\
.2 \\
.2 \\
.3 \\
.5 \\
1.0 \\
1.3 \\
1.4 \\
1.7\end{array}$ & $\begin{array}{r}0.0 \\
.1 \\
.1 \\
.2 \\
.3 \\
.5 \\
.7 \\
.9 \\
1.2\end{array}$ & $\begin{array}{r}0.0 \\
0.0 \\
0.0 \\
.1 \\
.1 \\
.2 \\
.4 \\
.5 \\
.9\end{array}$ & $\begin{array}{r}0.0 \\
0.0 \\
0.0 \\
0.0 \\
0.0 \\
.1 \\
.2 \\
.3 \\
.6\end{array}$ & $\begin{array}{r}0.0 \\
0.0 \\
0.0 \\
0.0 \\
0.0 \\
0.0 \\
.1 \\
.2 \\
.5\end{array}$ \\
\hline
\end{tabular}

MAGNITUDE AND PROBABILITY OF ANNUAL HIGH FLOW BASED ON PERIOD OF RECORD 1908-09, 1925 ,

$$
1927-28 \text {, 1931-85 }
$$

\begin{tabular}{|c|c|c|c|c|c|c|}
\hline \multirow{2}{*}{$\begin{array}{l}\text { PERIOD } \\
\text { (CON- } \\
\text { SECU- } \\
\text { TIVE } \\
\text { DAYS) }\end{array}$} & \multicolumn{6}{|c|}{$\begin{array}{l}\text { DISCHARGE, IN FT } 3 / S \text {, FOR INDICATED RECURRENCE } \\
\text { INTERVAL, IN YEARS, AND ANNUAL } \\
\text { EXCEEDANCE PROBABILITY, IN PERCENT }\end{array}$} \\
\hline & $\begin{array}{c}2 \\
508\end{array}$ & $\begin{array}{c}5 \\
208\end{array}$ & $\begin{array}{l}10 \\
108\end{array}$ & $\begin{array}{l}25 \\
48\end{array}$ & $\begin{array}{l}50 \\
28\end{array}$ & $\begin{array}{r}100 \\
18\end{array}$ \\
\hline $\begin{array}{r}1 \\
3 \\
7 \\
15 \\
30 \\
60 \\
90\end{array}$ & $\begin{array}{r}264 \\
155 \\
95 \\
61 \\
40 \\
26 \\
20\end{array}$ & $\begin{array}{r}811 \\
480 \\
311 \\
211 \\
142 \\
88 \\
63\end{array}$ & $\begin{array}{r}1390 \\
832 \\
566 \\
404 \\
283 \\
173 \\
121\end{array}$ & $\begin{array}{r}2390 \\
1450 \\
1050 \\
807 \\
599 \\
368 \\
250\end{array}$ & $\begin{array}{r}3340 \\
2050 \\
1560 \\
1260 \\
982 \\
608 \\
407\end{array}$ & $\begin{array}{r}4440 \\
2760 \\
2200 \\
1890 \\
1540 \\
965 \\
638\end{array}$ \\
\hline
\end{tabular}

DURATION TABLE OF DAILY MEAN FLOW FOR PERIOD OF RECORD 1908-09, 1925, 1927-28, 1931-85 DISCHARGE, IN FT ${ }^{3} / \mathrm{S}$, THAT WAS EQUALED OR EXCEEDED FOR INDICATED PERCENT OF TIME

\begin{tabular}{|c|c|c|c|c|c|c|c|c|c|c|c|c|c|c|}
\hline 58 & 108 & 158 & 208 & 258 & 308 & 408 & 508 & 608 & 708 & 758 & 808 & 858 & 908 & 958 \\
\hline 51 & 17 & 11 & 8.3 & 6.9 & 5.9 & 4.7 & 3.7 & 3.1 & 2.4 & 2.1 & 1.9 & 1.5 & 1.1 & 6 \\
\hline
\end{tabular}


ARKANSAS RIVER BASIN

07211500 CANADIAN RIVER NEAR TAYLOR SPRINGS, NM

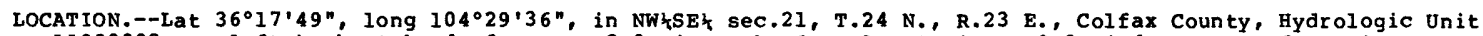
11080003 , on left bank at head of gorge, $2.0 \mathrm{mi}$ south of Taylor Springs, $2.3 \mathrm{mi}$ downstream from Cimarron River, $2.4 \mathrm{mi}$ upstream from Chico Creek, $7.1 \mathrm{mi}$ southeast of $\mathrm{Spr}$ inger, and at mile 847.9.

DRAINAGE AREA. $--2,850 \mathrm{mi}^{2}$.

PERIOD OF RECORD.--January 1940 to September 1958, annual maximum, water years 1959-63, June 1964 to current year. Water-year estimate for 1940 , published in wSP 1311 .

REVISED RECORDS.--WSP 1177: Drainage area. WSP 1281: 1941-42(P), 1945-47(M), $1948-50(P)$.

GAGE.--Water-stage recorder. Elevation of gage is 5,635 ft above National Geodetic Vertical Datum of 1929 , from topographic map. Prior to June 10, 1964, water-stage recorder at site $1.7 \mathrm{mi}$ downstream at different datum; operated as crest-stage gage at that site and datum during water years 1959-64.

REMARKS.--Diversions for irrigation of about 30,000 acres upstream from station.

AVERAGE DISCHARGE. -40 years (water years 1940-58, 1965-85), $78.7 \mathrm{ft} / \mathrm{s}, 57,020 \mathrm{acre-ft/yr}$.

EXTREMES FOR PERIOD OF RECORD.--Maximum discharge, 162,000 ft $3 / \mathrm{s}$, June 18, 1965, gage height, $47.4 \mathrm{ft}$, from floodmarks, from rating curve extended above $7,000 \mathrm{ft} / \mathrm{s}$ on bas is of slope-area measurement of peak flow; no flow at times some years.

EXTREMES OUTSIDE PERIOD OF RECORD, --Maximum flood prior to 1965 occurred Sept. 29, 1904, discharge published as $91,100 \mathrm{ft}^{3} / \mathrm{s}$ in WSP 842,847 .

STATISTICAL SUMMARIES

MEAN MONTHLY AND MEAN ANNUAL DISCHARGES $\begin{aligned} & 1940-58, \\ & 1965-85\end{aligned}$

\begin{tabular}{|c|c|c|c|c|c|c|}
\hline MONTH & $\begin{array}{l}\text { MINIMUH } \\
\left(\mathrm{FT}^{3} / \mathrm{S}\right)\end{array}$ & $\begin{array}{l}\text { MAXIMUH } \\
\left(\mathrm{FT}^{3} / \mathrm{S}\right)\end{array}$ & $\begin{array}{l}\text { MEAN } \\
\left(\mathrm{FT}^{3} / \mathrm{S}\right)\end{array}$ & $\begin{array}{l}\text { STAN- } \\
\text { DARD } \\
\text { DEVIA- } \\
\text { TION } \\
\left(\mathrm{FT}^{3} / \mathrm{S}\right)\end{array}$ & $\begin{array}{l}\text { COEFFI- } \\
\text { CIENT OF } \\
\text { VARI- } \\
\text { ATION }\end{array}$ & $\begin{array}{c}\text { PERCENT } \\
\text { OF } \\
\text { ANNUAL } \\
\text { RUNOF F }\end{array}$ \\
\hline $\begin{array}{l}\text { OCTOBER } \\
\text { NOVEMBER } \\
\text { DECEMBER } \\
\text { JANUARY } \\
\text { FEBRUARY } \\
\text { MARCH } \\
\text { APR IL } \\
\text { MAY } \\
\text { JUNE } \\
\text { JULY } \\
\text { AUGUST } \\
\text { SEPTEMBER }\end{array}$ & $\begin{array}{l}0.0 \\
.9 \\
1.1 \\
1.2 \\
1.0 \\
2.0 \\
1.4 \\
3.6 \\
2.7 \\
1.6 \\
4.7 \\
0.0\end{array}$ & $\begin{array}{r}451 \\
192 \\
105 \\
121 \\
186 \\
79 \\
2853 \\
2174 \\
2313 \\
509 \\
563 \\
1354\end{array}$ & $\begin{array}{r}42 \\
20 \\
17 \\
17 \\
23 \\
18 \\
118 \\
236 \\
135 \\
99 \\
123 \\
85\end{array}$ & $\begin{array}{r}97 \\
33 \\
22 \\
20 \\
31 \\
19 \\
452 \\
454 \\
371 \\
119 \\
117 \\
228\end{array}$ & $\begin{array}{l}2.33 \\
1.64 \\
1.28 \\
1.13 \\
1.38 \\
1.08 \\
3.83 \\
1.92 \\
2.75 \\
1.20 \\
.95 \\
2.68\end{array}$ & $\begin{array}{r}4.5 \\
2.2 \\
1.8 \\
1.9 \\
2.4 \\
1.9 \\
12.7 \\
25.3 \\
14.5 \\
10.6 \\
13.2 \\
9.1\end{array}$ \\
\hline ANNUAL & 7.6 & 564 & 79 & 106 & 1.35 & 100 \\
\hline
\end{tabular}

MAGNITUDE AND PROBABILITY OF ANNUAL LOW FLOW BASED ON PERIOD OF RECORD 1941-58, 1966-85

\begin{tabular}{|c|c|c|c|c|c|c|}
\hline \multirow{3}{*}{$\begin{array}{l}\text { PERIOD } \\
\text { (CON- } \\
\text { SECU- } \\
\text { TIVE } \\
\text { DAYS) }\end{array}$} & \multicolumn{6}{|c|}{$\begin{array}{l}\text { DISCHARGE, IN FT }{ }^{3} / S \text {, FOR INDICATED RECURRENCE } \\
\text { INTERVAL, IN YEARS, AND ANNUAL NON- } \\
\text { EXCEEDANCE PROBABILITY, IN PERCENT }\end{array}$} \\
\hline & & & & -1 & & \\
\hline & $\begin{array}{c}2 \\
508\end{array}$ & $\begin{array}{c}5 \\
208\end{array}$ & $\begin{array}{l}10 \\
108\end{array}$ & $\begin{array}{l}20 \\
58\end{array}$ & $\begin{array}{l}50 \\
28\end{array}$ & $\begin{array}{r}100 \\
18\end{array}$ \\
\hline $\begin{array}{r}1 \\
3 \\
7 \\
14 \\
30 \\
60 \\
90 \\
120 \\
183\end{array}$ & $\begin{array}{l}1.0 \\
1.0 \\
1.3 \\
2.1 \\
3.9 \\
6.8 \\
9.5 \\
10 \\
13\end{array}$ & $\begin{array}{r}0.0 \\
0.0 \\
0.0 \\
.4 \\
1.5 \\
3.1 \\
3.7 \\
4.3 \\
5.8\end{array}$ & $\begin{array}{r}0.0 \\
0.0 \\
0.0 \\
.1 \\
.9 \\
2.0 \\
2.1 \\
2.6 \\
3.7\end{array}$ & $\begin{array}{l}0.0 \\
0.0 \\
0.0 \\
0.0 \\
.5 \\
1.3 \\
1.3 \\
1.7 \\
2.6\end{array}$ & $\begin{array}{l}0.0 \\
0.0 \\
0.0 \\
0.0 \\
0.0 \\
0.0 \\
.7 \\
1.0 \\
1.7\end{array}$ & $\begin{array}{r}0.0 \\
0.0 \\
0.0 \\
0.0 \\
0.0 \\
0.0 \\
.4 \\
.7 \\
1.3\end{array}$ \\
\hline
\end{tabular}

MAGNITUDE AND PROBABILITY OF ANNUAL HIGH FLOW BASED ON PERIOD OF RECORD 1940-58, 1965-85

\begin{tabular}{|c|c|c|c|c|c|c|}
\hline \multirow{2}{*}{$\begin{array}{l}\text { PERIOD } \\
\text { (CON- } \\
\text { SECU- } \\
\text { TIVE } \\
\text { DAYS) }\end{array}$} & \multicolumn{6}{|c|}{$\begin{array}{l}\text { DISCHARGE, IN FT }{ }^{3 / S} \text {, FOR INDICATED RECURRENCE } \\
\text { INTERVAL, IN YEARS, AND ANNUAL } \\
\text { EXCEEDANCE PROBABILITY, IN PERCENT }\end{array}$} \\
\hline & $\begin{array}{c}2 \\
508\end{array}$ & $\begin{array}{c}5 \\
208\end{array}$ & $\begin{array}{l}10 \\
108\end{array}$ & $\begin{array}{l}25 \\
48\end{array}$ & $\begin{array}{l}50 \\
28\end{array}$ & $\begin{array}{r}100 \\
18\end{array}$ \\
\hline $\begin{array}{r}1 \\
3 \\
7 \\
15 \\
30 \\
60 \\
90\end{array}$ & $\begin{array}{r}1600 \\
907 \\
569 \\
377 \\
263 \\
179 \\
131\end{array}$ & $\begin{array}{r}4540 \\
2570 \\
1590 \\
1010 \\
683 \\
443 \\
320\end{array}$ & $\begin{array}{r}8450 \\
4790 \\
2870 \\
1750 \\
1150 \\
714 \\
513\end{array}$ & $\begin{array}{r}17400 \\
9870 \\
5650 \\
3230 \\
2030 \\
1190 \\
855\end{array}$ & $\begin{array}{r}28800 \\
16300 \\
8960 \\
4890 \\
2960 \\
1670 \\
1190\end{array}$ & $\begin{array}{r}46300 \\
26200 \\
13800 \\
7160 \\
4170 \\
2260 \\
1610\end{array}$ \\
\hline
\end{tabular}

DURATION TABLE OF DAILY MEAN FLOW FOR PERIOD OF RECORD 1940-58, 1965-85 DISCHARGE, IN FT $3 / S$, THAT WAS EQUALED OR EXCEEDED FOR INDICATED PERCENT OF TIME

\begin{tabular}{|c|c|c|c|c|c|c|c|c|c|c|c|c|c|c|}
\hline 58 & 108 & 158 & 208 & 258 & 308 & 408 & 508 & 608 & 708 & 758 & 808 & 858 & 908 & 958 \\
\hline 298 & 119 & 65 & 42 & 30 & 25 & 17 & 13 & 9.3 & 6.5 & 5.5 & 4.4 & 3.2 & 2.4 & 1.2 \\
\hline
\end{tabular}


ARKANSAS RIVER BASIN

07214000 CANADIAN RIVER NEAR ROY, NM

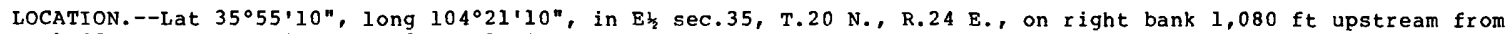
bridge on State Highway 120 and $9 \mathrm{miles}$ west of Roy.

DRAINAGE AREA.--4,066 $\mathrm{mi}^{2}$, of which $107 \mathrm{mi}^{2}$ is probably noncontributing.

PERIOD OF RECORD. - April 1936 to September 1965 (discontinued). Monthly discharge only for April 1936 , published in WSP 1311

GAGE. - Water-stage recorder. Datum of gage is $4,892.55 \mathrm{ft}$ above mean sea level (levels by Corps of Engineers). Prior to Oct. 9, 1942, Jan. 5, 1943 to Jan. 20,1945 and Aug. 6, 1945 to Apr. 30, 1946, at site $1,080 \mathrm{ft}$ downstream at datum $0.39 \mathrm{ft}$ higher. Oct. 10 , 1942 to Jan. 4, 1943, Jan. 21 to Aug. 5 , 1945 and May 1 , $1946 \mathrm{fo}$ Sept. 30,1958 , at present site at datum $1.00 \mathrm{ft}$ higher.

REMARKS.--Diversions for irrigation of about 30,000 acres above station.

EXTREMES FOR PERIOD OF RECORD. - Maximum discharge, that of June 18, 1965; no flow at times.

Prior to 1965 maximum flood known occurred Sept. 29 or 30, 1904, when peak near Taylor Springs was computed as $91,000 \mathrm{ft}^{3} / \mathrm{s}$ (see WSP 842,847 ).

STATISTICAL SUMMARIES

MEAN MONTHLY AND MEAN ANNUAL DISCHARGES 1937-65

\begin{tabular}{|c|c|c|c|c|c|c|}
\hline MONTH & $\begin{array}{l}\text { MINIMUM } \\
\left(\mathrm{FT}^{3} / \mathrm{S}\right)\end{array}$ & $\begin{array}{c}\text { MAXIMUM } \\
\left(\mathrm{FT}^{3} / \mathrm{S}\right)\end{array}$ & $\begin{array}{l}\text { MEAN } \\
\left(\mathrm{FT}^{3} / \mathrm{S}\right)\end{array}$ & $\begin{array}{l}\text { STAN- } \\
\text { DARD } \\
\text { DEVIA- } \\
\text { TION } \\
\left(\mathrm{FT}^{3} / \mathrm{S}\right)\end{array}$ & $\begin{array}{l}\text { COEFFI - } \\
\text { CIENT OF } \\
\text { VARI- } \\
\text { ATION }\end{array}$ & $\begin{array}{c}\text { PERCENT } \\
\text { OF } \\
\text { ANNUAL } \\
\text { RUNOFF }\end{array}$ \\
\hline $\begin{array}{l}\text { OCTOBER } \\
\text { NOVEMBER } \\
\text { DECEMBER } \\
\text { JANUARY } \\
\text { FEBRUARY } \\
\text { MARCH } \\
\text { APRIL } \\
\text { MAY } \\
\text { JUNE } \\
\text { JULY } \\
\text { AUGUST } \\
\text { SEPTEMBER }\end{array}$ & $\begin{array}{r}0.0 \\
0.0 \\
0.0 \\
0.0 \\
0.0 \\
.2 \\
.1 \\
.6 \\
.1 \\
1.1 \\
16 \\
.2\end{array}$ & $\begin{array}{r}546 \\
242 \\
127 \\
114 \\
271 \\
105 \\
3894 \\
2963 \\
2951 \\
552 \\
702 \\
2423\end{array}$ & $\begin{array}{r}86 \\
30 \\
22 \\
21 \\
38 \\
26 \\
181 \\
328 \\
263 \\
157 \\
223 \\
206\end{array}$ & $\begin{array}{r}146 \\
54 \\
30 \\
25 \\
58 \\
30 \\
718 \\
719 \\
598 \\
164 \\
164 \\
492\end{array}$ & $\begin{array}{l}1.71 \\
1.83 \\
1.33 \\
1.15 \\
1.52 \\
1.14 \\
3.97 \\
2.19 \\
2.27 \\
1.04 \\
.74 \\
2.39\end{array}$ & $\begin{array}{r}5.4 \\
1.9 \\
1.4 \\
1.4 \\
2.4 \\
1.7 \\
11.4 \\
20.7 \\
16.6 \\
9.9 \\
14.1 \\
13.0\end{array}$ \\
\hline ANNUAL & 12 & 770 & 132 & 165 & 1.25 & 100 \\
\hline
\end{tabular}

MAGNITUDE AND PROBABILITY OF ANNUAL LOW FLOW BASED ON PERIOD OF RECORD 1938-65

\begin{tabular}{|c|c|c|c|c|c|c|}
\hline \multirow{2}{*}{$\begin{array}{l}\text { PERIOD } \\
\text { (CON- } \\
\text { SECU- } \\
\text { TIVE } \\
\text { DAYS) }\end{array}$} & \multicolumn{6}{|c|}{$\begin{array}{l}\text { DISCHARGE, IN FT }{ }^{3 / S} \text {, FOR INDICATED RECURRENCE } \\
\text { INTERVAL, IN YEARS, AND ANNUAL, NON- } \\
\text { EXCEEDANCE PROBABILITY, IN PERCENT }\end{array}$} \\
\hline & $\begin{array}{c}2 \\
508\end{array}$ & $\begin{array}{c}5 \\
208\end{array}$ & $\begin{array}{l}10 \\
108\end{array}$ & $\begin{array}{l}20 \\
58\end{array}$ & $\begin{array}{l}50 \\
28\end{array}$ & $\begin{array}{r}100 \\
18\end{array}$ \\
\hline $\begin{array}{r}1 \\
3 \\
7 \\
14 \\
30 \\
60 \\
90 \\
120 \\
183\end{array}$ & $\begin{array}{r}0.0 \\
0.0 \\
.4 \\
.8 \\
2.9 \\
6.6 \\
10 \\
12 \\
17\end{array}$ & $\begin{array}{l}0.0 \\
0.0 \\
0.0 \\
0.0 \\
.2 \\
2.8 \\
4.6 \\
5.9 \\
8.3\end{array}$ & $\begin{array}{l}0.0 \\
0.0 \\
0.0 \\
0.0 \\
0.0 \\
1.8 \\
3.0 \\
4.2 \\
5.9\end{array}$ & $\begin{array}{l}0.0 \\
0.0 \\
0.0 \\
0.0 \\
0.0 \\
1.1 \\
1.9 \\
3.0 \\
4.3\end{array}$ & $\begin{array}{l}0.0 \\
0.0 \\
0.0 \\
0.0 \\
0.0 \\
0.0 \\
0.0 \\
0.0 \\
0.0\end{array}$ & $\begin{array}{l}-- \\
-- \\
-- \\
z- \\
-- \\
-- \\
--\end{array}$ \\
\hline
\end{tabular}

MAGNITUDE AND PROBABILITY OF ANNUAL HIGH FLOW BASED ON PERIOD OF RECORD 1937-65

\begin{tabular}{|c|c|c|c|c|c|c|}
\hline \multirow{2}{*}{$\begin{array}{l}\text { PERIOD } \\
\text { (CON- } \\
\text { SECU- } \\
\text { TIVE } \\
\text { DAYS) }\end{array}$} & \multicolumn{6}{|c|}{$\begin{array}{l}\text { DISCHARGE, IN FT'/S, FOR INDICATED RECURRENCE } \\
\text { INTERVAL, IN YEARS, AND ANNUAL } \\
\text { EXCEEDANCE 'PROBABILITY, IN PERCENT }\end{array}$} \\
\hline & $\begin{array}{c}2 \\
508\end{array}$ & $\begin{array}{c}5 \\
208\end{array}$ & $\begin{array}{l}10 \\
108\end{array}$ & $\begin{array}{l}25 \\
48\end{array}$ & $\begin{array}{l}50 \\
28\end{array}$ & $\begin{array}{r}100 \\
18\end{array}$ \\
\hline 1 & 3090 & 9470 & 18300 & 39200 & 66200 & -- \\
\hline 3 & 1780 & 5280 & 10100 & 21200 & 35600 & -- \\
\hline 7 & 1010 & 2940 & 5500 & 11300 & 18600 & - \\
\hline 15 & 632 & 1770 & 3180 & 6180 & 9720 & -- \\
\hline 30 & 426 & 1140 & 1970 & 3580 & 5330 & -- \\
\hline 60 & 282 & 707 & 1170 & & 3000 & -- \\
\hline 90 & 211 & 524 & 857 & 1470 & 2090 & -- \\
\hline
\end{tabular}

DURATION TABLE OF DAILY MEAN FLOW FOR PERIOD OF RECORD 1937-65 DISCHARGE, IN FT ${ }^{3} / 5$, THAT WAS EQUALED OR EXCEEDED FOR INDICATED PERCENT OF TIME

\begin{tabular}{|c|c|c|c|c|c|c|c|c|c|c|c|c|c|c|}
\hline 58 & 108 & 158 & 208 & 258 & 308 & 408 & 508 & 608 & 708 & 758 & 808 & 858 & 908 & 958 \\
\hline 453 & 197 & 114 & 71 & 48 & 37 & 24 & 17 & 12 & 7.8 & 6.2 & 4.7 & 2.6 & 1.5 & 1 \\
\hline
\end{tabular}


LOCATION.--Lat $36^{\circ} 06^{\prime} 37^{\prime \prime}$, long $105^{\circ} 22 \cdot 33^{\prime \prime}$, Mora County, in Mora Grant, on right bank $330 \mathrm{ft}$ downstream from bridge, $2.4 \mathrm{mi}$ south of Chacon, $4.5 \mathrm{mi}$ downstream from confluence of Luna and Lujan Creeks, $5.0 \mathrm{mi}$ north of Holman, 8.0 $\mathrm{mi}$ southwest of Guadalupita, and at mile 106.9 .

DRA INAGE AREA. $--57 \mathrm{mi}^{2}$.

PERIOD OF RECORD.--January 1953 to December 1973 (discontinued). Published as Rio Agua Negra near Holman prior to October 1965.

GAGE.--Water-stage recorder. Altitude of gage is $7,845 \mathrm{ft}$ from topographic map. Prior to Apr. 28 , 1972 , at site $500 \mathrm{ft}$ upstream at datum $7.86 \mathrm{ft}$ higher.

REMARKS.--Diversions for irrigation of about 1,600 acres above station.

AVERAGE DISCHARGE.--21 calendar years, $13.5 \mathrm{ft} / \mathrm{s}, 9,780$ acre-ft/yr; 20 calendar years (1954-73), $13.9 \mathrm{ft} / \mathrm{s}, 10,070$ acre-ft/yr.

EXTREMES FOR PERIOD OF RECORD.--Maximum discharge, 4,700 $\mathrm{ft}^{3} / \mathrm{s}$ July 22,1954 (gage height, $6.10 \mathrm{ft}$, site and datum then in use), from rating curve extended above $300 \mathrm{ft}^{3} / \mathrm{s}$ on basis of slope-area measurement of peak flow; minimum, about $0.06 \mathrm{ft} / \mathrm{s} \mathrm{Jan} .18,1967$, result of freezeup.

STATISTICAL SUMMARIES

MEAN MONTHLY AND MEAN ANNUAL DISCHARGES 1954-73

\begin{tabular}{|c|c|c|c|c|c|c|}
\hline MONTH & $\begin{array}{l}\text { MINI MUM } \\
\left(\mathrm{FT}^{3} / \mathrm{S}\right)\end{array}$ & $\begin{array}{c}\operatorname{MAXIMUM} \\
\left(\mathrm{FT}^{3} / \mathrm{S}\right)\end{array}$ & $\begin{array}{l}\text { MEAN } \\
\left(F^{3} / S\right)\end{array}$ & $\begin{array}{l}\text { STAN- } \\
\text { DARD } \\
\text { DEVIA- } \\
\text { TION } \\
\left(\mathrm{FT}^{3} / \mathrm{S}\right)\end{array}$ & $\begin{array}{l}\text { COEFFI - } \\
\text { CIENT OF } \\
\text { VARI - } \\
\text { ATION }\end{array}$ & $\begin{array}{c}\text { PERCENT } \\
\text { OF } \\
\text { ANNUAL } \\
\text { RUNOFF }\end{array}$ \\
\hline $\begin{array}{l}\text { OCTOBER } \\
\text { NOVEMBER } \\
\text { DECEMBER } \\
\text { JANUARY } \\
\text { FEBRUARY } \\
\text { MARCH } \\
\text { APRIL } \\
\text { MAY } \\
\text { JUNE } \\
\text { JULY } \\
\text { AUGUST } \\
\text { SEPTEMBER }\end{array}$ & $\begin{array}{r}.3 \\
1.7 \\
1.0 \\
1.2 \\
2.0 \\
1.9 \\
1.7 \\
1.1 \\
2.0 \\
2.6 \\
.4\end{array}$ & $\begin{array}{c}18 \\
20 \\
11 \\
12 \\
8.8 \\
11 \\
97 \\
201 \\
98 \\
35 \\
84 \\
40\end{array}$ & $\begin{array}{l}6.2 \\
6.2 \\
5.0 \\
4.5 \\
4.4 \\
6.3 \\
26 \\
43 \\
20 \\
11 \\
23 \\
11\end{array}$ & $\begin{array}{l}4.5 \\
4.6 \\
2.4 \\
2.4 \\
2.0 \\
3.1 \\
27 \\
55 \\
27 \\
8.8 \\
22 \\
11\end{array}$ & $\begin{array}{r}.72 \\
.74 \\
.48 \\
.53 \\
.46 \\
.48 \\
1.05 \\
1.29 \\
1.31 \\
.79 \\
.99 \\
1.00\end{array}$ & $\begin{array}{r}3.8 \\
3.7 \\
3.0 \\
2.7 \\
2.7 \\
3.8 \\
15.5 \\
25.7 \\
12.3 \\
6.7 \\
13.7 \\
6.4\end{array}$ \\
\hline NNUAL & 2.7 & 38 & 14 & 10 & .72 & 100 \\
\hline
\end{tabular}

MAGNITUDE AND PROBABILITY OF ANNUAL LOW FLOW BASED ON PERIOD OF RECORD 1955-73

\begin{tabular}{|c|c|c|c|c|c|c|}
\hline \multirow{2}{*}{$\begin{array}{l}\text { PERIOD } \\
\text { (CON- } \\
\text { SECU- } \\
\text { TIVE } \\
\text { DAYS) }\end{array}$} & \multicolumn{6}{|c|}{$\begin{array}{l}\text { DISCHARGE, IN FT }{ }^{3 / S} \text {, FOR INDICATED RECURRENCE } \\
\text { INTERVAL, IN YEARS, AND ANNUAL NON- } \\
\text { EXCEEDANCE PROBABILITY, IN PERCENT }\end{array}$} \\
\hline & $\begin{array}{c}2 \\
508\end{array}$ & $\begin{array}{c}5 \\
208\end{array}$ & $\begin{array}{l}10 \\
108\end{array}$ & $\begin{array}{l}20 \\
58\end{array}$ & $\begin{array}{l}50 \\
28\end{array}$ & $\begin{array}{r}100 \\
18\end{array}$ \\
\hline $\begin{array}{r}1 \\
3 \\
7 \\
14 \\
30 \\
60 \\
90 \\
120 \\
183\end{array}$ & $\begin{array}{l}1.5 \\
1.6 \\
1.9 \\
2.3 \\
2.9 \\
3.4 \\
3.7 \\
4.1 \\
4.8\end{array}$ & $\begin{array}{r}.5 \\
.7 \\
.9 \\
1.1 \\
1.4 \\
1.7 \\
1.9 \\
2.2 \\
2.7\end{array}$ & $\begin{array}{r}.3 \\
.4 \\
.6 \\
.7 \\
.8 \\
1.1 \\
1.3 \\
1.5 \\
1.9\end{array}$ & $\begin{array}{r}.1 \\
.3 \\
.4 \\
.4 \\
.5 \\
.7 \\
.9 \\
1.0 \\
1.4\end{array}$ & $\begin{array}{l}=- \\
-- \\
=- \\
z- \\
z- \\
z- \\
--\end{array}$ & $\begin{array}{l}=- \\
=- \\
-- \\
=- \\
=- \\
-- \\
--\end{array}$ \\
\hline
\end{tabular}

MAGNITUDE AND PROBABILITY OF ANNUAL HIGH FLOW BASED ON PERIOD OF RECORD 1954-73

\begin{tabular}{|c|c|c|c|c|c|c|}
\hline \multirow{2}{*}{$\begin{array}{l}\text { PERIOD } \\
\text { (CON- } \\
\text { SECU- } \\
\text { TIVE } \\
\text { DAYS) }\end{array}$} & \multicolumn{6}{|c|}{$\begin{array}{l}\text { DISCHARGE, IN FT } 3 / \mathrm{S} \text {, FOR INDICATED RECURRENCE } \\
\text { INTERVAL, IN YEARS, AND ANNUAL, } \\
\text { EXCEEDANCE PROBABILITY, IN PERCENT }\end{array}$} \\
\hline & 22 & $\begin{array}{c}5 \\
208\end{array}$ & $\begin{array}{l}10 \\
108\end{array}$ & $\begin{array}{l}25 \\
48\end{array}$ & $\begin{array}{l}50 \\
28\end{array}$ & $\begin{array}{r}100 \\
18\end{array}$ \\
\hline $\begin{array}{r}1 \\
3 \\
7 \\
15 \\
30 \\
60 \\
90\end{array}$ & $\begin{array}{r}107 \\
80 \\
64 \\
51 \\
40 \\
29 \\
23\end{array}$ & $\begin{array}{r}222 \\
175 \\
147 \\
119 \\
93 \\
67 \\
51\end{array}$ & $\begin{array}{r}322 \\
264 \\
225 \\
183 \\
142 \\
102 \\
77\end{array}$ & $\begin{array}{l}476 \\
407 \\
352 \\
286 \\
220 \\
159 \\
118\end{array}$ & $\begin{array}{l}-- \\
-- \\
-- \\
-- \\
--\end{array}$ & $\begin{array}{l}-- \\
-- \\
-- \\
-- \\
-- \\
--\end{array}$ \\
\hline
\end{tabular}

DURATION TABLE OF DAILY MEAN FLOW FOR PERIOD OF RECORD 1954-73 DISCHARGE, IN FT $3 / \mathrm{S}$, THAT WAS EQUALED OR EXCEEDED FOR INDICATED PERCENT OF TIME

\begin{tabular}{|c|c|c|c|c|c|c|c|c|c|c|c|c|c|}
\hline 58 & 108 & 158 & 208 & 258 & 308 & 408 & 508 & 608 & 708 & 758 & 808 & 858 & 908 \\
\hline 57 & 30 & 21 & 16 & 12 & 10 & 7.3 & 5.6 & 4.6 & 3.7 & 3.3 & 2.8 & 2.4 & 1.8 \\
\hline
\end{tabular}


ARKANSAS RIVER BASIN

07214800 RIO LA CASA NEAR CLEVELAND, NM

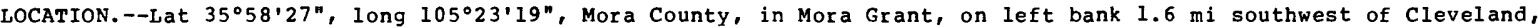
and $2.3 \mathrm{mi}$ upstream from mouth.

DRAINAGE AREA. $--23.0 \mathrm{mi}^{2}$

PERIOD OF RECORD.--May 1956 to September 1970 (discontinued). Prior to October 1964, published as Rio de la Casa near Cleveland.

GAGE.--Water-stage recorder. Altitude of gage is $7,635 \mathrm{ft}$ (from topographic map).

REMARKS.--Diversions for irrigation of about 100 acres above station.

AVERAGE DISCHARGE. --14 years, $14.4 \mathrm{ft}^{3} / \mathrm{s}, 10,430$ acre-ft/year.

EXTREMES FOR PERIOD OF RECORD.--Maximum discharge, 2,260 ft $3 / \mathrm{s}$ Aug. 6, 1959 , gage height, $6.00 \mathrm{ft}$, from rating curve extended above $170 \mathrm{ft}^{3} / \mathrm{s}$ on basis of slope-area measurement of peak flow; minimum, $0.08 \mathrm{ft} / \mathrm{s} 0 \mathrm{ct}$. 30 , curve 1958 .

STATISTICAL SUMMARIES

MEAN MONTHLY AND MEAN ANNUAL DISCHARGES 1957-70

\begin{tabular}{|c|c|c|c|c|c|c|}
\hline MONTH & $\begin{array}{l}\text { MINIMUM } \\
\left(\mathrm{FT}^{3} / \mathrm{S}\right)\end{array}$ & $\begin{array}{l}\text { MAXIMUM } \\
\left(\mathrm{FT}^{3} / \mathrm{S}\right)\end{array}$ & $\begin{array}{l}\text { MEAN } \\
\left(\mathrm{FT}^{3} / \mathrm{S}\right)\end{array}$ & $\begin{array}{l}\text { STAN- } \\
\text { DARD } \\
\text { DEVIA- } \\
\text { TION } \\
\text { (FT'S }\end{array}$ & $\begin{array}{l}\text { COEFFI- } \\
\text { CIENT OF } \\
\text { VARI- } \\
\text { ATION }\end{array}$ & $\begin{array}{c}\text { PERCENT } \\
\text { OF } \\
\text { ANNUAL } \\
\text { RUNOFF }\end{array}$ \\
\hline $\begin{array}{l}\text { OCTOBER } \\
\text { NOVEMBER } \\
\text { DECEMBER } \\
\text { JANUARY } \\
\text { FEBRUARY } \\
\text { MARCH } \\
\text { APRIL } \\
\text { MAY } \\
\text { JUNE } \\
\text { JULY } \\
\text { AUGUST } \\
\text { SEPTEMBER }\end{array}$ & $\begin{array}{r}.9 \\
.8 \\
.8 \\
1.6 \\
1.5 \\
2.3 \\
4.6 \\
7.3 \\
7.0 \\
3.7 \\
4.2 \\
3.9\end{array}$ & $\begin{array}{c}18 \\
13 \\
5.9 \\
4.5 \\
4.0 \\
9.9 \\
34 \\
63 \\
105 \\
42 \\
51 \\
19\end{array}$ & $\begin{array}{l}6.5 \\
5.2 \\
3.4 \\
2.8 \\
2.8 \\
4.6 \\
14 \\
36 \\
45 \\
17 \\
26 \\
9.2\end{array}$ & $\begin{array}{r}4.9 \\
3.8 \\
1.5 \\
.9 \\
.8 \\
2.2 \\
8.7 \\
15 \\
29 \\
10 \\
18 \\
4.6\end{array}$ & $\begin{array}{l}.75 \\
.72 \\
.43 \\
.33 \\
.28 \\
.49 \\
.62 \\
.42 \\
.65 \\
.60 \\
.68 \\
.50\end{array}$ & $\begin{array}{r}3.7 \\
3.0 \\
2.0 \\
1.6 \\
1.6 \\
2.7 \\
8.2 \\
21.1 \\
25.9 \\
9.7 \\
15.1 \\
5.4\end{array}$ \\
\hline ANNUAL & 6.0 & 24 & 14 & 5.3 & .37 & 100 \\
\hline
\end{tabular}

MAGNITUDE AND PROBABILITY OF ANNUAL LOW FLOW BASED ON PERIOD OF RECORD 1958-70

\begin{tabular}{|c|c|c|c|c|c|c|}
\hline \multirow{2}{*}{$\begin{array}{l}\text { PERIOD } \\
\text { (CON- } \\
\text { SECU- } \\
\text { TIVE } \\
\text { DAYS) }\end{array}$} & \multicolumn{6}{|c|}{$\begin{array}{l}\text { DISCHARGE, IN FT }{ }^{3} / S \text {, FOR INDICATED RECURRENCE } \\
\text { INTERVAL, IN YEARS, AND ANNUAL NON- } \\
\text { EXCEEDANCE PROBABILITY, IN PERCENT }\end{array}$} \\
\hline & $\begin{array}{c}2 \\
508\end{array}$ & $\begin{array}{c}5 \\
208\end{array}$ & $\begin{array}{l}10 \\
108\end{array}$ & $\begin{array}{l}20 \\
58\end{array}$ & $\begin{array}{l}50 \\
28\end{array}$ & $\begin{array}{r}100 \\
18\end{array}$ \\
\hline $\begin{array}{r}1 \\
3 \\
7 \\
14 \\
30 \\
60 \\
90 \\
120 \\
183\end{array}$ & $\begin{array}{l}1.9 \\
2.0 \\
2.2 \\
2.4 \\
2.6 \\
2.8 \\
2.9 \\
3.1 \\
3.8\end{array}$ & $\begin{array}{l}1.1 \\
1.3 \\
1.6 \\
1.7 \\
2.0 \\
2.2 \\
2.2 \\
2.4 \\
2.8\end{array}$ & $\begin{array}{r}.9 \\
1.0 \\
1.3 \\
1.5 \\
1.7 \\
1.9 \\
2.0 \\
2.1 \\
2.5\end{array}$ & $\begin{array}{r}.7 \\
.8 \\
1.0 \\
1.3 \\
1.5 \\
1.7 \\
1.7 \\
1.9 \\
2.2\end{array}$ & $\begin{array}{l}-- \\
-- \\
-- \\
-- \\
-- \\
-- \\
--\end{array}$ & \\
\hline
\end{tabular}

MAGNITUDE AND PROBABILITY OF ANNUAL HIGH FLOW BASED ON PERIOD OF RECORD 1957-70

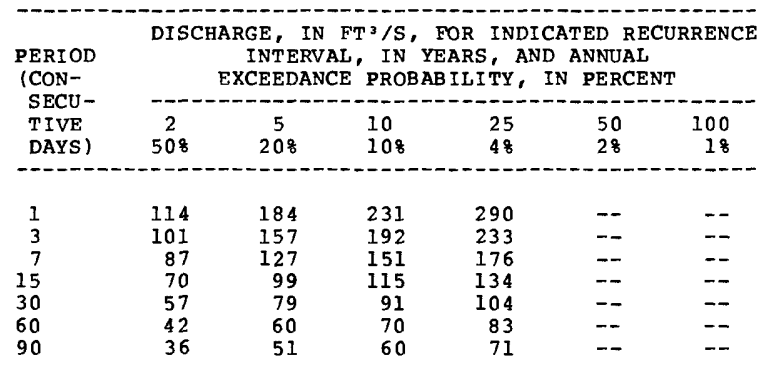

DURATION TABLE OF DAILY MEAN FLOW FOR PERIOD OF RECORD 1957-70 DISCHARGE, IN FT $3 / \mathrm{S}$, THAT WAS EOUALED OR EXCEEDED FOR INDICATED PERCENT OF TIME

\begin{tabular}{|c|c|c|c|c|c|c|c|c|c|c|c|c|c|c|}
\hline 58 & 108 & 158 & 208 & 258 & 308 & 408 & 508 & 608 & 708 & 758 & 808 & 858 & 908 & 958 \\
\hline 60 & 39 & 27 & 21 & 16 & 13 & 8.5 & 5.9 & 4.4 & 3.6 & 3.3 & 3.0 & 2.7 & 2.4 & \\
\hline
\end{tabular}


ARRANSAS RIVER BASIN

07215500 MORA RIVER AT LA CUEVA, NM

LOCATION.--Lat $35^{\circ} 56^{\prime} 27^{\prime}$, long $105^{\circ} 14^{\prime} 59^{\prime \prime}$, Mora County, Hydrologic Unit 11080004, in Mora Grant, on left bank 45 ft upstream from bridge on State Highway 3 at La Cueva, $0.3 \mathrm{mi}$ downstream from La Cueva damsite, and at mile 86.8 .

DRAINAGE AREA. $--173 \mathrm{mi}^{2}$.

PERIOD OF RECORD.--August 1903 to April 1905 (gage heights and discharge measurements only), May to December 1905, May 1906 to July 1911, April 1931 to current year. Monthly discharge only for some periods, published in wsp

1311. Records for February to April 1905, published in wSP 173, are unreliable and should not be used.

REVISED RECORDS.--WSP 857: 1937. WSP 1281: 1931 (M), 1932. WSP 1511: Drainage area. See also PERIOD OF RECORD.

GAGE. - Water-stage recorder. Elevation of gage is 7,000 ft above National Geodetic Vertical Datum of 1929 , from topographic map. Mar. 10, 1915 to June 4, 1921, water-stage recorder at site $2.8 \mathrm{mi}$ upstream at different datum. July 6,1921 to $\mathrm{Jan}$. 5, 1929, nonrecording gage or water-stage recorder at site $0.7 \mathrm{mi}$ downstream at datum about $14 \mathrm{ft}$ lower and Jan. 6 , 1929 to Apr. 1, 1972, water-stage recorder at site $0.7 \mathrm{mi}$ downstream at datum about 15 ft lower.

REMARKS.-Diversions upstream from station for irrigation of about 7,000 acres, part of which are downstream from station.

AVERAGE DISCHARGE. --58 years (water years 1907-10, 1932-85), 27.8 $\mathrm{ft}^{3} / \mathrm{s}, 20,140 \mathrm{acre}-\mathrm{ft} / \mathrm{yr}$.

EXTREMES FOR PERIOD OF RECORD (SINCE 1930).--Maximum discharge, 1,530 ft 3/s, Sept. 23, 1941, gage height, 7.58 ft, site and datum then in use, from rating curve extended above $400 \mathrm{ft}^{3} / \mathrm{s}$; no flow at times.

EXTREMES OUTSIDE PERIOD OF RECORD.--Flood of Sept. 29, 1904, may have exceeded 20,000 $\mathrm{ft} 3 / \mathrm{s}$; another major flood occurred June 11, 1913, but is believed less than that of 1904

STATISTICAL SUMMARIES

MEAN MONTHLY AND MEAN ANNUAL DISCHARGES 1907-10,

\begin{tabular}{|c|c|c|c|c|c|c|}
\hline MONTH & $\begin{array}{l}\text { MINIMUM } \\
\left(\mathrm{FT}^{3} / \mathrm{S}\right)\end{array}$ & $\begin{array}{c}\text { MAXIMUM } \\
\left(\mathrm{FT}^{3} / \mathrm{S}\right)\end{array}$ & $\begin{array}{l}\text { MEAN } \\
\left(\mathrm{FT}^{3} / \mathrm{S}\right)\end{array}$ & $\begin{array}{c}\text { STAN- } \\
\text { DARD } \\
\text { DEVIA- } \\
\text { TION } \\
\left(\mathrm{FT}^{3} / \mathrm{S}\right)\end{array}$ & $\begin{array}{l}\text { COEFFI - } \\
\text { CIENT OF } \\
\text { VARI - } \\
\text { ATION }\end{array}$ & $\begin{array}{c}\text { PERCENT } \\
\text { OF } \\
\text { ANNUAL } \\
\text { RUNOF F }\end{array}$ \\
\hline $\begin{array}{l}\text { OCTOBER } \\
\text { NOVEMBER } \\
\text { DECEMBER } \\
\text { JANUARY } \\
\text { FEBRUARY } \\
\text { MARCH } \\
\text { APRIL } \\
\text { MAY } \\
\text { JUNE } \\
\text { JULY } \\
\text { AUGUST } \\
\text { SEPTEMBER }\end{array}$ & $\begin{array}{r}.6 \\
.4 \\
.6 \\
0.0 \\
.5 \\
1.1 \\
2.1 \\
1.5 \\
1.1 \\
3.0 \\
1.4 \\
.5\end{array}$ & $\begin{array}{r}88 \\
61 \\
39 \\
22 \\
26 \\
44 \\
244 \\
555 \\
314 \\
142 \\
182 \\
83\end{array}$ & $\begin{array}{l}17 \\
11 \\
8.6 \\
7.7 \\
7.4 \\
11 \\
33 \\
77 \\
60 \\
32 \\
43 \\
27\end{array}$ & $\begin{array}{c}16 \\
12 \\
8.4 \\
6.1 \\
5.3 \\
9.5 \\
49 \\
102 \\
64 \\
29 \\
38 \\
20\end{array}$ & $\begin{array}{r}.95 \\
1.06 \\
.97 \\
.79 \\
.72 \\
.90 \\
1.48 \\
1.32 \\
1.07 \\
.93 \\
.88 \\
.75\end{array}$ & $\begin{array}{r}4.9 \\
3.2 \\
2.6 \\
2.3 \\
2.2 \\
3.2 \\
10.0 \\
23.1 \\
18.0 \\
9.4 \\
12.9 \\
8.0\end{array}$ \\
\hline NNALL & 3.1 & 113 & 28 & 22 & .78 & 100 \\
\hline
\end{tabular}

MAGNITUDE AND PROBABILITY OF ANNUAL LOW FLOW BASED ON PERIOD OF RECORD 1908-11, 1933-85

\begin{tabular}{|c|c|c|c|c|c|c|}
\hline \multirow{2}{*}{$\begin{array}{l}\text { PERIOD } \\
\text { (CON- } \\
\text { SECU- } \\
\text { TIVE } \\
\text { DAYS) }\end{array}$} & \multicolumn{6}{|c|}{$\begin{array}{l}\text { DISCHARGE, IN FT }{ }^{3} / S \text {, FOR INDICATED RECURRENCE } \\
\text { INTERVAL, IN YEARS, AND ANNUAL NON- } \\
\text { EXCEEDANCE PROBABILITY, IN PERCENT }\end{array}$} \\
\hline & $\begin{array}{c}2 \\
508\end{array}$ & $\begin{array}{c}5 \\
208\end{array}$ & $\begin{array}{l}10 \\
108\end{array}$ & $\begin{array}{l}20 \\
58\end{array}$ & $\begin{array}{l}50 \\
28\end{array}$ & $\begin{array}{r}100 \\
18\end{array}$ \\
\hline $\begin{array}{r}1 \\
3 \\
7 \\
14 \\
30 \\
60 \\
90 \\
120 \\
183\end{array}$ & $\begin{array}{l}1.2 \\
1.3 \\
1.5 \\
1.9 \\
2.5 \\
3.6 \\
4.3 \\
5.1 \\
7.6\end{array}$ & $\begin{array}{r}.3 \\
.4 \\
.6 \\
.7 \\
1.0 \\
1.4 \\
1.7 \\
2.1 \\
3.4\end{array}$ & $\begin{array}{r}.1 \\
.2 \\
.3 \\
.4 \\
.6 \\
.9 \\
1.0 \\
1.3 \\
2.2\end{array}$ & $\begin{array}{r}0.0 \\
0.0 \\
.2 \\
.2 \\
.4 \\
.6 \\
.7 \\
.8 \\
1.4\end{array}$ & $\begin{array}{r}0.0 \\
0.0 \\
0.0 \\
.1 \\
.1 \\
.4 \\
.4 \\
.5 \\
.9\end{array}$ & $\begin{array}{l}0.0 \\
0.0 \\
0.0 \\
0.0 \\
0.0\end{array}$ \\
\hline
\end{tabular}

MAGNITUDE AND PROBABILITY OF ANNUAL HIGH FLOW BASED ON PERIOD OF RECORD 1907-10, 1932-85

\begin{tabular}{|c|c|c|c|c|c|c|}
\hline \multirow{2}{*}{$\begin{array}{l}\text { PERIOD } \\
\text { (CON- } \\
\text { SECU- } \\
\text { TIVE } \\
\text { DAYS) }\end{array}$} & \multicolumn{6}{|c|}{$\begin{array}{l}\text { DISCHARGE, IN FT } 3 / \mathrm{S} \text {, FOR INDICATED RECURRENCE } \\
\text { INTERVAL, IN YEARS, AND ANNUAL } \\
\text { EXCEEDANCE PROBABILITY, IN PERCENT }\end{array}$} \\
\hline & $\begin{array}{c}2 \\
508\end{array}$ & $\begin{array}{c}5 \\
208\end{array}$ & $\begin{array}{l}10 \\
108\end{array}$ & $\begin{array}{l}25 \\
48\end{array}$ & $\begin{array}{l}50 \\
28\end{array}$ & $\begin{array}{r}100 \\
18\end{array}$ \\
\hline $\begin{array}{r}1 \\
3 \\
7 \\
15 \\
30 \\
60 \\
90\end{array}$ & $\begin{array}{r}222 \\
174 \\
139 \\
111 \\
86 \\
63 \\
50\end{array}$ & $\begin{array}{l}398 \\
333 \\
276 \\
226 \\
179 \\
133 \\
106\end{array}$ & $\begin{array}{l}510 \\
442 \\
374 \\
315 \\
254 \\
192 \\
153\end{array}$ & $\begin{array}{l}640 \\
577 \\
499 \\
435 \\
362 \\
279 \\
223\end{array}$ & $\begin{array}{l}727 \\
672 \\
590 \\
527 \\
450 \\
353 \\
283\end{array}$ & $\begin{array}{l}805 \\
760 \\
677 \\
620 \\
543 \\
433 \\
348\end{array}$ \\
\hline
\end{tabular}

DURATION TABLE OF DAILY MEAN FLOW FOR PERIOD OF RECORD 1907-10, 1932-85 DISCHARGE, IN FT'/S, THAT WAS EQUALED OR EXCEEDED FOR INDICATED PERCENT OF TIME

\begin{tabular}{|c|c|c|c|c|c|c|c|c|c|c|c|c|c|c|}
\hline 58 & 108 & 158 & 208 & 258 & 308 & 408 & 508 & 608 & 708 & 758 & 808 & 858 & 908 & 958 \\
\hline 113 & 70 & 49 & 35 & 27 & 21 & 16 & 11 & 8.1 & 5.5 & 4.3 & 3.2 & 2.2 & 1.5 & \\
\hline
\end{tabular}


ARKANSAS RIVER BASIN

07216500 MORA RIVER NEAR GOLONDRINAS, NM

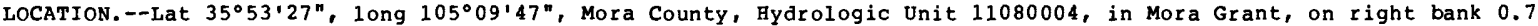
$\mathrm{mi}$ upstream from bridge on State Highway 160, $1.2 \mathrm{mi}$ east of Golondrinas, $1.9 \mathrm{mi}$ upstream from Coyote Creek, 4.7 mi downstream from Rito Cebolla, and at mile 75.8 .

DRAINAGE AREA. $--267 \mathrm{mi}^{2}$.

PERIOD OF RECORD,--March 1915 to May 1921, October 1921 to March 1922, May, August, September 1922, Ju1y 1923 to July 1924, December 1924 to current year. Monthly discharge only 1915-30, published in wSP 1311.

REVISED RECORDS.--WSP 1281: 1951(M). WSP 1311: 1935(M), 1937-38(M), 1940-42(M), 1949(M). WSP 1511: Drainage area. WSP 1731: $1958(\mathrm{M})$.

GAGE.--Water-stage recorder. Elevation of gage is 6,750 ft above National Geodetic Vertical Datum of 1929 , from topographic map. Mar. 10, 1915 to June 4, 1921, water-stage recorder at site $2.8 \mathrm{mi}$ upstream at different datum. July 6,1921 to Jan. 5, 1929, nonrecording gage or water-stage recorder at site $0.7 \mathrm{mi}$ downstream at datum about $14 \mathrm{ft}$ lower and Jan. 6, 1929 to Apr. 1, 1972, water-stage recorder at site $0.7 \mathrm{mi}$ downstream at datum about $15 \mathrm{ft}$ lower.

REMARKS.--Diversions for irrigation of about 12,000 acres upstream from station. Off-channel lakes make it possible to divert and store water during non-irrigation season.

AVERAGE DISCHARGE, - -68 years (water years 1916-20, 1927-85), 33.8 ft3/s, 24,490 acre-ft/yr.

EXTREMES FOR PERIOD OF RECORD.--Maximum discharge, 14,000 $\mathrm{ft}^{3} / \mathrm{s}$, Aug. 22, 1952, gage height, 14.4 $\mathrm{ft}$, site and datum then in use, from rating curve extended above $660 \mathrm{ft}^{3} / \mathrm{s}$ on basis of slope-area measurement of peak flow; no flow at times.

EXTREMES OUTSIDE PERIOD OF RECORD.--Floods of Sept. 29, 1904, and June 11, 1913, probably exceeded 25,000 ft $3 / \mathrm{s}$.

STATISTICAL SUMMARIES

MEAN MONTHLY AND MEAN ANNUAL DISCHARGES 1916-20

\begin{tabular}{|c|c|c|c|c|c|c|}
\hline MONTH & $\begin{array}{l}\text { MIN I MUM } \\
\left(\mathrm{FT}^{3} / \mathrm{S}\right)\end{array}$ & $\begin{array}{l}\text { MAXIMUM } \\
\left(\mathrm{FT}^{3} / \mathrm{S}\right)\end{array}$ & $\begin{array}{l}\text { MEAN } \\
\left(\mathrm{FT}^{3} / \mathrm{S}\right)\end{array}$ & $\begin{array}{c}\text { STAN- } \\
\text { DARD } \\
\text { DEVIA- } \\
\text { TION } \\
\left(\mathrm{FT}^{3} / \mathrm{S}\right)\end{array}$ & $\begin{array}{l}\text { COEFFI- } \\
\text { CIENT OF } \\
\text { VARI- } \\
\text { ATION }\end{array}$ & $\begin{array}{c}\text { PERCENT } \\
\text { OF } \\
\text { ANNUAL } \\
\text { RUNOFF }\end{array}$ \\
\hline OCTOBER & 2 & 119 & 21 & 23 & 1.06 & 5.2 \\
\hline NOVEMBER & .4 & 87 & 14 & 15 & 1.03 & 3.5 \\
\hline DECEMBER & .5 & 39 & 12 & 9.3 & .77 & 2.9 \\
\hline JANUARY & .7 & 30 & 12 & 7.5 & .64 & 2.9 \\
\hline FEBRUARY & .6 & 27 & 11 & 7.1 & .63 & 2.7 \\
\hline MARCH & .6 & 69 & 13 & 13 & .97 & 3.2 \\
\hline APRIL & .3 & 361 & 44 & 72 & 1.64 & 10.7 \\
\hline MAY & 1.0 & 661 & 90 & 117 & 1.30 & 22.0 \\
\hline JUNE & 0.0 & 377 & 68 & 77 & 1.13 & 16.7 \\
\hline JULY & 1.6 & 321 & 38 & 47 & 1.26 & 9.2 \\
\hline AUGUST & 0.0 & 307 & 55 & 57 & 1.04 & 13.3 \\
\hline SEPTEMBER & .3 & 103 & 32 & 26 & .83 & 7.7 \\
\hline NNUAL & 3.4 & 144 & 34 & 28 & .82 & 100 \\
\hline
\end{tabular}

MAGNITUDE AND PROBABILITY OF ANNUAL LOW FLOW BASED ON PERIOD OF RECORD 1917-20, 1927-85

\begin{tabular}{|c|c|c|c|c|c|c|}
\hline \multirow{2}{*}{$\begin{array}{l}\text { PERIOD } \\
\text { (CON- } \\
\text { SECU- } \\
\text { TIVE } \\
\text { DAYS) }\end{array}$} & \multicolumn{6}{|c|}{$\begin{array}{l}\text { DISCHARGE, IN } \mathrm{FT}^{3} / \mathrm{S} \text {, FOR INDICATED RECURRENCE } \\
\text { INTERVAL, IN YEARS, AND ANNUAL NON- } \\
\text { EXCEEDANCE PROBABILITY, IN PERCENT }\end{array}$} \\
\hline & $\begin{array}{c}2 \\
508\end{array}$ & $\begin{array}{c}5 \\
208\end{array}$ & $\begin{array}{l}10 \\
108\end{array}$ & $\begin{array}{l}20 \\
58\end{array}$ & $\begin{array}{l}50 \\
28\end{array}$ & $\begin{array}{r}100 \\
18\end{array}$ \\
\hline $\begin{array}{r}1 \\
3 \\
7 \\
14 \\
30 \\
60 \\
90 \\
120 \\
183\end{array}$ & $\begin{array}{r}1.3 \\
1.4 \\
1.7 \\
2.1 \\
2.9 \\
5.4 \\
6.8 \\
8.0 \\
11\end{array}$ & $\begin{array}{r}.4 \\
.4 \\
.5 \\
.7 \\
1.0 \\
2.2 \\
2.8 \\
3.5 \\
4.9\end{array}$ & $\begin{array}{r}.1 \\
.2 \\
.2 \\
.3 \\
.6 \\
1.3 \\
1.6 \\
2.1 \\
3.0\end{array}$ & $\begin{array}{r}0.0 \\
0.0 \\
0.0 \\
.1 \\
.3 \\
.8 \\
1.0 \\
1.4 \\
1.9\end{array}$ & $\begin{array}{r}0.0 \\
0.0 \\
0.0 \\
0.0 \\
.1 \\
.5 \\
.5 \\
.8 \\
1.1\end{array}$ & $\begin{array}{l}0.0 \\
0.0 \\
0.0 \\
0.0 \\
0.0 \\
.3 \\
.3 \\
.5 \\
.7\end{array}$ \\
\hline
\end{tabular}

MAGNITUDE AND PROBABILITY OF ANNUAL HIGH FLO BASED ON PERIOD OF RECORD 1916-20, 1927-85

\begin{tabular}{|c|c|c|c|c|c|c|}
\hline $\begin{array}{l}\text { PERIOD } \\
\text { (CON- } \\
\text { SECU- }\end{array}$ & DIS & $\begin{array}{l}\text { GE, } \\
\text { INTEI } \\
\text { CEED }\end{array}$ & $\begin{array}{l}T^{3} / \mathrm{S} \\
\text { IN } \\
\text { PRO }\end{array}$ & $\begin{array}{l}\text { OR INI } \\
\text { ARS, } \\
\text { ILITY, }\end{array}$ & $\begin{array}{l}\text { ATED } 5 \\
\text { ANNUA } \\
\text { N PERC }\end{array}$ & RRE \\
\hline $\begin{array}{l}\text { TIVE } \\
\text { DAYS) }\end{array}$ & $\begin{array}{c}2 \\
508\end{array}$ & $\begin{array}{c}5 \\
208\end{array}$ & $\begin{array}{l}10 \\
108\end{array}$ & $\begin{array}{l}25 \\
48\end{array}$ & $\begin{array}{l}50 \\
28\end{array}$ & $\begin{array}{r}100 \\
18\end{array}$ \\
\hline $\begin{array}{r}1 \\
3 \\
7 \\
15 \\
30 \\
60 \\
90\end{array}$ & $\begin{array}{r}314 \\
239 \\
182 \\
139 \\
104 \\
74 \\
59\end{array}$ & $\begin{array}{l}590 \\
448 \\
353 \\
286 \\
222 \\
160 \\
127\end{array}$ & $\begin{array}{l}801 \\
599 \\
480 \\
398 \\
317 \\
232 \\
186\end{array}$ & $\begin{array}{r}1090 \\
798 \\
648 \\
549 \\
451 \\
338 \\
274\end{array}$ & $\begin{array}{r}1320 \\
946 \\
774 \\
665 \\
558 \\
426 \\
349\end{array}$ & $\begin{array}{r}1560 \\
1090 \\
900 \\
781 \\
670 \\
521 \\
431\end{array}$ \\
\hline
\end{tabular}

DURATION TABLE OF DAILY MEAN FLOW FOR PERIOD OF RECORD 1916-20, 1927-85 DISCHARGE, IN FT'3/S, THAT WAS EQUALED OR EXCEEDED FOR INDICATED PERCENT OF TIME

\begin{tabular}{|c|c|c|c|c|c|c|c|c|c|c|c|c|c|c|}
\hline 58 & 108 & 158 & 208 & 258 & 308 & 408 & 508 & 608 & 708 & 758 & 808 & 858 & 908 & 958 \\
\hline 147 & 86 & 56 & 39 & 30 & 25 & 18 & 13 & 9.1 & 5.8 & 4.5 & 3.5 & 2.7 & 1.9 & 1. \\
\hline
\end{tabular}


ARKANSAS RIVER BASIN

07217000 COYOTE CREEK BELOW BLACK LAKE, NM

LOCATION.--Lat $36^{\circ} 16^{\prime} 20^{\prime \prime}$, long $105^{\circ} 14^{\prime} 50^{\prime \prime}$, in NWt sec.33, T.14 N., R.16 E., on right bank 150 ft downstream from road crossing, a quarter of a mile downstream from Black Lake, 2 miles south of Black Lake Village, and 12 miles road crossing, a qua
south of Agua Fria.

DRAINAGE AREA. $--48 \mathrm{mi}^{2}$.

PERIOD OF RECORD.--December 1952 to September 1963 (discontinued).

GAGE.--Water-stage recorder. Altitude of gage is $8,450 \mathrm{ft}$ (from topographic map).

REMARKS.--Small amount of regulation possible at open flume in outlet from Black Lake. Diversions for irrigation for irrigation of several hundred acres above station. Surface waste from one small ditch may enter stream between station and Black Lake.

AVERAGE DISCHARGE. - -10 years $(1953-63), 4.62 \mathrm{ft}^{3} / \mathrm{s}, 3,340$ acre-ft/year.

EXTREMES FOR PERIOD OF RECORD.--Maximum discharge, $913 \mathrm{ft} / \mathrm{s}$ June 6,1958 (gage height, $4.70 \mathrm{ft}$ ), from rating curve extended above $110 \mathrm{ft}^{3} / \mathrm{s}$ on basis of slope-area measurement of peak flow; no flow at times.

STATISTICAL SUMMARIFS

MEAN MONTHLY AND MEAN ANNUAL DISCHARGES 1954-63

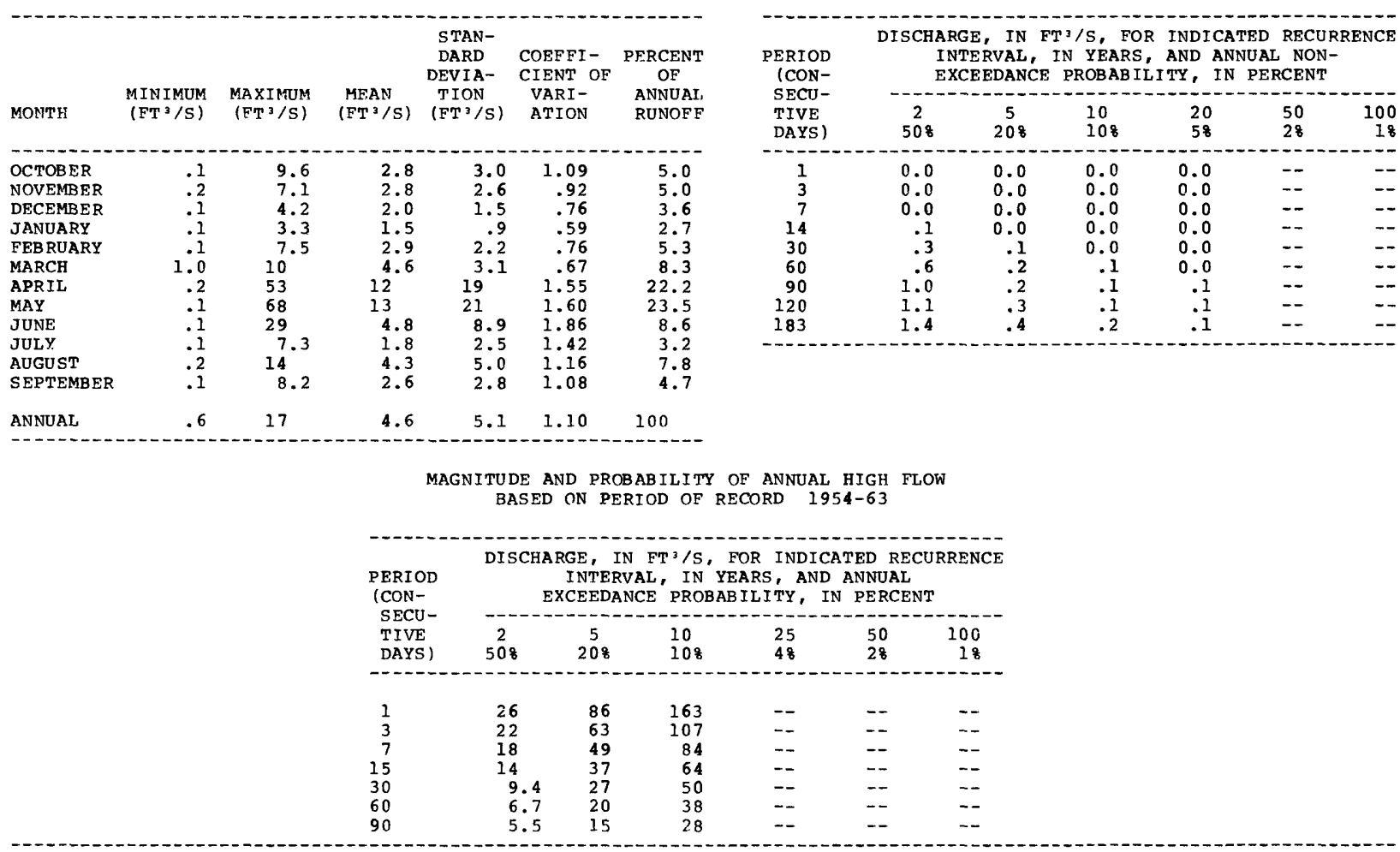

DURATION TABLE OF DAILY MEAN FLOW FOR PERIOD OF RECORD 1954-63

DISCHARGE, IN FT' ${ }^{3} / \mathrm{S}$, THAT WAS EQUALED OR EXCEEDED FOR INDICATED PERCENT OF TIME

\begin{tabular}{|c|c|c|c|c|c|c|c|c|c|c|c|c|c|c|}
\hline 58 & $10 \%$ & 158 & 208 & 258 & $30 \%$ & $40 \%$ & 508 & 608 & 708 & 758 & $80 \%$ & $85 \%$ & 908 & 958 \\
\hline 17 & 9.6 & 6.6 & 5.2 & 4.2 & 3.5 & 2.4 & 1.4 & 1.0 & .5 & .3 & .2 & 1 & .1 & .1 \\
\hline
\end{tabular}




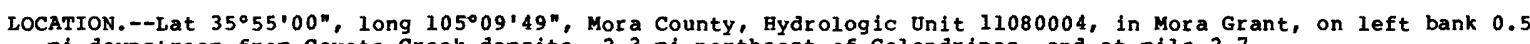
mi downstream from Coyote Creek damsite, $2.3 \mathrm{mi}$ northeast of Golondrinas, and at mile 2.7.

DRAINAGE AREA. $--215 \mathrm{mi}^{2}$.

PERIOD OF RECORD.--April 1928 to September 1930 (monthly discharge only, published in wSP 1311), October 1930 to current year.

REVISED RECORDS.--WSP 1281: 1939-40(M), 1941-42, 1945-47. WSP 1511: Drainage area.

GAGE.--Water-stage recorder. Elevation of gage is 6,785 ft above National Geodetic Vertical Datum of 1929 , from topographic map. Prior to Apr. 26, 1938, at site $0.4 \mathrm{mi}$ downstream at different datum (nonrecording gage prior to Apr. 20, 1929). Apr. 26, 1938 to Sept. 25, 1946, at site $139 \mathrm{ft}$ downstream at same datum.

REMÁRKS.--Diversions (including off-channel storage) for irrigation of about 4,000 acres upstream from station. AVERAGE DISCHARGE. --56 years (water years 1930-85), $11.7 \mathrm{ft} / \mathrm{s}, 8,480$ acre-ft/yr.

EXTREMES FOR PERIOD OF RECORD.--Maximum discharge, 4,050 $\mathrm{ft}^{3} / \mathrm{s}$, Aug. 17, 1961, gage height, $9.60 \mathrm{ft}$, from rating curve extended above $250 \mathrm{ft} / \mathrm{s}$ on basis of slope-area measurements at gage heights $5.54 \mathrm{ft}, 7.74 \mathrm{ft}$, and 9.60 $\mathrm{ft}$; maximum gage height, $10.1 \mathrm{ft}$, Aug. 30,1936 (site and datum then in use); no flow Aug. 4, 1945, Apr. 10, May 9. 10, 1956, Feb. 20, 1978 .

STATISTICAL SUMMARIES

MEAN MONTHLY AND MEAN ANNUAL DISCHARGES 1930-85

\begin{tabular}{|c|c|c|c|c|c|c|}
\hline MONTH & $\begin{array}{l}\text { MINIMUM } \\
(\mathrm{FT} 3 / \mathrm{S})\end{array}$ & $\begin{array}{c}\text { MAXIMUM } \\
\left(\mathrm{FT}^{3} / \mathrm{S}\right)\end{array}$ & $\begin{array}{l}\text { MEAN } \\
\left(\mathrm{FT}^{3} / \mathrm{S}\right)\end{array}$ & $\begin{array}{l}\text { STAN- } \\
\text { DARD } \\
\text { DEVIA- } \\
\text { TION } \\
\left(\mathrm{FT}^{3} / \mathrm{S}\right)\end{array}$ & $\begin{array}{l}\text { COEFFI- } \\
\text { CIENT OF } \\
\text { VARI- } \\
\text { ATION }\end{array}$ & $\begin{array}{c}\text { PERCENT } \\
\text { OF } \\
\text { ANNUAL } \\
\text { RUNOF F }\end{array}$ \\
\hline $\begin{array}{l}\text { OCTOBER } \\
\text { NOVEMBER } \\
\text { DECEMBER } \\
\text { JANUARY } \\
\text { FEBRUARY } \\
\text { MARCE } \\
\text { APRIL } \\
\text { MAY } \\
\text { JUNE } \\
\text { JULY } \\
\text { AUGUST } \\
\text { SEPTEMBER }\end{array}$ & $\begin{array}{r}.7 \\
1.7 \\
1.6 \\
1.6 \\
1.1 \\
1.0 \\
.3 \\
.5 \\
.2 \\
.8 \\
.8 \\
.7\end{array}$ & $\begin{array}{r}80 \\
54 \\
24 \\
16 \\
19 \\
44 \\
191 \\
219 \\
130 \\
67 \\
135 \\
47\end{array}$ & $\begin{array}{c}8.5 \\
7.7 \\
7.1 \\
6.8 \\
7.2 \\
8.3 \\
19 \\
29 \\
13 \\
8.9 \\
15 \\
9.5\end{array}$ & $\begin{array}{l}13 \\
8.5 \\
4.3 \\
3.4 \\
3.9 \\
7.7 \\
38 \\
45 \\
25 \\
12 \\
20 \\
9.7\end{array}$ & $\begin{array}{r}1.57 \\
1.10 \\
.61 \\
.50 \\
.54 \\
.92 \\
2.00 \\
1.59 \\
1.92 \\
1.29 \\
1.34 \\
1.03\end{array}$ & $\begin{array}{r}6.1 \\
5.5 \\
5.1 \\
4.9 \\
5.1 \\
6.0 \\
13.6 \\
20.4 \\
9.3 \\
6.4 \\
10.8 \\
6.8\end{array}$ \\
\hline ANNUAL & 2.3 & 53 & 12 & 11 & .95 & 100 \\
\hline
\end{tabular}

MAGNITUDE AND PROBABILITY OF ANNUAL LOW FLOM BASED ON PERIOD OF RECORD 1931-85

\begin{tabular}{|c|c|c|c|c|c|c|}
\hline \multirow{2}{*}{$\begin{array}{l}\text { PERIOD } \\
\text { (CON- } \\
\text { SECU- } \\
\text { TIVE } \\
\text { DAYS) }\end{array}$} & \multicolumn{6}{|c|}{$\begin{array}{l}\text { DISCHARGE, IN FT3/S, FOR INDICATED RECURRENCE } \\
\text { INTERVAL, IN YEARS, AND ANNUAL NON- } \\
\text { EXCEEDANCE PROBABILITY, IN PERCENT }\end{array}$} \\
\hline & $\begin{array}{c}2 \\
508\end{array}$ & $\begin{array}{c}5 \\
208\end{array}$ & $\begin{array}{l}10 \\
108\end{array}$ & $\begin{array}{l}20 \\
58\end{array}$ & $\begin{array}{l}50 \\
28\end{array}$ & $\begin{array}{r}100 \\
18\end{array}$ \\
\hline $\begin{array}{r}1 \\
3 \\
7 \\
14 \\
30 \\
60 \\
90 \\
120 \\
183\end{array}$ & $\begin{array}{r}.5 \\
.6 \\
.7 \\
.9 \\
1.3 \\
2.1 \\
2.7 \\
3.3 \\
4.6\end{array}$ & $\begin{array}{r}.2 \\
.2 \\
.3 \\
.4 \\
.6 \\
1.0 \\
1.4 \\
1.8 \\
2.8\end{array}$ & $\begin{array}{r}.1 \\
.1 \\
.2 \\
.3 \\
.4 \\
.7 \\
.9 \\
1.3 \\
2.2\end{array}$ & $\begin{array}{r}.1 \\
.1 \\
.2 \\
.2 \\
.3 \\
.5 \\
.7 \\
1.0 \\
1.8\end{array}$ & $\begin{array}{r}0.0 \\
.1 \\
.1 \\
.2 \\
.2 \\
.4 \\
.5 \\
.8 \\
1.4\end{array}$ & $\begin{array}{r}.1 \\
.2 \\
.2 \\
.3 \\
.4 \\
.6 \\
1.3\end{array}$ \\
\hline
\end{tabular}

MAGNITUDE AND PROBABILITY OF ANNUAL HIGH FLOW BASED ON PERIOD OF RECORD $1930-85$

\begin{tabular}{|c|c|c|c|c|c|c|}
\hline \multirow{2}{*}{$\begin{array}{l}\text { PERIOD } \\
\text { (CON- } \\
\text { SECU- } \\
\text { TIVE } \\
\text { DAYS) }\end{array}$} & \multicolumn{6}{|c|}{$\begin{array}{l}\text { DISCBARGE, IN FT3/S, FOR INDICATED RECURRENCE } \\
\text { INTERVAL, IN YEARS, AND ANNUAL } \\
\text { EXCEEDANCE' PROBAB ILITY, IN PERCENT }\end{array}$} \\
\hline & $\stackrel{2}{508}$ & $\begin{array}{c}5 \\
208\end{array}$ & $\begin{array}{l}10 \\
108\end{array}$ & $\begin{array}{l}25 \\
48\end{array}$ & $\begin{array}{l}50 \\
28\end{array}$ & $\begin{array}{r}100 \\
18\end{array}$ \\
\hline $\begin{array}{r}1 \\
3 \\
7 \\
15 \\
30 \\
60 \\
90\end{array}$ & $\begin{array}{r}122 \\
77 \\
54 \\
38 \\
29 \\
20 \\
16\end{array}$ & $\begin{array}{r}262 \\
180 \\
130 \\
95 \\
69 \\
48 \\
37\end{array}$ & $\begin{array}{r}39( \\
286 \\
211 \\
156 \\
113 \\
77 \\
58\end{array}$ & $\begin{array}{r}595 \\
477 \\
358 \\
268 \\
194 \\
132 \\
99\end{array}$ & $\begin{array}{l}781 \\
670 \\
508 \\
385 \\
278 \\
189 \\
140\end{array}$ & $\begin{array}{l}995 \\
915 \\
699 \\
535 \\
386 \\
264 \\
195\end{array}$ \\
\hline
\end{tabular}

DURATION TABLE OF DAILY MEAN FLOW FOR PERIOD OF RECORD 1930-85

DISCHARGE, IN FT ${ }^{3} / \mathrm{S}$, THAT WAS EQUALED OR EXCEEDED FOR INDICATED PERCENT OF TIME

\begin{tabular}{|c|c|c|c|c|c|c|c|c|c|c|c|c|c|c|}
\hline 58 & 108 & 158 & 208 & 258 & 308 & 408 & 508 & 608 & 708 & 758 & 808 & 858 & 908 & 958 \\
\hline 42 & 22 & 16 & 12 & 10 & 8.5 & 6.5 & 5.1 & 3.8 & 2.8 & 2.3 & 1.9 & 1.5 & 1.1 & \\
\hline
\end{tabular}


ARKANSAS RIVER BASIN

07220000 SAPELLO RIVER AT SAPELLO, NM

LOCATION, - Lat $35^{\circ} 46^{\prime} 11^{\prime \prime}$, long $105^{\circ} 15^{\prime} 05^{\prime \prime}$, San Miguel County, in Mora Grant, on downstream end of bridge pier nearest left bank, on State Highway 3, in Sapello, $0.5 \mathrm{mi}$ downstream from Manuelitas Creek, and at mile 20.3 .

DRAINAGE AREA. $--132 \mathrm{mi}^{2}$.

PERIOD OF RECORD. - May to October 1915, January 1916 to November 1918, February 1919 to May 1921 , JuIy to September 1921. July 1956 to December 1973 (discontinued). Monthly discharge only for some periods, published in wSP 1311. Gage heights and discharge measurements published under same name for August 1903 to March 1904 are for a site above Manuelitas Creek, and are not equivalent.

REVISED RECORDS, --WSP 1511: Drainage area. WSP 1731: $1956(\mathrm{M})$.

GAGE.--Water-stage recorder. Altitude of gage is 6,910 ft from topographic map. May 1915 to September 1921 , nonrecording gage at site $300 \mathrm{ft}$ upstream at different datum.

REMARRs.--Diversions above station for irrigation of about 4,200 acres. Sapello Canal diverts from right bank $500 \mathrm{ft}$ above station.

AVERAGE DISCHARGE, - -18 years $(1918,1957-73), 21.0 \mathrm{ft} / \mathrm{s}, 15,210 \mathrm{scre-ft/yr}$.

EXTREMES FOR PERIOD OF RECORD.--Maximum discharge, 6,420 ft'/s Aug. 5, 1966 (gage height, $7.50 \mathrm{ft}$ ), from rating curve extended above $350 \mathrm{ft} / \mathrm{s}$ on basis of computation of flow over dam at gage height $7.40 \mathrm{ft}$; maximum gage height, 10.6 ft Aug. 8, 1972; no flow at times.

The flood of June 11,1913 , reached a peak discharge of $11,400 \mathrm{ft}^{3} / \mathrm{s}$ at a site $3 \mathrm{mi}$ downstream.

STATISTICAL SUMMARIES

MEAN MONTHLY AND MEAN ANNUAL DISCHARGES 1918, 1957-73

MAGNITUDE AND PROBABILITY OF ANNUAL LOW FLOW BASED ON PERIOD OF RECORD 1958-73

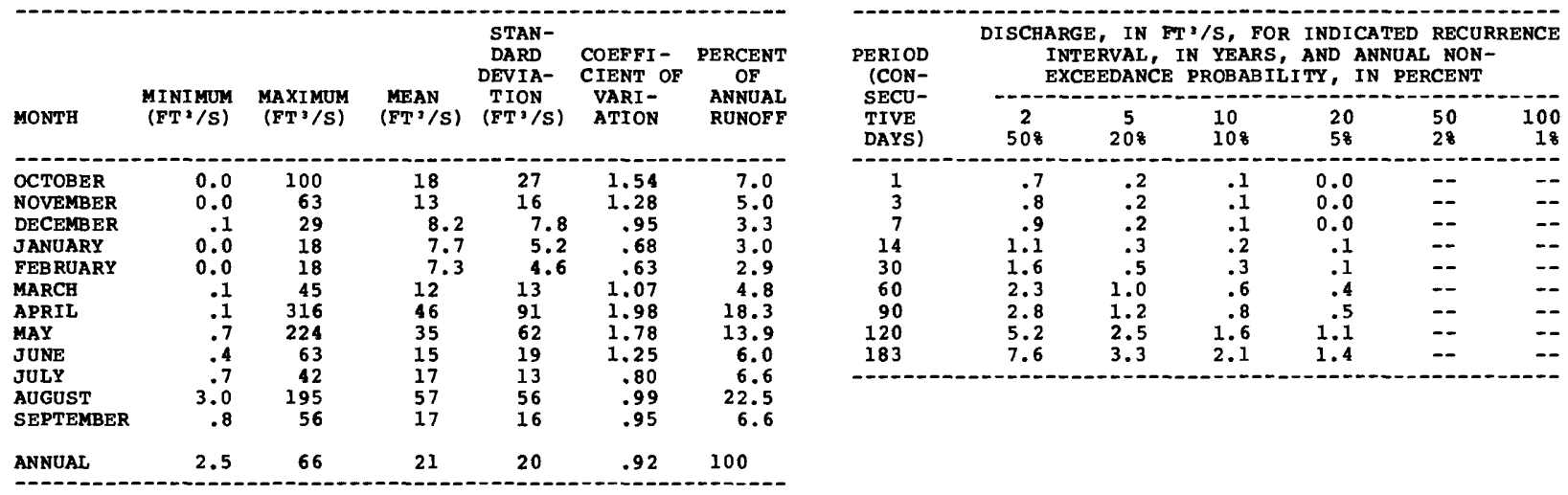

MAGNITUDE AND PROBABILITY OF ANNUAL HIGH FLOW BASED ON PERIOD OF RECORD 1918, 1957-73

\begin{tabular}{|c|c|c|c|c|c|c|}
\hline \multirow{2}{*}{$\begin{array}{l}\text { PERIOD } \\
\text { (CON- } \\
\text { SECU- } \\
\text { TIVE } \\
\text { DAYS) }\end{array}$} & \multicolumn{6}{|c|}{$\begin{array}{l}\text { DISCHARGE, IN FT }{ }^{3 / S} \text {, FOR INDICATED RECURRENCE } \\
\text { INTERVAL, IN YEARS, AND ANNUAL } \\
\text { EXCEEDANCE PROBABILI TY, IN PERCENT }\end{array}$} \\
\hline & $\begin{array}{c}2 \\
508\end{array}$ & $\begin{array}{c}5 \\
208\end{array}$ & $\begin{array}{l}10 \\
108\end{array}$ & $\begin{array}{l}25 \\
48\end{array}$ & $\begin{array}{l}50 \\
28\end{array}$ & $\begin{array}{r}100 \\
18\end{array}$ \\
\hline $\begin{array}{r}1 \\
3 \\
7 \\
15 \\
30 \\
60 \\
90\end{array}$ & $\begin{array}{r}317 \\
213 \\
140 \\
95 \\
70 \\
48 \\
36\end{array}$ & $\begin{array}{r}765 \\
517 \\
344 \\
234 \\
169 \\
112 \\
83\end{array}$ & $\begin{array}{r}1160 \\
770 \\
518 \\
353 \\
255 \\
170 \\
124\end{array}$ & $\begin{array}{r}1740 \\
1130 \\
767 \\
524 \\
381 \\
257 \\
188\end{array}$ & $\begin{array}{l}-- \\
-- \\
-- \\
-- \\
--\end{array}$ & $\begin{array}{l}-- \\
-- \\
-- \\
-- \\
-- \\
--\end{array}$ \\
\hline
\end{tabular}

DURATION TABLE OF DAILY MEAN FLOW FOR PERIOD OF RECORD 1918, 1957-73 DISCHARGE, IN FT'/S, THAT WAS EQUALED OR EXCEEDED FOR INDICATED PERCENT OF TIME

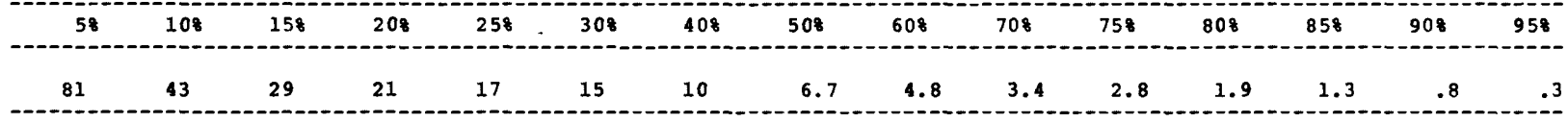


ARKANSAS RIVER BASIN

07221000 MORA RIVER NEAR SHOEMAKER, NM

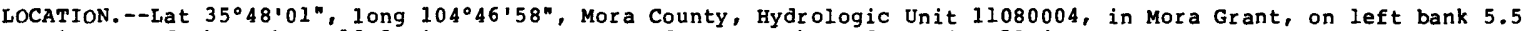
$\mathrm{mi}$ east of Shoemaker, $12.3 \mathrm{mi}$ upstream from Pedroso Creek, and at mile 39.4.

DRAINAGE AREA. - -1,104 $\mathrm{mi}^{2}$, of which $71 \mathrm{mi}^{2}$ is probably noncontributing.

PERIOD OF RECORD.--October 1914 to JuIy 1915, October 1915 to August 1918, May 1919 to July 1924 , September to November 1924, March to July 1925, June 1927 to current year. Prior to October 1930 monthly discharge only, published in WSP 1311.

REVISED RECORDS.--WSP 1117: Drainage area, WSP 1281: 1931(M), 1933-1934(M), 1937(M), 1938(P), 1939-40(M), 1941-42(P). WSP 1731: 1921, 1928, 1951(M). WRD NM-75-1: 1974. WRD NK-78-1: 1977 .

GAGE.--Water-stage recorder. Elevation of gage is 6,145 above National Geodetic vertical Datum of 1929 , from topographic map. Prior to oct. 10, 1934, at site 2,000 ft upstream at different datum.

REMARKS.--Diversions for irrigation of about 26,000 acres upstream from station. off-channel lakes make it possible to divert and store water during non-irrigation season.

AVERAGE DISCHARGE.--63 years (water year 1920-24, 1928-85), 55.3 ft $3 / \mathrm{s}, 40,060$ acre-ft/yr.

EXTREMES FOR PERIOD OF RECORD.--Maximum discharge, 15,000 ft $/ \mathrm{s}$, June 3, 1948, gage height, $12.79 \mathrm{ft}$, from rating curve extended above $2,800 \mathrm{ft}^{3} / \mathrm{s}$ on basis of slope-area measurements at gage heights $10.09 \mathrm{ft}$ and $12.79 \mathrm{ft}$; no flow at times.

EXTREMES OUTSIDE PERIOD OF RECORD.--Floods of Sept. 29, 1904, and June 11, 1913, probably exceeded 30,000 ft $3 / \mathrm{s}$,

STATISTICAL SUMMARIES

MEAN MONTHLY AND MEAN ANNUAL DISCHARGES 1920-24 1928-85

\begin{tabular}{|c|c|c|c|c|c|c|}
\hline MONTH & $\begin{array}{l}\text { MINIMUM } \\
\left(\mathrm{FT}^{3} / \mathrm{S}\right)\end{array}$ & $\begin{array}{l}\text { MAXIMUM } \\
\left(\mathrm{FT}^{3} / \mathrm{S}\right)\end{array}$ & $\begin{array}{l}\text { MEAN } \\
\left(\mathrm{FT}^{3} / \mathrm{S}\right)\end{array}$ & $\begin{array}{l}\text { STAN- } \\
\text { DARD } \\
\text { DEVIA- } \\
\text { TION } \\
\left(\mathrm{FT}^{3} / \mathrm{S}\right)\end{array}$ & $\begin{array}{l}\text { COEFFI- } \\
\text { CIENT OF } \\
\text { VARI- } \\
\text { ATION }\end{array}$ & $\begin{array}{c}\text { PERCENT } \\
\text { OF } \\
\text { ANNUAL } \\
\text { RUNOF F }\end{array}$ \\
\hline $\begin{array}{l}\text { OCTOBER } \\
\text { NOVEMBER } \\
\text { DECEMBER } \\
\text { JANUARY } \\
\text { FEBRUARY } \\
\text { MARCH } \\
\text { APRIL } \\
\text { MAY } \\
\text { JUNE } \\
\text { JULY } \\
\text { AUGUST } \\
\text { SEPTEMBER }\end{array}$ & $\begin{array}{r}0.0 \\
.3 \\
.6 \\
1.0 \\
.8 \\
.6 \\
.3 \\
1.6 \\
.4 \\
.3 \\
.1 \\
0.0\end{array}$ & $\begin{array}{r}326 \\
212 \\
134 \\
94 \\
62 \\
184 \\
842 \\
1437 \\
937 \\
700 \\
587 \\
359\end{array}$ & $\begin{array}{r}37 \\
26 \\
26 \\
26 \\
22 \\
22 \\
72 \\
124 \\
102 \\
59 \\
91 \\
56\end{array}$ & $\begin{array}{r}59 \\
36 \\
25 \\
20 \\
17 \\
32 \\
175 \\
231 \\
175 \\
105 \\
115 \\
75\end{array}$ & $\begin{array}{l}1.59 \\
1.35 \\
.99 \\
.76 \\
.77 \\
1.49 \\
2.44 \\
1.86 \\
1.72 \\
1.79 \\
1.26 \\
1.34\end{array}$ & $\begin{array}{r}5.6 \\
4.0 \\
3.9 \\
3.9 \\
3.3 \\
3.3 \\
10.8 \\
18.8 \\
15.4 \\
8.9 \\
13.8 \\
8.5\end{array}$ \\
\hline NNOAL, & 2.7 & 302 & 55 & 60 & 1.08 & 100 \\
\hline
\end{tabular}

MAGNITUDE AND PROBABILITY OF ANNUAL LOW FLOW BASED ON PERIOD OF RECORD 1921-24, 1929-85

\begin{tabular}{|c|c|c|c|c|c|c|}
\hline \multirow{2}{*}{$\begin{array}{l}\text { PERIOD } \\
\text { (CON- } \\
\text { SECU- } \\
\text { TIVE } \\
\text { DAYS) }\end{array}$} & \multicolumn{6}{|c|}{$\begin{array}{l}\text { DISCHARGE, IN FT3/S, FOR INDICATED RECURRENCE } \\
\text { INTERVAL, IN YEARS, AND ANNUAL NON- } \\
\text { EXCEEDANCE PROBABILITY, IN PERCENT }\end{array}$} \\
\hline & $\stackrel{2}{508}$ & $\begin{array}{c}5 \\
208\end{array}$ & $\begin{array}{l}10 \\
108\end{array}$ & $\begin{array}{l}20 \\
58\end{array}$ & $\begin{array}{l}50 \\
28\end{array}$ & $\begin{array}{r}100 \\
18\end{array}$ \\
\hline $\begin{array}{r}1 \\
3 \\
7 \\
14 \\
30 \\
60 \\
90 \\
120 \\
183\end{array}$ & $\begin{array}{r}1.2 \\
1.3 \\
1.4 \\
1.6 \\
2.4 \\
4.6 \\
7.1 \\
10 \\
14\end{array}$ & $\begin{array}{r}.3 \\
.3 \\
.4 \\
.5 \\
.7 \\
1.3 \\
2.2 \\
3.5 \\
5.2\end{array}$ & $\begin{array}{r}0.0 \\
0.0 \\
.1 \\
.1 \\
.3 \\
.6 \\
1.1 \\
1.9 \\
2.9\end{array}$ & $\begin{array}{r}0.0 \\
0.0 \\
0.0 \\
0.0 \\
0.0 \\
.3 \\
.6 \\
1.1 \\
1.7\end{array}$ & $\begin{array}{l}0.0 \\
0.0 \\
0.0 \\
0.0 \\
0.0 \\
.1 \\
.3 \\
.5 \\
.9\end{array}$ & $\begin{array}{l}0.0 \\
0.0 \\
0.0 \\
0.0 \\
0.0 \\
.1 \\
.2 \\
.3 \\
.6\end{array}$ \\
\hline
\end{tabular}

MAGNITUDE AND PROBABILITY OF ANNUAL HIGH FLOW BASED ON PERIOD OF RECORD 1920-24, 1928-85

\begin{tabular}{|c|c|c|c|c|c|c|}
\hline \multirow{2}{*}{$\begin{array}{l}\text { PERIOD } \\
\text { (CON- } \\
\text { SECU- } \\
\text { TIVE } \\
\text { DAYS) }\end{array}$} & \multicolumn{6}{|c|}{$\begin{array}{c}\text { DISCHARGE, IN FT } 3 \text { /S, FOR INDICATED RECURRENCE } \\
\text { INTERVAL, IN YEARS, AND ANNUAL } \\
\text { EXCEEDANCE PROBABILITY, IN PERCENT }\end{array}$} \\
\hline & $\begin{array}{c}2 \\
508\end{array}$ & $\begin{array}{c}5 \\
208\end{array}$ & $\begin{array}{l}10 \\
108\end{array}$ & $\begin{array}{l}25 \\
48\end{array}$ & $\begin{array}{l}50 \\
28\end{array}$ & $\begin{array}{r}100 \\
18\end{array}$ \\
\hline $\begin{array}{r}1 \\
3 \\
7 \\
15 \\
30 \\
60 \\
90\end{array}$ & $\begin{array}{r}786 \\
499 \\
333 \\
231 \\
168 \\
116 \\
89\end{array}$ & $\begin{array}{r}1840 \\
1160 \\
786 \\
563 \\
408 \\
283 \\
216\end{array}$ & $\begin{array}{r}2900 \\
1790 \\
1200 \\
861 \\
621 \\
436 \\
336\end{array}$ & $\begin{array}{r}4730 \\
2850 \\
1850 \\
1310 \\
941 \\
678 \\
533\end{array}$ & $\begin{array}{r}6500 \\
3850 \\
2430 \\
1700 \\
1210 \\
890 \\
712\end{array}$ & $\begin{array}{r}8690 \\
5050 \\
3070 \\
2120 \\
1500 \\
1130 \\
920\end{array}$ \\
\hline
\end{tabular}

DURATION TABLE OF DAILY MEAN FLOW FOR PERIOD OF RECORD 1920-24, 1928-85 DISCHARGE, IN FT'/S, THAT WAS EQUALED OR EXCEEDED FOR INDICATED PERCENT OF TIME

\begin{tabular}{|c|c|c|c|c|c|c|c|c|c|c|c|c|c|c|}
\hline 58 & 108 & 158 & 208 & 258 & 308 & 408 & 508 & 608 & 708 & 758 & 808 & 858 & 908 & 958 \\
\hline 233 & 118 & 77 & 58 & 45 & 36 & 23 & 14 & 7.7 & 4.4 & 3.5 & 2.7 & 2.1 & 1.6 & . 8 \\
\hline
\end{tabular}


LOCATION.--Lat $35^{\circ} 39^{\circ} 08^{\prime \prime}$, long $104^{\circ} 22^{\prime} 39^{\prime \prime}$, in SWł sec.34, T.17 N., R.24 E., San Miguel County, Hydrologic Unit 11080003 , on right bank $1,000 \mathrm{ft}$ downstream from bridge on state Highway $65,0.9 \mathrm{mi}$ upstream from Lagartija Creek, $3.2 \mathrm{mi}$ northeast of Sanchez, $10 \mathrm{mi}$ downstream from Mora River, $25 \mathrm{mi}$ southwest of Mosquero, and at mile 777.0.

DRAINAGE AREA. - -6,015 $\mathrm{mi}^{2}$, of which $303 \mathrm{mi}^{2}$ is probably noncontributing.

PERIOD OF RECORD.--May 1912 to December 1914, October 1935 to current year. Monthly discharge only for some periods, published in WSP 1311.

REVISED RECORDS.--WSP 1177: Drainage area. WSP 1281: 1939, 1940(P), 1942, 1946. WSP 1731: 1956-57(M). WDR NM-82: $1965(\mathrm{M}), 1979(\mathrm{M})$. The revised figures of discharge for September 1942, as published in wSP 1281, supersede those published in WSP 1311.

GAGE.- Water-stage recorder. Elevation of gage is 4,495 ft above National Geodetic Vertical Datum of 1929 , from topographic map. See WSP 2121 for history of changes prior to November 1966 . Supplemental water-stage recorder at site $0.6 \mathrm{mi}$ upstream used at various times since 1966 .

REMARKS.--Diversions for irrigation of about 56,000 acres upstream from station.

AVERAGE DISCHARGE.--51 years (water years 1913-14, 1937-85), $187 \mathrm{ft} 3 / \mathrm{s}, 135,480 \mathrm{acre}-\mathrm{ft} / \mathrm{yr}$.

EXTREMES FOR PERIOD OF RECORD.--Maximum discharge, 145,000 $\mathrm{ft}^{3} / \mathrm{s}$, June 18, 1965, gage height, about $36.6 \mathrm{ft}$, from floodmarks, present site and datum, from rating curve extended above $91,000 \mathrm{ft}^{3 / \mathrm{s}}$ on basis of slope-area measurement of peak flow; no flow at times.

EXTREMES OUTSIDE PERIOD OF RECORD.--The flood of Sept. 29, or 30,1904 , probably exceeded $100,000 \mathrm{ft} 3 / \mathrm{s}$, but is believed to have been less than the peak of June $18,1965$.

STATISTICAL SUMMARIES

MEAN MONTHLY AND MEAN ANNUAL DISChaRGeS $\begin{gathered}1913-14, \\ 1937-85\end{gathered}$

\begin{tabular}{|c|c|c|c|c|c|c|}
\hline MONTH & $\begin{array}{c}\text { MINIMUM } \\
\left(\mathrm{FT}^{3} / \mathrm{S}\right)\end{array}$ & $\begin{array}{c}\text { MAXIMUM } \\
\left(\mathrm{FT}^{3} / \mathrm{S}\right)\end{array}$ & $\begin{array}{l}\text { MEAN } \\
\left(\mathrm{FT}^{3} / \mathrm{S}\right)\end{array}$ & $\begin{array}{c}\text { STAN- } \\
\text { DARD } \\
\text { DEVIA- } \\
\text { TION } \\
\left(\mathrm{FT}^{3} / \mathrm{S}\right)\end{array}$ & $\begin{array}{l}\text { COEFFI- } \\
\text { CIENT OF } \\
\text { VARI- } \\
\text { ATION }\end{array}$ & $\begin{array}{c}\text { PERCENT } \\
\text { OF } \\
\text { ANNUAL } \\
\text { RUNOFF }\end{array}$ \\
\hline $\begin{array}{l}\text { OCTOBER } \\
\text { NOVEMBER } \\
\text { DECEMBER } \\
\text { JANUARY } \\
\text { FEBRUARY } \\
\text { MARCH } \\
\text { APRIL } \\
\text { MAY } \\
\text { JUNE } \\
\text { JULY } \\
\text { AUGUST } \\
\text { SEPTEMBER }\end{array}$ & $\begin{array}{l}0.0 \\
1.4 \\
2.0 \\
1.4 \\
1.5 \\
.7 \\
0.0 \\
0.0 \\
0.0 \\
0.0 \\
8.4 \\
1.0\end{array}$ & $\begin{array}{r}870 \\
506 \\
252 \\
183 \\
363 \\
261 \\
5573 \\
4721 \\
4260 \\
1129 \\
1173 \\
4079\end{array}$ & $\begin{array}{r}115 \\
52 \\
44 \\
47 \\
55 \\
46 \\
210 \\
395 \\
409 \\
250 \\
333 \\
255\end{array}$ & $\begin{array}{r}210 \\
83 \\
45 \\
39 \\
61 \\
58 \\
788 \\
828 \\
841 \\
246 \\
275 \\
610\end{array}$ & $\begin{array}{r}1.83 \\
1.61 \\
1.03 \\
.85 \\
1.10 \\
1.27 \\
3.75 \\
2.10 \\
2.06 \\
.98 \\
.83 \\
2.39\end{array}$ & $\begin{array}{l}5.2 \\
2.3 \\
2.0 \\
2.1 \\
2.5 \\
2.1 \\
9.5 \\
17.9 \\
18.5 \\
11.3 \\
15.1 \\
11.5\end{array}$ \\
\hline NNUAL & 20 & 1191 & 187 & 210 & 1.12 & 100 \\
\hline
\end{tabular}

MAGNITUDE AND PROBABILITY OF ANNUAL LOW FLOW BASED ON PERIOD OF RECORD 1914, $1937-85$

\begin{tabular}{|c|c|c|c|c|c|c|}
\hline \multirow{2}{*}{$\begin{array}{l}\text { PERIOD } \\
\text { (CON- } \\
\text { SECU- } \\
\text { TIVE } \\
\text { DAYS) }\end{array}$} & \multicolumn{6}{|c|}{$\begin{array}{l}\text { DISCHARGE, IN FT33/S, FOR INDICATED RECURRENCE } \\
\text { INTERVAL, IN YEARS, AND ANNUAL NON- } \\
\text { EXCEEDANCE PROBABILITY, IN PERCENT }\end{array}$} \\
\hline & $\begin{array}{c}2 \\
508\end{array}$ & $\begin{array}{c}5 \\
208\end{array}$ & $\begin{array}{l}10 \\
108\end{array}$ & $\begin{array}{l}20 \\
58\end{array}$ & $\begin{array}{l}50 \\
28\end{array}$ & $\begin{array}{r}100 \\
18\end{array}$ \\
\hline $\begin{array}{r}1 \\
3 \\
7 \\
14 \\
30 \\
60 \\
90 \\
120 \\
183\end{array}$ & $\begin{array}{r}.5 \\
.8 \\
1.0 \\
1.5 \\
4.2 \\
12 \\
19 \\
25 \\
33\end{array}$ & $\begin{array}{r}0.0 \\
0.0 \\
0.0 \\
0.0 \\
.5 \\
2.6 \\
6.0 \\
9.6 \\
14\end{array}$ & $\begin{array}{l}0.0 \\
0.0 \\
0.0 \\
0.0 \\
0.0 \\
.9 \\
3.0 \\
5.4 \\
8.4\end{array}$ & $\begin{array}{r}0.0 \\
0.0 \\
0.0 \\
0.0 \\
0.0 \\
.3 \\
1.5 \\
3.3 \\
5.6\end{array}$ & $\begin{array}{r}0.0 \\
0.0 \\
0.0 \\
0.0 \\
0.0 \\
0.0 \\
.7 \\
1.8 \\
3.5\end{array}$ & $\begin{array}{l}0.0 \\
0.0 \\
0.0 \\
.4 \\
1.2 \\
2.6\end{array}$ \\
\hline
\end{tabular}

MAGNITUDE AND PROBABILITY OF ANNUAL HIGH FLOM BASED ON PERIOD OF RECORD 1913-14, 1937-85

\begin{tabular}{|c|c|c|c|c|c|c|}
\hline $\begin{array}{l}\text { PERIOD } \\
\text { (CON- } \\
\text { SECU - }\end{array}$ & DIS & $\begin{array}{l}\text { GE, } \\
\text { INTE } \\
\text { CEED }\end{array}$ & $\begin{array}{l}\text { FT'/S } \\
L^{\prime}, I N \\
\text { E PROB }\end{array}$ & $\begin{array}{l}\text { OR IN } \\
\text { ARS, } \\
\text { ILITY }\end{array}$ & $\begin{array}{l}\text { CATED } \\
\text { IN ANNU } \\
\text { IN PER }\end{array}$ & URRENC \\
\hline $\begin{array}{l}\text { TIVE } \\
\text { DAYS) }\end{array}$ & $\begin{array}{c}2 \\
508\end{array}$ & $\begin{array}{c}5 \\
208\end{array}$ & $\begin{array}{l}10 \\
108\end{array}$ & $\begin{array}{l}25 \\
48\end{array}$ & $\begin{array}{l}50 \\
28\end{array}$ & $\begin{array}{r}100 \\
18\end{array}$ \\
\hline $\begin{array}{r}1 \\
3 \\
7 \\
15 \\
30 \\
60 \\
90\end{array}$ & $\begin{array}{r}3230 \\
2080 \\
1300 \\
881 \\
639 \\
451 \\
348\end{array}$ & $\begin{array}{r}9120 \\
5620 \\
3360 \\
2170 \\
1500 \\
1010 \\
769\end{array}$ & $\begin{array}{r}16700 \\
10100 \\
5840 \\
3570 \\
2360 \\
1520 \\
1150\end{array}$ & $\begin{array}{r}33600 \\
19900 \\
11000 \\
6220 \\
3850 \\
2340 \\
1750\end{array}$ & $\begin{array}{r}54200 \\
31600 \\
16900 \\
9020 \\
5280 \\
3080 \\
2290\end{array}$ & $\begin{array}{r}84900 \\
49100 \\
25400 \\
12700 \\
7050 \\
3930 \\
2900\end{array}$ \\
\hline
\end{tabular}

DURATION TABLE OF DAILY MEAN FLOW FOR PERIOD OF RECORD 1913-14, 1937-85 DISCHARGE, IN FT'3/S, THAT WAS EOUALED OR EXCEEDED FOR INDICATED PERCENT OF TIME

\begin{tabular}{|c|c|c|c|c|c|c|c|c|c|c|c|c|c|c|}
\hline 58 & 108 & 158 & 208 & 258 & 308 & 408 & 508 & 608 & 708 & 758 & 808 & 858 & 908 & 958 \\
\hline 760 & 344 & 209 & 140 & 104 & 81 & 53 & 36 & 25 & 16 & 12 & 8.7 & 5.8 & 3.2 & 9 \\
\hline
\end{tabular}


ARKANSAS RIVER BASIN

07222500 CONCHAS RIVER AT VARTADERO, NM

LOCATION.--Lat $35^{\circ} 24^{\prime} 10^{\prime \prime}$, long $104^{\circ} 26^{\prime} 35^{\prime \prime}$, in NEłNEł sec.36, T.14 N., R.23 E., San Miguel County, Hydrologic Unit 11080005 , on left bank $1.5 \mathrm{mi}$ northeast of variadero, $14 \mathrm{mi}$ west of Conchas Dam, and at $\mathrm{mile} 15.0$.

DRAINAGE AREA. $--523 \mathrm{mi}^{2}$, of which $130 \mathrm{mi}^{2}$ is probably noncontributing.

PERIOD OF RECORD.--October 1936 to current year.

REVISED RECORDS.--WSP 1281: 1937-39, 1941-47.

GAGE.--Water-stage recorder. Elevation of gage is 4,390 ft above National Geodetic vertical Datum of 1929 , from topographic map. Prior to Mar. 30, 1942, at site $1.5 \mathrm{mi}$ upstream at different datum. Mar. 30 , 1942 to May 18, 1950, at present site at datum $0.5 \mathrm{ft}$ higher.

REMARKS.--Diversions for irrigation of about 300 acres upstream from station.

AVERAGE DISCHARGE.--49 years (water years 1937-85), $14.1 \mathrm{ft} / \mathrm{s}, 10,220 \mathrm{acre}-\mathrm{ft} / \mathrm{yr}$.

EXTREMES FOR PERIOD OF RECORD.--Maximum discharge, 44,000 ft $3 / \mathrm{s}$, Sept. 1, 1942, gage height, $19.96 \mathrm{ft}$, present datum, from rating curve extended above $760 \mathrm{ft}^{3} / \mathrm{s}$ on basis of slope-area measurements at gage heights 10.5 ft and $19.96 \mathrm{ft}$, present datum; no flow many days.

STATISTICAL SUMMARIES

MEAN MONTHLY AND MEAN ANNUAL DISCHARGES 1937-85

\begin{tabular}{|c|c|c|c|c|c|c|}
\hline MONTH & $\begin{array}{c}\text { MINIMUM } \\
\left(\mathrm{FT}^{3} / \mathrm{S}\right)\end{array}$ & $\begin{array}{c}\text { MAXIMUM } \\
\left(\mathrm{FT}^{3} / \mathrm{S}\right)\end{array}$ & $\begin{array}{l}\text { MEAN } \\
\left(\mathrm{FT}^{3} / \mathrm{S}\right)\end{array}$ & $\begin{array}{l}\text { STAN- } \\
\text { DARD } \\
\text { DEVIA- } \\
\text { TION } \\
(\text { FT'/S) }\end{array}$ & $\begin{array}{l}\text { COEFFI- } \\
\text { CIENT OF } \\
\text { VARI- } \\
\text { ATION }\end{array}$ & $\begin{array}{c}\text { PERCENT } \\
\text { OF } \\
\text { ANNUAL } \\
\text { RUNOFF }\end{array}$ \\
\hline OCTOBER & 0.0 & 91 & 9.7 & 22 & 2.22 & 5.7 \\
\hline NOVEMBER & 0.0 & 15 & 1.3 & 2.9 & 2.30 & .8 \\
\hline DECEMBER & 0.0 & 11 & .9 & 2.3 & 2.40 & .6 \\
\hline JANUARY & 0.0 & 8.7 & .8 & 1.6 & 2.13 & .5 \\
\hline FEBRUARY & 0.0 & 6.2 & .7 & 1.4 & 1.97 & .4 \\
\hline MARCH & 0.0 & 14 & 1.0 & 2.6 & 2.52 & .6 \\
\hline APRIL & 0.0 & 64 & 3.4 & 11 & 3.07 & 2.0 \\
\hline MAY & 0.0 & 302 & 14 & 48 & 3.49 & 8.2 \\
\hline JUNE & 0.0 & 503 & 28 & 79 & 2.82 & 16.6 \\
\hline JULY & 0.0 & 144 & 33 & 38 & 1.15 & 19.4 \\
\hline AUGUST & .2 & 154 & 35 & 37 & 1.06 & 20.6 \\
\hline SEPTEMBER & 0.0 & 549 & 42 & 119 & 2.87 & 24.6 \\
\hline ANNUAL & .2 & 108 & 14 & 19 & 1.37 & 100 \\
\hline
\end{tabular}

MAGNITUDE AND PROBABILITY OF ANNUAL LOW FLOW BASED ON PERIOD OF RECORD $1938-85$

\begin{tabular}{|c|c|c|c|c|c|c|}
\hline \multirow{2}{*}{$\begin{array}{l}\text { PERIOD } \\
\text { (CON- } \\
\text { SECU- } \\
\text { TIVE } \\
\text { DAYS) }\end{array}$} & \multicolumn{6}{|c|}{$\begin{array}{l}\text { DISCHARGE, IN FT'/S, FOR INDICATED RECURRENCE } \\
\text { INTERVAL, IN YEARS, AND ANNUAL NON- } \\
\text { EXCEEDANCE PROBABILITY, IN PERCENT }\end{array}$} \\
\hline & $\begin{array}{c}2 \\
508\end{array}$ & $\begin{array}{c}5 \\
208\end{array}$ & $\begin{array}{l}10 \\
108\end{array}$ & $\begin{array}{l}20 \\
58\end{array}$ & $\begin{array}{l}50 \\
28\end{array}$ & $\begin{array}{r}100 \\
18\end{array}$ \\
\hline $\begin{array}{r}1 \\
3 \\
7 \\
14 \\
30 \\
60 \\
90 \\
120 \\
183\end{array}$ & $\begin{array}{r}0.0 \\
0.0 \\
0.0 \\
0.0 \\
0.0 \\
0.0 \\
.1 \\
.2 \\
.5\end{array}$ & $\begin{array}{r}0.0 \\
0.0 \\
0.0 \\
0.0 \\
0.0 \\
0.0 \\
0.0 \\
0.0 \\
.1\end{array}$ & $\begin{array}{l}0.0 \\
0.0 \\
0.0 \\
0.0 \\
0.0 \\
0.0 \\
0.0 \\
0.0 \\
0.0\end{array}$ & $\begin{array}{l}0.0 \\
0.0 \\
0.0 \\
0.0 \\
0.0 \\
0.0 \\
0.0 \\
0.0 \\
0.0\end{array}$ & $\begin{array}{l}0.0 \\
0.0 \\
0.0 \\
0.0 \\
0.0 \\
0.0 \\
0.0 \\
0.0 \\
0.0\end{array}$ & $\begin{array}{l}0.0 \\
0.0 \\
0.0 \\
0.0 \\
0.0 \\
0.0 \\
0.0 \\
0.0 \\
0.0\end{array}$ \\
\hline
\end{tabular}

MAGNITUDE AND PROBABILITY OF ANNUAL HIGH FLOW BASED ON PERIOD OF RECORD 1937-85

\begin{tabular}{|c|c|c|c|c|c|c|}
\hline \multirow{2}{*}{$\begin{array}{l}\text { PERIOD } \\
\text { (CON- } \\
\text { SECU- } \\
\text { TIVE } \\
\text { DAYS) }\end{array}$} & \multicolumn{6}{|c|}{$\begin{array}{l}\text { DISCHARGE, IN FT' } / S \text {, FOR INDICATED RECURRENCE } \\
\text { INTERVAL, IN YEARS, AND ANNUAL } \\
\text { EXCEEDANCE PROBABIIIT, IN PERCENT }\end{array}$} \\
\hline & $\begin{array}{c}2 \\
508\end{array}$ & $\begin{array}{c}5 \\
208\end{array}$ & $\begin{array}{l}10 \\
108\end{array}$ & $\begin{array}{l}25 \\
48\end{array}$ & $\begin{array}{l}50 \\
28\end{array}$ & $\begin{array}{r}100 \\
18\end{array}$ \\
\hline $\begin{array}{r}1 \\
3 \\
7 \\
15 \\
30 \\
60 \\
90\end{array}$ & $\begin{array}{r}635 \\
300 \\
150 \\
91 \\
58 \\
37 \\
27\end{array}$ & $\begin{array}{r}1630 \\
786 \\
395 \\
236 \\
147 \\
90 \\
67\end{array}$ & $\begin{array}{r}2780 \\
1360 \\
683 \\
394 \\
234 \\
137 \\
104\end{array}$ & $\begin{array}{r}5080 \\
2510 \\
1260 \\
686 \\
381 \\
211 \\
161\end{array}$ & $\begin{array}{r}7630 \\
3810 \\
1910 \\
985 \\
518 \\
275 \\
210\end{array}$ & $\begin{array}{r}11100 \\
5600 \\
2810 \\
1370 \\
681 \\
345 \\
265\end{array}$ \\
\hline
\end{tabular}

DURATION TABLE OF DAILY MEAN FLOW FOR PERIOD OF RECORD 1937-85 DISCHARGE, IN FT'S, THAT WAS EQUALED OR EXCEEDED FOR INDICATED PERCENT OF TIME

\begin{tabular}{|c|c|c|c|c|c|c|c|c|c|c|c|c|c|c|}
\hline 58 & 108 & 158 & 208 & 258 & 308 & 408 & 508 & 608 & 708 & 758 & 808 & 858 & 908 & 958 \\
\hline 32 & 9.5 & 4.2 & 2.3 & 1.3 & .9 & .3 & .1 & .1 & 0.0 & 0.0 & 0.0 & 0.0 & 0.0 & 0.0 \\
\hline
\end{tabular}


ARKANSAS RIVER BASIN

07224500 CANADIAN RIVER BELOW CONCHAS DAM, NM

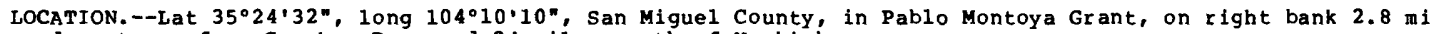

downstream from Conchas Dam, and 24 miles north of Newkirk.

DRAINAGE AREA. $--7,417 \mathrm{mi}^{2}$, of which $433 \mathrm{mi}^{2}$ is probably noncontributing.

PERIOD OF RECORD. --May 1936 to December 1938, January 1942 to september 1972 (discontinued). Water-year estimate for 1942 published in WSP 1311

REVISED RECORDS.--WSP 1177: Drainage area,

GAGE.--Water-stage recorder. Datum of gage is 4,021.90 ft above mean sea level. Prior to Dec. 13, 1941, at site $0.7 \mathrm{mi}$ downstream at datum $6.2 \mathrm{ft}$ higher.

REMARKS.-Flow regulated by Conchas Lake (see sta 07223500). Diversions above station for irrigation of about 90,000 acres, 36,000 of which are below station. Bell Ranch Canal (see sta 07223000) diverts directly from Conchas Dam and flumes from right to left bank just above river gage for irrigation of about 700 acres

on Bell Ranch. Conchas Canal (see sta 07223300) diverts directly from Conchas Dam and bypasses gage for

irrigation of about 35,000 acres around Tucumcari.

AVERAGE DISChARGE. --32 years $(1937-38,1943-72), 54.0 \mathrm{ft} / \mathrm{s}, 39,110 \mathrm{acre}-\mathrm{ft} / \mathrm{yr}$.

EXTREMES FOR PERIOD OF RECORD.--Maximum discharge, 73,000 $\mathrm{ft}^{3 / 5} \mathrm{June} 3,1937$ (may have been affected by construction work on Conchas Dam); maximum gage height, $20.34 \mathrm{ft}$ May 30, 1938, present datum (backwater from temporary construction dam); no flow at times.

Flood of Sept. 30, 1904 was estimated as $279,000 \mathrm{ft}^{3 / 5}$ by Corps of Engineers.

STATISTICAL SUMMARIES

MEAN MONTHLY AND MEAN ANNUAL DISCHARGES 1937-38, 1943-72 MAGNITUDE AND PROBABILITY OF ANNUAL LOW FLOW BASED ON PERIOD OF RECORD 1938, 1943-72

\begin{tabular}{|c|c|c|c|c|c|c|}
\hline MONTH & $\begin{array}{c}\text { MINI MUM } \\
\left(\mathrm{FT}^{\prime} 3 / \mathrm{S}\right)\end{array}$ & $\begin{array}{c}\text { MAXI MUM } \\
\left(\mathrm{FT}^{3} / \mathrm{S}\right)\end{array}$ & $\begin{array}{l}\text { MEAN } \\
\left(\mathrm{FT}^{3} / \mathrm{S}\right)\end{array}$ & $\begin{array}{l}\text { STAN- } \\
\text { DARD } \\
\text { DEVIA- } \\
\text { TION } \\
\left(\mathrm{FT}^{3} / \mathrm{S}\right)\end{array}$ & $\begin{array}{l}\text { COEFFI- } \\
\text { CIENT OF } \\
\text { VARI- } \\
\text { ATION }\end{array}$ & $\begin{array}{c}\text { PERCENT } \\
\text { OF } \\
\text { ANNUAL } \\
\text { RUNOFF }\end{array}$ \\
\hline $\begin{array}{l}\text { OCTOBER } \\
\text { NOVEMBER } \\
\text { DECEMBER } \\
\text { JANUARY } \\
\text { FEBRUARY } \\
\text { MARCH } \\
\text { APRIL } \\
\text { MAY } \\
\text { JUNE } \\
\text { JULY } \\
\text { AUGUST } \\
\text { SEPTEMBER }\end{array}$ & $\begin{array}{l}0.0 \\
0.0 \\
.1 \\
0.0 \\
0.0 \\
0.0 \\
0.0 \\
0.0 \\
.1 \\
.2 \\
.1 \\
.1\end{array}$ & $\begin{array}{r}701 \\
403 \\
146 \\
264 \\
338 \\
116 \\
6063 \\
2549 \\
3221 \\
650 \\
535 \\
4434\end{array}$ & $\begin{array}{r}59 \\
20 \\
13 \\
17 \\
19 \\
12 \\
198 \\
163 \\
207 \\
68 \\
71 \\
233\end{array}$ & $\begin{array}{r}176 \\
70 \\
26 \\
47 \\
59 \\
23 \\
1054 \\
517 \\
619 \\
143 \\
143 \\
819\end{array}$ & $\begin{array}{l}2.96 \\
3.56 \\
2.06 \\
2.70 \\
3.08 \\
1.94 \\
5.32 \\
3.17 \\
2.99 \\
2.11 \\
2.01 \\
3.52\end{array}$ & $\begin{array}{r}5.5 \\
1.8 \\
1.2 \\
1.6 \\
1.8 \\
1.1 \\
18.3 \\
15.1 \\
19.2 \\
6.3 \\
6.6 \\
21.6\end{array}$ \\
\hline ANNUAL & .1 & 485 & 54 & 107 & 1.99 & 100 \\
\hline
\end{tabular}

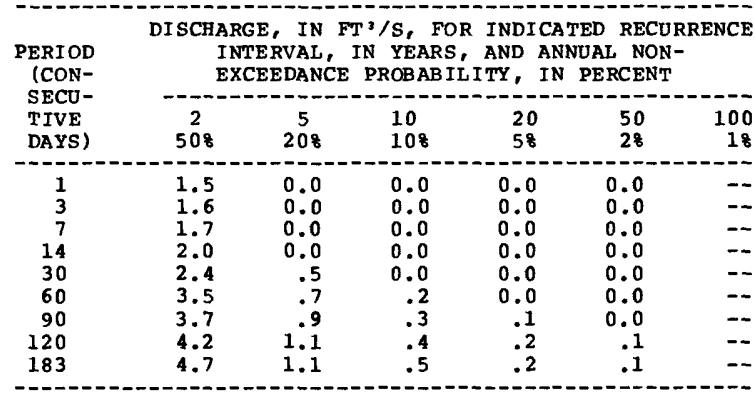

MAGNITUDE AND PROBABILITY OF ANNUAL HIGH FLOW BASED ON PERIOD OF RECORD 1937-38, 1943-72

\begin{tabular}{|c|c|c|c|c|c|c|}
\hline \multirow{2}{*}{$\begin{array}{l}\text { PERIOD } \\
\text { (CON- } \\
\text { SECU- } \\
\text { TIVE } \\
\text { DAYS) }\end{array}$} & \multicolumn{6}{|c|}{$\begin{array}{l}\text { DISCHARGE, IN FT } 3 / \mathrm{S} \text {, FOR INDICATED RECURRENCE } \\
\text { INTERVAL, IN YEARS, AND ANNUAL } \\
\text { EXCEEDANCE PROBABILITY, IN PERCENT }\end{array}$} \\
\hline & $\begin{array}{c}2 \\
508\end{array}$ & $\begin{array}{c}5 \\
208\end{array}$ & $\begin{array}{l}10 \\
108\end{array}$ & $\begin{array}{l}25 \\
48\end{array}$ & $\begin{array}{l}50 \\
28\end{array}$ & $\begin{array}{r}100 \\
18\end{array}$ \\
\hline $\begin{array}{r}1 \\
3 \\
7 \\
15 \\
30 \\
60 \\
90\end{array}$ & $\begin{array}{r}193 \\
102 \\
64 \\
41 \\
29 \\
21 \\
18\end{array}$ & $\begin{array}{r}1870 \\
1020 \\
619 \\
371 \\
242 \\
155 \\
125\end{array}$ & $\begin{array}{r}6440 \\
3740 \\
2250 \\
1280 \\
800 \\
474 \\
373\end{array}$ & $\begin{array}{r}25000 \\
16100 \\
9650 \\
5160 \\
3040 \\
1660 \\
1260\end{array}$ & $\begin{array}{r}61500 \\
43300 \\
25800 \\
13200 \\
7450 \\
3830 \\
2850\end{array}$ & $\begin{array}{l}-- \\
-- \\
-- \\
-- \\
-- \\
--\end{array}$ \\
\hline
\end{tabular}

DURATION TABLE OF DAILY MEAN FLOW FOR PERIOD OF RECORD 1937-38, 1943-72

DISCHARGE, IN FT $3 / \mathrm{S}$, THAT WAS EQUALED OR EXCEEDED FOR INDICATED PERCENT OF TIME

\begin{tabular}{|c|c|c|c|c|c|c|c|c|c|c|c|c|c|c|}
\hline 58 & 108 & 158 & 208 & 258 & 308 & 408 & 508 & 608 & 708 & 758 & 808 & 858 & 908 & 958 \\
\hline 74 & 19 & 13 & 11 & 9.1 & 7.8 & 5.6 & 4.2 & 3.4 & 2.8 & 2.5 & 2.1 & 1.3 & 1.1 & .3 \\
\hline
\end{tabular}




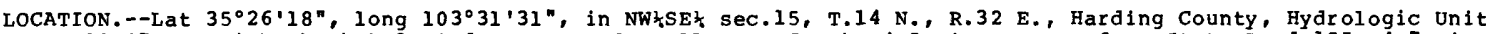
11090007 , on right bank $1.9 \mathrm{mi}$ downstream from Alamosa Creek, $4.5 \mathrm{mi}$ upstream from State Road 155, $4.7 \mathrm{mi}$ upstream from high-water $i$ ine of ute Reservoir, $8.2 \mathrm{mi}$ northwest of Logan, and at mile 10.0

DRAINAGE AREA. $--2,060 \mathrm{mi}^{2}$, of which $617 \mathrm{mi}^{2}$ is probably noncontributing.

PERIOD OF RECORD.--January 1912 to May 1914 (gage heights and discharge measurements only), January 1942 to current year. Records of discharge for August 1904 to June 1906, Apr 111909 to December 1911, published in wsP 307, are unreliable and should not be used.

REVISED RECORDS.--WSP 1281: 1942-48, 1950, 1951(P), WDR NM-81: 1965(P), 1967-68(M), 1969(P), 1971(M), 1972, 1975 (M) 1977,1979 . See also PERIOD OF RECORD.

GAGE.--Water-stage recorder. Elevation of gage is 3,815 ft above National Geodetic Vertical Datum of 1929 , from topographic map. See WSP 2121 for history of changes prior to Oct. 1, 1964 .

REMARKS.--Diversions for irrigation of a few hundred acres upstream from station.

AVERAGE DISCHARGE. -43 years (water years 1943-85), $23.2 \mathrm{ft}^{3} / \mathrm{s}, 16,810$ acre-ft/yr.

EXTREMES FOR PERIOD OF RECORD.--Maximum discharge, 24,500 $\mathrm{ft} / \mathrm{s}$, May 28, 1946, July 12, 1951, gage height, 8.4 ft, site and datum then in use, from rating curve extended above $7,700 \mathrm{ft} 3 / \mathrm{s}$ on basis of slope-area measurements at gage heights $5.2 \mathrm{ft}$ and $7.2 \mathrm{ft}$; maximum gage height, $9.94 \mathrm{ft}$, Aug. 11,1981 ; no flow most of time.

EXTREMES OUTSIDE PERIOD OF RECORD.--Flood of May 1, 1914, reached a stage of $22.95 \mathrm{ft}$, site and datum then in use. Another major flood reached a stage of $16.0 \mathrm{ft}, 1942$ datum, sometime in 1941 , from information furnished by U.S. Bureau of Reclamation; discharge, about $70,000 \mathrm{ft}^{3} / \mathrm{s}$.

STATISTICAL SUMMARIES

MEAN MONTHLY AND MEAN ANNUAL DISChaRges 1943-85

MAGNITUDE AND PROBABILITY OF ANNUAL LOW FLOW BASED ON PERIOD OF RECORD 1944-85

\begin{tabular}{|c|c|c|c|c|c|c|}
\hline MONTH & $\begin{array}{c}\text { MINIMUM } \\
\left(\mathrm{FT}^{3} / \mathrm{S}\right)\end{array}$ & $\frac{\operatorname{MAXIMUM}}{\left(\mathrm{FT}^{3} / \mathrm{S}\right)}$ & $\begin{array}{l}\text { MEAN } \\
\left(\mathrm{FT}^{3} / \mathrm{S}\right)\end{array}$ & $\begin{array}{l}\text { STAN- } \\
\text { DARD } \\
\text { DEVIA- } \\
\text { TION } \\
\left(\mathrm{FT}^{3} / \mathrm{S}\right)\end{array}$ & $\begin{array}{l}\text { COEFFI- } \\
\text { CIENT OF } \\
\text { VARI- } \\
\text { ATION }\end{array}$ & $\begin{array}{c}\text { PERCENT } \\
\text { OF } \\
\text { ANNUAL } \\
\text { RUNOFF }\end{array}$ \\
\hline $\begin{array}{l}\text { OCTOBER } \\
\text { NOVEMBER } \\
\text { DECEMBER } \\
\text { JANUARY } \\
\text { FEBRUARY } \\
\text { MARCH } \\
\text { APR IL } \\
\text { MAY } \\
\text { JUNE } \\
\text { JULY } \\
\text { AUGUST } \\
\text { SEPTEMBER }\end{array}$ & $\begin{array}{l}0.0 \\
0.0 \\
0.0 \\
0.0 \\
0.0 \\
0.0 \\
0.0 \\
0.0 \\
0.0 \\
0.0 \\
0.0 \\
0.0\end{array}$ & $\begin{array}{r}139 \\
93 \\
40 \\
28 \\
15 \\
24 \\
37 \\
351 \\
191 \\
317 \\
520 \\
261\end{array}$ & $\begin{array}{r}11 \\
3.4 \\
2.2 \\
2.0 \\
1.7 \\
1.7 \\
3.0 \\
47 \\
28 \\
65 \\
82 \\
30\end{array}$ & $\begin{array}{c}29 \\
14 \\
6.6 \\
5.9 \\
3.8 \\
4.9 \\
7.6 \\
86 \\
45 \\
86 \\
101 \\
54\end{array}$ & $\begin{array}{l}2.59 \\
4.22 \\
3.01 \\
3.00 \\
2.18 \\
2.94 \\
2.57 \\
1.86 \\
1.59 \\
1.34 \\
1.23 \\
1.83\end{array}$ & $\begin{array}{r}4.0 \\
1.2 \\
.8 \\
.7 \\
.6 \\
.6 \\
1.1 \\
16.8 \\
10.2 \\
23.4 \\
29.7 \\
10.8\end{array}$ \\
\hline ANNUAL & .1 & 57 & 23 & 18 & .76 & 100 \\
\hline
\end{tabular}

DISCHARGE, IN $\mathrm{FT}^{3} / \mathrm{S}$, FOR INDICATED RECURRENCE INTERVAL, IN YEARS, AND ANNUAL NON-

\begin{tabular}{|c|c|c|c|c|c|c|}
\hline \multirow{2}{*}{$\begin{array}{l}\text { PERIOD } \\
\text { (CON- } \\
\text { SECU- } \\
\text { TIVE } \\
\text { DAYS) }\end{array}$} & \multicolumn{6}{|c|}{$\begin{array}{l}\text { DISCHARGE, IN } \mathrm{FT}^{3} / \mathrm{S} \text {, FOR INDICATED RECURRENCE } \\
\text { INTERVAL, IN YEARS, AND ANNUAL NON- } \\
\text { EXCEEDANCE PROBABILITY, IN PERCENT }\end{array}$} \\
\hline & $\begin{array}{c}2 \\
508\end{array}$ & $\begin{array}{c}5 \\
208\end{array}$ & $\begin{array}{l}10 \\
108\end{array}$ & $\begin{array}{l}20 \\
58\end{array}$ & $\begin{array}{l}50 \\
28\end{array}$ & $\begin{array}{r}100 \\
18\end{array}$ \\
\hline $\begin{array}{r}1 \\
3 \\
7 \\
14 \\
30 \\
60 \\
90 \\
120 \\
183\end{array}$ & $\begin{array}{l}0.0 \\
0.0 \\
0.0 \\
0.0 \\
0.0 \\
0.0 \\
0.0 \\
0.0 \\
.2\end{array}$ & $\begin{array}{l}0.0 \\
0.0 \\
0.0 \\
0.0 \\
0.0 \\
0.0 \\
0.0 \\
0.0 \\
0.0\end{array}$ & $\begin{array}{l}0.0 \\
0.0 \\
0.0 \\
0.0 \\
0.0 \\
0.0 \\
0.0 \\
0.0 \\
0.0\end{array}$ & $\begin{array}{l}0.0 \\
0.0 \\
0.0 \\
0.0 \\
0.0 \\
0.0 \\
0.0 \\
0.0 \\
0.0\end{array}$ & $\begin{array}{l}0.0 \\
0.0 \\
0.0 \\
0.0 \\
0.0 \\
0.0 \\
0.0 \\
0.0 \\
0.0\end{array}$ & $\begin{array}{l}0.0 \\
0.0 \\
0.0 \\
0.0 \\
0.0 \\
0.0 \\
0.0 \\
0.0 \\
0.0\end{array}$ \\
\hline
\end{tabular}

MAGNITUDE AND PROBABILITY OF ANNUAL HIGH FLOW BASED ON PERIOD OF RECORD 1943-85

\begin{tabular}{|c|c|c|c|c|c|c|}
\hline \multirow{2}{*}{$\begin{array}{l}\text { PERIOD } \\
\text { (CON- } \\
\text { SECU- } \\
\text { TIVE } \\
\text { DAYS) }\end{array}$} & \multicolumn{6}{|c|}{$\begin{array}{l}\text { DISCHARGE, IN FT'/S, FOR INDICATED RECURRENCE } \\
\text { INTERVAL, IN YEARS, AND ANNUAL } \\
\text { EXCEEDANCE PROBABILITY, IN PERCENT }\end{array}$} \\
\hline & $\begin{array}{c}2 \\
508\end{array}$ & $\begin{array}{c}5 \\
208\end{array}$ & $\begin{array}{l}10 \\
108\end{array}$ & $\begin{array}{l}25 \\
48\end{array}$ & $\begin{array}{l}50 \\
28\end{array}$ & $\begin{array}{r}100 \\
18\end{array}$ \\
\hline $\begin{array}{r}1 \\
3 \\
7 \\
15 \\
30 \\
60 \\
90\end{array}$ & $\begin{array}{r}1380 \\
729 \\
375 \\
219 \\
127 \\
82 \\
63\end{array}$ & $\begin{array}{r}3270 \\
1660 \\
866 \\
491 \\
279 \\
178 \\
134\end{array}$ & $\begin{array}{r}4550 \\
2220 \\
1170 \\
648 \\
361 \\
228 \\
170\end{array}$ & $\begin{array}{r}5980 \\
2770 \\
1470 \\
796 \\
435 \\
271 \\
200\end{array}$ & $\begin{array}{r}6870 \\
3070 \\
1650 \\
874 \\
472 \\
291 \\
214\end{array}$ & $\begin{array}{r}7610 \\
3300 \\
1780 \\
930 \\
497 \\
304 \\
223\end{array}$ \\
\hline
\end{tabular}

DURATION TABLE OF DAILY MEAN FLOW FOR PERIOD OF RECORD 1943-85

DISCHARGE, IN FT3/S, THAT WAS EQUALED OR EXCEEDED FOR INDICATED PERCENT OF TIME

\begin{tabular}{|c|c|c|c|c|c|c|c|c|c|c|c|c|c|c|}
\hline 58 & 108 & 158 & 208 & 258 & 308 & 408 & 508 & 608 & 708 & 758 & 808 & 858 & 908 & 958 \\
\hline 73 & 19 & 6.3 & 2.5 & .9 & 0.0 & 0.0 & 0.0 & 0.0 & 0.0 & 0.0 & 0.0 & 0.0 & 0.0 & \\
\hline
\end{tabular}




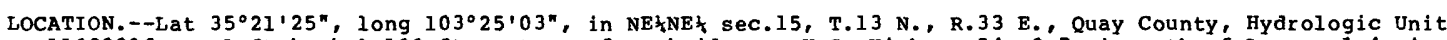
11080006 , on left bank $1,100 \mathrm{ft}$ upstream from bridge on U.S. Highway $54,0.7 \mathrm{mi}$ south of Logan, $1.4 \mathrm{mi}$ upstream from Chicago, Rock Island Pacific Railroad Co. bridge, $2.0 \mathrm{mi}$ downstream from Ute Dam, $4.3 \mathrm{mi}$ upstream from Revuelto Creek, and at mile 672.0 .

DRAINAGE AREA. - $-11,141 \mathrm{mi}^{2}$, of which $1,100 \mathrm{mi}^{2}$ is probably noncontributing.

PERIOD OF RECORD.--June 1904 to November 1905 (gage heights and discharge measurements only), December 1908 to September 1909 , February 1910, Apr il to JuIy 1910, August 1910 to September 1911 (gage heights and discharge
measurements only). October 1911 to May 1914, January to May 1924, September 1924 to July 1925 . January 1927 to measurements only), October 1911 to May 1914, January to May 1924, September 1924 to July $1925, J$ anuary 192 Records for December 1909, January 1910, and May to July 1934, published in wsp 267, 287 , and 762 are unreliable Records for December 1909 , January 1910 , and May to July 1934, published in WSP 26
and should not be used. published as South Canadian River June to September 1904.

REVISED RECORDS.--WSP 1087: 1935-36. WSP 1117: Drainage area. WSP 1281: 1912, 1932(M), 1934, 1945-47, 1949-50. WSP 1311: $1931(M)$. See also PERIOD OF RECORD.

GAGE.--Water-stage recorder. Datum of gage is $3,668.1 \mathrm{ft}$ above National Geodetic vertical Datum of 1929 . See wSP 1311 or 1731 for history of changes prior to oct. 1, 1934 .

REMARKS.--Flow regulated by Conchas Lake, $45 \mathrm{mi}$ upstream (station 07223500 ) and Ute Reservoir, 2 mi upstream (station 07226800). Diversions for irrigation of about 90,000 acres upstream from station.

AVERAGE DISCHARGE.--36 years (water years 1912-13, 1928-29, 1931-62), $306 \mathrm{ft} / \mathrm{s}, 221,700 \mathrm{acre-ft} / \mathrm{yr}$.

EXTREMES FOR PERIOD OF RECORD (SINCE 1925).--Maximum discharge, 219,000 ft3/s, Sept. 22, 1941, gage height, 29.3 $\mathrm{ft}$, from floodmarks, from rating curve extended above $75,000 \mathrm{ft}^{3} / \mathrm{s}$; no flow at times prior to completion of Ute Dam.

EXTREMES OUTSIDE PERIOD OF RECORD.--Maximum discharge, 278,000 ft $3 / \mathrm{s}$, Sept. 30, 1904, gage height, about $36.5 \mathrm{ft}$, site and datum used in 1909, from rating curve extended above $14,000 \mathrm{ft} / \mathrm{s}$, from Ninth Biennial Report of State Engineer.

STATISTICAL SUMMARIES (PERIOD BEFORE REGULATION OF UTE DAM)

MEAN MONTHLY AND MEAN ANNUAL DISCHARGES 1912-13,

1928-29, $1931-62$

\begin{tabular}{|c|c|c|c|c|c|c|}
\hline MONTH & $\begin{array}{c}\text { MIN IMUM } \\
\left(\mathrm{FT}^{3} / \mathrm{S}\right)\end{array}$ & $\begin{array}{l}\text { MAXIMUM } \\
\left(F^{3} / S\right)\end{array}$ & $\begin{array}{l}\text { MEAN } \\
\left(F^{3} / S\right)\end{array}$ & $\begin{array}{l}\text { STAN- } \\
\text { DARD } \\
\text { DEVIA- } \\
\text { TION } \\
(\text { FT' } / S \text { ) }\end{array}$ & $\begin{array}{l}\text { COEFFI - } \\
\text { CIENT OF } \\
\text { VARI - } \\
\text { ATION }\end{array}$ & $\begin{array}{c}\text { PERCENT } \\
\text { OF } \\
\text { ANNUAL } \\
\text { RUNOFF }\end{array}$ \\
\hline $\begin{array}{l}\text { OCTOBER } \\
\text { NOVEMBER } \\
\text { DECEMBER } \\
\text { JANUARY } \\
\text { FEBRUARY } \\
\text { MARCH } \\
\text { APRIL } \\
\text { MAY } \\
\text { JUNE } \\
\text { JULY } \\
\text { AUGUST } \\
\text { SEPTEMBER }\end{array}$ & $\begin{array}{l}0.0 \\
0.0 \\
0.0 \\
0.0 \\
0.0 \\
0.0 \\
0.0 \\
0.0 \\
0.0 \\
11 \\
23 \\
0.0\end{array}$ & $\begin{array}{r}4845 \\
663 \\
477 \\
388 \\
282 \\
274 \\
6930 \\
4109 \\
7407 \\
2255 \\
2054 \\
8027\end{array}$ & $\begin{array}{r}382 \\
51 \\
33 \\
44 \\
37 \\
30 \\
229 \\
539 \\
774 \\
433 \\
473 \\
581\end{array}$ & $\begin{array}{r}1022 \\
130 \\
80 \\
74 \\
54 \\
57 \\
1079 \\
933 \\
1518 \\
445 \\
468 \\
1492\end{array}$ & $\begin{array}{l}2.68 \\
2.55 \\
2.41 \\
1.70 \\
1.44 \\
1.91 \\
4.71 \\
1.73 \\
1.96 \\
1.03 \\
.99 \\
2.57\end{array}$ & $\begin{array}{r}10.6 \\
1.4 \\
.9 \\
1.2 \\
1.0 \\
.8 \\
6.4 \\
14.9 \\
21.5 \\
12.0 \\
13.1 \\
16.1\end{array}$ \\
\hline ANNUAL & 23 & 1819 & 306 & 410 & 1.34 & 100 \\
\hline
\end{tabular}

MAGNITUDE AND PROBABILITY OF ANNUAL LOW FLOW BASED ON PERIOD OF RECORD 1913-14, 1928-29, 1931-62

\begin{tabular}{|c|c|c|c|c|c|c|}
\hline \multirow{2}{*}{$\begin{array}{l}\text { PERIOD } \\
\text { (CON- } \\
\text { SECU- } \\
\text { TIVE } \\
\text { DAYS) }\end{array}$} & \multicolumn{6}{|c|}{$\begin{array}{l}\text { DISCHARGE, IN FT'/S, FOR INDICATED RECURRENCE } \\
\text { INTERVAL, IN YEARS, AND ANNUAL NON- } \\
\text { EXCEEDANCE PROBABILITY, IN PERCENT }\end{array}$} \\
\hline & $\begin{array}{c}2 \\
508\end{array}$ & $\begin{array}{c}5 \\
208\end{array}$ & $\begin{array}{l}10 \\
108\end{array}$ & $\begin{array}{l}20 \\
58\end{array}$ & $\begin{array}{l}50 \\
28\end{array}$ & $\begin{array}{r}100 \\
1 \%\end{array}$ \\
\hline $\begin{array}{r}1 \\
3 \\
7 \\
14 \\
30 \\
60 \\
90 \\
120 \\
183\end{array}$ & $\begin{array}{l}0.0 \\
0.0 \\
0.0 \\
0.0 \\
0.0 \\
2.0 \\
5.2 \\
7.3 \\
15\end{array}$ & $\begin{array}{r}0.0 \\
0.0 \\
0.0 \\
0.0 \\
0.0 \\
.1 \\
.8 \\
1.4 \\
3.5\end{array}$ & $\begin{array}{r}0.0 \\
0.0 \\
0.0 \\
0.0 \\
0.0 \\
0.0 \\
.3 \\
.5 \\
1.7\end{array}$ & $\begin{array}{r}0.0 \\
0.0 \\
0.0 \\
0.0 \\
0.0 \\
0.0 \\
.1 \\
.2 \\
.9\end{array}$ & $\begin{array}{l}0.0 \\
0.0 \\
0.0 \\
0.0 \\
0.0 \\
0.0 \\
0.0 \\
0.0 \\
.5\end{array}$ & $\begin{array}{l}0.0 \\
0.0 \\
0.0 \\
0.0 \\
0.0 \\
0.0 \\
0.0 \\
0.0 \\
.3\end{array}$ \\
\hline
\end{tabular}

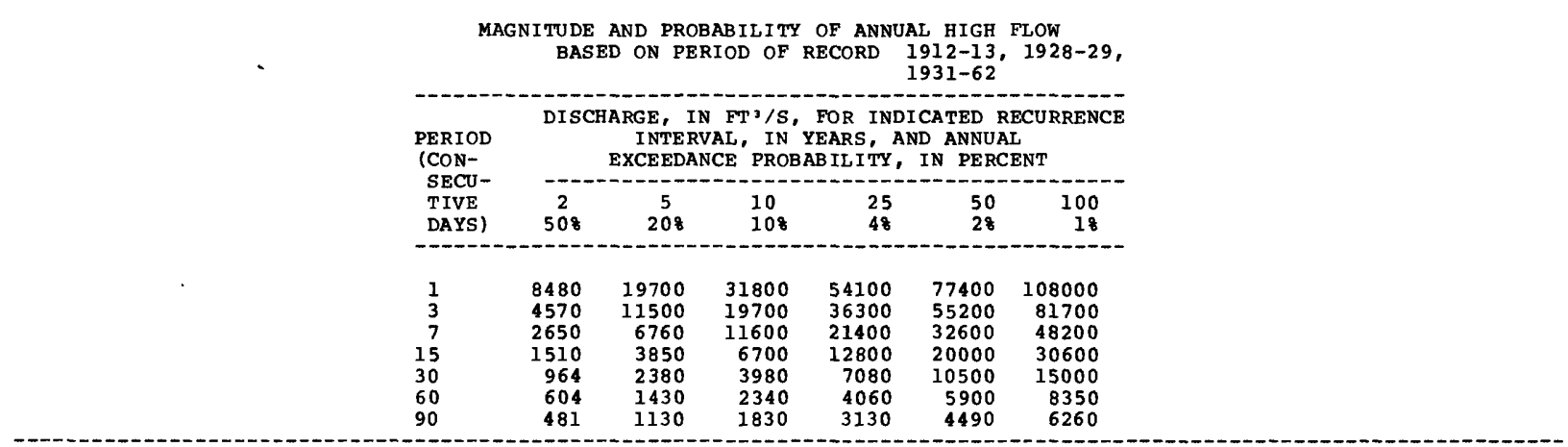

DURATION TABLE OF DAILY MEAN FLOW FOR PERIOD OF RECORD 1912-13, 1928-29, 1931-62 DISCHARGE, IN FT' ${ }^{3}$, THAT WAS EQUALED OR EXCEEDED FOR INDICATED PERCENT OF TIME

\begin{tabular}{|c|c|c|c|c|c|c|c|c|c|c|c|c|c|c|}
\hline 58 & 108 & 158 & 208 & 258 & 308 & 408 & 508 & 608 & 708 & 758 & 808 & 858 & 908 & 958 \\
\hline 981 & 411 & 207 & 126 & 83 & 56 & 28 & 14 & 5.8 & 2.0 & .9 & .1 & .1 & 0.0 & 0 \\
\hline
\end{tabular}


ARKANSAS RIVER BASIN

07227000 CANADIAN RIVER AT LOGAN, NM--Continued

STATISTICAL SUMMARIES (PERIOD AFTER COMPLETION OF UTE DAM)

MEAN MONTHLY AND MEAN ANNUAL DISCHARGES 1964-85

\begin{tabular}{|c|c|c|c|c|c|c|}
\hline MONTH & $\begin{array}{c}\text { MINIMUM } \\
\left(\mathrm{FT}^{3} / \mathrm{S}\right)\end{array}$ & $\begin{array}{c}\text { MAXIMUM } \\
\left(\mathrm{FT}^{3} / \mathrm{S}\right)\end{array}$ & $\begin{array}{l}\text { MEAN } \\
\left(\mathrm{FT}^{3} / \mathrm{S}\right)\end{array}$ & $\begin{array}{l}\text { STAN- } \\
\text { DARD } \\
\text { DEVIA- } \\
\text { TION } \\
\left(\mathrm{FT}^{3} / \mathrm{S}\right)\end{array}$ & $\begin{array}{l}\text { COEFFI- } \\
\text { CIENT OF } \\
\text { VARI- } \\
\text { ATION }\end{array}$ & $\begin{array}{c}\text { PERCENT } \\
\text { OF } \\
\text { ANNUAL } \\
\text { RUNOFF }\end{array}$ \\
\hline $\begin{array}{l}\text { OCTOBER } \\
\text { NOVEMBER } \\
\text { DECEMBER } \\
\text { JANUARY } \\
\text { FEBRUARY } \\
\text { MARCH } \\
\text { APRIL } \\
\text { MAY } \\
\text { JUNE } \\
\text { JULY } \\
\text { AUGUST } \\
\text { SEPTEMBER }\end{array}$ & $\begin{array}{l}1.3 \\
1.2 \\
1.2 \\
1.5 \\
1.5 \\
1.6 \\
1.7 \\
1.0 \\
.8 \\
1.0 \\
1.5 \\
1.4\end{array}$ & $\begin{array}{r}325 \\
287 \\
84 \\
62 \\
174 \\
11 \\
77 \\
10.0 \\
575 \\
608 \\
720 \\
838\end{array}$ & $\begin{array}{c}42 \\
23 \\
6.0 \\
7.1 \\
13 \\
2.8 \\
10 \\
2.7 \\
48 \\
85 \\
111 \\
105\end{array}$ & $\begin{array}{c}93 \\
62 \\
17 \\
16 \\
38 \\
2.0 \\
21 \\
1.8 \\
131 \\
166 \\
185 \\
207\end{array}$ & $\begin{array}{l}2.21 \\
2.74 \\
2.90 \\
2.21 \\
3.02 \\
.70 \\
2.05 \\
.65 \\
2.75 \\
1.96 \\
1.67 \\
1.97\end{array}$ & $\begin{array}{r}9.2 \\
5.0 \\
1.3 \\
1.6 \\
2.8 \\
.6 \\
2.3 \\
.6 \\
10.5 \\
18.6 \\
24.4 \\
23.1\end{array}$ \\
\hline ANNUAL & 1.6 & 145 & 38 & 44 & 1.15 & 100 \\
\hline
\end{tabular}

MAGNI TUDE AND PROBABILITY OF ANNUAL LOW FLOW BASED ON PERIOD OF RECORD 1965-85

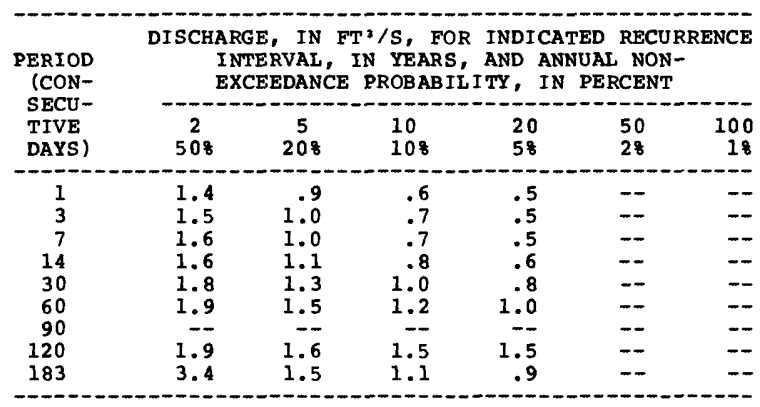

MAGNITUDE AND PROBABILITY OF ANNUAL HIGH FLOW BASED ON PERIOD OF RECORD 1964-85

\begin{tabular}{|c|c|c|c|c|c|c|}
\hline \multirow{2}{*}{$\begin{array}{l}\text { PERIOD } \\
\text { (CON- } \\
\text { SECU- } \\
\text { TIVE } \\
\text { DAYS) }\end{array}$} & \multicolumn{6}{|c|}{$\begin{array}{l}\text { DISCHARGE, IN FT' } / S, \text { FOR INDICATED RECURRENCE } \\
\text { INTERVAL, IN YEARS, AND ANNUAL } \\
\text { EXCEEDANCE PROBABILITY, IN PERCENT }\end{array}$} \\
\hline & ${ }_{508}^{2}$ & $\begin{array}{c}5 \\
208\end{array}$ & $\begin{array}{l}10 \\
108\end{array}$ & $\begin{array}{l}25 \\
48\end{array}$ & $\begin{array}{l}50 \\
28\end{array}$ & $\begin{array}{r}100 \\
18\end{array}$ \\
\hline $\begin{array}{r}1 \\
3 \\
7 \\
15 \\
30 \\
60 \\
90\end{array}$ & $\begin{array}{r}307 \\
224 \\
166 \\
126 \\
85 \\
59 \\
44\end{array}$ & $\begin{array}{r}1590 \\
1250 \\
897 \\
686 \\
453 \\
290 \\
212\end{array}$ & $\begin{array}{r}3620 \\
2850 \\
1940 \\
1460 \\
968 \\
610 \\
447\end{array}$ & $\begin{array}{r}8520 \\
6510 \\
4070 \\
2990 \\
2010 \\
1260 \\
941\end{array}$ & $\begin{array}{l}-- \\
-- \\
-- \\
-- \\
-\end{array}$ & $\begin{array}{l}-- \\
-- \\
-- \\
-- \\
--\end{array}$ \\
\hline
\end{tabular}

DURATION TABLE OF DAILY MEAN FLOW FOR PERIOD OF RECORD 1964-85

DISCHARGE, IN FT3/S, THAT WAS EQUALED OR EXCEEDED FOR INDICATED PERCENT OF TIME

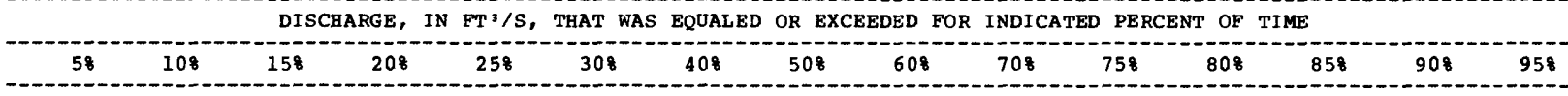

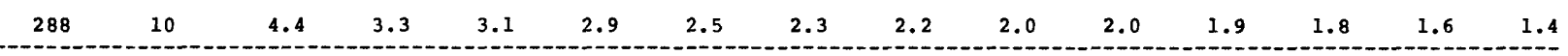




\section{ARKANSAS RIVER BASIN \\ 07227100 REVUELTO CREEK NEAR LOGAN, NM}

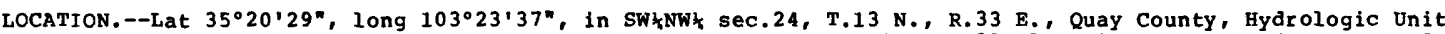
11080008 , on right bank $0.3 \mathrm{mi}$ upstream from bridge on state Highway $39,1.9 \mathrm{mi}$ southeast of Logan, and at mile 2.3 .

DRAINAGE AREA. $--786 \mathrm{mi}^{2}$.

PERIOD OF RECORD.--August 1959 to current year.

GAGE.--Water-stage recorder. Elevation of gage is 3,665 ft above National Geodetic vertical Datum of 1929 , from topographic map. Prior to Jan. 16, 1981, at site $320 \mathrm{ft}$ upstream at datum $0.56 \mathrm{ft}$ higher.

REMARKS.--LOW flows supplemented by surface and ground-water return from irrigation in vicinity of Tucumcari. AVERAGE DISCHARGE.--26 years (water years 1960-85), $44.1 \mathrm{ft}^{3} / \mathrm{s}, 31,950$ acre-ft/yr.

EXTREMES FOR PERIOD OF RECORD.--Maximum discharge, 26,700 ft'/s, July 9, 1960, gage height, 14.3 ft, site and datum then in use; no flow at times most years.

EXTREMES OUTSIDE PERIOD OF RECORD (1941-47).--Maximum discharge determined, about $13,400 \mathrm{ft} / \mathrm{s}$, Sept. 18, 1946, gage height, $9.04 \mathrm{ft}$, at site $180 \mathrm{ft}$ downstream at different datum, from unpublished records collected by U.S. Bureau of Reclamation.

A peak of $26,100 \mathrm{ft}^{3} / \mathrm{s}$, date unknown, gage height, $12.9 \mathrm{ft}$ at former site and datum, was measured by slopearea method in May 1957 .

STATISTICAL SUMMARIES

MEAN MONTHLY AND MEAN ANNUAL DISCHARGES 1960-85

\begin{tabular}{|c|c|c|c|c|c|c|}
\hline MONTH & $\begin{array}{c}\text { MINIMUM } \\
\left(\mathrm{FT}^{3} / \mathrm{S}\right)\end{array}$ & $\begin{array}{c}\text { MAXIMUM } \\
\left(\mathrm{FT}^{3} / \mathrm{S}\right)\end{array}$ & $\begin{array}{l}\text { MEAN } \\
\left(\mathrm{F}^{\prime} \mathrm{T}^{3} / \mathrm{S}\right)\end{array}$ & $\begin{array}{l}\text { STAN- } \\
\text { DARD } \\
\text { DEVIA- } \\
\text { TION } \\
\left(\mathrm{FT}^{3} / \mathrm{S}\right)\end{array}$ & $\begin{array}{l}\text { COEFFI- } \\
\text { CIENT OF } \\
\text { VARI- } \\
\text { ATION }\end{array}$ & $\begin{array}{c}\text { PERCENT } \\
\text { OF } \\
\text { ANNUAL } \\
\text { RUNOFF }\end{array}$ \\
\hline OCTOBER & & & & 60 & 70 & - \\
\hline OCTOBER & 0.0 & 320 & 38 & 68 & 1.79 & 7.2 \\
\hline $\begin{array}{l}\text { NOVEMBER } \\
\text { DCEBE }\end{array}$ & .1 & 34 & 7.4 & 9.1 & 1.23 & 1.4 \\
\hline DECEMBER & 0.0 & 129 & 9.5 & 25 & 2.65 & 1.8 \\
\hline JANUARY & 0.0 & 19 & 3.9 & 4.9 & 1.26 & .7 \\
\hline FEBRUARY & 0.0 & 43 & 6.2 & 10 & 1.61 & 1.2 \\
\hline MARCH & 0.0 & 52 & 6.0 & 11 & 1.82 & 1.1 \\
\hline APRIL & .3 & 346 & 26 & 67 & 2.55 & 5.0 \\
\hline MAY & .1 & 177 & 42 & 41 & .98 & 8.0 \\
\hline JUNE & .9 & 492 & 62 & 96 & 1.53 & 11.9 \\
\hline JULY & .4 & 1203 & 135 & 239 & 1.77 & 25.7 \\
\hline AUGUST & .9 & 575 & 118 & 135 & 1.14 & 22.4 \\
\hline SEPTEMBER & 1.7 & 515 & 71 & 104 & 1.46 & 13.5 \\
\hline ANNUAL & 4.7 & 204 & 44 & 39 & .89 & 100 \\
\hline
\end{tabular}

MAGNITUDE AND PROBABILITY OF ANNUAL LOW FLOW BASED ON PERIOD OF RECORD 1961-85

\begin{tabular}{|c|c|c|c|c|c|c|}
\hline \multirow{2}{*}{$\begin{array}{l}\text { PERIOD } \\
\text { (CON- } \\
\text { SECU- } \\
\text { TIVE } \\
\text { DAYS) }\end{array}$} & \multicolumn{6}{|c|}{$\begin{array}{l}\text { DISCHARGE, IN FT'/S, FOR INDICATED RECURRENCE } \\
\text { INTERVAL, IN YEARS, AND ANNUAL NON- } \\
\text { EXCEEDANCE PROBABILITY, IN PERCENT }\end{array}$} \\
\hline & $\begin{array}{c}2 \\
508\end{array}$ & $\begin{array}{c}5 \\
208\end{array}$ & $\begin{array}{l}10 \\
108\end{array}$ & $\begin{array}{l}20 \\
58\end{array}$ & $\begin{array}{l}50 \\
28\end{array}$ & $\begin{array}{r}10 \\
1\end{array}$ \\
\hline $\begin{array}{r}1 \\
3 \\
7 \\
14 \\
30 \\
60 \\
90 \\
120 \\
183\end{array}$ & $\begin{array}{r}0.0 \\
0.0 \\
0.0 \\
0.0 \\
.3 \\
.9 \\
1.5 \\
2.5 \\
6.4\end{array}$ & $\begin{array}{r}0.0 \\
0.0 \\
0.0 \\
0.0 \\
0.0 \\
.1 \\
.3 \\
.5 \\
1.8\end{array}$ & $\begin{array}{l}0.0 \\
0.0 \\
0.0 \\
0.0 \\
0.0 \\
0.0 \\
.1 \\
.2 \\
.8\end{array}$ & $\begin{array}{r}0.0 \\
0.0 \\
0.0 \\
0.0 \\
0.0 \\
0.0 \\
0.0 \\
.1 \\
.4\end{array}$ & $\begin{array}{l}0.0 \\
0.0 \\
0.0 \\
0.0 \\
0.0 \\
0.0 \\
0.0 \\
0.0 \\
.2\end{array}$ & \\
\hline
\end{tabular}

MAGNITUDE AND PROBABILITY OF ANNUAL HIGH FLOW BASED ON PERIOD OF RECORD 1960-85

\begin{tabular}{|c|c|c|c|c|c|c|}
\hline \multirow{2}{*}{$\begin{array}{l}\text { PERIOD } \\
\text { (CON- } \\
\text { SECU- } \\
\text { TIVE } \\
\text { DAYS) }\end{array}$} & \multicolumn{6}{|c|}{$\begin{array}{l}\text { DISCHARGE, IN FT }{ }^{3} / \mathrm{S} \text {, FOR INDICATED RECURRENCE } \\
\text { INTERVAL, IN YEARS, AND ANNUAL } \\
\text { EXCEEDANCE PROBABIL ITY, IN PERCENT }\end{array}$} \\
\hline & $\begin{array}{c}2 \\
508\end{array}$ & $\begin{array}{c}5 \\
208\end{array}$ & $\begin{array}{l}10 \\
108\end{array}$ & $\begin{array}{l}25 \\
48\end{array}$ & $\begin{array}{l}50 \\
28\end{array}$ & $\begin{array}{r}100 \\
18\end{array}$ \\
\hline $\begin{array}{r}1 \\
3 \\
7 \\
15 \\
30 \\
60 \\
90\end{array}$ & $\begin{array}{r}2000 \\
940 \\
505 \\
299 \\
182 \\
121 \\
94\end{array}$ & $\begin{array}{r}4660 \\
2260 \\
1150 \\
649 \\
373 \\
248 \\
192\end{array}$ & $\begin{array}{r}6900 \\
3480 \\
1740 \\
938 \\
525 \\
348 \\
269\end{array}$ & $\begin{array}{r}10100 \\
5410 \\
2650 \\
1350 \\
737 \\
490 \\
375\end{array}$ & $\begin{array}{r}12600 \\
7110 \\
3450 \\
1680 \\
905 \\
602 \\
459\end{array}$ & $\begin{array}{l}-- \\
-- \\
-- \\
-- \\
--\end{array}$ \\
\hline
\end{tabular}

DURATION TABLE OF DAILY MEAN FLOW FOR PERIOD OF RECORD 1960-85

DISCHARGE, IN FT'3/S, THAT WAS EQUALED OR EXCEEDED FOR INDICATED PERCENT OF TIME

\begin{tabular}{|c|c|c|c|c|c|c|c|c|c|c|c|c|c|c|}
\hline 58 & 108 & 158 & 208 & 258 & 308 & 408 & 508 & 608 & 708 & 758 & 808 & 858 & 908 & 958 \\
\hline 133 & 56 & 36 & 25 & 19 & 14 & 7.7 & 4.2 & 2.0 & .8 & .3 & .1 & 0.0 & 0.0 & 0.0 \\
\hline
\end{tabular}


RIO GRANDE BASIN

08254000 COSTILLA CREEK BELOW COSTILLA DAM, NM

LOCATION.--Lat $36^{\circ} 52 \cdot 26^{\prime \prime}$, long $105^{\circ} 16^{\prime} 47^{\circ}$, Taos County, Hydrologic Unit 13020101, in Sangre de Cristo Grant, on left bank $125 \mathrm{ft}$ downstream from Costilla Dam, $16 \mathrm{mi}$ southeast of Costilla, and at mile 34.7 .

DRAINAGE AREA. $--54.6 \mathrm{mi}^{2}$.

PERIOD OF RECORD.--April 1937 to current year (no winter records 1937-44, 1947-49). Monthly discharge only for some periods, published in WSP 1312. Prior to October 1951, published as "below reservoir near Costilla."

REVISED RECORDS. --WSP 1923: Drainage area.

GAGE.--Water-stage recorder and concrete control. Elevation of gage is 9,290 ft above National Geodetic Vertical Datum of 1929, from topographic map.

REMARKS.--Flow regulated by Costilla Reservoir (station 08253900). Diversions for irrigation of about 1,300 acres upstream from reservoir.

AVERAGE DISCHARGE.--39 years (water years 1945-47, 1950-85), $17.9 \mathrm{ft} / \mathrm{s}, 12,970 \mathrm{acre}-\mathrm{ft} / \mathrm{yr}$.

EXTREMES FOR PERIOD OF RECORD.--Maximum discharge, $301 \mathrm{ft}^{3} / \mathrm{s}$, June 19, 1979, gage height, $3.04 \mathrm{ft}$; no flow at times.

STATISTICAL SUMMARIES

MEAN MONTHLY AND MEAN ANNUAL DISChaRges $\begin{aligned} & 1945-47, \\ & 1950-85\end{aligned}$

\begin{tabular}{|c|c|c|c|c|c|c|}
\hline MONTH & $\begin{array}{l}\text { MINIMUM } \\
\left(\mathrm{FT}^{3} / \mathrm{S}\right)\end{array}$ & $\begin{array}{l}\text { MAXIMUM } \\
\left(\mathrm{FT}^{3} / \mathrm{S}\right)\end{array}$ & $\begin{array}{l}\text { MEAN } \\
\left(F^{3} T^{3} / S\right)\end{array}$ & $\begin{array}{l}\text { STAN- } \\
\text { DARD } \\
\text { DEVIA- } \\
\text { TION } \\
(\text { FT' } 3 / S)\end{array}$ & $\begin{array}{l}\text { COEFFI- } \\
\text { CIENT OF } \\
\text { VARI- } \\
\text { ATION }\end{array}$ & $\begin{array}{c}\text { PERCENT } \\
\text { OF } \\
\text { ANNUAL } \\
\text { RUNOFF }\end{array}$ \\
\hline $\begin{array}{l}\text { OCTOBER } \\
\text { NOVEMBER } \\
\text { DECEMBER } \\
\text { JANUARY } \\
\text { FEBRUARY } \\
\text { MARCH } \\
\text { APRIL } \\
\text { MAY } \\
\text { JUNE } \\
\text { JULY } \\
\text { AUGUST } \\
\text { SEPTEMBER }\end{array}$ & $\begin{array}{l}0.0 \\
0.0 \\
0.0 \\
0.0 \\
0.0 \\
0.0 \\
0.0 \\
0.0 \\
11 \\
17 \\
9.0 \\
2.4\end{array}$ & $\begin{array}{c}31 \\
11 \\
4.2 \\
2.8 \\
3.0 \\
6.0 \\
62 \\
173 \\
145 \\
172 \\
120 \\
84\end{array}$ & $\begin{array}{r}4.1 \\
.9 \\
.3 \\
.3 \\
.3 \\
.4 \\
3.4 \\
26 \\
64 \\
69 \\
52 \\
21\end{array}$ & $\begin{array}{r}6.2 \\
2.1 \\
.7 \\
.5 \\
.6 \\
1.1 \\
11 \\
35 \\
30 \\
33 \\
28 \\
14\end{array}$ & $\begin{array}{l}1.51 \\
2.39 \\
2.48 \\
2.12 \\
2.24 \\
2.63 \\
3.31 \\
1.38 \\
.47 \\
.47 \\
.54 \\
.67\end{array}$ & $\begin{array}{r}1.7 \\
.4 \\
.1 \\
.1 \\
.1 \\
.2 \\
1.4 \\
10.6 \\
26.7 \\
28.6 \\
21.5 \\
8.6\end{array}$ \\
\hline NNUAL & 8.6 & 37 & 18 & 7.0 & .39 & 100 \\
\hline
\end{tabular}

MAGNITUDE AND PROBABILITY OF ANNUAL LOW FLOW BASED ON PERIOD OF RECORD 1946-47, 1951-85

\begin{tabular}{|c|c|c|c|c|c|c|}
\hline \multirow{2}{*}{$\begin{array}{l}\text { PERIOD } \\
\text { (CON- } \\
\text { SECU- } \\
\text { TIVE } \\
\text { DAYS) }\end{array}$} & \multicolumn{6}{|c|}{$\begin{array}{l}\text { DISCHARGE, IN FT' }{ }^{3 / S,} \text { FOR INDICATED RECURRENCE } \\
\text { INTERVAL, IN YEARS, AND ANNUAL NON- } \\
\text { EXCEEDANCE PROBABILITY, IN PERCENT }\end{array}$} \\
\hline & $\begin{array}{c}2 \\
508\end{array}$ & $\begin{array}{c}5 \\
208\end{array}$ & $\begin{array}{l}10 \\
108\end{array}$ & $\begin{array}{l}20 \\
58\end{array}$ & $\begin{array}{l}50 \\
28\end{array}$ & $\begin{array}{r}100 \\
18\end{array}$ \\
\hline $\begin{array}{r}1 \\
3 \\
7 \\
14 \\
30 \\
60 \\
90 \\
120 \\
183\end{array}$ & $\begin{array}{r}0.0 \\
0.0 \\
0.0 \\
0.0 \\
0.0 \\
0.0 \\
0.0 \\
0.0 \\
.2\end{array}$ & $\begin{array}{l}0.0 \\
0.0 \\
0.0 \\
0.0 \\
0.0 \\
0.0 \\
0.0 \\
0.0 \\
0.0\end{array}$ & $\begin{array}{l}0.0 \\
0.0 \\
0.0 \\
0.0 \\
0.0 \\
0.0 \\
0.0 \\
0.0 \\
0.0\end{array}$ & $\begin{array}{l}0.0 \\
0.0 \\
0.0 \\
0.0 \\
0.0 \\
0.0 \\
0.0 \\
0.0 \\
0.0\end{array}$ & $\begin{array}{l}0.0 \\
0.0 \\
0.0 \\
0.0 \\
0.0 \\
0.0 \\
0.0 \\
0.0 \\
0.0\end{array}$ & $\begin{array}{l}0.0 \\
0.0 \\
0.0 \\
0.0 \\
0.0 \\
0.0 \\
0.0 \\
0.0 \\
0.0\end{array}$ \\
\hline
\end{tabular}

MAGNITUDE AND PROBABILITY OF ANNUAL HIGH FLOW BASED ON PERIOD OF RECORD 1945-47, 1950-85

\begin{tabular}{|c|c|c|c|c|c|c|}
\hline \multirow{2}{*}{$\begin{array}{l}\text { PERIOD } \\
\text { (CON- } \\
\text { SECU- } \\
\text { TIVE } \\
\text { DAYS) }\end{array}$} & \multicolumn{6}{|c|}{$\begin{array}{l}\text { DISCHARGE, IN FT } 3 \text { /S, FOR INDICATED RECURRENCE } \\
\text { INTERVAL, IN YEARS, AND ANNUAL } \\
\text { EXCEEDANCE PROBABILITY, IN PERCENT }\end{array}$} \\
\hline & 2 & $\begin{array}{c}5 \\
208\end{array}$ & $\begin{array}{l}10 \\
108\end{array}$ & $\begin{array}{l}25 \\
48\end{array}$ & $\begin{array}{l}50 \\
28\end{array}$ & $\begin{array}{r}100 \\
18\end{array}$ \\
\hline $\begin{array}{r}1 \\
3 \\
7 \\
15 \\
30 \\
60 \\
90\end{array}$ & $\begin{array}{r}123 \\
118 \\
87 \\
79 \\
72 \\
65 \\
56\end{array}$ & $\begin{array}{r}151 \\
145 \\
117 \\
106 \\
97 \\
86 \\
75\end{array}$ & $\begin{array}{r}168 \\
164 \\
142 \\
126 \\
114 \\
99 \\
88\end{array}$ & $\begin{array}{l}190 \\
188 \\
177 \\
155 \\
139 \\
117 \\
104\end{array}$ & $\begin{array}{l}206 \\
206 \\
206 \\
180 \\
158 \\
130 \\
115\end{array}$ & $\begin{array}{l}221 \\
225 \\
239 \\
206 \\
179 \\
143 \\
127\end{array}$ \\
\hline
\end{tabular}

DURATION TABLE OF DAILY MEAN FLOW FOR PERIOD OF RECORD 1945-47, 1950-85 DISCHARGE, IN FT' ${ }^{3} / \mathrm{S}$, THAT WAS EQUALED OR EXCEEDED FOR INDICATED PERCENT OF TIME

\begin{tabular}{|c|c|c|c|c|c|c|c|c|c|c|c|c|c|c|}
\hline 58 & 108 & 158 & 208 & 258 & 308 & 408 & 508 & 608 & 708 & 758 & 808 & 858 & 908 & 958 \\
\hline 97 & 71 & 51 & 34 & 21 & 12 & 1.8 & .4 & . 1 & . & 0.0 & 0.0 & 0.0 & 0.0 & 0.0 \\
\hline
\end{tabular}


RIO GRANDE BASIN

08255500 COSTILLA CREEK NEAR COSTILLA, NM

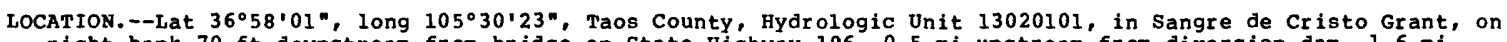
$r$ ight bank $70 \mathrm{ft}$ downstream from bridge on State Highway $196,0.5 \mathrm{mi}$ upstream from diversion dam, $1.6 \mathrm{mi}$ southeast of costilla, and at mile 15.9 .

DRA INAGE AREA. $--195 \mathrm{mi}^{2}$.

PERIOD OF RECORD,--March 1936 to current year (no winter records 1936-41, 1943). Monthly discharge for March 1943 and water-year estimate for 1943, published in WSP 1312 .

REVISED RECORDS.--WSP 1312: 1937-39(M).

GAGE.--Water-stage recorder. Concrete control since oct. 13, 1952. Elevation of gage is $7,900 \mathrm{ft}$ above National Geodetic Vertical Datum of 1929, from topographic map. Prior to June 18, 1944, at site $200 \mathrm{ft}$ downstream at different datum. June 18, 1944 to Sept. 30, 1964, at site 0.4 mi upstream at different datum.

REMARKS.--Flow regulated by Costilla Reservoir (station 08253900) $19 \mathrm{mi}$ upstream. Diversions for irrigation of about 2,000 acres upstream from station.

AVERAGE DISCHARGE.--25 years (water years 1961-85), $42.0 \mathrm{ft}^{3} / \mathrm{s}, 30,430 \mathrm{acre-ft} / \mathrm{yr}$.

EXTREMES FOR PERIOD OF RECORD.--Maximum discharge, $1,150 \mathrm{ft}^{3} / \mathrm{s}$, May 11, 1942 , gage height, $5.37 \mathrm{ft}$, site and datum then in use; minimum, $0.34 \mathrm{ft} / \mathrm{s}, \operatorname{Mar} .15,1969$, result of freezeup.

EXTREMES OUTSIDE PERIOD OF RECORD.--A major flood occurred in 1886, from information by local residents.

STATISTICAL SUMMARIES

MEAN MONTHLY AND MEAN ANNUAL DISCHARGES 1961-85

\begin{tabular}{|c|c|c|c|c|c|c|}
\hline MONTH & $\begin{array}{c}\text { MINIMUM } \\
\left(F T^{3} / S\right)\end{array}$ & $\begin{array}{l}\text { MAXIMUM } \\
\left(\mathrm{FT}^{3} / \mathrm{S}\right)\end{array}$ & $\begin{array}{l}\text { MEAN } \\
\left(\mathrm{FT}^{3} / \mathrm{S}\right)\end{array}$ & $\begin{array}{l}\text { STAN- } \\
\text { DARD } \\
\text { DEVIA- } \\
\text { TION } \\
\left(\text { FTI }^{3} / \mathrm{S}\right)\end{array}$ & $\begin{array}{l}\text { COEFFI- } \\
\text { CIENT OF } \\
\text { VARI- } \\
\text { ATION }\end{array}$ & $\begin{array}{c}\text { PERCENT } \\
\text { OF } \\
\text { ANNUAL } \\
\text { RUNOFF }\end{array}$ \\
\hline $\begin{array}{l}\text { OCTOBER } \\
\text { NOVEMBER } \\
\text { DECEMBER } \\
\text { JANUARY } \\
\text { FEBRUARY } \\
\text { MARCH } \\
\text { APRIL } \\
\text { MAY } \\
\text { JUNE } \\
\text { JULY } \\
\text { AUGUST } \\
\text { SEPTEMBER }\end{array}$ & $\begin{array}{l}4.9 \\
4.1 \\
3.7 \\
3.4 \\
3.4 \\
6.9 \\
15 \\
31 \\
48 \\
24 \\
17 \\
7.9\end{array}$ & $\begin{array}{r}45 \\
23 \\
19 \\
12 \\
13 \\
26 \\
124 \\
264 \\
342 \\
140 \\
137 \\
64\end{array}$ & $\begin{array}{c}13 \\
10 \\
7.6 \\
6.9 \\
8.0 \\
14 \\
45 \\
104 \\
113 \\
79 \\
65 \\
31\end{array}$ & $\begin{array}{l}9.3 \\
4.4 \\
3.3 \\
1.9 \\
2.3 \\
4.9 \\
29 \\
68 \\
72 \\
36 \\
32 \\
15\end{array}$ & $\begin{array}{l}.71 \\
.43 \\
.43 \\
.27 \\
.29 \\
.34 \\
.66 \\
.65 \\
.64 \\
.45 \\
.49 \\
.50\end{array}$ & $\begin{array}{r}2.6 \\
2.1 \\
1.5 \\
1.4 \\
1.6 \\
2.8 \\
9.0 \\
20.9 \\
22.7 \\
16.0 \\
13.1 \\
6.3\end{array}$ \\
\hline ANNUAL & 17 & 87 & 42 & 20 & .48 & 100 \\
\hline
\end{tabular}

MAGNITUDE AND PROBABILITY OF ANNUAL LOW FLOW BASED ON PERTOD OF RECORD $1962-85$

\begin{tabular}{|c|c|c|c|c|c|c|}
\hline \multirow{2}{*}{$\begin{array}{l}\text { PERIOD } \\
\text { (CON- } \\
\text { SECU- } \\
\text { TIVE } \\
\text { DAYS) }\end{array}$} & \multicolumn{6}{|c|}{$\begin{array}{l}\text { DISCHARGE, IN FT3/S, FOR INDICATED RECURRENCE } \\
\text { INTERVAL, IN YEARS, AND ANNUAL NON- } \\
\text { EXCEEDANCE PROBABILITY, IN PERCENT }\end{array}$} \\
\hline & $\begin{array}{c}2 \\
508\end{array}$ & $\begin{array}{c}5 \\
208\end{array}$ & $\begin{array}{l}10 \\
108\end{array}$ & $\begin{array}{l}20 \\
58\end{array}$ & $\begin{array}{l}50 \\
28\end{array}$ & $\begin{array}{r}100 \\
18\end{array}$ \\
\hline $\begin{array}{r}1 \\
3 \\
7 \\
14 \\
30 \\
60 \\
90 \\
120 \\
183\end{array}$ & $\begin{array}{l}3.7 \\
4.0 \\
4.4 \\
4.9 \\
5.4 \\
6.2 \\
6.9 \\
7.5 \\
9.2\end{array}$ & $\begin{array}{l}2.9 \\
3.1 \\
3.4 \\
3.7 \\
4.3 \\
4.9 \\
5.4 \\
5.9 \\
6.8\end{array}$ & $\begin{array}{l}2.5 \\
2.7 \\
3.0 \\
3.3 \\
3.8 \\
4.3 \\
4.7 \\
5.1 \\
5.9\end{array}$ & $\begin{array}{l}2.3 \\
2.4 \\
2.7 \\
2.9 \\
3.5 \\
3.8 \\
4.2 \\
4.6 \\
5.2\end{array}$ & $\begin{array}{l}2.0 \\
2.1 \\
2.4 \\
2.6 \\
3.2 \\
3.4 \\
3.7 \\
4.0 \\
4.5\end{array}$ & 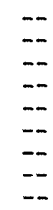 \\
\hline
\end{tabular}

MAGNITUDE AND PROBABILITY OF ANNUAL HIGH FLOW BASED ON PERIOD OF RECORD 1961-85

\begin{tabular}{|c|c|c|c|c|c|c|}
\hline \multirow{2}{*}{$\begin{array}{l}\text { PERIOD } \\
\text { (CON- } \\
\text { SECU- } \\
\text { TIVE } \\
\text { DAYS) }\end{array}$} & \multicolumn{6}{|c|}{$\begin{array}{l}\text { DISCHARGE, IN FT'3/S, FOR INDICATED RECURRENCE } \\
\text { INTERVAL, IN YEARS, AND ANNUAL } \\
\text { EXCEEDANCE PROBABILITY, IN PERCENT }\end{array}$} \\
\hline & $\begin{array}{c}2 \\
508\end{array}$ & $\begin{array}{c}5 \\
208\end{array}$ & $\begin{array}{l}10 \\
108\end{array}$ & $\begin{array}{l}25 \\
48\end{array}$ & $\begin{array}{l}50 \\
28\end{array}$ & $\begin{array}{r}100 \\
18\end{array}$ \\
\hline $\begin{array}{r}1 \\
3 \\
7 \\
15 \\
30 \\
60 \\
90\end{array}$ & $\begin{array}{r}155 \\
146 \\
126 \\
115 \\
106 \\
95 \\
88\end{array}$ & $\begin{array}{l}251 \\
237 \\
215 \\
193 \\
176 \\
153 \\
139\end{array}$ & $\begin{array}{l}335 \\
317 \\
294 \\
263 \\
238 \\
201 \\
178\end{array}$ & $\begin{array}{l}469 \\
444 \\
419 \\
376 \\
337 \\
275 \\
236\end{array}$ & $\begin{array}{l}592 \\
561 \\
535 \\
483 \\
428 \\
340 \\
284\end{array}$ & $\begin{array}{l}-- \\
m \\
-- \\
-- \\
--\end{array}$ \\
\hline
\end{tabular}

DURATION TABLE OF DAILY MEAN FLOW FOR PERIOD OF RECORD 1961-85

DISCHARGE, IN FT $3 / S$, THAT WAS EQUALED OR EXCEEDED FOR INDICATED PERCENT OF TIME

\begin{tabular}{|c|c|c|c|c|c|c|c|c|c|c|c|c|c|c|}
\hline 58 & 108 & 158 & 208 & 258 & 308 & 408 & 508 & 608 & 708 & 758 & 808 & 858 & 908 & 958 \\
\hline 149 & 110 & 90 & 75 & 61 & 45 & 26 & 17 & 12 & 9.0 & 8.2 & 7.4 & 6.7 & 5.8 & 4.5 \\
\hline
\end{tabular}


RIO GRANDE BASIN

08263000 LATIR CREEK NEAR CERRO, NM

LOCATION.--Lat $36^{\circ} 49^{\prime} 45^{\prime \prime}$, long $105^{\circ} 32^{\prime} 50^{\prime \prime}$, in SWłSW sec.15, T. 30 N., R.13 E. Taos County, in Carson National Forest, on right bank at mouth of canyon, $100 \mathrm{ft}$ upstream from heading of Cerro community ditch and 6.3 miles nor theast of Cerro.

DRAINAGE AREA. $--10.5 \mathrm{mi}^{2}$.

PERIOD OF RECORD.--June 1937 to September 1970 (discontinued). Monthly discharge only for some periods, published in WSP 1312. Records for April and May 1937, published in wsp 828, have been found to be unreliable (unknown portion of flow bypassing gage) and should not be used.

REVISED RECORDS.--WSP 1312: 1938(M). WRD N.Mex. 1968: Drainage area. See also PERIOD OF RECORD.

GAGE.-Water-stage recorder and Parshall flume. Altitude of gage is $8,280 \mathrm{ft}$ (from topographic map).

REMARKS. - -No diversion above station.

AVERAGE DISCHARGE. --25 years (water years 1946-70), $5.40 \mathrm{ft}{ }^{3} / \mathrm{s}(3,910 \mathrm{acre}-\mathrm{ft} / \mathrm{year}$ ).

EXTREMES FOR PFRIOD OF RECORD,--Maximum discharge, $126 \mathrm{ft}^{3} / \mathrm{s}$ June 18,1965 , from rating curve extended above 57

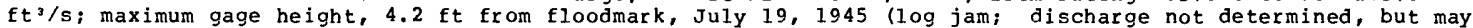

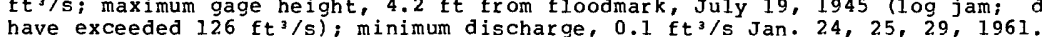

STATISTICAL SUMMARIES

MEAN MONTHLY AND MEAN ANNUAL DISCHARGES 1946-70

MAGNITUDE AND PROBABILITY OF ANNUAL LOW FLOW BASED ON PERIOD OF RECORD $1947-70$

\begin{tabular}{|c|c|c|c|c|c|c|}
\hline MONTH & $\begin{array}{l}\text { MINIMUM } \\
\left(\mathrm{FT}^{3} / \mathrm{S}\right)\end{array}$ & $\begin{array}{l}\text { MAXIMUM } \\
\left(\mathrm{FT}^{3} / \mathrm{S}\right)\end{array}$ & $\begin{array}{l}\text { MEAN } \\
\left(\mathrm{FT}^{3} / \mathrm{S}\right)\end{array}$ & $\begin{array}{l}\text { STAN- } \\
\text { DARD } \\
\text { DEVIA- } \\
\text { TION } \\
\left(\mathrm{FT}^{3} / \mathrm{S}\right)\end{array}$ & $\begin{array}{l}\text { COEFFI- } \\
\text { CIENT OF } \\
\text { VARI- } \\
\text { ATION }\end{array}$ & $\begin{array}{c}\text { PERCENT } \\
\text { OF } \\
\text { ANNUAL } \\
\text { RUNOF F }\end{array}$ \\
\hline $\begin{array}{l}\text { OCTOBER } \\
\text { NOVEMBER } \\
\text { DECEMBER } \\
\text { JANUARY } \\
\text { FEBRUARY } \\
\text { MARCH } \\
\text { APRIL } \\
\text { MAY } \\
\text { JUNE } \\
\text { JULY } \\
\text { AUGUST } \\
\text { SEPTEMBER }\end{array}$ & $\begin{array}{r}2.1 \\
1.9 \\
1.5 \\
.6 \\
1.5 \\
1.5 \\
1.8 \\
4.0 \\
3.4 \\
2.3 \\
3.0 \\
2.1\end{array}$ & $\begin{array}{r}6.4 \\
4.5 \\
3.0 \\
2.9 \\
3.1 \\
3.1 \\
7.2 \\
29 \\
42 . \\
21 \\
13 \\
7.6\end{array}$ & $\begin{array}{r}3.4 \\
2.8 \\
2.3 \\
2.0 \\
2.2 \\
2.3 \\
4.2 \\
11 \\
17 \\
7.5 \\
5.9 \\
4.5\end{array}$ & $\begin{array}{r}1.0 \\
.7 \\
.4 \\
.5 \\
.4 \\
.4 \\
1.3 \\
5.5 \\
11 \\
4.4 \\
2.3 \\
1.5\end{array}$ & $\begin{array}{l}.29 \\
.24 \\
.19 \\
.25 \\
.18 \\
.18 \\
.32 \\
.51 \\
.64 \\
.58 \\
.38 \\
.34\end{array}$ & $\begin{array}{r}5.2 \\
4.3 \\
3.5 \\
3.2 \\
3.4 \\
3.6 \\
6.5 \\
16.9 \\
2.5 .8 \\
11.6 \\
9.2 \\
6.9\end{array}$ \\
\hline NNUAL & 2.9 & 8.8 & 5.4 & 1.8 & .33 & 100 \\
\hline
\end{tabular}

\begin{tabular}{|c|c|c|c|c|c|c|}
\hline $\begin{array}{l}\text { PERIOD } \\
\text { (CON- } \\
\text { SECU- }\end{array}$ & \multicolumn{6}{|c|}{$\begin{array}{l}\text { INTERVAL, IN YEARS, AND ANNUAL NON- } \\
\text { EXCEEDANCE PROBABILITY, IN PERCENT }\end{array}$} \\
\hline $\begin{array}{l}\text { TIVE } \\
\text { DAYS) }\end{array}$ & $\begin{array}{c}2 \\
508\end{array}$ & $\begin{array}{c}5 \\
20 \%\end{array}$ & $\begin{array}{l}10 \\
108\end{array}$ & $\begin{array}{l}20 \\
58\end{array}$ & $\begin{array}{l}50 \\
28\end{array}$ & $\begin{array}{r}100 \\
1 \%\end{array}$ \\
\hline $\begin{array}{r}1 \\
3 \\
7 \\
14 \\
30 \\
60 \\
90 \\
120 \\
183\end{array}$ & $\begin{array}{l}1.5 \\
1.6 \\
1.8 \\
2.0 \\
2.0 \\
2.0 \\
2.0 \\
2.1 \\
2.4\end{array}$ & $\begin{array}{r}.9 \\
1.1 \\
1.3 \\
1.4 \\
1.5 \\
1.7 \\
1.7 \\
1.8 \\
2.0\end{array}$ & $\begin{array}{r}.6 \\
.8 \\
.9 \\
1.1 \\
1.2 \\
1.5 \\
1.6 \\
1.7 \\
1.9\end{array}$ & $\begin{array}{r}.4 \\
.6 \\
.7 \\
.8 \\
1.0 \\
1.4 \\
1.5 \\
1.6 \\
1.8\end{array}$ & $\begin{array}{r}.3 \\
.4 \\
.4 \\
.5 \\
.7 \\
1.2 \\
1.4 \\
1.5 \\
1.6\end{array}$ & \\
\hline
\end{tabular}

MAGNITUDE AND PROBABILITY OF ANNUAL HIGH FLOW BASED ON PERIOD OF RECORD $1946-70$

\begin{tabular}{|c|c|c|c|c|c|c|}
\hline \multirow{3}{*}{$\begin{array}{l}\text { PERIOD } \\
\text { (CON- } \\
\text { SECU- } \\
\text { TIVE } \\
\text { DAYS) }\end{array}$} & \multicolumn{6}{|c|}{$\begin{array}{l}\text { DISCHARGE, IN FT }{ }^{3} / \mathrm{S} \text {, FOR INDICATED RECURRENC } \\
\text { INTERVAL, IN YEARS, AND ANNUAL } \\
\text { EXCEEDANCE PROBABILITY, IN PERCENT }\end{array}$} \\
\hline & & & - & $--\infty$ & $--n$ & \\
\hline & $\begin{array}{c}2 \\
508\end{array}$ & $\begin{array}{c}5 \\
208\end{array}$ & $\begin{array}{l}10 \\
108\end{array}$ & $\begin{array}{l}25 \\
48\end{array}$ & $\begin{array}{l}50 \\
28\end{array}$ & $\begin{array}{r}100 \\
18\end{array}$ \\
\hline 1 & 24 & 40 & 54 & 73 & 89 & -- \\
\hline 3 & 22 & 37 & 50 & 67 & 82. & \\
\hline 7 & 20 & 35 & 46 & 62. & 75 & -- \\
\hline 15 & 18 & 31 & 41 & 55 & 66 & - \\
\hline 30 & 16 & 27 & 34 & 45 & 53 & \\
\hline 60 & 13 & 2.1 & 26 & 32. & 37 & - \\
\hline 90 & 11 & 17 & 2.1 & 26 & 30 & \\
\hline
\end{tabular}

DURATION TABLE OF DAILY MEAN FLOW FOR PERIOD OF RECORD 1946-70 DISCHARGE, IN FT ${ }^{3} / \mathrm{S}$, THAT WAS EQUALED OR EXCEEDED FOR INDICATED PERCENT OF TIME

\begin{tabular}{|c|c|c|c|c|c|c|c|c|c|c|c|c|c|c|}
\hline 58 & 108 & 158 & 208 & 258 & 308 & 408 & 508 & 608 & 708 & 758 & $80 \%$ & 858 & 908 & 958 \\
\hline 18 & 12. & 8.6 & 6.8 & 5.7 & 5.0 & 3.9 & 3.2 & 2.8 & 2.5 & 2.4 & 2.2 & 2.1 & 1.9 & 1. \\
\hline
\end{tabular}


RIO GRANDE BASIN

08263500 RIO GRANDE NEAR CERRO, NM

LOCATION.--Lat $36^{\circ} 44^{\prime} 24^{\prime \prime}$, long $105^{\circ} 40^{\prime} 59^{\prime \prime}$, in NWłNE sec.20, T.29 N., R.12 E., Taos County, Hydrologic Unit 13020101 , on left bank $4 \mathrm{mi}$ southwest of Cerro, $5.5 \mathrm{mi}$ northwest of Questa, $7.4 \mathrm{mi}$ upstream from Red River, and at mile $1,693.1$.

DRAINAGE AREA. $-8,440 \mathrm{mi}^{2}$, approximately, including 2,940 $\mathrm{mi}^{2}$ in closed basin in San Luis Valley, co.

PERIOD OF RECORD.--May 1948 to current year.

REVISED RECORDS. --WDR NM-80-1: 1978 (M).

GAGE.--Water-stage recorder. Elevation of gage is 7,110 ft above National Geodetic Vertical Datum of 1929 , from topographic map.

REMARKS.--Diversions upstream from station for irrigation of about 620,000 acres in Colorado and 7,000 acres in New Mexico.

AVERAGE DISCHARGE. --37 years (water years 1949-85), $429 \mathrm{ft} / \mathrm{s}, 310,800$ acre-ft/yr.

EXTREMES FOR PERIOD OF RECORD.--Maximum discharge, 9,740 ft/s, June 22, 1949, gage height, 15.78 ft; minimum, about $40 \mathrm{ft}^{3} / \mathrm{s}$, Sept. $10,11,1977$.

STATISTICAL SUMMARIES

MEAN MONTHLY AND MEAN ANNUAL DISCHARGES 1949-85

\begin{tabular}{|c|c|c|c|c|c|c|}
\hline MONTH & $\begin{array}{l}\text { MINIMUM } \\
\left(\mathrm{FT}^{3} / \mathrm{S}\right)\end{array}$ & $\begin{array}{c}\text { MAXIMUM } \\
\left(\mathrm{FT}^{3} / \mathrm{S}\right)\end{array}$ & $\begin{array}{l}\text { MEAN } \\
\left(\mathrm{FT} \mathrm{T}^{3} / \mathrm{S}\right)\end{array}$ & $\begin{array}{l}\text { STAN- } \\
\text { DARD } \\
\text { DEVIA- } \\
\text { TION } \\
\left(\mathrm{FT}^{3} / \mathrm{S}\right)\end{array}$ & $\begin{array}{l}\text { COEFFI- } \\
\text { CIENT OF } \\
\text { VARI- } \\
\text { ATION }\end{array}$ & $\begin{array}{c}\text { PERCENT } \\
\text { OF } \\
\text { ANNUAL } \\
\text { RUNOFF }\end{array}$ \\
\hline $\begin{array}{l}\text { OCTOBER } \\
\text { NOVEMBER } \\
\text { DECEMBER } \\
\text { JANUARY } \\
\text { FEBRUARY } \\
\text { MARCH } \\
\text { APRIL } \\
\text { MAY } \\
\text { JUNE } \\
\text { JULY } \\
\text { AUGUST } \\
\text { SEPTEMBER }\end{array}$ & $\begin{array}{r}53 \\
88 \\
100 \\
116 \\
140 \\
110 \\
107 \\
84 \\
58 \\
52 \\
48 \\
45\end{array}$ & $\begin{array}{r}720 \\
867 \\
522 \\
401 \\
517 \\
721 \\
2329 \\
3740 \\
4400 \\
2161 \\
957 \\
804\end{array}$ & $\begin{array}{r}191 \\
335 \\
272 \\
266 \\
324 \\
411 \\
460 \\
885 \\
1114 \\
469 \\
248 \\
172\end{array}$ & $\begin{array}{r}164 \\
225 \\
103 \\
75 \\
95 \\
162 \\
422 \\
907 \\
1203 \\
563 \\
235 \\
168\end{array}$ & $\begin{array}{r}.86 \\
.67 \\
.38 \\
.28 \\
.29 \\
.39 \\
.92 \\
1.02 \\
1.08 \\
1.20 \\
.95 \\
.98\end{array}$ & $\begin{array}{r}3.7 \\
6.5 \\
5.3 \\
5.2 \\
6.3 \\
8.0 \\
8.9 \\
17.2 \\
21.6 \\
9.1 \\
4.8 \\
3.3\end{array}$ \\
\hline NNUAL & 112 & 1187 & 429 & 258 & .60 & 100 \\
\hline
\end{tabular}

MAGNITUDE AND PROBABILITY OF ANNUAL LOW FLOW BASED ON PERIOD OF RECORD 1950-85

\begin{tabular}{|c|c|c|c|c|c|c|}
\hline \multirow{2}{*}{$\begin{array}{l}\text { PERIOD } \\
\text { (CON- } \\
\text { SECU- } \\
\text { TIVE } \\
\text { DAYS) }\end{array}$} & \multicolumn{6}{|c|}{$\begin{array}{l}\text { DISCHARGE, IN } \mathrm{FT}^{3} / \mathrm{S} \text {, FOR INDICATED RECURRENCE } \\
\text { INTERVAL, IN YEARS, AND ANNUAL NON- } \\
\text { EXCEEDANCE PROBABILITY, IN PERCENT }\end{array}$} \\
\hline & $\begin{array}{c}2 \\
508\end{array}$ & $\begin{array}{c}5 \\
208\end{array}$ & $\begin{array}{l}10 \\
108\end{array}$ & $\begin{array}{l}20 \\
58\end{array}$ & $\begin{array}{l}50 \\
28\end{array}$ & $\begin{array}{r}100 \\
18\end{array}$ \\
\hline $\begin{array}{r}1 \\
3 \\
7 \\
14 \\
30 \\
60 \\
90 \\
120 \\
183\end{array}$ & $\begin{array}{r}71 \\
72 \\
76 \\
80 \\
86 \\
100 \\
118 \\
134 \\
171\end{array}$ & $\begin{array}{r}51 \\
52 \\
54 \\
55 \\
59 \\
64 \\
71 \\
78 \\
100\end{array}$ & $\begin{array}{l}45 \\
45 \\
46 \\
48 \\
50 \\
53 \\
56 \\
60 \\
75\end{array}$ & $\begin{array}{l}41 \\
41 \\
42 \\
43 \\
44 \\
45 \\
46 \\
49 \\
60\end{array}$ & $\begin{array}{l}38 \\
38 \\
38 \\
38 \\
39 \\
39 \\
38 \\
39 \\
46\end{array}$ & $\begin{array}{l}-- \\
-- \\
-- \\
-- \\
-- \\
-- \\
--\end{array}$ \\
\hline
\end{tabular}

MAGNITUDE AND PROBABILITY OF ANNUAL HIGH FLO BASED ON PERIOD OF RECORD $1949-85$

\begin{tabular}{|c|c|c|c|c|c|c|}
\hline \multirow{2}{*}{$\begin{array}{l}\text { PERI OD } \\
\text { (CON- } \\
\text { SECU- }- \\
\text { TIVE } \\
\text { DAYS) }\end{array}$} & \multicolumn{6}{|c|}{$\begin{array}{l}\text { DISCHARGE, IN FT'/S, FOR INDICATED RECURRENC } \\
\text { INTERVAL, IN YEARS, AND ANNUAL } \\
\text { EXCEEDANCE' PROBABILITY, IN PERCENT }\end{array}$} \\
\hline & $\begin{array}{c}2 \\
508\end{array}$ & $\begin{array}{c}5 \\
208\end{array}$ & $\begin{array}{l}10 \\
108\end{array}$ & $\begin{array}{l}25 \\
48\end{array}$ & $\begin{array}{l}50 \\
28\end{array}$ & $\begin{array}{r}100 \\
18\end{array}$ \\
\hline $\begin{array}{r}1 \\
3 \\
7 \\
15 \\
30 \\
60 \\
90\end{array}$ & $\begin{array}{r}1490 \\
1420 \\
1290 \\
1110 \\
971 \\
809 \\
700\end{array}$ & $\begin{array}{l}3170 \\
3040 \\
2750 \\
2380 \\
2090 \\
1720 \\
1430\end{array}$ & $\begin{array}{r}4690 \\
4490 \\
4090 \\
3580 \\
3140 \\
571 \\
2090\end{array}$ & $\begin{array}{l}7120 \\
6800 \\
6240 \\
5550 \\
4880 \\
3970 \\
3170\end{array}$ & $\begin{array}{l}9320 \\
8880 \\
8200 \\
7400 \\
6510 \\
5290 \\
4150\end{array}$ & $\begin{array}{l}-- \\
-- \\
-- \\
-- \\
-- \\
-- \\
--\end{array}$ \\
\hline
\end{tabular}

DURATION TABLE OF DAILY MEAN FLOW FOR PERIOD OF RECORD 1949-85

DISCHARGE, IN FT'3/S, THAT WAS EOUALED OR EXCEEDED FOR INDICATED PERCENT OF TIME

\begin{tabular}{|c|c|c|c|c|c|c|c|c|c|c|c|c|c|c|}
\hline 58 & 108 & 158 & 208 & 258 & 308 & 408 & 508 & 608 & 708 & 758 & 808 & 858 & 908 & 958 \\
\hline 1580 & 898 & 633 & 518 & 446 & 387 & 311 & 253 & 193 & 143 & 124 & 107 & 91 & 76 & 61 \\
\hline
\end{tabular}


LOCATION.--Lat $36^{\circ} 37^{\prime} 30^{\prime \prime}$, long $105^{\circ} 23^{\prime} 20^{n}$, in NEt sec.36, T.28 N., R.14 E. (projected), on right bank 100 ft downstream from confluence of Middle and East Forks and 6 miles south of Red River.

DRAINAGE AREA. $--19.1 \mathrm{mi}^{2}$.

PERIOD OF RECORD.--July 1940 to September 1964, discontinued (no winter records 1956 to 1962 ). Prior to October 1947, published as "Rio Colorado near Red River."

GAGE.--Water-stage recorder. Datum of gage is $9,394.2 \mathrm{ft}$ above mean sea level (plane-table levels by Division of Water and Power)

REMARKS. - None.

AVERAGE DISCHARGE. --17 years $(1940-55,1962-64), 17.5 \mathrm{ft}^{3} / \mathrm{s}(12,670 \mathrm{acre}-\mathrm{ft} /$ year $)$.

EXTREMES FOR PERIOD OF RECORD.--Maximum discharge, $264 \mathrm{ft}^{3} / \mathrm{s}$ June 12, 1952 (gage height, $3.16 \mathrm{ft}$ ); maximum gage height recorded, $4.19 \mathrm{ft}$ during period Jan. 9 to May 11,1960 (ice jam); minimum daily discharge determined, 2.0 $\mathrm{ft}^{3} / \mathrm{s}$ Mar. 6-9, 1965, Jan. 12, 13, 1963, Dec. 13, 1963, Feb. 28, 1964 .

STATISTICAL SUMMARIES

MEAN MONTHLY AND MEAN ANNUAL DISChARGES 1944-55

\begin{tabular}{|c|c|c|c|c|c|c|}
\hline MONTH & $\begin{array}{c}\text { MINIMUM } \\
\left(\mathrm{FT}^{3} / \mathrm{S}\right)\end{array}$ & $\begin{array}{c}\text { MAXIMUM } \\
\left(\mathrm{FT}^{3} / \mathrm{S}\right)\end{array}$ & $\begin{array}{l}\operatorname{MEAN} \\
\left(\mathrm{FT}^{3} / \mathrm{S}\right)\end{array}$ & $\begin{array}{l}\text { STAN- } \\
\text { DARD } \\
\text { DEVIA- } \\
\text { TION } \\
\left(\mathrm{FT}^{3} / \mathrm{S}\right)\end{array}$ & $\begin{array}{l}\text { COEFFI- } \\
\text { CIENT OF } \\
\text { VARI- } \\
\text { ATION }\end{array}$ & $\begin{array}{c}\text { PERCENT } \\
\text { OF } \\
\text { ANNUAL } \\
\text { RUNOFF }\end{array}$ \\
\hline $\begin{array}{l}\text { OCTOBER } \\
\text { NOVEMBER } \\
\text { DECEMBER } \\
\text { JANUARY } \\
\text { FEBRUARY } \\
\text { MARCH } \\
\text { APRIL } \\
\text { MAY } \\
\text { JUNE } \\
\text { JULY } \\
\text { AUGUST } \\
\text { SEPTEMBER }\end{array}$ & $\begin{array}{l}4.5 \\
4.1 \\
3.2 \\
2.8 \\
2.4 \\
2.3 \\
4.9 \\
21 \\
16 \\
9.5 \\
7.7 \\
4.9\end{array}$ & $\begin{array}{r}12 \\
8.6 \\
6.7 \\
5.1 \\
5.0 \\
6.0 \\
16 \\
72 \\
136 \\
46 \\
28 \\
18\end{array}$ & $\begin{array}{l}7.3 \\
5.7 \\
4.7 \\
4.1 \\
3.9 \\
4.3 \\
11 \\
42 \\
67 \\
25 \\
15 \\
9.4\end{array}$ & $\begin{array}{r}1.9 \\
1.3 \\
1.0 \\
.7 \\
.9 \\
1.1 \\
3.7 \\
18 \\
36 \\
12 \\
5.7 \\
3.7\end{array}$ & $\begin{array}{l}.26 \\
.23 \\
.22 \\
.17 \\
.22 \\
.25 \\
.33 \\
.43 \\
.53 \\
.49 \\
.39 \\
.40\end{array}$ & $\begin{array}{r}3.7 \\
2.9 \\
2.4 \\
2.1 \\
2.0 \\
2.2 \\
5.5 \\
21.0 \\
33.7 \\
12.6 \\
7.4 \\
4.8\end{array}$ \\
\hline NUAL & 9.5 & 26 & 17 & 5.5 & .33 & 100 \\
\hline
\end{tabular}

MAGNITUDE AND PROBABILITY OF ANNUAL LOW FLOW BASED ON PERIOD OF RECORD 1945-55

\begin{tabular}{|c|c|c|c|c|c|c|}
\hline \multirow{2}{*}{$\begin{array}{l}\text { PERIOD } \\
\text { (CON- } \\
\text { SECU- } \\
\text { TIVE } \\
\text { DAYS) }\end{array}$} & \multicolumn{6}{|c|}{$\begin{array}{l}\text { DISCHARGE, IN } \text { FT }^{3 / S} \text {, FOR INDICATED RECURRENCE } \\
\text { INTERVAL, IN YEARS, AND ANNUAL NON- } \\
\text { EXCEEDANCE PROBABIL ITY, IN PERCENT }\end{array}$} \\
\hline & $\begin{array}{c}2 \\
508\end{array}$ & $\begin{array}{c}5 \\
208\end{array}$ & $\begin{array}{l}10 \\
108\end{array}$ & $\begin{array}{l}20 \\
58\end{array}$ & $\begin{array}{l}50 \\
28\end{array}$ & $\begin{array}{r}100 \\
1\end{array}$ \\
\hline $\begin{array}{r}1 \\
3 \\
7 \\
14 \\
30 \\
60 \\
90 \\
120 \\
183\end{array}$ & $\begin{array}{l}3.2 \\
3.4 \\
3.6 \\
3.7 \\
3.8 \\
3.9 \\
4.0 \\
4.2 \\
4.9\end{array}$ & $\begin{array}{l}2.6 \\
2.7 \\
2.8 \\
2.9 \\
3.0 \\
3.1 \\
3.3 \\
3.4 \\
4.0\end{array}$ & $\begin{array}{l}2.3 \\
2.4 \\
2.4 \\
2.5 \\
2.6 \\
2.7 \\
2.9 \\
3.0 \\
3.6\end{array}$ & $\begin{array}{l}2.0 \\
2.1 \\
2.1 \\
2.2 \\
2.2 \\
2.4 \\
2.5 \\
2.7 \\
3.3\end{array}$ & $\begin{array}{l}-- \\
-- \\
-- \\
-- \\
-- \\
-- \\
-- \\
--\end{array}$ & \\
\hline
\end{tabular}

MAGNITUDE AND PROBABILITY OF ANNUAL HIGH FLOW BASED ON PERIOD OF RECORD $1944-55$

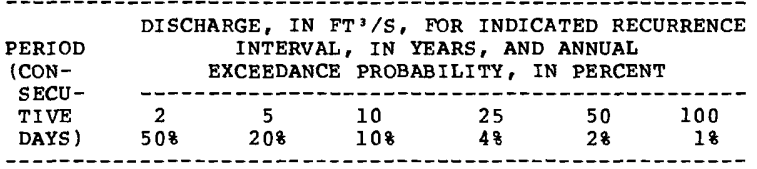

$\begin{array}{rrrrrrr}1 & 93 & 142 & 173 & -- & -- & -- \\ 3 & 89 & 137 & 169 & -- & -- & - \\ 7 & 83 & 128 & 157 & -- & -- & - \\ 15 & 76 & 117 & 142 & -- & -- & - \\ 30 & 67 & 101 & 121 & -- & -- & - \\ 60 & 53 & 78 & 94 & -- & -- & -- \\ 90 & 42 & 62 & 75 & -- & -- & --\end{array}$

DURATION TABLE OF DAILY MEAN FLOW FOR PERIOD OF RECORD 1944-55 DISCHARGE, IN $\mathrm{FT}^{3} / \mathrm{S}$, THAT WAS EQUALED OR EXCEEDED FOR INDICATED PERCENT OF TIME

\begin{tabular}{|c|c|c|c|c|c|c|c|c|c|c|c|c|c|c|}
\hline 58 & 108 & 158 & 208 & 258 & 308 & 408 & 508 & 608 & 708 & 758 & 808 & 858 & 908 & 958 \\
\hline 71 & 43 & 30 & 22 & 17 & 14 & 9.3 & 7.0 & 5.8 & 5.0 & 4.6 & 4.3 & 4.0 & 3.7 & \\
\hline
\end{tabular}


LOCATION.--Lat 36.42'12", long $105^{\circ} 34^{\prime} 04^{\prime \prime}$, in NEłSEk sec.32, T.29 N., R.13 E. (projected), Taos County, Hydrologic Unit 13020101 , in Carson National Forest, on left bank $1.3 \mathrm{mi}$ upstream from Cabresto Creek, $1.5 \mathrm{mi}$ east of Questa, and at mile 9.0 .

DRAINAGE AREA. $--113 \mathrm{mi}^{2}$.

PERIOD OF RECORD.--April to October 1910 and January to September 1911 (gage heights and discharge measurements only), October 1912 to March 1924, May 1924 to September 1925, January to March 1926, September 1926 to current year. Monthly discharge only for some periods, published in wSP 1312. Published as Rio Colorado above Questa $1910-11,1926-30$, and as Rio Colorado near Questa 1912-25, 1930-48.

REVISED RECORDS,--WSP 808: 1935. WSP 1392: 1913, 1932, 1941, 1947-48. wSP 1712: Drainage area.

GAGE.--Water-stage recorder. Wood or concrete control since Mar. 20, 1936. Datum of gage is $7,451.92$ ft above National Geodetic Vertical Datum of 1929. See WSP 1923 for history of changes prior to Oct. 4 , 1938.

REMARKS.--Diversions for irrigation of a few hundred acres upstream from station. Figures of discharge do not include flow in South ditch which diverts from left bank 1,500 ft upstream and bypasses gage for irrigation and stock water downstream. Since January 1966 surface-and ground-water diversions by Molybdenum Corp. of America (Molycorp) refinery $5.5 \mathrm{mi}$ upstream bypass gage in tailings pipelines on left bank and discharge into settling pond $3 \mathrm{mi}$ downstream. Effluent from this pond enters Red River as surface water and is included in discharge at Red River below Fish Hatchery, near Questa (station 08266820).

AVERAGE DISCHARGE.--56 years (water years 1925, 1931-85), $45.0 \mathrm{ft}$ / $/ \mathrm{s}, 32,600$ acre-ft/yr, prior to extensive upstream diversions by Molycorp.

20 years (water years 1966-85), 38.1 ft3/s, 27,600 acre-ft/yr.

EXTREMES FOR PERIOD OF RECORD (SINCE 1929).--Maximum discharge, $886 \mathrm{ft} / \mathrm{s}$, May 25, 1942, from rating curve extended above $450 \mathrm{ft} / \mathrm{s}$; maximum gage height, $5.80 \mathrm{ft}$, June 8,1979 ; minimum discharge, $0.60 \mathrm{ft} / \mathrm{s}, \mathrm{Jan}$. 21,1981, result of freezeup.

The maximum discharge of May 25, 1942, may have been equaled or exceeded by the peak of June 15, 1921.

STATISTICAL SUMMARIES

MEAN MONTHLY AND MEAN ANNUAL DISCHARgES 1925, 1931-85

\begin{tabular}{|c|c|c|c|c|c|c|c|c|c|c|c|c|c|}
\hline \multirow[b]{2}{*}{ MONTH } & \multirow{2}{*}{$\begin{array}{l}\text { MINIMUM } \\
\left(\mathrm{FT}^{3} / \mathrm{S}\right)\end{array}$} & \multirow{2}{*}{$\begin{array}{c}\text { MAXIMUM } \\
\left(\mathrm{FT}^{3} / \mathrm{S}\right)\end{array}$} & \multirow{2}{*}{$\begin{array}{l}\text { MEAN } \\
\left(\mathrm{FT}^{3} / \mathrm{S}\right)\end{array}$} & \multirow{2}{*}{$\begin{array}{l}\text { STAN- } \\
\text { DARD } \\
\text { DEVIA- } \\
\text { TION } \\
\left(\mathrm{FT}^{3} / \mathrm{S}\right)\end{array}$} & \multirow{2}{*}{$\begin{array}{l}\text { COEFFI- } \\
\text { CIENT OF } \\
\text { VARI- } \\
\text { ATION }\end{array}$} & \multirow{2}{*}{$\begin{array}{l}\text { PERCENT } \\
\text { OF } \\
\text { ANNUAL } \\
\text { RUNOFF }\end{array}$} & \multirow{2}{*}{$\begin{array}{l}\text { PERIOD } \\
\text { (CON- } \\
\text { SECU- } \\
\text { TIVE } \\
\text { DAYS) }\end{array}$} & \multicolumn{6}{|c|}{$\begin{array}{l}\text { DISCHARGE, IN FT }{ }^{3} / S \text {, FOR INDICATED RECURRENCE } \\
\text { INTERVAL, IN YEARS, AND ANNUAL NON- } \\
\text { EXCEEDANCE PROBAB IL ITY, IN PERCENT }\end{array}$} \\
\hline & & & & & & & & $\begin{array}{c}2 \\
508\end{array}$ & $\begin{array}{c}5 \\
208\end{array}$ & $\begin{array}{l}10 \\
108\end{array}$ & $\begin{array}{l}20 \\
58\end{array}$ & $\begin{array}{l}50 \\
28\end{array}$ & $\begin{array}{r}100 \\
18\end{array}$ \\
\hline $\begin{array}{l}\text { OCTOBER } \\
\text { NOVEMBER } \\
\text { DECEMBER } \\
\text { JANUARY } \\
\text { FEBRUARY } \\
\text { MARCH } \\
\text { APRIL } \\
\text { MAY } \\
\text { JUNE }\end{array}$ & $\begin{array}{l}7.9 \\
8.1 \\
3.9 \\
3.9 \\
4.8 \\
5.1 \\
9.7 \\
18 \\
23\end{array}$ & $\begin{array}{r}50 \\
40 \\
33 \\
23 \\
24 \\
35 \\
143 \\
407 \\
405\end{array}$ & $\begin{array}{r}23 \\
18 \\
15 \\
14 \\
15 \\
17 \\
45 \\
137 \\
144\end{array}$ & $\begin{array}{l}9.1 \\
6.4 \\
6.1 \\
5.3 \\
5.1 \\
6.1 \\
28 \\
93 \\
98\end{array}$ & $\begin{array}{l}.39 \\
.35 \\
.41 \\
.37 \\
.34 \\
.37 \\
.63 \\
.68 \\
.68\end{array}$ & $\begin{array}{r}4.2 \\
3.3 \\
2.7 \\
2.6 \\
2.7 \\
3.0 \\
8.1 \\
24.6 \\
25.9\end{array}$ & $\begin{array}{r}1 \\
3 \\
7 \\
14 \\
30 \\
60 \\
90 \\
120 \\
183\end{array}$ & $\begin{array}{l}7.8 \\
8.7 \\
9.8 \\
11 \\
12 \\
13 \\
14 \\
14 \\
16\end{array}$ & $\begin{array}{r}4.8 \\
5.4 \\
6.1 \\
7.1 \\
8.0 \\
8.7 \\
9.2 \\
9.7 \\
12\end{array}$ & $\begin{array}{l}3.6 \\
4.1 \\
4.6 \\
5.3 \\
6.0 \\
6.6 \\
7.0 \\
7.6 \\
9.4\end{array}$ & $\begin{array}{l}2.8 \\
3.2 \\
3.6 \\
4.1 \\
4.6 \\
5.1 \\
5.5 \\
6.0 \\
7.8\end{array}$ & $\begin{array}{l}2.1 \\
2.3 \\
2.6 \\
3.0 \\
3.3 \\
3.7 \\
4.1 \\
4.6 \\
6.2\end{array}$ & $\begin{array}{l}1.7 \\
1.9 \\
2.1 \\
2.4 \\
2.6 \\
3.0 \\
3.3 \\
3.7 \\
5.3\end{array}$ \\
\hline $\begin{array}{l}\text { JULY } \\
\text { AUGUST } \\
\text { SEPTEMBER }\end{array}$ & $\begin{array}{l}12 \\
12 \\
8.8\end{array}$ & $\begin{array}{r}172 \\
121 \\
53\end{array}$ & $\begin{array}{l}61 \\
39 \\
28\end{array}$ & $\begin{array}{l}38 \\
20 \\
11\end{array}$ & $\begin{array}{l}.62 \\
.52 \\
.40\end{array}$ & $\begin{array}{r}11.0 \\
6.9 \\
5.0\end{array}$ & & & & & & & \\
\hline ANNUAL & 12 & 98 & 45 & 21 & .47 & 100 & & & & & & & \\
\hline
\end{tabular}

MAGNITUDE AND PROBABILITY OF ANNUAL HIGH FLOW BASED ON PERIOD OF RECORD $1925,1931-85$

\begin{tabular}{|c|c|c|c|c|c|c|}
\hline \multirow{2}{*}{$\begin{array}{l}\text { PERIOD } \\
\text { (CON- } \\
\text { SECU- } \\
\text { TIVE } \\
\text { DAYS) }\end{array}$} & \multicolumn{6}{|c|}{$\begin{array}{c}\text { DISCHARGE, IN FT' }{ }^{3} \text { S, FOR INDICATED RECURREN } \\
\text { INTERVAL, IN YEARS, AND ANNUAL } \\
\text { EXCEEDANCE PROBABILI TY, IN PERCENT }\end{array}$} \\
\hline & $\begin{array}{c}2 \\
508\end{array}$ & $\begin{array}{c}5 \\
208\end{array}$ & $\begin{array}{l}10 \\
108\end{array}$ & $\begin{array}{l}25 \\
48\end{array}$ & $\begin{array}{l}50 \\
28\end{array}$ & $\begin{array}{r}100 \\
18\end{array}$ \\
\hline $\begin{array}{r}1 \\
3 \\
7 \\
15 \\
30 \\
60 \\
90\end{array}$ & $\begin{array}{r}196 \\
188 \\
175 \\
161 \\
146 \\
120 \\
99\end{array}$ & $\begin{array}{l}358 \\
346 \\
320 \\
287 \\
258 \\
209 \\
170\end{array}$ & $\begin{array}{l}485 \\
464 \\
428 \\
377 \\
337 \\
272 \\
219\end{array}$ & $\begin{array}{l}662 \\
625 \\
574 \\
495 \\
439 \\
353 \\
283\end{array}$ & $\begin{array}{l}806 \\
751 \\
687 \\
583 \\
515 \\
412 \\
330\end{array}$ & $\begin{array}{l}957 \\
880 \\
802 \\
670 \\
589 \\
470 \\
377\end{array}$ \\
\hline
\end{tabular}

DURATION TABLE OF DAILY MEAN FLOW FOR PERIOD OF RECORD 1925, 1931-85

DISCHARGE, IN FT $3 / S$, THAT WAS EQUALED OR EXCEEDED FOR INDICATED PERCENT OF TIME

\begin{tabular}{|c|c|c|c|c|c|c|c|c|c|c|c|c|c|c|}
\hline 58 & 108 & 158 & 208 & 258 & 308 & 408 & 508 & 608 & 708 & 758 & 808 & 858 & 908 & 958 \\
\hline 174 & 109 & 75 & 55 & 44 & 37 & 27 & 23 & 20 & 17 & 16 & 14 & 13 & 11 & \\
\hline
\end{tabular}


RIO GRANDE BASIN

08266000 CABRESTO CREEK NEAR QUESTA, NM

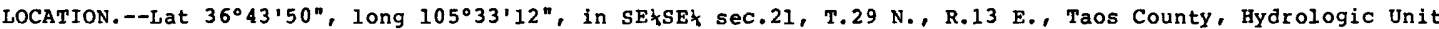
13020101 , in Carson National Forest, on $r i g h t$ bank $900 \mathrm{ft}$ downstream from Llano ditch heading, $2.6 \mathrm{mi}$ downstream

from Lake Fork, $3 \mathrm{mi}$ northeast of Questa, and at mile 3.5 .

DRAINAGE AREA. $--36.7 \mathrm{mi}^{2}$

PERIOD OF RECORD.--September 1943 to current year.

REVISED RECORDS.--WSP 1712: Drainage area.

GAGE.--Water-stage recorder and concrete control. Elevation of gage is $7,845 \mathrm{ft}$ above National Geodetic Vertical Datum of 1929, from river-profile map.

REMARKS. - Llano ditch (station 08265500), the only diversion upstream from station, diverts from right bank 900 ft upstream from gage for irrigation of about 800 acres downstream. Flow regulated by Cabresto Reservoir

(capacity, 732 acre-feet, after reconstruction in 1928) on Lake Fork 1 mi upstream from mouth. Present

capacity of Cabresto Reservoir is 1,100 acre-feet after further rehabilitation between 1959 and 1961.

AVERAGE DISCHARGE. -42 years (water years 1944-85), $10.3 \mathrm{ft}^{3} / \mathrm{s}, 7,460 \mathrm{acre}-\mathrm{ft} / \mathrm{yr}^{\circ}$.

EXTREMES FOR PERIOD OF RECORD.--Maximum discharge, $204 \mathrm{ft}^{3} / \mathrm{s}$, June 2, 1983, gage height, $4.82 \mathrm{ft} ; \mathrm{minimum,} 0.44$ $\mathrm{Et}^{3} / \mathrm{s}$, Dec. 2, 1950, result of freezeup.

EXTREMES OUTSIDE PERIOD OF RECORD.--The flood of May 25, 1942, may have exceeded the maximum of record.

STATISTICAL SUMMARIES

MEAN MONTHLY AND MEAN ANNUAL DISCHARGES 1944-85

\begin{tabular}{|c|c|c|c|c|c|c|}
\hline MONTH & $\begin{array}{c}\text { MINI MUM } \\
\left(\mathrm{FT}^{3} / \mathrm{S}\right)\end{array}$ & $\begin{array}{c}\text { MAXIMUM } \\
\left(\mathrm{FT}^{3} / \mathrm{S}\right)\end{array}$ & $\begin{array}{l}\text { MEAN } \\
\left(\mathrm{FT}^{3} / \mathrm{S}\right)\end{array}$ & $\begin{array}{l}\text { STAN- } \\
\text { DARD } \\
\text { DEVIA- } \\
\text { TION } \\
\left(\mathrm{FT}^{3} / \mathrm{S}\right)\end{array}$ & $\begin{array}{l}\text { COEFFI- } \\
\text { CIENT OF } \\
\text { VARI - } \\
\text { ATION }\end{array}$ & $\begin{array}{c}\text { PERCENT } \\
\text { OF } \\
\text { ANNUAL } \\
\text { RUNOF F }\end{array}$ \\
\hline $\begin{array}{l}\text { OCTOBER } \\
\text { NOVEMBER } \\
\text { DECEMBER } \\
\text { JANUARY } \\
\text { FEBRUARY } \\
\text { MARCH } \\
\text { APRIL } \\
\text { MAY } \\
\text { JUNE } \\
\text { JULY } \\
\text { AUGUST } \\
\text { SEPTEMBER }\end{array}$ & $\begin{array}{l}2.7 \\
2.7 \\
2.4 \\
2.3 \\
2.3 \\
3.0 \\
6.7 \\
7.9 \\
5.8 \\
4.6 \\
4.3 \\
2.9\end{array}$ & $\begin{array}{l}10 \\
9.8 \\
8.1 \\
6.5 \\
6.5 \\
7.7 \\
30 \\
97 \\
95 \\
27 \\
21 \\
14\end{array}$ & $\begin{array}{r}5.7 \\
4.8 \\
4.2 \\
4.1 \\
4.3 \\
5.0 \\
13 \\
33 \\
22 \\
11 \\
9.3 \\
7.0\end{array}$ & $\begin{array}{r}1.9 \\
1.5 \\
1.3 \\
1.0 \\
.9 \\
1.0 \\
5.4 \\
27 \\
21 \\
4.0 \\
3.5 \\
2.9\end{array}$ & $\begin{array}{l}.34 \\
.31 \\
.30 \\
.25 \\
.20 \\
.19 \\
.43 \\
.82 \\
.93 \\
.35 \\
.38 \\
.40\end{array}$ & $\begin{array}{r}4.6 \\
3.9 \\
3.4 \\
3.3 \\
3.5 \\
4.1 \\
10.2 \\
26.7 \\
17.9 \\
9.1 \\
7.6 \\
5.7\end{array}$ \\
\hline NNUAL & 5.3 & 23 & 10 & 4.5 & .44 & 100 \\
\hline
\end{tabular}

MAGNITUDE AND PROBABILITY OF ANNUAL LOW FLOW BASED ON PERIOD OF RECORD 1945-85

\begin{tabular}{|c|c|c|c|c|c|c|}
\hline \multirow{2}{*}{$\begin{array}{l}\text { PERIOD } \\
\text { (CON- } \\
\text { SECU- } \\
\text { TIVE } \\
\text { DAYS) }\end{array}$} & \multicolumn{6}{|c|}{$\begin{array}{l}\text { DISCHARGE, IN FT'/S, FOR INDICATED RECURRENCE } \\
\text { INTERVAL, IN YEARS, AND ANNUAL NON- } \\
\text { EXCEEDANCE PROBABILITY, IN PERCENT }\end{array}$} \\
\hline & $\begin{array}{c}2 \\
508\end{array}$ & $\begin{array}{c}5 \\
208\end{array}$ & $\begin{array}{l}10 \\
108\end{array}$ & $\begin{array}{l}20 \\
58\end{array}$ & $\begin{array}{l}50 \\
28\end{array}$ & \\
\hline $\begin{array}{r}1 \\
3 \\
7 \\
14 \\
30 \\
60 \\
90 \\
120 \\
183\end{array}$ & $\begin{array}{l}2.1 \\
2.4 \\
2.8 \\
3.1 \\
3.4 \\
3.7 \\
3.8 \\
4.0 \\
4.5\end{array}$ & $\begin{array}{l}1.5 \\
1.8 \\
2.2 \\
2.5 \\
2.8 \\
3.0 \\
3.2 \\
3.4 \\
3.7\end{array}$ & $\begin{array}{l}1.2 \\
1.5 \\
1.9 \\
2.2 \\
2.5 \\
2.8 \\
2.9 \\
3.1 \\
3.3\end{array}$ & $\begin{array}{l}1.0 \\
1.3 \\
1.7 \\
2.1 \\
2.3 \\
2.6 \\
2.7 \\
2.8 \\
3.1\end{array}$ & $\begin{array}{r}.9 \\
1.1 \\
1.5 \\
1.9 \\
2.2 \\
2.4 \\
2.5 \\
2.6 \\
2.8\end{array}$ & \\
\hline
\end{tabular}

MAGNITUDE AND PROBABILITY OF ANNUAL HIGH FLOW BASED ON PERIOD OF RECORD $1944-85$

\begin{tabular}{|c|c|c|c|c|c|c|}
\hline $\begin{array}{l}\text { PERIOD } \\
\text { (CON- }\end{array}$ & \multicolumn{6}{|c|}{$\begin{array}{c}\text { DISCHARGE, IN FT } 3 \text { /S, FOR INDICATED RECURRENCE } \\
\text { INTERVAL, IN YEARS, AND ANNUAL } \\
\text { EXCEEDANCE PROBABILITY, IN PERCENT }\end{array}$} \\
\hline $\begin{array}{l}\text { SECU- } \\
\text { TIVE } \\
\text { DAYS ) }\end{array}$ & $\begin{array}{c}2 \\
508\end{array}$ & $\begin{array}{c}5 \\
208\end{array}$ & $\begin{array}{l}10 \\
108\end{array}$ & $\begin{array}{l}25 \\
48\end{array}$ & $\begin{array}{l}50 \\
28\end{array}$ & $\begin{array}{r}100 \\
18\end{array}$ \\
\hline
\end{tabular}

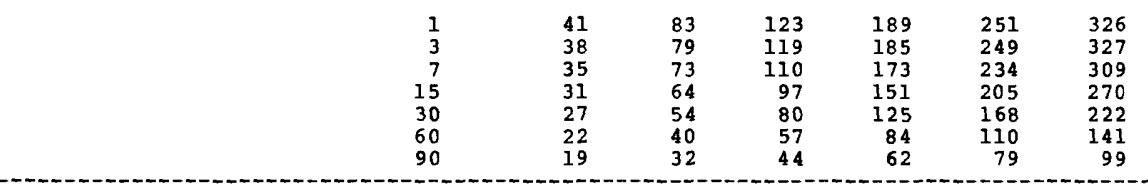

DURATION TABLE OF DAILY MEAN FLOW FOR PERIOD OF RECORD 1944-85

DISCHARGE, IN FT 3 /S, THAT WAS EQUALED OR EXCEEDED FOR INDICATED PERCENT OF TIME

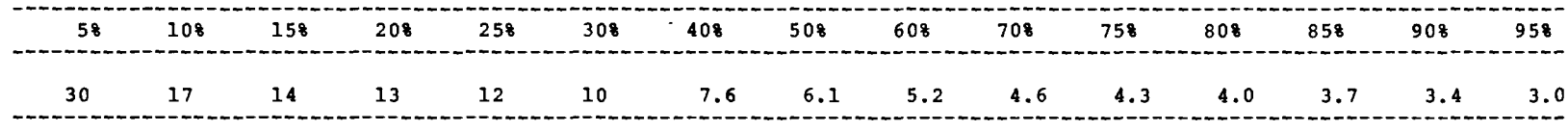


RIO GRANDE BASIN

08267000 RED RIVER AT MOUTH, NEAR QUESTA, NM

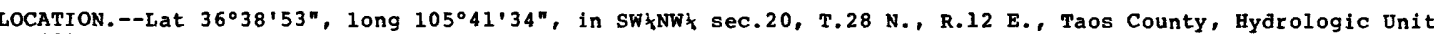
13020101, in Carson National Forest, on left bank $250 \mathrm{ft}$ upstream from Rio Grande, and $6.5 \mathrm{mi}$ southwest of Questa.

DRAINAGE AREA. $--190 \mathrm{mi}^{2}$.

PERIOD OF RECORD.--October 1950 to September 1978 (discontinued). Monthly discharge only for October and November 1950, published in WSP 1732 .

GAGE.--Water-stage recorder. Altitude of gage is 6,600 ft, from topographic map.

REMARKS.--Diversions for irrigation of about 3,000 acres above station.

AVERAGE DISCHARGE. --27 years (water years 1952-78), $76.0 \mathrm{ft} / \mathrm{s}, 55,040$ acre-ft/yr.

EXTREMES FOR PERIOD OF RECORD.--Maximum discharge, $730 \mathrm{ft}^{3} / \mathrm{s}$ Aug. 12, 1964, gage height, $6.05 \mathrm{ft} ; \mathrm{minimum}, 29 \mathrm{ft} / \mathrm{s}$ Feb. 13, 1965 .

STATISTICAL SUMMARIES

MEAN MONTHLY AND MEAN ANNUAL DISCHARGES 1952-78

\begin{tabular}{|c|c|c|c|c|c|c|}
\hline MONTH & $\begin{array}{l}\text { MINIMUM } \\
\left(\mathrm{FT}^{3} / \mathrm{S}\right)\end{array}$ & $\begin{array}{l}\text { MAXI MUM } \\
\left(\mathrm{FT}^{3} / \mathrm{S}\right)\end{array}$ & $\begin{array}{l}\text { MEAN } \\
\left(\mathrm{FT}^{3} / \mathrm{S}\right)\end{array}$ & $\begin{array}{c}\text { STAN- } \\
\text { DARD } \\
\text { DEVIA- } \\
\text { TION } \\
\left(\text { FT }^{3} / \mathrm{S}\right)\end{array}$ & $\begin{array}{l}\text { COEFFI- } \\
\text { CIENT OF } \\
\text { VARI - } \\
\text { ATION }\end{array}$ & $\begin{array}{c}\text { PERCENT } \\
\text { OF } \\
\text { ANNUAL, } \\
\text { RUNOFF }\end{array}$ \\
\hline & & & & -- & & -- \\
\hline OCTOBER & 43 & 79 & 57 & 9.8 & .17 & 6.3 \\
\hline NOVEMBER & 42 & 73 & 54 & 7.1 & .13 & 5.9 \\
\hline DECEMBER & 38 & 69 & 49 & 6.8 & .14 & 5.4 \\
\hline JANDARY & 41 & 60 & 50 & 5.7 & .11 & 5.5 \\
\hline FEB RUARY & 42 & 63 & 50 & 4.8 & .10 & 5.6 \\
\hline MARCH & 42 & 62 & 51 & 4.9 & .10 & 5.6 \\
\hline APRIL & 44 & 141 & 71 & 25 & .36 & 7.8 \\
\hline MAY & 52 & 388 & 145 & 82 & .56 & 16.0 \\
\hline JUNE & 49 & 370 & 154 & 86 & .56 & 17.0 \\
\hline JUL,Y & 46 & 158 & 88 & 33 & .37 & 9.7 \\
\hline AUGUST & 50 & 135 & 75 & 21 & .28 & 8.3 \\
\hline SEPTEMBER & 41 & 92 & 61 & 13 & .22 & 6.8 \\
\hline ANNUAL, & 48 & 119 & 76 & 19 & .25 & 100 \\
\hline
\end{tabular}

MAGNITUDE AND PROBABILITY OF ANNUAL LOW FLOW BASED ON PERIOD OF RECORD $1953-78$

\begin{tabular}{|c|c|c|c|c|c|c|}
\hline \multirow{2}{*}{$\begin{array}{c}\text { PERIOD } \\
\text { (CON- } \\
\text { SECU- } \\
\text { TIVE } \\
\text { DAYS) }\end{array}$} & \multicolumn{6}{|c|}{$\begin{array}{l}\text { DISCHARGE, IN FT'3/S, FOR INDICATED RECURRENCE } \\
\text { INTERVAL, IN YEARS, AND ANNUAL NON- } \\
\text { EXCEEDANCE PROBAB IL, ITY, IN PERCENT }\end{array}$} \\
\hline & $\begin{array}{c}2 \\
508\end{array}$ & $\begin{array}{c}5 \\
208\end{array}$ & $\begin{array}{l}10 \\
108\end{array}$ & $\begin{array}{l}20 \\
58\end{array}$ & $\begin{array}{l}50 \\
28\end{array}$ & $\begin{array}{r}100 \\
18\end{array}$ \\
\hline $\begin{array}{r}1 \\
3 \\
7 \\
14 \\
30 \\
60 \\
90 \\
120 \\
183\end{array}$ & $\begin{array}{l}40 \\
41 \\
43 \\
44 \\
46 \\
47 \\
48 \\
49 \\
51\end{array}$ & $\begin{array}{l}37 \\
38 \\
39 \\
41 \\
42 \\
43 \\
45 \\
45 \\
47\end{array}$ & $\begin{array}{l}36 \\
37 \\
38 \\
39 \\
40 \\
42 \\
43 \\
44 \\
45\end{array}$ & $\begin{array}{l}35 \\
35 \\
36 \\
37 \\
39 \\
40 \\
41 \\
42 \\
44\end{array}$ & $\begin{array}{l}34 \\
34 \\
35 \\
36 \\
38 \\
38 \\
40 \\
41 \\
42\end{array}$ & $\begin{array}{l}-- \\
-- \\
-- \\
-- \\
-- \\
-- \\
--\end{array}$ \\
\hline
\end{tabular}

MAGNITUDE AND PROBABILITY OF ANNUAL, HIGH FLOW BASED ON PERIOD OF RECORD 1952-78

\begin{tabular}{lcccccc} 
PERIOD & \multicolumn{1}{c}{ DISCHARGE, IN FT ${ }^{3}$ /S, FOR INDICATED RECURRENCE } \\
INTERVAL, IN YEARS, AND ANNUAL, \\
SECU-
\end{tabular}

DURATION TABLE OF DAILY MEAN FLOW FOR PERIOD OF RECORD 1952-78

DISCHARGE, IN FT'/S, THAT WAS EQUALED OR EXCEEDED FOR INDICATED PERCENT OF TIME

\begin{tabular}{|c|c|c|c|c|c|c|c|c|c|c|c|c|c|c|}
\hline 58 & 108 & 158 & 208 & 258 & 308 & 408 & 508 & 608 & 708 & 758 & 808 & 858 & 908 & 958 \\
\hline 181 & 131 & 104 & 87 & 77 & 70 & 61 & 57 & 54 & 51 & 50 & 48 & 47 & 45 & 43 \\
\hline
\end{tabular}


RIO GRANDE BASIN

08267500 RIO HONDO NEAR VALDEZ, NM

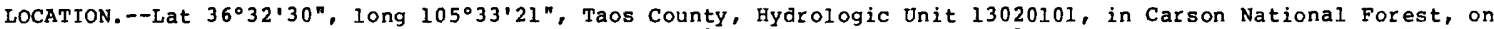
right bank $500 \mathrm{ft}$ upstream from first diversion, $1.6 \mathrm{mi}$ east of Valdez, $3.8 \mathrm{mi}$ downstream from South Fork, and at mile 9.2 .

DRA INAGE AREA. $--36.2 \mathrm{mi} 2$

PERIOD OF RECORD.--August 1934 to current year.

REVISED RECORDS.--WSP 1342: 1935. WSP 1712: Drainage area. WSP 1732: 1942 (M).

GAGE.--Water-stage recorder. Concrete control since Oct. 28, 1938. Elevation of gage is $7,650 \mathrm{ft}$ above National Geodetic vertical Datum of 1929, from topographic map. Prior to oct. 28, 1938, at datum $1.92 \mathrm{ft} 10 \mathrm{wer}$.

REMARKS.--No diversions upstream from station.

AVERAGE DISChARGE. -51 years, $34.9 \mathrm{ft}^{3} / \mathrm{s}, 25,280$ acre-ft/yr.

EXTREMES FOR PERIOD OF RECORD,--Maximum discharge, $541 \mathrm{ft}^{3} / \mathrm{s}$, May 13, 1941; maximum gage height, 4.81 ft, Jan. 5, 1970 (ice jam); minimum discharge, about I ft3/s, Jan. 27, 1942, result of freezeup.

STATISTICAL SUMMARIES

MEAN MONTHLY AND MEAN ANNUAL DISCHARGES 1935-85

\begin{tabular}{|c|c|c|c|c|c|c|}
\hline MONTH & $\begin{array}{l}\text { MINIMUM } \\
\text { (FT, } / \mathrm{S})\end{array}$ & $\begin{array}{c}\operatorname{MAXIMUM} \\
\left(\mathrm{FT}^{3} / \mathrm{S}\right)\end{array}$ & $\begin{array}{l}\text { MEAN } \\
\left(\mathrm{FT}^{3} / \mathrm{S}\right)\end{array}$ & $\begin{array}{l}\text { STAN- } \\
\text { DARD } \\
\text { DEVIA- } \\
\text { TION } \\
\left.\text { (FT' } \text { FT }^{3} / \mathrm{S}\right)\end{array}$ & $\begin{array}{l}\text { COEFFI- } \\
\text { CIENT OF } \\
\text { VARI - } \\
\text { ATION }\end{array}$ & $\begin{array}{c}\text { PERCENT } \\
\text { OF } \\
\text { ANNUAL } \\
\text { RUNOFF }\end{array}$ \\
\hline $\begin{array}{l}\text { OCTOBER } \\
\text { NOVEMBER } \\
\text { DECEMBER } \\
\text { JANUARY } \\
\text { FEBRUARY } \\
\text { MARCH } \\
\text { APRIL } \\
\text { MAY } \\
\text { JUNE } \\
\text { JULY } \\
\text { AUGUST } \\
\text { SEPTEMBER }\end{array}$ & $\begin{array}{l}11 \\
8.3 \\
7.5 \\
6.0 \\
6.1 \\
7.6 \\
11 \\
21 \\
26 \\
15 \\
11 \\
9.9\end{array}$ & $\begin{array}{r}44 \\
36 \\
23 \\
20 \\
17 \\
28 \\
92 \\
246 \\
299 \\
144 \\
60 \\
49\end{array}$ & $\begin{array}{r}18 \\
15 \\
12 \\
11 \\
11 \\
13 \\
33 \\
96 \\
113 \\
48 \\
29 \\
21\end{array}$ & $\begin{array}{r}5.9 \\
4.5 \\
2.9 \\
2.3 \\
2.0 \\
3.5 \\
17 \\
56 \\
70 \\
31 \\
12 \\
8.0\end{array}$ & $\begin{array}{l}.34 \\
.31 \\
.24 \\
.22 \\
.19 \\
.26 \\
.51 \\
.58 \\
.62 \\
.64 \\
.40 \\
.38\end{array}$ & $\begin{array}{r}4.2 \\
3.5 \\
2.9 \\
2.5 \\
2.5 \\
3.1 \\
7.9 \\
23.0 \\
27.0 \\
11.4 \\
6.8 \\
5.1\end{array}$ \\
\hline ANNUAL & 16 & 70 & 35 & 15 & .42 & 100 \\
\hline
\end{tabular}

MAGNITUDE AND PROBABILITY OF ANNUAL LOW FLOW BASED ON PERIOD OF RECORD 1936-85

\begin{tabular}{|c|c|c|c|c|c|c|}
\hline \multirow{2}{*}{$\begin{array}{l}\text { PERIOD } \\
\text { (CON- } \\
\text { SECU- } \\
\text { TIVE } \\
\text { DAYS) }\end{array}$} & \multicolumn{6}{|c|}{$\begin{array}{l}\text { DISCHARGE, IN FT } 3 / \mathrm{S} \text {, FOR INDICATED RECURRENCE } \\
\text { INTERVAL, IN YEARS, AND ANNUAL NON- } \\
\text { EXCEEDANCE PROBABILITY, IN PERCENT }\end{array}$} \\
\hline & $\begin{array}{c}2 \\
508\end{array}$ & $\begin{array}{c}5 \\
208\end{array}$ & $\begin{array}{l}10 \\
108\end{array}$ & $\begin{array}{l}20 \\
58\end{array}$ & $\begin{array}{l}50 \\
28\end{array}$ & $\begin{array}{r}100 \\
18\end{array}$ \\
\hline $\begin{array}{r}1 \\
3 \\
7 \\
14 \\
30 \\
60 \\
90 \\
120 \\
183\end{array}$ & $\begin{array}{l}7.7 \\
8.2 \\
8.8 \\
9.3 \\
9.7 \\
10 \\
10 \\
11 \\
12\end{array}$ & $\begin{array}{r}6.2 \\
6.7 \\
7.3 \\
7.9 \\
8.4 \\
8.8 \\
9.0 \\
9.4 \\
11\end{array}$ & $\begin{array}{l}5.5 \\
6.0 \\
6.6 \\
7.3 \\
7.9 \\
8.2 \\
8.4 \\
8.7 \\
9.8\end{array}$ & $\begin{array}{l}5.0 \\
5.5 \\
6.1 \\
6.8 \\
7.5 \\
7.7 \\
8.0 \\
8.2 \\
9.3\end{array}$ & $\begin{array}{l}4.5 \\
4.9 \\
5.5 \\
6.4 \\
7.1 \\
7.3 \\
7.5 \\
7.8 \\
8.8\end{array}$ & $\begin{array}{l}5.1 \\
6.1 \\
6.8 \\
7.0 \\
7.2 \\
7.5 \\
8.5\end{array}$ \\
\hline
\end{tabular}

MAGNITUDE AND PROBABILITY OF ANNUAL HIGH FTON BASED ON PERIOD OF RECORD 1935-85

\begin{tabular}{|c|c|c|c|c|c|c|}
\hline \multirow{2}{*}{$\begin{array}{l}\text { PERIOD } \\
\text { (CON- } \\
\text { SECU- } \\
\text { TIVE } \\
\text { DAYS) }\end{array}$} & \multicolumn{6}{|c|}{$\begin{array}{l}\text { DISCHARGE, IN FT' } 3 \text { /S, FOR INDICATED RECURRENCE } \\
\text { INTERVAL, IN YEARS, AND ANNUAL } \\
\text { EXCEEDANCE PROBABILITY, IN PERCENT }\end{array}$} \\
\hline & $\begin{array}{c}2 \\
508\end{array}$ & $\begin{array}{c}5 \\
208\end{array}$ & $\begin{array}{l}10 \\
108\end{array}$ & $\begin{array}{l}25 \\
48\end{array}$ & $\begin{array}{l}50 \\
28\end{array}$ & $\begin{array}{r}100 \\
18\end{array}$ \\
\hline $\begin{array}{r}1 \\
3 \\
7 \\
15 \\
30 \\
60 \\
90\end{array}$ & $\begin{array}{r}146 \\
141 \\
133 \\
124 \\
113 \\
93 \\
77\end{array}$ & $\begin{array}{l}247 \\
239 \\
223 \\
205 \\
187 \\
154 \\
125\end{array}$ & $\begin{array}{l}319 \\
309 \\
287 \\
262 \\
238 \\
197 \\
159\end{array}$ & $\begin{array}{l}414 \\
400 \\
371 \\
336 \\
305 \\
255 \\
204\end{array}$ & $\begin{array}{l}486 \\
470 \\
434 \\
392 \\
355 \\
299 \\
240\end{array}$ & $\begin{array}{l}559 \\
540 \\
498 \\
448 \\
406 \\
345 \\
276\end{array}$ \\
\hline
\end{tabular}

DURATION TABLE OF DAILY MEAN FLOW FOR PERIOD OF RECORD 1935-85

\begin{tabular}{|c|c|c|c|c|c|c|c|c|c|c|c|c|c|c|}
\hline 58 & 108 & 158 & 208 & 258 & 308 & 408 & 508 & 608 & 708 & 758 & 808 & 858 & 908 & 958 \\
\hline 128 & 85 & 59 & 44 & 34 & 29 & 22 & 18 & 15 & 13 & 12 & 12 & 11 & 9.6 & 8.6 \\
\hline
\end{tabular}


RIO GRANDE BASIN

08268500 ARROYO HONDO AT ARROYO HONDO, NM

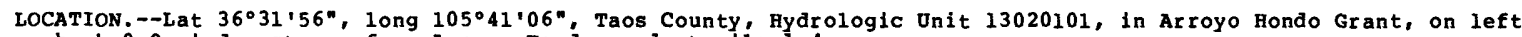
bank $0.9 \mathrm{mi}$ downstream from Arroyo Hondo, and at mile 1.4 .

DRAINAGE AREA. $--65.6 \mathrm{mi}^{2}$.

PERIOD OF RECORD.--April 1910 to June 1912 (discharge measurements and fragmentary gage-height record), July 1912 to December 1928 (fragmentary), and January 1932 to current year. Monthly discharge only for some periods,
published in WSP 1312. Statement in wSP 328 that there was no flow in January and much of February 1912 is erroneous. Published as Rio Fondo near Arroyo Hondo prior to 1928 , and as Rio Hondo at Arroyo Hondo $1928-65$. Discontinued October 1 , 1985.

REVISED RECORDS.--WSP 1342: 1915, 1932(M), 1934-38(M). WSP 1712: Drainage area. WSP 1732: 1926. See also PERIOD OF RECORD.

GAGE.--Water-stage recorder. Elevation of gage is 6,670 ft above National Geodetic Vertical Datum of 1929 , from topographic map. See WSP 1923 for history of changes prior to Sept. 11, 1963. Sept. 11, 1963 to Apr. 2, 1969, at site $25 \mathrm{ft}$ downstream on right bank at same datum.

REMARRS.--Diversions upstream from station for irrigation of about 2,500 acres, of which about 1,700 acres is a transbasin diversion to Rio Lucero.

AVERAGE DISCHARGE.--66 years (water years 1913-24, 1927-28, 1933-85), $27.2 \mathrm{ft} 3 / \mathrm{s}, 19,710 \mathrm{acre-ft} / \mathrm{yr}$.

EXTREMES FOR PERIOD OF RECORD (SINCE 1937).--Maximum discharge, 1,060 ft /s, July 19, 1948, gage height, 3.75 ft, from rating curve extended above $200 \mathrm{ft} / \mathrm{s}$; maximum gage height, $5.06 \mathrm{ft}$, June 8 , 1979, backwater from debris; minimum discharge, $3.3 \mathrm{ft}^{3 / \mathrm{s}}$, May 7,1977 .

Maximum gage height observed, $5.45 \mathrm{ft}$, site and datum then in use, Aug. 23, 1935; discharge uncertain, but probably exceeded $1,200 \mathrm{ft}^{3} / \mathrm{s}$. A minimum daily discharge of $3 \mathrm{ft}^{3} / \mathrm{s}$ occurred Oct. 19, 1912. Discharge not determined for the major floods of oct. 6, 1911, Sept. 1, 1932 and July 22, 1934.

STATISTICAL SUMMARIES MEAN MONTHLy AND MEAN ANNUAL DISchaRges $\begin{aligned} & 1913-24,1927-28, \\ & 1933-85\end{aligned}$

\begin{tabular}{|c|c|c|c|c|c|c|}
\hline MONTH & $\begin{array}{l}\text { MINIMUM } \\
\left(\text { FT }^{3} / S\right)\end{array}$ & $\begin{array}{l}\text { MAXIMUM } \\
\left(\mathrm{FT}^{3}{ }^{3} / \mathrm{S}\right)\end{array}$ & $\begin{array}{l}\text { MEAN } \\
\left(F^{3} / S\right)\end{array}$ & $\begin{array}{c}\text { STAN- } \\
\text { DARD } \\
\text { DEVIA- } \\
\text { TION } \\
\left(\text { FT }^{3} / \mathrm{S}\right)\end{array}$ & $\begin{array}{l}\text { COEFFI- } \\
\text { CIENT OF } \\
\text { VARI- } \\
\text { ATION }\end{array}$ & $\begin{array}{c}\text { PERCENT } \\
\text { OF } \\
\text { ANNUAL } \\
\text { RUNOFF }\end{array}$ \\
\hline остОВЕR & 4.0 & 44 & 13 & 7.5 & .57 & 3.9 \\
\hline NOVEMBER & 6.3 & 41 & 15 & 6.6 & .43 & 4.6 \\
\hline DECEMBER & 7.8 & 33 & 18 & 4.3 & .24 & 5.4 \\
\hline JANUAR & . & 30 & 17 & 3. & .20 & 5.0 \\
\hline FEB RUARY & 9.7 & 27 & 17 & 3.2 & .19 & 5.0 \\
\hline MARCH & 8.6 & 42 & 17 & 4.9 & .29 & 5.0 \\
\hline APR IL & 6.1 & 85 & 26 & 18 & .70 & 7.9 \\
\hline MAY & 4. & 277 & 75 & 65 & .8 & 22.8 \\
\hline JUNE & 5. & 293 & 81 & 73 & .90 & 24.5 \\
\hline JULY & 5. & 107 & 26 & 23 & .9 & 7.7 \\
\hline AUGUST & & 61 & 14 & 9.2 & .6 & 4.1 \\
\hline SEPTEMBER & & & 13 & 8.1 & & 3.8 \\
\hline NNUAL & 9.6 & 65 & 27 & 15 & .55 & 100 \\
\hline
\end{tabular}

MAGNITUDE AND PROBABILITY OF ANNUAL LOW FLOW BASED ON PERIOD OF RECORD 1914-25, 1927-28, $1933-85$

\begin{tabular}{|c|c|c|c|c|c|c|}
\hline \multirow{2}{*}{$\begin{array}{l}\text { PERIOD } \\
\text { (CON- } \\
\text { SECU- } \\
\text { TIVE } \\
\text { DAYS) }\end{array}$} & \multicolumn{6}{|c|}{$\begin{array}{l}\text { DISCHARGE, IN FT'/S, FOR INDICATED RECURRENCE } \\
\text { INTERVAL, IN YEARS, AND ANNUAL NON- } \\
\text { EXCEEDANCE PROBABILITY, IN PERCENT }\end{array}$} \\
\hline & $\begin{array}{c}2 \\
508\end{array}$ & $\begin{array}{c}5 \\
208\end{array}$ & $\begin{array}{l}10 \\
108\end{array}$ & $\begin{array}{l}20 \\
58\end{array}$ & $\begin{array}{l}50 \\
28\end{array}$ & $\begin{array}{r}100 \\
18\end{array}$ \\
\hline $\begin{array}{r}1 \\
3 \\
7 \\
14 \\
30 \\
60 \\
90 \\
120 \\
183\end{array}$ & $\begin{array}{r}6 . \\
6 . \\
6 . \\
7 . \\
7 . \\
9 . \\
10 \\
11 \\
13\end{array}$ & $\begin{array}{l}5 . \\
5 . \\
5 .\end{array}$ & 4.7 & $\begin{array}{l}4.5 \\
4.6 \\
4.8 \\
5.0 \\
5.8 \\
5.9 \\
6.0 \\
6.2 \\
7.0\end{array}$ & $\begin{array}{l}4 . \\
4 . \\
4 . \\
4 . \\
5 . \\
5 . \\
5 . \\
5 . \\
6 .\end{array}$ & $\begin{array}{l}4.1 \\
4.2 \\
4.5 \\
4.8 \\
5.1 \\
5.1 \\
5.2 \\
5.5\end{array}$ \\
\hline
\end{tabular}

MAGNITUDE AND PROBABILITY OF ANNUAL HIGH FLOW BASED ON PERIOD OF RECORD 1913-24, 1927-28, $1933-85$

\begin{tabular}{|c|c|c|c|c|c|c|}
\hline \multirow{2}{*}{$\begin{array}{l}\text { PERIOD } \\
\text { (CON- } \\
\text { SECU- } \\
\text { TIVE } \\
\text { DAYS) }\end{array}$} & \multicolumn{6}{|c|}{$\begin{array}{l}\text { DISCHARGE, IN FT'/S, FOR INDICATED RECURRENC } \\
\text { INTERVAL, IN YEARS, AND ANNUAL } \\
\text { EXCEEDANCE PROBABILITY, IN PERCENT }\end{array}$} \\
\hline & $\begin{array}{c}2 \\
508\end{array}$ & $\begin{array}{c}5 \\
208\end{array}$ & $\begin{array}{l}10 \\
108\end{array}$ & $\begin{array}{l}25 \\
48\end{array}$ & $\begin{array}{l}50 \\
28\end{array}$ & $\begin{array}{r}100 \\
18\end{array}$ \\
\hline $\begin{array}{r}1 \\
3 \\
7 \\
15 \\
30 \\
60 \\
90\end{array}$ & $\begin{array}{r}121 \\
113 \\
103 \\
90 \\
75 \\
59 \\
48\end{array}$ & $\begin{array}{r}240 \\
222 \\
204 \\
181 \\
155 \\
121 \\
94\end{array}$ & $\begin{array}{l}333 \\
307 \\
283 \\
254 \\
225 \\
176 \\
135\end{array}$ & $\begin{array}{l}463 \\
423 \\
393 \\
360 \\
331 \\
263 \\
201\end{array}$ & $\begin{array}{l}566 \\
515 \\
481 \\
446 \\
423 \\
341 \\
261\end{array}$ & $\begin{array}{l}673 \\
609 \\
572 \\
538 \\
526 \\
431 \\
331\end{array}$ \\
\hline
\end{tabular}

DURATION TABLE OF DA ILY MEAN FLOW FOR PERIOD OF RECORD 1913-24, 1927-28, 1933-85 DISCHARGE, IN FT'/S, THAT WAS EQUALED OR EXCEEDED FOR INDICATED PERCENT OF TIME

\begin{tabular}{|c|c|c|c|c|c|c|c|c|c|c|c|c|c|c|}
\hline 58 & 108 & 158 & 208 & 258 & 308 & 408 & 508 & 608 & 708 & 758 & 808 & 858 & 908 & 958 \\
\hline 103 & 54 & 36 & 27 & 23 & 21 & 18 & 16 & 14 & 12 & 11 & 9.3 & 8.3 & 7.4 & 6.5 \\
\hline
\end{tabular}


RIO GRANDE BASIN

08268700 RIO GRANDE NEAR ARROYO HONDO, NM

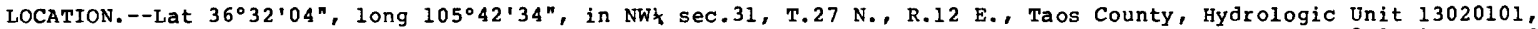
on right bank $350 \mathrm{ft}$ downstream from Arroyo Hondo, $400 \mathrm{ft}$ downstream from bridge on county road, $2.2 \mathrm{mi}$ west of Arroyo Hondo, $11.6 \mathrm{mi}$ northwest of Taos, and at mile $1,677.4$.

DRAINAGE AREA. - -8,760 $\mathrm{mi}^{2}$, approximately, including 2,940 $\mathrm{mi}^{2}$ in closed basin in san Luis Valley, CO.

PERIOD OF RECORD.--February 1963 to current year.

GAGE.--Water-stage recorder. Elevation of gage is $6,470 \mathrm{ft}$ above National Geodetic Vertical Datum of 1929 , from topographic map.

REMARKS.--Diversions upstream from station for irrigation of about 620,000 acres in colorado and 15,000 acres in New Mexico.

AVERAGE DISCHARGE.--22 years (water years 1964-85), $643 \mathrm{ft} 3 / \mathrm{s}, 465,900 \mathrm{acre}-\mathrm{ft} / \mathrm{yr}$.

EXTREMES FOR PERIOD OF RECORD.--Maximum discharge, 6,940 ft $3 / \mathrm{s}$, June 14, 1985, gage height, $8.08 \mathrm{ft}$; minimum, 136 $\mathrm{ft}^{3} / \mathrm{s}$, Aug. 2, 1963 .

STATISTICAL SUMMARIES

MEAN MONTHLy AND MEAN ANNUAL DischaRges 1964-85

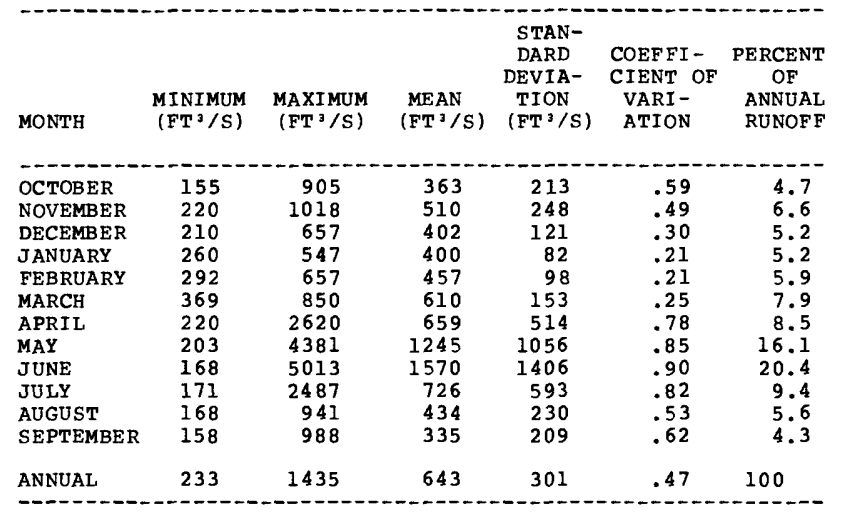

MAGNITUDE AND PROBABILITY OF ANNUAL LOW FLOW BASED ON PERIOD OF RECORD 1965-85

\begin{tabular}{|c|c|c|c|c|c|c|}
\hline \multirow{2}{*}{$\begin{array}{l}\text { PERIOD } \\
\text { (CON- } \\
\text { SECU- } \\
\text { TIVE } \\
\text { DAYS) }\end{array}$} & \multicolumn{6}{|c|}{$\begin{array}{l}\text { DISCHARGE, IN FT }{ }^{3 / S} \text {, FOR INDICATED RECURRENCE } \\
\text { INTERVAL, IN YEARS, AND ANNUAL NON- } \\
\text { EXCEEDANCE PROBAB ILITY, IN PERCENT }\end{array}$} \\
\hline & $\begin{array}{c}2 \\
508\end{array}$ & $\begin{array}{c}5 \\
208\end{array}$ & $\begin{array}{l}10 \\
108\end{array}$ & $\begin{array}{l}20 \\
58\end{array}$ & $\begin{array}{l}50 \\
28\end{array}$ & $\begin{array}{r}100 \\
18\end{array}$ \\
\hline $\begin{array}{r}1 \\
3 \\
7 \\
14 \\
30 \\
60 \\
90 \\
120 \\
183\end{array}$ & $\begin{array}{l}198 \\
201 \\
206 \\
210 \\
222 \\
245 \\
280 \\
304 \\
345\end{array}$ & $\begin{array}{l}160 \\
163 \\
165 \\
167 \\
175 \\
188 \\
208 \\
221 \\
248\end{array}$ & $\begin{array}{l}146 \\
149 \\
151 \\
152 \\
158 \\
167 \\
180 \\
189 \\
208\end{array}$ & $\begin{array}{l}137 \\
140 \\
142 \\
142 \\
147 \\
153 \\
160 \\
166 \\
181\end{array}$ & $\begin{array}{l}-- \\
-- \\
-- \\
-- \\
-- \\
\overline{-} \\
--\end{array}$ & $\begin{array}{l}-- \\
-- \\
-- \\
-- \\
-- \\
-- \\
--\end{array}$ \\
\hline
\end{tabular}

MAGNITUDE AND PROBABILITY OF ANNUAL HIGH FLOW BASED ON PERIOD OF RECORD 1964-85

\begin{tabular}{|c|c|c|c|c|c|c|}
\hline $\begin{array}{l}\text { PERIOD } \\
\text { (CON- }\end{array}$ & \multicolumn{6}{|c|}{$\begin{array}{l}\text { DISCHARGE, IN FT'/S, FOR INDICATED RECURRENCE } \\
\text { INTERVAL, IN YEARS, AND ANNUAL } \\
\text { EXCEEDANCE PROBABILITY, IN PERCENT }\end{array}$} \\
\hline $\begin{array}{l}\text { SECU- } \\
\text { TIVE } \\
\text { DAYS) }\end{array}$ & 208 & $\begin{array}{c}5 \\
208\end{array}$ & $\begin{array}{l}10 \\
108\end{array}$ & 25 & 28 & $\begin{array}{r}100 \\
18\end{array}$ \\
\hline $\begin{array}{r}1 \\
3 \\
7 \\
15 \\
30 \\
60 \\
90\end{array}$ & $\begin{array}{l}2000 \\
1930 \\
1780 \\
1600 \\
1420 \\
1200 \\
1040\end{array}$ & $\begin{array}{l}3730 \\
3630 \\
3350 \\
3020 \\
2700 \\
2270 \\
1920\end{array}$ & $\begin{array}{l}5090 \\
4970 \\
4600 \\
4170 \\
3770 \\
3180 \\
2660\end{array}$ & $\begin{array}{l}7010 \\
6840 \\
6380 \\
5850 \\
5370 \\
4570 \\
3770\end{array}$ & $\begin{array}{l}-- \\
=- \\
=- \\
-- \\
--\end{array}$ & $\begin{array}{l}-- \\
-- \\
-- \\
-- \\
--\end{array}$ \\
\hline
\end{tabular}

DURATION TABLE OF DAILY MEAN FLOW FOR PERIOD OF RECORD 1964-85 DISCHARGE, IN FT ${ }^{3} / \mathrm{S}$, THAT WAS EQUALED OR EXCEEDED FOR INDICATED PERCENT OF TIME

\begin{tabular}{|c|c|c|c|c|c|c|c|c|c|c|c|c|c|c|}
\hline 58 & 108 & 158 & 208 & $25 \%$ & 308 & 408 & 508 & 608 & 708 & 758 & 808 & 858 & 908 & 958 \\
\hline 2000 & 1260 & 917 & 743 & 662 & 597 & 498 & 430 & 374 & 306 & 276 & 250 & 226 & 204 & 180 \\
\hline
\end{tabular}




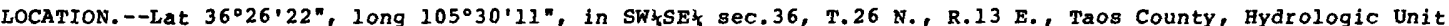
13020101 , in Taos Pueblo Grant, on right bank $2.3 \mathrm{mi}$ east of Taos Pueblo, $4.5 \mathrm{mi}$ northeast of Taos, $5.8 \mathrm{mi}$ upstream from Rio Lucero, and at mile 15.1 .

DRA INAGE AREA, $--66.6 \mathrm{mi}^{2}$.

PERIOD OF RECORD.--January 1911 to December 1916, January 1940 to December 1951, annual maximum, water years 1952-62, October 1962 (monthly discharge only), November 1962 to current year. Monthly discharge only for some periods, published in WSP 1312 .

REVISED RECORDS.--WSP 1312: 1911-12, 1914. WSP 1732: Drainage area,

GAGE. - Water-stage recorder. Concrete control since Nov, 20, 1962 . Elevation of gage is $7,380 \mathrm{ft}$ above National Geodetic vertical Datum of 1929 , from topographic map. See WSP 1923 for history of changes prior to Nov. 20 , Geode 1962 .

REMARKS. --No diversions upstream from station.

AVERAGE DISCHARGE.--34 years (water years 1915, 1941-51, 1964-85), 29.8 ft 3/s, 21,590 acre-ft/yr.

EXTREMES FOR PERIOD OF RECORD.--Maximum discharge, 1,050 $\mathrm{ft}^{3} / \mathrm{s}$, May 26, 1979, gage height, $3.42 \mathrm{ft}$, from rating curve extended above $370 \mathrm{ft}^{3} / \mathrm{s}$; maximum gage height, $3.90 \mathrm{ft}$, from floodmark, May 14, 1941, site and datum then in use; minimum discharge, about $0.9 \mathrm{ft} / \mathrm{s}, \mathrm{Jan} .9,1964$, result of freezeup.

STATISTICAL SUMMARIES MEAN MONTHLY AND MEAN ANNUAL DISCHARGES $\begin{aligned} & 1915,1941-51, \\ & 1964-85\end{aligned}$

\begin{tabular}{|c|c|c|c|c|c|c|}
\hline MONTH & $\begin{array}{c}\text { MINIMUM } \\
\left(\mathrm{FT}^{3} / \mathrm{S}\right)\end{array}$ & $\begin{array}{c}\text { MAXIMUM } \\
\left(\mathrm{FT}^{3} / \mathrm{S}\right)\end{array}$ & $\begin{array}{l}\text { MEAN } \\
\left(F T^{3} / S\right)\end{array}$ & $\begin{array}{l}\text { STAN- } \\
\text { DARD } \\
\text { DEVIA- } \\
\text { TION } \\
\text { (FT'/S) }\end{array}$ & $\begin{array}{l}\text { COEFFI- } \\
\text { CIENT OF } \\
\text { VARI- } \\
\text { ATION }\end{array}$ & $\begin{array}{c}\text { PERCENT } \\
\text { OF } \\
\text { ANNUAL } \\
\text { RUNOFF }\end{array}$ \\
\hline $\begin{array}{l}\text { OCTOBER } \\
\text { NOVEMBER } \\
\text { DECEMBER } \\
\text { JANUARY } \\
\text { FEBRUARY } \\
\text { MARCH } \\
\text { APRIL } \\
\text { MAY } \\
\text { JUNE } \\
\text { JULY } \\
\text { AUGUST } \\
\text { SEPTEMBER }\end{array}$ & $\begin{array}{l}4.8 \\
4.8 \\
4.1 \\
3.4 \\
3.6 \\
5.6 \\
13 \\
11 \\
8.6 \\
4.6 \\
4.5 \\
4.2\end{array}$ & $\begin{array}{r}19 \\
18 \\
12 \\
11 \\
13 \\
27 \\
155 \\
356 \\
268 \\
75 \\
32 \\
32\end{array}$ & $\begin{array}{r}9.2 \\
8.5 \\
7.4 \\
6.7 \\
7.2 \\
12 \\
46 \\
121 \\
75 \\
23 \\
15 \\
11\end{array}$ & $\begin{array}{r}3.4 \\
2.7 \\
2.0 \\
1.6 \\
1.8 \\
4.2 \\
31 \\
96 \\
65 \\
16 \\
7.0 \\
5.9\end{array}$ & $\begin{array}{l}.37 \\
.32 \\
.27 \\
.25 \\
.25 \\
.36 \\
.67 \\
.79 \\
.87 \\
.71 \\
.46 \\
.53\end{array}$ & $\begin{array}{r}2.7 \\
2.5 \\
2.2 \\
1.9 \\
2.1 \\
3.4 \\
13.5 \\
35.4 \\
21.9 \\
6.8 \\
4.5 \\
3.2\end{array}$ \\
\hline ANNUAL & 7.7 & 72 & 30 & 18 & .59 & 100 \\
\hline
\end{tabular}

MAGNITUDE AND PROBABILITY OF ANNUAL LOW FLOW BASED ON PERIOD OF RECORD 1941-51, 1964-85

\begin{tabular}{|c|c|c|c|c|c|c|}
\hline \multirow{2}{*}{$\begin{array}{l}\text { PERIOD } \\
\text { (CON- } \\
\text { SECU- } \\
\text { TIVE } \\
\text { DAYS) }\end{array}$} & \multicolumn{6}{|c|}{$\begin{array}{l}\text { DISCHARGE, IN FT }{ }^{3} / \mathrm{S} \text {, FOR INDICATED RECURRENCE } \\
\text { INTERVAL, IN YEARS, AND ANNUAL NON- } \\
\text { EXCEEDANCE PROBABILITY, IN PERCENT }\end{array}$} \\
\hline & $\begin{array}{c}2 \\
508\end{array}$ & $\begin{array}{c}5 \\
208\end{array}$ & $\begin{array}{l}10 \\
108\end{array}$ & $\begin{array}{l}20 \\
58\end{array}$ & 50 & $\begin{array}{r}100 \\
18\end{array}$ \\
\hline $\begin{array}{r}1 \\
3 \\
7 \\
14 \\
30 \\
60 \\
90 \\
120 \\
183\end{array}$ & $\begin{array}{l}4.4 \\
4.8 \\
5.3 \\
5.7 \\
6.1 \\
6.4 \\
6.7 \\
7.0 \\
7.5\end{array}$ & $\begin{array}{l}3.3 \\
3.7 \\
4.2 \\
4.5 \\
4.9 \\
5.1 \\
5.4 \\
5.6 \\
6.0\end{array}$ & $\begin{array}{l}2.8 \\
3.2 \\
3.7 \\
3.9 \\
4.3 \\
4.5 \\
4.7 \\
5.0 \\
5.3\end{array}$ & $\begin{array}{l}2.4 \\
2.8 \\
3.3 \\
3.5 \\
3.8 \\
4.1 \\
4.2 \\
4.5 \\
4.8\end{array}$ & $\begin{array}{l}2.1 \\
2.5 \\
3.0 \\
3.0 \\
3.3 \\
3.6 \\
3.7 \\
4.0 \\
4.4\end{array}$ & $\begin{array}{l}-- \\
-- \\
-- \\
-- \\
-- \\
-- \\
--\end{array}$ \\
\hline
\end{tabular}

MAGNITUDE AND PROBABILITY OF ANNUAL HIGH FLOW BASED ON PERIOD OF RECORD 1915, 1941-51, $1964-85$

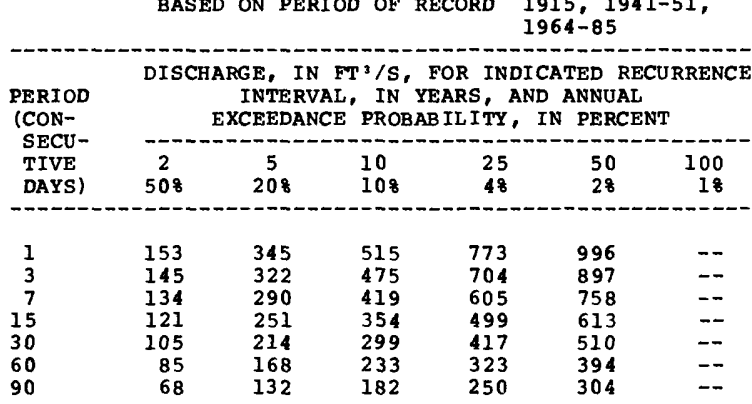

DURATION TABLE OF DAILY MEAN FLOW FOR PERIOD OF RECORD 1915, 1941-51, 1964-85 DISCHARGE, IN FT'/S, THAT WAS EQUALED OR EXCEEDED FOR INDICATED PERCENT OF TIME

\begin{tabular}{|c|c|c|c|c|c|c|c|c|c|c|c|c|c|c|}
\hline 58 & 108 & 158 & 208 & 258 & 308 & 408 & 508 & 608 & 708 & 758 & 808 & 858 & 908 & 958 \\
\hline 133 & 73 & 45 & 31 & 24 & 19 & 14 & 11 & 8.7 & 7.6 & 7.1 & 6.6 & 6.1 & 5.6 & \\
\hline
\end{tabular}


RIO GRANDE BASIN

08271000 RIO LUCERO NEAR ARROYO SECO, NM

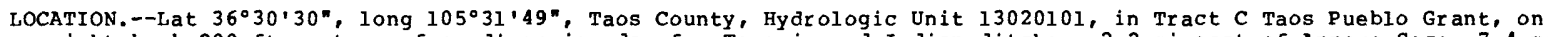
right bank $200 \mathrm{ft}$ upstream from diversion dam for Tenorio and Indian ditches, $2.2 \mathrm{mi}$ east of Arroyo Seco, $7.4 \mathrm{mi}$ northeast of Taos, and at mile 8.1 .

DRA INAGE AREA, $--16.6 \mathrm{mi}^{2}$.

PERIOD OF RECORD.--April to December 1910 (discharge measurements and occasional gage heights), January 19l1 to September 1915, March to December 1916 (fragmentary), October 1933 to December 1951, annual maximum, water years 1952-62, october 1962 (monthly discharge only), November 1962 to current year. Monthly discharge only for some periods, published in WSP 1312. Fragmentary records for October 1915 to February 1916, published in wSP 438, are unreliable and should not be used. Published as "near Taos," 1910-16.

REVISED RECORDS.--WSP 1512: 1912, 1916, 1949. WSP 1732: Drainage area. WDR NM-75-1: 1973. See also PERIOD OF RECORD.

GAGE.--Water-stage recorder. Concrete control since Nov. 21, 1962. Datum of gage is $8,051.44 \mathrm{ft}$ above National Geodetic Vertical Datum of 1929. See WSP 1923 for history of changes prior to Nov. $21,1962$.

REMARKS.--No diversions upstream from station.

AVERAGE DISCHARGE.--39 years (water years 1914-15, 1935-51, 1964-85), 22.0 ft 3/s, 15,940 acre-ft/yr.

EXTREMES FOR PERIOD OF RECORD.--Maximum discharge, $310 \mathrm{ft}^{3} / \mathrm{s}$, June 8,1979 , gage height, $2.33 \mathrm{ft}$; maximum gage height, $3.12 \mathrm{ft}$, May 13, 1941, datum then in use; minimum discharge, about $1.4 \mathrm{ft} / \mathrm{s}, \mathrm{Nov}$. $2,1951, \mathrm{result}$, of freezeup.

STATISTICAL SUMMARIES MEAN MONTHLY AND MEAN ANNUAL DISCHARGES $\begin{aligned} & 1914-15,1935-51 . \\ & 1964-85\end{aligned}$

\begin{tabular}{|c|c|c|c|c|c|c|}
\hline MONTH & $\begin{array}{c}\text { MINIMUM } \\
\left(\mathrm{FT}^{3} / \mathrm{S}\right)\end{array}$ & $\begin{array}{l}\text { MAXIMUM } \\
\left(\mathrm{FT}^{3} / \mathrm{S}\right)\end{array}$ & $\begin{array}{l}\text { MEAN } \\
\left(\mathrm{FT}^{3} / \mathrm{S}\right)\end{array}$ & $\begin{array}{l}\text { STAN- } \\
\text { DARD } \\
\text { DEVIA- } \\
\text { TION } \\
\left(\text { FT }^{3} / \mathrm{S}\right)\end{array}$ & $\begin{array}{l}\text { COEFFI- } \\
\text { CIENT OF } \\
\text { VARI- } \\
\text { ATION }\end{array}$ & $\begin{array}{c}\text { PERCENT } \\
\text { OF } \\
\text { ANNUAL } \\
\text { RUNOFF }\end{array}$ \\
\hline $\begin{array}{l}\text { OCTOBER } \\
\text { NOVEMBER } \\
\text { DECEMBER } \\
\text { JANUARY } \\
\text { FEBRUARY } \\
\text { MARCH } \\
\text { APRIL } \\
\text { MAY } \\
\text { JUNE } \\
\text { JULY } \\
\text { AUGUST } \\
\text { SEPTEMBER }\end{array}$ & $\begin{array}{r}6.3 \\
5.4 \\
4.3 \\
3.5 \\
3.5 \\
4.1 \\
8.8 \\
15 \\
14 \\
7.9 \\
6.6 \\
6.7\end{array}$ & $\begin{array}{c}28 \\
22 \\
14 \\
10 \\
9.0 \\
16 \\
48 \\
156 \\
178 \\
97 \\
38 \\
35\end{array}$ & $\begin{array}{l}11 \\
8.7 \\
6.9 \\
5.9 \\
5.7 \\
8.3 \\
22 \\
60 \\
72 \\
30 \\
18 \\
13\end{array}$ & $\begin{array}{l}4.3 \\
3.1 \\
1.9 \\
1.3 \\
1.3 \\
2.6 \\
9.1 \\
32 \\
46 \\
20 \\
7.6 \\
5.3\end{array}$ & $\begin{array}{l}.38 \\
.35 \\
.27 \\
.22 \\
.23 \\
.31 \\
.42 \\
.53 \\
.64 \\
.66 \\
.41 \\
.40\end{array}$ & $\begin{array}{r}4.2 \\
3.3 \\
2.6 \\
2.2 \\
2.2 \\
3.2 \\
8.3 \\
22.9 \\
27.6 \\
11.5 \\
7.0 \\
5.0\end{array}$ \\
\hline & 9.9 & 47 & 22 & 8.8 & .40 & 100 \\
\hline
\end{tabular}

MAGNITUDE AND PROBABILITY OF ANNUAL LOW FLOW BASED ON PERIOD OF RECORD 1915, 1936-51 $1964-85$

\begin{tabular}{|c|c|c|c|c|c|c|}
\hline $\begin{array}{l}\text { PERIOD } \\
\text { (CON- }\end{array}$ & \multicolumn{6}{|c|}{$\begin{array}{l}\text { DISCHARGE, IN FT }{ }^{3} / \mathrm{S} \text {, FOR INDICATED RECURRENCE } \\
\text { INTERVAL, IN YEARS, AND ANNUAL NON- } \\
\text { EXCEEDANCE PROBABILITY, IN PERCENT }\end{array}$} \\
\hline $\begin{array}{l}\text { SECU- } \\
\text { TIVE } \\
\text { DAYS) }\end{array}$ & $\begin{array}{c}2 \\
508\end{array}$ & $\begin{array}{c}5 \\
208\end{array}$ & $\begin{array}{l}10 \\
108\end{array}$ & $\begin{array}{l}20 \\
58\end{array}$ & 50 & 100 \\
\hline $\begin{array}{r}1 \\
3 \\
7 \\
14 \\
30 \\
60 \\
90 \\
120 \\
183\end{array}$ & $\begin{array}{l}4.5 \\
4.7 \\
4.9 \\
5.0 \\
5.2 \\
5.5 \\
5.7 \\
6.1 \\
7.2\end{array}$ & $\begin{array}{l}3.5 \\
3.7 \\
4.0 \\
4.2 \\
4.4 \\
4.6 \\
4.8 \\
5.1 \\
6.0\end{array}$ & $\begin{array}{l}3.0 \\
3.2 \\
3.5 \\
3.8 \\
4.0 \\
4.2 \\
4.4 \\
4.7 \\
5.5\end{array}$ & $\begin{array}{l}2.6 \\
2.8 \\
3.1 \\
3.5 \\
3.7 \\
3.9 \\
4.1 \\
4.4 \\
5.1\end{array}$ & $\begin{array}{l}2.1 \\
2.4 \\
2.8 \\
3.2 \\
3.4 \\
3.6 \\
3.8 \\
4.1 \\
4.8\end{array}$ & $\begin{array}{l}1.9 \\
2.2 \\
2.5 \\
3.0 \\
3.2 \\
3.4 \\
3.6 \\
3.9 \\
4.6\end{array}$ \\
\hline
\end{tabular}

MAGNITUDE AND PROBABILITY OF ANNUAL HIGH FLON BASED ON PERIOD OF RECORD 1914-15, 1935-51, 1964-85

\begin{tabular}{|c|c|c|c|c|c|c|}
\hline \multirow{2}{*}{$\begin{array}{l}\text { PERIOD } \\
\text { (CON- } \\
\text { SECU- } \\
\text { TIVE } \\
\text { DAYS) }\end{array}$} & \multicolumn{6}{|c|}{$\begin{array}{c}\text { DISCHARGE, IN FT } 3 / \mathrm{S} \text {, FOR INDICATED RECURRENC } \\
\text { INTERVAL, IN YEARS, AND ANNUAL } \\
\text { EXCEEDANCE' PROBABILITY, IN PERCENT }\end{array}$} \\
\hline & $\begin{array}{c}2 \\
508\end{array}$ & $\begin{array}{c}5 \\
208\end{array}$ & $\begin{array}{l}10 \\
108\end{array}$ & $\begin{array}{l}25 \\
48\end{array}$ & $\begin{array}{l}50 \\
28\end{array}$ & $\begin{array}{r}100 \\
18\end{array}$ \\
\hline $\begin{array}{r}1 \\
3 \\
7 \\
15 \\
30 \\
60 \\
90\end{array}$ & $\begin{array}{r}109 \\
104 \\
96 \\
87 \\
77 \\
63 \\
51\end{array}$ & $\begin{array}{r}178 \\
168 \\
153 \\
140 \\
125 \\
101 \\
81\end{array}$ & $\begin{array}{l}221 \\
207 \\
188 \\
172 \\
155 \\
126 \\
101\end{array}$ & $\begin{array}{l}270 \\
250 \\
227 \\
208 \\
191 \\
157 \\
125\end{array}$ & $\begin{array}{l}303 \\
278 \\
253 \\
232 \\
216 \\
179 \\
143\end{array}$ & $\begin{array}{l}332 \\
304 \\
277 \\
254 \\
239 \\
201 \\
160\end{array}$ \\
\hline
\end{tabular}

DURATION TABLE OF DAILY MEAN FLOW FOR PERIOD OF RECORD 1914-15, 1935-51, 1964-85 DISCHARGE, IN $\mathrm{FT}^{3} / \mathrm{S}$, THAT WAS EQUALED OR EXCEEDED FOR INDICATED PERCENT OF TIME

\begin{tabular}{|c|c|c|c|c|c|c|c|c|c|c|c|c|c|c|}
\hline 58 & 108 & 158 & 208 & 258 & 308 & 408 & 508 & 608 & 708 & 758 & 808 & 858 & 908 & 958 \\
\hline 86 & 54 & 38 & 29 & 23 & 19 & 14 & 11 & 8.9 & 7.3 & 6.8 & 6.3 & 5.8 & 5.3 & \\
\hline
\end{tabular}




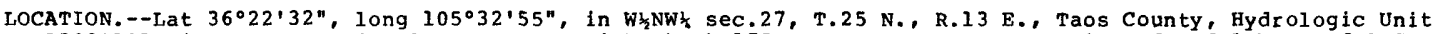

13020101 , in Carson National Forest, on right bank 175 ft upstream from Acequia Madre del Norte del Canon, 2.5 mi southeast of Taos, and at mile 5.0.

DRAINAGE AREA. $--71.7 \mathrm{mi} 2$.

PERIOD OF RECORD.--April to September 1910 (gage heights and discharge measurements only), October 1910 to June 1911 (discharge measurements only), October 1912 to September 1917, October 1927 to December 1928, October to November 1962 (monthly discharge only), December 1962 to September 1980 (discontinued).

REVISED RECORDS.--WSP 1512: 1914-15. WSP 1923: Drainage area.

GAGE.--Water-stage recorder. Concrete control since Dec. 13, 1962. Altitude of gage is $7,140 \mathrm{ft}$, from topographic map. See WSp 1923 for history of changes prior to Dec. 13, 1962.

REMARKS. --A few very small diversions above station for irrigation.

AVERAGE DISCHARGE. - -17 years (water years 1964-80), $5.70 \mathrm{ft}^{3} / \mathrm{s}, 4,130 \mathrm{acre}-\mathrm{ft} / \mathrm{yr}$.

EXTREMES FOR PERIOD OF RECORD (SINCE 1962).--Maximum discharge, $219 \mathrm{ft} / \mathrm{s}$ May 13, 1973, gage height, $2.38 \mathrm{ft}$ minimum, $0.02 \mathrm{ft} / \mathrm{s}$ part or all of each day Jan, 14-18, 1967, Sept. 15-19, 1972, Sept. 2, 4, 5, 8-13, 16, 19, oct. 7, 1978

EXTREMES OUTSIDE PERIOD OF RECORD.--A flood of undetermined magnitude occurred July $21,1921$.

STATISTICAL SUMMARIES

MEAN MONTHLY AND MEAN ANNUAL DISCHARGES 1964-80

\begin{tabular}{|c|c|c|c|c|c|c|}
\hline MONTH & $\begin{array}{l}\text { MIN I MUM } \\
\left(\mathrm{FTT}^{3} / \mathrm{S}\right)\end{array}$ & $\begin{array}{c}\text { MAXIMUM } \\
\left(\mathrm{FT}^{3} / \mathrm{S}\right)\end{array}$ & $\begin{array}{l}\text { MEAN } \\
\left(\mathrm{FT}^{3} / \mathrm{S}\right)\end{array}$ & $\begin{array}{l}\text { STAN- } \\
\text { DARD } \\
\text { DEVIA- } \\
\text { TION } \\
\left(\mathrm{FT}^{3} / \mathrm{S}\right)\end{array}$ & $\begin{array}{l}\text { COEFFI- } \\
\text { CIENT OF } \\
\text { VARI- } \\
\text { ATION }\end{array}$ & $\begin{array}{c}\text { PERCENT } \\
\text { OF } \\
\text { ANNUAI, } \\
\text { RUNOFF }\end{array}$ \\
\hline $\begin{array}{l}\text { OCTOBER } \\
\text { NOVEMBER } \\
\text { DECEMBER } \\
\text { JANUARY } \\
\text { FEBRUARY } \\
\text { MARCH } \\
\text { APRIL } \\
\text { MAY } \\
\text { JUNE } \\
\text { JULY } \\
\text { AUGUST } \\
\text { SEPTEMBER }\end{array}$ & $\begin{array}{r}.1 \\
.1 \\
0.0 \\
0.0 \\
.2 \\
.9 \\
2.8 \\
2.0 \\
.6 \\
.1 \\
.1 \\
0.0\end{array}$ & $\begin{array}{r}3.7 \\
4.2 \\
3.8 \\
3.8 \\
4.2 \\
7.9 \\
46 \\
115 \\
60 \\
15 \\
8.1 \\
4.2\end{array}$ & $\begin{array}{l}1.2 \\
1.4 \\
1.3 \\
1.3 \\
1.7 \\
3.7 \\
13 \\
29 \\
9.2 \\
2.8 \\
2.2 \\
1.3\end{array}$ & $\begin{array}{l}.9 \\
1.0 \\
1.0 \\
1.0 \\
1.0 \\
1.7 \\
10 \\
35 \\
15 \\
3.6 \\
2.0 \\
1.2\end{array}$ & $\begin{array}{r}.77 \\
.69 \\
.72 \\
.80 \\
.57 \\
.46 \\
.78 \\
1.20 \\
1.57 \\
1.28 \\
.91 \\
.88\end{array}$ & $\begin{array}{r}1.7 \\
2.0 \\
1.9 \\
1.9 \\
2.5 \\
5.4 \\
19.3 \\
42.3 \\
13.5 \\
4.1 \\
3.2 \\
1.9\end{array}$ \\
\hline ANNUAL & 1.3 & 20 & 5.7 & 5.2 & .91 & 100 \\
\hline
\end{tabular}

MAGNITUDE AND PROBABILITY OF ANNUAL LOW FLOW BASED ON PERIOD OF RECORD $1965-80$

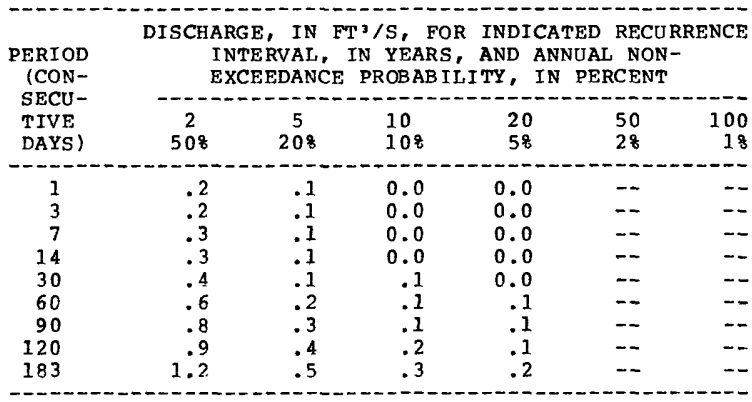

MAGNITUDE AND PROBABII.ITY OF ANNUAL HIGH FLOW BASED ON PERIOD OF RECORD $1964-80$

\begin{tabular}{|c|c|c|c|c|c|c|}
\hline \multirow{2}{*}{$\begin{array}{l}\text { PERIOD } \\
\text { (CON- } \\
\text { SECU- } \\
\text { TIVE } \\
\text { DAYS) }\end{array}$} & \multicolumn{6}{|c|}{$\begin{array}{l}\text { DISCHARGE, IN FT'/S, FOR INDICATED RECURRENC } \\
\text { INTERVAL, IN YEARS, AND ANNUAL } \\
\text { EXCEEDANCE PROBABILITY, IN PERCENT }\end{array}$} \\
\hline & $\begin{array}{c}2 \\
508\end{array}$ & $\begin{array}{c}5 \\
208\end{array}$ & $\begin{array}{l}10 \\
10 z\end{array}$ & $\begin{array}{l}25 \\
48\end{array}$ & $\begin{array}{l}50 \\
28\end{array}$ & $\begin{array}{r}100 \\
18\end{array}$ \\
\hline 1 & 26 & 68 & 116 & 207 & -- & -- \\
\hline 3 & 25 & 67 & 113 & 199 & -- & -- \\
\hline 7 & 23 & 62 & 103 & 180 & -- & -- \\
\hline 15 & 21 & 54 & 92 & 161 & -- & -- \\
\hline 30 & 18 & 45 & 74 & 129 & -- & -- \\
\hline 60 & 14 & 34 & 55 & 92 & -- & -- \\
\hline 90 & 11 & 26 & 41 & 68 & -- & -- \\
\hline
\end{tabular}

DURATION TABLE OF DAILY MEAN FLOW FOR PERIOD OF RECORD 1964-80

DISCHARGF, IN FT $3 / \mathrm{S}$, THAT WAS EQUALED OR EXCEEDFD FOR INDICATED PERCENT OF TIMF

\begin{tabular}{|c|c|c|c|c|c|c|c|c|c|c|c|c|c|c|}
\hline 58 & 108 & 158 & 208 & 258 & 308 & 408 & 508 & $60 \%$ & 708 & 758 & 808 & 858 & $90 z$ & $95 \%$ \\
\hline 23 & 12 & 7.5 & 5.1 & 4.1 & 3.3 & 2.5 & 1.9 & 1.4 & 1.0 & .8 & .7 & .5 & .3 & .1 \\
\hline
\end{tabular}




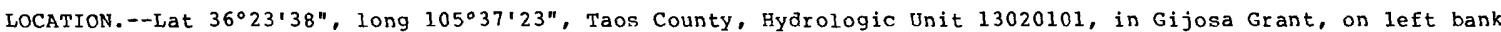

$1,100 \mathrm{ft}$ downstream from Rio Fernando de Taos, $1.6 \mathrm{mi}$ southwest of Ranchito, and at mile 7.9 .

DRAINAGE AREA, - - $199 \mathrm{mi}^{2}$.

PERIOD OF RECORD.--March 1957 to September 1980 (discontinued).

GAGE.--Water-stage recorder. Altitude of gage is 6,747 ft, from topographic map.

REMARKS.--Diversions for irrigation of about 9,000 acres above station.

AVERAGE DISCHARGE.--22 years (water years 1958-80), 31.0 $\mathrm{ft}^{3} / \mathrm{s}, 22,460$ acre-ft/yr.

EXTREMES FOR PERIOD OF RECORD.--Maximum discharge, 1,290 ft $3 / \mathrm{s}$ May 26, 1979, gage height, $4.82 \mathrm{ft}$; minimum, 0.21 $\mathrm{ft}^{3} / \mathrm{s}$ Aug. 24,1972 , result of regulation.

STATISTICAL SUMMARIES

MEAN MONTHLY AND MEAN ANNUAL DISCHARgES 1958-80

\begin{tabular}{|c|c|c|c|c|c|c|}
\hline MONTH & $\begin{array}{l}\text { MINIMUM } \\
\left(\mathrm{FT}^{3} / \mathrm{S}\right)\end{array}$ & $\begin{array}{c}\operatorname{MAXIMUM} \\
\left(\mathrm{FT}^{3} / \mathrm{S}\right)\end{array}$ & $\begin{array}{l}\text { MEAN } \\
\left(\mathrm{FT}^{3} / \mathrm{S}\right)\end{array}$ & $\begin{array}{l}\text { STAN- } \\
\text { DARD } \\
\text { DEVIA- } \\
\text { TION } \\
\left(\mathrm{FT}^{3} / \mathrm{S}\right)\end{array}$ & $\begin{array}{l}\text { COEFFI- } \\
\text { CIENT OF } \\
\text { VARI- } \\
\text { ATION }\end{array}$ & $\begin{array}{c}\text { PERCENT } \\
\text { OF } \\
\text { ANNUAL } \\
\text { RUNOFF }\end{array}$ \\
\hline $\begin{array}{l}\text { OCTOBER } \\
\text { NOVEMBER } \\
\text { DECEMBER } \\
\text { JANUARY } \\
\text { FFBRUARY } \\
\text { NIARCH } \\
\text { APRIL } \\
\text { MAY } \\
\text { JUNE } \\
\text { JULY } \\
\text { AUGUST } \\
\text { SEPTEMBER }\end{array}$ & $\begin{array}{r}4.0 \\
8.2 \\
10 \\
8.3 \\
12 \\
14 \\
4.7 \\
2.8 \\
2.6 \\
1.5 \\
1.7 \\
2.2\end{array}$ & $\begin{array}{r}45 \\
52 \\
35 \\
26 \\
33 \\
39 \\
150 \\
454 \\
461 \\
109 \\
33 \\
30\end{array}$ & $\begin{array}{r}15 \\
19 \\
18 \\
17 \\
20 \\
23 \\
51 \\
103 \\
64 \\
16 \\
12 \\
10\end{array}$ & $\begin{array}{r}9.9 \\
9.0 \\
5.8 \\
4.4 \\
4.8 \\
6.7 \\
41 \\
125 \\
103 \\
24 \\
10 \\
8.6\end{array}$ & $\begin{array}{r}.68 \\
.48 \\
.32 \\
.25 \\
.24 \\
.29 \\
.79 \\
1.21 \\
1.61 \\
1.51 \\
.86 \\
.82\end{array}$ & $\begin{array}{r}3.9 \\
5.1 \\
5.0 \\
4.7 \\
5.4 \\
6.3 \\
13.9 \\
28.0 \\
17.3 \\
4.3 \\
3.2 \\
2.8\end{array}$ \\
\hline ANNUAL & 9.2 & 108 & 31 & 24 & .78 & 100 \\
\hline
\end{tabular}

MAGNITUDE AND PROBABILITY OF ANNUAL LOW FLOW BASED ON PERIOD OF RECORD 1959-80

\begin{tabular}{|c|c|c|c|c|c|c|}
\hline \multirow{3}{*}{$\begin{array}{l}\text { PERIOD } \\
\text { (CON- } \\
\text { SECU - } \\
\text { TIVE } \\
\text { DAYS) }\end{array}$} & \multicolumn{6}{|c|}{$\begin{array}{l}\text { DISCHARGE, IN } \text { FT }^{3} / S \text {, FOR INDICATED RECURRENCE } \\
\text { INTERVAL, IN YEARS, AND ANNUAL NON- } \\
\text { EXCEEDANCE PROBABILITY, IN PERCENT }\end{array}$} \\
\hline & ---- & 5 & 10 & 20 & 50 & 100 \\
\hline & $50 \%$ & 208 & $10 \%$ & 58 & 28 & 18 \\
\hline 1 & 3.0 & 1.4 & 9 & 6 & -- & - \\
\hline 3 & 3.2 & 1.5 & 1.0 & .7 & - & \\
\hline 7 & 3.5 & 1.7 & 1.1 & .8 & -- & $\ldots$ \\
\hline 14 & 3.8 & 2.0 & 1.4 & 1.0 & -- & -- \\
\hline 30 & 4.5 & 2.4 & 1.8 & 3.4 & - & $\ldots$ \\
\hline 60 & 5.8 & 2.9 & 2.1 & 1.5 & -- & -- \\
\hline 90 & 6.7 & 3.4 & 2.4 & 1.7 & -- & - \\
\hline 120 & 7.7 & 4.0 & 2.8 & 2.1 & - &.- \\
\hline 183 & 11 & 5.8 & 4.0 & 2.9 & -- & -- \\
\hline
\end{tabular}

MAGNITUDE AND PROBABILITY OF ANNUAL HIGH FLOW BASED ON PERIOD OF RECORD 1958-80

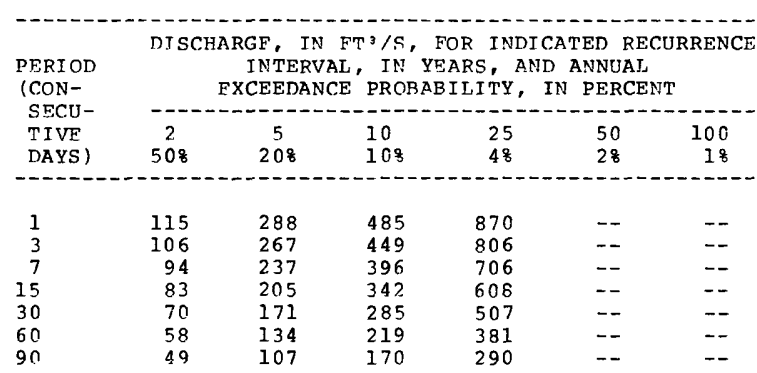

DURATION TABLE OF DAIJYY MEAN FIOW FOR PERIOD OF RECORD 1958-80 DISCHARGE, IN FT ${ }^{3} / S$, THAT WAS FQUALFD OR EXCFEDED FOR INDICATED PERCENT OF TIME

\begin{tabular}{|c|c|c|c|c|c|c|c|c|c|c|c|c|c|c|}
\hline 58 & 108 & 158 & 208 & 258 & $30 \%$ & 408 & 508 & 608 & 708 & $75 \%$ & $80 \%$ & 858 & $90 \%$ & 958 \\
\hline 105 & 56 & 39 & 30 & 26 & 24 & 20 & 18 & 15 & 12 & 9.7 & 7.2 & 5.3 & 4.0 & \\
\hline
\end{tabular}


RIO GRANDE BASIN

08275500 RIO GRANDE DEL RANCHO NEAR TALPA, NM

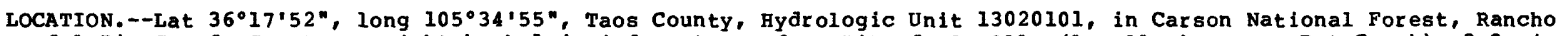
del Rio Grande Grant, on right bank $1.4 \mathrm{mi}$ downstream from Rito de la olla (locally known as Pot Creek), 3.2 mi south of Talpa, $4.3 \mathrm{mi}$ upstream from Rio Chiguito, and at mile 6.9.

DRAINAGE AREA. $--83 \mathrm{mi}^{2}$, approximately.

PERIOD OF RECORD.--October 1952 to September 1982 (discontinued). Prior to October 1955, published as Rio Grande del Rancho near Ranchos de Taos and October 1955 to September 1960 as Rio Grande de Ranchos near Talpa.

GAGE.--Water-stage recorder. Altitude of gage is $7,238 \mathrm{ft}$, from topographic map. Prior to Nov. 11, 1952, nonrecording gage at site $1,035 \mathrm{ft}$ downstream at lower datum. Nov. 11, 1952 to Nov. 5, 1968, water-stage recorder at site $1,000 \mathrm{ft}$ downstream at lower datum. Nov. 6, 1968 to Aug. 28, 1980, water-stage recorder at present. site on left bank at same datum.

REMARKS. - Minor diversions for irrigation above station.

AVERAGE DISCHARGE.--30 years (water years 1953-82), $19.7 \mathrm{ft} / \mathrm{s}, 14,270$ acre-ft/yr.

EXTREMES FOR PERIOD OF RECORD.--Maximum discharge, $497 \mathrm{ft} / \mathrm{s}$ May 21, 1973, gage height $3.87 \mathrm{ft}$; maximum gage height, $4.01 \mathrm{ft}$ Sept. 10, 1964, site and datum then in use; minimum discharge, $0.2 \mathrm{ft} / \mathrm{s} \mathrm{Jan}$. 5, $1955, \mathrm{result}$ of freezeup.

STATISTICAL SUMMARIES

MEAN MONTHLY AND MEAN ANNUAL DISCHARGES 1953-82

\begin{tabular}{|c|c|c|c|c|c|c|}
\hline MONTH & $\begin{array}{c}\text { MINIMUM } \\
\left(\mathrm{FT}^{2} / \mathrm{S}\right)\end{array}$ & $\begin{array}{c}\text { MAXIMUM } \\
(\mathrm{FT} \cdot / \mathrm{S})\end{array}$ & $\begin{array}{l}\text { MEAN } \\
\left(\mathrm{FT}^{3} / \mathrm{S}\right)\end{array}$ & $\begin{array}{c}\text { STAN- } \\
\text { DARD } \\
\text { DEVIA- } \\
\text { TION } \\
(\text { FT'/S) }\end{array}$ & $\begin{array}{l}\text { COEFFI - } \\
\text { CIENT OF } \\
\text { VARI- } \\
\text { ATION }\end{array}$ & $\begin{array}{c}\text { PERCENT } \\
\text { OF } \\
\text { ANNUAL } \\
\text { RUNOFF }\end{array}$ \\
\hline $\begin{array}{l}\text { OCTOBER } \\
\text { NOVEMBER } \\
\text { DECEMBER } \\
\text { JANUARY } \\
\text { FEBRUARY } \\
\text { MARCH } \\
\text { APRIL } \\
\text { MAY } \\
\text { JUNE } \\
\text { JULY } \\
\text { AUGUST } \\
\text { SEPTEMBER }\end{array}$ & $\begin{array}{r}2.1 \\
3.0 \\
3.0 \\
2.1 \\
2.7 \\
4.7 \\
9.6 \\
13 \\
6.4 \\
3.1 \\
2.3 \\
1.6\end{array}$ & $\begin{array}{c}14 \\
11 \\
10 \\
9.2 \\
8.8 \\
13 \\
92 \\
237 \\
171 \\
29 \\
36 \\
25\end{array}$ & $\begin{array}{c}6.7 \\
5.9 \\
5.3 \\
4.8 \\
5.2 \\
7.8 \\
30 \\
88 \\
49 \\
13 \\
12 \\
8.6\end{array}$ & $\begin{array}{l}2.8 \\
1.9 \\
1.6 \\
1.5 \\
1.4 \\
2.3 \\
20 \\
63 \\
45 \\
6.9 \\
8.5 \\
6.0\end{array}$ & $\begin{array}{l}. .43 \\
.32 \\
.31 \\
.32 \\
.27 \\
.29 \\
.67 \\
.72 \\
.91 \\
.53 \\
.69 \\
.69\end{array}$ & $\begin{array}{r}2.8 \\
2.5 \\
2.2 \\
2.0 \\
2.2 \\
3.3 \\
12.7 \\
37.1 \\
20.7 \\
5.5 \\
5.2 \\
3.7\end{array}$ \\
\hline ANNUAL & 6.0 & 42 & 20 & 11 & .54 & 100 \\
\hline
\end{tabular}

MAGNITUDE AND PROBABILITY OF ANNUAL LOW FLOW BASED ON PERIOD OF RECORD 1954-82

\begin{tabular}{|c|c|c|c|c|c|c|}
\hline \multirow{2}{*}{$\begin{array}{l}\text { PERIOD } \\
\text { (CON- } \\
\text { SECU- } \\
\text { TIVE } \\
\text { DAYS) }\end{array}$} & \multicolumn{6}{|c|}{$\begin{array}{l}\text { DISCHARGE, IN FT } 3 / \mathrm{S} \text {, FOR INDICATED RECURRENCE } \\
\text { INTERVAL, IN YEARS, AND ANNUAL NON- } \\
\text { EXCEEDANCE PROBABILITY, IN PERCENT }\end{array}$} \\
\hline & $\begin{array}{c}2 \\
50 \%\end{array}$ & $\begin{array}{c}5 \\
208\end{array}$ & $\begin{array}{l}10 \\
108\end{array}$ & $\begin{array}{l}20 \\
58\end{array}$ & $\begin{array}{l}50 \\
28\end{array}$ & $\begin{array}{r}100 \\
18\end{array}$ \\
\hline $\begin{array}{r}1 \\
3 \\
7 \\
14 \\
30 \\
60 \\
90 \\
120 \\
183\end{array}$ & $\begin{array}{l}3.1 \\
3.2 \\
3.6 \\
3.8 \\
4.1 \\
4.5 \\
4.7 \\
4.9 \\
5.4\end{array}$ & $\begin{array}{l}2.0 \\
2.2 \\
2.5 \\
2.7 \\
3.0 \\
3.3 \\
3.5 \\
3.7 \\
4.1\end{array}$ & $\begin{array}{l}1.5 \\
1.7 \\
1.9 \\
2.2 \\
2.4 \\
2.7 \\
3.0 \\
3.2 \\
3.5\end{array}$ & $\begin{array}{l}1.1 \\
1.3 \\
1.6 \\
1.8 \\
2.0 \\
2.3 \\
2.6 \\
2.8 \\
3.0\end{array}$ & $\begin{array}{r}.8 \\
1.0 \\
1.2 \\
1.4 \\
1.6 \\
1.9 \\
2.2 \\
2.4 \\
2.6\end{array}$ & \\
\hline
\end{tabular}

MAGNITUDE AND PROBABILITY OF ANNUAL HIGH FLOW BASED ON PERIOD OF RECORD 1953-82

\begin{tabular}{|c|c|c|c|c|c|c|}
\hline \multirow{2}{*}{$\begin{array}{l}\text { PERIOD } \\
\text { (CON- } \\
\text { SECU- } \\
\text { TIVE } \\
\text { DAYS) }\end{array}$} & \multicolumn{6}{|c|}{$\begin{array}{l}\text { DISCHARGE, IN FT } 3 / \text { S, FOR INDICATED RECURRENCE } \\
\text { INTERVAL, IN YEARS, AND ANNUAL } \\
\text { EXCEEDANCE PROBABILITY, IN PERCENT }\end{array}$} \\
\hline & $\begin{array}{c}2 \\
508\end{array}$ & $\begin{array}{c}5 \\
208\end{array}$ & $\begin{array}{l}10 \\
108\end{array}$ & $\begin{array}{l}25 \\
48\end{array}$ & $\begin{array}{l}50 \\
28\end{array}$ & $\begin{array}{r}100 \\
18\end{array}$ \\
\hline $\begin{array}{r}1 \\
3 \\
7 \\
15 \\
30 \\
60 \\
90\end{array}$ & $\begin{array}{r}116 \\
112 \\
105 \\
94 \\
79 \\
59 \\
46\end{array}$ & $\begin{array}{r}233 \\
223 \\
207 \\
184 \\
155 \\
113 \\
85\end{array}$ & $\begin{array}{l}319 \\
306 \\
282 \\
250 \\
212 \\
153 \\
115\end{array}$ & $\begin{array}{l}432 \\
413 \\
380 \\
336 \\
289 \\
209 \\
154\end{array}$ & $\begin{array}{l}515 \\
493 \\
452 \\
400 \\
349 \\
252 \\
185\end{array}$ & $\begin{array}{l}-- \\
-- \\
-- \\
-- \\
-- \\
-- \\
--\end{array}$ \\
\hline
\end{tabular}

DURATION TABLE OF DAILY MEAN FLOW FOR PERIOD OF RECORD 1953-82 DISCHARGE, IN FT'/S, THAT WAS EQUALED OR EXCEEDED FOR INDICATED PERCENT OF TIME

\begin{tabular}{|c|c|c|c|c|c|c|c|c|c|c|c|c|c|c|}
\hline 58 & 108 & 158 & 208 & 258 & 308 & 408 & 508 & 608 & 708 & 758 & 808 & 858 & 908 & 958 \\
\hline 88 & 46 & 28 & 20 & 15 & 13 & 9.2 & 7.4 & 6.3 & 5.4 & 5.0 & 4.6 & 4.2 & 3.7 & \\
\hline
\end{tabular}


LOCATION,--Lat $36^{\circ} 19^{\prime} 55^{\prime \prime}$, long $105^{\circ} 34^{\prime} 42^{\prime \prime}$, Taos County, Hydrologic Unit 13020101, in Carson National Forest, Rancho del Rio Grande Grant, on right bank $1 \mathrm{mi}$ southeast of Talpa, and at mile 2.1 .

DRAINAGE AREA. $--37.0 \mathrm{mi}^{2}$.

PERIOD OF RECORD.--March 1957 to September 1980 (discontinued).

GAGE.--Water-stage recorder. Altitude of gage is $7,223 \mathrm{ft}$, from topographic map.

REMARKS, - No diversions above station.

AVERAGE DISCHARGE, --23 years (water years $1958-80$ ), $8.41 \mathrm{ft}^{3} / \mathrm{s}, 6,090$ acre-ft/yr.

EXTREMES FOR PERIOD OF RECORD.--Maximum discharge, $309 \mathrm{ft} 3 / \mathrm{s}$ June 8, 1979, gage height, $2.81 \mathrm{ft}$; maximum gage height, $3.50 \mathrm{ft}$ May 20, 1973 (backwater from debris); minimum discharge, 0.16 ft3/s Jan. 31,1972 , result of freezeup.

STATISTICAL SUMMARIES

MEAN MONTHLY AND MEAN ANNUAL DISCHARGES 1958-80

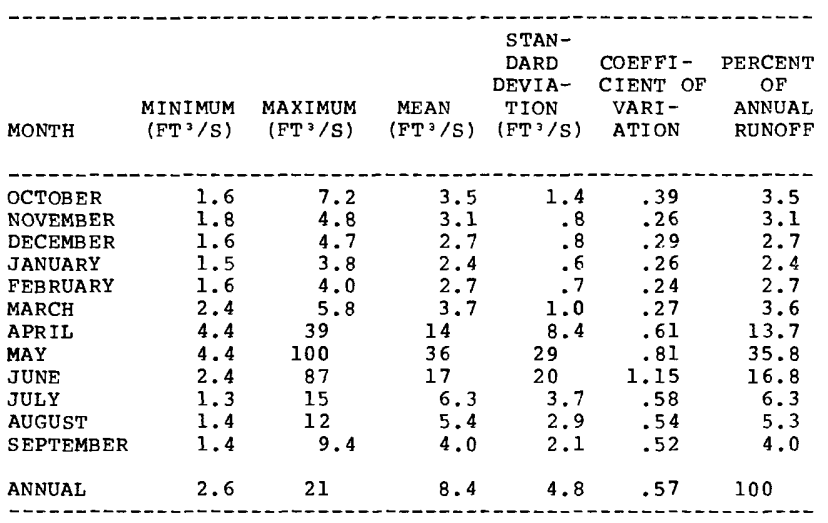

MAGNITUDE AND PROBABILITY OF ANNUAL LOW FLOW BASED ON PERIOD OF RECORD 1959-80

\begin{tabular}{|c|c|c|c|c|c|c|}
\hline $\begin{array}{l}\text { PERIOD } \\
\text { (CON- } \\
\text { SECU- }\end{array}$ & \multicolumn{6}{|c|}{$\begin{array}{l}\text { DISCHARGE, IN FT }{ }^{3} / S \text {, FOR INDICATED RECURRENCE } \\
\text { INTERVAL, IN YEARS, AND ANNUAL NON- } \\
\text { EXCEEDANCE PROBABILITY, IN PERCENT }\end{array}$} \\
\hline $\begin{array}{l}\text { TIVE } \\
\text { DAYS) }\end{array}$ & $\begin{array}{c}2 \\
508\end{array}$ & $\begin{array}{c}5 \\
208\end{array}$ & $\begin{array}{l}10 \\
108\end{array}$ & $\begin{array}{l}20 \\
58\end{array}$ & $\begin{array}{l}50 \\
28\end{array}$ & $\begin{array}{r}100 \\
18\end{array}$ \\
\hline $\begin{array}{r}1 \\
3 \\
7 \\
14 \\
30 \\
60 \\
90 \\
120 \\
183\end{array}$ & $\begin{array}{l}1.2 \\
1.4 \\
1.7 \\
1.8 \\
2.0 \\
2.2 \\
2.4 \\
2.5 \\
2.8\end{array}$ & $\begin{array}{r}.8 \\
1.0 \\
1.2 \\
1.3 \\
1.6 \\
1.7 \\
1.9 \\
2.0 \\
2.2\end{array}$ & $\begin{array}{r}.6 \\
.7 \\
.9 \\
1.1 \\
1.4 \\
1.5 \\
1.7 \\
1.8 \\
1.9\end{array}$ & $\begin{array}{r}.4 \\
.6 \\
.8 \\
1.0 \\
1.2 \\
1.4 \\
1.5 \\
1.6 \\
1.7\end{array}$ & $\begin{array}{l}-- \\
-- \\
-- \\
-- \\
-- \\
-- \\
-- \\
--\end{array}$ & - \\
\hline
\end{tabular}

MAGNITUDE AND PROBABILITY OF ANNUAL HIGH FLOW BASED ON PERIOD OF RECORD 1958-80

\begin{tabular}{|c|c|c|c|c|c|c|}
\hline $\begin{array}{l}\text { PERIOD } \\
\text { (CON- } \\
\text { SECU- }\end{array}$ & DIS & $\begin{array}{l}\text { EE, } \\
\text { NTE } \\
\text { CEED }\end{array}$ & $\begin{array}{l}\Gamma^{3} / \mathrm{S} \\
\text { IN } \\
\text { PRO }\end{array}$ & $\begin{array}{l}\text { R IN } \\
\text { RS, } \\
\text { LITY }\end{array}$ & $\begin{array}{l}\text { ED R } \\
\text { NNUA } \\
\text { PERC }\end{array}$ & RRENCE \\
\hline $\begin{array}{l}\text { TIVE } \\
\text { DAYS) }\end{array}$ & $\begin{array}{c}2 \\
508\end{array}$ & $\begin{array}{c}5 \\
20 \%\end{array}$ & $\begin{array}{l}10 \\
108\end{array}$ & $\begin{array}{l}25 \\
48\end{array}$ & $\begin{array}{l}50 \\
28\end{array}$ & $\begin{array}{r}100 \\
18\end{array}$ \\
\hline $\begin{array}{r}1 \\
3 \\
7 \\
15 \\
30 \\
60 \\
90\end{array}$ & $\begin{array}{l}42 \\
40 \\
38 \\
34 \\
30 \\
22 \\
18\end{array}$ & $\begin{array}{l}92 \\
89 \\
82 \\
73 \\
63 \\
45 \\
35\end{array}$ & $\begin{array}{r}136 \\
130 \\
119 \\
105 \\
90 \\
64 \\
48\end{array}$ & $\begin{array}{r}200 \\
188 \\
170 \\
151 \\
129 \\
91 \\
67\end{array}$ & $\begin{array}{l}-- \\
=- \\
-- \\
-- \\
-- \\
-- \\
--\end{array}$ & $\begin{array}{l}-- \\
-- \\
-- \\
-- \\
-- \\
-- \\
--\end{array}$ \\
\hline
\end{tabular}

DURATION TABLE OF DAILY MEAN FLOW FOR PERIOD OF RECORD 1958-80 DISCHARGE, IN FT $3 / S$, THAT WAS EQUALED OR EXCEEDED FOR INDICATED PERCENT OF TIME

\begin{tabular}{|c|c|c|c|c|c|c|c|c|c|c|c|c|c|c|}
\hline 58 & 108 & 158 & 208 & 258 & 308 & 408 & 508 & 608 & 708 & 758 & 808 & 858 & 908 & 958 \\
\hline 36 & 17 & 12 & 8.3 & 6.8 & 5.6 & 4.5 & 3.8 & 3.2 & 2.8 & 2.6 & 2.4 & 2.2 & 1.9 & 1 \\
\hline
\end{tabular}


RIO GRANDE BASIN

08276000 RIO PUEBLO DE TAOS AT LOS CORDOVAS, NM

LOCATION, - Lat $36^{\circ} 23^{\prime} 20^{\prime \prime}$, long $105^{\circ} 38^{\prime} 00^{\prime \prime}$, in SEl/2 sec.23, T.25 N., R.12 E., (projected), in Martinez Grant, on left bank $200 \mathrm{ft}$ downstream from Rio Grande de Ranchos, 0.2 mile downstream from Arroyo Seco, half a mile left bank $200 \mathrm{ft}$ downstream from Rio Grande de Ranchos,
northeast of Los Cordovas, and $31 / 2 \mathrm{miles}$ west of Taos.

DRAINAGE AREA. $--359 \mathrm{mi}^{2}$.

PERIOD OF RECORD.--April 1910 to January 1926, September 1926 to September 1965 (discontinued). Monthly discharge only for some periods, published in WSP 1312. Prior to October 1955, published as Rio Taos at Los Cordovas. All records include flow of Rio Grande de Ranchos.

GAGE.-Water-stage recorder. Datum of gage is 6,709.59 ft above mean sea level, datum of 1929 . Prior to Oct. 4 , 192I, staff gages at nearby sites at different datums. Oct. 4, 1921 to sept. 30, 1934, water-stage recorder at site $200 \mathrm{ft}$ upstream at datum about $1.26 \mathrm{ft}$ higher and Oct. 1, 1934 to Apr. 30, 1957, at site 200 ft upstream at datum $1.00 \mathrm{ft}$ higher.

REMARKS.--Diversions above station for irrigation of about 12,000 acres, a small part of which is below station. AVERAGE DISCHARGE. - -54 years $(1910-25,1926-65), 58.5 \mathrm{ft}^{3} / \mathrm{s}(42,350 \mathrm{acre-ft} /$ year $)$.

EXTREMES FOR PERIOD OF RECORD.--Maximum discharge, I,830 $\mathrm{ft}^{3 / \mathrm{s}}$ May 14, 1941 (gage height, $5.81 \mathrm{ft}$, site and datum then in use), from rating curve extended above $1,300 \mathrm{ft}^{3} / \mathrm{s}$ by logarithmic plotting; minimum, $0.8 \mathrm{ft}{ }^{3} / \mathrm{s}$ July 17 , 1951.

STATISTICAL SUMMARIES MEAN MONTHLY AND MEAN ANNUAL DISChARgES $\begin{array}{r}1911-25 \\ 1927-65\end{array}$
MAGNITUDE AND PROBABILITY OF ANNUAL LOW FLOW BASED ON PERIOD OF RECORD 1912-25, 1928-65

\begin{tabular}{|c|c|c|c|c|c|c|c|c|c|c|c|c|c|}
\hline \multirow[b]{2}{*}{ MONTH } & \multirow{2}{*}{$\begin{array}{l}\text { MINIMUM } \\
\left(\mathrm{FT}^{3} / \mathrm{S}\right)\end{array}$} & \multirow{2}{*}{$\begin{array}{c}\text { MAXIMUM } \\
\left(\mathrm{FT}^{3} / \mathrm{S}\right)\end{array}$} & \multirow{2}{*}{$\begin{array}{l}\text { MEAN } \\
\left(\mathrm{FT}^{3} / \mathrm{S}\right)\end{array}$} & \multirow{2}{*}{$\begin{array}{l}\text { STAN- } \\
\text { DARD } \\
\text { DEVIA- } \\
\text { TION } \\
\left(\mathrm{FT}^{3} / \mathrm{S}\right)\end{array}$} & \multirow{2}{*}{$\begin{array}{l}\text { COEFFI- } \\
\text { CIENT OF } \\
\text { VARI- } \\
\text { ATION }\end{array}$} & \multirow{2}{*}{$\begin{array}{l}\text { PERCENT } \\
\text { OF } \\
\text { ANNUAL } \\
\text { RUNOFF }\end{array}$} & \multirow{2}{*}{$\begin{array}{l}\text { PERIOD } \\
\text { (CON- } \\
\text { SECU- } \\
\text { TIVE } \\
\text { DAYS) }\end{array}$} & \multicolumn{6}{|c|}{ 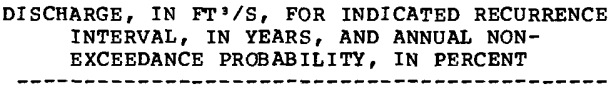 } \\
\hline & & & & & & & & $\begin{array}{c}2 \\
508\end{array}$ & $\begin{array}{c}5 \\
20 \%\end{array}$ & $\begin{array}{l}10 \\
108\end{array}$ & $\begin{array}{l}20 \\
58\end{array}$ & $\begin{array}{l}50 \\
28\end{array}$ & $\begin{array}{r}100 \\
18\end{array}$ \\
\hline $\begin{array}{l}\text { OCTOBER } \\
\text { NOVEMBER } \\
\text { DECEMBER } \\
\text { JANUARY } \\
\text { FEBRUARY } \\
\text { MARCH } \\
\text { APRIL } \\
\text { MAY } \\
\text { JUNE }\end{array}$ & $\begin{array}{l}3.9 \\
11 \\
15 \\
15 \\
17 \\
19 \\
16 \\
10 \\
4.1\end{array}$ & $\begin{array}{r}149 \\
75 \\
59 \\
53 \\
72 \\
89 \\
452 \\
1038 \\
480\end{array}$ & $\begin{array}{r}25 \\
29 \\
29 \\
29 \\
35 \\
43 \\
102 \\
230 \\
116\end{array}$ & $\begin{array}{r}22 \\
13 \\
9.3 \\
8.1 \\
11 \\
16 \\
82 \\
225 \\
121\end{array}$ & $\begin{array}{l}.90 \\
.46 \\
.33 \\
.28 \\
.32 \\
.36 \\
.80 \\
.98 \\
1.04\end{array}$ & $\begin{array}{r}3.6 \\
4.1 \\
4.1 \\
4.2 \\
5.0 \\
6.2 \\
14.6 \\
32.8 \\
16.6\end{array}$ & $\begin{array}{r}1 \\
3 \\
7 \\
14 \\
30 \\
60 \\
90 \\
120 \\
183\end{array}$ & $\begin{array}{l}4.9 \\
5.4 \\
6.0 \\
6.9 \\
8.6 \\
11 \\
12 \\
14 \\
18\end{array}$ & $\begin{array}{r}2.8 \\
3.2 \\
3.5 \\
4.0 \\
5.0 \\
6.0 \\
7.0 \\
8.1 \\
11\end{array}$ & $\begin{array}{l}2.1 \\
2.4 \\
2.6 \\
3.0 \\
3.8 \\
4.5 \\
5.2 \\
6.1 \\
8.9\end{array}$ & $\begin{array}{l}1.7 \\
2.0 \\
2.1 \\
2.4 \\
3.0 \\
3.5 \\
4.1 \\
4.7 \\
7.3\end{array}$ & $\begin{array}{l}1.3 \\
1.5 \\
1.7 \\
1.9 \\
2.4 \\
2.7 \\
3.1 \\
3.6 \\
5.8\end{array}$ & $\begin{array}{l}1.1 \\
1.3 \\
1.4 \\
1.6 \\
2.0 \\
2.3 \\
2.6 \\
3.0 \\
5.0\end{array}$ \\
\hline $\begin{array}{l}\text { JULY } \\
\text { AUGUST } \\
\text { SEPTEMBER }\end{array}$ & $\begin{array}{l}2.3 \\
3.4 \\
2.6\end{array}$ & $\begin{array}{r}148 \\
131 \\
73\end{array}$ & $\begin{array}{l}25 \\
19 \\
18\end{array}$ & $\begin{array}{l}32 \\
22 \\
15\end{array}$ & $\begin{array}{r}1.27 \\
1.11 \\
.81\end{array}$ & $\begin{array}{l}3.6 \\
2.8 \\
2.6\end{array}$ & & & & & & & \\
\hline ANNUAL & 15 & 204 & 59 & 38 & .66 & 100 & & & & & & & \\
\hline
\end{tabular}

MAGNITUDE AND PROBABILITY OF ANNUAL HIGH FLOW BASED ON PERIOD OF RECORD 1911-25, 1927-65

\begin{tabular}{|c|c|c|c|c|c|c|}
\hline \multirow{2}{*}{$\begin{array}{l}\text { PERIOD } \\
\text { (CON- } \\
\text { SECU- } \\
\text { TIVE } \\
\text { DAYS) }\end{array}$} & \multicolumn{6}{|c|}{$\begin{array}{l}\text { DISCHARGE, IN FT } 3 / S \text {, FOR INDICATED RECURRENCE } \\
\text { INTERVAL, IN YEARS, AND ANNUAL } \\
\text { EXCEEDANCE PROBABILITY, IN PERCENT }\end{array}$} \\
\hline & $\begin{array}{c}2 \\
508\end{array}$ & $\begin{array}{c}5 \\
208\end{array}$ & $\begin{array}{l}10 \\
108\end{array}$ & $\begin{array}{l}25 \\
48\end{array}$ & $\begin{array}{l}50 \\
28\end{array}$ & $\begin{array}{r}100 \\
18\end{array}$ \\
\hline $\begin{array}{r}1 \\
3 \\
7 \\
15 \\
30 \\
60 \\
90\end{array}$ & $\begin{array}{l}335 \\
309 \\
273 \\
235 \\
194 \\
146 \\
116\end{array}$ & $\begin{array}{l}700 \\
649 \\
573 \\
489 \\
405 \\
300 \\
230\end{array}$ & $\begin{array}{l}995 \\
923 \\
819 \\
700 \\
584 \\
434 \\
328\end{array}$ & $\begin{array}{r}1410 \\
1310 \\
1170 \\
1010 \\
851 \\
639 \\
479\end{array}$ & $\begin{array}{r}1750 \\
1620 \\
1460 \\
1260 \\
1080 \\
819 \\
613\end{array}$ & $\begin{array}{r}2090 \\
1950 \\
1770 \\
1530 \\
1330 \\
1020 \\
764\end{array}$ \\
\hline
\end{tabular}

DURATION TABLE OF DAILY MEAN FLOW FOR PERIOD OF RECORD 1911-25, 1927-65 DISCHARGE, IN FT ${ }^{3} / \mathrm{S}$, THAT WAS EQUALED OR EXCEEDED FOR INDICATED PERCENT OF TIME

\begin{tabular}{|c|c|c|c|c|c|c|c|c|c|c|c|c|c|c|}
\hline 58 & 108 & 158 & 208 & 258 & 308 & 408 & 508 & 608 & 708 & 758 & 808 & 858 & 908 & 958 \\
\hline 244 & 126 & 77 & 57 & 47 & 41 & 33 & 28 & 24 & 19 & 17 & 14 & 11 & 7.7 & \\
\hline
\end{tabular}


RIO GRANDE BASIN

08276300 RIO PUEBLO DE TAOS BELOW LOS CORDOVAS, NM

LOCATION.--Lat $36^{\circ} 22^{\prime} 39^{\prime \prime}$, long $105^{\circ} 40^{\prime} 05^{\prime \prime}$, Taos County, Hydrologic Unit 13020101, in Gijosa Grant, on left bank 1.9 $\mathrm{mi}$ southwest of Los Cordovas, $2.5 \mathrm{mi}$ downstream from Rio Grande del Rancho, and at mile 5.1 .

DRAINAGE AREA. $--380 \mathrm{mi}^{2}$.

PERIOD OF RECORD,--March 1957 to current year.

REVISED RECORDS.--WSP 1732: 1957(M). WSP 1923: 1957(P), 1958, WDR NM-81-1: 1979(P).

GAGE.--Water-stage recorder. Elevation of gage is 6,652 ft above National Geodetic Vertical Datum of 1929 , from topographic map. Prior to Sept. 4, 1984 at site $700 \mathrm{ft}$ downstream at same datum.

REMARKS.--Diversions for irrigation of about 12,000 acres upstream from station, of which about 1,700 acres are irrigated by water from Rio Hondo.

AVERAGE DISCHARGE, --28 years (water years 1958-85), 59.9 $\mathrm{ft}^{3} / \mathrm{s}, 43,400 \mathrm{acre}-\mathrm{ft} / \mathrm{yr}$.

EXTREMES FOR PERIOD OF RECORD.--Maximum discharge, 2,380 $\mathrm{ft}^{3} / \mathrm{s}$, Aug. 24, 1957, gage height, $5.80 \mathrm{ft} ; \mathrm{maximum} \mathrm{gage}$ height, $6.00 \mathrm{ft}$ (site then in use), July 30 , 1982, from rating curve extended above $900 \mathrm{ft} 3 / \mathrm{s} ; \mathrm{minimum}^{2} 1.9$ $\mathrm{ft}^{3} / \mathrm{s}$, July 31 , Aug. 1,1972 .

STATISTICAL SUMMARIES

MEAN MONTHLY AND MEAN ANNUAL DISCHARGES 1958-85

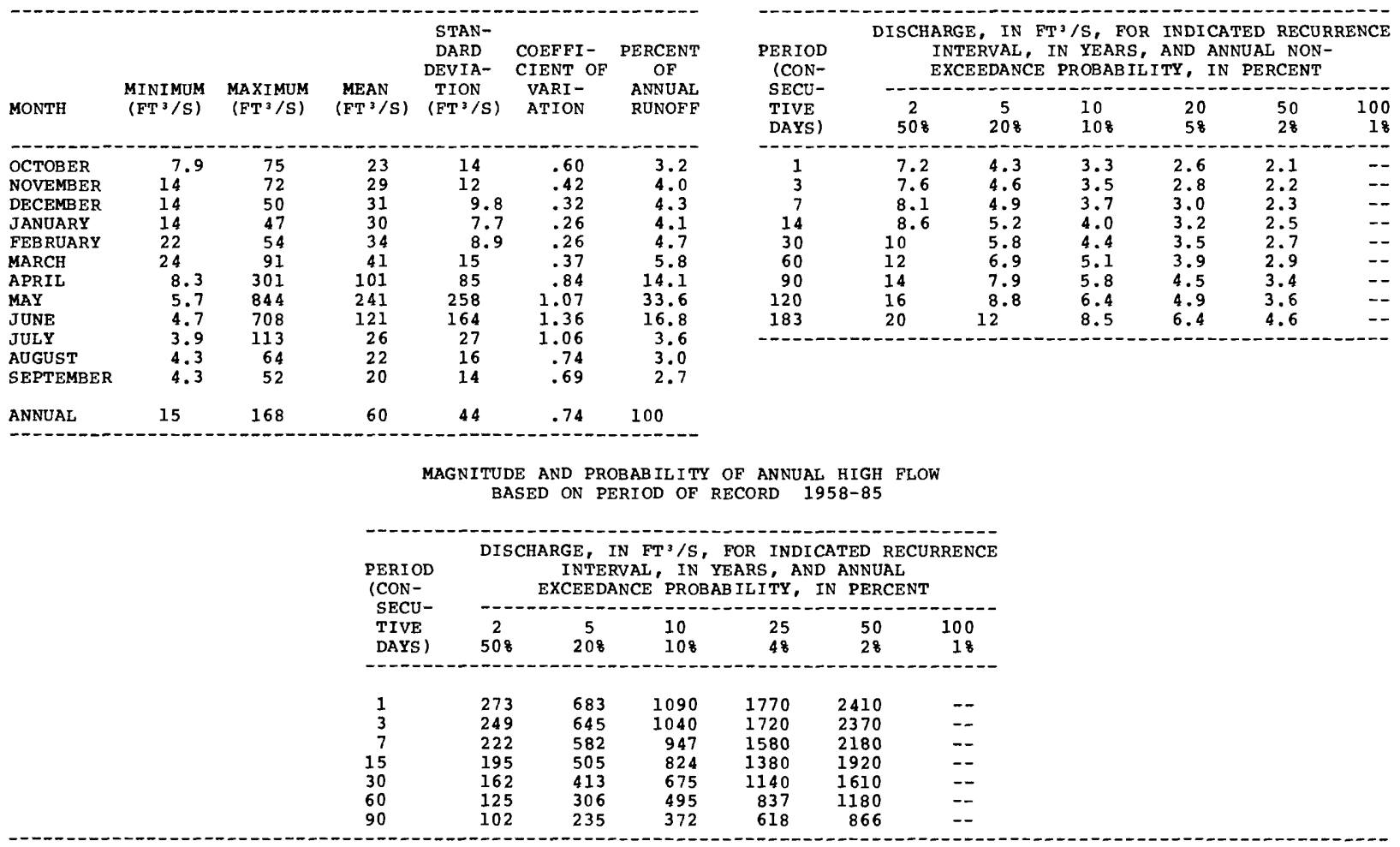

DURATION TABLE OF DAILY MEAN FLOW FOR PERIOD OF RECORD 1958-85

DISCHARGE, IN FT $3 / S$, THAT WAS EQUALED OR EXCEEDED FOR INDICATED PERCENT OF TIME

\begin{tabular}{|c|c|c|c|c|c|c|c|c|c|c|c|c|c|c|}
\hline 58 & 108 & 158 & 208 & 258 & 308 & 408 & 508 & 608 & 708 & 758 & 808 & 858 & 908 & 958 \\
\hline 261 & 106 & 65 & 51 & 44 & 40 & 34 & 29 & 25 & 21 & 18 & 15 & 12 & 8.2 & 6.0 \\
\hline
\end{tabular}


RIO GRANDE BASIN

08276500 RIO GRANDE BELOW TAOS JUNCTION BRIDGE, NEAR TAOS, NM (Surveillance network station)

LOCATION.--Lat $36^{\circ} 19^{\prime} 12^{\prime \prime}$, long $105^{\circ} 45^{\prime} 14^{\prime \prime}$, in NWłNEł sec.15, T.24 N., R.11 E., Taos County, Hydrologic Unit 13020101 , on left bank $1.7 \mathrm{mi}$ downstream from bridge on State Highway $96,2.0 \mathrm{mi}$ downstream from Rio Pueblo de Taos, 11.8 mi southwest of Taos, and at mile 1,657.7.

DRAINAGE AREA. $--9,730 \mathrm{mi}^{2}$, approximately, including $2,940 \mathrm{mi}^{2}$ in closed basin in san Luis Valley, co.

PERIOD OF RECORD.--July 1925 to current year. Prior to October 1930 monthly discharge only, published in wSP 1312. Published as "at Taos Junction Bridge, near Taos" prior to 1934.

REVISED RECORDS.--WSP 788: 1934 (M). WSP 828: Drainage area. WSP 1392: 1931-1932, 1935, 1937, 1945, 1950.

GAGE.-Water-stage recorder. Datum of gage is $6,050.3 \mathrm{ft}$ above National Geodetic Vertical Datum of 1929 . Prior to Apr. 14, 1934, at bridge $1.7 \mathrm{mi}$ upstream at different datum.

REMARKS.--Diversions upstream from station for irrigation of about 620,000 acres in colorado and 30,000 acres in New Mexico.

AVERAGE DISCHARGE.--60 years (water years 1926-85), $742 \mathrm{ft}^{3} / \mathrm{s}, 537,600$ acre-ft/yr.

EXTREMES FOR PERIOD OF RECORD.--Maximum discharge, 9,730 ft $3 / \mathrm{s}$, June 7, 1948 , gage height, $9.18 \mathrm{ft}$, and June 22 , 1949 , gage height, $9.23 \mathrm{ft}$; minimum, $155 \mathrm{ft}^{3} / \mathrm{s}$, Sept. $21,1956$.

EXTREMES OUTSIDE PERIOD OF RECORD.--Maximum flood since at least 1888, about $14,000 \mathrm{ft} 3 / \mathrm{s} \mathrm{June} 19$, 1903 , from records for Rio Grande at Embudo and estimated inflow. Other floods exceeding $10,000 \mathrm{ft} / \mathrm{s}$ occurred June 9 1905, May 28, 1920, and June 16, 1921, from comparison of records for stations near Lobatos and at Embuđo.

STATISTICAL SUMMARIES

MEAN MONTHLY AND MEAN ANNUAL DISCHARGES 1926-85

\begin{tabular}{|c|c|c|c|c|c|c|}
\hline MONTH & $\begin{array}{l}\text { MINIMUM } \\
\left(\mathrm{FT}^{2} / \mathrm{S}\right)\end{array}$ & $\begin{array}{c}\text { MAXIMUM } \\
\left(\mathrm{FT}^{3} / \mathrm{S}\right)\end{array}$ & $\begin{array}{l}\text { MEAN } \\
\left(\mathrm{FT}^{3} / \mathrm{S}\right)\end{array}$ & $\begin{array}{l}\text { STAN- } \\
\text { DARD } \\
\text { DEVIA- } \\
\text { TION } \\
\left(\mathrm{FT}^{3} / \mathrm{S}\right)\end{array}$ & $\begin{array}{l}\text { COEFFI - } \\
\text { CIENT OF } \\
\text { VARI- } \\
\text { ATION }\end{array}$ & $\begin{array}{c}\text { PERCENT } \\
\text { OF } \\
\text { ANNUAL } \\
\text { RUNOF F }\end{array}$ \\
\hline $\begin{array}{l}\text { OCTOBER } \\
\text { NOVEMBER } \\
\text { DECEMBER } \\
\text { JANUARY } \\
\text { FEBRUARY } \\
\text { MARCH } \\
\text { APRIL } \\
\text { MAY } \\
\text { JUNE } \\
\text { JULY } \\
\text { AUGUST } \\
\text { SEPTEMBER }\end{array}$ & $\begin{array}{l}171 \\
224 \\
243 \\
263 \\
290 \\
259 \\
250 \\
233 \\
188 \\
185 \\
184 \\
161\end{array}$ & $\begin{array}{r}1675 \\
1532 \\
1018 \\
748 \\
757 \\
1151 \\
3020 \\
5993 \\
6007 \\
2945 \\
1537 \\
2087\end{array}$ & $\begin{array}{r}412 \\
516 \\
482 \\
465 \\
530 \\
630 \\
809 \\
1774 \\
1789 \\
710 \\
415 \\
375\end{array}$ & $\begin{array}{r}301 \\
256 \\
136 \\
90 \\
108 \\
180 \\
584 \\
1515 \\
1634 \\
679 \\
281 \\
311\end{array}$ & $\begin{array}{l}.73 \\
.50 \\
.28 \\
.19 \\
.20 \\
.29 \\
.72 \\
.85 \\
.91 \\
.96 \\
.68 \\
.83\end{array}$ & $\begin{array}{r}4.6 \\
5.8 \\
5.4 \\
5.2 \\
6.0 \\
7.1 \\
9.1 \\
19.9 \\
20.1 \\
8.0 \\
4.7 \\
4.2\end{array}$ \\
\hline ANNUAL & 271 & 1840 & 742 & 366 & .49 & 100 \\
\hline
\end{tabular}

MAGNITUDE AND PROBABILITY OF ANNUAL LOW FLOW BASED ON PERIOD OF RECORD $1927-85$

\begin{tabular}{|c|c|c|c|c|c|c|}
\hline \multirow{2}{*}{$\begin{array}{l}\text { PERIOD } \\
\text { (CON- } \\
\text { SECU- } \\
\text { TIVE } \\
\text { DAYS) }\end{array}$} & \multicolumn{6}{|c|}{$\begin{array}{l}\text { DISCHARGE, IN FT'/S, FOR INDICATED RECURRENCE } \\
\text { INTERVAL, IN YEARS, AND ANNUAL NON- } \\
\text { EXCEEDANCE PROBABILITY, IN PERCENT }\end{array}$} \\
\hline & $\begin{array}{c}2 \\
508\end{array}$ & $\begin{array}{c}5 \\
208\end{array}$ & $\begin{array}{l}10 \\
108\end{array}$ & $\begin{array}{l}20 \\
58\end{array}$ & $\begin{array}{l}50 \\
28\end{array}$ & $\begin{array}{r}100 \\
18\end{array}$ \\
\hline $\begin{array}{r}1 \\
3 \\
7 \\
14 \\
30 \\
60 \\
90 \\
120 \\
183\end{array}$ & $\begin{array}{l}223 \\
225 \\
230 \\
236 \\
249 \\
272 \\
292 \\
312 \\
357\end{array}$ & $\begin{array}{l}187 \\
188 \\
191 \\
196 \\
205 \\
215 \\
226 \\
236 \\
266\end{array}$ & $\begin{array}{l}173 \\
174 \\
176 \\
180 \\
187 \\
195 \\
202 \\
209 \\
233\end{array}$ & $\begin{array}{l}164 \\
165 \\
167 \\
170 \\
176 \\
181 \\
186 \\
191 \\
210\end{array}$ & $\begin{array}{l}156 \\
157 \\
158 \\
161 \\
165 \\
168 \\
171 \\
174 \\
189\end{array}$ & $\begin{array}{l}151 \\
153 \\
153 \\
156 \\
159 \\
161 \\
163 \\
164 \\
177\end{array}$ \\
\hline
\end{tabular}

MAGNITUDE AND PROBABILITY OF ANNUAL HIGH FLOW BASED ON PERIOD OF RECORD $1926-85$

\begin{tabular}{|c|c|c|c|c|c|c|}
\hline \multirow{2}{*}{$\begin{array}{l}\text { PERIOD } \\
\text { (CON- } \\
\text { SECU- } \\
\text { TIVE } \\
\text { DAYS) }\end{array}$} & \multicolumn{6}{|c|}{$\begin{array}{l}\text { DISCHARGE, IN FT }{ }^{3} / S \text {, FOR INDICATED RECURRENCE } \\
\text { INTERVAL, IN YEARS, AND ANNUAL } \\
\text { EXCEEDANCE PROBABILITY, IN PERCENT }\end{array}$} \\
\hline & $\begin{array}{c}2 \\
508\end{array}$ & $\begin{array}{c}5 \\
208\end{array}$ & $\begin{array}{l}10 \\
108\end{array}$ & $\begin{array}{l}25 \\
48\end{array}$ & $\begin{array}{l}50 \\
28\end{array}$ & $\begin{array}{r}100 \\
18\end{array}$ \\
\hline $\begin{array}{r}1 \\
3 \\
7 \\
15 \\
30 \\
60 \\
90\end{array}$ & $\begin{array}{l}2560 \\
2460 \\
2250 \\
2000 \\
1750 \\
1440 \\
1230\end{array}$ & $\begin{array}{l}5160 \\
4980 \\
4590 \\
4070 \\
3570 \\
2890 \\
2340\end{array}$ & $\begin{array}{l}7340 \\
7100 \\
6590 \\
5850 \\
5160 \\
4160 \\
3300\end{array}$ & $\begin{array}{r}10600 \\
10300 \\
9630 \\
8560 \\
7600 \\
6150 \\
4780\end{array}$ & $\begin{array}{r}13300 \\
13000 \\
12200 \\
10900 \\
9750 \\
7910 \\
6090\end{array}$ & $\begin{array}{r}16300 \\
15900 \\
15200 \\
13600 \\
12200 \\
9940 \\
7580\end{array}$ \\
\hline
\end{tabular}

DURATION TABLE OF DAILY MEAN FLOW FOR PERIOD OF RECORD 1926-85 DISCHARGE, IN FT'/S, THAT WAS EQUALED OR EXCEEDED FOR INDICATED PERCENT OF TIME

\begin{tabular}{|c|c|c|c|c|c|c|c|c|c|c|c|c|c|c|}
\hline 58 & 108 & 158 & 208 & 258 & 308 & 408 & 508 & 608 & 708 & 758 & 808 & 858 & 908 & 958 \\
\hline 2590 & 1450 & 972 & 784 & 687 & 618 & 527 & 460 & 398 & 335 & 310 & 285 & 260 & 237 & 209 \\
\hline
\end{tabular}


RIO GRANDE BASIN

08279000 EMBUDO CREEK AT DIXON, NM

LOCATION.--Lat $36^{\circ} 12^{\prime} 39^{n}$, Iong $105^{\circ} 54^{\prime} 47^{\prime \prime}$, in NEsEl sec.19, T.23 N., R. 10 E., Rio Arriba County, Hydrologic Unit 13020101 , on right bank $750 \mathrm{ft}$ upstream from U.S. Highway $64,0.5 \mathrm{mi}$ upstream from mouth, $0.5 \mathrm{mi}$ east of Embudo Post office, and $1.7 \mathrm{mi}$ northwest of Dixon.

DRAINAGE AREA. $--305 \mathrm{mi}^{2}$.

PERIOD OF RECORD.--October 1923 to February 1926, October 1926 to September 1955, annual maximum, water years 1956-62, September 1962 to current year. Monthly discharge only for some periods, published in wSp 1312 . Figures of daily discharge for July 6-25, 1932, published in WSP 733, and maximum discharges for water years $1931-33$, 1935, 1937-38, 1941, are unreliable and should not be used.

REVISED RECORDS.--WSP 1512: 1931-32, 1941, 1947 (M). See al so PERIOD OF RECORD.

GAGE.--Water-stage recorder. Datum of gage is 5,858.60 ft above National Geodetic Vertical Datum of 1929 . Prior to Nov. 30, 1938, at site about $1 \mathrm{mi}$ upstream at different datum. Nov. 30, 1938 to Aug. 1 , 1941 , at site about $0.9 \mathrm{mi}$ upstream at datum about $59.9 \mathrm{ft}$ higher. Aug. 2, 1941 to Sept. 1, 1971, at site $750 \mathrm{ft}$ downstream at datum $9.10 \mathrm{ft}$ lower. April 1956 to Sept. 21, 1962, crest-stage gage.

REMARKS.--Diversions upstream from station for irrigation of about 6,500 acres, a small part of which are are downstream from gage.

AVERAGE DISCHARGE.--59 years (water years 1924-25, 1927-29, 1931-85), 80.7 $\mathrm{ft}^{3} / \mathrm{s}, 58,500 \mathrm{acre}-\mathrm{ft} / \mathrm{yr}$.

EXTREMES FOR PERIOD OF RECORD (SINCE 1941).--Maximum discharge, 4,200 ft3/s, Aug. 29, 1977, gage height, 7.10 ft, from rating curve extended above $1,600 \mathrm{ft}^{3} / \mathrm{s}$; maximum gage height, $7.6 \mathrm{ft}$, Aug. 4, 1967 ; minimum discharge, 0.06 $\mathrm{ft}^{3} / \mathrm{s}$, June $26,27,1950$.

STATISTICAL SUMMARIES

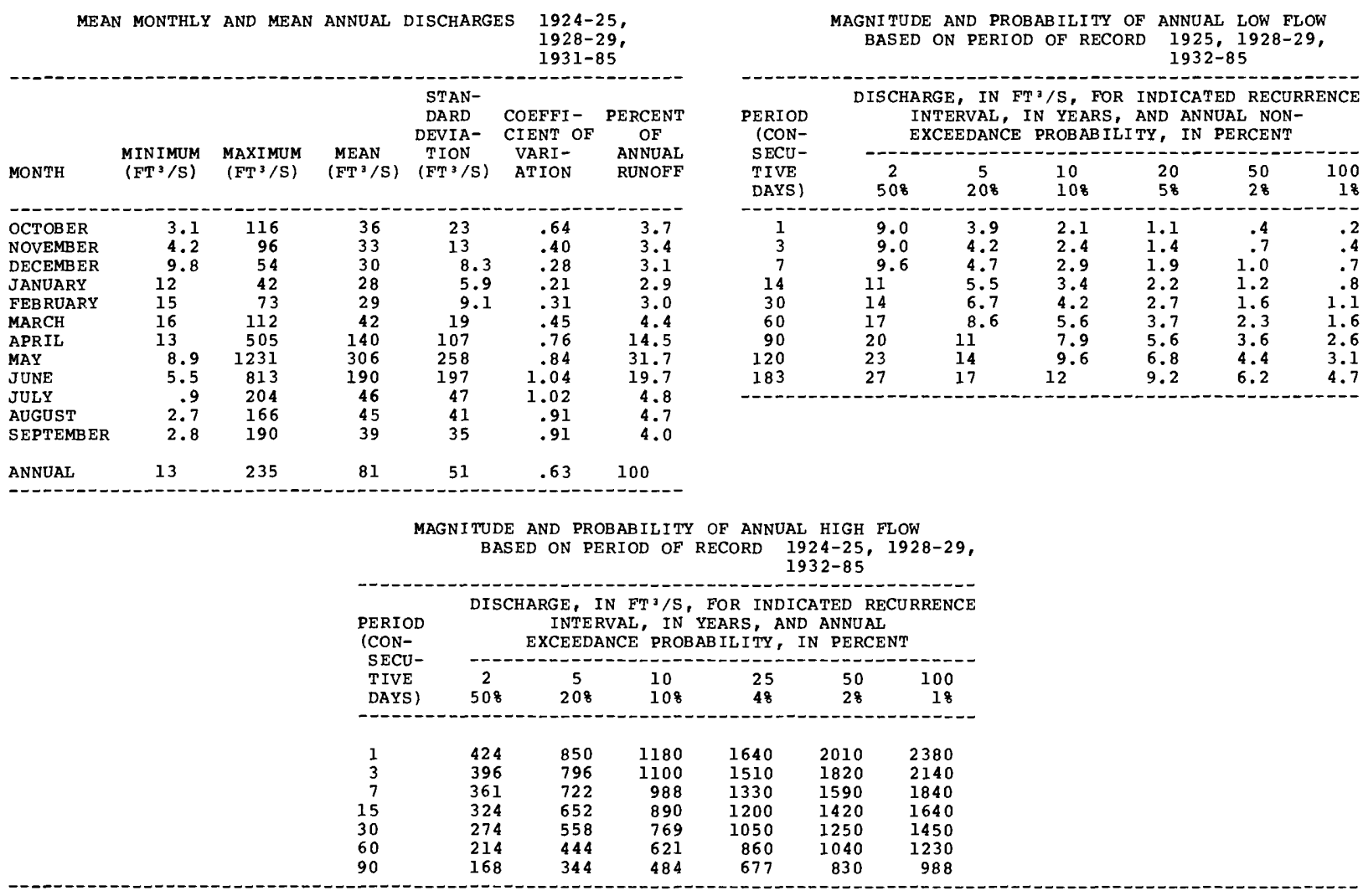

DURATION TABLE OF DAILY MEAN FLOW FOR PERIOD OF RECORD 1924-25, 1928-29, 1931-85

DISCHARGE, IN FT3/S, THAT WAS EQUALED OR EXCEEDED FOR INDICATED PERCENT OF TIME

\begin{tabular}{|c|c|c|c|c|c|c|c|c|c|c|c|c|c|c|}
\hline 58 & 108 & 158 & 208 & 258 & 308 & 408 & 508 & 608 & 708 & 758 & $80 \%$ & 858 & $90 \%$ & $95 \%$ \\
\hline 371 & 205 & 124 & 84 & 63 & 52 & 40 & 33 & 29 & 25 & 22 & 20 & 16 & 12 & 7. \\
\hline
\end{tabular}


RIO GRANDE BASIN

08279500 RIO GRANDE AT EMBUDO, NM

LOCATION.--Lat $36^{\circ} 12^{\prime} 20^{\prime \prime}$, long $105^{\circ} 577^{\prime \prime} 9^{\prime \prime}$, in SW3SW sec.23, T.23 N., R.9 E., Rio Arriba County, Hydrologic Unit 13020101 , on right bank $0.2 \mathrm{mi}$ downstream from bridge at Embudo, $2.8 \mathrm{mi}$ downstream from Embudo Creek, and at mile $1,643.1$

DRAINAGE AREA.--10,400 $\mathrm{mi}^{2}$, approximately, including $2,940 \mathrm{mi}^{2}$ in closed basin in San Luis Valley, co.

PERIOD OF RECORD.--January 1889 to current year. Monthly discharge only for some periods, published in wSP 1312. Figures of daily discharge for oct. 4 to Nov. 30,1896 , published in wSP 358 , are unreliable and should not be used.

REVISED RECORDS.--WSP 358: 1900-1902. WSP 828: Drainage area. WSP 878: 1915-16. WSP 1512: 1892-99, 1904, 1916, 1931-32, 1939, 1944-45, 1950. WSP 1712: 1903 (M). See also PERIOD OF RECORD.

GAGE.--Water-stage recorder. Datum of gage is 5,789.14 ft above National Geodetic Vertical Datum of 1929. Jan. 1 to Feb. 28,1889 , nonrecording gage 1.2 mi upstream at different datum. March 1889 to December 1903 , nonrecording gage 1,300 ft upstream at different datum. September 1912 to June 1914, water-stage recorder on downstream end of bridge pier at site $200 \mathrm{ft}$ upstream at present datum.

REMARKS.--Diversions upstream from station for irrigation about 620,000 acres in colorado and 40,000 acres in New Mexico. National Weather Service gage-height telemeter at station.

AVERAGE DISCHARGE. --86 years (water years 1890-93, 1895-1903, 1913-85), $929 \mathrm{ft}^{3} / \mathrm{s}, 673,100 \mathrm{acre-ft} / \mathrm{yr}$.

55 years (water years 1931-85), $807 \mathrm{ft}^{3} / \mathrm{s}, 584,700$ acre-ft/yr, subsequent to upstream development.

EXTREMES FOR PERIOD OF RECORD (1889-1903 AND SINCE 1911).--Maximum discharge, 16,200 ft3/s, June 19, 1903, gage height, about $15.9 \mathrm{ft}$; minimum daily, $130 \mathrm{ft} / \mathrm{s}$, June 30,1902 . A flood of about $14,000 \mathrm{ft} / \mathrm{s}$ occurred between May 20 and June 10,1905 , from a comparison of records for Lobatos and otowi Bridge. Another major flood occurred sept. 29 or 30,1904

STATISTICAL SUMMARIES

MEAN MONTHLY AND MEAN ANNUAL, DISCHARGES 1890-93, 1895-93, 1913-85

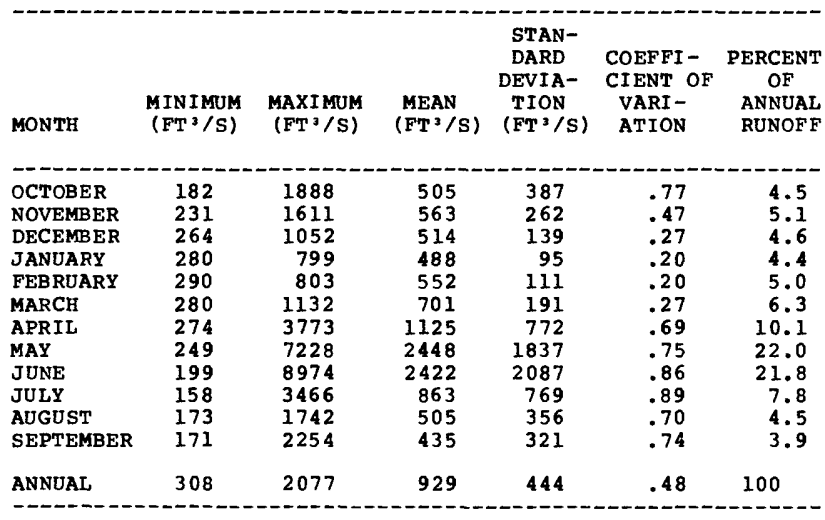

MAGNITUDE AND PROBABILITY OF ANNUAL LOW FLOW BASED ON PERIOD OF RECORD 1891-93, 1896-1904, $1914-85$

\begin{tabular}{|c|c|c|c|c|c|c|}
\hline \multirow{2}{*}{$\begin{array}{l}\text { PERIOD } \\
\text { (CON- } \\
\text { SECU- } \\
\text { TIVE } \\
\text { DAYS) }\end{array}$} & \multicolumn{6}{|c|}{$\begin{array}{l}\text { DISCHARGE, IN FT'/S, FOR INDICATED RECURRENCE } \\
\text { INTERVAL, IN YEARS, AND ANNUAL NON- } \\
\text { EXCEEDANCE PROBABILITY, IN PERCENT }\end{array}$} \\
\hline & $\begin{array}{c}2 \\
508\end{array}$ & $\begin{array}{c}5 \\
208\end{array}$ & $\begin{array}{l}10 \\
108\end{array}$ & $\begin{array}{l}20 \\
58\end{array}$ & $\begin{array}{l}50 \\
28\end{array}$ & $\begin{array}{r}100 \\
18\end{array}$ \\
\hline $\begin{array}{r}1 \\
3 \\
7 \\
14 \\
30 \\
60 \\
90 \\
120 \\
183\end{array}$ & $\begin{array}{l}244 \\
250 \\
257 \\
266 \\
286 \\
316 \\
341 \\
367 \\
413\end{array}$ & $\begin{array}{l}194 \\
198 \\
202 \\
209 \\
222 \\
238 \\
254 \\
271 \\
303\end{array}$ & $\begin{array}{l}174 \\
177 \\
181 \\
186 \\
197 \\
209 \\
221 \\
234 \\
261\end{array}$ & $\begin{array}{l}161 \\
164 \\
166 \\
171 \\
179 \\
188 \\
198 \\
208 \\
232\end{array}$ & $\begin{array}{l}148 \\
150 \\
152 \\
156 \\
162 \\
168 \\
176 \\
183 \\
205\end{array}$ & $\begin{array}{l}141 \\
143 \\
144 \\
148 \\
153 \\
157 \\
163 \\
168 \\
190\end{array}$ \\
\hline
\end{tabular}

MAGNITUDE AND PROBABILITY OF ANNUAL HIGH FLOW BASED ON PERIOD OF RECORD 1890-93, 1895-1903, 1913-85

\begin{tabular}{|c|c|c|c|c|c|c|}
\hline \multirow{2}{*}{$\begin{array}{l}\text { PERIOD } \\
\text { (CON- } \\
\text { SECU- } \\
\text { TIVE } \\
\text { DAYS) }\end{array}$} & \multicolumn{6}{|c|}{$\begin{array}{l}\text { DISCHARGE, IN FT } 3 / S \text {, FOR INDICATED RECURRENCE } \\
\text { INTERVAL, IN YEARS, AND ANNUAL } \\
\text { EXCEEDANCE PROBABILITY, IN PERCENT }\end{array}$} \\
\hline & $\begin{array}{c}2 \\
508\end{array}$ & $\begin{array}{c}5 \\
208\end{array}$ & $\begin{array}{l}10 \\
108\end{array}$ & $\begin{array}{l}25 \\
48\end{array}$ & $\begin{array}{l}50 \\
28\end{array}$ & $\begin{array}{r}100 \\
18\end{array}$ \\
\hline $\begin{array}{r}1 \\
3 \\
7 \\
15 \\
30 \\
60 \\
90\end{array}$ & $\begin{array}{l}3550 \\
3420 \\
3180 \\
2850 \\
2510 \\
2070 \\
1730\end{array}$ & $\begin{array}{l}7010 \\
6810 \\
6370 \\
5680 \\
4960 \\
4010 \\
3200\end{array}$ & $\begin{array}{l}9740 \\
9500 \\
8930 \\
7950 \\
6920 \\
5530 \\
4350\end{array}$ & $\begin{array}{r}13500 \\
13300 \\
12600 \\
11200 \\
9680 \\
7660 \\
5940\end{array}$ & $\begin{array}{r}16600 \\
16300 \\
15500 \\
13800 \\
11900 \\
9380 \\
7220\end{array}$ & $\begin{array}{r}19800 \\
19500 \\
18600 \\
16600 \\
14300 \\
11200 \\
8560\end{array}$ \\
\hline
\end{tabular}

DURATION TABLE OF DAILY MEAN FLOW FOR PERIOD OF RECORD 1890-93, 1895-1903, 1913-85 DISCHARGE, IN FT'3/S, THAT WAS EOUALED OR EXCEEDED FOR INDICATED PERCENT OF TIME

\begin{tabular}{|c|c|c|c|c|c|c|c|c|c|c|c|c|c|c|}
\hline 58 & 108 & 158 & 208 & 258 & 308 & 408 & 508 & 608 & 708 & 758 & 808 & 858 & 908 & 958 \\
\hline 3530 & 2030 & 1350 & 982 & 828 & 731 & 614 & 531 & 465 & 398 & 362 & 328 & 295 & 263 & 227 \\
\hline
\end{tabular}


RIO GRANDE BASIN

08281100 RIO GRANDE ABOVE SAN JUAN PUEBLO, NM

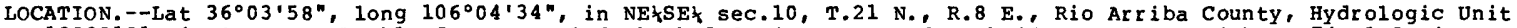
13020101 , in San Juan Pueblo Grant, on left bank $0.8 \mathrm{mi}$ upstream from bridge on $\mathrm{State} H i g h w a y, 74$, $1.0 \mathrm{mi}$

northwest of San Juan Pueblo, $1.8 \mathrm{mi}$ upstream from Rio Chama, $5.1 \mathrm{mi}$ north of Espanola, and at mile $1,630.1$.

DRAINAGE AREA. $--10,550 \mathrm{mi}^{2}$, approximately, including $2,940 \mathrm{mi}^{2}$ in closed basin in San Luis Valley, Co.

PERIOD OF RECORD.--March 1963 to current year.

GAGE.--Water-stage recorder. Elevation of gage is 5,630 ft above National Geodetic vertical Datum of 1929 , from topographic map.

REMARKS.--Diversions upstream from station for irrigation of about 620,000 acres in Colorado and 42,000 acres in New Mexico. San Juan lateral (station 08280100 - discontinued, Sept. 1984) and San Juan Pueblo ditch

(station 08280200), both on left bank, and Guique ditch (station 08280700), on right bank, bypass gage for

irrigation of several hundred acres downstream from station.

AVERAGE DISCHARGE. --22 years, $783 \mathrm{ft}^{3 / \mathrm{s}}, 567,300$ acre-ft/yr.

EXTREMES FOR PERIOD OF RECORD.--Maximum discharge, 8,220 $\mathrm{ft}^{3} / \mathrm{s}$, June 9,1979 , gage height, $6.94 \mathrm{ft}$; minimum, 92 $\mathrm{ft}^{3 / \mathrm{s}}$, Aug. I0-11, 1977 .

EXTREMES OUTSIDE PERIOD OF RECORD.--For years of outstanding floods see records for Rio Grande at Embudo (station 08279500 ).

STATISTICAL SUMMARIES

MEAN MONTHLY AND MEAN ANNUAL DISCHARGES 1964-85

\begin{tabular}{|c|c|c|c|c|c|c|}
\hline MONTH & $\begin{array}{l}\text { MINIMUM } \\
\left(\mathrm{FT}^{3} / \mathrm{S}\right)\end{array}$ & $\begin{array}{l}\text { MAXIMUM } \\
\left(\mathrm{FT}^{3} / \mathrm{S}\right)\end{array}$ & $\begin{array}{l}\text { MEAN } \\
\left(\mathrm{FT}^{3} / \mathrm{S}\right)\end{array}$ & $\begin{array}{l}\text { STAN- } \\
\text { DARD } \\
\text { DEVIA- } \\
\text { TION } \\
\left(\mathrm{FT}^{3} / \mathrm{S}\right)\end{array}$ & $\begin{array}{l}\text { COEFFI- } \\
\text { CIENT OF } \\
\text { VARI- } \\
\text { ATION }\end{array}$ & $\begin{array}{c}\text { PERCENT } \\
\text { OF } \\
\text { ANNUAL } \\
\text { RUNOFF }\end{array}$ \\
\hline \multicolumn{7}{|c|}{ 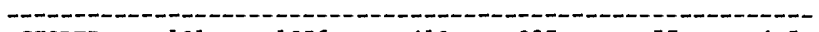 } \\
\hline NOVEMBER & 257 & 1213 & $\begin{array}{l}410 \\
586\end{array}$ & 266 & .01 & $\begin{array}{l}4.3 \\
6.2\end{array}$ \\
\hline DECEMBER & 278 & 811 & 493 & 135 & .27 & 5.2 \\
\hline JANUARY & 335 & 639 & 484 & 89 & .18 & 5.2 \\
\hline FEBRUARY & 369 & 727 & 540 & 102 & .19 & 5.8 \\
\hline MARCH & 439 & 1147 & 724 & 186 & .26 & 7.7 \\
\hline APRIL & 233 & 3266 & 856 & 663 & .77 & 9.1 \\
\hline MAY & 217 & 5066 & 1715 & 1433 & .84 & 18.3 \\
\hline JUNE & 142 & 5951 & 1877 & 1697 & .90 & 20.0 \\
\hline JULY & 165 & 2722 & 801 & 673 & .84 & 8.5 \\
\hline AUGUST & 173 & 1151 & 510 & 285 & .56 & 5.4 \\
\hline SEPTEMBER & 152 & 1155 & 387 & 241 & .62 & 4.1 \\
\hline NNUAL & 292 & 1645 & 783 & 377 & .48 & 100 \\
\hline
\end{tabular}

MAGNITUDE AND PROBABILITY OF ANNUAL LOW FLOW BASED ON PERIOD OF RECORD 1965-85

\begin{tabular}{|c|c|c|c|c|c|c|}
\hline \multirow{2}{*}{$\begin{array}{l}\text { PERIOD } \\
\text { (CON- } \\
\text { SECU- } \\
\text { TIVE } \\
\text { DAYS) }\end{array}$} & \multicolumn{6}{|c|}{$\begin{array}{l}\text { DISCHARGE, IN FT' } / S \text {, FOR INDICATED RECURRENCE } \\
\text { INTERVAL, IN YEARS, AND ANNUAL NON- } \\
\text { EXCEEDANCE PROBABILITY, IN PERCENT }\end{array}$} \\
\hline & $\begin{array}{c}2 \\
508\end{array}$ & $\begin{array}{c}5 \\
208\end{array}$ & $\begin{array}{l}10 \\
108\end{array}$ & $\begin{array}{l}20 \\
58\end{array}$ & $\begin{array}{l}50 \\
28\end{array}$ & $\begin{array}{r}100 \\
18\end{array}$ \\
\hline $\begin{array}{r}1 \\
3 \\
7 \\
14 \\
30 \\
60 \\
90 \\
120 \\
183\end{array}$ & $\begin{array}{l}209 \\
214 \\
222 \\
230 \\
251 \\
278 \\
317 \\
345 \\
398\end{array}$ & $\begin{array}{l}149 \\
154 \\
163 \\
170 \\
185 \\
202 \\
224 \\
242 \\
278\end{array}$ & $\begin{array}{l}127 \\
132 \\
142 \\
149 \\
161 \\
174 \\
188 \\
201 \\
229\end{array}$ & $\begin{array}{l}111 \\
117 \\
128 \\
135 \\
145 \\
155 \\
163 \\
174 \\
196\end{array}$ & $\begin{array}{l}-- \\
-- \\
-- \\
-- \\
-- \\
-- \\
-- \\
--\end{array}$ & $\begin{array}{l}-- \\
-- \\
-- \\
-- \\
-- \\
-- \\
-- \\
--\end{array}$ \\
\hline
\end{tabular}

MAGNITUDE AND PROBABILITY OF ANNUAL HIGH FLOW BASED ON PERIOD OF RECORD 1964-85

\begin{tabular}{|c|c|c|c|c|c|c|}
\hline \multirow{2}{*}{$\begin{array}{l}\text { PERIOD } \\
\text { (CON- } \\
\text { SECU- } \\
\text { TIVE } \\
\text { DAYS) }\end{array}$} & \multicolumn{6}{|c|}{$\begin{array}{l}\text { DISCHARGE, IN FT' }{ }^{3} \text { S, FOR INDICATED RECURRENCE } \\
\text { INTERVAL, IN YEARS, AND ANNUAL } \\
\text { EXCEEDANCE PROBABILITY, IN PERCENT }\end{array}$} \\
\hline & $\begin{array}{c}2 \\
508\end{array}$ & $\begin{array}{c}5 \\
208\end{array}$ & $\begin{array}{l}10 \\
108\end{array}$ & $\begin{array}{l}25 \\
48\end{array}$ & $\begin{array}{l}50 \\
28\end{array}$ & $\begin{array}{r}100 \\
18\end{array}$ \\
\hline $\begin{array}{r}1 \\
3 \\
7 \\
15 \\
30 \\
60 \\
90\end{array}$ & $\begin{array}{l}2410 \\
2330 \\
2170 \\
1970 \\
1750 \\
1470 \\
1280\end{array}$ & $\begin{array}{l}4560 \\
4480 \\
4190 \\
3820 \\
3400 \\
2870 \\
2410\end{array}$ & $\begin{array}{l}6350 \\
6280 \\
5880 \\
5380 \\
4820 \\
4080 \\
3380\end{array}$ & $\begin{array}{l}9030 \\
8950 \\
8420 \\
7760 \\
6990 \\
5950 \\
4880\end{array}$ & $\begin{array}{l}=- \\
=- \\
-- \\
=- \\
--\end{array}$ & $\begin{array}{l}-- \\
-- \\
-- \\
-- \\
-- \\
-- \\
--\end{array}$ \\
\hline
\end{tabular}

DURATION TABLE OF DAILY MEAN FLOW FOR PERIOD OF RECORD 1964-85

DISCHARGE, IN $\mathrm{FT}^{3} / \mathrm{S}$, THAT WAS EQUALED OR EXCEEDED FOR INDICATED PERCENT OF TIME

\begin{tabular}{|c|c|c|c|c|c|c|c|c|c|c|c|c|c|c|}
\hline 58 & 108 & 158 & 208 & 258 & 308 & 408 & 508 & 608 & 708 & 758 & 808 & 858 & 908 & 958 \\
\hline 2500 & 1570 & 1130 & 891 & 778 & 702 & 596 & 518 & 452 & 367 & 326 & 292 & 263 & 232 & 191 \\
\hline
\end{tabular}


RIO GRANDE BASIN

08283500 RIO CHAMA AT PARK VIEW, NM

LOCATrON, --Lat $36^{\circ} 44^{\prime} 15^{\prime \prime}$, long $106^{\circ} 34^{\prime} 40^{\prime \prime}$, in Tierra Amarilla Grant, at downstream end of bridge pier nearest right bank on state Bighway 51 , just below mouth of Rio Brazos and half a mile northwest of park view.

DRAINAGE AREA. $-\mathbf{4 0 5} \mathrm{mi}$ ?

PERIOD OF RECORD.--November 1912 to september 1916 and October 1930 to september 1955 (discontinued) in reports of Geological Survey. November 1912 to September 1916 and April 1925 to December 1931 in reports of state Engineer. All records include flow in Rio Brazos.

REVISED RECORDS, --WSP 1342: 1914-15, $1931(\mathrm{M}), 1946-47(\mathrm{M})$.

GAGE.--Water-stage recorder. Altitude of gage is $7,280 \mathrm{ft}$ (from river-profile map). Prior to July 21 , 1945 , intermittently at present or either of two other sites within $150 \mathrm{ft}$ of present gage at different datums.

REMARKS.--Diversions for irrigation of about 7,000 acres above station.

AVERAGE DISCHARGE.--27 years (water years 1914-15, 1931-55), $334 \mathrm{ft}^{3} / \mathrm{s}(241,900 \mathrm{acre}-\mathrm{ft} / \mathrm{year})$.

EXTREMES FOR PERIOD OF RECORD.--Maximum discharge, 8,530 ft3/s Apr. 16, 1937, from rating curve extended above

$3,800 \mathrm{ft} / \mathrm{s}$; maximum gage height, 8.12 $\mathrm{ft}$ May 26, 1941, site and datum then in use; minimum daily discharge, 1.3 $\mathrm{ft} / \mathrm{s}$ Aug. 15,16 , Oct. 27,1951 .

STATISTICAL SUMMARIES

MEAN MONTHLY AND MEAN ANNUAL DISCHARGES 1914-15, 1931-55

\begin{tabular}{|c|c|c|c|c|c|c|}
\hline MONTH & $\begin{array}{c}\text { MINIMUM } \\
\left(\mathrm{FT}^{3} / \mathrm{S}\right)\end{array}$ & $\begin{array}{c}\text { MAXIMUM } \\
\left(\mathrm{FT}^{3} / \mathrm{S}\right)\end{array}$ & $\begin{array}{l}\text { MEAN } \\
\left(\mathrm{FT}^{3} / \mathrm{S}\right)\end{array}$ & $\begin{array}{l}\text { STAN- } \\
\text { DARD } \\
\text { DEVIA- } \\
\text { TION } \\
\text { (FT' } 3 \text { /S) }\end{array}$ & $\begin{array}{l}\text { COEFFI- } \\
\text { CIENT OF } \\
\text { VARI - } \\
\text { ATION }\end{array}$ & $\begin{array}{c}\text { PERCENT } \\
\text { OF } \\
\text { ANNUAL } \\
\text { RUNOFF }\end{array}$ \\
\hline \multicolumn{7}{|c|}{ 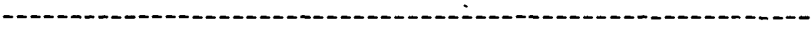 } \\
\hline OCTOBER & 5.2 & 380 & 75 & 77 & 1.03 & 1.9 \\
\hline NOVEMBER & 19 & 228 & 54 & 44 & .81 & 1.3 \\
\hline DECEMBER & 18 & 111 & 45 & 22 & .49 & 1.1 \\
\hline JANUARY & 19 & 100 & 45 & 18 & .40 & 1.1 \\
\hline FEB RUARY & 26 & 143 & 54 & 22 & .41 & 1.3 \\
\hline MARCH & 42 & 246 & 116 & 57 & .49 & 2.9 \\
\hline APRIL & 231 & 2710 & 916 & 564 & .62 & 22.9 \\
\hline MAY & 286 & 3814 & 1694 & 927 & .55 & 42.4 \\
\hline JUNE & 48 & 2391 & 736 & 627 & .85 & 18.4 \\
\hline JULY & 6.7 & 550 & 141 & 140 & .99 & 3.5 \\
\hline AUGUST & 9.5 & 189 & 66 & 47 & .71 & 1.7 \\
\hline SEPTEMBER & & & 58 & 40 & .69 & 1.4 \\
\hline NNUAL & 128 & 644 & 334 & 160 & .48 & 100 \\
\hline
\end{tabular}

MAGNITUDE AND PROBABILITY OF ANNUAL LOW FLOW BASED ON PERIOD OF RECORD 1915, 1932-55

\begin{tabular}{|c|c|c|c|c|c|c|}
\hline \multirow{2}{*}{$\begin{array}{l}\text { PERIOD } \\
\text { (CON- } \\
\text { SECU- } \\
\text { TIVE } \\
\text { DAYS) }\end{array}$} & \multicolumn{6}{|c|}{$\begin{array}{l}\text { DISCHARGE, IN FT'3/S, FOR INDICATED RECURRENCE } \\
\text { INTERVAL, IN YEARS, AND ANNUAL NON- } \\
\text { EXCEEDANCE PROBABILITY, IN PERCENT }\end{array}$} \\
\hline & $\begin{array}{c}2 \\
508\end{array}$ & $\begin{array}{c}5 \\
208\end{array}$ & $\begin{array}{l}10 \\
108\end{array}$ & $\begin{array}{l}20 \\
58\end{array}$ & $\begin{array}{l}50 \\
28\end{array}$ & $\begin{array}{r}100 \\
18\end{array}$ \\
\hline $\begin{array}{r}1 \\
3 \\
7 \\
14 \\
30 \\
60 \\
90 \\
120 \\
183\end{array}$ & $\begin{array}{l}14 \\
15 \\
17 \\
20 \\
24 \\
29 \\
34 \\
39 \\
42\end{array}$ & $\begin{array}{l}5.8 \\
6.3 \\
7.5 \\
9 . \\
12 \\
16 \\
20 \\
23 \\
26\end{array}$ & $\begin{array}{r}3.5 \\
3.7 \\
4.5 \\
5.8 \\
7.6 \\
11 \\
14 \\
16 \\
20\end{array}$ & $\begin{array}{c}2.2 \\
2.3 \\
2.8 \\
3.8 \\
5.1 \\
7.6 \\
10 \\
12 \\
17\end{array}$ & $\begin{array}{r}1.2 \\
1.3 \\
1.5 \\
2.2 \\
3.1 \\
5.0 \\
7.2 \\
8.1 \\
14\end{array}$ & $\begin{array}{l}-- \\
-- \\
-- \\
-- \\
-- \\
-- \\
--\end{array}$ \\
\hline
\end{tabular}

MAGNITUDE AND PROBABILI TY OF ANNUAL HIGH FTOW BASED ON PERIOD OF RECORD 1914-15, 1931-55

\begin{tabular}{|c|c|c|c|c|c|c|}
\hline \multirow{2}{*}{$\begin{array}{l}\text { PERIOD } \\
\text { (CON- } \\
\text { SECU- } \\
\text { TIVE } \\
\text { DAYS) }\end{array}$} & \multicolumn{6}{|c|}{$\begin{array}{l}\text { DISCHARGE, IN FT'/S, FOR INDICATED RECURRENCE } \\
\text { INTERVAL, IN YEARS, AND ANNUAL } \\
\text { EXCEEDANCE PROBABILITY, IN PERCENT }\end{array}$} \\
\hline & $\begin{array}{c}2 \\
508\end{array}$ & $\begin{array}{c}5 \\
208\end{array}$ & $\begin{array}{l}10 \\
108\end{array}$ & $\begin{array}{l}25 \\
48\end{array}$ & $\begin{array}{l}50 \\
28\end{array}$ & $\begin{array}{r}100 \\
18\end{array}$ \\
\hline $\begin{array}{r}1 \\
3 \\
7 \\
15 \\
30 \\
60 \\
90\end{array}$ & $\begin{array}{l}2950 \\
2790 \\
2530 \\
2220 \\
1850 \\
1360 \\
1030\end{array}$ & $\begin{array}{l}4320 \\
4020 \\
3630 \\
3270 \\
2760 \\
2080 \\
1580\end{array}$ & $\begin{array}{l}5240 \\
4830 \\
4350 \\
3970 \\
3370 \\
2570 \\
1960\end{array}$ & $\begin{array}{l}6420 \\
5840 \\
5260 \\
4870 \\
4150 \\
3200 \\
2440\end{array}$ & $\begin{array}{l}7290 \\
6580 \\
5940 \\
5530 \\
4720 \\
3670 \\
2800\end{array}$ & $\begin{array}{l}-- \\
-- \\
-- \\
-- \\
--\end{array}$ \\
\hline
\end{tabular}

DURATION TABLE OF DAILY MEAN FLOW FOR PERIOD OF RECORD 1914-15, 1931-55

\begin{tabular}{|c|c|c|c|c|c|c|c|c|c|c|c|c|c|c|}
\hline 58 & 108 & 158 & 208 & 258 & 308 & 408 & 508 & 608 & 708 & 758 & 808 & 858 & 908 & 958 \\
\hline 1910 & 1040 & 604 & 374 & 224 & 151 & 85 & 62 & 51 & 42 & 38 & 34 & 29 & 23 & 15 \\
\hline
\end{tabular}




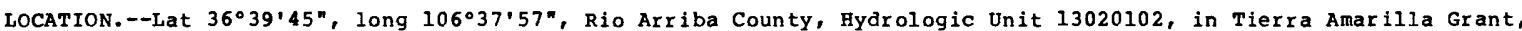
on right bank $0.7 \mathrm{mi}$ downstream from Rito de Tierra Amarilla, 3.1 southwest of La Puente, $6.7 \mathrm{mi}$ upstream from flow line of El vado Reservoir, and at mile 91.4 .

DRAINAGE AREA. $-480 \mathrm{mi}^{2}$, approximately.

PERIOD OF RECORD.--October 1955 to current year.

GAGE.--Water-stage recorder. Concrete control since Nov. 9, 1965. Elevation of gage is $7,083 \mathrm{ft}$ above National Geodetic Vertical Datum of 1929, from river profile map.

REMARKS.--Diversions for irrigation of about 10,300 acres upstream from station (1962 determination).

AVERAGE DISCHARGE.--30 years (water years 1956-85), $345 \mathrm{ft}^{3} / \mathrm{s}, 250,000 \mathrm{acre}-\mathrm{ft} / \mathrm{yr}$.

EXTREMES FOR PERIOD OF RECORD.--Maximum discharge, $11,200 \mathrm{ft} 3 / \mathrm{s}$, May 28 , 1979 , gage height, $6.35 \mathrm{ft}$, from rating curve extended above $5,400 \mathrm{ft} / \mathrm{s}$; maximum gage height, 6.46 ft, May 14,$1984 ; \mathrm{minimum}, 4.0 \mathrm{ft} / \mathrm{s}$, Sept. 19 , 1956 .

EXTREMES OUTSIDE PERIOD OF RECORD.--A discharge of about $9,000 \mathrm{ft} 3 / \mathrm{s}$ occurred Apr. 16, 1937, based on flow of Rio Chama at Los ojos (Park View) with allowance for tributary inflow. A peak on May 21 or 22, 1926, may have exceeded $10,000 \mathrm{ft}^{3} / \mathrm{s}$

STATISTICAL SUMMARIES

MEAN MONTHLY AND MEAN ANNUAL DISCHARGES 1956-85

\begin{tabular}{|c|c|c|c|c|c|c|}
\hline MONTH & $\begin{array}{l}\text { MINIMUM } \\
\left(\mathrm{FT}^{3} / \mathrm{S}\right)\end{array}$ & $\begin{array}{c}\text { MAXIMUM } \\
\left(\mathrm{FT}^{3} / \mathrm{S}\right)\end{array}$ & $\begin{array}{l}\text { MEAN } \\
\left(\mathrm{FT}^{3} / \mathrm{S}\right)\end{array}$ & $\begin{array}{l}\text { STAN- } \\
\text { DARD } \\
\text { DEVIA- } \\
\text { TION } \\
\left(\mathrm{FT}^{3} / \mathrm{S}\right)\end{array}$ & $\begin{array}{l}\text { COEFFI- } \\
\text { CIENT OF } \\
\text { VARI- } \\
\text { ATION }\end{array}$ & $\begin{array}{c}\text { PERCENT } \\
\text { OF } \\
\text { ANNUAL } \\
\text { RUNOFF }\end{array}$ \\
\hline $\begin{array}{l}\text { OCTOBER } \\
\text { NOVEMBER } \\
\text { DECEMBER } \\
\text { JANUARY } \\
\text { FEBRUARY } \\
\text { MARCH } \\
\text { APRIL } \\
\text { MAY } \\
\text { JUNE } \\
\text { JULY } \\
\text { AUGUST } \\
\text { SEPTEMBER }\end{array}$ & $\begin{array}{c}9.8 \\
25 \\
26 \\
16 \\
26 \\
50 \\
244 \\
123 \\
19 \\
9.2 \\
9.0 \\
8.0\end{array}$ & $\begin{array}{r}215 \\
169 \\
104 \\
85 \\
174 \\
445 \\
1846 \\
4195 \\
3091 \\
571 \\
352 \\
320\end{array}$ & $\begin{array}{r}77 \\
71 \\
56 \\
51 \\
67 \\
153 \\
787 \\
1794 \\
763 \\
133 \\
99 \\
76\end{array}$ & $\begin{array}{r}55 \\
39 \\
23 \\
18 \\
30 \\
93 \\
427 \\
1074 \\
769 \\
135 \\
87 \\
71\end{array}$ & $\begin{array}{l}.71 \\
.55 \\
.42 \\
.36 \\
.45 \\
.61 \\
.54 \\
.60 \\
1.01 \\
1.02 \\
.87 \\
.93\end{array}$ & $\begin{array}{r}1.9 \\
1.7 \\
1.4 \\
1.2 \\
1.6 \\
3.7 \\
19.1 \\
43.5 \\
18.5 \\
3.2 \\
2.4 \\
1.8\end{array}$ \\
\hline ANNUAL & 63 & 723 & 345 & 173 & .50 & 100 \\
\hline
\end{tabular}

MAGNITUDE AND PROBABILITY OF ANNUAL LOW FLOW BASED ON PERIOD OF RECORD 1957-85

\begin{tabular}{|c|c|c|c|c|c|c|}
\hline \multirow{2}{*}{$\begin{array}{l}\text { PERIOD } \\
\text { (CON- } \\
\text { SECU- } \\
\text { TIVE } \\
\text { DAYS) }\end{array}$} & \multicolumn{6}{|c|}{$\begin{array}{l}\text { DISCHARGE, IN FT'/S, FOR INDICATED RECURRENCE } \\
\text { INTERVAL, IN YEARS, AND ANNUAL NON- } \\
\text { EXCEEDANCE PROBABILITY, IN PERCENT }\end{array}$} \\
\hline & $\begin{array}{c}2 \\
508\end{array}$ & $\begin{array}{c}5 \\
208\end{array}$ & $\begin{array}{l}10 \\
108\end{array}$ & $\begin{array}{l}20 \\
58\end{array}$ & $\begin{array}{l}50 \\
28\end{array}$ & $\begin{array}{r}100 \\
18\end{array}$ \\
\hline $\begin{array}{r}1 \\
3 \\
7 \\
14 \\
30 \\
60 \\
90 \\
120 \\
183\end{array}$ & $\begin{array}{l}19 \\
20 \\
22 \\
25 \\
29 \\
37 \\
42 \\
47 \\
56\end{array}$ & $\begin{array}{l}9.7 \\
11 \\
12 \\
13 \\
16 \\
21 \\
25 \\
29 \\
36\end{array}$ & $\begin{array}{r}6.8 \\
7.5 \\
8.2 \\
9.5 \\
11 \\
15 \\
19 \\
22 \\
29\end{array}$ & $\begin{array}{r}5.0 \\
5.5 \\
6.1 \\
7.1 \\
8.3 \\
12 \\
15 \\
17 \\
24\end{array}$ & $\begin{array}{r}3 . \\
3 . \\
5 . \\
5 . \\
8 . \\
11 \\
13 \\
19\end{array}$ & $\begin{array}{l}-- \\
-- \\
-- \\
-- \\
-- \\
--\end{array}$ \\
\hline
\end{tabular}

MAGNITULE AND PROBABILITY OF ANNUAL HIGH FLOW BASED ON PERIOD OF RECORD 1956-85

\begin{tabular}{|c|c|c|c|c|c|c|}
\hline \multirow{2}{*}{$\begin{array}{l}\text { PERIOD } \\
\text { (CON- } \\
\text { SECU- } \\
\text { TIVE } \\
\text { DAYS) }\end{array}$} & \multicolumn{6}{|c|}{$\begin{array}{l}\text { DISCHARGE, IN FT }{ }^{3} / S \text {, FOR INDICATED RECURRENCE } \\
\text { INTERVAL, IN YEARS, AND ANNUAL } \\
\text { EXCEEDANCE PROBABILI TY, IN PERCENT }\end{array}$} \\
\hline & $\begin{array}{c}2 \\
508\end{array}$ & $\begin{array}{c}5 \\
208\end{array}$ & $\begin{array}{l}10 \\
108\end{array}$ & $\begin{array}{l}25 \\
48\end{array}$ & $\begin{array}{l}50 \\
28\end{array}$ & $\begin{array}{r}100 \\
18\end{array}$ \\
\hline $\begin{array}{r}1 \\
3 \\
7 \\
15 \\
30 \\
60 \\
90\end{array}$ & $\begin{array}{l}3000 \\
2860 \\
2600 \\
2270 \\
1890 \\
1390 \\
1050\end{array}$ & $\begin{array}{l}5040 \\
4790 \\
4340 \\
3720 \\
3040 \\
2230 \\
1690\end{array}$ & $\begin{array}{l}6500 \\
6150 \\
5540 \\
4670 \\
3750 \\
2740 \\
2080\end{array}$ & $\begin{array}{l}8420 \\
7890 \\
7080 \\
5830 \\
4580 \\
3320 \\
2510\end{array}$ & $\begin{array}{l}9890 \\
9200 \\
8220 \\
6650 \\
5130 \\
3700 \\
2800\end{array}$ & $\begin{array}{l}=- \\
-- \\
-- \\
-- \\
-- \\
--\end{array}$ \\
\hline
\end{tabular}

DURATION TABLE OF DAILY MEAN FLOW FOR PERIOD OF RECORD 1956-85

DISCHARGE, IN FT $3 / \mathrm{S}$, THAT WAS EQUALED OR EXCEEDED FOR INDICATED PERCENT OF TIME

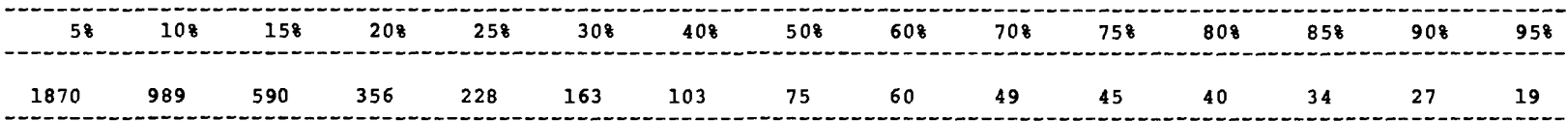


08284160 AZOTEA TUNNET AT OUTLET, NEAR CHAMA, NM

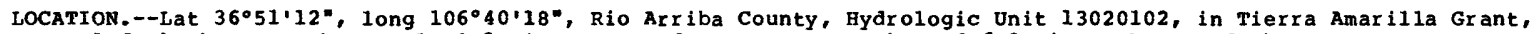
on left bank at south portal, $0.2 \mathrm{mi}$ upstrean from Azotea creek, and $6.2 \mathrm{mi}$ southwest of Chama.

PERIOD OF RECORD.--October 1970 to current year.

GAGE.--Water-stage recorder and Parshall flume. Datum of gage is 7,519.87 ft above National Geodetic Vertical Datum of 1929 (levels by U.S. Bureau of Reclamation).

REMARKS.--Records represent regulated diversions from Rio Blanco, Little Navajo River, and Navajo River in San Juan River Basin.

COOPERATION,--Records provided by U.S. Bureau of Reclamation.

AVERAGE DISCHARGE. --15 years (water years 1971-85), $138 \mathrm{ft}^{3} / \mathrm{s}, 99,980 \mathrm{acre}-\mathrm{ft} / \mathrm{yr}$.

EXTREMES FOR PERIOD OF RECORD.--Maximum discharge, 1,170 $\mathrm{ft}^{3} / \mathrm{s}$, May 17, 1978, gage height, $7.85 \mathrm{ft}$; no f1ow many days most years.

STATISTICAL SUMMARIES

MEAN MONTHLY AND MEAN ANNUAL DISCHARGES 1971-85

\begin{tabular}{|c|c|c|c|c|c|c|}
\hline MONTH & $\begin{array}{l}\text { MINI MUM } \\
\left(\mathrm{FT}^{3} / \mathrm{S}\right)\end{array}$ & $\begin{array}{c}\text { MAXIMUM } \\
(\text { FT'/S) }\end{array}$ & $\begin{array}{l}\text { MEAN } \\
\left(\mathrm{FT}^{3} / \mathrm{S}\right)\end{array}$ & $\begin{array}{l}\text { STAN- } \\
\text { DARD } \\
\text { DEVIA- } \\
\text { TION } \\
\text { (FT'S) }\end{array}$ & $\begin{array}{l}\text { COEFFI - } \\
\text { CIENT OF } \\
\text { VARI - } \\
\text { ATION }\end{array}$ & $\begin{array}{c}\text { PERCENT } \\
\text { OF } \\
\text { ANNUAL } \\
\text { RUNOFF }\end{array}$ \\
\hline $\begin{array}{l}\text { OCTOBER } \\
\text { NOVEMBER } \\
\text { DECEMBER } \\
\text { JANUARY } \\
\text { FEBRUARY } \\
\text { MARCH } \\
\text { APRIL } \\
\text { MAY } \\
\text { JUNE } \\
\text { JULY } \\
\text { AUGUST } \\
\text { SEPTEMBER }\end{array}$ & $\begin{array}{l}0.0 \\
0.0 \\
0.0 \\
0.0 \\
0.0 \\
0.0 \\
76 \\
68 \\
46 \\
7.7 \\
.6 \\
0.0\end{array}$ & $\begin{array}{r}272 \\
65 \\
34 \\
25 \\
21 \\
91 \\
633 \\
789 \\
1003 \\
543 \\
88 \\
35\end{array}$ & $\begin{array}{r}29 \\
8.0 \\
3.3 \\
2.3 \\
2.1 \\
15 \\
250 \\
529 \\
569 \\
188 \\
44 \\
16\end{array}$ & $\begin{array}{c}70 \\
19 \\
8.9 \\
6.5 \\
5.5 \\
26 \\
133 \\
249 \\
354 \\
200 \\
33 \\
13\end{array}$ & $\begin{array}{r}2.44 \\
2.39 \\
2.72 \\
2.82 \\
2.67 \\
1.72 \\
.53 \\
.47 \\
.62 \\
1.06 \\
.74 \\
.86\end{array}$ & $\begin{array}{r}1.7 \\
.5 \\
.2 \\
.1 \\
.1 \\
.9 \\
15.1 \\
32.0 \\
34.4 \\
11.4 \\
2.6 \\
.9\end{array}$ \\
\hline ANNUAL & 27 & 242 & 138 & 67 & .49 & 100 \\
\hline
\end{tabular}

MAGNI TUDE AND PROBABILITY OF ANNUAL LOW FLOW BASED ON PERIOD OF RECORD 1972-85

\begin{tabular}{|c|c|c|c|c|c|c|}
\hline \multirow{2}{*}{$\begin{array}{l}\text { PERIOD } \\
\text { (CON- } \\
\text { SECU- } \\
\text { TIVE } \\
\text { DAYS) }\end{array}$} & \multicolumn{6}{|c|}{$\begin{array}{l}\text { DISCHARGE, IN FT'/S, FOR INDICATED RECURRENCE } \\
\text { INTERVAL, IN YEARS, AND ANNUAL NON- } \\
\text { EXCEEDANCE PROBABILITY, IN PERCENT }\end{array}$} \\
\hline & $\begin{array}{c}2 \\
508\end{array}$ & $\begin{array}{c}5 \\
208\end{array}$ & $\begin{array}{l}10 \\
108\end{array}$ & $\begin{array}{l}20 \\
58\end{array}$ & 50 & $\begin{array}{r}100 \\
18\end{array}$ \\
\hline $\begin{array}{r}1 \\
3 \\
7 \\
14 \\
30 \\
60 \\
90 \\
120 \\
183\end{array}$ & $\begin{array}{r}0.0 \\
0.0 \\
0.0 \\
0.0 \\
.1 \\
.2 \\
.2 \\
.2 \\
1.1\end{array}$ & $\begin{array}{r}0.0 \\
0.0 \\
0.0 \\
0.0 \\
0.0 \\
0.0 \\
0.0 \\
0.0 \\
.1\end{array}$ & $\begin{array}{r}0.0 \\
0.0 \\
0.0 \\
0.0 \\
0.0 \\
0.0 \\
0.0 \\
0.0 \\
.1\end{array}$ & $\begin{array}{l}0.0 \\
0.0 \\
0.0 \\
0.0 \\
0.0 \\
0.0 \\
0.0 \\
0.0 \\
0.0\end{array}$ & $\begin{array}{l}-- \\
-- \\
-- \\
-- \\
-- \\
-- \\
--\end{array}$ & $\begin{array}{l}-- \\
-- \\
-- \\
-- \\
-- \\
-- \\
--\end{array}$ \\
\hline
\end{tabular}

MAGNITUDE AND PROBABILITY OF ANNUAL HIGH FLOW BASED ON PERIOD OF RECORD 1971-85

\begin{tabular}{|c|c|c|c|c|c|c|}
\hline \multirow{2}{*}{$\begin{array}{l}\text { PERIOD } \\
\text { (CON- } \\
\text { SECU- } \\
\text { TIVE } \\
\text { DAYS) }\end{array}$} & \multicolumn{6}{|c|}{$\begin{array}{l}\text { DISCHARGE, IN FT'/S, FOR INDICATED RECURRENCE } \\
\text { INTERVAL, IN YEARS, AND ANNUAL } \\
\text { EXCEEDANCE PROBABILITY, IN PERCENT }\end{array}$} \\
\hline & $\begin{array}{c}2 \\
508\end{array}$ & $\begin{array}{c}5 \\
208\end{array}$ & $\begin{array}{l}10 \\
108\end{array}$ & $\begin{array}{l}25 \\
48\end{array}$ & $\begin{array}{l}50 \\
28\end{array}$ & $\begin{array}{r}100 \\
18\end{array}$ \\
\hline $\begin{array}{r}1 \\
3 \\
7 \\
15 \\
30 \\
60 \\
90\end{array}$ & $\begin{array}{l}859 \\
823 \\
794 \\
757 \\
698 \\
588 \\
486\end{array}$ & $\begin{array}{r}1120 \\
1120 \\
1110 \\
1070 \\
1030 \\
902 \\
751\end{array}$ & $\begin{array}{r}1230 \\
1240 \\
1240 \\
1190 \\
1170 \\
1050 \\
873\end{array}$ & $\begin{array}{r}1320 \\
1340 \\
1340 \\
1270 \\
1280 \\
1180 \\
976\end{array}$ & $\begin{array}{l}-- \\
-- \\
-- \\
-- \\
--\end{array}$ & $\begin{array}{l}-- \\
-- \\
-- \\
-- \\
-- \\
--\end{array}$ \\
\hline
\end{tabular}

DURATION TABLE OF DAILY MEAN FLOW FOR PERIOD OF RECORD 1971-85

DISCHARGE, IN FT'/S, THAT WAS EOUALED OR EXCEEDED FOR INDICATED PERCENT OF TIME

\begin{tabular}{|c|c|c|c|c|c|c|c|c|c|c|c|c|c|c|}
\hline 58 & 108 & 158 & 208 & 258 & 308 & 408 & 508 & 608 & 708 & 758 & 808 & 858 & 908 & 958 \\
\hline 863 & 580 & 342 & 195 & 120 & 70 & 25 & 5.1 & .8 & .3 & .2 & 0.0 & 0.0 & 0.0 & 0.0 \\
\hline
\end{tabular}


RIO GRANDE BASIN

08284200 WILLOW CREEK ABOVE HERON RESERVOIR, NEAR LOS OJOS, NM

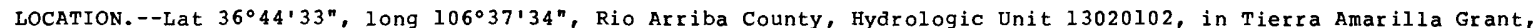
on right bank $200 \mathrm{ft}$ downstream from bridge, $0.2 \mathrm{mi}$ downstream from Iron Spring Creek, $3.3 \mathrm{mi}$ west of Los Ojos, and at mile 9.7 .

DRA INAGE AREA. $--112 \mathrm{mi}^{2}$.

PERIOD OF RECORD.--October and November 1962 (monthly discharge only), December 1962 to current year. published as "near Park View" prior to 1976.

GAGE.--Water-stage recorder. Concrete control since June 6, 1963. Datum of gage is $7,196.29 \mathrm{ft}$ above National Geodetic Vertical Datum of 1929 (levels by U.S. Bureau of Reclamation). Prior to Apr. 1, 1971, at site 900 ft downstream at lower datum.

REMARKS.--Records represent inflow to Heron Reservoir and since Nov, 17, 1970, include San Juan River water imported through Azotea tunnel (station 08284160).

COOPERATION.--Records provided by U.S. Bureau of Reclamation.

AVERAGE DISCHARGE.--22 years (water years 1964-85), $107 \mathrm{ft}^{3} / \mathrm{s}, 77,520$ acre-ft/yr, prior to completion of Azotea tunnel.

15 years (water years $1971-85$ ), $152 \mathrm{ft}^{3} / \mathrm{s}, 110,100$ acre-ft/yr.

EXTREMES FOR PERIOD OF RECORD.--Maximum discharge, 1,610 ft $3 / \mathrm{s}$, Mar. 12, 1985, gage height, $6.65 \mathrm{ft}$; no flow at times most years prior to 1971.

STATISTICAL SUMMARIES

MEAN MONTHLY AND MEAN ANNUAL DISCHARGES 1964-85

\begin{tabular}{|c|c|c|c|c|c|c|}
\hline MONTH & $\begin{array}{l}\text { MINIMUM } \\
\left(\mathrm{FT}^{3} / \mathrm{S}\right)\end{array}$ & $\begin{array}{l}\text { MAXIMUM } \\
\left(\mathrm{FT}^{3} / \mathrm{S}\right)\end{array}$ & $\begin{array}{l}\text { MEAN } \\
\left(\mathrm{FT}^{3} / \mathrm{S}\right)\end{array}$ & $\begin{array}{l}\text { STAN- } \\
\text { DARD } \\
\text { DEVIA- } \\
\text { TION } \\
\left(\mathrm{FT}^{3} / \mathrm{S}\right)\end{array}$ & $\begin{array}{l}\text { COEFFI- } \\
\text { CIENT OF } \\
\text { VARI- } \\
\text { ATION }\end{array}$ & $\begin{array}{c}\text { PERCENT } \\
\text { OF } \\
\text { ANNUAL } \\
\text { RUNOF }\end{array}$ \\
\hline \multicolumn{7}{|c|}{ 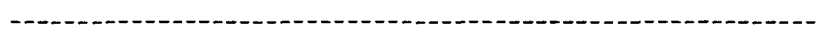 } \\
\hline OCTOBER & .1 & 266 & 21 & 57 & 2.75 & 1.6 \\
\hline NOVEMBER & .2 & 80 & 6.8 & 19 & 2.78 & .5 \\
\hline DECEMBER & .1 & 35 & 2.8 & 7.6 & 2.73 & .2 \\
\hline JANUARY & .1 & 25 & 2.9 & 6.0 & 2.04 & .2 \\
\hline FEBRUARY & .1 & 58 & 6.1 & 13 & 2.11 & .5 \\
\hline MARCH & .9 & 201 & 54 & 49 & .90 & 4.2 \\
\hline APRIL & 2.3 & 752 & 242 & 202 & .83 & 18.9 \\
\hline MAY & 1.6 & 813 & 370 & 331 & .89 & 28.9 \\
\hline JUNE & 2.2 & 997 & 390 & 396 & 1.02 & 30.5 \\
\hline JULY & 3.1 & 547 & 133 & 187 & 1.41 & 10.4 \\
\hline AUGUST & 1.4 & 107 & 38 & 34 & .90 & 3.0 \\
\hline SEPTEMBER & .1 & 38 & 13 & 14 & 1.03 & 1.0 \\
\hline ANNUAL & 3.4 & 267 & 107 & 91 & .85 & 100 \\
\hline
\end{tabular}

MAGNITUDE AND PROBABILITY OF ANNUAL LOW FLOW BASED ON PERIOD OF RECORD 1965-85

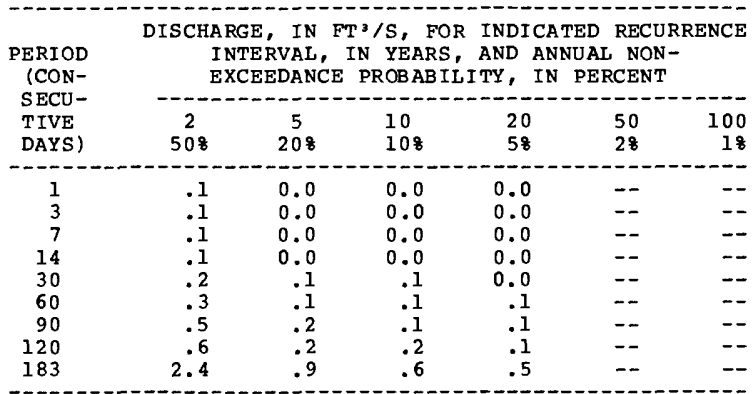

MAGNITUDE AND PROBABILITY OF ANNUAL HIGH FLOW BASED ON PERIOD OF RECORD 1964-85

\begin{tabular}{|c|c|c|c|c|c|c|}
\hline \multirow{2}{*}{$\begin{array}{l}\text { PERIOD } \\
\text { (CON- } \\
\text { SECU- } \\
\text { TIVE } \\
\text { DAYS) }\end{array}$} & \multicolumn{6}{|c|}{$\begin{array}{l}\text { DISCHARGE, IN FT } 3 / S \text {, FOR INDICATED RECURRENCE } \\
\text { INTERVAL, IN YEARS, AND ANNUAL } \\
\text { EXCEEDANCE PROBABILI TY, IN PERCENT }\end{array}$} \\
\hline & $\begin{array}{c}2 \\
508\end{array}$ & $\begin{array}{c}5 \\
208\end{array}$ & $\begin{array}{l}10 \\
108\end{array}$ & $\begin{array}{l}25 \\
48\end{array}$ & $\begin{array}{l}50 \\
28\end{array}$ & $\begin{array}{r}100 \\
18\end{array}$ \\
\hline $\begin{array}{r}1 \\
3 \\
7 \\
15 \\
30 \\
60 \\
90\end{array}$ & $\begin{array}{l}670 \\
571 \\
509 \\
428 \\
356 \\
265 \\
210\end{array}$ & $\begin{array}{r}1070 \\
1020 \\
1020 \\
971 \\
926 \\
792 \\
672\end{array}$ & $\begin{array}{l}1250 \\
1280 \\
1310 \\
1330 \\
1350 \\
1250 \\
1100\end{array}$ & $\begin{array}{l}1420 \\
1550 \\
1610 \\
1730 \\
1870 \\
1870 \\
1720\end{array}$ & $\begin{array}{l}-- \\
-- \\
-- \\
-- \\
-- \\
--\end{array}$ & $\begin{array}{l}-- \\
-- \\
-- \\
-- \\
-- \\
--\end{array}$ \\
\hline
\end{tabular}

DURATION TABLE OF DAILY MEAN FLOW FOR PERIOD OF RECORD 1964-85 DISCHARGE, IN FT3/S, THAT WAS EQUALED OR EXCEEDED FOR INDICATED PERCENT OF TIME

\begin{tabular}{|c|c|c|c|c|c|c|c|c|c|c|c|c|c|c|}
\hline $5 \%$ & 108 & 158 & 208 & 258 & 308 & 408 & 508 & 608 & 708 & 758 & 808 & 858 & 908 & $95 \%$ \\
\hline 770 & 382 & 200 & 120 & 66 & 37 & 11 & 3.5 & 1.5 & .7 & .5 & .3 & .2 & .2 & . \\
\hline
\end{tabular}


08284200 WILLOW CREER ABOVE HERON RESERVOIR, NEAR LOS OJOS, NM--COntinued STATISTICAL SUMMARIES (PERIOD AFTER COMPLETION OF AZOTEA TUNNEL)

MEAN MONTHLY AND MEAN ANNUAL DISCHARGES 1971-85

\begin{tabular}{|c|c|c|c|c|c|c|}
\hline MONTH & $\begin{array}{l}\text { MINIMUM } \\
\left(\mathrm{FT}^{3} / \mathrm{S}\right)\end{array}$ & $\begin{array}{c}\operatorname{MAXIMUM} \\
\left(\mathrm{FT}^{3} / \mathrm{S}\right)\end{array}$ & $\begin{array}{l}\text { MEAN } \\
\left(\mathrm{FT}^{3} / \mathrm{S}\right)\end{array}$ & $\begin{array}{l}\text { STAN- } \\
\text { DARD } \\
\text { DEVIA- } \\
\text { TION } \\
\left(\text { FT }^{3} / \mathrm{S}\right)\end{array}$ & $\begin{array}{l}\text { COEFFI- } \\
\text { CIENT OF } \\
\text { VARI- } \\
\text { ATION }\end{array}$ & $\begin{array}{c}\text { PERCENT } \\
\text { OF } \\
\text { ANNUAL } \\
\text { RUNOFF }\end{array}$ \\
\hline ОстовЕ & 4 & 266 & 30 & 68 & 2.27 & 1.6 \\
\hline NOVEMBER & .2 & $\begin{array}{r}260 \\
80\end{array}$ & 9.5 & $\begin{array}{l}08 \\
23\end{array}$ & 2.38 & $\begin{array}{r}1.6 \\
.5\end{array}$ \\
\hline DECEMBER & .1 & 35 & 3.7 & 9.1 & 2.46 & .2 \\
\hline JANUARY & .1 & 25 & 2.8 & 6.4 & 2.32 & .2 \\
\hline FEBRUARY & .2 & 58 & 8.0 & 15 & 1.91 & .4 \\
\hline MARCH & .9 & 201 & 61 & 52 & .84 & 3.4 \\
\hline APRIL & 76 & 752 & 331 & 181 & .55 & 18.2 \\
\hline MAY & 69 & 813 & 540 & 259 & .4 & 29.7 \\
\hline JUNE & 46 & 997 & 571 & 354 & .62 & 31.4 \\
\hline JULY & 10 & 547 & 193 & 200 & 1.04 & 10.6 \\
\hline AUGUST & 1.5 & 107 & 49 & 35 & .73 & 2.7 \\
\hline SEPTEMBER & .9 & 38 & 18 & 14 & .78 & 1.0 \\
\hline ANNUAL & 28 & 267 & 152 & 75 & .49 & 100 \\
\hline
\end{tabular}

MAGNITUDE AND PROBABILITY OF ANNUAL LOW FLOW BASED ON PERIOD OF RECORD 1972-85

\begin{tabular}{|c|c|c|c|c|c|c|}
\hline \multirow{2}{*}{$\begin{array}{l}\text { PERIOD } \\
\text { (CON- } \\
\text { SECU- } \\
\text { TIVE } \\
\text { DAYS) }\end{array}$} & \multicolumn{6}{|c|}{$\begin{array}{l}\text { DISCHARGE, IN FT'3/S, FOR INDICATED RECURRENCE } \\
\text { INTERVAL, IN YEARS, AND ANNUAL NON- } \\
\text { EXCFEDANCE PROBABILITY, IN PERCENT }\end{array}$} \\
\hline & $\begin{array}{c}2 \\
508\end{array}$ & $\begin{array}{c}5 \\
208\end{array}$ & $\begin{array}{l}10 \\
108\end{array}$ & $\begin{array}{l}20 \\
58\end{array}$ & $\begin{array}{l}50 \\
28\end{array}$ & $\begin{array}{r}100 \\
18\end{array}$ \\
\hline $\begin{array}{r}1 \\
3 \\
7 \\
14 \\
30 \\
60 \\
90 \\
120 \\
183\end{array}$ & $\begin{array}{r}.1 \\
.1 \\
.2 \\
.2 \\
.3 \\
.4 \\
.5 \\
.7 \\
3.7\end{array}$ & $\begin{array}{r}.1 \\
.1 \\
.1 \\
.1 \\
.1 \\
.2 \\
.2 \\
.2 \\
1.2\end{array}$ & $\begin{array}{r}0.0 \\
0.0 \\
.1 \\
.1 \\
.1 \\
.1 \\
.1 \\
.1 \\
.7\end{array}$ & $\begin{array}{r}0.0 \\
0.0 \\
0.0 \\
.1 \\
.1 \\
.1 \\
.1 \\
.1 \\
.5\end{array}$ & $\begin{array}{l}-- \\
-- \\
-- \\
-- \\
-- \\
-- \\
--\end{array}$ & $\begin{array}{l}=- \\
=- \\
=- \\
-- \\
-- \\
-- \\
--\end{array}$ \\
\hline
\end{tabular}

MAGNITUDE AND PROBABILITY OF ANNUAL HIGH FLOW BASED ON PERIOD OF RECORD 1971-85

\begin{tabular}{|c|c|c|c|c|c|c|}
\hline \multirow{2}{*}{$\begin{array}{l}\text { PERIOD } \\
\text { (CON- } \\
\text { SECU- } \\
\text { TIVE } \\
\text { DAYS) }\end{array}$} & \multicolumn{6}{|c|}{$\begin{array}{l}\text { DISCHARGE, IN FT } 3 / \mathrm{S}, \text { FOR INDICATED RECURRENCE } \\
\text { INTERVAL, IN YEARS, AND ANNUAL } \\
\text { EXCEEDANCE PROBABILITY, IN PERCENT }\end{array}$} \\
\hline & $\begin{array}{c}2 \\
508\end{array}$ & $\begin{array}{c}5 \\
208\end{array}$ & $\begin{array}{l}10 \\
108\end{array}$ & $\begin{array}{l}25 \\
48\end{array}$ & $\begin{array}{l}50 \\
28\end{array}$ & $\begin{array}{r}100 \\
18\end{array}$ \\
\hline $\begin{array}{r}1 \\
3 \\
7 \\
15 \\
30 \\
60 \\
90\end{array}$ & $\begin{array}{l}878 \\
836 \\
796 \\
759 \\
704 \\
594 \\
504\end{array}$ & $\begin{array}{r}1160 \\
1150 \\
1120 \\
1080 \\
1040 \\
913 \\
791\end{array}$ & $\begin{array}{r}1290 \\
1280 \\
1260 \\
1210 \\
1180 \\
1060 \\
924\end{array}$ & $\begin{array}{l}1400 \\
1390 \\
1370 \\
1310 \\
1300 \\
1190 \\
1040\end{array}$ & $\begin{array}{l}-- \\
-- \\
-- \\
-- \\
-- \\
--\end{array}$ & $\begin{array}{l}-- \\
-- \\
-- \\
-- \\
-- \\
--\end{array}$ \\
\hline
\end{tabular}

DURATION TABLE OF DAILY MEAN FLOW FOR PERIOD OF RECORD 1971-85

DISCHARGE, IN FT 3 /S, THAT WAS EOUALED OR EXCEEDED FOR INDICATED PERCENT OF TIME

\begin{tabular}{|c|c|c|c|c|c|c|c|c|c|c|c|c|c|c|}
\hline 58 & 108 & 158 & 208 & 258 & 308 & 408 & 508 & 608 & 708 & 758 & 808 & 858 & 908 & 958 \\
\hline 904 & 623 & 369 & 228 & 155 & 100 & 42 & 15 & 3.2 & 1.0 & .6 & .5 & .3 & .2 & .2 \\
\hline
\end{tabular}




\section{RIO GRANDE BASIN}

08284300 HORSE LAKE CREEK ABOVE HERON RESERVOIR, NEAR LOS OJOS, NM

LOCATION.--Lat 36 $42^{\prime} 24^{\prime \prime}$, long $106^{\circ} 44^{\prime} 42^{\prime \prime}$, Rio Arriba County, in Tierra Amarilla Grant, on right bank $3.7 \mathrm{mi}$ northwest of Heron Dam, $7.8 \mathrm{mi}$ downstream from Horse Lake, and $9.9 \mathrm{mi}$ west of Los Ojos.

DRAINAGE AREA. $--45 \mathrm{mi}^{2}$, approximately.

PERIOD OF RECORD.--October and November 1962 (monthly discharge only), December 1962 to current year.

GAGE.--Water-stage recorder. Concrete control since June 10, 1963. Datum of gage is $7,188.85 \mathrm{ft}$ above mean sea level (levels by Bureau of Reclamation). Prior to July 1, 1971, at site l, 100 ft upstream at higher datums.

REMARKS.--Diversions above station for irrigation of meadows and for off-channel stock tanks.

COOPERATION,--Records furnished by Bureau of Reclamation.

AVERAGE DISCHARGE.--12 years (water years 1963-73, 1985), $1.70 \mathrm{ft}^{3} / \mathrm{s}, 1,230 \mathrm{acre}-\mathrm{ft} / \mathrm{yr}$.

EXTREMES FOR PERIOD OF RECORD.--Maximum discharge, 3,960 $\mathrm{ft}^{3} / \mathrm{s} \mathrm{July} \mathrm{30,} 1968$ (gage height, $4.9 \mathrm{ft}$, site and datum

then in use), from rating curve extended above $37 \mathrm{ft}^{3} / \mathrm{s}$ on basis of slope-area measurements at gage heights 3.20
$\mathrm{ft}$ and $4.9 \mathrm{ft}$; no flow most of time.

STATISTICAL SUMMARIES

MEAN MONTHLY AND MEAN ANNUAL DISChARGES 1963-73, 1985

\begin{tabular}{|c|c|c|c|c|c|c|}
\hline \multirow{2}{*}{$\begin{array}{c}\text { PERIOD } \\
\text { (CON- } \\
\text { SECU- } \\
\text { TIVE } \\
\text { DAYS) }\end{array}$} & \multicolumn{6}{|c|}{$\begin{array}{l}\text { DISCHARGE, IN FT }{ }^{3 / S} \text {, FOR INDICATED RECURRENCE } \\
\text { INTERVAL, IN YEARS, AND ANNUAL NON- } \\
\text { EXCEEDANCE PROBAB ILITY, IN PERCENT }\end{array}$} \\
\hline & $\begin{array}{c}2 \\
508\end{array}$ & $\begin{array}{c}5 \\
208\end{array}$ & $\begin{array}{l}10 \\
108\end{array}$ & $\begin{array}{l}20 \\
58\end{array}$ & $\begin{array}{l}50 \\
28\end{array}$ & $\begin{array}{r}100 \\
18\end{array}$ \\
\hline 1 & - & -- & - & -2 & -- & $\ldots$ \\
\hline 3 & -- & -- & - & -- & -- & - \\
\hline 7 & -- & - & - & -- & -- & -- \\
\hline 14 & -- & -- & -- & -- & -- & -- \\
\hline 30 & -- & -- & - & -- & - & - \\
\hline 60 & -- & -- & -- & -- & - & -- \\
\hline 90 & 0.0 & 0.0 & 0.0 & 0.0 & -- & - \\
\hline 120 & 0.0 & 0.0 & 0.0 & 0.0 & -- & $\cdots$ \\
\hline 183 & .1 & 0.0 & 0.0 & 0.0 & -- & - \\
\hline
\end{tabular}

\begin{tabular}{|c|c|c|c|c|c|c|}
\hline MONTH & $\begin{array}{l}\text { MINI MUM } \\
\left(\mathrm{FT}^{3} / \mathrm{S}\right)\end{array}$ & $\begin{array}{l}\text { MAXIMUM } \\
\left(F^{3} / S\right)\end{array}$ & $\begin{array}{l}\text { MEAN } \\
\left(\mathrm{FT}^{3} / \mathrm{S}\right)\end{array}$ & $\begin{array}{l}\text { STAN- } \\
\text { DARD } \\
\text { DEVIA- } \\
\text { TION } \\
\left(\text { FT }^{3} / \mathrm{S}\right)\end{array}$ & $\begin{array}{l}\text { COEFFI- } \\
\text { CIENT OF } \\
\text { VARI- } \\
\text { ATION }\end{array}$ & $\begin{array}{c}\text { PERCENT } \\
\text { OF } \\
\text { ANNUAL, } \\
\text { RUNOFF }\end{array}$ \\
\hline $\begin{array}{l}\text { OCTOBER } \\
\text { NOVEMBER } \\
\text { DECEMBER } \\
\text { JANUARY } \\
\text { FEBRUARY } \\
\text { MARCH } \\
\text { APRIL } \\
\text { MAY } \\
\text { JUNE } \\
\text { JULY } \\
\text { AUGUST } \\
\text { SEPTEMBER }\end{array}$ & $\begin{array}{l}0.0 \\
0.0 \\
0.0 \\
0.0 \\
0.0 \\
0.0 \\
0.0 \\
0.0 \\
0.0 \\
0.0 \\
0.0 \\
0.0\end{array}$ & $\begin{array}{r}3.3 \\
1.2 \\
.4 \\
1.4 \\
2.4 \\
36 \\
41 \\
12 \\
6.6 \\
7.2 \\
13 \\
4.0\end{array}$ & $\begin{array}{r}.3 \\
.2 \\
.1 \\
.2 \\
.5 \\
7.7 \\
8.1 \\
1.4 \\
.5 \\
.8 \\
1.7 \\
.8\end{array}$ & $\begin{array}{r}.8 \\
.4 \\
.1 \\
.4 \\
.8 \\
9.3 \\
11 \\
2.8 \\
1.5 \\
1.9 \\
3.0 \\
1.2\end{array}$ & $\begin{array}{l}2.29 \\
1.79 \\
2.20 \\
2.60 \\
1.80 \\
1.21 \\
1.41 \\
1.93 \\
3.11 \\
2.34 \\
1.79 \\
1.55\end{array}$ & $\begin{array}{r}1.5 \\
1.1 \\
.2 \\
.7 \\
2.0 \\
34.7 \\
36.5 \\
6.5 \\
2.1 \\
3.6 \\
7.7 \\
3.5\end{array}$ \\
\hline ANNUAL & .1 & 8.4 & 1.7 & 2.3 & 1.33 & 100 \\
\hline
\end{tabular}

MAGNITUDE AND PROBABILITY OF ANNUAL HIGH FLOW BASED ON PERIOD OF RECORD $1963-73,1985$

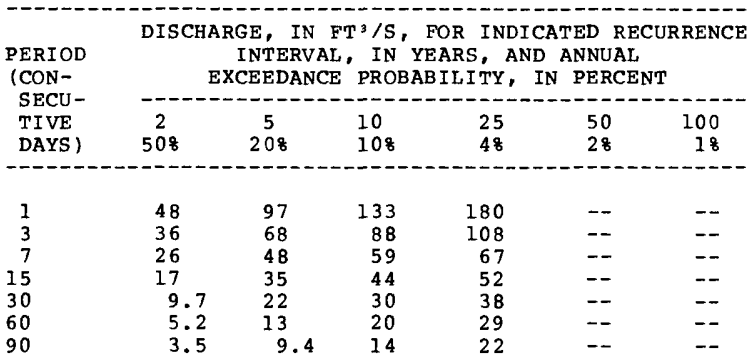

DURATION TABLE OF DAILY MEAN FLOW FOR PERIOD OF RECORD 1963-73,1985

DISCHARGE, IN FT"S, THAT WAS EQUALED OR EXCEEDED FOR INDICATED PERCENT OF TIME

\begin{tabular}{|c|c|c|c|c|c|c|c|c|c|c|c|c|c|c|}
\hline 58 & 108 & 158 & 208 & 258 & 308 & 408 & 508 & 608 & 708 & 758 & 808 & 858 & 908 & 958 \\
\hline 9.3 & 3.6 & 1.0 & .2 & .1 & 0.0 & 0.0 & 0.0 & 0. & 0.0 & 0.0 & 0.0 & 0.0 & 0.0 & 0.0 \\
\hline
\end{tabular}


LOCATION.--Lat $36^{\circ} 40^{\circ} 05^{\prime \prime}$, long $106^{\circ} 42^{\prime} 15^{\prime \prime}$, Rio Arriba County, in Tierra Amarilla Grant, at Heron damsite, 0.4 mi above mouth, $0.7 \mathrm{mi}$ downstream from Horse Lake Creek, and $8.6 \mathrm{miles}$ southwest of Park View.

DRAINAGE AREA. $--193 \mathrm{mi}^{2}$.

PERIOD OF RECORD.--May 1936 to September 1942 (no winter periods), October 1942 to February 1971 (discontinued). Monthly or yearly discharge only for some periods prior to 1943, published in wSP 1312 .

REVISED RECORDS.--WSP 858: 1936. WSP 1312: $1943(\mathrm{M}), 1949(\mathrm{M})$.

GAGE.--Water-stage recorders $0.7 \mathrm{mi}$ upstream on Horse Lake Creek at mouth and 3 mi upstrean on Willow Creek at steel bridge (construction of Heron Dam prevented collection of a record at Heron damsite subsequent to Nov. 8 , 1965). At Heron damsite, datum of gage at time of discontinuance was $6,944.99 \mathrm{ft}$ above mean sea level (Bureau of Reclamation datum). Prior to Oct. 1, 1937, at datum $0.79 \mathrm{ft}$ higher. Apr. 19, 1949 to Aug. 8, 1951, at different datums. Aug. 9, 1951 to Sept. 30, 1960, at datum $0.41 \mathrm{ft}$ higher.

REMARKS. --Small diversions above station for irrigation and stock tanks. Subsequent to Nov. 8, 1965 , published record is the composite of Horse Lake Creek at mouth and willow Creek at steel bridge pending construction of Heron Dam. Diversions through Azotea tunnel were made on Nov. 17, Dec. 7, 11, 1970.

AVERAGE DISCHARGE.--28 years (water years 1943-70), $14.0 \mathrm{ft}^{3} / \mathrm{s}(10,140 \mathrm{acre}-\mathrm{ft} /$ year).

EXTREMES FOR PERIOD OF RECORD.--Maximum discharge, 4,500 ft ${ }^{3 / s}$ Apr. 23, 1942 (gage height, $10.45 \mathrm{ft}^{2}$ site and datum then in use), from rating curve extended above $1,400 \mathrm{ft}^{3} / \mathrm{s}$ on basis of slope-area measurement of peak flow; no flow at times.

STATISTICAL SUMMARIES

MEAN MONTHLY AND MEAN ANNUAL DISCHARGES 1943-70

\begin{tabular}{|c|c|c|c|c|c|c|}
\hline MONTH & $\begin{array}{l}\text { MINIMUM } \\
\left(\mathrm{FT}^{3} / \mathrm{S}\right)\end{array}$ & $\begin{array}{l}\text { MAXIMUM } \\
\left(\mathrm{FT}^{3} / \mathrm{S}\right)\end{array}$ & $\begin{array}{l}\text { MEAN } \\
\left(\mathrm{FT}^{3} / \mathrm{S}\right)\end{array}$ & $\begin{array}{l}\text { STAN- } \\
\text { DARD } \\
\text { DEVIA- } \\
\text { TION } \\
\left(\mathrm{FT}^{3} / \mathrm{S}\right)\end{array}$ & $\begin{array}{l}\text { COEFFI- } \\
\text { CIENT OF } \\
\text { VARI- } \\
\text { ATION }\end{array}$ & $\begin{array}{c}\text { PERCENT } \\
\text { OF } \\
\text { ANNUAL } \\
\text { RUNOFF }\end{array}$ \\
\hline $\begin{array}{l}\text { OCTOBER } \\
\text { NOVEMBER } \\
\text { DECEMBER } \\
\text { JANUARY } \\
\text { FEBRUARY } \\
\text { MARCH } \\
\text { APRIL } \\
\text { MAY } \\
\text { JUNE } \\
\text { JULY } \\
\text { AUGUST } \\
\text { SEPTEMBER }\end{array}$ & $\begin{array}{r}0.0 \\
0.0 \\
0.0 \\
0.0 \\
0.0 \\
1.5 \\
2.1 \\
.2 \\
.2 \\
0.0 \\
.2 \\
0.0\end{array}$ & $\begin{array}{c}11 \\
11 \\
5.3 \\
30 \\
36 \\
164 \\
390 \\
71 \\
13 \\
18 \\
79 \\
20\end{array}$ & $\begin{array}{r}2.9 \\
2.3 \\
.8 \\
1.8 \\
5.3 \\
47 \\
72 \\
10 \\
4.7 \\
6.1 \\
13 \\
4.1\end{array}$ & $\begin{array}{r}2.8 \\
2.7 \\
1.2 \\
5.8 \\
9.1 \\
45 \\
94 \\
14 \\
3.7 \\
5.2 \\
17 \\
6.1\end{array}$ & $\begin{array}{r}.96 \\
1.15 \\
1.52 \\
3.30 \\
1.71 \\
.98 \\
1.31 \\
1.40 \\
.78 \\
.85 \\
1.33 \\
1.49\end{array}$ & $\begin{array}{r}1.7 \\
1.4 \\
.5 \\
1.0 \\
3.1 \\
27.4 \\
42.6 \\
6.1 \\
2.8 \\
3.6 \\
7.4 \\
2.4\end{array}$ \\
\hline ANNUAL & 1.5 & 48 & 14 & 11 & .78 & 100 \\
\hline
\end{tabular}

MAGNITUDE AND PROBABILITY OF ANNUAL LOW FLON BASED ON PERIOD OF RECORD 1944-70

\begin{tabular}{|c|c|c|c|c|c|c|}
\hline \multirow{2}{*}{$\begin{array}{l}\text { PERIOD } \\
\text { (CON- } \\
\text { SECU- } \\
\text { TIVE } \\
\text { DAYS) }\end{array}$} & \multicolumn{6}{|c|}{$\begin{array}{l}\text { DISCHARGE, IN } \text { FT }^{3} / \mathrm{S} \text {, FOR INDICATED RECURRENCE } \\
\text { INTERVAL, IN YEARS, AND ANNUAL NON- } \\
\text { EXCEEDANCE PROBABILITY, IN PERCENT }\end{array}$} \\
\hline & $\begin{array}{c}2 \\
508\end{array}$ & 5 & 10 & 20 & 50 & 100 \\
\hline $\begin{array}{r}1 \\
3 \\
7 \\
14 \\
30 \\
60 \\
90 \\
120 \\
183\end{array}$ & $\begin{array}{r}0.0 \\
0.0 \\
0.0 \\
0.0 \\
0.0 \\
.1 \\
.2 \\
.5 \\
1.4\end{array}$ & $\begin{array}{r}0.0 \\
0.0 \\
0.0 \\
0.0 \\
0.0 \\
0.0 \\
0.0 \\
.1 \\
.5\end{array}$ & $\begin{array}{r}0.0 \\
0.0 \\
0.0 \\
0.0 \\
0.0 \\
0.0 \\
0.0 \\
.1 \\
.3\end{array}$ & $\begin{array}{r}0.0 \\
0.0 \\
0.0 \\
0.0 \\
0.0 \\
0.0 \\
0.0 \\
0.0 \\
.2\end{array}$ & $\begin{array}{r}0.0 \\
0.0 \\
0.0 \\
0.0 \\
0.0 \\
0.0 \\
0.0 \\
0.0 \\
.1\end{array}$ & - \\
\hline
\end{tabular}

MAGNITUDE AND PROBABILITY OF ANNUAL HIGH FLOW BASED ON PERTOD OF RECORD 1943-70

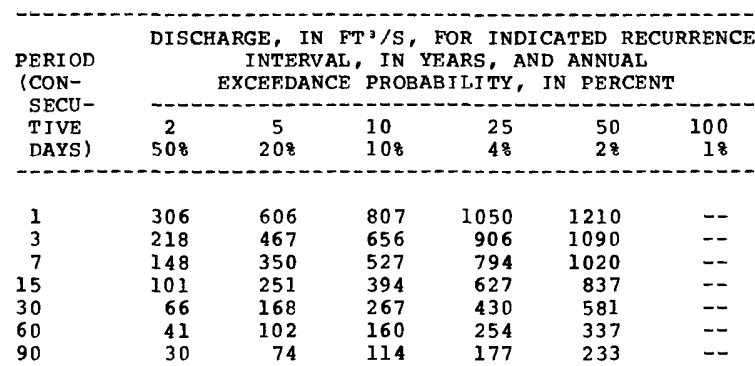

DURATION TABLF OF DAILY MEAN FLOW FOR PERIOD OF RECORD 1943-70

DISCHARGE, IN FT $3 / \mathrm{S}$, THAT WAS EOUALED OR EXCEEDED FOR INDICATEC PERCENT OF TIME

\begin{tabular}{|c|c|c|c|c|c|c|c|c|c|c|c|c|c|}
\hline 58 & 108 & 158 & 208 & 258 & 308 & 408 & 508 & 608 & 708 & 758 & 808 & 858 & $90 \%$ \\
\hline 64 & 24 & 13 & 8.7 & 6.3 & 4.9 & 3.4 & 1.9 & 1.1 & .4 & .1 & .1 & 0.0 & 0.0 \\
\hline
\end{tabular}


RIO GRANDE BASIN

08284520 WILLOW CREEK BELOW HERON DAM, NM

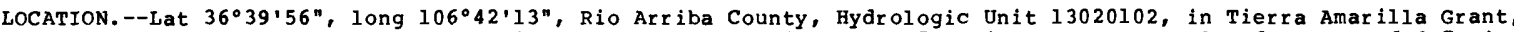
in outlet conduits of Heron Dam, $0.2 \mathrm{mi}$ upstream from Rio Chama, $5.1 \mathrm{mi}$ northeast of El Vado Dam, and $8.7 \mathrm{mi}$

southwest of Los Ojos.

DRAINAGE AREA, $--193 \mathrm{mi}^{2}$.

PERIOD OF RECORD.--January 1971 to current year.

GAGE.--Totalizing flowmeters in each of two outlet conduits in Heron Dam.

REMARKS.--Flow regulated by Heron Reservoir (station 08284510) since Oct. 21, 1970. Outlet conduits are 14 in. and 120 in. in diameter.

COOPERATION.--Records provided by U.S. Bureau of Reclamation.

AVERAGE DISCHARGE.--14 years (water years 1972-85), $119 \mathrm{ft}^{3} / \mathrm{s}, 86,220$ acre-ft/yr.

EXTREMES FOR PERIOD OF RECORD.--Maximum daily discharge, $2,780 \mathrm{ft} 3 / \mathrm{s}$, Dec. 18, 19, 1982; no flow many days each year.

STATISTICAL SUMMARIES

MEAN MONTHLY AND MEAN ANNUAL DISCHARGES 1972-85

\begin{tabular}{|c|c|c|c|c|c|c|}
\hline MONTH & $\begin{array}{l}\text { MINIMUM } \\
\left(\mathrm{FT}^{3} / \mathrm{S}\right)\end{array}$ & $\begin{array}{c}\text { MAXIMUM } \\
\left(\mathrm{FT}^{3} / \mathrm{S}\right)\end{array}$ & $\begin{array}{l}\text { MEAN } \\
\left(\mathrm{FT}^{3} / \mathrm{S}\right)\end{array}$ & $\begin{array}{l}\text { STAN- } \\
\text { DARD } \\
\text { DEVIA- } \\
\text { TION } \\
\left(\mathrm{FT}^{3} / \mathrm{S}\right)\end{array}$ & $\begin{array}{l}\text { COEFFI- } \\
\text { CIENT OF } \\
\text { VARI- } \\
\text { ATION }\end{array}$ & $\begin{array}{c}\text { PERCENT } \\
\text { OF } \\
\text { ANNUAL } \\
\text { RUNOFF }\end{array}$ \\
\hline оСТОВЕR & .3 & 94 & 18 & 23 & 1.30 & 1.3 \\
\hline NOVEMBER & 0.0 & 169 & 28 & 49 & 1.73 & 2.0 \\
\hline DECEMBER & 2.0 & 1290 & 548 & 353 & .64 & 38.8 \\
\hline JANUARY & 0. & 26 & 63 & 108 & 1.73 & 4.4 \\
\hline FEB RUARY & 0.0 & 354 & 54 & 115 & 2.11 & 3.8 \\
\hline MARCH & 13 & 591 & 146 & 169 & 1.16 & 10.3 \\
\hline APRIL & 2.2 & 834 & 231 & 231 & 1.00 & 16.3 \\
\hline MAY & 0.0 & 74 & 34 & 24 & .70 & 2.4 \\
\hline JUNE & 0.0 & 288 & 86 & 94 & 1.1 & 6.1 \\
\hline JULY & 5.6 & 422 & 108 & 127 & 1.1 & 7.6 \\
\hline AUGUST & 6.2 & 206 & 57 & 58 & 1.03 & 4.0 \\
\hline SEPTEMBER & 0.0 & 259 & 42 & 73 & 1.75 & 2.9 \\
\hline ANNUAL & 45 & 203 & 119 & 50 & .42 & 100 \\
\hline
\end{tabular}

MAGNITUDE AND PROBABILITY OF ANNUAL LOW FLOW BASED ON PERIOD OF RECORD $1973-85$

\begin{tabular}{|c|c|c|c|c|c|c|}
\hline \multirow{2}{*}{$\begin{array}{l}\text { PERIOD } \\
\text { (CON- } \\
\text { SECU- } \\
\text { TIVE } \\
\text { DAYS) }\end{array}$} & \multicolumn{6}{|c|}{$\begin{array}{l}\text { DISCHARGE, IN FT' }{ }^{3} / \mathrm{S} \text {, FOR INDICATED RECURRENCE } \\
\text { INTERVAL, IN YEARS, AND ANNUAL NON- } \\
\text { EXCEEDANCE PROBABILITY, IN PERCENT }\end{array}$} \\
\hline & $\begin{array}{c}2 \\
508\end{array}$ & $\begin{array}{c}5 \\
208\end{array}$ & $\begin{array}{l}10 \\
108\end{array}$ & $\begin{array}{l}20 \\
58\end{array}$ & $\begin{array}{l}50 \\
28\end{array}$ & $\begin{array}{r}100 \\
18\end{array}$ \\
\hline 1 & -- & -- & -- & - & -- & - \\
\hline 3 & - & - & - & -- & -- & - \\
\hline 7 & 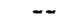 & -- & -- & $=$ & $\ldots$ & -- \\
\hline 14 & - & - & - & -- & - & -- \\
\hline 30 & $\sim$ & - & -- & -- & $\sim$ & $=$ \\
\hline 60 & 1.4 & 0.0 & 0.0 & 0.0 & -- & \\
\hline 90 & 7.2 & 3.1 & 1.9 & 0.0 & -- & \\
\hline 120 & 17 & 8.3 & 5.9 & 4.5 & -- & -- \\
\hline 183 & 38 & 17 & 10 & 7.0 & -- & -- \\
\hline
\end{tabular}

MAGNITUDE AND PROBABILITY OF ANNUAL BIGH FLOW BASED ON PERIOD OF RECORD $1972-85$

\begin{tabular}{|c|c|c|c|c|c|c|}
\hline \multirow{2}{*}{$\begin{array}{l}\text { PERIOD } \\
\text { (CON- } \\
\text { SECU- } \\
\text { TIVE } \\
\text { DAYS) }\end{array}$} & \multicolumn{6}{|c|}{$\begin{array}{l}\text { DISCHARGE, IN FT } \\
\text { INTERVAL, IN YER INDICATED RECURRENCE } \\
\text { EXCEEDANCE PROBABILITY, IN PERCENT }\end{array}$} \\
\hline & 22 & $\begin{array}{c}5 \\
208\end{array}$ & $\begin{array}{l}10 \\
108\end{array}$ & $\begin{array}{l}25 \\
48\end{array}$ & $\begin{array}{l}50 \\
28\end{array}$ & $\begin{array}{r}100 \\
18\end{array}$ \\
\hline $\begin{array}{r}1 \\
3 \\
7 \\
15 \\
30 \\
60 \\
90\end{array}$ & $\begin{array}{r}1510 \\
1470 \\
1370 \\
1000 \\
598 \\
334 \\
240\end{array}$ & $\begin{array}{r}2140 \\
2100 \\
1940 \\
1430 \\
925 \\
503 \\
359\end{array}$ & $\begin{array}{r}2450 \\
2390 \\
2190 \\
1630 \\
1140 \\
618 \\
444\end{array}$ & $\begin{array}{r}2720 \\
2650 \\
2400 \\
1810 \\
1410 \\
763 \\
556\end{array}$ & $\begin{array}{l}-- \\
-- \\
=- \\
-- \\
--\end{array}$ & $\begin{array}{l}-- \\
-- \\
-- \\
-- \\
--\end{array}$ \\
\hline
\end{tabular}

DURATION TABLE OF DAILY MEAN FLOW FOR PERIOD OF RECORD 1972-85 DISCHARGE, IN FT ${ }^{3 / S}$, THAT WAS EQUALED OR EXCEEDED FOR INDICATED PERCENT OF TIME

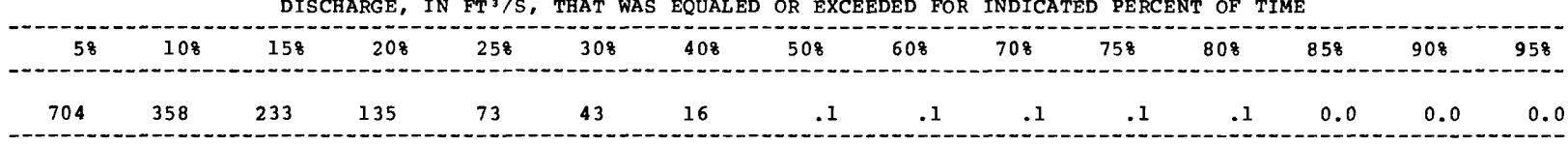


RIO GRANDE BASIN

08285500 RIO CHAMA BELOW EL VADO DAM, NM

LOCATION.--Lat 36 34'48", long $106^{\circ} 43^{\prime} 24^{\prime \prime}$, Rio Arriba County, Hydrologic Unit 13020102, in Tierra Amarilla Grant, on left bank $1.5 \mathrm{mi}$ downstream from E1 Vado Dam, $2.8 \mathrm{mi}$ upstream from Rio Nutrias, $13 \mathrm{mi}$ southwest of Tierra Amarilla, and at mile 76.2.

DRAINAGE AREA, - -877 $\mathrm{mi}^{2}$, of which about $100 \mathrm{mi}^{2}$ is probably noncontributing.

PERIOD OF RECORD,--October 1913 to November 1915, April to November 1916, March, April 1920, September 1920 to August 1924, October 1935 to current year. Monthly discharge only for some periods, published in wsp l312. August 1924, "Chober 1935 to current year. Monthly discharge only for some periods, published in wsp 1312. and as "at E1 Vado" 1920-24.

REVISED RECORDS.--WSP 1312: 1914, 1949. WSP 1392: 1949.

GAGE.--Water-stage recorder. Datum of gage is 6,696.12 ft above National Geodetic Vertical Datum of 1929 . Prior to October 1935, at site $1.5 \mathrm{mi}$ upstream at different datum. October 1935 to September 1938 at site $1.1 \mathrm{mi}$ upstream at datum $30.34 \mathrm{ft}$ higher.

REMARKS.-Flow regulated by El Vado Reservoir (station 08285000) since 1935. Flow affected by release of transmountain water from Heron Reservoir (station 08284510) since May 1971. Diversions for irrigation of about 10,600 acres upstream from station.

AVERAGE DISCHARGE.--34 years (water years 1937-70), $367 \mathrm{ft} / \mathrm{s}, 265,900$ acre-ft/yr.

35 years (water years 1936-70), $373 \mathrm{ft} / \mathrm{s}, 270,200$ acre-ft/yr, prior to release of transmountain water.

35 years (water years $1936-70), 373 \mathrm{ft} / \mathrm{s}, 270,200$ acre-ft/yr,
15 years (water years $1971-85), 461 \mathrm{ft} / \mathrm{s}, 334,000$ acre-ft/yr.

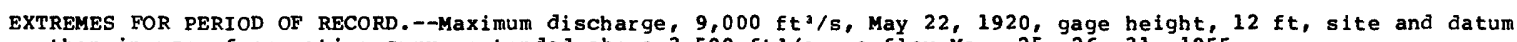
then in use, from rating curve extended above $3,500 \mathrm{ft} / \mathrm{s}$; no flow Mar. 25, 26, 31, 1955 .

Maximum discharge since construction of El vado Dam in $1935,6,010 \mathrm{ft} / \mathrm{s}$, May 17, 1941, gage height, 6.89 ft.

EXTREMES OUTSIDE PERIOD OF RECORD.--Flood of Oct. 4 or 5, 1911, was greater than floods in September 1904 and May 1920, from information hy local residents.

STATISTICAL SUMMARIES(PERIOD AFTER COMPLETION OF EL VADO DAM AND BEFORE REGULATION OF HERON DAM)

MEAN MONTHLY AND MEAN ANNUAL DISCHARGES 1937-70

MAGNITUDE AND PROBABILITY OF ANNUAL LOW FLOW BASED ON PERIOD OF RECORD $1937-70$

\begin{tabular}{|c|c|c|c|c|c|c|}
\hline MONTH & $\begin{array}{l}\text { MINIMUM } \\
\left(\mathrm{FT}^{3} / \mathrm{S}\right)\end{array}$ & $\begin{array}{l}\text { MAXIMUM } \\
\left(\mathrm{FT}^{3} / \mathrm{S}\right)\end{array}$ & $\begin{array}{l}\text { MEAN } \\
\left(\mathrm{FT}^{3} / \mathrm{S}\right)\end{array}$ & $\begin{array}{c}\text { STAN- } \\
\text { DARD } \\
\text { DEVIA- } \\
\text { TION } \\
(\text { FT'/S) }\end{array}$ & $\begin{array}{l}\text { COEFFI- } \\
\text { CIENT OF } \\
\text { VARI- } \\
\text { ATION }\end{array}$ & $\begin{array}{c}\text { PERCENT } \\
\text { OF } \\
\text { ANNUAL } \\
\text { RUNOF F }\end{array}$ \\
\hline остОВЕR & 8.0 & 610 & 131 & 127 & .97 & 2.9 \\
\hline NOVEMBER & 2.6 & 1270 & 265 & 350 & 1.32 & 6.0 \\
\hline DECEMBER & .9 & 926 & 124 & 233 & 1.88 & 2.8 \\
\hline JANUARY & .5 & 172 & 35 & 40 & 1.14 & .8 \\
\hline FEBRUARY & 2.0 & 1414 & 134 & 267 & 1.99 & 3.0 \\
\hline MARCH & 1.8 & 1280 & 248 & 323 & 1.30 & 5.6 \\
\hline APRIL & 4.0 & 1614 & 459 & 446 & .97 & 10.3 \\
\hline MAY & 16 & 3916 & 1045 & 850 & .81 & 23.5 \\
\hline JUNE & 143 & 2151 & 767 & 459 & .60 & 17.2 \\
\hline JULY & 61 & 1688 & 486 & 419 & .86 & 10.9 \\
\hline AUGUST & 18 & 1316 & 478 & 367 & .77 & 10.7 \\
\hline SEPTEMBER & 12 & 751 & 280 & 231 & .83 & 6.3 \\
\hline NNUAL & 148 & 823 & 367 & 170 & .46 & 100 \\
\hline
\end{tabular}

\begin{tabular}{|c|c|c|c|c|c|c|}
\hline \multirow{2}{*}{$\begin{array}{l}\text { PERIOD } \\
\text { (CON- } \\
\text { SECU- } \\
\text { TIVE } \\
\text { DAYS) }\end{array}$} & \multicolumn{6}{|c|}{$\begin{array}{l}\text { DISCHARGE, IN FT'3/S, FOR INDICATED RECURRENCE } \\
\text { INTERVAL, IN YEARS, AND ANNUAL NON- } \\
\text { EXCEEDANCE PROBABILITY, IN PERCENT }\end{array}$} \\
\hline & $\begin{array}{c}2 \\
508\end{array}$ & $\begin{array}{c}5 \\
208\end{array}$ & $\begin{array}{l}10 \\
108\end{array}$ & $\begin{array}{l}20 \\
58\end{array}$ & $\begin{array}{l}50 \\
28\end{array}$ & $\begin{array}{r}100 \\
18\end{array}$ \\
\hline $\begin{array}{r}1 \\
3 \\
7 \\
14 \\
30 \\
60 \\
90 \\
120 \\
183\end{array}$ & $\begin{array}{r}2.7 \\
3.4 \\
4.4 \\
6.4 \\
9.7 \\
13 \\
18 \\
37 \\
88\end{array}$ & $\begin{array}{r}.7 \\
.99 \\
1.3 \\
1.8 \\
2.8 \\
3.6 \\
5.8 \\
12 \\
46\end{array}$ & $\begin{array}{r}.3 \\
.4 \\
.7 \\
.9 \\
1.4 \\
1.7 \\
3.1 \\
6.4 \\
33\end{array}$ & $\begin{array}{r}.1 \\
.2 \\
.4 \\
.5 \\
.8 \\
.9 \\
1.9 \\
3.6 \\
25\end{array}$ & $\begin{array}{r}0.0 \\
.1 \\
.2 \\
.3 \\
.4 \\
.4 \\
1.0 \\
1.8 \\
19\end{array}$ & $\begin{array}{l}-- \\
-- \\
-- \\
-- \\
-- \\
-\end{array}$ \\
\hline
\end{tabular}

MAGNITUDE AND PROBABILITY OF ANNUAL HIGH FLOW BASED ON PERIOD OF RECORD $1937-70$

\begin{tabular}{|c|c|c|c|c|c|c|}
\hline \multirow{2}{*}{$\begin{array}{l}\text { PERIOD } \\
\text { (CON- } \\
\text { SECU- } \\
\text { TIVE } \\
\text { DAYS) }\end{array}$} & \multicolumn{6}{|c|}{$\begin{array}{l}\text { DISCHARGE, IN FT } 3 \text { /S, FOR INDICATED RECURRENC } \\
\text { INTERVAL, IN YEARS, AND ANNUAL } \\
\text { EXCEEDANCE PROBABILITY, IN PERCENT }\end{array}$} \\
\hline & $\begin{array}{c}2 \\
508\end{array}$ & $\begin{array}{c}5 \\
208\end{array}$ & $\begin{array}{l}10 \\
108\end{array}$ & $\begin{array}{l}25 \\
48\end{array}$ & $\begin{array}{l}50 \\
28\end{array}$ & $\begin{array}{r}100 \\
18\end{array}$ \\
\hline $\begin{array}{r}1 \\
3 \\
7 \\
15 \\
30 \\
60 \\
90\end{array}$ & $\begin{array}{r}1720 \\
1670 \\
1580 \\
1430 \\
1270 \\
997 \\
816\end{array}$ & $\begin{array}{l}2690 \\
2570 \\
2400 \\
2150 \\
1870 \\
1460 \\
1210\end{array}$ & $\begin{array}{l}3520 \\
3330 \\
3110 \\
2760 \\
2370 \\
1810 \\
1500\end{array}$ & $\begin{array}{l}4830 \\
4540 \\
4200 \\
3710 \\
3110 \\
2310 \\
1910\end{array}$ & $\begin{array}{l}6010 \\
5630 \\
5190 \\
4580 \\
3770 \\
2730 \\
2250\end{array}$ & $\begin{array}{l}-- \\
-- \\
-- \\
-- \\
-- \\
--\end{array}$ \\
\hline
\end{tabular}

DURATION TABLE OF DAILY MEAN FLOW FOR PERIOD OF RECORD 1937-70

DISCHARGE, IN FT $3 / \mathrm{S}$, THAT WAS EQUALED OR EXCEEDED FOR INDICATED PERCENT OF TIME

\begin{tabular}{|c|c|c|c|c|c|c|c|c|c|c|c|c|c|c|}
\hline 58 & 108 & 158 & 208 & 258 & 308 & 408 & 508 & 608 & 708 & 758 & 808 & 858 & 908 & 958 \\
\hline 1470 & 1130 & 938 & 726 & 548 & 414 & 181 & 181 & 54 & 32 & 21 & 14 & 9.6 & 4.9 & \\
\hline
\end{tabular}


RIO GRANDE BASIN

08285500 RIO CHAMA BELOW EI VADO DAM,-NM--Continued

STATISTICAL SUMMARIES (PERIOD AFTER COMPLETION OF HERON DAM)

MEAN MONTHLY AND MEAN ANNUAL DISCHARGES 1971-85

\begin{tabular}{|c|c|c|c|c|c|c|}
\hline MONTH & $\begin{array}{l}\text { MINIMUM } \\
\left(\mathrm{FT}^{3} / \mathrm{S}\right)\end{array}$ & $\begin{array}{c}\text { MAXI MUM } \\
\left(\mathrm{FT}^{3} / \mathrm{S}\right)\end{array}$ & $\begin{array}{l}\text { MEAN } \\
\left(\mathrm{FT}^{3} / \mathrm{S}\right)\end{array}$ & $\begin{array}{l}\text { STAN- } \\
\text { DARD } \\
\text { DEVIA- } \\
\text { TION } \\
\left(\mathrm{FT}{ }^{3} / \mathrm{S}\right)\end{array}$ & $\begin{array}{l}\text { COEFFI- } \\
\text { CIENT OF } \\
\text { VARI- } \\
\text { ATION }\end{array}$ & $\begin{array}{c}\text { PERCENT } \\
\text { OF } \\
\text { ANNUAL } \\
\text { RUNOFF }\end{array}$ \\
\hline $\begin{array}{l}\text { OCTOBER } \\
\text { NOVEMBER } \\
\text { DECEMBER } \\
\text { JANUARY } \\
\text { FEBRUARY } \\
\text { MARCH } \\
\text { APRII } \\
\text { MAY } \\
\text { JUNE } \\
\text { JULY } \\
\text { AUGUST } \\
\text { SEPTEMBER }\end{array}$ & $\begin{array}{r}37 \\
44 \\
63 \\
24 \\
17 \\
28 \\
33 \\
262 \\
186 \\
126 \\
54 \\
51\end{array}$ & $\begin{array}{r}453 \\
445 \\
1272 \\
387 \\
433 \\
962 \\
1876 \\
3412 \\
2184 \\
568 \\
574 \\
692\end{array}$ & $\begin{array}{r}169 \\
167 \\
370 \\
121 \\
117 \\
253 \\
704 \\
1741 \\
955 \\
342 \\
288 \\
280\end{array}$ & $\begin{array}{r}97 \\
142 \\
390 \\
124 \\
130 \\
247 \\
528 \\
1070 \\
549 \\
136 \\
160 \\
193\end{array}$ & $\begin{array}{r}.57 \\
.85 \\
1.05 \\
1.02 \\
1.11 \\
.98 \\
.75 \\
.61 \\
.57 \\
.40 \\
.56 \\
.69\end{array}$ & $\begin{array}{r}3.1 \\
3.0 \\
6.7 \\
2.2 \\
2.1 \\
4.6 \\
12.8 \\
31.6 \\
17.3 \\
6.2 \\
5.2 \\
5.1\end{array}$ \\
\hline NNUAL & 194 & 754 & 461 & 189 & .41 & 100 \\
\hline
\end{tabular}

MAGNITUDE AND PROBABILITY OF ANNUAL LOW FLOW BASED ON PERIOD OF RECORD 1972-85

\begin{tabular}{|c|c|c|c|c|c|c|}
\hline $\begin{array}{l}\text { PERIOD } \\
\text { (CON- } \\
\text { SECU- }\end{array}$ & \multicolumn{6}{|c|}{$\begin{array}{l}\text { DISCHARGE, IN } \text { FT }^{3} / S \text {, FOR INDICATED RECURRENCE } \\
\text { INTERVAL, IN YEARS, AND ANNUAL NON- } \\
\text { EXCEEDANCE PROBABILITY, IN PERCENT }\end{array}$} \\
\hline $\begin{array}{l}\text { TIVE } \\
\text { DAYS) }\end{array}$ & $\begin{array}{c}2 \\
508\end{array}$ & $\begin{array}{c}5 \\
208\end{array}$ & $\begin{array}{l}10 \\
108\end{array}$ & $\begin{array}{l}20 \\
58\end{array}$ & $\begin{array}{l}50 \\
28\end{array}$ & $\begin{array}{r}100 \\
18\end{array}$ \\
\hline $\begin{array}{r}1 \\
3 \\
7 \\
14 \\
30 \\
60 \\
90 \\
120 \\
183\end{array}$ & $\begin{array}{r}19 \\
20 \\
26 \\
34 \\
40 \\
54 \\
84 \\
119 \\
147\end{array}$ & $\begin{array}{l}14 \\
16 \\
18 \\
21 \\
24 \\
30 \\
44 \\
72 \\
92\end{array}$ & $\begin{array}{l}13 \\
14 \\
15 \\
17 \\
19 \\
23 \\
31 \\
56 \\
73\end{array}$ & $\begin{array}{l}12 \\
13 \\
13 \\
15 \\
17 \\
18 \\
23 \\
46 \\
60\end{array}$ & $\begin{array}{l}-- \\
-- \\
-- \\
-- \\
-- \\
-- \\
-- \\
--\end{array}$ & $\begin{array}{l}-- \\
-- \\
-- \\
-- \\
-- \\
-- \\
--\end{array}$ \\
\hline
\end{tabular}

MAGNITUDE AND PROBABILITY OF ANNUAL HIGH FLOW BASED ON PERIOD OF RECORD 1971-85

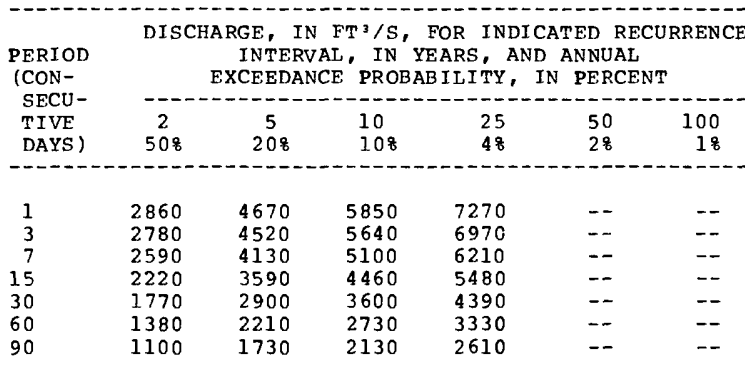

DURATION TABLE OF DAILY MEAN FLOW FOR PERIOD OF RECORD 1971-85 DISCHARGE, IN FT ${ }^{3} / \mathrm{S}$, THAT WAS EQUALED OR EXCEEDED FOR INDICATED PERCENT OF TIME

\begin{tabular}{|c|c|c|c|c|c|c|c|c|c|c|c|c|c|c|}
\hline 58 & 108 & 158 & 208 & 258 & 308 & 408 & 508 & 608 & 708 & 758 & 808 & 858 & 908 & 958 \\
\hline 2020 & 1210 & 873 & 695 & 562 & 444 & 287 & 163 & 103 & 74 & 62 & 49 & 39 & 30 & 25 \\
\hline
\end{tabular}


08286500 RIO CHAMA ABOVE ABIQUIU RESERVOIR, NM

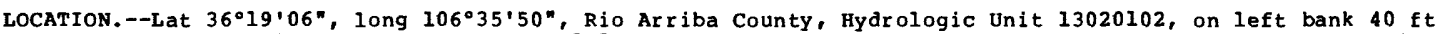
downstream from site of former bridge, $7.7 \mathrm{mi}$ downstream from Rio Gallina, 9 mi northwest of Youngsville, 15.6 mi upstream from Abiquiu Dam, $30.3 \mathrm{mi}$ downstream from El Vado Dam, and at mile 47.4 .

DRATNAGE AREA.- - 1,600 $\mathrm{mi} 2$, of which about $100 \mathrm{mi} 2$ is probably noncontributing.

PERIOD OF RECORD.--August 1961 to current year.

GAGE.--Water-stage recorder. Elevation of gage is 6,275 ft above National Geodetic Vertical Datum of 1929 , from topographic map.

REMARKS.--Flow regulated by El Vado Reservoir (station 08285000). Since May 1971 flow affected by release of transmountain water from Heron Reservoir (station 08284510). Diversions for irrigation of about 15,000 acres upstream from station.

AVERAGE DISCHARGE. --15 years (water years 1971-85), $493 \mathrm{ft}^{3} / \mathrm{s}, 357,200$ acre-ft/yr. water.

EXTREMES FOR PERIOD OF RECORD.--Maximum discharge, $6,680 \mathrm{ft}^{3} / \mathrm{s}$, May 8,1985 , gage height, $7.67 \mathrm{ft}$; maximum gage height, 8.70 ft, May 20, 1973; minimum, $7.5 \mathrm{ft}^{3} / \mathrm{s}$, Oct. 17, 18, 1963.

EXTREMES OUTSIDE PERIOD OF RECORD.--Major floods probably occurred on Sept. 29, 1904, Oct. 4 or 5 , 1911 , and May 22,1920 .

STATISTICAL SUMMARIES (PERIOD AFTER COMPLETION OF HERON DAM)

MEAN MONTHLY AND MEAN ANNUAL DISCHARGES 1971-85

\begin{tabular}{|c|c|c|c|c|c|c|}
\hline MONTH & $\begin{array}{l}\text { MINIMUM } \\
\left(\text { FT }^{3} / \mathrm{S}\right)\end{array}$ & $\begin{array}{l}\text { MAXIMUM } \\
\left(\mathrm{FT}^{3} / \mathrm{S}\right)\end{array}$ & $\begin{array}{l}\text { MEAN } \\
\left(\mathrm{FT}^{3} / \mathrm{S}\right)\end{array}$ & $\begin{array}{c}\text { STAN- } \\
\text { DARD } \\
\text { DEVIA- } \\
\text { TION } \\
\left(\mathrm{FT}^{3} / \mathrm{S}\right)\end{array}$ & $\begin{array}{l}\text { COEFFI- } \\
\text { CIENT OF } \\
\text { VARI - } \\
\text { ATION }\end{array}$ & $\begin{array}{c}\text { PERCENT } \\
\text { OF } \\
\text { ANNUAL } \\
\text { RUNOF F }\end{array}$ \\
\hline $\begin{array}{l}\text { OCTOBER } \\
\text { NOVBMBER } \\
\text { DECEMBER } \\
\text { JANUARY } \\
\text { FEBRUARY } \\
\text { MARCH } \\
\text { APRIL } \\
\text { MAY } \\
\text { JUNE } \\
\text { JULY } \\
\text { AUGUST } \\
\text { SEPTEMBER }\end{array}$ & $\begin{array}{r}40 \\
48 \\
74 \\
29 \\
30 \\
44 \\
106 \\
259 \\
185 \\
132 \\
86 \\
78\end{array}$ & $\begin{array}{r}487 \\
455 \\
1273 \\
404 \\
475 \\
1050 \\
1985 \\
3741 \\
2409 \\
579 \\
587 \\
724\end{array}$ & $\begin{array}{l}180 \\
170 \\
381 \\
134 \\
135 \\
303 \\
760 \\
1863 \\
1027 \\
354 \\
301 \\
288\end{array}$ & $\begin{array}{r}103 \\
137 \\
390 \\
122 \\
138 \\
260 \\
544 \\
1198 \\
634 \\
140 \\
155 \\
193\end{array}$ & $\begin{array}{r}.57 \\
.81 \\
1.02 \\
.91 \\
1.02 \\
.86 \\
.72 \\
.64 \\
.62 \\
.40 \\
.51 \\
.67\end{array}$ & $\begin{array}{r}3.1 \\
2.9 \\
6.5 \\
2.3 \\
2.3 \\
5.1 \\
12.9 \\
31.6 \\
17.4 \\
6.0 \\
5.1 \\
4.9\end{array}$ \\
\hline ANNUAL & 204 & 823 & 493 & 209 & .42 & 100 \\
\hline
\end{tabular}

MAGNITUDE AND PROBABILITY OF ANNUAL LOW FLOW BASED ON PERIOD OF RECORD 1972-85

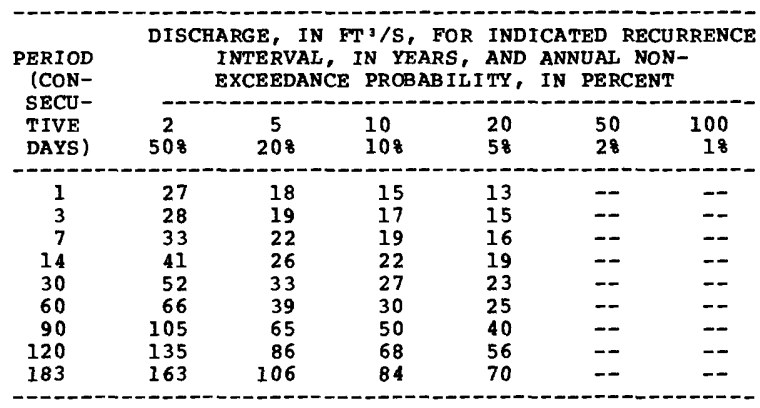

MAGNITUDE AND PROBABILITY OF ANNUAL HIGH FLOW BASED ON PERIOD OF RECORD $1971-85$

\begin{tabular}{|c|c|c|c|c|c|c|}
\hline \multirow{2}{*}{$\begin{array}{l}\text { PERIOD } \\
\text { (CON- } \\
\text { SECU- } \\
\text { TIVE } \\
\text { DAYS) }\end{array}$} & \multicolumn{6}{|c|}{$\begin{array}{l}\text { DISCHARGE, IN FT' } / S \text {, FOR INDICATED RECURRENCE } \\
\text { INTERVAL, IN YEARS, AND ANNUAL } \\
\text { EXCEEDANCE PROBABILITY, IN PERCENT }\end{array}$} \\
\hline & $\begin{array}{c}2 \\
508\end{array}$ & $\begin{array}{c}5 \\
208\end{array}$ & $\begin{array}{l}10 \\
108\end{array}$ & $\begin{array}{l}25 \\
48\end{array}$ & $\begin{array}{l}50 \\
28\end{array}$ & $\begin{array}{r}100 \\
18\end{array}$ \\
\hline $\begin{array}{r}1 \\
3 \\
7 \\
15 \\
30 \\
60 \\
90\end{array}$ & $\begin{array}{l}2930 \\
2850 \\
2700 \\
2330 \\
1850 \\
1440 \\
1160\end{array}$ & $\begin{array}{l}5000 \\
4870 \\
4540 \\
3980 \\
3170 \\
2390 \\
1870\end{array}$ & $\begin{array}{l}6460 \\
6270 \\
5780 \\
5110 \\
4030 \\
3010 \\
2340\end{array}$ & $\begin{array}{l}8340 \\
8040 \\
7300 \\
6520 \\
5060 \\
3760 \\
2920\end{array}$ & $\begin{array}{l}-- \\
-- \\
-- \\
-- \\
-- \\
--\end{array}$ & $\begin{array}{l}-- \\
-- \\
-- \\
-- \\
--\end{array}$ \\
\hline
\end{tabular}

DURATION TABLE OF DAILY MEAN FLOW FOR PERIOD OF RECORD 1971-85

DISCHARGE, IN FT'S/S, THAT WAS EQUALED OR EXCEEDED FOR INDICATED PERCENT OF TIME

\begin{tabular}{|c|c|c|c|c|c|c|c|c|c|c|c|c|c|c|}
\hline 58 & 108 & 158 & 208 & 258 & 308 & 408 & $50 \%$ & 608 & 708 & 758 & 808 & 858 & 908 & 958 \\
\hline 2140 & 1250 & 909 & 726 & 585 & 455 & 306 & 183 & 118 & 87 & 76 & 64 & 53 & 42 & 32 \\
\hline
\end{tabular}




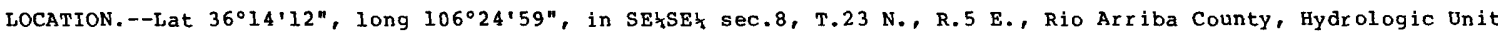
13020102 , on right bank $0.8 \mathrm{mi}$ downstream from Abiquiu Dam, $5.9 \mathrm{mi}$ northwest of Abiquiu, and at mile 31.3 . DRAINAGE AREA. $--2,147 \mathrm{mi}^{2}$, of which about $100 \mathrm{mi}^{2}$ is probably noncontributing.

PERIOD OF RECORD.--October 1961 to current year (monthly discharge only, October 1961).

GAGE,--Water-stage recorder. Concrete control since Jan. 25, 1966. Elevation of gage is $6,040 \mathrm{ft}$ above National Geodetic Vertical Datum of 1929, from topographic map. Prior to Jan. 25, 1966, at datum $1.60 \mathrm{ft}$ lower.

REMARRS.--Flow controlled by El Vado Reservoir (station 08285000) $46.4 \mathrm{mi}$ upstream and Abiquiu Reservoir (station $08286900) 0.8 \mathrm{mi}$ upstream. Since May 1971 flow affected by release of transmountain water from Heron

Reservoir (station 08284510) $54.5 \mathrm{mi}$ upstream. Diversions for irrigation of about 17,600 acres upstream

from station. U.S. Army Corps of Engineers gage-height telemeter at station.

AVERAGE DISCHARGE.--15 years (water years 1971-85), $497 \mathrm{ft}^{3} / \mathrm{s}, 360,100$ acre-ft/yr, prior to release of transmountain water.

EXTREMES FOR PERIOD OF RECORD.--Maximum discharge, 2,990 $\mathrm{ft}^{3} / \mathrm{s}$, July 1,1965 , gage height, $6.69 \mathrm{ft}$, datum then in use; maximum gage height, $7.29 \mathrm{ft}, \mathrm{Jan} .14,1967$ (backwater from ice); minimum discharge, about $0.5 \mathrm{ft}{ }^{3} / \mathrm{s}$,

Mar. 17, 1966, Jan. 28, 1972 .

STATISTICAL SUMMARIES (PERIOD AFTER COMPLETION OF HERON DAM)

MEAN MONTHLY AND MEAN ANNUAL DISCHARGES 1971-85

\begin{tabular}{|c|c|c|c|c|c|c|}
\hline MONTH & $\begin{array}{c}\text { MINIMUM } \\
\left(\mathrm{FT}^{3} / \mathrm{S}\right)\end{array}$ & $\begin{array}{c}\text { MAXIMUM } \\
\left(\mathrm{FT}^{3} / \mathrm{S}\right)\end{array}$ & $\begin{array}{l}\text { MEAN } \\
\left(\mathrm{FT}^{3} / \mathrm{S}\right)\end{array}$ & $\begin{array}{l}\text { STAN- } \\
\text { DARD } \\
\text { DEVIA- } \\
\text { TION } \\
\left(\mathrm{FT}^{3} / \mathrm{S}\right)\end{array}$ & $\begin{array}{l}\text { COEFFI- } \\
\text { CIENT OF } \\
\text { VARI- } \\
\text { ATION }\end{array}$ & $\begin{array}{c}\text { PERCENT } \\
\text { OF } \\
\text { ANNUAL } \\
\text { RUNOFF }\end{array}$ \\
\hline $\begin{array}{l}\text { OCTOBER } \\
\text { NOVEMBER } \\
\text { DECEMBER } \\
\text { JANUARY } \\
\text { FEBRUARY } \\
\text { MARCH } \\
\text { APRIL } \\
\text { MAY } \\
\text { JUNE } \\
\text { JULY } \\
\text { AUGUST } \\
\text { SEPTEMBER }\end{array}$ & $\begin{array}{r}45 \\
59 \\
44 \\
36 \\
38 \\
52 \\
111 \\
242 \\
184 \\
201 \\
98 \\
64\end{array}$ & $\begin{array}{r}555 \\
1181 \\
1308 \\
717 \\
264 \\
1046 \\
1894 \\
2055 \\
2418 \\
1488 \\
1084 \\
779\end{array}$ & $\begin{array}{r}208 \\
322 \\
387 \\
157 \\
114 \\
313 \\
753 \\
1148 \\
1144 \\
670 \\
407 \\
326\end{array}$ & $\begin{array}{r}121 \\
333 \\
416 \\
189 \\
70 \\
249 \\
502 \\
608 \\
735 \\
414 \\
246 \\
199\end{array}$ & $\begin{array}{l}.58 \\
1.03 \\
1.07 \\
1.20 \\
.62 \\
.80 \\
.67 \\
.53 \\
.64 \\
.62 \\
.60 \\
.61\end{array}$ & $\begin{array}{r}3.5 \\
5.4 \\
6.5 \\
2.6 \\
1.9 \\
5.3 \\
12.7 \\
19.3 \\
19.2 \\
11.3 \\
6.8 \\
5.5\end{array}$ \\
\hline NNUAL & 213 & 828 & 497 & 170 & .34 & 100 \\
\hline
\end{tabular}

MAGNITUDE AND PROBABILITY OF ANNUAL LOW FLOW BASED ON PERIOD OF RECORD 1972-85

\begin{tabular}{|c|c|c|c|c|c|c|}
\hline \multirow{4}{*}{$\begin{array}{l}\text { PERIOD } \\
\text { (CON- } \\
\text { SECU- } \\
\text { TIVE } \\
\text { DAYS) }\end{array}$} & \multicolumn{6}{|c|}{$\begin{array}{l}\text { DISCHARGE, IN } \mathrm{FT}^{3} / \mathrm{S} \text {, FOR INDICATED RECURRENCE } \\
\text { INTERVAL, IN YEARS, AND ANNUAL NON- } \\
\text { EXCEEDANCE PROBABILITY, IN PERCENT }\end{array}$} \\
\hline & & & & & & \\
\hline & 2 & 5 & 10 & 20 & 50 & 100 \\
\hline & 508 & 208 & 108 & 58 & 28 & 18 \\
\hline 1 & 18 & 13 & 11 & 10 & - & -- \\
\hline 3 & 21 & 16 & 14 & 13 & -- & -- \\
\hline 7 & 29 & 22 & 19 & 18 & - & -- \\
\hline 14 & 37 & 27 & 24 & 22 & -- & -- \\
\hline 30 & 53 & 39 & 34 & 30 & -- & -- \\
\hline 60 & 74 & 48 & 39 & 32 & -- & -- \\
\hline 90 & 112 & 68 & 51 & 41 & -- & -- \\
\hline 120 & 147 & 83 & 62 & 49 & -- & -- \\
\hline 183 & 183 & 106 & 80 & 64 & $\cdots$ & -- \\
\hline
\end{tabular}
BASED ON PERIOD OF RECORD 1971-85

\begin{tabular}{|c|c|c|c|c|c|c|}
\hline \multirow{2}{*}{$\begin{array}{l}\text { PERIOD } \\
\text { (CON- } \\
\text { SECU- } \\
\text { TIVE } \\
\text { DAYS) }\end{array}$} & \multicolumn{6}{|c|}{$\begin{array}{l}\text { DISCHARGE, IN FT } 3 / \text { S, FOR INDICATED RECURRENCE } \\
\text { INTERVAL, IN YEARS, AND ANNUAL } \\
\text { EXCEEDANCE PROBABILITY, IN PERCENT }\end{array}$} \\
\hline & $\begin{array}{c}2 \\
508\end{array}$ & $\begin{array}{c}5 \\
208\end{array}$ & $\begin{array}{l}10 \\
108\end{array}$ & $\begin{array}{l}25 \\
48\end{array}$ & $\begin{array}{l}50 \\
28\end{array}$ & $\begin{array}{r}100 \\
18\end{array}$ \\
\hline $\begin{array}{r}1 \\
3 \\
7 \\
15 \\
30 \\
60 \\
90\end{array}$ & $\begin{array}{l}1780 \\
1720 \\
1680 \\
1630 \\
1550 \\
1350 \\
1120\end{array}$ & $\begin{array}{l}2250 \\
2240 \\
2210 \\
2180 \\
2130 \\
1910 \\
1660\end{array}$ & $\begin{array}{l}2520 \\
2530 \\
2490 \\
2470 \\
2410 \\
2200 \\
1980\end{array}$ & $\begin{array}{l}2820 \\
2840 \\
2800 \\
2770 \\
2650 \\
2490 \\
2340\end{array}$ & $\begin{array}{l}-- \\
-- \\
-- \\
-- \\
--\end{array}$ & $\begin{array}{l}-- \\
-- \\
-- \\
-- \\
-- \\
--\end{array}$ \\
\hline
\end{tabular}

DURATION TABLE OF DAILY MEAN FLOW FOR PERIOD OF RECORD 1971-85

DISCHARGE, IN FT $3 / S$, THAT WAS EQUALED OR EXCEEDED FOR INDICATED PERCENT OF TIME

\begin{tabular}{|c|c|c|c|c|c|c|c|c|c|c|c|c|c|c|}
\hline 58 & 108 & 158 & 208 & 258 & 308 & 408 & 508 & 608 & 708 & 758 & 808 & 858 & 908 & 958 \\
\hline 1880 & 1490 & 1060 & 881 & 727 & 593 & 372 & 226 & 142 & 99 & 85 & 72 & 58 & 45 & 34 \\
\hline
\end{tabular}


RIO GRANDE BASIN

08287500 RIO CHAMA NEAR ABIQUIU, NM

LOCATION.--Lat $36^{\circ} 13^{\prime} 00^{\prime \prime}$, long $106^{\circ} 15^{\prime} 00^{\prime \prime}$, in Juan Jose Lobato Grant, at downstream end of bridge pier on State Highway $96,13 / 4 \mathrm{mi}$ upstream from El Rito Creek, $5 \mathrm{mi}$ downstream from Abiquiu, Rio Arriba County, $13.5 \mathrm{mi}$ downstream from Abiquiu Dam, and at mile 18.2 .

DRAINAGE AREA. - - 2,284 $\mathrm{mi}^{2}$, of which about $100 \mathrm{mi}^{2}$ is probably noncontributing.

PERIOD OF RECORD.--October 1941 to September 1967 (discontinued). Monthly discharge only for some periods, published in WSP 1312 .

GAGE.--Water-stage recorder. Datum of gage is $5,873.17 \mathrm{ft}$ above mean sea 1 evel, datum of 1929 .

REMARKS.--Flow regulated by El Vado Reservoir (see station 08286900) and Abiquiu Reservoir (see station 08286900). Diversions above station for irrigation of about 19,100 acres a few hundred of which is below station.

AVERAGE DISCHARGE.--21 years (water years 1942-62), $408 \mathrm{ft}^{3} / \mathrm{s}, 295,600 \mathrm{acre}-\mathrm{ft} / \mathrm{yr}$.

EXTREMES FOR PERIOD OF RECORD.--Maximum discharge, 7,870 $\mathrm{ft}^{3 / \mathrm{s}} \mathrm{July} \mathrm{28,} \mathrm{1952,} \mathrm{from} \mathrm{rating} \mathrm{curve} \mathrm{extended} \mathrm{above}$ $2,900 \mathrm{ft} 3 / \mathrm{s}$ by logarithmic plotting; maximum gage height, $6.38 \mathrm{ft}$ Aug. 5 , 1959 ; minimum daily discharge, 1 ft $3 / \mathrm{s}$
June 11,1947 .

STATISTICAL SUMMARIES (PERIOD BEFORE REGULATION OF ABIQUIU DAM)

MEAN MONTHLY AND MEAN ANNUAL DISCHARgeS 1942-62

\begin{tabular}{|c|c|c|c|c|c|c|}
\hline MONTH & $\begin{array}{l}\text { MINIMUM } \\
\left(\mathrm{FT}^{3} / \mathrm{S}\right)\end{array}$ & $\begin{array}{c}\text { MAXIMUM } \\
\left(\mathrm{FT}^{3} / \mathrm{S}\right)\end{array}$ & $\begin{array}{l}\text { MEAN } \\
\left(\mathrm{FT}^{3} / \mathrm{S}\right)\end{array}$ & $\begin{array}{l}\text { STAN- } \\
\text { DARD } \\
\text { DEVIA- } \\
\text { TION } \\
\left(\text { FT }^{3} / S\right)\end{array}$ & $\begin{array}{l}\text { COEFFI- } \\
\text { CIENT OF } \\
\text { VARI- } \\
\text { ATION }\end{array}$ & $\begin{array}{c}\text { PERCENT } \\
\text { OF } \\
\text { ANNUAL } \\
\text { RUNOF F }\end{array}$ \\
\hline $\begin{array}{l}\text { OCTOBER } \\
\text { NOVEMBER } \\
\text { DECEMBER } \\
\text { JANUARY } \\
\text { FEBRUARY } \\
\text { MARCH } \\
\text { APRIL } \\
\text { MAY } \\
\text { JUNE } \\
\text { JULY } \\
\text { AUGUST } \\
\text { SEPTEMBER }\end{array}$ & $\begin{array}{c}7.5 \\
15 \\
9.4 \\
12 \\
28 \\
23 \\
13 \\
150 \\
150 \\
62 \\
48 \\
4.1\end{array}$ & $\begin{array}{r}730 \\
1265 \\
794 \\
157 \\
1382 \\
1382 \\
2728 \\
3165 \\
2083 \\
1697 \\
1324 \\
818\end{array}$ & $\begin{array}{r}159 \\
295 \\
152 \\
49 \\
219 \\
319 \\
623 \\
1006 \\
793 \\
492 \\
483 \\
302\end{array}$ & $\begin{array}{l}167 \\
407 \\
242 \\
38 \\
322 \\
399 \\
659 \\
806 \\
482 \\
426 \\
339 \\
262\end{array}$ & $\begin{array}{r}1.05 \\
1.38 \\
1.59 \\
.79 \\
1.47 \\
1.25 \\
1.06 \\
.80 \\
.61 \\
.87 \\
.70 \\
.87\end{array}$ & $\begin{array}{r}3.3 \\
6.0 \\
3.1 \\
1.0 \\
4.5 \\
6.5 \\
12.7 \\
20.6 \\
16.2 \\
10.1 \\
9.9 \\
6.2\end{array}$ \\
\hline NNUAL & 179 & 1061 & 408 & 211 & .52 & 1.00 \\
\hline
\end{tabular}

MAGNITUDE AND PROBABILITY OF ANNUAL LOW FLOW BASED ON PERIOD OF RECORD 1943-62

\begin{tabular}{|c|c|c|c|c|c|c|}
\hline \multirow{2}{*}{$\begin{array}{l}\text { PERIOD } \\
\text { (CON- } \\
\text { SECU- } \\
\text { TIVE } \\
\text { DAYS) }\end{array}$} & \multicolumn{6}{|c|}{$\begin{array}{l}\text { DISCHARGE, IN FT'/S, FOR INDICATED RECURRENCE } \\
\text { INTERVAL, IN YEARS, AND ANNUAL NON- } \\
\text { FXCEEDANCE PROBABILITY, IN PERCENT }\end{array}$} \\
\hline & $\begin{array}{c}2 \\
508\end{array}$ & $\begin{array}{c}5 \\
208\end{array}$ & $\begin{array}{l}10 \\
108\end{array}$ & $\begin{array}{l}20 \\
58\end{array}$ & $\begin{array}{l}50 \\
28\end{array}$ & $\begin{array}{r}100 \\
18\end{array}$ \\
\hline $\begin{array}{r}1 \\
3 \\
7 \\
14 \\
30 \\
60 \\
90 \\
120 \\
183\end{array}$ & $\begin{array}{c}9.3 \\
10 \\
13 \\
18 \\
23 \\
26 \\
32 \\
50 \\
103\end{array}$ & $\begin{array}{r}3 . \\
4 . \\
5 . \\
7 . \\
9 . \\
15 \\
21 \\
30 \\
58\end{array}$ & $\begin{array}{r}2.0 \\
2.5 \\
3.0 \\
3.9 \\
5.7 \\
12 \\
18 \\
23 \\
44\end{array}$ & $\begin{array}{l}1.2 \\
1.5 \\
1.8 \\
2.3 \\
3.5 \\
10 \\
16 \\
20 \\
35\end{array}$ & $\begin{array}{l}-- \\
-- \\
-- \\
-- \\
-- \\
-- \\
--\end{array}$ & $\begin{array}{l}-- \\
-- \\
-- \\
-- \\
-- \\
-- \\
-\end{array}$ \\
\hline
\end{tabular}

MAGNITUDE AND PROBABILITY OF ANNUAL HIGH FLOW BASED ON PERIOD OF RECORD 1942-62

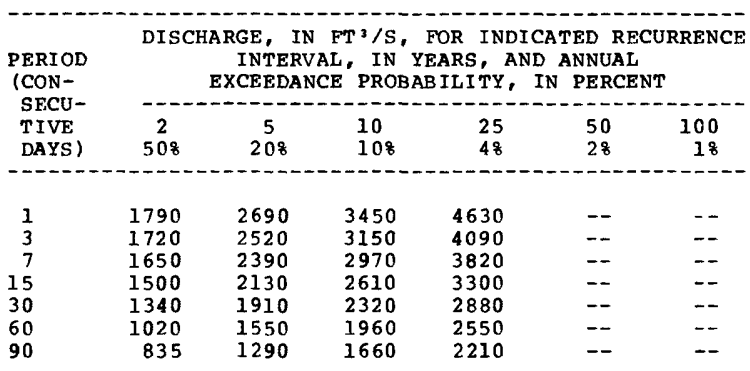

DURATION TABLF: OF DAILY MEAN FIOW FOR PERIOD OF RECORD 1942-62 DISCHARGE, IN FT $3 / 5$, THAT WAS EQUALED OR EXCEEDED FOR INDICATED PERCENT OF TIME

\begin{tabular}{|c|c|c|c|c|c|c|c|c|c|c|c|c|c|c|}
\hline 58 & 108 & 158 & 208 & 258 & 308 & 408 & 508 & 608 & 708 & 758 & 808 & 858 & 908 & 958 \\
\hline 1560 & 1230 & 996 & 765 & 609 & 474 & 239 & 123 & 72 & 49 & 40 & 32 & 26 & 20 & 14 \\
\hline
\end{tabular}


08288000 EL RITO CREEK NEAR EL RITO, NM

LOCATION.--Lat $36^{\circ} 23^{\prime} 30^{\prime \prime}$, long $106^{\circ} 14^{\prime} 20^{n}$, in NWs sec.19, T.25 N., R.7 E., on right bank three-quarters of a mi upstream from Carson National forest boundary and 3 miles northwest of El Rito.

DRAINAGE AREA. $--52 \mathrm{mi}^{2}$.

PERIOD OF RECORD.--May 1931 to September 1951 (no winter records 1937-41), discontinued.

GAGF. - Water-stage recorder. Altitude of gage is 6,950 ft (from topographic map). May 15, 1931 to May 3, 1934 and Apr. 29, 1937 to Apr. 5, 1938, about same site at different datum. May 4, 1934 to Apr. 28, 1937, at site about 200 ft upstream at datum $4.50 \mathrm{ft}$ higher.

REMARKS.--Diversion for irrigation of less than a hundred acres above station.

AVERAGE DISCHARGE.--18 years (water years 1932-50), $19.0 \mathrm{ft}^{3} / \mathrm{s}, 13,770$ acre-ft/yr.

EXTREMES FOR PERIOD OF RECORD.--Maximum discharge not determined; minimum daily recorded, that of July 9 , 10 , 12 , 1951.

STATISTICAL SUMMARIFS

MEAN MONTHLY AND MEAN ANNUAL DISCHARGES 1932-50

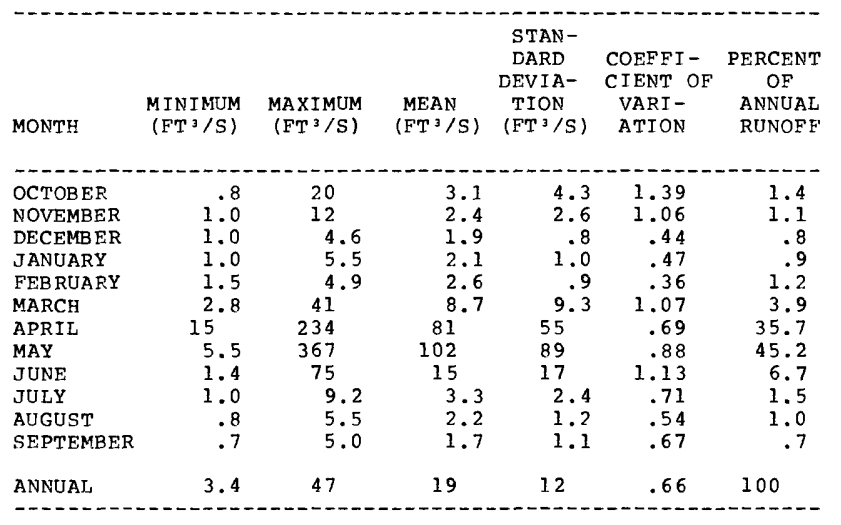

MAGNI TUDE AND PROBABILITY OF ANNUAL LOW FLOW BASED ON PERIOD OF RECORD $1933-50$

\begin{tabular}{|c|c|c|c|c|c|c|}
\hline $\begin{array}{l}\text { PERIOD } \\
\text { (CON- }\end{array}$ & \multicolumn{6}{|c|}{$\begin{array}{l}\text { DISCHARGE, IN } \mathrm{FT}^{3} / \mathrm{S} \text {, FOR INDICATED RECURRENCE } \\
\text { INTERVAL, IN YEARS, AND ANNUAL NON- } \\
\text { EXCEFDANCE PROBABILITY, IN PERCENT }\end{array}$} \\
\hline $\begin{array}{l}\text { TIVE } \\
\text { DAYS) }\end{array}$ & $\begin{array}{c}2 \\
508\end{array}$ & $\begin{array}{c}5 \\
208\end{array}$ & $\begin{array}{l}10 \\
108\end{array}$ & $\begin{array}{l}20 \\
58\end{array}$ & $\begin{array}{l}50 \\
28\end{array}$ & $\begin{array}{r}100 \\
18\end{array}$ \\
\hline $\begin{array}{r}1 \\
3 \\
7 \\
14 \\
30 \\
60 \\
90 \\
120 \\
183\end{array}$ & $\begin{array}{r}.5 \\
.6 \\
.7 \\
.8 \\
1.0 \\
1.2 \\
1.3 \\
1.4 \\
1.5\end{array}$ & $\begin{array}{r}.4 \\
.4 \\
.5 \\
.6 \\
.7 \\
.9 \\
1.1 \\
1.1 \\
1.1\end{array}$ & $\begin{array}{r}.3 \\
.3 \\
.4 \\
.5 \\
.6 \\
.9 \\
1.0 \\
1.0 \\
1.1\end{array}$ & $\begin{array}{r}.3 \\
.3 \\
.4 \\
.5 \\
.6 \\
.8 \\
.9 \\
1.0 \\
1.1\end{array}$ & $\begin{array}{l}-- \\
-- \\
-- \\
-- \\
-- \\
-- \\
--\end{array}$ & $\begin{array}{l}-- \\
-- \\
-- \\
-- \\
-- \\
-- \\
--\end{array}$ \\
\hline
\end{tabular}

MAGNITUDE AND PROBABILITY OF ANNUAL HIGH FLOW BASED ON PERIOD OF RECORD $1932-50$

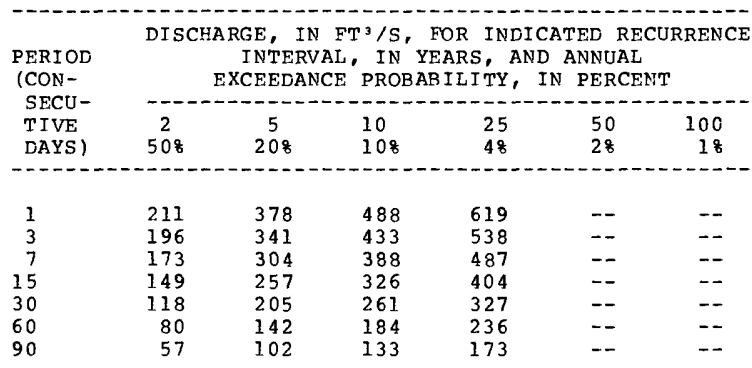

DURATION TABLE OF DAILY MEAN FLOW FOR PERIOD OF RECORD 1932-50

DISCHARGE, IN FT' ${ }^{3} / S$, THAT WAS EQUALED OR EXCEEDED FOR INDICATED PERCENT OF TIME

\begin{tabular}{|c|c|c|c|c|c|c|c|c|c|c|c|c|c|c|}
\hline 58 & 108 & 158 & 208 & 258 & 308 & 408 & 508 & 608 & 708 & 758 & 808 & 858 & 908 & 958 \\
\hline 112 & 51 & 24 & 13 & 7.2 & 5.2 & 3.4 & 2.5 & 2.0 & 1.7 & 1.5 & 1.4 & 1.2 & 1.0 & 8 \\
\hline
\end{tabular}




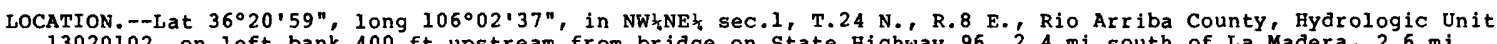
13020102 , on left bank $400 \mathrm{ft}$ upstream from bridge on State Highway $96,2.4 \mathrm{mi}$ south of La Madera, $2.6 \mathrm{mi}$ downstream from confluence of Rio Vallecitos and Rio Tusas, $3.1 \mathrm{mi}$ north of ojo Caliente, and at mile 19.9.

DRAINAGE AREA, $--419 \mathrm{mi}^{2}$.

PERIOD OF RECORD.--April 1932 to current year.

REVISED RECORDS.--WSP 1712: 1959.

GAGE.--Water-stage recorder. Datum of gage is $6,358.84 \mathrm{ft}$ above National Geodetic Vertical Datum of 1929 . Prior to Apr. 23, 1934, at site about $2.6 \mathrm{mi}$ upstream at different datum. Apr. 23, 1934 to Apr. 21, 1936, at datum $12.58 \mathrm{ft}$ lower and Apr. 22, 1936 to Oct. 26, 1956, at datum 13.84 ft lower, both at site 1,400 ft downstream. REMARKS. --Diversion upstream from station for irrigation of about 3,500 acres (1962 determination).

AVERAGE DISCHARGE.--53 years (water years 1933-85), 69.2 $\mathrm{ft}^{3} / \mathrm{s}, 50,140 \mathrm{acre}-\mathrm{ft} / \mathrm{yr}$.

EXTREMES FOR PERIOD OF RECORD.--Maximum discharge, 3,140 ft $/ \mathrm{s}$, Apr. 21, 1958, gage height, $6.42 \mathrm{ft}$, from rating curve extended above $1,300 \mathrm{ft}^{3} / \mathrm{s}$; maximum gage height, $7.25 \mathrm{ft}$, from floodmarks, June 19, I966; minimum discharge, $0.2 \mathrm{ft}^{3} / \mathrm{s}$, Aug. $17,1956$.

EXTREMES OUTSIDE PERIOD OF RECORD,--The flood of Apr. 21, 1958, may have been exceeded by a flood in May 1920, from information by local resident.

STATISTICAL SUMMARIES

MEAN MONTHLY AND MEAN ANNUAL DISCHARGES 1933-85

\begin{tabular}{|c|c|c|c|c|c|c|}
\hline MONTH & $\begin{array}{c}\text { MINIMUM } \\
\left(\mathrm{FT}^{3} / \mathrm{S}\right)\end{array}$ & $\begin{array}{c}\text { MAXI MUM } \\
\left(\mathrm{FT}^{3} / \mathrm{S}\right)\end{array}$ & $\begin{array}{l}\text { MEAN } \\
\left(\mathrm{FT}^{3} / \mathrm{S}\right)\end{array}$ & $\begin{array}{c}\text { STAN- } \\
\text { DARD } \\
\text { DEVIA- } \\
\text { TION } \\
\left(\mathrm{FT}^{3} / \mathrm{S}\right)\end{array}$ & $\begin{array}{l}\text { COEFFI- } \\
\text { CIENT OF } \\
\text { VARI- } \\
\text { ATION }\end{array}$ & $\begin{array}{c}\text { PERCENT } \\
\text { OF } \\
\text { ANNUAL } \\
\text { RUNOFF }\end{array}$ \\
\hline $\begin{array}{l}\text { OCTOBER } \\
\text { NOVEMBER } \\
\text { DECEMBER } \\
\text { JANUARY } \\
\text { FEBRUARY } \\
\text { MARCH } \\
\text { APRIL } \\
\text { MAY } \\
\text { JUNE } \\
\text { JULY } \\
\text { AUGUST } \\
\text { SEPTEMBER }\end{array}$ & $\begin{array}{l}4.0 \\
8.8 \\
11 \\
10 \\
12 \\
16 \\
45 \\
9.3 \\
5.1 \\
2.6 \\
3.1 \\
2.3\end{array}$ & $\begin{array}{r}54 \\
49 \\
32 \\
34 \\
56 \\
164 \\
979 \\
1256 \\
275 \\
33 \\
68 \\
30\end{array}$ & $\begin{array}{c}13 \\
16 \\
17 \\
18 \\
22 \\
52 \\
280 \\
326 \\
51 \\
9.8 \\
14 \\
9.8\end{array}$ & $\begin{array}{r}9.1 \\
8.3 \\
4.6 \\
4.6 \\
7.5 \\
34 \\
218 \\
283 \\
61 \\
6.8 \\
12 \\
5.8\end{array}$ & $\begin{array}{r}.68 \\
.51 \\
.28 \\
.26 \\
.34 \\
.65 \\
.78 \\
.87 \\
1.20 \\
.69 \\
.91 \\
.59\end{array}$ & $\begin{array}{r}1.6 \\
2.0 \\
2.0 \\
2.1 \\
2.7 \\
6.3 \\
33.8 \\
39.4 \\
6.1 \\
1.2 \\
1.6 \\
1.2\end{array}$ \\
\hline ANNUAL & 13 & 205 & 69 & 44 & .64 & 100 \\
\hline
\end{tabular}

MAGNITUDE AND PROBABILITY OF ANNUAL LOW FLOW BASED ON PERIOD OF RECORD 1934-85

\begin{tabular}{|c|c|c|c|c|c|c|}
\hline \multirow{2}{*}{$\begin{array}{l}\text { PERIOD } \\
\text { (CON- } \\
\text { SECU- } \\
\text { TIVE } \\
\text { DAYS) }\end{array}$} & \multicolumn{6}{|c|}{$\begin{array}{l}\text { DISCHARGE, IN FT } 3 / S \text {, FOR INDICATED RECURRENCE } \\
\text { INTERVAL, IN YEARS, AND ANNUAL NON- } \\
\text { EXCEEDANCE PROBABILITY, IN PERCENT }\end{array}$} \\
\hline & $\begin{array}{c}2 \\
508\end{array}$ & $\begin{array}{c}5 \\
208\end{array}$ & $\begin{array}{l}10 \\
108\end{array}$ & $\begin{array}{l}20 \\
58\end{array}$ & $\begin{array}{l}50 \\
28\end{array}$ & \\
\hline $\begin{array}{r}1 \\
3 \\
7 \\
14 \\
30 \\
60 \\
90 \\
120 \\
183\end{array}$ & $\begin{array}{r}3.2 \\
3.5 \\
3.8 \\
4.3 \\
5.0 \\
6.4 \\
7.7 \\
8.6 \\
11\end{array}$ & $\begin{array}{l}1.9 \\
2.2 \\
2.5 \\
3.0 \\
3.6 \\
4.3 \\
5.2 \\
6.0 \\
7.9\end{array}$ & $\begin{array}{l}1.4 \\
1.7 \\
2.0 \\
2.5 \\
3.0 \\
3.5 \\
4.3 \\
5.0 \\
6.8\end{array}$ & $\begin{array}{l}1.1 \\
1.3 \\
1.6 \\
2.1 \\
2.6 \\
3.0 \\
3.7 \\
4.3 \\
6.1\end{array}$ & $\begin{array}{l}.7 \\
1.0 \\
1.3 \\
1.7 \\
2.3 \\
2.5 \\
3.2 \\
3.7 \\
5.4\end{array}$ & \\
\hline
\end{tabular}

MAGNITUDE AND PROBABILITY OF ANNUAL HIGH FLOW BASED ON PERIOD OF RECORD 1933-85

\begin{tabular}{|c|c|c|c|c|c|c|}
\hline \multirow{2}{*}{$\begin{array}{l}\text { PERIOD } \\
\text { (CON- } \\
\text { SECU- } \\
\text { TIVE } \\
\text { DAYS) }\end{array}$} & \multicolumn{6}{|c|}{$\begin{array}{l}\text { DISCHARGE, IN FT'3/S, FOR INDICATED RECURRENCE } \\
\text { INTERVAL, IN YEARS, AND ANNUAL } \\
\text { EXCEEDANCE PROBABILITY, IN PERCENT }\end{array}$} \\
\hline & $\begin{array}{c}2 \\
508\end{array}$ & $\begin{array}{c}5 \\
208\end{array}$ & $\begin{array}{l}10 \\
108\end{array}$ & $\begin{array}{l}25 \\
48\end{array}$ & $\begin{array}{l}50 \\
28\end{array}$ & $\begin{array}{r}100 \\
18\end{array}$ \\
\hline $\begin{array}{r}1 \\
3 \\
7 \\
15 \\
30 \\
60 \\
90\end{array}$ & $\begin{array}{l}651 \\
599 \\
533 \\
454 \\
360 \\
251 \\
183\end{array}$ & $\begin{array}{r}1210 \\
1120 \\
1000 \\
856 \\
691 \\
481 \\
348\end{array}$ & $\begin{array}{r}1620 \\
1500 \\
1350 \\
1150 \\
941 \\
657 \\
476\end{array}$ & $\begin{array}{r}2180 \\
2010 \\
1800 \\
1530 \\
1280 \\
897 \\
652\end{array}$ & $\begin{array}{r}2600 \\
2400 \\
2150 \\
1810 \\
1530 \\
1080 \\
792\end{array}$ & $\begin{array}{r}3040 \\
2790 \\
2500 \\
2080 \\
1790 \\
1280 \\
938\end{array}$ \\
\hline
\end{tabular}

DURATION TABLE OF DAILY MEAN FLOW FOR PERIOD OF RECORD 1933-85 DISCHARGE, IN FT $3 / \mathrm{S}$, THAT WAS EQUALED OR EXCEEDED FOR INDICATED PERCENT OF TIME

\begin{tabular}{|c|c|c|c|c|c|c|c|c|c|c|c|c|c|c|}
\hline 58 & 108 & $15 \%$ & 208 & 258 & 308 & 408 & 508 & 608 & 708 & 758 & 808 & 858 & 908 & 958 \\
\hline 375 & 161 & 83 & 50 & 35 & 28 & 21 & 17 & 14 & 12 & 9.9 & 8.1 & 6.5 & 5.1 & \\
\hline
\end{tabular}


LOCATION. - Lat $36^{\circ} 04^{\prime} 26^{\prime \prime}$, long $106^{\circ} 06^{\prime} 40^{\prime \prime}$, in NEלNE sec.8, T.21 N., R. 8 E., Rio Arriba County, Hydrologic Unit 13020102 , in San Juan Pueblo Grant, at downstream end of pier nearest left bank of bridge on U.S. Highway 285 , $0.5 \mathrm{mi}$ west of Chamita, $2.5 \mathrm{mi}$ northwest of $\mathrm{San} J u a n$ Pueblo, and at mile 2.8.

DRAINAGE AREA. $--3,144 \mathrm{mi}^{2}$, of which about $100 \mathrm{mi}^{2}$ is probably noncontributing.

PERIOD OF RECORD.--October 1912 to current year. Monthly discharge only for some periods, published in wSP 1312. Published as Chama River near Chamita prior to 1928, and Chama River at Chamita 1929-30.

REVISED RECORDS.--WSP 1512: 1913-15, 1934, 1936. WSP 1632: 1929(M). wSP 1732: 1931(M). WSP 1923: Drainage area.

GAGE. - Water-stage recorder. Concrete control since Jan, 1, 1964. Datum of gage is $5.653 .61 \mathrm{ft}$ above National Geodetic Vertical Datum of 1929 . Prior to oct. 4, 1933, at railroad bridge $2.3 \mathrm{mi}$ downstream at different Geodetic Vertical Datum of 1929. Prior to Oct. 4 , 1933 , at railroad bridge 2.3 mi downstream at different
datums. Oct. 4, 1933 to Mar. 1, 1942 , at site $50 \mathrm{ft}$ downstream at datum $0.22 \mathrm{ft}$ higher. Mar. 2 , 1942 to datums. Oct. 4, 1933 to Mar. 1, 1942 , at site $50 \mathrm{ft}$ downs
Dec. 31, 1963, at site $200 \mathrm{ft}$ downstream, present datum.

REMARKS.--Diversions upstream from station for irrigation of about 27,600 acres. Chamita ditch (station 08289500), on left bank, and Hernandez ditch (station 08289800), on right bank, bypass gage for irrigation of several hundred acres downstream from station. Flow regulated by El Vado Reservoir (station 08285000) and Abiguiu Reservoir (station 08286900 ), $74.9 \mathrm{mi}$ and $29.3 \mathrm{mi}$ upstream respectively. Since May 1971 flow affected by release of transmountain water from Heron Reservoir (station 08284510) 83.0 mi upstream. National Weather Service gage-height telemeter at station.

AVERAGE DISCHARGE.--13 years (water years 1913-15, 1917-19, 1922, 1928, 1930-34), 561 ft?/s, 406,400 acre-ft/yr

EXTREMES FOR PERIOD OF RECORD,--Maximum discharge, 15,000 ft $3 / \mathrm{s}$, May 22, 1920, from rating curve extended above $2,300 \mathrm{ft}^{3} / \mathrm{s}$; maximum gage height, $10.45 \mathrm{ft}$. Aug. 22 , 1961 ; no flow at times.

EXTREMES OUTSIDE PERIOD OF RECORD.--The floods of Sept. 29, 1904, and Oct. 4 or 5, 1911, probably exceeded 15,000 $\mathrm{ft}^{3 / \mathrm{s}}$. Another major flood occurred in 1884, from newspaper accounts.

STATISTICAL SUMMARIES (PERIOD BEFORE REGULATION OF EU VADO DAM)

MEAN MONTHLY AND MEAN ANNUAL DISCHARGES 1913-15,

$$
1917-19,1922
$$

$1928,1930-34$

\begin{tabular}{|c|c|c|c|c|c|c|}
\hline MONTH & $\begin{array}{l}\text { MINIMUM } \\
\left(\mathrm{FT}^{3} / \mathrm{S}\right)\end{array}$ & $\begin{array}{l}\text { MAXIMUM } \\
\left(\mathrm{FT}^{3} / \mathrm{S}\right)\end{array}$ & $\begin{array}{l}\text { MEAN } \\
\left(\mathrm{FT}^{3} / \mathrm{S}\right)\end{array}$ & $\begin{array}{l}\text { STAN- } \\
\text { DARD } \\
\text { DEVIA- } \\
\text { TION } \\
\text { (FT'/S) }\end{array}$ & $\begin{array}{l}\text { COEFFI- } \\
\text { CIENT OF } \\
\text { VARI- } \\
\text { ATION }\end{array}$ & $\begin{array}{c}\text { PERCENT } \\
\text { OF } \\
\text { ANNUAL } \\
\text { RUNOFE }\end{array}$ \\
\hline $\begin{array}{l}\text { OCTOBER } \\
\text { NOVEMBER } \\
\text { DECEMBER } \\
\text { JANUARY } \\
\text { FEBRUARY } \\
\text { MARCH } \\
\text { APRIL } \\
\text { MAY } \\
\text { JUNE } \\
\text { JULY } \\
\text { AUGUST } \\
\text { SEPTEMBER }\end{array}$ & $\begin{array}{c}22 \\
58 \\
28 \\
45 \\
70 \\
150 \\
524 \\
297 \\
71 \\
25 \\
11 \\
5.5\end{array}$ & $\begin{array}{r}706 \\
247 \\
167 \\
150 \\
364 \\
1039 \\
4129 \\
4984 \\
3679 \\
1309 \\
350 \\
934\end{array}$ & $\begin{array}{r}213 \\
123 \\
98 \\
100 \\
159 \\
396 \\
1522 \\
2458 \\
1137 \\
372 \\
158 \\
168\end{array}$ & $\begin{array}{r}218 \\
63 \\
39 \\
33 \\
77 \\
232 \\
1021 \\
1215 \\
944 \\
337 \\
98 \\
237\end{array}$ & $\begin{array}{r}1.02 \\
.51 \\
.40 \\
.33 \\
.48 \\
.59 \\
.67 \\
.49 \\
.83 \\
.91 \\
.62 \\
1.41\end{array}$ & $\begin{array}{r}3.1 \\
1.8 \\
1.4 \\
1.4 \\
2.3 \\
5.7 \\
22.0 \\
35.6 \\
16.5 \\
5.4 \\
2.3 \\
2.4\end{array}$ \\
\hline NNUAL & 160 & 1130 & 561 & 278 & .50 & 100 \\
\hline
\end{tabular}

MAGNITUDE AND PROBABILITY OF ANNUAL LOW FLOW BASED ON PERIOD OF RECORD 1914-15, 1918-19, $1923,1928,1931-34$

DERIOD DISCHARGE, IN FT ${ }^{3} / \mathrm{S}$, FOR INDICATED RECURRENCE
INTERVAL, IN YEARS, AND ANNUAL NON-

$\begin{array}{ll}\text { PERIOD } & \text { INTERVAL, IN YEARS, AND ANNUAL NON- } \\ \text { (CON- } & \text { EXCEEDANCE PROBABILITY, IN PERCENT }\end{array}$

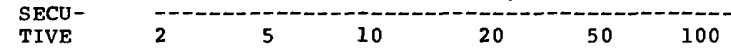

$\begin{array}{lcccccr}\text { TIVE } & 2 & 5 & 10 & 20 & 50 & 100 \\ \text { DAYS) } & 508 & 208 & 108 & 58 & 28 & 18\end{array}$

\begin{tabular}{rrrrrrr} 
DAYS) & 508 & 208 & 108 & 58 & 28 & 18 \\
\hline 1 & 12 & 0.0 & 0.0 & 0.0 & - & - \\
3 & 14 & 0.0 & 0.0 & 0.0 & -- & - \\
7 & 18 & 4.4 & 0.0 & 0.0 & -- & - \\
14 & 24 & 9.0 & 5.1 & 3.1 & -- & - \\
30 & 38 & 17 & 10 & 6.7 & -- & - \\
60 & 52 & 30 & 22 & 16 & -- & -- \\
90 & 66 & 45 & 37 & 31 & -- & -- \\
120 & 82 & 56 & 47 & 40 & -- & - \\
183 & 98 & 67 & 57 & 50 & - & - \\
\hline
\end{tabular}

MAGNITUDE AND PROBABILITY OF ANNUAL HIGH FLOW BASED ON PERIOD OF RECORD 1913-15, 1917-19 $1922,1928,1930-34$

\begin{tabular}{|c|c|c|c|c|c|c|}
\hline \multirow{2}{*}{$\begin{array}{l}\text { PERIOD } \\
\text { (CON- } \\
\text { SECU- } \\
\text { TIVE } \\
\text { DAYS) }\end{array}$} & \multicolumn{6}{|c|}{$\begin{array}{l}\text { DISCHARGE, IN FT } 3 / S \text {, FOR INDICATED RECURRENCE } \\
\text { INTERVAL, IN YEARS, AND ANNUAL } \\
\text { EXCEEDANCE PROBABILITY, IN PERCENT }\end{array}$} \\
\hline & $\begin{array}{c}2 \\
508\end{array}$ & $\begin{array}{c}5 \\
208\end{array}$ & $\begin{array}{l}10 \\
108\end{array}$ & $\begin{array}{l}25 \\
48\end{array}$ & $\begin{array}{l}50 \\
28\end{array}$ & $\begin{array}{r}100 \\
18\end{array}$ \\
\hline $\begin{array}{r}1 \\
3 \\
7 \\
15 \\
30 \\
60 \\
90\end{array}$ & $\begin{array}{l}3790 \\
3560 \\
3230 \\
3010 \\
2650 \\
2020 \\
1560\end{array}$ & $\begin{array}{l}5350 \\
5030 \\
4570 \\
4250 \\
3780 \\
3090 \\
2440\end{array}$ & $\begin{array}{l}6210 \\
5860 \\
5350 \\
4940 \\
4370 \\
3730 \\
3000\end{array}$ & $\begin{array}{l}7140 \\
6750 \\
6220 \\
5670 \\
4970 \\
4460 \\
3660\end{array}$ & $\begin{array}{l}-- \\
-- \\
-- \\
-- \\
-- \\
-- \\
--\end{array}$ & $\begin{array}{l}-- \\
-- \\
-- \\
-- \\
-- \\
--\end{array}$ \\
\hline
\end{tabular}

DURATION TABLE OF DAILY MEAN FLOW FOR PERIOD OF RECORD 1913-15, 1917-19, 1922, 1928, 1930-34 DISCHARGE, IN FT'/S, THAT WAS EQUALED OR EXCEEDED FOR INDICATED PERCENT OF TIME

\begin{tabular}{|c|c|c|c|c|c|c|c|c|c|c|c|c|c|c|}
\hline 58 & 108 & 158 & 208 & 258 & 308 & 408 & 508 & 608 & 708 & 758 & 808 & 858 & 908 & 958 \\
\hline 2830 & 1760 & 1140 & 730 & 515 & 390 & 229 & 159 & 125 & 95 & 83 & 72 & 58 & 42 & 16 \\
\hline
\end{tabular}


RIO GRANDE BASIN

08290000 RIO CHAMA NEAR CHAMITA, MM--Continued

STATISTICAL SUMMARIES (PERIOD BEFORE REGULATION OF ABIQUIU DAM)

MEAN MONTHLY AND MEAN ANNUAI, DISCHARGES 1936-62

\begin{tabular}{|c|c|c|c|c|c|c|}
\hline MONTH & $\begin{array}{c}\text { MINIMUM } \\
\left(\mathrm{FT}^{3} / \mathrm{S}\right)\end{array}$ & $\begin{array}{l}\text { MAXIMUM } \\
\left(\mathrm{FT}^{3} / \mathrm{S}\right)\end{array}$ & $\begin{array}{l}\text { MEAN } \\
(\mathrm{FT} 3 / \mathrm{S})\end{array}$ & $\begin{array}{l}\text { STAN- } \\
\text { DARD } \\
\text { DEVIA- } \\
\text { TION } \\
\text { (FT'/S) }\end{array}$ & $\begin{array}{l}\text { COEFFI- } \\
\text { CIENT OF } \\
\text { VARI- } \\
\text { ATION }\end{array}$ & $\begin{array}{c}\text { PERCENT } \\
\text { OF } \\
\text { ANNUAL } \\
\text { RUNOFF }\end{array}$ \\
\hline OCTOBER & 1.6 & 863 & 197 & 186 & 94 & 31 \\
\hline NOVEMBER & $21^{1.0}$ & $\begin{array}{r}863 \\
1236\end{array}$ & 282 & 365 & $\begin{array}{r}.94 \\
1.29\end{array}$ & 4.5 \\
\hline DECEMBER & 25 & 831 & 145 & 222 & 1.53 & 2.3 \\
\hline JANUARY & 30 & 246 & 69 & 45 & .65 & 1.1 \\
\hline FEBRUARY & 50 & 1362 & 237 & 288 & 1.22 & 3.8 \\
\hline MARCH & 45 & 1476 & 396 & 391 & .99 & 6.3 \\
\hline APRIL & 57 & 3273 & 1032 & 883 & .86 & 16.5 \\
\hline MAY & 188 & 6542 & 1555 & 1490 & .96 & 24.8 \\
\hline JUNE & 110 & 3153 & 899 & 643 & .72 & 14.3 \\
\hline JULY & 51 & 1712 & 554 & 413 & .75 & 8.8 \\
\hline AUGUST & 40 & 1373 & 559 & 358 & .64 & 8.9 \\
\hline SEPTEMBER & 2.3 & 802 & 345 & 257 & .74 & 5.5 \\
\hline ANNUAL & 197 & 1210 & 523 & 289 & .55 & 100 \\
\hline
\end{tabular}

MAGNITUDE AND PROBABILITY OF ANNUAL LOW FLOW BASED ON PERIOD OF RECORD 1937-62

\begin{tabular}{|c|c|c|c|c|c|c|}
\hline \multirow{2}{*}{$\begin{array}{l}\text { PERIOD } \\
\text { (CON- } \\
\text { SECU- } \\
\text { TIVE } \\
\text { DAYS) }\end{array}$} & \multicolumn{6}{|c|}{$\begin{array}{l}\text { DISCHARGE, IN FT } 3 / S \text {, FOR INDICATED RECURRENCE } \\
\text { INTERVAL, IN YEARS, AND ANNUAL NON- } \\
\text { EXCEEDANCE PROBABILITY, IN PERCENT }\end{array}$} \\
\hline & $\stackrel{2}{508}$ & $\begin{array}{c}5 \\
208\end{array}$ & $\begin{array}{l}10 \\
108\end{array}$ & $\begin{array}{l}20 \\
58\end{array}$ & $\begin{array}{l}50 \\
28\end{array}$ & $\begin{array}{r}100 \\
18\end{array}$ \\
\hline $\begin{array}{r}1 \\
3 \\
7 \\
14 \\
30 \\
60 \\
90 \\
120 \\
183\end{array}$ & $\begin{array}{r}8 . \\
11 \\
16 \\
24 \\
36 \\
45 \\
52 \\
72 \\
131\end{array}$ & $\begin{array}{r}3 \\
5 \\
9 \\
20 \\
31 \\
40 \\
75\end{array}$ & $\begin{array}{l}0.0 \\
0.0 \\
1.0 \\
2 . \\
3 . \\
12 \\
24 \\
30 \\
56\end{array}$ & $\begin{array}{r}0 . \\
0 . \\
. \\
1 . \\
7 . \\
20 \\
24 \\
45\end{array}$ & $\begin{array}{r}0.0 \\
0.0 \\
.1 \\
.2 \\
.4 \\
4.5 \\
16 \\
19 \\
35\end{array}$ & $\begin{array}{l}-- \\
-- \\
-- \\
-- \\
-- \\
-- \\
--\end{array}$ \\
\hline
\end{tabular}

MAGNITUDE AND PROBABILITY OF ANNUAL HIGH FLOW BASED ON PERIOD OF RECORD 1936-62

\begin{tabular}{|c|c|c|c|c|c|c|}
\hline \multirow{2}{*}{$\begin{array}{l}\text { PERIOD } \\
\text { (CON- } \\
\text { SECU- } \\
\text { TIVE } \\
\text { DAYS) }\end{array}$} & \multicolumn{6}{|c|}{$\begin{array}{c}\text { DISCHARGE, IN FT' } / S \text {, FOR INDICATED RECURRENCE } \\
\text { INTERVAL, IN YEARS, AND ANNUAL } \\
\text { EXCEEDANCE' PROBABILITY, IN PERCENT' }\end{array}$} \\
\hline & $\begin{array}{c}2 \\
508\end{array}$ & $\begin{array}{c}5 \\
208\end{array}$ & $\begin{array}{l}10 \\
108\end{array}$ & $\begin{array}{l}25 \\
48\end{array}$ & $\begin{array}{l}50 \\
28\end{array}$ & $\begin{array}{r}100 \\
18\end{array}$ \\
\hline $\begin{array}{r}1 \\
3 \\
7 \\
15 \\
30 \\
60 \\
90\end{array}$ & $\begin{array}{l}2280 \\
2130 \\
2010 \\
1810 \\
1610 \\
1280 \\
1050\end{array}$ & $\begin{array}{l}3860 \\
3610 \\
3410 \\
3080 \\
2720 \\
2180 \\
1770\end{array}$ & $\begin{array}{l}5220 \\
4910 \\
4670 \\
4210 \\
3690 \\
2960 \\
2370\end{array}$ & $\begin{array}{l}7350 \\
6970 \\
6690 \\
6050 \\
5210 \\
4160 \\
3290\end{array}$ & $\begin{array}{l}9280 \\
8860 \\
8570 \\
7770 \\
6610 \\
5240 \\
4090\end{array}$ & $\begin{array}{l}-- \\
-- \\
-- \\
-- \\
-- \\
-- \\
--\end{array}$ \\
\hline
\end{tabular}

DURATION TABLE OF DAILY MEAN FLOW FOR PERIOD OF RECORD 1936-62

DISCHARGE, IN FT'3/S, THAT WAS EQUALED OR EXCEEDED FOR INDICATED PERCENT OF TIME

\begin{tabular}{|c|c|c|c|c|c|c|c|c|c|c|c|c|c|c|}
\hline 58 & 108 & 158 & 208 & 258 & 308 & 408 & 508 & 608 & 708 & 758 & 808 & 858 & 908 & 958 \\
\hline 1860 & 1380 & 1110 & 907 & 724 & 564 & 351 & 194 & 107 & 71 & 59 & 49 & 39 & 29 & 15 \\
\hline
\end{tabular}


RIO GRANDE BASIN

08290000 RIO CHAMA NEAR CHAMITA, NM--Continued

STATISTICAL SUMMARIES (PERIOD AFTER COMPLETION OF HERON DAM)

MEAN MONTHLY AND MEAN ANNUAI, DISCHARGES

$1971-85$
MAGNI TUDE AND PROBABILITY OF ANNUAL LOW FLOW BASED ON PERIOD OF RECORD $1972-85$

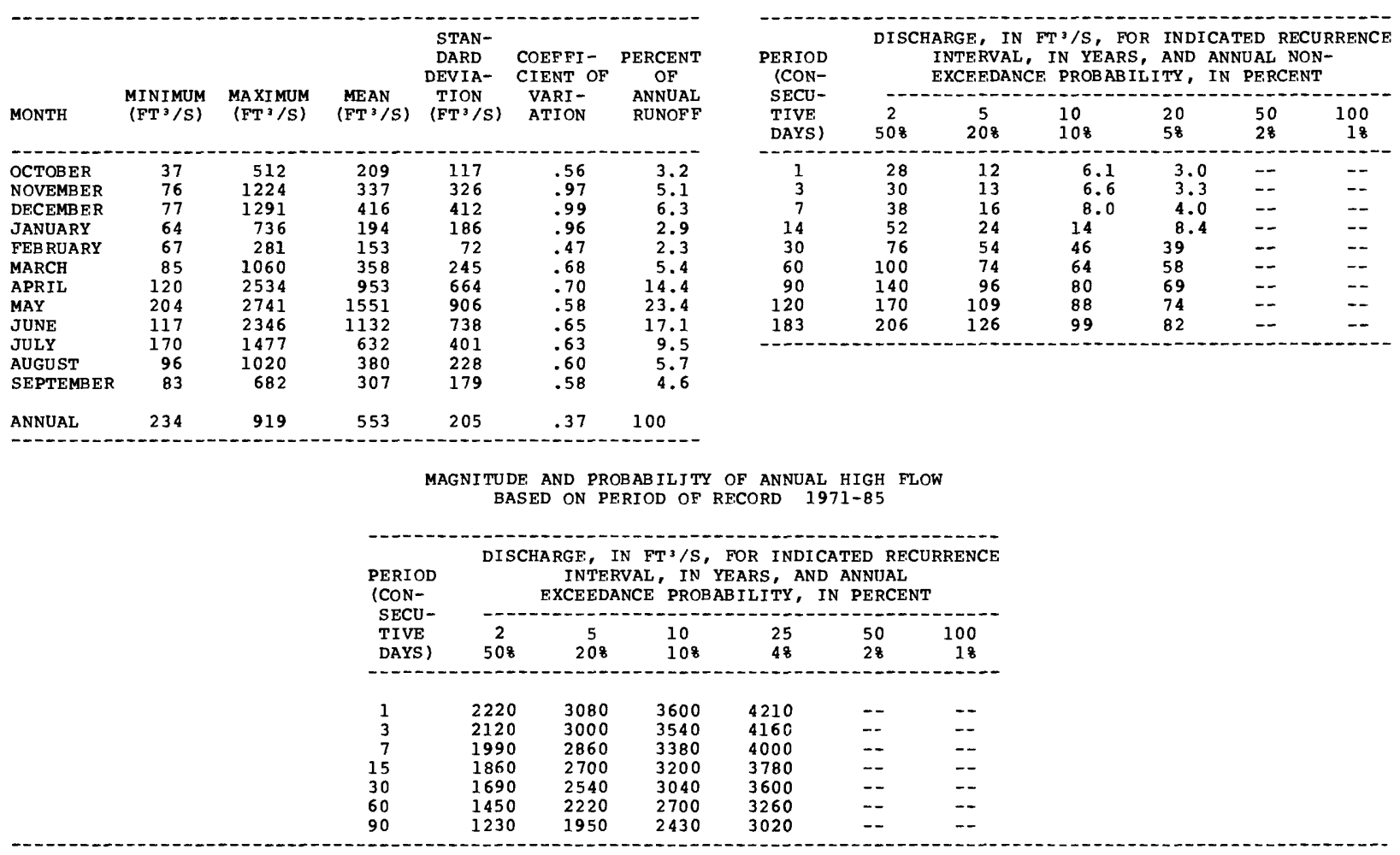

DURATION TABLE OF DAILY MEAN FLOW FOR PERIOD OF RECORD 1971-85

DISCHARGE, IN FT'3/S, THAT WAS EQUALED OR EXCEEDED FOR INDICATED PERCENT OF TIME

\begin{tabular}{|c|c|c|c|c|c|c|c|c|c|c|c|c|c|c|}
\hline 58 & 108 & 158 & 208 & 258 & 308 & 408 & 508 & 608 & 708 & 758 & 808 & 858 & 908 & 958 \\
\hline 2220 & 1630 & 1180 & 914 & 734 & 601 & 385 & 239 & 161 & 120 & 205 & 90 & 76 & 64 & 49 \\
\hline
\end{tabular}


RIO GRANDE BASIN

08291000 SANTA CRUZ RIVER AT CUNDIYO, NM

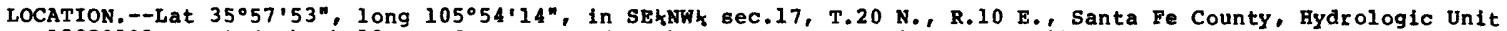
13020101 , on left bank $135 \mathrm{ft}$ downstream from bridge on state Highway 4,200 ft downstream from confiuence of Rio Medio and Rio Frijoles, $0.6 \mathrm{mi}$ northwest of Cundiyo, $1.8 \mathrm{mi}$ upstream from Santa Cruz Dam, and at mile 11.9.

DRAINAGE AREA. $--86 \mathrm{ml}^{2}$, approximately.

PERIOD OF RECORD,--October 1930 to current year. Monthly discharge only from some periods, published in wSP 1312. Prior to October 1953, published as Rio Santa cruz at cundiyo.

REVISED RECORDS.--WSP 1392: $1931(\mathrm{M}), 1932-33,1934-39(\mathrm{M}), 1942,1943(\mathrm{M})$.

GAGE.--Water-stage recorder. Concrete control since Jan. 3, 1954. Elevation of gage is 6,460 ft above National Geodetic Vertical Datum of 1929, from topographic map. Sept. 1 , 1930 to Aug. 12, 1932, water-stage recorder at site about 1 mi downstream at different datum. Aug. 13, 1932 to oct. 29, 1934, water-stage recorder at site 35 ft upstream at datum $0.42 \mathrm{ft}$ higher. Oct. 30,1934 to Jan. 2, 1954 , water-stage recorder at present site at datum $0.64 \mathrm{ft}$ lower.

REMARKS.--Diversions for irrigation of about 1,000 acres upstream from station.

AVERAGE DISCHARGE.--53 years (water years 1933-85), 30.2 ft3/s, 21,880 acre-ft/yr.

EXTREMES FOR PERIOD OF RECORD.--Maximum discharge, 2,420 ft'/s, Sept. 24, 1931, gage height, $7.80 \mathrm{ft}$, site and datum then in use, from rating curve extended above $170 \mathrm{ft} \% \mathrm{~s}$; minimum, $0.19 \mathrm{ft} / \mathrm{s}$, Max. 13, 1954, result of freezeup.

STATISTICAL SUMMARIES

MEAN MONTHLY AND MEAN ANNUAL DISCHARGES 1933-85

\begin{tabular}{|c|c|c|c|c|c|c|}
\hline MONTH & $\begin{array}{l}\text { MINIMUM } \\
\left(\mathrm{FT}^{3} / \mathrm{S}\right)\end{array}$ & $\begin{array}{l}\text { MAXIMUM } \\
\left(\mathrm{FT}^{1 / 8} / \mathrm{S}\right)\end{array}$ & $\begin{array}{l}\text { MEAN } \\
\left(F^{2} / S\right)\end{array}$ & $\begin{array}{l}\text { STAN- } \\
\text { DARD } \\
\text { DEVIA- } \\
\text { TION } \\
\left(\mathrm{FT}^{3} / \mathrm{S}\right)\end{array}$ & $\begin{array}{l}\text { COEFFI- } \\
\text { CIENT OF } \\
\text { VARI- } \\
\text { ATION }\end{array}$ & $\begin{array}{c}\text { PERCENT } \\
\text { OF } \\
\text { ANNUAL } \\
\text { RUNOFF }\end{array}$ \\
\hline \multicolumn{6}{|c|}{ 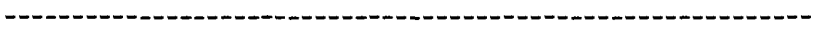 } & 4.0 \\
\hline NOVEMBER & 4.7 & 43 & 11 & 5.5 & .49 & 3.1 \\
\hline DECEMBER & 3.8 & 23 & 9.7 & 3.4 & .35 & 2.7 \\
\hline JANUARY & 4.8 & 18 & 8.9 & 2.4 & .27 & 2.5 \\
\hline FEBRUARY & 5.4 & 17 & 9.5 & 2.5 & .26 & 2.7 \\
\hline MARCH & 7.0 & 51 & 18 & 10 & .57 & 5.0 \\
\hline APRIL & 13 & 205 & 50 & 35 & .70 & 13.9 \\
\hline MAY & 16 & 329 & 95 & 71 & .75 & 26.6 \\
\hline JUNE & 7.1 & 294 & 73 & 67 & .91 & 20.5 \\
\hline JULY & 5.6 & 73 & 26 & 17 & .66 & 7.4 \\
\hline $\begin{array}{l}\text { AUGUST } \\
\text { SEPTEMBER }\end{array}$ & 4.6 & 69 & 24 & 15 & .63 & 6.6 \\
\hline & & & & & & \\
\hline ANNUAE & 8.9 & 75 & 30 & 17 & .57 & 100 \\
\hline
\end{tabular}

MAGNITUDE AND PROBABILITY OF ANNUAL LOW FLOW BASED ON PERIOD OF RECORD 1934-85

\begin{tabular}{|c|c|c|c|c|c|c|}
\hline \multirow{2}{*}{$\begin{array}{l}\text { PERIOD } \\
\text { (CON- } \\
\text { SECU- } \\
\text { TIVE } \\
\text { DAYS) }\end{array}$} & \multicolumn{6}{|c|}{$\begin{array}{l}\text { DISCHARGE, IN FT'/S, FOR INDICATED RECURRENCE } \\
\text { INTERVAL, IN YEARS, AND ANNUAL NON- } \\
\text { EXCEEDANCE PROBABIL ITY, IN PERCENT }\end{array}$} \\
\hline & ${ }_{508}^{2}$ & $\begin{array}{c}5 \\
208\end{array}$ & $\begin{array}{l}10 \\
108\end{array}$ & $\begin{array}{l}20 \\
58\end{array}$ & $\begin{array}{l}50 \\
28\end{array}$ & $\begin{array}{r}100 \\
1:\end{array}$ \\
\hline $\begin{array}{r}1 \\
3 \\
7 \\
14 \\
30 \\
60 \\
90 \\
120 \\
183\end{array}$ & $\begin{array}{r}4.8 \\
5.8 \\
6.8 \\
7.4 \\
8.0 \\
8.4 \\
8.7 \\
9.0 \\
10\end{array}$ & $\begin{array}{l}3.3 \\
4.3 \\
5.1 \\
5.5 \\
6.1 \\
6.5 \\
6.8 \\
7.1 \\
7.8\end{array}$ & $\begin{array}{l}2.6 \\
3.6 \\
4.3 \\
4.6 \\
5.2 \\
5.7 \\
6.0 \\
6.3 \\
6.9\end{array}$ & $\begin{array}{l}2.1 \\
3.1 \\
3.7 \\
3.9 \\
4.4 \\
5.0 \\
5.3 \\
5.7 \\
6.3\end{array}$ & $\begin{array}{l}1.7 \\
2.6 \\
3.1 \\
3.2 \\
3.7 \\
4.3 \\
4.7 \\
5.1 \\
5.8\end{array}$ & $\begin{array}{l}1.4 \\
2.3 \\
2.7 \\
2.7 \\
3.2 \\
3.8 \\
4.3 \\
4.7 \\
5.5\end{array}$ \\
\hline
\end{tabular}

MAGNITUDE AND PROBABILITY OF ANNUAL HIGH FLOW BASED ON PERIOD OF RECORD 1933-85

\begin{tabular}{|c|c|c|c|c|c|c|}
\hline \multirow{2}{*}{$\begin{array}{l}\text { PERIOD } \\
\text { (CON- } \\
\text { SECU- } \\
\text { TIVE } \\
\text { DAYS) }\end{array}$} & \multicolumn{6}{|c|}{$\begin{array}{l}\text { DISCHARGE, IN FT'/S, FOR INDICATED RECURRENCE } \\
\text { INTERVAL, IN YEARS, AND ANNUAL } \\
\text { EXCEEDANCE PROBABILITY, IN PERCENT }\end{array}$} \\
\hline & $\begin{array}{c}2 \\
508\end{array}$ & $\begin{array}{c}5 \\
208\end{array}$ & $\begin{array}{l}10 \\
108\end{array}$ & $\begin{array}{l}25 \\
48\end{array}$ & $\begin{array}{l}50 \\
28\end{array}$ & $\begin{array}{r}100 \\
1 \%\end{array}$ \\
\hline $\begin{array}{r}1 \\
3 \\
7 \\
15 \\
30 \\
60 \\
90\end{array}$ & $\begin{array}{r}118 \\
111 \\
104 \\
95 \\
85 \\
71 \\
59\end{array}$ & $\begin{array}{l}223 \\
209 \\
195 \\
178 \\
162 \\
133 \\
109\end{array}$ & $\begin{array}{l}315 \\
292 \\
269 \\
246 \\
224 \\
184 \\
149\end{array}$ & $\begin{array}{l}459 \\
417 \\
378 \\
344 \\
314 \\
258 \\
208\end{array}$ & $\begin{array}{l}588 \\
525 \\
469 \\
425 \\
389 \\
320 \\
257\end{array}$ & $\begin{array}{l}737 \\
647 \\
568 \\
513 \\
470 \\
388 \\
310\end{array}$ \\
\hline
\end{tabular}

DURATION T'ABLE OF DAILY MEAN FLOW FOR PERIOD OF RECORD 1933-85 DISCHARGE, IN FT'/S, THAT WAS EQUALED OR EXCEEDED FOR INDICATED PERCENT OF TIME

\begin{tabular}{|c|c|c|c|c|c|c|c|c|c|c|c|c|c|c|}
\hline $5 \%$ & 108 & $15 \%$ & $20 \%$ & 258 & $30 \%$ & 408 & 508 & $60 \%$ & 708 & $75 \%$ & 802 & 858 & 908 & 958 \\
\hline 110 & 72 & 51 & 38 & 29 & 24 & 18 & 14 & 12 & 10 & 9.4 & 8.6 & 7.9 & 7.2 & 6.0 \\
\hline
\end{tabular}


RIO GRANDE BASIN

08294300 RIO NAMBE AT NAMBE FALLS, NEAR NAMBE, NM

LOCATION.--Lat $35^{\circ} 50^{\prime} 46^{\prime \prime}$, long $105^{\circ} 54^{\prime} 29^{\prime \prime}$, in NWłSWł sec.29, T.19 N., R.10 E., Santa Fe County, Hydrologic Unit 13020101 , in Nambe Indian Reservation, on left bank $800 \mathrm{ft}$ downstream from Nambe Falls, 1,100 ft downstream from Nambe Falls Dam, $2.4 \mathrm{mi}$ upstream from Rio En Medio, $4.2 \mathrm{mi}$ southeast of Nambe Pueblo and $5.2 \mathrm{mi}$ southeast of Nambe.

DRAINAGE AREA. $--34.2 \mathrm{mi}^{2}$.

PERIOD OF RECORD.--March 1963 to December 1978 (discontinued).

REVISED RECORDS.--WDR NM-77-1: Drainage area.

GAGE.-Water-stage recorder and concrete control. Datum of gage is $6,513.68 \mathrm{ft}$ above National Geodetic Vertical Datum, 1929 (levels by Bureau of Reclamation).

REMARKS.--Flow regulated by Nambe Falls Reservoir (station 08294200) since Feb. 22, 1976. Outlet conduits are one 6-in and two 12-in diameter pipes. No diversions above station.

COOPERATION.--Records furnished by Bureau of Reclamation.

AVERAGE DISCHARGE.--15 years (water years 1964-78), $10.7 \mathrm{ft}^{3} / \mathrm{s}, 7,750$ acre-ft/yr.

EXTREMES FOR PERIOD OF RECORD.--Maximum discharge, 1,090 $\mathrm{ft}^{3} / \mathrm{s}$ Aug. 8, 1967, gage height, about $6.0 \mathrm{ft}$, from floodmarks, from rating curve extended above $44 \mathrm{ft}^{3 / \mathrm{s}}$ on bas is of field estimate of peak flow; minimum daily, $0.30 \mathrm{ft} / \mathrm{s}$ Aug. 21 , 1977 .

STATISTICAL SUMMARIES

MEAN MONTHLY AND MEAN ANNUAL DISCHARGES 1964-78

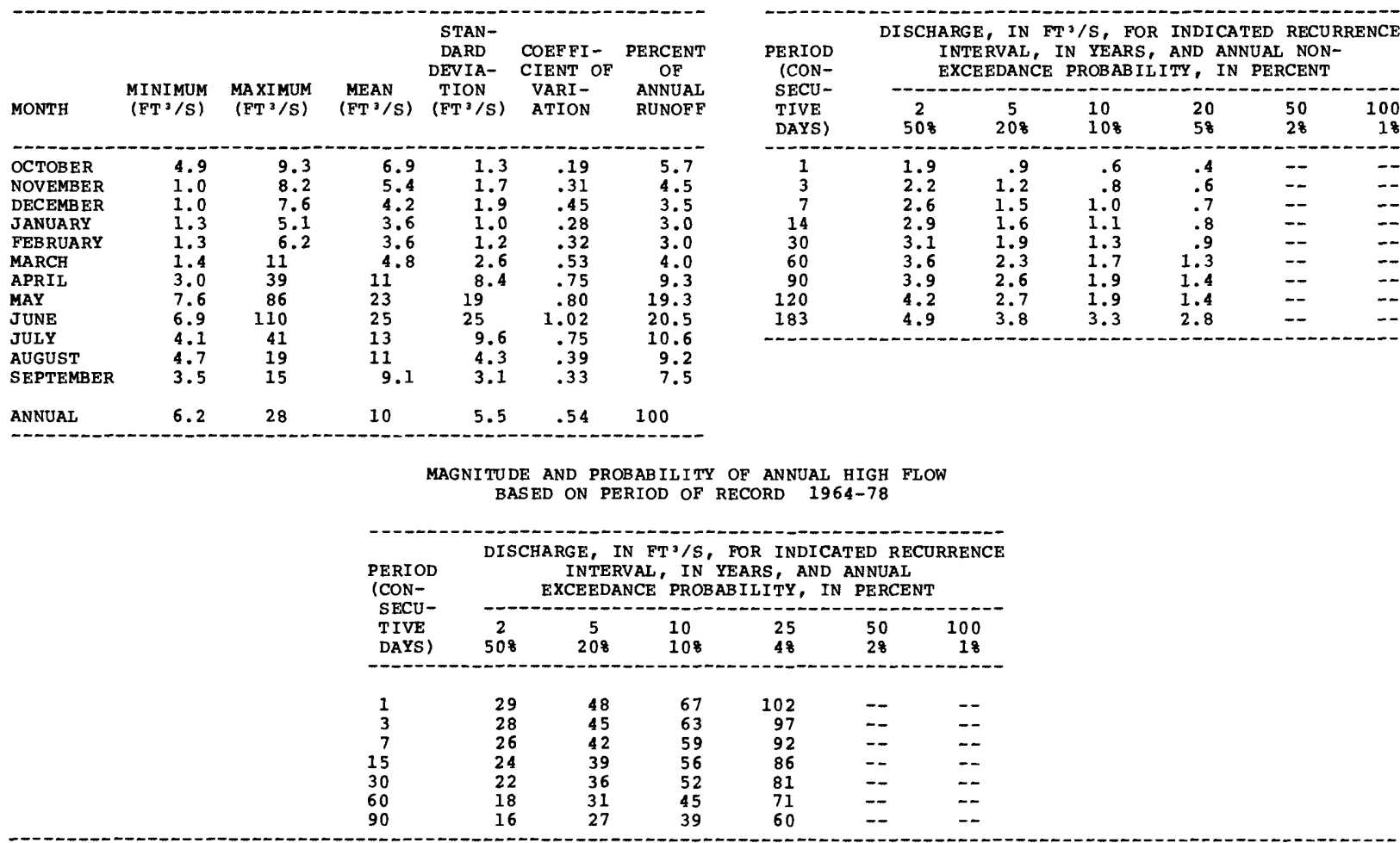

DURATION TABLE OF DAILY MEAN FLOW FOR PERIOD OF RECORD 1964-78

\begin{tabular}{|c|c|c|c|c|c|c|c|c|c|c|c|c|c|c|}
\hline 58 & 108 & 158 & 208 & 258 & 308 & 408 & 508 & 608 & 708 & 758 & 808 & 858 & 908 & 958 \\
\hline 28 & 21 & 17 & 14 & 12 & 9.8 & 7.9 & 6.6 & 5.6 & 4.8 & 4.4 & 4.0 & 3.7 & 3.3 & \\
\hline
\end{tabular}


RIO GRANDE BASIN

08295000 RIO NAMBE NEAR NAKBE, NM

LOCATION,--Lat $35^{\circ} 52^{\prime}$, long $105^{\circ} 57^{\prime}$, in sec.24, T. 19 N., R.9 E., on right bank, in Nambe Pueblo Grant, 1,000 ft downstream from diversion dam for Nambe Canal, $2 \frac{1}{2} \mathrm{i}$ southeast of Nambe, and $6 \frac{1}{2} \mathrm{~m}$ upstream from Rio Tesuque.

DRA INAGE AREA. $--37.0 \mathrm{mi} 2$.

PERIOD OF RECORD.--October 1932 to September 1951.

GAGE.--Water-stage recorder and concrete control. Altitude of gage is 6,200 ft (from topographic map).

REMARKS.--Nambe Canal diverts water 1,000 ft upstream for irrigation of about 200 acres below station.

AVERAGE DISCHARGE.--19 years (water years 1933-51), $10.7 \mathrm{ft} / \mathrm{s}, 7,750 \mathrm{acre}-\mathrm{ft} / \mathrm{yr}$.

EXTREMES FOR PERIOD OF RECORD.--Maximum discharge, $878 \mathrm{ft} / \mathrm{s}$ Aug. 23,1935 (gage height, 6.43 ft), from rating curve extended above $78 \mathrm{ft}^{3} / \mathrm{s}$ by logarithmic plotting; no flow for several days in October 1934.

STATISTICAL SUMMARIES

MEAN MONTHLY AND MEAN ANNUAL DISCHARGES 1933-51

\begin{tabular}{|c|c|c|c|c|c|c|}
\hline MONTH & $\begin{array}{c}\text { MINI MUM } \\
\left(\mathrm{FT}^{3} / \mathrm{S}\right)\end{array}$ & $\begin{array}{l}\text { MAXIMUM } \\
\left(F^{3} / \mathrm{S}\right)\end{array}$ & $\begin{array}{l}\text { MEAN } \\
\left(F T^{3} / S\right)\end{array}$ & $\begin{array}{l}\text { STAN- } \\
\text { DARD } \\
\text { DEVIA- } \\
\text { TION } \\
(\text { FT } 3 / \mathrm{S})\end{array}$ & $\begin{array}{l}\text { COEFFI- } \\
\text { CIENT OF } \\
\text { VARI - } \\
\text { ATION }\end{array}$ & $\begin{array}{c}\text { PERCENT } \\
\text { OF } \\
\text { ANNUAL } \\
\text { RUNOF F }\end{array}$ \\
\hline OCTOBER & 2.1 & 27 & 7.3 & 5.2 & .72 & 5.8 \\
\hline NOVEMBER & 12 & 20 & 53 & 38 & 71 & \\
\hline DECEIBER & 8 & 10 & 3.9 & 19 & 48 & 4.2 \\
\hline JANUARY & .7 & 7.3 & 3.6 & 1.3 & .35 & 3.1 \\
\hline FEBRUARY & 1.0 & 5.3 & 3.4 & 1.0 & .30 & 2.7 \\
\hline MARCH & 1.3 & 8.1 & 4.8 & 2.0 & .41 & 3.8 \\
\hline APRIL & 3.4 & 55 & 15 & 11 & .76 & 11.6 \\
\hline MAY & 4.7 & 106 & 32 & 25 & .80 & 25.0 \\
\hline JUNE & 3.3 & 96 & 25 & 24 & .95 & 19.8 \\
\hline JULY & 1.4 & 45 & 10 & 9.8 & .95 & 8.1 \\
\hline AUGUST & 2.5 & 21 & 8.6 & 5.1 & .60 & 6.8 \\
\hline SEPTEMBER & 2.3 & 23 & 8.0 & & .67 & 6.3 \\
\hline ANNUAL & 3.2 & 29 & 11 & 6.5 & .62 & 100 \\
\hline
\end{tabular}

MAGNITUDE AND PROBABILITY OF ANNUAL LOW FLON BASED ON PERIOD OF RECORD 1934-51

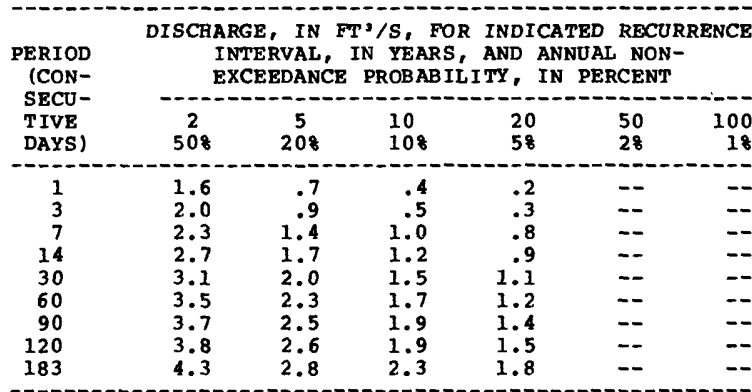

MAGNITUDE AND PROBABILITY OF ANNUAL HIGH FLOW BASED ON PERIOD OF RECORD 1933-51

\begin{tabular}{|c|c|c|c|c|c|c|}
\hline \multirow{2}{*}{$\begin{array}{l}\text { PERIOD } \\
\text { (CON- } \\
\text { SECU- } \\
\text { TIVE } \\
\text { DAYS) }\end{array}$} & \multicolumn{6}{|c|}{$\begin{array}{l}\text { DISCHARGE, IN FT'/S, FOR INDICATED RECURRENCE } \\
\text { INTERVAL, IN YEARS, AND ANNUAL } \\
\text { EXCEEDANCE PROBAB ILI TY, IN PERCENT }\end{array}$} \\
\hline & $\stackrel{2}{508}$ & $\begin{array}{c}5 \\
208\end{array}$ & $\begin{array}{l}10 \\
108\end{array}$ & $\begin{array}{l}25 \\
48\end{array}$ & $\begin{array}{l}50 \\
28\end{array}$ & $\begin{array}{r}100 \\
18\end{array}$ \\
\hline $\begin{array}{r}1 \\
3 \\
7 \\
15 \\
30 \\
60 \\
90\end{array}$ & $\begin{array}{l}40 \\
36 \\
34 \\
31 \\
28 \\
24 \\
20\end{array}$ & $\begin{array}{l}69 \\
64 \\
62 \\
57 \\
53 \\
45 \\
37\end{array}$ & $\begin{array}{l}96 \\
89 \\
85 \\
79 \\
74 \\
63 \\
51\end{array}$ & $\begin{array}{r}142 \\
130 \\
119 \\
111 \\
104 \\
89 \\
73\end{array}$ & $\begin{array}{l}-- \\
-- \\
-- \\
-- \\
--\end{array}$ & $\begin{array}{l}-- \\
=- \\
-- \\
-- \\
-- \\
--\end{array}$ \\
\hline
\end{tabular}

DURATION TABLE OF DAILY MEAN FLOW FOR PERIOD OF RECORD 1933-5I DISCHARGE, IN FT $3 / \mathrm{S}$, THAT WAS EQUALED OR EXCEEDED FOR INDICATED PERCENT OF TIME

\begin{tabular}{|c|c|c|c|c|c|c|c|c|c|c|c|c|c|c|}
\hline 58 & 108 & 158 & 208 & 258 & 308 & 408 & 508 & 608 & 708 & 758 & 808 & 858 & 908 & 958 \\
\hline 37 & 25 & 18 & 14 & 11 & 9.1 & 6.6 & 5.3 & 4.5 & 3.8 & 3.5 & 3.2 & 2.9 & 2.5 & \\
\hline
\end{tabular}


RIO GRANDE BASIN

08302500 TESUQUE CREEK ABOVE DIVERSIONS, NEAR SANTA FE, NM

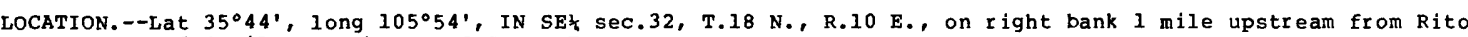
Tesuque and 4 miles nor theast of Santa $\mathrm{Fe}$.

DRAINAGE AREA. $--11 \mathrm{mi}^{2}$, approximately.

PERIOD OF RECORD.--March 1936 to January 1952 (discontinued) in reports of Geological survey. May to October 1919 in reports of state Engineer.

GAGE.--Water-stage recorder and concrete control. Altitude of gage is $7,100 \mathrm{ft}$ (from topographic map). May to October 1919 at site $175 \mathrm{ft}$ downstream at datum 6.00 ft lower.

REMARKS.- One diversion for irrigation of about 2 acres above station.

AVERAGE DISCHARGE.--15 years (water years 1937-51), $3.21 \mathrm{ft}^{3} / \mathrm{s}, 2,330 \mathrm{acre}-\mathrm{ft} / \mathrm{yr}$.

EXTREMES FOR PERIOD OF RECORD.--Maximum discharge, $425 \mathrm{ft} / \mathrm{s}$ July 19, 1938 (gage height, $4.2 \mathrm{ft}, \mathrm{from}$ floodmark), from rating curve extended above $10 \mathrm{ft}^{3} / \mathrm{s}$ on basis of slope-area determination at gage height $4.0 \mathrm{ft}$; no $\mathrm{flow}$ Aug. $25,31,1950$, July 11,1951

STATISTICAL SUMMARIES

MEAN MONTHLY AND MEAN ANNUAL DISChaRGES 1937-51

\begin{tabular}{|c|c|c|c|c|c|c|}
\hline MONTH & $\begin{array}{c}\text { MINIMUM } \\
\left(\mathrm{FT}^{3} / \mathrm{S}\right)\end{array}$ & $\begin{array}{c}\operatorname{MAXIMUM} \\
\left(\mathrm{FT}^{3} / \mathrm{S}\right)\end{array}$ & $\begin{array}{l}\text { MEAN } \\
\left(\mathrm{FT}^{3} / \mathrm{S}\right)\end{array}$ & $\begin{array}{l}\text { STAN- } \\
\text { DARD } \\
\text { DEVIA- } \\
\text { TION } \\
\left(\text { FT }^{3} / \mathrm{S}\right)\end{array}$ & $\begin{array}{l}\text { COEFFI - } \\
\text { CIENT OF } \\
\text { VARI - } \\
\text { ATION }\end{array}$ & $\begin{array}{c}\text { PERCENT } \\
\text { OF } \\
\text { ANNUAL } \\
\text { RUNOFF }\end{array}$ \\
\hline OCTOBER & .4 & 10 & 2.0 & 2.4 & 1.17 & 5.3 \\
\hline NOVEMBE $R$ & .4 & 6.6 & $\begin{array}{l}2.0 \\
1.5\end{array}$ & $\begin{array}{l}2.4 \\
1.5\end{array}$ & $\begin{array}{r}1.17 \\
.98\end{array}$ & 3.9 \\
\hline DECEMBE R & .4 & 2.7 & 1.1 & .5 & .48 & 2.8 \\
\hline JANUA RY & .4 & 2.0 & 1.0 & .4 & .36 & 2.6 \\
\hline FEBRUARY & .5 & 2.0 & 1.0 & .4 & .35 & 2.7 \\
\hline MARCH & .4 & 4.3 & 2.1 & 1.2 & .57 & 5.4 \\
\hline APRIL & .8 & 26 & 6.3 & 6.1 & .97 & 16.4 \\
\hline MAY & .8 & 37 & 11 & 9.6 & .89 & 28.1 \\
\hline JUNE & .5 & 27 & 7.0 & 6.7 & .96 & 18.2 \\
\hline JULY & .3 & 7.7 & 2.5 & 2.1 & .87 & 6.4 \\
\hline AUGUST & .3 & 3.7 & 1.7 & 1.1 & .61 & 4.5 \\
\hline SEPTEMBER & .4 & 4.4 & 1.5 & .9 & .64 & 3.9 \\
\hline ANNUAL & .7 & 8.1 & 3.2 & 2.2 & .68 & 100 \\
\hline
\end{tabular}

MAGNITUDE AND PROBABILITY OF ANNUAL LOW FLOW BASED ON PERIOD OF RECORD 1938-51

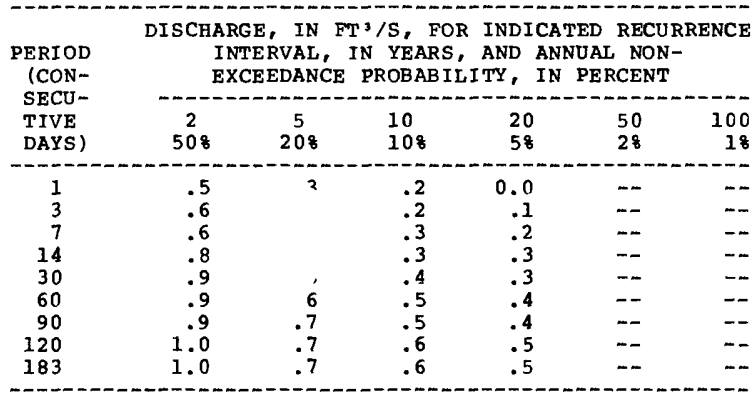

MAGNITUDE AND PROBABILITY OF ANNUAL HIGH FLOW BASED ON PERIOD OF RECORD 1937-51

\begin{tabular}{|c|c|c|c|c|c|c|}
\hline \multirow{2}{*}{$\begin{array}{l}\text { PERIOD } \\
\text { (CON- } \\
\text { SECU- } \\
\text { TIVE } \\
\text { DAYS) }\end{array}$} & \multicolumn{6}{|c|}{$\begin{array}{l}\text { DISCHARGE, IN FT }{ }^{3} / \mathrm{S} \text {, FOR INDICATED RECURRENCE } \\
\text { INTERVAL, IN YEARS, AND ANNUAL } \\
\text { EXCEEDANCE PROBABILITY, IN PERCENT }\end{array}$} \\
\hline & $\begin{array}{c}2 \\
508\end{array}$ & $\begin{array}{c}5 \\
208\end{array}$ & & $\begin{array}{l}25 \\
48\end{array}$ & 28 & $\begin{array}{r}100 \\
18\end{array}$ \\
\hline
\end{tabular}

$\begin{array}{rrrrrrr}1 & 13 & 28 & 41 & 59 & -- & -- \\ 3 & 12 & 25 & 37 & 54 & -- & - \\ 7 & 11 & 24 & 34 & 49 & -- & - \\ 15 & 10 & 22 & 32 & 45 & -- & - \\ 30 & 9.0 & 19 & 27 & 39 & -- & - \\ 60 & 7.7 & 16 & 22 & 31 & -- & - \\ 90 & 6.5 & 13 & 18 & 25 & -- & -\end{array}$

DURATION TABLE OF DAILY MEAN FLOW FOR PERIOD OF RECORD 1937-51 DISCHARGE, IN FT'/S, THAT WAS EQUALED OR EXCEEDED FOR INDICATED PERCENT OF TIME

\begin{tabular}{|c|c|c|c|c|c|c|c|c|c|c|c|c|c|c|}
\hline 58 & 108 & 158 & 208 & 258 & 308 & 408 & 508 & 608 & 708 & 758 & 808 & 858 & 908 & 958 \\
\hline 13 & 8.0 & 5.6 & 4.1 & 3.1 & 2.5 & 1.8 & 1.4 & 1.2 & 1.0 & .9 & .8 & .7 & .6 & .5 \\
\hline
\end{tabular}




\section{RIO GRANDE BASIN}

08313000 RIO GRANDE AT OTOWI BRIDGE, NEAR SAN ILDEFONSO, NM

(National stream-quality accounting network, surveillance network, and radiochemical network station)

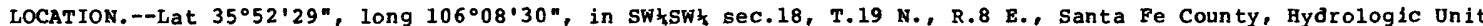
13020101 , in San Ildefonso pueblo Grant, near right bank on downstream end of pier of former railway bridge, 400 ft downstream from bridge on State Highway $4,1.8 \mathrm{mi}$ southwest of San Ildefonso pueblo, $2.5 \mathrm{mi}$ downstream from ft downstream from bridge on State Highway $4,1.8 \mathrm{mi}$ southwest
Pojoaque River, $6.8 \mathrm{mi}$ west of Pojoaque, and at mile $1,614.2$.

DRAINAGE AREA.--14,300 $\mathrm{mi}^{2}$, approximately, including 2,940 $\mathrm{mi} 2$ in closed basin in san Luis Valley, co.

PERIOD OF RECORD.--February 1895 to December 1905, June 1909 to current year. Monthly discharge only for some periods, published in WSP 1312. In early reports this record was published as "at Water Tank," as "at Rio Grande," and as "near Buckman."

REVISED RECORDS.--WSP 828: Drainage area. WSP 1512: 1895-99, 1904-6, 1911-12, 1914, 1931(M), 1935. WSP 1712: $1904(M)$.

GAGE.-Water-stage recorder. Datum of gage is $5,488.48 \mathrm{ft}$ above National Geodetic Vertical Datum of 1929 . See wSP 1312 , 1732, or 1923 for history of changes prior to June $1,1910$.

REMARRS.- Considerable regulation by Heron Reservoir (station 08284510), El Vado Reservoir (station 08285000) and Abiquiu Reservoir (station 08286900) on Rio Chama, which can contribute a major portion of the total flow. Flow affected by release of transmountain water from Heron Reservoir since May 1971. Diversions upstream from station for irrigation of about 620,000 acres in Colorado and 75,000 acres in New Mexico. Gage-height telemeter at station.

AVERAGE DISCHARGE. - -59 years (water years 1896-1905, 1910-14, 1919-62), 1,495 ft/s, 1,083,000 acre-ft/yr.

EXTREMES FOR PERIOD OF RECORD,--Maximum discharge, 24,400 ft $/ \mathrm{s}$, May 23, 1920 ; maximum gage height, $14.5 \mathrm{ft}$, Sept. 29, 1904, present site and datum; minimum daily discharge, $60 \mathrm{ft} 3 / \mathrm{s}, \mathrm{Ju} 2 \mathrm{y} 4,5,1902$.

EXTREMES OUTSIDE PERIOD OF RECORD.--The $1920 \mathrm{flood}$ is greatest since at least 1884 and probably since 1741 ; information from $W$. H. Yeo's file on floods.

STATISTICAL SUMMARIES (PERIOD BEFORE REGULATION OF ABIQUIU DAM)

MEAN MONTHLY AND MEAN ANNUAL DISChARGES 1896-1905, $1910-14$ $1919-62$

\begin{tabular}{|c|c|c|c|c|c|c|}
\hline MONTH & $\begin{array}{l}\text { MINIMUM } \\
\left(\mathrm{FT}^{3} / \mathrm{S}\right)\end{array}$ & $\begin{array}{l}\text { MAXI MUM } \\
\left(\mathrm{FT}^{3} / \mathrm{S}\right)\end{array}$ & $\begin{array}{l}\text { MEAN } \\
\left(\mathrm{FT}^{3} / \mathrm{S}\right)\end{array}$ & $\begin{array}{l}\text { STAN- } \\
\text { DARD } \\
\text { DEVIA- } \\
\text { TION } \\
\text { (FT'/S) }\end{array}$ & $\begin{array}{l}\text { COEFFI - } \\
\text { CIENT OF } \\
\text { VARI - } \\
\text { ATION }\end{array}$ & $\begin{array}{c}\text { PERCENT } \\
\text { OF } \\
\text { ANNUAL } \\
\text { RUNOFF }\end{array}$ \\
\hline $\begin{array}{l}\text { OCTOBER } \\
\text { NOVEMBER } \\
\text { DECEMBER } \\
\text { JANUARY } \\
\text { FEBRUARY } \\
\text { MARCH } \\
\text { APRIL } \\
\text { MAY } \\
\text { JUNE } \\
\text { JULY } \\
\text { AUGUST } \\
\text { SEPTEMBER }\end{array}$ & $\begin{array}{l}168 \\
281 \\
313 \\
376 \\
445 \\
401 \\
366 \\
433 \\
274 \\
179 \\
165 \\
191\end{array}$ & $\begin{array}{r}5475 \\
2421 \\
1959 \\
1326 \\
2191 \\
3251 \\
7329 \\
14590 \\
11920 \\
5692 \\
3308 \\
3411\end{array}$ & $\begin{array}{r}769 \\
862 \\
757 \\
646 \\
780 \\
1154 \\
2296 \\
4395 \\
3386 \\
1317 \\
899 \\
754\end{array}$ & $\begin{array}{r}756 \\
469 \\
351 \\
159 \\
251 \\
520 \\
1571 \\
3288 \\
2753 \\
1069 \\
548 \\
563\end{array}$ & $\begin{array}{l}.98 \\
.54 \\
.46 \\
.25 \\
.32 \\
.45 \\
.68 \\
.75 \\
.81 \\
.81 \\
.61 \\
.75\end{array}$ & $\begin{array}{r}4.3 \\
4.8 \\
4.2 \\
3.6 \\
4.3 \\
6.4 \\
12.7 \\
24.4 \\
18.8 \\
7.3 \\
5.0 \\
4.2\end{array}$ \\
\hline NNUAL & 498 & 3322 & 1495 & 710 & .47 & 100 \\
\hline
\end{tabular}

MAGNITUDE AND PROBABILITY OF ANNUAL LOW FLOW BASED ON PERIOD OF RECORD 1897-1905, 1911-14, $1920-62$

\begin{tabular}{|c|c|c|c|c|c|c|}
\hline \multirow{2}{*}{$\begin{array}{l}\text { PERIOD } \\
\text { (CON- } \\
\text { SECU- } \\
\text { TIVE } \\
\text { DAYS) }\end{array}$} & \multicolumn{6}{|c|}{$\begin{array}{l}\text { DISCHARGE, IN FT'/S, FOR INDICATED RECURRENCE } \\
\text { INTERVAL, IN YEARS, AND ANNUAL NON- } \\
\text { EXCEEDANCE PROBABILITY, IN PERCENT }\end{array}$} \\
\hline & $\begin{array}{c}2 \\
508\end{array}$ & $\begin{array}{c}5 \\
208\end{array}$ & $\begin{array}{l}10 \\
108\end{array}$ & $\begin{array}{l}20 \\
58\end{array}$ & $\begin{array}{l}50 \\
28\end{array}$ & $\begin{array}{r}100 \\
18\end{array}$ \\
\hline $\begin{array}{r}1 \\
3 \\
7 \\
14 \\
30 \\
60 \\
90 \\
120 \\
183\end{array}$ & $\begin{array}{l}279 \\
290 \\
310 \\
340 \\
398 \\
450 \\
512 \\
526 \\
595\end{array}$ & $\begin{array}{l}173 \\
180 \\
194 \\
216 \\
263 \\
309 \\
364 \\
379 \\
430\end{array}$ & $\begin{array}{l}131 \\
136 \\
148 \\
168 \\
208 \\
252 \\
303 \\
320 \\
369\end{array}$ & $\begin{array}{l}103 \\
107 \\
117 \\
135 \\
170 \\
212 \\
259 \\
278 \\
329\end{array}$ & $\begin{array}{r}77 \\
80 \\
88 \\
104 \\
135 \\
173 \\
217 \\
238 \\
291\end{array}$ & $\begin{array}{r}63 \\
65 \\
72 \\
87 \\
115 \\
151 \\
192 \\
214 \\
269\end{array}$ \\
\hline
\end{tabular}

MAGNITUDE AND PROBABILITY OF ANNUAL HIGH FLOW BASED ON PERIOD OF RECORD 1896-1905, 1910-14, 1919-62

\begin{tabular}{|c|c|c|c|c|c|c|}
\hline \multirow{2}{*}{$\begin{array}{l}\text { PERIOD } \\
\text { (CON- } \\
\text { SECU- } \\
\text { TIVE } \\
\text { DAYS) }\end{array}$} & \multicolumn{6}{|c|}{$\begin{array}{c}\text { DISCHARGE, IN FT } 3 / \mathrm{S} \text {, FOR INDICATED RECURRENCE } \\
\text { INTERVAL, IN YEARS, AND ANNUAL } \\
\text { EXCEEDANCE PROBABILITY, IN PERCENT }\end{array}$} \\
\hline & $\begin{array}{c}2 \\
508\end{array}$ & $\begin{array}{c}5 \\
208\end{array}$ & $\begin{array}{l}10 \\
108\end{array}$ & $\begin{array}{l}25 \\
48\end{array}$ & $\begin{array}{l}50 \\
28\end{array}$ & $\begin{array}{r}100 \\
18\end{array}$ \\
\hline $\begin{array}{r}1 \\
3 \\
7 \\
15 \\
30 \\
60 \\
90\end{array}$ & $\begin{array}{l}6150 \\
5720 \\
5260 \\
4790 \\
4260 \\
3870 \\
3190\end{array}$ & $\begin{array}{r}11000 \\
10300 \\
9620 \\
8780 \\
7860 \\
7010 \\
5620\end{array}$ & $\begin{array}{r}14600 \\
13900 \\
13100 \\
11900 \\
10600 \\
9320 \\
7380\end{array}$ & $\begin{array}{r}19500 \\
18800 \\
18000 \\
16400 \\
14500 \\
12400 \\
9700\end{array}$ & $\begin{array}{l}23300 \\
22800 \\
22000 \\
20100 \\
17700 \\
14800 \\
11500\end{array}$ & $\begin{array}{l}27400 \\
27000 \\
26300 \\
24000 \\
21000 \\
17200 \\
13200\end{array}$ \\
\hline
\end{tabular}

DURATION TABLE OF DAILY MEAN FLOW FOR PERIOD OF RECORD 1896-1905, 1910-14, 1919-62 DISCHARGE, IN FT'/S, THAT WAS EQUALED OR EXCEEDED FOR INDICATED PERCENT OF TIME

\begin{tabular}{|c|c|c|c|c|c|c|c|c|c|c|c|c|c|c|}
\hline 58 & 108 & 158 & 208 & 258 & 308 & 408 & 508 & 608 & 708 & 758 & 808 & $85 \%$ & 908 & 958 \\
\hline 6120 & 3680 & 2520 & 1880 & 1500 & 1250 & 954 & 779 & 666 & 570 & 523 & 471 & 414 & 348 & 268 \\
\hline
\end{tabular}


RIO GRANDE BASIN

08313000 RIO GRANDE AT OTOWI BRIDGE, NM--Continued

STATISTICAL SUMMARIES (PERIOD AFTER COMPLETION OF HERON DAM)

MEAN MONTHLY AND MEAN ANNUAL DISCHARGES 1971-85

MAGNITUDE AND PROBABILITY OF ANNUAL LOW FLOW BASED ON PERIOD OF RECORD 1972-85

\begin{tabular}{|c|c|c|c|c|c|c|}
\hline MONTH & $\begin{array}{l}\text { MINIMUM } \\
\left(\text { FT }^{3} / \mathrm{S}\right)\end{array}$ & $\begin{array}{l}\text { MAXI MUM } \\
\left(\mathrm{FT}^{3} / \mathrm{S}\right)\end{array}$ & $\begin{array}{l}\text { MEAN } \\
\left(\mathrm{FT}^{3} / \mathrm{S}\right)\end{array}$ & $\begin{array}{c}\text { STAN- } \\
\text { DARD } \\
\text { DEVIA- } \\
\text { TION } \\
\left(\text { FT }^{3} / \mathrm{S}\right)\end{array}$ & $\begin{array}{l}\text { COEFFI- } \\
\text { CIENT OF } \\
\text { VARI - } \\
\text { ATION }\end{array}$ & $\begin{array}{c}\text { PERCENT } \\
\text { OF } \\
\text { ANNUAL } \\
\text { RUNOFF }\end{array}$ \\
\hline \multicolumn{7}{|c|}{ n-n } \\
\hline OCTOBER & 361 & 1026 & 665 & 200 & .30 & 3.8 \\
\hline NOVFMBER & 401 & 1618 & 949 & 397 & .42 & 5.4 \\
\hline DECEMBF, R & 450 & 1959 & 959 & 466 & .49 & 5.4 \\
\hline JANUARY & 436 & 1326 & 718 & 242 & .34 & 4.1 \\
\hline FEB RUARY & 500 & 1021 & 727 & 158 & .22 & 4.1 \\
\hline MARCH & 612 & 2346 & 1170 & 450 & .38 & 6.6 \\
\hline APRII. & 489 & 6412 & 2017 & 1528 & .76 & 11.5 \\
\hline MAY & 433 & 8390 & 3718 & 2639 & .71 & 21.1 \\
\hline JUNE & 470 & 7914 & 3476 & 2489 & .72 & 19.7 \\
\hline JULY & 394 & 3579 & 1592 & 1043 & .66 & 9.0 \\
\hline AUGUST & 391 & 1612 & 891 & 309 & .35 & 5.1 \\
\hline SEPTEMBER & 263 & 1547 & 721 & 341 & .47 & 4.1 \\
\hline ANNUAL & 602 & 2672 & 1469 & 658 & .45 & 100 \\
\hline
\end{tabular}

\begin{tabular}{|c|c|c|c|c|c|c|}
\hline \multirow{2}{*}{$\begin{array}{l}\text { PERIOD } \\
\text { (CON- }- \\
\text { SECU- } \\
\text { TIVF } \\
\text { DAYS) }\end{array}$} & \multicolumn{6}{|c|}{$\begin{array}{l}\text { DISCHARGE, IN FT }{ }^{3 / S} \text {, FOR INDICATED RECURRENCE } \\
\text { INTERVAL, IN YEARS, AND ANNUAL NON- } \\
\text { EXCEEDANCE PROBABILITY, IN PERCENT }\end{array}$} \\
\hline & $\begin{array}{c}2 \\
508\end{array}$ & $\begin{array}{c}5 \\
208\end{array}$ & $\begin{array}{l}10 \\
108\end{array}$ & $\begin{array}{l}20 \\
58\end{array}$ & $\begin{array}{l}50 \\
28\end{array}$ & $\begin{array}{r}100 \\
18\end{array}$ \\
\hline $\begin{array}{r}1 \\
3 \\
7 \\
14 \\
30 \\
60 \\
90 \\
120 \\
183\end{array}$ & $\begin{array}{l}282 \\
290 \\
318 \\
367 \\
457 \\
516 \\
577 \\
628 \\
688\end{array}$ & $\begin{array}{l}217 \\
226 \\
249 \\
278 \\
353 \\
402 \\
445 \\
468 \\
512\end{array}$ & $\begin{array}{l}194 \\
203 \\
225 \\
244 \\
308 \\
354 \\
390 \\
403 \\
439\end{array}$ & $\begin{array}{l}179 \\
188 \\
209 \\
220 \\
275 \\
320 \\
350 \\
357 \\
387\end{array}$ & $\begin{array}{l}-- \\
-- \\
-- \\
-- \\
-- \\
-- \\
--\end{array}$ & $\begin{array}{l}-- \\
-- \\
-- \\
=- \\
-- \\
-- \\
-- \\
--\end{array}$ \\
\hline
\end{tabular}

MAGNITUDE AND PROBABIL.ITY OF ANNUAL HIGH FLON BASED ON PERIOD OF RECORD 1971-85

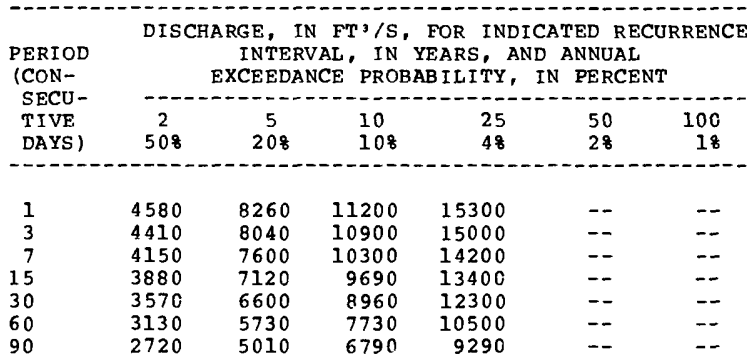

DURATION TABLE OF DAILY MEAN FLOW FOR PERIOD OF RECORD 1971-85

DISCHARGE, IN FT $3 / \mathrm{S}$, THAT WAS EQUALED OR EXCEEDED FOR INDICATED PERCENT OF TIME

\begin{tabular}{|c|c|c|c|c|c|c|c|c|c|c|c|c|c|c|}
\hline 58 & 108 & 158 & 208 & 258 & 308 & 408 & 508 & 608 & 708 & 758 & 808 & 858 & 908 & 958 \\
\hline 5360 & 3710 & 2340 & 1700 & 1450 & 1270 & 1030 & 867 & 749 & 641 & 587 & 536 & 469 & 414 & 334 \\
\hline
\end{tabular}


RIO GRANDE BASIN

08314500 RIO GRANDE AT COCHITI, NM

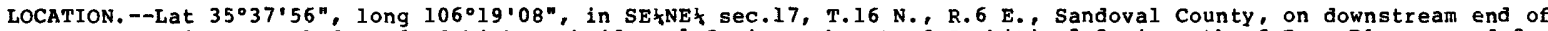
concrete pier near left end of highway bridge, $1.5 \mathrm{mi}$ northeast of Cochiti, $3.2 \mathrm{mi}$ north of Pena Blanca, and 8 miles upstream from Galisteo Creek.

DRAINAGE AREA. - - 14,600 $\mathrm{mi}^{2}$, approximately (includes 2,940 $\mathrm{mi}^{2}$ in closed basin in san Luis Valley, Co.).

PERIOD OF RECORD,--October 1924 to September 1970 (discontinued). Monthly discharge only for some periods, published in WSP 1312. Published as "near Cochiti" prior to 1928.

REVISED RECORDS. --WSP 1312: 1925-29. WSP 1512: 1931-33, 1935, 1939-40(M).

GAGE.--Water-stage recorder. Datum of gage is 5,224.70 ft above mean sea level, datum of 1929 . Prior to July 16 , 1925. staff gage $1 \mathrm{mi}$ upstream at different datum. July 16, 1925 to Jan. 28, 1947, at or near right abutment of bridge at same datum. Jan. 28 to May 15, 1947, $600 \mathrm{ft}$ upstream at same datum.

REMARKS.--Diversions above station for irrigation of about 620,000 acres in Colorado and about 81,000 acres in New Mexico. Cochiti eastside main canal, on left bank, and sili main canal, on right bank, bypass gage for irrigation of about 6,000 acres below station.

AVERAGE DISCHARGE. --44 years (water years 1927-70), 1,301 $\mathrm{ft}^{3} / \mathrm{s}, 942,600$ acre-ft/yr.

EXTREMES FOR PERIOD OF RECORD,--Maximum discharge, 23,400 ft3/s May 15, 1941 (gage height, 10.93 ft); minimum, 0.7 $\mathrm{ft}^{3} / \mathrm{s}$ Aug. $10,11,1934$

The flood of May 23, 1920, probably exceeded 23,400 ft3/s, and is likely the highest since 1905.

STATISTICAL SUMMARIES (PERIOD BEFORE REGULATION OF HERON DAM)

MEAN MONTHLY AND MEAN ANNUAL DISCHARgeS 1927-70

\begin{tabular}{|c|c|c|c|c|c|c|c|c|c|c|c|c|c|}
\hline \multirow[b]{2}{*}{ MONTH } & \multirow{2}{*}{$\begin{array}{l}\text { MI N IMUM } \\
\left(\mathrm{FT}^{3} / \mathrm{S}\right)\end{array}$} & \multirow{2}{*}{$\begin{array}{c}\text { MAXIMUM } \\
\left(\mathrm{FT}^{3} / \mathrm{S}\right)\end{array}$} & \multirow{2}{*}{$\begin{array}{l}\text { MEAN } \\
\left(F^{*} T^{3} / S\right)\end{array}$} & \multirow{2}{*}{$\begin{array}{l}\text { STAN- } \\
\text { DARD } \\
\text { DEVIA- } \\
\text { TION } \\
\left(\text { FT }^{3} / \mathrm{S}\right)\end{array}$} & \multirow{2}{*}{$\begin{array}{l}\text { COEFFI- } \\
\text { CIENT OF } \\
\text { VARI- } \\
\text { ATION }\end{array}$} & \multirow{2}{*}{$\begin{array}{l}\text { PERCENT } \\
\text { OF } \\
\text { ANNUAL } \\
\text { RUNOFF }\end{array}$} & \multirow{2}{*}{$\begin{array}{l}\text { PERIOD } \\
\text { (CON- } \\
\text { SECU- } \\
\text { TIVE } \\
\text { DAYS) }\end{array}$} & \multicolumn{6}{|c|}{$\begin{array}{l}\text { DISCHARGE, IN FT'3/S, FOR INDICATED RECURRENCE } \\
\text { INTERVAL, IN YEARS, AND ANNUAL NON- } \\
\text { EXCEEDANCE PROBABILITY, IN PERCENT }\end{array}$} \\
\hline & & & & & & & & $\begin{array}{c}2 \\
508\end{array}$ & $\begin{array}{c}5 \\
208\end{array}$ & $\begin{array}{l}10 \\
108\end{array}$ & $\begin{array}{l}20 \\
58\end{array}$ & $\begin{array}{l}50 \\
28\end{array}$ & $\begin{array}{r}100 \\
18\end{array}$ \\
\hline $\begin{array}{l}\text { OCTOHER } \\
\text { NOVEMBER } \\
\text { DECEMBER } \\
\text { JANUARY } \\
\text { FEB RUARY } \\
\text { MARCH } \\
\text { APRIL } \\
\text { MAY } \\
\text { JUNE }\end{array}$ & $\begin{array}{r}76 \\
254 \\
357 \\
394 \\
450 \\
378 \\
267 \\
487 \\
148\end{array}$ & $\begin{array}{r}2864 \\
2798 \\
1861 \\
1032 \\
2162 \\
2194 \\
7387 \\
15330 \\
10110\end{array}$ & $\begin{array}{r}578 \\
876 \\
764 \\
650 \\
827 \\
1011 \\
1948 \\
3667 \\
2677\end{array}$ & $\begin{array}{r}536 \\
562 \\
348 \\
124 \\
291 \\
493 \\
1526 \\
3157 \\
2269\end{array}$ & $\begin{array}{l}.93 \\
.64 \\
.46 \\
.19 \\
.35 \\
.49 \\
.78 \\
.86 \\
.85\end{array}$ & $\begin{array}{r}3.7 \\
5.6 \\
4.9 \\
4.2 \\
5.3 \\
6.5 \\
12.5 \\
23.5 \\
17.2\end{array}$ & $\begin{array}{r}1 \\
3 \\
7 \\
14 \\
30 \\
60 \\
90 \\
120 \\
183\end{array}$ & $\begin{array}{l}189 \\
215 \\
265 \\
279 \\
337 \\
401 \\
464 \\
495 \\
595\end{array}$ & $\begin{array}{r}61 \\
75 \\
117 \\
138 \\
175 \\
223 \\
278 \\
308 \\
386\end{array}$ & $\begin{array}{r}26 \\
33 \\
62 \\
87 \\
115 \\
155 \\
203 \\
234 \\
302\end{array}$ & $\begin{array}{r}11 \\
14 \\
33 \\
57 \\
78 \\
111 \\
152 \\
184 \\
244\end{array}$ & $\begin{array}{l}3.5 \\
4.7 \\
14 \\
33 \\
48 \\
74 \\
107 \\
138 \\
189\end{array}$ & $\begin{array}{r}1.5 \\
2.0 \\
7.7 \\
22 \\
34 \\
55 \\
83 \\
113 \\
159\end{array}$ \\
\hline $\begin{array}{l}\text { JULY } \\
\text { AUGUST } \\
\text { SEPTEMBER }\end{array}$ & $\begin{array}{r}71 \\
81 \\
116\end{array}$ & $\begin{array}{l}4276 \\
3217 \\
2894\end{array}$ & $\begin{array}{r}1067 \\
876 \\
664\end{array}$ & $\begin{array}{r}1004 \\
632 \\
539\end{array}$ & $\begin{array}{l}.94 \\
.72 \\
.81\end{array}$ & $\begin{array}{l}6.8 \\
5.6 \\
4.3\end{array}$ & & & & & & & \\
\hline NNUAL & 455 & 3298 & 1301 & 676 & .52 & 100 & & & & & & & \\
\hline
\end{tabular}

MAGNITUDE AND PROBABILITY OF ANNUAL HIGH FLOW BASED ON PERIOD OF RECORD $1927-70$

\begin{tabular}{|c|c|c|c|c|c|c|}
\hline \multirow{2}{*}{$\begin{array}{l}\text { PERIOD } \\
\text { (CON- } \\
\text { SECU- } \\
\text { TIVE } \\
\text { DAYS) }\end{array}$} & \multicolumn{6}{|c|}{$\begin{array}{c}\text { DISCHARGE, IN FT }{ }^{3} / S \text {, FOR INDICATED RECURRENCE } \\
\text { INTERVAL, IN YEARS, AND ANNUAL } \\
\text { EXCEEDANCE PROBABILITY, IN PERCENT }\end{array}$} \\
\hline & $\begin{array}{c}2 \\
508\end{array}$ & $\begin{array}{c}5 \\
208\end{array}$ & $\begin{array}{l}10 \\
108\end{array}$ & $\begin{array}{l}25 \\
48\end{array}$ & $\begin{array}{l}50 \\
28\end{array}$ & $\begin{array}{r}100 \\
18\end{array}$ \\
\hline $\begin{array}{r}1 \\
3 \\
7 \\
15 \\
30 \\
60 \\
90\end{array}$ & $\begin{array}{l}5160 \\
4780 \\
4430 \\
3990 \\
3520 \\
2830 \\
2360\end{array}$ & $\begin{array}{l}9210 \\
8610 \\
8080 \\
7290 \\
6510 \\
5260 \\
4300\end{array}$ & $\begin{array}{r}12300 \\
11600 \\
11000 \\
9980 \\
8940 \\
7250 \\
5870\end{array}$ & $\begin{array}{r}16700 \\
16000 \\
15400 \\
13900 \\
12500 \\
10200 \\
8170\end{array}$ & $\begin{array}{l}20200 \\
19700 \\
19000 \\
17300 \\
15500 \\
12700 \\
10100\end{array}$ & $\begin{array}{l}23900 \\
23600 \\
23000 \\
21000 \\
18900 \\
15400 \\
12200\end{array}$ \\
\hline
\end{tabular}

DURATION TABLE OF DAILY MEAN FLOW FOR PERIOD OF RECORD 1927-70

DISCHARGE, IN FT3/S, THAT WAS EQUALED OR EXCEEDED FOR INDICATED PERCENT OF TIME

\begin{tabular}{|c|c|c|c|c|c|c|c|c|c|c|c|c|c|c|}
\hline 58 & 108 & 158 & 208 & 258 & 308 & 408 & 508 & 608 & 708 & 758 & 808 & 858 & 908 & 958 \\
\hline 4650 & 2920 & 2060 & 1650 & 1340 & 1120 & 900 & 738 & 639 & 537 & 483 & 429 & 355 & 275 & 181 \\
\hline
\end{tabular}


RIO GRANDE BASIN

08316000 SANTA FE RIVER NEAR SANTA FE, NM

LOCATION.--Lat $35^{\circ} 41^{\prime} 12^{\prime \prime}$, long $105^{\circ} 50^{\prime} 35^{\prime \prime}$, in NEłSE sec.23, T.17 N., R.10 E., Santa Fe County, Hydrologic Unit 13020201 , in Santa Fe National Forest, on left bank $0.4 \mathrm{mi}$ downstream from McClure Dam, $5.3 \mathrm{mi}$ east of Santa Fe, and at mile 36.6 .

DRAINAGE AREA. $--18.2 \mathrm{mi}^{2}$.

PERIOD OF RECORD.--June 1910, January 1913 to current year. Monthly discharge only for some periods, published in WSP 1312. Prior to October 1953, published as Santa Fe Creek near Santa Fe.

REVISED RECORDS.--WSP 1512: 1933, 1936-37(M), 1942, drainage area. WSP 1732: 1923, 1925. WDR NM-75-1: 1927.

GAGE.--Water-stage recorder and concrete control. Elevation of gage is 7,718 ft above National Geodetic Vertical Datum of 1929, from topographic map. See WSP 1312 for history of changes prior to oct. 1 , 1947.

REMARKS.--Flow regulated by Mcclure Reservoir (station 08315500), completed in 1926, raised in 1935 and again in 1947 .

AVERAGE DISCHARGE, - -69 years (water years 1914-27, 1931-85), 8.06 ft $3 / \mathrm{s}, 5,840 \mathrm{acre}-\mathrm{ft} / \mathrm{yr}$.

EXTREMES FOR PERIOD OF RECORD.--Maximum discharge, $1,500 \mathrm{ft}^{3} / \mathrm{s}$, Aug. 14, 1921, gage height, $5.17 \mathrm{ft}$, site and datum then in use, from rating curve extended above $150 \mathrm{ft} / \mathrm{s}$; minimum, $0.05 \mathrm{ft} / \mathrm{s}, \mathrm{Apr}$. 7, 8, 1981 .

EXTREMES OUTSIDE PERIOD OF RECORD.--Peaks which probably exceeded 1,000 ft $3 / \mathrm{s}$ occurred Aug. 19, 1872, and Sept. 29 or 30,1904 . Without regulation the flood of Sept. 23,1929 , might have exceeded $1,500 \mathrm{ft} / \mathrm{s}$.

STATISTICAL SUMMARIES

MEAN MONTHLY AND MEAN ANNUAL DISCHARGES 1914-27

\begin{tabular}{|c|c|c|c|c|c|c|}
\hline MONTH & $\begin{array}{c}\text { MIN IMUM } \\
\left(\mathrm{FT}^{3} / \mathrm{S}\right)\end{array}$ & $\begin{array}{l}\text { MAXIMUM } \\
\left(\mathrm{FT}^{3} / \mathrm{S}\right)\end{array}$ & $\begin{array}{l}\text { MEAN } \\
\left(\mathrm{FT}^{3} / \mathrm{S}\right)\end{array}$ & $\begin{array}{l}\text { STAN- } \\
\text { DARD } \\
\text { DEVIA- } \\
\text { TION } \\
\left(\mathrm{FT}^{3} / \mathrm{S}\right)\end{array}$ & $\begin{array}{l}\text { COEFFI- } \\
\text { CIENT OF } \\
\text { VARI- } \\
\text { ATION }\end{array}$ & $\begin{array}{c}\text { PERCENT } \\
\text { OF } \\
\text { ANNUAL } \\
\text { RUNOFF }\end{array}$ \\
\hline OCTOBER & .6 & 23 & 4.6 & 3.6 & .79 & 4.8 \\
\hline NOVEMBER & .3 & 14 & 3.0 & 2.2 & .74 & 3.1 \\
\hline DECEMBER & .3 & 7.2 & 2.6 & 1.6 & .64 & 2.7 \\
\hline JANUARY & .5 & 6.9 & 2.4 & 1.4 & .57 & 2.4 \\
\hline FEB RUARY & .4 & 14 & 2.6 & 2.0 & .78 & 2.7 \\
\hline MARCH & .3 & 30 & 4. & 4.7 & 1.00 & 4.8 \\
\hline APRIL & .2 & 69 & 13 & 14 & 1.13 & 13.3 \\
\hline MAY & .5 & 93 & 23 & 21 & .89 & 24.2 \\
\hline JUNE & .7 & 75 & 17 & 14 & .84 & 17.6 \\
\hline JULY & 1.1 & 56 & 9.1 & 8.6 & .95 & 9.4 \\
\hline AUGUST & .8 & 74 & 8. & 9.3 & 1.13 & 8.5 \\
\hline SEPTEMBER & .9 & 36 & 6.3 & 5.6 & .89 & 6.5 \\
\hline NNUAL & 1.9 & 26 & 8.0 & 5.2 & .65 & 100 \\
\hline
\end{tabular}

MAGNITUDE AND PROBABILITY OF ANNUAL LOW FLOW BASED ON PERIOD OF RECORD 1915-27, 1931-85

\begin{tabular}{|c|c|c|c|c|c|c|}
\hline \multirow{2}{*}{$\begin{array}{l}\text { PERIOD } \\
\text { (CON- } \\
\text { SECU- } \\
\text { TIVE } \\
\text { DAYS) }\end{array}$} & \multicolumn{6}{|c|}{$\begin{array}{l}\text { DISCHARGE, IN FT }{ }^{3 / S} \text {, FOR INDICATED RECURRENCE } \\
\text { INTERVAL, IN YEARS, AND ANNUAL NON- } \\
\text { EXCEEDANCE PROBABILITY, IN PERCENT }\end{array}$} \\
\hline & $\begin{array}{c}2 \\
508\end{array}$ & $\begin{array}{c}5 \\
208\end{array}$ & $\begin{array}{l}10 \\
108\end{array}$ & $\begin{array}{l}20 \\
58\end{array}$ & $\begin{array}{l}50 \\
28\end{array}$ & \\
\hline $\begin{array}{r}1 \\
3 \\
7 \\
14 \\
30 \\
60 \\
90 \\
120 \\
183\end{array}$ & $\begin{array}{r}.9 \\
.9 \\
1.0 \\
1.1 \\
1.2 \\
1.5 \\
1.8 \\
2.0 \\
2.6\end{array}$ & $\begin{array}{r}.4 \\
.5 \\
.5 \\
.6 \\
.7 \\
.9 \\
1.1 \\
1.2 \\
1.6\end{array}$ & $\begin{array}{r}.3 \\
.3 \\
.3 \\
.4 \\
.5 \\
.7 \\
.8 \\
.9 \\
1.2\end{array}$ & $\begin{array}{r}.2 \\
.2 \\
.2 \\
.3 \\
.4 \\
.5 \\
.6 \\
.7 \\
1.0\end{array}$ & $\begin{array}{l}.1 \\
.1 \\
.2 \\
.2 \\
.2 \\
.4 \\
.5 \\
.6 \\
.8\end{array}$ & \\
\hline
\end{tabular}

MAGNITUDE AND PROBABILITY OF ANNUAL HIGH FLOW BASED ON PERIOD OF RECORD 1914-27, 1931-85

\begin{tabular}{lcccccc} 
PERIOD & \multicolumn{2}{c}{ DISCHARGE, IN FT $3 / S$, FOR INDICATED RECURRENCE } \\
INTERVAL, IN YEARS, AND ANNUAL \\
(CON-
\end{tabular}

DURATION TABLE OF DAILY MEAN FLOW FOR PERIOD OF RECORD 1914-27, 1931-85 DISCHARGE, IN FT ${ }^{3} / S$, THAT WAS EQUALED OR EXCEEDED FOR INDICATED PERCENT OF TIME

\begin{tabular}{|c|c|c|c|c|c|c|c|c|c|c|c|c|c|c|}
\hline 58 & 108 & 158 & 208 & 258 & $30 \%$ & 408 & 508 & 608 & 708 & 758 & 808 & 858 & 908 & $95 \%$ \\
\hline 33 & 18 & 13 & 10 & 8.4 & 7.2 & 5.3 & 4.1 & 3.0 & 2.2 & 1.5 & 1.6 & 1.3 & 1.1 & \\
\hline
\end{tabular}


RIO GRANDE BASIN

08317200 SANTA FE RIVER ABOVE COCHITI LAKE, NM

LOCATION, --Lat $35^{\circ} 32^{\prime} 49^{\prime \prime}$, long $106^{\circ} 13^{\prime} 4^{\prime \prime}$, in NWł sec.8, T.15 N., R.7 E., Santa Fe County, Hydrologic Unit 13020201 in Mesita de Juana Lopez Grant, on right bank at foot of La Bajada Hill, 5.0 mi upstream from Cochiti Dam, 6.3

mi east of Pena Blanca, and at mile 7.9 .

DRA INAGE AREA. $--231 \mathrm{mi}^{2}$.

PERIOD OF RECORD.--March 1970 to current year.

GAGE.--Water-stage recorder. Elevation of gage is 5,505 ft above National Geodetic Vertical Datum of 1929 , from topograph ic map.

REMARKs.--Surface and ground-water diversions and returns for municipal supply of city of Santa Fe in upper part of basin. Diversions for irrigation of about 400 acres upstream from station.

AVERAGE DISCHARGE. --15 years (water years 1971-85), $9.56 \mathrm{ft} 3 / \mathrm{s}, 6,920 \mathrm{acre}-\mathrm{ft} / \mathrm{yr}$.

EXTREMES FOR PERIOD OF RECORD,--Maximum discharge, 11,400 ft $3 / \mathrm{s}$, July 26, 1971, gage height, $9.58 \mathrm{ft}$, from rating curve extended above $160 \mathrm{ft}^{3} / \mathrm{s}$ on basis of slope-area measurements at gage heights $5.69 \mathrm{ft}$ and $9.58 \mathrm{ft}$; no flow July $16-18,1971$.

STATISTICAL SUMMARIES

MEAN MONTHLY AND MEAN ANNUAL DISCHARGES 1971-85

\begin{tabular}{|c|c|c|c|c|c|c|}
\hline MONTH & $\begin{array}{l}\text { MINIMUM } \\
\left(\mathrm{FT}^{3} / \mathrm{S}\right)\end{array}$ & $\begin{array}{c}\text { MAXIMUM } \\
\left(\mathrm{FT}^{3} / \mathrm{S}\right)\end{array}$ & $\begin{array}{l}\text { MEAN } \\
\left(\mathrm{FT}^{3} / \mathrm{S}\right)\end{array}$ & $\begin{array}{l}\text { STAN- } \\
\text { DARD } \\
\text { DEVIA- } \\
\text { TION } \\
\text { (FT'/S) }\end{array}$ & $\begin{array}{l}\text { COEFFI- } \\
\text { CIENT OF } \\
\text { VARI- } \\
\text { ATION }\end{array}$ & $\begin{array}{c}\text { PERCENT } \\
\text { OF } \\
\text { ANNUAL } \\
\text { RUNOF F }\end{array}$ \\
\hline $\begin{array}{l}\text { OCTOBER } \\
\text { NOVEMBER } \\
\text { DECEMBER } \\
\text { JANUARY } \\
\text { FEBRUARY } \\
\text { MARCH } \\
\text { APRIL } \\
\text { MAY } \\
\text { JUNE } \\
\text { JULY } \\
\text { AUGUST } \\
\text { SEPTEMBER }\end{array}$ & $\begin{array}{l}4.0 \\
5.5 \\
6.8 \\
6.5 \\
7.2 \\
6.2 \\
3.6 \\
2.3 \\
1.2 \\
2.3 \\
2.1 \\
3.1\end{array}$ & $\begin{array}{l}11 \\
10 \\
11 \\
11 \\
11 \\
19 \\
46 \\
69 \\
75 \\
28 \\
10 \\
15\end{array}$ & $\begin{array}{l}6.3 \\
7.6 \\
8.6 \\
9.3 \\
9.3 \\
9.2 \\
10 \\
19 \\
16 \\
7.8 \\
5.6 \\
6.4\end{array}$ & $\begin{array}{l}1.8 \\
1.3 \\
1.1 \\
1.3 \\
1.0 \\
3.1 \\
11 \\
23 \\
22 \\
6.2 \\
2.6 \\
3.3\end{array}$ & $\begin{array}{l}.29 \\
.17 \\
.12 \\
.14 \\
.11 \\
.33 \\
1.06 \\
1.24 \\
1.37 \\
.80 \\
.46 \\
.52\end{array}$ & $\begin{array}{r}5.5 \\
6.7 \\
7.5 \\
8.1 \\
8.1 \\
8.1 \\
9.0 \\
16.1 \\
13.7 \\
6.8 \\
4.9 \\
5.6\end{array}$ \\
\hline NNU & 6.1 & 19 & 9.6 & 4.6 & .48 & 100 \\
\hline
\end{tabular}

MAGNITUDE AND PROBABILITY OF ANNUAL LOW FLOW BASED ON PERIOD OF RECORD 1972-85

\begin{tabular}{|c|c|c|c|c|c|c|}
\hline \multirow{2}{*}{$\begin{array}{l}\text { PERIOD } \\
\text { (CON- } \\
\text { SECU- } \\
\text { TIVE } \\
\text { DAYS) }\end{array}$} & \multicolumn{6}{|c|}{$\begin{array}{l}\text { DISCHARGE, IN FT3/S, FOR INDICATED RECURRENCE } \\
\text { INTERVAL, IN YEARS, AND ANNUAL NON- } \\
\text { EXCEEDANCE PROBABIL ITY, IN PERCENT }\end{array}$} \\
\hline & $\begin{array}{c}2 \\
508\end{array}$ & $\begin{array}{c}5 \\
208\end{array}$ & $\begin{array}{l}10 \\
108\end{array}$ & $\begin{array}{l}20 \\
58\end{array}$ & $\begin{array}{l}50 \\
28\end{array}$ & $\begin{array}{r}100 \\
18\end{array}$ \\
\hline 1 & 1.0 & .6 & .4 & 0.0 & -- & 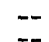 \\
\hline $\begin{array}{l}3 \\
7\end{array}$ & 1.3 & 8 & 6 & 0.0 & -- & - \\
\hline 14 & 2.0 & 1.1 & .7 & .4 & - & - \\
\hline 30 & 2.4 & 1.7 & 1.4 & 1.2 & -- & - \\
\hline 60 & 3.0 & 2.2 & 1.8 & 1.5 & -- & -- \\
\hline $\begin{array}{r}90 \\
120\end{array}$ & 3.7 & 2.8 & 2.4 & 2.1 & - & $=$ \\
\hline 183 & $\begin{array}{l}4.4 \\
5.6\end{array}$ & 4.9 & $\begin{array}{l}3.4 \\
4.5\end{array}$ & 4.2 & - & - \\
\hline
\end{tabular}

MAGNITUDE AND PROBABILITY OF ANNUAL HIGH FLOW BASED ON PERIOD OF RECORD $1971-85$

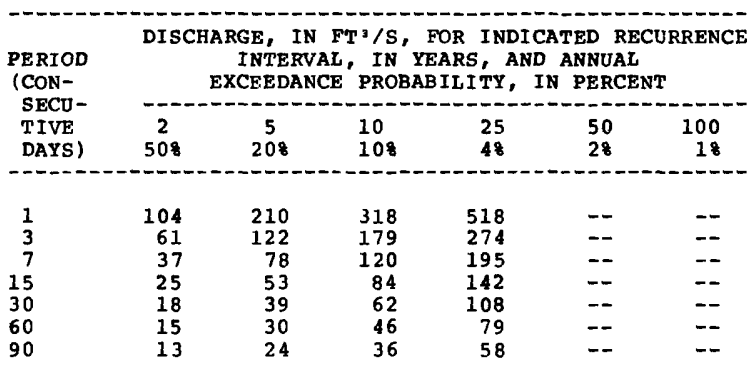

DURATION TABLE OF DAILY MEAN FLOW FOR PERIOD OF RECORD 1971-85

DISCHARGE, IN FT'3/S, THAT WAS EQUALED OR EXCEEDED FOR INDICATED PERCENT OF TIME

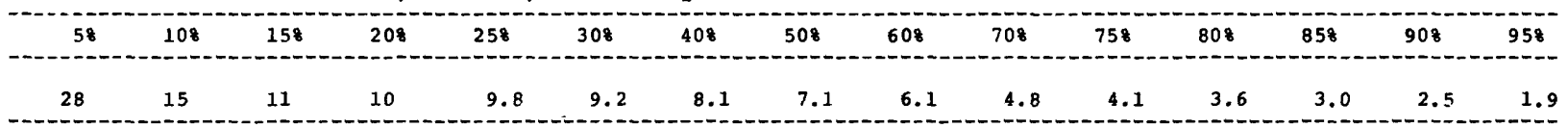


08317400 RIO GRANDE BELOW COCHITI DAM, NM

LOCATION.--Lat $35^{\circ} 37^{\prime} 05^{\prime \prime}$, long $106^{\circ} 19^{\prime} 24^{n}$, in SW $\frac{1}{4} \mathrm{NE}_{4}$ sec.17, T.16 N., R. 6 E., Sandoval County, Hydrologic Unit 13020201 , in Pueblo de Cochiti Grant, on right bank $320 \mathrm{ft}$ upstream from bridge on State Highway 22 , 700 ft downstream from Cochiti Dam, 1.4 mi northeast of Cochiti Pueblo, and at mile 1,587.6.

DRAINAGE AREA.--14,900 $\mathrm{mi}^{2}$, approximately, including 2,940 $\mathrm{mi}^{2}$ in closed basin in san Luis Valley, Co.

PERIOD OF RECORD.--October 1970 to current year.

GAGE.--Water-stage recorder. Datum of gage is $5,226.08 \mathrm{ft}$ above National Geodetic Vertical Datum of 1929

(U.S. Army Corps of Engineers bench mark). Prior to Nov. 14, 1973, at site 2.4 mi downstream at elevation 5,210

$\mathrm{ft}$, from topographic map. Nov, 14, 1973 to Jan. 8, 1976, at site $320 \mathrm{ft}$ downstream at datum $1.79 \mathrm{ft}$ lower.

REMARKS.--Discharges include flow of Santa Fe River which is intercepted by Cochiti Dam and released through the combined outlet works. Flow regulated by Cochiti Dam since Nov. 12, 1973. Diversions upstream from station for irrigation of about 620,000 acres in Colorado and about 81,000 acres in New Mexico. Cochiti eastside main canal, on left bank, and sili main canal, on right bank, head at Cochiti Dam and bypass gage for irrigation of about 6,000 acres downstream from station.

AVERAGE DISCHARGE.--12 years (water years 1974-85), 1,378 $\mathrm{ft}^{3} / \mathrm{s}, 998,400$ acre-ft/yr.

EXTREMES FOR PERIOD OF RECORD.--Maximum discharge 10,300 $\mathrm{ft}^{3} / \mathrm{s}$, July 26, 1971, gage height, $7.90 \mathrm{ft}$, site and datum then in use, from rating curve extended above $2,600 \mathrm{ft}^{3} / \mathrm{s} ; \mathrm{minimum}, 0.51 \mathrm{ft}^{3 / 5}, \mathrm{Aug} .3-5,1977, \mathrm{Aug} .27-28$,

1978 , result of regulation.

EXTREMES OUTSIDE PERIOD OF RECORD. - The flood of May 15, 1941, reached a discharge of $23,400 \mathrm{ft} 3 / \mathrm{s}$ at a nearby site upstream from mouth of Santa Fe River. The flood of May 23, 1920, probably exceeded 23,400 $\mathrm{ft} 3 / \mathrm{s}$, and is $1 \mathrm{ikely}$ the highest since 1905 .

\section{STATISTICAL SUMMARIES}

MEAN MONTHLY AND MEAN ANNUAL DISChARgES 1974-85

\begin{tabular}{|c|c|c|c|c|c|c|}
\hline MONTH & $\begin{array}{c}\text { MINIMUM } \\
\left(\mathrm{FT}^{3} / \mathrm{S}\right)\end{array}$ & $\frac{\operatorname{MAXIMUM}}{\left(\mathrm{FT}^{3} / \mathrm{S}\right)}$ & $\begin{array}{l}\text { MEAN } \\
\left(\mathrm{FT}^{3} / \mathrm{S}\right)\end{array}$ & $\begin{array}{l}\text { STAN- } \\
\text { DARD } \\
\text { DEVIA- } \\
\text { TION } \\
\left(\mathrm{FT}^{3} / \mathrm{S}\right)\end{array}$ & $\begin{array}{l}\text { COEFFI- } \\
\text { CIENT OF } \\
\text { VARI- } \\
\text { ATION }\end{array}$ & $\begin{array}{c}\text { PERCENT } \\
\text { OF } \\
\text { ANNUAL, } \\
\text { RUNOF F }\end{array}$ \\
\hline \multicolumn{7}{|c|}{ 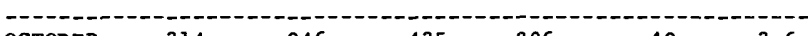 } \\
\hline OСTOBER & 214 & 846 & 425 & 206 & .48 & 2.6 \\
\hline NOVEMBER & 368 & 1485 & 821 & 376 & .46 & 5.0 \\
\hline DECEMBER & 461 & 1688 & 953 & 449 & .47 & 5.8 \\
\hline JANUARY & 428 & 1301 & 725 & 286 & .39 & 4.4 \\
\hline FEBRUARY & 493 & 1119 & 716 & 188 & .26 & 4.3 \\
\hline MARCH & 438 & 2184 & 1001 & 499 & .50 & 6.1 \\
\hline APRIL & 281 & 6320 & 1991 & 1694 & .85 & 12.0 \\
\hline MAY & 432 & 6101 & 3542 & 2187 & .62 & 21.4 \\
\hline JUNE & 739 & 6205 & 3384 & 2163 & .64 & 20.5 \\
\hline JULY & 467 & 5643 & 1760 & 1534 & .87 & 10.7 \\
\hline AUGUST & 447 & 993 & 681 & 186 & .27 & $4 \cdot 1$ \\
\hline SEPTEMBER & 121 & 1337 & 525 & 329 & .63 & 3.2 \\
\hline ANNUAL & 452 & 2336 & 1378 & 634 & .46 & 100 \\
\hline
\end{tabular}

MAGNITUDE AND PROBABILITY OF ANNUAL LOW FLOW BASED ON PERIOD OF RECORD 1975-85

\begin{tabular}{|c|c|c|c|c|c|c|}
\hline $\begin{array}{l}\text { PERIOD } \\
\text { (CON- }\end{array}$ & \multicolumn{6}{|c|}{$\begin{array}{l}\text { DISCHARGE, IN FT } 3 / S \text {, FOR INDICATED RECURRENCE } \\
\text { INTERVAL, IN YEARS, AND ANNUAL NON- } \\
\text { EXCEEDANCE PROBABILITY, IN PERCENT }\end{array}$} \\
\hline $\begin{array}{l}\text { TIVE } \\
\text { DAYS) }\end{array}$ & $\begin{array}{c}2 \\
508\end{array}$ & $\begin{array}{c}5 \\
208\end{array}$ & $\begin{array}{l}10 \\
108\end{array}$ & $\begin{array}{l}20 \\
58\end{array}$ & $\begin{array}{l}50 \\
28\end{array}$ & $\begin{array}{r}100 \\
18\end{array}$ \\
\hline $\begin{array}{r}1 \\
3 \\
7 \\
14 \\
30 \\
60 \\
90 \\
120 \\
183\end{array}$ & $\begin{array}{r}75 \\
80 \\
116 \\
177 \\
273 \\
340 \\
419 \\
492 \\
593\end{array}$ & $\begin{array}{r}15 \\
37 \\
63 \\
93 \\
175 \\
231 \\
307 \\
365 \\
430\end{array}$ & $\begin{array}{r}4 . \\
24 \\
47 \\
66 \\
139 \\
191 \\
265 \\
316 \\
365\end{array}$ & $\begin{array}{r}1 . \\
16 \\
37 \\
50 \\
114 \\
164 \\
235 \\
281 \\
319\end{array}$ & $\begin{array}{l}-- \\
-- \\
-- \\
-- \\
-- \\
-- \\
--\end{array}$ & $\begin{array}{l}-- \\
-- \\
-- \\
-- \\
-- \\
-- \\
-- \\
--\end{array}$ \\
\hline
\end{tabular}

MAGNITUDE AND PROBABILITY OF ANNUAL HIGH FLOW BASED ON PERIOD OF RECORD 1974-85

\begin{tabular}{|c|c|c|c|c|c|c|}
\hline $\begin{array}{l}\text { PERIOD } \\
\text { (CON - } \\
\text { SECU - }\end{array}$ & DIS & $\begin{array}{l}\text { RGE, } \\
\text { INTE } \\
\text { XCEED }\end{array}$ & $\begin{array}{l}\mathrm{T}^{3} / \mathrm{S} \\
\text { IN } \\
\text { PRO }\end{array}$ & $\begin{array}{l}\text { IN } \\
\text { TY }\end{array}$ & $\begin{array}{l}\text { ED R } \\
\text { NNUA } \\
\text { PERC }\end{array}$ & U \\
\hline $\begin{array}{l}\text { TIVE } \\
\text { DAYS) }\end{array}$ & $\begin{array}{c}2 \\
508\end{array}$ & $\begin{array}{c}5 \\
208\end{array}$ & $\begin{array}{l}10 \\
10 \text { 영 }\end{array}$ & $\begin{array}{l}25 \\
48\end{array}$ & $\begin{array}{l}50 \\
28\end{array}$ & $\begin{array}{r}100 \\
18\end{array}$ \\
\hline $\begin{array}{r}1 \\
3 \\
7 \\
15 \\
30 \\
60 \\
90\end{array}$ & $\begin{array}{l}4340 \\
4200 \\
4070 \\
3920 \\
3690 \\
3290 \\
2850\end{array}$ & $\begin{array}{l}7090 \\
6960 \\
6820 \\
6630 \\
6370 \\
5680 \\
5010\end{array}$ & $\begin{array}{l}8840 \\
8740 \\
8620 \\
8400 \\
8140 \\
7240 \\
6480\end{array}$ & $\begin{array}{l}-- \\
-- \\
-- \\
-- \\
-- \\
--\end{array}$ & $\begin{array}{l}-- \\
-- \\
-- \\
-- \\
-- \\
-- \\
--\end{array}$ & $\begin{array}{l}-- \\
-- \\
-- \\
-- \\
-- \\
--\end{array}$ \\
\hline
\end{tabular}

DURATION TABLE OF DAILY MEAN FLOW FOR PERIOD OF RECORD 1974-85 DISCHARGE, IN $\mathrm{FT}^{3} / \mathrm{S}$, THAT WAS EQUALED OR EXCEEDED FOR INDICATED PERCENT OF TIME

\begin{tabular}{|c|c|c|c|c|c|c|c|c|c|c|c|c|c|c|}
\hline 58 & 108 & 158 & 208 & 258 & 308 & 408 & 508 & $60 \%$ & 708 & 758 & 80 응 & $85 \%$ & 908 & 958 \\
\hline 5570 & 3930 & 2630 & 1720 & 1380 & 1190 & 918 & 759 & 654 & 548 & 493 & 438 & 370 & 282 & 161 \\
\hline
\end{tabular}


RIO GRANDE BASIN

08317950 GALISTEO CREEK BELOW GALISTEO DAM, NM

LOCATION. --Lat $35^{\circ} 27.53^{\prime \prime}$, long $106^{\circ} 12^{\prime} 4^{\prime \prime}$, in NEłNE sec. 8, T.14 N., R. 7 E. , Santa Fe County, Hydrologic Unit

13020201 , in Mesita de Juana Lopez Grant, on $r$ ight bank $0.4 \mathrm{mi}$ downstream from Galisteo Dam, $5.3 \mathrm{mi}$ northwest of

Cerrillos, and at mile 11.4 .

DRAINAGE AREA. $--597 \mathrm{mi}^{2}$.

PERIOD OF RECORD.--March 1970 to current year.

GAGE.--Water-stage recorder. Elevation of gage is 5,450 ft above National Geodetic Vertical Datum of 1929 , from topographic map. Prior to Dec. 21, 1981, at site 1,200 ft downstream at different datum.

REMARKS. - Flow regulated by Galisteo Reservoir $0.4 \mathrm{mi}$ upstream. Diversions for irrigation of about 50 acres upstream from station.

AVERAGE DISCHARGE.--15 years (water years 1971-85), $6.33 \mathrm{ft} 3 / \mathrm{s}, 4,590 \mathrm{acre}-\mathrm{ft} / \mathrm{yr}$.

EXTREMES FOR PERIOD OF RECORD. -Maximum discharge, 2,000 ft ${ }^{3} / \mathrm{s}$ July 27, 1971, gage height, $7.00 \mathrm{ft}$; maximum gage height, $7.33 \mathrm{ft}$ July 20,1971 ; no flow for many days each year.

STATISTICAL SUMMARIES

MEAN MONTHLY AND MEAN ANNUAL DISCHARGES 1971-85

\begin{tabular}{|c|c|c|c|c|c|c|}
\hline MONTH & $\begin{array}{l}\text { MINIMUM } \\
\left(\mathrm{FT}^{3} / \mathrm{S}\right)\end{array}$ & $\begin{array}{l}\text { MAXIMUM } \\
\left(\mathrm{FT}^{3} / \mathrm{S}\right)\end{array}$ & $\begin{array}{l}\text { MEAN } \\
\left(\mathrm{FT}^{3} / \mathrm{S}\right)\end{array}$ & $\begin{array}{l}\text { STAN- } \\
\text { DARD } \\
\text { DEVIA- } \\
\text { TION } \\
\left(\mathrm{FT}^{3} / \mathrm{S}\right)\end{array}$ & $\begin{array}{l}\text { COEFFI- } \\
\text { CIENT OF } \\
\text { VARI- } \\
\text { ATION }\end{array}$ & $\begin{array}{c}\text { PERCENT } \\
\text { OF } \\
\text { ANNUAL } \\
\text { RUNOFF }\end{array}$ \\
\hline $\begin{array}{l}\text { OCTOBER } \\
\text { NOVEMBER } \\
\text { DECEMBER } \\
\text { JANUARY } \\
\text { FEBRUARY } \\
\text { MARCH } \\
\text { APRIL } \\
\text { MAY } \\
\text { JUNE } \\
\text { JULY } \\
\text { AUGUST } \\
\text { SEPTEMBER }\end{array}$ & $\begin{array}{l}0.0 \\
0.0 \\
0.0 \\
0.0 \\
0.0 \\
0.0 \\
0.0 \\
0.0 \\
0.0 \\
.1 \\
1.4 \\
0.0\end{array}$ & $\begin{array}{r}29 \\
3.0 \\
5.9 \\
2.4 \\
7.0 \\
10 \\
24 \\
32 \\
30 \\
110 \\
45 \\
52\end{array}$ & $\begin{array}{l}4.9 \\
1.0 \\
1.2 \\
1.2 \\
1.9 \\
2.1 \\
3.4 \\
4.0 \\
5.8 \\
23 \\
16 \\
12\end{array}$ & $\begin{array}{l}8.4 \\
1.0 \\
1.5 \\
.6 \\
1.8 \\
2.8 \\
7.2 \\
8.5 \\
9.8 \\
30 \\
13 \\
17\end{array}$ & $\begin{array}{l}1.72 \\
1.00 \\
1.28 \\
.51 \\
.95 \\
1.34 \\
2.08 \\
2.11 \\
1.69 \\
1.32 \\
.81 \\
1.41\end{array}$ & $\begin{array}{l}6.5 \\
1.3 \\
1.6 \\
1.5 \\
2.5 \\
2.7 \\
4.6 \\
5.3 \\
7.7 \\
29.8 \\
20.8 \\
15.8\end{array}$ \\
\hline NNUAL & 1.3 & 13 & 6.3 & 3.7 & .58 & 100 \\
\hline
\end{tabular}

MAGNITUDE AND PROBABILITY OF ANNUAL LOW FLOW BASED ON PERIOD OF RECORD 1972-85

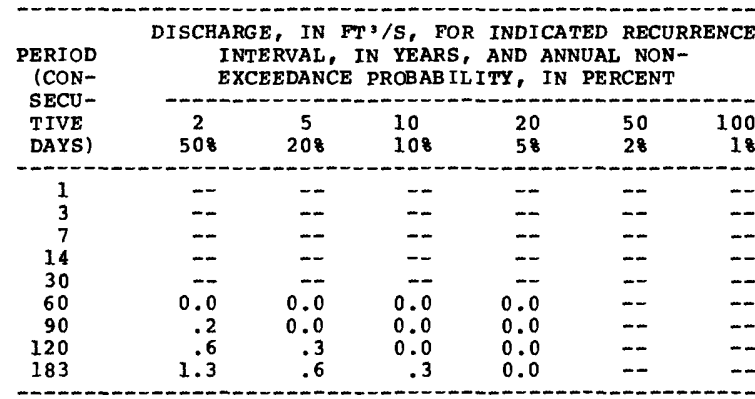

MAGNITUDE AND PROBABILITY OF ANNUAL HIGH FLOW BASED ON PERIOD OF RECORD 1971-85

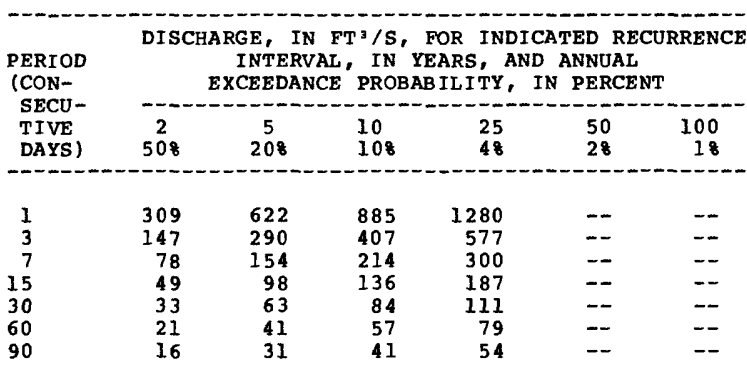

DURATION TABLE OF DAILY MEAN FLOW FOR PERIOD OF RECORD 1971-85 DISCHARGE, IN FT'3/S, THAT WAS EQUALED OR EXCEEDED FOR INDICATED PERCENT OF TIME

\begin{tabular}{|c|c|c|c|c|c|c|c|c|c|c|c|c|c|c|}
\hline 58 & 108 & 158 & 208 & 258 & 308 & 408 & 508 & 608 & 708 & 758 & 808 & 858 & 908 & 958 \\
\hline 21 & 7.0 & 3.2 & 2.1 & 1.5 & 1.2 & .6 & .3 & 0.0 & 0.0 & 0.0 & 0.0 & 0.0 & 0.0 & \\
\hline
\end{tabular}


RIO GRANDE BASIN

08318000 GALISTEO CREEK AT DOMINGO, NM

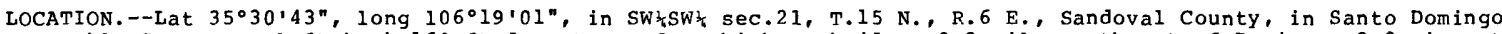
Pueblo Grant, on left bank $160 \mathrm{ft}$ downstream from highway bridge, $0.2 \mathrm{mile}$ northeast of Domingo, $2.8 \mathrm{mi}$ east of Santo Domingo Pueblo, and 4 miles upstream from mouth.

DRAINAGE AREA. $--640 \mathrm{mi}^{2}$, approximately.

PERIOD OF RECORD.--October 1941 to June 1971 (discontinued).

REVISED RECORDS.--WSP 1148: 1942, 1943(M), 1944, 1945(M), 1946-47. WRD N. MEX. $1970: 1967$.

GAGE. - Water-stage recorder. Datum of gage is 5,255.50 ft above mean sea level, datum of 1929 . Prior to July 20 , 1956, at site $160 \mathrm{ft}$ upstream at same datum.

REMARKS.--Flow regulated by Galisteo Dam, 8.5 miles upstream. Diversions for irrigation of about 50 acres above station.

AVERAGE DISCHARGE. - -29 years (water years 1942-70), $10.2 \mathrm{ft} / \mathrm{s}, 7,390$ acre-ft/yr.

EXTREMES FOR PERIOD OF RECORD,--Maximum discharge, 22,800 $\mathrm{ft}^{3} / \mathrm{s}$ Aug. 2, 1966 , (gage height, $8.08 \mathrm{ft}$ inside, $10.4 \mathrm{ft}$ from floodmarks), from rating curve extended above $1,000 \mathrm{ft}^{3} / \mathrm{s}$ on basis of field estimate of peak flow; no flow most of time.

The floods of Sept. 23, 1929 and Aug. 20, 1935, probably exceeded 20,000 ft $3 / \mathrm{s}$. Discharge for the flood of Aug. 20, 1935, was estimated as $24,300 \mathrm{ft}^{3} / \mathrm{s}$ by $\mathrm{H}$. W. Yeo.

STATISTICAL SUMMARIES

MEAN MONTHLY AND MEAN ANNUAL DISCHARGES 1942-70

\begin{tabular}{|c|c|c|c|c|c|c|}
\hline MONTH & $\begin{array}{l}\text { MINIMUM } \\
\left(\mathrm{FT}^{3} / \mathrm{S}\right)\end{array}$ & $\begin{array}{l}\text { MAXIMUM } \\
\left(\mathrm{FT}^{3} / \mathrm{S}\right)\end{array}$ & $\begin{array}{l}\text { MEAN } \\
\left(\mathrm{FT}^{3} / \mathrm{S}\right)\end{array}$ & $\begin{array}{c}\text { STAN- } \\
\text { DARD } \\
\text { DEVIA- } \\
\text { TION } \\
\left(\mathrm{FT}^{3} / \mathrm{S}\right)\end{array}$ & $\begin{array}{l}\text { COEFFI- } \\
\text { CIENT OF } \\
\text { VARI- } \\
\text { ATION }\end{array}$ & $\begin{array}{c}\text { PERCENT } \\
\text { OF } \\
\text { ANNUAI } \\
\text { RUNOF F }\end{array}$ \\
\hline OCTOBER & 0.0 & 146 & 8.9 & 28 & 3.13 & 7.4 \\
\hline NOVEMBFR & 0.0 & 7.8 & .6 & I. 6 & 2.69 & .5 \\
\hline DECEMBER & 0.0 & 2.7 & .4 & .7 & 1.64 & .4 \\
\hline JANUARY & 0.0 & 2.4 & .3 & .5 & 1.82 & .2 \\
\hline FEBRUARY & 0.0 & 1.1 & .1 & .3 & 2.00 & .1 \\
\hline MARCH & 0.0 & 7.9 & .4 & 1.5 & 3.75 & .3 \\
\hline APRIL & 0.0 & 66 & 2.9 & 12 & 4.23 & 2.4 \\
\hline MAY & 0.0 & 26 & 2.1 & 5.2 & 2.50 & 1.7 \\
\hline JUNE & 0.0 & 65 & 8.6 & 16 & 1.86 & 7.2 \\
\hline JULY & 0.0 & 91 & 27 & 28 & 1.04 & 22.6 \\
\hline AUGUST & .4 & 232 & 50 & 49 & .98 & 41.9 \\
\hline SEPTEMBER & 0.0 & 143 & 18 & 33 & 1.82 & 15.1 \\
\hline ANNUAL & 1.5 & 28 & 10 & 6.8 & .67 & 100 \\
\hline
\end{tabular}

MAGNITUDE AND PROBABILITY OF ANNUAL LOW FLOW BASED ON PERIOD OF RECORD 1943-70

\begin{tabular}{|c|c|c|c|c|c|c|}
\hline $\begin{array}{l}\text { PERIOD } \\
\text { (CON- }\end{array}$ & \multicolumn{6}{|c|}{$\begin{array}{l}\text { DISCHARGE, IN FT } 3 / \text { S, FOR INDICATED RECURRENCE } \\
\text { INTERVAL, IN YEARS, AND ANNUAL NON- } \\
\text { EXCEEDANCE PROBABILITY, IN PERCENT }\end{array}$} \\
\hline TIVE & 2 & 5 & 10 & 20 & 50 & \\
\hline DAYS) & $50 \%$ & 208 & 108 & 58 & 28 & \\
\hline & & & & & & \\
\hline 1 & 0.0 & 0.0 & 0.0 & 0.0 & 0.0 & \\
\hline 3 & 0.0 & 0.0 & 0.0 & 0.0 & 0.0 & \\
\hline 7 & 0.0 & 0.0 & 0.0 & 0.0 & 0.0 & \\
\hline 14 & 0.0 & 0.0 & 0.0 & 0.0 & 0.0 & \\
\hline 30 & 0.0 & 0.0 & 0.0 & 0.0 & 0.0 & \\
\hline 60 & 0.0 & 0.0 & 0.0 & 0.0 & 0.0 & \\
\hline 90 & 0.0 & 0.0 & 0.0 & 0.0 & 0.0 & \\
\hline 120 & .1 & 0.0 & 0.0 & 0.0 & 0.0 & \\
\hline 183 & .3 & .1 & 0.0 & 0.0 & 0.0 & \\
\hline
\end{tabular}

MAGNITUDE AND PROBABILITY OF ANNUAL HIGH FLOW BASED ON PERIOD OF RECORD $1942-70$

DISCHARGF, IN FT
PERT $/ S$, FOR INDICATEN RECURRENCF
(CON-

DURATION TABLE OF DAILY MEAN FLOW FOR PERIOD OF RECORD 1942-70 DISCHARGE, IN FT ${ }^{3 / S}$, THAT WAS FQUALED OR EXCEEDED FOR INDICATED PERCENT OF TIME

\begin{tabular}{|c|c|c|c|c|c|c|c|c|c|c|c|c|c|c|}
\hline 58 & 108 & I58 & 208 & 258 & 308 & 408 & 508 & 608 & 708 & 758 & 808 & 858 & 908 & 958 \\
\hline 21 & 3.0 & 1.3 & .4 & .1 & 0.0 & 0.0 & 0.0 & 0.0 & 0.0 & 0.0 & 0.0 & 0.0 & 0.0 & 0.0 \\
\hline
\end{tabular}


RIO GRANDE BASIN

08319000 RIO GRANDE AT SAN FELIPE, NM

(Surveillance network station)

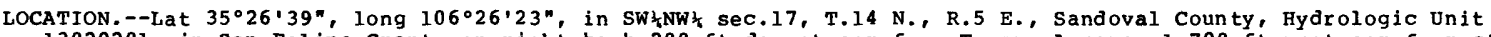

13020201 , in San Felipe Grant, on right bank $200 \mathrm{ft}$ downstream from Tonque Arroyo, 1,700 ft upstream from steel

highway bridge, $0.8 \mathrm{mi}$ upstream from San Felipe Pueblo, $11 \mathrm{mi}$ northeast of Bernalillo, and at $\mathrm{mile} 1,572.7$.

DRAINAGE AREA.--16,100 $\mathrm{mi}^{2}$, approximately, including 2,940 $\mathrm{mi}^{2}$ in closed basin in San Luis Valley, Co.

PERIOD OF RECORD.--October 1925 to current year. Monthly discharge only for some periods, published in wSP 1312.

REVISED RECORDS.--WSP 1312: 1926-30, WSP 1392: 1937(M), WSP 1512: 1931-32, 1933(M), 1934-36, 1938(M).

GAGE.--Water-stage recorder. Datum of gage is $5,115,73 \mathrm{ft}$ above National Geodetic Vertical Datum of 1929 . Prior to Sept. 27, 1957, at site 1,800 ft downstream at datum $5.35 \mathrm{ft}$ lower, except period May 16 , 1945 to Sept. 30 , 1946 when it was $5.94 \mathrm{ft}$ lower than present datum.

REMARKS.--Flow completely regulated since November 1973 by Cochiti Dam (station 08317300 ) $17 \mathrm{mi}$ upstream. Prior to November 1973 some regulation of flow by El Vado Reservoir (station 08285000 ) and Abiquiu Reservoir

(station 08286900 ). Since May 1971 flow affected by release of transmountain water from Heron Reservoir

(station 08284510). Diversions for irrigation of about 705,000 acres upstream from station, some of which

is irrigated downstream by cochiti eastside main canal and San Felipe eastside acequia, which bypass station.

AVERAGE DISCHARGE. --33 years (water years 1928, 1931-62), 1,394 ft3/s, 1,010,000 acre-ft/yr.

EXTREMES FOR PERIOD OF RECORD.--Maximum discharge, 27,300 $\mathrm{ft}^{3 / \mathrm{s}}$, June 26,1937 , gage height, $11.13 \mathrm{ft}$ site and datum then in use, from rating curve extended above $15,000 \mathrm{ft} / \mathrm{s}$; minimum, 32 ft/s, July 7,1934 ,

EXTREMES OUTSIDE PERIOD OF RECORD,--Other major floods occurred in 1874, 1884 , and 1904.

STATISTICAL SUMMARIES (PERIOD BEFORE REGULATION OF ABIQUIU DAM)

MEAN MONTHLY AND MEAN ANNUAL DISCHARGES 1928, 1931-62

MAGNITUDE AND PROBABILITY OF ANNUAL LOW FLOW BASED ON PERIOD OF RECORD $1932-62$

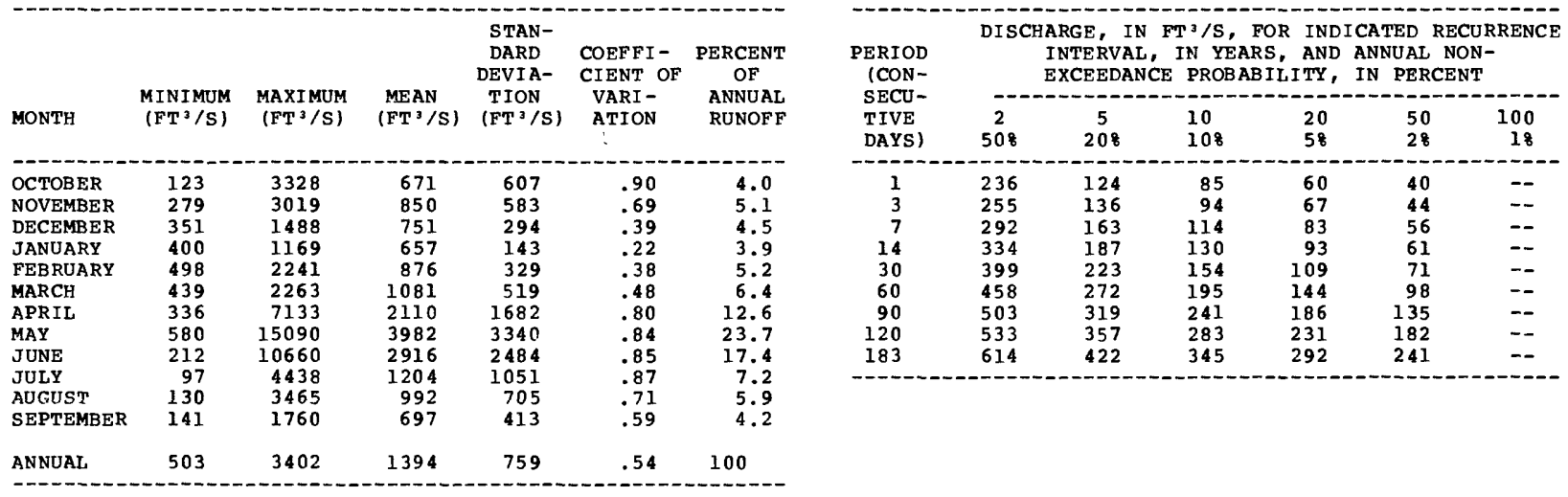

MAGNITUDE AND PROBABILITY OF ANNUAL HIGH FLOW BASED ON PERIOD OF RECORD 1928, 1931-62

\begin{tabular}{|c|c|c|c|c|c|c|}
\hline \multirow{2}{*}{$\begin{array}{l}\text { PERIOD } \\
\text { (CON- } \\
\text { SECU- } \\
\text { TIVE } \\
\text { DAYS) }\end{array}$} & \multicolumn{6}{|c|}{$\begin{array}{l}\text { DISCHARGE, IN FT' } 3 \text {, FOR INDICATED RECURRENC } \\
\text { INTERVAL, IN YEARS, AND ANNUAL } \\
\text { EXCEEDANCE PROBABILITY, IN PERCENT }\end{array}$} \\
\hline & $\begin{array}{c}2 \\
508\end{array}$ & $\begin{array}{c}5 \\
208\end{array}$ & $\begin{array}{l}10 \\
108\end{array}$ & $\begin{array}{l}25 \\
48\end{array}$ & $\begin{array}{l}50 \\
28\end{array}$ & $\begin{array}{r}100 \\
18\end{array}$ \\
\hline $\begin{array}{r}1 \\
3 \\
7 \\
15 \\
30 \\
60 \\
90\end{array}$ & $\begin{array}{l}5790 \\
5160 \\
4690 \\
4280 \\
3750 \\
3010 \\
2520\end{array}$ & $\begin{array}{r}10500 \\
9600 \\
8880 \\
8100 \\
7190 \\
5790 \\
4700\end{array}$ & $\begin{array}{r}14100 \\
13100 \\
12300 \\
11200 \\
10000 \\
8120 \\
6500\end{array}$ & $\begin{array}{r}18900 \\
17900 \\
17300 \\
15800 \\
14200 \\
11600 \\
9150\end{array}$ & $\begin{array}{l}22600 \\
21800 \\
21400 \\
19600 \\
17700 \\
14600 \\
11400\end{array}$ & $\begin{array}{l}-- \\
-- \\
-- \\
-- \\
--\end{array}$ \\
\hline
\end{tabular}

DURATION TABLE OF DAILY MEAN FLOW FOR PERIOD OF RECORD 1928, 1931-62 DISCHARGE, IN $\mathrm{FT}^{3} / \mathrm{S}$, THAT WAS EODALED OR EXCEEDED FOR INDICATED PERCENT OF TIME

\begin{tabular}{|c|c|c|c|c|c|c|c|c|c|c|c|c|c|c|}
\hline 58 & 108 & 158 & 208 & 258 & 308 & 408 & 508 & 608 & 708 & 758 & 808 & 858 & 908 & 958 \\
\hline 5180 & 2990 & 2130 & 1670 & 1380 & 1200 & 921 & 755 & 660 & 572 & 529 & 472 & 408 & 333 & 238 \\
\hline
\end{tabular}


RIO GRANDE BASIN

08319000 RIO GRANDE AT SAN FELIPE, NM--Continued

STATISTICAL SUMMARIES (PERIOD BEFORE REGULATION OF COCHITI DAM AND AFTER COMPLETION OF ABIOUIU DAM)

MEAN MONTHLY AND MEAN ANNUAL, DISCHARGES 1964-73

\begin{tabular}{|c|c|c|c|c|c|c|}
\hline MONTH & $\begin{array}{l}\text { MINIMUM } \\
\left(\mathrm{FT}^{3} / \mathrm{S}\right)\end{array}$ & $\begin{array}{c}\operatorname{MAXIMUM} \\
\left(\mathrm{FT}^{3} / \mathrm{S}\right)\end{array}$ & $\begin{array}{l}\text { MEAN } \\
\left(\mathrm{FT}^{3} / \mathrm{S}\right)\end{array}$ & $\begin{array}{l}\text { STAN- } \\
\text { DARD } \\
\text { DEVIA- } \\
\text { TION } \\
\left(\mathrm{FT}^{3} / \mathrm{S}\right)\end{array}$ & $\begin{array}{l}\text { COEFFI- } \\
\text { CIENT OF } \\
\text { VARI - } \\
\text { ATION }\end{array}$ & $\begin{array}{c}\text { PERCENT } \\
\text { OF } \\
\text { ANNUAL } \\
\text { RUNOFF }\end{array}$ \\
\hline $\begin{array}{l}\text { OCTOBER } \\
\text { NOVEMBER } \\
\text { DECEMBER } \\
\text { JANUARY } \\
\text { FEBRUARY } \\
\text { MARCH } \\
\text { APRIL } \\
\text { MAY } \\
\text { JUNE } \\
\text { JULY } \\
\text { AUGUST } \\
\text { SEPTEMBER }\end{array}$ & $\begin{array}{l}190 \\
553 \\
358 \\
441 \\
484 \\
520 \\
493 \\
366 \\
411 \\
264 \\
428 \\
216\end{array}$ & $\begin{array}{l}1430 \\
1751 \\
1886 \\
915 \\
1031 \\
1665 \\
2473 \\
5671 \\
5111 \\
3374 \\
2344 \\
1114\end{array}$ & $\begin{array}{r}606 \\
1211 \\
850 \\
722 \\
793 \\
1011 \\
1384 \\
2506 \\
2207 \\
1105 \\
1077 \\
649\end{array}$ & $\begin{array}{r}418 \\
400 \\
456 \\
139 \\
159 \\
311 \\
747 \\
1683 \\
1761 \\
1130 \\
677 \\
343\end{array}$ & $\begin{array}{l}.69 \\
.33 \\
.54 \\
.19 \\
.20 \\
.31 \\
.54 \\
.67 \\
.80 \\
1.02 \\
.63 \\
.53\end{array}$ & $\begin{array}{r}4.3 \\
8.6 \\
6.0 \\
5.1 \\
5.6 \\
7.2 \\
9.8 \\
17.7 \\
15.6 \\
7.6 \\
7.6 \\
4.6\end{array}$ \\
\hline ANNUAL & 503 & 1978 & 1178 & 451 & .38 & 100 \\
\hline
\end{tabular}

MAGNITUDF: AND PROBABILITY OF ANNUAL LOW FLOW BASED ON PERIOD OF RECORD 1965-73

\begin{tabular}{|c|c|c|c|c|c|c|}
\hline \multirow{2}{*}{$\begin{array}{l}\text { PERIOD } \\
\text { (CON- } \\
\text { SECU- } \\
\text { TIVE } \\
\text { DAYS) }\end{array}$} & \multicolumn{6}{|c|}{$\begin{array}{l}\text { DISCHARGE, IN FT }{ }^{3} / S \text {, FOR INDICATED RECURRENCE } \\
\text { INTERVAL, IN YEARS, AND ANNUAL NON- } \\
\text { EXCEEDANCE PROBABILITY, IN PERCENT }\end{array}$} \\
\hline & $\begin{array}{c}2 \\
508\end{array}$ & $\begin{array}{c}5 \\
208\end{array}$ & $\begin{array}{l}10 \\
108\end{array}$ & $\begin{array}{l}20 \\
58\end{array}$ & $\begin{array}{l}50 \\
28\end{array}$ & $\begin{array}{r}100 \\
18\end{array}$ \\
\hline $\begin{array}{r}1 \\
3 \\
7 \\
14 \\
30 \\
60 \\
90 \\
120 \\
183\end{array}$ & $\begin{array}{l}149 \\
173 \\
200 \\
230 \\
308 \\
412 \\
550 \\
588 \\
695\end{array}$ & $\begin{array}{r}96 \\
114 \\
136 \\
161 \\
214 \\
281 \\
381 \\
404 \\
487\end{array}$ & $\begin{array}{r}80 \\
95 \\
116 \\
140 \\
184 \\
234 \\
311 \\
333 \\
403\end{array}$ & $\begin{array}{r}71 \\
84 \\
104 \\
127 \\
165 \\
203 \\
263 \\
285 \\
344\end{array}$ & $\begin{array}{l}-- \\
-- \\
-- \\
-- \\
-- \\
-- \\
--\end{array}$ & $\begin{array}{l}-- \\
-- \\
-- \\
-- \\
-- \\
-- \\
-- \\
--\end{array}$ \\
\hline
\end{tabular}

MAGNITUDE AND PROBABILITY OF ANNUAL HIGH FLOW BASED ON PERIOD OF RECORD 1964-73

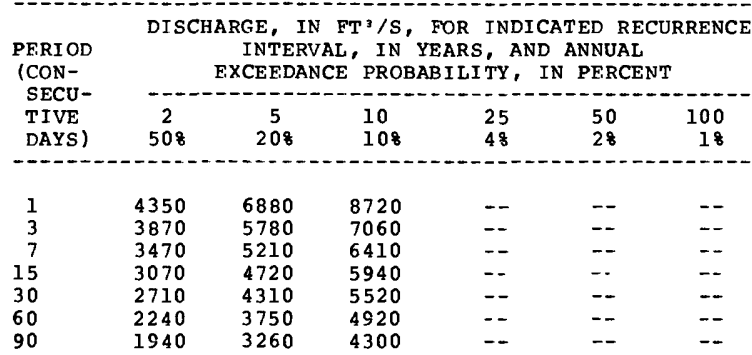

DURATION TABLE OF DAILY MEAN FLOW FOR PERIOD OF RECORD 1964-73

DISCHARGE, IN FT ${ }^{3} / \mathrm{S}$, THAT WAS FQUALED OR FXCEFDED FOR INDICATED PERCENT OF TIME

\begin{tabular}{|c|c|c|c|c|c|c|c|c|c|c|c|c|c|c|}
\hline 58 & 108 & 158 & 208 & 258 & 308 & 408 & 508 & 608 & 708 & 758 & 808 & 858 & 908 & 958 \\
\hline 3740 & 2630 & 1960 & 1630 & 1350 & 1160 & 952 & 823 & 720 & 608 & 520 & 450 & 379 & 303 & 212 \\
\hline
\end{tabular}


RIO GRANDE BASIN

08319000 RIO GRANDE AT SAN FELIPE, NM--Continued

STATISTICAL SUMMARIES (PERIOD AFTER COMPLETION OF COCHITI DAM)

MEAN MONTHLY AND MEAN ANNUAL DISCHARGES

1974-85

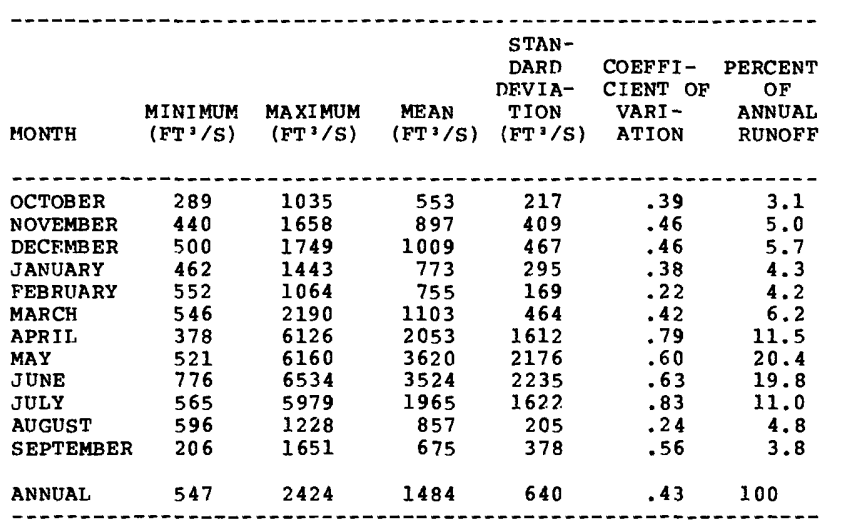

MAGNITUDE AND PROBABILITY OF ANNUAL LOW FLOW BASED ON PERIOD OF RECORD 1975-85

\begin{tabular}{|c|c|c|c|c|c|c|}
\hline \multirow{2}{*}{$\begin{array}{l}\text { PERIOD } \\
\text { (CON- } \\
\text { SECU- } \\
\text { TIVE } \\
\text { DAYS) }\end{array}$} & \multicolumn{6}{|c|}{$\begin{array}{l}\text { DISCHARGE, IN FT }{ }^{3 / S} \text {, FOR INDJCATED RECURRENCE } \\
\text { INTERVAL, IN YEARS, AND ANNUAL NON- } \\
\text { EXCEEDANCE PROBABILITY, IN PERCENT }\end{array}$} \\
\hline & $\begin{array}{c}2 \\
508\end{array}$ & $\begin{array}{c}5 \\
208\end{array}$ & $\begin{array}{l}10 \\
108\end{array}$ & $\begin{array}{l}20 \\
58\end{array}$ & $\begin{array}{l}50 \\
28\end{array}$ & $\begin{array}{r}100 \\
18\end{array}$ \\
\hline $\begin{array}{r}1 \\
3 \\
7 \\
14 \\
30 \\
60 \\
90 \\
120 \\
183\end{array}$ & $\begin{array}{l}167 \\
178 \\
226 \\
294 \\
407 \\
473 \\
548 \\
609 \\
691\end{array}$ & $\begin{array}{l}111 \\
129 \\
161 \\
203 \\
289 \\
345 \\
416 \\
459 \\
518\end{array}$ & $\begin{array}{r}90 \\
111 \\
138 \\
170 \\
239 \\
292 \\
360 \\
397 \\
446\end{array}$ & $\begin{array}{r}76 \\
99 \\
122 \\
148 \\
202 \\
255 \\
319 \\
354 \\
394\end{array}$ & $\begin{array}{l}-- \\
-- \\
-- \\
-- \\
-- \\
-- \\
-- \\
--\end{array}$ & $\begin{array}{l}-- \\
-- \\
-- \\
-- \\
-- \\
-- \\
-- \\
-- \\
--\end{array}$ \\
\hline
\end{tabular}

MAGNITUDE AND PROBABII,ITY OF ANNUAL HIGH FLOW BASED ON PERIOD OF RECORD $1974-85$

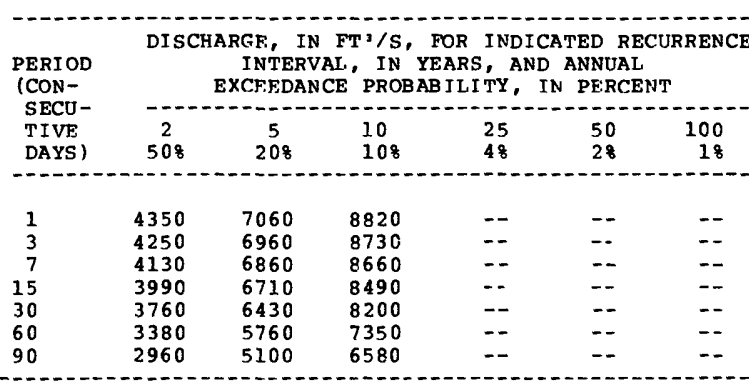

DURATION TABLE OF DAILY MEAN FLOW FOR PERIOD OF RECORD 1974-85

DISCHARGE, IN FT ${ }^{3} / \mathrm{S}$, THAT WAS EQUALED OR EXCEEDED FOR INDICATED PERCENT OF TIME

\begin{tabular}{|c|c|c|c|c|c|c|c|c|c|c|c|c|c|c|}
\hline 58 & 108 & 158 & 208 & 258 & 308 & 408 & 508 & 608 & 708 & 758 & 808 & 858 & 908 & 958 \\
\hline 5870 & 4020 & 2670 & 1790 & 1530 & 1340 & 1040 & 868 & 751 & 633 & 571 & 515 & 455 & 384 & 267 \\
\hline
\end{tabular}


RIO GRANDE BASIN

08321500 JEMEZ RIVER BELOW EAST FORK, NEAR JEMEZ SPRINGS, NM

LOCATION. --Lat $35^{\circ} 49^{\prime} 39^{\prime \prime}$, long $106^{\circ} 38^{\prime} 52^{\prime \prime}$, in NWt sec.5, T.18 N., R. 3 E., Sandoval County, Hydrologic Unit 13020202 , on left bank $0.4 \mathrm{mi}$ downstream from East Fork and boundary of Santa Fe National Forest, $5.3 \mathrm{mi}$ northeast of Jemez Springs, and at mile 43.0 .

DRA INAGE AREA, $--173 \mathrm{mi}^{2}$.

PERIOD OF RECORD. --July 1949 to October 1950 (gaged separately upstream from East Fork), May 1951 to September 1957 (irrigation seasons only), March 1958 to September 1976, JuIY 1981 to current year.

REVISED RECORDS.--WSP 1512: 1951-54(M), 1955, 1956(M). WSP 1712: Drainage area.

GAGE.-Water-stage recorder. Datum of gage is $6,703 \mathrm{ft}$ above National Geodetic Vertical Datum of 1929 (plane-table survey by Topographic Division, U.S. Geological Survey, 1952). Prior to May 1951, at sites 3,000 ft upstream, at different datums and on separate channels.

REMARKS. --No diversion upstream from station.

AVERAGE DISCHARGE,--22 years (water years 1959-76, 1982-85), 33.0 ft3/s, 23,910 acre-ft/yr

EXTREMES FOR PERIOD OF RECORD,--Maximum discharge recorded, 2,500 ft $3 / \mathrm{s}$, Apr. 21 , 1958 , gage height, $7.35 \mathrm{ft}$, from rating curve extended above $1,100 \mathrm{ft} / \mathrm{s}$ on basis of slope-area and contracted-opening measurements of peak flow; minimum, $0.91 \mathrm{ft}^{3 / \mathrm{s}}$, Jan. 24 , 1969, result of freezeup.

STATISTICAL SUMMARIES

MEAN MONTHLY AND MEAN ANNUAL DISCHARgES 1982-85,

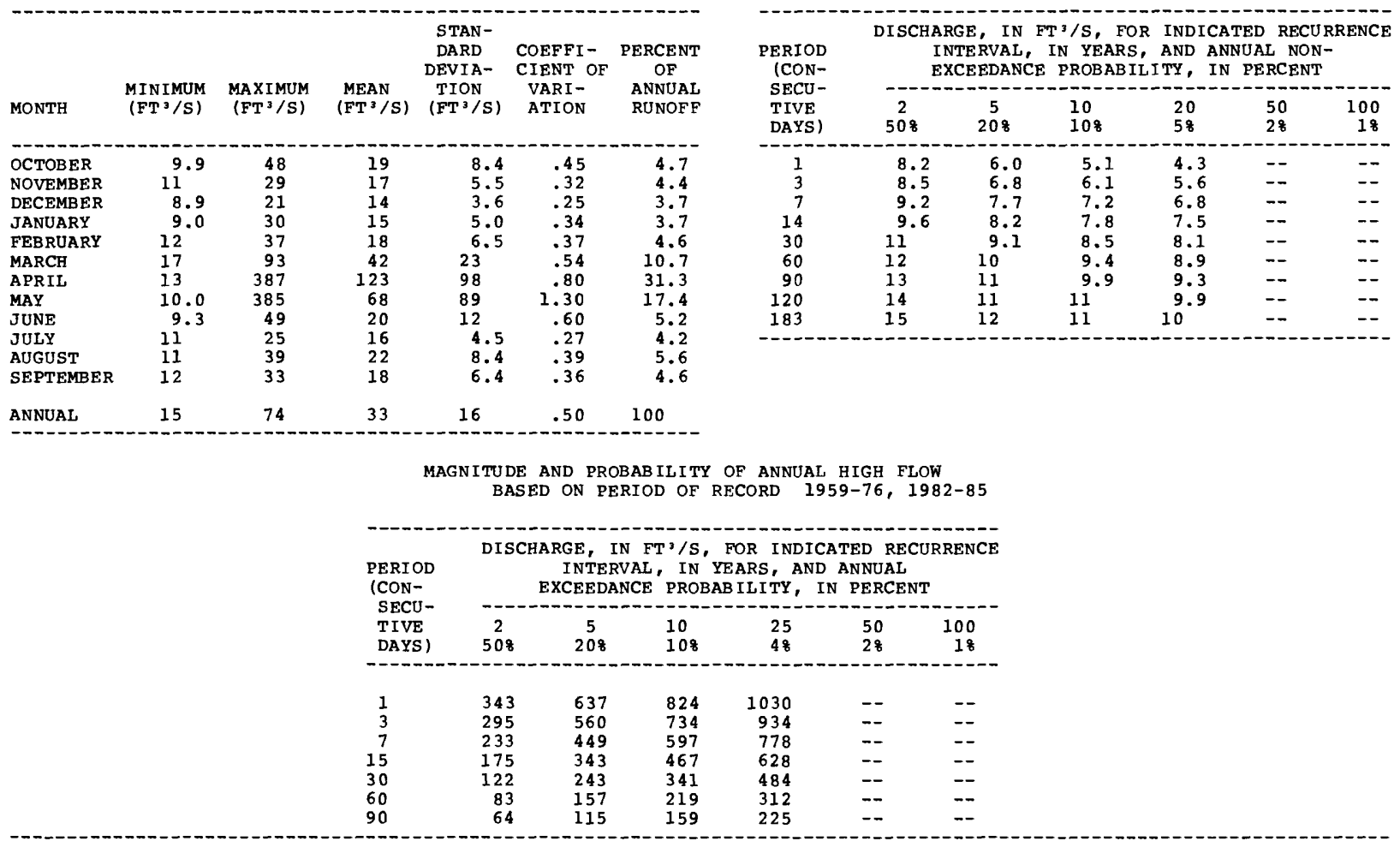

DURATION TABLE OF DAILY MEAN FLOW FOR PERIOD OF RECORD 1959-76, 1982-85

DISCHARGE, IN FT' $3 / S$, THAT WAS EOUALED OR EXCEEDED FOR INDICATED PERCENT OF TIME

\begin{tabular}{|c|c|c|c|c|c|c|c|c|c|c|c|c|c|c|}
\hline 58 & 10 응 & 158 & $20 \%$ & 258 & 308 & 408 & 50 응 & $60 \%$ & 708 & 758 & $80 \%$ & $85 \%$ & 908 & 958 \\
\hline 108 & 55 & 36 & 29 & 25 & 23 & 19 & 17 & 15 & 14 & 13 & 12 & 12 & 11 & 9.7 \\
\hline
\end{tabular}


RIO GRANDE BASIN

08323000 RIO GUADALUPE AT BOX CANYON, NEAR JENEZ, NM

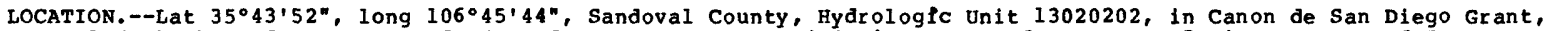
on left bank at downstream end of Guadalupe Box Canyon, $4.8 \mathrm{mi}$ upstream from mouth, $5 \mathrm{mi}$ southwest of Jemez Springs, and $7 \mathrm{mi}$ north of Jemez.

DRAINAGE AREA. $--235 \mathrm{mi}^{2}$.

PERIOD OF RECORD.--November 1938 to September 1942, August 1949 to September 1950, (monthly discharge only for November, December 1938 and August 1949 published in WSP 1312), May 1951 to September I957 (irrigation seasons only), May 1958 to September 1976, July 1981 to current year. Prior to 1951 published as "08323500 Rio Guadalupe near Jemez Springs".

REVISED RECORDS.--WSP 1712: Drainage area.

GAGE.--Water-stage recorder. Datum of gage is $6,015 \mathrm{ft}$ above National Geodetic Vertical Datum of 1929 (plane-table survey by Topographic Division, U.S. Geological Survey, 1952). Prior to 1951 at site $2.4 \mathrm{mi}$ downstream at lower datums.

REMARKS.--Flow regulated to some extent since October 1958 by San Gregorio Reservoir on Clear Creek, 24 mi upstream (capacity, 345 acre-ft), and by transmountain diversion into Rio puerco Basin for irrigation of about 300 acres in vicinity of cuba.

AVERAGE DISCHARGE.--22 years (water years 1959-76, 1982-1985), $42.0 \mathrm{ft} / \mathrm{s}, 30,430 \mathrm{acre-ft} / \mathrm{yr}$.

EXTREMES FOR PERIOD OF RECORD.--Maximum discharge, 3,190 $\mathrm{ft}^{3} / \mathrm{s}$, May 13 or 14, 1941, gage height, $8.4 \mathrm{ft}$, from floodmarks, site and datum in use June 1941 to september 1942, from rating curve extended above 1,000 $\mathrm{ft}^{3} / \mathrm{s}$; minimum, $2.8 \mathrm{ft}^{3} / \mathrm{s}$, Dec. 9,1967

STATISTICAL SUMMARIES

MEAN MONTHLY AND MEAN ANNUAL DISCHARGES $\begin{array}{r}1959-76 \\ 1982-85\end{array}$

\begin{tabular}{|c|c|c|c|c|c|c|}
\hline MONTH & $\begin{array}{c}\text { MINIMUM } \\
\left(\mathrm{FT}^{3} / \mathrm{S}\right)\end{array}$ & $\begin{array}{c}\text { MAXIMUM } \\
\left(\mathrm{FT}^{3} / \mathrm{S}\right)\end{array}$ & $\begin{array}{l}\text { MEAN } \\
\left(\mathrm{FT}^{3} / \mathrm{S}\right)\end{array}$ & $\begin{array}{c}\text { STAN- } \\
\text { DARD } \\
\text { DEVIA- } \\
\text { TION } \\
\left(\mathrm{FT}^{3} / \mathrm{S}\right)\end{array}$ & $\begin{array}{l}\text { COEFFI- } \\
\text { CIENT OF } \\
\text { VARI- } \\
\text { ATION }\end{array}$ & $\begin{array}{c}\text { PERCENT } \\
\text { OF } \\
\text { ANNUAL } \\
\text { RUNOFF }\end{array}$ \\
\hline $\begin{array}{l}\text { OCTOBER } \\
\text { NOVEMBER } \\
\text { DECEMBER } \\
\text { JANUARY } \\
\text { FEBRUARY } \\
\text { MARCH } \\
\text { APRIL } \\
\text { MAY } \\
\text { JUNE } \\
\text { JULY } \\
\text { AUGUST } \\
\text { SEPTEMBER }\end{array}$ & $\begin{array}{l}5.9 \\
6.2 \\
6.3 \\
5.8 \\
6.3 \\
11 \\
30 \\
15 \\
6.3 \\
5.3 \\
6.7 \\
4.8\end{array}$ & $\begin{array}{r}33 \\
34 \\
20 \\
33 \\
24 \\
85 \\
404 \\
654 \\
174 \\
31 \\
45 \\
23\end{array}$ & $\begin{array}{r}13 \\
14 \\
12 \\
12 \\
13 \\
33 \\
133 \\
181 . \\
43 \\
15 \\
18 \\
13\end{array}$ & $\begin{array}{c}7.0 \\
6.7 \\
4.0 \\
5.7 \\
4.6 \\
19 \\
95 \\
168 \\
46 \\
7.7 \\
9.5 \\
4.7\end{array}$ & $\begin{array}{r}.52 \\
.49 \\
.34 \\
.49 \\
.34 \\
.56 \\
.71 \\
.93 \\
1.06 \\
.50 \\
.52 \\
.37\end{array}$ & $\begin{array}{r}2.7 \\
2.7 \\
2.4 \\
2.3 \\
2.7 \\
6.6 \\
26.6 \\
36.2 \\
8.6 \\
3.1 \\
3.7 \\
2.6\end{array}$ \\
\hline NNUAL & 15 & 104 & 42 & 27 & .64 & 100 \\
\hline
\end{tabular}

MAGNITUDE AND PROBABILITY OF ANNUAL LOW FLOW BASED ON PERIOD OF RECORD 1960-76, 1983-85

\begin{tabular}{|c|c|c|c|c|c|c|}
\hline \multirow{2}{*}{$\begin{array}{l}\text { PERIOD } \\
\text { (CON- } \\
\text { SECU- } \\
\text { TIVE } \\
\text { DAYS) }\end{array}$} & \multicolumn{6}{|c|}{$\begin{array}{l}\text { DISCHARGE, IN FT } 3 / \mathrm{S} \text {, FOR INDICATED RECURRENCF } \\
\text { INTERVAL, IN YEARS, AND ANNUAL NON- } \\
\text { EXCEEDANCE PROBABILITY, IN PERCENT }\end{array}$} \\
\hline & $\begin{array}{c}2 \\
508\end{array}$ & $\begin{array}{c}5 \\
208\end{array}$ & $\begin{array}{l}10 \\
108\end{array}$ & $\begin{array}{l}20 \\
58\end{array}$ & $\begin{array}{l}50 \\
28\end{array}$ & $\begin{array}{r}100 \\
18\end{array}$ \\
\hline $\begin{array}{r}1 \\
3 \\
7 \\
14 \\
30 \\
60 \\
90 \\
120 \\
183\end{array}$ & $\begin{array}{r}5.7 \\
6.0 \\
6.3 \\
6.6 \\
7.5 \\
8.7 \\
9.7 \\
10 \\
11\end{array}$ & $\begin{array}{l}4.2 \\
4.4 \\
4.7 \\
4.9 \\
5.6 \\
6.5 \\
7.4 \\
7.8 \\
8.7\end{array}$ & $\begin{array}{l}3.6 \\
3.8 \\
4.0 \\
4.2 \\
4.9 \\
5.7 \\
6.5 \\
6.9 \\
7.6\end{array}$ & $\begin{array}{l}3.1 \\
3.3 \\
3.5 \\
3.7 \\
4.4 \\
5.1 \\
5.8 \\
6.2 \\
6.8\end{array}$ & $\begin{array}{l}= \\
=- \\
=- \\
-- \\
-\end{array}$ & $\begin{array}{l}-- \\
-- \\
-- \\
-- \\
-- \\
-- \\
--\end{array}$ \\
\hline
\end{tabular}

MAGNITUDE AND PROBABILITY OF ANNUAL HIGH FLOW BASED ON PERIOD OF RECORD 1959-76, 1982-85

\begin{tabular}{|c|c|c|c|c|c|c|}
\hline \multirow{2}{*}{$\begin{array}{l}\text { PERIOD } \\
\text { (CON- } \\
\text { SECU- } \\
\text { TIVE } \\
\text { DAYS) }\end{array}$} & \multicolumn{6}{|c|}{$\begin{array}{l}\text { DISCHARGE, IN FT' } 3 \text { /S, FOR INDICATED RECURRENCE } \\
\text { INTERVAL, IN YEARS, AND ANNUAL } \\
\text { EXCEEDANCE PROBABILITY, IN PERCENT }\end{array}$} \\
\hline & $\begin{array}{c}2 \\
508\end{array}$ & $\begin{array}{c}5 \\
208\end{array}$ & $\begin{array}{l}10 \\
108\end{array}$ & $\begin{array}{l}25 \\
48\end{array}$ & $\begin{array}{l}50 \\
28\end{array}$ & $\begin{array}{r}100 \\
18\end{array}$ \\
\hline $\begin{array}{r}1 \\
3 \\
7 \\
15 \\
30 \\
60 \\
90\end{array}$ & $\begin{array}{r}269 \\
249 \\
221 \\
190 \\
161 \\
125 \\
95\end{array}$ & $\begin{array}{l}512 \\
487 \\
440 \\
386 \\
329 \\
250 \\
187\end{array}$ & $\begin{array}{l}726 \\
697 \\
633 \\
560 \\
474 \\
358 \\
265\end{array}$ & $\begin{array}{r}1060 \\
1020 \\
935 \\
831 \\
697 \\
521 \\
383\end{array}$ & $\begin{array}{l}-- \\
-- \\
-- \\
-- \\
-- \\
--\end{array}$ & $\begin{array}{l}-- \\
=- \\
-- \\
-- \\
-- \\
--\end{array}$ \\
\hline
\end{tabular}

DURATION TABLE OF DAILY MEAN FLOW FOR PERIOD OF RECORD 1959-76, 1982-85 DISCHARGE, IN FT3/S, THAT WAS EQUALED OR EXCEEDED FOR INDICATED PERCENT OF TIME

\begin{tabular}{|c|c|c|c|c|c|c|c|c|c|c|c|c|c|c|}
\hline 58 & 108 & 158 & 208 & 258 & 308 & 408 & 508 & 608 & 708 & 758 & 808 & 858 & 90 능 & 958 \\
\hline 203 & 95 & 55 & 38 & 29 & 24 & 18 & 15 & 13 & 11 & 9.7 & 8.8 & 8.0 & 7.2 & \\
\hline
\end{tabular}


RIO GRANDE BASIN

08324000 JEMEZ RIVER NEAR JEMEZ, NM

LOCATION.--Lat 35०39'42", long 106 $44^{\prime} 34^{\prime \prime}$, Sandoval County, Hydrologic Unit 13020202, in Canon de San Diego Grant, on left bank $0.7 \mathrm{mi}$ downstream from Rio Guadalupe, $3.5 \mathrm{mi}$ north of Jemez, and at $\mathrm{mile} 29.5$.

DRAINAGE AREA. $--470 \mathrm{mi}^{2}$.

PERIOD OF RECORD.--June 1936 to May 1941, August 1949 to October 1950, May 1951 to September 1952 (irrigation seasons only), March 1953 to current year. Monthly discharge only for some periods, published in wsp 1732 Published as Jemez Creek near Jemez, 1936-41.

REVISED RECORDS. --WSP 1712: Drainage area. WSP 1923, 1957-58.

GAGE.--Water-stage recorder. Concrete control since Dec. 6, 1965. Datum of gage is $5,622 \mathrm{ft}$ above National Geodetic Vertical Datum of 1929 (plane-table survey by Topographic Division, U.S. Geological Survey 1952). June 22 , 1936 to Mar. 11, 1937 , at site $60 \mathrm{ft}$ upstream at datum $0.50 \mathrm{ft}$ higher. Mar. 12, 1937, to July 8 , 1938, at 22,1936 to Mar. 11,1937 , at site 60 ft upstream at datum 0.50 ft higher. Mar. 12 , 1937 , to July 8 , 1938 ,
present site at datum $0.7 \mathrm{ft}$ higher. July 9, 1938 , to May 6,1941 , at site 60 ft upstream at datum 0.70 ft higher.

REMARKS.--Diversions for irrigation of about 300 acres upstream from station.

AVERAGE DISCHARGE.--37 years (water years 1937-40, 1950, 1954-85), 74.9 ft3/s, 54,260 acre-ft/yr.

EXTREMES FOR PERIOD OF RECORD.--Maximum discharge, 5,900 $\mathrm{ft}^{3} / \mathrm{s}$, Apr. 21, 1958, from rating curve extended above $2,200 \mathrm{ft}^{3} / \mathrm{s}$ on basis of contracted-opening measurement of peak flow; maximum gage height, $10.10 \mathrm{ft}, \mathrm{July} 15$, 1985, present datum; minimum, $1.2 \mathrm{ft}^{3} / \mathrm{s}$, July 25,1981 .

EXTREMES OUTSIDE PERIOD OF RECORD.--Maximum flood since at least 1890 occurred between May 6 and 15 , 1941 , after gaco was destroyed (discharge probably exceeded $6,000 \mathrm{ft} / \mathrm{s}$ ), from information by local residents.

STATISTICAL SUMMARIES

MEAN MONTHLY AND MEAN ANNUAJ DISCHARgES $1937-40,1950$,

MAGNITUDE AND PROBABILITY OF ANNUAL LOW FLOW $1954-85$

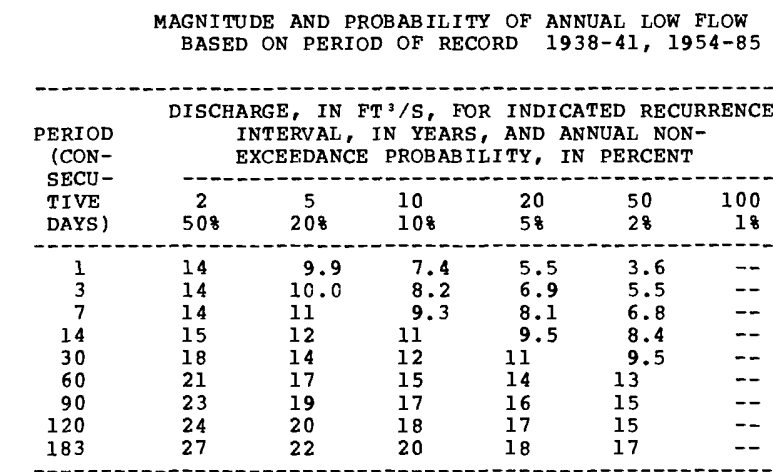

\begin{tabular}{|c|c|c|c|c|c|c|}
\hline MONTH & $\begin{array}{l}\text { MINIMUM } \\
\left(\mathrm{FT}^{3} / \mathrm{S}\right)\end{array}$ & $\begin{array}{l}\text { MAXIMUM } \\
\left(\mathrm{FT}^{3} / \mathrm{S}\right)\end{array}$ & $\begin{array}{l}\text { MEAN } \\
\left(\mathrm{FT}^{3} / \mathrm{S}\right)\end{array}$ & $\begin{array}{l}\text { STAN- } \\
\text { DARD } \\
\text { DEVIA- } \\
\text { TION } \\
\left(\mathrm{FT}^{3} / \mathrm{S}\right)\end{array}$ & $\begin{array}{l}\text { COEFFI- } \\
\text { CIENT OF } \\
\text { VARI- } \\
\text { ATION }\end{array}$ & $\begin{array}{c}\text { PERCENT } \\
\text { OF } \\
\text { ANNUAL } \\
\text { RUNOFF }\end{array}$ \\
\hline OCTOBER & 15 & 91 & 35 & 20 & .57 & 3.9 \\
\hline NOVEMBER & 18 & 103 & 34 & 17 & .51 & 3.8 \\
\hline DECEMBER & 17 & 51 & 27 & 7.7 & .28 & 3.0 \\
\hline JANUARY & 17 & 42 & 27 & 5.7 & .22 & 3.0 \\
\hline FEB RUARY & 20 & 57 & 34 & 9.7 & .29 & 3.8 \\
\hline MARCH & 32 & 221 & 81 & 45 & .56 & 9.0 \\
\hline APRIL & 43 & 961 & 266 & 224 & .84 & 29.7 \\
\hline MAY & 23 & 1118 & 229 & 236 & 1.03 & 25.5 \\
\hline JUNE & 12 & 274 & 61 & 62 & 1.01 & 6.8 \\
\hline JULY & 15 & 63 & 30 & 12 & .39 & 3.4 \\
\hline AUGUST & 15 & 121 & 42 & 25 & .60 & 4.6 \\
\hline SEPTEMBER & 11 & 110 & 31 & 18 & .57 & 3.5 \\
\hline ANNUAL & 29 & 189 & 75 & 45 & .60 & 100 \\
\hline
\end{tabular}

MAGNITUDE AND PROBABILITY OF ANNUAL HIGH FLOW BASED ON PERIOD OF RECORD $1937-40,1950$ $1954-85$

\begin{tabular}{|c|c|c|c|c|c|c|}
\hline \multirow{3}{*}{$\begin{array}{l}\text { PERIOD } \\
\text { (CON- } \\
\text { SECU- } \\
\text { TIVE } \\
\text { DAYS) }\end{array}$} & \multicolumn{6}{|c|}{$\begin{array}{c}\text { DISCHARGE, IN FT }{ }^{3} / \mathrm{S} \text {, FOR INDICATED RECURRFNCE } \\
\text { INTERVAL, IN YEARS, AND ANNUAL } \\
\text { EXCEEDANCE PROBABILITY, IN PERCENT }\end{array}$} \\
\hline & & & -1 & & & \\
\hline & $\begin{array}{c}2 \\
508\end{array}$ & $\begin{array}{c}5 \\
208\end{array}$ & $\begin{array}{l}10 \\
108\end{array}$ & $\begin{array}{l}25 \\
4 \%\end{array}$ & $\begin{array}{l}50 \\
28\end{array}$ & $\begin{array}{r}100 \\
18\end{array}$ \\
\hline 1 & 446 & 912 & 1370 & 2180 & 2980 & -- \\
\hline 3 & 390 & 814 & 1230 & 1970 & 2710 & -- \\
\hline 7 & 333 & 705 & 1080 & 1730 & 2380 & -- \\
\hline & 280 & 585 & 885 & 1410 & 1920 & -- \\
\hline & 240 & 497 & 746 & 1170 & 1590 & -- \\
\hline & 193 & 382 & 554 & 831 & 1090 & -- \\
\hline & 151 & 290 & 414 & 611 & 791 & -- \\
\hline
\end{tabular}

DURATION TABLE OF DAILY MEAN FLOW FOR PERIOD OF RECORD 1937-40, 1950, 1954-85 DISCHARGE, IN $\mathrm{FT}^{3} / \mathrm{S}$, THAT WAS EQUALED OR EXCEEDED FOR INDICATED PERCENT OF TIME

\begin{tabular}{|c|c|c|c|c|c|c|c|c|c|c|c|c|c|c|}
\hline 58 & 108 & 158 & 208 & 258 & 308 & 408 & 508 & 608 & 708 & 758 & 808 & 858 & $90 \%$ & 958 \\
\hline 299 & 160 & 104 & 74 & 59 & 49 & 38 & 32 & 28 & 24 & 23 & 21 & 20 & 18 & 15 \\
\hline
\end{tabular}


RIO GRANDE BASIN

08329000 JEMEZ RIVER BELOW JEMEZ CANYON DAM, NM

LOCATION,--Lat $35^{\circ} 23124^{\prime \prime}$, long $106^{\circ} 32 \cdot 03^{\prime \prime}$, in NEt sec.5, T.13 N., R.4 E., Sandoval County, Hydrologic Unit 13020202 , on right bank $0.8 \mathrm{mi}$ downstream from Jemez Canyon Dam, $2.0 \mathrm{mi}$ upstream from mouth, and $6 \mathrm{mi}$ north of Bernalilio.

DRAINAGE AREA. $--1,038 \mathrm{~m}^{2}$.

PERIOD OF RECORD.--March 1936 to January 1938, March 1943 to current year. Published as "Jemez Creek" prior to 1948, and as "near Bernal11lo" prior to 1954 .

REVISED RECORDS.--WSP 1178: 1949. WSP 1212: 1950. WSP 1512: 1936, 1943, 1945, 1947-48, 1949(M), 1950. WSP 1732: Drainage area.

GAGE.--Water-stage recorder. Datum of gage is 5,095.60 ft above National Geodetic Vertical Datum of 1929 (U.S. Army Corps of Engineers bench mark). Prior to Apr. 24, 1951, at site $0.8 \mathrm{mi}$ upstream at datum 24.51 ft higher. Apr. 24, 1951 , to June 25, 1958, at site $37 \mathrm{ft}$ upstream at datum $4.40 \mathrm{ft}$ above present datum. higher. Apr. 24, 1951 , to June 25, 1958 , at site 37 ft upstream at datum 4 , 40 ft above present datum. vertical Datum of 1929 (U.S. Army Corps of Engineers bench mark) used at times since January 1953 .

REMARRS. --Subsequent to October 1953, flow at this station can be completely regulated by Jemez Canyon Reservoir (station 08328500 ). However, reservolr is designed essentially for desilting and flood control rather than storage. Diversions for irrigation of about 3,000 acres upstream from station.

AVERAGE DISCHARGE.--11 years (water years 1937, 1944-53), 49.0 ft $/ \mathrm{s}, 35,500$ acre-ft/yr.

EXTREMES FOR PERIOD OF RECORD.--Maximum discharge, 16,300 $\mathrm{ft}^{3} / \mathrm{s}$, Aug. 29, 1943, gage height, 5.62 ft, site and datum then in use, from rating curve extended above $3,000 \mathrm{ft} / \mathrm{s}$; no flow for many days.

EXTREMES OUTSIDE PERIOD OF RECORD,--A flood in 1900 was probably less than $16,000 \mathrm{ft} / \mathrm{s}$, but highest observed outside period of record.

STATISTICAL SUMMARIES (PERIOD BEFORE REGULATION OF JEMEZ CANYON DAM)

MEAN MONTHLY AND MEAN ANNUAL DISCHARGES 1937, 1944-53

MAGNITUDE AND PROBABILITY OF ANNUAL LOW FLOW BASED ON PERIOD OF RECORD 1944-53

\begin{tabular}{|c|c|c|c|c|c|c|}
\hline MONTH & $\begin{array}{c}\text { MINIMUM } \\
\left(\mathrm{FT}^{3} / \mathrm{S}\right)\end{array}$ & $\begin{array}{c}\text { MAXIMUM } \\
\left(\mathrm{FT}^{3} / \mathrm{S}\right)\end{array}$ & $\begin{array}{l}\text { MEAN } \\
\left(\mathrm{FT}^{2} / \mathrm{S}\right)\end{array}$ & $\begin{array}{l}\text { STAN- } \\
\text { DARD } \\
\text { DEVIA- } \\
\text { TION } \\
\left(\mathrm{FT}^{3} / \mathrm{S}\right)\end{array}$ & $\begin{array}{l}\text { COEFFI- } \\
\text { CIENT OF } \\
\text { VARI- } \\
\text { ATION }\end{array}$ & $\begin{array}{c}\text { PERCENT } \\
\text { OF } \\
\text { ANNUAL } \\
\text { RUNOFF }\end{array}$ \\
\hline $\begin{array}{l}\text { OCTOBER } \\
\text { NOVEMBER } \\
\text { DECEMBER } \\
\text { JANUARY } \\
\text { FEBRUARY } \\
\text { HARCH } \\
\text { APRIL } \\
\text { MAY } \\
\text { JUNE } \\
\text { JULY } \\
\text { AUGUST } \\
\text { SEPTEMBER }\end{array}$ & $\begin{array}{r}.1 \\
4.3 \\
6.8 \\
3.1 \\
14 \\
20 \\
5.6 \\
3.4 \\
0.0 \\
0.0 \\
.1 \\
0.0\end{array}$ & $\begin{array}{r}106 \\
51 \\
33 \\
40 \\
62 \\
67 \\
578 \\
390 \\
91 \\
37 \\
87 \\
23\end{array}$ & $\begin{array}{r}20 \\
19 \\
18 \\
16 \\
28 \\
39 \\
234 \\
144 \\
17 \\
10 \\
21 \\
4.8\end{array}$ & $\begin{array}{c}32 \\
15 \\
8.8 \\
11 \\
14 \\
15 \\
208 \\
137 \\
26 \\
12 \\
24 \\
7.1\end{array}$ & $\begin{array}{r}1.56 \\
.78 \\
.49 \\
.67 \\
.49 \\
.39 \\
.89 \\
.95 \\
1.53 \\
1.22 \\
1.14 \\
1.49\end{array}$ & $\begin{array}{r}3.6 \\
3.3 \\
3.2 \\
2.8 \\
4.8 \\
6.9 \\
40.9 \\
25.2 \\
3.0 \\
1.8 \\
3.7 \\
.8\end{array}$ \\
\hline ANNUAL & 11 & 104 & 49 & 34 & .70 & 100 \\
\hline
\end{tabular}

\begin{tabular}{|c|c|c|c|c|c|c|}
\hline \multirow{2}{*}{$\begin{array}{l}\text { PERIOD } \\
\text { (CON- } \\
\text { SECU- } \\
\text { TIVE } \\
\text { DAYS) }\end{array}$} & \multicolumn{6}{|c|}{$\begin{array}{l}\text { DISCHARGE, IN FT3/S, FOR INDICATED RECURRENCE } \\
\text { INTERVAL, IN YEARS, AND ANNUAL NON- } \\
\text { EXCEEDANCE PROBABILITY, IN PERCENT }\end{array}$} \\
\hline & $\begin{array}{c}2 \\
508\end{array}$ & $\begin{array}{c}5 \\
208\end{array}$ & $\begin{array}{l}10 \\
108\end{array}$ & $\begin{array}{l}20 \\
58\end{array}$ & $\begin{array}{l}50 \\
28\end{array}$ & $\begin{array}{r}100 \\
18\end{array}$ \\
\hline 1 & -- & -- & - & - & -- & \\
\hline 3 & -- & -- & -- & - & - & \\
\hline 7 & -- & -- & - & - & -- & \\
\hline 14 & -- & -- & - & $\cdots$ & - & \\
\hline 30 & -- & -- & -- & - & -- & \\
\hline 60 & 2.4 & 1.0 & .6 & .4 & - & \\
\hline 90 & 2.4 & 1.0 & .6 & .4 & - & \\
\hline 120 & 7.3 & 2.9 & 1.3 & .5 & -- & \\
\hline 183 & 8.3 & 6.1 & 5.4 & 5.1 & -- & \\
\hline
\end{tabular}

MAGNITUDE AND PROBABILITY OF ANNUAL HIGH FLOW BASED ON PERIOD OF RECORD 1937, $1944-53$

\begin{tabular}{|c|c|c|c|c|c|c|}
\hline \multirow{2}{*}{$\begin{array}{l}\text { PERIOD } \\
\text { (CON- } \\
\text { SECU- } \\
\text { TIVE } \\
\text { DAYS) }\end{array}$} & \multicolumn{6}{|c|}{$\begin{array}{l}\text { DISCHARGE, IN FT }{ }^{3} / \mathrm{S}, \text { FOR INDICATED RECURRENC } \\
\text { INTERVAL, IN YEARS, AND ANNUAL } \\
\text { EXCEEDANCE PROBAB ILITY, IN PERCENT }\end{array}$} \\
\hline & $\begin{array}{c}2 \\
508\end{array}$ & $\begin{array}{c}5 \\
208\end{array}$ & $\begin{array}{l}10 \\
108\end{array}$ & $\begin{array}{l}25 \\
48\end{array}$ & $\begin{array}{l}50 \\
28\end{array}$ & $\begin{array}{r}100 \\
18\end{array}$ \\
\hline $\begin{array}{r}1 \\
3 \\
7 \\
15 \\
30 \\
60 \\
90\end{array}$ & $\begin{array}{l}691 \\
469 \\
324 \\
258 \\
193 \\
144 \\
108\end{array}$ & $\begin{array}{r}1130 \\
959 \\
759 \\
602 \\
469 \\
336 \\
242\end{array}$ & $\begin{array}{r}1340 \\
1300 \\
1130 \\
898 \\
730 \\
509 \\
361\end{array}$ & $\begin{array}{l}-- \\
-- \\
-- \\
-- \\
--\end{array}$ & $\begin{array}{l}-- \\
-- \\
-- \\
-- \\
--\end{array}$ & $\begin{array}{l}- \\
- \\
-- \\
-- \\
--\end{array}$ \\
\hline
\end{tabular}

DURATION TABLE OF DAILY MEAN FLOW FOR PERIOD OF RECORD 1937, 1944-53 DISCHARGE, IN FT'/S, THAT WAS EQUALED OR EXCEFDED FOR INDICATED PERCENT OF TIME

\begin{tabular}{|c|c|c|c|c|c|c|c|c|c|c|c|c|c|c|}
\hline 58 & 108 & 158 & 208 & 258 & 308 & 408 & 508 & 608 & 708 & 758 & 808 & 858 & 908 & 958 \\
\hline 275 & 102 & 61 & 46 & 37 & 29 & 20 & 12 & 5.8 & 1.1 & .1 & 1 & . I & 0.0 & 0.0 \\
\hline
\end{tabular}


FIO GRANDF; BASIN

08329000 JFMEZ RJVER BFI,OW JEMEZ, CANYON DAM, NM--COntinued

STATISTICAL SUMMARIES (PFRION AFTER COMPLETION OF JEMEZ CANYON DAM)

MEAN MONTHLY AND MEAN ANNUAI, DISCHARgES 1954-85

\begin{tabular}{|c|c|c|c|c|c|c|}
\hline MONTH & $\begin{array}{l}\text { MINTMUM } \\
\left(\mathrm{FT}^{3} / \mathrm{S}\right)\end{array}$ & $\begin{array}{c}\text { MAXI MUM } \\
\left(\mathrm{FT}^{3} / \mathrm{S}\right)\end{array}$ & $\begin{array}{l}\text { MEAN } \\
\left(\mathrm{FT}^{3} / \mathrm{S}\right)\end{array}$ & $\begin{array}{l}\text { STAN- } \\
\text { DARD } \\
\text { DEVIA- } \\
\text { TION } \\
\left(\mathrm{FT}^{3} / \mathrm{S}\right)\end{array}$ & $\begin{array}{l}\text { COEFFI- } \\
\text { CIENT OF } \\
\text { VARI- } \\
\text { ATION }\end{array}$ & $\begin{array}{c}\text { PFRCENT } \\
\text { OF } \\
\text { ANNUAL } \\
\text { RUNOFF }\end{array}$ \\
\hline \multicolumn{7}{|c|}{ n-n } \\
\hline OCTOBER & 0.0 & 178 & 28 & 45 & 1.64 & 3.6 \\
\hline NOVEMBER & 3.8 & 179 & 28 & 31 & 1.10 & 3.7 \\
\hline DECEMBER & .2 & 50 & 19 & 10 & .53 & 2.4 \\
\hline JANUARY & .3 & 51 & 23 & 9.7 & .43 & 2.9 \\
\hline FEBRUARY & .3 & 57 & 27 & 12 & .44 & 3.6 \\
\hline MARCH & 14 & 250 & 59 & 46 & .77 & 7.7 \\
\hline APRII, & 14 & 772 & 188 & 162 & .86 & 24.6 \\
\hline MAY & 0.0 & 968 & 211 & 245 & 1.16 & 27.6 \\
\hline JUNE & 0.0 & 988 & 95 & 200 & 2.10 & 12.5 \\
\hline JUI.Y & 0.0 & 137 & 21 & 29 & 1.43 & 2.7 \\
\hline AUGUS?' & .2 & 187 & 46 & 47 & 1.02 & 6.0 \\
\hline SEPTFMBFR & 0.0 & 99 & 19 & 27 & 1.42 & 2.5 \\
\hline NNUAL & 18 & 178 & 64 & 45 & .71 & 100 \\
\hline
\end{tabular}

MAGNITUDE AND PROBABILITY OF ANNUAL LOW FLOW BASFD ON PERIOD OF RECORD $1955-85$

\begin{tabular}{|c|c|c|c|c|c|c|}
\hline \multirow{4}{*}{$\begin{array}{l}\text { PERIOD } \\
\text { (CON- } \\
\text { SECU- } \\
\text { TIVE } \\
\text { DAYS) }\end{array}$} & \multicolumn{6}{|c|}{$\begin{array}{l}\text { DISCHARGE, IN } \mathrm{FT}^{3} / \mathrm{S} \text {, FOR INDICATED RECURRENCE } \\
\text { INTERVAL, IN YEARS, AND ANNUAL NON- } \\
\text { EXCEEDANCE PROBABILITY, IN PERCENT }\end{array}$} \\
\hline & & & & & & \\
\hline & 2 & 5 & 10 & 20 & 50 & 100 \\
\hline & $50 \%$ & 208 & 108 & 58 & 28 & 18 \\
\hline 1 & 0.0 & 0.0 & 0.0 & 0.0 & 0.0 & -- \\
\hline 3 & 0.0 & 0.0 & 0.0 & 0.0 & 0.0 & -- \\
\hline 7 & 0.0 & 0.0 & 0.0 & 0.0 & 0.0 & -- \\
\hline 14 & 0.0 & 0.0 & 0.0 & 0.0 & 0.0 & -- \\
\hline 30 & 0.0 & 0.0 & 0.0 & 0.0 & 0.0 & - \\
\hline 60 & 1.1 & .1 & 0.0 & 0.0 & 0.0 & - \\
\hline 90 & 5.5 & 1.3 & .4 & 0.0 & 0.0 & - \\
\hline 120 & 7.9 & 3.5 & 2.3 & 1.6 & 1.0 & - \\
\hline 183 & 14 & 8.8 & 7.2 & 6.1 & 5.2 & - \\
\hline
\end{tabular}

MAGNITUDE AND PROBABILITY OF ANNUAL HIGH FLOW BASED ON PERIOD OF RECORD 1954-85

\begin{tabular}{|c|c|c|c|c|c|c|}
\hline \multirow{3}{*}{$\begin{array}{l}\text { PERIOD } \\
\text { (CON- } \\
\text { SECU- } \\
\text { TIVE } \\
\text { DAYS) }\end{array}$} & \multicolumn{6}{|c|}{$\begin{array}{l}\text { DISCHARGE, IN FT'/S, FOR INDICATED RECURRENCE } \\
\text { INTERVAL, IN YEARS, AND ANNUAL } \\
\text { EXCEEDANCE' PROBABILITY, IN PERCENT }\end{array}$} \\
\hline & 2 & 5 & 10 & 25 & & 100 \\
\hline & 508 & $20 \%$ & $10 \%$ & 48 & $\begin{array}{l}50 \\
28\end{array}$ & $\begin{array}{r}100 \\
18\end{array}$ \\
\hline 1 & 688 & 1290 & 1780 & 2520 & 3150 & -- \\
\hline 3 & 534 & 1060 & 1520 & 2260 & 2930 & $\ldots$ \\
\hline 7 & 383 & 813 & 1230 & 1950 & 2650 & - \\
\hline 15 & 285 & 612 & 929 & 1470 & 2000 & -- \\
\hline 30 & 223 & 478 & 720 & 1130 & 1510 & -- \\
\hline 60 & 168 & 360 & 543 & 850 & 1140 & -- \\
\hline 90 & 129 & 270 & 402 & 620 & 825 & -. \\
\hline
\end{tabular}

DURATION TABLE OF DAILY MEAN FIOW FOR PERIOD OF RECORD 1954-85

DISCHARGE, IN FT ${ }^{3} / \mathrm{S}$, THAT WAS EQUALED OR EXCEEDED FOR INDICATED PERCENT OF TIME

\begin{tabular}{|c|c|c|c|c|c|c|c|c|c|c|c|c|c|c|}
\hline 58 & 108 & 158 & 208 & 258 & 308 & 408 & 508 & 608 & 708 & 758 & $80 \%$ & 858 & $90 \%$ & 958 \\
\hline 307 & 149 & 90 & 61 & 46 & 38 & 27 & 19 & 13 & 4.3 & 1.4 & .3 & 0.0 & 0.0 & 0.0 \\
\hline
\end{tabular}


RIO GRANDE BASIN

08329500 RIO GRANDE NEAR BERNALILLO, NM

LOCATION.--Lat $35^{\circ} 17^{\prime} 05^{\prime \prime}$, long $106^{\circ} 35^{\prime} 45^{\prime \prime}$, Sandoval County, on right bank 2 mi northwest of Sandia Pueblo, 3 mi southwest of Bernalilio, $3.5 \mathrm{mi}$ downstream from state Highway 44 , and $8.5 \mathrm{miles}$ downstream from Jemez River.

DRAINAGE AREA. - -17,300 $\mathrm{mi}^{2}$, approximately (includes 2,940 $\mathrm{mi}^{2}$ in closed basin in San Luis Valley, Co.).

PERIOD OF RECORD.--May 1941 to September 1969 (discontinued). Monthly discharge only for some periods, published in WSP 1312 .

REVISED RECORDS.-WSP 1312: 1943 (M). WSP 1923: 1953-54.

GAGE. - Water-stage recorder. Datum of gage is 5,030.57 ft above mean sea level, datum of 1929 . Supplemental water-stage recorder at a site 1,900 ft downstream used alternately 1953-58, 1961, 1964 , 1966 at the same datum 1953-55, variable $1956-58$ and $1.26 \mathrm{ft}$ lower than primary gage in 1961 .

REMARKS.--Diversions above station for irrigation of about 710,000 acres, some of which is below station. Possible regulation by operation of two reservoirs on Rio Chama and flood-and-silt detention reservoir on Jemez River (see sta 08328500 ).

AVERAGE DISCBARGE.--21 years (water years 1942-62), 1,132 ft $3 / \mathrm{s}, 820,100$ acre-ft/yr.

EXTREMES FOR PERIOD OF RECORD,--Maximum discharge, 25,400 ft $3 / \mathrm{s}$ May 16, 1941 ; maximum gage height, $6.83 \mathrm{ft}$ Sept. 20, 1941, no flow at times.

Other major floods occurred as follows (based primarily on records for station at San Felipe); Sept. 23 , 1929, about 23,000 ft $/ \mathrm{s}$; Aug. 21, 1935, about 22,000 ft/s; June 26, 1937, about 27,000 $\mathrm{ft} / \mathrm{s}$.

STATISTICAL SUMMARIES (PERIOD BEFORE REGUIATION OF ABIQUIU DAM)

MEAN MONTHLY AND MEAN ANNUAL DISChARGES 1942-62

\begin{tabular}{|c|c|c|c|c|c|c|}
\hline MONTH & $\begin{array}{l}\text { M I NIMUN } \\
\left(\mathrm{F}^{*} * / \mathrm{S}\right)\end{array}$ & $\begin{array}{l}\text { MAXIMUM } \\
\left(\mathrm{FT}^{3} / \mathrm{S}\right)\end{array}$ & $\begin{array}{l}\text { MEAN } \\
\left(\mathrm{FT}^{3} / \mathrm{S}\right)\end{array}$ & $\begin{array}{l}\text { STAN- } \\
\text { DARD } \\
\text { DEVIA- } \\
\text { TION } \\
\left.\text { (FT' }{ }^{3} / \mathrm{S}\right)\end{array}$ & $\begin{array}{l}\text { COEFFI- } \\
\text { CIENT OF } \\
\text { VARI- } \\
\text { ATION }\end{array}$ & $\begin{array}{c}\text { PERCENT } \\
\text { OF } \\
\text { ANNUAL } \\
\text { RUNOFF }\end{array}$ \\
\hline $\begin{array}{l}\text { OCTOBER } \\
\text { NOVEMBER } \\
\text { DECEMBER } \\
\text { JANUARY } \\
\text { FEBRUARY } \\
\text { MARCH } \\
\text { APRIL } \\
\text { MAY } \\
\text { JUNE } \\
\text { JULY } \\
\text { AUGUST } \\
\text { SEPTEMBER }\end{array}$ & $\begin{array}{r}12 \\
177 \\
284 \\
367 \\
470 \\
259 \\
133 \\
311 \\
52 \\
40 \\
31 \\
.3\end{array}$ & $\begin{array}{r}3200 \\
2800 \\
1498 \\
1059 \\
2167 \\
2021 \\
8341 \\
11160 \\
7176 \\
4498 \\
3599 \\
1607\end{array}$ & $\begin{array}{r}413 \\
804 \\
738 \\
624 \\
844 \\
808 \\
1802 \\
3222 \\
2444 \\
845 \\
680 \\
363\end{array}$ & $\begin{array}{r}687 \\
703 \\
353 \\
152 \\
385 \\
499 \\
1913 \\
3050 \\
2391 \\
1077 \\
759 \\
384\end{array}$ & $\begin{array}{r}1.66 \\
.87 \\
.48 \\
.24 \\
.46 \\
.62 \\
1.06 \\
.95 \\
.98 \\
1.27 \\
1.12 \\
1.06\end{array}$ & $\begin{array}{r}3.0 \\
5.9 \\
5.4 \\
4.6 \\
6.2 \\
5.9 \\
13.3 \\
23.7 \\
18.0 \\
6.2 \\
5.0 \\
2.7\end{array}$ \\
\hline ANNUAL & 373 & 3403 & 1132 & 763 & .67 & 100 \\
\hline
\end{tabular}

MAGNITUDE AND PROBABILITT OF ANNUAL HIGH FLOW BASED ON PERIOD OF RECORD 1942-62

\begin{tabular}{|c|c|c|c|c|c|c|}
\hline \multirow{2}{*}{$\begin{array}{l}\text { PERIOD } \\
\text { (CON- } \\
\text { SECU- } \\
\text { TIVE } \\
\text { DAYS) }\end{array}$} & \multicolumn{6}{|c|}{$\begin{array}{l}\text { DISCHARGE, IN FT } 3 / \mathrm{S} \text {, FOR INDICATED RECURRENCE } \\
\text { INTERVAL, IN YEARS, AND ANNUAL } \\
\text { EXCEEDANCE PROBABILITY, IN PERCENT }\end{array}$} \\
\hline & $\begin{array}{c}2 \\
508\end{array}$ & $\begin{array}{c}5 \\
208\end{array}$ & $\begin{array}{l}10 \\
108\end{array}$ & $\begin{array}{l}25 \\
48\end{array}$ & $\begin{array}{l}50 \\
28\end{array}$ & $\begin{array}{r}100 \\
18\end{array}$ \\
\hline $\begin{array}{r}1 \\
3 \\
7 \\
15 \\
30 \\
60 \\
90\end{array}$ & $\begin{array}{l}4990 \\
4290 \\
3920 \\
3520 \\
3030 \\
2350 \\
1960\end{array}$ & $\begin{array}{l}9540 \\
8510 \\
7780 \\
7030 \\
6240 \\
4980 \\
4040\end{array}$ & $\begin{array}{r}13400 \\
12100 \\
11100 \\
10000 \\
9050 \\
7380 \\
5940\end{array}$ & $\begin{array}{r}19100 \\
17600 \\
16100 \\
14700 \\
13400 \\
11300 \\
9010\end{array}$ & $\begin{array}{l}-- \\
-- \\
-- \\
-- \\
--\end{array}$ & $\begin{array}{l}-- \\
-- \\
-- \\
-- \\
--\end{array}$ \\
\hline
\end{tabular}

DURATION TABLE OF DAILY MEAN FLOW FOR PERIOD OF RECORD 1942-62

DISCHARGE, IN FT $3 / S$, THAT WAS EOUALED OR EXCEEDED FOR INDICATED PERCENT OF TIME

\begin{tabular}{|c|c|c|c|c|c|c|c|c|c|c|c|c|c|c|}
\hline 58 & 108 & 158 & 208 & 258 & 308 & 408 & 508 & 608 & 708 & 758 & 808 & 858 & 908 & 958 \\
\hline 4570 & 2700 & 1910 & 1450 & 1140 & 935 & 715 & 599 & 486 & 372 & 308 & 236 & 150 & 66 & 17 \\
\hline
\end{tabular}


LOCATION.--Lat $35^{\circ} 05^{\prime} 21^{\prime \prime}$, long $106^{\circ} 40^{\prime} 48^{\prime \prime}$, Bernalillo County, Hydrologic Unit 13020203, in Atrisco Grant, on downstream side of Central Ave. bridge in Albuquerque, and at mile $1,540.0$.

DRAINAGE AREA,--17,440 $\mathrm{mi}^{2}$, approximately, including $2,940 \mathrm{mi}^{2}$ in closed basin in San Luis Valley, co.

PERIOD OF RECORD.--October 1941 to current year. Monthly discharge only for some periods, published in wSP 1312 .

REVISED RECORDS.--WSP 1312: 1946 (M).

GAGE.--Water-stage recorder. Datum of gage is 4,946.16 ft above National Geodetic Vertical Datum of 1929 . Prior to sept. 18, 1947, at various sites at datum about $2.00 \mathrm{ft}$ higher; Sept. 18, 1947, to Apr. 12, 1959, at site 550 ft to the left of present site; Apr. 13, 1959, to June 29, 1960, at site $150 \mathrm{ft}$ to right of present site. Supplemental water-stage recorders at sites $75 \mathrm{ft}$ and $150 \mathrm{ft}$ to $\mathrm{right}$ of present site used at various times since 1964 .

REMARKS.--Flow completely regulated since November 1973 by Cochiti Dam (station 08317300) 50 mi upstream. Possible requlation by operation of reservoirs on Rio Chama and by flood-and-silt-detention reservoirs on Galisteo Creek and Jemez River (stations 08285000, 08286900, 08317900, 08328500). Since May 1971 flow affected by release of transmountain water from Heron Reservoir (station 08284510). Diversions upstream from station for irrigation of about 718,000 acres, several hundred of which are downstream from station. National Weather Service gage height telemeter at station.

COOPERATION,--Records for Albuquerque Riverside drain and Arenal, Armijo, and Atrisco canals provided by Middle Rio Grande Conservancy District.

AVERAGE DISCHARGE.--20 years (water years 1943-62), 1,004 ft3/s, 773,800 acre-ft/yr, prior to closure of Abiquiu Dam.

12 years (water years 1974-85), 1,352 ft $3 / \mathrm{s}, 979,500$ acre-ft/yr, since closure of Cochiti Dam.

EXTREMES FOR PERIOD OF RECORD.--Maximum discharge, 25,000 $\mathrm{ft}^{3} / \mathrm{s}$, Apr. 24, 1942, from rating curve extended above $13,900 \mathrm{ft} 3 / \mathrm{s}$; maximum gage height, $7.82 \mathrm{ft}$, Aug. 10,1967 ; no flow at times.

STATISTICAL SUMMARIES (PERIOD BEFORE REGULATION OF ABIQUIU DAM)

MEAN MONTHLY AND MEAN ANNUAL DISCHARGES 1943-62

\begin{tabular}{|c|c|c|c|c|c|c|}
\hline MONTH & $\begin{array}{c}\text { MINIMUM } \\
\left(\mathrm{FT}^{3} / \mathrm{S}\right)\end{array}$ & $\begin{array}{c}\text { MAXIMUM } \\
\left(\mathrm{FT}^{3} / \mathrm{S}\right)\end{array}$ & $\begin{array}{l}\text { MEAN } \\
\left(\mathrm{FT}^{3} / \mathrm{S}\right)\end{array}$ & $\begin{array}{l}\text { STAN- } \\
\text { DARD } \\
\text { DEVIA- } \\
\text { TION } \\
\left(\text { FT }^{3} / \mathrm{S}\right)\end{array}$ & $\begin{array}{l}\text { COEFFI- } \\
\text { CIENT OF } \\
\text { VARI - } \\
\text { ATION }\end{array}$ & $\begin{array}{c}\text { PERCENT } \\
\text { OF } \\
\text { ANNUAL } \\
\text { RUNOFF }\end{array}$ \\
\hline OCTOBER & 0.0 & 1260 & 263 & 288 & 1.10 & 2,2 \\
\hline NOVEMBER & 66 & 2211 & 724 & 640 & $\begin{array}{r}1.10 \\
.88\end{array}$ & $\begin{array}{l}2.2 \\
6.0\end{array}$ \\
\hline DECEMBER & 270 & 1557 & 735 & 357 & .49 & 6.1 \\
\hline JANUARY & 406 & 806 & 619 & 117 & .19 & 5.1 \\
\hline FEB RUARY & 473 & 2146 & 862 & 390 & .45 & 7.1 \\
\hline MARCH & 167 & 2103 & 749 & 491 & .66 & 6.2 \\
\hline APRIL & 55 & 4166 & 1436 & 1274 & .89 & 11.9 \\
\hline MAY & 226 & 8831 & 2840 & 2639 & .93 & 23.5 \\
\hline JUNE & 109 & 6114 & 2157 & 2244 & 1.04 & 17.9 \\
\hline JULY & $8 B$ & 4183 & 746 & 1053 & 1.41 & 6.2 \\
\hline AUGUST & 28 & 3687 & 630 & 801 & 1.27 & 5.2 \\
\hline SEPTEMBER & 16 & 1615 & 299 & 362 & 1.21 & 2.5 \\
\hline ANNUAL & 333 & 2190 & 1004 & 597 & .59 & 100 \\
\hline
\end{tabular}

MAGNITUDE AND PROBABILITY OF ANNUAL LOW FLOW BASED ON PERIOD OF RECORD 1944-62

\begin{tabular}{|c|c|c|c|c|c|c|}
\hline \multirow{3}{*}{$\begin{array}{l}\text { PERI OD } \\
\text { (CON- } \\
\text { SECU- } \\
\text { TIVE } \\
\text { DAYS) }\end{array}$} & \multicolumn{6}{|c|}{$\begin{array}{l}\text { DISCHARGE, IN FT } 3 / S \text {, FOR INDICATED RECURRENCE } \\
\text { INTERVAL, IN YEARS, AND ANNUAL NON- } \\
\text { EXCEEDANCE PROBABILITY, IN PERCENT }\end{array}$} \\
\hline & & $-\infty$ & & $-\cdots$ & -- & \\
\hline & $\begin{array}{c}2 \\
508\end{array}$ & $\begin{array}{c}5 \\
208\end{array}$ & $\begin{array}{l}10 \\
108\end{array}$ & $\begin{array}{l}20 \\
58\end{array}$ & $\begin{array}{l}50 \\
28\end{array}$ & $\begin{array}{r}100 \\
18\end{array}$ \\
\hline 1 & 25 & 1. & 0.0 & 0. & -- & -- \\
\hline 3 & 25 & 3. & 0. & 0. & - & -. \\
\hline 7 & 34 & 6. & 2.7 & 0.0 & - & -- \\
\hline 14 & 50 & 18 & 9.4 & 0. & - & -- \\
\hline 30 & 140 & 22 & 3.8 & .5 & -- & -- \\
\hline 60 & 156 & 49 & 24 & 12 & -- & -- \\
\hline 90 & 223 & 94 & 55 & 33 & -- & -- \\
\hline 120 & 260 & 129 & 85 & 59 & - & -- \\
\hline 183 & 379 & 203 & 140 & 100 & -- & -- \\
\hline
\end{tabular}

MAGNITUDE AND PROBABILITY OF ANNUAL HIGH FLOW BASED ON PERIOD OF RECORD 1943-62

\begin{tabular}{|c|c|c|c|c|c|c|}
\hline $\begin{array}{l}\text { PERIOD } \\
\text { (CON- } \\
\text { SECU- }\end{array}$ & \multicolumn{6}{|c|}{$\begin{array}{l}\text { DISCHARGE, IN } \mathrm{FT}^{3} / \mathrm{S} \text {, FOR INDICATED RECURRENCE } \\
\text { INTERVAL, IN YEARS, AND ANNUAL } \\
\text { EXCEEDANCE PROBABILITY, IN PERCENT }\end{array}$} \\
\hline $\begin{array}{l}\text { TIVE } \\
\text { DAYS) }\end{array}$ & $\begin{array}{c}2 \\
508\end{array}$ & $\begin{array}{c}5 \\
208\end{array}$ & $\begin{array}{l}10 \\
108\end{array}$ & $\begin{array}{l}25 \\
48\end{array}$ & $\begin{array}{l}50 \\
28\end{array}$ & $\begin{array}{r}100 \\
18\end{array}$ \\
\hline $\begin{array}{r}1 \\
3 \\
7 \\
15 \\
30 \\
60 \\
90\end{array}$ & $\begin{array}{l}4510 \\
3940 \\
3650 \\
3270 \\
2790 \\
2160 \\
1790\end{array}$ & $\begin{array}{l}8370 \\
7710 \\
7190 \\
6570 \\
5710 \\
4480 \\
3630\end{array}$ & $\begin{array}{r}11400 \\
10800 \\
10200 \\
9400 \\
8270 \\
6540 \\
5230\end{array}$ & $\begin{array}{r}15700 \\
15500 \\
14600 \\
13700 \\
12200 \\
9770 \\
7740\end{array}$ & $\begin{array}{l}=- \\
=- \\
=- \\
-- \\
--\end{array}$ & $\begin{array}{l}-- \\
-- \\
-- \\
-- \\
-- \\
--\end{array}$ \\
\hline
\end{tabular}

DURATION TABLE OF DAILY MEAN FLOW FOR PERIOD OF RECORD 1943-62 DISCHARGE, IN FT ${ }^{3} / \mathrm{S}$, THAT WAS EQUALED OR EXCEEDED FOR INDICATED PERCENT OF TIME

\begin{tabular}{|c|c|c|c|c|c|c|c|c|c|c|c|c|c|c|}
\hline 58 & 108 & 158 & 208 & 258 & 308 & $40 \%$ & 508 & 608 & 708 & 758 & 808 & 858 & 908 & 958 \\
\hline 4160 & 2520 & 1750 & 1270 & 973 & 861 & 667 & 553 & 434 & 304 & 231 & 158 & 98 & 59 & 26 \\
\hline
\end{tabular}


RIO GRANDE BASIN

08330000 RIO GRANDE AT ALBUQUERQUE, NM--Continued

STATISTICAL SUMMARIES (PERIOD BEFORE REGULATION OF COCHITI DAM AND AFTER COMPLETION OF ABIQUID DAM)

MEAN MONTHLY AND MEAN ANNUAL DISChaRges 1964-73

\begin{tabular}{|c|c|c|c|c|c|c|}
\hline MONTH & $\begin{array}{c}\text { MINIMUM } \\
\left(\mathrm{FT}^{3} / \mathrm{S}\right)\end{array}$ & $\begin{array}{c}\text { MAXJMUM } \\
\left(\mathrm{FT}^{3} / \mathrm{S}\right)\end{array}$ & $\begin{array}{l}\text { MEAN } \\
\left(\mathrm{FT}^{3} / \mathrm{S}\right)\end{array}$ & $\begin{array}{l}\text { STAN- } \\
\text { DARD } \\
\text { DEVIA- } \\
\text { TION } \\
\text { (FT' } / S \text { ) }\end{array}$ & $\begin{array}{l}\text { COEFFI- } \\
\text { CIENT OF } \\
\text { VARI- } \\
\text { ATION }\end{array}$ & $\begin{array}{c}\text { PERCENT } \\
\text { OF } \\
\text { ANNUAL } \\
\text { RUNOFF }\end{array}$ \\
\hline \multicolumn{7}{|c|}{ 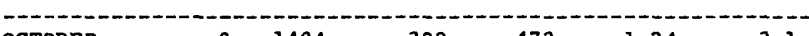 } \\
\hline OCTOBER & .9 & 1404 & 382 & 472 & 1.24 & 3.1 \\
\hline NOVEMBER & 444 & 1829 & 1196 & 476 & .40 & 9.8 \\
\hline DECEMBER & 305 & 2054 & 863 & 516 & .60 & 7.1 \\
\hline JANUARY & 392 & 1016 & 708 & 167 & .24 & 5.8 \\
\hline FEBRUARY & 453 & 1084 & 788 & 184 & .23 & 6.5 \\
\hline MARCH & 279 & 1569 & 868 & 376 & .43 & 7.1 \\
\hline APRIL & 152 & 2350 & 1229 & 821 & .67 & 10.1 \\
\hline MAY & 28 & 6290 & 2334 & 1961 & .84 & 19.2 \\
\hline JONE & 3.6 & 5284 & 1927 & 1901 & .99 & 15.8 \\
\hline JULY & .9 & 3015 & 752 & 1138 & 1.51 & 6.2 \\
\hline AUGUST & 70 & 2029 & 768 & 711 & .93 & 6.3 \\
\hline SEPTEMBER & 13 & 843 & 360 & 311 & .86 & 3.0 \\
\hline ANNUAL & 290 & 1962 & 1015 & 498 & .49 & 100 \\
\hline
\end{tabular}

MAGNI TUDE AND PROBABILITY OF ANNUAL LOW FLOW BASED ON PERIOD OF RECORD $1965-73$

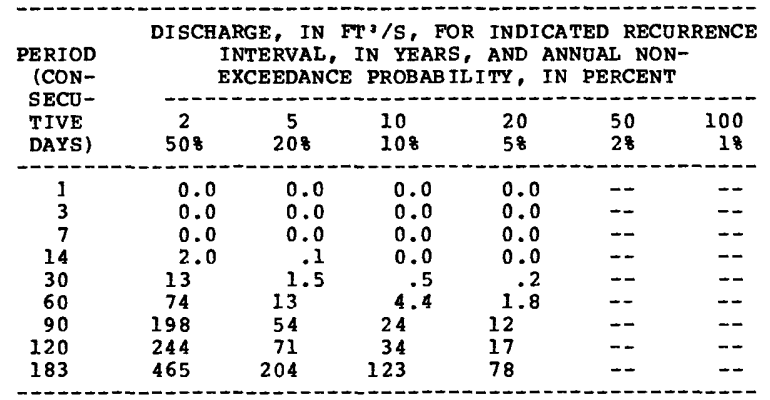

MAGNITUDE AND PROBABILITY OF ANNUAL HIGH FI,OW BASED ON PERIOD OF RECORD 1964-73

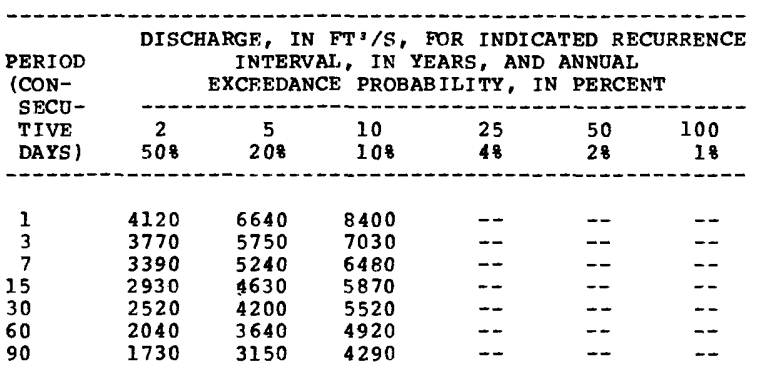

DURATION TABLE OF DAILY MEAN FLOW FOR PERIOD OF RECORD 1964-73

DISCHARSE, IN FT'S, THAT WAS EQUALED OR EXCEEDED FOR INDICATED PERCENT OF TIME

\begin{tabular}{|c|c|c|c|c|c|c|c|c|c|c|c|c|c|c|}
\hline 58 & 108 & 158 & $20 \%$ & 258 & 308 & $40 \%$ & 508 & 608 & 708 & 758 & 808 & 858 & 908 & 958 \\
\hline 3680 & 2520 & 1900 & 1540 & 1240 & 1050 & 841 & 688 & 563 & 375 & 282 & 175 & 55 & 9.1 & \\
\hline
\end{tabular}


RIO GRANDE BASIN

08330000 RIO GRANDE AT ALBUQUERQUE, NM--Continued

STATISTICAL SUMMARIES (PERIOD AFTER COMPLETION OF COCHITI DAM)

MEAN MONTHLY AND MEAN ANNUAL DISCHARGES

$1974-85$

\begin{tabular}{|c|c|c|c|c|c|c|}
\hline MONTH & $\begin{array}{l}\text { MINIMUM } \\
\left(\mathrm{FT}^{3} / \mathrm{S}\right)\end{array}$ & $\begin{array}{l}\text { MAXI MUM } \\
\left(\mathrm{FT}^{3} / \mathrm{S}\right)\end{array}$ & $\begin{array}{l}\text { MEAN } \\
\left(\mathrm{FT}^{3} / \mathrm{S}\right)\end{array}$ & $\begin{array}{l}\text { STAN- } \\
\text { DARD } \\
\text { DEVIA- } \\
\text { TION } \\
\text { (FT3/S) }\end{array}$ & $\begin{array}{l}\text { COEFFI- } \\
\text { CIENT OF } \\
\text { VARI- } \\
\text { ATION }\end{array}$ & $\begin{array}{c}\text { PERCENT } \\
\text { OF } \\
\text { ANNUAL } \\
\text { RUNOFF }\end{array}$ \\
\hline $\begin{array}{l}\text { OCTOBER } \\
\text { NOVEMBER } \\
\text { DECEMBER } \\
\text { JANUARY } \\
\text { FEBRUARY } \\
\text { MARCH } \\
\text { APRIL } \\
\text { MAY } \\
\text { JUNE } \\
\text { JULY } \\
\text { AUGUST } \\
\text { SEPTEMBER }\end{array}$ & $\begin{array}{r}38 \\
401 \\
480 \\
486 \\
590 \\
480 \\
137 \\
148 \\
445 \\
287 \\
278 \\
51\end{array}$ & $\begin{array}{r}666 \\
1723 \\
1736 \\
1487 \\
1031 \\
2287 \\
6343 \\
6203 \\
6113 \\
5439 \\
1001 \\
1495\end{array}$ & $\begin{array}{r}261 \\
911 \\
1050 \\
824 \\
778 \\
1057 \\
1995 \\
3577 \\
3193 \\
1572 \\
570 \\
419\end{array}$ & $\begin{array}{r}194 \\
432 \\
471 \\
301 \\
154 \\
548 \\
1748 \\
2290 \\
2285 \\
1543 \\
230 \\
415\end{array}$ & $\begin{array}{l}.74 \\
.47 \\
.45 \\
.37 \\
.20 \\
.52 \\
.88 \\
.64 \\
.72 \\
.98 \\
.40 \\
.99\end{array}$ & $\begin{array}{r}1.6 \\
5.6 \\
6.5 \\
5.1 \\
4.8 \\
6.5 \\
12.3 \\
22.1 \\
19.7 \\
9.7 \\
3.5 \\
2.6\end{array}$ \\
\hline ANNUAL & 356 & 2295 & 1352 & 668 & .49 & 100 \\
\hline
\end{tabular}

MAGNITUDE AND PROBABILITY OF ANNUAL LOW FLOW BASED ON PERIOD OF RECORD 1975-85

\begin{tabular}{|c|c|c|c|c|c|c|}
\hline \multirow{2}{*}{$\begin{array}{l}\text { PERIOD } \\
\text { (CON- } \\
\text { SECU- } \\
\text { TIVE } \\
\text { DAYS) }\end{array}$} & \multicolumn{6}{|c|}{$\begin{array}{l}\text { DISCHARGE, IN FT'3/S, FOR INDICATED RECURRENCE } \\
\text { INTERVAL, IN YEARS, AND ANNUAL NON- } \\
\text { EXCEEDANCE PROBABILITY, IN PERCENT }\end{array}$} \\
\hline & $\begin{array}{c}2 \\
508\end{array}$ & $\begin{array}{c}5 \\
208\end{array}$ & $\begin{array}{l}10 \\
108\end{array}$ & 20 & 50 & 100 \\
\hline $\begin{array}{r}1 \\
3 \\
7 \\
14 \\
30 \\
60 \\
90 \\
120 \\
183\end{array}$ & $\begin{array}{l}13 \\
13 \\
20 \\
40 \\
109 \\
218 \\
303 \\
391 \\
543\end{array}$ & $\begin{array}{r}2 . \\
5 \\
9 . \\
38 \\
97 \\
185 \\
254 \\
349\end{array}$ & $\begin{array}{r}1 . \\
4 \\
21 \\
61 \\
144 \\
205 \\
275\end{array}$ & $\begin{array}{r}0 \\
0 \\
0 \\
2 \\
13 \\
41 \\
117 \\
172 \\
225\end{array}$ & $\begin{array}{l}-- \\
-- \\
-- \\
-- \\
-- \\
-- \\
--\end{array}$ & $\begin{array}{l}-- \\
-- \\
-- \\
-- \\
-- \\
-- \\
--\end{array}$ \\
\hline
\end{tabular}

MAGNITUDE AND PROBABILITY OF ANNUAL HIGH FLON BASED ON PERIOD OF RECORD 1974-85

\begin{tabular}{|c|c|c|c|c|c|c|}
\hline \multirow{2}{*}{$\begin{array}{l}\text { PERIOD } \\
\text { (CON- } \\
\text { SECU- } \\
\text { TIVE } \\
\text { DAYS) }\end{array}$} & \multicolumn{6}{|c|}{$\begin{array}{c}\text { DISCHARGE, IN FT } 3 / S \text {, FOR INDICATED RECURRENCE } \\
\text { INTERVAL, IN YEARS, AND ANNUAL } \\
\text { EXCEEDANCE PROBABILITY, IN PERCENT }\end{array}$} \\
\hline & $\begin{array}{c}2 \\
508\end{array}$ & $\begin{array}{c}5 \\
208\end{array}$ & $\begin{array}{l}10 \\
108\end{array}$ & $\begin{array}{l}25 \\
48\end{array}$ & $\begin{array}{l}50 \\
28\end{array}$ & $\begin{array}{r}100 \\
18\end{array}$ \\
\hline $\begin{array}{r}1 \\
3 \\
7 \\
15 \\
30 \\
60 \\
90\end{array}$ & $\begin{array}{l}4820 \\
4640 \\
4390 \\
4140 \\
3910 \\
3390 \\
2920\end{array}$ & $\begin{array}{l}7630 \\
7480 \\
7220 \\
6900 \\
6590 \\
5790 \\
5070\end{array}$ & $\begin{array}{l}9400 \\
9250 \\
8960 \\
8520 \\
8080 \\
7190 \\
6450\end{array}$ & $\begin{array}{l}-- \\
-- \\
-- \\
-- \\
--\end{array}$ & $\begin{array}{l}-- \\
-- \\
-- \\
-- \\
--\end{array}$ & $\begin{array}{l}-- \\
-- \\
-- \\
-- \\
-- \\
--\end{array}$ \\
\hline
\end{tabular}

DURATION TABLE OF DAILY MEAN FLOW FOR PERIOD OF RECORD 1974-85

DISCHARGE, IN FT'S/S, THAT WAS EQUALED OR EXCEEDED FOR INDICATED PERCENT OF TIME

\begin{tabular}{|c|c|c|c|c|c|c|c|c|c|c|c|c|c|c|}
\hline 58 & 108 & 158 & 208 & 258 & 308 & 408 & 508 & 608 & 708 & 758 & 808 & 858 & 908 & 958 \\
\hline 5500 & 3890 & 2680 & 1880 & 1490 & 1240 & 932 & 741 & 605 & 480 & 419 & 347 & 262 & 128 & 36 \\
\hline
\end{tabular}


RIO GRANDE BASIN

08331500 RIO GRANDE NEAR BELEN, NM

LOCATION.--Lat $34^{\circ} 39^{\prime} 10^{\prime \prime}$, long $106^{\circ} 44^{\prime} 10^{\prime \prime}$, in Tome claim, on left bank 300 ft downstream from highway bridge on State Highway 6 and 2 miles east of Belen.

DRAINAGE AREA.--18,230 $\mathrm{mi}^{2}$, approximately (includes 2,940 $\mathrm{mi}^{2}$ in closed basin in San Luis Valley, co).

PERIOD OF RECORD.--January 1942 to June 1957 (discontinued).

GAGE.--Water-stage recorder. Datum of gage is 4,797.32 ft above mean sea level, datum of 1929 . Prior to Apr. 9, 1953, at bridge 300 ft upstream at same datum.

REMARKS.--Diversions for irrigation of about 725,000 acres above station. Station is bypassed by 1 canal, 3 ditches, and 1 riverside drain.

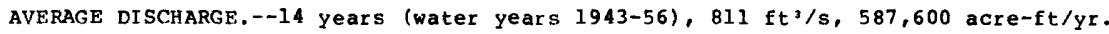

EXTREMES FOR PERIOD OF RECORD. - Maximum cischarge, 23,100 ft/s Apr. 24, 1942 (gage height, $5.05 \mathrm{ft}$ ), from rating curve extended above $12,500 \mathrm{ft}^{3} / \mathrm{s}$ by logarithmic plotting; minimum daily, that of 0ct. 10, 11, 14, 15, 1956.

STATISTICAL. SUMMARIES

MEAN MONTHLY AND MEAN ANNUAL DISCHARGES 1943-56

\begin{tabular}{|c|c|c|c|c|c|c|}
\hline MONTH & $\begin{array}{l}\text { MINIMUM } \\
\left(\mathrm{FT}^{3} / \mathrm{S}\right)\end{array}$ & $\begin{array}{c}\text { MAXIMUM } \\
\left(\mathrm{FT}^{3} / \mathrm{S}\right)\end{array}$ & $\begin{array}{l}\text { MEAN } \\
\left(\mathrm{FT}^{3} / \mathrm{S}\right)\end{array}$ & $\begin{array}{l}\text { STAN- } \\
\text { DARD } \\
\text { DEVIA- } \\
\text { TION } \\
\text { (FT'/S) }\end{array}$ & $\begin{array}{l}\text { COEFFI - } \\
\text { CIENT OF } \\
\text { VARI- } \\
\text { ATION }\end{array}$ & $\begin{array}{c}\text { PERCENT } \\
\text { OF } \\
\text { ANNUAL } \\
\text { RUNOFF }\end{array}$ \\
\hline $\begin{array}{l}\text { OCTOBER } \\
\text { NOVEMBER } \\
\text { DECEMBER } \\
\text { JANUARY } \\
\text { FEB RUARY } \\
\text { MARCH } \\
\text { APRII } \\
\text { MAY } \\
\text { JUNE } \\
\text { JULY } \\
\text { AUGUST } \\
\text { SEPTEMBER }\end{array}$ & $\begin{array}{r}14 \\
26 \\
154 \\
339 \\
469 \\
147 \\
69 \\
114 \\
101 \\
46 \\
35 \\
21\end{array}$ & $\begin{array}{r}665 \\
1753 \\
1636 \\
864 \\
1981 \\
1798 \\
2585 \\
6661 \\
5899 \\
2327 \\
1253 \\
465\end{array}$ & $\begin{array}{r}196 \\
431 \\
702 \\
652 \\
851 \\
635 \\
830 \\
2298 \\
2062 \\
567 \\
362 \\
174\end{array}$ & $\begin{array}{r}204 \\
465 \\
373 \\
149 \\
371 \\
456 \\
792 \\
2415 \\
2395 \\
739 \\
343 \\
150\end{array}$ & $\begin{array}{r}1.04 \\
1.08 \\
.53 \\
.23 \\
.44 \\
.72 \\
.95 \\
1.05 \\
1.16 \\
1.30 \\
.95 \\
.86\end{array}$ & $\begin{array}{r}2.0 \\
4.4 \\
7.2 \\
6.7 \\
8.7 \\
6.5 \\
8.5 \\
23.5 \\
21.1 \\
5.8 \\
3.7 \\
1.8\end{array}$ \\
\hline NNUAL & 253 & 1592 & 811 & 560 & .69 & 100 \\
\hline
\end{tabular}

MAGNITUDE AND PROBABILITY OF ANNUAL LOW FLOW BASED ON PERIOD OF RECORD $1944-57$

\begin{tabular}{|c|c|c|c|c|c|c|}
\hline \multirow{2}{*}{$\begin{array}{l}\text { PERIOD } \\
\text { (CON- } \\
\text { SECU- } \\
\text { TIVE } \\
\text { DAYS) }\end{array}$} & \multicolumn{6}{|c|}{$\begin{array}{l}\text { DISCHARGE, IN FT3/S, FOR INDICATED RECURRENCE } \\
\text { INTERVAL, IN YEARS, AND ANNUAL NON- } \\
\text { EXCEEDANCE PROBABIL ITY, IN PERCENT } \\
\end{array}$} \\
\hline & $\begin{array}{c}2 \\
508\end{array}$ & $\begin{array}{c}5 \\
208\end{array}$ & $\begin{array}{l}10 \\
108\end{array}$ & $\begin{array}{l}20 \\
58\end{array}$ & $\begin{array}{l}50 \\
28\end{array}$ & $\begin{array}{r}100 \\
18\end{array}$ \\
\hline $\begin{array}{r}1 \\
3 \\
7 \\
14 \\
30 \\
60 \\
90 \\
120 \\
183\end{array}$ & $\begin{array}{r}50 \\
51 \\
55 \\
59 \\
69 \\
92 \\
128 \\
160 \\
234\end{array}$ & $\begin{array}{r}24 \\
24 \\
24 \\
26 \\
30 \\
38 \\
53 \\
67 \\
105\end{array}$ & $\begin{array}{l}15 \\
16 \\
16 \\
18 \\
20 \\
24 \\
32 \\
41 \\
66\end{array}$ & $\begin{array}{l}11 \\
11 \\
11 \\
13 \\
14 \\
16 \\
21 \\
27 \\
43\end{array}$ & $\begin{array}{l}= \\
- \\
z- \\
z- \\
z \\
-\end{array}$ & $\begin{array}{l}\because- \\
\because- \\
z- \\
\approx \\
\approx\end{array}$ \\
\hline
\end{tabular}

MAGNITUDE AND PROBABILITY OF ANNUAL HIGH FLOW BASED ON PERIOD OF RECORD 1943-56

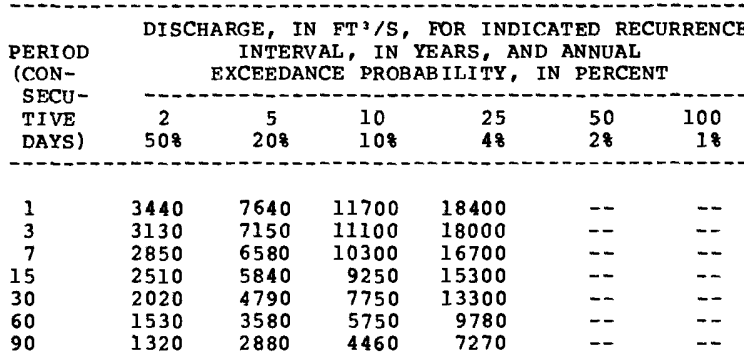

DURATION TABLE OF DAILY MEAN FLOW FOR PERIOD OF RECORD 1943-56 DISCHARGE, IN FT'/S, THAT WAS EQUALED OR EXCEEDED FOR INDICATED PERCENT OF TIME

\begin{tabular}{|c|c|c|c|c|c|c|c|c|c|c|c|c|c|c|}
\hline 58 & 108 & 158 & 208 & 258 & 308 & 408 & 508 & 608 & 708 & 758 & 808 & 858 & 908 & 958 \\
\hline 2960 & 1860 & 1180 & 944 & 808 & 726 & 580 & 445 & 293 & 168 & 115 & 90 & 74 & 58 & 42 \\
\hline
\end{tabular}


08331990 RIO GRANDE CONVEYANCE CHANNEL NEAR BERNARDO, NM

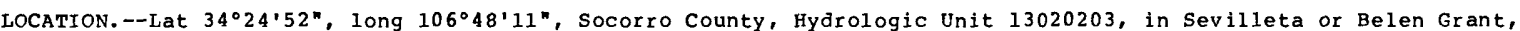
$0.2 \mathrm{mi}$ south of U.S. Highway $60,1.8 \mathrm{mi}$ east of Bernardo, about $3 \mathrm{mi}$ upstream from $\mathrm{floodway,} \mathrm{and} 4 \mathrm{mi}$ upstream from Rio Puerco.

PERIOD OF RECORD.--June 1936 to September 1937, October 1964 to current year. Ju1y 1943 to September 1964 , included in composite flow of "Rio Grande near Bernardo". October 1960 to September 1964, monthly acre-feet published in WSP 1923 (daily records available in district files). Beginning October 1952, flow in conveyance channel represents controlled diversion from Rio Grande. Prior to October 1952, records called "San Francisco Riverside drain near Bernardo", are not equivalent.

GAGE.--Water-stage recorder and concrete control. Datum of gage is $4,720.00 \mathrm{ft}$ above National Geodetic Vertical Datum of 1929. Prior to October 1964, 0.2 mi upstream at various datums.

REMARKS.--Conveyance channel is 1 of 4 channels (stations 08332010, 08332030, and 08332050) carrying flow in valley cross section. Original design and plan was for conveyance channel to carry flow up to about $2,000 \mathrm{ft}^{3} / \mathrm{s}$. For combined monthly flow in acre-ft of this channel, floodway, Bernardo interior drain and Lower San Juan Riverside drain.

AVERAGE DISCHARGE. - -10 years (water years 1953-62), $462 \mathrm{ft}^{3} / \mathrm{s}, 334,700$ acre-ft/yr.

EXTREMES FOR PERIOD OF RECORD.--Maximum daily discharge, 2,220 ft $3 / \mathrm{s}$, Apr. 22, 1958 ; no flow many days most years.

STATISTICAL SUMMARIES (PERIOD BEFORE COMPLETION OF ABIQUIU DAM)

MEAN MONTHLY AND MEAN ANNUAL DISCHARGES 1953-62

\begin{tabular}{|c|c|c|c|c|c|c|}
\hline MONTH & $\begin{array}{l}\text { MINIMUM } \\
\left(\mathrm{FT}^{3} / \mathrm{S}\right)\end{array}$ & $\begin{array}{c}\text { MAXIMUM } \\
\left(\mathrm{FT}^{3} / \mathrm{S}\right)\end{array}$ & $\begin{array}{l}\text { MEAN } \\
\left(\mathrm{FT}^{3} / \mathrm{S}\right)\end{array}$ & $\begin{array}{l}\text { STAN- } \\
\text { DARD } \\
\text { DEVIA- } \\
\text { TION } \\
\left(\mathrm{FT}^{3} / \mathrm{S}\right)\end{array}$ & $\begin{array}{l}\text { COEFFI- } \\
\text { CIENT OF } \\
\text { VARI- } \\
\text { ATION }\end{array}$ & $\begin{array}{c}\text { PERCENT } \\
\text { OF } \\
\text { ANNUAL } \\
\text { RUNOF F }\end{array}$ \\
\hline $\begin{array}{l}\text { OCTOBER } \\
\text { NOVEMBER } \\
\text { DECEMBER } \\
\text { JANUARY } \\
\text { FEBRUARY } \\
\text { MARCH } \\
\text { APRIL } \\
\text { MAY } \\
\text { JUNE } \\
\text { JULY } \\
\text { AUGUST } \\
\text { SEPTEMBER }\end{array}$ & $\begin{array}{r}.4 \\
9.9 \\
7.6 \\
8.2 \\
134 \\
203 \\
51 \\
309 \\
46 \\
.9 \\
2.2 \\
0.0\end{array}$ & $\begin{array}{r}790 \\
1413 \\
1200 \\
768 \\
880 \\
1025 \\
1597 \\
1724 \\
1354 \\
1625 \\
1752 \\
982\end{array}$ & $\begin{array}{l}159 \\
571 \\
643 \\
540 \\
611 \\
484 \\
567 \\
778 \\
519 \\
213 \\
286 \\
182\end{array}$ & $\begin{array}{l}230 \\
532 \\
396 \\
221 \\
208 \\
262 \\
472 \\
484 \\
463 \\
498 \\
538 \\
304\end{array}$ & $\begin{array}{l}1.45 \\
.93 \\
.62 \\
.41 \\
.34 \\
.54 \\
.83 \\
.62 \\
.89 \\
2.34 \\
1.88 \\
1.67\end{array}$ & $\begin{array}{r}2.9 \\
10.3 \\
11.6 \\
9.7 \\
11.0 \\
8.7 \\
10.2 \\
14.0 \\
9.3 \\
3.8 \\
5.2 \\
3.3\end{array}$ \\
\hline ANNUAL & 165 & 864 & 462 & 217 & .47 & 100 \\
\hline
\end{tabular}

MAGNITUDE AND PROBABILITY OF ANNUAL LOW FLOW BASED ON PERIOD OF RECORD 1954-62

\begin{tabular}{|c|c|c|c|c|c|c|}
\hline \multirow{2}{*}{$\begin{array}{l}\text { PERIOD } \\
\text { (CON- } \\
\text { SECU- } \\
\text { TIVE } \\
\text { DAYS) }\end{array}$} & \multicolumn{6}{|c|}{$\begin{array}{l}\text { DISCHARGE, IN FT'/S, FOR INDICATED RECURRENCE } \\
\text { INTERVAL, IN YEARS, AND ANNUAL NON- } \\
\text { EXCEEDANCE PROBABILITY, IN PERCENT }\end{array}$} \\
\hline & $\begin{array}{c}2 \\
508\end{array}$ & $\begin{array}{c}5 \\
208\end{array}$ & $\begin{array}{l}10 \\
108\end{array}$ & $\begin{array}{l}20 \\
58\end{array}$ & $\begin{array}{l}50 \\
28\end{array}$ & $\begin{array}{r}100 \\
18\end{array}$ \\
\hline $\begin{array}{r}1 \\
3 \\
7 \\
14 \\
30 \\
60 \\
90 \\
120 \\
183\end{array}$ & $\begin{array}{r}.5 \\
.5 \\
.8 \\
1.7 \\
2.9 \\
14 \\
31 \\
55 \\
167\end{array}$ & $\begin{array}{l}0.0 \\
0.0 \\
0.0 \\
0.0 \\
0.0 \\
1.2 \\
3.8 \\
12 \\
50\end{array}$ & $\begin{array}{r}0.0 \\
0.0 \\
0.0 \\
0.0 \\
0.0 \\
0.0 \\
1.2 \\
5.5 \\
24\end{array}$ & $\begin{array}{r}0.0 \\
0.0 \\
0.0 \\
0.0 \\
0.0 \\
0.0 \\
.4 \\
2.8 \\
13\end{array}$ & $\begin{array}{l}-- \\
-- \\
-- \\
-- \\
-- \\
-- \\
--\end{array}$ & $\begin{array}{l}-- \\
-- \\
-- \\
-- \\
-- \\
-- \\
-- \\
--\end{array}$ \\
\hline
\end{tabular}

MAGNITUDE AND PROBABILTTY OF ANNUAL HIGH FLON BASED ON PERIOD OF RECORD 1953-62

\begin{tabular}{|c|c|c|c|c|c|c|}
\hline \multirow{3}{*}{$\begin{array}{l}\text { PERIOD } \\
\text { (CON- } \\
\text { SECU- } \\
\text { TIVE } \\
\text { DAYS) }\end{array}$} & \multicolumn{6}{|c|}{$\begin{array}{l}\text { DISCHARGE, IN FT }{ }^{3 / S} \text {, FOR INDICATED RECURRENCE } \\
\text { INTERVAL, IN YEARS, AND ANNUAL } \\
\text { EXCEEDANCE PROBABILITY, IN PERCENT }\end{array}$} \\
\hline & 2 & 5 & 10 & 25 & 50 & 100 \\
\hline & 508 & 208 & 108 & 48 & 28 & 18 \\
\hline 1 & 1640 & 1940 & 2070 & -- & -- & -- \\
\hline 3 & 1460 & 1840 & 2040 & -- & -- & -. \\
\hline 7 & 1360 & 1750 & 1980 & -- & -- & -- \\
\hline 15 & 1200 & 1660 & 1960 & -- & -- & -- \\
\hline 30 & 1050 & 1520 & 1840 & -- & -- & -- \\
\hline 60 & 931 & 1390 & 1700 & -- & - & -- \\
\hline 90 & 857 & 1260 & 1510 & -- & -- & -- \\
\hline
\end{tabular}

DURATION TABLE OF DAILY MEAN FLOW FOR PERIOD OF RECORD 1953-62 DISCHARGE, IN FT $3 / \mathrm{S}$, THAT WAS EQUALED OR EXCEEDED FOR INDICATED PERCENT OF TIME

\begin{tabular}{|c|c|c|c|c|c|c|c|c|c|c|c|c|c|c|}
\hline 58 & 108 & 158 & 208 & 258 & 308 & 408 & 508 & 608 & 708 & 758 & 808 & 858 & 908 & 958 \\
\hline 1490 & 1190 & 984 & 825 & 720 & 626 & 506 & 345 & 182 & 81 & 46 & 26 & 12 & 4.8 & \\
\hline
\end{tabular}


RIO GRANDE BASIN

08331990 RIO GRANDE CONVEYANCE CHANNEL NEAR BERNARDO, NM--Continued STATISTICAL SUMMARIES (PERIOD BEFORE REGULATION OF COCHITI DAM AND AFTER COMPLETION OF ABIQUIU DAM)

MEAN MONTHLY AND MEAN ANNUAL DISChaRgES 1964-73

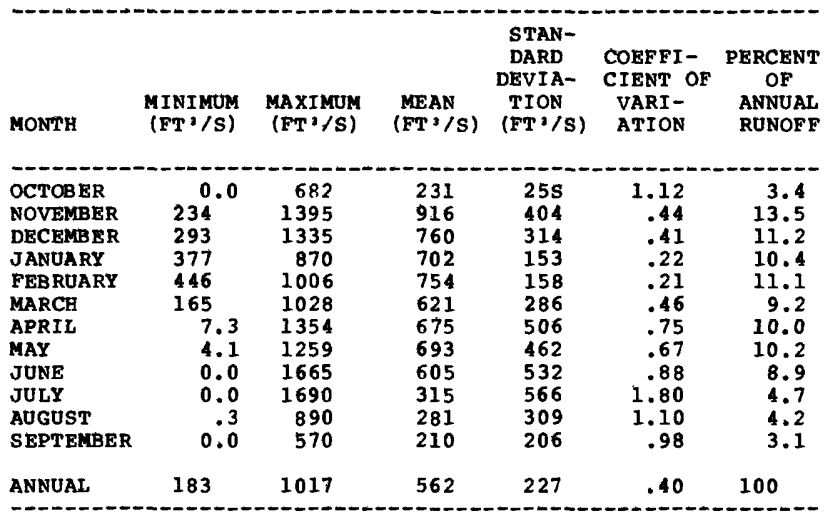

MAGNITUDE AND PROBABILITY OF ANNUAL LOW FLON BASED ON PERIOD OF RECORD 1965-73

\begin{tabular}{|c|c|c|c|c|c|c|}
\hline \multirow{2}{*}{$\begin{array}{l}\text { PERIOD } \\
\text { (CON- } \\
\text { SECU- } \\
\text { TIVE } \\
\text { DAYS) }\end{array}$} & \multicolumn{6}{|c|}{$\begin{array}{l}\text { DISCHARGE, IN FT'/S, FOR INDICATED RECURRENCE } \\
\text { INTERVAL, IN YEARS, AND ANNUAL NON- } \\
\text { EXCEEDANCE PROBABILITY, IN PERCENT }\end{array}$} \\
\hline & ${ }^{2} 0^{2}$ & $\begin{array}{c}5 \\
208\end{array}$ & $\begin{array}{l}10 \\
108\end{array}$ & $\begin{array}{l}20 \\
58\end{array}$ & $\begin{array}{l}50 \\
28\end{array}$ & $\begin{array}{r}100 \\
18\end{array}$ \\
\hline $\begin{array}{r}1 \\
3 \\
7 \\
14 \\
30 \\
60 \\
90 \\
120 \\
183\end{array}$ & $\begin{array}{r}0.0 \\
0.0 \\
0.0 \\
0.0 \\
0.0 \\
9.2 \\
58 \\
61 \\
221\end{array}$ & $\begin{array}{l}0.0 \\
0.0 \\
0.0 \\
0.0 \\
0.0 \\
0.0 \\
1.1 \\
4.2 \\
95\end{array}$ & $\begin{array}{r}0.0 \\
0.0 \\
0.0 \\
0.0 \\
0.0 \\
0.0 \\
0.0 \\
. .7\end{array}$ & $\begin{array}{r}0.0 \\
0.0 \\
0.0 \\
0.0 \\
0.0 \\
0.0 \\
0.0 \\
.1\end{array}$ & $\begin{array}{l}-- \\
=- \\
-- \\
-- \\
-- \\
--\end{array}$ & $\begin{array}{l}=- \\
=- \\
=- \\
=- \\
=-\end{array}$ \\
\hline
\end{tabular}

MAGNITUDE AND PROBABILITY OF ANNUAL HIGH FLON BASED ON PERIOD OF RECORD 1964-73

\begin{tabular}{|c|c|c|c|c|c|c|}
\hline \multirow{2}{*}{$\begin{array}{l}\text { PERIOD } \\
\text { (CON- } \\
\text { SECU- } \\
\text { TIVE } \\
\text { DAYS) }\end{array}$} & \multicolumn{6}{|c|}{$\begin{array}{l}\text { DISCHARGE, IN ET'/S, FOR INDICATED RECURRENCE } \\
\text { INTERVAL, IN YEARS, AND ANNUAL } \\
\text { EXCEEDANCE PROBABILITY, IN PERCENT }\end{array}$} \\
\hline & $\begin{array}{c}2 \\
508\end{array}$ & $\begin{array}{c}5 \\
208\end{array}$ & $\begin{array}{l}10 \\
108\end{array}$ & $\begin{array}{l}25 \\
48\end{array}$ & $\begin{array}{l}50 \\
28\end{array}$ & $\begin{array}{r}100 \\
18\end{array}$ \\
\hline $\begin{array}{r}1 \\
3 \\
7 \\
15 \\
30 \\
60 \\
90\end{array}$ & $\begin{array}{l}1650 \\
1560 \\
1480 \\
1380 \\
1220 \\
1100 \\
1020\end{array}$ & $\begin{array}{l}1820 \\
1750 \\
1680 \\
1610 \\
1480 \\
1390 \\
1290\end{array}$ & $\begin{array}{l}1930 \\
1850 \\
1790 \\
1710 \\
1600 \\
1500 \\
1390\end{array}$ & $\begin{array}{l}=- \\
=- \\
=- \\
=- \\
--\end{array}$ & $\begin{array}{l}=- \\
=- \\
=- \\
--\end{array}$ & $\begin{array}{l}\cdots \\
- \\
-- \\
-- \\
--\end{array}$ \\
\hline
\end{tabular}

DURATION TABLE OF DAILY MEAN FLOW FOR PERIOD OF RECORD 1964-73

DISCHARGE, IN FT'/S, THAT WAS EQUALED OR EXCEEDED FOR INDICATED PERCENT OF TIME

\begin{tabular}{|c|c|c|c|c|c|c|c|c|c|c|c|c|c|c|}
\hline 58 & 108 & 158 & 208 & 258 & 308 & 408 & 508 & 608 & 708 & 758 & 808 & 858 & 908 & 958 \\
\hline 1500 & 1290 & 1140 & 995 & 908 & 840 & 703 & 543 & 336 & 137 & 68 & 14 & .1 & 0.0 & 0.0 \\
\hline
\end{tabular}


RIO GRANDE BASIN

08331990 RIO GRANDE CONVEYANCE CHANNEL NEAR BERNARDO, NM--Continued STATISTICAL SUMMARIES (PERIOD AFTER COMPLETION OF COCHITI DAM)

MAGNITUDE AND PROBABILITY OF ANNUAL LOW FLOW BASED ON PERIOD OF RECORD 1975-85

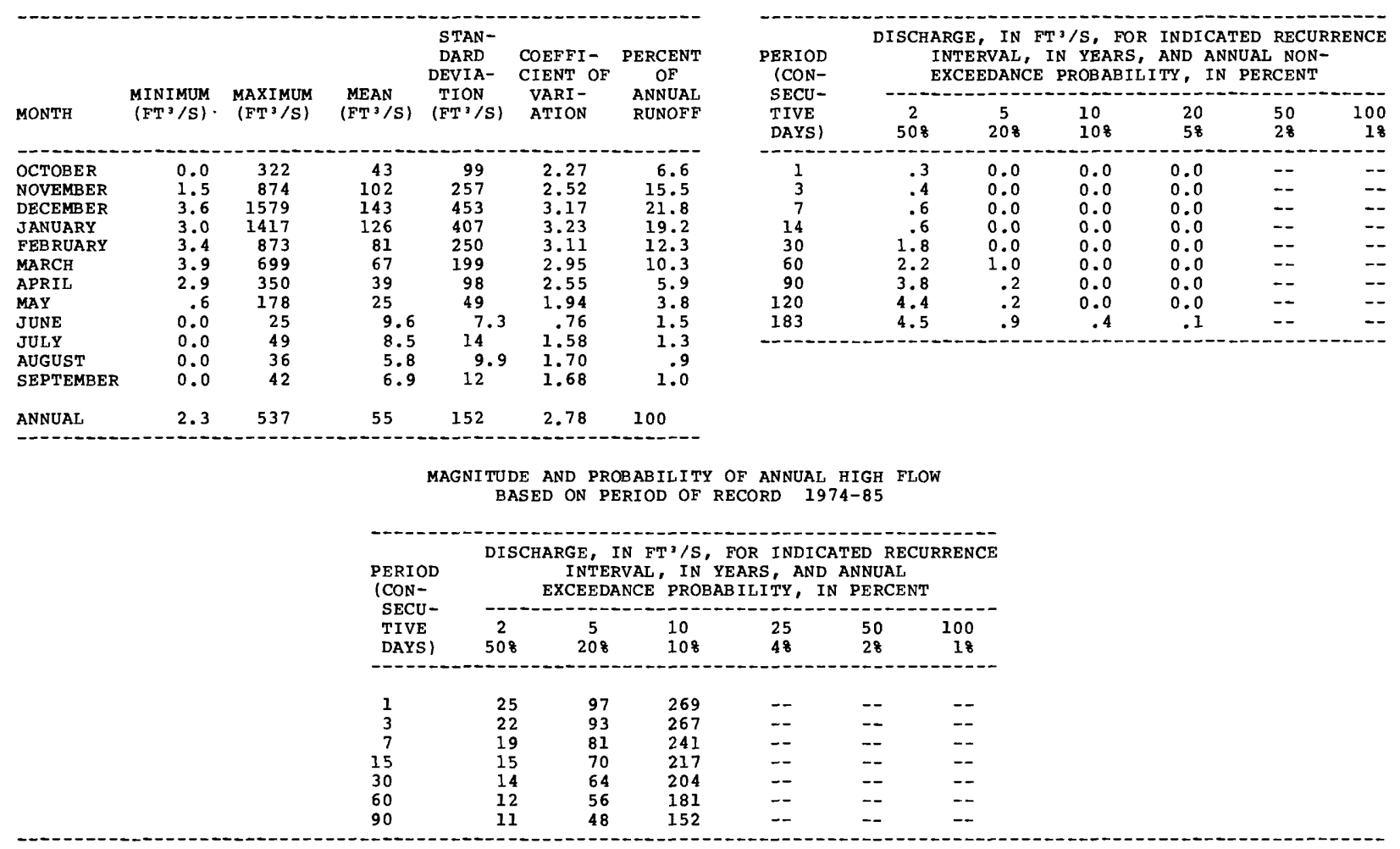

DURATION TABLE OF DAILY MEAN FLOW FOR PERIOD OF RECORD 1974-85

DISCHARGE, IN FT $3 / S$, THAT WAS EQUALED OR EXCEEDED FOR INDICATED PERCENT OF TIME

\begin{tabular}{|c|c|c|c|c|c|c|c|c|c|c|c|c|c|c|}
\hline 58 & 108 & 158 & 208 & 258 & 308 & 408 & 508 & 608 & 708 & 758 & 808 & 858 & 908 & 958 \\
\hline 265 & 29 & 18 & 13 & 11 & 9.9 & 7.7 & 6.0 & 5.0 & 4.0 & 3.5 & 2.9 & 2.2 & .7 & \\
\hline
\end{tabular}


RIO GRANDE BASIN

08332000 RIO GRANDE NEAR BERNARDO, NM

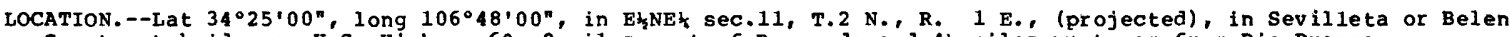
Grant, at bridge on U.S. Highway 60,2 miles east of Bernardo and $4 \frac{1}{2}$ miles upstream from Rio Puerco.

DRAINAGE AREA. - - 19,230 $\mathrm{mi}^{2}$, approximately (includes 2,940 $\mathrm{mi}^{2}$ in closed basin San Luis Valley, Co.).

PERIOD OF RECORD.--June 1936 to January 1939, October 1941 to September 1964. Month1y discharge on1y October 1942 to June 1943 published in wSP 1312. Prior to October 1951, flow of Bernardo interior drain was included only when it carried river overflow; since that date entire flow is included.

GAGE.--Water-stage recorders. There are 3 gages, 1 on a conveyance channel designed to carry 2,000 ft $3 / s$, 5 miles downstream from heading, formerly San Francisco Riverside drain, 1 on the floodway (former river channel) which will now carry water when total flow exceeds about $1,700 \mathrm{ft}^{3} / \mathrm{s}$, and 1 on Bernardo interior drain. Datum of conveyance channel gage is 4,720.00 ft above mean sea level, datum of 1929, leveling of 1951 . Prior to October 1952, main gage was on river channel (the present floodway gage, present datum) at datum 4.722 .55 ft above mean sea level, datum of 1929, leveling of 1951 . Datum of Bernardo interior drain supplementary recording gage is 4,713.99 $\mathrm{ft}$ above mean sea level, datum of 1929 , leveling of 1951 .

REMARKS.--Records represent total discharge of the $r$ iver and are a summation of discharge in $r i v e r$ channel (now called floodway), conveyance channel, and Bernardo interior drain. Flow in La Joya Eastside drain along left side of floodway is not included in the composite. Diversions for irrigation of about 740,000 acres above station.

AVERAGE DISCHARGE.--22 years (water years 1937-38, 1942, 1944-62), 1,093 ft3/s, 791,900 acre-ft/yr.

COOPERATION.--Records for La Joya Eastside drain are furnished by Bureau of Reclamation.

EXTREMES FOR PERIOD OF RECORD. --Maximum discharge, 21,100 ft $3 / \mathrm{s}$ Apr. 25, 1942 (gage height, $6.90 \mathrm{ft}$ ); no flow at times.

ST'ATISTICAL SUMMARIES (PERIOD BEFORE REGULATION OF ABIQUIU DAM)

MEAN MONTHLY AND MEAN ANNUAL DISCHARGES $\begin{aligned} & 1937-38,1942, \\ & 1944-62\end{aligned}$

MAGNITUDE AND PROBABILITY OF ANNUAL LOW FLOW BASED ON PERIOD OF RECORD 1938, 1945-62

\begin{tabular}{|c|c|c|c|c|c|c|}
\hline MONTH & $\begin{array}{l}\text { MINIMUM } \\
\left(\mathrm{FT}^{3} / \mathrm{S}\right)\end{array}$ & $\begin{array}{c}\text { MAXIMUM } \\
\left(\mathrm{FT}^{3} / \mathrm{S}\right)\end{array}$ & $\begin{array}{l}\text { MEAN } \\
\left(\mathrm{FT}^{3} / \mathrm{S}\right)\end{array}$ & $\begin{array}{c}\text { STAN- } \\
\text { DARD } \\
\text { DEVIA- } \\
\text { TION } \\
\left(\mathrm{FT}^{3} / \mathrm{S}\right)\end{array}$ & $\begin{array}{l}\text { COEFFI- } \\
\text { CIENT OF } \\
\text { VARI- } \\
\text { ATION }\end{array}$ & $\begin{array}{c}\text { PERCENT } \\
\text { OF } \\
\text { ANNUAL } \\
\text { RUNOF F }\end{array}$ \\
\hline $\begin{array}{l}\text { OCTOBER } \\
\text { NOVEMBER } \\
\text { DECEMBER } \\
\text { JANUARY } \\
\text { FEBRUARY } \\
\text { MARCH } \\
\text { APRIL } \\
\text { MAY } \\
\text { JUNE } \\
\text { JULY } \\
\text { AUGUST } \\
\text { SEPTEMBER }\end{array}$ & $\begin{array}{l}11^{.4} \\
150 \\
367 \\
498 \\
150 \\
50 \\
98 \\
51 \\
4.4 \\
7.6 \\
0.0\end{array}$ & $\begin{array}{r}2857 \\
2728 \\
1682 \\
1148 \\
1962 \\
1907 \\
7863 \\
10610 \\
7052 \\
4024 \\
3235 \\
1516\end{array}$ & $\begin{array}{r}415 \\
758 \\
803 \\
670 \\
874 \\
791 \\
1696 \\
3323 \\
2352 \\
682 \\
452 \\
333\end{array}$ & $\begin{array}{r}625 \\
703 \\
389 \\
152 \\
340 \\
514 \\
1992 \\
3205 \\
2383 \\
981 \\
674 \\
445\end{array}$ & $\begin{array}{r}1.51 \\
.93 \\
.48 \\
.23 \\
.39 \\
.65 \\
1.17 \\
.96 \\
1.01 \\
1.44 \\
1.49 \\
1.34\end{array}$ & $\begin{array}{r}3.2 \\
5.8 \\
6.1 \\
5.1 \\
6.6 \\
6.0 \\
12.9 \\
25.3 \\
17.9 \\
5.2 \\
3.4 \\
2.5\end{array}$ \\
\hline NNUAL & 237 & 3251 & 1093 & 780 & .71 & 100 \\
\hline
\end{tabular}

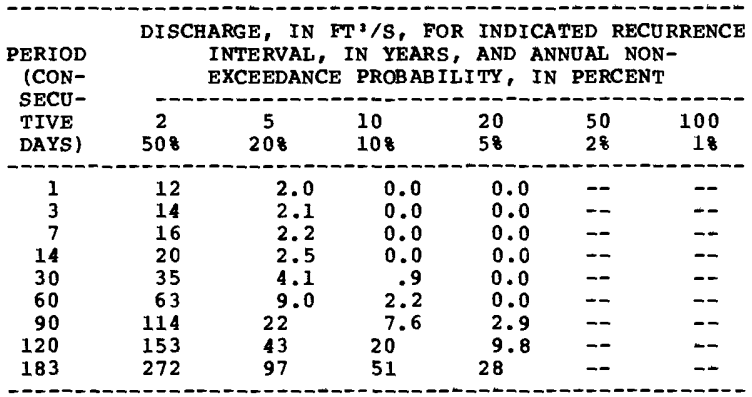

MAGNITUDE AND PROBABILITY OF ANNUAL HIGH FLOW BASED ON PERIOD OF RECORD $1937-38,1942$, $1944-62$

\begin{tabular}{|c|c|c|c|c|c|c|}
\hline \multirow{2}{*}{$\begin{array}{l}\text { PERIOD } \\
\text { (CON- } \\
\text { SECU- } \\
\text { TIVE } \\
\text { DAYS) }\end{array}$} & \multicolumn{6}{|c|}{$\begin{array}{l}\text { DISCHARGE, IN FT } 3 / \mathrm{S} \text {, FOR INDICATED RECURRENCE } \\
\text { INTERVAL, IN YEARS, AND ANNUAL } \\
\text { EXCEEDANCE PROBABILITY, IN PERCENT }\end{array}$} \\
\hline & $\begin{array}{c}2 \\
508\end{array}$ & $\begin{array}{c}5 \\
208\end{array}$ & $\begin{array}{l}10 \\
108\end{array}$ & $\begin{array}{l}25 \\
48\end{array}$ & $\begin{array}{l}50 \\
28\end{array}$ & $\begin{array}{r}100 \\
18\end{array}$ \\
\hline $\begin{array}{r}1 \\
3 \\
7 \\
15 \\
30 \\
60 \\
90\end{array}$ & $\begin{array}{l}4790 \\
4480 \\
4100 \\
3620 \\
3100 \\
2470 \\
2060\end{array}$ & $\begin{array}{l}9940 \\
9380 \\
8550 \\
7580 \\
6670 \\
5400 \\
4300\end{array}$ & $\begin{array}{r}14100 \\
13400 \\
12200 \\
10900 \\
9710 \\
7960 \\
6230\end{array}$ & $\begin{array}{r}20100 \\
19100 \\
17300 \\
15600 \\
14200 \\
11900 \\
9140\end{array}$ & $\begin{array}{l}=- \\
=- \\
=- \\
=- \\
--\end{array}$ & $\begin{array}{l}-- \\
-- \\
-- \\
-- \\
-- \\
=-\end{array}$ \\
\hline
\end{tabular}

DURATION TABLE OF DAILY MEAN FLOW FOR PERIOD OF RECORD 1937-38, 1942, 1944-62 DISCHARGE, IN FT ${ }^{3} / \mathrm{S}$, THAT WAS EQUALED OR EXCEEDED FOR INDICATED PERCENT OF TIME

\begin{tabular}{|c|c|c|c|c|c|c|c|c|c|c|c|c|c|c|}
\hline 58 & 108 & 158 & 208 & 258 & 308 & 408 & 508 & 608 & 708 & 758 & 808 & 858 & 908 & 958 \\
\hline 4700 & 2660 & 1870 & 1390 & 1070 & 876 & 701 & 561 & 409 & 221 & 148 & 91 & 52 & 28 & 8.3 \\
\hline
\end{tabular}


RIO GRANDE BASIN

08332010 RIO GRANDE FLOODWAY NEAR BERNARDO, NM

LOCATION,--Lat $34^{\circ} 25^{\prime} 01^{\prime \prime}$, long $106^{\circ} 48^{\prime} 00^{\prime \prime}$, Socorro County, Hydrologic unit 13020203, in Belen or Sevilleta Grant, on downstream side of bridge on U.s. Highway $60,5 \mathrm{mi}$ downstream from heading of conveyarice channel, 2 mi east of Bernardo, and at mile 1,487.2.

DRAINAGE AREA, $--19,230 \mathrm{mi}^{2}$, approximately, including 2,940 $\mathrm{mi}^{2}$ in closed basin in san Luis Valley, Co.

PERIOD OF RECORD. --June 1936 to January 1939, October 1941 to current year. Monthly discharge only October 1942 to June 1943 published in WSP 1312 , and October 1960 to September 1964 , published in wSP 1923 (daily records available in district files). Published as "Rio Grande near Bernardo" prior to October 1964 . Prior to October 1952, flow of Bernardo interior drain was included only when it carried river overflow, the entire flow has been included from October 1952 to September 1964. Flow in the conveyance channel, formerly San Francisco Riverside drain, has been included in record prior to october 1964.

GAGE.--Water-stage recorder. Datum of gage is 4,722.55 ft above National Geodetic Vertical Datum of 1929 .

REMARKS.--Since November 1973 flow completely regulated by Cochiti Dam (station 08317300 ) 100 mi upstream. Floodway is 1 of 4 channels (stations 08331990,08332030 , and 08332050 ) carrying flow in valley cross section. Diversions for irrigation of about 740,000 acres upstream from station.

AVERAGE DISCHARGE.--10 years (water years 1964-73), $272 \mathrm{ft}^{3} / \mathrm{s}, 197,100$ acre-ft/yr. Includes flow of floodway, conveyance channel, and Bernardo interior drain.

EXTREMES FOR PERIOD OF RECORD (1936-39 AND SINCE 1941).--Maximum discharge, 21,000 ft3/s, Apr. 25, 1942, gage height, $6.90 \mathrm{ft}$; no flow for many days most years.

STATISTICAL SUMMARIES (PERIOD BEFORE REgULATION OF COCHITI DAM AND AFTER COMPLETION OF ABIQUIU DAM) MEAN MONTHLY AND MEAN ANNUAL DISCHARgES 1964-73

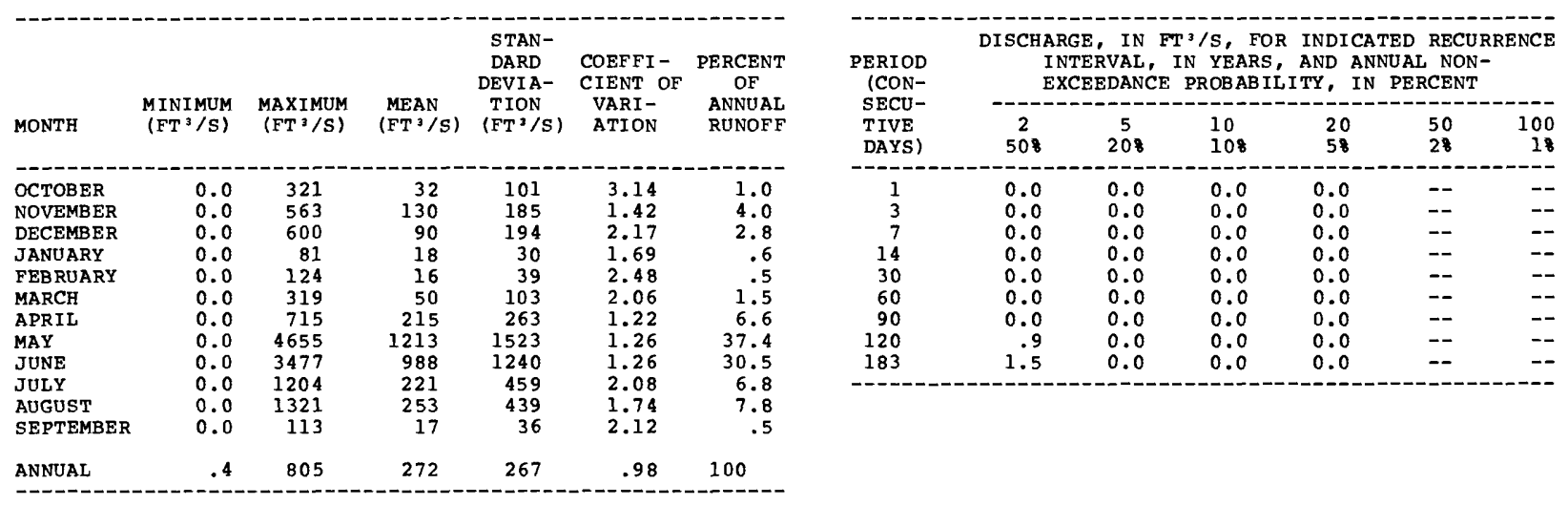

MAGNITUDE AND PROBABILITY OF ANNUAL HIGH FLOW BASED ON PERIOD OF RECORD $1964-73$

\begin{tabular}{|c|c|c|c|c|c|c|}
\hline \multirow{2}{*}{$\begin{array}{l}\text { PERIOD } \\
\text { (CON- } \\
\text { SECU- } \\
\text { TIVE } \\
\text { DAYS) }\end{array}$} & \multicolumn{6}{|c|}{$\begin{array}{l}\text { DISCHARGE, IN FT } 3 / S \text {, FOR INDICATED RECURRENCE } \\
\text { INTERVAL, IN YEARS, AND ANNUAL } \\
\text { EXCEEDANCE PROBABILITY, IN PERCENT }\end{array}$} \\
\hline & $\begin{array}{c}2 \\
508\end{array}$ & $\begin{array}{c}5 \\
208\end{array}$ & $\begin{array}{l}10 \\
108\end{array}$ & $\begin{array}{l}25 \\
48\end{array}$ & $\begin{array}{l}50 \\
28\end{array}$ & $\begin{array}{r}100 \\
18\end{array}$ \\
\hline $\begin{array}{r}1 \\
3 \\
7 \\
15 \\
30 \\
60 \\
90\end{array}$ & $\begin{array}{r}2760 \\
2480 \\
2140 \\
1610 \\
1190 \\
734 \\
583\end{array}$ & $\begin{array}{l}5320 \\
4740 \\
4460 \\
3900 \\
3390 \\
2500 \\
2000\end{array}$ & $\begin{array}{l}6450 \\
5560 \\
5320 \\
4900 \\
4560 \\
3670 \\
2940\end{array}$ & $\begin{array}{l}-- \\
-- \\
-- \\
-- \\
-- \\
--\end{array}$ & $\begin{array}{l}-- \\
-- \\
-- \\
-- \\
-- \\
--\end{array}$ & $\begin{array}{l}-- \\
-- \\
-- \\
-- \\
-- \\
--\end{array}$ \\
\hline
\end{tabular}

DURATION TABLE OF DAILY MEAN FLOW FOR PERIOD OF RECORD 1964-73

DISCHARGE, IN FT'/S, THAT WAS EQUALED OR EXCEEDED FOR INDICATED PERCENT OF TIME

\begin{tabular}{|c|c|c|c|c|c|c|c|c|c|c|c|c|c|c|}
\hline 58 & 108 & 158 & 208 & 258 & 308 & 408 & 508 & 608 & 708 & 758 & 808 & 858 & 908 & 958 \\
\hline 2080 & 864 & 363 & 110 & 7.2 & .3 & .3 & .2 & .2 & .1 & .1 & .1 & .1 & 0.0 & 0. \\
\hline
\end{tabular}


RIO GRANDE BASIN

08332010 RIO GRANDE FLOODWAY NEAR BERNARDO, NH--Continued STATISTICAL SUMMARIES (PERIOD AFTER COMPLETION OF COCHITI DAM)

MAGNITUDE AND PROBABILITY OF ANNUAL LOW FLOW BASED ON PERIOD OF RECORD 1975-85

\begin{tabular}{|c|c|c|c|c|c|c|}
\hline MONTH & $\begin{array}{l}\text { MINIMUM } \\
\left(\mathrm{FT}^{2} / \mathrm{S}\right)\end{array}$ & $\begin{array}{c}\text { MAXIMUM } \\
\left(\mathrm{FT}^{3} / \mathrm{S}\right)\end{array}$ & $\begin{array}{l}\text { MEAN } \\
\left(\mathrm{FT}^{3} / \mathrm{S}\right)\end{array}$ & $\begin{array}{c}\text { STAN- } \\
\text { DARD } \\
\text { DEVIA- } \\
\text { TION } \\
\left(\mathrm{FT}^{3} / \mathrm{S}\right)\end{array}$ & $\begin{array}{l}\text { COEFFI- } \\
\text { CIENT OF } \\
\text { VARI- } \\
\text { ATION }\end{array}$ & $\begin{array}{c}\text { PERCENT } \\
\text { OF } \\
\text { ANNUAL } \\
\text { RUNOFF }\end{array}$ \\
\hline \multicolumn{7}{|l|}{ OCTOBER } \\
\hline NOVEMBER & 3.7 & 1683 & 805 & 521 & .65 & 6.1 \\
\hline DECEMBER & 117 & 1765 & 898 & 499 & .56 & 6.8 \\
\hline JANUARY & 37 & 1265 & 691 & 319 & .46 & 5.2 \\
\hline FEBRUARY & 0.0 & 1054 & 655 & 285 & .44 & 4.9 \\
\hline MARCH & 0.0 & 1910 & 758 & 551 & & 5.7 \\
\hline APRIL & 0.0 & 5160 & 1478 & 1551 & 1.05 & 11.1 \\
\hline MAY & 0.0 & 5746 & 3242 & 2292 & .71 & 24.4 \\
\hline JUNE & 0.0 & 6178 & 2731 & 2345 & .86 & 20.6 \\
\hline JULY & 0.0 & 5261 & 1251 & 1573 & 1.26 & 9.4 \\
\hline AUGUST & 0.0 & 769 & 297 & 243 & .82 & 2.2 \\
\hline SEPTEMBER & 0.0 & 1179 & 291 & 380 & 1.31 & 2.2 \\
\hline ANNUAL & 13 & 2111 & 1107 & 720 & .65 & 100 \\
\hline
\end{tabular}

\begin{tabular}{|c|c|c|c|c|c|c|}
\hline \multirow{2}{*}{$\begin{array}{l}\text { PERIOD } \\
\text { (CON- } \\
\text { SECU- } \\
\text { TIVE } \\
\text { DAYS) }\end{array}$} & \multicolumn{6}{|c|}{$\begin{array}{l}\text { DISCHARGE, IN FT } 3 / S \text {, FOR INDICATED RECURRENCE } \\
\text { INTERVAL, IN YEARS, AND ANNUAL NON- } \\
\text { EXCEEDANCE PROBABILITY, IN PERCENT }\end{array}$} \\
\hline & $\begin{array}{c}2 \\
508\end{array}$ & $\begin{array}{c}5 \\
208\end{array}$ & $\begin{array}{l}10 \\
108\end{array}$ & $\begin{array}{l}20 \\
58\end{array}$ & $\begin{array}{l}50 \\
28\end{array}$ & $\begin{array}{r}100 \\
18\end{array}$ \\
\hline $\begin{array}{r}1 \\
3 \\
7 \\
14 \\
30 \\
60 \\
90 \\
120 \\
183\end{array}$ & $\begin{array}{r}0.0 \\
0.0 \\
0.0 \\
0.0 \\
23 \\
103 \\
53 \\
127 \\
346\end{array}$ & $\begin{array}{l}0.0 \\
0.0 \\
0.0 \\
0.0 \\
0.0 \\
0.0 \\
111^{.8} \\
59\end{array}$ & $\begin{array}{l}0.0 \\
0.0 \\
0.0 \\
0.0 \\
0.0 \\
0.0 \\
0.0 \\
.4 \\
3.9\end{array}$ & $\begin{array}{l}0.0 \\
0.0 \\
0.0 \\
0.0 \\
0.0 \\
0.0 \\
0.0 \\
0.0 \\
0.0\end{array}$ & $\begin{array}{l}-- \\
=- \\
=- \\
=- \\
=- \\
=- \\
--\end{array}$ & 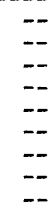 \\
\hline
\end{tabular}

MAGNITUDE AND PROBABILITY OF ANNUAL HIGH FLOW BASED ON PERIOD OF RECORD 1974-85

\begin{tabular}{|c|c|c|c|c|c|c|}
\hline \multirow{2}{*}{$\begin{array}{l}\text { PERIOD } \\
\text { (CON- } \\
\text { SECU- } \\
\text { TIVE } \\
\text { DAYS) }\end{array}$} & \multicolumn{6}{|c|}{$\begin{array}{l}\text { DISCHARGE, IN FT }{ }^{3} / \mathrm{S} \text {, FOR INDICATED RECURRENCE } \\
\text { INTERVAL, IN YEARS, AND ANNUAL } \\
\text { EXCEEDANCE PROBABILITY, IN PERCENT }\end{array}$} \\
\hline & $\begin{array}{c}2 \\
508\end{array}$ & $\begin{array}{c}5 \\
208\end{array}$ & $\begin{array}{l}10 \\
108\end{array}$ & $\begin{array}{l}25 \\
48\end{array}$ & $\begin{array}{l}50 \\
28\end{array}$ & $\begin{array}{r}100 \\
18\end{array}$ \\
\hline $\begin{array}{r}1 \\
3 \\
7 \\
15 \\
30 \\
60 \\
90\end{array}$ & $\begin{array}{l}4600 \\
4350 \\
4150 \\
3830 \\
3510 \\
3190 \\
2780\end{array}$ & $\begin{array}{l}8470 \\
8070 \\
7710 \\
7290 \\
6850 \\
6030 \\
5260\end{array}$ & $\begin{array}{r}10500 \\
10100 \\
9530 \\
9070 \\
8470 \\
7180 \\
6210\end{array}$ & $\begin{array}{l}=- \\
=- \\
=- \\
=- \\
=-\end{array}$ & $\begin{array}{l}-- \\
-- \\
-- \\
-- \\
--\end{array}$ & $\begin{array}{l}-- \\
-- \\
-- \\
-- \\
--\end{array}$ \\
\hline
\end{tabular}

DURATION TABLE OF DAILY MEAN FLOW FOR PERIOD OF RECORD 1974-85

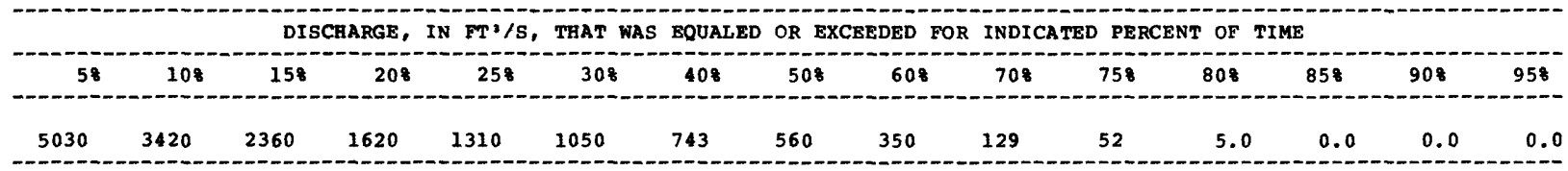


RIO GRANDE BASIN

08334000 RIO PUERCO ABOVE ARROYO CHICO, NEAR GUADALUPE, NM

LOCATION.--Lat $35^{\circ} 38^{\prime} 08^{\prime \prime}$, long $107^{\circ} 09^{\prime} 56^{\prime \prime}$, in SW $\frac{1}{4}$ sec. 21, T.16 N., R. 3 w., Sandoval County, Hydrologic Unit 13020204 , on right bank $1.6 \mathrm{mi}$ upstream from Arroyo Chico, $5.5 \mathrm{mi}$ northeast of village of Guadalupe, and at mile 106.8 .

DRAINAGE AREA. $--420 \mathrm{mi}^{2}$, approximately.

PERIOD OF RECORD.--July 1951 to current year.

GAGE.--Water-stage recorder. Datum of gage is $5,950 \mathrm{ft}$ above National Geodetic Vertical Datum of 1929 . Prior to July 14,1966 at datum $1.01 \mathrm{ft}$ higher.

REMARKS.--Diversions for irrigation of about 3,700 acres upstream from station in past years, but present diversion negligible.

AVERAGE DISCHARGE.--34 years (water years 1952-85), $13.6 \mathrm{ft}^{3} / \mathrm{s}, 9,850 \mathrm{acre}-\mathrm{ft} / \mathrm{yr}$.

EXTREMES FOR PERIOD OF RECORD.--Maximum discharge, 6,940 $\mathrm{ft}^{3} / \mathrm{s}$ July 29,1967 , gage height, $13.53 \mathrm{ft}$, from rating curve extended above $1,300 \mathrm{ft}^{3} / \mathrm{s}$ on basis of slope-area measurements at gage heights $7.75 \mathrm{ft}$ and $10.60 \mathrm{ft}$; no flow for many days most years.

EXTREMES OUTSIDE PERIOD OF RECORD.--Flood of June 29, 1943, probably exceeded 5,000 $\mathrm{ft}^{3} / \mathrm{s}$ based on records for stations upstream and downstream.

STATISTICAL SUMMARIES

MEAN MONTHLY AND MEAN ANNUAL DISCHARges 1952-85

\begin{tabular}{|c|c|c|c|c|c|c|}
\hline MONTH & $\begin{array}{l}\text { MINI MUM } \\
\left(\mathrm{FT}^{3} / \mathrm{S}\right)\end{array}$ & $\begin{array}{l}\text { MAXI MUM } \\
\left(\mathrm{FT}^{3} / \mathrm{S}\right)\end{array}$ & $\begin{array}{l}\text { MEAN } \\
\left(\mathrm{FT}^{3} / \mathrm{S}\right)\end{array}$ & $\begin{array}{l}\text { STAN- } \\
\text { DARD } \\
\text { DEVIA- } \\
\text { TION } \\
\left(\text { FT }^{3} / S\right)\end{array}$ & $\begin{array}{l}\text { COEFFI- } \\
\text { CIENT OF } \\
\text { VARI- } \\
\text { ATION }\end{array}$ & $\begin{array}{c}\text { PERCENT } \\
\text { OF } \\
\text { ANNUAL } \\
\text { RUNOFF }\end{array}$ \\
\hline $\begin{array}{l}\text { OCTOBER } \\
\text { NOVEMBER } \\
\text { DECEMBER } \\
\text { JANUARY } \\
\text { FEBRUARY } \\
\text { MARCH } \\
\text { APRIL } \\
\text { MAY } \\
\text { JUNE } \\
\text { JULY } \\
\text { AUGUST } \\
\text { SEPTEMBER }\end{array}$ & $\begin{array}{l}0.0 \\
0.0 \\
0.0 \\
0.0 \\
0.0 \\
0.0 \\
0.0 \\
0.0 \\
0.0 \\
0.0 \\
0.0 \\
0.0\end{array}$ & $\begin{array}{r}129 \\
17 \\
8.8 \\
24 \\
79 \\
161 \\
99 \\
236 \\
98 \\
78 \\
101 \\
90\end{array}$ & $\begin{array}{l}8.3 \\
2.5 \\
1.3 \\
1.8 \\
11 \\
18 \\
18 \\
37 \\
13 \\
15 \\
25 \\
12\end{array}$ & $\begin{array}{l}23 \\
4.9 \\
2.3 \\
4.4 \\
20 \\
32 \\
25 \\
48 \\
22 \\
17 \\
25 \\
19\end{array}$ & $\begin{array}{l}2.75 \\
1.95 \\
1.86 \\
2.40 \\
1.77 \\
1.83 \\
1.37 \\
1.30 \\
1.66 \\
1.13 \\
1.00 \\
1.63\end{array}$ & $\begin{array}{r}5.1 \\
1.5 \\
.8 \\
1.1 \\
6.9 \\
10.8 \\
11.2 \\
22.7 \\
8.1 \\
9.0 \\
15.5 \\
7.2\end{array}$ \\
\hline ANNUAL & 1.1 & 49 & 14 & 10 & .75 & 100 \\
\hline
\end{tabular}

MAGNITUDE AND PROBABILITY OF ANNUAL LOW FLOW BASED ON PERIOD OF RECORD 1953-85

\begin{tabular}{|c|c|c|c|c|c|c|}
\hline \multirow{2}{*}{$\begin{array}{l}\text { PERIOD } \\
\text { (CON- } \\
\text { SECU- } \\
\text { TIVE } \\
\text { DAYS) }\end{array}$} & \multicolumn{6}{|c|}{$\begin{array}{l}\text { DISCHARGE, IN } F^{3} / \mathrm{S}, \text { FOR INDICATED RECURRENCE } \\
\text { INTERVAL, IN YEARS, AND ANNUAL NON- } \\
\text { EXCEEDANCE PROBABILITY, IN PERCENT }\end{array}$} \\
\hline & $\begin{array}{c}2 \\
508\end{array}$ & $\begin{array}{c}5 \\
208\end{array}$ & $\begin{array}{l}10 \\
108\end{array}$ & $\begin{array}{l}20 \\
58\end{array}$ & $\begin{array}{l}50 \\
28\end{array}$ & $\begin{array}{r}100 \\
18\end{array}$ \\
\hline $\begin{array}{r}1 \\
3 \\
7 \\
14 \\
30 \\
60 \\
90 \\
120 \\
183\end{array}$ & $\begin{array}{r}0.0 \\
0.0 \\
0.0 \\
0.0 \\
0.0 \\
0.0 \\
.1 \\
.3 \\
1.3\end{array}$ & $\begin{array}{l}0.0 \\
0.0 \\
0.0 \\
0.0 \\
0.0 \\
0.0 \\
0.0 \\
0.0 \\
.2\end{array}$ & $\begin{array}{l}0.0 \\
0.0 \\
0.0 \\
0.0 \\
0.0 \\
0.0 \\
0.0 \\
0.0 \\
0.0\end{array}$ & $\begin{array}{l}0.0 \\
0.0 \\
0.0 \\
0.0 \\
0.0 \\
0.0 \\
0.0 \\
0.0 \\
0.0\end{array}$ & $\begin{array}{l}0.0 \\
0.0 \\
0.0 \\
0.0 \\
0.0 \\
0.0 \\
0.0 \\
0.0 \\
0.0\end{array}$ & $\begin{array}{l}-- \\
-- \\
-- \\
-- \\
-- \\
-- \\
-- \\
--\end{array}$ \\
\hline
\end{tabular}

MAGNITUDE AND PROBABILITY OF ANNUAL HIGH FLOW BASED ON PERIOD OF RECORD 1952-B5

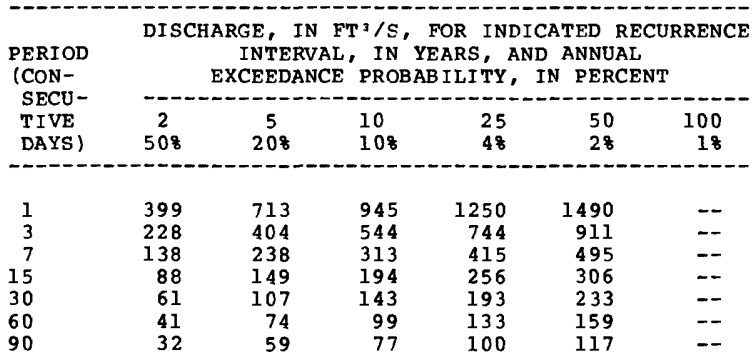

DURATION TABLE OF DAILY MEAN FLOW FOR PERIOD OF RECORD 1952-85

DISCHARGE, IN FT $3 / \mathrm{S}$, THAT WAS EQUALED OR EXCEEDED FOR INDICATED PERCENT OF TIME

\begin{tabular}{|c|c|c|c|c|c|c|c|c|c|c|c|c|c|c|}
\hline 58 & 108 & 158 & 208 & 258 & 308 & 408 & 508 & 608 & 708 & 758 & 808 & 858 & 908 & 958 \\
\hline 70 & 37 & 20 & 11 & 5.6 & 3. & .6 & .1 & 0.0 & 0.0 & 0.0 & 0.0 & 0.0 & 0.0 & 0.0 \\
\hline
\end{tabular}


RIO GRANDE BASIN

08340500 ARROYO CHICO NEAR GUADALUPE, NM

LOCATION,--Lat $35^{\circ} 35^{\prime} 33^{\prime \prime}$, long $107^{\circ} 11^{\prime} 19^{\prime \prime}$, in NE sec.30, T.16 N., R.3 w., Sandoval County, Hydrologic Unit 13020205 , on left bank $0.2 \mathrm{mi}$ upstream from mouth, 4.1 mi northwest of Guadalupe, and $5.5 \mathrm{mi}$ southwest of Cabezon.

DRAINAGE AREA. $--1,390 \mathrm{mi}^{2}$, approximately.

PERIOD OF RECORD.--November 1943 to current year.

REVISED RECORDS. --WSP 1282: $1944-50$.

GAGE.--Water-stage recorder and concrete control. Datum of gage is 5,920 ft above National Geodetic Vertical Datum of 1929. Prior to June 2l, 1968 at site $500 \mathrm{ft}$ upstream at datum $2.00 \mathrm{ft}$ higher.

REMARKS.--Diversions for irrigation of about 100 acres upstream from station.

AVERAGE DISCHARGE. -42 years (water years 1944-85), $21.1 \mathrm{ft}^{3} / \mathrm{s}, 15,290 \mathrm{acre}-\mathrm{ft} / \mathrm{yr}^{\mathrm{r}}$.

EXTREMES FOR PERIOD OP RECORD. --Maximum discharge, 15,200 $\mathrm{ft}^{3} / \mathrm{s}$, Sept, 12, 1972, gage height, $17.5 \mathrm{ft} f \mathrm{fom}$

floodmarks, from rating curve extended above $2,900 \mathrm{ft} / \mathrm{s}$ on bas is of slope-area measurements at gage heights

$11.6 \mathrm{ft}$ and $14.8 \mathrm{ft}$; no flow at times.

STATISTICAL SUMMARIES

MEAN MONTHLY AND MEAN ANNUAL DISCHARGES 1944-85

\begin{tabular}{|c|c|c|c|c|c|c|}
\hline MONTH & $\begin{array}{l}\text { MINIMUM } \\
\left(\mathrm{FT}^{3} / \mathrm{S}\right)\end{array}$ & $\begin{array}{l}\text { MAXIMUM } \\
\left(\mathrm{FT}^{3} / \mathrm{S}\right)\end{array}$ & $\begin{array}{l}\text { MEAN } \\
\left(\mathrm{FT}^{3} / \mathrm{S}\right)\end{array}$ & $\begin{array}{l}\text { STAN- } \\
\text { DARD } \\
\text { DEVIA- } \\
\text { TION } \\
\left(\mathrm{FT}^{3} / \mathrm{S}\right)\end{array}$ & $\begin{array}{l}\text { COEFFI- } \\
\text { CIENT OF } \\
\text { VARI- } \\
\text { ATION }\end{array}$ & $\begin{array}{c}\text { PERCENT } \\
\text { OF } \\
\text { ANNUAL } \\
\text { RUNOFF }\end{array}$ \\
\hline $\begin{array}{l}\text { OCTOBER } \\
\text { NOVEMBER } \\
\text { DECEMBER } \\
\text { JANUARY } \\
\text { PEBRUARY } \\
\text { MARCH } \\
\text { APRIL } \\
\text { MAY } \\
\text { JUNE } \\
\text { JULY } \\
\text { AUGUST } \\
\text { SEPTEMBER }\end{array}$ & $\begin{array}{l}0.0 \\
0.0 \\
0.0 \\
0.0 \\
0.0 \\
0.0 \\
0.0 \\
0.0 \\
0.0 \\
0.0 \\
.6 \\
0.0\end{array}$ & $\begin{array}{r}131 \\
20 \\
15 \\
27 \\
210 \\
169 \\
75 \\
12 \\
44 \\
218 \\
577 \\
230\end{array}$ & $\begin{array}{c}19 \\
3.0 \\
1.6 \\
2.4 \\
13 \\
12 \\
4.2 \\
2.5 \\
4.2 \\
41 \\
106 \\
42\end{array}$ & $\begin{array}{c}29 \\
4.6 \\
3.6 \\
5.9 \\
34 \\
29 \\
12 \\
3.7 \\
9.4 \\
51 \\
126 \\
56\end{array}$ & $\begin{array}{l}1.54 \\
1.55 \\
2.18 \\
2.46 \\
2.55 \\
2.33 \\
2.95 \\
1.52 \\
2.22 \\
1.26 \\
1.19 \\
1.32\end{array}$ & $\begin{array}{l}7.5 \\
1.2 \\
.6 \\
1.0 \\
5.3 \\
4.9 \\
1.7 \\
1.0 \\
1.7 \\
16.2 \\
42.2 \\
16.9\end{array}$ \\
\hline GNUAL & 3.8 & 62 & 21 & 14 & .64 & 100 \\
\hline
\end{tabular}

MAGNITUDE AND PROBABILITY OF ANNUAL LOW FLOW BASED ON PERIOD OF RECORD 1945-85

\begin{tabular}{|c|c|c|c|c|c|c|}
\hline \multirow{2}{*}{$\begin{array}{l}\text { PERIOD } \\
\text { (CON- } \\
\text { SECU- } \\
\text { TIVE } \\
\text { DAYS) }\end{array}$} & \multicolumn{6}{|c|}{$\begin{array}{l}\text { DISCHARGE, IN FT } 3 / \mathrm{S} \text {, FOR INDICATED RECURRENCE } \\
\text { INTERVAL, IN YEARS, AND ANNUAL NON- } \\
\text { EXCEEDANCE PROBABILITY, IN PERCENT }\end{array}$} \\
\hline & $\begin{array}{c}2 \\
508\end{array}$ & $\begin{array}{c}5 \\
208\end{array}$ & $\begin{array}{l}10 \\
108\end{array}$ & $\begin{array}{l}20 \\
58\end{array}$ & $\begin{array}{l}50 \\
28\end{array}$ & $\begin{array}{r}100 \\
18\end{array}$ \\
\hline $\begin{array}{r}1 \\
3 \\
7 \\
14 \\
30 \\
60 \\
90 \\
120 \\
183\end{array}$ & $\begin{array}{r}0.0 \\
0.0 \\
0.0 \\
0.0 \\
0.0 \\
0.0 \\
0.0 \\
.3 \\
2.4\end{array}$ & $\begin{array}{r}0.0 \\
0.0 \\
0.0 \\
0.0 \\
0.0 \\
0.0 \\
0.0 \\
0.0 \\
.4\end{array}$ & $\begin{array}{r}0.0 \\
0.0 \\
0.0 \\
0.0 \\
0.0 \\
0.0 \\
0.0 \\
0.0 \\
.1\end{array}$ & $\begin{array}{l}0.0 \\
0.0 \\
0.0 \\
0.0 \\
0.0 \\
0.0 \\
0.0 \\
0.0 \\
0.0\end{array}$ & $\begin{array}{l}0.0 \\
0.0 \\
0.0 \\
0.0 \\
0.0 \\
0.0 \\
0.0 \\
0.0 \\
0.0\end{array}$ & $\begin{array}{l}0.0 \\
0.0 \\
0.0 \\
0.0 \\
0.0 \\
0.0 \\
0.0 \\
0.0 \\
0.0\end{array}$ \\
\hline
\end{tabular}

MAGNITUDE AND PROBABIIITY OF ANNUAL HTGH FLOW BASED ON PERIOD OF RECORD 1944-85

\begin{tabular}{|c|c|c|c|c|c|c|}
\hline \multirow{2}{*}{$\begin{array}{l}\text { PERIOD } \\
\text { (CON- } \\
\text { SECU- } \\
\text { TIVE } \\
\text { DAYS) }\end{array}$} & \multicolumn{6}{|c|}{$\begin{array}{c}\text { DISCHARGE, IN FT' } / S \text {, FOR INDICATED RECUR } \\
\text { INTERVAL, IN YEARS, AND ANNUAL } \\
\text { EXCEEDANCE PROBABILITY, IN PERCENT }\end{array}$} \\
\hline & $\begin{array}{c}2 \\
508\end{array}$ & $\begin{array}{c}5 \\
208\end{array}$ & $\begin{array}{l}10 \\
108\end{array}$ & $\begin{array}{l}25 \\
48\end{array}$ & $\begin{array}{l}50 \\
28\end{array}$ & $\begin{array}{r}100 \\
18\end{array}$ \\
\hline $\begin{array}{r}1 \\
3 \\
7 \\
15 \\
30 \\
60 \\
90\end{array}$ & $\begin{array}{r}1140 \\
597 \\
322 \\
184 \\
119 \\
77 \\
56\end{array}$ & $\begin{array}{r}1980 \\
1090 \\
601 \\
345 \\
231 \\
144 \\
102\end{array}$ & $\begin{array}{r}2580 \\
1450 \\
826 \\
478 \\
324 \\
195 \\
138\end{array}$ & $\begin{array}{r}3350 \\
1940 \\
1150 \\
673 \\
464 \\
266 \\
189\end{array}$ & $\begin{array}{r}3920 \\
2320 \\
1420 \\
838 \\
583 \\
323 \\
230\end{array}$ & $\begin{array}{r}4490 \\
2710 \\
1710 \\
1020 \\
715 \\
383 \\
273\end{array}$ \\
\hline
\end{tabular}

DURATION TABLE OF DAILY MEAN FLOW FOR PERIOD OF RECORD 1944-85

DISCHARGE, IN FT $3 / \mathrm{S}$, THAT WAS EOUALED OR EXCEEDED FOR INDYCATED PERCENT OF TIME

\begin{tabular}{|c|c|c|c|c|c|c|c|c|c|c|c|c|c|c|}
\hline 58 & 108 & 158 & 208 & 258 & 308 & 408 & 508 & 608 & 708 & 758 & 808 & 858 & 908 & 958 \\
\hline 73 & 20 & 9.2 & 5.5 & 2.9 & 1.4 & .2 & 0.0 & 0.0 & 0.0 & 0.0 & 0.0 & 0.0 & 0.0 & \\
\hline
\end{tabular}


RIO GRANDE BASIN

08342000 BLUEWATER CREEK NEAR BLUEWATER, NM

LOCATION.--Lat $35^{\circ} 17 \cdot 40^{\circ}$, long $108^{\circ} 01^{\prime} 40^{\prime}$, in Whst sec.5, T.12 N., R.11 W., Valencia County, on left bank, 3.5 mi northwest of Bluewater viliage, and $8 \mathrm{mi}$ downstream from Bluewater Dam.

DRAINAGE AREA. $--209 \mathrm{mi}^{2}$.

PERIOD OF RECORD.--July 1912 to August 1915, April 1916 to June 1919, water years 1919-22, 1924, 1926 (annual maximum). January 1927 to December 1972 (discontinued). Monthly discharge only for some periods, published in WSP 1312. Figures of daily discharge for July 20-23, 1912, published in wsP 358 , have been found to be unreliable and should not be used.

REVISED RECORDS.--WSP 1512: 1912-15, 1931, 1943(M), 1945(M). WSP 1312: 1915, 1917-18, 1929. WSP 1732: 1931(M). GAGE.--Water-stage recorder. Altitude of gage is $6,720 \mathrm{ft}$ (by barometer). See WSP 1732 or $1923 \mathrm{for}$ history of changes prior to Mar. 18, 1939.

REMARKS.--Flow regulated by Bluewater Lake (see sta 08341400 ).

AVERAGE DISCHARGE.--12 years (water years 1961-72), $5.00 \mathrm{ft} / \mathrm{s}, 3,620$ acre-ft/yr.

years $(1953-72), 3.52 \mathrm{ft}^{3} / \mathrm{s}(2,550$ acre-ft year $)$.

EXTREMES FOR PERIOD OF RECORD. --Maximum discharge, about 4,000 $\mathrm{ft}^{3} / \mathrm{s}$, during period Ju1y 12-19, 1919 (gage height, $13.5 \mathrm{ft}$, from floodmarks, site and datum in use Mar. 4, 1918 to June 28, 1919); no flow at times.

$3.5 \mathrm{ft}$, from floodmarks, site and datum in use Mar. 4, 1918 to June 28, 1919); no flow at times. (stage and discharge not determined). For other major floods during period 1919-26, see WSP 1732, p. 429, and WSP $1682, P 410$.

STATISTICAL SUMMARIES

MEAN MONTHLY AND MEAN ANNUAL DISCHARGES 1961-72

\begin{tabular}{|c|c|c|c|c|c|c|}
\hline MONTH & $\begin{array}{l}\text { MINIMUM } \\
\left(\mathrm{FT}^{3} / \mathrm{S}\right)\end{array}$ & $\begin{array}{l}\text { MAXIMUM } \\
\left(\mathrm{FT}^{3} / \mathrm{S}\right)\end{array}$ & $\begin{array}{l}\text { MEAN } \\
\left(\mathrm{FT}^{3} / \mathrm{S}\right)\end{array}$ & $\begin{array}{l}\text { STAN- } \\
\text { DARD } \\
\text { DEVIA- } \\
\text { TION } \\
\left(\mathrm{FT}^{3} / \mathrm{S}\right)\end{array}$ & $\begin{array}{l}\text { COEFFI- } \\
\text { CIENT OF } \\
\text { VARI- } \\
\text { ATION }\end{array}$ & $\begin{array}{c}\text { PERCENT } \\
\text { OF } \\
\text { ANNUAL } \\
\text { RUNOFF }\end{array}$ \\
\hline $\begin{array}{l}\text { OCTOBER } \\
\text { NOVEMBER } \\
\text { DECEMBER } \\
\text { JANUARY } \\
\text { FEBRUARY } \\
\text { MARCH } \\
\text { APRIL } \\
\text { MAY } \\
\text { JUNE } \\
\text { JULY } \\
\text { AUGUST } \\
\text { SEPTEMBER }\end{array}$ & $\begin{array}{l}.7 \\
.9 \\
.7 \\
.6 \\
.8 \\
.7 \\
.8 \\
.9 \\
.6 \\
.6 \\
.5 \\
.5\end{array}$ & $\begin{array}{l}3.6 \\
2.4 \\
1.9 \\
2.2 \\
2.0 \\
2.7 \\
5.3 \\
27 \\
32 \\
23 \\
26 \\
17\end{array}$ & $\begin{array}{r}1.9 \\
1.5 \\
1.4 \\
1.4 \\
1.3 \\
1.4 \\
2.1 \\
9.8 \\
13 \\
11 \\
9.9 \\
4.5\end{array}$ & $\begin{array}{r}1.1 \\
.5 \\
.4 \\
.5 \\
.3 \\
.5 \\
1.4 \\
9.1 \\
12 \\
9.4 \\
8.4 \\
5.3\end{array}$ & $\begin{array}{l}.56 \\
.30 \\
.28 \\
.32 \\
.26 \\
.40 \\
.65 \\
.93 \\
.92 \\
.84 \\
.85 \\
1.18\end{array}$ & $\begin{array}{r}3.2 \\
2.6 \\
2.3 \\
2.4 \\
2.2 \\
2.3 \\
3.5 \\
16.5 \\
22.0 \\
19.0 \\
16.6 \\
7.6\end{array}$ \\
\hline NNU & .9 & 11 & 5.0 & 3.6 & .73 & 100 \\
\hline
\end{tabular}

MAGNITUDE AND PROBABILITY OF ANNUAL LOW FLOW BASED ON PERIOD OF RECORD 1962-72

\begin{tabular}{|c|c|c|c|c|c|c|}
\hline \multirow{2}{*}{$\begin{array}{l}\text { PERIOD } \\
\text { (CON- } \\
\text { SECU- } \\
\text { TIVE } \\
\text { DAYS) }\end{array}$} & \multicolumn{6}{|c|}{$\begin{array}{l}\text { DISCHARGE, IN FT }{ }^{3 / S} \text {, FOR INDICATED RECURRENCE } \\
\text { INTERVAL, IN YEARS, AND ANNUAL NON- } \\
\text { EXCEEDANCE PROBABILITY, IN PERCENT }\end{array}$} \\
\hline & $\begin{array}{c}2 \\
508\end{array}$ & $\begin{array}{c}5 \\
208\end{array}$ & $\begin{array}{l}10 \\
108\end{array}$ & $\begin{array}{l}20 \\
58\end{array}$ & $\begin{array}{l}50 \\
28\end{array}$ & \\
\hline $\begin{array}{r}1 \\
3 \\
7 \\
14 \\
30 \\
60 \\
90 \\
120 \\
183\end{array}$ & $\begin{array}{r}.7 \\
.7 \\
.8 \\
.9 \\
1.0 \\
1.0 \\
1.1 \\
1.2 \\
1.4\end{array}$ & $\begin{array}{r}.4 \\
.5 \\
.6 \\
.6 \\
.7 \\
.8 \\
.8 \\
.9 \\
1.0\end{array}$ & $\begin{array}{l}.3 \\
.4 \\
.5 \\
.5 \\
.6 \\
.7 \\
.7 \\
.8 \\
.9\end{array}$ & $\begin{array}{l}.3 \\
.3 \\
.4 \\
.4 \\
.5 \\
.6 \\
.6 \\
.7 \\
.7\end{array}$ & $\begin{array}{l}-- \\
-- \\
-- \\
-- \\
-- \\
-- \\
--\end{array}$ & \\
\hline
\end{tabular}

MAGNITUDE AND PROBABILITY OF ANNUAL HIGH FLOW BASED ON PERIOD OF RECORD $1961-72$

\begin{tabular}{|c|c|c|c|c|c|c|}
\hline $\begin{array}{l}\text { PERI OD } \\
\text { (CON- }\end{array}$ & & $\begin{array}{l}\text { GE, I } \\
\text { INTER } \\
\text { CEEDA }\end{array}$ & $\begin{array}{l}\mathrm{T}^{3} / \mathrm{S} \\
\text { IN } \\
\text { PROE }\end{array}$ & $\begin{array}{l}\text { IND } \\
\text { S, } \\
\text { ITY, }\end{array}$ & $\begin{array}{l}\text { TED R } \\
\text { ANNUA } \\
\text { PERC }\end{array}$ & JRRENC \\
\hline $\begin{array}{l}\text { TIVE } \\
\text { DAYS) }\end{array}$ & $\begin{array}{c}2 \\
508\end{array}$ & $\begin{array}{c}5 \\
208\end{array}$ & $\begin{array}{l}10 \\
108\end{array}$ & $\begin{array}{l}25 \\
48\end{array}$ & $\begin{array}{l}50 \\
28\end{array}$ & $\begin{array}{r}100 \\
18\end{array}$ \\
\hline
\end{tabular}

$\begin{array}{rrrrrrr}1 & 18 & 34 & 44 & 57 & -- & -- \\ 3 & 14 & 32 & 48 & 70 & -- & -- \\ 7 & 12 & 31 & 48 & 73 & -- & -- \\ 15 & 11 & 28 & 45 & 73 & -- & -- \\ 30 & 9.5 & 26 & 41 & 68 & -- & -- \\ 60 & 8.3 & 23 & 37 & 60 & -- & -- \\ 90 & 7.8 & 21 & 34 & 55 & -- & --\end{array}$

DURATION TABLE OF DAILY MEAN FLOW FOR PERIOD OF RECORD 1961-72

DISCHARGE, IN FT $3 / \mathrm{S}$, THAT WAS EQUALED OR EXCEEDED FOR INDICATED PERCENT OF TIME

\begin{tabular}{|c|c|c|c|c|c|c|c|c|c|c|c|c|c|c|}
\hline 58 & 108 & 158 & 208 & 258 & 308 & 408 & 508 & 608 & 708 & 758 & 808 & 858 & 908 & 958 \\
\hline 25 & 19 & 13 & 5.7 & 3.2 & 2.5 & 2.0 & 1.7 & 1.4 & 1.2 & 1.1 & 1.0 & .9 & .8 & \\
\hline
\end{tabular}


RIO GRANDE BASIN

08343000 RIO SAN JOSE AT GRANTS, NM

LOCATION. --Lat $35^{\circ} 09^{\prime} 16^{\mathrm{n}}$, long $107^{\circ} 52^{\prime} 1^{\prime \prime}$, in SWtwWł sec.26, T.11 N., R.10 w., Cibola County, Bydrologic Unit 13020207 , on right bank at bridge on El Morro St., $0.2 \mathrm{mi}$ south of Santa Fe Ave. in Grants, and at mile 67.8 .

DRAINAGE AREA. $--1,020 \mathrm{mi}^{2}$, approximately.

PERIOD OF RECORD.--October 1912 to February 1914, June 1914, October 1914 to February 1915, May 1915 to June 1921, September 1921 to June 1923, October 1923 to May 1926, September to December 1926, May 1949 to September 1966, June 1968 to current year. Monthly discharge only for some periods published in wsp 1312. Prior to October 1967, published as "Bluewater Creek at Grants".

REVISED RECORDS. --WSP 1512: 1913-14. WSP 1712: Drainage area.

GAGE.--Water-stage recorder. Datum of gage is $6,468.34 \mathrm{ft}$ above National Geodetic Vertical Datum of 1929 (levels by U. S. Army Corps of Engineers). See WSP 1732 or 1923 for history of changes prior to Jan. 1 , 1926.

REMARKS.--Flow slightly regulated by Bluewater Lake (station 08341400 ) 24 mi upstream. Diversions and groundwater withdrawals for irrigation of about 4,500 acres upstream from station.

AVERAGR DISCHARGE. -45 years (water years 1913, 1916-20, 1922, 1924-25, 1950-85), 2.90 ft $3 / \mathrm{s}, 2,100$ acre-ft/yr.

EXTREMES FOR PERIOD OF RECORD (1950-66 AND SINCE 1968).--Maximum discharge recorded, 1,760 ft $3 / \mathrm{s}, \mathrm{Aug}$. 28, 1952, gage height, $5.35 \mathrm{ft}$, from rating curve extended above $300 \mathrm{ft} / \mathrm{s}$ on basis of velocity-area studies; no flow for long periods.

EXTREMES OUTSIDE PERIOD OF RECORD.--Maximum flood observed occurred Sept. 6 or 7, 1909, when Bluewater Dam washed out. A flood in July 1919 probably exceeded the one in 1952 .

STATISTICAL SUMMARIES

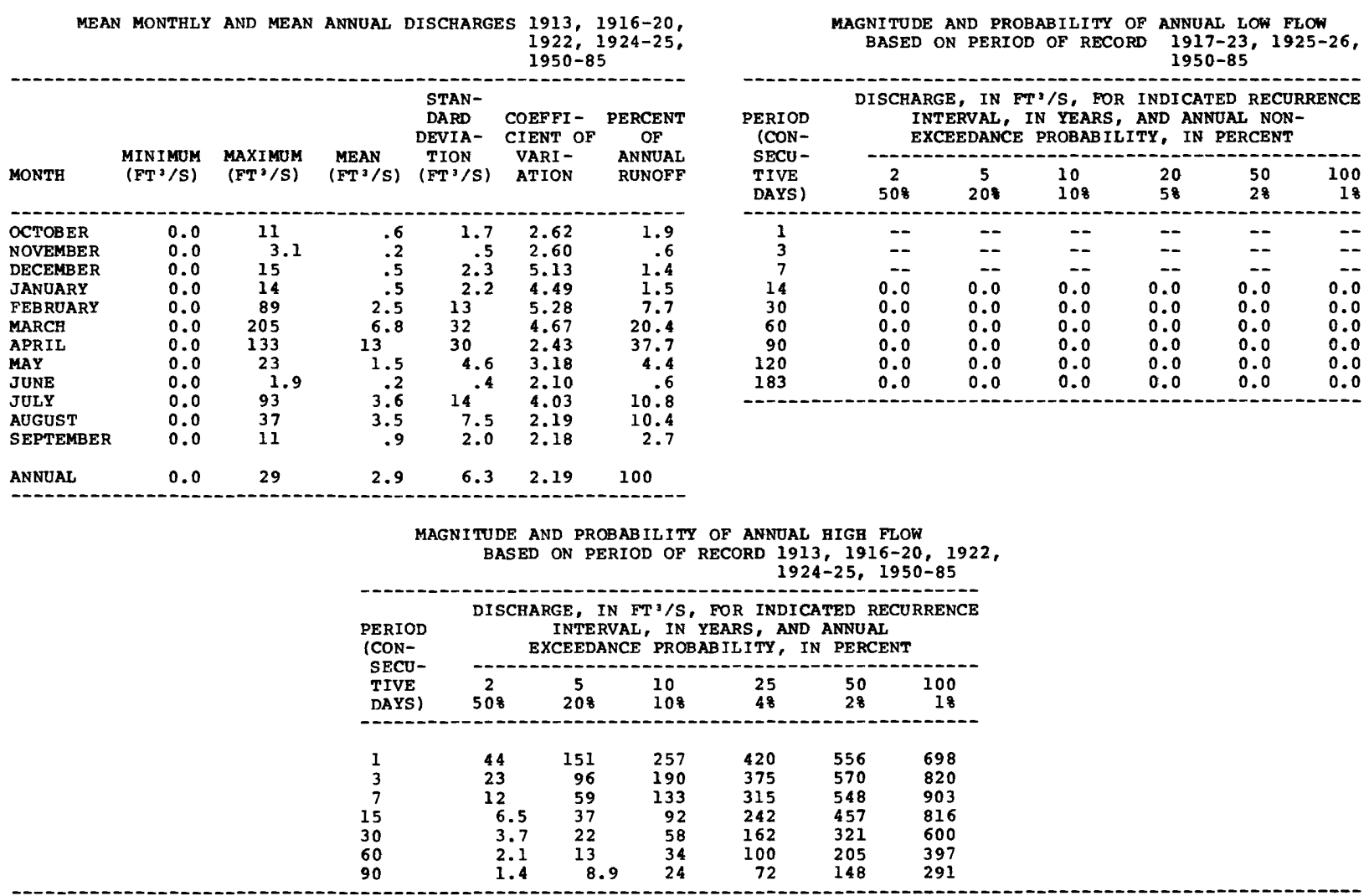

DURATION TABLE OF DAILY MEAN FLOW FOR PERIOD OF RECORD 1913, 1916-20, 1922

$1924-25,1950-85$

\begin{tabular}{|c|c|c|c|c|c|c|c|c|c|c|c|c|c|c|}
\hline & & DIS & GE, & $\mathrm{FT}^{3} / \mathrm{S}$ & HAT WA & EQUALE & SR EXC & ED $F$ & INDIC & PER & OF & & & \\
\hline 58 & 108 & 158 & 208 & 258 & 308 & 408 & 508 & 608 & 708 & 758 & 808 & 858 & 908 & 958 \\
\hline 2.7 & 1.0 & .3 & .2 & .1 & 0.0 & 0.0 & 0.0 & 0.0 & 0.0 & 0.0 & 0.0 & 0.0 & 0.0 & 0 \\
\hline
\end{tabular}


RIO GRANDE BASIN

08343500 RIO SAN JOSE NEAR GRANTS, NM

LOCATION, - Lat $35^{\circ} 04^{\prime} 27^{\prime \prime}$, long $107^{\circ} 45^{\prime} 01^{\prime \prime}$, in SEsSEl sec. 23 , T.10 N., R. 9 w. , Cibola County, Hydrologic Unit 13020207 , on $\mathrm{r}$ ight bank at west boundary of Acoma Pueblo Grant, $8.5 \mathrm{mi}$ southeast of Grants, and at mile 57.4 . DRAINAGE AREA.--2,300 $\mathrm{mi}^{2}$, approximately, of which $1,130 \mathrm{mi}^{2}$ does not contribute directly to surface runoff. PERIOD OF RECORD.--June 1936 to current year. Prior to October 1955, published as "San Jose River near Grants". REVISED RECORDS.--WSP 898: 1936-39(M). WSP 1512: 1943. WSP 1712: Drainage area.

GAGE.--Water-stage recorder and concrete control. Datum of gage is 6,269.47 ft above National Geodetic Vertical Datum of 1929 .

REMARKS.--Flow slightly regulated by Bluewater Lake (station 08341400), 34 mi upstream. Diversions and ground-water withdrawal for irrigation of about 5,100 acres upstream from station.

AVERAGE DISCHARGE. -49 years (water years 1937-85), $6.81 \mathrm{ft} 3 / \mathrm{s}, 4.930 \mathrm{acre}-\mathrm{ft} / \mathrm{yr}$.

EXTREMES FOR PERIOD OF RECORD.--Maximum discharge, 1,400 ft $3 / \mathrm{s}$, Sept. 20, 1963 , gage height, $4.87 \mathrm{ft}$, from rating curve extended above $450 \mathrm{ft} / \mathrm{s}$ on basis of slope-area measurements at gage heights $3.19 \mathrm{ft}$ and $4.87 \mathrm{ft}$; minimum, $1.9 \mathrm{ft}^{3} / \mathrm{s}$, Feb. 21,1973 .

EXTREMES OUTSIDE PERIOD OF RECORD, - Maximum flood probably occurred Sept. 6 or 7, 1909, following destruction of Bluewater dam. The peak of Sept. 20, 1963 may have been exceeded by those of July 1919, August and September 1929 , and August 1935 .

STATISTICAL SUMMARIES

MEAN MONTHLY AND MEAN ANNUAL DISCHARGES 1937-85

\begin{tabular}{|c|c|c|c|c|c|c|}
\hline MONTH & $\begin{array}{l}\text { MINIMUM } \\
\left(\mathrm{FT}^{3} / \mathrm{S}\right)\end{array}$ & $\begin{array}{l}\text { MAXIMUM } \\
\left(\mathrm{FT}^{3} / \mathrm{S}\right)\end{array}$ & $\begin{array}{l}\text { MEAN } \\
\left(\mathrm{FT}{ }^{3} / \mathrm{S}\right)\end{array}$ & $\begin{array}{l}\text { STAN- } \\
\text { DARD } \\
\text { DEVIA- } \\
\text { TION } \\
\left(\mathrm{FT}^{3} / \mathrm{S}\right)\end{array}$ & $\begin{array}{l}\text { COEFFI- } \\
\text { CIENT OF } \\
\text { VARI- } \\
\text { ATION }\end{array}$ & $\begin{array}{c}\text { PERCENT } \\
\text { OF } \\
\text { ANNUAL } \\
\text { RUNOFF }\end{array}$ \\
\hline $\begin{array}{l}\text { OCTOBER } \\
\text { NOVEMBER } \\
\text { DECEMBER } \\
\text { JANUARY } \\
\text { JEBRUARY } \\
\text { MARCH } \\
\text { APRIL } \\
\text { MAY } \\
\text { JUNE } \\
\text { JULY } \\
\text { AUGUST } \\
\text { SEPTEMBER }\end{array}$ & $\begin{array}{l}4.0 \\
4.1 \\
4.0 \\
3.8 \\
4.5 \\
4.0 \\
3.7 \\
4.0 \\
3.7 \\
3.6 \\
4.7 \\
4.2\end{array}$ & $\begin{array}{c}17 \\
9.8 \\
7.8 \\
11 \\
12 \\
11 \\
91 \\
128 \\
10 \\
24 \\
53 \\
25\end{array}$ & $\begin{array}{r}5.9 \\
5.4 \\
5.3 \\
5.7 \\
5.9 \\
5.8 \\
9.1 \\
8.9 \\
5.5 \\
7.1 \\
10 \\
7.0\end{array}$ & $\begin{array}{r}2.3 \\
1.0 \\
.9 \\
1.4 \\
1.5 \\
1.6 \\
15 \\
18 \\
1.3 \\
4.0 \\
9.3 \\
4.0\end{array}$ & $\begin{array}{r}.39 \\
.18 \\
.17 \\
.25 \\
.25 \\
.27 \\
1.68 \\
2.05 \\
.24 \\
.57 \\
.92 \\
.56\end{array}$ & $\begin{array}{r}7.2 \\
6.6 \\
6.5 \\
7.0 \\
7.2 \\
7.1 \\
11.2 \\
10.9 \\
6.7 \\
8.7 \\
12.4 \\
8.6\end{array}$ \\
\hline NNUAL & 4.4 & 19 & 6.8 & 2.7 & .40 & 100 \\
\hline
\end{tabular}

MAGNITUDE AND PROBABILITY OF ANNUAL LOW FLOW BASED ON PERIOD OF RECORD 1938-85

\begin{tabular}{|c|c|c|c|c|c|c|}
\hline \multirow{2}{*}{$\begin{array}{l}\text { PERIOD } \\
\text { (CON- } \\
\text { SECU- } \\
\text { TIVE } \\
\text { DAYS) }\end{array}$} & \multicolumn{6}{|c|}{$\begin{array}{l}\text { DISCHARGE, IN FT } 3 / \text { S, FOR INDICATED RECURRENCE } \\
\text { INTERVAL, IN YEARS, AND ANNUAL NON- } \\
\text { EXCEEDANCE PROBABIL ITY, IN PERCENT }\end{array}$} \\
\hline & $\begin{array}{c}2 \\
508\end{array}$ & $\begin{array}{c}5 \\
208\end{array}$ & $\begin{array}{l}10 \\
108\end{array}$ & $\begin{array}{l}20 \\
58\end{array}$ & $\begin{array}{l}50 \\
28\end{array}$ & \\
\hline $\begin{array}{r}1 \\
3 \\
7 \\
14 \\
30 \\
60 \\
90 \\
120 \\
183\end{array}$ & $\begin{array}{l}3.9 \\
4.0 \\
4.1 \\
4.2 \\
4.4 \\
4.6 \\
4.8 \\
4.9 \\
5.3\end{array}$ & $\begin{array}{l}3.5 \\
3.6 \\
3.7 \\
3.8 \\
4.0 \\
4.2 \\
4.4 \\
4.5 \\
4.8\end{array}$ & $\begin{array}{l}3.2 \\
3.3 \\
3.5 \\
3.6 \\
3.8 \\
4.0 \\
4.2 \\
4.3 \\
4.6\end{array}$ & $\begin{array}{l}3.0 \\
3.1 \\
3.3 \\
3.4 \\
3.7 \\
3.9 \\
4.1 \\
4.2 \\
4.4\end{array}$ & $\begin{array}{l}2.8 \\
2.9 \\
3.0 \\
3.2 \\
3.4 \\
3.7 \\
3.9 \\
4.0 \\
4.3\end{array}$ & \\
\hline
\end{tabular}

MAGNITUDE AND PROBABILITY OF ANNUAL HIGH FLOW BASED ON PERIOD OF RECORD 1937-85

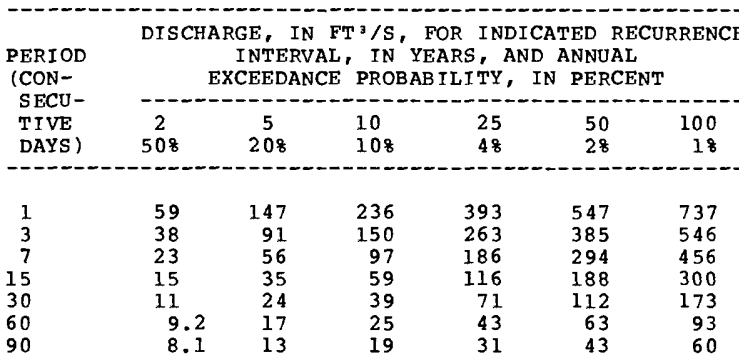

DURATION TABLE OF DAILY MEAN FLOW FOR PERIOD OF RECORD 1937-85 DISCHARGE, IN FT ${ }^{3} / \mathrm{S}$, THAT WAS EQUALED OR EXCEEDED FOR INDICATED PERCENT OF TIME

\begin{tabular}{|c|c|c|c|c|c|c|c|c|c|c|c|c|c|c|}
\hline 58 & 108 & 158 & 208 & 258 & 308 & 408 & 508 & 608 & 708 & 758 & 808 & 858 & 908 & 958 \\
\hline 9.5 & 7.6 & 6.7 & 6.4 & 6.0 & 5.8 & 5.5 & 5.3 & 5.0 & 4.8 & 4.7 & 4.6 & 4.5 & 4.4 & 4 \\
\hline
\end{tabular}


RIO GRANDE BASIN

08351500 RIO SAN JOSE AT CORREO, NM

LOCATION.--Lat $34^{\circ} 58^{\prime} 03^{\prime \prime}$, long $107^{\circ} 10^{\prime} 10^{\circ}$, in NEł sec.32, T.9 N., R.3 W., Cibola County, Hydrologic Unit 13020207, on left bank $0.3 \mathrm{mi}$ downstream from state Highway $6,1.2 \mathrm{mi}$ northeast of Correo, and $13 \mathrm{mi}$ upstream from mouth.

DRAINAGE AREA. - -3,660 $\mathrm{mi}^{2}$, approximately, of which about 1,130 $\mathrm{mi}^{2}$ does not contribute directly to surface runoff. PERIOD OF RECORD.--April 1943 to current year. Prior to October 1955, published as "San Jose River at Correo".

GAGE.--Water-stage recorder and concrete control. Datum of gage is 5,474.88 ft above National Geodetic Vertical Datum of 1929. Oct. 1, 1958 to Sept. 30, 1975, water-stage recorder at site 1 mi upstream at datum $17.55 \mathrm{ft}$ higher.

REMARKS.-Flow regulated to some extent since 1927 by Bluewater Lake (station 08341400 ) $79 \mathrm{mi}$ upstream.

AVERAGE DISCHARGE.--42 years (water years 1944-85), $11.1 \mathrm{ft}^{3} / \mathrm{s}, 8,040$ acre-ft/yr.

EXTREMES FOR PERIOD OF RECORD.--Maximum discharge, $7,150 \mathrm{ft} 3 / \mathrm{s}$, Aug. 11, 1955; maximum gage height, $20.7 \mathrm{ft}$, Aug. 22, 1958, backwater from dam (present datum); no flow for many days.

EXTREMES OUTSIDE PERIOD OF RECORD.--A flood which probably occurred Aug. 21, 1935, reached a stage of 15.4 ft, from floodmarks, (discharge, about $11,000 \mathrm{ft}^{3} / \mathrm{s}$ ), but was probably exceeded by the flood of Sept. 23 , 1929 (discharge not determined), based on study of records for Rio Puerco at Rio Puerco.

STATISTICAL SUMMARIES

MEAN MONTHLY AND MEAN ANNUAL DISCHARGES 1944-85

\begin{tabular}{|c|c|c|c|c|c|c|}
\hline MONTH & $\begin{array}{l}\text { MINIMUM } \\
\left(\mathrm{FT}^{3} / \mathrm{S}\right)\end{array}$ & $\begin{array}{c}\text { MAXIMUM } \\
\left(\mathrm{FT}^{3} / \mathrm{S}\right)\end{array}$ & $\begin{array}{l}\text { MEAN } \\
\left(\mathrm{FT}^{3} / \mathrm{S}\right)\end{array}$ & $\begin{array}{l}\text { STAN- } \\
\text { DARD } \\
\text { DEVIA- } \\
\text { TION } \\
\left(\mathrm{FT}^{3} / \mathrm{S}\right)\end{array}$ & $\begin{array}{l}\text { COEFFI- } \\
\text { CIENT OF } \\
\text { VARI - } \\
\text { ATION }\end{array}$ & $\begin{array}{c}\text { PERCENT } \\
\text { OF } \\
\text { ANNUAL } \\
\text { RUNOFF }\end{array}$ \\
\hline \multicolumn{7}{|c|}{ 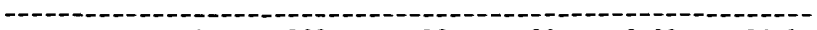 } \\
\hline OCTOBER & 0.0 & 123 & 13 & 28 & 2.08 & 10.1 \\
\hline NOVEMBER & 0.0 & 19 & 2.4 & 3.6 & 1.54 & 1.8 \\
\hline DECEMBER & 0.0 & 9.7 & 1.9 & 2.5 & 1.33 & 1.4 \\
\hline JANUARY & 0.0 & 10 & 3.4 & 3.0 & .87 & 2.6 \\
\hline FEB RUARY & 0.0 & 14 & 4.5 & 3.3 & .74 & 3.4 \\
\hline MARCH & 0.0 & 39 & 4.7 & 7.2 & 1.52 & 3.6 \\
\hline APR IL & 0.0 & 83 & 5.8 & 17 & 2.98 & 4.4 \\
\hline MAY & 0.0 & 33 & 4.5 & 9.6 & 2.16 & 3.4 \\
\hline JUNE & 0.0 & 33 & 2.6 & 7.1 & 2.79 & 1.9 \\
\hline JULY & 0.0 & 170 & 17 & $30^{\circ}$ & 1.77 & 12.7 \\
\hline AUGUST & 0. & 363 & 51 & 73 & 1.43 & 38.6 \\
\hline SEPTEMBER & 0.0 & 126 & 21 & 28 & 1.30 & 16.2 \\
\hline ANNUAL & 1.5 & 40 & 11 & 8.9 & .80 & 100 \\
\hline
\end{tabular}

MAGNITUDE AND PROBABILITY OF ANNUAL HIGH FLOW BASED ON PERIOD OF RECORD $1944-85$

\begin{tabular}{|c|c|c|c|c|c|c|}
\hline \multirow{2}{*}{$\begin{array}{l}\text { PERIOD } \\
\text { (CON- } \\
\text { SECU- } \\
\text { TIVE } \\
\text { DAYS) }\end{array}$} & \multicolumn{6}{|c|}{$\begin{array}{l}\text { DISCHARGE, IN FT } 3 / \mathrm{S}, \text { FOR INDICATED RECURRENCE } \\
\text { INTERVAL, IN YEARS, AND ANNUAL } \\
\text { EXCEEDANCE PROBABILITY, IN PERCENT }\end{array}$} \\
\hline & $\stackrel{2}{508}$ & $\begin{array}{c}5 \\
208\end{array}$ & $\begin{array}{l}10 \\
108\end{array}$ & $\begin{array}{l}25 \\
48\end{array}$ & $\begin{array}{l}50 \\
28\end{array}$ & $\begin{array}{r}100 \\
18\end{array}$ \\
\hline $\begin{array}{r}1 \\
3 \\
7 \\
15 \\
30 \\
60 \\
90\end{array}$ & $\begin{array}{r}496 \\
274 \\
152 \\
88 \\
55 \\
33 \\
24\end{array}$ & $\begin{array}{r}1070 \\
579 \\
314 \\
197 \\
122 \\
73 \\
52\end{array}$ & $\begin{array}{r}1530 \\
816 \\
435 \\
289 \\
177 \\
106 \\
74\end{array}$ & $\begin{array}{r}2180 \\
1140 \\
592 \\
420 \\
254 \\
157 \\
108\end{array}$ & $\begin{array}{r}2690 \\
1380 \\
709 \\
525 \\
317 \\
200 \\
136\end{array}$ & $\begin{array}{r}3210 \\
1630 \\
822 \\
636 \\
382 \\
246 \\
167\end{array}$ \\
\hline
\end{tabular}

DURATION TABLE OF DAILY MEAN FLOW FOR PERIOD OF RECORD 1944-85

DISCHARGE, IN FT $3 / S$, THAT WAS EOUALED OR EXCEEDED FOR INDICATED PERCENT OF TIME

\begin{tabular}{|c|c|c|c|c|c|c|c|c|c|c|c|c|c|c|}
\hline 58 & 108 & 158 & 208 & 258 & 308 & 408 & 508 & 608 & 708 & 758 & 808 & 858 & 908 & 958 \\
\hline & 11 & 6.9 & 5. & 4. & 3.1 & . & 0.0 & 0. & 0. & 0.0 & 0. & 0. & 0. & \\
\hline
\end{tabular}


RIO GRANDE BASIN

08352500 RIO PUERCO AT RIO PUERCO, NM

LOCATION.--Lat 34\%47'38", long $106^{\circ} 59^{\prime} 20^{\prime \prime}$, in Nws sec.31, T.7 N., R.I W., Valencia County, Hydrologic Unit 13020204 , in San Clemente Grant, on downstream end of pier nearest left abutment of the Atchison, Topeka and Santa Fe Railway Co. bridge, $7 \mathrm{mi}$ downstream from Rio San Jose, and at mile 36.2 .

DRAINAGE AREA. $--6,590 \mathrm{mi}^{2}$, approximately, of which at least $1,130 \mathrm{mi}$ does not contribute directly to surface $r$ unoff.

PERIOD OF RECORD.--June 1909 to December 1912 (records fragmentary, gage heights only), March 1934 to December 1976. Records for January 1913 to December 1914 published in WSP 358,388 , and 408 have been found to be unreliable and should not be used.

REVISED RECORDS.--WSP 1512: 1937 (calendar year figures only), 1941, 1944. WSP 1712: 1958. WSP 1732: Drainage area. See also PERIOD OF RECORD.

GAGE.--Water-stage recorder and concrete control. Datum of gage is 5,008.59 ft above mean sea level.

REMARKS.--Diversions for irrigation of about 11,500 acres above station (includes 3,700 acres irrigated partly or entirely from wells).

AVERAGE DISCHARGE. -42 years (water years 1935-76), $57.0 \mathrm{ft} 3 / \mathrm{s}, 41,300$ acre- $\mathrm{ft} / \mathrm{yr}$.

EXTREMES FOR PERIOD OF RECORD.--Maximum discharge, 28,000 $\mathrm{ft}^{3} / \mathrm{s}$ Aug. 21, 1935, gage height, $7.24 \mathrm{ft}$, by computation of peak flow over dam; no flow many days.

EXTREMES OUTSIDE PERIOD OF RECORD.--The damaging flood of Sept. 23, 1929, is the greatest since about 1880; it reached a stage of $18 \mathrm{ft}$ conditions prior to destruction of railroad bridge. Discharge, $37,700 \mathrm{ft} / \mathrm{s}$, by weir formula, from reports of state Engineer. The flood of Aug. 12, 1929, reached a stage of about 16 ft (discharge $31,300 \mathrm{ft}^{3} / \mathrm{s}$, by weir formula, from reports of State Engineer). A flood on Oct. 4, 1913, reached a stage of 9.5 ft (discharge not determined) prior to construction of the concrete control.

STATISTICAL SUMMARIES

MEAN MONTHLY AND MEAN ANNUAL DISCHARGES 1935-76

\begin{tabular}{|c|c|c|c|c|c|c|}
\hline MONT'H & $\begin{array}{c}\text { MINIMUM } \\
(\mathrm{FT} 3 / \mathrm{S})\end{array}$ & $\begin{array}{c}\operatorname{MAXIMUM} \\
\left(\mathrm{FT}^{3} / \mathrm{S}\right)\end{array}$ & $\begin{array}{l}\text { MEAN } \\
\left(\mathrm{FT}^{3} / \mathrm{S}\right)\end{array}$ & $\begin{array}{l}\text { STAN- } \\
\text { DARD } \\
\text { DEVIA- } \\
\text { TION } \\
\left(\mathrm{FT}^{3} / \mathrm{S}\right)\end{array}$ & $\begin{array}{l}\text { COEFFI- } \\
\text { CIENT OF } \\
\text { VARI - } \\
\text { ATION }\end{array}$ & $\begin{array}{c}\text { PERCENT } \\
\text { OF } \\
\text { ANNUAL } \\
\text { RUNOF F }\end{array}$ \\
\hline $\begin{array}{l}\text { OCTOBER } \\
\text { NOVEMBER } \\
\text { DECEMBER } \\
\text { JANUARY } \\
\text { FFBRUARY } \\
\text { MARCH } \\
\text { APRIL } \\
\text { MAY } \\
\text { JUNE } \\
\text { JULY } \\
\text { AUGUST } \\
\text { SEPTEMBER }\end{array}$ & $\begin{array}{l}0.0 \\
0.0 \\
0.0 \\
0.0 \\
0.0 \\
0.0 \\
0.0 \\
0.0 \\
0.0 \\
0.0 \\
5.4 \\
0.0\end{array}$ & $\begin{array}{r}566 \\
76 \\
14 \\
43 \\
193 \\
290 \\
191 \\
990 \\
219 \\
396 \\
1161 \\
461\end{array}$ & $\begin{array}{l}63 \\
5.8 \\
1.1 \\
3.9 \\
19 \\
25 \\
18 \\
53 \\
20 \\
86 \\
260 \\
120\end{array}$ & $\begin{array}{c}119 \\
13 \\
2.3 \\
7.6 \\
40 \\
57 \\
37 \\
154 \\
38 \\
109 \\
275 \\
149\end{array}$ & $\begin{array}{l}1.89 \\
2.28 \\
2.09 \\
1.94 \\
2.10 \\
2.29 \\
2.10 \\
2.91 \\
1.96 \\
1.27 \\
1.06 \\
1.24\end{array}$ & $\begin{array}{r}9.4 \\
.9 \\
.2 \\
.6 \\
2.8 \\
3.7 \\
2.6 \\
7.9 \\
2.9 \\
12.7 \\
38.6 \\
17.8\end{array}$ \\
\hline ANNUAE & 7.8 & 197 & 57 & 41 & .72 & 100 \\
\hline
\end{tabular}

MAGNITUDE AND PROBABIEITY OF ANNUAL LOW FLOW BASED ON PERIOD OF RECORD $1936-76$

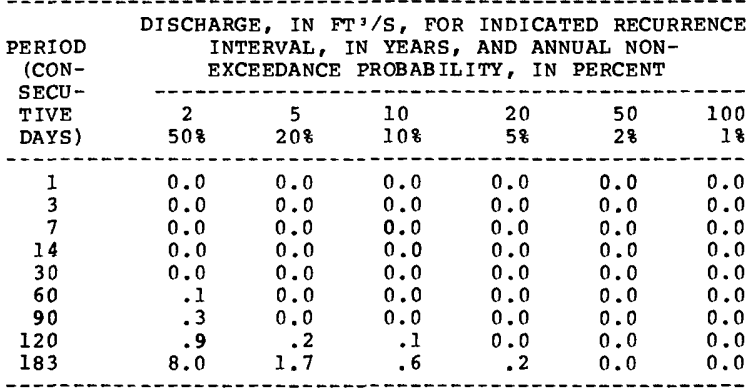

MAGNITUDE AND PROBABILITY OF ANNUAL HIGH FLON BASED ON PERIOD OF RECORD 1935-76

\begin{tabular}{|c|c|c|c|c|c|c|}
\hline \multirow{3}{*}{$\begin{array}{l}\text { PERIOD } \\
\text { (CON- } \\
\text { SECU- } \\
\text { TIVE } \\
\text { DAYS) }\end{array}$} & \multicolumn{6}{|c|}{$\begin{array}{l}\text { DISCHARGE, IN } \mathrm{FT}^{3 / S} \text {, FOR INDICATED RECURRENCE } \\
\text { INTERVAL, IN YEARS, AND ANNUAL } \\
\text { EXCEEDANCE PROBAB ILITY, IN PERCENT }\end{array}$} \\
\hline & 2 & 5 & 10 & 25 & 50 & 100 \\
\hline & 508 & 208 & 108 & 48 & 28 & 18 \\
\hline 1 & 370 & 4270 & 5790 & 8010 & 986 & \\
\hline 3 & 1400 & 2450 & 3240 & 4320 & 5170 & 6050 \\
\hline 7 & 820 & 1390 & 1800 & 2320 & 2710 & 3090 \\
\hline 15 & 485 & 870 & 1160 & 1570 & 1890 & 2220 \\
\hline 30 & 321 & 596 & 807 & 1100 & 1330 & 1570 \\
\hline 60 & 200 & 375 & 514 & 711 & 873 & 1050 \\
\hline 90 & 143 & 266 & 364 & 507 & 625 & 754 \\
\hline
\end{tabular}

DURATION TABLE OF DAILY MEAN FLOW FOR PERIOD OF RECORD 1935-76

DISCHARGF, IN FT'3/S, THAT WAS EQUALED OR EXCEEDED FOR INDICATED PERCENT OF TIME

\begin{tabular}{|c|c|c|c|c|c|c|c|c|c|c|c|c|c|c|}
\hline 58 & $10 \%$ & 158 & 208 & 258 & 308 & 408 & 508 & 608 & 708 & 758 & 808 & 858 & 908 & 958 \\
\hline 244 & 80 & 33 & 16 & 8.5 & 5.0 & 1.7 & .5 & 0.0 & 0.0 & 0.0 & 0.0 & 0.0 & 0.0 & 0.0 \\
\hline
\end{tabular}


RIO GRANDE BASIN

08353000 RIO PUERCO NEAR BERNARDO, NM

LOCATION.--Lat $34^{\circ} 24^{\prime} 33^{\prime \prime}$, Iong 106 51.09", In SBł sec.8, T.2 N., R.I E., Socorro County, Hydrologic Unit 13020204, on bridge on former $\mathrm{D} . \mathrm{s}$. Highway 85 and $0.2 \mathrm{mi}$ upstream from Interstate Highway $25,1.2 \mathrm{mi}$ southwest of Bernardo, $3 \mathrm{mi}$ upstream from mouth, and $18 \mathrm{mi}$ south of Belen.

DRAINAGE AREA.- -7,350 mi2, approximately, of which at least $1,130 \mathrm{mi}$ does not contribute directly to surface runoff.

PERIOD OF RECORD.--November 1939 to current year. Fragmentary gage height record and footnotes concerning no flow for the period September 1910 to August 1914, published in wSP 358 and 388 , are in error and should not be used.

REVISED RECORDS.--WSP 1512: 1941-42, 1944-45, 1946 (P), 1947-49. wSP 1632: 1957. wSP 1732: Drainage area. See also PERIOD OF RECORD.

GAGE.- Water-stage recorder. Datum of gage is $4,722.34 \mathrm{ft}$ above National Geodetic vertical Datum of 1929 . Prior to Jan. 24, 1969, at datum $3.10 \mathrm{ft}$ higher.

REMARKS.--Diversions for irrigation of about 11,500 acres upstream from station (includes 3,700 acres irrigated wholly or partly from wells).

AVERAGE DISCHARGB. --45 years (water years 1941-85), $45.2 \mathrm{ft} / \mathrm{s}, 32,750 \mathrm{acre}-\mathrm{ft} / \mathrm{yr}$

BXTREMES FOR PERIOD OF RECORD.--Maximum discharge, $18,800 \mathrm{ft} 3 / 8$, Sept. 23 , 1941 , from rating curve extended above $7,800 \mathrm{ft} / \mathrm{s}$; maximum gage height, $16.9 \mathrm{ft}$, present datum, Aug. 12, 1955; no flow for extended periods.

EXTREMES OUTSIDE PERIOD OF RBCORD.--The greatest flood since about 1880 occurred Sept. 23, 1929, from information by local residents (discharge, about $35,000 \mathrm{ft} / \mathrm{s}$, estimated on basis of peak at Rio Puerco). Another flood occurred Aug. 12, 1929 (discharge, 30,600 ft $/ 8$, by slope-area method, from reports of State Engineer).

STATISTICAL SUMMARIES

MEAN MONTHLY AND MEAN ANNUAL DISCHARGES 1941-85

\begin{tabular}{|c|c|c|c|c|c|c|}
\hline MONTH & $\begin{array}{l}\text { MINIMUM } \\
\left(F^{2} / / S\right)\end{array}$ & $\begin{array}{l}\text { MAXIMUM } \\
(\mathrm{FT}: / \mathrm{S})\end{array}$ & $\begin{array}{l}\text { MEAN } \\
\left(\mathrm{FT}^{3} / \mathrm{S}\right)\end{array}$ & $\begin{array}{l}\text { STAN- } \\
\text { DARD } \\
\text { DEVIA- } \\
\text { TION } \\
(\text { FT'/S) }\end{array}$ & $\begin{array}{l}\text { COEFFI- } \\
\text { CIENT OF } \\
\text { VARI- } \\
\text { ATION }\end{array}$ & $\begin{array}{c}\text { PERCENT } \\
\text { OF } \\
\text { ANNUAL } \\
\text { RUNOFF }\end{array}$ \\
\hline $\begin{array}{l}\text { OCTOBER } \\
\text { NOVEMBER } \\
\text { DECEMBER } \\
\text { JANUARY } \\
\text { FEBRUARY } \\
\text { MARCH } \\
\text { APRIL } \\
\text { MAY } \\
\text { JUNE } \\
\text { JULY } \\
\text { AUGUST } \\
\text { SEPTEMBER }\end{array}$ & $\begin{array}{l}0.0 \\
0.0 \\
0.0 \\
0.0 \\
0.0 \\
0.0 \\
0.0 \\
0.0 \\
0.0 \\
0.0 \\
2.3 \\
0.0\end{array}$ & $\begin{array}{r}586 \\
79 \\
27 \\
19 \\
142 \\
208 \\
179 \\
885 \\
203 \\
362 \\
922 \\
584\end{array}$ & $\begin{array}{r}58 \\
6.1 \\
.9 \\
1.3 \\
14 \\
20 \\
15 \\
47 \\
20 \\
64 \\
201 \\
92\end{array}$ & $\begin{array}{r}116 \\
15 \\
4.2 \\
3.9 \\
32 \\
47 \\
34 \\
134 \\
41 \\
99 \\
233 \\
133\end{array}$ & $\begin{array}{l}2.01 \\
2.41 \\
4.51 \\
2.90 \\
2.27 \\
2.34 \\
2.24 \\
2.87 \\
2.04 \\
1.55 \\
1.16 \\
1.45\end{array}$ & $\begin{array}{r}10.7 \\
1.1 \\
.2 \\
.2 \\
2.7 \\
3.7 \\
2.8 \\
8.7 \\
3.7 \\
11.8 \\
37.3 \\
17.0\end{array}$ \\
\hline NNUAL & 5.5 & 171 & 45 & 35 & .77 & 100 \\
\hline
\end{tabular}

MAGNITUDE AND PROBABILITY OF ANNUAL LOW FLOW BASED ON PBRIOD OF RECORD 1942-85

\begin{tabular}{|c|c|c|c|c|c|c|}
\hline \multirow{2}{*}{$\begin{array}{l}\text { PERIOD } \\
\text { (CON- } \\
\text { SECU- } \\
\text { TIVE } \\
\text { DAYS) }\end{array}$} & \multicolumn{6}{|c|}{$\begin{array}{l}\text { DISCHARGE, IN FT'3/S, FOR INDICATED RECURRENCE } \\
\text { INTERVAL, IN YEARS, AND ANNUAL NON- } \\
\text { EXCEEDANCE PROBABILITY, IN PERCENT }\end{array}$} \\
\hline & $\begin{array}{c}2 \\
508\end{array}$ & $\begin{array}{c}5 \\
208\end{array}$ & $\begin{array}{l}10 \\
108\end{array}$ & $\begin{array}{l}20 \\
5\end{array}$ & $\begin{array}{l}50 \\
28\end{array}$ & $\begin{array}{r}100 \\
18\end{array}$ \\
\hline $\begin{array}{r}1 \\
3 \\
7 \\
14 \\
30 \\
60 \\
90 \\
120 \\
183\end{array}$ & $\begin{array}{l}0.0 \\
0.0 \\
0.0 \\
0.0 \\
0.0 \\
0.0 \\
0.0 \\
0.0 \\
4.0\end{array}$ & $\begin{array}{l}0.0 \\
0.0 \\
0.0 \\
0.0 \\
0.0 \\
0.0 \\
0.0 \\
0.0 \\
.2\end{array}$ & $\begin{array}{l}0.0 \\
0.0 \\
0.0 \\
0.0 \\
0.0 \\
0.0 \\
0.0 \\
0.0 \\
0.0\end{array}$ & $\begin{array}{l}0.0 \\
0.0 \\
0.0 \\
0.0 \\
0.0 \\
0.0 \\
0.0 \\
0.0 \\
0.0\end{array}$ & $\begin{array}{l}0.0 \\
0.0 \\
0.0 \\
0.0 \\
0.0 \\
0.0 \\
0.0 \\
0.0 \\
0.0\end{array}$ & $\begin{array}{l}0.0 \\
0.0 \\
0.0 \\
0.0 \\
0.0 \\
0.0 \\
0.0 \\
0.0 \\
0.0\end{array}$ \\
\hline
\end{tabular}

MAGNITUDE AND PROBABILITY OF ANNUAL HIGH FLON BASED ON PERIOD OF RECORD 1941-85

\begin{tabular}{|c|c|c|c|c|c|c|}
\hline \multirow{2}{*}{$\begin{array}{l}\text { PERIOD } \\
\text { (CON- } \\
\text { SECU- } \\
\text { TIVE } \\
\text { DAYS) }\end{array}$} & \multicolumn{6}{|c|}{$\begin{array}{l}\text { DISCHARGE, IN FT'/S, FOR INDICATED RECURRENCE } \\
\text { INTERVAL, IN YEARS, AND ANNUAL } \\
\text { EXCEEDANCE' PROBABILITY, IN PERCENT }\end{array}$} \\
\hline & $\begin{array}{c}2 \\
508\end{array}$ & $\begin{array}{c}5 \\
208\end{array}$ & $\begin{array}{l}10 \\
108\end{array}$ & $\begin{array}{l}25 \\
48\end{array}$ & $\begin{array}{l}50 \\
28\end{array}$ & $\begin{array}{r}100 \\
18\end{array}$ \\
\hline $\begin{array}{r}1 \\
3 \\
7 \\
15 \\
30 \\
60 \\
90\end{array}$ & $\begin{array}{r}1670 \\
1110 \\
652 \\
391 \\
251 \\
155 \\
111\end{array}$ & $\begin{array}{r}3140 \\
2050 \\
1200 \\
746 \\
493 \\
303 \\
215\end{array}$ & $\begin{array}{r}4330 \\
2770 \\
1600 \\
1010 \\
686 \\
421 \\
302\end{array}$ & $\begin{array}{r}6050 \\
3760 \\
2120 \\
1360 \\
961 \\
588 \\
432\end{array}$ & $\begin{array}{r}7480 \\
4540 \\
2510 \\
1620 \\
1180 \\
724 \\
544\end{array}$ & $\begin{array}{r}9040 \\
5350 \\
2900 \\
1890 \\
1420 \\
867 \\
669\end{array}$ \\
\hline
\end{tabular}

DURATION TABLE OF DAILY MEAN FLOW FOR PERIOD OF RECORD 1941-85

DISCHARGE, IN FT'/S, THAT WAS EQUALED OR EXCEEDED FOR INDICATED PERCENT OF TIME

\begin{tabular}{|c|c|c|c|c|c|c|c|c|c|c|c|c|c|c|}
\hline 58 & 108 & 158 & 208 & 258 & 308 & 408 & 508 & 608 & 708 & 758 & 808 & 858 & 908 & 958 \\
\hline 192 & 65 & 27 & 11 & 3.7 & .5 & 0.0 & 0.0 & 0.0 & 0.0 & 0.0 & 0.0 & 0. & 0.0 & 0. \\
\hline
\end{tabular}


RIO GRANDE BASIN

08354000 RIO SALADO NEAR SAN ACACIA, NM

LOCATION.--Lat 34\%17'50", long $106^{\circ} 53^{\prime} 59^{\prime \prime}$, in NWł sec.24, T.1 N., R.1 W., Socorro County, Hydrologic Unit 13020209, at former bridge site $0.3 \mathrm{mi}$ upstream from bridge on Interstate Highway $25,3.1 \mathrm{mi}$ upstream from mouth, $2.9 \mathrm{mi}$ north of San Acacia, and $15 \mathrm{mi}$ north of Socorro.

DRAINAGE AREA. $--1,380 \mathrm{mi}^{2}$, approximately.

PERIOD OF RECORD.--Oct ober 1947 to September 1984 .

REVISED RECORDS.--WSP 1512: 1948-49, 1955. WSP 1632: 1953.

GAGE.- Water-stage recorder. Altitude of gage is 4,765 ft from topographic map. Prior to Sept. 14, 1966, at site $1.7 \mathrm{mi}$ downstream at different datum.

REMARKS.--Diversions for irrigation of about 100 acres above station.

AVERAGE DISCHARGE.--37 years (water years 1948-84), 14.3 $\mathrm{ft}^{3} / \mathrm{s}, 10,360 \mathrm{acre}-\mathrm{ft} / \mathrm{yr}$.

EXTREMES FOR PERIOD OF RECORD.--Maximum discharge, $36,200 \mathrm{ft}$ /s July 31,1965 , gage height, $5.54 \mathrm{ft}$, from

floodmarks, present site and datum, from rating curve extended above $900 \mathrm{ft}^{3} / \mathrm{s}$ on basis of slope-area

measurements of peak flow; no flow most of time.

EXTREMES OUTSIDE PERIOD OF RECORD.--Another flood occurred Aug. 12, 1929 (discharge, 27,400 ft $3 / \mathrm{s}$, by slope-area method), from reports of State Engineer.

STATISTICAL SUMMARIES

MEAN MONTHLY AND MEAN ANNUAL DISCHARGES 1948-84

\begin{tabular}{|c|c|c|c|c|c|c|}
\hline MONTH & $\begin{array}{c}\text { M INIMUM } \\
\left(\mathrm{FT}^{3} / \mathrm{S}\right)\end{array}$ & $\begin{array}{c}\text { MAXI MUM } \\
\left(\mathrm{FT}{ }^{3} / \mathrm{S}\right)\end{array}$ & $\begin{array}{l}\text { MEAN } \\
\left\langle\mathrm{FT}^{3} / \mathrm{S}\right\rangle\end{array}$ & $\begin{array}{l}\text { STAN- } \\
\text { DARD } \\
\text { DEVIA- } \\
\text { TION } \\
\text { (FT'/S) }\end{array}$ & $\begin{array}{l}\text { COEFFI- } \\
\text { CIENT OF } \\
\text { VARI- } \\
\text { ATION }\end{array}$ & $\begin{array}{c}\text { PERCENT } \\
\text { OF } \\
\text { ANNUAL } \\
\text { RUNOFF }\end{array}$ \\
\hline & & & & & & 7 \\
\hline OCTOBER & 0.0 & 230 & 13 & 40 & 3.01 & 7.8 \\
\hline NOVEMBER & 0.0 & 37 & 1.0 & 6.0 & 6.00 & .6 \\
\hline DECEMBE R & 0.0 & .8 & 0.0 & .1 & 6.50 & 0.0 \\
\hline JANUARY & 0. & 0.0 & 0.0 & 0.0 & -- & 0.0 \\
\hline FEBRUARY & 0. & 1.0 & 0.0 & .2 & 5.33 & 0.0 \\
\hline MARCH & 0.0 & .1 & 0.0 & 0.0 & -- & 0.0 \\
\hline APRIL & 0. & 2.4 & .1 & .4 & 5.13 & 0.0 \\
\hline MAY & 0.0 & 5.1 & .3 & 1.1 & 4.00 & .2 \\
\hline JUNE & 0.0 & 43 & 3.3 & 8.1 & 2.45 & 1.9 \\
\hline JULY & 0.0 & 185 & 26 & 39 & 1.49 & 15.5 \\
\hline AUGUST & .1 & 451 & 78 & 89 & 1.13 & 46.1 \\
\hline SEPTEMBER & 0.0 & 429 & 47 & 81 & 1.70 & 27.8 \\
\hline NNUAL & .4 & 89 & 14 & 15 & 1.08 & 100 \\
\hline
\end{tabular}

MAGNITUDE AND PROBABILITY OF ANNUAL LOW FLOW BASED ON PERIOD OF RECORD 1949-84

\begin{tabular}{|c|c|c|c|c|c|c|}
\hline \multirow{2}{*}{$\begin{array}{l}\text { PERIOD } \\
\text { (CON- } \\
\text { SECU- } \\
\text { TIVE } \\
\text { DAYS) }\end{array}$} & \multicolumn{6}{|c|}{$\begin{array}{l}\text { DISCHARGE, IN FT }{ }^{3} / S \text {, FOR INDICATED RECURRENCE } \\
\text { INTERVAL, IN YEARS, AND ANNUAL NON- } \\
\text { EXCEEDANCE PROBABIL ITY, IN PERCENT }\end{array}$} \\
\hline & $\begin{array}{c}2 \\
508\end{array}$ & $\begin{array}{c}5 \\
208\end{array}$ & $\begin{array}{l}10 \\
108\end{array}$ & $\begin{array}{l}20 \\
58\end{array}$ & $\begin{array}{l}50 \\
28\end{array}$ & $\begin{array}{r}100 \\
18\end{array}$ \\
\hline $\begin{array}{r}1 \\
3 \\
7 \\
14 \\
30 \\
60 \\
90 \\
120 \\
183\end{array}$ & $\begin{array}{r}0.0 \\
0.0 \\
0.0 \\
0.0 \\
0.0 \\
0.0 \\
0.0 \\
0.0 \\
.1\end{array}$ & $\begin{array}{l}0.0 \\
0.0 \\
0.0 \\
0.0 \\
0.0 \\
0.0 \\
0.0 \\
0.0 \\
0.0\end{array}$ & $\begin{array}{l}0.0 \\
0.0 \\
0.0 \\
0.0 \\
0.0 \\
0.0 \\
0.0 \\
0.0 \\
0.0\end{array}$ & $\begin{array}{l}0.0 \\
0.0 \\
0.0 \\
0.0 \\
0.0 \\
0.0 \\
0.0 \\
0.0 \\
0.0\end{array}$ & $\begin{array}{l}0.0 \\
0.0 \\
0.0 \\
0.0 \\
0.0 \\
0.0 \\
0.0 \\
0.0 \\
0.0\end{array}$ & \\
\hline
\end{tabular}

MAGNITUDE AND PROBABILITY OF ANNUAL HIGH FLOW BASED ON PERIOD OF RECORD 1948-84

\begin{tabular}{|c|c|c|c|c|c|c|}
\hline \multirow{2}{*}{$\begin{array}{l}\text { PERIOD } \\
\text { (CON- } \\
\text { SECU- } \\
\text { TIVE } \\
\text { DAYS) }\end{array}$} & \multicolumn{6}{|c|}{$\begin{array}{l}\text { DISCHARGE, IN FT' } 3 / \mathrm{S} \text {, FOR INDICATED RECURRENCE } \\
\text { INTERVAL, IN YEARS, AND ANNUAL } \\
\text { EXCEEDANCE PROBABILI TY, IN PERCENT }\end{array}$} \\
\hline & $\begin{array}{c}2 \\
508\end{array}$ & $\begin{array}{c}5 \\
208\end{array}$ & $\begin{array}{l}10 \\
108\end{array}$ & $\begin{array}{l}25 \\
48\end{array}$ & $\begin{array}{l}50 \\
28\end{array}$ & $\begin{array}{r}100 \\
18\end{array}$ \\
\hline $\begin{array}{r}1 \\
3 \\
7 \\
15 \\
30 \\
60 \\
90\end{array}$ & $\begin{array}{r}750 \\
417 \\
215 \\
130 \\
82 \\
51 \\
37\end{array}$ & $\begin{array}{r}1620 \\
957 \\
513 \\
319 \\
209 \\
128 \\
91\end{array}$ & $\begin{array}{r}2270 \\
1380 \\
747 \\
470 \\
310 \\
189 \\
131\end{array}$ & $\begin{array}{r}3120 \\
1930 \\
1060 \\
671 \\
443 \\
268 \\
181\end{array}$ & $\begin{array}{r}3750 \\
2330 \\
1290 \\
821 \\
541 \\
325 \\
216\end{array}$ & $\begin{array}{l}-- \\
=- \\
=- \\
-- \\
--\end{array}$ \\
\hline
\end{tabular}

DURATION TABLE OF DAILY MEAN FLOW FOR PERIOD OF RECORD 1948-84

DISCHARGE, IN FT'S/S, THAT WAS EQUALED OR EXCEEDED FOR INDICATED PERCENT OF TIME

\begin{tabular}{|c|c|c|c|c|c|c|c|c|c|c|c|c|c|c|}
\hline 58 & 108 & 158 & 208 & 258 & 308 & 408 & $50 \%$ & 608 & 708 & 758 & 808 & 858 & 908 & 958 \\
\hline 26 & 1.0 & 0.0 & 0.0 & 0.0 & 0.0 & 0.0 & 0.0 & 0.0 & 0.0 & 0.0 & 0.0 & 0.0 & 0.0 & 0. \\
\hline
\end{tabular}


RIO GRANDE BASIN

08354500 SOCORRO MAIN CANAL NORTH AT SAN ACACIA, NM

LOCATION.--Lat $34^{\circ} 15^{\prime} 17^{\prime \prime}$, long $106^{\circ} 53^{\prime} 4^{\prime \prime}$, in SEłNWł sec.1, T.1 S., R.1 w., Socorro County, Hydrologic Unit 13020203 , on right bank at San Acacia, and $0.5 \mathrm{mi}$ downstream from point of diversion.

PERIOD OF RECORD.--April 1936 to September 1964 (monthly discharge only), october 1964 to current year.

REVISED RECORDS.--WSP 1242: 1951.

GAGE.--Water-stage recorder. Datum of gage is $4,660.16 \mathrm{ft}$ above National Geodetic vertical Datum of 1929 . Prior to Mar. 8, 1958, at site $300 \mathrm{ft}$ upstream (in old channel) at datum $0.42 \mathrm{ft}$ lower.

REMARKS.--This canal is 1 of 3 channels (stations 08354800,08354900 ) carrying flow in valley cross section.

Canal diverts water from right bank of Rio Grande for irrigation of about 8,000 acres. Alamillo Acequia and

3 other smaller ditches divert water from canal upstream from station for irrigation of about 400 acres.

Discharge records collected at the canal heading from October 1964 to september 1965 indicate that 7,770 acre-ft

or 98 of the initial canal flow was diverted before reaching the regular gaging station.

AVERAGE DISCHARGE.--49 years (water years 1937-85), $88 \mathrm{ft}^{3} / \mathrm{s}, 63,760$ acre-ft/yr.

EXTREMES FOR PERIOD OF RECORD.--Maximum daily discharge, $274 \mathrm{ft}^{3} / \mathrm{s}$, June 22 , 1980 ; no flow at times.

STATISTICAL SUMMARIES

MEAN MONTHLY AND MEAN ANNUAL DISCHARGES 1937-85

MAGNITUDE AND PROBABILITY OF ANNUAL LOW FLOW BASED ON PERIOD OF RECORD $1938-85$

\begin{tabular}{|c|c|c|c|c|c|c|}
\hline MONTH & $\begin{array}{l}\text { MINIMUM } \\
\left(\mathrm{FT}^{3} / \mathrm{S}\right)\end{array}$ & $\begin{array}{c}\operatorname{MAXIMUM} \\
\left(\mathrm{FT}^{3} / \mathrm{S}\right)\end{array}$ & $\begin{array}{l}\text { MEAN } \\
\left(\mathrm{FT}^{3} / \mathrm{S}\right)\end{array}$ & $\begin{array}{c}\text { STAN- } \\
\text { DARD } \\
\text { DEVIA- } \\
\text { TION } \\
(\text { FT'/S) }\end{array}$ & $\begin{array}{l}\text { COEFFI- } \\
\text { CIENT OF } \\
\text { VARI- } \\
\text { ATION }\end{array}$ & $\begin{array}{c}\text { PERCENT } \\
\text { OF } \\
\text { ANNUAL } \\
\text { RUNOFF }\end{array}$ \\
\hline остОВЕR & 0.0 & 176 & 90 & 46 & .51 & 8.5 \\
\hline NOVEMBER & 0.0 & 128 & 27 & 36 & 1.30 & 2.6 \\
\hline DECEMBER & 0.0 & 79 & 9.7 & 18 & 1.84 & .9 \\
\hline JANUARY & 0. & 71 & 8.3 & 19 & 2.30 & .8 \\
\hline FEBRUARY & 0.0 & 101 & 15 & 26 & 1.74 & 1.4 \\
\hline MARCH & 35 & 191 & 118 & 41 & .34 & 11.2 \\
\hline APR IL & 78 & 239 & 158 & 40 & .25 & 15.0 \\
\hline MAY & 46 & 226 & 153 & 47 & .31 & 14.5 \\
\hline JUNE & 34 & 253 & 145 & 57 & .39 & 13.8 \\
\hline JULY & 8.8 & 244 & 126 & 57 & .45 & 12.0 \\
\hline AUGUST & 29 & 202 & 116 & 43 & .37 & 11.0 \\
\hline SEPTEMBER & 0.0 & & 89 & 46 & .52 & 8.4 \\
\hline NNL & 43 & 136 & 88 & 23 & .26 & 100 \\
\hline
\end{tabular}

\begin{tabular}{|c|c|c|c|c|c|c|}
\hline \multirow{2}{*}{$\begin{array}{l}\text { PERIOD } \\
\text { (CON- } \\
\text { SECU- } \\
\text { TIVE } \\
\text { DAYS) }\end{array}$} & \multicolumn{6}{|c|}{ 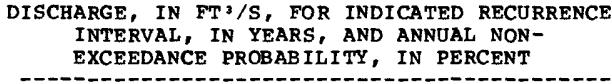 } \\
\hline & $\begin{array}{c}2 \\
508\end{array}$ & $\begin{array}{c}5 \\
208\end{array}$ & $\begin{array}{l}10 \\
108\end{array}$ & $\begin{array}{l}20 \\
58\end{array}$ & $\begin{array}{l}50 \\
28\end{array}$ & $\begin{array}{r}100 \\
18\end{array}$ \\
\hline $\begin{array}{r}1 \\
3 \\
7 \\
14 \\
30 \\
60 \\
90 \\
120 \\
183\end{array}$ & $\begin{array}{r}0.0 \\
0.0 \\
0.0 \\
0.0 \\
0.0 \\
0.0 \\
.7 \\
2.8\end{array}$ & $\begin{array}{l}0.0 \\
0.0 \\
0.0 \\
0.0 \\
0.0 \\
0.0 \\
0.0 \\
0.0 \\
20\end{array}$ & $\begin{array}{r}0.0 \\
0.0 \\
0.0 \\
0.0 \\
0.0 \\
0.0 \\
0.0 \\
0.0 \\
12\end{array}$ & $\begin{array}{l}0.0 \\
0.0 \\
0.0 \\
0.0 \\
0.0 \\
0.0 \\
0.0 \\
0.0 \\
6.6\end{array}$ & $\begin{array}{l}0.0 \\
0.0 \\
0.0 \\
0.0 \\
0.0 \\
0.0 \\
0.0 \\
0.0 \\
3.2\end{array}$ & $\begin{array}{l}0.0 \\
0.0 \\
0.0 \\
0.0 \\
0.0 \\
0.0 \\
0.0 \\
0.0 \\
1.8\end{array}$ \\
\hline
\end{tabular}

MAGNITUDE AND PROBABILITY OF ANNUAL HIGH FLON BASED ON PERIOD OF RECORD 1937-85

\begin{tabular}{|c|c|c|c|c|c|c|}
\hline \multirow{2}{*}{$\begin{array}{l}\text { PERIOD } \\
\text { (CON- } \\
\text { SECU- } \\
\text { TIVE } \\
\text { DAYS) }\end{array}$} & \multicolumn{6}{|c|}{$\begin{array}{l}\text { DISCHARGE, IN FT' }{ }^{3 / S} \text {, FOR INDICATED RECURRENCE } \\
\text { INTERVAL, IN YEARS, AND ANNUAL } \\
\text { EXCEEDANCE PROBABILITY, IN PERCENT }\end{array}$} \\
\hline & $\begin{array}{c}2 \\
508\end{array}$ & $\begin{array}{c}5 \\
208\end{array}$ & $\begin{array}{l}10 \\
108\end{array}$ & $\begin{array}{l}25 \\
48\end{array}$ & $\begin{array}{l}50 \\
28\end{array}$ & $\begin{array}{r}100 \\
18\end{array}$ \\
\hline $\begin{array}{r}1 \\
3 \\
7 \\
15 \\
30 \\
60 \\
90\end{array}$ & $\begin{array}{l}216 \\
209 \\
202 \\
195 \\
186 \\
176 \\
164\end{array}$ & $\begin{array}{l}247 \\
238 \\
232 \\
226 \\
219 \\
208 \\
198\end{array}$ & $\begin{array}{l}263 \\
254 \\
249 \\
243 \\
236 \\
225 \\
216\end{array}$ & $\begin{array}{l}281 \\
271 \\
266 \\
261 \\
255 \\
241 \\
235\end{array}$ & $\begin{array}{l}293 \\
283 \\
278 \\
273 \\
267 \\
251 \\
248\end{array}$ & $\begin{array}{l}304 \\
293 \\
288 \\
284 \\
277 \\
260 \\
259\end{array}$ \\
\hline
\end{tabular}

DURATION TABLE OF DAILY MEAN FLOW FOR PERIOD OF RECORD 1937-85 DISCHARGE, IN FT'3/S, THAT WAS EQUALED OR EXCEEDED FOR INDICATED PERCENT OF TIME

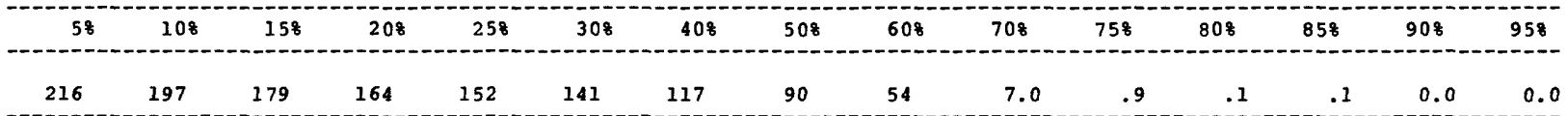


RIO GRANDE BASIN

08354800 RTO GRANDE CONVEYANCE CHANNEL AT SAN ACACIA, NM (Surveillance network)

LOCATION.--Lat $34^{\circ} 14^{\prime} 54^{\prime \prime}$, long $106^{\circ} 54^{\prime} 04^{\prime \prime}$, in SW sec.l, T.1 S., R.1 W., Socorro County, Hydrologic Unit 13020203 , on right bank $75 \mathrm{ft}$ upstream from railway crossing, $0.5 \mathrm{mi}$ south of San Acacia, and $1.2 \mathrm{mi}$ downstream from San Acacia diversion dam.

PERIOD OF RECORD.--October 1958 to september 1964 included in composite flow of station "08355000 Rio Grande at San Acacia," October 1960 to September 1964 (monthly discharge published in wSP 1923 with records for station 08355000 ), October 1964 to current year. Daily records 1958-64 are available in files at district office.

GAGE.--Water-stage recorder. Datum of gage is 4,652.50 ft above National Geodetic Vertical Datum of 1929 (levels by U.S. Bureau of Reclamation).

REMARKS.--Conveyance channel, constructed in 1958, is 1 of 3 channels (stations 08354500, 08354900) carrying flow in valley cross section. Original design and plan was for conveyance channel to carry all flows up to about $2,000 \mathrm{ft}^{3} / \mathrm{s}$.

AVERAGE DISCHARGE.--10 years (water years 1964-73), $635 \mathrm{ft}^{3} / \mathrm{s}, 460,100 \mathrm{acre}-\mathrm{ft} / \mathrm{yr}$.

EXTREMES FOR PERIOD OF RECORD.--Maximum daily discharge, 1,950 $\mathrm{ft}^{3 / 5}$, May 12, 13 , 1966 ; no flow at times.

STATISTICAL SUMMARIES (PERIOD BEFORE REGULATION OF COCHITI DAM AND AFTER COMPLETION OF ABIQUIU DAM) MEAN MONTHLy AND MEAN ANNUAL DISChARges 1964-73

\begin{tabular}{|c|c|c|c|c|c|c|}
\hline MONTH & $\begin{array}{l}\text { MINIMUM } \\
\left(\mathrm{FT}^{3} / \mathrm{S}\right)\end{array}$ & $\begin{array}{c}\text { MAXIMUM } \\
\left(\mathrm{FT}^{3} / \mathrm{S}\right)\end{array}$ & $\begin{array}{l}\text { MEAN } \\
\left(\mathrm{FT}^{3} / \mathrm{S}\right)\end{array}$ & $\begin{array}{l}\text { STAN- } \\
\text { DARD } \\
\text { DEVIA- } \\
\text { TION } \\
\left(\mathrm{FT}^{3} / \mathrm{S}\right)\end{array}$ & $\begin{array}{l}\text { COEFFI- } \\
\text { CIENT OF } \\
\text { VARI- } \\
\text { ATION }\end{array}$ & $\begin{array}{c}\text { PERCENT } \\
\text { OF } \\
\text { ANNUAL } \\
\text { RUNOFF }\end{array}$ \\
\hline $\begin{array}{l}\text { OCTOBER } \\
\text { NOVEMBER } \\
\text { DECEMBER } \\
\text { JANUARY } \\
\text { FEBRUARY } \\
\text { MARCH } \\
\text { APRIL } \\
\text { MAY } \\
\text { JUNE } \\
\text { JULY } \\
\text { AUGUST } \\
\text { SEPTEMBER }\end{array}$ & $\begin{array}{l}228^{.3} \\
296 \\
397 \\
480 \\
153 \\
9.0 \\
1.1 \\
1.4 \\
4.2 \\
3.9 \\
3.8\end{array}$ & $\begin{array}{r}708 \\
1644 \\
1823 \\
961 \\
1060 \\
1240 \\
1491 \\
1548 \\
1456 \\
1492 \\
829 \\
633\end{array}$ & $\begin{array}{r}274 \\
1037 \\
879 \\
758 \\
817 \\
625 \\
688 \\
874 \\
675 \\
339 \\
363 \\
307\end{array}$ & $\begin{array}{l}297 \\
499 \\
457 \\
171 \\
161 \\
337 \\
570 \\
644 \\
552 \\
514 \\
274 \\
251\end{array}$ & $\begin{array}{r}1.08 \\
.48 \\
.52 \\
.23 \\
.20 \\
.54 \\
.83 \\
.74 \\
.82 \\
1.52 \\
.75 \\
.82\end{array}$ & $\begin{array}{r}3.6 \\
13.6 \\
11.5 \\
9.9 \\
10.7 \\
8.2 \\
9.0 \\
11.4 \\
8.8 \\
4.4 \\
4.8 \\
4.0\end{array}$ \\
\hline ANNUAL & 207 & 1033 & 635 & 244 & .38 & 100 \\
\hline
\end{tabular}

MAGNITUDE AND PROBABILITY OF ANNUAL LOW FLOW BASED ON PERIOD OF RECORD $1965-73$

\begin{tabular}{|c|c|c|c|c|c|c|}
\hline \multirow{2}{*}{$\begin{array}{l}\text { PERIOD } \\
\text { (CON- } \\
\text { SECU- } \\
\text { TIVE } \\
\text { DAYS) }\end{array}$} & \multicolumn{6}{|c|}{$\begin{array}{l}\text { DISCHARGE, IN FT3/S, FOR INDICATED RECURRENCE } \\
\text { INTERVAL, IN YEARS, AND ANNUAL NON- } \\
\text { EXCEEDANCE PROBABILITY, IN PERCENT }\end{array}$} \\
\hline & $\begin{array}{c}2 \\
508\end{array}$ & $\begin{array}{c}5 \\
208\end{array}$ & $\begin{array}{l}10 \\
108\end{array}$ & $\begin{array}{l}20 \\
58\end{array}$ & $\begin{array}{l}50 \\
28\end{array}$ & \\
\hline $\begin{array}{r}1 \\
3 \\
7 \\
14 \\
30 \\
60 \\
90 \\
120 \\
183\end{array}$ & $\begin{array}{r}0.0 \\
0.0 \\
.3 \\
.6 \\
2.9 \\
25 \\
60 \\
111 \\
288\end{array}$ & $\begin{array}{c}0.0 \\
0.0 \\
0.0 \\
0.0 \\
.4 \\
5.6 \\
13 \\
28 \\
160\end{array}$ & $\begin{array}{r}0.0 \\
0.0 \\
0.0 \\
0.0 \\
.2 \\
2.7 \\
5.6 \\
12 \\
120\end{array}$ & $\begin{array}{r}0.0 \\
0.0 \\
0.0 \\
0.0 \\
.1 \\
1.5 \\
2.7 \\
5.4 \\
95\end{array}$ & $\begin{array}{l}-- \\
-- \\
-- \\
-- \\
-- \\
-- \\
--\end{array}$ & \\
\hline
\end{tabular}

MAGNITUDE AND PROBABILITY OF ANNUAL HIGH FLOW BASED ON PERIOD OF RECORD $1964-73$

\begin{tabular}{|c|c|c|c|c|c|c|}
\hline $\begin{array}{l}\text { PERIOD } \\
\text { (CON- } \\
\text { SECU- }\end{array}$ & \multicolumn{6}{|c|}{$\begin{array}{l}\text { DISCHARGE, IN FT }{ }^{3} / \mathrm{S} \text {, FOR INDICATED RECURRENCE } \\
\text { INTERVAL, IN YEARS, AND ANNUAL } \\
\text { EXCEEDANCE PROBABILITY, IN PERCENT }\end{array}$} \\
\hline $\begin{array}{l}\text { TIVE } \\
\text { DAYS) }\end{array}$ & $\begin{array}{c}2 \\
508\end{array}$ & $\begin{array}{c}5 \\
208\end{array}$ & $\begin{array}{l}10 \\
108\end{array}$ & $\begin{array}{l}25 \\
48\end{array}$ & $\begin{array}{l}50 \\
28\end{array}$ & $\begin{array}{r}100 \\
18\end{array}$ \\
\hline 1 & 1820 & 1890 & 1910 & -- & -- & - \\
\hline 3 & 1780 & 1870 & 1880 & -- & - & -- \\
\hline 7 & 1640 & 1780 & 1820 & -- & -- & -- \\
\hline 15 & 1570 & 1760 & 1810 & -- & -- & -- \\
\hline 30 & 1470 & 1730 & 1810 & -- & -- & - \\
\hline 60 & & 1620 & 1730 & -- & -- & -- \\
\hline 90 & 1180 & 1480 & 1580 & -- & - & -- \\
\hline
\end{tabular}

DURATION TABLE OF DAILY MEAN FLOW FOR PERIOD OF RECORD 1964-73 DISCHARGE, IN FT ${ }^{3} / \mathrm{S}$, THAT WAS EQUALED OR EXCEEDED FOR INDICATED PERCENT OF TIME

\begin{tabular}{|c|c|c|c|c|c|c|c|c|c|c|c|c|c|c|}
\hline 58 & 108 & 158 & 208 & 258 & 308 & 408 & 508 & 608 & 708 & 758 & 808 & 858 & 908 & 958 \\
\hline 1730 & 1560 & 1390 & 1200 & 1050 & 943 & 743 & 585 & 366 & 156 & 74 & 19 & 4.6 & 2.3 & \\
\hline
\end{tabular}


RIO GRANDE BASIN

08354800 RIO GRANDE CONVEYANCE CHANNEL AT SAN ACACIA, NM--Continued

STATISTICAL SUMMARIES (PERIOD AFTER COMPLETION OF COCHITI DAM)

MEAN MONTHLY AND MEAN ANNUAL DISCHARGES 1974-85

\begin{tabular}{|c|c|c|c|c|c|c|}
\hline MONTH & $\begin{array}{l}\text { MINIMUM } \\
\left(\mathrm{FT}^{3} / \mathrm{S}\right)\end{array}$ & $\underset{\left(\mathrm{FT}^{3} / \mathrm{S}\right)}{\operatorname{MAXIMUM}}$ & $\begin{array}{l}\text { MEAN } \\
\left(\mathrm{FT}^{3} / \mathrm{S}\right)\end{array}$ & $\begin{array}{l}\text { STAN- } \\
\text { DARD } \\
\text { DEVIA- } \\
\text { TION } \\
\left(\mathrm{FT}^{3} / \mathrm{S}\right)\end{array}$ & $\begin{array}{l}\text { COEFFI- } \\
\text { CIENT OF } \\
\text { VARI- } \\
\text { ATION }\end{array}$ & $\begin{array}{c}\text { PERCENT } \\
\text { OF } \\
\text { ANNUAL } \\
\text { RUNOFF }\end{array}$ \\
\hline OCTOBER & 1.0 & 765 & 144 & 226 & 1.57 & 2.4 \\
\hline NOVEMBER & 4 & 1630 & 741 & 533 & 72 & \\
\hline DECEMBER & & 1733 & 947 & 605 & 64 & \\
\hline JANUARY & 1.0 & 1513 & 733 & 434 & .59 & 10.4 \\
\hline FEBRUARY & .3 & 1065 & 630 & 406 & .64 & 10.7 \\
\hline MARCH & 4.9 & 1066 & 493 & 382 & .77 & 8.4 \\
\hline APR IL & .3 & 1506 & 387 & 500 & 1.29 & 6.6 \\
\hline MAY & .2 & 1663 & 663 & 743 & 1.12 & 11.2 \\
\hline JUNE & .1 & 1580 & 532 & 687 & 1.29 & 9.0 \\
\hline JULY & & 1522 & 326 & 469 & 1.44 & 5.5 \\
\hline AUGUST & .2 & 705 & 198 & 240 & 1.21 & 3.4 \\
\hline SEPTEMBER & 0.0 & 377 & 102 & 126 & 1.24 & 1.7 \\
\hline ANNUAL & 3.3 & 974 & 491 & 308 & .63 & 100 \\
\hline
\end{tabular}

MAGNITUDE AND PROBABILITY OF ANNUAL LOW FLOW BASED ON PERIOD OF RECORD 1975-85

\begin{tabular}{|c|c|c|c|c|c|c|}
\hline \multirow{2}{*}{$\begin{array}{l}\text { PERIOD } \\
\text { (CON- } \\
\text { SECU- } \\
\text { TIVE } \\
\text { DAYS) }\end{array}$} & \multicolumn{6}{|c|}{$\begin{array}{l}\text { DISCHARGE, IN FT }{ }^{3} / S \text {, FOR INDICATED RECURRENCE } \\
\text { INTERVAL, IN YEARS, AND ANNUAL NON- } \\
\text { EXCEEDANCE PROBABILITY, IN PERCENT }\end{array}$} \\
\hline & $\begin{array}{c}2 \\
50 \%\end{array}$ & $\begin{array}{c}5 \\
208\end{array}$ & $\begin{array}{l}10 \\
108\end{array}$ & $\begin{array}{l}20 \\
58\end{array}$ & $\begin{array}{l}50 \\
28\end{array}$ & $\begin{array}{r}100 \\
18\end{array}$ \\
\hline $\begin{array}{r}1 \\
3 \\
7 \\
14 \\
30 \\
60 \\
90 \\
120 \\
183\end{array}$ & $\begin{array}{r}.3 \\
.5 \\
.7 \\
1.0 \\
3.2 \\
9.3 \\
16 \\
44 \\
111\end{array}$ & $\begin{array}{r}.1 \\
.1 \\
.2 \\
.2 \\
.7 \\
1.3 \\
2.1 \\
4.9 \\
12\end{array}$ & $\begin{array}{r}0.0 \\
0.0 \\
.1 \\
.1 \\
.3 \\
.5 \\
.7 \\
1.4 \\
3.1\end{array}$ & $\begin{array}{r}0.0 \\
0.0 \\
0.0 \\
.1 \\
.2 \\
.2 \\
.3 \\
.4 \\
.9\end{array}$ & $\begin{array}{l}-- \\
-- \\
=- \\
=- \\
-- \\
--\end{array}$ & $\begin{array}{l}-- \\
-- \\
-- \\
-- \\
-- \\
--\end{array}$ \\
\hline
\end{tabular}

MAGNITUDE AND PROBABILITY OF ANNUAL HIGH FLOW BASED ON PERIOD OF RECORD 1974-85

\begin{tabular}{|c|c|c|c|c|c|c|}
\hline \multirow{2}{*}{$\begin{array}{l}\text { PERIOD } \\
\text { (CON- } \\
\text { SECU- } \\
\text { TIVE } \\
\text { DAYS) }\end{array}$} & \multicolumn{6}{|c|}{$\begin{array}{l}\text { DISCHARGE, IN FT'/S, FOR INDICATED RECURRENCE } \\
\text { INTERVAL, IN YEARS, AND ANNUAL } \\
\text { EXCEEDANCE' PROBABILITY, IN PERCENT }\end{array}$} \\
\hline & $\begin{array}{c}2 \\
508\end{array}$ & $\begin{array}{c}5 \\
208\end{array}$ & $\begin{array}{l}10 \\
108\end{array}$ & $\begin{array}{l}25 \\
48\end{array}$ & $\begin{array}{l}50 \\
28\end{array}$ & $\begin{array}{r}100 \\
18\end{array}$ \\
\hline $\begin{array}{r}1 \\
3 \\
7 \\
15 \\
30 \\
60 \\
90\end{array}$ & $\begin{array}{r}1390 \\
1320 \\
1240 \\
1160 \\
1080 \\
1010 \\
894\end{array}$ & $\begin{array}{l}2940 \\
3040 \\
2970 \\
2820 \\
2720 \\
2620 \\
2340\end{array}$ & $\begin{array}{l}3520 \\
3730 \\
3680 \\
3520 \\
3440 \\
3350 \\
3000\end{array}$ & $\begin{array}{l}-- \\
-- \\
-- \\
-- \\
--\end{array}$ & $\begin{array}{l}-- \\
-- \\
-- \\
-- \\
--\end{array}$ & $\begin{array}{l}-- \\
-- \\
-- \\
-- \\
--\end{array}$ \\
\hline
\end{tabular}

DURATION TABLE OF DAILY MEAN FLOW FOR PERIOD OF RECORD 1974-85

DISCHARGE, IN FT $3 / \mathrm{S}$, THAT WAS EQUALED OR EXCEEDED FOR INDICATED PERCENT OF TIME

\begin{tabular}{|c|c|c|c|c|c|c|c|c|c|c|c|c|c|c|}
\hline 58 & 108 & 158 & 208 & 258 & 308 & 408 & 508 & 608 & 708 & 758 & 808 & 858 & 908 & 958 \\
\hline 1700 & 1490 & 1290 & 1060 & 857 & 742 & 520 & 225 & 24 & 6.2 & 4.2 & 3.0 & 2.0 & .9 & 3 \\
\hline
\end{tabular}




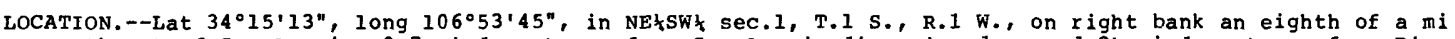
southeast of San Acacia, $0.7 \mathrm{mi}$ downstream from San Acacia diversion dam, and $2 \hbar_{2} \mathrm{mi}$ downstream from $\mathrm{Rio}$ Salado.

DRAINAGE AREA.--26,770 $\mathrm{mi}^{2}$, approximately (includes $2,940 \mathrm{mi}^{2}$ in closed basin in $\mathrm{San}$ Luis Valley, co.).

PERIOD OF RECORD.--April 1936 to september 1964.

GAGE.--Water-stage recorders. There are two recorders here, one on the conveyance channel designed to carry 2,000 $\mathrm{ft}^{3} / \mathrm{s}, 0.8 \mathrm{mi}$ below heading established February 1959 at elevation of $4,652.5 \mathrm{ft}$ above mean sea level (from U.S.B.R. specifications). The other recorder is $0.7 \mathrm{mile}$ downstream form heading on floodway or main channel at elevation of 4,658.10 ft above mean sea level, datum of 1929 . Prior to Mar. 19, $1953 \mathrm{floodway}$ gage at site 0.5

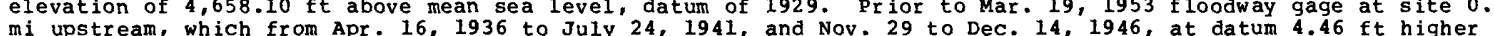
mi upstream, which from Apr. 16,1936 to July 24,1941 , and Nov. 29 to Dec. 14, 1946 , at datum 4.46 ft higher datum. Prior to about October 1958, all flow in floodway.

REMARKS.--Record is composite of main stem and conveyance channel (constructed in 1985, headgates opened May 18, 1959). Diversions above station for irrigation of about 760,000 acres; this includes Socorro Main Canal North, which bypasses station and irrigates about 8,000 acres.

AVERAGE DISCHARGE. --26 years (water years 1937-62), 1,121 $\mathrm{ft}^{3} / \mathrm{s}, 812,200 \mathrm{acre}-\mathrm{ft} / \mathrm{yr}$.

EXTREMES FOR PERIOD OF RECORD.--Maximum discharge, 27,400 $\mathrm{ft}^{3} / \mathrm{s}$ Aug. 5 , 1936 (gage height, $10.75 \mathrm{ft}$, site and datum then in use); no flow at times.

STATISTICAL SUMMARIES (PERIOD BEFORE REGULATION OF ABIQUIU DAM)

MEAN MONTHLY AND MEAN ANNUAL DISCHARGES 1937-62

MAGNITUDE AND PROBABILITY OF ANNUAL LOW FLOW BASED ON PERIOD OF RECORD 1938-62

\begin{tabular}{|c|c|c|c|c|c|c|}
\hline MONTH & $\begin{array}{l}\text { MINI MUM } \\
\left(\mathrm{FT}^{3} / \mathrm{S}\right)\end{array}$ & $\begin{array}{c}\text { MAXIMUM } \\
\left(\mathrm{FT}^{3} / \mathrm{S}\right)\end{array}$ & $\begin{array}{l}\text { MEAN } \\
\left(\mathrm{FT}^{3} / \mathrm{S}\right)\end{array}$ & $\begin{array}{l}\text { STAN- } \\
\text { DARD } \\
\text { DEVIA- } \\
\text { TION } \\
\left(\mathrm{FT}^{3} / \mathrm{S}\right)\end{array}$ & $\begin{array}{l}\text { COEFFI- } \\
\text { CIENT OF } \\
\text { VARI- } \\
\text { ATION }\end{array}$ & $\begin{array}{c}\text { PERCENT } \\
\text { OF } \\
\text { ANNUAL } \\
\text { RUNOF F }\end{array}$ \\
\hline \multicolumn{7}{|l|}{-ー-ーーー-ー- } \\
\hline $\begin{array}{l}\text { OCTOBER } \\
\text { NOVEMBER }\end{array}$ & $\begin{array}{l}0.0 \\
3.5\end{array}$ & $\begin{array}{l}3386 \\
2701\end{array}$ & $\begin{array}{l}447 \\
687\end{array}$ & $\begin{array}{l}700 \\
681\end{array}$ & $\begin{array}{r}1.57 \\
.99\end{array}$ & 3.3 \\
\hline DECEMBER & 158 & 1596 & 800 & 360 & .45 & 6.0 \\
\hline JANUARY & 372 & 1118 & 702 & 144 & .21 & 5.2 \\
\hline FEBRUARY & 509 & 1971 & 880 & 331 & .38 & 6.5 \\
\hline MARCH & 112 & 2087 & 824 & 582 & .71 & 6.1 \\
\hline APR IL & 4.5 & 7615 & 1515 & 1761 & 1.16 & 11.3 \\
\hline MAY & 30 & 16370 & 3399 & 3971 & 1.17 & 25.3 \\
\hline JUNE & 6.8 & 10020 & 2292 & 2728 & 1.19 & 17.1 \\
\hline JULY & 3.2 & 4084 & 818 & 1127 & 1.38 & 6.1 \\
\hline AUGUST & 5.0 & 4055 & 661 & 799 & 1.21 & 4.9 \\
\hline SEPTEMBER & 0.0 & 1715 & 416 & 489 & 1.18 & 3.1 \\
\hline AN NUAL & 248 & 3354 & 1121 & 843 & .75 & 100 \\
\hline
\end{tabular}

\begin{tabular}{|c|c|c|c|c|c|c|}
\hline \multirow{2}{*}{$\begin{array}{l}\text { PERIOD } \\
\text { (CON- } \\
\text { SECU- } \\
\text { TIVE } \\
\text { DAYS) }\end{array}$} & \multicolumn{6}{|c|}{$\begin{array}{l}\text { DISCHARGE, IN FT3/S, FOR INDICATED RECURRENCE } \\
\text { INTERVAL, IN YEARS, AND ANNUAL NON- } \\
\text { EXCEEDANCE PROBABILITY, IN PERCENT }\end{array}$} \\
\hline & $\begin{array}{c}2 \\
508\end{array}$ & $\begin{array}{c}5 \\
208\end{array}$ & $\begin{array}{l}10 \\
108\end{array}$ & $\begin{array}{l}20 \\
58\end{array}$ & $\begin{array}{l}50 \\
28\end{array}$ & $\begin{array}{r}100 \\
18\end{array}$ \\
\hline $\begin{array}{r}1 \\
3 \\
7 \\
14 \\
30 \\
60 \\
90 \\
120 \\
183\end{array}$ & $\begin{array}{r}1.7 \\
2.1 \\
3.4 \\
7.7 \\
31 \\
102 \\
183 \\
241 \\
380\end{array}$ & $\begin{array}{r}0.0 \\
0.0 \\
0.0 \\
0.0 \\
1.4 \\
8.6 \\
32 \\
88 \\
173\end{array}$ & $\begin{array}{r}0.0 \\
0.0 \\
0.0 \\
0.0 \\
. .1 \\
1.2 \\
10 \\
48 \\
108\end{array}$ & $\begin{array}{r}0.0 \\
0.0 \\
0.0 \\
0.0 \\
0.0 \\
.1 \\
3.4 \\
29 \\
71\end{array}$ & $\begin{array}{l}0.0 \\
0.0 \\
0.0 \\
0.0 \\
0.0 \\
0.0 \\
15 \\
.8 \\
43\end{array}$ & - \\
\hline
\end{tabular}

MAGNITUDE AND PROBABILITY OF ANNUAL HIGH FLOW BASED ON PERIOD OF RECORD 1937-62

\begin{tabular}{|c|c|c|c|c|c|c|}
\hline \multirow{2}{*}{$\begin{array}{l}\text { PERIOD } \\
\text { (CON- } \\
\text { SECU- } \\
\text { TIVE } \\
\text { DAYS) }\end{array}$} & \multicolumn{6}{|c|}{$\begin{array}{l}\text { DISCHARGE, IN FT } 3 / \mathrm{S}, \text { FOR INDICATED RECURRENCE } \\
\text { INTERVAL, IN YEARS, AND ANNUAL } \\
\text { EXCEEDANCE PROBABILITY, IN PERCENT }\end{array}$} \\
\hline & $\begin{array}{c}2 \\
508\end{array}$ & $\begin{array}{c}5 \\
208\end{array}$ & $\begin{array}{l}10 \\
108\end{array}$ & $\begin{array}{l}25 \\
48\end{array}$ & $\begin{array}{l}50 \\
28\end{array}$ & $\begin{array}{r}100 \\
18\end{array}$ \\
\hline $\begin{array}{r}1 \\
3 \\
7 \\
15 \\
30 \\
60 \\
90\end{array}$ & $\begin{array}{l}5840 \\
4970 \\
4120 \\
3540 \\
2960 \\
2320 \\
1920\end{array}$ & $\begin{array}{r}10600 \\
9570 \\
8530 \\
7600 \\
6580 \\
5170 \\
4130\end{array}$ & $\begin{array}{r}14200 \\
13200 \\
12300 \\
11300 \\
9980 \\
7920 \\
6240\end{array}$ & $\begin{array}{r}19300 \\
18400 \\
18000 \\
17000 \\
15500 \\
12600 \\
9800\end{array}$ & $\begin{array}{l}23400 \\
22500 \\
22900 \\
22200 \\
20700 \\
17000 \\
13200\end{array}$ & $\begin{array}{l}-- \\
-- \\
-- \\
-- \\
-- \\
-- \\
--\end{array}$ \\
\hline
\end{tabular}

DURATION TABLE OF DAILY MEAN FLOW FOR PERIOD OF RECORD 1937-62

DISCHARGE, IN FT $3 / S$, THAT WAS EQUALED OR EXCEEDED FOR INDICATED PERCENT OF TIME

\begin{tabular}{|c|c|c|c|c|c|c|c|c|c|c|c|c|c|c|}
\hline 58 & 108 & 158 & 208 & 258 & 308 & 408 & 508 & 608 & 708 & 758 & 808 & 858 & 908 & 958 \\
\hline 4800 & 2660 & 1890 & 1400 & 1110 & 941 & 721 & 586 & 414 & 227 & 138 & 63 & 19 & 4.4 & 1.0 \\
\hline
\end{tabular}


08358300 RIO GRANDE CONVEYANCE CHANNEL AT SAN MARCIAL, NM

(National stream-quality accounting network, surveillance network, and radiochemical network station)

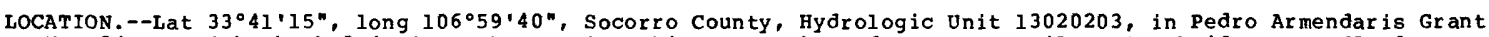
No. 34 , on right bank $0.4 \mathrm{mi}$ northwest of Atchison, Topeka and Santa Fe Railway Co. bridge over floodway channel, $1.0 \mathrm{mi}$ southwest of former site of San Marcial, $3.5 \mathrm{mi}$ downstream from railroad bridge near Tiffany siding, and $51 \mathrm{mi}$ downstream from heading at San Acacia.

PERIOD OF RECORD.--October 1958 to September 1959 , October 1969 to current year. Prior to October 1964 monthly discharge only published with record for Rio Grande at San Marcial (station 08358500).

GAGE.--Water-stage recorder. Datum of gage is $4,454.00 \mathrm{ft}$ above National Geodetic vertical Datum of 1929 (levels by U.S. Bureau of Reclamation). Prior to Apr. 29, 1958, at datum $4.19 \mathrm{ft}$ higher.

REMARKs.--Estimated daily discharges: Nov. 19-27, Dec. 12-25, Dec. 30 to Jan. 5, Jan. 9-21, and Aug. 21 to Sept.4. Water-discharge records good except for estimated daily discharges, which are poor. Original design and plan was for conveyance channel to carry all flows up to about $2,000 \mathrm{ft}^{3} / \mathrm{s}$. Conveyance channel is 1 of 2 channels (station 08358400) carrying flow in valley cross section. For combined monthly flow in acre-ft of this channel and floodway see tabulation below daily table for station 08358400 .

AVERAGE DISCHARGE. --10 years (water years 1953-62), $296 \mathrm{ft}^{3} / \mathrm{s}, 214,500$ acre-ft/yr.

EXTREMES FOR PERIOD OF RECORD (SINCE 1954).--Maximum daily discharge, 2,200 ft3/s, May 14, 1966; no flow at times.

STATISTICAL SUMMARIES

MEAN MONTHLY AND MEAN ANNUAL DISCHARGES 1953-62

\begin{tabular}{|c|c|c|c|c|c|c|}
\hline MONTH & $\begin{array}{l}\text { MINIMUM } \\
\left(F^{3} / S\right)\end{array}$ & $\begin{array}{l}\text { MAXIMUM } \\
\left(\mathrm{FT}^{3} / \mathrm{S}\right)\end{array}$ & $\begin{array}{l}\text { MEAN } \\
\left(\mathrm{FT}^{3} / \mathrm{S}\right)\end{array}$ & $\begin{array}{l}\text { STAN- } \\
\text { DARD } \\
\text { DEVIA- } \\
\text { TION } \\
\left(\mathrm{FT}^{3} / \mathrm{S}\right)\end{array}$ & $\begin{array}{l}\text { COEFFI- } \\
\text { CIENT OF } \\
\text { VARI- } \\
\text { ATION }\end{array}$ & $\begin{array}{c}\text { PERCENT } \\
\text { OF } \\
\text { ANNUAL } \\
\text { RUNOFF }\end{array}$ \\
\hline $\begin{array}{l}\text { OCTOBER } \\
\text { NOVEMBER } \\
\text { DECEMBER } \\
\text { JANUARY } \\
\text { FEB RUARY } \\
\text { MARCH } \\
\text { APRIL } \\
\text { MAY } \\
\text { JUNE } \\
\text { JULY } \\
\text { AUGUST } \\
\text { SEPTEMBER }\end{array}$ & $\begin{array}{r}4.6 \\
6.5 \\
.4 \\
.4 \\
.4 \\
.4 \\
.6 \\
.4 \\
.3 \\
1.9 \\
25 \\
5.5\end{array}$ & $\begin{array}{r}271 \\
1430 \\
1250 \\
780 \\
1243 \\
771 \\
1149 \\
1172 \\
1409 \\
1074 \\
643 \\
431\end{array}$ & $\begin{array}{l}117 \\
305 \\
314 \\
321 \\
386 \\
348 \\
373 \\
505 \\
328 \\
206 \\
207 \\
148\end{array}$ & $\begin{array}{l}91 \\
445 \\
381 \\
265 \\
369 \\
264 \\
361 \\
424 \\
441 \\
314 \\
186 \\
147\end{array}$ & $\begin{array}{r}.78 \\
1.46 \\
1.21 \\
.83 \\
.96 \\
.76 \\
.97 \\
.84 \\
1.34 \\
1.52 \\
.90 \\
.99\end{array}$ & $\begin{array}{r}3.3 \\
8.6 \\
8.8 \\
9.0 \\
10.8 \\
9.8 \\
10.5 \\
14.2 \\
9.2 \\
5.8 \\
5.8 \\
4.2\end{array}$ \\
\hline ANNUAL & 43 & 738 & 296 & 211 & .71 & 100 \\
\hline
\end{tabular}

MAGNITUDE AND PROBABILITY OF ANNUAL LOW FLON BASED ON PERIOD OF RECORD $1954-62$

\begin{tabular}{|c|c|c|c|c|c|c|}
\hline $\begin{array}{l}\text { PERIOD } \\
\text { (CON- }\end{array}$ & \multicolumn{6}{|c|}{$\begin{array}{l}\text { DISCHARGE, IN FT'/S, FOR INDICATED RECURRENCE } \\
\text { INTERVAL, IN YEARS, AND ANNUAL NON- } \\
\text { EXCEEDANCE PROBABILITY, IN PERCENT }\end{array}$} \\
\hline $\begin{array}{l}\text { SECU- } \\
\text { TIVE } \\
\text { DAYS) }\end{array}$ & $\begin{array}{c}2 \\
508\end{array}$ & $\begin{array}{c}5 \\
208\end{array}$ & $\begin{array}{l}10 \\
108\end{array}$ & $\begin{array}{l}20 \\
58\end{array}$ & $\begin{array}{l}50 \\
28\end{array}$ & $\begin{array}{r}100 \\
18\end{array}$ \\
\hline $\begin{array}{r}1 \\
3 \\
7 \\
14 \\
30 \\
60 \\
90 \\
120 \\
183\end{array}$ & $\begin{array}{r}1.1 \\
1.3 \\
1.2 \\
1.5 \\
3.2 \\
8.8 \\
24 \\
30 \\
97\end{array}$ & $\begin{array}{r}0.0 \\
0.0 \\
0.0 \\
.1 \\
.5 \\
1.0 \\
3.1 \\
9.6 \\
39\end{array}$ & $\begin{array}{r}0.0 \\
0.0 \\
0.0 \\
0.0 \\
.3 \\
.9 \\
2.9\end{array}$ & $\begin{array}{r}0.0 \\
0.0 \\
0.0 \\
0.0 \\
.1 \\
.1 \\
.3 \\
1 . .7\end{array}$ & $\begin{array}{l}-- \\
-- \\
-- \\
-- \\
-- \\
-- \\
--\end{array}$ & $\begin{array}{l}-- \\
-- \\
-- \\
-- \\
-- \\
-- \\
-- \\
--\end{array}$ \\
\hline
\end{tabular}

MAGNITUDE AND PROBABILITY OF ANNUAL HIGH FLOW BASED ON PERIOD OF RECORD 1953-62

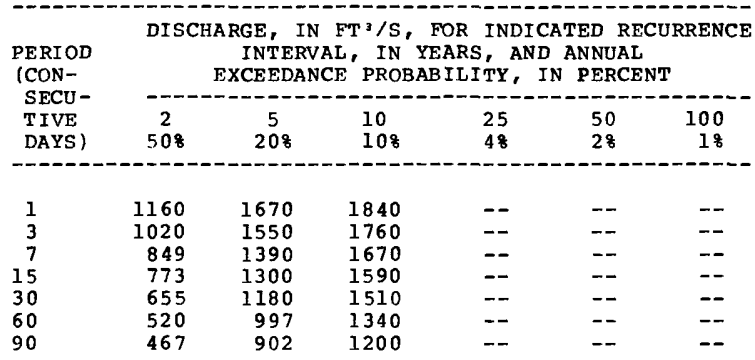

DURATION TABLE OF DAILY MEAN FLOW FOR PERIOD OF RECORD 1953-62 DISCHARGE, IN FT' $3 / \mathrm{S}$, THAT WAS EQUALED OR EXCEEDED FOR INDICATED PERCENT OF TIME

\begin{tabular}{|c|c|c|c|c|c|c|c|c|c|c|c|c|c|c|}
\hline 58 & 108 & 158 & 208 & $25 z$ & 308 & $40 \%$ & 508 & 608 & 708 & 758 & 808 & $85 \%$ & 908 & 958 \\
\hline 1130 & 824 & 643 & 519 & 433 & 354 & 247 & 171 & 90 & 45 & 28 & 17 & 7.6 & 1.8 & .4 \\
\hline
\end{tabular}


RIO GRANDE BASIN

08358300 RIO GRANDE CONVEYANCE CHANNEL AT SAN MARCIAL, MM--Continued STATISTICAL SUMAARIES (PERIOD BEFORE REGULATION OF COCHITI DAM AND AFTER COMPLETION OF ABIQUIU DAM)

MEAN MONTHLY AND MEAN ANNUAL DISCHARGES 1964-73

\begin{tabular}{|c|c|c|c|c|c|c|}
\hline MONTH & $\begin{array}{l}\text { MINIMUM } \\
\left(\mathrm{FT}^{3} / \mathrm{S}\right)\end{array}$ & $\begin{array}{c}\text { MAX XIMUM } \\
\left(\mathrm{FT}^{3} / \mathrm{S}\right)\end{array}$ & $\begin{array}{l}\text { MEAN } \\
\left(\mathrm{FT}^{3} / \mathrm{S}\right)\end{array}$ & $\begin{array}{l}\text { STAN- } \\
\text { DARD } \\
\text { DEVIA- } \\
\text { TION } \\
(\text { FT } 3 / 5)\end{array}$ & $\begin{array}{l}\text { COEFFI- } \\
\text { CIENT OF } \\
\text { VARI- } \\
\text { ATION }\end{array}$ & $\begin{array}{c}\text { PERCENT } \\
\text { OF } \\
\text { ANNUAL } \\
\text { RUNOFF }\end{array}$ \\
\hline \multicolumn{7}{|c|}{ 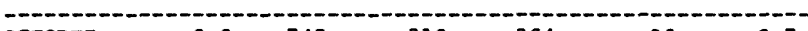 } \\
\hline OCTOBER & 0.0 & 745 & 310 & 304 & .98 & 3.7 \\
\hline NOVEMBER & 214 & 1729 & 1037 & 531 & .51 & 12.2 \\
\hline DECEMBER & 291 & 1880 & 911 & 495 & .54 & 10.7 \\
\hline JANUARY & 375 & 1071 & 777 & 206 & .27 & 9.2 \\
\hline FEB RUARY & 467 & 1079 & 819 & 181 & .22 & 9.7 \\
\hline MARCH & 255 & 1394 & 732 & 342 & .47 & 8.6 \\
\hline APRIL & 77 & 1679 & 792 & 582 & .73 & 9.3 \\
\hline MAY & 37 & 1782 & 989 & 696 & .70 & 11.7 \\
\hline JUNE & 24 & 1652 & 816 & 639 & .78 & 9.6 \\
\hline JULY & 38 & 1690 & 459 & 570 & 1.24 & 5.4 \\
\hline AUGUST & 145 & 986 & 458 & 281 & .61 & 5.4 \\
\hline SEPTEMBER & 29 & 730 & 383 & 306 & .80 & 4.5 \\
\hline ANNUAL & 223 & 1137 & 706 & 275 & .39 & 100 \\
\hline
\end{tabular}

MAGNTTUDE AND PROBABILITY OF ANNUAL LOW FLOW BASED ON PERIOD OF RECORD 1965-73

\begin{tabular}{|c|c|c|c|c|c|c|}
\hline \multirow{2}{*}{$\begin{array}{l}\text { PERIOD } \\
\text { (CON- } \\
\text { SECU- } \\
\text { TIVE } \\
\text { DAYS) }\end{array}$} & \multicolumn{6}{|c|}{$\begin{array}{l}\text { DISCHARGE, IN FT'/S, FOR INDICATED RECURRENCE } \\
\text { INTERVAL, IN YEARS, AND ANNUAL NON- } \\
\text { EXCEEDANCE PROBABILITY, IN PERCENT }\end{array}$} \\
\hline & $\begin{array}{c}2 \\
508\end{array}$ & $\begin{array}{c}5 \\
208\end{array}$ & $\begin{array}{l}10 \\
108\end{array}$ & $\begin{array}{l}20 \\
58\end{array}$ & $\begin{array}{l}50 \\
28\end{array}$ & $\begin{array}{r}100 \\
18\end{array}$ \\
\hline $\begin{array}{r}1 \\
3 \\
7 \\
14 \\
30 \\
60 \\
90 \\
120 \\
183\end{array}$ & $\begin{array}{r}13 \\
13 \\
15 \\
18 \\
16 \\
57 \\
123 \\
156 \\
358\end{array}$ & $\begin{array}{r}0 . \\
0 . \\
0 . \\
0 . \\
17 \\
47 \\
82 \\
196\end{array}$ & $\begin{array}{r}0.0 \\
0.0 \\
0.0 \\
0.0 \\
0.0 \\
9.1 \\
29 \\
62 \\
144\end{array}$ & $\begin{array}{r}0.0 \\
0.0 \\
0.0 \\
0.0 \\
0.0 \\
5.6 \\
19 \\
50 \\
111\end{array}$ & $\begin{array}{l}-- \\
\overline{--} \\
-- \\
-- \\
-- \\
--\end{array}$ & $\begin{array}{l}-- \\
=- \\
-- \\
=- \\
\overline{-} \\
--\end{array}$ \\
\hline
\end{tabular}

MAGNITUDE AND PROBABILITY OF ANNUAL HIGA FLOW BASED ON PERIOD OF RECORD 1964-73

\begin{tabular}{|c|c|c|c|c|c|c|}
\hline \multirow{2}{*}{$\begin{array}{l}\text { PERIOD } \\
\text { (CON- } \\
\text { SECU- } \\
\text { TIVE } \\
\text { DAYS) }\end{array}$} & \multicolumn{6}{|c|}{$\begin{array}{l}\text { DISCHARGE, IN FT'/S, FOR INDICATED RECURRENCE } \\
\text { INTERVAL, IN YEARS, AND ANNUAL } \\
\text { EXCEEDANCE PROBABILITY, IN PERCENT }\end{array}$} \\
\hline & 508 & $\begin{array}{c}5 \\
208\end{array}$ & 10 & $\begin{array}{l}25 \\
48\end{array}$ & $\begin{array}{l}50 \\
28\end{array}$ & $\begin{array}{r}100 \\
18\end{array}$ \\
\hline $\begin{array}{r}1 \\
3 \\
7 \\
15 \\
30 \\
60 \\
90\end{array}$ & $\begin{array}{l}1980 \\
1920 \\
1720 \\
1660 \\
1560 \\
1370 \\
1260\end{array}$ & $\begin{array}{l}2100 \\
2050 \\
1940 \\
1900 \\
1900 \\
1770 \\
1640\end{array}$ & $\begin{array}{l}2130 \\
2080 \\
2020 \\
1980 \\
2010 \\
1920 \\
1790\end{array}$ & $\begin{array}{l}-- \\
-- \\
-- \\
-- \\
--\end{array}$ & $\begin{array}{l}=- \\
=- \\
-- \\
--\end{array}$ & $\begin{array}{l}-- \\
-- \\
-- \\
-- \\
--\end{array}$ \\
\hline
\end{tabular}

DURATION TABLE OF DAILY MEAN FLOW FOR PERIOD OF RECORD 1964-73 DISCHARGE, IN FT $3 / 5$, THAT WAS EQUALED OR EXCEEDED FOR INDICATED PERCENT OF TIME

\begin{tabular}{|c|c|c|c|c|c|c|c|c|c|c|c|c|c|c|}
\hline 58 & 108 & 158 & 208 & 258 & 308 & 408 & 508 & 608 & 708 & 758 & 808 & 858 & 908 & 958 \\
\hline 1950 & 1690 & 1470 & 1270 & 1110 & 956 & 776 & 630 & 439 & 281 & 196 & 135 & 78 & 32 & \\
\hline
\end{tabular}


RIO GRANDE BASIN

08358300 RIO GRANDE CONVEYANCE CHANNEL AT SAN MARCIAL, NM--Continued STATISTICAL SUMMARIES (PERIOD AFTER COMPLETION OF COCHITI DAM)

MEAN MONTHLY AND MEAN ANNUAL DISCHARGES 1974-85

\begin{tabular}{|c|c|c|c|c|c|c|}
\hline MONTH & $\begin{array}{l}\text { MINTMUM } \\
\left(F T^{3} / S\right)\end{array}$ & $\begin{array}{l}\text { MAXIMUM } \\
(F T 3 / S)\end{array}$ & $\begin{array}{l}\text { MEAN } \\
\left(F T^{2} / S\right)\end{array}$ & $\begin{array}{l}\text { STAN- } \\
\text { DARD } \\
\text { DEVIA- } \\
\text { TION } \\
(F T, / S)\end{array}$ & $\begin{array}{l}\text { COEFFI- } \\
\text { CIENT OF } \\
\text { VARI- } \\
\text { ATION }\end{array}$ & $\begin{array}{c}\text { PERCENT } \\
\text { OF } \\
\text { ANNUAL } \\
\text { RUNOFF }\end{array}$ \\
\hline $\begin{array}{l}\text { OCTOBER } \\
\text { NOVEMBER } \\
\text { DECEMBER } \\
\text { JANUARY } \\
\text { FEBRUARY } \\
\text { MARCH } \\
\text { APRIL } \\
\text { MAY } \\
\text { JUNE } \\
\text { JULY } \\
\text { AUGUST } \\
\text { SEPTEMBER }\end{array}$ & $\begin{array}{l}0.0 \\
0.0 \\
0.0 \\
0.0 \\
0.0 \\
0.0 \\
0.0 \\
0.0 \\
0.0 \\
0.0 \\
0.0 \\
0.0\end{array}$ & $\begin{array}{r}759 \\
1400 \\
1658 \\
1558 \\
1112 \\
1035 \\
893 \\
1376 \\
1632 \\
1108 \\
440 \\
249\end{array}$ & $\begin{array}{r}151 \\
339 \\
437 \\
319 \\
267 \\
252 \\
236 \\
287 \\
291 \\
222 \\
109 \\
86\end{array}$ & $\begin{array}{r}231 \\
520 \\
630 \\
563 \\
454 \\
383 \\
328 \\
405 \\
480 \\
337 \\
134 \\
99\end{array}$ & $\begin{array}{l}1.53 \\
1.53 \\
1.44 \\
1.76 \\
1.70 \\
1.52 \\
1.39 \\
1.41 \\
1.65 \\
1.52 \\
1.23 \\
1.15\end{array}$ & $\begin{array}{r}5.0 \\
11.3 \\
14.6 \\
10.6 \\
8.9 \\
8.4 \\
7.9 \\
9.6 \\
9.7 \\
7.4 \\
3.6 \\
2.9\end{array}$ \\
\hline ANNUAL & 1.4 & 819 & 250 & 291 & 1.16 & 100 \\
\hline
\end{tabular}

MAGNITUDE AND PROBABILITY OF ANNUAL LOW FLOW BASED ON PERIOD OF RECORD 1975-85

\begin{tabular}{|c|c|c|c|c|c|c|}
\hline \multirow{2}{*}{$\begin{array}{l}\text { PERIOD } \\
\text { (CON- } \\
\text { SECU- } \\
\text { TIVE }\end{array}$} & \multicolumn{6}{|c|}{$\begin{array}{l}\text { DISCEARGE, IN FT'/S, FOR INDICATED RECURRENCE } \\
\text { INTERVAL, IN YEARS, AND ANNUAL NON- } \\
\text { EXCEEDANCE PROBABIL ITY, IN PERCENT }\end{array}$} \\
\hline & 2 & 5 & 10 & 20 & 50 & 100 \\
\hline DAYS) & 508 & 208 & 108 & 58 & 28 & 18 \\
\hline $\begin{array}{r}1 \\
3 \\
7 \\
14 \\
30 \\
60 \\
90 \\
120 \\
183\end{array}$ & $\begin{array}{r}0.0 \\
0.0 \\
.1 \\
.1 \\
.2 \\
.6 \\
1.1 \\
2.3 \\
7.6\end{array}$ & $\begin{array}{l}0.0 \\
0.0 \\
0.0 \\
0.0 \\
0.0 \\
0.0 \\
0.0 \\
0.0 \\
0.0\end{array}$ & $\begin{array}{l}0 . \\
0 . \\
0 . \\
0 . \\
0 . \\
0 . \\
0 . \\
0 . \\
0 .\end{array}$ & $\begin{array}{l}0.0 \\
0.0 \\
0.0 \\
0.0 \\
0.0 \\
0.0 \\
0.0 \\
0.0 \\
0.0\end{array}$ & $\begin{array}{l}-- \\
-- \\
-- \\
-- \\
-- \\
-- \\
--\end{array}$ & $\begin{array}{l}=- \\
=- \\
=- \\
=- \\
=- \\
=\end{array}$ \\
\hline
\end{tabular}

MAGNITUDE AND PROBABILITY OF ANNUAL HIGH FLON BASED ON PERIOD OF RECORD 1974-85

\begin{tabular}{|c|c|c|c|c|c|c|}
\hline \multirow{2}{*}{$\begin{array}{l}\text { PERIOD } \\
\text { (CON- } \\
\text { SECU- } \\
\text { TIVE } \\
\text { DAYS) }\end{array}$} & \multicolumn{6}{|c|}{$\begin{array}{l}\text { DISCHARGE, IN FT' }{ }^{3} \text { S, FOR INDICATED RECURRENCE } \\
\text { INTERVAL, IN YEARS, AND ANNUAL } \\
\text { EXCEEDANCE' PROBABILITY, IN PERCENT }\end{array}$} \\
\hline & $\begin{array}{c}2 \\
508\end{array}$ & $\begin{array}{c}5 \\
208\end{array}$ & $\begin{array}{l}10 \\
108\end{array}$ & $\begin{array}{l}25 \\
48\end{array}$ & $\begin{array}{l}50 \\
28\end{array}$ & $\begin{array}{r}100 \\
18\end{array}$ \\
\hline $\begin{array}{r}1 \\
3 \\
7 \\
15 \\
30 \\
60 \\
90\end{array}$ & $\begin{array}{l}398 \\
386 \\
374 \\
346 \\
313 \\
266 \\
224\end{array}$ & $\begin{array}{l}1400 \\
1360 \\
1330 \\
1290 \\
1270 \\
1170 \\
1020\end{array}$ & $\begin{array}{l}2510 \\
2460 \\
2400 \\
2350 \\
2350 \\
2180 \\
1930\end{array}$ & $\begin{array}{l}=- \\
=- \\
=- \\
=- \\
=-\end{array}$ & $\begin{array}{l}=- \\
=- \\
=- \\
=- \\
=\end{array}$ & $\begin{array}{l}-- \\
=- \\
=- \\
=- \\
=- \\
--\end{array}$ \\
\hline
\end{tabular}

DURATION TABLE OF DAILY MEAN FLOW FOR PERIOD OF RECORD 1974-85 DISCBARGE, IN FT/S, THAT WAS EQUALED OR EXCEEDED FOR INDICATED PERCENT OF TIME

\begin{tabular}{|c|c|c|c|c|c|c|c|c|c|c|c|c|c|c|}
\hline 58 & 108 & 158 & 208 & 258 & 308 & 408 & 508 & 608 & 708 & 758 & 808 & 858 & 908 & 958 \\
\hline 1390 & 951 & 672 & 375 & 254 & 199 & 109 & 19 & 2.9 & .4 &. & 0.0 & 0.0 & 0.0 & 0.0 \\
\hline
\end{tabular}




\section{RIO GRANDE BASIN}

08358400 RIO GRANDE FLOODWAY AT SAN MARCIAL, NM

(National stream-quality accounting network, surveillance network, and radiochemical network station)

LOCATION.--Lat 33040'50", long 106 59.30", Socorro County, Hydrologic Unit 13020203, in Pedro Armendaris Grant No. 33 , on pier of the Atchison, Topeka, and Santa Fe Railway Co. bridge, 1.1 mi downstream from former site of San Marcial, $18.5 \mathrm{mi}$ southwest of San Antonio, and at mile 1,425.2.

DRAINAGE AREA.--27,700 $\mathrm{mi}^{2}$, approximately, including 2,940 $\mathrm{mi}^{2}$ in closed basin in San Luis Valley, co.

PERIOD OF RECORD.--October 1964 to current year. Records collected at this site January 1895 to September 1964 represented total flow of the river and were published as Rio Grande at San Marcial (station 08358500 ). Records of daily discharge for floodway only April 1950 to September 1964 are available in files of district office.

GAGE.--Water-stage recorder. Datum of gage is 4,455.19 ft above National Geodetic Vertical Datum of 1929 .

REMARRs.--Floodway is 1 of 2 channels (station 08358300 ) carrying flow in valley cross section. Prior to 1950 all flow was in floodway channel. Normal plan is for floodway to carry flow when capacity of conveyance channel (about 2,000 $\mathrm{ft}^{3} / \mathrm{s}$ ) is exceeded. Diversions for irrigation of about 775,000 acres upstream from station (includes about 13,800 acre-ft diverted from conveyance channel, as based on weekly measurements, data provided by U.S. Bureau of Reclamation).

AVERAGE DISCHARGE.--13 years (water years 1950-62), $533 \mathrm{ft} / \mathrm{s}, 386,200$ acre-ft/yr.

Total flow of river.--90 years (water years 1895-1985), 1,248 $\mathrm{ft}^{3} / \mathrm{s}, 904,200$ acre-ft/yr.

EXTREMES FOR PERIOD OF RECORD.--Maximum discharge, since January 1895 about $50,000 \mathrm{ft} / \mathrm{s}$ Oct. 11 , 1904 ; no flow at times.

STATISTICAL SUMMARIES (PERIOD BEFORE REGULATION OF ABIOUIU DAM)

MEAN MONTHLY AND MEAN ANNUAL DIscharges 1950-62

\begin{tabular}{|c|c|c|c|c|c|c|}
\hline MONTH & $\begin{array}{c}\text { MINIMUM } \\
\left(\mathrm{FT}^{3} / \mathrm{S}\right)\end{array}$ & $\begin{array}{l}\text { MAXI MUM } \\
(\mathrm{FT} \cdot / \mathrm{S})\end{array}$ & $\begin{array}{l}\text { MEAN } \\
(\text { FT }: / S)\end{array}$ & $\begin{array}{l}\text { STAN- } \\
\text { DARD } \\
\text { DEVIA- } \\
\text { TION } \\
(\text { FT 3/S) }\end{array}$ & $\begin{array}{l}\text { COEFFI- } \\
\text { CIENT OF } \\
\text { VARI- } \\
\text { ATION }\end{array}$ & $\begin{array}{c}\text { PERCENT } \\
\text { OF } \\
\text { ANNUAL } \\
\text { RUNOFF }\end{array}$ \\
\hline $\begin{array}{l}\text { OCTOBER } \\
\text { NOVEMBER } \\
\text { DECEMBER } \\
\text { JANUARY } \\
\text { FEB RUARY } \\
\text { MARCH } \\
\text { APRIL } \\
\text { MAY } \\
\text { JUNE } \\
\text { JULY } \\
\text { AUGUST } \\
\text { SEPTEMBER }\end{array}$ & $\begin{array}{l}0.0 \\
0.0 \\
0.0 \\
0.0 \\
0.0 \\
0.0 \\
0.0 \\
0.0 \\
0.0 \\
0.0 \\
0.0 \\
0.0\end{array}$ & $\begin{array}{l}540 \\
1604 \\
1795 \\
1347 \\
1168 \\
1467 \\
4764 \\
5323 \\
5338 \\
4655 \\
1831 \\
1152\end{array}$ & $\begin{array}{r}105 \\
266 \\
310 \\
280 \\
292 \\
328 \\
719 \\
1679 \\
1365 \\
547 \\
292 \\
203\end{array}$ & $\begin{array}{r}158 \\
442 \\
496 \\
412 \\
402 \\
462 \\
1120 \\
1821 \\
1722 \\
1029 \\
424 \\
318\end{array}$ & $\begin{array}{l}1.50 \\
1.66 \\
1.60 \\
1.47 \\
1.38 \\
1.41 \\
1.56 \\
1.08 \\
1.26 \\
1.88 \\
1.45 \\
1.57\end{array}$ & $\begin{array}{r}1.6 \\
4.2 \\
4.9 \\
4.4 \\
4.6 \\
5.1 \\
11.3 \\
26.3 \\
21.4 \\
8.6 \\
4.6 \\
3.2\end{array}$ \\
\hline NNUAL & 1.2 & 1956 & 533 & 576 & 1.08 & 100 \\
\hline
\end{tabular}

MAGNITUDE AND PROBABILITY OF ANNUAL LOW FLOW BASED ON PERIOD OF RECORD 1951-62

\begin{tabular}{|c|c|c|c|c|c|c|}
\hline \multirow{2}{*}{$\begin{array}{l}\text { PERIOD } \\
\text { (CON- } \\
\text { SECU- } \\
\text { TIVE } \\
\text { DAYS) }\end{array}$} & \multicolumn{6}{|c|}{$\begin{array}{l}\text { DISCHARGE, IN FT'/S, FOR INDICATED RECURRENCE } \\
\text { INTERVAL, IN YEARS, AND ANNUAL NON- } \\
\text { EXCEEDANCE PROBABILITY, IN PERCENT }\end{array}$} \\
\hline & $\begin{array}{c}2 \\
508\end{array}$ & $\begin{array}{c}5 \\
208\end{array}$ & $\begin{array}{l}10 \\
108\end{array}$ & $\begin{array}{l}20 \\
58\end{array}$ & $\begin{array}{l}50 \\
28\end{array}$ & $\begin{array}{r}100 \\
18\end{array}$ \\
\hline $\begin{array}{r}1 \\
3 \\
7 \\
14 \\
30 \\
60 \\
90 \\
120 \\
183\end{array}$ & $\begin{array}{r}0.0 \\
0.0 \\
0.0 \\
0.0 \\
0.0 \\
0.0 \\
0.0 \\
9.3 \\
39\end{array}$ & $\begin{array}{l}0.0 \\
0.0 \\
0.0 \\
0.0 \\
0.0 \\
0.0 \\
0.0 \\
0.0 \\
2.1\end{array}$ & $\begin{array}{l}0.0 \\
0.0 \\
0.0 \\
0.0 \\
0.0 \\
0.0 \\
0.0 \\
0.0 \\
0.0\end{array}$ & $\begin{array}{l}0.0 \\
0.0 \\
0.0 \\
0.0 \\
0.0 \\
0.0 \\
0.0 \\
0.0 \\
0.0\end{array}$ & $\begin{array}{l}0.0 \\
0.0 \\
0.0 \\
0.0 \\
0.0 \\
0.0 \\
0.0 \\
0.0 \\
0.0\end{array}$ & \\
\hline
\end{tabular}

MAGNITUDE AND PROBABILITY OF ANNUAL HIGE FLON BASED ON PERIOD OF RECORD $1960-85$

\begin{tabular}{|c|c|c|c|c|c|c|}
\hline \multirow{2}{*}{$\begin{array}{l}\text { PERIOD } \\
\text { (CON- } \\
\text { SECU- } \\
\text { TIVE } \\
\text { DAYS) }\end{array}$} & \multicolumn{6}{|c|}{$\begin{array}{l}\text { DISCHARGE, IN FT'/S, FOR INDICATED RECURRENCE } \\
\text { INTERVAL, IN YEARS, AND ANNUAL } \\
\text { EXCEEDANCE PROBABILITY, IN PERCENT }\end{array}$} \\
\hline & $\begin{array}{c}2 \\
508\end{array}$ & $\begin{array}{c}5 \\
208\end{array}$ & $\begin{array}{l}10 \\
108\end{array}$ & $\begin{array}{l}25 \\
48\end{array}$ & $\begin{array}{l}50 \\
28\end{array}$ & $\begin{array}{r}100 \\
18\end{array}$ \\
\hline $\begin{array}{r}1 \\
3 \\
7 \\
15 \\
30 \\
60 \\
90\end{array}$ & $\begin{array}{r}3100 \\
2770 \\
2260 \\
1790 \\
1410 \\
1120 \\
893\end{array}$ & $\begin{array}{l}6020 \\
5870 \\
5550 \\
5000 \\
4500 \\
2450 \\
2000\end{array}$ & $\begin{array}{l}7530 \\
7550 \\
7530 \\
7170 \\
6830 \\
3820 \\
3140\end{array}$ & $\begin{array}{l}8870 \\
9060 \\
9420 \\
9430 \\
9450 \\
6210 \\
5110\end{array}$ & $\begin{array}{r}9540 \\
9830 \\
10400 \\
10700 \\
11000 \\
8680 \\
7150\end{array}$ & $\begin{array}{l}=- \\
-- \\
=- \\
=- \\
--\end{array}$ \\
\hline
\end{tabular}

DURATION TABLE OF DAILY MEAN FLOW FOR PERIOD OF RECORD 1950-62 DISCHARGE, IN FT $3 / \mathrm{S}$, THAT WAS EOUALED OR EXCEEDED FOR INDICATED PERCENT OF TIME

\begin{tabular}{|c|c|c|c|c|c|c|c|c|c|c|c|c|c|c|}
\hline 58 & 108 & 158 & 208 & 258 & 308 & 408 & 508 & 608 & 708 & 758 & 808 & 858 & 908 & 958 \\
\hline 2440 & 1410 & 946 & 681 & 497 & 353 & 97 & 4.4 & .1 & .1 & .1 & 0.0 & 0.0 & 0.0 & \\
\hline
\end{tabular}


08358400 RIO GRANDE FLOODWAY AT SAN MARCIAL, NM--Continued

STATISTICAL SUMMARIES (PERIOD BEFORE REGULATION OF COCHITI DAM AND AFTER COMPLETION OF ABIQUIU DAM)

MEAN MONTHLY AND MEAN ANNUAL DISCHARGES 1964-73

\begin{tabular}{|c|c|c|c|c|c|c|}
\hline MONTH & $\begin{array}{l}\text { MINIMUM } \\
\left(\mathrm{FT}^{3} / \mathrm{S}\right)\end{array}$ & $\begin{array}{l}\text { MAXIMUM } \\
\left(\mathrm{FT}^{\prime} \cdot / \mathrm{S}\right)\end{array}$ & $\begin{array}{l}\text { MEAN } \\
\left(\mathrm{FT}^{3} / \mathrm{S}\right)\end{array}$ & $\begin{array}{l}\text { STAN- } \\
\text { DARD } \\
\text { DEVIA- } \\
\text { TION } \\
\text { (FT'/S) }\end{array}$ & $\begin{array}{l}\text { COEFFI- } \\
\text { CIENT OF } \\
\text { VARI- } \\
\text { ATION }\end{array}$ & $\begin{array}{c}\text { PERCENT } \\
\text { OF } \\
\text { ANNUAL } \\
\text { RUNOFF }\end{array}$ \\
\hline $\begin{array}{l}\text { OCTOBER } \\
\text { NOVEMBER } \\
\text { DECEMBER } \\
\text { JANUARY } \\
\text { FEBRUARY } \\
\text { MARCH } \\
\text { APRIL } \\
\text { MAY } \\
\text { JUNE } \\
\text { JULY } \\
\text { AUGUST } \\
\text { SEPTEMBER }\end{array}$ & $\begin{array}{l}0.0 \\
0.0 \\
0.0 \\
0.0 \\
0.0 \\
0.0 \\
0.0 \\
0.0 \\
0.0 \\
0.0 \\
.8 \\
0.0\end{array}$ & $\begin{array}{c}540 \\
101 \\
21 \\
0.0 \\
2.1 \\
76 \\
277 \\
3642 \\
2841 \\
833 \\
1831 \\
213\end{array}$ & $\begin{array}{r}87 \\
11 \\
2.1 \\
0.0 \\
.2 \\
8.5 \\
63 \\
708 \\
680 \\
180 \\
346 \\
65\end{array}$ & $\begin{array}{r}175 \\
32 \\
6.5 \\
0.0 \\
.7 \\
24 \\
103 \\
1181 \\
973 \\
334 \\
596 \\
77\end{array}$ & $\begin{array}{l}2.02 \\
2.90 \\
3.16 \\
3.19 \\
2.79 \\
1.64 \\
1.67 \\
1.43 \\
1.86 \\
1.72 \\
1.17\end{array}$ & $\begin{array}{r}4.0 \\
.5 \\
.1 \\
0.0 \\
0.0 \\
.4 \\
2.9 \\
32.9 \\
31.6 \\
8.4 \\
16.1 \\
3.0\end{array}$ \\
\hline NNUAL & 1.2 & 671 & 180 & 220 & 1.22 & 100 \\
\hline
\end{tabular}

MAGNITUDE AND PROBABILITY OF ANNUAL LOW FLOW BASED ON PERIOD OF RECORD 1965-73

\begin{tabular}{|c|c|c|c|c|c|c|}
\hline \multirow{2}{*}{$\begin{array}{l}\text { PERIOD } \\
\text { (CON- } \\
\text { SECU- } \\
\text { TIVE } \\
\text { DAYS) }\end{array}$} & \multicolumn{6}{|c|}{$\begin{array}{l}\text { DISCBARGE, IN FT'/S, FOR INDICATED RECURRENCE } \\
\text { INTERVAL, IN YEARS, AND ANNUAL NON- } \\
\text { EXCEEDANCE PROBABIL ITY, IN PERCENT }\end{array}$} \\
\hline & $\begin{array}{c}2 \\
508\end{array}$ & $\begin{array}{c}5 \\
208\end{array}$ & $\begin{array}{l}10 \\
108\end{array}$ & $\begin{array}{l}20 \\
58\end{array}$ & $\begin{array}{l}50 \\
28\end{array}$ & $\begin{array}{r}100 \\
18\end{array}$ \\
\hline $\begin{array}{r}1 \\
3 \\
7 \\
14 \\
30 \\
60 \\
90 \\
120 \\
183\end{array}$ & $\begin{array}{l}0.0 \\
0.0 \\
0.0 \\
0.0 \\
0.0 \\
0.0 \\
0.0 \\
0.0 \\
3.1\end{array}$ & $\begin{array}{l}0.0 \\
0.0 \\
0.0 \\
0.0 \\
0.0 \\
0.0 \\
0.0 \\
0.0 \\
0.0\end{array}$ & $\begin{array}{l}0.0 \\
0.0 \\
0.0 \\
0.0 \\
0.0 \\
0.0 \\
0.0 \\
0.0 \\
0.0\end{array}$ & $\begin{array}{l}0.0 \\
0.0 \\
0.0 \\
0.0 \\
0.0 \\
0.0 \\
0.0 \\
0.0 \\
0.0\end{array}$ & $\begin{array}{l}=- \\
=- \\
=- \\
=- \\
-- \\
-- \\
--\end{array}$ & \\
\hline
\end{tabular}

MAGNITUDE AND PROBABILITY OF ANNUAL HIGH FLOW BASED ON PERIOD OF RECORD 1964-73

\begin{tabular}{|c|c|c|c|c|c|c|}
\hline \multirow{2}{*}{$\begin{array}{l}\text { PERIOD } \\
\text { (CON- } \\
\text { SECU- } \\
\text { TIVE } \\
\text { DAYS) }\end{array}$} & \multicolumn{6}{|c|}{$\begin{array}{l}\text { DISCHARGE, IN FT }{ }^{3} / \mathrm{S} \text {, FOR INDICATED RECUF } \\
\text { INTERVAL, IN YEARS, AND ANNUAL } \\
\text { EXCEEDANCE PROBABILITY, IN PERCENT }\end{array}$} \\
\hline & $\begin{array}{c}2 \\
508\end{array}$ & $\begin{array}{c}5 \\
208\end{array}$ & $\begin{array}{l}10 \\
108\end{array}$ & $\begin{array}{l}25 \\
48\end{array}$ & $\begin{array}{l}50 \\
28\end{array}$ & $\begin{array}{r}100 \\
18\end{array}$ \\
\hline $\begin{array}{r}1 \\
3 \\
7 \\
15 \\
30 \\
60 \\
90\end{array}$ & $\begin{array}{r}2220 \\
1750 \\
1140 \\
754 \\
489 \\
287 \\
206\end{array}$ & $\begin{array}{l}5410 \\
5070 \\
4010 \\
3010 \\
2260 \\
1500 \\
1150\end{array}$ & $\begin{array}{l}7600 \\
7690 \\
6730 \\
5390 \\
4370 \\
3160 \\
2520\end{array}$ & $\begin{array}{l}-- \\
-- \\
-- \\
-\end{array}$ & $\begin{array}{l}-- \\
-- \\
-- \\
-- \\
--\end{array}$ & $\begin{array}{l}-- \\
-- \\
=- \\
--\end{array}$ \\
\hline
\end{tabular}

DURATION TABLE OF DAILY MEAN FLOW FOR PERIOD OF RECORD 1964-73

DISCHARGE, IN FT/S, THAT WAS EQUALED OR EXCEEDED FOR INDICATED PERCENT OF TIME

\begin{tabular}{|c|c|c|c|c|c|c|c|c|c|c|c|c|c|c|}
\hline 58 & 108 & 158 & 208 & 258 & 308 & 408 & 508 & 608 & 708 & 758 & 808 & 858 & 908 & 958 \\
\hline 1370 & 442 & 80 & 5.6 & .1 & .1 & .1 & 1 & .1 & 0.0 & 0.0 & 0.0 & 0.0 & 0.0 & 0 . \\
\hline
\end{tabular}


RIO GRANDE BASIN

08358400 RIO GRANDE FLOODWAY AT SAN MARCIAL, NM--Continued STATISTICAL SUMMARIES (PERIOD AFTER COMPLETION OF COCHITI DAM)

MEAN MONTHLY AND MEAN ANNUAL DISCHARGES

1974-85
MAGNITUDE AND PROBABILITY OF ANNUAL LOW FLOW BASED ON PERIOD OF RECORD 1975-85

\begin{tabular}{|c|c|c|c|c|c|c|}
\hline \multirow{2}{*}{$\begin{array}{l}\text { PERIOD } \\
\text { (CON- } \\
\text { SECU- } \\
\text { TIVE } \\
\text { DAYS) }\end{array}$} & \multicolumn{6}{|c|}{$\begin{array}{l}\text { INTERVAL, IN YEARS, AND ANNUAL NON- } \\
\text { EXCEEDANCE PROBABILITY, IN PERCENT }\end{array}$} \\
\hline & $\begin{array}{c}2 \\
508\end{array}$ & $\begin{array}{c}5 \\
208\end{array}$ & $\begin{array}{l}10 \\
108\end{array}$ & $\begin{array}{l}20 \\
58\end{array}$ & $\begin{array}{l}50 \\
28\end{array}$ & \\
\hline $\begin{array}{r}1 \\
3 \\
7 \\
14 \\
30 \\
60 \\
90 \\
120 \\
183\end{array}$ & $\begin{array}{r}0.0 \\
0.0 \\
0.0 \\
0.0 \\
0.0 \\
13 \\
30 \\
90 \\
239\end{array}$ & $\begin{array}{r}0.0 \\
0.0 \\
0.0 \\
0.0 \\
0.0 \\
0.0 \\
.1 \\
.1\end{array}$ & $\begin{array}{r}0.0 \\
0.0 \\
0.0 \\
0.0 \\
0.0 \\
0.0 \\
0.0 \\
0.0 \\
13\end{array}$ & $\begin{array}{l}0.0 \\
0.0 \\
0.0 \\
0.0 \\
0.0 \\
0.0 \\
0.0 \\
0.0 \\
4.1\end{array}$ & $\begin{array}{l}=- \\
\overline{--} \\
\overline{--} \\
\overline{--} \\
\overline{-}\end{array}$ & \\
\hline
\end{tabular}

DURATION TABLE OF DAILY MEAN FLOW FOR PERIOD OF RECORD 1974-85

DISCHARGE, IN FT3/S, THAT WAS EQUALED OR EXCEEDED FOR INDICATED PERCENT OF TIME

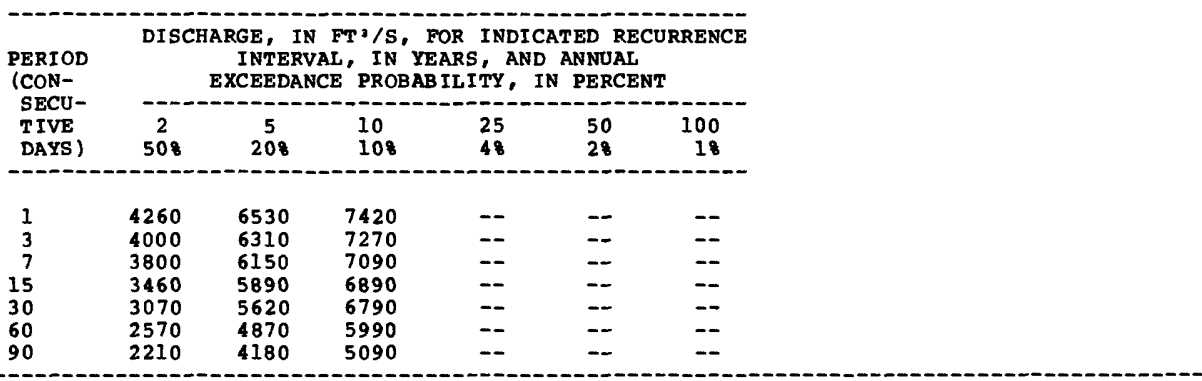

\begin{tabular}{|c|c|c|c|c|c|c|c|c|c|c|c|c|c|c|}
\hline 58 & 108 & 158 & 208 & 258 & 308 & 408 & 508 & 608 & 708 & 758 & 808 & 858 & 908 & 958 \\
\hline 4420 & 2870 & 1950 & 1430 & 1140 & 912 & 624 & 464 & 229 & 64 & 9.8 & 0.0 & 0.0 & 0.0 & .0 \\
\hline
\end{tabular}


RIO GRANDE BASIN

08358500 RIO GRANDE AT SAN MARCIAI, NM

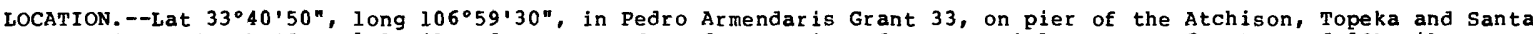
Fe Railway Co. bridge, I.l miles downstream from former site of San Marcial, Socorro County, and $18 \frac{1}{2}$ miles southwest of San Antonio.

DRAINAGE AREA.--27,700 $\mathrm{mi}^{2}$, approximately (includes $2,940 \mathrm{mi}^{2}$ in closed basin in san Luis Valley, co).

PERIOD OF RECORD.--January 1895 to September 1964 .

GAGE.- Water-stage recorder(river channel now called floodway). Datum of gage is 4,455.19 $\mathrm{ft}$ above mean sea level, datum of 1929. Prior to June 25, 1943, floodway had water-stage recorder, inverted rod, inclined staff, and wire-weight gages at several sites within $2 \mathrm{mi}$ of present site at various datums.

Conveyance channel: Water-stage recorder. Datum of gage is 4,454.00 $\mathrm{ft}$ above mean sea level (levels by Bureau of Reclamation). Prior to Apr. 29, 1958, at datum 4.19 ft higher. Apr. 14, 1950 to Feb. 28, 1954, bypass flow forming composite, measured at Tiffany Channel, 4 miles upstream; prior to 1950 all flow through floodway.

REMARKS.--Record is composite of floodway and conveyance channel (flows below $2,000 \mathrm{ft}^{3} / \mathrm{s}$ generally routed through conveyance channel). Discharge measurements generally made once a week on each channel.

Diversions for irrigation of about 775,000 acres above station (includes about 13,800 acre-ft diverted from conveyance channel, as based on weekly measurements, data furnished by Bureau of Reclamation).

AVERAGE DISCHARGE.--5I years (water years 190I-03, 1905-08, 1914-15, 1917-20, 1925-62), 1,277 ft3/s, 925,200 acre-ft/yr. EXTREMES FOR PERIOD OF RECORD.--Maximum discharge, about $50,000 \mathrm{ft} / \mathrm{s}$ Oct. 11 , 1904 ; no flow at times.

STATISTICAL SUMMARIES (PERIOD BeFore REgUlation OF ABIQUiU DAM)

MEAN MONTHLY AND MEAN ANNUAL DISCHARGES 1901-03, $1905-08,1914-15$ $1917-20,1925-62$

\begin{tabular}{|c|c|c|c|c|c|c|}
\hline MONTH & $\begin{array}{c}\text { MIN IMUM } \\
\left(\mathrm{FT}^{3} / \mathrm{S}\right)\end{array}$ & $\begin{array}{c}\text { MAXIMUM } \\
\left(\mathrm{FT}^{3} / \mathrm{S}\right)\end{array}$ & $\begin{array}{l}\text { MEAN } \\
\left(E T^{3} / S\right)\end{array}$ & $\begin{array}{l}\text { STAN- } \\
\text { DARD } \\
\text { DEVIA- } \\
\text { TION } \\
\left(\mathrm{FT}^{3} / \mathrm{S}\right)\end{array}$ & $\begin{array}{l}\text { COEFFI- } \\
\text { CIENT OF } \\
\text { VARI- } \\
\text { ATION }\end{array}$ & $\begin{array}{c}\text { PERCENT } \\
\text { OF } \\
\text { ANNUAL } \\
\text { RUNOF }\end{array}$ \\
\hline $\begin{array}{l}\text { OCTOBER } \\
\text { NOVEMBER } \\
\text { DECEMBER } \\
\text { JANUARY } \\
\text { FEBRUARY } \\
\text { MARCH } \\
\text { APRIL } \\
\text { MAY } \\
\text { JUNE } \\
\text { JULY } \\
\text { AUGUST } \\
\text { SEPTEMBER }\end{array}$ & $\begin{array}{r}0.0 \\
39 \\
39 \\
224 \\
314 \\
129 \\
46 \\
49 \\
.6 \\
0.0 \\
0.0 \\
0.0\end{array}$ & $\begin{array}{r}6630 \\
2871 \\
1551 \\
1180 \\
1861 \\
3544 \\
7257 \\
16160 \\
12000 \\
5346 \\
4468 \\
5178\end{array}$ & $\begin{array}{r}604 \\
577 \\
653 \\
645 \\
787 \\
923 \\
1756 \\
4034 \\
3151 \\
1067 \\
714 \\
554\end{array}$ & $\begin{array}{r}1090 \\
553 \\
342 \\
187 \\
296 \\
612 \\
1584 \\
3677 \\
3319 \\
1281 \\
860 \\
904\end{array}$ & $\begin{array}{r}1.80 \\
.96 \\
.52 \\
.29 \\
.38 \\
.66 \\
.90 \\
.91 \\
1.05 \\
1.20 \\
1.20 \\
1.63\end{array}$ & $\begin{array}{r}3.9 \\
3.7 \\
4.2 \\
4.2 \\
5.1 \\
6.0 \\
11.4 \\
26.1 \\
20.4 \\
6.9 \\
4.6 \\
3.6\end{array}$ \\
\hline ANNUAL & 184 & 3922 & 1277 & 871 & .68 & 100 \\
\hline
\end{tabular}

MAGNITUDE AND PROBABILITY OF ANNUAL HIGH FLOW BASED ON PERTOD OF RECORD 1901-03, 1905-08, $1914-15,1917-20,1925-62$

\begin{tabular}{|c|c|c|c|c|c|c|}
\hline $\begin{array}{l}\text { PERIOD } \\
\text { (CON- } \\
\text { SECU- }\end{array}$ & \multicolumn{6}{|c|}{$\begin{array}{l}\text { DISCHARGE, IN FT' }{ }^{3 / S} \text {, FOR INDICATED RECURRENCE } \\
\text { INTERVAL, IN YEARS, AND ANNUAL } \\
\text { EXCEEDANCE PROBABILI TY, IN PERCENT }\end{array}$} \\
\hline $\begin{array}{l}\text { TIVE } \\
\text { DAYS) }\end{array}$ & $\begin{array}{c}2 \\
508\end{array}$ & $\begin{array}{c}5 \\
208\end{array}$ & $\begin{array}{l}10 \\
108\end{array}$ & $\begin{array}{l}25 \\
48\end{array}$ & $\begin{array}{l}50 \\
28\end{array}$ & $\begin{array}{r}100 \\
18\end{array}$ \\
\hline $\begin{array}{r}1 \\
3 \\
7 \\
15 \\
30 \\
60 \\
90\end{array}$ & $\begin{array}{l}7420 \\
6440 \\
5430 \\
4590 \\
3870 \\
3010 \\
2420\end{array}$ & $\begin{array}{r}13800 \\
12100 \\
10700 \\
9300 \\
8010 \\
6260 \\
4940\end{array}$ & $\begin{array}{r}18300 \\
16200 \\
14800 \\
13100 \\
11300 \\
8960 \\
7010\end{array}$ & $\begin{array}{l}24200 \\
21500 \\
20500 \\
18400 \\
16000 \\
12900 \\
10000\end{array}$ & $\begin{array}{l}28600 \\
25500 \\
24900 \\
22800 \\
19700 \\
16100 \\
12500\end{array}$ & $\begin{array}{l}32900 \\
29500 \\
29500 \\
27300 \\
23600 \\
19600 \\
15200\end{array}$ \\
\hline
\end{tabular}

DURATION TABLE OF DAILY MEAN FIOW FOR PERIOD OF RECORD 1901-03, 1905-08, 1914-15, 1917-20, 2925-62

DISCHARGE, IN FT ${ }^{3} / S$, THAT WAS EQUALED OR EXCEEDED FOR INDICATED PERCENT OF TIME

\begin{tabular}{|c|c|c|c|c|c|c|c|c|c|c|c|c|c|c|}
\hline 58 & 108 & 158 & 208 & $25 \%$ & 308 & 408 & 508 & 608 & 708 & 758 & 808 & 858 & 908 & 958 \\
\hline 5730 & 3410 & 2170 & 1580 & 1240 & 978 & 774 & 600 & 442 & 264 & 185 & 113 & 54 & 19 & \\
\hline
\end{tabular}


RIO GRANDE BASIN

08360000 ALAMOSA CREEK NEAR MONTICELLO, NM

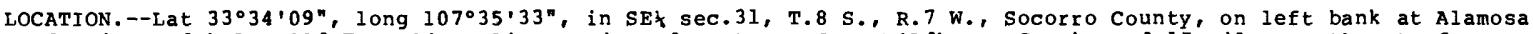
damsite and below 0ld Fort Ojo Caliente, just downstream from wildhorse Creek, and $15 \mathrm{miles}$ northwest of Monticello.

DRA INAGE AREA. $--403 \mathrm{mi}^{2}$.

PERIOD OF RECORD.--October to December 1929, May 1931 to April 1942, July 1956 to June 1958 (annual maximum only), July 1958 to September 1971 (discontinued). Monthly discharge only for some periods, published in wsp 1312. Prior to 1966 published as Alamosa River.

REVISED RECORDS.--WSP 1562: 1931, 1932-34(M), 1935-36, 1937-38(M), 1940-41(M).

GAGE.--Water-stage recorder and crest-stage gage. Datum of gage is $6,142.04 \mathrm{ft}$ above mean sea level. Prior to August 17, 1930, at different datum. May 2, 1931 to December 14, 1939, at datum 0.20 ft lower. July 16 , 1956 to July 27,1958 , crest-stage only.

REMARKS.--No diversion above station. Entire normal flow diverted below station for irrigation.

AVERAGE DISCHARGE.--23 years (water years 1932-41, 1959-71), 8.27 ft3/s, 5,990 acre-ft/yr.

EXTREMES FOR PERIOD OF RECORD.--Maximum discharge, $10,800 \mathrm{ft} 3 / \mathrm{s}$ Aug. 13,1964 (gage height, $14.04 \mathrm{ft}$ ), from rating curve extended above $390 \mathrm{ft} / \mathrm{s}$ on basis of slope-area measurements at gage heights 6.66 and $12.0 \mathrm{ft}$; minimum, $5.0 \mathrm{ft}^{3 / \mathrm{s}}$ on several days in June, July and August 1969

A flood in 1895 exceeded all other floods at this location from information by local residents. A flood in August 1943 was the highest since 1917 .

STATISTICAL SUMMARIES

MEAN MONTHLY AND MEAN ANNUAL DISCHARGES $\begin{aligned} & 1932-41 \\ & 1959-71\end{aligned}$

\begin{tabular}{|c|c|c|c|c|c|c|}
\hline MONTH & $\begin{array}{c}\text { MINIMUM } \\
\left(\mathrm{FT}^{3} / \mathrm{S}\right)\end{array}$ & $\begin{array}{l}\text { MAXIMUM } \\
\left(\mathrm{FT}^{3} / \mathrm{S}\right)\end{array}$ & $\begin{array}{l}\text { MEAN } \\
\left(\mathrm{FT}^{3} / \mathrm{S}\right)\end{array}$ & $\begin{array}{c}\text { STAN- } \\
\text { DARD } \\
\text { DEVIA- } \\
\text { TION } \\
(\text { FT'/S) }\end{array}$ & $\begin{array}{l}\text { COEFFI- } \\
\text { CIENT OF } \\
\text { VARI- } \\
\text { ATION }\end{array}$ & $\begin{array}{c}\text { PERCENT } \\
\text { OF } \\
\text { ANNUAL } \\
\text { RUNOFF }\end{array}$ \\
\hline $\begin{array}{l}\text { OCTOBER } \\
\text { NOVEMBER } \\
\text { DECEMBER } \\
\text { JANUARY } \\
\text { FEBRUARY } \\
\text { MARCH } \\
\text { APRIL } \\
\text { MAY } \\
\text { JUNE } \\
\text { JULY } \\
\text { AUGUST } \\
\text { SEPTEMBER }\end{array}$ & $\begin{array}{l}6.2 \\
6.1 \\
6.5 \\
6.1 \\
6.0 \\
5.8 \\
5.3 \\
5.8 \\
5.4 \\
5.4 \\
6.3 \\
6.7\end{array}$ & $\begin{array}{l}12 \\
9.2 \\
8.3 \\
7.8 \\
9.5 \\
8.5 \\
8.3 \\
15 \\
16 \\
19 \\
50 \\
19\end{array}$ & $\begin{array}{l}7.5 \\
7.3 \\
7.3 \\
7.1 \\
7.1 \\
6.9 \\
6.9 \\
7.4 \\
7.8 \\
10 \\
14 \\
10\end{array}$ & $\begin{array}{r}1.3 \\
.7 \\
.5 \\
.5 \\
.7 \\
.6 \\
.7 \\
2.1 \\
2.3 \\
3.5 \\
9.5 \\
3.0\end{array}$ & $\begin{array}{l}.17 \\
.09 \\
.07 \\
.07 \\
.10 \\
.08 \\
.10 \\
.28 \\
.29 \\
.35 \\
.69 \\
.30\end{array}$ & $\begin{array}{l}7.6 \\
7.4 \\
7.3 \\
7.1 \\
7.1 \\
7.0 \\
7.0 \\
7.5 \\
7.8 \\
10.1 \\
13.8 \\
10.3\end{array}$ \\
\hline NNUAL & 6.4 & 13 & 8.3 & 1.3 & .15 & 100 \\
\hline
\end{tabular}

MAGNITUDF AND PROBABILITY OF ANNUAL LOW FLOW BASED ON PERIOD OF RECORD 1933-42, 1960-71

\begin{tabular}{|c|c|c|c|c|c|c|}
\hline $\begin{array}{l}\text { PERIOD } \\
\text { (CON- } \\
\text { SECU- }\end{array}$ & $\mathrm{DISCH}$ & $\begin{array}{l}\text { IN } \\
\text { RVAL } \\
\text { EDAN }\end{array}$ & $\begin{array}{l}\text { IS, } \\
\text { YEA } \\
\text { ROBA }\end{array}$ & $\begin{array}{l}\text { INDI } \\
\text { AND } \\
\text { TY, }\end{array}$ & $\begin{array}{r}\text { REC } \\
\mathrm{NC} \\
\mathrm{RCEl}\end{array}$ & ENCE \\
\hline $\begin{array}{l}\text { TIVE } \\
\text { DAYS) }\end{array}$ & $\begin{array}{c}2 \\
508\end{array}$ & $\begin{array}{c}5 \\
208\end{array}$ & $\begin{array}{l}10 \\
108\end{array}$ & $\begin{array}{l}20 \\
58\end{array}$ & $\begin{array}{l}50 \\
28\end{array}$ & $\begin{array}{r}100 \\
18\end{array}$ \\
\hline $\begin{array}{r}1 \\
3 \\
7 \\
14 \\
30 \\
60 \\
90 \\
120 \\
183\end{array}$ & $\begin{array}{l}5.8 \\
5.9 \\
6.0 \\
6.1 \\
6.3 \\
6.5 \\
6.7 \\
7.0 \\
7.1\end{array}$ & $\begin{array}{l}5.5 \\
5.5 \\
5.6 \\
5.7 \\
5.9 \\
6.1 \\
6.3 \\
6.5 \\
6.7\end{array}$ & $\begin{array}{l}5.3 \\
5.4 \\
5.5 \\
5.6 \\
5.7 \\
5.9 \\
6.0 \\
6.2 \\
6.5\end{array}$ & $\begin{array}{l}5.2 \\
5.3 \\
5.4 \\
5.5 \\
5.6 \\
5.7 \\
5.9 \\
5.9 \\
6.4\end{array}$ & $\begin{array}{l}- \\
- \\
- \\
- \\
- \\
- \\
-\end{array}$ & $\begin{array}{l}= \\
=- \\
=- \\
-- \\
= \\
--\end{array}$ \\
\hline
\end{tabular}

MAGNITUDE AND PROBABIJITY OF ANNUAL HIGH FLOW BASED ON PERIOD OF RECORD 1932-41, 1959-71

DISCHARGE, IN FT
PERIOD
(CON-

DURATION TABLE OF DAILY MEAN FLOW FOR PERIOD OF RECORD 1932-41, 1959-71 DISCHARGE, IN FT $3 / \mathrm{S}$, THAT WAS EQUALED OR EXCEEDED FOR INDICATED PERCENT OF TIME

\begin{tabular}{|c|c|c|c|c|c|c|c|c|c|c|c|c|c|c|}
\hline 58 & 108 & 158 & 208 & 258 & 308 & 408 & 508 & 608 & 708 & 758 & 808 & 858 & 908 & 958 \\
\hline 11 & 8.7 & 8.3 & 7.9 & 7.7 & 7.6 & 7.3 & 7.1 & 6.9 & 6.7 & 6.6 & 6.4 & 6.2 & 6.1 & \\
\hline
\end{tabular}


LOCATION,--Lat $33^{\circ} 08^{\prime} 54^{n}$, long $107^{\circ} 12^{\prime} 22^{n}$, Sierra County, Hydrologic unit 13030101, in Pedro Armendaris Grant, on left bank $1.0 \mathrm{mi}$ downstream from dam, $1.5 \mathrm{mi}$ upstream from Cuchillo Negro River, and at mile $1,382.2$.

DRAINAGE AREA. - -29,450 $\mathrm{mi}^{2}$, approximately, including 2,940 $\mathrm{mi}^{2}$ in closed basin in San Luis Valley, co.

PERIOD OF RECORD.--January 1915 to current year. Monthly or annual discharge only for some periods, published in WSP 1732. Figures of daily discharge, published in wSP 458 for october to December 1916, are unreliable.

REVISED RECORDS,--WSP 1562: 1920, WSP 1632: Drainage area. WSP 1732: 1917, 1920. See also PERIOD OF RECORD.

GAGE.--Water-stage recorder. Datum of gage is $4,241.09 \mathrm{ft}$ above National Geodetic Vertical Datum of 1929 . Prior to Mar. 24, 1980 at datum $1.0 \mathrm{ft}$ higher. See wSP 1732 for history of changes prior to Apr. 24 , 1942.

REMARKS.--Flow regulated by Elephant Butte Reservoir (station 08360500 ). Diversion for irrigation of about 800,000 acres upstream from station.

AVERAGE DISCHARGE. - -69 years (water years 1917-85), $968 \mathrm{ft}^{3} / \mathrm{s}, 701,300 \mathrm{acre-ft/yr}$.

EXTREMES FOR PERIOD OF RECORD.--Maximum daily discharge, 8,220 ft3/s, May 22, 1942 ; no flow at times prior to 1929, Mar. 2-4, 1979.

STATISTICAL SUMMARIES

MEAN MONTHLY AND MEAN ANNUAL DISCHARges 1917-85

\begin{tabular}{|c|c|c|c|c|c|c|}
\hline MONTH & $\begin{array}{c}\text { MINIMUM } \\
\left(\mathrm{FT}^{3} / \mathrm{S}\right)\end{array}$ & $\begin{array}{c}\text { MAXIMUM } \\
\left(\mathrm{FT}^{3} / \mathrm{S}\right)\end{array}$ & $\begin{array}{l}\text { MEAN } \\
\left(\mathrm{FT}^{3} / \mathrm{S}\right)\end{array}$ & $\begin{array}{l}\text { STAN - } \\
\text { DARD } \\
\text { DEVIA- } \\
\text { TION } \\
\left(\mathrm{FT}^{3} / \mathrm{S}\right)\end{array}$ & $\begin{array}{l}\text { COEFFI- } \\
\text { CIENT OF } \\
\text { VARI- } \\
\text { ATION }\end{array}$ & $\begin{array}{c}\text { PERCENT } \\
\text { OF } \\
\text { ANNUAL } \\
\text { RUNOFF }\end{array}$ \\
\hline $\begin{array}{l}\text { OCTOBER } \\
\text { NOVEMBER } \\
\text { DECEMBER } \\
\text { JANUARY } \\
\text { FEB RUARY } \\
\text { MARCH } \\
\text { APRIL } \\
\text { MAY } \\
\text { JUNE } \\
\text { JULY } \\
\text { AUGUST } \\
\text { SEPTEMBER }\end{array}$ & $\begin{array}{c}2.7 \\
1.3 \\
2.9 \\
0.0 \\
3.4 \\
17 \\
188 \\
8.3 \\
284 \\
673 \\
155 \\
2.7\end{array}$ & $\begin{array}{l}1785 \\
2662 \\
1872 \\
1553 \\
1920 \\
2097 \\
2717 \\
7601 \\
6098 \\
3431 \\
2623 \\
2169\end{array}$ & $\begin{array}{r}333 \\
290 \\
324 \\
328 \\
664 \\
1073 \\
1484 \\
1520 \\
1713 \\
1668 \\
1415 \\
790\end{array}$ & $\begin{array}{l}463 \\
449 \\
442 \\
430 \\
482 \\
477 \\
513 \\
981 \\
731 \\
523 \\
557 \\
554\end{array}$ & $\begin{array}{l}1.39 \\
1.55 \\
1.36 \\
1.31 \\
.73 \\
.44 \\
.35 \\
.65 \\
.43 \\
.31 \\
.39 \\
.70\end{array}$ & $\begin{array}{r}2.9 \\
2.5 \\
2.8 \\
2.8 \\
5.7 \\
9.2 \\
12.8 \\
13.1 \\
14.8 \\
14.4 \\
12.2 \\
6.8\end{array}$ \\
\hline JNUAI & 253 & 2665 & 968 & 350 & .36 & 100 \\
\hline
\end{tabular}

MAGNITUDE AND PROBABILTTY OF ANNUAL LOW FLOW BASED ON PERIOD OF RECORD 1918-85

\begin{tabular}{|c|c|c|c|c|c|c|}
\hline \multirow{2}{*}{$\begin{array}{l}\text { PERIOD } \\
\text { (CON- } \\
\text { SECU- } \\
\text { TIVE } \\
\text { DAYS) }\end{array}$} & \multicolumn{6}{|c|}{$\begin{array}{l}\text { DISCHARGE, IN FT }{ }^{3} / \mathrm{S} \text {, FOR INDICATED RECURRENCE } \\
\text { INTERVAL, IN YEARS, AND ANNUAL NON- } \\
\text { EXCEEDANCE PROBABILITY, IN PERCENT }\end{array}$} \\
\hline & $\begin{array}{c}2 \\
508\end{array}$ & $\begin{array}{c}5 \\
208\end{array}$ & $\begin{array}{l}10 \\
108\end{array}$ & $\begin{array}{l}20 \\
58\end{array}$ & $\begin{array}{l}50 \\
28\end{array}$ & \\
\hline $\begin{array}{r}1 \\
3 \\
7 \\
14 \\
30 \\
60 \\
90 \\
120 \\
183\end{array}$ & $\begin{array}{c}3.7 \\
4.7 \\
5.3 \\
5.9 \\
10 \\
29 \\
51 \\
81 \\
309\end{array}$ & $\begin{array}{l}.8 \\
1.1 \\
1.1 \\
1.4 \\
2.9 \\
6.4 \\
11 \\
16 \\
82\end{array}$ & $\begin{array}{r}0.0 \\
.6 \\
.5 \\
.8 \\
1.8 \\
3.0 \\
4.6 \\
6.3 \\
33\end{array}$ & $\begin{array}{r}0.0 \\
0.0 \\
.2 \\
.4 \\
1.3 \\
1.6 \\
2.3 \\
2.9 \\
13\end{array}$ & $\begin{array}{r}0.0 \\
0.0 \\
0.0 \\
0.0 \\
.8 \\
.6 \\
1.1 \\
1.1 \\
4.4\end{array}$ & \\
\hline
\end{tabular}

MAGNITUDE AND PROBABILITY OF ANNUAL HIGH FLOW BASED ON PERTOD OF RECORD 1917-85

\begin{tabular}{|c|c|c|c|c|c|c|}
\hline \multirow{2}{*}{$\begin{array}{l}\text { PERIOD } \\
\text { (CON- } \\
\text { SECU- } \\
\text { TIVE } \\
\text { DAYS) }\end{array}$} & \multicolumn{6}{|c|}{$\begin{array}{l}\text { DISCHARGE, IN FT'/S, FOR INDICATED RECURRENCE } \\
\text { INTERVAL, IN YEARS, AND ANNUAL } \\
\text { EXCEEDANCE PROBABILITY, IN PERCENT }\end{array}$} \\
\hline & $\begin{array}{c}2 \\
508\end{array}$ & $\begin{array}{c}5 \\
208\end{array}$ & $\begin{array}{l}10 \\
10 \%\end{array}$ & $\begin{array}{l}25 \\
48\end{array}$ & $\begin{array}{l}50 \\
28\end{array}$ & $\begin{array}{r}100 \\
18\end{array}$ \\
\hline $\begin{array}{r}1 \\
3 \\
7 \\
15 \\
30 \\
60 \\
90\end{array}$ & $\begin{array}{l}2060 \\
2020 \\
1980 \\
1930 \\
1850 \\
1790 \\
1740\end{array}$ & $\begin{array}{l}2610 \\
2570 \\
2540 \\
2490 \\
2430 \\
2350 \\
2260\end{array}$ & $\begin{array}{l}3110 \\
3060 \\
3030 \\
2970 \\
2870 \\
2730 \\
2570\end{array}$ & $\begin{array}{l}3890 \\
3840 \\
3780 \\
3700 \\
3510 \\
3210 \\
2940\end{array}$ & $\begin{array}{l}4600 \\
4540 \\
4440 \\
4340 \\
4040 \\
3570 \\
3180\end{array}$ & $\begin{array}{l}5440 \\
5360 \\
5220 \\
5080 \\
4620 \\
3930 \\
3420\end{array}$ \\
\hline
\end{tabular}

DURATION TABLE OF DAILY MEAN FLOW FOR PERIOD OF RECORD 1917-85

DISCHARGE, IN FT $3 / S$, THAT WAS EOUALED OR EXCEEDED FOR INDICATED PERCENT OF TIME

\begin{tabular}{|c|c|c|c|c|c|c|c|c|c|c|c|c|c|c|}
\hline 58 & 108 & 158 & 208 & 258 & 308 & 408 & 508 & 608 & 708 & 758 & 808 & 858 & 908 & 958 \\
\hline 2480 & 2210 & 1930 & 1770 & 1620 & 1470 & 1210 & 979 & 681 & 144 & 18 & 12 & 7.7 & 5.2 & \\
\hline
\end{tabular}


RIO GRANDE BASIN

08362500 RIO GRANDE BELOW CABALLO DAM, NM

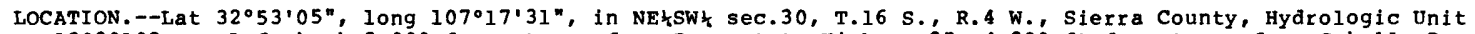

13030102 , on left bank $2,000 \mathrm{ft}$ upstream from Intergtate Highway 25, 4,200 ft downstream from Caballo Dam, 1.2

mi downstream from Apache Canyon, $1.3 \mathrm{mi}$ upstream from Percha diversion dam, $3 \mathrm{mi}$ northeast of Arrey, $5 \mathrm{mi}$ south of Caballo, and at mile $1,355.6$.

DRAINAGE AREA. - -30,700 $\mathrm{mi}^{2}$, approximately, including $2,940 \mathrm{mi}^{2}$ in closed basin in san Luis Valley, Co.

PERIOD OF RECORD.--January 1938 to current year.

GAGE.--Water-stage recorder. Datum of gage is $4,140.9 \mathrm{ft}$ above National Geodetic vertical Datum of 1929 . Prior to Oct. 7, 1938, at datum $7.0 \mathrm{ft}$ higher, Oct. 7-12, 1938, at datum 6.0 ft higher, and Oct. 13, 1938, to Dec. 31, 1945, at datum $5.0 \mathrm{ft}$ higher than present datum.

REMARRS.--Flow regulated by Caballo Reservoir (station 08362000) capacity, 344,000 acre-ft, 1958 survey and

Elephant Butte Reservoir (station 08360500) capacity, 2,109,000 acre-ft, 1974 survey. Diversions for irrigation

of about 800,000 acres upstream from station. Figures of daily discharge do not include Bonita ditch which

diverts from Caballo Dam and bypasses station for irrigation below.

COOPERATION.--Records provided by U.S. Bureau of Reclamation.

AVERAGE DISCHARGE.--25 years (water years 1961-85), $764 \mathrm{ft}^{3} / \mathrm{s}, 553,500$ acre-ft/yr.

EXTREMES FOR PERIOD OF RECORD.--Maximum daily discharge, 7,650 ft $3 / \mathrm{s}$, May 20, 1942; minimum daily, 0.1 ft $3 / \mathrm{s}$, Oct. 31 to Nov. 14, 1954, Nov. 7 to Dec. 31, 1955, Feb. 15-29, 1972 .

STATISTICAL SUMMARIES

MEAN MONTHLY AND MEAN ANNUAL DISCHARGES 1961-85

\begin{tabular}{|c|c|c|c|c|c|c|}
\hline MONTH & $\begin{array}{c}\text { MINIMUM } \\
\left(\mathrm{FT}^{3} / \mathrm{S}\right)\end{array}$ & $\begin{array}{c}\text { MAXIMUM } \\
\left(\mathrm{FT}^{3} / \mathrm{S}\right)\end{array}$ & $\begin{array}{l}\text { MEAN } \\
\left(\mathrm{FT}^{3} / \mathrm{S}\right)\end{array}$ & $\begin{array}{c}\text { STAN- } \\
\text { DARD } \\
\text { DEVIA- } \\
\text { TION } \\
\left(\mathrm{FT}^{3} / \mathrm{S}\right)\end{array}$ & $\begin{array}{l}\text { COEFFI- } \\
\text { CIENT OF } \\
\text { VARI- } \\
\text { ATION }\end{array}$ & $\begin{array}{c}\text { PERCENT } \\
\text { OF } \\
\text { ANNUAL } \\
\text { RUNOFF }\end{array}$ \\
\hline $\begin{array}{l}\text { OCTOBER } \\
\text { NOVEMBER } \\
\text { DECEMBER } \\
\text { JANUARY } \\
\text { FEBRUARY } \\
\text { MARCH } \\
\text { APRIL } \\
\text { MAY } \\
\text { JUNE } \\
\text { JULY } \\
\text { AUGUST } \\
\text { SEPTEMBER }\end{array}$ & $\begin{array}{r}.9 \\
.7 \\
.7 \\
.8 \\
510^{.2} \\
428 \\
1.4 \\
425 \\
671 \\
681 \\
114\end{array}$ & $\begin{array}{r}59 \\
3.2 \\
3.3 \\
342 \\
433 \\
2158 \\
1581 \\
1770 \\
2039 \\
2315 \\
2306 \\
1397\end{array}$ & $\begin{array}{r}4.9 \\
1.5 \\
1.4 \\
26 \\
104 \\
1452 \\
1053 \\
1026 \\
1577 \\
1651 \\
1521 \\
697\end{array}$ & $\begin{array}{l}12.6 \\
73^{.6} \\
142 \\
445 \\
310 \\
496 \\
419 \\
401 \\
415 \\
322\end{array}$ & $\begin{array}{r}2.43 \\
.40 \\
.43 \\
2.82 \\
1.37 \\
.31 \\
.29 \\
.48 \\
.27 \\
.24 \\
.27 \\
.46\end{array}$ & $\begin{array}{r}.1 \\
0.0 \\
0.0 \\
.3 \\
1.1 \\
15.9 \\
11.6 \\
11.3 \\
17.3 \\
18.1 \\
16.7 \\
7.6\end{array}$ \\
\hline INNUAL & 284 & 936 & 764 & 178 & .23 & 100 \\
\hline
\end{tabular}

MAGNITUDE AND PROBABILITY OF ANNUAL LOW FLOW BASED ON PERIOD OF RECORD $1962-85$

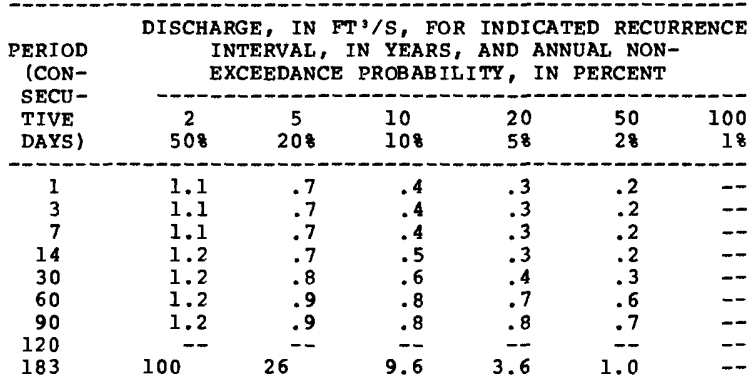

MAGNITUDE AND PROBABILITY OF ANNUAL HIGH FIOW BASED ON PERIOD OF RECORD 1961-85

\begin{tabular}{|c|c|c|c|c|c|c|}
\hline \multirow{2}{*}{$\begin{array}{l}\text { PERIOD } \\
\text { (CON- } \\
\text { SECU- } \\
\text { TIVE } \\
\text { DAYS) }\end{array}$} & \multicolumn{6}{|c|}{$\begin{array}{l}\text { DISCHARGE, IN FT' }{ }^{3} \text {, FOR INDICATED RECURRENCE } \\
\text { INTERVAL, IN YEARS, AND ANNUAL } \\
\text { EXCEEDANCE PROBABILITY, IN PERCENT }\end{array}$} \\
\hline & $\begin{array}{c}2 \\
508\end{array}$ & $\begin{array}{c}5 \\
208\end{array}$ & $\begin{array}{l}10 \\
108\end{array}$ & $\begin{array}{l}25 \\
48\end{array}$ & $\begin{array}{l}50 \\
28\end{array}$ & $\begin{array}{r}100 \\
18\end{array}$ \\
\hline $\begin{array}{r}1 \\
3 \\
7 \\
15 \\
30 \\
60 \\
90\end{array}$ & $\begin{array}{l}2510 \\
2460 \\
2370 \\
2260 \\
2060 \\
1800 \\
1720\end{array}$ & $\begin{array}{l}2840 \\
2780 \\
2680 \\
2550 \\
2310 \\
2050 \\
1960\end{array}$ & $\begin{array}{l}2980 \\
2910 \\
2810 \\
2660 \\
2390 \\
2130 \\
2030\end{array}$ & $\begin{array}{l}3100 \\
3020 \\
2910 \\
2740 \\
2440 \\
2180 \\
2080\end{array}$ & $\begin{array}{l}3160 \\
3070 \\
2960 \\
2770 \\
2460 \\
2200 \\
2090\end{array}$ & $\begin{array}{l}-- \\
-- \\
-- \\
-- \\
-- \\
--\end{array}$ \\
\hline
\end{tabular}

DURATION TABLE OF DAILY MEAN FLOW FOR PERIOD OF RECORD 1961-85

DISCHARGE, IN FT/S, THAT WAS EQUALED OR EXCEEDED FOR INDICATED PERCENT OF TIME

\begin{tabular}{|c|c|c|c|c|c|c|c|c|c|c|c|c|c|c|}
\hline 58 & 108 & 158 & $208^{\circ}$ & 258 & 308 & 408 & 508 & 608 & 708 & 758 & 808 & 858 & 908 & 958 \\
\hline 2230 & 2010 & 1780 & 1600 & 1450 & 1310 & 993 & 603 & 2.8 & 1.7 & 1.5 & 1.4 & 1.3 & 1.1 & 1.0 \\
\hline
\end{tabular}


08377900 RIO MORA NEAR TERRERO, NM (Hydrologic bench-mark station) LOCATION.--Lat $35^{\circ} 46^{\prime} 38^{\circ}$, long $105^{\circ} 39^{\prime} 27^{\prime \prime}$, in EłsE sec.22, T.18 N., R.12 E., San Miguel County, Hydrologic Unit
13060001 , in Santa Fe National Forest, on left bank $450 \mathrm{ft}$ upstream from br idge on State Highway 63 , 600 ft upstream from mouth, and $2.6 \mathrm{mi}$ north of Terrero.

DRAINAGE AREA. $--53.2 \mathrm{mi}^{2}$.

PERIOD OF RECORD.--October 1963 to current year.

GAGE.--Water-stage recorder and concrete control. Elevation of gage is 7,890 ft above National Geodetic Vertical Datum of 1929 , from topographic map.

REMARRs.--About 90 percent of the drainage is in the Pecos wilderness Area and not subject to development, watershed management, or the building of highways; there is limited cattle grazing by permit.

AVERAGE DISCHARGE.-22 years (water years 1964-85), $31.1 \mathrm{ft} / \mathrm{s}, 22,530 \mathrm{acre}-\mathrm{ft} / \mathrm{yr}$.

EXTREMES FOR PERIOD OF RECORD.--Maximum discharge, $820 \mathrm{ft}^{2} / \mathrm{s}$, June 8 , 1979, gage height, $4.15 \mathrm{ft}$; minimum determined, $0.90 \mathrm{ft}^{3} / \mathrm{s}$, Jan. 12-14, 1964, but may have been less during periods of ice effect.

EXTREMES OUTSIDE PERIOD OF RECORD.--Greatest flood since 1886 probab1y occurred Sept. 29,1904 (based on statement for Pecos River near Pecos and history of that flood period).

STATISTICAL SUMMARIES

MEAN MONTHLY AND MEAN ANNUAL DISCHARGES 1964-85

\begin{tabular}{|c|c|c|c|c|c|c|}
\hline MONTH & $\begin{array}{c}\text { MINIMUM } \\
\left(\mathrm{PT}^{3} / \mathrm{S}\right)\end{array}$ & $\begin{array}{l}\text { MAXIMUM } \\
\left(\mathrm{FT}^{3} / \mathrm{S}\right)\end{array}$ & $\begin{array}{l}\text { MEAN } \\
\left(\mathrm{FT}^{3} / \mathrm{S}\right)\end{array}$ & $\begin{array}{c}\text { STAN- } \\
\text { DARD } \\
\text { DEVIA- } \\
\text { TION } \\
\left(\text { FT }^{3} / \mathrm{S}\right)\end{array}$ & $\begin{array}{l}\text { COEFFI- } \\
\text { CIENT OF } \\
\text { VARI- } \\
\text { ATION }\end{array}$ & $\begin{array}{c}\text { PERCENT } \\
\text { OF } \\
\text { ANNUAL } \\
\text { RUNOF F }\end{array}$ \\
\hline $\begin{array}{l}\text { OCTOBER } \\
\text { NOVEMBER } \\
\text { DECEMBER } \\
\text { JANUARY } \\
\text { FEBRUARY } \\
\text { MARCH } \\
\text { APRIL } \\
\text { MAY } \\
\text { JUNE } \\
\text { JULY } \\
\text { AUGUST } \\
\text { SEPTEMBER }\end{array}$ & $\begin{array}{l}5.7 \\
4.6 \\
4.2 \\
1.7 \\
2.4 \\
3.4 \\
11 \\
14 \\
8.3 \\
8.6 \\
11 \\
6.9\end{array}$ & $\begin{array}{c}24 \\
18 \\
13 \\
9.0 \\
10.0 \\
21 \\
88 \\
319 \\
256 \\
61 \\
118 \\
73\end{array}$ & $\begin{array}{r}13 \\
9.4 \\
6.9 \\
5.3 \\
5.4 \\
9.5 \\
33 \\
114 \\
82 \\
26 \\
43 \\
25\end{array}$ & $\begin{array}{l}6.0 \\
4.1 \\
2.5 \\
1.8 \\
1.8 \\
4.7 \\
20 \\
81 \\
76 \\
16 \\
30 \\
17\end{array}$ & $\begin{array}{l}.47 \\
.43 \\
.36 \\
.34 \\
.34 \\
.50 \\
.62 \\
.71 \\
.93 \\
.59 \\
.70 \\
.66\end{array}$ & $\begin{array}{r}3.4 \\
2.5 \\
1.9 \\
1.4 \\
1.5 \\
2.6 \\
8.8 \\
30.7 \\
22.0 \\
7.1 \\
11.4 \\
6.8\end{array}$ \\
\hline NNUAL & 12 & 65 & 31 & 16 & .51 & 100 \\
\hline
\end{tabular}

MAGNITUDE AND PROBABILITY OF ANNUAL LOW FLOW BASED ON PERIOD OF RECORD 1965-85

\begin{tabular}{|c|c|c|c|c|c|c|}
\hline \multirow{2}{*}{$\begin{array}{l}\text { PERIOD } \\
\text { (CON- } \\
\text { SECU- } \\
\text { TIVE } \\
\text { DAYS) }\end{array}$} & \multicolumn{6}{|c|}{$\begin{array}{l}\text { DISCHARGE, IN FT }{ }^{3} / \mathrm{S} \text {, FOR INDICATED RECURRENCE } \\
\text { INTERVAL, IN YEARS, AND ANNUAL NON- } \\
\text { EXCEEDANCE PROBABILITY, IN PERCENT }\end{array}$} \\
\hline & $\begin{array}{c}2 \\
508\end{array}$ & $\begin{array}{c}5 \\
208\end{array}$ & $\begin{array}{l}10 \\
108\end{array}$ & $\begin{array}{l}20 \\
58\end{array}$ & $\begin{array}{l}50 \\
28\end{array}$ & \\
\hline $\begin{array}{r}1 \\
3 \\
7 \\
14 \\
30 \\
60 \\
90 \\
120 \\
183\end{array}$ & $\begin{array}{l}3.7 \\
3.9 \\
4.1 \\
4.3 \\
4.7 \\
5.0 \\
5.5 \\
5.9 \\
7.7\end{array}$ & $\begin{array}{l}2.8 \\
2.9 \\
3.2 \\
3.5 \\
3.8 \\
4.0 \\
4.3 \\
4.6 \\
5.8\end{array}$ & $\begin{array}{l}2.4 \\
2.5 \\
2.8 \\
3.1 \\
3.4 \\
3.6 \\
3.9 \\
4.1 \\
5.0\end{array}$ & $\begin{array}{l}2.1 \\
2.2 \\
2.5 \\
2.8 \\
3.1 \\
3.3 \\
3.5 \\
3.8 \\
4.5\end{array}$ & $\begin{array}{l}-- \\
-- \\
-- \\
-- \\
z- \\
--\end{array}$ & 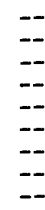 \\
\hline
\end{tabular}

MAGNITUDE AND PROBABILITY OF ANNUAL HIGH FLON BASED ON PERIOD OF RECORD 1964-85

\begin{tabular}{|c|c|c|c|c|c|c|}
\hline \multirow{2}{*}{$\begin{array}{l}\text { PERIOD } \\
\text { (CON- } \\
\text { SECU- } \\
\text { TIVE } \\
\text { DAYS) }\end{array}$} & \multicolumn{6}{|c|}{$\begin{array}{l}\text { DISCHARGE, IN FT' }{ }^{3} \text { /S, FOR INDICATED RECURRENCE } \\
\text { INTERVAL, IN YEARS, AND ANNUAL } \\
\text { EXCEEDANCE PROBABILITY, IN PERCENT }\end{array}$} \\
\hline & $\begin{array}{c}2 \\
508\end{array}$ & ${ }_{208}^{5}$ & $\begin{array}{l}10 \\
108\end{array}$ & $\begin{array}{l}25 \\
48\end{array}$ & $\begin{array}{l}50 \\
28\end{array}$ & $\begin{array}{r}100 \\
18\end{array}$ \\
\hline $\begin{array}{r}1 \\
3 \\
7 \\
15 \\
30 \\
60 \\
90\end{array}$ & $\begin{array}{r}191 \\
179 \\
165 \\
145 \\
117 \\
85 \\
67\end{array}$ & $\begin{array}{l}325 \\
306 \\
278 \\
249 \\
204 \\
149 \\
117\end{array}$ & $\begin{array}{l}428 \\
404 \\
363 \\
329 \\
274 \\
202 \\
158\end{array}$ & $\begin{array}{l}572 \\
543 \\
480 \\
441 \\
377 \\
283 \\
219\end{array}$ & $\begin{array}{l}-- \\
-- \\
-- \\
-- \\
-- \\
--\end{array}$ & $\begin{array}{l}-- \\
-- \\
-- \\
-- \\
-- \\
--\end{array}$ \\
\hline
\end{tabular}

DURATION TABLE OF DAILY MEAN FLOW FOR PERIOD OF RECORD 1964-85 DISCHARGE, IN FT3/S, THAT WAS EQUALED OR EXCEEDED FOR INDICATED PERCENT OF TIME

\begin{tabular}{|c|c|c|c|c|c|c|c|c|c|c|c|c|c|c|}
\hline 58 & 108 & 158 & 208 & 258 & 308 & 408 & 508 & 608 & 708 & 758 & 808 & 858 & 908 & 958 \\
\hline 131 & 75 & 51 & 38 & 30 & 24 & 17 & 13 & 9.3 & 7.4 & 6.5 & 5.8 & 5.2 & 4.6 & \\
\hline
\end{tabular}


RIO GRANDE BASIN

08378500 PECOS RIVER NEAR PECOS, NM

LOCATION.--Lat $35^{\circ} 42^{\prime} 30^{\prime}$, long $105^{\circ} 40^{\prime} 55^{\prime}$, in NEłNEł sec.17, T.17 N., R.12 E. , San Miguel County, Hydrologic Unit 13060001 , in Santa Fe National Forest, on left bank $30 \mathrm{ft}$ downstream from bridge on private road, 270 ft

upstream from Indian Creek, $2.4 \mathrm{mi}$ downstream from Holy Ghost Creek, $9.0 \mathrm{mi}$ north of Pecos, and at mile 896.6 .

DRAINAGE AREA. $--189 \mathrm{mi}^{2}$.

PERIOD OF RECORD.--August 1919 to current year. Monthly discharge only for some periods, published in wSP 1312. Published as "near Cowles" 1919-25, "at Irvins Ranch" 1926-29, and as "at Irvins Ranch near Pecos" $1930-39$.

REVISED RECORDS.--WSP 898: Drainage area. WSP 1312: $1932(\mathrm{M})$.

GAGE.--Water-stage recorder. Datum of gage is $7,502.94 \mathrm{ft}$ above National Geodetic Vertical Datum of 1929 . Prior to oct. 27,1977 , at site $30 \mathrm{ft}$ upstream at same datum.

REMARKS.--Diversions for irrigation of about 75 acres, 1959 determinations, upstream from station.

National Weather Service satellite telemeter at station.

AVERAGE DISCHARGE.--57 years (water years 1920, 1924, 1931-85) 99,3 ft3/s, 71,950 acre-ft/yr.

EXTREMES FOR PERIOD OF RECORD,--Maximum discharge, about 4,500 ft $/ \mathrm{s}$, Sept, 21 or 22,1929 , gage height, $6.2 \mathrm{ft}$, from floodmark, from rating curve extended above $1,600 \mathrm{ft} 3 / \mathrm{s}$; minimum, $2.0 \mathrm{ft} / \mathrm{s}, \mathrm{Mar}$. ig, 1971 , result of freezeup.

EXTREMES OUTSIDE PERIOD OF RECORD.--Flood of Sept. 29, 1904, was greatest since 1886, from information by local residents.

STATISTICAL SUMMARIES

MEAN MONTHLY AND MEAN ANNUAL DISChaRgeS 1920, 1924, $1931-85$

\begin{tabular}{|c|c|c|c|c|c|c|}
\hline MONTH & $\begin{array}{l}\text { MINIMUM } \\
\left(\mathrm{FT}^{3} / \mathrm{S}\right)\end{array}$ & $\begin{array}{c}\text { MAXIMUM } \\
(\mathrm{FT} 3 / \mathrm{S})\end{array}$ & $\begin{array}{l}\text { MEAN } \\
\left(\mathrm{FT}^{3} / \mathrm{S}\right)\end{array}$ & $\begin{array}{l}\text { STAN- } \\
\text { DARD } \\
\text { DEVIA- } \\
\text { TION } \\
\left(\mathrm{FT}^{3} / \mathrm{S}\right)\end{array}$ & $\begin{array}{l}\text { COEFFI- } \\
\text { CIENT OF } \\
\text { VARI- } \\
\text { ATION }\end{array}$ & $\begin{array}{c}\text { PERCENT } \\
\text { OF } \\
\text { ANNUAL } \\
\text { RUNOFF }\end{array}$ \\
\hline $\begin{array}{l}\text { OCTOBER } \\
\text { NOVEMBER } \\
\text { DECEMBER } \\
\text { JANUARY } \\
\text { JANBRUARY } \\
\text { FARCH } \\
\text { MARRIL } \\
\text { MAY } \\
\text { MUNE } \\
\text { JUNLY } \\
\text { JULGUST } \\
\text { AUGUST } \\
\text { SEPTEMBER }\end{array}$ & $\begin{array}{l}12 \\
12 \\
9.5 \\
11 \\
15 \\
18 \\
40 \\
44 \\
29 \\
21 \\
20 \\
11\end{array}$ & $\begin{array}{r}217 \\
138 \\
62 \\
50 \\
42 \\
81 \\
366 \\
1158 \\
950 \\
299 \\
402 \\
284\end{array}$ & $\begin{array}{r}50 \\
37 \\
29 \\
25 \\
26 \\
38 \\
132 \\
330 \\
242 \\
92 \\
106 \\
72\end{array}$ & $\begin{array}{c}32 \\
19 \\
11 \\
7.6 \\
6.5 \\
14 \\
75 \\
224 \\
201 \\
55 \\
71 \\
49\end{array}$ & $\begin{array}{l}.64 \\
.53 \\
.38 \\
.30 \\
.25 \\
.38 \\
.57 \\
.68 \\
.83 \\
.60 \\
.67 \\
.67\end{array}$ & $\begin{array}{r}4.3 \\
3.1 \\
2.5 \\
2.1 \\
2.2 \\
3.2 \\
11.2 \\
28.0 \\
20.5 \\
7.8 \\
9.0 \\
6.1\end{array}$ \\
\hline NNUAL & 31 & 267 & 99 & 49 & .50 & 100 \\
\hline
\end{tabular}

MAGNITUDE AND PROBABILITY OF ANNUAL LOW FLON BASED ON PERIOD OF RECORD 1924, 1931-85

\begin{tabular}{|c|c|c|c|c|c|c|}
\hline \multirow{2}{*}{$\begin{array}{l}\text { PERIOD } \\
\text { (CON- } \\
\text { SECU- } \\
\text { TIVE } \\
\text { DAYS) }\end{array}$} & \multicolumn{6}{|c|}{$\begin{array}{l}\text { DISCHARGE, IN FT'/S, FOR INDICATED RECURRENCE } \\
\text { INTERVAL, IN YEARS, AND ANNUAL NON- } \\
\text { EXCEEDANCE PROBABILITY, IN PERCENT }\end{array}$} \\
\hline & 508 & 208 & $\begin{array}{l}10 \\
108\end{array}$ & $\begin{array}{l}20 \\
58\end{array}$ & $\begin{array}{l}50 \\
28\end{array}$ & $\begin{array}{r}100 \\
18\end{array}$ \\
\hline $\begin{array}{r}1 \\
3 \\
7 \\
14 \\
30 \\
60 \\
90 \\
120 \\
183\end{array}$ & $\begin{array}{l}16 \\
17 \\
19 \\
20 \\
22 \\
23 \\
24 \\
26 \\
30\end{array}$ & $\begin{array}{l}12 \\
13 \\
14 \\
16 \\
17 \\
19 \\
19 \\
20 \\
23\end{array}$ & $\begin{array}{l}9.6 \\
11 \\
12 \\
13 \\
15 \\
16 \\
17 \\
18 \\
20\end{array}$ & $\begin{array}{l}8 . \\
9 . \\
10 \\
12 \\
14 \\
15 \\
16 \\
16 \\
19\end{array}$ & $\begin{array}{r}6 \\
7 \\
8 \\
10 \\
12 \\
13 \\
14 \\
15 \\
17\end{array}$ & $\begin{array}{l}9 \\
11 \\
12 \\
13 \\
14 \\
16\end{array}$ \\
\hline
\end{tabular}

MAGNITUDE AND PROBABILITY OF ANNUAL HIGH FLOW BASED ON PERIOD OF RECORD 1920, 1924, 1931-85

\begin{tabular}{|c|c|c|c|c|c|c|}
\hline \multirow{2}{*}{$\begin{array}{l}\text { PERIOD } \\
\text { (CON- } \\
\text { SECU- } \\
\text { TIVE } \\
\text { DAYS) }\end{array}$} & \multicolumn{6}{|c|}{$\begin{array}{l}\text { DISCHARGE, IN FT'/S, FOR INDICATED RECURRENCE } \\
\text { INTERVAL, IN YEARS, AND ANNUAL } \\
\text { EXCEEDANCE PROBABILITY, IN PERCENT }\end{array}$} \\
\hline & $\begin{array}{c}2 \\
508\end{array}$ & $\begin{array}{c}5 \\
208\end{array}$ & $\begin{array}{l}10 \\
108\end{array}$ & $\begin{array}{l}25 \\
48\end{array}$ & $\begin{array}{l}50 \\
28\end{array}$ & $\begin{array}{r}100 \\
18\end{array}$ \\
\hline $\begin{array}{r}1 \\
3 \\
7 \\
15 \\
30 \\
60 \\
90\end{array}$ & $\begin{array}{l}500 \\
469 \\
427 \\
378 \\
330 \\
261 \\
210\end{array}$ & $\begin{array}{l}870 \\
813 \\
742 \\
660 \\
579 \\
454 \\
362\end{array}$ & $\begin{array}{r}1120 \\
1050 \\
959 \\
858 \\
755 \\
594 \\
472\end{array}$ & $\begin{array}{r}1440 \\
1340 \\
1230 \\
1110 \\
984 \\
781 \\
619\end{array}$ & $\begin{array}{r}1670 \\
1540 \\
1430 \\
1300 \\
1160 \\
924 \\
733\end{array}$ & $\begin{array}{r}1900 \\
1740 \\
1620 \\
1480 \\
1330 \\
1070 \\
849\end{array}$ \\
\hline
\end{tabular}

DURATION TABLE OF DAILY MEAN FLOW FOR PERIOD OF RECORD 1920, G1924,1931-85

DISCHARGE, IN FT'J/S, THAT WAS EOUALED OR EXCEEDED FOR INDICATED PERCENT OF TIME

\begin{tabular}{|c|c|c|c|c|c|c|c|c|c|c|c|c|c|c|}
\hline 58 & 108 & 158 & 208 & 258 & 308 & 408 & 508 & 608 & 708 & 758 & 808 & 858 & 908 & 958 \\
\hline 388 & 243 & 165 & 125 & 99 & 80 & 59 & 46 & 37 & 31 & 28 & 26 & 24 & 22 & 19 \\
\hline
\end{tabular}




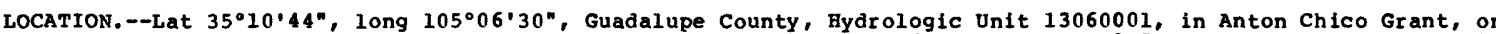
right bank $2.1 \mathrm{mi}$ upstream from Canon Blanco, $2.3 \mathrm{mi}$ southeast of Anton Chico, $9.7 \mathrm{mi}$ downstream from Tecolote Creek, and at mile 808.0 .

DRAINAGE AREA.--1,050 mi2, approximately (contributing area).

PERIOD OF RECORD.--April 1910 to May 1916, October 1916 to September 1924, August to December 1925 , January 1927 to current year. Monthly discharge only for some periods, published in wsp 1312.

REVISED RECORDS.--WSP 1342: 1951(M), 1952-53. WSP 1512: 1912-14, 1931, 1933(M), 1935-36(K), 1938(P), 1939-40, 41-42(P), $1945(M), 1946(P)$. WSP 1712: $1942(P)$.

GAGE.--Water-stage recorder. Elevation of gage is 5,130 ft above National Geodetic Vertical Datum of 1929 , from river-profile map. See WSP 1732 for history of changes prior to June 21, 1951.

REMARKS.--Diversions upstream from station for irrigation of about 4,900 acres, 1959 determinations, upstream and downstream from station. Acequia del Bodo Juan Paiz (see table below) diverts water about $8 \mathrm{mi}$ upstream from gage and bypasses this station on left bank: ditch flow not included in record measurements made at point opposite regular gage. A portion of this flow may be returned to the river about $5.0 \mathrm{mi}$ downstream.

AVERAGE DISCHARGE. - -61 years (1911-14, 1929-85), $124 \mathrm{ft}^{3} / \mathrm{s}, 89,840 \mathrm{acre}-\mathrm{ft} / \mathrm{yr}$.

EXTREMES FOR PERIOD OF RECORD.--Maximum discharge, 40,300 ft $/ \mathrm{s}$, June 1, 1937, gage height, $20.34 \mathrm{ft}$, from floodmarks, at site and datum then in use, by slope-area measurement; no flow at times.

EXTREMES OUTSIDE PERIOD OF RECORD.--The greatest flood since 1879 occurred Sept. 29, 1904, discharge about 73,000 $\mathrm{ft}^{3 / \mathrm{s}}$, from information by a local resident.

STATISTICAL SUMMARIES

MEAN MONTHLY AND MEAN ANNUAL DISChARGES $\begin{aligned} & 1911-14 \\ & 1929-85\end{aligned}$

\begin{tabular}{|c|c|c|c|c|c|c|}
\hline MONYH & $\begin{array}{l}\text { MINIMUM } \\
\left(\mathrm{FT}^{3} / \mathrm{S}\right)\end{array}$ & $\begin{array}{l}\text { MAXIMUM } \\
\left(\mathrm{FT}^{3} / \mathrm{S}\right)\end{array}$ & $\begin{array}{l}\text { MEAN } \\
\left(F^{\prime \prime} T^{\prime} / S^{\prime}\right)\end{array}$ & $\begin{array}{l}\text { STAN- } \\
\text { DARD } \\
\text { DEVIA- } \\
\text { TION } \\
\left(\mathrm{FT}^{3} / \mathrm{S}\right)\end{array}$ & $\begin{array}{l}\text { COEFFI- } \\
\text { CIENT OF } \\
\text { VARI- } \\
\text { ATION }\end{array}$ & $\begin{array}{c}\text { PERCENT } \\
\text { OF } \\
\text { ANNUAL } \\
\text { RUNOF F }\end{array}$ \\
\hline & & & & & ----- & $--\infty$ \\
\hline OCTOBER & 0.0 & 500 & 67 & 97 & 1.46 & 4.4 \\
\hline NOVEMBER & 0.0 & 279 & 38 & 46 & 1.21 & 2.5 \\
\hline DECEMBER & 0.0 & 103 & 27 & 20 & .74 & 1.8 \\
\hline JANUARY & 1.8 & 78 & 25 & 15 & .62 & 1.6 \\
\hline FEB RUARY & .9 & 150 & 25 & 22 & .87 & 1.7 \\
\hline MARCH & .3 & 331 & 59 & 63 & 1.05 & 3.9 \\
\hline APRIL & 1.5 & 854 & 190 & 197 & 1.04 & 12.6 \\
\hline MAY & 2.9 & 2031 & 371 & 355 & .96 & 24.5 \\
\hline JUNE & 4.2 & 1150 & 269 & 268 & 1.00 & 17.8 \\
\hline JULY & 3.8 & 507 & 140 & 112 & .80 & 9.3 \\
\hline AUGUST & 13 & 837 & 187 & 157 & .84 & 12.4 \\
\hline SEPTEMBER & 0.0 & 679 & 116 & 140 & 1.21 & 7.7 \\
\hline ANNUAL & 23 & 489 & 124 & 86 & .70 & 100 \\
\hline
\end{tabular}

MAGNITUDE AND PROBABILITY OF ANNUAL LOW FLON BASED ON PERIOD OF RECORD 1912-14, 1916 $1930-85$

\begin{tabular}{|c|c|c|c|c|c|c|}
\hline \multirow{2}{*}{$\begin{array}{l}\text { PERIOD } \\
\text { (CON- } \\
\text { SECU- } \\
\text { TIVE } \\
\text { DAYS) }\end{array}$} & \multicolumn{6}{|c|}{$\begin{array}{l}\text { DISCHARGE, IN FT'/S, FOR INDICATED RECURRENCE } \\
\text { INTERVAL, IN YEARS, AND ANNUAL NON- } \\
\text { EXCEEDANCE PROBABIL ITY, IN PERCENT }\end{array}$} \\
\hline & $\begin{array}{c}2 \\
508\end{array}$ & $\begin{array}{c}5 \\
208\end{array}$ & $\begin{array}{l}10 \\
108\end{array}$ & $\begin{array}{l}20 \\
58\end{array}$ & $\begin{array}{l}50 \\
28\end{array}$ & \\
\hline $\begin{array}{r}1 \\
3 \\
7 \\
14 \\
30 \\
60 \\
90 \\
120 \\
183\end{array}$ & $\begin{array}{l}1.2 \\
1.7 \\
2.9 \\
4.1 \\
8.4 \\
12 \\
15 \\
18 \\
31\end{array}$ & $\begin{array}{r}.1 \\
.2 \\
.4 \\
.8 \\
2.2 \\
5.5 \\
8.1 \\
10 \\
13\end{array}$ & $\begin{array}{r}0.0 \\
0.0 \\
.1 \\
.3 \\
.9 \\
3.4 \\
5.7 \\
7.4 \\
7.1\end{array}$ & $\begin{array}{r}0.0 \\
0.0 \\
0.0 \\
.1 \\
.3 \\
2.2 \\
4.1 \\
5.6 \\
4.1\end{array}$ & $\begin{array}{l}0.0 \\
0.0 \\
0.0 \\
0.0 \\
0.0 \\
.8 \\
2.0 \\
3.1 \\
2.1\end{array}$ & \\
\hline
\end{tabular}

MAGNITUDE AND PROBABILITY OF ANNUAL HIGH FLOW BASED ON PERIOD OF RECORD 1911-14, 1929-85

\begin{tabular}{|c|c|c|c|c|c|c|}
\hline \multirow{2}{*}{$\begin{array}{l}\text { PERIOD } \\
\text { (CON- } \\
\text { SECU- } \\
\text { TIVE } \\
\text { DAYS) }\end{array}$} & \multicolumn{6}{|c|}{$\begin{array}{l}\text { DISCHARGE, IN FT'/S, FOR INDICATED RECURRENC } \\
\text { INTERVAL, IN YEARS, AND ANNUAL } \\
\text { EXCEEDANCE PROBABILITY, IN PERCENT }\end{array}$} \\
\hline & $\begin{array}{c}2 \\
508\end{array}$ & $\begin{array}{c}5 \\
208\end{array}$ & $\begin{array}{l}10 \\
108\end{array}$ & $\begin{array}{l}25 \\
48\end{array}$ & $\begin{array}{l}50 \\
28\end{array}$ & $\begin{array}{r}100 \\
18\end{array}$ \\
\hline $\begin{array}{r}1 \\
3 \\
7 \\
15 \\
30 \\
60 \\
90\end{array}$ & $\begin{array}{r}1460 \\
935 \\
652 \\
505 \\
419 \\
317 \\
260\end{array}$ & $\begin{array}{r}2560 \\
1530 \\
1060 \\
854 \\
720 \\
561 \\
462\end{array}$ & $\begin{array}{r}3450 \\
2020 \\
1400 \\
1140 \\
958 \\
754 \\
617\end{array}$ & $\begin{array}{r}4780 \\
2770 \\
1930 \\
1580 \\
1300 \\
1030 \\
832\end{array}$ & $\begin{array}{l}5920 \\
3420 \\
2410 \\
1960 \\
1590 \\
1260 \\
1000\end{array}$ & $\begin{array}{l}7190 \\
4170 \\
2960 \\
2390 \\
1900 \\
1510 \\
1190\end{array}$ \\
\hline
\end{tabular}

DURATION TABLE OF DAILY MEAN FLOW FOR PERIOD OF RECORD 1911-14, 1929-85

DISCHARGE, IN FT $3 / \mathrm{S}$, THAT WAS EQUALED OR EXCEEDED FOR INDICATED PFRCENT OF TIME

\begin{tabular}{|c|c|c|c|c|c|c|c|c|c|c|c|c|c|}
\hline 58 & 108 & 158 & 208 & 258 & 308 & 408 & 508 & 608 & 708 & 758 & 808 & 858 & 908 \\
\hline 551 & 350 & 243 & 173 & 123 & 93 & 55 & 38 & 27 & 19 & 15 & 12 & 8.5 & 5.1 \\
\hline
\end{tabular}


RIO GRANDE BASIN

08380500 GALLINAS CREEK NEAR MONTEZUMA, NM

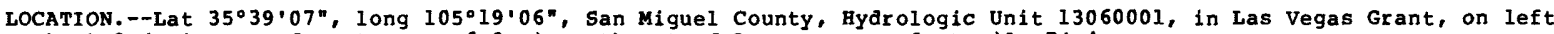
bank $2.4 \mathrm{mi}$ west of Montezuma, $6.9 \mathrm{mi}$ northwest of Las Vegas, and at mile 74.4 .

DRAINAGE AREA. $--84 \mathrm{mi}^{2}$, approximately.

PERIOD OF RECORD.--March to September 1915, June 1916 to current year. Monthly discharge only for some periods, published in WSP 1312. Prior to October 1964, published as Gallinas River near Montezuma.

REVISED RECORDS. --WSP 898: Drainage area. WSP 1562: 1951(P), 1952(M), 1955(P), 1957. WSP 1632: 1931-32, $1933(\mathrm{M}), 1934,1935(\mathrm{M}), 1938,1939-40(\mathrm{M}), 1941-42,1945,1949-50(\mathrm{~K})$

GAGE.-Water-stage recorder. Elevation of gage is $6,875 \mathrm{ft}$ above National Geodetic Vertical Datum of 1929 , from topographic map. Prior to Sept. 21, 1934, at different datum.

REMARKS.--Diversions for Irrigation of about 80 acres, 1959 determination, upstream from station.

AVERAGE DISCHARGE.--59 years (water years 1927-85), $18.3 \mathrm{ft} / \mathrm{s}, 13,260 \mathrm{acre}-\mathrm{ft} / \mathrm{yr}$.

EXTREMES FOR PERIOD OF RECORD.--Maximum discharge, $7,120 \mathrm{ft}^{3} / \mathrm{s}$, Aug. 2, 1966, gage height, $9.7 \mathrm{ft}, \mathrm{from}$ floodmarks, from rating curve extended above $500 \mathrm{ft} / \mathrm{s}$ on basis of slope-area measurements at gage heights $5.25 \mathrm{ft}$, $8.25 \mathrm{ft}$ from rating curve extended above $500 \mathrm{ft} / \mathrm{s}$ on basis of slope-area measurements at gage height
and $9.7 \mathrm{ft}$; minimum, $0.20 \mathrm{ft} / \mathrm{s}$, Oct. 6-9, 1922, Sept. 21, Oct. 9-14, 1956, Dec. 13, 1964 .

EXTREMES OUTSIDE PERIOD OF RECORD.--The greatest flood since about 1900 occurred the night of Sept. 29 , 1904 (discharge not determined), from information by local residents and G. B. Monk's report on floods.

STATISTICAL SUMMARIES

MEAN MONTHLY AND MEAN ANNUAL DISCHARGES 1927-85

\begin{tabular}{|c|c|c|c|c|c|c|}
\hline MONTH & $\begin{array}{c}\text { MINIMUM } \\
\left(\mathrm{FT}^{3} / \mathrm{S}\right)\end{array}$ & $\begin{array}{c}\operatorname{MAXI} M U M \\
\left(\mathrm{FT}^{3} / \mathrm{S}\right)\end{array}$ & $\begin{array}{l}\text { MEAN } \\
\left(\mathrm{FT}^{3} / \mathrm{S}\right)\end{array}$ & $\begin{array}{l}\text { STAN- } \\
\text { DARD } \\
\text { DEVIA- } \\
\text { TION } \\
\text { (FT'/S) }\end{array}$ & $\begin{array}{l}\text { COEFFI - } \\
\text { CIENT OF } \\
\text { VARI- } \\
\text { ATION }\end{array}$ & $\begin{array}{c}\text { PERCENT } \\
\text { OF } \\
\text { ANNUAL } \\
\text { RUNOF F }\end{array}$ \\
\hline $\begin{array}{l}\text { OCTOBER } \\
\text { NOVEMBER } \\
\text { DECEMBER } \\
\text { JANUARY } \\
\text { FEBRUARY } \\
\text { MARCH } \\
\text { APRIL } \\
\text { MAY } \\
\text { JUNE } \\
\text { JULY } \\
\text { AUGUST } \\
\text { SEPTEMBER }\end{array}$ & $\begin{array}{r}.4 \\
.5 \\
.8 \\
1.8 \\
1.5 \\
2.4 \\
3.1 \\
2.0 \\
.7 \\
1.2 \\
1.1 \\
.4\end{array}$ & $\begin{array}{r}108 \\
58 \\
21 \\
13 \\
14 \\
44 \\
184 \\
380 \\
119 \\
78 \\
159 \\
141\end{array}$ & $\begin{array}{l}12 \\
9.0 \\
6.2 \\
5.0 \\
5.4 \\
11 \\
35 \\
54 \\
21 \\
15 \\
28 \\
19\end{array}$ & $\begin{array}{l}18 \\
11 \\
4.1 \\
2.4 \\
2.9 \\
9.4 \\
40 \\
66 \\
25 \\
14 \\
30 \\
25\end{array}$ & $\begin{array}{r}1.48 \\
1.16 \\
.67 \\
.47 \\
.54 \\
.86 \\
1.13 \\
1.23 \\
1.19 \\
.94 \\
1.07 \\
1.32\end{array}$ & $\begin{array}{r}5.6 \\
4.1 \\
2.8 \\
2.3 \\
2.4 \\
5.0 \\
15.9 \\
24.3 \\
9.6 \\
6.6 \\
12.8 \\
8.6\end{array}$ \\
\hline NNUAL & 2.5 & 81 & 18 & 15 & .80 & 100 \\
\hline
\end{tabular}

MAGNITUDE AND PROBABILITY OF ANNUAL IOW FLOW BASED ON PERIOD OF RECORD $1928-85$

\begin{tabular}{|c|c|c|c|c|c|c|}
\hline \multirow{2}{*}{$\begin{array}{l}\text { PERIOD } \\
\text { (CON- } \\
\text { SECU- } \\
\text { TIVE } \\
\text { DAYS) }\end{array}$} & \multicolumn{6}{|c|}{$\begin{array}{l}\text { DISCHARGE, IN FT'3/S, FOR INDICATED RECURRENCE } \\
\text { INTERVAL, IN YEARS, AND ANNUAL NON- } \\
\text { EXCEEDANCE PROBABILITY, IN PERCENT }\end{array}$} \\
\hline & $\begin{array}{c}2 \\
508\end{array}$ & $\begin{array}{c}5 \\
208\end{array}$ & $\begin{array}{l}10 \\
108\end{array}$ & $\begin{array}{l}20 \\
58\end{array}$ & $\begin{array}{l}50 \\
28\end{array}$ & $\begin{array}{r}100 \\
18\end{array}$ \\
\hline $\begin{array}{r}1 \\
3 \\
7 \\
14 \\
30 \\
60 \\
90 \\
120 \\
183\end{array}$ & $\begin{array}{l}2.1 \\
2.2 \\
2.4 \\
2.7 \\
3.2 \\
3.8 \\
4.3 \\
4.7 \\
5.5\end{array}$ & $\begin{array}{l}1.2 \\
1.3 \\
1.4 \\
1.5 \\
1.9 \\
2.3 \\
2.6 \\
2.9 \\
3.1\end{array}$ & $\begin{array}{r}.8 \\
.9 \\
1.0 \\
1.1 \\
1.3 \\
1.7 \\
1.9 \\
2.1 \\
2.4\end{array}$ & $\begin{array}{r}.6 \\
.7 \\
.7 \\
.8 \\
1.0 \\
1.2 \\
1.4 \\
1.6 \\
1.9\end{array}$ & $\begin{array}{r}.4 \\
.5 \\
.5 \\
.6 \\
.7 \\
.9 \\
1.0 \\
1.1 \\
1.5\end{array}$ & $\begin{array}{r}.3 \\
.8 \\
.9 \\
1.3\end{array}$ \\
\hline
\end{tabular}

MAGNITUDE AND PROBABILITY OF ANNUAL RIGR FLOW BASED ON PERIOD OF RECORD $1927-85$

\begin{tabular}{|c|c|c|c|c|c|c|}
\hline \multirow{2}{*}{$\begin{array}{l}\text { PERIOD } \\
\text { (CON- } \\
\text { SECU- } \\
\text { TIVE } \\
\text { DAYS) }\end{array}$} & \multicolumn{6}{|c|}{$\begin{array}{l}\text { DISCHARGE, IN FT' }{ }^{3} \text {, , FOR INDICATED RECURRENC) } \\
\text { INTERVAL, IN YEARS, AND ANNUAL } \\
\text { EXCEEDANCE PROBAB ILITY, IN PERCENT }\end{array}$} \\
\hline & $\begin{array}{c}2 \\
508\end{array}$ & $\begin{array}{c}5 \\
208\end{array}$ & $\begin{array}{l}10 \\
108\end{array}$ & $\begin{array}{l}25 \\
48\end{array}$ & $\begin{array}{l}50 \\
28\end{array}$ & $\begin{array}{r}100 \\
18\end{array}$ \\
\hline $\begin{array}{r}1 \\
3 \\
7 \\
15 \\
30 \\
60 \\
90\end{array}$ & $\begin{array}{r}152 \\
125 \\
99 \\
75 \\
58 \\
42 \\
33\end{array}$ & $\begin{array}{r}346 \\
264 \\
202 \\
154 \\
118 \\
84 \\
64\end{array}$ & $\begin{array}{r}523 \\
374 \\
278 \\
216 \\
165 \\
117 \\
90\end{array}$ & $\begin{array}{l}799 \\
525 \\
375 \\
301 \\
230 \\
163 \\
127\end{array}$ & $\begin{array}{r}1040 \\
641 \\
447 \\
367 \\
281 \\
200 \\
157\end{array}$ & $\begin{array}{r}1310 \\
759 \\
517 \\
435 \\
333 \\
238 \\
190\end{array}$ \\
\hline
\end{tabular}

DURATION TABLE OF DAILY MEAN FLOW FOR PERIOD OF RECORD 1927-85

DISCHARGE, IN FT $3 / \mathrm{S}$, THAT WAS EQUALED OR EXCEEDED FOR INDICATED PERCENT OF TIME

\begin{tabular}{|c|c|c|c|c|c|c|c|c|c|c|c|c|c|c|}
\hline 58 & 108 & 158 & 208 & 258 & 308 & 408 & 508 & 608 & 708 & 758 & 808 & 858 & 908 & 958 \\
\hline 72 & 43 & 30 & 22 & 17 & 14 & 9.2 & 7.0 & 5.4 & 4.4 & 3.9 & 3.5 & 3.1 & 2.6 & \\
\hline
\end{tabular}


RIO GRANDE BASIN

08381000 GALLINAS CREEK AT MONTEZUMA, NM

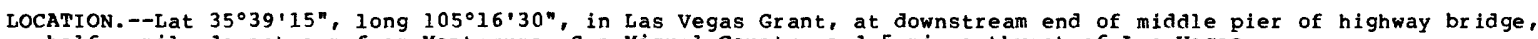
half a mile downstream from Montezuma, San Miguel County, and $5 \mathrm{mi}$ northwest of Las Vegas.

DRAINAGE AREA. $--87 \mathrm{mi}^{2}$, approximately.

PERIOD OF RECORD.--August 1903 to September 1904 (gage heights only), October 1904 to May 1912 , October 1912 to September 1966. Yearly estimate for water year 1912 (incomplete) and monthly discharge only for some periods, published in wSP 1312. Figures of daily discharge for the periods Oct. 8-22, 1904 and Jan. 1 to Feb. 20 , 1906, published in WSP 177 and 213, respectively, have been found to be unreliable and should not be used. Prior to october 1964 published as Gallinas River at Montezuma.

GAGE.--Water-stage recorder. Altitude of gage is 6,675 ft (from topographic map). Prior to Mar. 24, 1926, staff gage at site a quarter of a mile upstream. Gages at various datums prior to Sept. 3, 1942 .

REMARKS.--Flow partly regulated at low flows by reservoirs owned by Public Service Co. of New Mexico. Diversions above station for irrigation of about 180 acres (1959 determination) and for Las vegas city water supply.

AVERAGE DISCHARGE.--57 years (water years 1907-11, 1914-15, 1917-66), 18.0 $\mathrm{ft}^{3} / \mathrm{s}, 13,040 \mathrm{acre}-\mathrm{ft}_{\mathrm{yr}}$.

EXTREMES FOR PERIOD OF RECORD.--Maximum discharge, 9,140 ft $3 / \mathrm{s}$ Aug. 4, 1957 (gage height, $11.8 \mathrm{ft}$, from floodmark), from rating curve extended above $450 \mathrm{ft}^{3} / \mathrm{s}$ on basis of slope-area measurement of peak flow; no flow at times in $1934,1956-57,1963$.

Flood of Sept. 30,1904 , is the highest since about 1900 (discharge, $11,600 \mathrm{ft}^{3} / \mathrm{s}$ by slope-area method), from G. B. Monk's report on floods and WSP 147 .

STATISTICAL SUMMARIES

MEAN MONTHLY AND MEAN ANNUAL DISCHARGES 1907-11, 1914-15, $1914-15$
$1917-66$

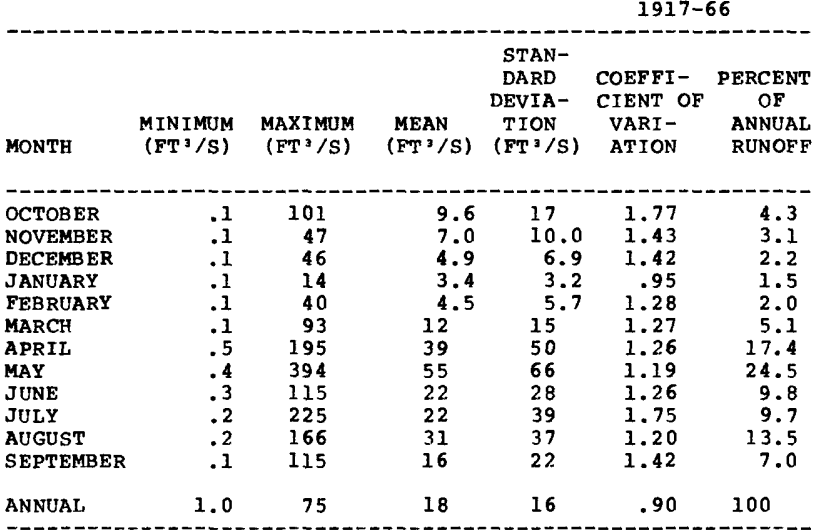

MAGNITUDE AND PROBABILITY OF ANNUAL LOW FLOW BASED ON PERIOD OF RECORD 1907-12, 1914-16, $1918-66$

\begin{tabular}{|c|c|c|c|c|c|c|}
\hline \multirow{2}{*}{$\begin{array}{l}\text { PERIOD } \\
\text { (CON- } \\
\text { SECU- } \\
\text { TIVE } \\
\text { DAYS) }\end{array}$} & \multicolumn{6}{|c|}{$\begin{array}{l}\text { DISCHARGE, IN FT'3/S, FOR INDICATED RECURRENCE } \\
\text { INTERVAL, IN YEARS, AND ANNUAL NON- } \\
\text { EXCEEDANCE PROBABILITY, IN PERCENT }\end{array}$} \\
\hline & $\begin{array}{c}2 \\
508\end{array}$ & $\begin{array}{c}5 \\
208\end{array}$ & $\begin{array}{l}10 \\
108\end{array}$ & $\begin{array}{l}20 \\
58\end{array}$ & $\begin{array}{l}50 \\
28\end{array}$ & \\
\hline $\begin{array}{r}1 \\
3 \\
7 \\
14 \\
30 \\
60 \\
90 \\
120 \\
183\end{array}$ & $\begin{array}{r}.4 \\
.5 \\
.5 \\
.7 \\
1.0 \\
1.4 \\
1.8 \\
2.1 \\
3.2\end{array}$ & $\begin{array}{r}.2 \\
.2 \\
.2 \\
.3 \\
.4 \\
.5 \\
.6 \\
.8 \\
1.1\end{array}$ & $\begin{array}{l}.1 \\
.1 \\
.1 \\
.2 \\
.2 \\
.3 \\
.4 \\
.4 \\
.6\end{array}$ & $\begin{array}{r}0.0 \\
0.0 \\
.1 \\
.1 \\
.2 \\
.2 \\
.2 \\
.3 \\
.4\end{array}$ & $\begin{array}{r}0.0 \\
0.0 \\
0.0 \\
.1 \\
.1 \\
.1 \\
.1 \\
.1 \\
.1\end{array}$ & 0. \\
\hline
\end{tabular}

MAGNITUDE AND PROBABILITY OF ANNUAL HIGH FLON BASED ON PERIOD OF RECORD 1907-11, 1914-15, $1917-66$

\begin{tabular}{|c|c|c|c|c|c|c|}
\hline $\begin{array}{l}\text { PERIOD } \\
\text { (CON- }\end{array}$ & & $\begin{array}{l}\text { GE, } \\
\text { INTE } \\
\text { CEED }\end{array}$ & PROE & $\begin{array}{l}\text { R IND } \\
\text { RS, A } \\
\text { LI TY, }\end{array}$ & $\begin{array}{l}\text { TED } \\
\text { ANNU } \\
\text { PERC }\end{array}$ & \\
\hline $\begin{array}{l}\text { TIVE } \\
\text { DAYS) }\end{array}$ & $\begin{array}{c}2 \\
508\end{array}$ & $\begin{array}{c}5 \\
208\end{array}$ & $\begin{array}{l}10 \\
108\end{array}$ & $\begin{array}{l}25 \\
48\end{array}$ & $\begin{array}{l}50 \\
28\end{array}$ & $\begin{array}{r}100 \\
18\end{array}$ \\
\hline $\begin{array}{r}1 \\
3 \\
7 \\
15 \\
30 \\
60 \\
90\end{array}$ & $\begin{array}{r}196 \\
148 \\
114 \\
84 \\
66 \\
47 \\
36\end{array}$ & $\begin{array}{r}404 \\
298 \\
227 \\
171 \\
136 \\
95 \\
74\end{array}$ & $\begin{array}{l}544 \\
398 \\
301 \\
230 \\
184 \\
128 \\
101\end{array}$ & $\begin{array}{l}709 \\
513 \\
386 \\
299 \\
241 \\
168 \\
134\end{array}$ & $\begin{array}{l}818 \\
589 \\
440 \\
345 \\
280 \\
195 \\
157\end{array}$ & $\begin{array}{l}915 \\
655 \\
488 \\
386 \\
315 \\
219 \\
179\end{array}$ \\
\hline
\end{tabular}

DURATION TABLE OF DAILY MEAN FLOW FOR PERIOD OF RECORD 1907-11, 1914-15, 1917-66

DISCHARGE, IN FT ${ }^{3} / \mathrm{S}$, THAT WAS EQUALED OR EXCEEDED FOR INDICATED PERCENT OF TIHE

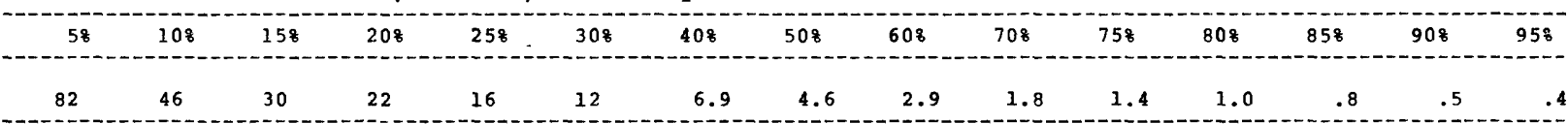


LOCATION.--Lat $35^{\circ} 28^{\prime} 15^{\prime \prime}$, long $105^{\circ} 09^{\prime} 35^{\prime \prime}$, in Las Vegas Grant, on right bank $0.8 \mathrm{mi}$ upstream from ford on Lourdes-Romeroville road, $1.2 \mathrm{mi}$ northwest of Lourdes, San Miquel County, 2.8 miles downstream from Pagosa Lourdes-Romeroville road, 1.2 mi northwest
Canyon, and 9 miles south of Las Vegas.

DRAINAGE AREA, $--313 \mathrm{mi}^{2}$.

PERIOD OF RECORD.--June 1951 to September 1963.

GAGE.--Water-stage recorder. Datum of gage is $5,928 \mathrm{ft}$ above mean sea level (levels from plane-table bench mark). REMARKS.--Diversions for irrigation of about 6,600 acres (1959 determination) above station.

AVERAGE DISCHARgE.--12 years (water years 1952-63), $14.5 \mathrm{ft}^{3} / \mathrm{s}, 10,500 \mathrm{acre-ft} / \mathrm{yr}$.

EXTREIES FOR PERIOD OF RECORD.--Maximum discharge, 6,680 ft*/s Aug. 17, 1961 (gage height $9.40 \mathrm{ft}$ ), from rating curve extended above $2,100 \mathrm{ft} / \mathrm{s}$ on basis of slope-area measurement of peak flow; no flow June 17, July $2-10$, curve extended above 2,100 ft/s on basis of slope-area measurement of peak flow; no flow June 17, July 2-10, Flood 1937 .

STATISTICAL SUMMARIES

MEAN MONTHLY AND MEAN ANNUAL DISChARGES 1952-63

\begin{tabular}{|c|c|c|c|c|c|c|}
\hline MONTH & $\begin{array}{l}\text { MINIMUM } \\
\left(\mathrm{FT}^{3} / \mathrm{S}\right)\end{array}$ & $\begin{array}{c}\text { MAXIMUM } \\
(\text { FT } 3 / S)^{-S /}\end{array}$ & $\begin{array}{l}\text { MEAN } \\
(\mathrm{FT} 3 / \mathrm{S})\end{array}$ & $\begin{array}{l}\text { STAN- } \\
\text { DARD } \\
\text { DEVIA- } \\
\text { TION } \\
\left.\text { (FT }{ }^{3} / \mathrm{S}\right)\end{array}$ & $\begin{array}{l}\text { COEFFI- } \\
\text { CIENT OF } \\
\text { VARI- } \\
\text { ATION }\end{array}$ & $\begin{array}{c}\text { PERCENT } \\
\text { OF } \\
\text { ANNUAL } \\
\text { RUNOF F }\end{array}$ \\
\hline $\begin{array}{l}\text { OCTOBER } \\
\text { NOVEMBER } \\
\text { DECEMBER } \\
\text { JANUARY } \\
\text { FEBRUARY } \\
\text { MARCH } \\
\text { APRIL } \\
\text { MAY } \\
\text { JUNE } \\
\text { JULY } \\
\text { AUGUST } \\
\text { SEPTEMBER }\end{array}$ & $\begin{array}{r}.4 \\
.8 \\
.9 \\
1.2 \\
1.1 \\
1.3 \\
1.0 \\
1.5 \\
1.1 \\
8.4 \\
9.7 \\
.4\end{array}$ & $\begin{array}{c}82 \\
11 \\
9.3 \\
13 \\
7.5 \\
35 \\
248 \\
184 \\
30 \\
46 \\
188 \\
46\end{array}$ & $\begin{array}{r}18 \\
5.8 \\
5.0 \\
4.9 \\
4.1 \\
6.4 \\
24 \\
22 \\
11 \\
16 \\
42 \\
15\end{array}$ & $\begin{array}{r}23 \\
3.2 \\
2.9 \\
3.7 \\
2.5 \\
9.3 \\
71 \\
52 \\
9.9 \\
11 \\
50 \\
14\end{array}$ & $\begin{array}{r}1.28 \\
.55 \\
.58 \\
.76 \\
.60 \\
1.46 \\
2.93 \\
2.38 \\
.91 \\
.69 \\
1.20 \\
.94\end{array}$ & $\begin{array}{r}10.4 \\
3.4 \\
2.9 \\
2.8 \\
2.4 \\
3.7 \\
14.0 \\
12.5 \\
6.3 \\
9.0 \\
24.3 \\
8.4\end{array}$ \\
\hline ANNUAL & 4.6 & 54 & 15 & 14 & .96 & 100 \\
\hline
\end{tabular}

MAGNITUDE AND PROBABILITY OF ANNUAL LOW FLOW BASED ON PERIOD OF RECORD 1953-63

\begin{tabular}{|c|c|c|c|c|c|c|}
\hline \multirow{2}{*}{$\begin{array}{l}\text { PERIOD } \\
\text { (CON- } \\
\text { SECU- } \\
\text { TIVE } \\
\text { DAYS) }\end{array}$} & \multicolumn{6}{|c|}{$\begin{array}{l}\text { DISCHARGE, IN FT }{ }^{3} / S \text {, FOR INDICATED RECURRENCE } \\
\text { INTERVAL, IN YEARS, AND ANNUAL NON- } \\
\text { EXCEEDANCE PROBABIL I TY, IN PERCENT }\end{array}$} \\
\hline & $\begin{array}{c}2 \\
508\end{array}$ & $\begin{array}{c}5 \\
208\end{array}$ & $\begin{array}{l}10 \\
108\end{array}$ & $\begin{array}{l}20 \\
58\end{array}$ & $\begin{array}{l}50 \\
28\end{array}$ & $\begin{array}{r}100 \\
18\end{array}$ \\
\hline $\begin{array}{r}1 \\
3 \\
7 \\
14 \\
30 \\
60 \\
90 \\
120 \\
183\end{array}$ & $\begin{array}{r}.8 \\
.99 \\
1.2 \\
1.3 \\
1.9 \\
2.9 \\
3.0 \\
3.9 \\
6.6\end{array}$ & $\begin{array}{r}.1 \\
.2 \\
.3 \\
.4 \\
.8 \\
1.2 \\
1.4 \\
1.8 \\
3.0\end{array}$ & $\begin{array}{r}0.0 \\
0.0 \\
0.0 \\
.2 \\
.4 \\
.7 \\
.9 \\
1.1 \\
1.8\end{array}$ & $\begin{array}{r}0.0 \\
0.0 \\
0.0 \\
.1 \\
.3 \\
.5 \\
.6 \\
.7 \\
1.1\end{array}$ & $\begin{array}{l}-+ \\
-- \\
-- \\
-- \\
-- \\
--\end{array}$ & 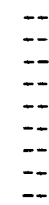 \\
\hline
\end{tabular}

MAGNITUDE AND PROBABILITY OF ANNUAL HIGH FLOW BASED ON PERIOD OF RECORD 1952-63

\begin{tabular}{|c|c|c|c|c|c|c|}
\hline \multirow{2}{*}{$\begin{array}{l}\text { PERIOD } \\
\text { (CON- } \\
\text { SECU- } \\
\text { TIVE } \\
\text { DAYS) }\end{array}$} & \multicolumn{6}{|c|}{$\begin{array}{l}\text { DISCHARGE, IN FT'/S, FOR INDICATED RECURRENCE } \\
\text { INTERVAL, IN YEARS, AND ANNUAL } \\
\text { EXCEEDANCE PROBABILITY, IN PERCENT }\end{array}$} \\
\hline & $\begin{array}{c}2 \\
508\end{array}$ & $\begin{array}{c}5 \\
208\end{array}$ & $\begin{array}{l}10 \\
108\end{array}$ & $\begin{array}{l}25 \\
48\end{array}$ & $\begin{array}{l}50 \\
28\end{array}$ & $\begin{array}{r}100 \\
18\end{array}$ \\
\hline $\begin{array}{r}1 \\
3 \\
7 \\
15 \\
30 \\
60 \\
90\end{array}$ & $\begin{array}{r}466 \\
228 \\
123 \\
73 \\
44 \\
30 \\
23\end{array}$ & $\begin{array}{r}854 \\
397 \\
258 \\
168 \\
107 \\
68 \\
50\end{array}$ & $\begin{array}{r}1140 \\
524 \\
386 \\
267 \\
179 \\
112 \\
81\end{array}$ & $\begin{array}{r}1510 \\
697 \\
601 \\
449 \\
324 \\
204 \\
141\end{array}$ & $\begin{array}{l}-- \\
-- \\
-- \\
-- \\
--\end{array}$ & $\begin{array}{l}-- \\
=- \\
-- \\
-- \\
--\end{array}$ \\
\hline
\end{tabular}

DURATION TABLE OF DAILY MEAN FLOW FOR PERIOD OF RECORD 1952-63

DISCHARGE, IN FT:/S, THAT WAS EQUALED OR EXCEEDED FOR INDICATED PERCENT OF TIME

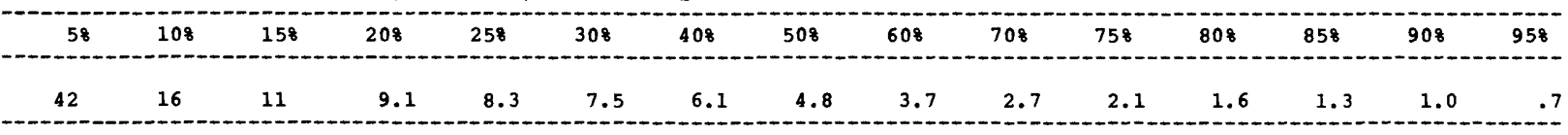


RIO GRANDE BASIN

08382500 GALLINAS RIVER NEAR COLONIAS, NM

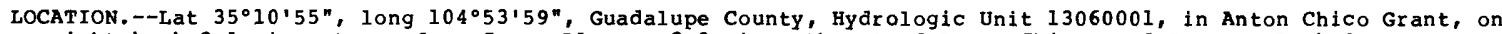
right bank $2.1 \mathrm{mi}$ upstream from Canon Blanco, $2.3 \mathrm{mi}$ southeast of Anton Chico, and Prestun Beck Grants, on $\mathrm{right}$ bank $2.3 \mathrm{mi}$ south of San Miguel-Guadalupe County line, $2.4 \mathrm{mi}$ upstream from mouth, $5.8 \mathrm{mi}$ northwest of Colonias, and $9.0 \mathrm{mi}$ east of Dilia. Mouth at Pecos River mile 789.2 .

DRAINAGE AREA, $--610 \mathrm{mi}^{2}$, approximately.

PERIOD OF RECORD.--January 1951 to current year.

GAGE.-Water-stage recorder. Elevation of gage is 4,944 ft above National Geodetic Vertical Datum of 1929 , from topograph ic map.

REMARKS.--Diversions for irrigation of about 7,000 acres, 1959 determination, upstream from station.

AVERAGE DISCHARGE.--34 years (water years 1952-85), $15.6 \mathrm{ft}^{3} / \mathrm{s}, 11,300 \mathrm{acre-ft/yr}$.

EXTREMES FOR PERIOD OF RECORD.--Maximum discharge, $13,700 \mathrm{ft}^{3} / \mathrm{s}$, July 11 , 1982 , gage height, $19.67 \mathrm{ft}$, from rating curve extended above $1,900 \mathrm{ft}^{3} / \mathrm{s}$ on basis of slope-area measurements at gage heights $8.64 \mathrm{ft}, 12.74 \mathrm{ft}, 16.65$ $\mathrm{ft}$, and $27.2 \mathrm{ft}$; no flow most of time.

EXTREMES OUTSIDE PERIOD OF RECORD,--Flood of about June 1, 1937, reached a stage of about $27.2 \mathrm{ft}$; discharge determined as $26,700 \mathrm{ft} / \mathrm{s}$ by slope-area measurement made in 1951 . A flood of about the same magnitude occurred Sept. $29-30,1904$.

STATISTICAL SUMMARIES

MEAN MONTHLY AND MEAN ANNUAL DISCHARGES 1952-85

\begin{tabular}{|c|c|c|c|c|c|c|}
\hline MONTH & $\begin{array}{c}\text { MINIMUM } \\
\left(\mathrm{FT}^{3} / \mathrm{S}\right)\end{array}$ & $\begin{array}{c}\operatorname{MAXIMUM} \\
\left(\mathrm{FT}^{3} / \mathrm{S}\right)\end{array}$ & $\begin{array}{l}\text { MEAN } \\
\left(F T^{3} / S\right)\end{array}$ & $\begin{array}{c}\text { STAN- } \\
\text { DARD } \\
\text { DEVIA- } \\
\text { TION } \\
\left(\text { FT }^{3} / \mathrm{S}\right)\end{array}$ & $\begin{array}{l}\text { COEFFI- } \\
\text { CIENT OF } \\
\text { VARI- } \\
\text { ATION }\end{array}$ & $\begin{array}{c}\text { PERCENT } \\
\text { OF } \\
\text { ANNUAL } \\
\text { RUNOFF }\end{array}$ \\
\hline $\begin{array}{l}\text { OCTOBER } \\
\text { NOVEMBER } \\
\text { DECEMBER } \\
\text { JANUARY } \\
\text { FEBRUARY } \\
\text { MARCH } \\
\text { APRIL } \\
\text { MAY } \\
\text { JUNE } \\
\text { JULY } \\
\text { AUGUST } \\
\text { SEPTEMBER }\end{array}$ & $\begin{array}{l}0.0 \\
0.0 \\
0.0 \\
0.0 \\
0.0 \\
0.0 \\
0.0 \\
0.0 \\
0.0 \\
0.0 \\
1.1 \\
0.0\end{array}$ & $\begin{array}{c}166 \\
29 \\
12 \\
8.2 \\
6.6 \\
48 \\
269 \\
261 \\
64 \\
151 \\
222 \\
178\end{array}$ & $\begin{array}{l}13 \\
2.9 \\
1.5 \\
1.1 \\
1.0 \\
3.1 \\
17 \\
17 \\
14 \\
34 \\
61 \\
20\end{array}$ & $\begin{array}{l}32 \\
5.9 \\
2.4 \\
2.1 \\
1.7 \\
9.2 \\
62 \\
53 \\
17 \\
40 \\
58 \\
34\end{array}$ & $\begin{array}{l}2.46 \\
2.02 \\
1.65 \\
1.94 \\
1.78 \\
2.97 \\
3.65 \\
3.16 \\
1.27 \\
1.16 \\
.95 \\
1.65\end{array}$ & $\begin{array}{r}7.0 \\
1.6 \\
.8 \\
.6 \\
.5 \\
1.7 \\
9.2 \\
9.0 \\
7.4 \\
18.4 \\
32.8 \\
11.0\end{array}$ \\
\hline ANNUAL & .9 & 67 & 16 & 14 & .90 & 100 \\
\hline
\end{tabular}

MAGNITUDE AND PROBABILITY OF ANNUAL LOW FLOW BASED ON PERIOD OF RECORD 1953-85

\begin{tabular}{|c|c|c|c|c|c|c|}
\hline \multirow{2}{*}{$\begin{array}{l}\text { PERIOD } \\
\text { (CON- } \\
\text { SECU- } \\
\text { TIVE } \\
\text { DAYS) }\end{array}$} & \multicolumn{6}{|c|}{$\begin{array}{l}\text { DISCHARGE, IN FT'3/S, FOR INDICATED RECURRENCE } \\
\text { INTERVAL, IN YEARS, AND ANNUAL NON- } \\
\text { EXCEEDANCE PROBABILITY, IN PERCENT }\end{array}$} \\
\hline & $\begin{array}{c}2 \\
508\end{array}$ & $\begin{array}{c}5 \\
208\end{array}$ & $\begin{array}{l}10 \\
108\end{array}$ & $\begin{array}{l}20 \\
58\end{array}$ & $\begin{array}{l}50 \\
28\end{array}$ & $\begin{array}{r}100 \\
18\end{array}$ \\
\hline $\begin{array}{r}1 \\
3 \\
7 \\
14 \\
30 \\
60 \\
90 \\
120 \\
183\end{array}$ & $\begin{array}{r}0.0 \\
0.0 \\
0.0 \\
0.0 \\
0.0 \\
0.0 \\
0.0 \\
.2 \\
1.0\end{array}$ & $\begin{array}{l}0.0 \\
0.0 \\
0.0 \\
0.0 \\
0.0 \\
0.0 \\
0.0 \\
0.0 \\
0.0\end{array}$ & $\begin{array}{l}0.0 \\
0.0 \\
0.0 \\
0.0 \\
0.0 \\
0.0 \\
0.0 \\
0.0 \\
0.0\end{array}$ & $\begin{array}{l}0.0 \\
0.0 \\
0.0 \\
0.0 \\
0.0 \\
0.0 \\
0.0 \\
0.0 \\
0.0\end{array}$ & $\begin{array}{l}0.0 \\
0.0 \\
0.0 \\
0.0 \\
0.0 \\
0.0 \\
0.0 \\
0.0 \\
0.0\end{array}$ & $\begin{array}{l}-- \\
-- \\
-- \\
-- \\
-- \\
-- \\
--\end{array}$ \\
\hline
\end{tabular}

MAGNITUDE AND PROBABILITY OF ANNUAL HIGH FLOW BASED ON PERIOD OF RECORD 1952-85

\begin{tabular}{|c|c|c|c|c|c|c|}
\hline \multirow{2}{*}{$\begin{array}{l}\text { PERIOD } \\
\text { (CON- } \\
\text { SECU- } \\
\text { TIVE } \\
\text { DAYS) }\end{array}$} & \multicolumn{6}{|c|}{$\begin{array}{l}\text { DISCHARGE, IN FT }{ }^{3} / \mathrm{S}, \text { FOR INDICATED RECURRENCE } \\
\text { INTERVAL, IN YEARS, AND ANNUAL } \\
\text { EXCEEDANCE PROBABILITY, IN PERCENT }\end{array}$} \\
\hline & $\begin{array}{c}2 \\
508\end{array}$ & $\begin{array}{c}5 \\
208\end{array}$ & $\begin{array}{l}10 \\
108\end{array}$ & $\begin{array}{l}25 \\
48\end{array}$ & $\begin{array}{l}50 \\
28\end{array}$ & $\begin{array}{r}100 \\
18\end{array}$ \\
\hline $\begin{array}{r}1 \\
3 \\
7 \\
15 \\
30 \\
60 \\
90\end{array}$ & $\begin{array}{r}691 \\
371 \\
208 \\
125 \\
81 \\
53 \\
41\end{array}$ & $\begin{array}{r}1290 \\
688 \\
399 \\
250 \\
165 \\
110 \\
82\end{array}$ & $\begin{array}{r}1660 \\
888 \\
526 \\
336 \\
222 \\
148 \\
109\end{array}$ & $\begin{array}{r}2070 \\
1110 \\
675 \\
440 \\
290 \\
192 \\
140\end{array}$ & $\begin{array}{r}2320 \\
1260 \\
775 \\
511 \\
335 \\
221 \\
160\end{array}$ & $\begin{array}{l}-- \\
-- \\
-- \\
-- \\
-- \\
-- \\
--\end{array}$ \\
\hline
\end{tabular}

DURATION TABLE OF DAILY MEAN FLOW FOR PERIOD OF RECORD 1952-85 DISCHARGE, IN FT ${ }^{3} / S$, THAT WAS EQUALED OR EXCEEDED FOR INDICATED PERCENT OF TIME

\begin{tabular}{|c|c|c|c|c|c|c|c|c|c|c|c|c|c|c|}
\hline 58 & 108 & 158 & 208 & 258 & 308 & 408 & 508 & 608 & 708 & 758 & 808 & 858 & 908 & 958 \\
\hline 66 & 20 & 7.6 & 4.4 & 2.8 & 1.7 & 0.0 & 0.0 & 0.0 & 0.0 & 0.0 & 0.0 & 0.0 & 0.0 & 0.0 \\
\hline
\end{tabular}


RIO GRANDE BASIN

08382730 LOS ESTEROS CREER ABOVE SANTA ROSA LARE, NM

LOCATION.--Lat 35०05'42", long $104^{\circ} 39^{\prime} 49^{\prime \prime}$, Guadalupe County, Fydrologic Unit 13060001 in Preston-Beck Grant, on left bank, $3.7 \mathrm{mi}$ upstream from mouth, $4.9 \mathrm{mi}$ north-northeast of Santa Rosa Dam, and $10.4 \mathrm{mi}$ north-northeast of Santa Rosa. Mouth at Pecos River mile 763.0 .

DRAINAGE AREA. $--65.6 \mathrm{mi}^{2}$.

PERIOD OF RECORD.--July 1973 to current year. Prior to October 1979, published as "above Los Esteros Reservoir." GAGE.--Water-stage recorder and concrete control. Elevation of gage is 4,767 ft above National Geodetic Vertical Datum of 1929, from topographic map.

REMARRS.--No known diversions or groundwater withdrawals for irrigation upstream from station.

AVERAGE DISCHARGE.--12 years (water years 1974-85), $1.26 \mathrm{ft} 3 / \mathrm{s}, 913 \mathrm{acre-ft} / \mathrm{yr}$.

EXTREMES FOR PERIOD OF RECORD.--Maximum discharge, 3,900 ft3/s, July 24, 1976, gage height, $9.3 \mathrm{ft}$, from rating curve extended above $70 \mathrm{ft}^{3} / \mathrm{s}$ on basis of velocity-area studies and slope-area measurements at gage heights 6.5 $\mathrm{ft}$ and $9.3 \mathrm{ft}$; no flow most of time.

EXTREMES OUPSIDE PERIOD OF RECORD.--A flood of unknown date reached a discharge of about $6,800 \mathrm{ft} 3 / \mathrm{s}$, gage height, $11.6 \mathrm{ft}$, from floodmarks, from rating curve extended as explained above.

STATISTICAL SUMMARIES

MEAN MONTHLY AND MEAN ANNUAL DISCHARGES 1974-85

\begin{tabular}{|c|c|c|c|c|c|c|}
\hline MONTH & $\begin{array}{c}\text { MINIMUM } \\
\left(\mathrm{FT}^{3} / \mathrm{S}\right)\end{array}$ & $\begin{array}{l}\text { MAXIMUM } \\
\left(\mathrm{FT}^{3} / \mathrm{S}\right)\end{array}$ & $\begin{array}{l}\text { MEAN } \\
\left(\mathrm{FT}^{3} / \mathrm{S}\right)\end{array}$ & $\begin{array}{l}\text { STAN- } \\
\text { DARD } \\
\text { DEVIA- } \\
\text { TION } \\
\left(\text { FT }^{3} / \mathrm{S}\right)\end{array}$ & $\begin{array}{l}\text { COEFFI- } \\
\text { CIENT OF } \\
\text { VARI- } \\
\text { ATION }\end{array}$ & $\begin{array}{c}\text { PERCENT } \\
\text { OF } \\
\text { ANNUAL } \\
\text { RUNOFF }\end{array}$ \\
\hline OCTOBER & 0.0 & 1.5 & .2 & .4 & 2.26 & 1.3 \\
\hline NOVEMBE $R$ & 0.0 & 2.3 & .2 & .7 & 3.25 & 1.3 \\
\hline DECEMBER & 0.0 & 0.0 & 0.0 & 0.0 & -- & 0.0 \\
\hline JANUARY & 0.0 & 0.0 & 0.0 & 0.0 & -- & 0.0 \\
\hline FEBROARY & 0.0 & 0.0 & 0.0 & 0.0 & -- & 0.0 \\
\hline MARCH & 0.0 & 0.0 & 0.0 & 0.0 & -- & 0.0 \\
\hline APRIL & 0.0 & 1.6 & .1 & .5 & 3.46 & .9 \\
\hline MAY & 0.0 & 1.7 & .2 & .5 & 2.29 & 1.4 \\
\hline JUNE & 0.0 & 11 & 1.6 & 3.0 & 1.86 & 10.9 \\
\hline JULY & 0.0 & 34 & 4.1 & 9.7 & 2.34 & 27.7 \\
\hline AUGUST & 0.0 & 48 & 7.3 & 13 & 1.81 & 48.8 \\
\hline SEPTEMBER & 0.0 & 6.8 & 1.2 & 1.9 & 1.67 & 7.8 \\
\hline ANNUAL & .1 & 5.4 & 1.3 & 1.7 & 1.33 & 100 \\
\hline
\end{tabular}

MAGNITUDE AND PROBABILITY OF ANNUAL LOW FLOW BASED ON PERIOD OF RECORD 1975-85

\begin{tabular}{|c|c|c|c|c|c|c|}
\hline \multirow{2}{*}{$\begin{array}{l}\text { PERIOD } \\
\text { (CON- } \\
\text { SECU- } \\
\text { TIVE } \\
\text { DAYS) }\end{array}$} & \multicolumn{6}{|c|}{$\begin{array}{l}\text { DISCHARGE, IN FT'3/S, FOR INDICATED RECURRENCE } \\
\text { INTERVAL, IN YEARS, AND ANNUAL NON- } \\
\text { EXCEEDANCE PROBABIL ITY, IN PERCENT }\end{array}$} \\
\hline & $\begin{array}{c}2 \\
508\end{array}$ & $\begin{array}{c}5 \\
208\end{array}$ & $\begin{array}{l}10 \\
108\end{array}$ & 20 & $\begin{array}{l}50 \\
28\end{array}$ & $\begin{array}{r}100 \\
18\end{array}$ \\
\hline $\begin{array}{r}1 \\
3 \\
7 \\
14 \\
30 \\
60 \\
90 \\
120 \\
183\end{array}$ & $\begin{array}{l}0.0 \\
0.0 \\
0.0 \\
0.0 \\
0.0 \\
0.0 \\
0.0 \\
0.0 \\
0.0\end{array}$ & $\begin{array}{l}0.0 \\
0.0 \\
0.0 \\
0.0 \\
0.0 \\
0.0 \\
0.0 \\
0.0 \\
0.0\end{array}$ & $\begin{array}{l}0.0 \\
0.0 \\
0.0 \\
0.0 \\
0.0 \\
0.0 \\
0.0 \\
0.0 \\
0.0\end{array}$ & $\begin{array}{l}0.0 \\
0.0 \\
0.0 \\
0.0 \\
0.0 \\
0.0 \\
0.0 \\
0.0 \\
0.0\end{array}$ & $\begin{array}{l}-- \\
-- \\
-- \\
-- \\
-- \\
-- \\
--\end{array}$ & $\begin{array}{l}=- \\
-- \\
-- \\
-- \\
-- \\
-- \\
--\end{array}$ \\
\hline
\end{tabular}

MAGNITUDE AND PROBABILITY OF ANNUAL HIGH FLOW BASED ON PERIOD OF RECORD 1974-85

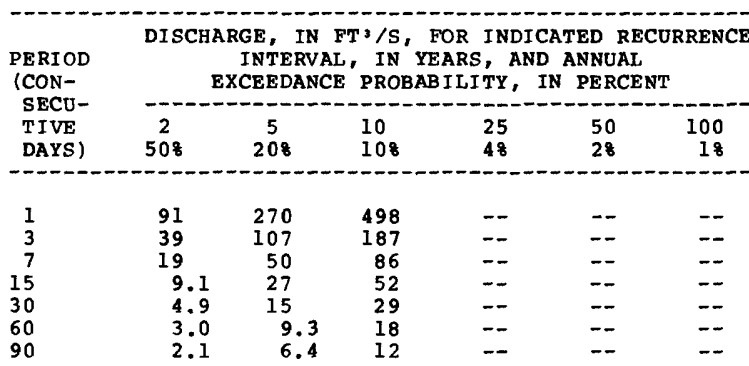

DURATION TABLE OF DAILY MEAN FLOW FOR PERIOD OF RECORD 1974-85 DISCHARGE, IN FT ${ }^{3} / \mathrm{S}$, THAT WAS EQUALED OR EXCEEDED FOR INDICATED PERCENT OF TIME

\begin{tabular}{|c|c|c|c|c|c|c|c|c|c|c|c|c|c|c|}
\hline 58 & 108 & 158 & 208 & 258 & 308 & 408 & 508 & 608 & 708 & 758 & 808 & 858 & 908 & 958 \\
\hline .2 & 0.0 & 0.0 & 0.0 & 0.0 & 0.0 & 0. & 0.0 & 0. & 0.0 & 0.0 & 0. & 0. & 0.0 & 0.0 \\
\hline
\end{tabular}


RIO GRANDE BASIN

08382760 LOS ESTEROS CREEK TRIBUTARY ABOVE SANTA ROSA LARE, NM

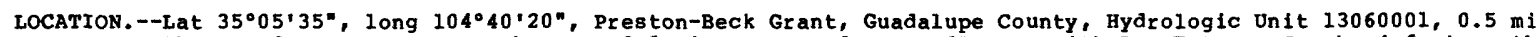
west-southwest of Los Esteros Creek gage, $0.8 \mathrm{mi}$ upstream from confluence with Los Esteros Creek, $4.6 \mathrm{mi}$ northnortheast of Santa Rosa Dam, and $10.2 \mathrm{mi}$ north-northeast of Santa Rosa.

DRAINAGE AREA. $--13.7 \mathrm{mi}^{2}$.

PERIOD OF RECORD.--July 1973 to current year. Prior to October 1979, published as "above Los Esteros Reservoir." GAGE.--Water-stage recorder and concrete control. Elevation of gage is 4,758 ft above National Geodetic Vertical Datum of 1929, from-topographic map.

REMARKs.--No known diversions or groundwater withdrawals for irrigation upstream from station.

AVERAGE DISCHARGE.--12 years (water years 1974-85), $0.31 \mathrm{ft} / \mathrm{s}, 225 \mathrm{acre}-\mathrm{ft} / \mathrm{yr}$.

EXTREMES FOR PERIOD OF RECORD.--Maximum discharge, 7,400 ft'/s, Aug. 29, 1977, gage height, $7.80 \mathrm{ft}$, from rating curve extended above $0.5 \mathrm{ft}^{3} / \mathrm{s}$ on basis of velocity-area studies and slope-area measurement at gage height 7.80 $f t$; no flow most of the time.

STATISTICAL SUMMARIES

MEAN MONTHLY AND MEAN ANNUAL DISCHARGES 1974-85

\begin{tabular}{|c|c|c|c|c|c|c|}
\hline MONTH & $\begin{array}{l}\text { MINIMUM } \\
\left(\mathrm{FT}^{2} / \mathrm{S}\right)\end{array}$ & $\begin{array}{c}\text { MAXIMUM } \\
\left(\mathrm{FT}^{3} / \mathrm{S}\right)\end{array}$ & $\begin{array}{l}\text { MEAN } \\
\left(\mathrm{FT}^{3} / \mathrm{S}\right)\end{array}$ & $\begin{array}{l}\text { STAN- } \\
\text { DARD } \\
\text { DEVIA- } \\
\text { TION } \\
\left(F^{3} / \mathrm{S}\right)\end{array}$ & $\begin{array}{l}\text { COEFFI - } \\
\text { CIENT OF } \\
\text { VARI- } \\
\text { ATION }\end{array}$ & $\begin{array}{c}\text { PERCENT } \\
\text { OF } \\
\text { ANNUAL } \\
\text { RUNOFF }\end{array}$ \\
\hline $\begin{array}{l}\text { OCTOBER } \\
\text { NOVEMBER } \\
\text { DECEMBER } \\
\text { JANUARY } \\
\text { FEBRUARY } \\
\text { MARCH } \\
\text { APRIL } \\
\text { MAY } \\
\text { JUNE } \\
\text { JULY } \\
\text { AUGUST } \\
\text { SEPTEMBER }\end{array}$ & $\begin{array}{l}0.0 \\
0.0 \\
0.0 \\
0.0 \\
0.0 \\
0.0 \\
0.0 \\
0.0 \\
0.0 \\
0.0 \\
0.0 \\
0.0\end{array}$ & $\begin{array}{r}0.0 \\
0.0 \\
0.0 \\
0.0 \\
0.0 \\
0.0 \\
0.0 \\
.7 \\
1.0 \\
4.2 \\
28 \\
.5\end{array}$ & $\begin{array}{r}0.0 \\
0.0 \\
0.0 \\
0.0 \\
0.0 \\
0.0 \\
0.0 \\
.1 \\
.1 \\
.4 \\
2.9 \\
.1\end{array}$ & $\begin{array}{r}0.0 \\
0.0 \\
0.0 \\
0.0 \\
0.0 \\
0.0 \\
0.0 \\
.2 \\
.3 \\
1.2 \\
8.1 \\
.2\end{array}$ & $\begin{array}{r}-- \\
-- \\
-- \\
-- \\
-- \\
-\overline{-} \\
3.17 \\
2.31 \\
2.70 \\
2.76 \\
2.14\end{array}$ & $\begin{array}{r}0.0 \\
0.0 \\
0.0 \\
0.0 \\
0.0 \\
0.0 \\
0.0 \\
1.6 \\
3.6 \\
12.1 \\
80.8 \\
1.9\end{array}$ \\
\hline ANNUAL & 0.0 & 2.6 & .3 & .7 & 2.35 & 100 \\
\hline
\end{tabular}

MAGNITUDE AND PROBABILITY OF ANNUAL LOW FLOW BASED ON PERIOD OF RECORD 1975-85

\begin{tabular}{|c|c|c|c|c|c|c|}
\hline \multirow{2}{*}{$\begin{array}{l}\text { PERIOD } \\
\text { (CON- } \\
\text { SECU- } \\
\text { TIVE } \\
\text { DAYS) }\end{array}$} & \multicolumn{6}{|c|}{$\begin{array}{l}\text { DISCHARGE, IN FT'/S, FOR INDICATED RECURRENCE } \\
\text { INTERVAL, IN YEARS, AND ANNUAL NON- } \\
\text { EXCEEDANCE PROBABILITY, IN PERCENT }\end{array}$} \\
\hline & $\begin{array}{c}2 \\
508\end{array}$ & $\begin{array}{c}5 \\
208\end{array}$ & $\begin{array}{l}10 \\
108\end{array}$ & $\begin{array}{l}20 \\
58\end{array}$ & $\begin{array}{l}50 \\
28\end{array}$ & $\begin{array}{r}100 \\
18\end{array}$ \\
\hline $\begin{array}{r}1 \\
3 \\
7 \\
14 \\
30 \\
60 \\
90 \\
120 \\
183\end{array}$ & $\begin{array}{l}0.0 \\
0.0 \\
0.0 \\
0.0 \\
0.0 \\
0.0 \\
0.0 \\
0.0 \\
0.0\end{array}$ & $\begin{array}{l}0.0 \\
0.0 \\
0.0 \\
0.0 \\
0.0 \\
0.0 \\
0.0 \\
0.0 \\
0.0\end{array}$ & $\begin{array}{l}0.0 \\
0.0 \\
0.0 \\
0.0 \\
0.0 \\
0.0 \\
0.0 \\
0.0 \\
0.0\end{array}$ & $\begin{array}{l}0.0 \\
0.0 \\
0.0 \\
0.0 \\
0.0 \\
0.0 \\
0.0 \\
0.0 \\
0.0\end{array}$ & $\begin{array}{l}-- \\
-- \\
-- \\
-- \\
-- \\
--\end{array}$ & $\begin{array}{l}=- \\
-- \\
\overline{-} \\
\overline{-} \\
\overline{-} \\
\overline{-}\end{array}$ \\
\hline
\end{tabular}

MAGNITUDE AND PROBABILITY OF ANNUAL HIGH FLOW BASED ON PERIOD OF RECORD 1974-85

\begin{tabular}{|c|c|c|c|c|c|c|}
\hline $\begin{array}{l}\text { PERIOD } \\
\text { (CON- }\end{array}$ & & $\begin{array}{l}\text { GE, } \\
\text { INTE } \\
\text { CEED }\end{array}$ & $\begin{array}{l}I^{3} / \mathrm{S} \\
\text { IN } \\
\text { PRO }\end{array}$ & $\begin{array}{l}\text { INI } \\
\text { I TY }\end{array}$ & $\begin{array}{l}\text { TED } \\
\text { ANNU } \\
\text { PER }\end{array}$ & 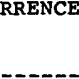 \\
\hline $\begin{array}{l}\text { TIVE } \\
\text { DAYS) }\end{array}$ & $\begin{array}{c}2 \\
508\end{array}$ & $\begin{array}{c}5 \\
208\end{array}$ & $\begin{array}{l}10 \\
108\end{array}$ & $\begin{array}{l}25 \\
48\end{array}$ & $\begin{array}{l}50 \\
28\end{array}$ & $\begin{array}{r}100 \\
18\end{array}$ \\
\hline
\end{tabular}

$\begin{array}{rrrrrrr}1 & & & & & & \\ 3 & 6.4 & 56 & 184 & - & -- & - \\ 7 & 2.8 & 23 & 72 & - & - & - \\ 15 & 1.2 & 10 & 32 & - & - \\ 30 & .6 & 5.1 & 16 & - & - \\ 60 & .4 & 3.0 & 9.1 & - & -- & - \\ 90 & 0.0 & 0.0 & 0.0 & -- & -- & -\end{array}$

DURATION TABLE OF DAILY MEAN FLOW FOR PERIOD OF RECORD 1974-85

DISCHARGE, IN FT ${ }^{3} / S$, THAT WAS EQUALED OR EXCEEDED FOR INDICATED PERCENT OF TIME

\begin{tabular}{|c|c|c|c|c|c|c|c|c|c|c|c|c|c|c|}
\hline 58 & 108 & 158 & 208 & 258 & 308 & 408 & 508 & 608 & 708 & 758 & 808 & 858 & 908 & 958 \\
\hline 0.0 & 0.0 & 0.0 & 0. & 0.0 & 0.0 & 0.0 & 0.0 & 0.0 & 0.0 & 0.0 & 0.0 & 0.0 & 0.0 & \\
\hline
\end{tabular}


LOCATION.--Lat $34^{\circ} 56^{\circ} 36^{\prime \prime}$, long $104^{\circ} 41^{\prime} 55^{\prime \prime}$, in NWłSEł sec.3, T. 8 N., R.21 E., Guadalupe County, Hydrologic Unit 13060001 , on left bank, $0.4 \mathrm{mi}$ downstream from bridge on 0.5 . Highway I-40, $0.6 \mathrm{mi}$ upstream from bridge on o.s. Highway I-40 (Business) in Santa Rosa, $1.9 \mathrm{mi}$ upstream from El Rito Creek, and at mile 748.4

DRAINAGE AREA. - - 2,650 $\mathrm{mi}^{2}$, approximately (contributing area).

PERIOD OF RECORD.--May 1903 to December 1905 (gage heights only), January to December 1906, February 1910 to Ju1y 1911, September 1912 to December 1924, March to May 1927, July 1927, January 1928 to current year. Monthly discharge only for some periods, published in wsp 1312. Figures of daily discharge for Apr. 5-20, May 4-7, 11, Aug. 13, 16-18, 24, Sept. 7-9, 11, 13, 19, 21, 23, 25, 27, Oct. 1-31, Nov. 3, 4, 9, 11, 20, 22, 1910, and Feb. to Mar. 31, June 1 to July 31, 1911, published in wSP 358 are unreliable and should not be used.

REVISED RECORDS.--WSP 1512: 1913-15. SEe alsO PERIOD OF RECORD.

GAGE.- Water-stage recorder and partial concrete control. Elevation of gage is $4,537.56 \mathrm{ft}$ above National Geodetic Vertical Datum of 1929. For history of changes prior to Sept. 13, 1967, see WSP 2123.

REMARKS.--Flow regulated by Santa Rosa Lake (station 08382810) since April 1980. Diversions for irrigation of about 12,000 acres, 1959 determination, upstream from station.

AVERAGE DISCHARGE, - -53 years (1913-14, 1929-79), $126 \mathrm{ft}^{3} / \mathrm{s}, 91,290$ acre-ft/yr, prior to completion of Santa Rosa Dam.

6 years $(1980-1985), 83.1 \mathrm{ft}^{3} / \mathrm{s}, 60,210$ acre-ft/yr, since completion of Santa Rosa Dam.

EXTREMES FOR PERIOD OF RECORD.--Maximum discharge, 55,200 ft $3 / \mathrm{s}$, June 2,1937 , gage height, $25.7 \mathrm{ft}$, site and datum then in use, from rating curve extended above $32,000 \mathrm{ft} / \mathrm{s}$; minimum, $0.28 \mathrm{ft} / \mathrm{s}, \mathrm{Jan}$. 7, 1971. The flood of then in use, from rating curve extended above $32,000 \mathrm{ft} / \mathrm{s} ;$ minimum, $0.28 \mathrm{ft} / \mathrm{s}, \mathrm{Jan}$. 7, 1971. The flood of datum then in use, discharge, $45,000 \mathrm{ft} / \mathrm{s}$, by Kutter's formula. Flood of June 9, 1903 , reached a stage of 21.1 datum then in use, discharge, $45,000 \mathrm{ft} / \mathrm{s}$, by Rutter's formula. Flood of June 9, $1903, \mathrm{reac}$
$\mathrm{ft}$, same site and datum as in 1904 , discharge, $34,000 \mathrm{ft} / \mathrm{s}$, by comparison with $1904 \mathrm{f} 100 \mathrm{~d}$ $\mathrm{ft}$, same site and datum as in 1904 , discharge, $34,000 \mathrm{ft} / \mathrm{s}$, by comparison with 1904 f100d, $\mathrm{f}^{3}$, Since completion of Santa Rosa Dam in 1980 , maximum discharge, 7,
$\mathrm{ft}$; minimum daily, $2.0 \mathrm{ft} / \mathrm{s}$, July $23-25,31$, and Aug. 1, 12, 1985 .

STATISTICAL SUMMARIES (PERIOD BEFORE REGULATION OF SANTA ROSA DAM)

MEAN MONTHLY AND MEAN ANNUAL DISCHARGES 1913-14,
MAGNITUDE AND PROBABILITY OF ANNUAL LOW FLOW BASED ON PERIOD OF RECORD 1914, 1929-79

\begin{tabular}{|c|c|c|c|c|c|c|c|c|c|c|c|c|c|}
\hline \multirow[b]{2}{*}{ MONTH } & \multirow{2}{*}{$\begin{array}{c}\text { MINIMUM } \\
\left(\mathrm{FT}^{3} / \mathrm{S}\right)\end{array}$} & \multirow{2}{*}{$\begin{array}{l}\text { MAXIMUM } \\
\left(\mathrm{FT}^{3} / \mathrm{S}\right)\end{array}$} & \multirow{2}{*}{$\begin{array}{l}\text { MEAN } \\
\left(\text { FT }^{3} / \mathrm{S}\right)\end{array}$} & \multirow[t]{2}{*}{$\begin{array}{l}\text { STAN- } \\
\text { DARD } \\
\text { DEVIA- } \\
\text { TION } \\
\left(\text { FT }^{3} / \mathrm{S}\right)\end{array}$} & \multirow{2}{*}{$\begin{array}{l}\text { COEFFI- } \\
\text { CIENT OF } \\
\text { VARI- } \\
\text { ATION }\end{array}$} & \multirow{2}{*}{$\begin{array}{l}\text { PERCENT } \\
\text { OF } \\
\text { ANNUAL } \\
\text { RUNOFF }\end{array}$} & \multirow{2}{*}{$\begin{array}{l}\text { PERIOD } \\
\text { (CON- } \\
\text { SECU- } \\
\text { TIVE } \\
\text { DAYS) }\end{array}$} & \multicolumn{6}{|c|}{$\begin{array}{l}\text { DISCHARGE, IN FT }{ }^{3} / S \text {, FOR INDICATED RECURRENCE } \\
\text { INTERVAL, IN YEARS, AND ANNUAL NON- } \\
\text { EXCEEDANCE PROBABILITY, IN PERCENT }\end{array}$} \\
\hline & & & & & & & & $\begin{array}{c}2 \\
508\end{array}$ & $\begin{array}{c}5 \\
208\end{array}$ & $\begin{array}{l}10 \\
108\end{array}$ & $\begin{array}{l}20 \\
58\end{array}$ & $\begin{array}{l}50 \\
28\end{array}$ & $\begin{array}{r}100 \\
18\end{array}$ \\
\hline $\begin{array}{l}\text { OCTOBER } \\
\text { NOVEMBER } \\
\text { DECEMBER } \\
\text { JANUARY } \\
\text { FEBROARY } \\
\text { MARCH } \\
\text { APR IL } \\
\text { MAY } \\
\text { JUNE }\end{array}$ & $\begin{array}{l}5.5 \\
7.5 \\
7.6 \\
6.9 \\
6.3 \\
6.8 \\
6.4 \\
9.7 \\
8.6\end{array}$ & $\begin{array}{r}806 \\
273 \\
82 \\
61 \\
51 \\
166 \\
1061 \\
2452 \\
1693\end{array}$ & $\begin{array}{r}94 \\
32 \\
23 \\
21 \\
20 \\
26 \\
123 \\
289 \\
238\end{array}$ & $\begin{array}{r}155 \\
38 \\
13 \\
10 \\
8.9 \\
27 \\
213 \\
424 \\
339\end{array}$ & $\begin{array}{r}1.66 \\
1.19 \\
.55 \\
.51 \\
.46 \\
1.00 \\
1.73 \\
1.47 \\
1.42\end{array}$ & $\begin{array}{r}6.2 \\
2.1 \\
1.5 \\
1.4 \\
1.3 \\
1.8 \\
8.2 \\
19.3 \\
15.9\end{array}$ & $\begin{array}{r}1 \\
3 \\
7 \\
14 \\
30 \\
60 \\
90 \\
120 \\
183\end{array}$ & $\begin{array}{l}9.9 \\
11 \\
12 \\
13 \\
15 \\
16 \\
17 \\
18 \\
25\end{array}$ & $\begin{array}{l}5.3 \\
6.1 \\
7.2 \\
8.2 \\
9.8 \\
11 \\
12 \\
13 \\
15\end{array}$ & $\begin{array}{l}3.7 \\
4.5 \\
5.6 \\
6.5 \\
7.9 \\
9.4 \\
10 \\
11 \\
12\end{array}$ & $\begin{array}{r}2.7 \\
3.5 \\
4.6 \\
5.4 \\
6.6 \\
8.1 \\
8.8 \\
9.4\end{array}$ & $\begin{array}{l}1.9 \\
2.6 \\
3.6 \\
4.3 \\
5.3 \\
6.8 \\
7.6 \\
8.1 \\
9.3\end{array}$ & $\begin{array}{l}1.5 \\
2.1 \\
3.1 \\
3.7 \\
4.7 \\
6.1 \\
6.9 \\
7.4 \\
8.6\end{array}$ \\
\hline $\begin{array}{l}\text { JULY } \\
\text { AUGUST } \\
\text { SEPTEMBER }\end{array}$ & $\begin{array}{l}11 \\
23 \\
5.8\end{array}$ & $\begin{array}{l}1002 \\
1205 \\
1909\end{array}$ & $\begin{array}{l}187 \\
257 \\
190\end{array}$ & $\begin{array}{l}191 \\
222 \\
358\end{array}$ & $\begin{array}{r}1.02 \\
.86 \\
1.88\end{array}$ & $\begin{array}{l}12.5 \\
17.2 \\
12.7\end{array}$ & & & & & & & \\
\hline ANNUAL & 25 & 663 & 126 & 112 & .89 & 100 & & & & & & & \\
\hline
\end{tabular}

MAGNITUDE AND PROBABILITY OF ANNUAL HIGH FLOW BASED ON PERIOD OF RECORD 1913-14, 1929-79

\begin{tabular}{|c|c|c|c|c|c|c|}
\hline \multirow{2}{*}{$\begin{array}{l}\text { PERIOD } \\
\text { (CON- } \\
\text { SECU- } \\
\text { TIVE } \\
\text { DAYS) }\end{array}$} & \multicolumn{6}{|c|}{$\begin{array}{l}\text { DISCHARGE, IN FT } 3 / \mathrm{S} \text {, FOR INDICATED RECURRENCE } \\
\text { INTERVAL, IN YEARS, AND ANNUAL } \\
\text { EXCEEDANCE PROBABILITY, IN PERCENT }\end{array}$} \\
\hline & $\stackrel{2}{508}$ & $\begin{array}{c}5 \\
208\end{array}$ & $\begin{array}{l}10 \\
108\end{array}$ & $\begin{array}{l}25 \\
48\end{array}$ & $\begin{array}{l}50 \\
28\end{array}$ & $\begin{array}{r}100 \\
18\end{array}$ \\
\hline $\begin{array}{r}1 \\
3 \\
7 \\
15 \\
30 \\
60 \\
90\end{array}$ & $\begin{array}{r}3010 \\
1700 \\
1010 \\
655 \\
454 \\
305 \\
246\end{array}$ & $\begin{array}{r}6030 \\
3280 \\
1870 \\
1230 \\
849 \\
586 \\
468\end{array}$ & $\begin{array}{r}8780 \\
4670 \\
2600 \\
1730 \\
1180 \\
833 \\
655\end{array}$ & $\begin{array}{r}13200 \\
6870 \\
3740 \\
2500 \\
1680 \\
1220 \\
937\end{array}$ & $\begin{array}{r}17300 \\
8840 \\
4750 \\
3200 \\
2120 \\
1570 \\
1180\end{array}$ & $\begin{array}{r}22100 \\
11100 \\
5910 \\
4000 \\
2610 \\
1980 \\
1450\end{array}$ \\
\hline
\end{tabular}

DURATION TABLE OF DAILY MEAN FLOW FOR PERIOD OF RECORD 1913-14, 1929-79 DISCHARGE, IN FT'/S, THAT WAS EQUALED OR EXCEEDED FOR INDICATED PERCENT OF TIME

\begin{tabular}{|c|c|c|c|c|c|c|c|c|c|c|c|c|c|c|}
\hline 58 & 108 & 158 & 208 & 258 & 308 & 408 & 508 & 608 & 708 & 758 & 808 & 858 & 908 & 958 \\
\hline 503 & 273 & 168 & 105 & 64 & 46 & 32 & 25 & 21 & 17 & 16 & 14 & 13 & 11 & 8. \\
\hline
\end{tabular}


RIO GRANDE BASIN

08383500 PECOS RIVER NEAR PUERTO DE LUNA, NM (Survelllance network station)

LOCATION.--Lat $34^{\circ} 43^{\prime} 48^{\prime \prime}$, long $104^{\circ} 31^{\prime} 28^{\prime \prime}$, in NEłSEłNWt sec.20, T.6 N., R.23 E., Guadalupe County, Hydrologic Unit

13060001 , on left bank $9.0 \mathrm{mi}$ southeast of Puerto de Luna, $17.5 \mathrm{mi}$ upstream from Sumner Dam, and at mile 719.5 .

DRAINAGE AREA. $--3,970 \mathrm{ml}^{2}$, approximately (contributing area).

PERIOD OF RECORD. - April 1938 to current year.

REVISED RECORDS.--WSP 1512: 1939.

GAGE.-Water-stage recorder and concrete control. Elevation of gage is 4,311.34 ft above National Geodetic Vertical Datum of 1929. Prior to Apr. 15, 1954, at datum 1.0 ft higher.

REMARKS.--Flow regulated by Santa Rosa Lake (station 08382810) since April 1980. Diversions for irrigation of about 12,500 acres, 1959 determination, upstream from station. Discharge represents inflow to Lake Sumner.

AVERAGE DISCHARGE, -42 years $(1938-79), 209 \mathrm{ft} / \mathrm{s}, 151,400$ acre-ft/yr, prior to completion of Santa Rosa Dam. 6 years $(1980-85), 166 \mathrm{ft}^{3} / \mathrm{s}, 120,300$ acre- $\mathrm{ft} / \mathrm{yr}$, since completion of Santa Rosa Dam.

EXTRRMES FOR PERIOD OF RECORD. --Maximum discharge, 48,600 ft $3 / \mathrm{s}$, Sept. 1, 1942, gage helght, $17.00 \mathrm{ft}$, from rating

curve extended above $7,400 \mathrm{ft} / \mathrm{s}$ on basis of flow "at Santa Rosa"; minimum, $11 \mathrm{ft} / \mathrm{s}$, Jan. 31 , 1951 . Since completion of Santa Rosa Dam in 1980 , maximum discharge, $10,900 \mathrm{ft} / \mathrm{s}, \mathrm{June} 10,1982$, gage height, 7,44
$\mathrm{ft}$; minimum, $41 \mathrm{ft} / \mathrm{s}$, Aug. 13, 14, 1985.

EXTREMES OUTSIDE PERIOD OF RECORD.--Maximum flood since at least 1886 occurred June 2, 1937, when peak at Santa Rosa was $55,200 \mathrm{ft}^{2 / \mathrm{s}}$ and peak inflow to Lake sumner was about 75,000 ft/s. Flood of July 24 , 1895 , was reported as "highest in 10 years." Other major floods occurred on June 9, 1903, Sept. 30, 1904, and May 1, 1914.

STATISTICAL SUMMARIES (PERIOD BEFORE REGULATION OF SANTA ROSA DAM)

MEAN MONTHLY AND MEAN ANNUAL DISCHARGES 1939-79

MAGNITUDE AND PROBABILITY OF ANNUAL LOW FLOW BASED ON PERIOD OF RECORD 1940-79

\begin{tabular}{|c|c|c|c|c|c|c|}
\hline MONTH & $\begin{array}{l}\text { MINIMUM } \\
(\mathrm{FT} ; / \mathrm{S})\end{array}$ & $\begin{array}{c}\text { MAXIMUM } \\
(\mathrm{FT}, / \mathrm{S})\end{array}$ & $\begin{array}{l}\text { MEAN } \\
\left(\mathrm{FT}^{3} / \mathrm{S}\right)\end{array}$ & $\begin{array}{l}\text { STAN- } \\
\text { DARD } \\
\text { DEVIA- } \\
\text { TION } \\
\text { (PT'/S) }\end{array}$ & $\begin{array}{l}\text { COEFFI- } \\
\text { CIENT OF } \\
\text { VARI- } \\
\text { ATION }\end{array}$ & $\begin{array}{c}\text { PERCENT } \\
\text { OF } \\
\text { ANNUAL } \\
\text { RUNOFF }\end{array}$ \\
\hline OCTOBER & 62 & 1174 & 189 & 220 & 1.16 & 7.6 \\
\hline NOVEMBER & $\begin{array}{l}62 \\
73\end{array}$ & $\begin{array}{r}11 / 4 \\
379\end{array}$ & $\begin{array}{l}189 \\
116\end{array}$ & 50 & $\begin{array}{r}1.16 \\
.43\end{array}$ & $\begin{array}{l}7.6 \\
4.6\end{array}$ \\
\hline DECEMBER & 75 & 158 & 106 & 17 & .16 & 4.2 \\
\hline JANUARY & 77 & 147 & 103 & 14 & .14 & 4.1 \\
\hline FEBRUARY & 80 & 144 & 101 & 13 & .13 & 4.0 \\
\hline MARCH & 80 & 232 & 104 & 29 & .28 & 4.2 \\
\hline APRIL & 67 & 1084 & 193 & 230 & 1.19 & 7.7 \\
\hline MAY & 68 & 2563 & 342 & 478 & 1.40 & 13.7 \\
\hline JUNE & 60 & 1687 & 290 & 309 & 1.07 & 11.6 \\
\hline JULY & 69 & 978 & 290 & 211 & .73 & 11.6 \\
\hline AUGUST & 78 & 1389 & 373 & 260 & .70 & 14.9 \\
\hline SEPTEMBER & 58 & 2342 & 290 & 465 & 1.60 & 11.6 \\
\hline ANNUAC & 100 & 798 & 209 & 133 & .64 & 100 \\
\hline
\end{tabular}

\begin{tabular}{|c|c|c|c|c|c|c|}
\hline \multirow{2}{*}{$\begin{array}{l}\text { PERIOD } \\
\text { (CON- } \\
\text { SECU- } \\
\text { TIVE } \\
\text { DAYS) }\end{array}$} & \multicolumn{6}{|c|}{$\begin{array}{l}\text { DISCHARGE, IN FT'/S, FOR INDICATED RECURRENCE } \\
\text { INTERVAL, IN YEARS, AND ANNUAL NON- } \\
\text { EXCEEDANCE PROBABILITY, IN PERCENT' }\end{array}$} \\
\hline & $\begin{array}{c}2 \\
508\end{array}$ & $\begin{array}{c}5 \\
208\end{array}$ & $\begin{array}{l}10 \\
108\end{array}$ & $\begin{array}{l}20 \\
58\end{array}$ & $\begin{array}{l}50 \\
28\end{array}$ & $\begin{array}{r}100 \\
18\end{array}$ \\
\hline $\begin{array}{r}1 \\
3 \\
7 \\
14 \\
30 \\
60 \\
90 \\
120 \\
183\end{array}$ & $\begin{array}{r}63 \\
65 \\
69 \\
74 \\
82 \\
90 \\
94 \\
98 \\
101\end{array}$ & $\begin{array}{l}50 \\
52 \\
56 \\
61 \\
69 \\
77 \\
81 \\
86 \\
90\end{array}$ & $\begin{array}{l}44 \\
46 \\
51 \\
56 \\
63 \\
71 \\
75 \\
80 \\
89\end{array}$ & $\begin{array}{l}40 \\
42 \\
46 \\
51 \\
58 \\
66 \\
70 \\
76 \\
88\end{array}$ & $\begin{array}{l}36 \\
38 \\
42 \\
47 \\
54 \\
61 \\
65 \\
72 \\
88\end{array}$ & $\begin{array}{l}33 \\
35 \\
39 \\
44 \\
51 \\
58 \\
62 \\
69 \\
88\end{array}$ \\
\hline
\end{tabular}

MAGNITUDE AND PROBABILITY OF ANNUAL HIGH FLON BASED ON PERIOD OF RECORD 1939-79

\begin{tabular}{|c|c|c|c|c|c|c|}
\hline \multirow{2}{*}{$\begin{array}{l}\text { PERIOD } \\
\text { (CON- } \\
\text { SECU- } \\
\text { TIVE } \\
\text { DAYS) }\end{array}$} & \multicolumn{6}{|c|}{$\begin{array}{l}\text { DISCHARGE, IN FT' }{ }^{3} \text {, F, FOR INDICATED RECURRENCE } \\
\text { INTERVAL, IN YEARS, AND ANNUAL } \\
\text { EXCEEDANCE PROBABILITY, IN PERCENT }\end{array}$} \\
\hline & $\begin{array}{c}2 \\
508\end{array}$ & $\begin{array}{c}5 \\
208\end{array}$ & $\begin{array}{l}10 \\
108\end{array}$ & $\begin{array}{l}25 \\
48\end{array}$ & $\begin{array}{l}50 \\
28\end{array}$ & $\begin{array}{r}100 \\
18\end{array}$ \\
\hline $\begin{array}{r}1 \\
3 \\
7 \\
15 \\
30 \\
60 \\
90\end{array}$ & $\begin{array}{r}2890 \\
1790 \\
1120 \\
763 \\
563 \\
396 \\
324\end{array}$ & $\begin{array}{r}5660 \\
3240 \\
1920 \\
1340 \\
975 \\
677 \\
543\end{array}$ & $\begin{array}{r}8580 \\
4690 \\
2650 \\
1870 \\
1340 \\
931 \\
741\end{array}$ & $\begin{array}{r}14100 \\
7310 \\
3830 \\
2730 \\
1910 \\
1350 \\
1070\end{array}$ & $\begin{array}{r}20000 \\
10000 \\
4950 \\
3540 \\
2440 \\
1750 \\
1370\end{array}$ & $\begin{array}{r}27900 \\
13600 \\
6310 \\
4520 \\
3060 \\
2230 \\
1750\end{array}$ \\
\hline
\end{tabular}

DURATION TABLE OF DAILY MEAN FLOW FOR PERIOD OF RECORD 1939-79 DISCHARGE, IN ET $3 / \mathrm{S}$, THAT WAS EQUALED OR EXCEEDED FOR INDICATED PERCENT OF TIME

\begin{tabular}{|c|c|c|c|c|c|c|c|c|c|c|c|c|c|c|}
\hline 58 & 108 & 158 & 208 & 258 & 308 & 408 & 508 & 608 & 708 & 758 & 808 & 858 & 908 & 958 \\
\hline 653 & 348 & 242 & 183 & 147 & 128 & 112 & 105 & 99 & 92 & 89 & 85 & 80 & 75 & 67 \\
\hline
\end{tabular}


LOCATION.--Lat $34^{\circ} 36^{\prime} 15^{\prime \prime}$, long $104^{\circ} 23^{\prime} 14^{\prime \prime}$, in lot 1, sec.2, T.4 N., R.24 E., DeBaca County, Hydrologic Unit 13060003 , on left bank 1,200 ft downstream from Sumner Dam, $2.9 \mathrm{mi}$ upstream from Salado Creek, $4.6 \mathrm{mi}$ northeast of Guadalupe, $12.2 \mathrm{mi}$ northwest of Fort Sumner, and at mile 701.7 .

DRAINAGE AREA. $--4,390 \mathrm{mi}^{2}$, approximately (contributing area).

PERIOD OF RECORD.--October 1912 to April 1926, August 1926 to current year. Monthly discharge only for some periods, published in WSP 1312. October 1944 to September 1974, published as "below Alamogordo Dam." Prior to October 1944, published as near Guadalupe.

REVISED RECORDS.--WSP 1512: 1932, WSP 1632: 1942. WSP 1712: 1944.

GAGE.--Water-stage recorder and Parshall flume, with concrete control above top of flume. Elevation of gage is 4,142.67 ft above National Geodetic Vertical Datum of 1929 (U.S. Bureau of Reclamation bench mark). Prior to Sept. 10, 1936, at site 1.5 mi upstream at different datum. Sept. 14, 1936 to Mar. 8, 1941, and June 11 to Sept. 21, 1941, at site $0.2 \mathrm{mi}$ downstream at different datums.

REMARKS.--Flow completely regulated by Santa Rosa Lake (station 08382810) beginning April 1980 and Lake Sumner (station 08484000 ) $0.3 \mathrm{mi}$ upstream beginning August 1937. Diversion for irrigation of about 12,500 acres, 1959 determination, upstream from station.

AVERAGE DISCHARGE. - -21 years $(1913-20,1922,1925,1927-37), 240 \mathrm{ft} 3 / \mathrm{s}, 173,900$ acre-ft/yr.

EXTREMES FOR PERIOD OF RECORD.--Maximum discharge, $42,800 \mathrm{ft}^{3} / \mathrm{s}$, Sept. 1, 1942, by computation of flow over spillway and through outlet gates of Sumner Dam by U.S. Bureau of Reclamation; maximum gage height, 13.58 ft, Sept. 22, 1941; no flow at times.

Flood of June 2, 1937, about $75,000 \mathrm{ft}^{3} / \mathrm{s}$ at site $1.5 \mathrm{mi}$ upstream, from peak inflow to Lake Sumner.

STATISTICAL SUMMARIES (PERIOD BEFORE REGULATION OF SUMNER DAM) MEAN MONTHLY AND MEAN ANNUAL DISCHARGES $\begin{aligned} & 1913-20,1922 \\ & 1922,1927-37\end{aligned}$

\begin{tabular}{|c|c|c|c|c|c|c|}
\hline MONTH & $\begin{array}{l}\text { MINIMUM } \\
\left(\mathrm{FT}^{3} / \mathrm{S}\right)\end{array}$ & $\begin{array}{l}\text { MAXIMUM } \\
\left(\mathrm{FT}^{3} / \mathrm{S}\right)\end{array}$ & $\begin{array}{l}\text { MEAN } \\
\left(\mathrm{FT}^{3} / \mathrm{S}\right)\end{array}$ & $\begin{array}{l}\text { STAN- } \\
\text { DARD } \\
\text { DEVIA- } \\
\text { TION } \\
\left(\mathrm{FT}^{3} / \mathrm{S}\right)\end{array}$ & $\begin{array}{l}\text { COEFFI- } \\
\text { CIENT OF } \\
\text { VARI- } \\
\text { ATION }\end{array}$ & $\begin{array}{c}\text { PERCENT } \\
\text { OF } \\
\text { ANNUAL } \\
\text { RUNOF F }\end{array}$ \\
\hline $\begin{array}{l}\text { OCTOBER } \\
\text { NOVEMBER } \\
\text { DECEMBER } \\
\text { JANUARY } \\
\text { FEBRUARY } \\
\text { MARCH } \\
\text { APRIL } \\
\text { MAY } \\
\text { JUNE } \\
\text { JULY } \\
\text { AUGUST } \\
\text { SEPTEMBER }\end{array}$ & $\begin{array}{r}72 \\
74 \\
78 \\
76 \\
74 \\
68 \\
73 \\
76 \\
73 \\
74 \\
107 \\
59\end{array}$ & $\begin{array}{r}676 \\
120 \\
126 \\
150 \\
112 \\
393 \\
1212 \\
1637 \\
2905 \\
1536 \\
677 \\
562\end{array}$ & $\begin{array}{r}177 \\
98 \\
97 \\
96 \\
92 \\
111 \\
247 \\
501 \\
471 \\
372 \\
368 \\
222\end{array}$ & $\begin{array}{c}145 \\
12 \\
13 \\
16 \\
8.1 \\
77 \\
310 \\
413 \\
615 \\
379 \\
184 \\
150\end{array}$ & $\begin{array}{r}.82 \\
.12 \\
.13 \\
.17 \\
.09 \\
.69 \\
1.26 \\
.82 \\
1.31 \\
1.02 \\
.50 \\
.68\end{array}$ & $\begin{array}{r}6.2 \\
3.4 \\
3.4 \\
3.4 \\
3.2 \\
3.9 \\
8.7 \\
17.6 \\
16.5 \\
13.0 \\
12.9 \\
7.8\end{array}$ \\
\hline ANNUAL & 112 & 602 & 240 & 119 & .50 & 100 \\
\hline
\end{tabular}

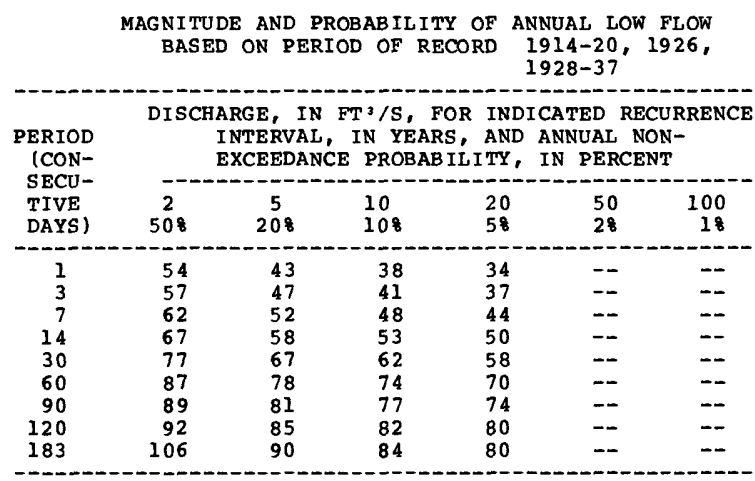

MAGNITUDE AND PROBABILITY OF ANNUAL HIGH FLOW BASED ON PERIOD OF RECORD $1913-20,1922,1925$,

\begin{tabular}{|c|c|c|c|c|c|c|}
\hline \multirow{2}{*}{$\begin{array}{l}\text { PERIOD } \\
\text { (CON- } \\
\text { SECU- } \\
\text { TIVE } \\
\text { DAYS) }\end{array}$} & \multicolumn{6}{|c|}{$\begin{array}{l}\text { DISCHARGE, IN FT }{ }^{3 / S} \text {, FOR INDICATED RECURRENCE } \\
\text { INTERVAL, IN YEARS, AND ANNUAL } \\
\text { EXCEEDANCE PROBABILITY, IN PERCENT }\end{array}$} \\
\hline & $\begin{array}{c}2 \\
508\end{array}$ & $\begin{array}{c}5 \\
208\end{array}$ & $\begin{array}{l}10 \\
108\end{array}$ & $\begin{array}{l}25 \\
48\end{array}$ & $\begin{array}{l}50 \\
28\end{array}$ & $\begin{array}{r}100 \\
18\end{array}$ \\
\hline $\begin{array}{r}1 \\
3 \\
7 \\
15 \\
30 \\
60 \\
90\end{array}$ & $\begin{array}{r}3610 \\
2160 \\
1360 \\
919 \\
699 \\
520 \\
431\end{array}$ & $\begin{array}{r}7340 \\
4060 \\
2430 \\
1590 \\
1190 \\
863 \\
726\end{array}$ & $\begin{array}{r}11100 \\
5960 \\
3480 \\
2290 \\
1630 \\
1150 \\
976\end{array}$ & $\begin{array}{r}17700 \\
9360 \\
5340 \\
3570 \\
2360 \\
1580 \\
1360\end{array}$ & $\begin{array}{l}-- \\
-- \\
-- \\
-- \\
--\end{array}$ & $\begin{array}{l}-- \\
-- \\
-- \\
-- \\
--\end{array}$ \\
\hline
\end{tabular}

DURATION TABLE OF DAILY MEAN FLOW FOR PERIOD OF RECORD 1913-20, 1922, 1925, 1927-37 DISCHARGE, IN FT'3/S, THAT WAS EQUALED OR EXCEEDED FOR INDICATED PERCENT OF TIME

\begin{tabular}{|c|c|c|c|c|c|c|c|c|c|c|c|c|c|c|}
\hline 58 & 108 & 158 & 208 & 258 & 308 & 408 & 508 & 608 & 708 & 758 & 808 & 858 & 908 & 958 \\
\hline 876 & 526 & 356 & 261 & 196 & 151 & 116 & 107 & 98 & 88 & 84 & 79 & 74 & 69 & 64 \\
\hline
\end{tabular}


08384500 PECOS RIVER BELOW SUMNER DAM, NM--Continued

STATISTICAL SUMMARIES (PERIOD BEFORE REGULATION OF SANTA ROSA DAM AND AFTER COMPLETION OF SUMNER DAM)

MEAN MONTHLY AND MEAN ANNUAL DISCHARGES 1938-79

\begin{tabular}{|c|c|c|c|c|c|c|}
\hline MONTH & $\begin{array}{l}\text { M IN I MUM } \\
\left(\mathrm{FT}^{3} / \mathrm{S}\right)\end{array}$ & $\begin{array}{l}\text { MAXIMUM } \\
\left(\mathrm{FT}^{3} / \mathrm{S}\right)\end{array}$ & $\begin{array}{l}\text { MEAN } \\
\left(\mathrm{FT}^{3} / \mathrm{S}\right)\end{array}$ & $\begin{array}{l}\text { STAN- } \\
\text { DARD } \\
\text { DEVIA- } \\
\text { TION } \\
\left(\mathrm{FT}^{3} / \mathrm{S}\right)\end{array}$ & $\begin{array}{l}\text { COEFFI - } \\
\text { CIENT OF } \\
\text { VARI- } \\
\text { ATION }\end{array}$ & $\begin{array}{c}\text { PERCENT } \\
\text { OF } \\
\text { ANNUAL } \\
\text { RUNOFF }\end{array}$ \\
\hline $\begin{array}{l}\text { OCTOBER } \\
\text { NOVEMBER } \\
\text { DECEMBER } \\
\text { JANUARY } \\
\text { FEBRUARY } \\
\text { MARCH } \\
\text { APRIL } \\
\text { MAY } \\
\text { JUNE } \\
\text { JULY } \\
\text { AUGUST } \\
\text { SEPTEMBER }\end{array}$ & $\begin{array}{r}30 \\
.2 \\
.2 \\
.2 \\
.2 \\
2.1 \\
46 \\
62 \\
62 \\
69 \\
72 \\
37\end{array}$ & $\begin{array}{r}1184 \\
910 \\
170 \\
143 \\
193 \\
605 \\
1317 \\
1404 \\
2282 \\
894 \\
796 \\
2789\end{array}$ & $\begin{array}{r}120 \\
43 \\
15 \\
20 \\
16 \\
274 \\
284 \\
290 \\
411 \\
317 \\
297 \\
282\end{array}$ & $\begin{array}{r}181 \\
145 \\
33 \\
35 \\
36 \\
212 \\
270 \\
349 \\
381 \\
220 \\
195 \\
525\end{array}$ & $\begin{array}{l}1.51 \\
3.36 \\
2.26 \\
1.73 \\
2.26 \\
.77 \\
.95 \\
1.20 \\
.93 \\
.69 \\
.66 \\
1.86\end{array}$ & $\begin{array}{r}5.1 \\
1.8 \\
.6 \\
.9 \\
.7 \\
11.6 \\
12.0 \\
12.2 \\
17.3 \\
13.4 \\
12.5 \\
11.9\end{array}$ \\
\hline ANNUAL & 92 & 710 & 198 & 113 & .57 & 100 \\
\hline
\end{tabular}

MAGNITUDE AND PROBABILITY OF ANNUAL LOW FLOW BASED ON PERIOD OF RECORD 1939-79

\begin{tabular}{|c|c|c|c|c|c|c|}
\hline \multirow{2}{*}{$\begin{array}{l}\text { PERIOD } \\
\text { (CON- } \\
\text { SECU- } \\
\text { TIVE } \\
\text { DAYS) }\end{array}$} & \multicolumn{6}{|c|}{$\begin{array}{l}\text { DISCHARGE, IN FT } 3 \text {, , FOR INDICATED RECURRENCE } \\
\text { INTERVAL, IN YEARS, AND ANNUAL NON- } \\
\text { EXCEEDANCE PROBABILITY, IN PERCENT }\end{array}$} \\
\hline & $\begin{array}{c}2 \\
508\end{array}$ & $\begin{array}{c}5 \\
208\end{array}$ & $\begin{array}{l}10 \\
108\end{array}$ & $\begin{array}{l}20 \\
58\end{array}$ & $\begin{array}{l}50 \\
28\end{array}$ & $\begin{array}{r}100 \\
18\end{array}$ \\
\hline $\begin{array}{r}1 \\
3 \\
7 \\
14 \\
30 \\
60 \\
90 \\
120 \\
183\end{array}$ & $\begin{array}{r}.3 \\
.6 \\
.9 \\
1.0 \\
1.2 \\
1.7 \\
3.0 \\
4.9 \\
31\end{array}$ & $\begin{array}{r}0.0 \\
0.0 \\
.1 \\
.2 \\
.3 \\
.5 \\
.7 \\
1.3 \\
19\end{array}$ & $\begin{array}{r}0.0 \\
0.0 \\
0.0 \\
.1 \\
.2 \\
.3 \\
.4 \\
.7\end{array}$ & $\begin{array}{r}0.0 \\
0.0 \\
0.0 \\
0.0 \\
.1 \\
.2 \\
.2 \\
15^{.4}\end{array}$ & $\begin{array}{r}0.0 \\
0.0 \\
0.0 \\
0.0 \\
.1 \\
.1 \\
.1 \\
.2\end{array}$ & $\begin{array}{r}0.0 \\
0.0 \\
0.0 \\
0.0 \\
0.0 \\
.1 \\
.1 \\
13\end{array}$ \\
\hline
\end{tabular}

MAGNITUDE AND PROBABILITY OF ANNUAL HIGH FLOW BASED ON PERIOD OF RECORD 1938-79

\begin{tabular}{|c|c|c|c|c|c|c|}
\hline \multirow{2}{*}{$\begin{array}{c}\text { PERIOD } \\
\text { (CON- } \\
\text { SECU- } \\
\text { TIVE } \\
\text { DAYS) }\end{array}$} & \multicolumn{6}{|c|}{$\begin{array}{l}\text { DISCHARGE, IN FT'/S, FOR INDICATED RECURRENCE } \\
\text { INTERVAL, IN YEARS, AND ANNUAL } \\
\text { EXCEEDANCE 'PROBAB ILITY, IN PERCENT }\end{array}$} \\
\hline & $\begin{array}{c}2 \\
508\end{array}$ & $\begin{array}{c}5 \\
208\end{array}$ & $\begin{array}{l}10 \\
108\end{array}$ & $\begin{array}{l}25 \\
48\end{array}$ & $\begin{array}{l}50 \\
28\end{array}$ & $\begin{array}{r}100 \\
18\end{array}$ \\
\hline $\begin{array}{r}1 \\
3 \\
7 \\
15 \\
30 \\
60 \\
90\end{array}$ & $\begin{array}{r}1330 \\
1290 \\
1210 \\
1010 \\
722 \\
474 \\
393\end{array}$ & $\begin{array}{r}2490 \\
2190 \\
1770 \\
1380 \\
1030 \\
686 \\
562\end{array}$ & $\begin{array}{r}4270 \\
3400 \\
2430 \\
1810 \\
1310 \\
880 \\
705\end{array}$ & $\begin{array}{r}9100 \\
6250 \\
3770 \\
2660 \\
1790 \\
1200 \\
926\end{array}$ & $\begin{array}{r}16500 \\
10000 \\
5290 \\
3600 \\
2250 \\
1500 \\
1130\end{array}$ & $\begin{array}{r}30100 \\
16300 \\
7480 \\
4910 \\
2820 \\
1880 \\
1360\end{array}$ \\
\hline
\end{tabular}

DURATION TABLE OF DAILY MEAN FLOW FOR PERIOD OF RECORD 1938-79

DISCHARGE, IN FT 3 /S, THAT WAS EQUALED OR EXCEEDED FOR INDICATED PERCENT OF TIME

\begin{tabular}{|c|c|c|c|c|c|c|c|c|c|c|c|c|c|c|}
\hline 58 & 108 & 158 & 208 & 258 & 308 & 408 & 508 & 608 & 708 & 758 & 808 & 858 & 908 & 958 \\
\hline 1140 & 731 & 165 & 108 & 103 & 98 & 88 & 78 & 59 & 7.8 & 4.0 & 2.4 & 1.5 & .7 & \\
\hline
\end{tabular}


08386000 PECOS RIVER NEAR ACME, NM

(Surveillance network station)

LOCATION.--Lat $33^{\circ} 32^{\prime} 10^{\prime \prime}$, long $104^{\circ} 22^{\prime} 34^{\prime \prime}$, in SW $\frac{1}{4} W_{\frac{1}{4}}$ sec.14, T.9 S., R. 25 E. Chaves County, Hydrologic Unit 13060007 , on right bank $3.0 \mathrm{mi}$ downstream from U.s. Highway $70,3.7 \mathrm{mi}$ downstream from Salt Creek, $4.7 \mathrm{mi}$ southwest of Acme, $14 \mathrm{mi}$ northeast of Roswell, and at mile 585.3 .

DRAINAGE AREA. - - 11, $380 \mathrm{mi}^{2}$, approximately (contributing area).

PERIOD OF RECORD.--September 1921 to June 1923, July 1937 to current year. Monthly discharge only for some periods, published in WSP 1312.

GAGE.--Water-stage recorder. Elevation of gage is 3,507 ft above National Geodetic vertical Datum of 1929 , from topographic map. Prior to Nov. 1, 1938, at site on highway bridge $3 \mathrm{mi}$ upstream at various datums. $\mathrm{since}$ oct. 25, 1963, supplemental water-stage recorder at site opposite base gage at same datum.

REMARKS.--Flow regulated by Santa Rosa Lake (station 08382810) since April 1980 and by Lake Sumner (station $08384000)$ since August 1937. Diversions for irrigation of about 20,000 acres, 1959 determination upstream from station.

AVERAGE DISCHARGE. -42 years $(1938-79), 185 \mathrm{ft}^{3} / \mathrm{s}, 134,000$ acre-ft/yr.

EXTREMES FOR PERIOD OF RECORD,--Maximum discharge, 45,000 $\mathrm{ft}^{3} / \mathrm{s}$, Sept, 23, 1941, gage height, 13.71 ft, from rating curve extended above $27,000 \mathrm{ft}^{3} / \mathrm{s}$; no flow at times.

EXTREMES OUTSIDE PERIOD OF RECORD.--The flood of May 28, 1937, reached a discharge of 53,000 $\mathrm{ft} 3 / \mathrm{s}, \mathrm{gage}$ height, $14.82 \mathrm{ft}$, from floodmarks, site and datum then in use, by slope-area method, but may have been exceeded by the flood of Oct. 1, 1904 .

STATISTICAL SUMMARIES (PERIOD BEFORE REGULATION OF SANTA ROSA DAM AND AFTER COMPLETION OF SUMNER DAM) MEAN MONTHLY AND MEAN ANNUAL DISChARGES 1938-79

MAGNITUDE AND PROBABILITY OF ANNUAL LOW FLOW BASED ON PERTOD OF RECORD 1939-79

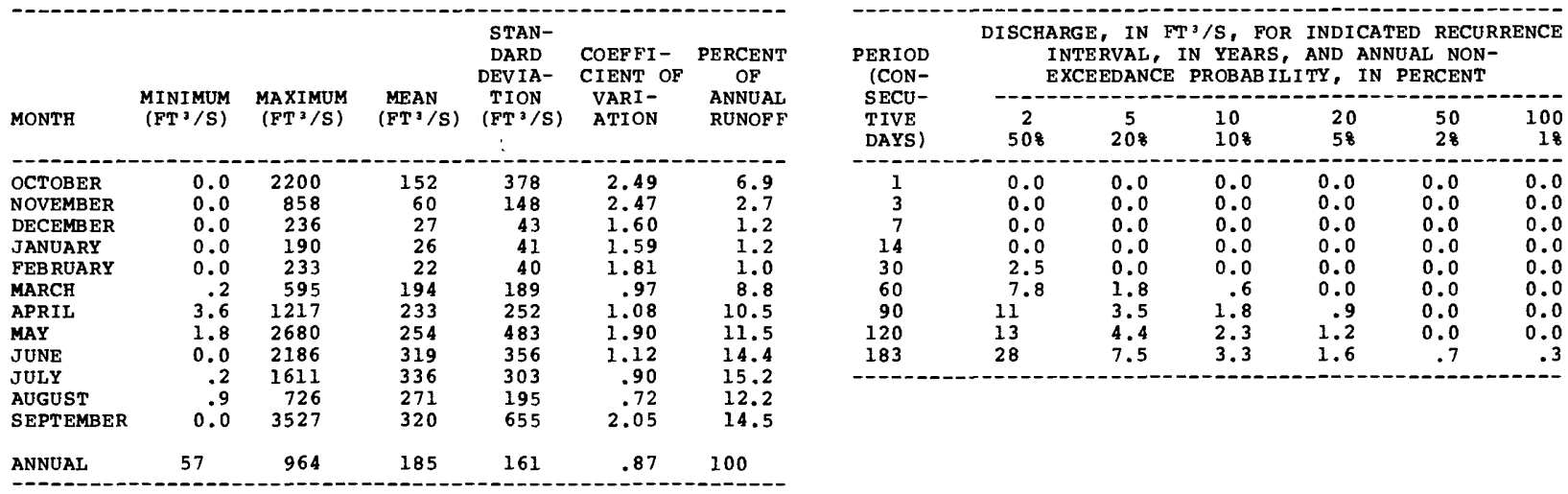

MAGNITUDE AND PROBABILITY OF ANNUAL HIGH FLOW BASED ON PERIOD OF RECORD $1938-79$

\begin{tabular}{|c|c|c|c|c|c|c|}
\hline \multirow{2}{*}{$\begin{array}{l}\text { PERIOD } \\
\text { (CON- } \\
\text { SECU- } \\
\text { TIVE } \\
\text { DAYS) }\end{array}$} & \multicolumn{6}{|c|}{$\begin{array}{l}\text { DISCHARGE, IN ET }{ }^{3} / \mathrm{S} \text {, FOR INDICATED RECURRENCE } \\
\text { INTERVAL, IN YEARS, AND ANNUAL } \\
\text { EXCEEDANCE PROBABILITY, IN PERCENT }\end{array}$} \\
\hline & $\begin{array}{c}2 \\
508\end{array}$ & $\begin{array}{c}5 \\
208\end{array}$ & $\begin{array}{l}10 \\
108\end{array}$ & $\begin{array}{l}25 \\
48\end{array}$ & $\begin{array}{l}50 \\
28\end{array}$ & $\begin{array}{r}100 \\
18\end{array}$ \\
\hline $\begin{array}{r}1 \\
3 \\
7 \\
15 \\
30 \\
60 \\
90\end{array}$ & $\begin{array}{r}2360 \\
1680 \\
1220 \\
922 \\
641 \\
426 \\
333\end{array}$ & $\begin{array}{r}5450 \\
3410 \\
2110 \\
1440 \\
990 \\
667 \\
527\end{array}$ & $\begin{array}{r}9430 \\
5560 \\
3170 \\
2040 \\
1330 \\
904 \\
717\end{array}$ & $\begin{array}{r}18500 \\
10400 \\
5380 \\
3240 \\
1930 \\
1320 \\
1050\end{array}$ & $\begin{array}{c}29900 \\
16400 \\
8020 \\
4600 \\
2530 \\
6.8 \\
1390\end{array}$ & $\begin{array}{r}47900 \\
25800 \\
11900 \\
6540 \\
3300 \\
2290 \\
1820\end{array}$ \\
\hline
\end{tabular}

DURATION TABLE OF DAILY MEAN FLOW FOR PERIOD OF RECORD 1938-79

DISCHARGE, IN FT ${ }^{3 / S}$, THAT WAS EQUALED OR EXCEEDED FOR INDICATED PERCENT OF TIME

\begin{tabular}{|c|c|c|c|c|c|c|c|c|c|c|c|c|c|c|}
\hline 58 & 108 & 158 & 208 & 258 & 308 & 408 & 508 & 608 & 708 & 758 & 808 & 858 & 908 & 958 \\
\hline 1030 & 660 & 278 & 132 & 81 & 57 & 32 & 20 & 14 & 8.3 & 6.0 & 3.7 & 1.4 & 0.0 & 0.0 \\
\hline
\end{tabular}


LOCATION. --Lat $33^{\circ} 19^{\prime} 43^{\prime \prime}$, Iong $105^{\circ} 36^{\prime} 34^{\prime \prime}$, in SWłSEłNEł sec.30, T.11 S., R.14 E., Lincoln County, Hydrologic Unit 13060008 , on right upstream bridge abutment on road leading to Ruidoso Downs Race Track, 0.2 mi north of 0.5 . Highway $70,1.1 \mathrm{mi}$ east of the Ho1lywood Post office, $1.8 \mathrm{mi}$ downstream from Gavilan Canyon, $2.8 \mathrm{mi}$ downstream from Carrizo Creek, and at mile 23.4. Due to construction work, a temporary gage was installed Mar. 28, 1985, $0.95 \mathrm{mi}$ upstream at different datum and used for the remainder of the water year.

DRAINAGE AREA. $--120 \mathrm{mi}^{2}$, approximate1y.

PERIOD OF RECORD.--March 1953 to current year.

GAGE.--Water-stage recorder. Elevation of gage is $6,365,42 \mathrm{ft}$ above National Geodetic Vertical Datum of 1929. Prior to Oct. 14, 1961 , at datum $0.30 \mathrm{ft}$ higher. Oct. 14, 1961 to Mar. 8, 1962 at datum $0.60 \mathrm{ft}$ higher. Mar. 9, 1962 to June 18,1965 , at datum 1.0 ft higher.

REMARKS.--Village of Ruidoso diverts from right bank 7.0 mi upstream for municipal use and return on this water as effluent from sewage disposal plant downstream from the gage.

AVERAGE DISCHARGE.--32 years (1954-1985), $17.0 \mathrm{ft} / \mathrm{s}, 12,320$ acre-ft/yr, for period when sewage disposal plant effluent was discharged upstream from gage.

EXTREMES FOR PERIOD OF RECORD.--Maximum discharge, 2,120 ft $3 / \mathrm{s}$, Aug, 11, 1984 , gage height, $9.68 \mathrm{ft}$, from rating curve extended above $510 \mathrm{ft} / \mathrm{s}$, on basis of slope-area measurement of peak flow; maximum gage height, $10.05 \mathrm{ft}$

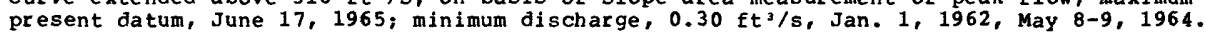

EXTREMES OUTSIDE PERIOD OF RECORD.--The flood of Sept. 29, 1941, is probably the highest since at 1east 1904 (discharge not determined).

STATISTICAL SUMMARIES

MEAN MONTHLY AND MEAN ANNUAL DISCHARGES 1954-85

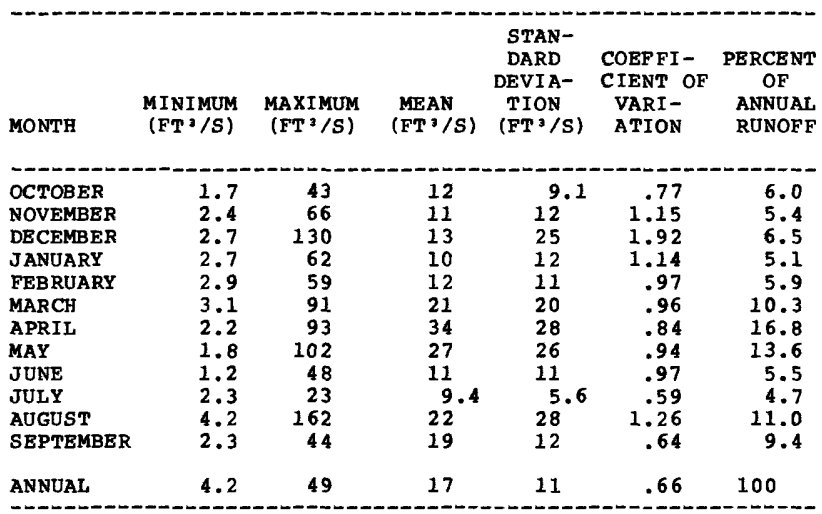

MAGNTTUDE AND PROBABILITY OF ANNUAL LOW FLOW BASED ON PERTOD OF RECORD 1955-85

MAGNITUDE AND PROBABILITY OF ANNUAL HIGH FLON BASED ON PERIOD OF RECORD 1954-85

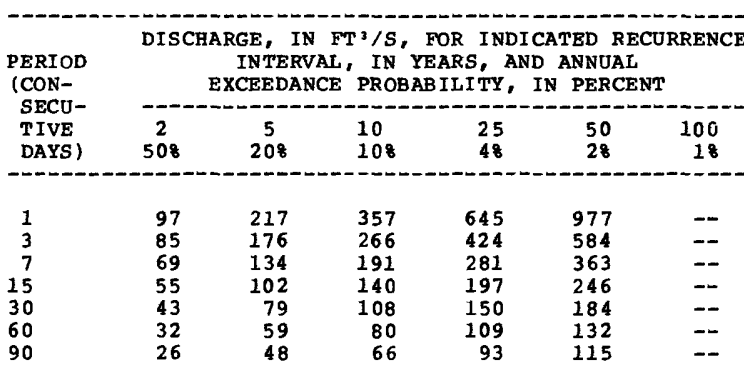

DURATION TABLE OF DAILY MEAN FLOW FOR PERIOD OF RECORD 1954-85

DISCHARGE, IN FT ${ }^{3} / S$, THAT WAS EQUALED OR EXCEEDED FOR INDICATED PERCENT OF TIME

\begin{tabular}{|c|c|c|c|c|c|c|c|c|c|c|c|c|c|c|}
\hline 58 & 108 & 158 & 208 & 258 & 308 & 408 & 508 & 608 & 708 & 758 & 808 & 858 & 908 & 958 \\
\hline 59 & 40 & 28 & 22 & 18 & 16 & 12 & 9.3 & 7.4 & 5.8 & 5.0 & 4.2 & 3.5 & 2.9 & \\
\hline
\end{tabular}




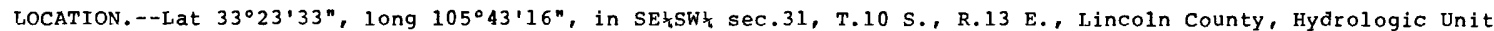
13060008 , in Lincoln National Forest at $\mathrm{right}$ bank, $100 \mathrm{ft}$ downstream from culvert under State Road No. 532 , 0.1 $\mathrm{mi}$ downstream from South Fork, and $2.4 \mathrm{mi}$ west of Alto. Mouth at Rio Ruidoso mile 11.3 .

DRAINAGE AREA. $--8.14 \mathrm{mi}^{2}$.

PERIOD OF RECORD.--August 1969 to December 1980.

GAGE. - Water-stage recorder and concrete control. Altitude of gage is 7,600 ft, from topographic map.

REMARKS.--Some water is stored in small unregulated recreational ponds on the Mescalero Apache Indian Reservation upstream.

AVERAGE DISCHARGE. -11 years (water years 1970-80), 3.14 $\mathrm{ft}^{3} / \mathrm{s}, 2,270$ acre-ft/yr.

EXTREMES FOR PERIOD OF RECORD.--Maximum discharge, $206 \mathrm{ft}^{3} / \mathrm{s} \mathrm{Dec.} \mathrm{19,} \mathrm{1978,} \mathrm{gage} \mathrm{height,} 3.79 \mathrm{ft}, \mathrm{from}$ rating curve extended above $21 \mathrm{ft}^{3 / \mathrm{s}}$; minimum, $0.05 \mathrm{ft} / \mathrm{s}$ June 30 , July $3,4,1974$.

STATISTICAL SUMMARIES

MEAN MONTHLY AND MEAN ANNUAL DISCHARGES 1970-80

\begin{tabular}{|c|c|c|c|c|c|c|}
\hline MONTH & $\begin{array}{l}\text { MINIMUM } \\
\left(\mathrm{FT}^{3} / \mathrm{S}\right)\end{array}$ & $\begin{array}{c}\text { MAXIMUM } \\
\left(\mathrm{FT}^{3} / \mathrm{S}\right)\end{array}$ & $\begin{array}{l}\text { MEAN } \\
\left(\mathrm{FT}^{3} / \mathrm{S}\right)\end{array}$ & $\begin{array}{l}\text { STAN- } \\
\text { DARD } \\
\text { DEVIA- } \\
\text { TION } \\
\left(\mathrm{FT}^{3} / \mathrm{S}\right)\end{array}$ & $\begin{array}{l}\text { COEFFI - } \\
\text { CIENT OF } \\
\text { VARI- } \\
\text { ATION }\end{array}$ & $\begin{array}{c}\text { PERCENT } \\
\text { OF } \\
\text { ANNUAL } \\
\text { RUNOFF }\end{array}$ \\
\hline $\begin{array}{l}\text { OCTOBER } \\
\text { NOVEMBER } \\
\text { DECEMBER } \\
\text { JANUARY } \\
\text { FEBRUARY } \\
\text { MARCH } \\
\text { APRIL } \\
\text { MAY } \\
\text { JUNE } \\
\text { JULY } \\
\text { AUGUST } \\
\text { SEPTEMBER }\end{array}$ & $\begin{array}{r}.4 \\
.3 \\
.2 \\
.3 \\
.4 \\
.3 \\
.3 \\
.2 \\
.1 \\
.1 \\
1.3 \\
.4\end{array}$ & $\begin{array}{l}14 \\
17 \\
20 \\
7.9 \\
8.2 \\
11 \\
14 \\
16 \\
5.9 \\
4.6 \\
5.8 \\
9.3\end{array}$ & $\begin{array}{l}3.1 \\
3.0 \\
2.6 \\
2.0 \\
2.6 \\
3.9 \\
5.6 \\
4.7 \\
1.7 \\
1.5 \\
2.7 \\
3.9\end{array}$ & $\begin{array}{l}4.2 \\
4.9 \\
5.4 \\
2.3 \\
2.6 \\
3.5 \\
4.8 \\
4.5 \\
1.8 \\
1.2 \\
1.5 \\
3.1\end{array}$ & $\begin{array}{r}1.35 \\
1.64 \\
2.11 \\
1.15 \\
.97 \\
.89 \\
.86 \\
.94 \\
1.06 \\
.84 \\
.56 \\
.81\end{array}$ & $\begin{array}{r}8.4 \\
8.0 \\
6.9 \\
5.4 \\
7.1 \\
10.4 \\
15.0 \\
12.7 \\
4.7 \\
4.0 \\
7.2 \\
10.4\end{array}$ \\
\hline ANNUAL & .4 & 8.5 & 3.1 & 2.4 & .77 & 100 \\
\hline
\end{tabular}

MAGNITUDE AND PROBABILITY OF ANNUAL LOW FLOW BASED ON PERIOD OF RECORD $1971-80$

\begin{tabular}{|c|c|c|c|c|c|c|}
\hline \multirow{2}{*}{$\begin{array}{l}\text { PERIOD } \\
\text { (CON- } \\
\text { SECU- } \\
\text { TIVE } \\
\text { DAYS) }\end{array}$} & \multicolumn{6}{|c|}{$\begin{array}{l}\text { DISCHARGE, IN FT'3/S, FOR INDICATED RECURRENCE } \\
\text { INTERVAL, IN YEARS, AND ANNUAL NON- } \\
\text { EXCEEDANCE PROBABILITY, IN PERCENT }\end{array}$} \\
\hline & $\begin{array}{c}2 \\
508\end{array}$ & $\begin{array}{c}5 \\
208\end{array}$ & $\begin{array}{l}10 \\
108\end{array}$ & $\begin{array}{l}20 \\
58\end{array}$ & $\begin{array}{l}50 \\
28\end{array}$ & $\begin{array}{r}100 \\
18\end{array}$ \\
\hline $\begin{array}{r}1 \\
3 \\
7 \\
14 \\
30 \\
60 \\
90 \\
120 \\
183\end{array}$ & $\begin{array}{r}.2 \\
.2 \\
.3 \\
.3 \\
.4 \\
.4 \\
.6 \\
.7 \\
1.1\end{array}$ & $\begin{array}{l}.1 \\
.1 \\
.1 \\
.2 \\
.2 \\
.2 \\
.3 \\
.3 \\
.6\end{array}$ & $\begin{array}{l}.1 \\
.3 \\
.1 \\
.1 \\
.1 \\
.1 \\
.2 \\
.2 \\
.4\end{array}$ & $\begin{array}{l}.1 \\
.1 \\
.1 \\
.1 \\
.1 \\
.1 \\
.1 \\
.2 \\
.3\end{array}$ & $\begin{array}{l}-- \\
-- \\
-- \\
-- \\
-- \\
--\end{array}$ & $\begin{array}{l}-- \\
-- \\
-- \\
-- \\
-- \\
-- \\
--\end{array}$ \\
\hline
\end{tabular}

MAGNITUDE AND PROBABILITY OF ANNUAL HIGH FLOW BAS OND ONTD OF RECORD $1970-80$

\begin{tabular}{|c|c|c|c|c|c|c|}
\hline $\begin{array}{l}\text { PERIOD } \\
\text { (CON- } \\
\text { SFCI- }\end{array}$ & & $\begin{array}{l}\text { GE, } \\
\text { INTE } \\
\text { CEED }\end{array}$ & $\begin{array}{l}\mathrm{T}^{3} / \mathrm{S} \\
\text { IN } \\
\text { PRO }\end{array}$ & $\begin{array}{l}\text { IN } \\
\text { TY }\end{array}$ & $\begin{array}{l}\text { ED } \\
\text { VNU } \\
\text { PER }\end{array}$ & RRENCE \\
\hline $\begin{array}{l}\text { TIVE } \\
\text { DAYS \} }\end{array}$ & $\begin{array}{c}2 \\
508\end{array}$ & $\begin{array}{c}5 \\
208\end{array}$ & $\begin{array}{l}10 \\
108\end{array}$ & $\begin{array}{l}25 \\
48\end{array}$ & $\begin{array}{l}50 \\
28\end{array}$ & $\begin{array}{r}100 \\
18\end{array}$ \\
\hline
\end{tabular}

$\begin{array}{rrrrrrr}1 & 24 & 61 & 102 & -- & -- & -- \\ 3 & 19 & 43 & 67 & -- & -- & - \\ 7 & 14 & 28 & 41 & -- & - & - \\ 15 & 11 & 20 & 27 & -- & -- & - \\ 30 & 8.6 & 16 & 20 & -- & -- & - \\ 60 & 6.6 & 12 & 15 & -- & -- & - \\ 90 & 5.3 & 9.6 & 12 & -- & -\end{array}$

DURATION TABLE OF DAILY MEAN FLOW FOR PERIOD OF RECORD 1970-80

DISCHARGE, IN FT ${ }^{3} / \mathrm{S}$, THAT WAS EOUALED OR EXCEEDED FOR INDICATED PERCENT OF TIME

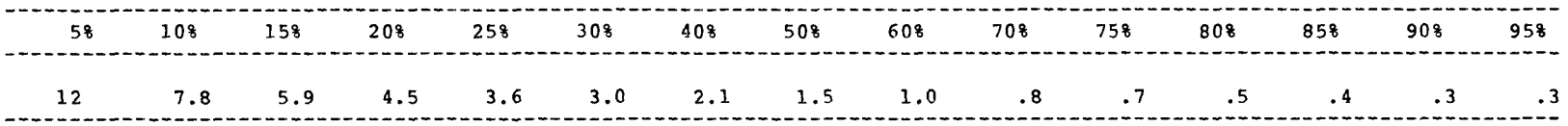


LOCATION.--Lat $33^{\circ} 23^{\prime} 29^{\prime \prime}$, long $105^{\circ} 36^{\prime} 39^{\prime \prime}$, in SWłSE 13060008 , left bank $200 \mathrm{ft}$ north of Lincoln National Forest boundary, 500 ft northeast of windmill, and 4.0 $\mathrm{mi}$ east of Alto. Mouth at Rio Ruidoso mile 11.3 .

DRATNAGE AREA. $--15.7 \mathrm{mi}^{2}$.

PERIOD OF RECORD.--October 1969 to December 1980.

GAGE.--Water-stage recorder and concrete control. Altitude of gage is 6,840 ft, from topographic map.

REMARKS.--Discharge at this station is affected by Alto Reservoir and municipal water supply diversions for Ruidoso and Capitan.

AVERAGE DISCHARGE. --11 years (water years 1970-80), $1.73 \mathrm{ft}^{3} / \mathrm{s}, 1,250$ acre-ft/yr.

EXTREMES FOR PERIOD OF RECORD.--Maximum discharge, $72 \mathrm{ft}^{3} / \mathrm{s}$ Sept, 16, 1976, gage height, $2.03 \mathrm{ft}$; from rating curve extended above $22 \mathrm{ft}^{3} / \mathrm{s}$; no flow most of time.

STATISTICAL SUMMARIES

MEAN MONTHLY AND MEAN ANNUAL DISCHARGES 1970-80

\begin{tabular}{|c|c|c|c|c|c|c|}
\hline MONTH & $\begin{array}{l}\text { MINIMUM } \\
\left(\mathrm{FT}^{3} / \mathrm{S}\right)\end{array}$ & $\begin{array}{c}\text { MAXIMUM } \\
\left(\mathrm{FT}^{3} / \mathrm{S}\right)\end{array}$ & $\begin{array}{l}\text { MEAN } \\
\left(\mathrm{FT}^{3} / \mathrm{S}\right)\end{array}$ & $\begin{array}{c}\text { STAN- } \\
\text { DARD } \\
\text { DEVIA- } \\
\text { TION } \\
\left(\mathrm{FT}^{3} / \mathrm{S}\right)\end{array}$ & $\begin{array}{l}\text { COEFFI- } \\
\text { CIENT OF } \\
\text { VARI - } \\
\text { ATION }\end{array}$ & $\begin{array}{c}\text { PERCENT } \\
\text { OF } \\
\text { ANNUAL } \\
\text { RUNOFF }\end{array}$ \\
\hline OCTOBER & 0.0 & 13 & 1.6 & 3.8 & 2.42 & 7.8 \\
\hline NOVEMBER & 0.0 & 15 & 2.2 & 4.6 & 2.15 & 10.6 \\
\hline DECEMBER & 0.0 & 18 & 1.7 & 5.1 & 3.10 & 8.1 \\
\hline JANUARY & 0.0 & 6.8 & 1.0 & 2.1 & 2.15 & 4.8 \\
\hline FEBRUARY & 0.0 & 7.6 & 1.5 & 3.0 & 1.96 & 7.5 \\
\hline MARCH & 0.0 & 9.8 & 2.4 & 4.1 & 1.72 & 11.6 \\
\hline APRIL & 0.0 & 13 & 3.7 & 5.0 & 1.37 & 18.1 \\
\hline MAY & 0.0 & 13 & 2.9 & 4.0 & 1.40 & 14.1 \\
\hline JUNE & 0.0 & 4.1 & .6 & 1.3 & 2.15 & 3.1 \\
\hline JULY & 0.0 & 1.9 & .4 & .7 & 1.78 & 2.0 \\
\hline AUGUST & 0.0 & 4.1 & & 1.6 & 1.86 & 4.2 \\
\hline SEPTEMBER & 0.0 & 8.8 & 1.7 & 2.9 & 1.76 & 8.1 \\
\hline ANNUAL & 0.0 & 7.1 & 1.7 & 2.4 & 1.40 & 100 \\
\hline
\end{tabular}

MAGNITUDE AND PROBABILITY OF ANNUAL LOW FLOW BASED ON PERIOD OF RECORD 1971-80

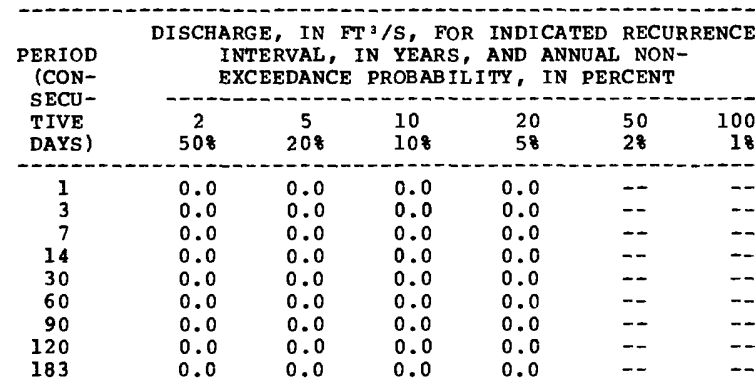

MAGNITUDE AND PROBABILITY OF ANNUAL HIGH FLOW BASED ON PERIOD OF RECORD $1970-80$

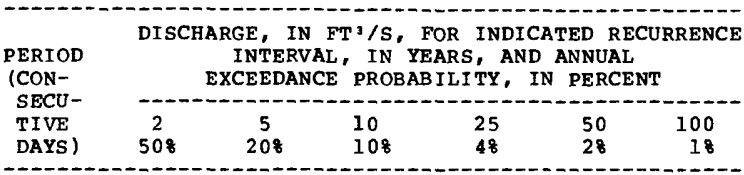

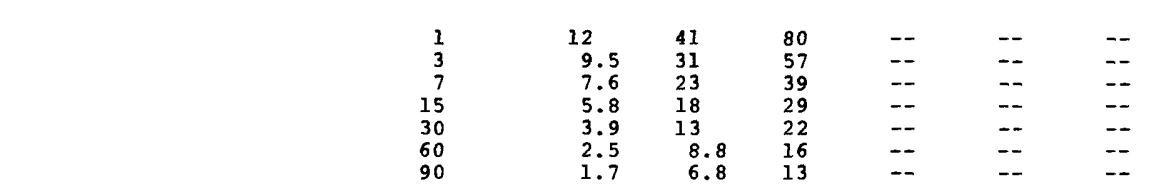

DURATION TABLE OF DAILY MEAN FLOW FOR PERIOD OF RECORD 1970-80

DISCHARGE, IN FT $3 / 5$, THAT WAS EQUALED OR EXCEEDED FOR INDICATED PERCENT OF TIME

\begin{tabular}{|c|c|c|c|c|c|c|c|c|c|c|c|c|c|c|}
\hline 58 & 108 & 158 & 208 & 258 & 308 & 408 & 508 & 608 & 708 & 758 & $80 \%$ & 858 & 908 & 958 \\
\hline 11 & 6.8 & 3.5 & 1.6 & .6 & .2 & 0.0 & 0.0 & 0.0 & 0.0 & 0.0 & 0.0 & 0.0 & 0.0 & 0.0 \\
\hline
\end{tabular}


RIO GRANDE BASIN

08388000 RIO RUIDOSO AT HONDO, NM

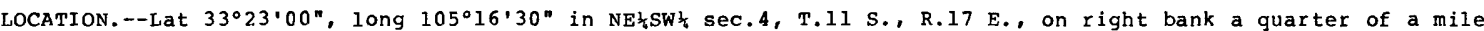
upstream from confluence with Rio Bonito and half a mile southwest of Hondo.

DRAINAGE AREA. $--290 \mathrm{mi}^{2}$ (revised).

PERIOD OF RECORD.--October 1930 to September 1955 (discontinued).

GAGE.--Water-stage recorder. Datum of gage is 5,181.38 ft above mean sea level, datum of 1929.

REMARKS.--Diversions for irrigation of about 1,700 acres above station.

AVERAGE DISCHARGF.--2.5 years (water years 1931-55), $19.0 \mathrm{ft}^{3 / s}, 13,760$ acre-ft/yr.

EXTREMES FOR PERIOD OF RECORD.--Maximum discharge $12,400 \mathrm{ft}^{3} / \mathrm{s}$ Sept. 29,1941 (gage height, $21.13 \mathrm{ft}$, from floodmark), from rating curve extended above $3,700 \mathrm{ft}^{3 / \mathrm{s}}$ on basis of velocity-area studies; no flow Aug. 15 , 16 , 1935 , June $2-7,1950$.

STATISTICAL SUMMARIES

MEAN MONTHLY AND MEAN ANNUAI, DISCHARGES 1931-55

\begin{tabular}{|c|c|c|c|c|c|c|}
\hline MONTH & $\begin{array}{c}\text { MINIMUM } \\
\left(\mathrm{FT}^{3} / \mathrm{S}\right)\end{array}$ & $\begin{array}{c}\text { MAXIMUM } \\
\left(\mathrm{FT}^{3} / \mathrm{S}\right)\end{array}$ & $\begin{array}{l}\text { MEAN } \\
\left(\mathrm{FT}^{3} / \mathrm{S}\right)\end{array}$ & $\begin{array}{c}\text { STAN- } \\
\text { DARD } \\
\text { DEVIA- } \\
\text { TION } \\
\left(\mathrm{FT}^{3} / \mathrm{S}\right)\end{array}$ & $\begin{array}{l}\text { COEFFI- } \\
\text { CIENT OF } \\
\text { VARI- } \\
\text { ATION }\end{array}$ & $\begin{array}{c}\text { PERCENT } \\
\text { OF } \\
\text { ANNUAL } \\
\text { RUNOF }\end{array}$ \\
\hline OCTOBER & .7 & 307 & 25 & 60 & 2.42 & 10.9 \\
\hline NOVEMBER & .7 & 105 & 12 & 20 & 1.77 & 5.1 \\
\hline DECEMBER & 1.0 & 59 & 9.1 & 12 & 1.28 & 4.0 \\
\hline JANUARY & .9 & 48 & 9.2 & 10 & 1.10 & 4.0 \\
\hline FEBRUARY & 1.0 & 39 & 10 & 9.0 & .90 & 4.4 \\
\hline MARCH & .7 & 70 & 17 & 16 & .99 & 7.3 \\
\hline APR IL & .6 & 96 & 25 & 32 & 1.25 & 11.2 \\
\hline MAY & .5 & 365 & 33 & 74 & 2.23 & 14.6 \\
\hline JUNE & .8 & 100 & 12 & 21 & 1.77 & 5.2 \\
\hline JULY & .4 & 94 & 18 & 23 & 1.28 & 7.8 \\
\hline AUGUST & .7 & 123 & 19 & 26 & 1.35 & 8.5 \\
\hline SEPTEMBER & .4 & 524 & 39 & 104 & 2.69 & 17.0 \\
\hline ANNUAL & 2.8 & 114 & 19 & 25 & 1.29 & 100 \\
\hline
\end{tabular}

MAGNITUDE AND PROBABILITY OF ANNUAL LOW FLOW BASED ON PERIOD OF RECORD $1932-55$

\begin{tabular}{|c|c|c|c|c|c|c|}
\hline $\begin{array}{l}\text { PERIOD } \\
\text { (CON- }\end{array}$ & \multicolumn{6}{|c|}{$\begin{array}{l}\text { INTERVAL, IN YEARS, AND ANNUAL NON- } \\
\text { EXCEEDANCE. PROBABILITY, IN PERCENT }\end{array}$} \\
\hline $\begin{array}{l}\text { SECU- } \\
\text { TIVE } \\
\text { DAYS) }\end{array}$ & $\begin{array}{c}2 \\
508\end{array}$ & $\begin{array}{c}5 \\
208\end{array}$ & $\begin{array}{l}10 \\
108\end{array}$ & 20 & $\begin{array}{l}50 \\
28\end{array}$ & $\begin{array}{r}100 \\
18\end{array}$ \\
\hline $\begin{array}{r}1 \\
3 \\
7 \\
14 \\
30 \\
60 \\
90 \\
120 \\
183\end{array}$ & $\begin{array}{r}.4 \\
.4 \\
.7 \\
.8 \\
1.1 \\
1.9 \\
2.5 \\
3.2 \\
5.4\end{array}$ & $\begin{array}{r}.2 \\
.2 \\
.2 \\
.4 \\
.5 \\
.8 \\
1.0 \\
1.0 \\
2.2\end{array}$ & $\begin{array}{r}.1 \\
.1 \\
.1 \\
.3 \\
.4 \\
.5 \\
.7 \\
.8 \\
1.4\end{array}$ & $\begin{array}{r}0.0 \\
.1 \\
.1 \\
.2 \\
.3 \\
.4 \\
.5 \\
.6 \\
1.0\end{array}$ & $\begin{array}{r}0.0 \\
0.0 \\
0.0 \\
.2 \\
.3 \\
.3 \\
.4 \\
.4 \\
.7\end{array}$ & - \\
\hline
\end{tabular}

NAGNITUDE AND PROBABILITY OF ANNUAL HIGH FLOW BASED ON PERIOD OF RECORD 1931-55

\begin{tabular}{|c|c|c|c|c|c|c|}
\hline $\begin{array}{l}\text { PERIOD } \\
\text { (CON- }\end{array}$ & \multicolumn{6}{|c|}{$\begin{array}{l}\text { DISCHARGE, IN FT }{ }^{3} / \mathrm{S}, \text { FOR INDICATED RECURRENCE } \\
\text { INTERVAI, IN YEARS, AND ANNUAL } \\
\text { EXCEEDANCE PROBABILITY, IN PERCENT }\end{array}$} \\
\hline $\begin{array}{l}\text { SECU- } \\
\text { TIVE } \\
\text { DAYS) }\end{array}$ & 508 & $\begin{array}{c}5 \\
2.08\end{array}$ & $\begin{array}{l}10 \\
108\end{array}$ & 25 & $\begin{array}{r}50 \\
28\end{array}$ & $\begin{array}{r}100 \\
18\end{array}$ \\
\hline $\begin{array}{r}1 \\
3 \\
7 \\
15 \\
30 \\
60 \\
90\end{array}$ & $\begin{array}{r}136 \\
90 \\
66 \\
48 \\
38 \\
27 \\
23\end{array}$ & $\begin{array}{r}433 \\
273 \\
185 \\
127 \\
91 \\
63 \\
53\end{array}$ & $\begin{array}{r}871 \\
541 \\
349 \\
234 \\
157 \\
106 \\
87\end{array}$ & $\begin{array}{r}1970 \\
1220 \\
748 \\
489 \\
301 \\
196 \\
155\end{array}$ & $\begin{array}{r}3490 \\
2180 \\
1280 \\
826 \\
476 \\
303 \\
232\end{array}$ & $\begin{array}{l}-- \\
-- \\
-- \\
-- \\
-- \\
-- \\
--\end{array}$ \\
\hline
\end{tabular}

DURATION TABLE OF DAIL, MEAN FLOW FOR PERIOD OF RECORD 1931-55

DISCHARGE, IN FT $3 / 5$, THAT WAS EQUALED OR EXCEEDED FOR INDICATED PERCENT OF TIME

\begin{tabular}{|c|c|c|c|c|c|c|c|c|c|c|c|c|c|c|}
\hline 58 & 108 & 158 & 208 & 258 & 308 & 408 & 508 & 608 & 708 & 758 & 808 & 858 & 908 & 958 \\
\hline 67 & 39 & 28 & 23 & 18 & 15 & 10 & 6.7 & 3.7 & 1.9 & 1.4 & 1.1 & .9 & .8 & \\
\hline
\end{tabular}


LOCATION.--Lat $33^{\circ} 23^{\prime} 20^{\prime \prime}$, long $105^{\circ} 16^{\prime} 30^{\prime \prime}$, in NEłNWł sec.4, T.11 S., R.17 E., near center of span on downstream side of bridge on v.s. Highway 70 at Hondo, half a mile upstream from confluence with Rio Ruidoso.

DRAINAGE AREA. $--295 \mathrm{mi}^{2}$, revised (contributing area).

PERIOD OF RECORD.--October 1930 to September 1955 (discontinued).

REVISED RECORDS.--WSP 1212: 1950.

GAGE.--Water-stage recorder and concrete control. Datum of gage is 5,205.17 ft above mean sea level, datum of 1929. Prior to Feb. 4, 1933, at site a quarter mile upstream at different datum. (Datum lowered 0.23 ft oct. 10, 1931).

REMARKS.--Diversions for irrigation of about 1,700 acres above station.

AVERAGE DISCHARGE.--25 years (water years 1931-55), $10.3 \mathrm{ft} / \mathrm{s}, 7,460 \mathrm{acre}-\mathrm{ft} / \mathrm{yr}$.

EXTREMES FOR PERIOD OF RECORD.--Maximum discharge, 11,000 ft $3 / \mathrm{s}$ Sept. 28 or 29 , 1941 (gage height, $20.92 \mathrm{ft}, \mathrm{from}$ floodmark), from rating curve extended above $220 \mathrm{ft} / \mathrm{s}$ on basis of slope-area determination at gage height 19.0 ft; no flow at times.

STATISTICAL SUMMARIES

MEAN MONTHLY AND MEAN ANNUAL DISChARGES 1931-55

\begin{tabular}{|c|c|c|c|c|c|c|}
\hline MONTH & $\begin{array}{l}\text { MINIMUM } \\
\left(\mathrm{FT}^{3} / \mathrm{S}\right)\end{array}$ & $\begin{array}{c}\text { MAXIMUM } \\
\left(\mathrm{FT}^{3} / \mathrm{S}\right)\end{array}$ & $\begin{array}{l}\text { MEAN } \\
\left(\mathrm{FT}^{3} / \mathrm{S}\right)\end{array}$ & $\begin{array}{l}\text { STAN- } \\
\text { DARD } \\
\text { DEVIA- } \\
\text { TION } \\
\left(\mathrm{FT}^{3} / \mathrm{S}\right)\end{array}$ & $\begin{array}{l}\text { COEFFI- } \\
\text { CIENT OF } \\
\text { VARI- } \\
\text { ATION }\end{array}$ & $\begin{array}{c}\text { PERCENT } \\
\text { OF } \\
\text { ANNUAL } \\
\text { RUNOFF }\end{array}$ \\
\hline 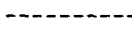 & $-\infty---n$ & $-\infty-$ & $-\infty$ & & $-n-n$ & - \\
\hline OCTOBER & 0.0 & 230 & 13 & 46 & 3.61 & 10.2 \\
\hline NOVEMBER & 0.0 & 79 & 5.2 & 16 & 2.99 & 4.2 \\
\hline DECEMBFR & 0.0 & 39 & 2.4 & 7.7 & 3.21 & 1.9 \\
\hline JANUARY & 0.0 & 30 & 1.6 & 6.0 & 3.72 & 1.3 \\
\hline FEB RUARY & 0.0 & 26 & 1.5 & 5.3 & 3.51 & 1.2 \\
\hline MARCH & 0.0 & 18 & 1.9 & 4.7 & 2.46 & 1.5 \\
\hline APRII, & 0.0 & 87 & 9.2 & 20 & 2.12 & 7.5 \\
\hline MAY & 0.0 & 175 & 18 & 40 & 2.26 & 14.2 \\
\hline JUNE & 0.0 & 36 & 7.0 & 12 & 1.64 & 5.7 \\
\hline JULY & 1.0 & 46 & 15 & 13 & .86 & 11.8 \\
\hline AUGUST & .8 & 55 & 16 & 13 & .79 & 13.3 \\
\hline SEPTEMBER & 0.0 & 424 & 34 & 83 & 2.48 & 27.1 \\
\hline ANNUAL & 1.6 & 59 & 10 & 15 & 1.46 & 100 \\
\hline
\end{tabular}

MAGNITUDE AND PROBABILITY OF ANNUAL LOW FLOW BASED ON PERIOD OF RECORD 1932-55

\begin{tabular}{|c|c|c|c|c|c|c|}
\hline \multirow{2}{*}{$\begin{array}{l}\text { PERIOD } \\
\text { (CON- } \\
\text { SECU- } \\
\text { TIVE } \\
\text { DAYS) }\end{array}$} & \multicolumn{6}{|c|}{$\begin{array}{l}\text { DISCHARGE, IN FT3/S, FOR INDICATED RECURRENCE } \\
\text { INTERVAL, IN YEARS, AND ANNUAL NON- } \\
\text { EXCEEDANCE PROBABILITY, IN PERCENT }\end{array}$} \\
\hline & $\begin{array}{c}2 \\
508\end{array}$ & $\stackrel{5}{5}^{08}$ & $\begin{array}{l}10 \\
10 \%\end{array}$ & $\begin{array}{l}20 \\
58\end{array}$ & $\begin{array}{l}50 \\
28\end{array}$ & \\
\hline $\begin{array}{r}1 \\
3 \\
7 \\
14 \\
30 \\
60 \\
90 \\
120 \\
183\end{array}$ & $\begin{array}{r}0.0 \\
0.0 \\
0.0 \\
0.0 \\
0.0 \\
0.0 \\
0.0 \\
.1 \\
.7\end{array}$ & $\begin{array}{l}0.0 \\
0.0 \\
0.0 \\
0.0 \\
0.0 \\
0.0 \\
0.0 \\
0.0 \\
.1\end{array}$ & $\begin{array}{l}0.0 \\
0.0 \\
0.0 \\
0.0 \\
0.0 \\
0.0 \\
0.0 \\
0.0 \\
.1\end{array}$ & $\begin{array}{l}0.0 \\
0.0 \\
0.0 \\
0.0 \\
0.0 \\
0.0 \\
0.0 \\
0.0 \\
0.0\end{array}$ & $\begin{array}{r}0.0 \\
0.0 \\
0.0 \\
0.0 \\
0.0 \\
0.0 \\
0.0 \\
0.0 \\
0.0\end{array}$ & \\
\hline
\end{tabular}

MAGNITUDE AND PROBABILITY OF ANNUAL HIGH FLOW BASED ON PERIOD OF RECORD 1931-55

\begin{tabular}{|c|c|c|c|c|c|c|}
\hline $\begin{array}{l}\text { PERIOD } \\
\text { (CON- } \\
\text { SECU- }\end{array}$ & \multicolumn{6}{|c|}{$\begin{array}{l}\text { DISCHARGE, IN } \mathrm{FT}^{3} / \mathrm{S}, \text { FOR INDICATED RECURRENCE } \\
\text { INTERVAL, IN YEARS, AND ANNUAL } \\
\text { EXCEEDANCE PROBABILI TY, IN PERCENT }\end{array}$} \\
\hline $\begin{array}{l}\text { TIVE } \\
\text { DAYS ) }\end{array}$ & $\begin{array}{c}2 \\
508\end{array}$ & $\begin{array}{c}5 \\
208\end{array}$ & $\begin{array}{l}10 \\
108\end{array}$ & $\begin{array}{l}25 \\
48\end{array}$ & $\begin{array}{l}50 \\
28\end{array}$ & $\begin{array}{r}100 \\
18\end{array}$ \\
\hline $\begin{array}{r}1 \\
3 \\
7 \\
15 \\
30 \\
60 \\
90\end{array}$ & $\begin{array}{r}241 \\
117 \\
72 \\
43 \\
29 \\
20 \\
16\end{array}$ & $\begin{array}{r}621 \\
310 \\
184 \\
113 \\
72 \\
47 \\
38\end{array}$ & $\begin{array}{r}1090 \\
558 \\
318 \\
200 \\
122 \\
78 \\
62\end{array}$ & $\begin{array}{r}2080 \\
1110 \\
594 \\
390 \\
224 \\
141 \\
105\end{array}$ & $\begin{array}{r}3270 \\
1790 \\
908 \\
621 \\
340 \\
212 \\
151\end{array}$ & $\begin{array}{l}-- \\
-- \\
-- \\
-- \\
--\end{array}$ \\
\hline
\end{tabular}

DURATION TABLE OF DAILY MEAN FLOW FOR PERIOD OF RECORD 1931-55

DISCHARGE, IN FT'3/S, THAT WAS EQUALED OR EXCEEDED FOR INDICATED PERCENT OF TIME

\begin{tabular}{|c|c|c|c|c|c|c|c|c|c|c|c|c|c|c|}
\hline 58 & 108 & 158 & 208 & 258 & $30 z$ & 408 & 508 & 608 & 708 & 758 & 808 & 858 & 908 & 958 \\
\hline 42 & 21 & 11 & 6.6 & 4.4 & 2.9 & .9 & .1 & .1 & .1 & .1 & 0.0 & 0.0 & 0.0 & \\
\hline
\end{tabular}


RIO GRANDE BASIN

08390500 RIO HONDO AT DIAMOND A RANCH, NEAR ROSWELL, NM

LOCATION, $-33^{\circ} 20^{\prime} 57^{\prime \prime}$, long $104^{\circ} 51^{\prime} 05^{\prime \prime}$, in NENE sec.20, T.11 S, R.21 E., Chaves County, Hydrologic Unit 13060008, on right bank $15 \mathrm{ft}$ downstream from county road bridge at Diamond A Ranch, $1.3 \mathrm{mi}$ south of U.S. Highway $70-380$, $13 \mathrm{mi}$ upstream from Two Rivers Reservoir, $21 \mathrm{mi}$ upstream from mouth of Rocky Arroyo, $18 \mathrm{mi}$ west of Roswell, and at mile 44.7

DRAINAGE AREA. - $-947 \mathrm{mi}^{2}$, contributing area.

PERIOD OF RECORD.--May 1908 to August 1909, May 1939 to current year. Monthly discharge only for 1908-9, published in Technical Report No. 7, State of New Mexico, State Engineer office, Streamflow and Reservoir Content $1888-1954$

REVISED RECORDS.--WSP 1392: Drainage area. WSP 1512: 1939-40(P), 1941, 1942-43(P), $1946(P)$.

GAGE.--Water-stage recorder and concrete control. Elevation of gage is $4,190 \mathrm{ft}$ above National Geodetic Vertical Datum of 1929, from topographic map. Prior to Nov, 11, 1965 at site on left bank at same datum.

REMARKS. - Diversions and groundwater withdrawals upstream from station for irrigation upstream and downstream from station of about 6,500 acres, 1959 determination.

AVERAGE DISCHARGE. -46 years $(1940-85) 22.3 \mathrm{ft}^{3} / \mathrm{s}, 16,160$ acre-ft/yr.

EXTREMES FOR PERIOD OF RECORD.--Maximum discharge, 54,800 ft $3 / \mathrm{s}$, June 18, 1965, gage height, $26.40 \mathrm{ft}$, from rating curve extended above $3,100 \mathrm{ft} / \mathrm{s}$, on basis of slope-area measurement of peak flow; maximum gage height, 28.78 $\mathrm{ft}$, Sept. 22, 1941; no flow most of the time.

EXTREMES OUTSIDE PERIOD OF RECORD.--A flood on June 1, 1937, reached a discharge of $24,900 \mathrm{ft}^{3} / \mathrm{s}$ at Riverside about $13 \mathrm{mi}$ upstream. Other major floods occurred Oct. 31, 1901, Sept. 29, 30, 1904 and July $25,1905$.

STATISTICAL SUMMARIES

MEAN MONTHLY AND MEAN ANNUAL DISCHARGES 1940-85

\begin{tabular}{|c|c|c|c|c|c|c|}
\hline MONTH & $\begin{array}{l}\text { MINIMUM } \\
\left(\mathrm{FT}^{3} / \mathrm{S}\right)\end{array}$ & $\begin{array}{c}\text { MAXIMUM } \\
\left(\mathrm{FT}^{3} / \mathrm{S}\right)\end{array}$ & $\begin{array}{l}\text { MEAN } \\
\left(\mathrm{FT}^{3} / \mathrm{S}\right)\end{array}$ & $\begin{array}{c}\text { STAN- } \\
\text { DARD } \\
\text { DEVIA- } \\
\text { TION } \\
\text { (FT3/S) }\end{array}$ & $\begin{array}{l}\text { COEFFI- } \\
\text { CIENT OF } \\
\text { VARI- } \\
\text { ATION }\end{array}$ & $\begin{array}{c}\text { PERCENT } \\
\text { OF } \\
\text { ANNUAL } \\
\text { RUNOFF }\end{array}$ \\
\hline $\begin{array}{l}\text { OCTOBER } \\
\text { NOVEMBER } \\
\text { DECEMBER } \\
\text { JANUARY } \\
\text { FEBRUARY } \\
\text { MARCH } \\
\text { APRIL } \\
\text { MAY } \\
\text { JUNE } \\
\text { JULY } \\
\text { AUGUST } \\
\text { SEPTEMBER }\end{array}$ & $\begin{array}{l}0.0 \\
0.0 \\
0.0 \\
0.0 \\
0.0 \\
0.0 \\
0.0 \\
0.0 \\
0.0 \\
0.0 \\
0.0 \\
0.0\end{array}$ & $\begin{array}{r}458 \\
199 \\
222 \\
160 \\
96 \\
106 \\
165 \\
519 \\
182 \\
163 \\
241 \\
1090\end{array}$ & $\begin{array}{l}23 \\
14 \\
16 \\
11 \\
7.8 \\
8.0 \\
22 \\
28 \\
18 \\
28 \\
38 \\
53\end{array}$ & $\begin{array}{r}71 \\
34 \\
44 \\
29 \\
19 \\
20 \\
42 \\
80 \\
42 \\
45 \\
52 \\
163\end{array}$ & $\begin{array}{l}3.01 \\
2.53 \\
2.83 \\
2.55 \\
2.46 \\
2.47 \\
1.93 \\
2.85 \\
2.34 \\
1.63 \\
1.37 \\
3.05\end{array}$ & $\begin{array}{r}8.8 \\
5.1 \\
5.9 \\
4.3 \\
2.9 \\
3.0 \\
8.2 \\
10.5 \\
6.7 \\
10.4 \\
14.3 \\
20.0\end{array}$ \\
\hline ANNUAL & 1.3 & 181 & 22 & 33 & 1.49 & 100 \\
\hline
\end{tabular}

MAGNITUDE AND PROBABILITY OF ANNUAL LOW FLOW BASED ON PERIOD OF RECORD $1941-85$

\begin{tabular}{|c|c|c|c|c|c|c|}
\hline \multirow{2}{*}{$\begin{array}{l}\text { PERIOD } \\
\text { (CON- } \\
\text { SECU- } \\
\text { TIVE } \\
\text { DAYS) }\end{array}$} & \multicolumn{6}{|c|}{$\begin{array}{l}\text { DISCHARGE, IN FT'/S, FOR INDICATED RECURRENCE } \\
\text { INTERVAL, IN YEARS, AND ANNUAL NON- } \\
\text { EXCEEDANCE PROBABILITY, IN PERCENT }\end{array}$} \\
\hline & $\begin{array}{c}2 \\
508\end{array}$ & $\begin{array}{c}5 \\
208\end{array}$ & $\begin{array}{l}10 \\
108\end{array}$ & $\begin{array}{l}20 \\
58\end{array}$ & $\begin{array}{l}50 \\
28\end{array}$ & $\begin{array}{r}100 \\
18\end{array}$ \\
\hline $\begin{array}{r}1 \\
3 \\
7 \\
14 \\
30 \\
60 \\
90 \\
120 \\
183\end{array}$ & $\begin{array}{r}0.0 \\
0.0 \\
0.0 \\
0.0 \\
0.0 \\
0.0 \\
0.0 \\
.2 \\
1.5\end{array}$ & $\begin{array}{l}0.0 \\
0.0 \\
0.0 \\
0.0 \\
0.0 \\
0.0 \\
0.0 \\
0.0 \\
0.0\end{array}$ & $\begin{array}{l}0.0 \\
0.0 \\
0.0 \\
0.0 \\
0.0 \\
0.0 \\
0.0 \\
0.0 \\
0.0\end{array}$ & $\begin{array}{l}0.0 \\
0.0 \\
0.0 \\
0.0 \\
0.0 \\
0.0 \\
0.0 \\
0.0 \\
0.0\end{array}$ & $\begin{array}{l}0.0 \\
0.0 \\
0.0 \\
0.0 \\
0.0 \\
0.0 \\
0.0 \\
0.0 \\
0.0\end{array}$ & $\begin{array}{l}0.0 \\
0.0 \\
0.0 \\
0.0 \\
0.0 \\
0.0 \\
0.0 \\
0.0 \\
0.0\end{array}$ \\
\hline
\end{tabular}

MAGNITUDE AND PROBABILITY OF ANNUAL HIGH FLOW BASED ON PERIOD OF RECORD $1940-85$

\begin{tabular}{|c|c|c|c|c|c|c|}
\hline \multirow{2}{*}{$\begin{array}{l}\text { PERIOD } \\
\text { (CON- } \\
\text { SECU- } \\
\text { TIVE } \\
\text { DAYS) }\end{array}$} & \multicolumn{6}{|c|}{$\begin{array}{l}\text { DISCHARGE, IN FT'3/S, FOR INDICATED RECURRENCE } \\
\text { INTERVAL, IN YEARS, AND ANNUAL } \\
\text { EXCEEDANCE PROBABILITY, IN PERCENT }\end{array}$} \\
\hline & $\begin{array}{c}2 \\
508\end{array}$ & $\begin{array}{c}5 \\
208\end{array}$ & $\begin{array}{l}10 \\
108\end{array}$ & $\begin{array}{l}25 \\
48\end{array}$ & $\begin{array}{l}50 \\
28\end{array}$ & $\begin{array}{r}100 \\
18\end{array}$ \\
\hline $\begin{array}{r}1 \\
3 \\
7 \\
15 \\
30 \\
60 \\
90\end{array}$ & $\begin{array}{r}489 \\
267 \\
165 \\
107 \\
74 \\
46 \\
35\end{array}$ & $\begin{array}{r}1270 \\
685 \\
405 \\
265 \\
178 \\
112 \\
84\end{array}$ & $\begin{array}{r}2160 \\
1160 \\
661 \\
436 \\
281 \\
177 \\
135\end{array}$ & $\begin{array}{r}3880 \\
2090 \\
1130 \\
755 \\
455 \\
286 \\
228\end{array}$ & $\begin{array}{r}5730 \\
3090 \\
1620 \\
1090 \\
619 \\
390 \\
321\end{array}$ & $\begin{array}{r}8210 \\
4450 \\
2240 \\
1520 \\
817 \\
514 \\
438\end{array}$ \\
\hline
\end{tabular}

DURATION TABLE OF DAILY MEAN FLOW FOR PERIOD OF RECORD 1940-85 DISCHARGE, IN FT $3 / S$, THAT WAS EQUALED OR EXCEEDED FOR INDICATED PERCENT OF TIME

\begin{tabular}{|c|c|c|c|c|c|c|c|c|c|c|c|c|c|c|}
\hline 58 & 108 & 158 & 208 & 258 & 308 & 408 & 508 & 608 & 708 & 758 & 808 & 858 & 908 & 958 \\
\hline 101 & 51 & 28 & 18 & 9.8 & 4.2 & 0.0 & 0.0 & 0.0 & 0.0 & 0.0 & 0.0 & 0.0 & 0.0 & 0 \\
\hline
\end{tabular}




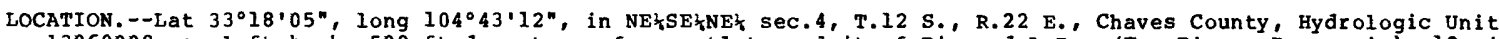
13060008 , on left bank, $500 \mathrm{ft}$ downstream from outlet conduit of Diamond A Dam (Two Rivers Reservoir), $13 \mathrm{mi}$ southwest of Roswell, and at mile 33.3 .

DRAINAGE AREA.- $-963 \mathrm{mi}^{2}$, contributing area.

PERIOD OF RECORD.--October 1963 to current year.

GAGE.--Water-stage recorder and concrete control. Elevation of gage is $3,949.68 \mathrm{ft}$ above National Geodetic Vertical Datum of 1929 (u.S. Army Corps of Engineers bench mark).

REMARKS.--Diversions and ground-water withdrawals for irrigation of about 6,500 acres, 1959 determination, upstream from station. This record represents the outflow from Two Rivers Reservoir through Diamond $A$ Dam. Flow from reservoir can also be discharged into Rocky Arroyo through Rocky Dam.

AVERAGE DISCHARGE.--22 years (water years 1964-85), $11.0 \mathrm{ft}^{3} / \mathrm{s}, 7,970$ acre-ft/yr.

EXTREMES FOR PERIOD OF RECORD.--Maximum discharge, $659 \mathrm{ft}^{3} / \mathrm{s}$, July 29, 1965, gage height, 4.91 ft; no flow most of time.

STATISTICAL SUMMARIES

MEAN MONTHLY AND MEAN ANNUAL DISChARGES 1964-85

\begin{tabular}{|c|c|c|c|c|c|c|}
\hline MONTH & $\begin{array}{c}\text { MINIMUM } \\
\left(\mathrm{FT}^{3} / \mathrm{S}\right)\end{array}$ & $\begin{array}{c}\text { MAXIMUM } \\
\left(\mathrm{FT}^{3} / \mathrm{S}\right)\end{array}$ & $\underset{\left(F T^{3} / \mathrm{S}\right)}{\operatorname{MEAN}}$ & $\begin{array}{l}\text { STAN- } \\
\text { DARD } \\
\text { DEVIA- } \\
\text { TION } \\
\left(\mathrm{FT}^{3} / \mathrm{S}\right)\end{array}$ & $\begin{array}{l}\text { COEFFI- } \\
\text { CIENT OF } \\
\text { VARI- } \\
\text { ATION }\end{array}$ & $\begin{array}{c}\text { PERCENT } \\
\text { OF } \\
\text { ANNUAL } \\
\text { RUNOFF }\end{array}$ \\
\hline $\begin{array}{l}\text { OCTOBER } \\
\text { NOVEMBER } \\
\text { DECEMBER } \\
\text { JANUARY } \\
\text { FEBRUARY } \\
\text { MARCH } \\
\text { APRIL } \\
\text { MAY } \\
\text { JUNE } \\
\text { JULY } \\
\text { AUGUST } \\
\text { SEPTEMBER }\end{array}$ & $\begin{array}{l}0.0 \\
0.0 \\
0.0 \\
0.0 \\
0.0 \\
0.0 \\
0.0 \\
0.0 \\
0.0 \\
0.0 \\
0.0 \\
0.0\end{array}$ & $\begin{array}{r}71 \\
70 \\
118 \\
128 \\
80 \\
104 \\
89 \\
108 \\
52 \\
46 \\
137 \\
104\end{array}$ & $\begin{array}{r}6.9 \\
7.7 \\
9.2 \\
11 \\
7.2 \\
8.5 \\
14 \\
13 \\
6.0 \\
4.9 \\
22 \\
21\end{array}$ & $\begin{array}{l}17 \\
18 \\
28 \\
32 \\
21 \\
25 \\
26 \\
28 \\
13 \\
12 \\
34 \\
33\end{array}$ & $\begin{array}{l}2.43 \\
2.31 \\
3.08 \\
2.96 \\
2.86 \\
2.89 \\
1.88 \\
2.11 \\
2.08 \\
2.36 \\
1.57 \\
1.56\end{array}$ & $\begin{array}{r}5.3 \\
5.9 \\
7.0 \\
8.2 \\
5.5 \\
6.5 \\
10.5 \\
10.2 \\
4.6 \\
3.7 \\
16.4 \\
16.3\end{array}$ \\
\hline NNUAL & .2 & 50 & 11 & 13 & 1.16 & 100 \\
\hline
\end{tabular}

MAGNITUDE AND PROBABILITY OF ANNUAL LOW FLOW BASED ON PERIOD OF RECORD 1965-85

\begin{tabular}{|c|c|c|c|c|c|c|}
\hline \multirow{2}{*}{$\begin{array}{l}\text { PERIOD } \\
\text { (CON- } \\
\text { SECU- } \\
\text { TIVE } \\
\text { DAYS) }\end{array}$} & \multicolumn{6}{|c|}{$\begin{array}{l}\text { DISCHARGE, IN FT' }{ }^{3 / S} \text {, FOR INDICATED RECURRENCE } \\
\text { INTERVAL, IN YEARS, AND ANNUAL NON- } \\
\text { EXCEEDANCE PROBAB IL ITY, IN PERCENT }\end{array}$} \\
\hline & $\begin{array}{c}2 \\
508\end{array}$ & $\begin{array}{c}5 \\
208\end{array}$ & $\begin{array}{l}10 \\
108\end{array}$ & $\begin{array}{l}20 \\
58\end{array}$ & $\begin{array}{l}50 \\
28\end{array}$ & $\begin{array}{r}100 \\
18\end{array}$ \\
\hline $\begin{array}{r}1 \\
3 \\
7 \\
14 \\
30 \\
60 \\
90 \\
120 \\
183\end{array}$ & $\begin{array}{r}0.0 \\
0.0 \\
0.0 \\
0.0 \\
0.0 \\
0.0 \\
0.0 \\
0.0 \\
.5\end{array}$ & $\begin{array}{l}0.0 \\
0.0 \\
0.0 \\
0.0 \\
0.0 \\
0.0 \\
0.0 \\
0.0 \\
0.0\end{array}$ & $\begin{array}{l}0.0 \\
0.0 \\
0.0 \\
0.0 \\
0.0 \\
0.0 \\
0.0 \\
0.0 \\
0.0\end{array}$ & $\begin{array}{l}0.0 \\
0.0 \\
0.0 \\
0.0 \\
0.0 \\
0.0 \\
0.0 \\
0.0 \\
0.0\end{array}$ & $\begin{array}{l}-- \\
-- \\
-- \\
-- \\
-- \\
-- \\
--\end{array}$ & \\
\hline
\end{tabular}

MAGNITUDE AND PROBABILITY OF ANNUAL HIGH FLOW BASED ON PERIOD OF RECORD $1964-85$

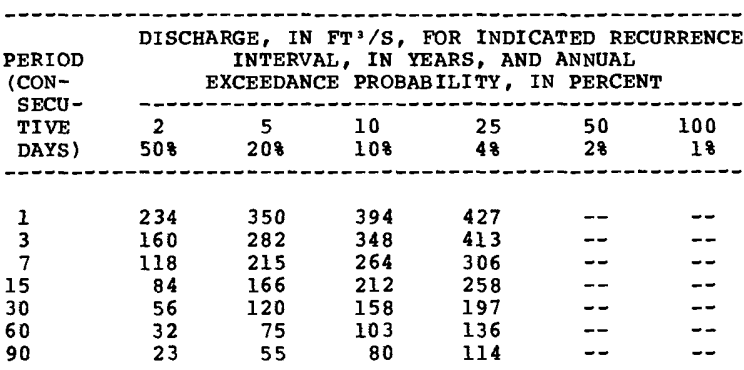

DURATION TABLE OF DAILY MEAN FLOW FOR PERIOD OF RECORD 1964-85

DISCHARGE, IN FT ${ }^{3} / S$, THAT WAS EQUALED OR EXCEEDED FOR INDICATED PERCENT OF TIME

\begin{tabular}{|c|c|c|c|c|c|c|c|c|c|c|c|c|c|c|}
\hline 58 & 108 & 158 & 208 & 258 & 308 & 408 & 508 & 608 & 708 & 758 & 808 & 858 & 908 & 958 \\
\hline 77 & 35 & 13 & 1.4 & 0.0 & 0. & 0.0 & 0. & 0. & 0.0 & 0. & 0. & 0. & 0.0 & \\
\hline
\end{tabular}




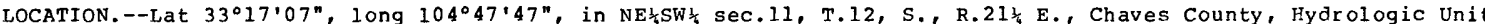
13060008 , on left bank, $2.1 \mathrm{mi}$ upstream from mouth of Buchnan Draw, 5.2 mi upstream from Rocky Dam, (Two Rivers Reservoir), and $17 \mathrm{mi}$ southwest of Roswell.

DRAINAGE AREA. $--31 \mathrm{mi}^{2}$, approximately.

PERIOD OF RECORD.--May 1963 to september 1980.

GAGE.--Water-stage recorder and concrete control. Datum of gage is $4,059.17 \mathrm{ft}$ Corps of Engineers datum. Prior to Dec. 7, 1968, at site on opposite bank at datum $3.72 \mathrm{ft}$ lower.

REMARKS.--Flow past station represents inflow to Two Rivers Reservoir.

AVERAGE DISCHARGE.--17 years (water years 1964-80), $0.90 \mathrm{ft}^{3} / \mathrm{s}, 652$ acre-ft/yr.

EXTREMES FOR PERIOD OF RECORD.--Maximum discharge, 12,000 $\mathrm{ft}^{3} / \mathrm{s} \mathrm{July} \mathrm{5,} \mathrm{1968,} \mathrm{gage} \mathrm{height,} 11.53 \mathrm{ft}$, from floodmarks, present datum, from rating curve extended above $350 \mathrm{ft} / \mathrm{s}$ on basis of slope-area measurements at gage heights $5.92 \mathrm{ft}, 7.14 \mathrm{ft}$, and $11.53 \mathrm{ft}$, present datum; no flow most of time.

STATISTICAL SUMMARIFS

MEAN MONTHLY AND MEAN ANNUAL DISCHARGFS 1964-80

\begin{tabular}{|c|c|c|c|c|c|c|}
\hline MONTH & $\begin{array}{c}\text { MINIMUM } \\
\left(\mathrm{FT}^{3} / \mathrm{S}\right)\end{array}$ & $\begin{array}{l}\text { MAXIMUM } \\
\left(\mathrm{FT}^{3} / \mathrm{S}\right)\end{array}$ & $\begin{array}{l}\text { MEAN } \\
\left(\mathrm{FT}^{3} / \mathrm{S}\right)\end{array}$ & $\begin{array}{l}\text { STAN- } \\
\text { DARD } \\
\text { DEVIA- } \\
\text { TION } \\
\left(\text { FT }^{3} / \mathrm{S}\right)\end{array}$ & $\begin{array}{l}\text { COEFFI- } \\
\text { CIENT OF } \\
\text { VARI- } \\
\text { ATION }\end{array}$ & $\begin{array}{c}\text { PERCENT } \\
\text { OF } \\
\text { ANNUAL } \\
\text { RUNOFF }\end{array}$ \\
\hline \multicolumn{7}{|c|}{ 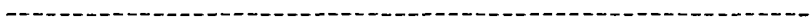 } \\
\hline OCTOBER & 0.0 & 23 & 1.4 & 5.7 & 4.06 & 13.2 \\
\hline NOVEMBER & 0.0 & 0.0 & 0.0 & 0.0 & -- & 0.0 \\
\hline DECEMBER & 0.0 & 0.0 & 0.0 & 0.0 & -- & 0.0 \\
\hline JANUARY & 0.0 & 0.0 & 0.0 & 0.0 & -- & 0.0 \\
\hline FEB RUARY & 0.0 & 0.0 & 0.0 & 0.0 & -- & 0.0 \\
\hline MARCH & 0.0 & 0.0 & 0.0 & 0.0 & -- & 0.0 \\
\hline APR IL & 0.0 & 1.4 & .1 & .3 & 4.25 & .8 \\
\hline MAY & 0.0 & 0.0 & 0.0 & 0.0 & -- & 0.0 \\
\hline JUNE & 0.0 & 1.4 & .1 & .4 & 3.00 & 1.1 \\
\hline JULY & 0.0 & 26 & 2.0 & 6.4 & 3.20 & 19.0 \\
\hline AUGUST & 0.0 & 26 & 2.9 & 6.5 & 2.29 & 26.9 \\
\hline SEPTEMBER & 0.0 & 23 & 4.1 & 7.9 & 1.91 & 39.0 \\
\hline NNUAL & .1 & 4.2 & .9 & 1.2 & 1.34 & 100 \\
\hline
\end{tabular}

MAGNITUDE AND PROBABIJ, ITY OF ANNUAL LOW FLOW BASED ON PERIOD OF RECORD 1965-80

\begin{tabular}{|c|c|c|c|c|c|c|}
\hline \multirow{3}{*}{$\begin{array}{l}\text { PERIOD } \\
\text { (CON- } \\
\text { SECU- } \\
\text { TIVE } \\
\text { DAYS) }\end{array}$} & \multicolumn{6}{|c|}{$\begin{array}{l}\text { DISCHARGF, IN FT }{ }^{3} / S, \text { FOR INDICATED RECURRENCE } \\
\text { INTERVAI, IN YEARS, AND ANNUAL NON- } \\
\text { EXCEEDANCE PROBABIL ITY, IN PERCENT }\end{array}$} \\
\hline & 2 & 5 & 10 & 20 & 50 & 100 \\
\hline & $50 \%$ & $20 \%$ & 108 & 58 & 28 & 18 \\
\hline$I$ & 0.0 & 0.0 & 0.0 & 0.0 & -- & -- \\
\hline 3 & 0.0 & 0.0 & 0.0 & 0.0 & -- & \\
\hline 7 & 0.0 & 0.0 & 0.0 & 0.0 & -- & -- \\
\hline 14 & 0.0 & 0.0 & 0.0 & 0.0 & -- & - \\
\hline 30 & 0.0 & 0.0 & 0.0 & 0.0 & -- & -- \\
\hline 60 & 0.0 & 0.0 & 0.0 & 0.0 & - & -2 \\
\hline 90 & 0.0 & 0.0 & 0.0 & 0.0 & -- & \\
\hline 120 & 0.0 & 0.0 & 0.0 & 0.0 & - & -- \\
\hline 183 & 0.0 & 0.0 & 0.0 & 0.0 & -- & - \\
\hline
\end{tabular}

MAGNITUDE AND PROBABILITY OF ANNUAL HIGH FLOW BASED ON PERIOD OF RECORD 1964-80

\begin{tabular}{|c|c|c|c|c|c|c|}
\hline $\begin{array}{l}\text { PERIOD } \\
\text { (CON- }\end{array}$ & \multicolumn{6}{|c|}{$\begin{array}{l}\text { DISCHARGE, IN FT }{ }^{3} / \mathrm{S} \text {, FOR INDICATED RECURRENCE } \\
\text { INTERVAL, IN YEARS, AND ANNUAL } \\
\text { EXCEEDANCE PROBABILITY, IN PERCENT }\end{array}$} \\
\hline $\begin{array}{l}\text { TIVE } \\
\text { DAYS }\end{array}$ & $\begin{array}{c}2 \\
508\end{array}$ & $\begin{array}{c}5 \\
208\end{array}$ & $\begin{array}{l}10 \\
108\end{array}$ & $\begin{array}{l}25 \\
48\end{array}$ & $\begin{array}{l}50 \\
28\end{array}$ & $\begin{array}{r}100 \\
1 \%\end{array}$ \\
\hline 1 & 81 & 306 & 565 & 1000 & -- & -- \\
\hline 3 & 31 & 118 & 220 & 397 & -- & -- \\
\hline 7 & 13 & 52 & 96 & 171 & - & -- \\
\hline 15 & 7.5 & 32 & 61 & 112 & - & $=-$ \\
\hline 30 & 3.8 & 16 & 31 & 56 & -- & -- \\
\hline 60 & 2.0 & 8.6 & 17 & 31 & -- & -- \\
\hline 90 & 1.3 & 5.7 & 11 & 21 & -- & -- \\
\hline
\end{tabular}

DURATION TABLE OF DAILY MEAN FLOW FOR PERIOD OF RECORD 1964-80 DISCHARGF, IN FT' $3 / S$, THAT WAS EQUALED OR EXCEEDED FOR INDICATED PERCENT OF TIME

\begin{tabular}{|c|c|c|c|c|c|c|c|c|c|c|c|c|c|c|}
\hline 58 & 108 & 158 & $20 \%$ & 258 & 308 & 408 & $50 \%$ & $60 \%$ & 708 & $75 \%$ & 808 & $85 \%$ & $90 \%$ & 958 \\
\hline 0.0 & 0.0 & 0.0 & 0.0 & 0.0 & 0.0 & 0.0 & 0.0 & 0.0 & 0.0 & 0.0 & 0.0 & 0.0 & 0.0 & \\
\hline
\end{tabular}




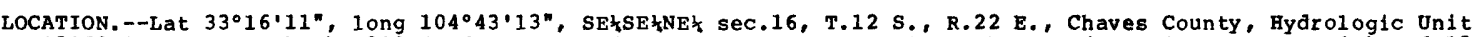
13060008 , on left bank, $300 \mathrm{ft}$ downstream from outlet structure in Rocky Dam (Two Rivers Reservoir) and $13.5 \mathrm{mi}$ southwest of Roswell.

DRAINAGE AREA.--64 $\mathrm{mi}^{2}$, approximately.

PERIOD OF RECORD.--May 1963 to September 1980.

GAGE.--Water-stage recorder and concrete control. Datum of gage is 3,935.66 ft National Geodetic Vertical Datum of 1929 (Corps of engineers benchmark). Prior to Jan. 12, 1972, at site $1.4 \mathrm{mi}$ downstream at datum $28.76 \mathrm{ft} 10 \mathrm{we}$.

REMARKS.--This record represents the outflow from Two River Reservoir through Rocky Dam. Outlet conduits in Rocky Dam have fixed openings.

AVERAGE DISCHARGE. - -17 years (water years 1964-80), $1.80 \mathrm{ft}^{3} / \mathrm{s}, 1,300$ acre-ft/yr.

EXTREMES FOR PERIOD OF RECORD.--Maximum discharge, $548 \mathrm{ft}^{3 / \mathrm{s}}$ Aug. 21, 1966, gage height, $4.57 \mathrm{ft}$, site and datum then in use, from rating curve extended above $260 \mathrm{ft}^{3} / \mathrm{s}$; no flow most of time.

STATISTICAL SUMMARIES

MEAN MONThLY AND MEAN ANNUAL DISChaRges 1964-80

\begin{tabular}{|c|c|c|c|c|c|c|}
\hline MONTH & $\begin{array}{l}\text { MINIMUM } \\
\left(\mathrm{FT}^{3} / \mathrm{S}\right)\end{array}$ & $\begin{array}{c}\text { MAXIMUM } \\
\left(\mathrm{FT}^{3} / \mathrm{S}\right)\end{array}$ & $\begin{array}{l}\text { MEAN } \\
\left\langle\mathrm{FT}^{3} / \mathrm{S}\right)\end{array}$ & $\begin{array}{l}\text { STAN- } \\
\text { DARD } \\
\text { DEVIA- } \\
\text { TION } \\
\left(\mathrm{FT}^{3} / \mathrm{S}\right)\end{array}$ & $\begin{array}{l}\text { COEFFI- } \\
\text { CIENT OF } \\
\text { VARI- } \\
\text { ATION }\end{array}$ & $\begin{array}{c}\text { PERCENT } \\
\text { OF } \\
\text { ANNUAL } \\
\text { RUNOFF }\end{array}$ \\
\hline $\begin{array}{l}\text { OCTOBER } \\
\text { NOVEMBER } \\
\text { DECEMBER } \\
\text { JANUARY } \\
\text { FEBRUARY } \\
\text { MARCH } \\
\text { APRIL } \\
\text { MAY } \\
\text { JUNE } \\
\text { JULY } \\
\text { AUGUST } \\
\text { SEPTEMBER }\end{array}$ & $\begin{array}{l}0.0 \\
0.0 \\
0.0 \\
0.0 \\
0.0 \\
0.0 \\
0.0 \\
0.0 \\
0.0 \\
0.0 \\
0.0 \\
0.0\end{array}$ & $\begin{array}{l}19 \\
0.0 \\
57 \\
0.0 \\
0.0 \\
0.0 \\
0.0 \\
0.0 \\
67 \\
32 \\
28 \\
26\end{array}$ & $\begin{array}{l}1.1 \\
0.0 \\
3.3 \\
0.0 \\
0.0 \\
0.0 \\
0.0 \\
0.0 \\
4.2 \\
3.3 \\
3.7 \\
5.3\end{array}$ & $\begin{array}{r}4.7 \\
0.0 \\
14 \\
0.0 \\
0.0 \\
0.0 \\
0.0 \\
0.0 \\
16 \\
8.7 \\
7.2 \\
8.5\end{array}$ & $\begin{array}{r}4.14 \\
=- \\
4.11 \\
-- \\
-- \\
-- \\
-- \\
-\overline{3} \\
2.88 \\
1.64 \\
1.91 \\
1.60\end{array}$ & $\begin{array}{r}5.4 \\
0.0 \\
15.9 \\
0.0 \\
0.0 \\
0.0 \\
0.0 \\
0.0 \\
20.0 \\
15.7 \\
17.9 \\
25.3\end{array}$ \\
\hline ANNUAL & 0.0 & 8.8 & 1.8 & 2.4 & 1.37 & 100 \\
\hline
\end{tabular}

MAGNITUDE AND PROBABILITY OF ANNUAL LOW FLOW BASED ON PERIOD OF RECORD 1965-80

\begin{tabular}{|c|c|c|c|c|c|c|}
\hline \multirow{2}{*}{$\begin{array}{l}\text { PERIOD } \\
\text { (CON- } \\
\text { SECU- } \\
\text { TIVE } \\
\text { DAYS) }\end{array}$} & \multicolumn{6}{|c|}{$\begin{array}{l}\text { DISCHARGE, IN FT'/S, FOR INDICATED RECURRENCE } \\
\text { INTERVAL, IN YEARS, AND ANNUAL NON- } \\
\text { EXCEEDANCE PROBABILITY, IN PERCENT }\end{array}$} \\
\hline & $\begin{array}{c}2 \\
508\end{array}$ & ${ }^{5} 0^{2}$ & $\begin{array}{l}10 \\
108\end{array}$ & $\begin{array}{l}20 \\
58\end{array}$ & $\begin{array}{l}50 \\
28\end{array}$ & $\begin{array}{r}100 \\
18\end{array}$ \\
\hline 1 & $=$ & - & -- & -- & -- & \\
\hline 3 & - & -- & - & - & -- & \\
\hline 7 & -- & - & -- & - & - & \\
\hline 14 & - & - & -- & -- & -- & \\
\hline 30 & -- & -- & -- & -- & -- & \\
\hline 60 & 0.0 & 0.0 & 0.0 & 0.0 & - & \\
\hline 90 & -- & - & - & - & -- & \\
\hline 120 & 0.0 & 0.0 & 0.0 & 0.0 & -- & \\
\hline 183 & 0.0 & 0.0 & 0.0 & 0.0 & -- & \\
\hline
\end{tabular}

MAGNITUDE AND PROBABILITY OF ANNUAL HIGH FLOW BASED ON PERIOD OF RECORD 1964-80

\begin{tabular}{|c|c|c|c|c|c|c|}
\hline \multirow{2}{*}{$\begin{array}{l}\text { PERIOD } \\
\text { (CON- } \\
\text { SECU- } \\
\text { TIVE } \\
\text { DAYS) }\end{array}$} & \multicolumn{6}{|c|}{$\begin{array}{l}\text { DISCHARGE, IN FT } 3 \text { /S, FOR INDICATED RECURRENCE } \\
\text { INTERVAL, IN YEARS, AND ANNUAL } \\
\text { EXCEEDANCE PROBABILITY, IN PERCENT }\end{array}$} \\
\hline & $\begin{array}{c}2 \\
508\end{array}$ & $\begin{array}{c}5 \\
208\end{array}$ & & $\begin{array}{l}25 \\
48\end{array}$ & $\begin{array}{l}50 \\
28\end{array}$ & $\begin{array}{r}100 \\
18\end{array}$ \\
\hline
\end{tabular}

$\begin{array}{rrrrrrr}1 & 101 & 271 & 311 & 325 & -- & -- \\ 3 & 65 & 217 & 263 & 284 & -- & - \\ 7 & 34 & 139 & 181 & 204 & -- & - \\ 15 & 18 & 68 & 104 & 142 & -- & - \\ 30 & 9.7 & 36 & 54 & 73 & -- & - \\ 60 & 4.9 & 19 & 31 & 43 & -- & - \\ 90 & 3.3 & 13 & 22 & 32 & -- & -\end{array}$

DURATION TABLE OF DAILY MEAN FLOW FOR PERIOD OF RECORD 1964-80

DISCHARGE, IN FT $3 / \mathrm{S}$, THAT WAS EOUALED OR EXCEEDED FOR INDICATED PERCENT OF TIME

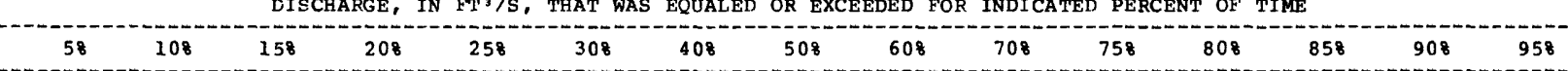


08393600 NORTH SPRING RIVER AT ROSWELL, NM

LOCATION.--Lat $33^{\circ} 23^{\prime} 47^{\prime \prime}$, long $104^{\circ} 32^{\prime} 5^{\prime \prime}$, in NWłWłSEł sec.31, T.10 S., R. 24 E., Chaves County, Hydrologic Unit 13060008 , on left bank, in Roswell Municipal Golf Course, 2,400 ft upstream from Montana Avenue, and 2 blocks north of West Second Street, Roswell.

DRAINAGE AREA. - - $19.5 \mathrm{mi}^{2}$.

PERIOD OF RECORD.--May 1958 to December 1977.

GAGE.--Water-stage recorder. Altitude of gage is 3,575 ft, from topographic map.

REMARKS.--No diversions above station.

AVERAGE DISCHARGE.--19 years (water years 1959-77), $0.004 \mathrm{ft} / \mathrm{s}, 2.90 \mathrm{acre}-\mathrm{ft} / \mathrm{yr}$.

EXTREMES FOR PERIOD OF RECORD.--Maximum discharge, $387 \mathrm{ft} 3 / \mathrm{s}$ June 13, 1964, gage height, 4.65 ft, from rating curve extended above $80 \mathrm{ft}^{3 / \mathrm{s}}$ on basis of slope-area measurement; no flow most of time.

STATISTICAL SUMMARIES

MEAN MONTHLY AND MEAN ANNUAL DISCHARGES 1959-77

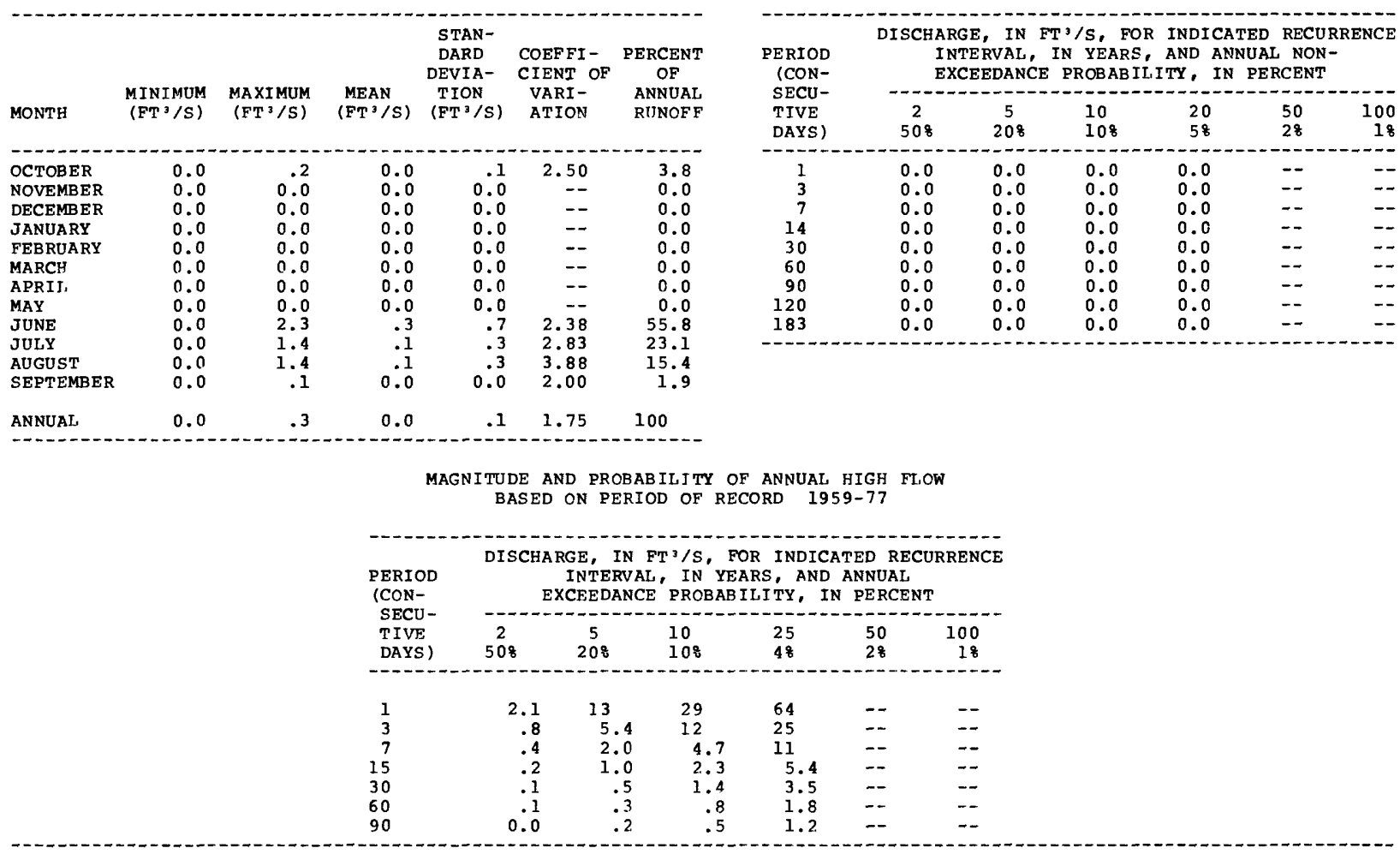

DURATION TABLE OF DAILY MEAN FLOW FOR PERIOD OF RECORD 1959-77

DISCHARGF, IN FT $3 / S$, THAT WAS FOUALED OR EXCEEDED FOR INDICATED PERCENT OF TIME

\begin{tabular}{|c|c|c|c|c|c|c|c|c|c|c|c|c|c|c|}
\hline 58 & 108 & 158 & 208 & 258 & 308 & 408 & 508 & 608 & 708 & 758 & 808 & 858 & $90 \frac{8}{8}$ & 958 \\
\hline 0.0 & 0.0 & 0.0 & 0.0 & 0.0 & 0.0 & 0.0 & 0.0 & 0. & 0.0 & 0.0 & 0. & 0.0 & 0.0 & 0. \\
\hline
\end{tabular}


08394100 PECOS RIVER NEAR HAGERMAN, NM

LOCATION.--Lat $33^{\circ} 10^{\circ} O B^{\prime \prime}$, long $104^{\circ} 18^{\prime} 24^{\prime \prime}$, in SEkSWkEk sec.23, T.13 S., R.26 E., Chaves County, Hydrologic Onit 13060007 , on left bank $3.4 \mathrm{mi}$ upstream from Rio Felix, $4.9 \mathrm{mi}$ north of Hagerman, and at mile 544.6 .

DRAINAGE AREA. $--13,630 \mathrm{mi}^{2}$, approximately (contributing area).

PERIOD OF RECORD,--February 1968 to December 1981 (operated as a low-flow station only).

GAGE.--Water-stage recorder. Elevation of gage is 3,390 ft above National Geodetic Vertical Datum of 1929 , from topographic map.

REMARKS.--Flow regulated by Santa Rosa Lake (station 08382810) since April 1980, by Lake Sumner (station 08384000) since August 1937 and by Two Rivers Reservoir (station 08390600 ) since July 1963. Diversions and ground-water withdrawals for irrigations of about 80,000 acres upstream from station.

AVERAGE DISCHARGE.--21 years (water years 1959-79), $164 \mathrm{ft} / \mathrm{s}, 118,800$ acre-ft/yr.

EXTREMES FOR PERIOD OF RECORD. --Maximum daily discharge determined, 3,700 ft $3 / \mathrm{s}$, Sept. 11, 1969; no flow at times in $1971,1974,1976,1977$.

STATISTICAL SUMMARIES (PERIOD BEFORE REGULATION OF SANTA ROSA DAM)

MEAN MONTHLY AND MEAN ANNUAL DISCHARGES 1969-79

\begin{tabular}{|c|c|c|c|c|c|c|}
\hline MONTH & $\begin{array}{l}\text { MINIMUM } \\
\text { (FT, } / S \text { ) }\end{array}$ & $\begin{array}{c}\text { MAXIMUM } \\
\left(\mathrm{FT}^{3} / \mathrm{S}\right)\end{array}$ & $\begin{array}{l}\text { MEAN } \\
\left(F^{3} / S\right)\end{array}$ & $\begin{array}{l}\text { STAN- } \\
\text { DARD } \\
\text { DEVIA- } \\
\text { TION } \\
(\mathrm{FT}: / \mathrm{S})\end{array}$ & $\begin{array}{l}\text { COEFFI- } \\
\text { CIENT OF } \\
\text { VARI- } \\
\text { ATION }\end{array}$ & $\begin{array}{c}\text { PERCENT } \\
\text { OF } \\
\text { ANNUAL } \\
\text { RUNOFF }\end{array}$ \\
\hline $\begin{array}{l}\text { OCTOBER } \\
\text { NOVEMBER } \\
\text { DECEMBER } \\
\text { JANUARY } \\
\text { FEBRUARY } \\
\text { MARCH } \\
\text { APRIL } \\
\text { MAY } \\
\text { JUNE } \\
\text { JULY } \\
\text { AUGUST } \\
\text { SEPTEMBER }\end{array}$ & $\begin{array}{l}21 \\
32 \\
35 \\
32 \\
24 \\
29 \\
21 \\
13 \\
4.7 \\
2.9 \\
14 \\
10\end{array}$ & $\begin{array}{r}284 \\
92 \\
128 \\
102 \\
61 \\
424 \\
522 \\
358 \\
628 \\
333 \\
454 \\
723\end{array}$ & $\begin{array}{r}87 \\
58 \\
53 \\
49 \\
41 \\
186 \\
209 \\
140 \\
272 \\
202 \\
224 \\
150\end{array}$ & $\begin{array}{r}84 \\
22 \\
27 \\
21 \\
11 \\
165 \\
190 \\
144 \\
255 \\
121 \\
186 \\
228\end{array}$ & $\begin{array}{r}.97 \\
.38 \\
.51 \\
.42 \\
.27 \\
.89 \\
.91 \\
1.03 \\
.94 \\
.60 \\
.83 \\
1.52\end{array}$ & $\begin{array}{r}5.2 \\
3.4 \\
3.2 \\
2.9 \\
2.5 \\
11.1 \\
12.5 \\
8.4 \\
16.3 \\
12.1 \\
13.4 \\
9.0\end{array}$ \\
\hline NNUAL & 106 & 236 & 164 & 56 & .34 & 100 \\
\hline
\end{tabular}

MAGNITUDE AND PROBABILITY OF ANNUAL LOW FLON BASED ON PERIOD OF RECORD 1970-79

\begin{tabular}{|c|c|c|c|c|c|c|}
\hline \multirow{2}{*}{$\begin{array}{l}\text { PERIOD } \\
\text { (CON- } \\
\text { SECU- } \\
\text { TIVE } \\
\text { DAYS) }\end{array}$} & \multicolumn{6}{|c|}{$\begin{array}{l}\text { DISCHARGE, IN FT'3 } 3 \text {, FOR INDICATED RECURRENCE } \\
\text { INTERVAL, IN YEARS, AND ANNUAL NON- } \\
\text { EXCEEDANCE PROBABILITY, IN PERCENT }\end{array}$} \\
\hline & ${ }_{508}^{2}$ & $\begin{array}{c}5 \\
208\end{array}$ & $\begin{array}{l}10 \\
108\end{array}$ & $\begin{array}{l}20 \\
58\end{array}$ & $\begin{array}{l}50 \\
28\end{array}$ & \\
\hline $\begin{array}{r}1 \\
3 \\
7 \\
14 \\
30 \\
60 \\
90 \\
120 \\
183\end{array}$ & $\begin{array}{l}4.6 \\
4.1 \\
5.9 \\
7.7 \\
19 \\
26 \\
31 \\
36 \\
43\end{array}$ & $\begin{array}{r}0.0 \\
.5 \\
2.0 \\
3.6 \\
12 \\
18 \\
23 \\
28 \\
31\end{array}$ & $\begin{array}{r}0.0 \\
.1 \\
1.1 \\
2.4 \\
9.1 \\
15 \\
20 \\
24 \\
27\end{array}$ & $\begin{array}{r}0.0 \\
0.0 \\
.6 \\
1.7 \\
7.2 \\
14 \\
19 \\
22 \\
24\end{array}$ & $\begin{array}{l}-- \\
-- \\
-- \\
-- \\
-- \\
-- \\
--\end{array}$ & \\
\hline
\end{tabular}

MAGNITUDE AND PROBABILITY OF ANNUAL HIGH FLON BASED ON PERIOD OF RECORD 1969-79

\begin{tabular}{|c|c|c|c|c|c|c|}
\hline \multirow{2}{*}{$\begin{array}{l}\text { PERIOD } \\
\text { (CON- } \\
\text { SECU- } \\
\text { TIVE } \\
\text { DAYS) }\end{array}$} & \multicolumn{6}{|c|}{$\begin{array}{l}\text { DISCHARGE, IN FT'/S, FOR INDICATED RECURRENC } \\
\text { INTERVAL, IN YEARS, AND ANNUAL } \\
\text { EXCEEDANCE PROBABILITY, IN PERCENT }\end{array}$} \\
\hline & $\begin{array}{c}2 \\
508\end{array}$ & $\begin{array}{c}5 \\
208\end{array}$ & $\begin{array}{l}10 \\
108\end{array}$ & $\begin{array}{l}25 \\
48\end{array}$ & $\begin{array}{l}50 \\
28\end{array}$ & $\begin{array}{r}100 \\
18\end{array}$ \\
\hline $\begin{array}{r}1 \\
3 \\
7 \\
15 \\
30 \\
60 \\
90\end{array}$ & $\begin{array}{r}2120 \\
1690 \\
1100 \\
894 \\
715 \\
477 \\
384\end{array}$ & $\begin{array}{r}3370 \\
2120 \\
1230 \\
1000 \\
806 \\
583 \\
513\end{array}$ & $\begin{array}{r}4220 \\
2310 \\
1300 \\
1080 \\
833 \\
624 \\
576\end{array}$ & $\begin{array}{l}-- \\
-- \\
-- \\
-- \\
--\end{array}$ & $\begin{array}{l}-- \\
-- \\
-- \\
-- \\
-- \\
--\end{array}$ & $\begin{array}{l}-- \\
-- \\
-- \\
-- \\
--\end{array}$ \\
\hline
\end{tabular}

DURATION TABLE OF DAILY MEAN FLOW FOR PERIOD OF RECORD 1969-79

DISCHARGE, IN FT'/S, THAT WAS EQUALED OR EXCEEDED FOR INDICATED PERCENT OF TIME

\begin{tabular}{|c|c|c|c|c|c|c|c|c|c|c|c|c|c|c|}
\hline 58 & 108 & 158 & 208 & 258 & 308 & 408 & 508 & 608 & 708 & 758 & 808 & 858 & 908 & 958 \\
\hline 897 & 626 & 273 & 159 & 110 & 84 & 61 & 47 & 38 & 32 & 29 & 25 & 21 & 17 & 13 \\
\hline
\end{tabular}


RIO GRANDE BASIN

08394500 RIO FELIX AT OLD HIGHWAY BRIDGE, NEAR HAGERMAN, NM

LOCATION, - Lat $33^{\circ} 07 \cdot 30^{\prime \prime}$, Iong $104^{\circ} 20^{\prime} 40^{\prime \prime}$, in SWłSW 13060009 , near left bank on downstream side of abandoned bridge pier, 0.6 mi upstream from alternate U.S. Highway $285,1.3 \mathrm{mi}$ northwest of Hagerman, and $2.7 \mathrm{mi}$ upstream from mouth. Mouth at Pecos River mile 541.4 .

DRAINAGE AREA. $--932 \mathrm{mi}^{2}$, contributing area.

PERIOD OF RECORD. --April 1939 to current year. March 1932 to April 1939 at site 1 mi downstream; records for periods of low flow not equivalent, owing to inflow between sites.

REVISED RECORDS.--WSP 928: 1940 (M). WSP 1562: 1939-40, 1941 (M).

GAGE.--Water-stage recorder. Elevation of gage is 3,403.40 ft above National Geodetic Vertical Datum of 1929. REMARKS.--Diversions for irrigation of about 350 acres, 1959 determination, upatream from station.

AVERAGE DISCHARGE. - 46 years (water years 1940-85), $13.6 \mathrm{ft}^{3} / \mathrm{s}, 9,850$ acre-ft/yr.

EXTREMES FOR PERIOD OF RECORD.--Maximum discharge, 74,000 ft $3 / \mathrm{s}$, Oct. 7, 1954, gage height, $27.5 \mathrm{ft}$, from floodmarks, from rating curve extended above $12,000 \mathrm{ft} / \mathrm{s}$ on basis of slope-area measurement at point $5.5 \mathrm{mi}$

upstream from gage (adjusted for channel storage); no flow for many periods.
Flood in 1954 is the highest since 1894 (information from local residents).

EXTREMES OUTSIDE PERIOD OF RECORD.--Flood of Oct. 1, 1904, is probably second highest since 1894; another major flood occurred in April 1915 .

STATISTICAL SUMMARIES

MEAN MONTHLY AND MEAN ANNUAL DISCHARGES 1940-85

\begin{tabular}{|c|c|c|c|c|c|c|}
\hline MONTH & $\begin{array}{l}\text { MINIMUM } \\
\left(\mathrm{FT}^{3} / \mathrm{S}\right)\end{array}$ & $\begin{array}{l}\text { MAXIMUM } \\
\left(F T^{3} / S\right)\end{array}$ & $\begin{array}{l}\text { MEAN } \\
\left(\mathrm{FT}^{3} / \mathrm{S}\right)\end{array}$ & $\begin{array}{l}\text { STAN- } \\
\text { DARD } \\
\text { DEVIA- } \\
\text { TION } \\
\left(\mathrm{FT}^{3} / \mathrm{S}\right)\end{array}$ & $\begin{array}{l}\text { COEFFI- } \\
\text { CIENT OF } \\
\text { VARI - } \\
\text { ATION }\end{array}$ & $\begin{array}{c}\text { PERCENT } \\
\text { OF } \\
\text { ANNUAL } \\
\text { RUNOF F }\end{array}$ \\
\hline $\begin{array}{l}\text { OCTOBER } \\
\text { NOVEMBER } \\
\text { DECEMBER } \\
\text { JANUARY } \\
\text { FEBRUARY } \\
\text { MARCH } \\
\text { APRIL } \\
\text { MAY } \\
\text { JUNE } \\
\text { JULY } \\
\text { AUGUST } \\
\text { SEPTEMBER }\end{array}$ & $\begin{array}{l}0.0 \\
0.0 \\
0.0 \\
0.0 \\
0.0 \\
0.0 \\
0.0 \\
0.0 \\
0.0 \\
0.0 \\
0.0 \\
0.0\end{array}$ & $\begin{array}{r}687 \\
61 \\
37 \\
45 \\
44 \\
12 \\
33 \\
173 \\
182 \\
231 \\
302 \\
705\end{array}$ & $\begin{array}{r}28 \\
5.1 \\
3.1 \\
2.9 \\
3.3 \\
.9 \\
1.5 \\
11 \\
14 \\
24 \\
22 \\
47\end{array}$ & $\begin{array}{r}105 \\
12 \\
7.2 \\
7.5 \\
8.6 \\
2.1 \\
5.5 \\
31 \\
33 \\
49 \\
52 \\
138\end{array}$ & $\begin{array}{l}3.79 \\
2.25 \\
2.35 \\
2.62 \\
2.64 \\
2.29 \\
3.56 \\
2.81 \\
2.43 \\
2.02 \\
2.40 \\
2.92\end{array}$ & $\begin{array}{r}17.1 \\
3.2 \\
1.9 \\
1.8 \\
2.0 \\
.6 \\
.9 \\
6.7 \\
8.4 \\
15.0 \\
13.3 \\
29.2\end{array}$ \\
\hline ANNUAL & 0.0 & 87 & 14 & 19 & 1.37 & 100 \\
\hline
\end{tabular}

MAGNITUDE AND PROBABILITY OF ANNUAL LOW FLOW BASED ON PERIOD OF RECORD 1941-85

\begin{tabular}{|c|c|c|c|c|c|c|}
\hline \multirow{2}{*}{$\begin{array}{l}\text { PERIOD } \\
\text { (CON- } \\
\text { SECU- } \\
\text { TIVE } \\
\text { DAYS) }\end{array}$} & \multicolumn{6}{|c|}{$\begin{array}{l}\text { DISCHARGE, IN FT }{ }^{3 / S} \text {, FOR INDICATED RECURRENCE } \\
\text { INTERVAL, IN YEARS, AND ANNUAL NON- } \\
\text { EXCEEDANCE PROBABILITY, IN PERCENT }\end{array}$} \\
\hline & $\begin{array}{c}2 \\
508\end{array}$ & $\begin{array}{c}5 \\
208\end{array}$ & $\begin{array}{l}10 \\
108\end{array}$ & $\begin{array}{l}20 \\
58\end{array}$ & $\begin{array}{l}50 \\
28\end{array}$ & $\begin{array}{r}100 \\
18\end{array}$ \\
\hline $\begin{array}{r}1 \\
3 \\
7 \\
14 \\
30 \\
60 \\
90 \\
120 \\
183\end{array}$ & $\begin{array}{l}0.0 \\
0.0 \\
0.0 \\
0.0 \\
0.0 \\
0.0 \\
0.0 \\
0.0 \\
1.0\end{array}$ & $\begin{array}{l}0.0 \\
0.0 \\
0.0 \\
0.0 \\
0.0 \\
0.0 \\
0.0 \\
0.0 \\
0.0\end{array}$ & $\begin{array}{l}0.0 \\
0.0 \\
0.0 \\
0.0 \\
0.0 \\
0.0 \\
0.0 \\
0.0 \\
0.0\end{array}$ & $\begin{array}{l}0.0 \\
0.0 \\
0.0 \\
0.0 \\
0.0 \\
0.0 \\
0.0 \\
0.0 \\
0.0\end{array}$ & $\begin{array}{l}0.0 \\
0.0 \\
0.0 \\
0.0 \\
0.0 \\
0.0 \\
0.0 \\
0.0 \\
0.0\end{array}$ & $\begin{array}{l}0.0 \\
0.0 \\
0.0 \\
0.0 \\
0.0 \\
0.0 \\
0.0 \\
0.0 \\
0.0\end{array}$ \\
\hline
\end{tabular}

AGNITUDE AND PROBABILITY OF ANNUAL HIGH FLOW BASED ON PERIOD OF RECORD 1940-85

\begin{tabular}{|c|c|c|c|c|c|c|}
\hline \multirow{2}{*}{$\begin{array}{l}\text { PERIOD } \\
\text { (CON- } \\
\text { SECU- } \\
\text { TIVE } \\
\text { DAYS) }\end{array}$} & \multicolumn{6}{|c|}{$\begin{array}{l}\text { DISCHARGE, IN FT'/S, FOR INDICATED RECURRENC } \\
\text { INTERVAL, IN YEARS, AND ANNUAL } \\
\text { EXCEEDANCE PROBABILITY, IN PERCENT }\end{array}$} \\
\hline & $\begin{array}{c}2 \\
508\end{array}$ & $\begin{array}{c}5 \\
208\end{array}$ & $\begin{array}{l}10 \\
108\end{array}$ & $\begin{array}{l}25 \\
48\end{array}$ & $\begin{array}{l}50 \\
28\end{array}$ & $\begin{array}{r}100 \\
18\end{array}$ \\
\hline $\begin{array}{r}1 \\
3 \\
7 \\
15 \\
30 \\
60 \\
90\end{array}$ & $\begin{array}{r}1020 \\
359 \\
192 \\
108 \\
64 \\
36 \\
27\end{array}$ & $\begin{array}{r}3190 \\
1360 \\
658 \\
338 \\
185 \\
103 \\
74\end{array}$ & $\begin{array}{r}4200 \\
2460 \\
1120 \\
547 \\
281 \\
154 \\
108\end{array}$ & $\begin{array}{r}4870 \\
4310 \\
1830 \\
842 \\
401 \\
215 \\
145\end{array}$ & $\begin{array}{r}5100 \\
6000 \\
2430 \\
1070 \\
484 \\
256 \\
168\end{array}$ & $\begin{array}{r}5220 \\
7930 \\
3060 \\
1300 \\
560 \\
292 \\
188\end{array}$ \\
\hline
\end{tabular}

DURATION TABLE OF DAILY MEAN FLOW FOR PERIOD OF RECORD 1940-85

DISCHARGE, IN FT'/S, THAT WAS EQUALED OR EXCEEDED FOR INDICATED PERCENT OF TIME

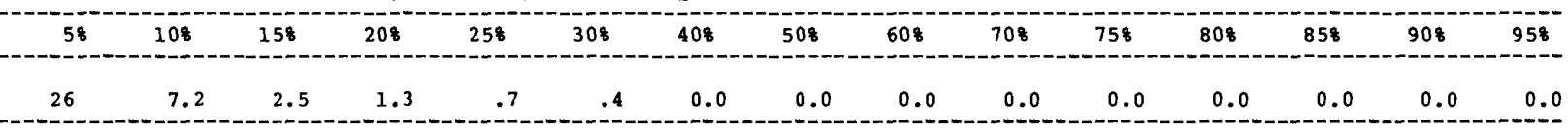


RIO GRANDE BASIN

08395500 PECOS RIVER NEAR LAKE ARTHUR, NM

LOCATION. --Lat 32.59'18", long $104^{\circ} 19^{\prime} 20^{\prime \prime}$, in SWHEk sec.27, T.15 S., R. 26 E. , Chaves County, Hydrologic Unit 1306007 , on left bank $400 \mathrm{ft}$ upstream from county bridge, $2.5 \mathrm{mi}$ east of Lake Arthur, $7 \mathrm{mi}$ upstream from cottonwood Creek, $11 \mathrm{mi}$ northeast of Artesia, and at mile 522.0.

DRAINAGE AREA.--14,760 $\mathrm{mi}^{2}$, approximately (contributing area).

PERIOD OF RECORD. --August 1938 to current year.

GAGE.--Water-stage recorder and rock control. Elevation of gage is 3,327.07 ft above National Geodetic Vertical Datum of 1929 .

REMARKS.--Flow regulated by Santa Rosa Lake (station 08382810) since April 1980, by Lake Sumner (station 08384000) since August 1937, and by Two Rivers Reservoir (station 08390600) since July 1963. Diversions and ground-water withdrawals for irrigation of about 124,000 acres, 1959 determination, upstream from station.

AVERAGE DISCHARGE.--41 years (water years 1939-79), $235 \mathrm{ft}^{3} / \mathrm{s}, 170,300$ acre-ft/yr.

EXTREMES FOR PERIOD OF RECORD.--Maximum discharge, 49,600 $\mathrm{ft}^{3} / \mathrm{s}$, Sept. 24, 1941, gage height, $21.90 \mathrm{ft}$, from $\mathrm{rating}$ curve extended above $16,100 \mathrm{ft}^{3} / \mathrm{s}$ on basis of slope-area measurement at gage height $21.77 \mathrm{ft}$; no $\mathrm{flow}$ at times in $1947,1953-4,1962,1964$.

EXTREMES OUTSIDE PERIOD OF RECORD.--Flood of May 30,1937 , reached a stage of $21.77 \mathrm{ft}$, discharge, $51,500 \mathrm{ft} / \mathrm{s}$, on basis of slope-area measurement of peak flow.

STATISTICAL SUMMARIES (PERIOD BEFORE REGULATION OF SANTA ROSA DAM)

MEAN MONTHLY AND MEAN ANNUAL DISCHARGES 1939-79

\begin{tabular}{|c|c|c|c|c|c|c|}
\hline MONTH & $\begin{array}{l}\text { M INIMUM } \\
\left(\mathrm{FT}^{3} / \mathrm{S}\right)\end{array}$ & $\begin{array}{c}\text { MAXIMUM } \\
\left(\mathrm{FT}^{3} / \mathrm{S}\right)\end{array}$ & $\begin{array}{l}\text { MEAN } \\
\left(\mathrm{FT}^{3} / \mathrm{S}\right)\end{array}$ & $\begin{array}{l}\text { STAN- } \\
\text { DARD } \\
\text { DEVIA- } \\
\text { TION } \\
\left(\mathrm{FT}^{3} / \mathrm{S}\right)\end{array}$ & $\begin{array}{l}\text { COEFFI- } \\
\text { CIENT OF } \\
\text { VARI- } \\
\text { ATION }\end{array}$ & $\begin{array}{c}\text { PE RCENT } \\
\text { OF } \\
\text { ANNUAL } \\
\text { RUNOF F }\end{array}$ \\
\hline $\begin{array}{l}\text { OCTOBER } \\
\text { NOVEMBER } \\
\text { DECEMBER } \\
\text { JANUARY } \\
\text { FEBRUARY } \\
\text { MARCH } \\
\text { APRIL } \\
\text { MAY } \\
\text { JUNE } \\
\text { JULY } \\
\text { AUGUST } \\
\text { SEPTEMBER }\end{array}$ & $\begin{array}{l}3.9 \\
32 \\
30 \\
35 \\
27 \\
17 \\
7.4 \\
12 \\
4.8 \\
1.0 \\
.4 \\
1.3\end{array}$ & $\begin{array}{r}3701 \\
983 \\
546 \\
451 \\
446 \\
682 \\
1308 \\
3673 \\
2436 \\
1521 \\
913 \\
5407\end{array}$ & $\begin{array}{r}278 \\
129 \\
98 \\
93 \\
81 \\
202 \\
251 \\
304 \\
322 \\
360 \\
285 \\
410\end{array}$ & $\begin{array}{r}692 \\
200 \\
87 \\
78 \\
68 \\
177 \\
259 \\
614 \\
390 \\
331 \\
213 \\
908\end{array}$ & $\begin{array}{r}2.49 \\
1.55 \\
.89 \\
.83 \\
.84 \\
.88 \\
1.03 \\
2.02 \\
1.21 \\
.92 \\
.75 \\
2.21\end{array}$ & $\begin{array}{r}9.9 \\
4.6 \\
3.5 \\
3.3 \\
2.9 \\
7.2 \\
8.9 \\
10.8 \\
11.4 \\
12.8 \\
10.1 \\
14.6\end{array}$ \\
\hline ANNUAL & 62 & 1314 & 235 & 226 & .96 & 100 \\
\hline
\end{tabular}

MAGNITUDE AND PROBABILITY OF ANNUAL LOW FLOW BASED ON PERIOD OF RECORD 1940-79

\begin{tabular}{|c|c|c|c|c|c|c|}
\hline \multirow{2}{*}{$\begin{array}{l}\text { PERI OD } \\
\text { (CON- } \\
\text { SECU- } \\
\text { TIVE } \\
\text { DAYS) }\end{array}$} & \multicolumn{6}{|c|}{$\begin{array}{l}\text { DISCHARGE, IN FT }{ }^{3} / S \text {, FOR INDICATED RECURRENCE } \\
\text { INTERVAL, IN YEARS, AND ANNUAL NON- } \\
\text { EXCEEDANCE PROBABILITY, IN PERCENT }\end{array}$} \\
\hline & $\begin{array}{c}2 \\
508\end{array}$ & $\begin{array}{c}5 \\
208\end{array}$ & $\begin{array}{l}10 \\
108\end{array}$ & $\begin{array}{l}20 \\
58\end{array}$ & $\begin{array}{l}50 \\
28\end{array}$ & $\begin{array}{r}100 \\
18\end{array}$ \\
\hline $\begin{array}{r}1 \\
3 \\
7 \\
14 \\
30 \\
60 \\
90 \\
120 \\
183\end{array}$ & $\begin{array}{c}1.8 \\
2.3 \\
3.4 \\
5.7 \\
16 \\
37 \\
57 \\
62 \\
67\end{array}$ & $\begin{array}{r}.4 \\
.6 \\
.9 \\
1.6 \\
4.6 \\
13 \\
25 \\
30 \\
39\end{array}$ & $\begin{array}{r}0.0 \\
.3 \\
.4 \\
.8 \\
2.2 \\
6.5 \\
14 \\
19 \\
32\end{array}$ & $\begin{array}{r}0.0 \\
.1 \\
.2 \\
.4 \\
1.1 \\
3.4 \\
8.2 \\
13 \\
28\end{array}$ & $\begin{array}{r}0.0 \\
0.0 \\
.1 \\
.2 \\
.5 \\
1.5 \\
4.1 \\
7.8 \\
25\end{array}$ & $\begin{array}{r}0.0 \\
0.0 \\
.1 \\
.1 \\
.3 \\
.8 \\
2.5 \\
5.4 \\
23\end{array}$ \\
\hline
\end{tabular}

MAGNITUDE AND PROBABILITY OF ANNUAL HIGH FLON BASED ON PERIOD OF RECORD 1939-79

\begin{tabular}{|c|c|c|c|c|c|c|}
\hline \multirow{2}{*}{$\begin{array}{l}\text { PERIOD } \\
\text { (CON- } \\
\text { SECU- } \\
\text { TIVE } \\
\text { DAYS) }\end{array}$} & \multicolumn{6}{|c|}{$\begin{array}{l}\text { DISCHARGE, IN FT" /S, FOR INDICATED RECURRENC } \\
\text { INTERVAL, IN YEARS, AND ANNUAL } \\
\text { EXCEEDANCE PROBABILITY, IN PERCENT }\end{array}$} \\
\hline & $\begin{array}{c}2 \\
508\end{array}$ & $\begin{array}{c}5 \\
208\end{array}$ & $\begin{array}{l}10 \\
108\end{array}$ & $\begin{array}{l}25 \\
48\end{array}$ & $\begin{array}{l}50 \\
28\end{array}$ & $\begin{array}{r}100 \\
18\end{array}$ \\
\hline $\begin{array}{r}1 \\
3 \\
7 \\
15 \\
30 \\
60 \\
90\end{array}$ & $\begin{array}{r}2810 \\
1970 \\
1330 \\
923 \\
638 \\
440 \\
349\end{array}$ & $\begin{array}{r}6280 \\
4250 \\
2560 \\
1600 \\
1100 \\
752 \\
596\end{array}$ & $\begin{array}{r}10400 \\
7200 \\
4130 \\
2440 \\
1620 \\
1080 \\
855\end{array}$ & $\begin{array}{r}19100 \\
13900 \\
7690 \\
4290 \\
2670 \\
1720 \\
1340\end{array}$ & $\begin{array}{r}29400 \\
22600 \\
12300 \\
6600 \\
3870 \\
2400 \\
1860\end{array}$ & $\begin{array}{r}44400 \\
36200 \\
19500 \\
10200 \\
5600 \\
3340 \\
2560\end{array}$ \\
\hline
\end{tabular}

DURATION TABLE OF DAILY MEAN FLOW FOR PERIOD OF RECORD 1939-79

DISCHARGE, IN FT'/S, THAT WAS EQUALED OR EXCEEDED FOR INDICATED PERCENT OF TIME

\begin{tabular}{|c|c|c|c|c|c|c|c|c|c|c|c|c|c|c|}
\hline 58 & 108 & 158 & 208 & 258 & 308 & 408 & 508 & 608 & 708 & 758 & 808 & 858 & 908 & 958 \\
\hline 948 & 647 & 364 & 209 & 149 & 125 & 90 & 68 & 52 & 40 & 34 & 28 & 20 & 13 & 5.1 \\
\hline
\end{tabular}


RIO GRANDE BASIN

08396000 COTTONWOOD CREEK NEAR LAKE ARTHUR, NK

LOCATION,--Lat $32^{\circ} 54^{\prime} 5^{\prime \prime}$, long $104^{\circ} 22^{\prime} 00^{\prime \prime}$, in SWłSEł sec.15, T.16 S., R. 26 E. on right bank 75 ft upstream from road bridge, 1/ miles upstream from mouth, and 6 miles south of town of Lake Arthur.

DRAINAGE AREA,--199 $\mathrm{mi}^{2}$ (contributing area).

PERIOD OF RECORD.--March 1932 to January 6, 1965 (discontinued). All figures of discharge above 150 ft $3 / \mathrm{s}$ prior to June 1937 and daily discharges Sept. 24-30, 1932, June 13, 14, Sept. 5, 1935 and May 29, 1937, as published in previous Water supply Papers have been found to be unreliable and should not be used.

GAGE.--Water-stage recorder (digital) and concrete control. Datum of gage is $3,316.3 \mathrm{ft}$ above mean sea leve1 (river-profile survey). At site $75 \mathrm{ft}$ downstream at different datum Mar. 7 , 1932 to Mar. 28 , 1935, and at datum $1.36 \mathrm{ft}$ and $2.10 \mathrm{ft}$ lower than present datum Mar. 29, 1935 to sept. 30, 1936, and Oct. 1 , 1936 to Aug. 29 , 1938, respectively. Aug. 30, 1938 to May 22, 1948, at present site at datum $1.54 \mathrm{ft} 10 \mathrm{fer}$.

REMARKS.--Diversions and ground-water withdrawals for irrigation of about 4,500 acres (1959 determination) above station. Capacity of original excavated channel at and above gage has been progressively reduced by salt-cedar growth, blow sand, and bank erosion. Since 1957 a compacted earth plug in channel (forms pond for pump diversion) 1 mile above gage has reduced low-flow record to leakage through or under plug, ground-water inflow, and irrigation return entering channel in the $1-m i l e$ reach above gage. Higher sustained discharges originating irrigation return entering channel in the l-mile reach above gage. Higher sustained discharges originating Pecos River flood plain, most of over-bank flow remaining ponded or bypassing gage. Low-fiow record represents contribution to Pecos River, based on comparative discharge measurements of March ig61.

AVERAGE DISCHARGE. --30 years (water years 1934, 1936-64), $5.40 \mathrm{ft} / \mathrm{s}, 3,910 \mathrm{acre-ft} / \mathrm{yr}$.

EXTREMES FOR PERIOD OF RECORD.--Maximum discharge not determined, occurred June 13, 1935; maximum gage height, 12.0 ft May 30, 1937, present datum, from floodmarks (backwater from Pecos River); no flow at times.

STATISTICAL SUMMARIES

MEAN MONTHLY AND MEAN ANNUAL DISChARGES 1934, 1936-64

\begin{tabular}{|c|c|c|c|c|c|c|}
\hline MONTH & $\begin{array}{l}\text { MINIMUM } \\
\left(\mathrm{FT}^{3} / \mathrm{S}\right)\end{array}$ & $\begin{array}{l}\text { MAXIMUM } \\
\left(\mathrm{FT}^{2} / \mathrm{S}\right)\end{array}$ & $\begin{array}{l}\text { MEAN } \\
\left(\mathrm{FT}^{3} / \mathrm{S}\right)\end{array}$ & $\begin{array}{c}\text { STAN- } \\
\text { DARD } \\
\text { DEVIA- } \\
\text { TION } \\
\left(\mathrm{FT}^{3} / \mathrm{S}\right)\end{array}$ & $\begin{array}{l}\text { COEFFI- } \\
\text { CIENT OF } \\
\text { VARI- } \\
\text { ATION }\end{array}$ & $\begin{array}{c}\text { PERCENT } \\
\text { OF } \\
\text { ANNUAL } \\
\text { RUNOF F }\end{array}$ \\
\hline $\begin{array}{l}\text { OCTOBER } \\
\text { NOVEMBER } \\
\text { DECEMBER } \\
\text { JANUARY } \\
\text { FEBRUARY } \\
\text { MARCH } \\
\text { APRIL } \\
\text { MAY } \\
\text { JUNE } \\
\text { JULY } \\
\text { AUGUST } \\
\text { SEPTEMBER }\end{array}$ & $\begin{array}{l}.1 \\
.2 \\
.2 \\
.2 \\
.3 \\
.3 \\
.2 \\
.2 \\
.2 \\
.1 \\
.1 \\
.1\end{array}$ & $\begin{array}{r}54 \\
41 \\
32 \\
31 \\
22 \\
14 \\
17 \\
100 \\
36 \\
32 \\
19 \\
52\end{array}$ & $\begin{array}{l}7.0 \\
7.1 \\
7.5 \\
7.4 \\
6.2 \\
4.9 \\
4.1 \\
6.4 \\
5.3 \\
4.2 \\
2.5 \\
4.4\end{array}$ & $\begin{array}{r}11 \\
8.8 \\
7.8 \\
7.4 \\
5.1 \\
3.8 \\
4.1 \\
17 \\
7.8 \\
6.2 \\
3.7 \\
10.0\end{array}$ & $\begin{array}{r}1.50 \\
1.24 \\
1.04 \\
.99 \\
.83 \\
.78 \\
1.01 \\
2.72 \\
1.48 \\
1.48 \\
1.48 \\
2.28\end{array}$ & $\begin{array}{r}10.4 \\
10.6 \\
11.2 \\
11.1 \\
9.2 \\
7.4 \\
6.1 \\
9.6 \\
7.9 \\
6.3 \\
3.8 \\
6.5\end{array}$ \\
\hline & .3 & 22 & 5.4 & 5.6 & 1.02 & 100 \\
\hline
\end{tabular}

MAGNITUDE AND PROBABILITY OF ANNUAL LOW FLOW BASED ON PERIOD OF RECORD 1934-35, 1937-64

\begin{tabular}{|c|c|c|c|c|c|c|}
\hline \multirow{2}{*}{$\begin{array}{l}\text { PERIOD } \\
\text { (CON- } \\
\text { SECU- } \\
\text { TIVE } \\
\text { DAYS) }\end{array}$} & \multicolumn{6}{|c|}{$\begin{array}{l}\text { DISCHARGE, IN FT } 3 / \text { S, FOR INDICATED RECURRENCE } \\
\text { INTERVAL, IN YEARS, AND ANNUAL NON- } \\
\text { EXCEEDANCE PROBABIL ITY, IN PERCENT }\end{array}$} \\
\hline & $\begin{array}{c}2 \\
508\end{array}$ & $\begin{array}{c}5 \\
208\end{array}$ & $\begin{array}{l}10 \\
108\end{array}$ & $\begin{array}{l}20 \\
58\end{array}$ & $\begin{array}{l}50 \\
28\end{array}$ & $\begin{array}{r}100 \\
18\end{array}$ \\
\hline $\begin{array}{r}1 \\
3 \\
7 \\
14 \\
30 \\
60 \\
90 \\
120 \\
183\end{array}$ & $\begin{array}{r}.2 \\
.2 \\
.3 \\
.4 \\
.5 \\
.8 \\
1.0 \\
1.3 \\
1.9\end{array}$ & $\begin{array}{r}0.0 \\
.1 \\
.1 \\
.2 \\
.2 \\
.3 \\
.3 \\
.4 \\
.6\end{array}$ & $\begin{array}{r}0.0 \\
.1 \\
.1 \\
.1 \\
.1 \\
.1 \\
.2 \\
.2 \\
.3\end{array}$ & $\begin{array}{r}0.0 \\
0.0 \\
.1 \\
.1 \\
.1 \\
.1 \\
.1 \\
.1 \\
.2\end{array}$ & $\begin{array}{r}0.0 \\
0.0 \\
0.0 \\
0.0 \\
.1 \\
.1 \\
.1 \\
.1 \\
.1\end{array}$ & $\begin{array}{l}=- \\
-- \\
-- \\
-- \\
-- \\
-- \\
--\end{array}$ \\
\hline
\end{tabular}

MAGNITUDE AND PROBABILITY OF ANNUAL HIGH FLOW BASED ON PERIOD OF RECORD 1934, 1936-64

\begin{tabular}{|c|c|c|c|c|c|c|}
\hline \multirow{2}{*}{$\begin{array}{l}\text { PERIOD } \\
\text { (CON- } \\
\text { SECU- } \\
\text { TIVE } \\
\text { DAYS) }\end{array}$} & \multicolumn{6}{|c|}{$\begin{array}{l}\text { DISCHARGE, IN FT'/S, FOR INDICATED RECURRENC } \\
\text { INTERVAL, IN YEARS, AND ANNUAL } \\
\text { EXCEEDANCE' PROBABILITY, IN PERCENT }\end{array}$} \\
\hline & $\begin{array}{c}2 \\
508\end{array}$ & $\begin{array}{c}5 \\
208\end{array}$ & $\begin{array}{l}10 \\
108\end{array}$ & $\begin{array}{l}25 \\
48\end{array}$ & $\begin{array}{l}50 \\
28\end{array}$ & $\begin{array}{r}100 \\
18\end{array}$ \\
\hline $\begin{array}{r}1 \\
3 \\
7 \\
15 \\
30 \\
60 \\
90\end{array}$ & $\begin{array}{r}39 \\
28 \\
18 \\
13 \\
9.2 \\
6.9 \\
6.0\end{array}$ & $\begin{array}{r}123 \\
84 \\
51 \\
33 \\
24 \\
18 \\
16\end{array}$ & $\begin{array}{r}208 \\
139 \\
83 \\
53 \\
38 \\
29 \\
25\end{array}$ & $\begin{array}{r}345 \\
226 \\
137 \\
84 \\
62 \\
46 \\
39\end{array}$ & $\begin{array}{r}466 \\
302 \\
185 \\
111 \\
83 \\
62 \\
52\end{array}$ & $\begin{array}{l}-- \\
-- \\
-- \\
-- \\
--\end{array}$ \\
\hline
\end{tabular}

DURATION TABLE OF DAILY MEAN FLOW FOR PERIOD OF RECORD 1934, 1936-64 DISCHARGE, IN FT' ${ }^{3} / \mathrm{S}$, THAT WAS EQUALED OR EXCEEDED FOR INDICATED PERCENT OF TIME

\begin{tabular}{|c|c|c|c|c|c|c|c|c|c|c|c|c|c|c|}
\hline 58 & 108 & 158 & 208 & 258 & 308 & 408 & 508 & 608 & 708 & 758 & 808 & 858 & 908 & 958 \\
\hline 19 & 13 & 9.6 & 8.2 & 6.8 & 5.5 & 3.3 & 2.1 & 1.4 & .9 & .6 & .4 & .3 & .2 & 1 \\
\hline
\end{tabular}


RIO GRANDE BASIN

08396500 PECOS RIVER NEAR ARTESIA, NM

(Surveillance program station)

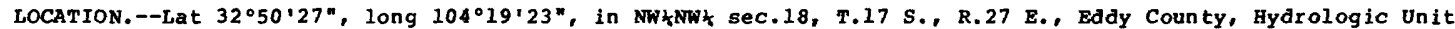
13060007 , on left bank $250 \mathrm{ft}$ upstream from briđge on State Highway $83,4.3 \mathrm{mi}$ east of Artesia, $7.0 \mathrm{mi}$ upstrean from Rio Penasco, $17 \mathrm{mi}$ upstream from McMillan Dam, and at mile 503.9 .

DRAINAGE AREA.--15,300 $\mathrm{mi}^{2}$, approximately (contributing area).

PERIOD OF RECORD.--September 1905 to June 1909, August 1909 to current year. Monthly discharge only for some periods, published in WSP 1312 and 1712. Records for Aug, 22-31, 1934, and October 1936 to April 1937, published in WSP 763 and 828 , respectively are not reliable and should not be used. Prior to February 1936 . published as "near Dayton."

REVISED RECORDS.-WSP 1312 and 1512: 1913, 1915, 1917-18(M), 1920, 1923, 1931-36. WSP 1712: 1906(M), 1908-11(M), 1919, 1921-23(M), 1929, 1931-32(M), 1935-36(M), 1937, 1939(M), 1941(M). See also PERIOD OF RECORD.

GAGE.-Water-stage recorder. Elevation of gage is 3,291.92 ft above National Geodetic Vertical Datum of 1929 (U.S. Bureau of Reclamation bench mark). See WSP 1923 or 2123 for history of changes prior to Apr. 5 , 1941 . Apr. 5 . 1941 to Apr. 2, 1981 , water-stage recorder at site $250 \mathrm{ft}$ downstream at same datum.

REMARKS.--Flow regulated by Santa Rosa Lake (station 08382810) since April 1980, by Lake Sumner (station 08384000) since August 1937, and by Two Rivers Reservoir (station 08390600) since July 1963. Diversions and ground-water withdrawals for irrigation of about 154,000 acres, 1959 determination, upstream from station.

AVERAGE DISCHARGE, --36 years, $(1906-08,1910-37), 379 \mathrm{ft}^{2} / \mathrm{s}, 274,600 \mathrm{acre}-\mathrm{ft} / \mathrm{yr}$.

EXTREMES FOR PERIOD OF RECORD.--Maximum discharge probably occurred May 30, 1937, when a discharge of 51,500 ft $3 / \mathrm{s}$ was measured by slope-area method at a point $15 \mathrm{mi}$ upstream, gage height, $14.7 \mathrm{ft}$, site and datum then in use; no flow at times in 1934, 1946-47, 1953-54, 1957, 1964-65.

EXTREMES OUTSIDE PERIOD OF RECORD.--Greatest flood since at least 1893 occurred oct. 2, 1904, discharge not determined; the peak inflow to Lake MCMillan, which includes Rio Penasco and Fourmile Draw, was estimated at $82,000 \mathrm{ft}^{3} / \mathrm{s}$. The second highest flood occurred July 25, 1905, discharge below Rio Penasco, $50,300 \mathrm{ft} / \mathrm{s}$, based on gain in storage and spill from Lake McMillan. The floods in August 1893 and October 1904 damaged McMillan Dam and washed out Avalon Dam.

STATISTICAL SUMMARIES (PERIOD BEFORE REGULATION OF SUMNER DAM)

MEAN MONTHLY AND MEAN ANNUAL DISChARGES 1906-08,
MAGNITUDE AND PROBABILITY OF ANNUAL LOW FLOW BASED ON PERIOD OF RECORD 1907-09, 1911-37

\begin{tabular}{|c|c|c|c|c|c|c|}
\hline MONTH & $\begin{array}{l}\text { MINIMUM } \\
\left(F^{3} / S\right)\end{array}$ & $\begin{array}{l}\text { NAXIMUM } \\
\left(F T^{3} / S\right)\end{array}$ & $\begin{array}{l}\text { MEAN } \\
\left(\mathrm{FT}^{3} / \mathrm{S}\right)\end{array}$ & $\begin{array}{l}\text { STAN- } \\
\text { DARD } \\
\text { DEVIA- } \\
\text { TION } \\
(\text { FT } 3 / S)\end{array}$ & $\begin{array}{l}\text { COEFFI- } \\
\text { CIENT OF } \\
\text { VARI- } \\
\text { ATION }\end{array}$ & $\begin{array}{c}\text { PERCENT } \\
\text { OF } \\
\text { ANNUAL } \\
\text { RUNOF F }\end{array}$ \\
\hline \multicolumn{7}{|c|}{ 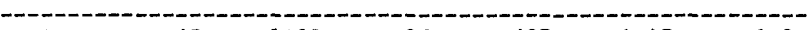 } \\
\hline OCTOBER & 45 & 1839 & 365 & 427 & 1.17 & 8.2 \\
\hline NOVEMBER & 72 & 648 & 246 & 124 & .50 & 5.5 \\
\hline DECEMBER & 130 & 657 & 293 & 122 & .42 & 6.5 \\
\hline JANUARY & 147 & 469 & 296 & 83 & .28 & 6.6 \\
\hline FEBRUARY & 161 & 395 & 246 & 61 & .25 & 5.5 \\
\hline MARCH & 79 & 2067 & 222 & 340 & 1.53 & 5.0 \\
\hline APRIL & 49 & 3696 & 326 & 651 & 2.00 & 7.3 \\
\hline MAY & 44 & 2971 & 545 & 586 & 1.08 & 12.2 \\
\hline JUNE & 48 & 3495 & 598 & 768 & 1.28 & 13.4 \\
\hline JULY & 9.4 & 1472 & 445 & 402 & .90 & 9.9 \\
\hline AUGUST & $4 I$ & 1561 & 478 & 386 & .81 & 10.7 \\
\hline SEPTEMBER & 73 & 2831 & 417 & 545 & 1.31 & 9.3 \\
\hline NNUAL & 143 & 998 & 379 & 189 & .50 & 100 \\
\hline
\end{tabular}

\begin{tabular}{|c|c|c|c|c|c|c|}
\hline \multirow{2}{*}{$\begin{array}{l}\text { PERIOD } \\
\text { (CON- } \\
\text { SECU- } \\
\text { TIVE } \\
\text { DAYS) }\end{array}$} & \multicolumn{6}{|c|}{$\begin{array}{l}\text { DISCHARGE, IN FT3/S, FOR INDICATED RECURRENCE } \\
\text { INTERVAL, IN YEARS, AND ANNUAL NON- } \\
\text { EXCEEDANCE PROBABIL ITY, IN PERCENT }\end{array}$} \\
\hline & $\begin{array}{c}2 \\
508\end{array}$ & $\begin{array}{c}5 \\
208\end{array}$ & $\begin{array}{l}10 \\
108\end{array}$ & $\begin{array}{l}20 \\
58\end{array}$ & $\begin{array}{l}50 \\
28\end{array}$ & $\begin{array}{r}100 \\
18\end{array}$ \\
\hline $\begin{array}{r}1 \\
3 \\
7 \\
14 \\
30 \\
60 \\
90 \\
120 \\
183\end{array}$ & $\begin{array}{r}41 \\
42 \\
46 \\
54 \\
77 \\
122 \\
162 \\
185 \\
218\end{array}$ & $\begin{array}{r}19 \\
21 \\
25 \\
32 \\
45 \\
75 \\
108 \\
129 \\
163\end{array}$ & $\begin{array}{r}11 \\
13 \\
17 \\
22 \\
31 \\
56 \\
84 \\
104 \\
140\end{array}$ & $\begin{array}{r}5 \\
71 \\
11 \\
16 \\
22 \\
43 \\
68 \\
86 \\
124\end{array}$ & $\begin{array}{r}2 . \\
3 . \\
6 \\
10 \\
14 \\
31 \\
52 \\
68 \\
109\end{array}$ & $\begin{array}{l}-- \\
-- \\
-- \\
-- \\
=- \\
=- \\
--\end{array}$ \\
\hline
\end{tabular}

MAGNI TUDE AND PROBABILITY OF ANNUAL HIGH FLO BASED ON PERIOD OF RECORD 1906-08, 1910-37

\begin{tabular}{|c|c|c|c|c|c|c|}
\hline \multirow{2}{*}{$\begin{array}{l}\text { PERIOD } \\
\text { (CON- } \\
\text { SECU- } \\
\text { TIVE } \\
\text { DAYS) }\end{array}$} & \multicolumn{6}{|c|}{$\begin{array}{l}\text { DISCHARGE, IN FT'/S, FOR INDICATED RECURRENCE } \\
\text { INTERVAL, IN YEARS, AND ANNUAL } \\
\text { EXCEEDANCE PROBABILI TY, IN PERCENT }\end{array}$} \\
\hline & $\begin{array}{c}2 \\
508\end{array}$ & $\begin{array}{c}5 \\
208\end{array}$ & $\begin{array}{l}10 \\
108\end{array}$ & $\begin{array}{l}25 \\
48\end{array}$ & $\begin{array}{l}50 \\
28\end{array}$ & $\begin{array}{r}100 \\
18\end{array}$ \\
\hline $\begin{array}{r}1 \\
3 \\
7 \\
15 \\
30 \\
60 \\
90\end{array}$ & $\begin{array}{r}5830 \\
3750 \\
2380 \\
1570 \\
1090 \\
744 \\
604\end{array}$ & $\begin{array}{r}14000 \\
8610 \\
4980 \\
3080 \\
1970 \\
1270 \\
1020\end{array}$ & $\begin{array}{r}22800 \\
13900 \\
7690 \\
4570 \\
2780 \\
1710 \\
1370\end{array}$ & $\begin{array}{r}38800 \\
24200 \\
12700 \\
7200 \\
4130 \\
2400 \\
1890\end{array}$ & $\begin{array}{r}55300 \\
35200 \\
17900 \\
9840 \\
5410 \\
3020 \\
2350\end{array}$ & $\begin{array}{l}-- \\
-- \\
-- \\
-- \\
--\end{array}$ \\
\hline
\end{tabular}

DURATION TABLE OF DAILY MEAN FLOW FOR PERIOD OF RECORD 1906-08, 1910-37 DISCHARGE, IN FT ${ }^{3} / S$, THAT WAS EQUALED OR EXCEEDED FOR INDICATED PERCENT OF TIME

$\begin{array}{cccccccccccc}58 & 108 & 158 & 208 & 258 & 308 & 408 & 508 & 608 & 708 & 758 & 808 \\ 1090 & 699 & 514 & 403 & 354 & 305 & 258 & 215 & 174 & 139 & 123 & 106\end{array}$


RIO GRANDE BASIN

08396500 PECOS RIVER NEAR ARTESIA, NM--Continued

STATISTICAL SUMMARIES (PERIOD BEFORE REGULATION OF SANTA ROSA DAM AND AFTER COMPLETION OF SUMNER DAM)

MEAN MONTHLY AND MEAN ANNUAL DISCHARGES 1938-79

\begin{tabular}{|c|c|c|c|c|c|c|}
\hline MONTH & $\begin{array}{l}\text { MINIMUM } \\
\left(\mathrm{FT}^{3} / \mathrm{S}\right)\end{array}$ & $\begin{array}{c}\text { MAXIMUM } \\
\left(\mathrm{FT}^{3} / \mathrm{S}\right)\end{array}$ & $\begin{array}{l}\text { MEAN } \\
\left(\mathrm{FT}^{3} / \mathrm{S}\right)\end{array}$ & $\begin{array}{l}\text { STAN- } \\
\text { DARD } \\
\text { DEVIA- } \\
\text { TION } \\
\left(\mathrm{FT}^{3} / \mathrm{S}\right)\end{array}$ & $\begin{array}{l}\text { COEFFI- } \\
\text { CIENT OF } \\
\text { VARI- } \\
\text { ATION }\end{array}$ & $\begin{array}{c}\text { PERCENT } \\
\text { OF } \\
\text { ANNUAL } \\
\text { RUNOFF }\end{array}$ \\
\hline $\begin{array}{l}\text { OCTOBER } \\
\text { NOVEMBER } \\
\text { DECEMBER } \\
\text { JANUARY } \\
\text { FEBRUARY } \\
\text { MARCH } \\
\text { APRIL } \\
\text { MAY } \\
\text { JUNE } \\
\text { JULY } \\
\text { AUGUST } \\
\text { SEPTEMBER }\end{array}$ & $\begin{array}{l}2.3 \\
32 \\
34 \\
35 \\
29 \\
22 \\
11 \\
16 \\
5.4 \\
.8 \\
.1 \\
.3\end{array}$ & $\begin{array}{r}4203 \\
1240 \\
614 \\
499 \\
504 \\
768 \\
1292 \\
3834 \\
2524 \\
1453 \\
880 \\
5704\end{array}$ & $\begin{array}{r}284 \\
143 \\
108 \\
103 \\
90 \\
208 \\
248 \\
303 \\
327 \\
354 \\
274 \\
408\end{array}$ & $\begin{array}{r}731 \\
232 \\
98 \\
86 \\
76 \\
179 \\
257 \\
633 \\
397 \\
321 \\
198 \\
931\end{array}$ & $\begin{array}{r}2.57 \\
1.62 \\
.91 \\
.84 \\
.85 \\
.86 \\
1.04 \\
2.09 \\
1.21 \\
.91 \\
.72 \\
2.28\end{array}$ & $\begin{array}{r}10.0 \\
5.0 \\
3.8 \\
3.6 \\
3.2 \\
7.3 \\
8.7 \\
10.6 \\
11.5 \\
12.4 \\
9.6 \\
14.3\end{array}$ \\
\hline NNUAL & 65 & 1378 & 238 & 238 & 1.00 & 100 \\
\hline
\end{tabular}

MAGNITUDE AND PROBABILITY OF ANNUAL LOW FLOW BASED ON PERIOD OF RECORD 1939-79

\begin{tabular}{|c|c|c|c|c|c|c|}
\hline \multirow{2}{*}{$\begin{array}{l}\text { PERIOD } \\
\text { (CON- } \\
\text { SECU- } \\
\text { TIVE } \\
\text { DAYS) }\end{array}$} & \multicolumn{6}{|c|}{$\begin{array}{l}\text { DISCHARGE, IN FT3/S, FOR INDICATED RECURRENCE } \\
\text { INTERVAL, IN YEARS, AND ANNUAL NON- } \\
\text { EXCEEDANCE PROBABILITY, IN PERCENT }\end{array}$} \\
\hline & $\begin{array}{c}2 \\
508\end{array}$ & $\begin{array}{c}5 \\
208\end{array}$ & $\begin{array}{l}10 \\
108\end{array}$ & $\begin{array}{l}20 \\
58\end{array}$ & $\begin{array}{l}50 \\
28\end{array}$ & $\begin{array}{r}100 \\
18\end{array}$ \\
\hline $\begin{array}{r}1 \\
3 \\
7 \\
14 \\
30 \\
60 \\
90 \\
120 \\
183\end{array}$ & $\begin{array}{l}1.7 \\
2.1 \\
3.1 \\
6.9 \\
16 \\
48 \\
65 \\
68 \\
72\end{array}$ & $\begin{array}{r}: \\
4 . \\
13 \\
25 \\
31 \\
41\end{array}$ & $\begin{array}{l}0.0 \\
0.0 \\
0.0 \\
0.0 \\
1.8 \\
5.3 \\
13 \\
19 \\
33\end{array}$ & $\begin{array}{r}0.0 \\
0.0 \\
0.0 \\
0.0 \\
.8 \\
2.1 \\
6.2 \\
12 \\
28\end{array}$ & $\begin{array}{r}0.0 \\
0.0 \\
0.0 \\
0.0 \\
0.0 \\
.7 \\
2.5 \\
6.7 \\
24\end{array}$ & 23 \\
\hline
\end{tabular}

MAGNITUDE AND PROBABILITY OF ANNUAL HIGH FLOW BASED ON PERIOD OF RECORD $1938-79$

\begin{tabular}{|c|c|c|c|c|c|c|}
\hline \multirow{2}{*}{$\begin{array}{c}\text { PERIOD } \\
\text { (CON- } \\
\text { SECU- } \\
\text { TIVE } \\
\text { DAYS) }\end{array}$} & \multicolumn{6}{|c|}{$\begin{array}{l}\text { DISCHARGE, IN FT }{ }^{3 / S} \text {, FOR INDICATED RECURRENCE } \\
\text { INTERVAL, IN YEARS, AND ANNUAL } \\
\text { EXCEEDANCE PROBABILITY, IN PERCENT }\end{array}$} \\
\hline & $\begin{array}{c}2 \\
508\end{array}$ & $\begin{array}{c}5 \\
208\end{array}$ & $\begin{array}{l}10 \\
108\end{array}$ & $\begin{array}{l}25 \\
48\end{array}$ & $\begin{array}{l}50 \\
28\end{array}$ & $\begin{array}{r}100 \\
18\end{array}$ \\
\hline $\begin{array}{r}1 \\
3 \\
7 \\
15 \\
30 \\
60 \\
90\end{array}$ & $\begin{array}{r}2440 \\
1810 \\
1260 \\
586 \\
614 \\
426 \\
340\end{array}$ & $\begin{array}{r}5280 \\
3890 \\
2420 \\
1550 \\
1070 \\
737 \\
588\end{array}$ & $\begin{array}{r}8910 \\
6650 \\
3950 \\
2390 \\
1600 \\
1080 \\
855\end{array}$ & $\begin{array}{r}17100 \\
13200 \\
7540 \\
4290 \\
2690 \\
1750 \\
1370\end{array}$ & $\begin{array}{r}27600 \\
22000 \\
12300 \\
6680 \\
3970 \\
2490 \\
1930\end{array}$ & $\begin{array}{r}44000 \\
36300 \\
20000 \\
10500 \\
5850 \\
3540 \\
2700\end{array}$ \\
\hline
\end{tabular}

DURATION TABLE OF DAILY MEAN FLOW FOR PERIOD OF RECORD 1938-79

DISCHARGE, IN FT $3 / \mathrm{S}$, THAT WAS EOUALED OR EXCEEDED FOR INDICATED PERCENT OF TIME

\begin{tabular}{|c|c|c|c|c|c|c|c|c|c|c|c|c|c|c|}
\hline 58 & 108 & 158 & 208 & 258 & 308 & 408 & 508 & 608 & 708 & 758 & 808 & 858 & 908 & 958 \\
\hline 946 & 634 & 382 & 215 & 162 & 135 & 97 & 74 & 57 & 43 & 37 & 30 & 21 & 13 & 5.7 \\
\hline
\end{tabular}


RIO GRANDE BASIN

08398500 RIO PENASCO AT DAYTON, NM

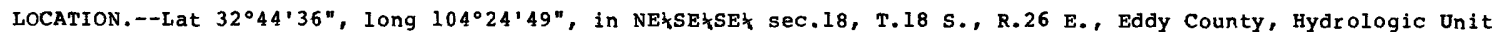
13060010 , on left bank $1.2 \mathrm{mi}$ upstream from U.S. Highway $285,1.9$ mi northwest of old Dayton railway station, $5.6 \mathrm{mi}$ upstream from mouth, and $7.0 \mathrm{mi}$ south of Artesia. Mouth at Pecos River mile 496.4 .

DRAINAGE AREA. $--1,060 \mathrm{mi}^{2}$, approximately.

PERIOD OF RECORD.--April 1951 to current year. Prior to October 1953, published as "near Dayton." REVISED RECORDS.--WSP 1242: 1951(M). WSP 1512: 1956. WSP 1923: 1955.

GAGE.--Water-stage recorder and rock and concrete control. Elevation of gage is $3,385.19 \mathrm{ft}$ above National Geodetic Vertical Datum of 1929. Prior to May 9, 1968, at site 2.4 mi downstream, at datum 44.30 ft 10wer. May 9, 1968 to June 12, 1975, at present site at datum $1.98 \mathrm{ft}$ higher.

REMARKS.--Diversions and ground-water withdrawals for irrigation of about 3,000 acres, 1959 determination, upstream from station.

AVERAGE DISCHARGE.--34 years (water years 1952-85), $5.34 \mathrm{ft}^{3} / \mathrm{s}, 3,870 \mathrm{acre}-\mathrm{ft} / \mathrm{yr}$.

EXTREMES FOR PERIOD OF RECORD.--Maximum discharge, $29,800 \mathrm{ft}^{3} / \mathrm{s}$, Aug, 23, 1966, gage height, $16.4 \mathrm{ft}, \mathrm{from}$ floodmarks, present site and datum, from rating curve extended above $6,000 \mathrm{ft} / \mathrm{s}$ on basis of slope-area measurements at gage heights $6.82 \mathrm{ft}$ and $7.90 \mathrm{ft}$ at previous site and datum; no flow most of time.

EXTREMES OUTSIDE PERIOD OF RECORD.--Flood of about Sept. 22, 1941, reached a stage of about 9 ft from floodmark, previous site and datum, discharge not determined, Peak discharge at discontinued station "near Dunken" (station 08397600 ), about $60 \mathrm{mi}$ upstream, was $70,000 \mathrm{ft}^{3} / \mathrm{s}$, determined in 1956 from rating curve extended above a slope-area measurement of $36,300 \mathrm{ft}^{3} / \mathrm{s}$ for peak of oct. 6 or 7, 1954 .

STATISTICAL SUMMARIES

MEAN MONTHLY AND MEAN ANNUAL DISCHARGES 1952-85

\begin{tabular}{|c|c|c|c|c|c|c|}
\hline MONTH & $\begin{array}{c}\text { MINIMUM } \\
\left(\mathrm{FT}^{3} / \mathrm{S}\right)\end{array}$ & $\begin{array}{c}\text { MAXIMUM } \\
\left(\mathrm{FT}^{3} / \mathrm{S}\right)\end{array}$ & $\begin{array}{l}\text { MEAN } \\
\left(\mathrm{FT}^{3} / \mathrm{S}\right)\end{array}$ & $\begin{array}{l}\text { STAN- } \\
\text { DARD } \\
\text { DEVIA- } \\
\text { TION } \\
\left(\mathrm{FT}^{3} / \mathrm{S}\right)\end{array}$ & $\begin{array}{l}\text { COEFFI- } \\
\text { CIENT OF } \\
\text { VARI- } \\
\text { ATION }\end{array}$ & $\begin{array}{c}\text { PERCENT } \\
\text { OF } \\
\text { ANNUAL, } \\
\text { RUNOF F }\end{array}$ \\
\hline \multicolumn{7}{|l|}{$---1--1$} \\
\hline $\begin{array}{l}\text { OCTOBER } \\
\text { NOVEMBER }\end{array}$ & $\begin{array}{l}0.0 \\
0.0\end{array}$ & $\begin{array}{r}201 \\
73\end{array}$ & $\begin{array}{l}7.1 \\
2.1\end{array}$ & $\begin{array}{l}35 \\
13\end{array}$ & $\begin{array}{l}4.85 \\
5.84\end{array}$ & $\begin{array}{r}11.2 \\
3.4\end{array}$ \\
\hline DECEMBER & 0.0 & 0.0 & 0.0 & 0.0 & -- & 0.0 \\
\hline JANUARY & 0.0 & 0.0 & 0.0 & 0.0 & -- & 0.0 \\
\hline FEBRUARY & 0.0 & 0.0 & 0.0 & 0.0 & -- & 0.0 \\
\hline MARCH & 0.0 & 0.0 & 0.0 & 0.0 & -- & 0.0 \\
\hline APRIL & 0.0 & .7 & 0.0 & .1 & 4.00 & 0.0 \\
\hline MAY & 0.0 & 41 & 1.7 & 7.3 & 4.19 & 2.7 \\
\hline JUNE & 0.0 & 19 & 2.0 & 4.6 & 2.23 & 3.2 \\
\hline JULY & 0.0 & 221 & 13 & 40 & 3.17 & 19.8 \\
\hline AUGUST & 0.0 & 328 & 23 & 65 & 2.86 & 35.6 \\
\hline SEPTEMBER & 0.0 & 372 & 15 & 64 & 4.24 & 23.9 \\
\hline ANNUAL & 0.0 & 31 & 5.3 & 8.6 & 1.61 & 100 \\
\hline
\end{tabular}

MAGNITUDE AND PROBABILITY OF ANNUAL LOW FLOW BASED ON PERIOD OF RECORD 1953-85

\begin{tabular}{|c|c|c|c|c|c|c|}
\hline \multirow{2}{*}{$\begin{array}{c}\text { PERIOD } \\
\text { (CON- } \\
\text { SECU- } \\
\text { TIVE } \\
\text { DAYS) }\end{array}$} & \multicolumn{6}{|c|}{$\begin{array}{l}\text { DISCHARGE, IN FT }{ }^{3 / S}, \text { FOR INDICATED RECURRENCE } \\
\text { INTERVAL, IN YEARS, AND ANNUAL NON- } \\
\text { EXCEEDANCE PROBABILITY, IN PERCENT }\end{array}$} \\
\hline & $\begin{array}{c}2 \\
508\end{array}$ & $\begin{array}{c}5 \\
208\end{array}$ & $\begin{array}{l}10 \\
108\end{array}$ & $\begin{array}{l}20 \\
58\end{array}$ & $\begin{array}{l}50 \\
28\end{array}$ & $\begin{array}{r}100 \\
18\end{array}$ \\
\hline $\begin{array}{r}1 \\
3 \\
7 \\
14 \\
30 \\
60 \\
90 \\
120 \\
183\end{array}$ & $\begin{array}{l}0.0 \\
0.0 \\
0.0 \\
0.0 \\
0.0 \\
0.0 \\
0.0 \\
0.0 \\
0.0\end{array}$ & $\begin{array}{l}0.0 \\
0.0 \\
0.0 \\
0.0 \\
0.0 \\
0.0 \\
0.0 \\
0.0 \\
0.0\end{array}$ & $\begin{array}{l}0.0 \\
0.0 \\
0.0 \\
0.0 \\
0.0 \\
0.0 \\
0.0 \\
0.0 \\
0.0\end{array}$ & $\begin{array}{l}0.0 \\
0.0 \\
0.0 \\
0.0 \\
0.0 \\
0.0 \\
0.0 \\
0.0 \\
0.0\end{array}$ & $\begin{array}{l}0.0 \\
0.0 \\
0.0 \\
0.0 \\
0.0 \\
0.0 \\
0.0 \\
0.0 \\
0.0\end{array}$ & $\begin{array}{l}-- \\
-- \\
-- \\
-- \\
-- \\
--\end{array}$ \\
\hline
\end{tabular}

MAGNITUDE AND PROBABILITY OF ANNUAL HIGH FLOW BASED ON PERIOD OF RECORD 1952-85

\begin{tabular}{|c|c|c|c|c|c|c|}
\hline \multirow{2}{*}{$\begin{array}{l}\text { PERIOD } \\
\text { (CON- } \\
\text { SECU- } \\
\text { TIVE } \\
\text { DAYS) }\end{array}$} & \multicolumn{6}{|c|}{$\begin{array}{l}\text { DISCHARGE, IN FT } 3 / \mathrm{S} \text {, FOR INDICATED RECURRENCE } \\
\text { INTERVAL, IN YEARS, AND ANNUAL } \\
\text { EXCEEDANCE PROBABILITY, IN PERCENT }\end{array}$} \\
\hline & $\begin{array}{c}2 \\
508\end{array}$ & $\begin{array}{c}5 \\
208\end{array}$ & $\begin{array}{l}10 \\
108\end{array}$ & $\begin{array}{l}25 \\
48\end{array}$ & $\begin{array}{l}50 \\
28\end{array}$ & $\begin{array}{r}100 \\
18\end{array}$ \\
\hline $\begin{array}{r}1 \\
3 \\
7 \\
15 \\
30 \\
60 \\
90\end{array}$ & $\begin{array}{r}429 \\
177 \\
78 \\
39 \\
21 \\
11 \\
7.1\end{array}$ & $\begin{array}{r}1990 \\
832 \\
373 \\
181 \\
94 \\
49 \\
32\end{array}$ & $\begin{array}{r}3490 \\
1490 \\
680 \\
325 \\
165 \\
86 \\
57\end{array}$ & $\begin{array}{r}5450 \\
2410 \\
1120 \\
525 \\
261 \\
133 \\
91\end{array}$ & $\begin{array}{r}6840 \\
3090 \\
1460 \\
674 \\
330 \\
167 \\
116\end{array}$ & $\begin{array}{l}-- \\
-- \\
-- \\
-- \\
-- \\
--\end{array}$ \\
\hline
\end{tabular}

DURATION TABLE OF DAILY MEAN FLOW FOR PERIOD OF RECORD 1952-85 DISCHARGE, IN FT ${ }^{3} / \mathrm{S}$, THAT WAS EQUALED OR EXCEEDED FOR INDICATED PERCENT OF TIME

\begin{tabular}{|c|c|c|c|c|c|c|c|c|c|c|c|c|c|c|}
\hline 58 & 108 & 158 & 208 & 258 & 308 & 408 & 508 & 608 & 708 & 758 & 808 & 858 & 908 & 958 \\
\hline 0.0 & 0.0 & 0.0 & 0.0 & 0. & 0.0 & 0.0 & 0.0 & 0.0 & 0.0 & 0.0 & 0.0 & 0.0 & 0.0 & 0. \\
\hline
\end{tabular}




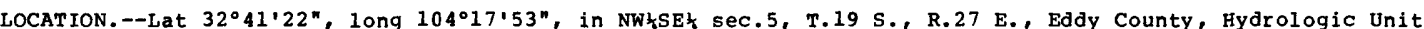
13060011 , on left bank $3.0 \mathrm{mi}$ upstream from high-water line of Lake McMillan, $6.0 \mathrm{mi}$ northeast of Lakewood, 7.0 $\mathrm{mi}$ northeast of gates in McMilian Dam, $12 \mathrm{mi}$ southeast of Artesia, and at mile 492.1 .

PERIOD OF RECORD.--May 1950 to current year. Prior to October 1954, published as Kaiser Lake-McMillan Channel near Lakewood.

GAGE.--Water-stage recorder. Elevation of gage is 3,268.53 ft above National Geodetic vertical survey of 1929 (U.S. Bureau of Reclamation bench mark). Prior to Mar. 23, 1955, at site $3.0 \mathrm{mi}$ downstream at datum $7.83 \mathrm{ft}$ lower. Mar. 23, 1955 to Sept. 30, 1963, at present site at datum 2.00 ft higher.

REMARKS.--Flow regulated by Santa Rosa Lake (station 08382810) since April 1980, by Lake Sumner (station 08384000) since August 1937, and by Two Rivers Reservoir (station 08390600) since July 1963. Diversions and ground-water withdrawals for irrigation of about 170,000 acres, 1959 determination, upstream from station. Above about 1,500 $\mathrm{ft} 3 / \mathrm{s}$ flow will begin bpassing station and depending on the magnitude and duration of flow, may reach Lake McMilian (station 08400500 ).

AVERAGE DISCHARGE.--28 years (water years 1951-55, 1957-79), $151 \mathrm{ft}^{3} / \mathrm{s}, 109,400 \mathrm{acre}-\mathrm{ft} / \mathrm{yr}$.

EXTREMES FOR PERIOD OF RECORD.--Maximum daily discharge, 2,920 $\mathrm{ft}^{3} / \mathrm{s}$, July 12, 1960 ; no flow at times in most years.

STATISTICAL SUMMARIES (PERIOD BEFORE REGULATION OF SANTA ROSA DAM)

MEAN MONTHLY AND MEAN ANNUAL DISCHARGES $\begin{aligned} & 1951-55, \\ & 1957-79\end{aligned}$

\begin{tabular}{|c|c|c|c|c|c|c|}
\hline MONTH & $\begin{array}{l}\text { MINIMUM } \\
\left(\mathrm{FT}^{3} / \mathrm{S}\right)\end{array}$ & $\begin{array}{l}\operatorname{MAXIMUM} \\
\left(\mathrm{FT}^{3} / \mathrm{S}\right)\end{array}$ & $\begin{array}{l}\text { MEAN } \\
\left(\mathrm{FT}^{3} / \mathrm{S}\right)\end{array}$ & $\begin{array}{l}\text { STAN- } \\
\text { DARD } \\
\text { DEVIA- } \\
\text { TION } \\
\left(\mathrm{FT}^{3} / \mathrm{S}\right)\end{array}$ & $\begin{array}{l}\text { COEFFI- } \\
\text { CIENT OF } \\
\text { VARI- } \\
\text { ATION }\end{array}$ & $\begin{array}{c}\text { PERCENT } \\
\text { OF } \\
\text { ANNUAL } \\
\text { RUNOFF }\end{array}$ \\
\hline \multicolumn{7}{|c|}{ 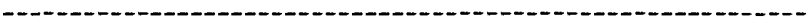 } \\
\hline OCTOBER & 0.0 & 695 & 119 & 170 & 1.43 & 6.6 \\
\hline NOVEMBER & 26 & 216 & 75 & 48 & .64 & 4.2 \\
\hline DECEMBER & 29 & 197 & 71 & 42 & .58 & 4.0 \\
\hline JANUARY & 31 & 20 & 68 & 36 & .53 & 3.8 \\
\hline FEB RUARY & 25 & 181 & 60 & 33 & .56 & 3.3 \\
\hline MARCH & 19 & 402 & 169 & 134 & .79 & 9.4 \\
\hline APRIL & 8.1 & 451 & 152 & 139 & .91 & 8.4 \\
\hline MAY & 15 & 1220 & 197 & 301 & 1.53 & 10.9 \\
\hline JUNE & 1.9 & 511 & 206 & 167 & .81 & 11.4 \\
\hline JULY & .2 & 886 & 271 & 196 & .72 & 15.0 \\
\hline AUGUST & 0.0 & 600 & 261 & 168 & .64 & 14.5 \\
\hline SEPTEMBER & & 624 & 157 & 199 & 1.27 & 8.7 \\
\hline NNU & 64 & 303 & 151 & 56 & .37 & 100 \\
\hline
\end{tabular}

MAGNITUDE AND PROBABILITY OF ANNUAL LOW FLOW BASED ON PERIOD OF RECORD 1952-55, 1957-79

MAGNITUDE AND PROBABILITY OF ANNUAL HIGH FLOW BASED ON PERIOD OF RECORD 1951-55, 1957-79

\begin{tabular}{|c|c|c|c|c|c|c|}
\hline \multirow{2}{*}{$\begin{array}{l}\text { PERIOD } \\
\text { (CON- } \\
\text { SECU- } \\
\text { TIVE } \\
\text { DAYS) }\end{array}$} & \multicolumn{6}{|c|}{$\begin{array}{c}\text { DISCHARGE, IN FT'/S, FOR INDICATED RECURRENCE } \\
\text { INTERVAL, IN YEARS, AND ANNUAL } \\
\text { EXCEEDANCE' PROBABILITY, IN PERCENT }\end{array}$} \\
\hline & $\begin{array}{c}2 \\
508\end{array}$ & $\begin{array}{c}5 \\
208\end{array}$ & 10 & 48 & $\begin{array}{l}50 \\
28\end{array}$ & 100 \\
\hline $\begin{array}{r}1 \\
3 \\
7 \\
15 \\
30 \\
60 \\
90\end{array}$ & $\begin{array}{r}1550 \\
1290 \\
1000 \\
766 \\
556 \\
367 \\
290\end{array}$ & $\begin{array}{r}2030 \\
1770 \\
1360 \\
1010 \\
772 \\
526 \\
420\end{array}$ & $\begin{array}{r}2290 \\
2080 \\
1630 \\
1180 \\
920 \\
644 \\
516\end{array}$ & $\begin{array}{r}2580 \\
2500 \\
2000 \\
1420 \\
1110 \\
809 \\
648\end{array}$ & $\begin{array}{r}2770 \\
2810 \\
2300 \\
1610 \\
1260 \\
942 \\
755\end{array}$ & $\begin{array}{l}-- \\
-- \\
-- \\
--\end{array}$ \\
\hline
\end{tabular}

DURATION TABLE OF DAILY MEAN FLOW FOR PERIOD OF RECORD 1951-55, 1957-79 DISCHARGE, IN FT $3 / S$, THAT WAS EQUALED OR EXCEEDED FOR INDICATED PERCENT OF TIME

\begin{tabular}{|c|c|c|c|c|c|c|c|c|c|c|c|c|c|}
\hline 58 & 108 & 158 & 208 & 258 & 308 & 408 & 508 & 608 & 708 & 758 & 808 & 858 & 908 \\
\hline
\end{tabular}


LOCATION.--Lat $32^{\circ} 40^{\prime} 20^{\prime \prime}$, long $104^{\circ} 22^{\prime} 07^{\prime \prime}$, in SWłNwłsł sec.10, T.19 S., R.26 E., Eddy County, Hydrologic Unit 13060011 , in left side of channel $360 \mathrm{ft}$ downstream from ford on Lakewood-Dayton road, $1.9 \mathrm{mi}$ downstream from U.S. Highway $285,2,8 \mathrm{mi}$ north of Lakewood, $3.8 \mathrm{mi}$ upstream from mouth, and $11.5 \mathrm{mi}$ south of Artesia. Mouth at Pecos River mile 490.6 .

DRAINAGE AREA. - $-265 \mathrm{mi}^{2}$, approximately.

PERIOD OF RECORD.--October 1951 to current year.

REVISED RECORDS. --WRD 1968: 1967.

GAGE.--Water-stage recorder. Elevation of gage is $3,299.14 \mathrm{ft}$ above National Geodetic Vertical Datum of 1929.

Oct. 1 , 1951 to June 19, 1962, at site $1.8 \mathrm{mi}$ upstrean at datum $30.61 \mathrm{ft}$ higher. June 19 , 1962 to Oct. 12 , 1966 at site $410 \mathrm{ft}$ upstream at datum $6.08 \mathrm{ft}$ higher.

REMARKS. --No surface diversions upstream from station.

AVERAGE DISCHARGE.--34 years (water years 1952-85), 3.69 ft3/s, 2.670 acre-ft/yr.

EXTREMES FOR PERIOD OF RECORD,--Maximum discharge, 29,300 ft 3/s, Aug. 23, 1966, gage height, $19.9 \mathrm{ft}$, from floodmarks, present datum, from rating curve extended above $5,000 \mathrm{ft} / \mathrm{s}$ on basis of slope-area measurement of peak flow; no flow most of time.

The flood of Aug. 23, 1966 (information from local resident) is believed to be the greatest since at least 1920 .

STATISTICAL SUMMARIES

MEAN MONTHLY AND MEAN ANNUAL DISCHARGES 1952-85

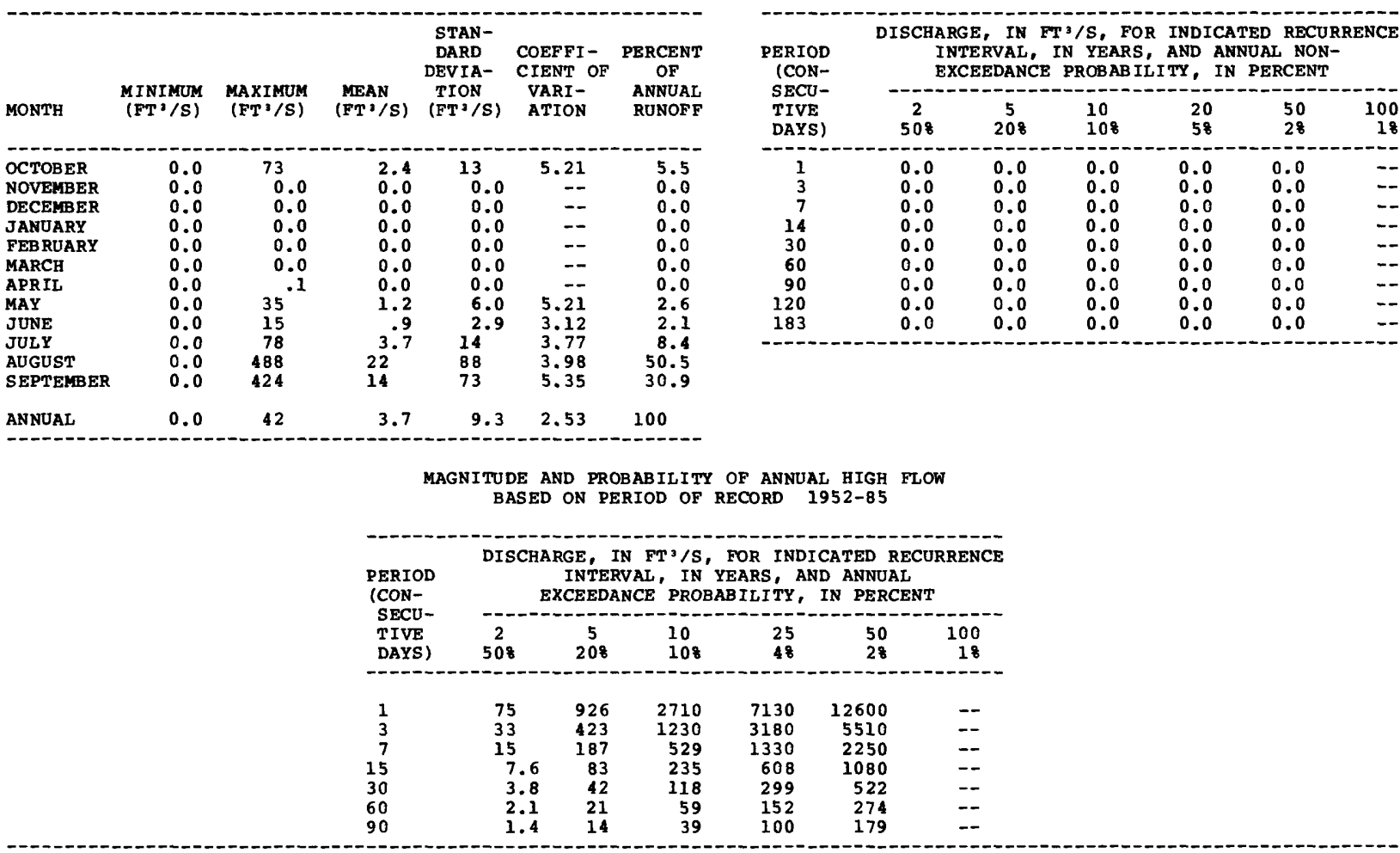

DURATION TABLE OF DAILY MEAN FLOW FOR PERIOD OF RECORD 1952-85

DISCHARGE, IN FT'3/S, THAT WAS EOUALED OR EXCEEDED FOR INDICATED PERCENT OF TIME

\begin{tabular}{|c|c|c|c|c|c|c|c|c|c|c|c|c|c|c|}
\hline 58 & 108 & 158 & 208 & 258 & 308 & 408 & 508 & 608 & 708 & 758 & 808 & 858 & 908 & 958 \\
\hline 0.0 & 0.0 & 0.0 & 0.0 & 0.0 & 0.0 & 0.0 & 0.0 & 0.0 & 0.0 & 0. & 0.0 & 0. & 0.0 & 0.0 \\
\hline
\end{tabular}




\section{RIO GRANDE BASIN}

08401000 PECOS RIVER BELOW MCMILLAN DAM, NM

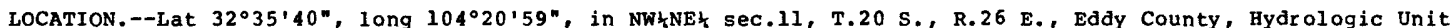
13060011 , on left bank $700 \mathrm{ft}$ downstream from gates in McMillan Dam, $3.4 \mathrm{mi}$ southeast of Lakewood, and at mile 484.1 .

DRAINAGE AREA.--16,990 $\mathrm{mi}^{2}$, approximately (contributing area).

PERIOD OF RECORD.--January 1906 to March 1908, January 1909 to December 1911, August 1939 to December 1940 , December 1946 to current year (January 1906, and January 1910 to December 1911, gage heights and discharge

REVISED RECORDS. --WSP 1512: 1909.

GAGE.--Water-stage recorder and rock control. Elevation of gage is 3,238.21 ft above National Geodetic Vertical Datum of 1929 (U.S. Bureau of Reclamation bench mark). See WSP 1732 for history of changes prior to Mar. 12 ,
1957 . Supplemental water-stage recorders on McMillan Dam spillways No. 1 and 2, Apr. 6 , 1960, to Sept. 30 , 1957. Supplemental water-stage recorders on McMillan Dam spillways No. 1 and 2, Apr.6, 1960, to Sept. 30 , 1970.

REMARKS.--Flow completely regulated by Lake McMillan (station 08400500). Flow also regulated by several other reservoirs. Discharge figures do not include flow, if any, over Lake McMillan spillways No. 1 and 2 which enters the Pecos River downstream from this gage. Diversions and ground-water withdrawals for irrigation of about 171,000 acres, 1959 determination, upstream from station.

AVERAGE DISCHARGE, -40 years $(1907,1940,1948-85), 95.8 \mathrm{ft}^{3 / \mathrm{s}}, 69,410 \mathrm{acre-ft} / \mathrm{yr}^{\mathrm{r}}$.

EXTREMES FOR PERIOD OF RECORD.--Maximum discharge, 16,500 ft3/s, Aug. 23, 1966, includes flow of spillways; no flow many days.

EXTREMES OUTSIDE PERIOD OF RECORD,- Flood of oct. 2, 1904, may have reached $60,000 \mathrm{ft}^{3} / \mathrm{s}$. The $\mathrm{flood}$ of Aug. 3 , 1893, damaged McMillan Dam, then under construction, and destroyed Avalon Dam; this flood was described as "highest in 50 years" at Carlsbad.

STATISTICAL SUMMARIES

MEAN MONTHLY AND MEAN ANNUAL DISChaRges 1907,1940

$$
1948-85
$$

\begin{tabular}{|c|c|c|c|c|c|c|}
\hline MONTH & $\begin{array}{c}\text { MINIMUM } \\
\left(\mathrm{FT}^{3} / \mathrm{S}\right)\end{array}$ & $\begin{array}{c}\text { MAXIMUM } \\
(\mathrm{FT} 3 / \mathrm{S})\end{array}$ & $\begin{array}{l}\text { MEAN } \\
\left(\mathrm{FT}^{3} / \mathrm{S}\right)\end{array}$ & $\begin{array}{l}\text { STAN- } \\
\text { DARD } \\
\text { DEVIA- } \\
\text { TION } \\
\left(\text { FT }^{3} / \mathrm{S}\right)\end{array}$ & $\begin{array}{l}\text { COEFFI- } \\
\text { CIENT OF } \\
\text { VARI- } \\
\text { ATION }\end{array}$ & $\begin{array}{c}\text { PERCENT } \\
\text { OF } \\
\text { ANNUAL } \\
\text { RUNOF F }\end{array}$ \\
\hline $\begin{array}{l}\text { OCTOBER } \\
\text { NOVEMBER } \\
\text { DECEMBER } \\
\text { JANUARY } \\
\text { FEB RUARY } \\
\text { MARCH } \\
\text { APRIL } \\
\text { MAY } \\
\text { JUNE } \\
\text { JULY } \\
\text { AUGUST } \\
\text { SEPTEMBER }\end{array}$ & $\begin{array}{r}0.0 \\
0.0 \\
0.0 \\
0.0 \\
0.0 \\
0.0 \\
24 \\
.4 \\
0.0 \\
.0 \\
0.0 \\
0.0\end{array}$ & $\begin{array}{r}1862 \\
430 \\
582 \\
364 \\
169 \\
160 \\
288 \\
1059 \\
417 \\
712 \\
978 \\
455\end{array}$ & $\begin{array}{r}111 \\
22 \\
29 \\
23 \\
13 \\
33 \\
196 \\
121 \\
119 \\
169 \\
178 \\
141\end{array}$ & $\begin{array}{r}292 \\
70 \\
103 \\
72 \\
32 \\
34 \\
54 \\
182 \\
85 \\
109 \\
149 \\
113\end{array}$ & $\begin{array}{l}2.63 \\
3.27 \\
3.54 \\
3.18 \\
2.50 \\
1.04 \\
.28 \\
1.50 \\
.72 \\
.64 \\
.84 \\
.80\end{array}$ & $\begin{array}{l}9.6 \\
1.9 \\
2.5 \\
1.9 \\
1.1 \\
2.9 \\
17.0 \\
10.5 \\
10.3 \\
14.6 \\
15.4 \\
12.2\end{array}$ \\
\hline ANNUAL & 42 & 252 & 96 & 48 & .50 & 100 \\
\hline
\end{tabular}

MAGNITUDE AND PROBABILITY OF ANNUAL LOW FLOW BASED ON PERIOD OF RECORD 1908, 1948-85

\begin{tabular}{|c|c|c|c|c|c|c|}
\hline \multirow{2}{*}{$\begin{array}{c}\text { PERIOD } \\
\text { (CON- } \\
\text { SECU- } \\
\text { TIVE } \\
\text { DAYS) }\end{array}$} & \multicolumn{6}{|c|}{$\begin{array}{l}\text { DISCHARGE, IN FT'3/S, FOR INDICATED RECURRENCE } \\
\text { INTERVAL, IN YEARS, AND ANNUAL NON- } \\
\text { EXCEEDANCE PROBABILITY, IN PERCENT }\end{array}$} \\
\hline & $\begin{array}{c}2 \\
508\end{array}$ & $\begin{array}{c}5 \\
208\end{array}$ & 108 & 20 & 50 & $\begin{array}{r}100 \\
18\end{array}$ \\
\hline $\begin{array}{r}1 \\
3 \\
7 \\
14 \\
30 \\
60 \\
90 \\
120 \\
183\end{array}$ & $\begin{array}{r}0.0 \\
0.0 \\
0.0 \\
0.0 \\
0.0 \\
0.0 \\
0.0 \\
0.0 \\
13\end{array}$ & $\begin{array}{l}0.0 \\
0.0 \\
0.0 \\
0.0 \\
0.0 \\
0.0 \\
0.0 \\
0.0 \\
1.1\end{array}$ & $\begin{array}{l}0.0 \\
0.0 \\
0.0 \\
0.0 \\
0.0 \\
0.0 \\
0.0 \\
0.0 \\
0.0\end{array}$ & $\begin{array}{l}0.0 \\
0.0 \\
0.0 \\
0.0 \\
0.0 \\
0.0 \\
0.0 \\
0.0 \\
0.0\end{array}$ & $\begin{array}{l}0.0 \\
0.0 \\
0.0 \\
0.0 \\
0.0 \\
0.0 \\
0.0 \\
0.0 \\
0.0\end{array}$ & $\begin{array}{l}0.0 \\
0.0 \\
0.0 \\
0.0 \\
0.0 \\
0.0 \\
0.0 \\
0.0 \\
0.0\end{array}$ \\
\hline
\end{tabular}

MAGNITUDE AND PROBABILITY OF ANNUAL HIGH FLOW BASED ON PERIOD OF RECORD $1907,1940,1948-85$

\begin{tabular}{|c|c|c|c|c|c|c|}
\hline \multirow{2}{*}{$\begin{array}{l}\text { PERIOD } \\
\text { (CON- } \\
\text { SECU- } \\
\text { TIVE } \\
\text { DAYS) }\end{array}$} & \multicolumn{6}{|c|}{$\begin{array}{l}\text { DISCHARGE, IN FT } 3 / S \text {, FOR INDICATED RECURRENCE } \\
\text { INTERVAL, IN YEARS, AND ANNUAL } \\
\text { EXCEEDANCE PROBABILITY, IN PERCENT }\end{array}$} \\
\hline & $\begin{array}{c}2 \\
508\end{array}$ & $\begin{array}{c}5 \\
208\end{array}$ & $\begin{array}{l}10 \\
108\end{array}$ & $\begin{array}{l}25 \\
48\end{array}$ & $\begin{array}{l}50 \\
28\end{array}$ & $\begin{array}{r}100 \\
18\end{array}$ \\
\hline $\begin{array}{r}1 \\
3 \\
7 \\
15 \\
30 \\
60 \\
90\end{array}$ & $\begin{array}{l}774 \\
682 \\
553 \\
422 \\
298 \\
212 \\
180\end{array}$ & $\begin{array}{r}1870 \\
1590 \\
1150 \\
770 \\
496 \\
327 \\
272\end{array}$ & $\begin{array}{r}3340 \\
2810 \\
1880 \\
1150 \\
700 \\
438 \\
354\end{array}$ & $\begin{array}{r}6820 \\
5680 \\
3460 \\
1880 \\
1080 \\
633 \\
486\end{array}$ & $\begin{array}{r}11400 \\
9460 \\
5390 \\
2690 \\
1480 \\
827 \\
609\end{array}$ & $\begin{array}{r}18800 \\
15600 \\
8300 \\
3820 \\
2010 \\
1070 \\
756\end{array}$ \\
\hline
\end{tabular}

DURATION TABLE OF DAILY MEAN FLOW FOR PERIOD OF RECORD 1907, 1940, 1948-85 DISCHARGE, IN FT $3 / S$, THAT WAS EQUALED OR EXCEEDED FOR INDICATED PERCENT OF TIME

\begin{tabular}{|c|c|c|c|c|c|c|c|c|c|c|c|c|c|c|}
\hline 58 & 108 & 158 & 208 & 258 & 308 & 408 & 508 & 608 & 708 & 758 & 808 & 858 & 908 & 958 \\
\hline 74 & 270 & 221 & 173 & 138 & 105 & 42 & 1.1 & .1 & 0.0 & 0.0 & 0.0 & 0.0 & 0.0 & 0.0 \\
\hline
\end{tabular}


LOCATION,--Lat $32^{\circ} 35^{\prime} 19^{\prime \prime}$, long $104^{\circ} 25^{\prime} 17^{\prime \prime}$, in SEłSEłNWł sec.7, T.20 S., R.26 E., Eddy County, Hydrologic Unit 13060011 , on downstream 8 ide of center pier of bridge on U.S. Highway $285,0.4 \mathrm{mi}$ south of Seven Rivers, $2.6 \mathrm{mi}$ upstream from mouth, and $4.0 \mathrm{mi}$ southwest of Lakewood. Mouth at Pecos River mile 480.9 .

DRA INAGE AREA. $-220 \mathrm{mi}^{2}$, approximately.

PERIOD OF RECORD.--October 1963 to current year.

GAGE.--Water-stage recorder. Elevation of gage is 3,276 ft above National Geodetic Vertical Datum of 1929 , from topographic map. Prior to July 8, 1965, at site $400 \mathrm{ft}$ upstream at datum $0.52 \mathrm{ft}$ higher.

REMARRS.--No surface diversions upstream from station, ground-water withdrawals for 240 acres, upstream from station.

AVERAGE DISCHARGE. --22 years, $4.29 \mathrm{ft} / \mathrm{s}, 3,110$ acre-ft/yr.

EXTREMES FOR PERIOD OF RECORD.--Maximum discharge, 25,500 ft $3 / \mathrm{s}$, May 30, 1965, gage height, $20.0 \mathrm{ft}$, from floodmarks, present site and datum, from rating curve extended above $5,700 \mathrm{ft} / \mathrm{s}$ on basis of slope-area measurements at gage heights $18.15 \mathrm{ft}$ and $20.0 \mathrm{ft}$; no flow most of time.

EXTREMES OUTSIDE PERIOD OF RECORD.--Maximum discharge since at least 1941, about $30,000 \mathrm{ft} / \mathrm{s}, \mathrm{gage}$ height, 22.8 $\mathrm{ft}$, from old debris on left bank, former site and datum, from rating curve extended above $5,700 \mathrm{ft} 3 / \mathrm{s}$ on basis of slope-area measurement at gage height $21.8 \mathrm{ft}$. Probable date of flood, 0ct. 7, 1954.

STATISTICAL SUMMARIES

MEAN MONTHLY AND MEAN ANNUAL DISCHARGES 1964-85

\begin{tabular}{|c|c|c|c|c|c|c|}
\hline MONTH & $\begin{array}{c}\text { MINIMUM } \\
\left(\mathrm{FT}^{3} / \mathrm{S}\right)\end{array}$ & $\begin{array}{c}\text { MAXIMUM } \\
\left(\mathrm{FT}^{3} / \mathrm{S}\right)\end{array}$ & $\begin{array}{l}\text { MEAN } \\
\left(\mathrm{FT}^{3} / \mathrm{S}\right)\end{array}$ & $\begin{array}{l}\text { STAN- } \\
\text { DARD } \\
\text { DEVIA- } \\
\text { TION } \\
\left(\mathrm{FT}^{3} / \mathrm{S}\right)\end{array}$ & $\begin{array}{l}\text { COEFFI- } \\
\text { CIENT OF } \\
\text { VARI - } \\
\text { ATION }\end{array}$ & $\begin{array}{c}\text { PERCENT } \\
\text { OF } \\
\text { ANNUAL } \\
\text { RUNOF F }\end{array}$ \\
\hline $\begin{array}{l}\text { OCTOBER } \\
\text { NOVEMBER } \\
\text { DECEMBER } \\
\text { JANUARY } \\
\text { FEBRUARY } \\
\text { MARCH } \\
\text { APRIL } \\
\text { MAY } \\
\text { JUNE } \\
\text { JULY } \\
\text { AUGUST } \\
\text { SEPTEMBER }\end{array}$ & $\begin{array}{l}0.0 \\
0.0 \\
0.0 \\
0.0 \\
0.0 \\
0.0 \\
0.0 \\
0.0 \\
0.0 \\
0.0 \\
0.0 \\
0.0\end{array}$ & $\begin{array}{c}16 \\
2.1 \\
0.0 \\
0.0 \\
0.0 \\
0.0 \\
.2 \\
84 \\
12 \\
28 \\
369 \\
237\end{array}$ & $\begin{array}{l}1.3 \\
.1 \\
0.0 \\
0.0 \\
0.0 \\
0.0 \\
0.0 \\
4.0 \\
1.6 \\
2.7 \\
26 \\
16\end{array}$ & $\begin{array}{r}3.9 \\
.4 \\
0.0 \\
0.0 \\
0.0 \\
0.0 \\
0.0 \\
18 \\
3.6 \\
6.6 \\
83 \\
52\end{array}$ & $\begin{array}{r}2.93 \\
4.89 \\
-- \\
-- \\
-- \\
4.00 \\
4.51 \\
2.31 \\
2.43 \\
3.24 \\
3.27\end{array}$ & $\begin{array}{l}2.6 \\
.2 \\
0.0 \\
0.0 \\
0.0 \\
0.0 \\
0.0 \\
7.8 \\
3.0 \\
5.3 \\
50.0 \\
31.1\end{array}$ \\
\hline NNUAL & 0.0 & 32 & 4.3 & 8.0 & 1.86 & 100 \\
\hline
\end{tabular}

MAGNITUDE AND PROBABILITY OF ANNUAL LOW FLOW BASED ON PERIOD OF RECORD 1965-85

\begin{tabular}{|c|c|c|c|c|c|c|}
\hline \multirow{2}{*}{$\begin{array}{l}\text { PERIOD } \\
\text { (CON- } \\
\text { SECU- } \\
\text { TIVE } \\
\text { DAYS) }\end{array}$} & \multicolumn{6}{|c|}{$\begin{array}{l}\text { DISCHARGE, IN FT } 3 / S \text {, FOR INDICATED RECURRENCE } \\
\text { INTERVAL, IN YEARS, AND ANNUAL NON- } \\
\text { EXCEEDANCE PROBABIL, ITY, IN PERCENT }\end{array}$} \\
\hline & $\begin{array}{c}2 \\
508\end{array}$ & $\begin{array}{c}5 \\
208\end{array}$ & $\begin{array}{l}10 \\
108\end{array}$ & $\begin{array}{l}20 \\
58\end{array}$ & $\begin{array}{l}50 \\
28\end{array}$ & $\begin{array}{r}100 \\
18\end{array}$ \\
\hline $\begin{array}{r}1 \\
3 \\
7 \\
14 \\
30 \\
60 \\
90 \\
120 \\
183\end{array}$ & $\begin{array}{l}0.0 \\
0.0 \\
0.0 \\
0.0 \\
0.0 \\
0.0 \\
0.0 \\
0.0 \\
0.0\end{array}$ & $\begin{array}{l}0.0 \\
0.0 \\
0.0 \\
0.0 \\
0.0 \\
0.0 \\
0.0 \\
0.0 \\
0.0\end{array}$ & $\begin{array}{l}0.0 \\
0.0 \\
0.0 \\
0.0 \\
0.0 \\
0.0 \\
0.0 \\
0.0 \\
0.0\end{array}$ & $\begin{array}{l}0.0 \\
0.0 \\
0.0 \\
0.0 \\
0.0 \\
0.0 \\
0.0 \\
0.0 \\
0.0\end{array}$ & $\begin{array}{l}-- \\
-- \\
-- \\
-- \\
-- \\
-- \\
--\end{array}$ & $\begin{array}{l}-- \\
-- \\
-- \\
-- \\
-- \\
--\end{array}$ \\
\hline
\end{tabular}

MAGNITUDE AND PROBABILITY OF ANNUAL HIGH FLON BASED ON PERIOD OF RECORD 1964-85

\begin{tabular}{|c|c|c|c|c|c|c|}
\hline \multirow{2}{*}{$\begin{array}{l}\text { PERIOD } \\
\text { (CON- } \\
\text { SECU- } \\
\text { TIVE } \\
\text { DAYS) }\end{array}$} & \multicolumn{6}{|c|}{$\begin{array}{l}\text { DISCHARGE, IN FT'3/S, FOR INDICATED RECURRENCE } \\
\text { INTERVAL, IN YEARS, AND ANNUAL } \\
\text { EXCEEDANCE PROBABILITY, IN PERCENT }\end{array}$} \\
\hline & ${ }_{508}^{2}$ & $\begin{array}{c}5 \\
208\end{array}$ & $\begin{array}{l}10 \\
108\end{array}$ & $\begin{array}{l}25 \\
48\end{array}$ & $\begin{array}{l}50 \\
28\end{array}$ & $\begin{array}{r}100 \\
18\end{array}$ \\
\hline $\begin{array}{r}1 \\
3 \\
7 \\
15 \\
30 \\
60\end{array}$ & $\begin{array}{r}103 \\
45 \\
20 \\
9.8 \\
5.0 \\
2.7\end{array}$ & $\begin{array}{r}1000 \\
468 \\
210 \\
100 \\
50 \\
27 \\
70\end{array}$ & $\begin{array}{r}3090 \\
1480 \\
666 \\
311 \\
156 \\
81 \\
58\end{array}$ & $\begin{array}{r}9760 \\
4830 \\
2160 \\
980 \\
488 \\
252\end{array}$ & $\begin{array}{l}=- \\
-- \\
-- \\
--\end{array}$ & $\begin{array}{l}-- \\
-- \\
-- \\
-- \\
--\end{array}$ \\
\hline
\end{tabular}

DURATION TABLE OF DAILY MEAN FLOW FOR PERIOD OF RECORD 1964-85 DISCHARGE, IN FT ${ }^{3} / \mathrm{S}$, THAT WAS EQUALED OR EXCEEDED FOR INDICATED PERCENT OF TIME

\begin{tabular}{|c|c|c|c|c|c|c|c|c|c|c|c|c|c|c|}
\hline 58 & 108 & 158 & 208 & 258 & 308 & 408 & 508 & 608 & 708 & 758 & 808 & 858 & 908 & 958 \\
\hline 0.0 & 0.0 & 0.0 & 0.0 & 0.0 & 0.0 & 0.0 & 0.0 & 0.0 & 0.0 & 0.0 & 0.0 & 0.0 & 0.0 & \\
\hline
\end{tabular}


LOCATION, --Lat $32^{\circ} 30^{\prime} 23^{\prime \prime}$, Iong $104^{\circ} 22^{\prime} 28^{\prime \prime}$, in SEłSEł sec.3, T. 21 S., R. 25 E., Eddy County, Hydrologic Unit

13060011 , at downstream end of bridge pier nearest left bank on U.S. Highway $285,2.1$ mi upstream from mouth and $10 \mathrm{mi}$ nor thwest of Carlsbad. Mouth at Pecos River mile 475.2.

DRAINAGE AREA.--285 mi, approximately.

PERIOD OF RECORD.--October 1963 to current year.

GAGE.--Water-stage recorder. Elevation of gage is 3,250 ft above National Geodetic Vertical Datum of 1929 , from topographic map.

REMARKS.--Diversions for irrigation of 220 acres, upstream from station.

AVERAGE DISCHARGE.--22 years (water years 1964-85), $7.49 \mathrm{ft} / \mathrm{s}, 5,430 \mathrm{acre}-\mathrm{ft} / \mathrm{yr}$.

EXTREMES FOR PERIOD OF RECORD.--Maximum discharge, 31,600 ft $3 / \mathrm{s}$, Aug. 23, 1966, gage height, $15.35 \mathrm{ft}$, from rating curve extended above $8,500 \mathrm{ft}^{3 / \mathrm{s}}$ on basis of slope-area measurement of peak flow; no flow most of time.

EXTREMES OUTSIDE PERIOD OF RECORD.--Since about 1941 the maximum discharge probably occurred oct. 7 , 1954, discharge $63,600 \mathrm{ft}^{3} / \mathrm{s}$, gage height, $19.2 \mathrm{ft}$, from highwater marks on downstream end of bridge pier, by slope-area measurement at site $5 \mathrm{mi}$ upstream.

STATISTICAL SUMMARIES

MEAN MONTHLY AND MEAN ANNUAL DISCHARGES 1964-85

\begin{tabular}{|c|c|c|c|c|c|c|}
\hline MONTH & $\begin{array}{c}\text { MINI MUM } \\
\left(\mathrm{FT}^{3} / \mathrm{S}\right)\end{array}$ & $\frac{\operatorname{MAXIMUM}}{\left(F \mathrm{~T}^{3} / \mathrm{S}\right)}$ & $\begin{array}{l}\text { MEAN } \\
\left(\mathrm{FT}^{3} / \mathrm{S}\right)\end{array}$ & $\begin{array}{l}\text { STAN- } \\
\text { DARD } \\
\text { DEVIA- } \\
\text { TION } \\
\text { (FT3/S) }\end{array}$ & $\begin{array}{l}\text { COEFFI- } \\
\text { CIENT OF } \\
\text { VARI- } \\
\text { ATION }\end{array}$ & $\begin{array}{c}\text { PERCENT } \\
\text { OF } \\
\text { ANNUAL } \\
\text { RUNOFF }\end{array}$ \\
\hline $\begin{array}{l}\text { OCTOBER } \\
\text { NOVEMBER } \\
\text { DECEMBER } \\
\text { JANUARY } \\
\text { FEBRUARY } \\
\text { MARCH } \\
\text { APRIL } \\
\text { MAY } \\
\text { JUNE } \\
\text { JULY } \\
\text { AUGUST } \\
\text { SEPTEMBER }\end{array}$ & $\begin{array}{l}0.0 \\
0.0 \\
0.0 \\
0.0 \\
0.0 \\
0.0 \\
0.0 \\
0.0 \\
0.0 \\
0.0 \\
0.0 \\
0.0\end{array}$ & $\begin{array}{r}185 \\
7.7 \\
.6 \\
0.0 \\
0.0 \\
0.0 \\
1.5 \\
38 \\
55 \\
19 \\
616 \\
335\end{array}$ & $\begin{array}{r}14 \\
.4 \\
0.0 \\
0.0 \\
0.0 \\
0.0 \\
.1 \\
3.4 \\
3.9 \\
3.2 \\
36 \\
28\end{array}$ & $\begin{array}{r}44 \\
1.6 \\
.1 \\
0.0 \\
0.0 \\
0.0 \\
.3 \\
11 \\
12 \\
5.9 \\
132 \\
82\end{array}$ & $\begin{array}{r}3.15 \\
4.21 \\
4.00 \\
-- \\
-- \\
-- \\
3.78 \\
3.24 \\
3.07 \\
1.84 \\
3.68 \\
2.90\end{array}$ & $\begin{array}{r}15.7 \\
.4 \\
0.0 \\
0.0 \\
0.0 \\
0.0 \\
.1 \\
3.8 \\
4.4 \\
3.6 \\
40.3 \\
31.6\end{array}$ \\
\hline INUAL & 0.0 & 54 & 7.5 & 13 & 1.71 & 100 \\
\hline
\end{tabular}

MAGNITUDE AND PROBABILITY OF ANNUAL LOW FLON BASED ON PERIOD OF RECORD 1965-85

\begin{tabular}{|c|c|c|c|c|c|c|}
\hline \multirow{2}{*}{$\begin{array}{l}\text { PERIOD } \\
\text { (CON- } \\
\text { SECU- } \\
\text { TIVE } \\
\text { DAYS) }\end{array}$} & \multicolumn{6}{|c|}{$\begin{array}{l}\text { DISCHARGE, IN FT' } 3 / S \text {, FOR INDICATED RECURRENCE } \\
\text { INTERVAL, IN YEARS, AND ANNUAL NON- } \\
\text { EXCEEDANCE PROBABILITY, IN PERCENT }\end{array}$} \\
\hline & $\begin{array}{c}2 \\
508\end{array}$ & $\begin{array}{c}5 \\
208\end{array}$ & $\begin{array}{l}10 \\
108\end{array}$ & $\begin{array}{l}20 \\
58\end{array}$ & $\begin{array}{l}50 \\
28\end{array}$ & $\begin{array}{r}100 \\
18\end{array}$ \\
\hline $\begin{array}{r}1 \\
3 \\
7 \\
14 \\
30 \\
60 \\
90 \\
120 \\
183\end{array}$ & & $\begin{array}{l}0.0 \\
0.0 \\
0.0 \\
0.0 \\
0.0 \\
0.0 \\
0.0 \\
0.0 \\
0.0\end{array}$ & $\begin{array}{l}0.0 \\
0.0 \\
0.0 \\
0.0 \\
0.0 \\
0.0 \\
0.0 \\
0.0 \\
0.0\end{array}$ & $\begin{array}{l}0.0 \\
0.0 \\
0.0 \\
0.0 \\
0.0 \\
0.0 \\
0.0 \\
0.0 \\
0.0\end{array}$ & $\begin{array}{l}-- \\
-- \\
-- \\
-- \\
-- \\
-\end{array}$ & $\begin{array}{l}-- \\
-- \\
-- \\
-- \\
-- \\
-- \\
--\end{array}$ \\
\hline
\end{tabular}

MAGNITUDE AND PROBABILITY OF ANNUAL HIGH FLOW BASED ON PERTOD OF RECORD 1964-85

\begin{tabular}{|c|c|c|c|c|c|c|}
\hline \multirow{2}{*}{$\begin{array}{l}\text { PERI OD } \\
\text { (CON- } \\
\text { SECU- } \\
\text { TIVE } \\
\text { DAYS) }\end{array}$} & \multicolumn{6}{|c|}{$\begin{array}{l}\text { DISCHARGE, IN FT' } / S \text {, FOR INDICATED RECURRENC } \\
\text { INTERVAL, IN YEARS, AND ANNUAL } \\
\text { EXCEEDANCE PROBABILITY, IN PERCENT }\end{array}$} \\
\hline & $\begin{array}{c}2 \\
508\end{array}$ & $\begin{array}{c}5 \\
208\end{array}$ & $\begin{array}{l}10 \\
108\end{array}$ & $\begin{array}{l}25 \\
48\end{array}$ & $\begin{array}{l}50 \\
28\end{array}$ & $\begin{array}{r}100 \\
18\end{array}$ \\
\hline $\begin{array}{r}1 \\
3 \\
7 \\
15 \\
30 \\
60 \\
90\end{array}$ & $\begin{array}{r}403 \\
157 \\
73 \\
34 \\
17 \\
8.9 \\
6.1\end{array}$ & $\begin{array}{r}2980 \\
1230 \\
558 \\
258 \\
133 \\
70 \\
48\end{array}$ & $\begin{array}{r}6130 \\
2660 \\
1190 \\
561 \\
292 \\
150 \\
104\end{array}$ & $\begin{array}{r}10900 \\
5030 \\
2190 \\
1060 \\
559 \\
298 \\
194\end{array}$ & $\begin{array}{l}-- \\
-- \\
-- \\
-- \\
--\end{array}$ & $\begin{array}{l}-- \\
-- \\
-- \\
-- \\
--\end{array}$ \\
\hline
\end{tabular}

DURATION TABLE OF DAILY MEAN FLOW FOR PERIOD OF RECORD 1964-85

DISCHARGE, IN FT ${ }^{3} / \mathrm{S}$, THAT WAS EQUALED OR EXCEEDED FOR INDICATED PERCENT OF TIME

\begin{tabular}{|c|c|c|c|c|c|c|c|c|c|c|c|c|c|c|}
\hline 58 & 108 & $15 \%$ & 208 & 258 & 308 & 408 & 508 & 608 & 708 & 758 & $80 \%$ & $85 \%$ & 908 & 958 \\
\hline .0 & 0.0 & 0.0 & 0.0 & 0.0 & 0.0 & 0.0 & 0.0 & 0.0 & 0.0 & 0.0 & 0.0 & 0.0 & 0.0 & \\
\hline
\end{tabular}




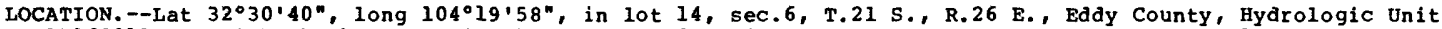
13060011 , on right bank at damsite 3 of Carlsbad project of Bureau of Reclamation, about 1 mi upstream from flow line of Lake Avalon, $1.3 \mathrm{mi}$ downstream from Rocky Arroyo, $8.0 \mathrm{mi}$ northwest of Carisbad, and at mile 473.8 .

DRAINAGE AREA.- $-17,980 \mathrm{mi}^{2}$, approximately (contributing area).

PERIOD OF RECORD.--August 1939 to December 1940, August 1944 to current year.

REVISED RECORDS.--WSP 1512: 1946-47(M), 1948 (P), 1949, 1950(P). WSP 1712: Drainage area.

GAGE.--Elevation of gage is $3,171.31 \mathrm{ft}$ above National Geodetic Vertical Datum of 1929 (levels by $\mathrm{U} . \mathrm{S}$. Bureau of Reclamation). Prior to Aug. 10, 1944, at site 1,000 ft downstream, at datum $1.00 \mathrm{ft}$ higher. Aug. 10 , 1944 to Dec. 31,1966 , at present datum $1.00 \mathrm{ft}$ higher.

REMARKS. -Flow regulated by Lake McMillan (station 08400500 ) since 1893 and by several other reservoirs. Diversions and ground-water withdrawals for irrigation of about 173,000 acres, 1959 determination, upstream from station. Discharge represents inflow to Lake Avalon.

AVERAGE DISCHARGE. -42 years $(1940,1945-85), 155 \mathrm{ft}^{3} / \mathrm{s}, 112,300 \mathrm{acre}-\mathrm{ft} / \mathrm{yr}$.

EXTREMES FOR PERIOD OF RECORD.--Maximum discharge, 69,000 ft 3/s, Aug. 23, 1966, gage height, $21.32 \mathrm{ft}$, present datum, from floodmark, from rating curve extended above $25,000 \mathrm{ft} / \mathrm{s}$ on basis of slope-area measurement at gage height $19.53 \mathrm{ft}$; minimum, $4.3 \mathrm{ft} 3 / \mathrm{s}$, Aug. 5 , 1954.

EXTREMES OUTSIDE PERIOD OF RECORD.--Peaks which probably exceeded 40,000 $\mathrm{ft}^{3} / \mathrm{s}$ occurred in Aug. 1893, Oct. 2, 1904, July 25, 1905, Apr. 17, 1915, Aug. 7, 1916, and May 30, 1937, based primarily on records for station "at Carisbad." Peak of May 22, 1941, was estimated at $60,000 \mathrm{ft} / \mathrm{s}$. Floods of 1893 and 1904 originated upstream from McMillan Dam and contributed to the two failures of Avalon Dam.

STATISTICAL SUMMARIES

MEAN MONTHLY AND MEAN ANNUAL DISCHARGES 1940, 1945-85

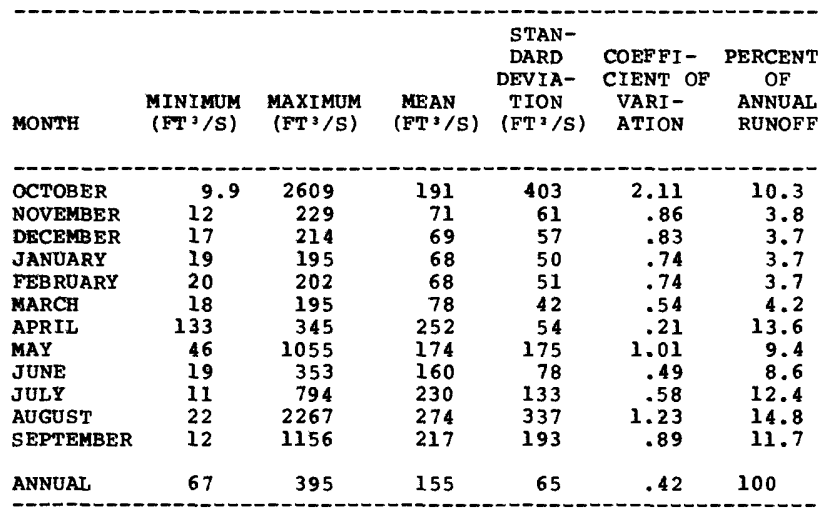

MAGNITUDE AND PROBABILITY OF ANNUAL LOW FLOW BASED ON PERIOD OF RECORD 1946-85

\begin{tabular}{|c|c|c|c|c|c|c|}
\hline \multirow{2}{*}{$\begin{array}{l}\text { PERIOD } \\
\text { (CON- } \\
\text { SECU- } \\
\text { TIVE } \\
\text { DAYS) }\end{array}$} & \multicolumn{6}{|c|}{$\begin{array}{l}\text { DISCHARGE, IN FT3/S, FOR INDICATED RECURRENCE } \\
\text { INTERVAL, IN YEARS, AND ANNUAL NON- } \\
\text { EXCEEDANCE PROBAB ILITY, IN PERCENT }\end{array}$} \\
\hline & $\begin{array}{c}2 \\
508\end{array}$ & $\begin{array}{c}5 \\
208\end{array}$ & $\begin{array}{l}10 \\
108\end{array}$ & $\begin{array}{l}20 \\
58\end{array}$ & $\begin{array}{l}50 \\
28\end{array}$ & $\begin{array}{r}100 \\
18\end{array}$ \\
\hline $\begin{array}{r}1 \\
3 \\
7 \\
14 \\
30 \\
60 \\
90 \\
120 \\
183\end{array}$ & $\begin{array}{l}21 \\
22 \\
23 \\
25 \\
28 \\
33 \\
38 \\
41 \\
57\end{array}$ & $\begin{array}{l}13 \\
13 \\
14 \\
15 \\
17 \\
22 \\
24 \\
25 \\
33\end{array}$ & $\begin{array}{l}9.2 \\
9.7 \\
10 \\
11 \\
13 \\
18 \\
20 \\
20 \\
26\end{array}$ & $\begin{array}{r}6 . \\
7 . \\
7 . \\
8 . \\
11 \\
16 \\
17 \\
17 \\
21\end{array}$ & $\begin{array}{r}4 \\
5 \\
5 \\
6 \\
8 \\
14 \\
15 \\
15 \\
17\end{array}$ & $\begin{array}{l}3.7 \\
4.0 \\
4.3 \\
5.4 \\
7.4 \\
13 \\
13 \\
13 \\
15\end{array}$ \\
\hline
\end{tabular}

MAGNITUDE AND PROBABILITY OF ANNUAL HIGH FLOW BASED ON PERIOD OF RECORD 1940, 1945-85

\begin{tabular}{|c|c|c|c|c|c|c|}
\hline \multirow{2}{*}{$\begin{array}{l}\text { PERIOD } \\
\text { (CON- } \\
\text { SECU- } \\
\text { TIVE } \\
\text { DAYS) }\end{array}$} & \multicolumn{6}{|c|}{$\begin{array}{l}\text { DISCHARGE, IN FT } 3 / S \text {, FOR INDICATED RECUR } \\
\text { INTERVAL, IN YEARS, AND ANNUAL } \\
\text { EXCEEDANCE PROBABILITY, IN PERCENT }\end{array}$} \\
\hline & $\begin{array}{c}2 \\
508\end{array}$ & $\begin{array}{c}5 \\
208\end{array}$ & $\begin{array}{l}10 \\
108\end{array}$ & $\begin{array}{l}25 \\
48\end{array}$ & $\begin{array}{l}50 \\
28\end{array}$ & $\begin{array}{r}100 \\
18\end{array}$ \\
\hline $\begin{array}{r}1 \\
3 \\
7 \\
15 \\
30 \\
60 \\
90\end{array}$ & $\begin{array}{r}1600 \\
981 \\
681 \\
501 \\
367 \\
283 \\
248\end{array}$ & $\begin{array}{r}4190 \\
2390 \\
1520 \\
975 \\
649 \\
455 \\
382\end{array}$ & $\begin{array}{r}7460 \\
4240 \\
2600 \\
1530 \\
955 \\
619 \\
498\end{array}$ & $\begin{array}{r}14600 \\
8450 \\
5080 \\
2690 \\
1550 \\
901 \\
681\end{array}$ & $\begin{array}{r}23300 \\
13800 \\
8280 \\
4070 \\
2200 \\
1180 \\
848\end{array}$ & $\begin{array}{r}36300 \\
22300 \\
13300 \\
6090 \\
3110 \\
1530 \\
1040\end{array}$ \\
\hline
\end{tabular}

DURATION TABLE OF DAILY MEAN FLOW FOR PERIOD OF RECORD 1940, 1945-85

DISCHARGE, IN FT $3 / S$, THAT WAS EQUALED OR EXCEEDED FOR INDICATED PERCENT OF TIME

\begin{tabular}{|c|c|c|c|c|c|c|c|c|c|c|c|c|c|c|}
\hline 58 & 108 & 158 & 208 & 258 & 308 & 408 & 508 & 608 & 708 & 758 & 808 & 858 & 908 & 958 \\
\hline 417 & 321 & 264 & 225 & 195 & 171 & 124 & 88 & 56 & 41 & 36 & 32 & 28 & 24 & 20 \\
\hline
\end{tabular}


RIO GRANDE BASIN

08404000 PECOS RIVER BELOW AVALON DAM, NM

LOCATION.--Lat $32^{\circ} 28^{\prime} 55^{\prime \prime}$, long $104^{\circ} 15^{\prime} 47^{\prime \prime}$, in SWłSWłNEł sec.14, T.21 S., R.26 E., Eddy County, Hydrologic Unit 13060011 , on right bank 4,800 ft downstream from Avalon Dam, 4.5 mi northwest of Carlsbad, and at mile 466.3 .

DRAINAGE AREA. $--18,080 \mathrm{mi}^{2}$, approximately (contributing area).

PERIOD OF RECORD.--January 1906 to March 1907, (published as "at Avalon"), June 1951 to current year.

GAGE.--Water-stage recorder. Elevation of gage is 3,130 ft above National Geodetic vertical Datum of 1929 , from topographic map. January 1906 to March 1907 nonrecording gage at site $0.5 \mathrm{mi}$ upstream at different datum.

REMARKS.-Flow completely regulated by Lake Avalon (station 08403800) since 1891 . Flow also regulated by several other reservoirs. Diversions and ground-water withdrawals upstream from station for irrigation of about 198,000 acres, 1959 determination. Station bypassed by Carlsbad Main Canal (station 08403500).

AVERAGE DISCHARGE. -34 years (water years 1952-85), $29.7 \mathrm{ft}^{3} / \mathrm{s}, 21,520$ acre-ft/yr.

EXTREMES FOR PERIOD OF RECORD.--Maximum discharge, 55,500 $\mathrm{ft}^{3} / \mathrm{s}$, Aug. 23,1966 , gage height, $26.4 \mathrm{ft}$, from floodmarks, from rating curve extended above $33,000 \mathrm{ft} / \mathrm{s}$ on basis of computation of peak flow over Tansill Dam $5.8 \mathrm{mi}$ downstream; no flow most of time.

EXTREMES OUTSIDE PERIOD OF RECORD.--Flood of Oct. 2, 1904, caused in part, by failure of Avalon Dam, probably exceeded $90,000 \mathrm{ft} / \mathrm{s}$ and is probably the greatest flood since 1842. A major flood occurred Aug. 3 , 1893, and was described as "greatest in 50 years"; it damaged McMilian Dam, then under construction, and washed out the original Avalon Dam. Another major flood occurred Aug. 7, 1916, discharge 70,000 ft:s at site 6.5 mi downstream.

STATISTICAL SUMMARIES

MEAN MONTHLY AND MEAN ANNUAL DISCHARGES 1952-85

\begin{tabular}{|c|c|c|c|c|c|c|}
\hline MONTH & $\begin{array}{l}\text { MINIMUM } \\
(\mathrm{FT}, / \mathrm{S})\end{array}$ & $\begin{array}{c}\text { MAXIMUM } \\
\left(\mathrm{FT}^{3} / \mathrm{S}\right)\end{array}$ & $\begin{array}{l}\text { MEAN } \\
(\text { FT } 3 / \mathrm{S})\end{array}$ & $\begin{array}{l}\text { STAN- } \\
\text { DARD } \\
\text { DEVIA- } \\
\text { TION } \\
\text { (FT'/S) }\end{array}$ & $\begin{array}{l}\text { COEFFI- } \\
\text { CIENT OF } \\
\text { VARI- } \\
\text { ATION }\end{array}$ & $\begin{array}{c}\text { PERCENT } \\
\text { OF } \\
\text { ANNUAL } \\
\text { RUNOFF }\end{array}$ \\
\hline \multicolumn{7}{|c|}{ 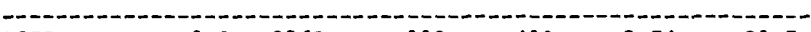 } \\
\hline OCTOBER & 0.0 & 2365 & 112 & 419 & 3.74 & 31.7 \\
\hline NOVEMBER & 0.0 & 117 & 11 & 30 & 2.65 & 3.2 \\
\hline DECEMBER & 0.0 & 159 & 11 & 32 & 2.93 & 3.1 \\
\hline JANUARY & 0.0 & 82 & 6.7 & 17 & 2.62 & 1.9 \\
\hline FEB RUARY & 0.0 & 111 & 6.3 & 21 & 3.36 & 1.8 \\
\hline MARCH & 0.0 & 6.7 & .6 & 1.6 & 2.73 & .2 \\
\hline APRIL & 0.0 & .1 & 0.0 & 0.0 & -- & 0.0 \\
\hline MAY & 0.0 & 739 & 39 & 152 & 3.94 & 10.9 \\
\hline JUNE & 0.0 & 53 & 3.4 & 12 & 3.58 & 1.0 \\
\hline JULY & 0.0 & 595 & 18 & 102 & 5.73 & 5.0 \\
\hline AUGUST & 0.0 & 2034 & 82 & 358 & 4.38 & 23.2 \\
\hline & & & & & & \\
\hline ANNUAL & 0.0 & 206 & 30 & 50 & 1.67 & 100 \\
\hline
\end{tabular}

MAGNITUDE AND PROBABILITY OF ANNUAL LOW FLON BASED ON PERIOD OF RECORD 1953-85

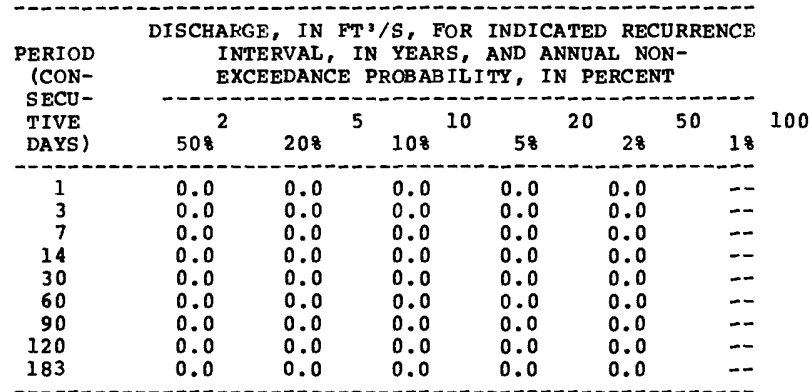

MAGNITUDE AND PROBABILITY OF ANNUAL HIGH FLOW BASED ON PERIOD OF RECORD 1952-85

\begin{tabular}{|c|c|c|c|c|c|c|}
\hline \multirow{2}{*}{$\begin{array}{l}\text { PERIOD } \\
\text { (CON- } \\
\text { SECU- } \\
\text { TIVE } \\
\text { DAYS) }\end{array}$} & \multicolumn{6}{|c|}{$\begin{array}{l}\text { DISCHARGE, IN FT }{ }^{3} / S \text {, FOR INDICATED RECURRENCE } \\
\text { INTERVAL, IN YEARS, AND ANNUAL } \\
\text { EXCEEDANCE PROBAB ILI T, IN PERCENT }\end{array}$} \\
\hline & $\stackrel{2}{508}^{2}$ & $\begin{array}{c}5 \\
208\end{array}$ & $\begin{array}{l}10 \\
108\end{array}$ & $\begin{array}{l}25 \\
48\end{array}$ & $\begin{array}{l}50 \\
28\end{array}$ & $\begin{array}{r}100 \\
18\end{array}$ \\
\hline $\begin{array}{r}1 \\
3 \\
7 \\
15 \\
30 \\
60 \\
90\end{array}$ & $\begin{array}{c}303 \\
156 \\
83 \\
46 \\
26 \\
14 \\
9.4\end{array}$ & $\begin{array}{r}4690 \\
2840 \\
1660 \\
959 \\
545 \\
292 \\
206\end{array}$ & $\begin{array}{r}12600 \\
7970 \\
4780 \\
2730 \\
1540 \\
824 \\
580\end{array}$ & $\begin{array}{r}28300 \\
18200 \\
11100 \\
6220 \\
3450 \\
1840 \\
1280\end{array}$ & $\begin{array}{r}43800 \\
28200 \\
17200 \\
9540 \\
5230 \\
2770 \\
1920\end{array}$ & $\begin{array}{l}-- \\
-- \\
-- \\
-- \\
-- \\
--\end{array}$ \\
\hline
\end{tabular}

DURATION TABLE OF DAILY MEAN FLOW FOR PERIOD OF RECORD 1952-85 DISCHARGE, IN FT $3 / S$, THAT WAS EQUALED OR EXCEEDED FOR INDICATED PERCENT OF TIME

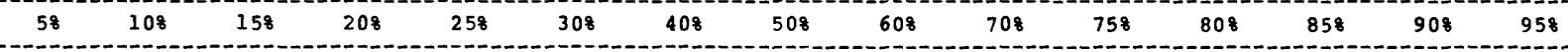


RIO GRANDE BASIN

08405000 PECOS RIVER AT CARLSBAD, NM

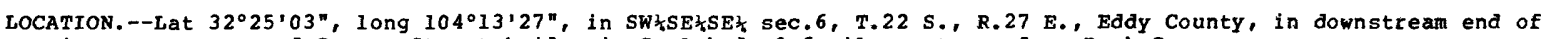
pier near center of Greene street bridge in Carlsbad, 0.6 mile upstream from Dark Canyon.

DRAINAGE AREA.--18,100 $\mathrm{mi}^{2}$, approximately (contributing area).

PERIOD OF RECORD.--May 1903 to March 1908 (January 1907 to March 1908, gage heights and discharge measurements only), May 1914 to September 1915, and April 1920 to December 1969. Monthly discharge only for some periods, published in WSP 1312. Records (except maximum discharges) for October 1915 to March 1920 , published in WSP $438,458,478$, and 508 , have been found to be unreliable and should not be used.

REVISED RECORDS.--WSP 1632: 1903-6, 1936, 1938. See also PERIOD OF RECORD.

GAGE.--Water-stage recorder. Datum of gage is 3,080.28 ft above mean sea level, datum of 1929 . Prior to June 1 , 1920, staff gages in immediate vicinity of present site, and at different datums prior to Jan. 6 , 1938 .

REMARKS.--Flow regulated by Lake Sumner, Lake McMil1an and Lake Avalon (sta 08384000,08400500 ,

08403800 ) and at low stages by power plant above station. Gage is bypassed on left bank by Carlsbad main canal east which irrigates several hundred acres adjacent to and below gage site, and on $r$ ight bank by Carlsbad main canal south, which with supplemental ground-water withdrawals irrigates about 23,000 acres below. This bypass flow is not presently gaged. Diversions and ground-water withdrawals above station for irrigation of about 198,000 acres (1959 determination).

AVERAGE DISCHARGE.--52 years (water years 1904, 1906, 1915, 1921-69), 200 ft3/s, 144,900 acre-ft/yr.

EXTREMES FOR PERIOD OF RECORD.--Maximum discharge probab1y exceeded 90,000 ft $/ \mathrm{s}$ Oct. 2, 1904 (gage height, 23.44

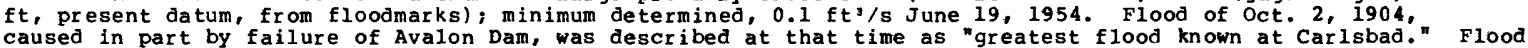
in August 1893 is probably second highest, and was described as "greatest in 50 years"; it damaged McMilian Dam and washed out the original Avalon Dam.

STATISTICAL SUMMARIES

MEAN MONTHLY AND MEAN ANNUAL DISCHARGES 1904, 1906 $1915,1921-69$
MAGNITUDE AND PROBABILITY OF ANNUAL LOW FLOW BASED ON PERIOD OF RECORD $1906,1921-69$

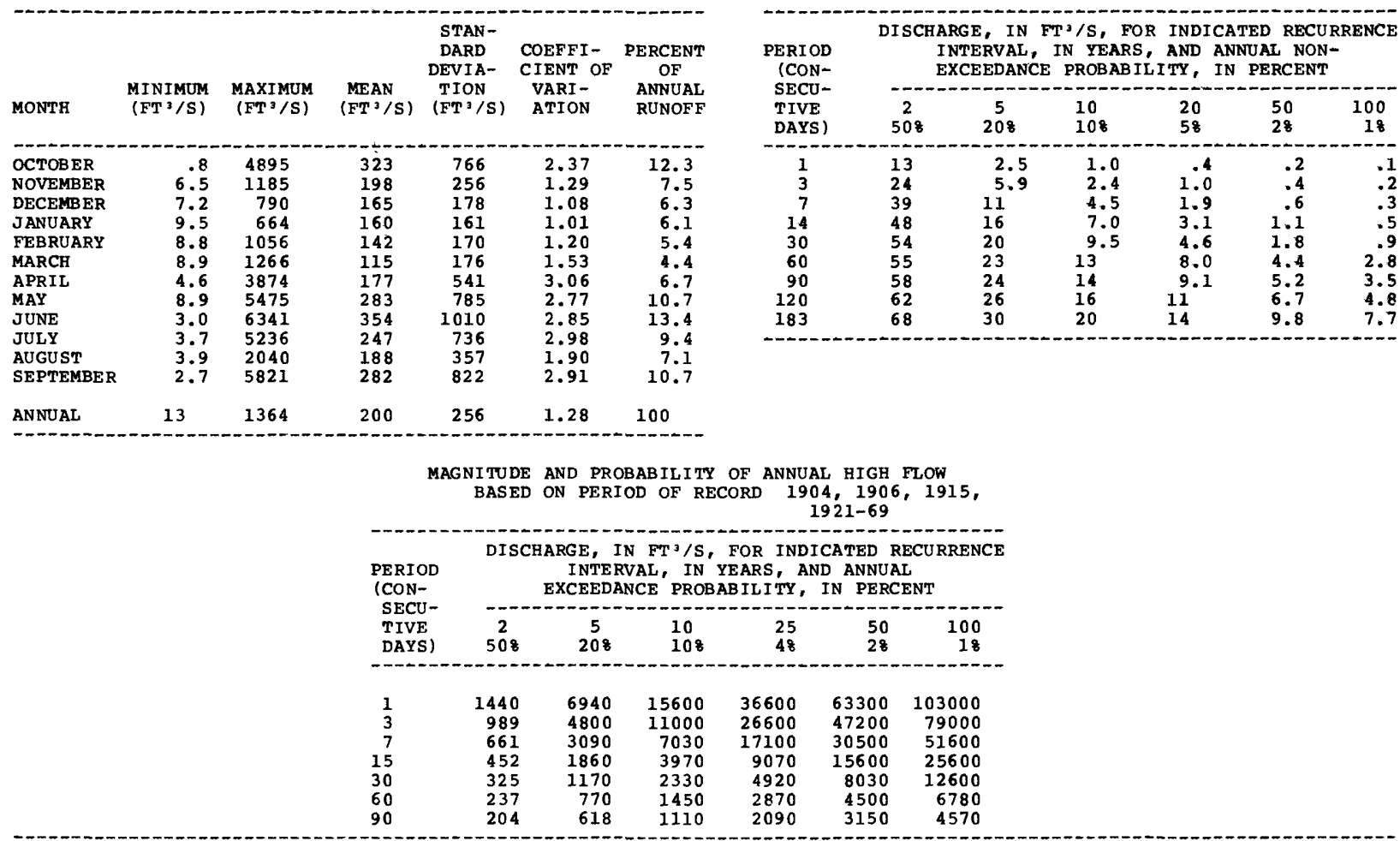

DURATION TABLE OF DAILY MEAN FLOW FOR PERIOD OF RECORD 1904, 1906, 1915, 1921-69

DISCHARGE, IN FT ${ }^{3} / \mathrm{S}$, THAT WAS EQUALED OR EXCEEDED FOR INDICATED PERCENT OF TIME

\begin{tabular}{|c|c|c|c|c|c|c|c|c|c|c|c|c|c|c|}
\hline 58 & 108 & 158 & 208 & 258 & 308 & 408 & 508 & 608 & 708 & 758 & 808 & 858 & 908 & 958 \\
\hline 484 & 281 & 203 & 154 & 126 & 106 & 91 & 76 & 61 & 46 & 38 & 32 & 25 & 18 & 10 \\
\hline
\end{tabular}


RIO GRANDE BASIN

08405150 DARK CANYON DRAW AT CARLSBAD, NM

LOCATION.--Lat $32^{\circ} 24^{\prime} 24^{\prime \prime}$, long $104^{\circ} 13^{\prime} 34^{\prime \prime}$, in NENWhEk sec.7, T.22 S., R.27 E., Eddy County, Hydrologic Unit 13060011 , on downstream side of U.S. Highway $62-285$ (Canal street) bridge in Carlsbad, and $0.6 \mathrm{mi}$ upstream from mouth. Mouth at Pecos River mile 459.2.

DRAINAGE AREA. $--450 \mathrm{mi}^{2}$, approximately.

PERIOD OF RECORD.--January 1973 to current year.

GAGE.--Water-stage recorder. Elevation of gage is 3,088.21 ft above National Geodetic vertical Datum of 1929 .

REMARKS. --A Soil Conservation Service flood control project on Hackberry Draw, an upstream tributary, has some effect on flood peaks and flow duration. Ground-water withdrawals upstream from station for irrigation of approximately 2,100 acres, 1973 determination and for municipal supply for carlsbad.

AVERAGE DISCHARGE.--12 years (water years 1974-85), $7.27 \mathrm{ft}^{3} / \mathrm{s}, 5,270 \mathrm{acre}-\mathrm{ft} / \mathrm{yr}$.

EXTREMES FOR PERIOD OF RECORD.--Maximum discharge, 27,000 fts/s, sept. 26, 1980, gage height, 12.10 ft, from rating curve extended above $7,100 \mathrm{ft} / \mathrm{s}$; no flow most of time.

EXTREMES OUTSIDE PERIOD OF RECORD.--The flood of Aug. 23, 1966, reached a discharge of $66,000 \mathrm{ft} / \mathrm{s}$ as determined by slope-area measurement at site $1.2 \mathrm{mi}$ upstream. Another flood of approximately the same magnitude occur red sept. 20, 1941 .

Other major peaks occurred July 17, 1906, July 24, 1908, July 24, 1911, Apr. 18, 1915, Aug. 8, 1916, Sept. 15, 1919, Aug. 4, 1925, and May 23, 1941.

STATISTICAL SUMMARIES

MEAN MONTHLY AND MEAN ANNUAL DISCHARGES 1974-85

\begin{tabular}{|c|c|c|c|c|c|c|}
\hline MONTH & $\begin{array}{l}\text { MINIMUM } \\
(\mathrm{FT} \% / \mathrm{S})\end{array}$ & $\begin{array}{c}\text { MAXIMUM } \\
\left(\mathrm{FT}^{3} / \mathrm{S}\right)\end{array}$ & $\begin{array}{l}\text { MEAN } \\
\left(\mathrm{FT}^{3} / \mathrm{S}\right)\end{array}$ & $\begin{array}{l}\text { STAN- } \\
\text { DARD } \\
\text { DEVIA- } \\
\text { TION } \\
\text { (FT: } 1 \text { S) }\end{array}$ & $\begin{array}{l}\text { COEFFI - } \\
\text { CIENT OF } \\
\text { VARI- } \\
\text { ATION }\end{array}$ & $\begin{array}{c}\text { PERCENT } \\
\text { OF } \\
\text { ANNUAL } \\
\text { RUNOFF }\end{array}$ \\
\hline $\begin{array}{l}\text { OCTOBER } \\
\text { NOVEMBER } \\
\text { DECEMBER } \\
\text { JANUARY } \\
\text { FEBRUARY } \\
\text { MARCH } \\
\text { APRIL } \\
\text { MAY } \\
\text { JUNE } \\
\text { JULY } \\
\text { AUGUST } \\
\text { SEPTEMBER }\end{array}$ & $\begin{array}{l}0.0 \\
0.0 \\
0.0 \\
0.0 \\
0.0 \\
0.0 \\
0.0 \\
0.0 \\
0.0 \\
0.0 \\
0.0 \\
0.0\end{array}$ & $\begin{array}{c}196 \\
20 \\
0.0 \\
0.0 \\
0.0 \\
0.0 \\
0.0 \\
8.8 \\
5.0 \\
12 \\
162 \\
331\end{array}$ & $\begin{array}{r}16 \\
1.6 \\
0.0 \\
0.0 \\
0.0 \\
0.0 \\
0.0 \\
.7 \\
.4 \\
1.0 \\
14 \\
54\end{array}$ & $\begin{array}{r}57 \\
5.7 \\
0.0 \\
0.0 \\
0.0 \\
0.0 \\
0.0 \\
2.5 \\
1.4 \\
3.6 \\
47 \\
107\end{array}$ & $\begin{array}{r}3.47 \\
3.47 \\
-- \\
-- \\
-- \\
-- \\
-. \\
3.48 \\
3.51 \\
3.47 \\
3.47 \\
1.99\end{array}$ & $\begin{array}{r}18.6 \\
1.9 \\
0.0 \\
0.0 \\
0.0 \\
0.0 \\
0.0 \\
.8 \\
.5 \\
1.2 \\
15.4 \\
61.6\end{array}$ \\
\hline ANNUAL & 1.1 & 27 & 7.3 & 9.1 & 1.26 & 100 \\
\hline
\end{tabular}

MAGNITUDE AND PROBABILITY OF ANNUAL LOW FLOW BASED ON PERIOD OF RECORD 1975-85

\begin{tabular}{|c|c|c|c|c|c|c|}
\hline \multirow{2}{*}{$\begin{array}{l}\text { PERIOD } \\
\text { (CON- } \\
\text { SECU- } \\
\text { TIVE } \\
\text { DAYS) }\end{array}$} & \multicolumn{6}{|c|}{$\begin{array}{l}\text { DISCHARGE, IN FT }{ }^{3} / S \text {, FOR INDICATED RECURRENCE } \\
\text { INTERVAL, IN YEARS, AND ANNUAL NON- } \\
\text { EXCEEDANCE PROBABILITY, IN PERCENT }\end{array}$} \\
\hline & $\begin{array}{c}2 \\
508\end{array}$ & $\begin{array}{c}5 \\
208\end{array}$ & $\begin{array}{l}10 \\
108\end{array}$ & $\begin{array}{l}20 \\
58\end{array}$ & $\begin{array}{l}50 \\
28\end{array}$ & $\begin{array}{r}100 \\
18\end{array}$ \\
\hline $\begin{array}{r}1 \\
3 \\
7 \\
14 \\
30 \\
60 \\
90 \\
120 \\
183\end{array}$ & $\begin{array}{l}0.0 \\
0.0 \\
0.0 \\
0.0 \\
0.0 \\
0.0 \\
0.0 \\
0.0 \\
0.0\end{array}$ & $\begin{array}{l}0.0 \\
0.0 \\
0.0 \\
0.0 \\
0.0 \\
0.0 \\
0.0 \\
0.0 \\
0.0\end{array}$ & $\begin{array}{l}0.0 \\
0.0 \\
0.0 \\
0.0 \\
0.0 \\
0.0 \\
0.0 \\
0.0 \\
0.0\end{array}$ & $\begin{array}{l}0.0 \\
0.0 \\
0.0 \\
0.0 \\
0.0 \\
0.0 \\
0.0 \\
0.0 \\
0.0\end{array}$ & $\begin{array}{l}-- \\
=- \\
=- \\
=- \\
-- \\
--\end{array}$ & - \\
\hline
\end{tabular}

MAGNITUDE AND PROBABILITY OF ANNUAL HIGH FLOW BASED ON PERIOD OF RECORD 1974-85

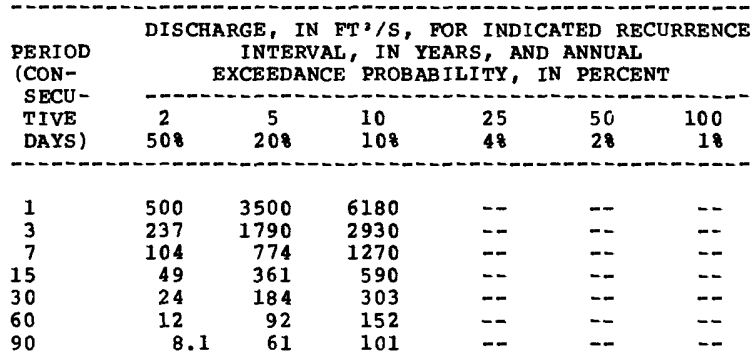

DURATION TABLE OF DAILY MEAN FLOW FOR PERIOD OF RECORD 1974-85 DISCHARGE, IN FT $3 / \mathrm{S}$, THAT WAS EQUALEL OK FXCEEDED FOR INDICATED PERCENT OF TIME

\begin{tabular}{|c|c|c|c|c|c|c|c|c|c|c|c|c|c|c|}
\hline 58 & 08 & 158 & 208 & 258 & 308 & 408 & 508 & 608 & 708 & 758 & 808 & 858 & 908 & 958 \\
\hline .1 & .1 & 0.0 & 0. & 0. & 0.0 & 0. & 0.0 & 0. & 0. & 0. & 0. & 0. & 0.0 & \\
\hline
\end{tabular}


RIO GRANDE BASIN

08405200 PECOS RIVER BELOW DARK CANYON DRAW, AT CARLSBAD, NM

LOCATION,--Lat $32^{\circ} 24^{\prime} 37^{\prime \prime}$, long $104^{\circ} 12^{\prime} 58^{\prime \prime}$, in NEłSW lNW $_{4}$ sec.8, T.22 S., R. 27 E., Eday County, Hydrologic Unit 13060011 , on left bank, $700 \mathrm{ft}$ downstream from mouth of Dark Canyon Draw, $0.3 \mathrm{mi}$ downstream from Lower Tansill Dam and Bataan recreational area, $0.8 \mathrm{mi}$ downstream from bridge on 0.5 . Highway $62-180$ in Carlsbad, and at mile 459.1.

DRAINAGE AREA. - - $18,550 \mathrm{mi}^{2}$, approximately, contributing area.

PERIOD OF RECORD.--January 1970 to current year.

GAGE.--Water-stage recorder and concrete control. Elevation of gage is 3,075.19 ft above National Geodetic Vertical Datum of 1929.

REMARKS.--Flow regulated by Lake Avalon (station 08403800) since 1891 and by several other reservoirs and up to Nov. 1982 at low stages by power plant. Power Plant discontinued operation Nov. 1982. Gage is bypassed on Nov. 1982 at low stages by power plant. Power Plant discontinued operation Nov. 1982. Gage is bypassed on gage site and on $r$ ight bank by Carlsbad Main Canal South, which with supplemental ground-water withdrawals irrigates about 23,000 acres downstream. Diversions and ground-water withdrawals upstream from station for irrigation of about 198,000 acres, 1959 determination.

AVERAGE DISCHARGE.--15 years (water years 1971-85), $45.7 \mathrm{ft}^{3} / \mathrm{s}, 33,110 \mathrm{acre}-\mathrm{ft} / \mathrm{yr}$.

EXTREMES FOR PERIOD OF RECORD.--Maximum discharge, 28,200 ft $3 / \mathrm{s}$, Sept. 26, 1980, gage height, $14.60 \mathrm{ft}$, from floodmarks, from rating curve extended above $12,000 \mathrm{ft}^{3} / \mathrm{s}$; no flow at times.

EXTREMES OUTSIDE PERIOD OF RECORD.--The flood of Aug. 23, 1966, reached a stage of about $22 \mathrm{ft}$, discharge not determined. (For dates of other historical floods see station 08404000. )

STATISTICAL SUMMARIES

MEAN MONTHLY AND MEAN ANNUAL DISCHARges 1971-85

\begin{tabular}{|c|c|c|c|c|c|c|}
\hline MONTH & $\begin{array}{l}\text { MINI MUM } \\
\left(\mathrm{FT}^{3} / \mathrm{S}\right)\end{array}$ & $\begin{array}{c}\text { MAXIMUM } \\
\left(\mathrm{FT}^{3} / \mathrm{S}\right)\end{array}$ & $\begin{array}{l}\text { MEAN } \\
\left(\mathrm{FT}^{3} / \mathrm{S}\right)\end{array}$ & $\begin{array}{l}\text { STAN- } \\
\text { DARD } \\
\text { DEVIA- } \\
\text { TION } \\
\left(\text { FT }^{3} / \mathrm{S}\right)\end{array}$ & $\begin{array}{l}\text { COEFFI- } \\
\text { CIENT OF } \\
\text { VARI - } \\
\text { ATION }\end{array}$ & $\begin{array}{c}\text { PERCENT } \\
\text { OF } \\
\text { ANNUAL } \\
\text { RUNOFF }\end{array}$ \\
\hline остОВЕR & 9.1 & 728 & 67 & 183 & 2.72 & 12.3 \\
\hline $\begin{array}{l}\text { OCTOBER } \\
\text { NOVEMBER }\end{array}$ & $\begin{array}{l}9.1 \\
8.1\end{array}$ & $\begin{array}{l}728 \\
160\end{array}$ & $\begin{array}{l}67 \\
32\end{array}$ & $\begin{array}{r}183 \\
39\end{array}$ & $\begin{array}{l}2.72 \\
1.25\end{array}$ & $\begin{array}{r}12.3 \\
5.7\end{array}$ \\
\hline DECEMBER & 9.6 & 109 & 25 & 24 & .98 & 4.5 \\
\hline JANUARY & 9.8 & 202 & 28 & 24 & .87 & 5.0 \\
\hline FEBRUARY & 11 & 90 & 31 & 24 & .79 & 5.6 \\
\hline MARCH & 6.0 & 54 & 24 & 15 & .62 & 4.4 \\
\hline APRIL & .1 & 36 & 16 & 11 & .65 & 3.0 \\
\hline MAY & 1.1 & 702 & 60 & 178 & 2.96 & 11.0 \\
\hline JUNE & .3 & 79 & 17 & 20 & 1.23 & 3.0 \\
\hline JULY & .1 & 40 & 12 & 13 & 1.06 & 2.2 \\
\hline AUGUST & .2 & 674 & 54 & 172 & 3.18 & 9.9 \\
\hline SEPTEMBER & 3.2 & 1252 & 183 & 347 & 1.90 & 33.4 \\
\hline ANNUAL & 11 & 117 & 46 & 35 & .78 & 100 \\
\hline
\end{tabular}

MAGNITUDE AND PROBABILITY OF ANNUAL LOW FLOW BASED ON PERIOD OF RECORD 1972-85

\begin{tabular}{|c|c|c|c|c|c|c|}
\hline \multirow{2}{*}{$\begin{array}{l}\text { PERIOD } \\
\text { (CON- } \\
\text { SECU- } \\
\text { TIVE } \\
\text { DAYS) }\end{array}$} & \multicolumn{6}{|c|}{$\begin{array}{l}\text { DISCHARGE, IN FT }{ }^{3} / \mathrm{S} \text {, FOR INDICATED RECURRENCE } \\
\text { INTERVAL, IN YEARS, AND ANNUAL NON- } \\
\text { EXCEEDANCE PROBABILITY, IN PERCENT }\end{array}$} \\
\hline & $\begin{array}{c}2 \\
508\end{array}$ & $\begin{array}{c}5 \\
208\end{array}$ & $\begin{array}{l}10 \\
108\end{array}$ & $\begin{array}{l}20 \\
58\end{array}$ & $\begin{array}{l}50 \\
28\end{array}$ & $\begin{array}{r}100 \\
18\end{array}$ \\
\hline $\begin{array}{r}1 \\
3 \\
7 \\
14 \\
30 \\
60 \\
90 \\
120 \\
183\end{array}$ & $\begin{array}{r}.1 \\
.1 \\
.2 \\
.9 \\
1.4 \\
3.6 \\
5.5 \\
6.8 \\
15\end{array}$ & $\begin{array}{r}0.0 \\
0.0 \\
0.0 \\
0.0 \\
.2 \\
.9 \\
1.7 \\
2.6 \\
7.3\end{array}$ & $\begin{array}{r}0.0 \\
0.0 \\
0.0 \\
0.0 \\
.1 \\
.4 \\
.8 \\
1.4 \\
5.4\end{array}$ & $\begin{array}{r}0.0 \\
0.0 \\
0.0 \\
0.0 \\
0.0 \\
.2 \\
.4 \\
.8 \\
4.3\end{array}$ & $\begin{array}{l}=- \\
=- \\
=- \\
=- \\
=- \\
=-\end{array}$ & $\begin{array}{l}-- \\
-- \\
-- \\
-- \\
-- \\
-- \\
--\end{array}$ \\
\hline
\end{tabular}

MAGNITUDE AND PROBABILITY OF ANNUAL HIGH FLOW BASED ON PERIOD OF RECORD 1971-85

\begin{tabular}{|c|c|c|c|c|c|c|}
\hline \multirow{2}{*}{$\begin{array}{l}\text { PERIOD } \\
\text { (CON- } \\
\text { SECU- } \\
\text { TIVE } \\
\text { DAYS) }\end{array}$} & \multicolumn{6}{|c|}{$\begin{array}{l}\text { DISCHARGE, IN FT }{ }^{3 / S} \text {, FOR INDICATED RECURRENCE } \\
\text { INTERVAL, IN YEARS, AND ANNUAL } \\
\text { EXCEEDANCE PROBABILI TY, IN PERCENT }\end{array}$} \\
\hline & ${ }^{2} 0^{2}$ & $\begin{array}{c}5 \\
208\end{array}$ & $\begin{array}{l}10 \\
108\end{array}$ & $\begin{array}{l}25 \\
48\end{array}$ & $\begin{array}{l}50 \\
28\end{array}$ & $\begin{array}{r}100 \\
18\end{array}$ \\
\hline $\begin{array}{r}1 \\
3 \\
7 \\
15 \\
30 \\
60 \\
90\end{array}$ & $\begin{array}{r}905 \\
550 \\
336 \\
216 \\
144 \\
94 \\
74\end{array}$ & $\begin{array}{r}4980 \\
2850 \\
1630 \\
907 \\
522 \\
283 \\
198\end{array}$ & $\begin{array}{r}12300 \\
6860 \\
3760 \\
1930 \\
1020 \\
505 \\
336\end{array}$ & $\begin{array}{r}33000 \\
17800 \\
9200 \\
4350 \\
2100 \\
940 \\
595\end{array}$ & $\begin{array}{l}-- \\
-- \\
-- \\
--\end{array}$ & $\begin{array}{l}=- \\
=- \\
=- \\
-- \\
--\end{array}$ \\
\hline
\end{tabular}

DURATION TABLE OF DAILY MEAN FLOW FOR PERIOD OF RECORD 1971-85 DISCBARGE, IN FT's, THAT WAS EQUALED OR EXCEEDED FOR INDICATED PERCENT OF TIME

\begin{tabular}{|c|c|c|c|c|c|c|c|c|c|c|c|c|c|c|}
\hline 58 & 108 & 158 & 208 & 258 & 308 & 408 & 508 & 608 & 708 & 758 & 808 & 858 & 908 & 958 \\
\hline 75 & 41 & 33 & 28 & 26 & 23 & 19 & 16 & 14 & 10 & 8.6 & 6.4 & 4.0 & 1.1 & .2 \\
\hline
\end{tabular}


RIO GRANDE BASIN

08405500 BLACK RIVER ABOVE MALAGA, NM

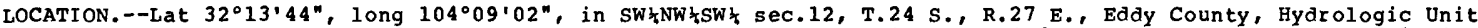
13060011 , on right bank $0.6 \mathrm{mi}$ upstream from Black River diversion dam, $4.6 \mathrm{mi}$ west of Malaga, and $7.1 \mathrm{mi}$ upstream from mouth. Mouth at Pecos River mile 436.3.

DRA INAGE AREA. $--343 \mathrm{mi}^{2}$.

PERIOD OF RECORD.--March to December 1940, December 1946 to current year.

REVISED RECORDS.--WSP 1632: 1948, 1949-50(P).

GAGE.--Water-stage recorder and concrete contro1. Elevation of gage is 3,070 ft above National Geodetic Vertical Datum of 1929, from topographic map. March to December 1940 water-stage recorder and Cippoletti weir at site $0.3 \mathrm{mi}$ downstream at different datum.

REMARKS.--Diversions and ground-water withdrawals for irrigation of about 1,000 acres, 1959 determination, upstream from station.

AVERAGE DISCHARGE. --38 years $(1948-85), 12.9 \mathrm{ft}^{3} / \mathrm{s}, 9,350 \mathrm{acre}-\mathrm{ft} / \mathrm{yr}^{2}$.

EXTREMES FOR PERIOD OF RECORD.--Maximum discharge, 74,600 ft $3 / \mathrm{s}$, Aug. 23, 1966, gage height, $21.7 \mathrm{ft}$, from floodmarks, from rating curve extended above $5,900 \mathrm{ft} / \mathrm{s}$ on basis of slope-area measurements at gage heights $12.60 \mathrm{ft}$ and $21.7 \mathrm{ft}$; minimum, $0.51 \mathrm{ft}^{3} / \mathrm{s}$, June 1,1983 .

The flood of Aug. 23, 1966, exceeded the previous maximum stage which occurred in 1908 by about 1.0 ft, from information by local resident.

EXTREMES OUTSIDE PERIOD OF RECORD.--Flood of Sept. 20 or 21,1941 , reached a stage of $19.0 \mathrm{ft}$, present site and datum, determined in $1947 \mathrm{from}$ well defined floodmarks, discharge, 33,000 ft $3 / \mathrm{s}$, from rating curve extended above $1,400 \mathrm{ft}^{3} / \mathrm{s}$ on basis of slope-area measurements at gage heights $8.41 \mathrm{ft}$ and $12.60 \mathrm{ft}$.

STATISTICAL SUMMARIES

MEAN MONTHLY AND MEAN ANNUAL DISCHARGES 1948-85

\begin{tabular}{|c|c|c|c|c|c|c|}
\hline MONTH & $\begin{array}{l}\text { MINI MUM } \\
\left(\mathrm{FT}^{3} / \mathrm{S}\right)\end{array}$ & $\begin{array}{c}\operatorname{MAXIMUM} \\
\left(\mathrm{FT}^{3} / \mathrm{S}\right)\end{array}$ & $\begin{array}{l}\text { MEAN } \\
\left(\mathrm{FT}^{3} / \mathrm{S}\right)\end{array}$ & $\begin{array}{c}\text { STAN- } \\
\text { DARD } \\
\text { DEVIA- } \\
\text { TION } \\
\text { (ET'/S) }\end{array}$ & $\begin{array}{l}\text { COEFFI- } \\
\text { CIENT OF } \\
\text { VARI- } \\
\text { ATION }\end{array}$ & $\begin{array}{c}\text { PERCENT } \\
\text { OF } \\
\text { ANNUAL } \\
\text { RUNOFF }\end{array}$ \\
\hline ОСТОВЕR & 2.5 & 80 & 13 & 19 & 1.39 & 8.7 \\
\hline NOVEMBER & 10 & 33 & $\log ^{8}$ & +30 & 57 & \\
\hline DECEMBER & 38 & 15 & 89 & 3 & 35 & 5.7 \\
\hline $\begin{array}{l}\text { JANUARY } \\
\text { JA }\end{array}$ & 2.8 & 18 & 9.7 & 3.6 & .37 & 5.8 \\
\hline FEB RUARY & 4.1 & 16 & 9.1 & 2.9 & .32 & 5.9 \\
\hline MARCH & 2.0 & 13 & 5.7 & 2.6 & .46 & 3.7 \\
\hline APR IL & 4.7 & 56 & 10 & 7.9 & .78 & 6.6 \\
\hline MAY & 4.3 & 106 & 13 & 17 & 1.32 & 8.3 \\
\hline JUNE & 2.8 & 60 & 13 & 13 & 1.02 & 8.5 \\
\hline JULY & 3.1 & 111 & 14 & 19 & 1.35 & 9.2 \\
\hline AUGUST & 3.3 & 553 & 28 & 89 & 3.20 & 18.0 \\
\hline SEPTEMBER & 3.4 & 121 & 21 & 26 & 1.27 & 13.4 \\
\hline ANNUAL & 6.8 & 58 & 13 & 8.5 & .66 & 100 \\
\hline
\end{tabular}

MAGNITUDE AND PROBABILITY OF ANNUAL LOW FLOW BASED ON PERIOD OF RECORD 1949-85

\begin{tabular}{|c|c|c|c|c|c|c|}
\hline \multirow{2}{*}{$\begin{array}{l}\text { PERIOD } \\
\text { (CON- } \\
\text { SECU- } \\
\text { TIVE } \\
\text { DAYS) }\end{array}$} & \multicolumn{6}{|c|}{$\begin{array}{l}\text { DISCHARGE, IN FT' }{ }^{3} / \mathrm{S} \text {, FOR INDICATED RECURRENCE } \\
\text { INTERVAL, IN YEARS, AND ANNUAL NON- } \\
\text { EXCEEDANCE PROBABIL ITY, IN PERCENT }\end{array}$} \\
\hline & $\begin{array}{c}2 \\
508\end{array}$ & $\begin{array}{c}5 \\
208\end{array}$ & $\begin{array}{l}10 \\
108\end{array}$ & $\begin{array}{l}20 \\
58\end{array}$ & $\begin{array}{l}50 \\
28\end{array}$ & $\begin{array}{r}100 \\
18\end{array}$ \\
\hline $\begin{array}{r}1 \\
3 \\
7 \\
14 \\
30 \\
60 \\
90 \\
120 \\
183\end{array}$ & $\begin{array}{l}2.3 \\
2.5 \\
2.8 \\
3.2 \\
3.8 \\
5.2 \\
5.8 \\
6.5 \\
7.9\end{array}$ & $\begin{array}{l}1.6 \\
1.7 \\
1.9 \\
2.2 \\
2.7 \\
3.9 \\
4.5 \\
5.1 \\
5.9\end{array}$ & $\begin{array}{l}1.3 \\
1.4 \\
1.6 \\
1.8 \\
2.2 \\
3.2 \\
3.9 \\
4.4 \\
5.2\end{array}$ & $\begin{array}{l}1.1 \\
1.2 \\
1.3 \\
1.5 \\
1.8 \\
2.7 \\
3.4 \\
3.9 \\
4.6\end{array}$ & $\begin{array}{l}.9 \\
1.0 \\
1.1 \\
1.2 \\
1.5 \\
2.2 \\
2.9 \\
3.4 \\
4.1\end{array}$ & $\begin{array}{l}\overline{-} \\
\overline{-} \\
\overline{-}\end{array}$ \\
\hline
\end{tabular}

MAGNITUDE AND PROBABILITY OF ANNUAL HIGH FLOW BASED ON PERIOD OF RECORD 1948-85

\begin{tabular}{|c|c|c|c|c|c|c|}
\hline \multirow{2}{*}{$\begin{array}{l}\text { PERIOD } \\
\text { (CON- } \\
\text { SECU- } \\
\text { TIVE } \\
\text { DAYS) }\end{array}$} & \multicolumn{6}{|c|}{$\begin{array}{l}\text { DISCHARGE, IN FT }{ }^{3} / S \text {, FOR INDICATED RECURRENCE } \\
\text { INTERVAL, IN YEAR, AND ANNUAL } \\
\text { EXCEEDANCE 'PROBABILITY, IN PERCENT }\end{array}$} \\
\hline & $\begin{array}{c}2 \\
508\end{array}$ & $\begin{array}{c}5 \\
208\end{array}$ & $\begin{array}{l}10 \\
108\end{array}$ & $\begin{array}{l}25 \\
48\end{array}$ & $\begin{array}{l}50 \\
28\end{array}$ & $\begin{array}{r}100 \\
18\end{array}$ \\
\hline $\begin{array}{r}1 \\
3 \\
7 \\
15 \\
30 \\
60 \\
90\end{array}$ & $\begin{array}{r}400 \\
185 \\
92 \\
51 \\
31 \\
21 \\
17\end{array}$ & $\begin{array}{r}1290 \\
558 \\
256 \\
126 \\
69 \\
41 \\
31\end{array}$ & $\begin{array}{r}2380 \\
1010 \\
451 \\
214 \\
113 \\
64 \\
46\end{array}$ & $\begin{array}{r}4570 \\
1930 \\
841 \\
391 \\
203 \\
109 \\
75\end{array}$ & $\begin{array}{r}6960 \\
2960 \\
1270 \\
590 \\
306 \\
159 \\
108\end{array}$ & $\begin{array}{l}-- \\
-- \\
-- \\
-- \\
-- \\
--\end{array}$ \\
\hline
\end{tabular}

DURATION TABLE OF DAILY MEAN FLOW FOR PERIOD OF RECORD 1948-85 DISCHARGE, IN FT"/S, THAT WAS EQUALED OR EXCEEDED FOR INDICATED PERCENT OF TIME

\begin{tabular}{|c|c|c|c|c|c|c|c|c|c|c|c|c|c|c|}
\hline 58 & 108 & 158 & 208 & 258 & 308 & 408 & 508 & 608 & 708 & 758 & 808 & 858 & 908 & 958 \\
\hline 17 & 14 & 13 & 11 & 10 & 9.4 & 8.3 & 7.3 & 6.5 & 5.7 & 5.3 & 4.9 & 4.4 & 3.8 & 3.0 \\
\hline
\end{tabular}


RIO GRANDE BASIN

08406500 PECOS RIVER NEAR MALAGA, NM

LOCATION, - Lat $32^{\circ} 12^{\prime} 26^{\prime \prime}$, long $104^{\circ} 01^{\prime} 22^{\prime \prime}$, in SWłNWłNEt sec.19, T.24 S., R.29 E., Bddy County, Hydrologic Unit 13060011, on right bank $3.1 \mathrm{mi}$ southeast of Malaga, $4.3 \mathrm{mi}$ downstream from Black River, and at mile 432.2 .

DRAINAGE AREA.--19,190 mi2, approximately (contributing area).

PERIOD OF RECORD,--May 1920 to current year. Monthly discharge only for some periods, published in wSP 1312.

REVISED RECORDS. - WSP 1632: 1925, 1932-37.

GAGE.--Water-stage recorder. Elevation of gage is 2,895.64 ft above National Geodetic vertical Datum of 1929 . May 1, 1920 to Mar. 24, 1949; at datum $3 \mathrm{ft}$ higher.

REMARKS. - Flow regulated by many reservoirs and diversion dams. Diversions and ground-water withdrawals upstream from station for irrigation of about 202,000 acres, 1959 determination. Harroun canal bypasses gage on left bank and irrigates approximately 1,000 acres adjacent to and downstream from gage. This bypass is not gaged.

AVERAGE DISCHARGE. -53 years $(1921-25,1938-85), 172 \mathrm{ft} / \mathrm{s}, 124,600$ acre-ft/yr

49 years $(1938-85), 166 \mathrm{ft}^{3 / \mathrm{s}}, 120,300$ acre-ft/yr.

EXTREMES FOR PERIOD OF RECORD. --Maximum discharge, 120,000 ft/s, Aug. 23, 1966, gage height, 42.1 ft, from floodmarks, from rating curve extended above $36,000 \mathrm{ft}^{3} / \mathrm{s}$ on basis of slope-area measurement of peak
flow; minimum, $3.7 \mathrm{ft} / \mathrm{s}$, Oct. 20,1976 .

EXTREMES OUTSIDE PERIOD OF RECORD.--A major flood occurred in 1904, discharge not determined. Flood of Aug. 7, 1916, reached a discharge of $70,000 \mathrm{ft} / \mathrm{s}$ at Carlsbad, $27 \mathrm{mi}$ upstream. Flood in September $1919 \mathrm{reached}$ a 8 tage of $29.4 \mathrm{ft}$, present datum, discharge, $40,400 \mathrm{ft}^{3} / \mathrm{s}$.

STATISTICAL SUMMARIES

MEAN MONTHLY AND MEAN ANNUAL DISCHARGES $\begin{array}{r}1921-25 \\ 1938-85\end{array}$

\begin{tabular}{|c|c|c|c|c|c|c|}
\hline MONTH & $\begin{array}{l}\text { MINIMUM } \\
(\mathrm{FT} \times / \mathrm{S})\end{array}$ & $\begin{array}{c}\text { MAXIMUM } \\
\left(\mathrm{FT}^{3} / \mathrm{S}\right)\end{array}$ & $\begin{array}{l}\text { MEAN } \\
\left(\mathrm{FT}^{3} / \mathrm{S}\right)\end{array}$ & $\begin{array}{c}\text { STAN- } \\
\text { DARD } \\
\text { DEVIA- } \\
\text { TION } \\
\left(\mathrm{FT}^{3} / \mathrm{S}\right)\end{array}$ & $\begin{array}{l}\text { COEFFI- } \\
\text { CIENT OF } \\
\text { VARI- } \\
\text { ATION }\end{array}$ & $\begin{array}{c}\text { PERCENT } \\
\text { OF } \\
\text { ANNUAL } \\
\text { RUNOFF }\end{array}$ \\
\hline $\begin{array}{l}\text { OCTOBER } \\
\text { NOVEMBER } \\
\text { DECEMBER } \\
\text { JANUARY } \\
\text { FEBRUARY } \\
\text { MARCB } \\
\text { APRIL } \\
\text { MAY } \\
\text { JUNE } \\
\text { JULY } \\
\text { AUGUST } \\
\text { SEPTEMBER }\end{array}$ & $\begin{array}{l}8.5 \\
7.8 \\
7.9 \\
11 \\
12 \\
9.4 \\
8.8 \\
7.9 \\
8.9 \\
6.7 \\
6.2 \\
8.3\end{array}$ & $\begin{array}{r}5302 \\
1338 \\
822 \\
738 \\
557 \\
380 \\
697 \\
6887 \\
6639 \\
1171 \\
4200 \\
6975\end{array}$ & $\begin{array}{r}315 \\
158 \\
132 \\
128 \\
108 \\
80 \\
64 \\
247 \\
312 \\
120 \\
211 \\
321\end{array}$ & $\begin{array}{r}822 \\
241 \\
147 \\
138 \\
108 \\
78 \\
97 \\
942 \\
1056 \\
242 \\
609 \\
976\end{array}$ & $\begin{array}{l}2.61 \\
1.53 \\
1.11 \\
1.08 \\
1.00 \\
.97 \\
1.53 \\
3.81 \\
3.38 \\
2.02 \\
2.89 \\
3.04\end{array}$ & $\begin{array}{r}14.3 \\
7.2 \\
6.0 \\
5.8 \\
4.9 \\
3.6 \\
2.9 \\
11.3 \\
14.2 \\
5.5 \\
9.6 \\
14.6\end{array}$ \\
\hline ANNUAL & 17 & 1652 & 172 & 272 & 1.58 & 100 \\
\hline
\end{tabular}

MAGNITUDE AND PROBABILITY OF ANNUAL LOW FLON BASED ON PERIOD OF RECORD 1922-25, 1938-85

\begin{tabular}{|c|c|c|c|c|c|c|}
\hline \multirow{2}{*}{$\begin{array}{c}\text { PERIOD } \\
\text { (CON- } \\
\text { SECU- } \\
\text { TIVE } \\
\text { DAYS) }\end{array}$} & \multicolumn{6}{|c|}{$\begin{array}{l}\text { DISCHARGE, IN } 1 / \text {, FOR INDICATED RECURRENCE } \\
\text { INTERVAL, IN YEARS, AND ANNUAL, NON- } \\
\text { EXCEEDANCE PROBABILITY, IN PERCENT }\end{array}$} \\
\hline & $\begin{array}{c}2 \\
508\end{array}$ & $\begin{array}{c}5 \\
208\end{array}$ & $\begin{array}{l}10 \\
108\end{array}$ & $\begin{array}{l}20 \\
58\end{array}$ & $\begin{array}{l}50 \\
28\end{array}$ & $\begin{array}{r}100 \\
18\end{array}$ \\
\hline $\begin{array}{r}1 \\
3 \\
7 \\
14 \\
30 \\
60 \\
90 \\
120 \\
183\end{array}$ & $\begin{array}{l}17 \\
18 \\
20 \\
21 \\
24 \\
30 \\
35 \\
41 \\
56\end{array}$ & $\begin{array}{l}9.2 \\
9.5 \\
10 \\
11 \\
12 \\
14 \\
16 \\
19 \\
26\end{array}$ & $\begin{array}{r}6.9 \\
7.2 \\
7.6 \\
7.9 \\
8.5 \\
9.8 \\
11 \\
13 \\
19\end{array}$ & $\begin{array}{c}5.5 \\
5.7 \\
6.0 \\
6.3 \\
6.6 \\
7.5 \\
8.3 \\
10 \\
14\end{array}$ & $\begin{array}{r}4.4 \\
4.6 \\
4.8 \\
4.9 \\
5.0 \\
5.7 \\
6.2 \\
7.6 \\
11\end{array}$ & \\
\hline
\end{tabular}

MAGNITUDE AND PROBABILITY OF ANNUAL, HIGH FLON BASED ON PERIOD OF RECORD 1921-25, 1938-85

\begin{tabular}{|c|c|c|c|c|c|c|}
\hline \multirow{2}{*}{$\begin{array}{l}\text { PERIOD } \\
\text { (CON- } \\
\text { SECU- } \\
\text { TIVE } \\
\text { DAYS) }\end{array}$} & \multicolumn{6}{|c|}{$\begin{array}{l}\text { DISCHARGE, IN FT'/S, FOR INDICATED RECURRENC } \\
\text { INTERVAL, IN YEARS, AND ANNUAL } \\
\text { EXCEEDANCE PROBABILITY, IN PERCENT }\end{array}$} \\
\hline & $\begin{array}{c}2 \\
508\end{array}$ & $\begin{array}{c}5 \\
208\end{array}$ & $\begin{array}{l}10 \\
108\end{array}$ & $\begin{array}{l}25 \\
48\end{array}$ & $\begin{array}{l}50 \\
28\end{array}$ & $\begin{array}{r}100 \\
18\end{array}$ \\
\hline $\begin{array}{r}1 \\
3 \\
7 \\
15 \\
30 \\
60 \\
90\end{array}$ & $\begin{array}{r}1860 \\
1080 \\
643 \\
414 \\
292 \\
204 \\
170\end{array}$ & $\begin{array}{r}7360 \\
4470 \\
2640 \\
1520 \\
944 \\
597 \\
466\end{array}$ & $\begin{array}{r}15000 \\
9550 \\
5760 \\
3160 \\
1840 \\
1120 \\
845\end{array}$ & $\begin{array}{r}32100 \\
21800 \\
13600 \\
7150 \\
3920 \\
2290 \\
1680\end{array}$ & $\begin{array}{r}52200 \\
37400 \\
24200 \\
12400 \\
6540 \\
3760 \\
2690\end{array}$ & $\begin{array}{r}80900 \\
61100 \\
41100 \\
20500 \\
10500 \\
5970 \\
4200\end{array}$ \\
\hline
\end{tabular}

DURATION TABLE OF DAILY MEAN FLOW FOR PERIOD OF RECORD 1921-25, 1938-85 DISCHARGE, IN FT $3 / \mathrm{S}$, THAT WAS EOUALED OR EXCEEDED FOR INDICATED PERCENT OF TIME

\begin{tabular}{|c|c|c|c|c|c|c|c|c|c|c|c|c|c|c|}
\hline 58 & 108 & 158 & 208 & 258 & 308 & 408 & 508 & 608 & 708 & 758 & 808 & 858 & 908 & 958 \\
\hline 368 & 245 & 184 & 146 & 125 & 104 & 73 & 54 & 40 & 29 & 25 & 21 & 18 & 14 & 11 \\
\hline
\end{tabular}


RIO GRANDE BASIN

08407000 PECOS RIVER AT PIERCE CANYON CROSSING, NEAR MALAGA, NM

LOCATION.--Lat $32^{\circ} 11^{\prime} 19^{n}$, long $103^{\circ} 58^{\prime} 43^{n}$, in SWhSW $h_{\text {NW }}$ sec.27, T.24 S., R. 29 E. Eddy County, Hydrologic Unit 13060011 , on right bank $550 \mathrm{ft}$ upstream from Pierce Canyon Crossing, and $6.0 \mathrm{mi}$ southeast of Malaga, and at mile 13060011
425.7 .

DRAINAGE AREA.--19,260 $\mathrm{mi}^{2}$, approximately (contributing area).

PERIOD OF RECORD.--July 1938 to September 1941, August 1951 to current year.

REVISED RECORDS.--WSP 898: 1938 (M). WSP 1712: 1959.

GAGE.--Water-stage recorder. Elevation of gage is $2,889.18 \mathrm{ft}$ above National Geodetic Vertical Datum of 1929 . July 1938 to Sept. 1941 at datum $1.19 \mathrm{ft}$ higher.

REMARKs.--Flow requlated by many reservoirs and diversion dams. Diversions and ground-water withdrawals upstream from station for irrigation of about 202,000 acres, 1959 determination.

AVERAGE DISCHARGE. --36 years $(1939-40,1952-85), 85 \mathrm{ft}^{3} / \mathrm{s}, 61,580 \mathrm{acre}-\mathrm{ft} / \mathrm{yr}$.

EXTREMES FOR PERIOD OF RECORD.--Maximum daily discharge determined, 65,000 ft/s, Aug. 23, 1966, maximum gage height, $31.6 \mathrm{ft}$, Aug. 23, i966, from floodmarks; minimum discharge, $0.54 \mathrm{ft}^{3} / \mathrm{s}, \mathrm{May} 30,1965$.

STATISTICAL SUMMARIES

MEAN MONTHLY AND MEAN ANNUAL DISChARGES $\begin{array}{r}1939-40, \\ 1952-85\end{array}$

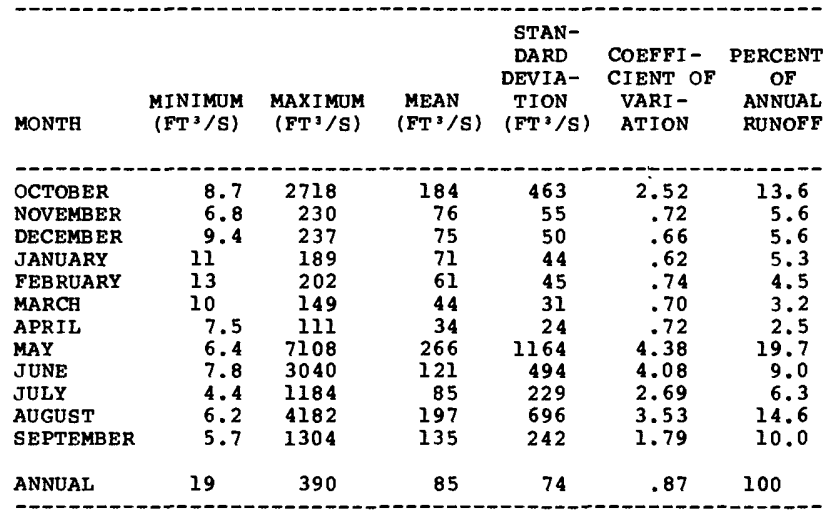

MAGNITUDE AND PROBABILITY OF ANNUAL LOW FLOW BASED ON PERIOD OF RECORD 1940-41, 1953-85

\begin{tabular}{|c|c|c|c|c|c|c|}
\hline \multirow{2}{*}{$\begin{array}{l}\text { PERIOD } \\
\text { (CON- } \\
\text { SECU- } \\
\text { TIVE } \\
\text { DAYS) }\end{array}$} & \multicolumn{6}{|c|}{$\begin{array}{l}\text { DISCHARGE, IN FT }{ }^{3} / S \text {, FOR INDICATED RECURRENCE } \\
\text { INTERVAL, IN YEARS, AND ANNUAL NON- } \\
\text { EXCEEDANCE PROBABILITY, IN PERCENT }\end{array}$} \\
\hline & $\begin{array}{c}2 \\
508\end{array}$ & $\begin{array}{c}5 \\
208\end{array}$ & $\begin{array}{l}10 \\
108\end{array}$ & 20 & $\begin{array}{l}50 \\
28\end{array}$ & $\begin{array}{r}100 \\
18\end{array}$ \\
\hline $\begin{array}{r}1 \\
3 \\
7 \\
14 \\
30 \\
60 \\
90 \\
120 \\
183\end{array}$ & $\begin{array}{l}9.1 \\
9.8 \\
11 \\
12 \\
14 \\
17 \\
21 \\
25 \\
40\end{array}$ & $\begin{array}{r}4.5 \\
4.9 \\
5.5 \\
6.6 \\
7.8 \\
10 \\
12 \\
15 \\
22\end{array}$ & $\begin{array}{r}3.1 \\
3.4 \\
3.9 \\
4.9 \\
5.9 \\
7.9 \\
9.0 \\
11 \\
16\end{array}$ & $\begin{array}{r}2.3 \\
2.5 \\
2.9 \\
3.8 \\
4.8 \\
6.6 \\
7.2 \\
9.5 \\
13\end{array}$ & $\begin{array}{l}1.6 \\
1.8 \\
2.2 \\
3.0 \\
3.8 \\
5.4 \\
5.7 \\
7.9 \\
9.7\end{array}$ & 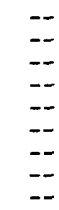 \\
\hline
\end{tabular}

MAGNITUDE AND PROBABILITY OF ANNUAL HIGH FLON BASED ON PERIOD OF RECORD 1939-40, 1952-85

\begin{tabular}{|c|c|c|c|c|c|c|}
\hline \multirow{2}{*}{$\begin{array}{l}\text { PERIOD } \\
\text { (CON- } \\
\text { SECU- } \\
\text { TIVE } \\
\text { DAYS) }\end{array}$} & \multicolumn{6}{|c|}{$\begin{array}{l}\text { DISCHARGE, IN FT } 3 / \mathrm{S} \text {, FOR INDICATED RECURRENCE } \\
\text { INTERVAL, IN YEARS, AND ANNUAL } \\
\text { EXCEEDANCE PROBABILITY, IN PERCENT }\end{array}$} \\
\hline & $\stackrel{2}{508}$ & $\begin{array}{c}5 \\
208\end{array}$ & $\begin{array}{l}10 \\
108\end{array}$ & $\begin{array}{l}25 \\
48\end{array}$ & $\begin{array}{l}50 \\
28\end{array}$ & $\begin{array}{r}100 \\
18\end{array}$ \\
\hline $\begin{array}{r}1 \\
3 \\
7 \\
15 \\
30 \\
60 \\
90\end{array}$ & $\begin{array}{r}1200 \\
717 \\
429 \\
273 \\
192 \\
135 \\
115\end{array}$ & $\begin{array}{r}5230 \\
3070 \\
1730 \\
970 \\
584 \\
349 \\
267\end{array}$ & $\begin{array}{r}11800 \\
6930 \\
3850 \\
2030 \\
1130 \\
6117 \\
445\end{array}$ & $\begin{array}{r}29100 \\
17200 \\
9520 \\
4740 \\
2400 \\
1200 \\
809\end{array}$ & $\begin{array}{r}53100 \\
31800 \\
17600 \\
8460 \\
4060 \\
1900 \\
1230\end{array}$ & $\begin{array}{l}-- \\
-- \\
-- \\
-- \\
--\end{array}$ \\
\hline
\end{tabular}

DURATION TABLE OF DAILY MEAN FLOW FOR PERIOD OF RECORD 1939-40, 1952-85 DISCHARGE, IN $\mathrm{FT}^{3} / \mathrm{S}$, THAT WAS EQUALED OR EXCEEDED FOR INDICATED PERCENT OF TIME

\begin{tabular}{|c|c|c|c|c|c|c|c|c|c|c|c|c|c|c|}
\hline 58 & 108 & 158 & 208 & 258 & $30 \%$ & 408 & 508 & 608 & 708 & 758 & 808 & 858 & 908 & 958 \\
\hline 170 & 115 & 87 & 72 & 64 & 57 & 46 & 37 & 29 & 23 & 20 & 18 & 15 & 12 & 8. \\
\hline
\end{tabular}


08407500 PECOS RIVER AT RED BLUFF, NM

(National stream-quality accounting network station)

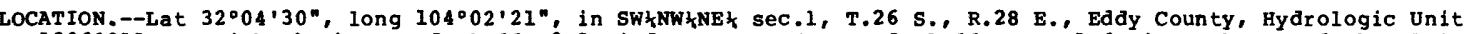
13060011 , on right bank at Red Bluff, 0.2 mi downstream from Red Bluff Draw, 1.6 mi northwest of the El Paso Natural Gas (Pecos River) compressor station, $5.2 \mathrm{mi}$ north of the New Mexico-Texas State 1 ine, $5.5 \mathrm{mi}$ upstream from Delaware River, and at mile 411.2 .

DRAINAGE AREA.--19,540 $\mathrm{mi}^{2}$, approximately (contributing area).

PERIOD OF RECORD.--october 1937 to current year.

GAGE.--Water-stage recorder. Elevation of gage is 2,850.05 ft above National Geodetic vertical Datum of 1929 .

REMARKS.--Flow regulated by many reservoirs and diversion dams. Diversions and ground-water withdrawals upstream from station for irrigation of about 202,000 acres, 1959 determination.

AVERAGE DISCHARGE. --48 years $(1938-85), 161 \mathrm{ft} / \mathrm{s}, 116,600$ acre-ft/yr.

EXTREMES FOR PERIOD OF RECORD.--Maximum discharge, 111,000 $\mathrm{ft}^{3} / \mathrm{s}$, Aug. 23, 1966, gage height, $33.32 \mathrm{ft}$, from rating curve extended above $32,000 \mathrm{ft} / \mathrm{s}$ on basis of slope-area measurement of peak flow; minimum, $0.19 \mathrm{ft} / \mathrm{s}$, Aug. 1 , 1966 .

EXTREMES OUTSIDE PERIOD OF RECORD.--Flood in October 1904 reached a stage of $28.0 \mathrm{ft}$, from information by Panhandle and Santa Fe Railway Co. (For dates of other historical floods see stations 08404000, 08406500.)

STATISTICAL SUMMARIES

MEAN MONTHLY AND MEAN ANNUAL DISCHARGES 1938-85

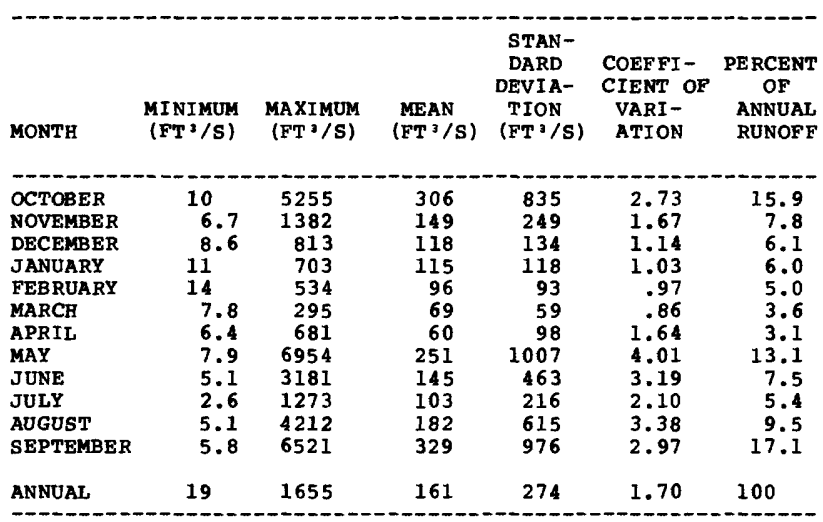

MAGNITUDE AND PROBABILITY OF ANNUAL LOW FLOW BASED ON PERIOD OF RECORD 1939-85

\begin{tabular}{|c|c|c|c|c|c|c|}
\hline \multirow{2}{*}{$\begin{array}{l}\text { PERIOD } \\
\text { (CON- } \\
\text { SECU- } \\
\text { TIVE } \\
\text { DAYS) }\end{array}$} & \multicolumn{6}{|c|}{$\begin{array}{l}\text { DISCHARGE, IN FT }{ }^{3 / S} \text {, FOR INDICATED RECURRENCE } \\
\text { INTERVAL, IN YEARS, AND ANNUAL NON- } \\
\text { EXCE EDANCE PROBABIL ITY, IN PERCENT }\end{array}$} \\
\hline & $\begin{array}{c}2 \\
508\end{array}$ & $\begin{array}{c}5 \\
208\end{array}$ & $\begin{array}{l}10 \\
108\end{array}$ & $\begin{array}{l}20 \\
58\end{array}$ & $\begin{array}{l}50 \\
28\end{array}$ & $\begin{array}{r}100 \\
18\end{array}$ \\
\hline $\begin{array}{r}1 \\
3 \\
7 \\
14 \\
30 \\
60 \\
90 \\
120 \\
183\end{array}$ & $\begin{array}{l}12 \\
14 \\
16 \\
17 \\
20 \\
26 \\
31 \\
37 \\
53\end{array}$ & $\begin{array}{r}3.7 \\
4.3 \\
5.3 \\
6.5 \\
8.2 \\
12 \\
14 \\
18 \\
26\end{array}$ & $\begin{array}{r}1.8 \\
2.1 \\
2.7 \\
3.7 \\
5.1 \\
8.0 \\
9.7 \\
12 \\
19\end{array}$ & $\begin{array}{r}.9 \\
1.1 \\
1.5 \\
2.3 \\
3.5 \\
5.9 \\
7.2 \\
9.5 \\
16\end{array}$ & $\begin{array}{r}.4 \\
.5 \\
1.8 \\
2.3 \\
4.3 \\
5.2 \\
7.2 \\
13\end{array}$ & $\begin{array}{r}.2 \\
.3 \\
.5 \\
.9 \\
1.6 \\
3.4 \\
4.3 \\
6.0 \\
11\end{array}$ \\
\hline
\end{tabular}

MAGNITUDE AND PROBABILITY OF ANNUAL HIGH FLOW BASED ON PERIOD OF RECORD 1938-85

\begin{tabular}{|c|c|c|c|c|c|c|}
\hline \multirow{2}{*}{$\begin{array}{l}\text { PERIOD } \\
\text { (CON- } \\
\text { SECU- } \\
\text { TIVE } \\
\text { DAYS) }\end{array}$} & \multicolumn{6}{|c|}{$\begin{array}{l}\text { DISCHARGE, IN FT } 3 / S^{\prime} \text { FOR INDICATED RECURRENCE } \\
\text { INTERVAL, IN YEARS, AND ANNUAL } \\
\text { EXCEEDANCE PROBABILITY, IN PERCENT }\end{array}$} \\
\hline & $\begin{array}{c}2 \\
508\end{array}$ & $\begin{array}{c}5 \\
208\end{array}$ & $\begin{array}{l}10 \\
108\end{array}$ & $\begin{array}{l}25 \\
48\end{array}$ & $\begin{array}{l}50 \\
28\end{array}$ & $\begin{array}{r}100 \\
18\end{array}$ \\
\hline $\begin{array}{r}1 \\
3 \\
7 \\
15 \\
30 \\
60 \\
90\end{array}$ & $\begin{array}{r}1760 \\
1130 \\
674 \\
413 \\
278 \\
190 \\
157\end{array}$ & $\begin{array}{r}6460 \\
4230 \\
2490 \\
1410 \\
864 \\
537 \\
416\end{array}$ & $\begin{array}{r}13100 \\
8750 \\
5210 \\
2870 \\
1680 \\
999 \\
747\end{array}$ & $\begin{array}{r}28300 \\
19600 \\
12000 \\
6430 \\
3620 \\
2060 \\
1490\end{array}$ & $\begin{array}{r}47100 \\
33400 \\
20900 \\
11100 \\
6130 \\
3410 \\
2400\end{array}$ & $\begin{array}{r}75100 \\
54800 \\
35300 \\
18600 \\
10100 \\
5490 \\
3780\end{array}$ \\
\hline
\end{tabular}

DURATION TABLE OF DAILY MEAN FLOW FOR PERIOD OF RECORD 1938-85 DISCHARGE, IN FT $3 / 5$, THAT WAS EQUALED OR EXCEEDED FOR INDICATED PERCENT OF TIME

\begin{tabular}{|c|c|c|c|c|c|c|c|c|c|c|c|c|c|c|}
\hline 58 & 108 & 158 & 208 & 258 & 308 & 408 & 508 & 608 & 708 & 758 & 808 & 858 & 908 & 958 \\
\hline 335 & 212 & 163 & 134 & 110 & 91 & 69 & 53 & 40 & 30 & 26 & 21 & 17 & 13 & 8.5 \\
\hline
\end{tabular}


RIO GRANDE BASIN

08408500 DELAWARE RIVER NEAR RED BLUFF, NM

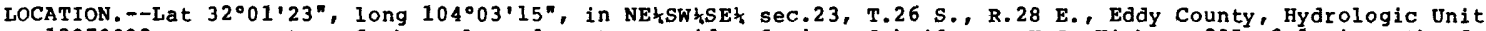
13070002 , near center of channel on downstream side of pier of bridge on U.S. Highway 285 , $2.1 \mathrm{mi}$ north of the New Mexico-Texas state line, $3.6 \mathrm{mi}$ southwest of Red Bluff, $3.7 \mathrm{mi}$ upstream from mouth and $14 \mathrm{mi}$ south of

Malaga. Mouth at Pecos River mile 405.6 .

DRAINAGE AREA. $--689 \mathrm{mi}^{2}$.

PERIOD OF RECORD,--April 1912 to September 1913, May 1914 to June 1915, october 1937 to current year. Published as "near Malaga" 1912-13, and as "near Angeles, Tex." 1914-15.

GAGE.-Water-stage recorder. Elevation of gage is 2,900.66 ft above National Geodetic Vertical Datum of 1929 (U.S. Boundary Commission post). Prior to May 1914, at site $3.0 \mathrm{mi}$ upstream at different datum. May 1914 to June 1915 at site $2.5 \mathrm{mi}$ downstream at different datum.

REMARKS.--One small upstream diversion.

AVERAGE DISCHARGE. -48 years $(1938-85), 12.9 \mathrm{ft}^{3} / \mathrm{s}, 9,350 \mathrm{acre}-\mathrm{ft} / \mathrm{yr}$.

EXTREMES FOR PERIOD OF RECORD.--Maximum discharge, 81,400 ft $/ \mathrm{s}$, Oct. 2, 1955, gage height, $27.0 \mathrm{ft}, \mathrm{from}$

floodmarks, from rating curve extended above $6,500 \mathrm{ft} / \mathrm{s}$ on basis of slope-area measurements at gage heights

$12.84 \mathrm{ft}$, $17.55 \mathrm{ft}$, and $27.0 \mathrm{ft}$; no flow many days most years.

Maximum discharge since at least 1911 is that of oct. 2, 1955

STATISTICAL SUMMARIES

MEAN MONTHLY AND MEAN ANNUAL DISCHARGES 1938-85

\begin{tabular}{|c|c|c|c|c|c|c|}
\hline MONTH & $\begin{array}{l}\text { MINIMUM } \\
\left(\mathrm{FT}^{3} / \mathrm{S}\right)\end{array}$ & $\begin{array}{l}\text { MAXI MUM } \\
(\mathrm{FT} \cdot / \mathrm{S})\end{array}$ & $\begin{array}{l}\text { MEAN } \\
\left(\mathrm{FT}^{3} / \mathrm{S}\right)\end{array}$ & $\begin{array}{l}\text { STAN- } \\
\text { DARD } \\
\text { DEVIA- } \\
\text { TION } \\
\left(\mathrm{FT}^{3} / \mathrm{S}\right)\end{array}$ & $\begin{array}{l}\text { COEFFI - } \\
\text { CIENT OF } \\
\text { VARI - } \\
\text { ATION }\end{array}$ & $\begin{array}{c}\text { PERCENT } \\
\text { OF } \\
\text { ANNUAL } \\
\text { RUNOFF }\end{array}$ \\
\hline $\begin{array}{l}\text { OCTOBER } \\
\text { NOVEMBER } \\
\text { DECEMBER } \\
\text { JANUARY } \\
\text { FEBRUARY } \\
\text { MARCH } \\
\text { APRII } \\
\text { MAY } \\
\text { JUNE } \\
\text { JULY } \\
\text { AUGUST } \\
\text { SEPTEMBER }\end{array}$ & $\begin{array}{r}0.0 \\
0.0 \\
.2 \\
.4 \\
.1 \\
.7 \\
.2 \\
0.0 \\
0.0 \\
0.0 \\
0.0 \\
0.0\end{array}$ & $\begin{array}{c}748 \\
19 \\
7.8 \\
7.6 \\
7.0 \\
6.7 \\
135 \\
233 \\
281 \\
166 \\
326 \\
303\end{array}$ & $\begin{array}{l}34 \\
3.6 \\
3.1 \\
3.2 \\
3.0 \\
2.6 \\
6.4 \\
11 \\
20 \\
16 \\
26 \\
26\end{array}$ & $\begin{array}{r}115 \\
3.1 \\
1.6 \\
1.6 \\
1.6 \\
1.5 \\
20 \\
34 \\
45 \\
28 \\
55 \\
49\end{array}$ & $\begin{array}{r}3.37 \\
.86 \\
.51 \\
.51 \\
.52 \\
.56 \\
3.10 \\
3.10 \\
2.29 \\
1.71 \\
2.17 \\
1.90\end{array}$ & $\begin{array}{r}22.2 \\
2.3 \\
2.0 \\
2.1 \\
2.0 \\
1.7 \\
4.1 \\
7.1 \\
12.7 \\
10.6 \\
16.6 \\
16.6\end{array}$ \\
\hline ANNUAL & 1.9 & 66 & 13 & 13 & .98 & 100 \\
\hline
\end{tabular}

MAGNITUDE AND PROBABILITY OF ANNUAL LOW FLOW BASED ON PERIOD OF RECORD 1939-85

\begin{tabular}{|c|c|c|c|c|c|c|}
\hline \multirow{2}{*}{$\begin{array}{l}\text { PERIOD } \\
\text { (CON- } \\
\text { SECU- } \\
\text { TIVE } \\
\text { DAYS) }\end{array}$} & \multicolumn{6}{|c|}{$\begin{array}{l}\text { DISCHARGE, IN FT }{ }^{3} / \mathrm{S} \text {, FOR INDICATED RECURRENCE } \\
\text { INTERVAL, IN YEARS, AND ANNUAL NON- } \\
\text { EXCEEDANCE PROBABILITY, IN PERCENT }\end{array}$} \\
\hline & $\begin{array}{c}2 \\
508\end{array}$ & $\begin{array}{c}5 \\
208\end{array}$ & $\begin{array}{l}10 \\
108\end{array}$ & $\begin{array}{l}20 \\
58\end{array}$ & $\begin{array}{l}50 \\
28\end{array}$ & $\begin{array}{r}100 \\
18\end{array}$ \\
\hline $\begin{array}{r}1 \\
3 \\
7 \\
14 \\
30 \\
60 \\
90 \\
120 \\
183\end{array}$ & $\begin{array}{r}0.0 \\
0.0 \\
0.0 \\
0.0 \\
.2 \\
.9 \\
1.5 \\
1.9 \\
3.3\end{array}$ & $\begin{array}{r}0.0 \\
0.0 \\
0.0 \\
0.0 \\
0.0 \\
.2 \\
.5 \\
.9 \\
1.6\end{array}$ & $\begin{array}{r}0.0 \\
0.0 \\
0.0 \\
0.0 \\
0.0 \\
.1 \\
.3 \\
.6 \\
1.1\end{array}$ & $\begin{array}{l}0.0 \\
0.0 \\
0.0 \\
0.0 \\
0.0 \\
0.0 \\
.1 \\
.4 \\
.8\end{array}$ & $\begin{array}{r}0.0 \\
0.0 \\
0.0 \\
0.0 \\
0.0 \\
0.0 \\
0.0 \\
.3 \\
.5\end{array}$ & $\begin{array}{r}0.0 \\
0.0 \\
0.0 \\
0.0 \\
0.0 \\
0.0 \\
0.0 \\
.2 \\
.4\end{array}$ \\
\hline
\end{tabular}

MAGNITUDE AND PROBABILITY OF ANNUAL HIGH FLON BASED ON PERIOD OF RECORD 1938-85

\begin{tabular}{|c|c|c|c|c|c|c|}
\hline \multirow{2}{*}{$\begin{array}{l}\text { PERIOD } \\
\text { (CON- } \\
\text { SECU- } \\
\text { TIVE } \\
\text { DAYS) }\end{array}$} & \multicolumn{6}{|c|}{$\begin{array}{l}\text { DISCHARGE, IN FT'/S, FOR INDICATED RECURRENC } \\
\text { INTERVAL, IN YEAR,, AND ANNUAL } \\
\text { EXCEEDANCE PROBABILITY, IN PERCENT }\end{array}$} \\
\hline & $\begin{array}{c}2 \\
508\end{array}$ & $\begin{array}{c}5 \\
208\end{array}$ & $\begin{array}{l}10 \\
108\end{array}$ & $\begin{array}{l}25 \\
48\end{array}$ & $\begin{array}{l}50 \\
28\end{array}$ & $\begin{array}{r}100 \\
18\end{array}$ \\
\hline $\begin{array}{r}1 \\
3 \\
7 \\
15 \\
30 \\
60 \\
90\end{array}$ & $\begin{array}{r}717 \\
329 \\
168 \\
89 \\
51 \\
31 \\
22\end{array}$ & $\begin{array}{r}2220 \\
973 \\
473 \\
241 \\
130 \\
75 \\
53\end{array}$ & $\begin{array}{r}4180 \\
1770 \\
831 \\
413 \\
217 \\
120 \\
86\end{array}$ & $\begin{array}{r}8460 \\
3450 \\
1540 \\
745 \\
385 \\
201 \\
144\end{array}$ & $\begin{array}{r}13600 \\
5390 \\
2320 \\
1100 \\
562 \\
282 \\
204\end{array}$ & $\begin{array}{r}21100 \\
8130 \\
3380 \\
1570 \\
797 \\
384 \\
280\end{array}$ \\
\hline
\end{tabular}

DURATION TABLE OF DAILY MEAN FLOW FOR PERIOD OF RECORD 1938-85 DISCHARGE, IN FT $3 / S$, THAT WAS EQUALED OR EXCEEDED FOR INDICATED PERCENT OF TIME

\begin{tabular}{|c|c|c|c|c|c|c|c|c|c|c|c|c|c|c|}
\hline $5 \%$ & 108 & 158 & 208 & 258 & 308 & 408 & 508 & 608 & 708 & 758 & 808 & 858 & 908 & 958 \\
\hline 18 & 7.1 & 5.7 & 4.6 & 4.0 & 3.4 & 2.8 & 2.2 & 1.7 & 1.2 & .8 & .5 & .2 & 0.0 & 0. \\
\hline
\end{tabular}


MIMBRES RIVER BASIN

08477000 MIMBRES RIVER NEAR MIMBRES, NM

LOCATION.--Lat $32^{\circ} 52^{\prime} 28^{\prime \prime}$, long $107^{\circ} 59^{\prime} 05^{\prime \prime}$, in SElNW sec.33, T.16 S., R.11 W., Grant County, Hydrologic Unit

13030202, on left bank $0.7 \mathrm{mi}$ downstream from Bear Canyon, $1.5 \mathrm{mi}$ northwest of Mimbres, and at mile 74.8 .

DRAINAGE AREA. $--152 \mathrm{mi}^{2}$.

PERIOD OF RECORD.--June 1921 to September 1930 (fragmentary), october 1930 to September 1976 (destroyed by flood of September 1976). Monthly discharge only for some periods, published in wSP 1312.

REVISED RECORDS.--WSP 1282: Drainage area, WSP 1512: 1931, 1933(M), 1935(M), 1938, 1939-40(M), 1941, 1942-43(M), $1944,1945(\mathrm{M}), 1946,1947(\mathrm{M})$.

GAGE.--Water-stage recorder. Datum of gage is 5,972 ft above mean sea level. Prior to Sept.12, 1923 , at site 10 $\mathrm{ft}$ downstream at datum $0.3 \mathrm{ft}$ higher. Sept. 12, 1923 to Jan. 17, 1934, at datum of $0.1 \mathrm{ft}$ lower.

REMARKS.--Some regulation by Bear Canyon Reservoir $1.3 \mathrm{mi}$ upstream, capacity, 700 acre-ft. Diversions for irrigation of about 300 acres above station.

AVERAGE DISCHARGE. --46 years (water years 1931-76), $11.2 \mathrm{ft} / \mathrm{s}, 8,110$ acre- $\mathrm{ft} / \mathrm{yr}$.

EXTREMES FOR PERIOD OF RECORD.--Maximum discharge, 3,370 ft $3 / \mathrm{s}$ oct. 20, 1972 , gage height, $7.49 \mathrm{ft}$, from rating curve extended above $600 \mathrm{ft} / \mathrm{s}$ on basis of slope-area measurements at gage heights $6.20 \mathrm{ft}$ and $7.49 \mathrm{ft}$; minimum, $0.7 \mathrm{ft}^{3} / \mathrm{s}$ Aug. 10,1951 .

STATISTICAL SUMMARIES

MEAN MONTHLY AND MEAN ANNUAI. DISCHARGES 1931-76

\begin{tabular}{|c|c|c|c|c|c|c|}
\hline MONTH & $\begin{array}{l}\text { MINIMUM } \\
\left(\mathrm{FT}^{3} / \mathrm{S}\right)\end{array}$ & $\begin{array}{c}\text { MAXIMUM } \\
\left(\mathrm{FT}^{3} / \mathrm{S}\right)\end{array}$ & $\begin{array}{l}\text { MEAN } \\
\left(F T^{3} / S\right)\end{array}$ & $\begin{array}{c}\text { STAN- } \\
\text { DARD } \\
\text { DEVIA- } \\
\text { TION } \\
\left(\mathrm{FT}^{3} / \mathrm{S}\right)\end{array}$ & $\begin{array}{l}\text { COEFFI- } \\
\text { CIENT OF } \\
\text { VARI- } \\
\text { ATION }\end{array}$ & $\begin{array}{c}\text { PERCENT } \\
\text { OF } \\
\text { ANNUAL } \\
\text { RUNOFF }\end{array}$ \\
\hline \multicolumn{6}{|l|}{$--1-1-1$} & 7.5 \\
\hline NOVEMBER & 2.1 & $\begin{array}{r}111 \\
25\end{array}$ & 7.2 & 3.9 & .55 & 5.3 \\
\hline DECEMBE R & 2.0 & 66 & 8.1 & 9.1 & 1.12 & 6.0 \\
\hline JANUARY & 1.9 & 52 & 8.6 & 9.2 & 1.06 & 6.4 \\
\hline FEBRUARY & 1.8 & 63 & 12 & 15 & 1.21 & 8.9 \\
\hline MARCH & 1.9 & 109 & 21 & 30 & 1.43 & 15.4 \\
\hline APRIL & 1.8 & 93 & 19 & 24 & 1.27 & 13.7 \\
\hline MAY & 1.9 & 66 & 11 & 14 & 1.27 & 8.3 \\
\hline JUNE & 1.7 & 12 & 5.5 & 3.0 & .55 & 4.1 \\
\hline JULY & 1.6 & 14 & 6.8 & 3.5 & .52 & 5.0 \\
\hline AUGUST & 2.4 & 49 & 13 & 12 & .91 & 9.5 \\
\hline SEPTEMBER & 1.5 & 105 & 14 & 17 & 1.27 & 10.0 \\
\hline ANNUAL & 2.7 & 37 & 11 & 8.7 & .77 & 100 \\
\hline
\end{tabular}

MAGNITUDE AND PROBABILITY OF ANNUAL LOW FLOW BASED ON PERIOD OF RECORD 1932-76

\begin{tabular}{|c|c|c|c|c|c|c|}
\hline \multirow{2}{*}{$\begin{array}{c}\text { PERIOD } \\
\text { (CON- } \\
\text { SECU- } \\
\text { TIVE } \\
\text { DAYS) }\end{array}$} & \multicolumn{6}{|c|}{$\begin{array}{l}\text { DISCHARGE, IN FT'3/S, FOR INDICATED RECURRENCE } \\
\text { INTERVAL, IN YEARS, AND ANNUAL NON- } \\
\text { EXCEEDANCE PROBABILITY, IN PERCENT }\end{array}$} \\
\hline & $\begin{array}{c}2 \\
508\end{array}$ & $\begin{array}{c}5 \\
208\end{array}$ & $\begin{array}{l}10 \\
108\end{array}$ & $\begin{array}{l}20 \\
58\end{array}$ & $\begin{array}{l}50 \\
28\end{array}$ & \\
\hline $\begin{array}{r}1 \\
3 \\
7 \\
14 \\
30 \\
60 \\
90 \\
120 \\
183\end{array}$ & $\begin{array}{l}1.8 \\
1.9 \\
2.0 \\
2.3 \\
2.7 \\
3.2 \\
3.6 \\
4.0 \\
5.5\end{array}$ & $\begin{array}{l}1.3 \\
1.4 \\
1.5 \\
1.7 \\
1.9 \\
2.2 \\
2.5 \\
2.8 \\
3.6\end{array}$ & $\begin{array}{l}1.2 \\
1.2 \\
1.4 \\
1.4 \\
1.6 \\
1.9 \\
2.1 \\
2.3 \\
2.9\end{array}$ & $\begin{array}{l}1.0 \\
1.1 \\
1.2 \\
1.3 \\
1.4 \\
1.6 \\
1.8 \\
2.0 \\
2.4\end{array}$ & $\begin{array}{r}.99 \\
1.0 \\
1.1 \\
1.1 \\
1.2 \\
1.4 \\
1.5 \\
1.7 \\
1.9\end{array}$ & \\
\hline
\end{tabular}

MAGNITUDE AND PROBABILITY OF ANNUAL HIGH FLOW BASED ON PERIOD OF RECORD 1931-76

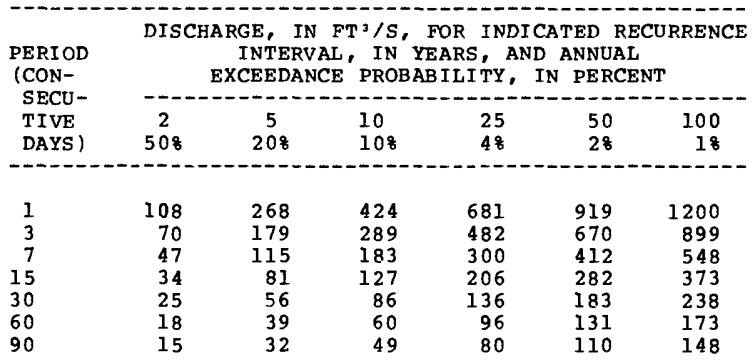

DURATION TABLE OF DAILY MEAN FLOW FOR PERIOD OF RECORD 1931-76

DISCHARGE, IN FT' ${ }^{3 / S}$, THAT WAS EOUALED OR EXCEEDED FOR INDICATED PERCENT OF TIME

\begin{tabular}{|c|c|c|c|c|c|c|c|c|c|c|c|c|c|c|}
\hline 58 & 108 & 158 & 208 & 258 & 308 & 408 & 508 & 608 & 708 & 758 & 808 & $85 \%$ & 908 & 958 \\
\hline 40 & 20 & 14 & 12 & 9.6 & 8.7 & 7.0 & 5.9 & 4.9 & 4.0 & 3.5 & 3.0 & 2.6 & 2.3 & 1 \\
\hline
\end{tabular}


LOCATION.--Lat $32^{\circ} 35^{\prime} 10^{\prime \prime}$, long $107^{\circ} 55^{\prime} 10^{\prime \prime}$, in NWt sec.7, T. $20 \mathrm{~s} .$, R.10 w., on right bank 6 mi northeast of Faywood Hot Springs, $10 \mathrm{mi}$ northeast of Faywood, and $12 \mathrm{miles}$ upstream from San Vicente Arroyo.

DRAINAGE AREA. $--440 \mathrm{mi}^{2}$.

PERIOD OF RECORD.--January 1909 to May 1914, January 1916 to December 1917, October 1920 to May 1921 , October to September 1930, all fragmentary. October 1930 to September 1955, October 1963 to September 1968 (discontinued). Monthly discharge only for some periods, published in wSP 1312. Records for August and September 1934, published in WSP 763 , have been found to be unreliable and should not be used.

GAGE.--Water-stage recorder. Datum of gage is 5,033 ft above mean sea level, datum of 1929 . Prior to Aug. 16, 1909, chain gage $300 \mathrm{ft}$ upstream at different datums. Aug. 16, 1909 to Sept. 25, 1920, water-stage recorder at site $500 \mathrm{ft}$ upstream at different datums (datum lowered $0.5 \mathrm{ft} \mathrm{Jan} .21,1915$, and $1.0 \mathrm{ft} \mathrm{Jan}$. 1 , 1916).

Water-stage recorder at present site since Sept. 26, 1920, at datums $2.0 \mathrm{ft}$ higher Sept. 26 , 1920 to Sept. 30 , 1942 , and 1 ft higher Oct. 1 , 1942 to Sept. 30, 1949.

REMARKS.--Diversions for irrigation of 1,750 acres above station.

AVERAGE DISCHARGE. - -37 years (water years 1935-68), $15.0 \mathrm{ft} 3 / \mathrm{s}, 10,870$ acre- $\mathrm{ft} / \mathrm{yr}$

EXTREMES FOR PERIOD OF RECORD.--Maximum discharge, 20,000 ft3/s Aug. 4, 1939 (gage height, $12 \mathrm{ft}$, present site and datum), from rating curve extended above $600 \mathrm{ft}^{3} / \mathrm{s}$ on basis of slope-area measurements at gage heights 7.80 and $11.7 \mathrm{ft}$; no flow at times.

STATISTICAL SUMMARIES

MEAN MONTHLY AND MEAN ANNUAJ, DISCHARGES 1931-33,
MAGNITUDE AND PROBABILITY OF ANNUAL LOW FLOW BASED ON PERIOD OF RECORD 1932-34, 1936-68

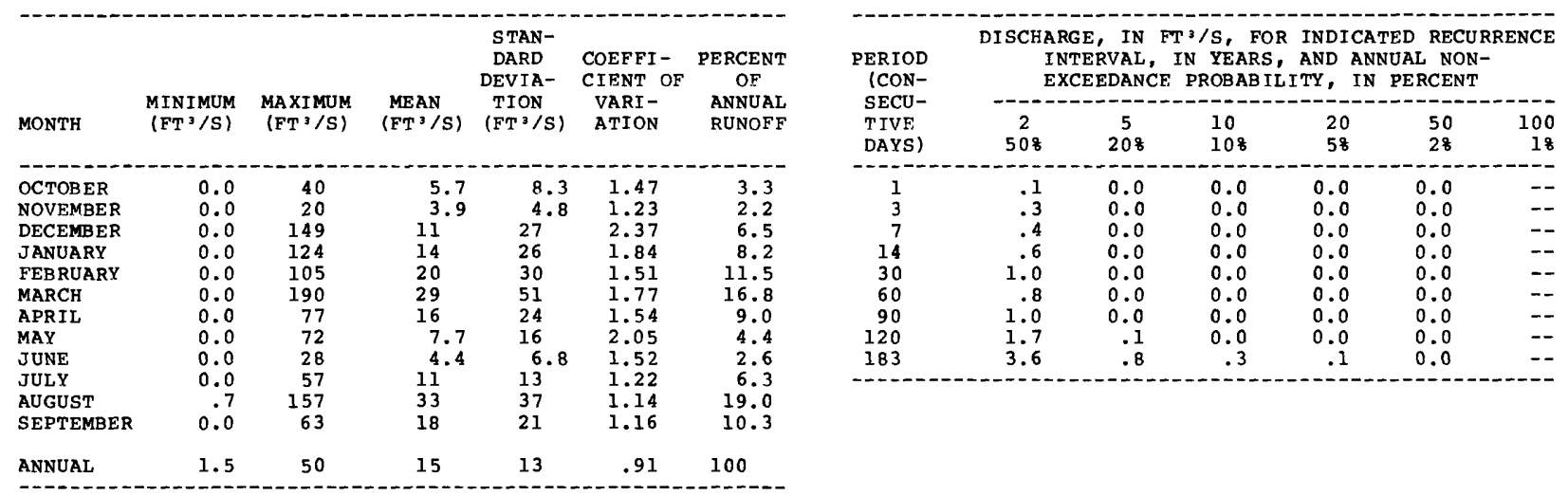

MAGNITUDE AND PROBABILITY OF ANNUAL HIGH FLOW BASED ON PERIOD OF RECORD 1931-33, 1935-68

\begin{tabular}{|c|c|c|c|c|c|c|}
\hline \multirow{2}{*}{$\begin{array}{l}\text { PERIOD } \\
\text { (CON- } \\
\text { SECU- } \\
\text { TIVE } \\
\text { DAYS) }\end{array}$} & \multicolumn{6}{|c|}{$\begin{array}{l}\text { DISCHARGE, IN FT'/S, FOR INDICATED RECURRENCF } \\
\text { INTERVAL, IN YFARS, AND ANNUAL } \\
\text { EXCEEDANCE PROBABILITX, IN PERCENT }\end{array}$} \\
\hline & $\begin{array}{c}2 \\
508\end{array}$ & $\begin{array}{c}5 \\
208\end{array}$ & $\begin{array}{l}10 \\
108\end{array}$ & $\begin{array}{l}25 \\
48\end{array}$ & $\begin{array}{l}50 \\
28\end{array}$ & $\begin{array}{r}100 \\
18\end{array}$ \\
\hline $\begin{array}{r}1 \\
3 \\
7 \\
15 \\
30 \\
60 \\
90\end{array}$ & $\begin{array}{r}395 \\
212 \\
127 \\
81 \\
54 \\
34 \\
25\end{array}$ & $\begin{array}{r}938 \\
489 \\
266 \\
164 \\
109 \\
74 \\
56\end{array}$ & $\begin{array}{r}1450 \\
740 \\
387 \\
236 \\
155 \\
109 \\
86\end{array}$ & $\begin{array}{r}2270 \\
1130 \\
573 \\
345 \\
222 \\
166 \\
135\end{array}$ & $\begin{array}{r}3010 \\
1470 \\
734 \\
440 \\
277 \\
216 \\
181\end{array}$ & $\begin{array}{l}-- \\
-- \\
-- \\
-- \\
-- \\
--\end{array}$ \\
\hline
\end{tabular}

DURATION TABLE OF DAILX MEAN FLOW FOR PERIOD OF RECORD 1931-33, 1935-68

DISCHARGE, IN FT $3 / \mathrm{S}$, THAT WAS EQUALED OR EXCEFDED FOR INDICATED PERCENT OF TIME

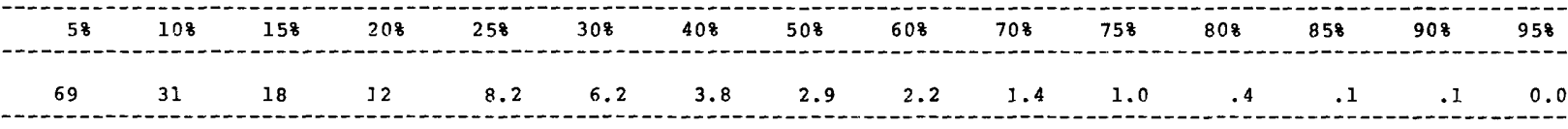


LOCATION. --Lat $32^{\circ} 46^{\prime} 15^{\prime \prime}$, long $108^{\circ} 16^{\prime} 30^{\prime \prime}$, in NWłsEz sec.3, T.18 S., R.14 w. (projected), on left bank in silver city, $800 \mathrm{ft}$ upstream from bridge at Broadway street and $1,300 \mathrm{ft}$ downstream from confluence of $\mathrm{Silva}$ Creek and Pinos Altos creek.

DRAINAGE AREA. $--26.5 \mathrm{mi}^{2}$.

PERIOD OF RECORD.--August 1953 to September 1965 (discontinued).

GAGE.--Water-stage recorder and concrete control. Datum of gage is $5,862.58 \mathrm{ft}$ above mean sea level, datum of 1929. Prior to May 25, 1958 , at site $500 \mathrm{ft}$ downstream at datum $17.99 \mathrm{ft}$ lower.

REMARRS. - No diversion above station.

AVERAGE DISCHARGE.--12 years (water years 1954-65), $0.79 \mathrm{ft}^{3} / \mathrm{s}, 572 \mathrm{acre-ft/yr}$.

EXTREMES FOR PERIOD OF RECORD.--Maximum discharge, 4,680 $\mathrm{ft} / \mathrm{s}$ Aug. 16, 1963 (gage height, $8.30 \mathrm{ft}$ in gage wel1, $10.0 \mathrm{ft}$ from outside gage); no flow for many days in most years.

Maximum flood known occurred July 21, 1895 (discharge probably exceeded $10,000 \mathrm{ft}^{3} / \mathrm{s}$ ), from newspaper

accounts. A peak of $6,800 \mathrm{ft}^{3 / \mathrm{s}}$ was measured by slope-area method from old floodmarks found in 1956 (probably

occurred sept. 9,1938 ).

STATISTICAL SUMMARIES

MEAN MONTHLY AND MEAN ANNUAL DISCHARGES 1954-65

\begin{tabular}{|c|c|c|c|c|c|c|}
\hline MONTH & $\begin{array}{l}\text { MINIMUM } \\
\left(\mathrm{FTI}^{3} / \mathrm{S}\right)\end{array}$ & $\begin{array}{c}\operatorname{MAXIMUM} \\
\left(\mathrm{FT}^{3} / \mathrm{S}\right)\end{array}$ & $\begin{array}{l}\text { MEAN } \\
\left(\mathrm{FT}^{3} / \mathrm{S}\right)\end{array}$ & $\begin{array}{l}\text { STAN- } \\
\text { DARD } \\
\text { DEVIA- } \\
\text { TION } \\
\left(\mathrm{FT}^{3} / \mathrm{S}\right)\end{array}$ & $\begin{array}{l}\text { COEFFI- } \\
\text { CIENT OF } \\
\text { VARI- } \\
\text { ATION: }\end{array}$ & $\begin{array}{c}\text { PERCENT } \\
\text { OF } \\
\text { ANNUAL } \\
\text { RUNOFF }\end{array}$ \\
\hline $\begin{array}{l}\text { OCTOBER } \\
\text { NOVEMBER } \\
\text { DECEMBER } \\
\text { JANUARY } \\
\text { FEBROARY } \\
\text { MARCH } \\
\text { APRIL } \\
\text { MAY } \\
\text { JUNE } \\
\text { JULY } \\
\text { AUGUST } \\
\text { SEPTEMBER }\end{array}$ & $\begin{array}{l}0.0 \\
0.0 \\
0.0 \\
0.0 \\
0.0 \\
0.0 \\
0.0 \\
0.0 \\
0.0 \\
.1 \\
.3 \\
0.0\end{array}$ & $\begin{array}{r}4.1 \\
.5 \\
.7 \\
2.5 \\
.2 \\
1.4 \\
.1 \\
.1 \\
2.5 \\
7.6 \\
14 \\
3.3\end{array}$ & $\begin{array}{l}.6 \\
.1 \\
.2 \\
.3 \\
.1 \\
.2 \\
0.0 \\
0.0 \\
.4 \\
2.7 \\
3.8 \\
1.0\end{array}$ & $\begin{array}{r}1.3 \\
.2 \\
.2 \\
.7 \\
.1 \\
.4 \\
.1 \\
0.0 \\
.9 \\
2.4 \\
3.8 \\
1.2\end{array}$ & $\begin{array}{l}1.95 \\
1.88 \\
1.35 \\
2.16 \\
1.14 \\
1.86 \\
1.50 \\
1.33 \\
2.02 \\
.89 \\
.99 \\
1.13\end{array}$ & $\begin{array}{r}6.7 \\
.8 \\
1.8 \\
3.4 \\
.7 \\
2.3 \\
.4 \\
.3 \\
4.6 \\
28.5 \\
39.6 \\
10.8\end{array}$ \\
\hline NNUAL & .3 & 1.7 & .8 & .4 & .45 & 100 \\
\hline
\end{tabular}

MAGNITUDE AND PROBABILITY OF ANNUAL LOW FLOW BASED ON PERIOD OF RPCORD $1955-65$

\begin{tabular}{|c|c|c|c|c|c|c|}
\hline \multirow{2}{*}{$\begin{array}{l}\text { PERIOD } \\
\text { (CON- } \\
\text { SECU- } \\
\text { TIVE } \\
\text { DAYS) }\end{array}$} & \multicolumn{6}{|c|}{$\begin{array}{l}\text { DISCHARGE, IN FT' }{ }^{3} / S \text {, FOR INDICATED RECURRENCE } \\
\text { INTERVAL, IN YEARS, AND ANNUAL NON- } \\
\text { EXCEEDANCE PROBABILITY, IN PERCENT }\end{array}$} \\
\hline & $\begin{array}{c}2 \\
508\end{array}$ & $\begin{array}{c}5 \\
208\end{array}$ & $\begin{array}{l}10 \\
108\end{array}$ & $\begin{array}{l}20 \\
58\end{array}$ & $\begin{array}{l}50 \\
28\end{array}$ & $\begin{array}{r}10 \\
1\end{array}$ \\
\hline $\begin{array}{r}1 \\
3 \\
7 \\
14 \\
30 \\
60 \\
90 \\
120 \\
183\end{array}$ & $\begin{array}{l}0.0 \\
0.0 \\
0.0 \\
0.0 \\
0.0 \\
0.0 \\
0.0 \\
0.0 \\
.1\end{array}$ & $\begin{array}{l}0.0 \\
0.0 \\
0.0 \\
0.0 \\
0.0 \\
0.0 \\
0.0 \\
0.0 \\
0.0\end{array}$ & $\begin{array}{l}0.0 \\
0.0 \\
0.0 \\
0.0 \\
0.0 \\
0.0 \\
0.0 \\
0.0 \\
0.0\end{array}$ & $\begin{array}{l}0.0 \\
0.0 \\
0.0 \\
0.0 \\
0.0 \\
0.0 \\
0.0 \\
0.0 \\
0.0\end{array}$ & $\begin{array}{l}-- \\
-- \\
-- \\
-- \\
-- \\
-- \\
--\end{array}$ & \\
\hline
\end{tabular}

MAGNITUDE AND PROBABILITY OF ANNUAL HIGH FLOW BASED ON PERIOD OF RECORD $1954-65$

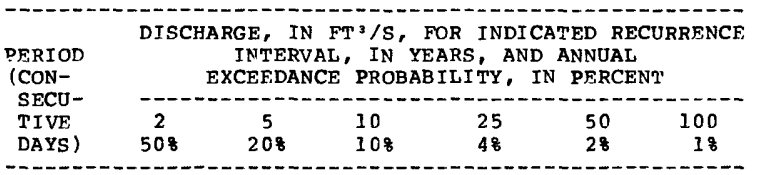

$\begin{array}{rrrrrrr}1 & 83 & 117 & 139 & -- & -- & - \\ 3 & 35 & 50 & 60 & -- & -- & - \\ 7 & 18 & 26 & 31 & -- & -- & - \\ 15 & 9.0 & 13 & 16 & -- & -- & - \\ 30 & 5.6 & 8.6 & 11 & -- & -- & - \\ 60 & 3.3 & 5.0 & 6.3 & -- & -- & - \\ 90 & 2.3 & 3.5 & 4.6 & -- & -- & -\end{array}$

DURATION TABLE OF DAII.Y MEAN FIOW FOR PERION OF RECORD 1954-65

DISCHARGE, IN FT'3/S, THAT WAS EOUALED OR FXCFEDED FOR INDICATED PERCENT OF TIME

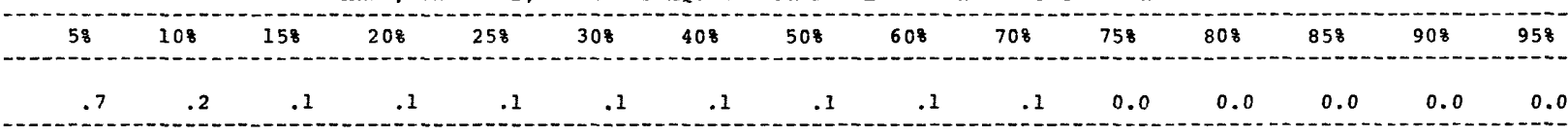


08481500 TULAROSA CREEK NEAR BENT, NM

(National stream-quality accounting network station)

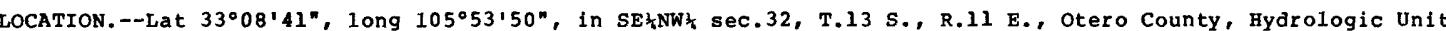
13044503 , on right bank $50 \mathrm{ft}$ downstream from old U.S. Highway 70 bridge, $2.6 \mathrm{mi}$ west of Bent, and $8.5 \mathrm{mi}$ northeast of Tularosa, and at mile 19.4 .

DRAINAGE AREA.--120 $\mathrm{mi}^{2}$, approximately.

PERIOD OF RECORD.--December 1947 to current year. Prior to october 1982 published as "Rio Tularosa near Bent." REVISED RECORDS.--WSP 1312: $1949(\mathrm{M})$.

GAGE.--Water-stage recorder. Elevation of gage is 5,450 ft above National Geodetic Vertical Datum of 1929 , from topographic map. Since Jan. 20, 1983, supplemental water-stage recorder at site $200 \mathrm{ft}$ upstream and at datum $9.70 \mathrm{ft}$ higher.

REMARRS.--Diversions for irrigation of about 1,000 acres, 1959 determination, upstream from station.

AVERAGE DISCHARGE.--37 years (water years 1949-85), $10.6 \mathrm{ft}^{3} / \mathrm{s}, 7,680 \mathrm{acre}-\mathrm{ft} / \mathrm{yr}$.

EXTREMES FOR PERIOD OF RECORD.--Maximum discharge, $4.280 \mathrm{ft} 3 / \mathrm{s}$, June 18,1965 , gage height, $5.02 \mathrm{ft}$, from rating curve extended above $160 \mathrm{ft}^{3} / \mathrm{s}$ on basis of slope-area measurement of peak flow; no flow May 14 , 1955, result of unusual regulation.

EXTREMES OUTSIDE PERIOD OF RECORD, - A major flood probably occurred Sept. 3, 1938, when a peak of $9,640 \mathrm{ft} 3 / \mathrm{s}$ was computed for station approximately $6 \mathrm{mi}$ downstream near Tularosa. Another flood may have occurred July 2 , 1914.

STATISTICAL SUMMARIES

MEAN MONTHLY AND MEAN ANNUAL DISCHARGES 1949-85

\begin{tabular}{|c|c|c|c|c|c|c|}
\hline MONTH & $\begin{array}{l}\text { MINIMUM } \\
\left(\mathrm{FT}^{3} / \mathrm{S}\right)\end{array}$ & $\begin{array}{c}\text { MAXIMUM } \\
\left(\mathrm{FT}^{3} / \mathrm{S}\right)\end{array}$ & $\begin{array}{l}\text { MEAN } \\
\left(\mathrm{FT}^{3} / \mathrm{S}\right)\end{array}$ & $\begin{array}{l}\text { STAN- } \\
\text { DARD } \\
\text { DEVIA- } \\
\text { TION } \\
\text { (FT'/S) }\end{array}$ & $\begin{array}{l}\text { COEFFI- } \\
\text { CIENT OF } \\
\text { VARI- } \\
\text { ATION }\end{array}$ & $\begin{array}{c}\text { PERCENT } \\
\text { OF } \\
\text { ANNUAL } \\
\text { RUNOFF }\end{array}$ \\
\hline $\begin{array}{l}\text { OCTOBER } \\
\text { NOVEMBER } \\
\text { DECEMBER } \\
\text { JANUARY } \\
\text { FEBRUARY } \\
\text { MARCH } \\
\text { APRIL } \\
\text { MAY } \\
\text { JUNE } \\
\text { JULY } \\
\text { AUGUST } \\
\text { SEPTEMBER }\end{array}$ & $\begin{array}{l}6.9 \\
7.7 \\
6.9 \\
7.4 \\
8.1 \\
7.7 \\
7.7 \\
5.8 \\
5.1 \\
4.2 \\
4.9 \\
5.7\end{array}$ & $\begin{array}{l}16 \\
17 \\
18 \\
20 \\
20 \\
19 \\
22 \\
20 \\
18 \\
18 \\
35 \\
21\end{array}$ & $\begin{array}{l}10 \\
11 \\
11 \\
12 \\
12 \\
11 \\
11 \\
9.6 \\
8.5 \\
9.4 \\
11 \\
10\end{array}$ & $\begin{array}{l}2.1 \\
2.2 \\
2.4 \\
2.6 \\
2.5 \\
2.6 \\
3.0 \\
2.9 \\
2.7 \\
3.3 \\
5.2 \\
3.1\end{array}$ & $\begin{array}{l}.21 \\
.20 \\
.21 \\
.22 \\
.21 \\
.23 \\
.28 \\
.30 \\
.32 \\
.35 \\
.46 \\
.30\end{array}$ & $\begin{array}{l}7.9 \\
8.7 \\
9.0 \\
9.1 \\
9.2 \\
9.0 \\
8.6 \\
7.5 \\
6.7 \\
7.4 \\
8.8 \\
8.1\end{array}$ \\
\hline NNUAL & 8.2 & 19 & 11 & 2.4 & .23 & 100 \\
\hline
\end{tabular}

MAGNITUDE AND PROBABILITY OF ANNUAL LOW FLON BASED ON PERIOD OF RECORD 1950-85

\begin{tabular}{|c|c|c|c|c|c|c|}
\hline \multirow{2}{*}{$\begin{array}{l}\text { PERIOD } \\
\text { (CON- } \\
\text { SECU- } \\
\text { TIVE } \\
\text { DAYS) }\end{array}$} & \multicolumn{6}{|c|}{$\begin{array}{l}\text { DISCHARGE, IN FT } 3 / \text { S, FOR INDICATED RECURRENCE } \\
\text { INTERVAL, IN YEARS, AND ANNUAL NON- } \\
\text { EXCEEDANCE PROBABILITY, IN PERCENT }\end{array}$} \\
\hline & $\begin{array}{c}2 \\
508\end{array}$ & $\begin{array}{c}5 \\
208\end{array}$ & $\begin{array}{l}10 \\
108\end{array}$ & $\begin{array}{l}20 \\
58\end{array}$ & $\begin{array}{l}50 \\
28\end{array}$ & $\begin{array}{r}100 \\
18\end{array}$ \\
\hline $\begin{array}{r}1 \\
3 \\
7 \\
14 \\
30 \\
60 \\
90 \\
120 \\
183\end{array}$ & $\begin{array}{l}3.2 \\
3.7 \\
4.7 \\
5.7 \\
6.5 \\
7.5 \\
8.0 \\
8.4 \\
9.1\end{array}$ & $\begin{array}{l}2.3 \\
2.7 \\
3.4 \\
4.4 \\
5.2 \\
6.1 \\
6.6 \\
7.0 \\
7.7\end{array}$ & $\begin{array}{l}1.9 \\
2.3 \\
2.9 \\
3.8 \\
4.7 \\
5.6 \\
6.0 \\
6.3 \\
7.1\end{array}$ & $\begin{array}{l}1.7 \\
2.0 \\
2.5 \\
3.4 \\
4.3 \\
5.1 \\
5.5 \\
5.9 \\
6.6\end{array}$ & $\begin{array}{l}1.5 \\
1.7 \\
2.1 \\
2.9 \\
3.8 \\
4.6 \\
5.0 \\
5.4 \\
6.2\end{array}$ & \\
\hline
\end{tabular}

MAGNITUDE AND PROBABILITY OF ANNUAL HIGH FLON BASED ON PERIOD OF RECORD 1949-85

\begin{tabular}{|c|c|c|c|c|c|c|}
\hline \multirow{2}{*}{$\begin{array}{l}\text { PERIOD } \\
\text { (CON- } \\
\text { SECU- } \\
\text { TIVE } \\
\text { DAYS) }\end{array}$} & \multicolumn{6}{|c|}{$\begin{array}{l}\text { DISCHARGE, IN FT'3/S, FOR INDICATED RECURRENC } \\
\text { INTERVAL, IN YEARS, AND ANNUAL } \\
\text { EXCEEDANCE PROBABILITY, IN PERCENT }\end{array}$} \\
\hline & $\begin{array}{c}2 \\
508\end{array}$ & $\begin{array}{c}5 \\
208\end{array}$ & $\begin{array}{l}10 \\
108\end{array}$ & $\begin{array}{l}25 \\
48\end{array}$ & $\begin{array}{l}50 \\
28\end{array}$ & $\begin{array}{r}100 \\
18\end{array}$ \\
\hline $\begin{array}{r}1 \\
3 \\
7 \\
15 \\
30 \\
60 \\
90\end{array}$ & $\begin{array}{l}39 \\
23 \\
17 \\
15 \\
13 \\
12 \\
12\end{array}$ & $\begin{array}{l}77 \\
36 \\
24 \\
19 \\
17 \\
15 \\
14\end{array}$ & $\begin{array}{r}120 \\
52 \\
32 \\
23 \\
20 \\
17 \\
16\end{array}$ & $\begin{array}{r}212 \\
85 \\
47 \\
31 \\
24 \\
19 \\
18\end{array}$ & $\begin{array}{r}320 \\
123 \\
64 \\
39 \\
28 \\
22 \\
20\end{array}$ & $\begin{array}{l}\ddot{-} \\
\ddot{z} \\
z- \\
--\end{array}$ \\
\hline
\end{tabular}

DURATION TABLE OF DAILY MEAN FLOW FOR PERIOD OF RECORD 1949-85

DISCHARGE, IN FT $3 / 5$, THAT WAS EQUALED OR EXCEEDED FOR INDICATED PERCENT OF TIME

\begin{tabular}{|c|c|c|c|c|c|c|c|c|c|c|c|c|c|c|}
\hline 58 & 108 & 158 & 208 & 258 & 308 & 408 & 508 & 608 & 708 & 758 & 808 & 858 & 908 & 958 \\
\hline 17 & 16 & 15 & 14 & 13 & 12 & 12 & 11 & 9.6 & 8.7 & 8.1 & 7.5 & 6.9 & 6.2 & \\
\hline
\end{tabular}


SAN JUAN RIVER BASIN

09355500 SAN JUAN RIVER NEAR ARCHULETA, NM

LOCATION.--Lat $36^{\circ} 48^{\prime} 05^{\prime}$, long $10^{\circ} 41^{\prime} 51^{\prime \prime}$, in N sec.20, T.30 N., R.8 w., San Juan County, Hydrologic Unit 14080101, on left bank $0.5 \mathrm{mi}$ upstream from Gobernador Canyon, $0.8 \mathrm{mi}$ northeast of Archuleta, $7.2 \mathrm{mi}$ downstream from Navajo Dam, and at mile 291.4.

DRAINAGE AREA. $--3,260 \mathrm{mi}^{2}$, approximately.

PERIOD OF RECORD.--December 1954 to current year.

REVISED RECORDS.--The annual runoff for the 1958 water year as published in table 2 , wSP 1733 , is 455,000 acre-ft. The correct value is $1,455,000$ acre-ft.

GAGE.--Water-stage recorder. Elevation of gage is $5,653 \mathrm{ft}$ above National Geodetic vertical Datum of 1929 , from river-profile survey. Prior to Dec. 29, 1959, at site $5.0 \mathrm{mi}$ upstream at elevation $55 \mathrm{ft}$ higher. Dec. 29 , 1959 to Nov. 15, 1964, at site $0.4 \mathrm{mi}$ upstream at elevation $5 \mathrm{ft}$ higher. Prior to Nov. 28, 1966, at elevation $2.0 \mathrm{ft}$ higher.

REMARRs.--Flow completely regulated by Navajo Reservoir (station 09355100) 7 mi upstream except for minor inflow from $30 \mathrm{mi}^{2}$ intervening drainage area. Highwater diversions through Azotea tunnel (station 08284160 ) into Rio Grande Basin began in March 1971. Diversions for irrigation of about 47,000 acres upstream from station. Releases from Navajo Reservoir, beginning in January 1976, for use on Navajo Indian Irrigation Project bypass gage in tunnel on left bank.

AVERAGE DISCHARGF.--22 years (water years 1964-85), 1,221 ft3/s, 884,600 acre-ft/yr.

23 years (water years $1963-85$ ), $1,180 \mathrm{ft} / \mathrm{s}, 854,900$ acre-ft/yr, since closure of Navajo Dam.

EXTREMES FOR PERIOD OF RECORD.--Maximum discharge, 18,900 $\mathrm{ft}^{3} / \mathrm{s}$, July 27, 1957, gage height, 11.00 ft, site and datum then in use; minimum determined, $8 \mathrm{ft}^{3} / \mathrm{s}$, Feb. 28, 1963. Maximum discharge since construction of Navajo Dam in $1962,6,500 \mathrm{ft}^{3} / \mathrm{s}$, June 20,1965 , gage height, $4.57 \mathrm{ft}$.

STATISTICAL SUMMARIES (PERIOD AFTER REGULATION OF NAVAJO DAM)

MEAN MONTHLY AND MEAN ANNUAL DISCHARGES 1964-85

\begin{tabular}{|c|c|c|c|c|c|c|}
\hline MONTH & $\begin{array}{l}\text { MINIMUM } \\
\left(\mathrm{FT}^{3} / \mathrm{S}\right)\end{array}$ & $\begin{array}{c}\text { MAXIMUM } \\
\left(\mathrm{FT}^{3} / \mathrm{S}\right)\end{array}$ & $\begin{array}{l}\text { MEAN } \\
\left(\mathrm{FT}^{3} / \mathrm{S}\right)\end{array}$ & $\begin{array}{l}\text { STAN- } \\
\text { DARD } \\
\text { DEVIA- } \\
\text { TION } \\
\left(\mathrm{FT}^{3} / \mathrm{S}\right)\end{array}$ & $\begin{array}{l}\text { COEFFI- } \\
\text { CIENT OF } \\
\text { VARI- } \\
\text { ATION }\end{array}$ & $\begin{array}{c}\text { PERCENT } \\
\text { OF } \\
\text { ANNUAL } \\
\text { RUNOF F }\end{array}$ \\
\hline $\begin{array}{l}\text { OCTOBER } \\
\text { NOVEMBER } \\
\text { DECEMBER } \\
\text { JANUARY } \\
\text { FEBRUARY } \\
\text { MARCH } \\
\text { APRIL } \\
\text { MAY } \\
\text { JUNE } \\
\text { JULY } \\
\text { AUGUST } \\
\text { SEPTEMBER }\end{array}$ & $\begin{array}{l}350 \\
306 \\
344 \\
283 \\
229 \\
207 \\
244 \\
279 \\
300 \\
320 \\
472 \\
439\end{array}$ & $\begin{array}{l}2131 \\
3018 \\
2886 \\
2733 \\
2362 \\
2618 \\
4758 \\
4962 \\
5169 \\
5126 \\
3508 \\
2674\end{array}$ & $\begin{array}{l}974 \\
937 \\
1118 \\
1215 \\
1274 \\
1172 \\
1381 \\
1602 \\
1536 \\
1325 \\
1087 \\
1028\end{array}$ & $\begin{array}{r}563 \\
695 \\
681 \\
689 \\
680 \\
689 \\
1186 \\
1305 \\
1419 \\
1252 \\
718 \\
536\end{array}$ & $\begin{array}{l}.58 \\
.74 \\
.61 \\
.57 \\
.53 \\
.59 \\
.86 \\
.81 \\
.92 \\
.94 \\
.66 \\
.52\end{array}$ & $\begin{array}{r}6.6 \\
6.4 \\
7.6 \\
8.3 \\
8.7 \\
8.0 \\
9.4 \\
10.9 \\
10.5 \\
9.0 \\
7.4 \\
7.0\end{array}$ \\
\hline ANNUAL & 515 & 2431 & 1221 & 522 & .43 & 100 \\
\hline
\end{tabular}

MAGNITUDE AND PROBABILITY OF ANNUAL LOW FLOW BASED ON PERIOD OF RECORD $1965-85$

\begin{tabular}{|c|c|c|c|c|c|c|}
\hline \multirow{2}{*}{$\begin{array}{l}\text { PERIOD } \\
\text { (CON- } \\
\text { SECU- } \\
\text { TIVE } \\
\text { DAYS) }\end{array}$} & \multicolumn{6}{|c|}{$\begin{array}{l}\text { DISCHARGE, IN FT'3/S, FOR INDICATED RECURRENC } \\
\text { INTERVAL, IN YEARS, AND ANNUAL NON- } \\
\text { EXCEEDANCE PROBABILITY, IN PERCENT }\end{array}$} \\
\hline & $\begin{array}{c}2 \\
508\end{array}$ & $\begin{array}{c}5 \\
208\end{array}$ & $\begin{array}{l}10 \\
108\end{array}$ & $\begin{array}{l}20 \\
58\end{array}$ & $\begin{array}{l}50 \\
28\end{array}$ & $\begin{array}{r}100 \\
18\end{array}$ \\
\hline $\begin{array}{r}1 \\
3 \\
7 \\
14 \\
30 \\
60 \\
90 \\
120 \\
183\end{array}$ & $\begin{array}{l}372 \\
387 \\
408 \\
443 \\
508 \\
580 \\
617 \\
659 \\
769\end{array}$ & $\begin{array}{l}253 \\
292 \\
308 \\
333 \\
363 \\
398 \\
419 \\
445 \\
520\end{array}$ & $\begin{array}{l}197 \\
250 \\
263 \\
287 \\
306 \\
332 \\
348 \\
371 \\
430\end{array}$ & $\begin{array}{l}156 \\
218 \\
231 \\
253 \\
267 \\
289 \\
302 \\
322 \\
370\end{array}$ & $\begin{array}{l}-- \\
-- \\
-- \\
-- \\
-- \\
-- \\
--\end{array}$ & $\begin{array}{l}-- \\
-- \\
-- \\
-- \\
-- \\
--\end{array}$ \\
\hline
\end{tabular}

MAGNITUDE AND PROBABILITY OF ANNUAL HIGH FLOW BASED ON PERIOD OF RECORD $1964-85$

\begin{tabular}{|c|c|c|c|c|c|c|}
\hline \multirow{2}{*}{$\begin{array}{l}\text { PERIOD } \\
\text { (CON- } \\
\text { SECU- } \\
\text { TIVE } \\
\text { DAYS) }\end{array}$} & \multicolumn{6}{|c|}{$\begin{array}{l}\text { DISCHARGE, IN FT } 3 / \mathrm{S} \text {, FOR INDICATED RECURRENC } \\
\text { INTERVAL, IN YEARS, AND ANNUAL } \\
\text { EXCEEDANCE PROBAB IL I TY, IN PERCENT }\end{array}$} \\
\hline & $\begin{array}{c}2 \\
508\end{array}$ & $\begin{array}{c}5 \\
208\end{array}$ & $\begin{array}{l}10 \\
108\end{array}$ & $\begin{array}{l}25 \\
48\end{array}$ & $\begin{array}{l}50 \\
28\end{array}$ & $\begin{array}{r}100 \\
18\end{array}$ \\
\hline $\begin{array}{r}1 \\
3 \\
7 \\
15 \\
30 \\
60 \\
90\end{array}$ & $\begin{array}{l}2330 \\
2370 \\
2350 \\
2320 \\
2230 \\
2030 \\
1860\end{array}$ & $\begin{array}{l}3630 \\
3670 \\
3620 \\
3520 \\
3330 \\
3100 \\
2910\end{array}$ & $\begin{array}{l}4680 \\
4620 \\
4530 \\
4350 \\
4070 \\
3830 \\
3650\end{array}$ & $\begin{array}{l}6240 \\
5910 \\
5740 \\
5440 \\
5020 \\
4780 \\
4600\end{array}$ & $\begin{array}{l}- \\
-- \\
-- \\
-- \\
-\end{array}$ & $\begin{array}{l}-- \\
-- \\
-- \\
-- \\
--\end{array}$ \\
\hline
\end{tabular}

DURATION TABLE OF DAILY MEAN FLOW FOR PERIOD OF RECORD 1964-85

DISCHARGE, IN FT3/S, THAT WAS EOUALED OR EXCEEDED FOR INDICATED PERCENT OF TIME

\begin{tabular}{|c|c|c|c|c|c|c|c|c|c|c|c|c|c|c|}
\hline 58 & 108 & 158 & 208 & 258 & 308 & 408 & 508 & 608 & 708 & 758 & 808 & 858 & 908 & 958 \\
\hline 3080 & 2340 & 2020 & 1840 & 1680 & 1550 & 1230 & 919 & 636 & 517 & 497 & 478 & 454 & 401 & 336 \\
\hline
\end{tabular}


SAN JUAN RIVER BASIN

09356500 SAN JUAN RIVER NEAR BLANCO, NM

LOCATION,--Lat $36^{\circ} 43^{\prime} 50^{\prime \prime}$, long $107^{\circ} 48^{\prime} 50^{\prime \prime}$, in NEł sec.18, T.29 N., R.9 w., on left bank half a mile upstream from highway bridge, I mile upstream from Canon Largo, and lis miles east of Blanco.

DRAINAGE AREA. $--3,560 \mathrm{mi}^{2}$, approximately.

PERIOD OF RECORD.--June 1907 to November 1908, January to December 1909, January to October 1910, (gage heights only), October 1927 to January 1955 (discontinued). Monthly discharge only for some periods, published in wsp
1313 . Published as "at Turley" $1907-8$.

GAGE.--Water-stage recorder. Altitude of gage is 5,540 ft (from river-profile map). Prior to Nov. 30 , 1908 , staff gage at site 4 miles upstream at different datum. Dec. 9, 1908 to Sept. 27, 1909, chain gage and Sept. 28 , 1909 to oct. 31, 1910, staff gage, at site half a mile downstream at different datums. Oct. 1 , 1927 to Dec. 20 , 1950, water-stage recorder at present site at datum $1.0 \mathrm{ft}$ higher.

REMARKS.--Diversions above station for irrigation of about 52,000 acres, of which 4,500 are below station.

AVERAGE DISCHARGE.--24 years (water years 1931-54), 1,376 ft3/s, 996,900 acre-ft/yr.

EXTREMES FOR PERIOD OF RECORD.--Maximum discharge, 25,000 ft3/s Aug. 11, 1929 (gage height, $9.70 \mathrm{ft}$, present

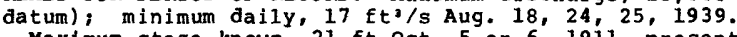

Maximum stage known, 21 ft oct. 5 or 6, 19il, present datum. Flood of Sept. 6, 1909, reached a stage of about $11 \mathrm{ft}$, site and datum then in use (discharge probably exceeded that of 1929). Flood of June 29, 1927, was of about the same magnitude as those of 1909 and 1929.

STATISTICAL SUMMARIES

MEAN MONTHLY AND MEAN ANNUAL DISCHARGES 1931-54

MAGNITUDE AND PROBABILITY OF ANNUAL LOW FLOW BASED ON PERIOD OF RECORD 1932-54

\begin{tabular}{|c|c|c|c|c|c|c|}
\hline MONTH & $\begin{array}{l}\text { MINIMUM } \\
\left(\mathrm{FT}^{3} / \mathrm{S}\right)\end{array}$ & $\begin{array}{c}\text { MAXIMUM } \\
\left(\mathrm{FT}^{3} / \mathrm{S}\right)\end{array}$ & $\begin{array}{l}\text { MEAN } \\
\left(\mathrm{FT}^{3} / \mathrm{S}\right)\end{array}$ & $\begin{array}{c}\text { STAN- } \\
\text { DARD } \\
\text { DEVIA- } \\
\text { TION } \\
\left(\mathrm{FT}^{3} / \mathrm{S}\right)\end{array}$ & $\begin{array}{l}\text { COEFFI- } \\
\text { CIENT OF } \\
\text { VARI- } \\
\text { ATION }\end{array}$ & $\begin{array}{c}\text { PERCENT } \\
\text { OF } \\
\text { ANNUAL } \\
\text { RUNOFF }\end{array}$ \\
\hline $\begin{array}{l}\text { OCTOBER } \\
\text { NOVEMBER } \\
\text { DECEMBER } \\
\text { JANUARY } \\
\text { FEBRUARY } \\
\text { MARCH } \\
\text { APRIL } \\
\text { MAY } \\
\text { JUNE } \\
\text { JULY } \\
\text { AUGUST } \\
\text { SEPTEMBER }\end{array}$ & $\begin{array}{r}125 \\
119 \\
136 \\
126 \\
174 \\
296 \\
465 \\
1166 \\
255 \\
116 \\
90 \\
121\end{array}$ & $\begin{array}{r}4181 \\
1388 \\
788 \\
681 \\
1035 \\
2420 \\
6904 \\
12750 \\
9501 \\
4967 \\
1619 \\
1477\end{array}$ & $\begin{array}{r}626 \\
340 \\
267 \\
273 \\
399 \\
959 \\
3001 \\
4290 \\
3885 \\
1267 \\
616 \\
568\end{array}$ & $\begin{array}{r}790 \\
246 \\
126 \\
111 \\
200 \\
559 \\
1970 \\
2629 \\
2766 \\
1207 \\
403 \\
343\end{array}$ & $\begin{array}{l}1.26 \\
.72 \\
.47 \\
.41 \\
.50 \\
.58 \\
.66 \\
.61 \\
.71 \\
.95 \\
.65 \\
.60\end{array}$ & $\begin{array}{r}3.8 \\
2.1 \\
1.6 \\
1.7 \\
2.4 \\
5.8 \\
18.2 \\
26.0 \\
23.6 \\
7.7 \\
3.7 \\
3.4\end{array}$ \\
\hline NNUAL & 458 & 3248 & 1376 & 752 & .55 & 100 \\
\hline
\end{tabular}

\begin{tabular}{|c|c|c|c|c|c|c|}
\hline \multirow{2}{*}{$\begin{array}{l}\text { PERIOD } \\
\text { (CON- } \\
\text { SECU- } \\
\text { TIVE } \\
\text { DAYS) }\end{array}$} & \multicolumn{6}{|c|}{$\begin{array}{l}\text { DISCHARGE, IN FT'/S, FOR INDICATED RECURRENCE } \\
\text { INTERVAL, IN YEARS, AND ANNUAL NON- } \\
\text { EXCEEDANCE PROBABILITY, IN PERCENT }\end{array}$} \\
\hline & $\begin{array}{c}2 \\
508\end{array}$ & $\begin{array}{c}5 \\
208\end{array}$ & $\begin{array}{l}10 \\
108\end{array}$ & $\begin{array}{l}20 \\
58\end{array}$ & $\begin{array}{l}50 \\
28\end{array}$ & $\begin{array}{r}100 \\
18\end{array}$ \\
\hline $\begin{array}{r}1 \\
3 \\
7 \\
14 \\
30 \\
60 \\
90 \\
120 \\
183\end{array}$ & $\begin{array}{l}100 \\
108 \\
126 \\
145 \\
171 \\
201 \\
229 \\
259 \\
305\end{array}$ & $\begin{array}{r}48 \\
53 \\
65 \\
76 \\
113 \\
145 \\
175 \\
199 \\
228\end{array}$ & $\begin{array}{r}31 \\
35 \\
43 \\
51 \\
92 \\
124 \\
155 \\
178 \\
205\end{array}$ & $\begin{array}{r}21 \\
24 \\
30 \\
35 \\
78 \\
109 \\
142 \\
164 \\
192\end{array}$ & $\begin{array}{r}13 \\
15 \\
19 \\
23 \\
65 \\
96 \\
129 \\
151 \\
182\end{array}$ & $\begin{array}{l}=- \\
=- \\
=- \\
-- \\
=- \\
=-\end{array}$ \\
\hline
\end{tabular}

MAGNITUDE AND PROBABILITY OF ANNUAL HIGH FLOW BASED ON PERIOD OF RECORD 1931-54

\begin{tabular}{|c|c|c|c|c|c|c|}
\hline \multirow{2}{*}{$\begin{array}{l}\text { PERIOD } \\
\text { (CON- } \\
\text { SECU- } \\
\text { TIVE } \\
\text { DAYS) }\end{array}$} & \multicolumn{6}{|c|}{$\begin{array}{l}\text { DISCHARGE, IN FT' } 3 \text { /S, FOR INDICATED RECURRENCE } \\
\text { INTERVAL, IN YEARS, AND ANNUAL } \\
\text { EXCEEDANCE PROBABILITY, IN PERCENT }\end{array}$} \\
\hline & $\begin{array}{c}2 \\
508\end{array}$ & $\begin{array}{c}5 \\
208\end{array}$ & $\begin{array}{l}10 \\
108\end{array}$ & $\begin{array}{l}25 \\
48\end{array}$ & $\begin{array}{l}50 \\
28\end{array}$ & $\begin{array}{r}100 \\
18\end{array}$ \\
\hline $\begin{array}{r}1 \\
3 \\
7 \\
15 \\
30 \\
60 \\
90\end{array}$ & $\begin{array}{l}6970 \\
6410 \\
5710 \\
5210 \\
4580 \\
3830 \\
3240\end{array}$ & $\begin{array}{r}11700 \\
10500 \\
9300 \\
8510 \\
7380 \\
6310 \\
5480\end{array}$ & $\begin{array}{r}15200 \\
13500 \\
12000 \\
11000 \\
9410 \\
8170 \\
7180\end{array}$ & $\begin{array}{r}20100 \\
17600 \\
15800 \\
14400 \\
12100 \\
10700 \\
9540\end{array}$ & $\begin{array}{l}24000 \\
20900 \\
18900 \\
17100 \\
14200 \\
12800 \\
11400\end{array}$ & $\begin{array}{l}-- \\
=- \\
=- \\
-- \\
=- \\
--\end{array}$ \\
\hline
\end{tabular}

DURATION TABLE OF DAILY MEAN FLOW FOR PERIOD OF RECORD 1931-54

DISCHARGE, IN FT $3 / S$, THAT WAS EQUALED OR EXCEEDED FOR INDICATED PERCENT OF TIME

\begin{tabular}{|c|c|c|c|c|c|c|c|c|c|c|c|c|c|c|}
\hline 58 & 108 & 158 & 208 & 258 & 308 & 408 & 508 & 608 & 708 & 758 & 808 & 858 & 908 & 958 \\
\hline 6100 & 4050 & 2800 & 2020 & 1470 & 1110 & 688 & 477 & 357 & 289 & 261 & 236 & 211 & 182 & 132 \\
\hline
\end{tabular}


09363500 ANIMAS RIVER NEAR CEDAR HILL, NM

LOCATION.--Lat $37^{\circ} 02 \cdot 17^{\prime \prime}$, long $107^{\circ} 52 \cdot 25^{\prime \prime}$, in sec.7, T.32 N., R.9 W., La Plata County, Colorado, Hydrologic Unit

14080104 , on right bank $0.8 \mathrm{mi}$ downstream from Florida River, $2.5 \mathrm{mi}$ upstream from Colorado-New Mexico state

line, $8.5 \mathrm{mi}$ north of Cedar Hill, and at mile 32.9 .

DRAINAGE AREA. $--1,090 \mathrm{mi}^{2}$, approximately.

PERIOD OF RECORD,--October 1933 to current year. Monthly discharge only for October and November l933, published in WSP 1313.

REVISED RECORDS.--WSP 1563: 1940 and 1946 (monthly figures only).

GAGE.--Water-stage recorder. Elevation of gage is 5,960 ft above National Geodetic Vertical Datum of 1929 , from topographic map. Prior to Sept. 14, 1937, at datum between $1.52 \mathrm{ft}$ and $1.36 \mathrm{ft}$ higher. Sept. 15, 1937, to Sept. 30, 1946, at datum $1.36 \mathrm{ft}$ higher.

REMARRs.--Diversions for irrigation of about 20,000 acres upstream from station. During water years $1944-49$, Twin Rocks Canal diverted upstream from station for irrigation downstream. Slight regulation by Lemon Dam about

$30 \mathrm{mi}$ upstream on Florida River since November 1963 (capacity, 40,100 acre-ft).

AVERAGE DISCHARGE.--51 years (water years 1935-85), $924 \mathrm{ft}^{3} / \mathrm{s}, 669,400 \mathrm{acre}-\mathrm{ft} / \mathrm{yr}^{\mathrm{r}}$.

EXTREMES FOR PERIOD OF RECORD.--Maximum discharge, 13,100 ft/s, June 19, 1949, gage height, $11.45 \mathrm{ft}$; minimum, 63 $\mathrm{ft}^{3} / \mathrm{s}, \mathrm{Jan} .21,1935$.

EXTREMES OUTSIDE PERIOD OF RECORD.--A major flood occurred in october 1911 at this location.

STATISTICAL SUMMARIES

MEAN MONTHLY AND MEAN ANNUAL DISCHARGES 1935-85

\begin{tabular}{|c|c|c|c|c|c|c|}
\hline MONTH & $\begin{array}{c}\text { MINIMUM } \\
\left(\mathrm{FT}^{3} / \mathrm{S}\right)\end{array}$ & $\begin{array}{c}\text { MAXIMUM } \\
\left(F T^{3} / S\right)\end{array}$ & $\begin{array}{l}\text { MEAN } \\
\left(\mathrm{FT}^{3} / \mathrm{S}\right)\end{array}$ & $\begin{array}{l}\text { STAN- } \\
\text { DARD } \\
\text { DEVIA- } \\
\text { TION } \\
\text { (FT' } 3 / S \text { ) }\end{array}$ & $\begin{array}{l}\text { COEFFI- } \\
\text { CIENT OF } \\
\text { VARI- } \\
\text { ATION }\end{array}$ & $\begin{array}{c}\text { PERCENT } \\
\text { OF } \\
\text { ANNUAL } \\
\text { RUNOFF }\end{array}$ \\
\hline $\begin{array}{l}\text { OCTOBER } \\
\text { NOVEMBER } \\
\text { DECEMBER } \\
\text { JANUARY } \\
\text { FEBRUARY } \\
\text { MARCH } \\
\text { APRIL } \\
\text { MAY } \\
\text { JUNE } \\
\text { JULY } \\
\text { AUGUST } \\
\text { SEPTEMBER }\end{array}$ & $\begin{array}{l}169 \\
158 \\
159 \\
169 \\
151 \\
141 \\
273 \\
449 \\
458 \\
223 \\
232 \\
155\end{array}$ & $\begin{array}{r}2479 \\
1069 \\
542 \\
388 \\
455 \\
768 \\
2192 \\
5686 \\
6145 \\
3710 \\
1681 \\
1922\end{array}$ & $\begin{array}{r}453 \\
324 \\
256 \\
235 \\
247 \\
393 \\
1080 \\
2514 \\
3023 \\
1273 \\
608 \\
501\end{array}$ & $\begin{array}{r}358 \\
151 \\
72 \\
51 \\
57 \\
154 \\
535 \\
1088 \\
1578 \\
861 \\
295 \\
313\end{array}$ & $\begin{array}{l}.79 \\
.47 \\
.28 \\
.22 \\
.23 \\
.39 \\
.50 \\
.43 \\
.52 \\
.68 \\
.49 \\
.62\end{array}$ & $\begin{array}{r}4.2 \\
3.0 \\
2.3 \\
2.2 \\
2.3 \\
3.6 \\
9.9 \\
23.0 \\
27.7 \\
11.7 \\
5.6 \\
4.6\end{array}$ \\
\hline NNUAL & 340 & 1713 & 924 & 334 & .36 & 100 \\
\hline
\end{tabular}

MAGNITUDE AND PROBABILITY OF ANNUAL LOW FLOW BASED ON PERIOD OF RECORD $1935-85$

\begin{tabular}{|c|c|c|c|c|c|c|}
\hline \multirow{2}{*}{$\begin{array}{l}\text { PERIOD } \\
\text { (CON- } \\
\text { SECU- } \\
\text { TIVE } \\
\text { DAYS) }\end{array}$} & \multicolumn{6}{|c|}{$\begin{array}{l}\text { DISCHARGE, IN FT }{ }^{3 / S} \text {, FOR INDICATED RECURRENCE } \\
\text { INTERVAL, IN YEARS, AND ANNUAL NON- } \\
\text { EXCEEDANCE PROBABILITY, IN PERCENT }\end{array}$} \\
\hline & $\begin{array}{c}2 \\
508\end{array}$ & $\begin{array}{c}5 \\
208\end{array}$ & $\begin{array}{l}10 \\
108\end{array}$ & $\begin{array}{l}20 \\
58\end{array}$ & $\begin{array}{l}50 \\
28\end{array}$ & $\begin{array}{r}100 \\
18\end{array}$ \\
\hline $\begin{array}{r}1 \\
3 \\
7 \\
14 \\
30 \\
60 \\
90 \\
120 \\
183\end{array}$ & $\begin{array}{l}162 \\
171 \\
182 \\
194 \\
207 \\
217 \\
226 \\
237 \\
272\end{array}$ & $\begin{array}{l}134 \\
143 \\
155 \\
166 \\
177 \\
187 \\
194 \\
203 \\
227\end{array}$ & $\begin{array}{l}122 \\
132 \\
144 \\
154 \\
164 \\
175 \\
182 \\
190 \\
213\end{array}$ & $\begin{array}{l}113 \\
123 \\
136 \\
146 \\
154 \\
167 \\
173 \\
181 \\
205\end{array}$ & $\begin{array}{l}103 \\
115 \\
129 \\
137 \\
145 \\
158 \\
165 \\
173 \\
199\end{array}$ & $\begin{array}{r}98 \\
110 \\
124 \\
132 \\
139 \\
154 \\
161 \\
168 \\
196\end{array}$ \\
\hline
\end{tabular}

MAGNITUDE AND PROBABILITY OF ANNUAL HIGH FLOW BASED ON PERIOD OF RECORD $1935-85$

\begin{tabular}{|c|c|c|c|c|c|c|}
\hline \multirow{2}{*}{$\begin{array}{l}\text { PERIOD } \\
\text { (CON- } \\
\text { SECU- } \\
\text { TIVE } \\
\text { DAYS) }\end{array}$} & \multicolumn{6}{|c|}{$\begin{array}{l}\text { DISCHARGE, IN FT } 3 / \mathrm{S} \text {, FOR INDICATED RECURRENC } \\
\text { INTERVAL, IN YEARS, AND ANNUAL } \\
\text { EXCEEDANCE PROBABILITY, IN PERCENT }\end{array}$} \\
\hline & $\begin{array}{c}2 \\
508\end{array}$ & $\begin{array}{c}5 \\
208\end{array}$ & $\begin{array}{l}10 \\
108\end{array}$ & $\begin{array}{l}25 \\
48\end{array}$ & $\begin{array}{l}50 \\
28\end{array}$ & $\begin{array}{r}100 \\
18\end{array}$ \\
\hline $\begin{array}{r}1 \\
3 \\
7 \\
15 \\
30 \\
60 \\
90\end{array}$ & $\begin{array}{l}5100 \\
4840 \\
4400 \\
3880 \\
3460 \\
2790 \\
2290\end{array}$ & $\begin{array}{l}7340 \\
6850 \\
6210 \\
5480 \\
4850 \\
3990 \\
3310\end{array}$ & $\begin{array}{l}8760 \\
8100 \\
7330 \\
6480 \\
5670 \\
4710 \\
3950\end{array}$ & $\begin{array}{r}10500 \\
9590 \\
8670 \\
7670 \\
6580 \\
5550 \\
4710\end{array}$ & $\begin{array}{r}11700 \\
10600 \\
9620 \\
8520 \\
7190 \\
6110 \\
5240\end{array}$ & $\begin{array}{r}12900 \\
11600 \\
10500 \\
9330 \\
7750 \\
6640 \\
5750\end{array}$ \\
\hline
\end{tabular}

DURATION TABLE OF DAILY MEAN FLOW FOR PERIOD OF RECORD 1935-85

DISCHARGE, IN FT $3 / 5$, THAT WAS EOUALED OR EXCEEDED FOR INDICATED PERCENT OF TIME

\begin{tabular}{|c|c|c|c|c|c|c|c|c|c|c|c|c|c|c|}
\hline 58 & 108 & 158 & 208 & 258 & 308 & 408 & 508 & 608 & 708 & 758 & 808 & 858 & 908 & 958 \\
\hline 3620 & 2460 & 1800 & 1310 & 981 & 780 & 535 & 396 & 322 & 273 & 255 & 239 & 223 & 207 & 186 \\
\hline
\end{tabular}


SAN JUAN RIVER BASIN

09364500 ANIMAS RIVER AT FARMINGTON, NM

(National stream-quality accounting network station)

LOCATION,--Lat $36^{\circ} 43^{\prime} 17^{\prime \prime}$, long $108^{\circ} 12^{\prime} 05^{\prime \prime}$, in SWłSWł sec.15, T.29 N., R.13 w., San Juan County, Hydrologic Unit 14080104, in Boyd City Park, on right bank $900 \mathrm{ft}$ upstream from bridge on Miller Ave., $0.4 \mathrm{mi}$ downstream from bridge on U.S. Highway 64 in Farmington, and $1.5 \mathrm{mi}$ upstream from mouth.

DRAINAGE AREA. $--1,360 \mathrm{mi}^{2}$, approximately.

PERIOD OF RECORD.--June 1904 to October 1905 (published as "near Farmington"), September 1912 to current year. Monthly discharge only for some periods, published in wSP 1313.

REVISED RECORDS.--WSP 1243: 1931. WSP 1313: 1913.

GAGE.--Water-stage recorder. Elevation of gage is 5,280 ft above National Geodetic Vertical Datum of 1929 , from topographic map. Prior to Nov. 1, 1905, non-recording gage at old bridge 0.1 mi upstream at different datum. Sept. 17, 1912, to Oct. 4, 1938, water-stage recorder at site $0.8 \mathrm{mi}$ downstream at lower datums (datum lowered $2.0 \mathrm{ft}$ Aug. 15, 1927, and raised $0.2 \mathrm{ft}$ Dec. 16, 1929). Oct. 5, 1938 to Nov. 1 , 1973 at site $900 \mathrm{ft}$ downstream at datum $1.74 \mathrm{ft}$ lower.

REMARKS.--Diversions for irrigation of about 30,000 acres upstream from station.

AVERAGE DISCHARGE.--62 years (water years 1914, 1920-25, 1931-85), 881 $\mathrm{ft}^{3} / \mathrm{s}, 638,300 \mathrm{acre-ft/yr}$.

EXTREMES FOR PERIOD OF RECORD.--Maximum discharge, about 25,000 fts/s, June 29, 1927, gage height, 8.5 ft, site and datum then in use, from rating curve extended above $10,000 \mathrm{ft} / \mathrm{s} ; \mathrm{minimum,} 1.0 \mathrm{ft} / \mathrm{s}, \mathrm{Aug}$. 11 , 1972 .

EXTREMES OUTSIDE PERIOD OF RECORD.--Maximum flood occurred Oct. 6, 1911 , when a stage of about $16.5 \mathrm{ft}$ was reached (datum in use Oct. 1938 to Nov. 1973). Flood of sept. 6, 1909, reached a stage of 11.1 ft, 1904-5 site and datum (discharge, about $19,000 \mathrm{ft}^{3} / \mathrm{s}$ ).

STATISTICAL SUMMARIES

MEAN MONTHLY AND MEAN ANNUAL DISCHARGES $\begin{aligned} & 1914,1920-25, \\ & 1931-85\end{aligned}$

MAGNITUDE AND PROBABILITY OF ANNUAL LOW FLOW BASED ON PERIOD OF RECORD 1921-25, 1932-85

\begin{tabular}{|c|c|c|c|c|c|c|}
\hline MONTH & $\begin{array}{l}\text { MINIMUM } \\
\left(\mathrm{FT}^{3} / \mathrm{S}\right)\end{array}$ & $\begin{array}{c}\text { MAXIMUM } \\
\left(\mathrm{FT}^{3} / \mathrm{S}\right)\end{array}$ & $\begin{array}{l}\text { MEAN } \\
\left(\mathrm{FT}^{3} / \mathrm{S}\right)\end{array}$ & $\begin{array}{l}\text { STAN- } \\
\text { DARD } \\
\text { DEVIA- } \\
\text { TION } \\
\left(\mathrm{FT}^{3} / \mathrm{S}\right)\end{array}$ & $\begin{array}{l}\text { COEFFI- } \\
\text { CIENT OF } \\
\text { VARI- } \\
\text { ATION }\end{array}$ & $\begin{array}{c}\text { PERCENT } \\
\text { OF } \\
\text { ANNUAL } \\
\text { RUNOFF }\end{array}$ \\
\hline OCTOBER & 87 & 2726 & 421 & 377 & .90 & 4.0 \\
\hline NOVEMBER & 152 & 1140 & 335 & 158 & .47 & 3.2 \\
\hline DECEMBER & 174 & 565 & 285 & 83 & .29 & 2.7 \\
\hline JANUARY & 163 & 554 & 269 & 69 & .26 & 2.5 \\
\hline FEBRUARY & 162 & 675 & 292 & 88 & .30 & 2.8 \\
\hline MARCH & 112 & 875 & 432 & 186 & .43 & 4.1 \\
\hline APRIL, & 54 & 2489 & 998 & 554 & .56 & 9.5 \\
\hline MAY & 195 & 6126 & 2437 & 1229 & .50 & 23.1 \\
\hline JUNE & 235 & 6930 & 3040 & 1684 & .55 & 28.8 \\
\hline JULY & 46 & 3609 & 1136 & 873 & .77 & 10.8 \\
\hline AUGUST & 50 & 1971 & 488 & 381 & .78 & 4.6 \\
\hline SEPTEMBER & 11 & 2182 & 424 & 394 & .93 & 4.0 \\
\hline NN & 239 & 1733 & 881 & 377 & .43 & 100 \\
\hline
\end{tabular}

\begin{tabular}{|c|c|c|c|c|c|c|}
\hline $\begin{array}{l}\text { PERIOD } \\
\text { (CON- }\end{array}$ & DISC & $\begin{array}{l}\text { GE, I } \\
\text { TERVA } \\
\text { CEEDA }\end{array}$ & $\begin{array}{l}T^{3} / \mathrm{S}, \\
\text { IN YE } \\
\text { PROB }\end{array}$ & $\begin{array}{l}\text { IND } \\
\text { AND } \\
\text { I TY, }\end{array}$ & $\begin{array}{l}\text { TED R } \\
\text { NUAL } \\
\text { PERC }\end{array}$ & RRENC \\
\hline SECU- & & $\cdots-$ & $\ldots$ & $\cdots$ & $-\cdots$ & ---1 \\
\hline TIVE & 2 & 5 & 10 & 20 & 50 & 100 \\
\hline DAYS ) & 508 & $20 \%$ & $10 \%$ & 58 & 28 & 18 \\
\hline 1 & 74 & 23 & 11 & 5.7 & 2. & 1.4 \\
\hline 3 & 79 & 26 & 13 & 7.2 & 3. & 2.0 \\
\hline 7 & 91 & 33 & 18 & 10 & 5.3 & 3.3 \\
\hline 14 & 112 & 48 & 28 & 17 & 9. & 6.4 \\
\hline 30 & 157 & 77 & 48 & 31 & 18 & 12 \\
\hline 60 & 205 & 122 & 86 & 61 & 40 & 29 \\
\hline 90 & 238 & 164 & 127 & 99 & 73 & 58 \\
\hline 120 & 252 & 187 & 156 & 133 & 109 & 95 \\
\hline 183 & 268 & 203 & 179 & 163 & 148 & 139 \\
\hline
\end{tabular}

MAGNITUDE AND PROBABILITY OF ANNUAL HIGH FLOW BASED ON PERIOD OF RECORD 1914, 1920-25, $1931-85$

\begin{tabular}{|c|c|c|c|c|c|c|}
\hline $\begin{array}{l}\text { PERIOD } \\
\text { (CON- }\end{array}$ & DIS & $\begin{array}{l}\text { RGE, } \\
\text { INTE } \\
\text { XCEED }\end{array}$ & $\begin{array}{l}T^{3} / \mathrm{S} \\
\text { IN } \\
\text { PRO }\end{array}$ & $\begin{array}{l}\text { FOR IN } \\
\text { EARS, } \\
\text { BILI TY }\end{array}$ & $\begin{array}{l}\text { CATED } \\
\text { D ANNU } \\
\text { IN PER }\end{array}$ & \\
\hline $\begin{array}{l}\text { TIVE } \\
\text { DAYS }\end{array}$ & $\begin{array}{c}2 \\
508\end{array}$ & $\begin{array}{c}5 \\
208\end{array}$ & $\begin{array}{l}10 \\
10 \%\end{array}$ & $\begin{array}{l}25 \\
48\end{array}$ & $\begin{array}{l}50 \\
28\end{array}$ & $\begin{array}{r}100 \\
18\end{array}$ \\
\hline $\begin{array}{r}1 \\
3 \\
7 \\
15 \\
30 \\
60 \\
90\end{array}$ & $\begin{array}{l}5090 \\
4780 \\
4270 \\
3730 \\
3290 \\
2660 \\
2150\end{array}$ & $\begin{array}{l}7560 \\
7000 \\
6300 \\
5530 \\
4890 \\
4010 \\
3300\end{array}$ & $\begin{array}{l}9110 \\
8360 \\
7570 \\
6670 \\
5850 \\
4820 \\
4020\end{array}$ & $\begin{array}{r}11000 \\
9940 \\
9080 \\
8040 \\
6940 \\
5720 \\
4860\end{array}$ & $\begin{array}{r}12300 \\
11000 \\
10100 \\
9000 \\
7670 \\
6310 \\
5450\end{array}$ & $\begin{array}{r}13500 \\
12000 \\
11100 \\
9920 \\
8340 \\
6850 \\
6000\end{array}$ \\
\hline
\end{tabular}

DURATION TABLE OF DAILY MEAN FLOW FOR PERIOD OF RECORD 1914, 1920-25, 1931-85 DISCHARGE, IN $\mathrm{FT}^{3} / \mathrm{S}$, THAT WAS EOUALED OR EXCEEDED FOR INDICATED PERCENT OF TIME

\begin{tabular}{|c|c|c|c|c|c|c|c|c|c|c|c|c|c|c|}
\hline $5 \%$ & 108 & 158 & 208 & 258 & $30 \%$ & 408 & 508 & 608 & 708 & $75 \%$ & 808 & 858 & 908 & 958 \\
\hline 3610 & 2380 & 1690 & 1230 & 921 & 718 & 481 & 368 & 302 & 262 & 242 & 222 & 201 & 181 & 123 \\
\hline
\end{tabular}


SAN JUAN RIVER BASIN

09365000 SAN JUAN RIVER AT FARMINGTON, NM

LOCATION. --Lat $36^{\circ} 43^{\circ} 22^{\mathrm{n}}$, long $108^{\circ} 13^{\prime} 30^{\mathrm{n}}$, in NWłEk sec.17, T.29 N., R.13 w., San Juan County, Hydrologic Unit 14080105 , on left bank $360 \mathrm{ft}$ downstream from highway bridge on State Highway 371 in Farmington, $4,000 \mathrm{ft}$ downstream from Animas River, $2.3 \mathrm{mi}$ upstream from La Plata River, and at mile 251.4 .

DRAINAGE AREA. $-7,240 \mathrm{mi}^{2}$, approximately.

PERIOD OF RECORD.--June to December 1904, January 1905 to September 1906 (gage heights and discharge measurements only), September 1912 to current year. Monthly discharge only for some periods, published in wSP 1313. Discharge records for January to December 1905, published in wSP 175, are unreliable and should not be used.

REVISED RECORDS.--WSP 1119: Drainage area. WSP 1243: 1938. WSP 1313: 1905, 1914. See also PERIOD OF RECORD.

GAGE.--Water-stage recorder. Datum of gage is 5,230.37 ft above National Geodetic Vertical Datum of 1929 . See wSP 1313 or 1733 for history of changes prior to Nov. 19, 1933.

REMARKS.--Since June 1962 flow is partly controlled by operation of Navajo Reservoir (station 09355100 ) $50 \mathrm{mi}$ upstream. Diversions upstream from station for irrigation of about 86,000 acres, 4,000 of which is irrigated upstream. Diversions upstream from station for irrigation of about 86,000 acres, 4 , 00 of which is irrigated record. At times this ditch may be supplied partly or entirely by diversion from San Juan River downstream from this station. National Weather Service gage-height telemeter at station.

AVERAGE DISCHARGE. - -32 years (water years 1931-62), 2,205 $\mathrm{ft}^{3} / \mathrm{s}, 1,598,000 \mathrm{acre}-\mathrm{ft} / \mathrm{yr}$.

EXTREMES FOR PERIOD OF RECORD.--Maximum discharge, about $68,000 \mathrm{ft} 3 / \mathrm{s}$, June 29,1927 , gage height, $10.2 \mathrm{ft}, \mathrm{site}$ and datum then in use, from rating curve extended above $37,000 \mathrm{ft}^{3} / \mathrm{s} ; \mathrm{minimum}, 14 \mathrm{ft} 3 / \mathrm{s}, \mathrm{Aug} .22,1939$.

EXTREMES OUTSIDE PERIOD OF RECORD.--Maximum flood occurred Oct. 6, 1911. Flood of Sept. 6, 1909, reached a stage of about $12.3 \mathrm{ft}$, site and datum in use May to September 1906.

STATISTICAL SUMMARIES (PERIOD BEFORE REGULATION OF NAVAJO DAM)

MEAN MONTHLY AND MEAN ANNUAL DISCHARGES 1931-62

MAGNITUDE AND PROBABILITY OF ANNUAL LOW FLOW BASED ON PERIOD OF RECORD 1932-62

\begin{tabular}{|c|c|c|c|c|c|c|}
\hline MONTH & $\begin{array}{l}\text { MINIMUM } \\
\left(\mathrm{FT}^{3} / \mathrm{S}\right)\end{array}$ & $\begin{array}{c}\text { MAXIMUM } \\
\left(\mathrm{FT}^{3} / \mathrm{S}\right)\end{array}$ & $\begin{array}{l}\text { MEAN } \\
\left(\mathrm{FT}^{3} / \mathrm{S}\right)\end{array}$ & $\begin{array}{l}\text { STAN- } \\
\text { DARD } \\
\text { DEVIA- } \\
\text { TION } \\
\left(\mathrm{FT}^{3} / \mathrm{S}\right)\end{array}$ & $\begin{array}{l}\text { COEFFI- } \\
\text { CIENT OF } \\
\text { VARI - } \\
\text { ATION }\end{array}$ & $\begin{array}{c}\text { PERCENT } \\
\text { OF } \\
\text { ANNUAL } \\
\text { RUNOF F }\end{array}$ \\
\hline $\begin{array}{l}\text { OCTOBER } \\
\text { NOVEMBER } \\
\text { DECEMBER } \\
\text { JANUARY } \\
\text { FEBRUARY } \\
\text { MARCH } \\
\text { APRIL } \\
\text { MAY } \\
\text { JUNE } \\
\text { JULY } \\
\text { AUGUST } \\
\text { SEPTEMBER }\end{array}$ & $\begin{array}{r}286 \\
315 \\
362 \\
375 \\
444 \\
453 \\
702 \\
2067 \\
517 \\
192 \\
166 \\
170\end{array}$ & $\begin{array}{r}7271 \\
2729 \\
1403 \\
1121 \\
1931 \\
4287 \\
9133 \\
18830 \\
14990 \\
8639 \\
4938 \\
2620\end{array}$ & $\begin{array}{r}1106 \\
722 \\
568 \\
556 \\
773 \\
1464 \\
3942 \\
6395 \\
6592 \\
2222 \\
1145 \\
959\end{array}$ & $\begin{array}{r}1223 \\
468 \\
229 \\
153 \\
356 \\
915 \\
2559 \\
3679 \\
4136 \\
2174 \\
958 \\
654\end{array}$ & $\begin{array}{l}1.11 \\
.65 \\
.40 \\
.28 \\
.46 \\
.63 \\
.65 \\
.58 \\
.63 \\
.98 \\
.84 \\
.68\end{array}$ & $\begin{array}{r}4.2 \\
2.7 \\
2.1 \\
2.1 \\
2.9 \\
5.5 \\
14.9 \\
24.2 \\
24.9 \\
8.4 \\
4.3 \\
3.6\end{array}$ \\
\hline ANNUAL & 843 & 5054 & 2205 & 1090 & .49 & 100 \\
\hline
\end{tabular}

\begin{tabular}{|c|c|c|c|c|c|c|}
\hline \multirow{2}{*}{$\begin{array}{l}\text { PERIOD } \\
\text { (CON- } \\
\text { SECU- } \\
\text { TIVE } \\
\text { DAYS) }\end{array}$} & \multicolumn{6}{|c|}{$\begin{array}{l}\text { DISCHARGE, IN FT }{ }^{3} / S \text {, FOR INDICATED RECURRENCE } \\
\text { INTERVAL, IN YEARS, AND ANNUAL NON- } \\
\text { EXCEEDANCE PROBABILITY, IN PERCENT }\end{array}$} \\
\hline & $\begin{array}{c}2 \\
508\end{array}$ & $\begin{array}{c}5 \\
208\end{array}$ & $\begin{array}{l}10 \\
108\end{array}$ & $\begin{array}{l}20 \\
58\end{array}$ & $\begin{array}{l}50 \\
28\end{array}$ & $\begin{array}{r}100 \\
18\end{array}$ \\
\hline $\begin{array}{r}1 \\
3 \\
7 \\
14 \\
30 \\
60 \\
90 \\
120 \\
183\end{array}$ & $\begin{array}{l}235 \\
243 \\
266 \\
306 \\
370 \\
457 \\
490 \\
536 \\
599\end{array}$ & $\begin{array}{l}109 \\
115 \\
129 \\
155 \\
229 \\
312 \\
376 \\
424 \\
460\end{array}$ & $\begin{array}{r}66 \\
72 \\
81 \\
100 \\
172 \\
249 \\
331 \\
383 \\
418\end{array}$ & $\begin{array}{r}41 \\
46 \\
53 \\
67 \\
133 \\
203 \\
299 \\
356 \\
393\end{array}$ & $\begin{array}{r}23 \\
26 \\
31 \\
40 \\
98 \\
160 \\
268 \\
332 \\
373\end{array}$ & $\begin{array}{l}-- \\
-- \\
-- \\
-- \\
-- \\
-- \\
-- \\
--\end{array}$ \\
\hline
\end{tabular}

MAGNITUDE AND PROBABILITY OF ANNUAL HIGH FLOW BASED ON PERIOD OF RECORD 1931-62

\begin{tabular}{|c|c|c|c|c|c|c|}
\hline \multirow{2}{*}{$\begin{array}{l}\text { PERIOD } \\
\text { (CON- } \\
\text { SECU- } \\
\text { TIVE } \\
\text { DAYS) }\end{array}$} & \multicolumn{6}{|c|}{$\begin{array}{l}\text { DISCHARGE, IN FT' }{ }^{3} / \mathrm{S}, \text { FOR INDICATED RECURRENCI } \\
\text { INTERVAL, IN YEARS, AND ANNUAL } \\
\text { EXCEEDANCE PROBABILITY, IN PERCENT }\end{array}$} \\
\hline & $\begin{array}{c}2 \\
508\end{array}$ & $\begin{array}{c}5 \\
208\end{array}$ & $\begin{array}{l}10 \\
108\end{array}$ & $\begin{array}{l}25 \\
48\end{array}$ & $\begin{array}{l}50 \\
28\end{array}$ & $\begin{array}{r}100 \\
18\end{array}$ \\
\hline $\begin{array}{r}1 \\
3 \\
7 \\
15 \\
30 \\
60 \\
90\end{array}$ & $\begin{array}{r}10900 \\
10200 \\
9230 \\
8270 \\
7360 \\
6050 \\
5110\end{array}$ & $\begin{array}{r}17300 \\
15800 \\
14300 \\
12900 \\
11300 \\
9540 \\
8230\end{array}$ & $\begin{array}{l}22100 \\
20000 \\
18100 \\
16300 \\
14200 \\
12100 \\
10500\end{array}$ & $\begin{array}{l}28800 \\
25700 \\
23500 \\
21200 \\
18200 \\
15700 \\
13700\end{array}$ & $\begin{array}{l}34300 \\
30300 \\
27800 \\
25100 \\
21300 \\
18500 \\
16200\end{array}$ & $\begin{array}{l}-- \\
-- \\
-- \\
-- \\
-- \\
--\end{array}$ \\
\hline
\end{tabular}

DURATION TABLE OF DAILY MEAN FLOW FOR PERIOD OF RECORD 1931-62 DISCHARGE, IN FT'/S, THAT WAS EQUALED OR EXCEEDED FOR INDICATED PERCENT OF TIME

\begin{tabular}{|c|c|c|c|c|c|c|c|c|c|c|c|c|c|c|}
\hline 58 & 108 & 158 & 208 & 258 & $30 \%$ & 408 & 508 & 608 & 708 & 758 & $80 \%$ & 858 & 908 & 958 \\
\hline 9250 & 6130 & 4430 & 3280 & 2430 & 1820 & 1180 & 851 & 670 & 564 & 519 & 478 & 437 & 382 & 284 \\
\hline
\end{tabular}


SAN JUAN RIVER BASIN

09365000 SAN JUAN RIVER AT FARMINGTON, NM--Continued STATISTICAL SUMMARIES (PERIOD AFTER COMPLETION OF NAVAJO DAM)

MEAN MONTHLY AND MEAN ANNUAL DISCHARGES 1964-85

\begin{tabular}{|c|c|c|c|c|c|c|}
\hline MONTH & $\begin{array}{l}\text { MINIMUM } \\
\left(\mathrm{FT}^{3} / \mathrm{S}\right)\end{array}$ & $\begin{array}{c}\text { MAXIMUM } \\
\left(\mathrm{FT}^{3} / \mathrm{S}\right)\end{array}$ & $\begin{array}{l}\text { MEAN } \\
\left(\mathrm{FT}^{3} / \mathrm{S}\right)\end{array}$ & $\begin{array}{l}\text { STAN- } \\
\text { DARD } \\
\text { DEVIA- } \\
\text { TION } \\
\left(\mathrm{FT}^{3} / \mathrm{S}\right)\end{array}$ & $\begin{array}{l}\text { COEFFI- } \\
\text { CIENT OF } \\
\text { VARI- } \\
\text { ATION }\end{array}$ & $\begin{array}{c}\text { PERCENT } \\
\text { OF } \\
\text { ANNUAL } \\
\text { RUNOFF }\end{array}$ \\
\hline $\begin{array}{l}\text { OCTOBER } \\
\text { NOVEMBER } \\
\text { DECEMBER } \\
\text { JANUARY } \\
\text { FEBRUARY } \\
\text { MARCH } \\
\text { APRIL } \\
\text { MAY } \\
\text { JUNE } \\
\text { JULY } \\
\text { AUGUST } \\
\text { SEPTEMBER }\end{array}$ & $\begin{array}{l}409 \\
425 \\
504 \\
476 \\
374 \\
349 \\
391 \\
576 \\
983 \\
512 \\
440 \\
478\end{array}$ & $\begin{array}{l}2785 \\
3532 \\
3381 \\
3184 \\
2733 \\
3323 \\
6844 \\
8840 \\
9803 \\
6856 \\
3768 \\
3331\end{array}$ & $\begin{array}{l}1359 \\
1242 \\
1444 \\
1558 \\
1641 \\
1669 \\
2145 \\
3643 \\
4196 \\
2350 \\
1498 \\
1380\end{array}$ & $\begin{array}{r}708 \\
751 \\
730 \\
698 \\
721 \\
787 \\
1673 \\
2151 \\
2584 \\
1881 \\
857 \\
867\end{array}$ & $\begin{array}{l}.52 \\
.60 \\
.51 \\
.45 \\
.44 \\
.47 \\
.78 \\
.59 \\
.62 \\
.80 \\
.57 \\
.63\end{array}$ & $\begin{array}{r}5.6 \\
5.1 \\
6.0 \\
6.5 \\
6.8 \\
6.9 \\
8.9 \\
15.1 \\
17.4 \\
9.7 \\
6.2 \\
5.7\end{array}$ \\
\hline NNUAL & 859 & 3633 & 2011 & 820 & .41 & 100 \\
\hline
\end{tabular}

MAGNITUDE AND PROBABILITY OF ANNUAL LOW FLON BASED ON PERIOD OF RECORD $1965-85$

\begin{tabular}{|c|c|c|c|c|c|c|}
\hline \multirow{2}{*}{$\begin{array}{l}\text { PERIOD } \\
\text { (CON- } \\
\text { SECU- } \\
\text { TIVE } \\
\text { DAYS) }\end{array}$} & \multicolumn{6}{|c|}{$\begin{array}{l}\text { DISCHARGE, IN } \text { FT }^{3} / \mathrm{S} \text {, FOR INDICATED RECURRENCE } \\
\text { INTERVAL, IN YEARS, AND ANNUAL NON- } \\
\text { EXCEEDANCE PROBABILITY, IN PERCENT }\end{array}$} \\
\hline & $\begin{array}{c}2 \\
508\end{array}$ & $\begin{array}{c}5 \\
208\end{array}$ & $\begin{array}{l}10 \\
108\end{array}$ & $\begin{array}{l}20 \\
58\end{array}$ & $\begin{array}{l}50 \\
28\end{array}$ & $\begin{array}{r}100 \\
18\end{array}$ \\
\hline $\begin{array}{r}1 \\
3 \\
7 \\
14 \\
30 \\
60 \\
90 \\
120 \\
183\end{array}$ & $\begin{array}{r}435 \\
472 \\
526 \\
592 \\
684 \\
812 \\
906 \\
1010 \\
1180\end{array}$ & $\begin{array}{l}305 \\
324 \\
352 \\
399 \\
468 \\
551 \\
613 \\
680 \\
827\end{array}$ & $\begin{array}{l}258 \\
272 \\
292 \\
332 \\
392 \\
458 \\
509 \\
560 \\
690\end{array}$ & $\begin{array}{l}227 \\
239 \\
253 \\
287 \\
342 \\
396 \\
440 \\
480 \\
596\end{array}$ & $\begin{array}{l}=- \\
=- \\
=- \\
-- \\
-- \\
-- \\
--\end{array}$ & $\begin{array}{l}-- \\
-- \\
-- \\
-- \\
-- \\
-- \\
--\end{array}$ \\
\hline
\end{tabular}

MAGNITUDE AND PROBABILITY OF ANNUAL HIGH FLOW BASED ON PERIOD OF RECORD 1964-85

\begin{tabular}{|c|c|c|c|c|c|c|}
\hline \multirow{2}{*}{$\begin{array}{l}\text { PERIOD } \\
\text { (CON- } \\
\text { SECU- } \\
\text { TIVE } \\
\text { DAYS) }\end{array}$} & \multicolumn{6}{|c|}{$\begin{array}{l}\text { DISCHARGE, IN FT3/S, FOR INDICATED RECURRENC } \\
\text { INTERVAL, IN YEARS, AND ANNUAL } \\
\text { EXCEEDANCE PROBABILITY, IN PERCENT }\end{array}$} \\
\hline & $\begin{array}{c}2 \\
508\end{array}$ & $\begin{array}{c}5 \\
208\end{array}$ & $\begin{array}{l}10 \\
108\end{array}$ & $\begin{array}{l}25 \\
48\end{array}$ & $\begin{array}{l}50 \\
28\end{array}$ & $\begin{array}{r}100 \\
18\end{array}$ \\
\hline $\begin{array}{r}1 \\
3 \\
7 \\
15 \\
30 \\
60 \\
90\end{array}$ & $\begin{array}{l}6390 \\
5860 \\
5410 \\
4890 \\
4240 \\
3640 \\
3240\end{array}$ & $\begin{array}{l}9460 \\
8860 \\
8300 \\
7570 \\
6710 \\
5800 \\
5150\end{array}$ & $\begin{array}{r}11500 \\
10900 \\
10300 \\
9400 \\
8490 \\
7410 \\
6600\end{array}$ & $\begin{array}{r}14000 \\
13600 \\
12800 \\
11700 \\
10900 \\
9640 \\
8620\end{array}$ & $\begin{array}{l}-- \\
-- \\
-- \\
-- \\
-- \\
--\end{array}$ & $\begin{array}{l}-- \\
-- \\
-- \\
=- \\
-- \\
--\end{array}$ \\
\hline
\end{tabular}

DURATION TABLE OF DAILY MEAN FLOW FOR PERIOD OF RECORD 1964-85

DISCHARGE, IN FT ${ }^{3} / \mathrm{S}$, THAT WAS EQUALED OR EXCEEDED FOR INDICATED PERCENT OF TIME

\begin{tabular}{|c|c|c|c|c|c|c|c|c|c|c|c|c|c|c|}
\hline 58 & 108 & 158 & 208 & 258 & 308 & 408 & 508 & 608 & 708 & 758 & 808 & 858 & 908 & 958 \\
\hline 5740 & 3970 & 3260 & 2810 & 2430 & 2200 & 1910 & 1570 & 1260 & 921 & 822 & 732 & 638 & 547 & 448 \\
\hline
\end{tabular}


SAN JUAN RIVER BASIN

09366500 LA PLATA RIVER AT COLORADO-NEW MEXICO STATE LINE

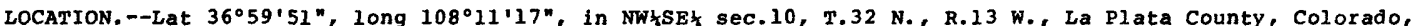
Hydrologic Unit 14080105 , on right bank at Colorado-New Mexico State line, 0.2 mi downstream from Ponds Arroyo, and $4.8 \mathrm{mi}$ north of La Plata, NM.

DRAINAGE AREA. $--331 \mathrm{mi}^{2}$.

PERIOD OF RECORD.--January 1920 to current year. Monthly discharge only for some periods, published in wSP 1313. REVISED RECORDS.--WSP 1313: $1934(\mathrm{M}), 1936(\mathrm{M})$.

GAGE.--Water-stage recorder. Datum of gage is 5,975.15 ft above National Geodetic Vertical Datum of 1929 . See WSP 1713 or 1733 for history of changes prior to Mar. 17, 1934.

REMARKS.--Diversions upstream from station for irrigation of about 15,000 acres, most of which are upstream from station.

COOPERATION.--Records collected and computed by Colorado Division of Water Resources and reviewed by Geological Survey.

AVERAGE DISCHARGE. --65 years (water years 1921-85), 35.6 ft $/ \mathrm{s}, 25,790$ acre-ft/yr.

EXTREMES FOR PERIOD OF RECORD.--Maximum discharge, 4,750 $\mathrm{ft}^{3} / \mathrm{s}$, Aug. 24, 1927, gage height, $11.36 \mathrm{ft}$, present datum, from rating curve extended above $750 \mathrm{ft} / \mathrm{s}$ on basis of slope-area measurement of peak flow; no flow at times in many years.

STATISTICAL SUMMARIES

MEAN MONTHLY AND MEAN ANNUAL DISCHARGES 1921-85

\begin{tabular}{|c|c|c|c|c|c|c|}
\hline MONTH & $\begin{array}{c}\text { MINIMUM } \\
\left(\mathrm{FT}^{3} / \mathrm{S}\right)\end{array}$ & $\begin{array}{l}\text { MAXIMUM } \\
\left(\mathrm{FT}^{3} / \mathrm{S}\right)\end{array}$ & $\begin{array}{l}\text { MEAN } \\
\left(\mathrm{FT}^{3} / \mathrm{S}\right)\end{array}$ & $\begin{array}{l}\text { STAN- } \\
\text { DARD } \\
\text { DEVIA- } \\
\text { TION } \\
\text { (FT'/S) }\end{array}$ & $\begin{array}{l}\text { COEFFI- } \\
\text { CIENT OF } \\
\text { VARI- } \\
\text { ATION }\end{array}$ & $\begin{array}{c}\text { PERCENT } \\
\text { OF } \\
\text { ANNUAL } \\
\text { RUNOFF }\end{array}$ \\
\hline \multicolumn{6}{|l|}{ 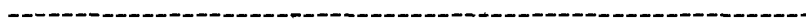 } & 3.2 \\
\hline NOVEMBER & 1.0 & 99 & 11 & 13 & 1.25 & 2.5 \\
\hline DECBMBER & 1.2 & 34 & 11 & 7.6 & .67 & 2.6 \\
\hline JANUARY & .8 & 38 & 11 & 6.9 & .62 & 2.6 \\
\hline FEBRUARY & 3.0 & 54 & 16 & 9.9 & .62 & 3.7 \\
\hline MARCH & .6 & 97 & 32 & 25 & .78 & 7.6 \\
\hline APRIL & 3.1 & 364 & 108 & 99 & .92 & 25.3 \\
\hline MAY & 5.3 & 506 & 113 & 102 & .90 & 26.5 \\
\hline JUNE & 1.9 & 306 & 68 & 68 & 1.01 & 15.8 \\
\hline JULY & 0.0 & 99 & 20 & 21 & 1.01 & 4.8 \\
\hline $\begin{array}{l}\text { AUGUST } \\
\text { P }\end{array}$ & 0.0 & $\begin{array}{r}65 \\
126\end{array}$ & 12 & 13 & 1.07 & 2.9 \\
\hline & & & & & & \\
\hline ANNUAL & 4.4 & 109 & 36 & 25 & .69 & 100 \\
\hline
\end{tabular}

MAGNITUDE AND PROBABILITY OF ANNUAL LOW FLOW BASED ON PERIOD OF RECORD 1922-85

\begin{tabular}{|c|c|c|c|c|c|c|}
\hline \multirow{2}{*}{$\begin{array}{l}\text { PERIOD } \\
\text { (CON- } \\
\text { SECU- } \\
\text { TIVE } \\
\text { DAYS) }\end{array}$} & \multicolumn{6}{|c|}{$\begin{array}{l}\text { DISCHARGE, IN FT'3/S, FOR INDICATED RECURRENCE } \\
\text { INTERVAL, IN YEARS, AND ANNUAL NON- } \\
\text { EXCEEDANCE PROBABILITY, IN PERCENT }\end{array}$} \\
\hline & $\begin{array}{c}2 \\
508\end{array}$ & $\begin{array}{c}5 \\
208\end{array}$ & $\begin{array}{l}10 \\
108\end{array}$ & $\begin{array}{l}20 \\
58\end{array}$ & $\begin{array}{l}50 \\
28\end{array}$ & \\
\hline $\begin{array}{r}1 \\
3 \\
7 \\
14 \\
30 \\
60 \\
90 \\
120 \\
183\end{array}$ & $\begin{array}{r}0.0 \\
.1 \\
.5 \\
1.1 \\
2.1 \\
3.9 \\
5.2 \\
5.6 \\
6.9\end{array}$ & $\begin{array}{r}0.0 \\
0.0 \\
0.0 \\
.2 \\
.5 \\
1.3 \\
1.9 \\
2.5 \\
3.5\end{array}$ & $\begin{array}{r}0.0 \\
0.0 \\
0.0 \\
0.0 \\
.1 \\
.5 \\
.9 \\
1.6 \\
2.5\end{array}$ & $\begin{array}{r}0.0 \\
0.0 \\
0.0 \\
0.0 \\
0.0 \\
.2 \\
.5 \\
1.0 \\
1.9\end{array}$ & $\begin{array}{r}0.0 \\
0.0 \\
0.0 \\
0.0 \\
0.0 \\
0.0 \\
.2 \\
.6 \\
1.3\end{array}$ & $\begin{array}{r}0.0 \\
0.0 \\
0.0 \\
0.0 \\
0.0 \\
0.0 \\
.1\end{array}$ \\
\hline
\end{tabular}

MAGNITUDE AND PROBABILITY OF ANNUAL HIGH FLOW BASED ON PERIOD OF RECORD 1921-85

\begin{tabular}{|c|c|c|c|c|c|c|}
\hline \multirow{2}{*}{$\begin{array}{l}\text { PERIOD } \\
\text { (CON- } \\
\text { SECU- } \\
\text { TIVE } \\
\text { DAYS) }\end{array}$} & \multicolumn{6}{|c|}{$\begin{array}{c}\text { DISCHARGE, IN FT'/S, FOR INDICATED RECURRENCE } \\
\text { INTERVAL, IN YEARS, AND ANNUAL } \\
\text { EXCEEDANCE PROBABILITY, IN PERCENT }\end{array}$} \\
\hline & $\begin{array}{c}2 \\
508\end{array}$ & $\begin{array}{c}5 \\
208\end{array}$ & $\begin{array}{l}10 \\
108\end{array}$ & $\begin{array}{l}25 \\
48\end{array}$ & $\begin{array}{l}50 \\
28\end{array}$ & $\begin{array}{r}100 \\
18\end{array}$ \\
\hline $\begin{array}{r}1 \\
3 \\
7 \\
15 \\
30 \\
60 \\
90\end{array}$ & $\begin{array}{r}299 \\
224 \\
184 \\
145 \\
116 \\
91 \\
76\end{array}$ & $\begin{array}{l}542 \\
422 \\
353 \\
287 \\
231 \\
181 \\
149\end{array}$ & $\begin{array}{l}735 \\
590 \\
500 \\
415 \\
333 \\
260 \\
210\end{array}$ & $\begin{array}{r}1010 \\
848 \\
731 \\
618 \\
496 \\
381 \\
303\end{array}$ & $\begin{array}{r}1240 \\
1080 \\
937 \\
804 \\
643 \\
488 \\
383\end{array}$ & $\begin{array}{r}1490 \\
1330 \\
1170 \\
1020 \\
815 \\
610 \\
472\end{array}$ \\
\hline
\end{tabular}

DURATION TABLE OF DAILY MEAN FLOW FOR PERIOD OF RECORD 1921-85 DISCHARGE, IN FT'/S, THAT WAS EQUALED OR EXCEEDED FOR INDICATED PERCENT OF TIME

\begin{tabular}{|c|c|c|c|c|c|c|c|c|c|c|c|c|c|c|}
\hline 58 & 108 & 158 & 208 & 258 & 308 & 408 & 508 & 608 & 708 & 758 & 808 & 858 & 908 & 958 \\
\hline 160 & 88 & 63 & 45 & 32 & 24 & 16 & 12 & 8.0 & 5.2 & 4.3 & 3.3 & 2.4 & 1.5 & 3 \\
\hline
\end{tabular}


SAN JUAN RIVER BASIN

09367500 LA PLATA RIVER NEAR FARMINGTON, NM

LOCATION.--Lat $36^{\circ} 44^{\prime} 23^{\prime \prime}$, long $108^{\circ} 14^{\prime} 51^{\prime \prime}$, in NEłSW sec.7, T.29 N., R.13 w. San Juan County, Hydrologic Unit 14080105 , on $r$ ight bank $1,300 \mathrm{ft}$ upstream from bridge on U.S. Highway 550 in Farmington, and 1,800 ft upstream from mouth.

DRAINAGE AREA. $--583 \mathrm{mi}^{2}$.

PERIOD OF RECORD.--March 1938 to current year.

REVISED RECORDS.--WSP 1243: 1944-45. WSP 1313: 1943-44(M), 1946-50(M). WSP 1733: 1951 (M).

GAGE.-Water-stage recorder. Elevation of gage is 5,210 ft above National Geodetic Vertical Datum of 1929 , from river-profile map. Prior to July 28,1978 at elevation $1.0 \mathrm{ft}$ higher.

REMARRS.--Diversions for irrigation of about 24,000 acres upstream from station.

AVERAGE DISCHARGE. --47 years (water years 1939-85), $27.8 \mathrm{ft}^{3} / \mathrm{s}, 10,140 \mathrm{acre-ft} / \mathrm{yr}$.

EXTREMES FOR PERIOD OF RECORD.--Maximum discharge, that of sept. 10, 1939, "discharge not determined", gage height,

$6.03 \mathrm{ft}$, site and datum then in use; no flow for long periods in some years.

Major floods occurred sept. 5 or 6,1909 , and oct. 5 or 6, 1911 .

STATISTICAL SUMMARIES

MEAN MONTHLY AND MEAN ANNUAL DISCHARGES 1939-85

\begin{tabular}{|c|c|c|c|c|c|c|}
\hline MONTH & $\begin{array}{l}\text { MINIMUM } \\
\left(\mathrm{FT}^{3} / \mathrm{S}\right)\end{array}$ & $\begin{array}{l}\text { MAXIMUM } \\
\left(\mathrm{FT}^{3} / \mathrm{S}\right)\end{array}$ & $\begin{array}{l}\text { MEAN } \\
\left(\mathrm{FT}^{3} / \mathrm{S}\right)\end{array}$ & $\begin{array}{l}\text { STAN- } \\
\text { DARD } \\
\text { DEVIA- } \\
\text { TION } \\
\left(\mathrm{FT}^{3} / \mathrm{S}\right)\end{array}$ & $\begin{array}{l}\text { COEFFI- } \\
\text { CIENT OF } \\
\text { VARI- } \\
\text { ATION }\end{array}$ & $\begin{array}{c}\text { PERCENT } \\
\text { OF } \\
\text { ANNUAL } \\
\text { RUNOFF }\end{array}$ \\
\hline $\begin{array}{l}\text { OCTOBER } \\
\text { NOVEMBER } \\
\text { DECEMBER } \\
\text { JANUARY } \\
\text { FEBRUARY } \\
\text { MARCH } \\
\text { APRIL } \\
\text { MAY } \\
\text { JUNE } \\
\text { JULY } \\
\text { AUGUST } \\
\text { SEPTEMBER }\end{array}$ & $\begin{array}{l}0.0 \\
0.0 \\
0.0 \\
0.0 \\
1.0 \\
.0 \\
0.0 \\
0.0 \\
0.0 \\
0.0 \\
0.0 \\
0.0\end{array}$ & $\begin{array}{r}537 \\
116 \\
49 \\
100 \\
89 \\
111 \\
408 \\
783 \\
252 \\
47 \\
65 \\
170\end{array}$ & $\begin{array}{l}22 \\
9.2 \\
11 \\
16 \\
22 \\
29 \\
85 \\
73 \\
36 \\
8.0 \\
12 \\
11\end{array}$ & $\begin{array}{r}79 \\
18 \\
12 \\
16 \\
19 \\
32 \\
116 \\
143 \\
68 \\
11 \\
15 \\
28\end{array}$ & $\begin{array}{l}3.68 \\
1.95 \\
1.02 \\
1.02 \\
.89 \\
1.12 \\
1.36 \\
1.97 \\
1.88 \\
1.37 \\
1.23 \\
2.44\end{array}$ & $\begin{array}{r}6.5 \\
2.7 \\
3.4 \\
4.8 \\
6.5 \\
8.5 \\
25.6 \\
21.7 \\
10.8 \\
2.4 \\
3.7 \\
3.4\end{array}$ \\
\hline INU? & .5 & 134 & 28 & 32 & 1.16 & 100 \\
\hline
\end{tabular}

MAGNITUDE AND PROBABILITY OF ANNUAL LOW FLOW BASED ON PERIOD OF RECORD $1940-85$

\begin{tabular}{|c|c|c|c|c|c|c|}
\hline \multirow{2}{*}{$\begin{array}{l}\text { PERIOD } \\
\text { (CON- } \\
\text { SECU- } \\
\text { TIVE } \\
\text { DAYS) }\end{array}$} & \multicolumn{6}{|c|}{$\begin{array}{l}\text { DISCHARGE, IN FT } 3 / S \text {, FOR INDICATED RECURRENCE } \\
\text { INTERVAL, IN YEARS, AND ANNUAL NON- } \\
\text { EXCEEDANCE PROBABILITY, IN PERCENT }\end{array}$} \\
\hline & $\begin{array}{c}2 \\
508\end{array}$ & $\begin{array}{c}5 \\
208\end{array}$ & $\begin{array}{l}10 \\
108\end{array}$ & $\begin{array}{l}20 \\
58\end{array}$ & $\begin{array}{l}50 \\
28\end{array}$ & $\begin{array}{r}100 \\
18\end{array}$ \\
\hline $\begin{array}{r}1 \\
3 \\
7 \\
14 \\
30 \\
60 \\
90 \\
120 \\
183\end{array}$ & $\begin{array}{r}0.0 \\
0.0 \\
0.0 \\
0.0 \\
.1 \\
.5 \\
1.1 \\
1.7 \\
4.0\end{array}$ & $\begin{array}{r}0.0 \\
0.0 \\
0.0 \\
0.0 \\
0.0 \\
0.0 \\
.2 \\
.4 \\
1.4\end{array}$ & $\begin{array}{r}0.0 \\
0.0 \\
0.0 \\
0.0 \\
0.0 \\
0.0 \\
.1 \\
.1 \\
.8\end{array}$ & $\begin{array}{l}0.0 \\
0.0 \\
0.0 \\
0.0 \\
0.0 \\
0.0 \\
0.0 \\
0.0 \\
.5\end{array}$ & $\begin{array}{r}0.0 \\
0.0 \\
0.0 \\
0.0 \\
0.0 \\
0.0 \\
0.0 \\
0.0 \\
.3\end{array}$ & $\begin{array}{r}0.0 \\
0.0 \\
0.0 \\
0.0 \\
0.0 \\
0.0 \\
0.0 \\
0.0 \\
.2\end{array}$ \\
\hline
\end{tabular}

MAGNITUDE AND PROBABILITY OF ANNUAL HIGH FLOW BASED ON PERIOD OF RECORD 1939-85

\begin{tabular}{|c|c|c|c|c|c|c|}
\hline \multirow{2}{*}{$\begin{array}{l}\text { PERIOD } \\
\text { (CON- } \\
\text { SECU- } \\
\text { TIVE } \\
\text { DAYS) }\end{array}$} & \multicolumn{6}{|c|}{$\begin{array}{l}\text { DISCHARGE, IN FT' }{ }^{3} / \mathrm{S} \text {, FOR INDICATED RECURRENCE } \\
\text { INTERVAL, IN YEARS, AND ANNUAL } \\
\text { EXCEEDANCE } \text { PROBABILITY, IN PERCENT }\end{array}$} \\
\hline & $\begin{array}{c}2 \\
508\end{array}$ & $\begin{array}{c}5 \\
208\end{array}$ & $\begin{array}{l}10 \\
108\end{array}$ & $\begin{array}{l}25 \\
48\end{array}$ & $\begin{array}{l}50 \\
28\end{array}$ & $\begin{array}{r}100 \\
18\end{array}$ \\
\hline $\begin{array}{r}1 \\
3 \\
7 \\
15 \\
30 \\
60 \\
90\end{array}$ & $\begin{array}{r}335 \\
227 \\
156 \\
103 \\
72 \\
48 \\
38\end{array}$ & $\begin{array}{l}712 \\
510 \\
380 \\
279 \\
205 \\
142 \\
112\end{array}$ & $\begin{array}{r}1040 \\
758 \\
592 \\
470 \\
354 \\
250 \\
196\end{array}$ & $\begin{array}{r}1520 \\
1130 \\
934 \\
818 \\
634 \\
458 \\
352\end{array}$ & $\begin{array}{r}1930 \\
1460 \\
1240 \\
1170 \\
926 \\
675 \\
511\end{array}$ & $\begin{array}{r}2380 \\
1810 \\
1600 \\
1620 \\
1300 \\
957 \\
713\end{array}$ \\
\hline
\end{tabular}

DURATION TABLE OF DAILY MEAN FLOW FOR PERIOD OF RECORD 1939-85

DISCHARGE, IN FT $3 / S$, THAT WAS EQUALED OR EXCEEDED FOR INDICATED PERCENT OF TIME

\begin{tabular}{|c|c|c|c|c|c|c|c|c|c|c|c|c|c|c|}
\hline 58 & 108 & $15 \%$ & 208 & 258 & 308 & 408 & 508 & 608 & 708 & 758 & 808 & 858 & 908 & 958 \\
\hline 143 & 62 & 36 & 25 & 18 & 13 & 7.0 & 3.5 & 1.6 & .9 & .4 & 0.0 & 0.0 & 0.0 . & 0.0 \\
\hline
\end{tabular}


SAN JUAN RIVER BASIN

09368000 SAN JUAN RIVER AT SHIPROCK, NM

(National stream-quality accounting network, surveillance network, and radiochemical network station)

LOCATION.--Lat $36^{\circ} 47^{\prime} 32^{\prime \prime}$, long $108^{\circ} 43^{\circ} 54^{\prime \prime}$, in NWt sec.27, T. 30 N., R.18 w., San Juan County, Hydrologic Unit

14080105, on left bank $3 \mathrm{mi}$ west of Shiprock, $6 \mathrm{mi}$ downstream from Chaco River, and at mile 215.0 .

DRA INAGE AREA. - - 12,900 $\mathrm{mi}^{2}$, approximately.

PERIOD OF RECORD.--January to October 1911, February 1927 to current year. Monthly or yearly discharge only for some periods, published in wSP 1313.

REVISED RECORDS.--WSP 1243: 1931, 1934-38, 1951. WSP 1313: 1911, 1933. WDR NM-78-1: 1977.

GAGE.-Water-stage recorder. Datum of gage is 4,848.68 ft above National Geodetic Vertical Datum of 1929 (river-profile survey). Prior to Apr. 6, 1922, nonrecording gage and Apr. 7, 1922, to Oct. 25, 1933, water-stage recorder, at site $3 \mathrm{mi}$ upstream at different datum. Oct. 26, 1933, to Sept. 30, 1936, water-stage recorder at present site at datum $3.31 \mathrm{ft}$ higher and Oct. 1, 1936, to Sept. 30, 1952, at datum $1.77 \mathrm{ft}$ higher. supplementary water-stage recorders at nearby sites, same datum, used at times.

REMARKS.--Since 1962 flow partly regulated by Navajo Reservoir (station 09355100). Diversions for irrigation of about 118,000 acres upstream from station. Ungaged canals bypass station on both $r$ ight and left bank, though some of bypass flow is returned to river downstream from gage.

AVERAGE DISCHARGE.--28 years (water years 1935-62), 2,309 $\mathrm{ft}^{3} / \mathrm{s}, 1,673,000 \mathrm{acre}-\mathrm{ft} / \mathrm{yr}$.

EXTREMES FOR PERIOD OF RECORD (SINCE 1927).--Maximum discharge, about 80,000 ft3/s, Aug. 11, 1929, gage height, 5.7 $\mathrm{ft}$, site and datum then in use; minimum daily, $8 \mathrm{ft} / \mathrm{s}$, Aug. $25,26,1939$.

EXTREMES OUTSIDE PERIOD OF RECORD.--Maximum flood occurred oct. 6, 1911, and reached a stage of 22 ft, site and datum then in use.

STATISTICAL SUMMARIES (PERIOD BEFORE REGULATION OF NAVAJO DAM)

MEAN MONTHLY AND MEAN ANNUAL DISCHARGES 1935-62

MAGNITUDE AND PROBABILITY OF ANNUAL LOW FLOW BASED ON PERIOD OF RECORD 1936-62

\begin{tabular}{|c|c|c|c|c|c|c|}
\hline MONTH & $\begin{array}{c}\text { MINIMUM } \\
\left(\mathrm{FT}^{3} / \mathrm{S}\right)\end{array}$ & $\begin{array}{c}\text { MAXIMUM } \\
\left(\mathrm{FT}^{3} / \mathrm{S}\right)\end{array}$ & $\begin{array}{l}\text { MEAN } \\
\left(\mathrm{FT}^{3} / \mathrm{S}\right)\end{array}$ & $\begin{array}{l}\text { STAN- } \\
\text { DARD } \\
\text { DEVIA- } \\
\text { TION } \\
\text { (FT3/S) }\end{array}$ & $\begin{array}{l}\text { COEFFI- } \\
\text { CIENT OF } \\
\text { VARI- } \\
\text { ATION }\end{array}$ & $\begin{array}{c}\text { PERCENT } \\
\text { OF } \\
\text { ANNUAL } \\
\text { RUNOFF }\end{array}$ \\
\hline $\begin{array}{l}\text { OCTOBER } \\
\text { NOVEMBER } \\
\text { DECEMBER } \\
\text { JANUARY } \\
\text { FEBRUARY } \\
\text { MARCH } \\
\text { APR IL } \\
\text { MAY } \\
\text { JUNE } \\
\text { JULY } \\
\text { AUGUST } \\
\text { SEPTEMBER }\end{array}$ & $\begin{array}{r}247 \\
365 \\
386 \\
437 \\
489 \\
534 \\
602 \\
2038 \\
1922 \\
199 \\
126 \\
44\end{array}$ & $\begin{array}{r}8370 \\
2812 \\
1445 \\
1066 \\
1821 \\
4443 \\
9275 \\
19790 \\
15540 \\
8869 \\
5171 \\
3329\end{array}$ & $\begin{array}{r}1234 \\
835 \\
657 \\
612 \\
856 \\
1527 \\
4069 \\
6483 \\
6812 \\
2284 \\
1243 \\
1082\end{array}$ & $\begin{array}{r}1500 \\
507 \\
251 \\
161 \\
351 \\
907 \\
2512 \\
3785 \\
4291 \\
2253 \\
1091 \\
883\end{array}$ & $\begin{array}{l}1.22 \\
.61 \\
.38 \\
.26 \\
.41 \\
.59 \\
.62 \\
.58 \\
.63 \\
.99 \\
.88 \\
.82\end{array}$ & $\begin{array}{r}4.5 \\
3.0 \\
2.4 \\
2.2 \\
3.1 \\
5.5 \\
14.7 \\
23.4 \\
24.6 \\
8.2 \\
4.5 \\
3.9\end{array}$ \\
\hline ANNUAL & 861 & 5324 & 2309 & 1107 & .48 & 100 \\
\hline
\end{tabular}

DISCHARGE, IN FT' $3 / \mathrm{S}$, FOR INDICATED RECURRENC

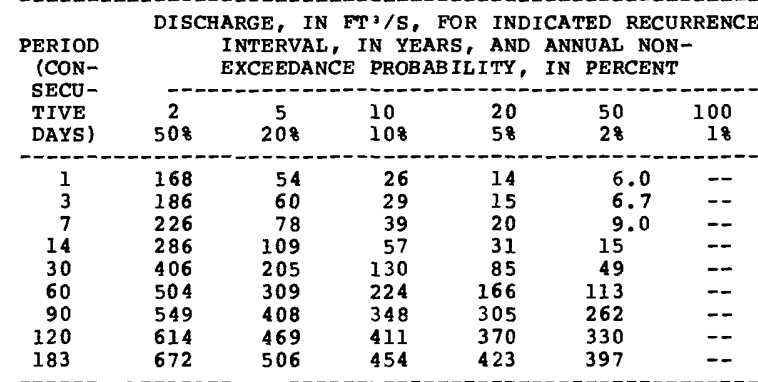

MAGNITUDE AND PROBABILITY OF ANNUAL HIGH FLOW BASED ON PERIOD OF RECORD 1935-62

\begin{tabular}{|c|c|c|c|c|c|c|}
\hline \multirow{2}{*}{$\begin{array}{l}\text { PERIOD } \\
\text { (CON- } \\
\text { SECU- } \\
\text { TIVE } \\
\text { DAYS) }\end{array}$} & \multicolumn{6}{|c|}{$\begin{array}{l}\text { DISCHARGE, IN FT }{ }^{3 / S} \text {, FOR INDICATED RECURRENCE } \\
\text { INTERVAL, IN YEARS, AND ANNUAL, } \\
\text { EXCEEDANCE PROBABILI TY, IN PERCENT }\end{array}$} \\
\hline & $\begin{array}{c}2 \\
508\end{array}$ & $\begin{array}{c}5 \\
208\end{array}$ & $\begin{array}{l}10 \\
108\end{array}$ & $\begin{array}{l}25 \\
48\end{array}$ & $\begin{array}{l}50 \\
28\end{array}$ & $\begin{array}{r}100 \\
18\end{array}$ \\
\hline $\begin{array}{r}1 \\
3 \\
7 \\
15 \\
30 \\
60 \\
90\end{array}$ & $\begin{array}{r}11800 \\
11000 \\
9550 \\
8420 \\
7470 \\
6200 \\
5280\end{array}$ & $\begin{array}{r}19500 \\
17200 \\
14900 \\
13200 \\
11700 \\
9840 \\
8500\end{array}$ & $\begin{array}{l}25500 \\
21900 \\
18900 \\
16800 \\
14800 \\
12600 \\
10800\end{array}$ & $\begin{array}{l}34300 \\
28200 \\
24500 \\
21900 \\
19100 \\
16300 \\
14000\end{array}$ & $\begin{array}{l}41600 \\
33200 \\
29000 \\
26200 \\
22500 \\
19300 \\
16500\end{array}$ & $\begin{array}{l}-- \\
-- \\
-- \\
-- \\
-- \\
--\end{array}$ \\
\hline
\end{tabular}

DURATION TABLE OF DAILY MEAN FLOW FOR PERIOD OF RECORD 1935-62 DISCHARGE, IN FT $3 / 5$, THAT WAS EQUALED OR EXCEEDED FOR INDICATED PERCENT OF TIME

\begin{tabular}{|c|c|c|c|c|c|c|c|c|c|c|c|c|c|c|}
\hline 58 & 108 & 158 & 208 & 258 & 308 & 408 & 508 & 608 & 708 & 758 & 808 & 858 & 908 & 958 \\
\hline 9410 & 6300 & 4590 & 3450 & 2570 & 1950 & 1260 & 919 & 732 & 624 & 576 & 525 & 474 & 415 & 263 \\
\hline
\end{tabular}


SAN JUAN RIVER BASIN

09368000 SAN JUAN RIVER AT SHIPROCK, NM--Continued

STATISTICAL SUMMARIES (PERIOD AFTER COMPLETION OF NAVAJO DAM)

MEAN MONTHLY AND MEAN ANNUAL DISCHARGES 1964-85

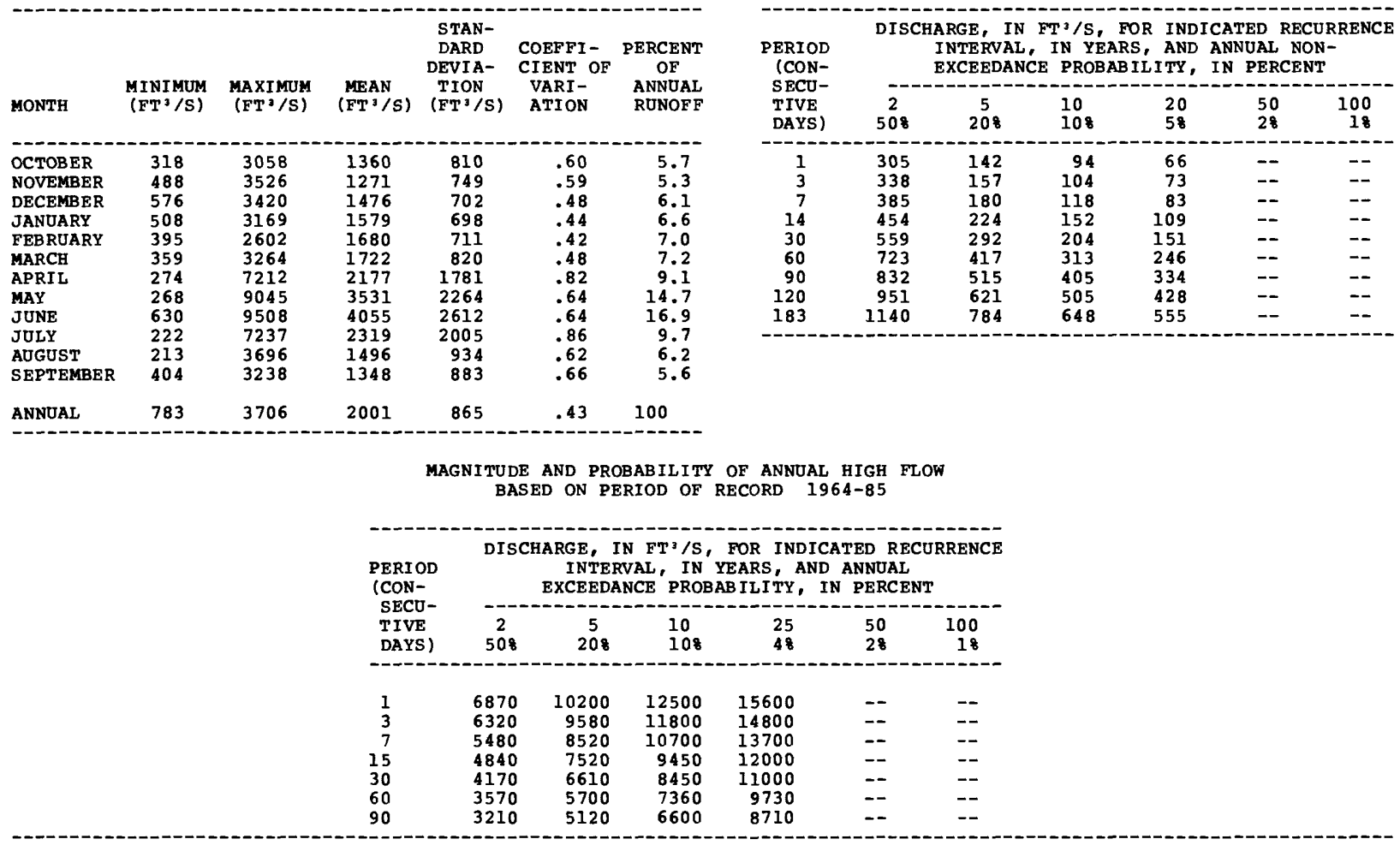

DURATION TABLE OF DAILY MEAN FLOW FOR PERIOD OF RECORD 1964-85

DISCHARGE, IN FT $3 / \mathrm{S}$, THAT WAS EQUALED OR EXCEEDED FOR INDICATED PERCENT OF TIME

\begin{tabular}{|c|c|c|c|c|c|c|c|c|c|c|c|c|c|c|}
\hline 58 & 108 & 158 & 208 & 258 & 308 & 408 & 508 & 608 & 708 & 758 & 808 & 858 & 908 & 958 \\
\hline 5780 & 4040 & 3330 & 2840 & 2500 & 2250 & 1920 & 1550 & 1200 & 891 & 804 & 708 & 602 & 479 & 337 \\
\hline
\end{tabular}


LOCATION.--Lat $35^{\circ} 16^{\prime} 57^{\prime \prime}$, long $108^{\circ} 33^{\prime} 10^{\prime \prime}$, in NWłSW sec.8, T.12 N., R.16 w., McKin1ey County, Hydrologic Unit 15020004 , on Zuni Indian Reservation, on left bank at mouth of Nutria Canyon, 0.9 mi upstream from Nutria

Diversion Dam, $1.3 \mathrm{mi}$ northeast of Upper Nutria, and $10.4 \mathrm{mi}$ northwest of Ramah.

DRAINAGE AREA. $--71.4 \mathrm{mi}^{2}$.

PERIOD OF RECORD.--October 1969 to current year.

REVISED RECORDS.--WDR NM-78-1: 1977.

GAGE.--Water-stage recorder and concrete contro1. Concrete contro1 raised 1.0 ft June 6 , 1975 . Contro1 raised $2.35 \mathrm{ft}$ June 28, 1984. Elevation of gage is $6,860 \mathrm{ft}$ above National Geodetic Vertical Datum of 1929 , from topographic map.

REMARKS. - No diversion above station.

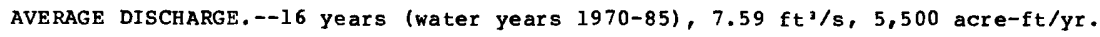

EXTREMES FOR PERIOD OF RECORD.--Maximum discharge, $782 \mathrm{ft} / \mathrm{s}$, Apr. 14, 1973 , gage height, $5.58 \mathrm{ft}$, datum then in

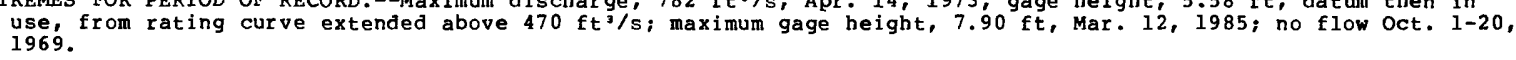

STATISTICAL SUMMARIES

MEAN MONTHLY AND MEAN ANNUAL DISCHARGES 1970-85

\begin{tabular}{|c|c|c|c|c|c|c|}
\hline MONTH & $\begin{array}{c}\text { MINIMUM } \\
\left(\mathrm{FT}^{3} / \mathrm{S}\right)\end{array}$ & $\begin{array}{c}\operatorname{MAXIMUM} \\
\left(\mathrm{FT}^{\top} \mathrm{T}^{3} / \mathrm{S}\right)\end{array}$ & $\begin{array}{l}\text { MEAN } \\
\left(\mathrm{FT}^{3} / \mathrm{S}\right)\end{array}$ & $\begin{array}{c}\text { STAN- } \\
\text { DARD } \\
\text { DEVIA- } \\
\text { TION } \\
\left(\text { FT }^{3} / \mathrm{S}\right)\end{array}$ & $\begin{array}{l}\text { COEFFI- } \\
\text { CIENT OF } \\
\text { VARI- } \\
\text { ATION }\end{array}$ & $\begin{array}{c}\text { PERCENT } \\
\text { OF } \\
\text { ANNUAL } \\
\text { RUNOFF }\end{array}$ \\
\hline $\begin{array}{l}\text { OCTOBER } \\
\text { NOVEMBER } \\
\text { DECEMBER } \\
\text { JANUARY } \\
\text { FEBRUARY } \\
\text { MARCH } \\
\text { APRIL } \\
\text { MAY } \\
\text { JUNE } \\
\text { JULY } \\
\text { AUGUST } \\
\text { SEPTEMBER }\end{array}$ & $\begin{array}{r}0.0 \\
0.0 \\
0.0 \\
.1 \\
.1 \\
.1 \\
.1 \\
.1 \\
0.0 \\
0.0 \\
0.0 \\
0.0\end{array}$ & $\begin{array}{r}2.4 \\
2.6 \\
3.8 \\
4.4 \\
25 \\
100 \\
187 \\
34 \\
1.3 \\
3.5 \\
3.0 \\
1.9\end{array}$ & $\begin{array}{r}.5 \\
.3 \\
1.0 \\
.7 \\
3.9 \\
31 \\
47 \\
5.0 \\
.4 \\
.6 \\
.9 \\
.3\end{array}$ & $\begin{array}{r}.8 \\
.6 \\
1.4 \\
1.1 \\
6.5 \\
35 \\
68 \\
9.0 \\
.5 \\
1.1 \\
1.0 \\
.5\end{array}$ & $\begin{array}{l}1.58 \\
1.88 \\
1.34 \\
1.59 \\
1.70 \\
1.13 \\
1.44 \\
1.79 \\
1.20 \\
1.70 \\
1.10 \\
1.55\end{array}$ & $\begin{array}{r}.5 \\
.4 \\
1.1 \\
.8 \\
4.2 \\
33.8 \\
51.3 \\
5.5 \\
.4 \\
.7 \\
1.0 \\
.4\end{array}$ \\
\hline ANNUAL & .1 & 22 & 7.6 & 8.6 & 1.14 & 100 \\
\hline
\end{tabular}

MAGNI TUDE AND PROBABILITY OF ANNUAL LOW FLOW BASED ON PERIOD OF RECORD 1971-85

\begin{tabular}{|c|c|c|c|c|c|c|}
\hline \multirow{2}{*}{$\begin{array}{l}\text { PERIOD } \\
\text { (CON- } \\
\text { SECU- } \\
\text { TIVE } \\
\text { DAYS) }\end{array}$} & \multicolumn{6}{|c|}{$\begin{array}{l}\text { DISCHARGE, IN FT } 3 / S \text {, FOR INDICATED RECURRENCE } \\
\text { INTERVAL, IN YEARS, AND ANNUAL NON- } \\
\text { EXCEEDANCE PROBAB ILITY, IN PERCENT }\end{array}$} \\
\hline & $\begin{array}{c}2 \\
508\end{array}$ & $\begin{array}{c}5 \\
208\end{array}$ & $\begin{array}{l}10 \\
108\end{array}$ & $\begin{array}{l}20 \\
58\end{array}$ & $\begin{array}{l}50 \\
28\end{array}$ & $\begin{array}{r}100 \\
18\end{array}$ \\
\hline $\begin{array}{r}1 \\
3 \\
7 \\
14 \\
30 \\
60 \\
90 \\
120 \\
183\end{array}$ & $\begin{array}{r}0.0 \\
0.0 \\
0.0 \\
0.0 \\
0.0 \\
.1 \\
.1 \\
.1 \\
.2\end{array}$ & $\begin{array}{r}0.0 \\
0.0 \\
0.0 \\
0.0 \\
0.0 \\
0.0 \\
0.0 \\
.1 \\
.1\end{array}$ & $\begin{array}{r}0.0 \\
0.0 \\
0.0 \\
0.0 \\
0.0 \\
0.0 \\
0.0 \\
0.0 \\
.1\end{array}$ & $\begin{array}{r}0.0 \\
0.0 \\
0.0 \\
0.0 \\
0.0 \\
0.0 \\
0.0 \\
0.0 \\
.1\end{array}$ & $\begin{array}{l}-- \\
-- \\
-- \\
-- \\
-- \\
-- \\
--\end{array}$ & $\begin{array}{l}-- \\
-- \\
-- \\
-- \\
-- \\
-- \\
--\end{array}$ \\
\hline
\end{tabular}

MAGNITUDE AND PROBABILITY OF ANNUAL HIGH FLOW BASED ON PERIOD OF RECORD 1970-85

\begin{tabular}{|c|c|c|c|c|c|c|}
\hline \multirow{2}{*}{$\begin{array}{l}\text { PERIOD } \\
\text { (CON- } \\
\text { SECU- } \\
\text { TIVE } \\
\text { DAYS) }\end{array}$} & \multicolumn{6}{|c|}{$\begin{array}{l}\text { DISCHARGE, IN FT } 3 / S \text {, FOR INDICATED RECURRENCE } \\
\text { INTERVAL, IN YEARS, AND ANNUAL } \\
\text { EXCEEDANCE PROBABILITY, IN PERCENT }\end{array}$} \\
\hline & $\begin{array}{c}2 \\
508\end{array}$ & $\begin{array}{c}5 \\
208\end{array}$ & $\begin{array}{l}10 \\
108\end{array}$ & $\begin{array}{l}25 \\
48\end{array}$ & $\begin{array}{l}50 \\
28\end{array}$ & $\begin{array}{r}100 \\
18\end{array}$ \\
\hline $\begin{array}{r}1 \\
3 \\
7 \\
15 \\
30 \\
60 \\
90\end{array}$ & $\begin{array}{c}92 \\
62 \\
40 \\
29 \\
19 \\
11 \\
8.1\end{array}$ & $\begin{array}{r}291 \\
239 \\
184 \\
147 \\
102 \\
65 \\
46\end{array}$ & $\begin{array}{l}506 \\
461 \\
387 \\
325 \\
232 \\
152 \\
108\end{array}$ & $\begin{array}{l}883 \\
895 \\
825 \\
721 \\
534 \\
359 \\
256\end{array}$ & $\begin{array}{l}-- \\
-- \\
-- \\
-- \\
-- \\
--\end{array}$ & $\begin{array}{l}-- \\
-- \\
-- \\
-- \\
-- \\
-- \\
--\end{array}$ \\
\hline
\end{tabular}

DURATION TABLE OF DAILY MEAN FLOW FOR PERIOD OF RECORD 1970-85 DISCHARGE, IN FT'/S, THAT WAS EQUALED OR EXCEEDED FOR INDICATED PERCENT OF TIME

\begin{tabular}{|c|c|c|c|c|c|c|c|c|c|c|c|c|c|c|}
\hline 58 & 108 & 158 & 208 & 258 & $30 \%$ & 408 & 508 & 608 & 708 & 758 & 808 & 858 & 908 & 958 \\
\hline 41 & 10 & 3.4 & 1.4 & .7 &. & . & .2 &. & .1 & . & . & .1 & 1 & 0.0 \\
\hline
\end{tabular}




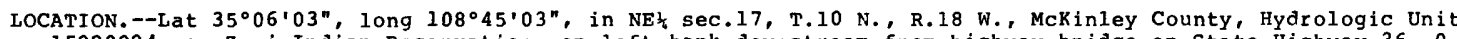
15020004 , on Zuni Indian Reservation, on left bank downstream from highway bridge on State Highway 36 , 0.8 mi upstream from flow line of Black Rock Reservoir, $2.3 \mathrm{mi}$ northeast of Black Rock, and $5.9 \mathrm{mi}$ northeast of $\mathrm{Zuni}$ Puehlo.

DRAINAGE AREA. --810 $\mathrm{mi}^{2}$, approximately.

PERIOD OF RECORD.--October 1969 to current year. Prior to October 1974 published as "above Zuni Reservoir".

GAGE.--Water-stage recorder, crest-stage gage and concrete control. Elevation of gage is $6,480 \mathrm{ft}$ above National Geodetic Vertical Datum of 1929 , from topographic map.

REMARKS.--No diversion above station.

AVERAGE DISCHARGE.--16 years (water years 1970-85), $14.9 \mathrm{ft}^{3} / \mathrm{s}, 10,800 \mathrm{acre}-\mathrm{ft} / \mathrm{yr}$.

EXTREMES FOR PERIOD OF RECORD.--Maximum discharge, 5,200 ft $3 / \mathrm{s}$, Aug. 4, 1974, gage height, 6.61 ft, from rating curve extended above $670 \mathrm{ft}^{3} / \mathrm{s}$ on basis of slope-area measurements at gage heights $4.05 \mathrm{ft}$, $3.94 \mathrm{ft}$, $5.16 \mathrm{ft}$, and $6.61 \mathrm{ft}$; no flow for many days.

STATISTICAL SUMMARIES

MEAN MONTHLY AND MEAN ANNUAL, DISCHARGES 1970-85

\begin{tabular}{|c|c|c|c|c|c|c|}
\hline MONTH & $\begin{array}{l}\text { MINIMUM } \\
\left(\mathrm{FT}^{3} / \mathrm{S}\right)\end{array}$ & $\begin{array}{l}\text { MAXIMUM } \\
\left(\mathrm{FT}^{3} / \mathrm{S}\right)\end{array}$ & $\begin{array}{l}\text { MEAN } \\
\left(\mathrm{FT}^{3} / \mathrm{S}\right)\end{array}$ & $\begin{array}{l}\text { STAN- } \\
\text { DARD } \\
\text { DEVIA- } \\
\text { TION } \\
\left(\mathrm{FT}^{3} / \mathrm{S}\right)\end{array}$ & $\begin{array}{l}\text { COEFFI- } \\
\text { CIENT OF } \\
\text { VARI- } \\
\text { ATION }\end{array}$ & $\begin{array}{c}\text { PERCENT } \\
\text { OF } \\
\text { ANNUAL } \\
\text { RUNOFF }\end{array}$ \\
\hline $\begin{array}{l}\text { OCTOBER } \\
\text { NOVEMBER } \\
\text { DECEMBER } \\
\text { JANUARY } \\
\text { FEBRUARY } \\
\text { MARCH } \\
\text { APRIL } \\
\text { MAY } \\
\text { JUNE } \\
\text { JULY } \\
\text { AUGUST } \\
\text { SEPTEMBER }\end{array}$ & $\begin{array}{r}0.0 \\
0.0 \\
0.0 \\
.1 \\
.3 \\
.7 \\
0.0 \\
0.0 \\
0.0 \\
0.0 \\
.5 \\
0.0\end{array}$ & $\begin{array}{c}13 \\
14 \\
5.9 \\
4.7 \\
73 \\
263 \\
308 \\
65 \\
2.0 \\
26 \\
24 \\
18\end{array}$ & $\begin{array}{r}2.5 \\
1.8 \\
1.5 \\
1.5 \\
12 \\
54 \\
79 \\
8.7 \\
.3 \\
4.7 \\
10.0 \\
4.0\end{array}$ & $\begin{array}{r}3.5 \\
3.3 \\
1.6 \\
1.4 \\
21 \\
81 \\
126 \\
18 \\
.5 \\
6.7 \\
8.8 \\
5.3\end{array}$ & $\begin{array}{l}1.41 \\
1.88 \\
1.06 \\
.88 \\
1.83 \\
1.49 \\
1.60 \\
2.02 \\
2.04 \\
1.43 \\
.88 \\
1.32\end{array}$ & $\begin{array}{r}1.4 \\
1.0 \\
.8 \\
.9 \\
6.4 \\
30.1 \\
44.0 \\
4.9 \\
.1 \\
2.6 \\
5.6 \\
2.2\end{array}$ \\
\hline ANNUAL & 1.4 & 47 & 15 & 18 & 1.19 & 100 \\
\hline
\end{tabular}

MAGNITUDE AND PROBABILITY OF ANNUAL LOW FLOW BASED ON PERIOD OF RECORD 1971-85

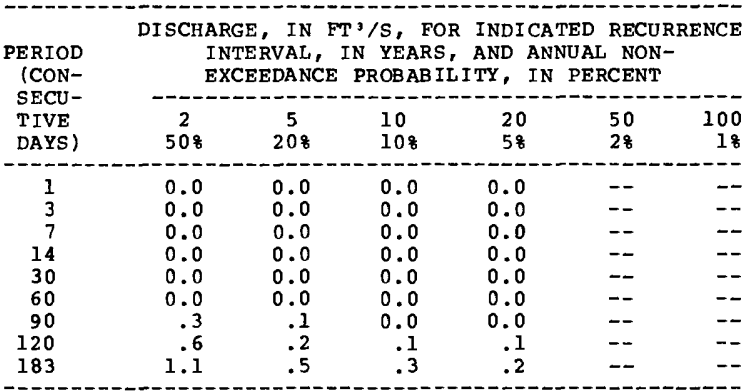

MAGNITUDE AND PROBABILITY OF ANNUAL HIGH FLOW BASED ON PERIOD OF RECORD 1970-85

\begin{tabular}{|c|c|c|c|c|c|c|}
\hline \multirow{2}{*}{$\begin{array}{l}\text { PERIOD } \\
\text { (CON- } \\
\text { SECU- } \\
\text { TIVE } \\
\text { DAYS) }\end{array}$} & \multicolumn{6}{|c|}{$\begin{array}{l}\text { DISCHARGE, IN FT' } 3 / \text {, FOR INDICATED RECURRENCE } \\
\text { INTERVAL, IN YEARS, AND ANNUAL } \\
\text { EXCEEDANCE PROBABILITY, IN PERCENT }\end{array}$} \\
\hline & $\begin{array}{c}2 \\
508\end{array}$ & $\begin{array}{c}5 \\
208\end{array}$ & $\begin{array}{l}10 \\
108\end{array}$ & $\begin{array}{l}25 \\
48\end{array}$ & $\begin{array}{l}50 \\
28\end{array}$ & $\begin{array}{r}100 \\
18\end{array}$ \\
\hline $\begin{array}{r}1 \\
3 \\
7 \\
15 \\
30 \\
60 \\
90\end{array}$ & $\begin{array}{r}312 \\
165 \\
95 \\
59 \\
38 \\
26 \\
18\end{array}$ & $\begin{array}{r}697 \\
463 \\
303 \\
202 \\
136 \\
94 \\
68\end{array}$ & $\begin{array}{r}1050 \\
816 \\
578 \\
414 \\
286 \\
198 \\
146\end{array}$ & $\begin{array}{r}1610 \\
1520 \\
1190 \\
945 \\
667 \\
460 \\
344\end{array}$ & $\begin{array}{l}-- \\
-- \\
-- \\
-- \\
--\end{array}$ & $\begin{array}{l}-- \\
-- \\
-- \\
-- \\
-- \\
--\end{array}$ \\
\hline
\end{tabular}

DURATION TABLE OF DAILY MEAN FLOW FOR PERIOD OF RECORD 1970-85 DISCHARGE, IN FT'S, THAT WAS EQUALED OR EXCEEDED FOR INDICATED PERCENT OF TIME

\begin{tabular}{|c|c|c|c|c|c|c|c|c|c|c|c|c|c|c|}
\hline 58 & 108 & 158 & 208 & 258 & 308 & 408 & 508 & 608 & 708 & 758 & 808 & 858 & $90 \%$ & 958 \\
\hline 79 & 17 & 7.9 & 4.3 & 2.7 & 2.1 & 1.3 & .8 & .5 & .1 & 0.0 & 0.0 & 0.0 & 0.0 & \\
\hline
\end{tabular}




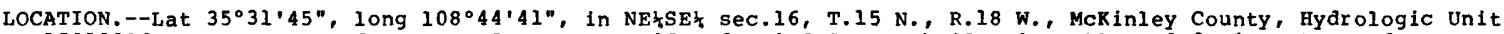
15020006 , near center of span on downstream side of Third street bridge in Gallup, $0.8 \mathrm{~m} i$ upstream from Gamerco Wash, $3.5 \mathrm{mi}$ downstream from Hogback, and $4.9 \mathrm{mi}$ downstream from South Fork.

DRAINAGE AREA. $--558 \mathrm{mi}^{2}$.

\section{WATER DISCHARGE RECORDS}

PERIOD OF RECORD.--June 1940 to July 1946, June 1957 to August 1977 (annual maximum only), September 1977 to September 1982 (discontinued).

GAGE.--Water-stage recorder. Altitude of gage is 6,480 ft from topographic map. Prior to September 1977 at site $2,000 \mathrm{ft}$ upstream at different datum.

REMARKS.--No diversion above station.

AVERAGE DISCHARGE.--10 years (water years 1941-45, 1978-82), $9.42 \mathrm{ft}^{3} / \mathrm{s}, 6,820 \mathrm{acre}-\mathrm{ft} / \mathrm{yr}$.

EXTREMES FOR PERIOD OF RECORD.--Maximum discharge, 12,000 $\mathrm{ft}^{3} / \mathrm{s} \mathrm{July} \mathrm{17,} \mathrm{1972,} \mathrm{gage} \mathrm{height,} 15.3 \mathrm{ft}$ site and datum then in use; no flow at times.

STATISTICAL SUMMARIES

MEAN MONTHLY AND MEAN ANNUAL Discharges 1941-45. $1978-82$

\begin{tabular}{|c|c|c|c|c|c|c|}
\hline MONTH & $\begin{array}{l}\text { MINIMUM } \\
\left(\mathrm{FT}^{3} / \mathrm{S}\right)\end{array}$ & $\begin{array}{l}\text { MAXIMUM } \\
\left(\mathrm{FT}^{3} / \mathrm{S}\right)\end{array}$ & $\begin{array}{l}\text { MEAN } \\
\left(\mathrm{FT}^{3} / \mathrm{S}\right)\end{array}$ & $\begin{array}{l}\text { STAN- } \\
\text { DARD } \\
\text { DEVIA- } \\
\text { TION } \\
\left(\text { FT }^{3} / S\right)\end{array}$ & $\begin{array}{l}\text { COEFFI- } \\
\text { CIENT OF } \\
\text { VARI- } \\
\text { ATION }\end{array}$ & $\begin{array}{c}\text { PFRCENT } \\
\text { OF } \\
\text { ANNUAI } \\
\text { RUNOF }\end{array}$ \\
\hline
\end{tabular}

MAGNITUDE AND PROBABILITY OF ANNUAL LOW FLOW BASED ON PERIOD OF RECORD $1942-46,1979-82$

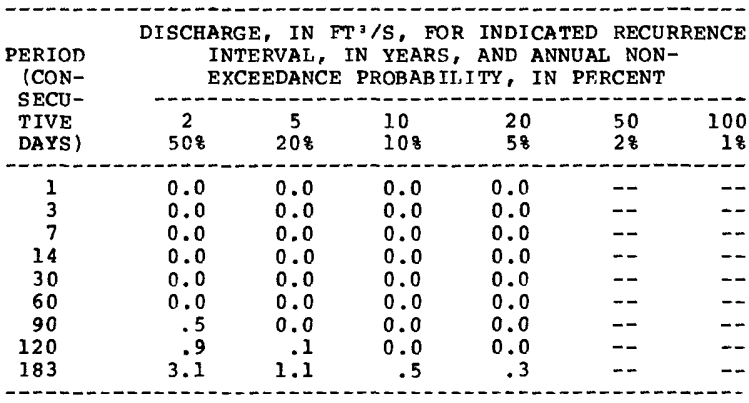

$\begin{array}{llll}9.4 & 5.7 \quad .61 \quad 100\end{array}$

MAGNITUDE AND PROBABILITY OF ANNUAL HIGH FLOW BASED ON PERIOD OF RECORD $1941-45,1978-82$

\begin{tabular}{|c|c|c|c|c|c|c|}
\hline $\begin{array}{l}\text { PERIOD } \\
\text { (CON- }\end{array}$ & & $\begin{array}{l}\text { RGE, } \\
\text { INTEF } \\
\text { CEED }\end{array}$ & $\begin{array}{l}T^{3} / \mathrm{S}, \\
\text { IN } \\
\text { PROB }\end{array}$ & $\begin{array}{l}\text { INI } \\
\mathrm{ST}^{\prime}{ }^{\prime}\end{array}$ & $\begin{array}{l}\text { ED } \\
\text { NNUP } \\
\text { PERC }\end{array}$ & RRENCE \\
\hline $\begin{array}{l}\text { TIVE } \\
\text { DAYS) }\end{array}$ & $\begin{array}{c}2 \\
508\end{array}$ & $\begin{array}{c}5 \\
208\end{array}$ & $\begin{array}{l}10 \\
10 \%\end{array}$ & $\begin{array}{l}25 \\
48\end{array}$ & $\begin{array}{l}50 \\
28\end{array}$ & $\begin{array}{r}100 \\
18\end{array}$ \\
\hline
\end{tabular}

$\begin{array}{rrrrrrr}1 & 210 & 448 & 631 & -- & -- & -- \\ 3 & 143 & 248 & 310 & -- & -- & - \\ 7 & 89 & 139 & 164 & -- & -- & - \\ 15 & 57 & 92 & 112 & -- & -- & - \\ 30 & 37 & 63 & 80 & -- & -- & - \\ 60 & 26 & 47 & 64 & -- & -- & - \\ 90 & 19 & 35 & 48 & -- & -- & -\end{array}$

DURATION TABLE OF DAILY MEAN FLOW FOR PERIOD OF RECORD 1941-45, 1978-82

DISCHARGE, IN FT'/S, THAT WAS EQUALED OR EXCEEDED FOR INDICATED PERCENT OF TIME

\begin{tabular}{|c|c|c|c|c|c|c|c|c|c|c|c|c|c|c|}
\hline 58 & 108 & 158 & 208 & $25 \%$ & 308 & 408 & 508 & 608 & 708 & 758 & 808 & 858 & 908 & 958 \\
\hline 45 & 22 & 12. & 9.0 & 6.8 & 5.5 & 3.9 & 2.8 & 1.2 & .1 & 0.0 & 0.0 & 0.0 & 0.0 & \\
\hline
\end{tabular}


GILA RIVER BASIN

09430500 GILA RIVER NEAR GILA, NM

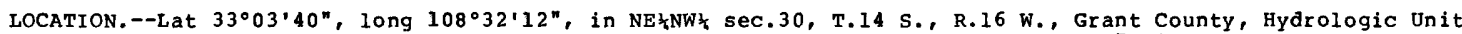
15040001 , on left bank at Hooker damsite, $1.6 \mathrm{mi}$ upstream from Mogollon Creek, 7 mi northeast of Gila, and at mile 572.5 .

DRAINAGE AREA. $--1,864 \mathrm{mi}^{2}$.

PERIOD OF RECORD.--April to December 1914, December 1927 to current year. Monthly discharge only December 1927 to September 1930, published in WSP 1313.

REVISED RECORDS:--WSP 1283: Drainage area. WSP 1313: 1944 (M), 1949 (M). WDR NM-78-1: 1977.

GAGE:--Water-stage recorder. Datum of gage is 4,655.8 ft above National Geodetic vertical Datum of 1929 , (river-profile survey). Prior to Dec. 31, 1928, at site $5 \mathrm{mi}$ upstream at different datum. Dec. 31 , 1928, to Jan. 7, 1942, at site $200 \mathrm{ft}$ upstream at same datum.

REMARKS.--Diversions for irrigation of about 500 acres upstream from station. National Weather Service gage height and rain gage satellite telemeter at station.

AVERAGE DISCHARGE.--57 years (water years 1929-85), $148 \mathrm{ft}^{3} / \mathrm{s}, 107,200 \mathrm{acre}-\mathrm{ft} / \mathrm{yr}$.

EXTREMES FOR PERIOD OF RECORD.--Maximum discharge, 35,200 $\mathrm{ft}^{3} / \mathrm{s}$, Dec. 28, 1984, gage height, $13.0 \mathrm{ft}$, from

floodmark, from rating curve extended above $7,000 \mathrm{ft}^{3} / \mathrm{s}$ on basis of slope-area measurement at gage height 12.5

$\mathrm{ft}$; maximum gage height, $17.2 \mathrm{ft}$ from floodmark, Sept. 29, 194l; minimum, 14 ft3/s, July 15, 1971.

EXTREMES OUTSIDE PERIOD OF RECORD.--Other major floods occurred in November 1905, December 1906 , and January 1916.

STATISTICAL SUMMARIES

MEAN MONTHLY AND MEAN ANNUAL DISCHARGES 1929-85

\begin{tabular}{|c|c|c|c|c|c|c|}
\hline MONTH & $\begin{array}{l}\text { M INI MUM } \\
\left(\text { FT }^{3} / \mathrm{S}\right)\end{array}$ & $\begin{array}{c}\text { MAXIMUM } \\
\left(E^{3} / S\right)\end{array}$ & $\begin{array}{l}\text { MEAN } \\
\left(\mathrm{FT}^{3} / \mathrm{S}\right)\end{array}$ & $\begin{array}{l}\text { STAN- } \\
\text { DARD } \\
\text { DEVIA- } \\
\text { TION } \\
\left(\text { FT }^{3} / \mathrm{S}\right)\end{array}$ & $\begin{array}{l}\text { COEFFI- } \\
\text { CIENT OF } \\
\text { VARI- } \\
\text { ATION }\end{array}$ & $\begin{array}{c}\text { PERCENT } \\
\text { OF } \\
\text { ANNUAI, } \\
\text { RUNOF F }\end{array}$ \\
\hline $\begin{array}{l}\text { OCTOBER } \\
\text { NOVEMBER } \\
\text { DECEMBER } \\
\text { JANUARY } \\
\text { FEBRUARY } \\
\text { MARCH } \\
\text { APR IL } \\
\text { MAY } \\
\text { JUNE } \\
\text { JULY } \\
\text { AUGUST } \\
\text { SEPTEMBER }\end{array}$ & $\begin{array}{l}29 \\
48 \\
50 \\
50 \\
51 \\
54 \\
49 \\
38 \\
24 \\
22 \\
38 \\
24\end{array}$ & $\begin{array}{r}994 \\
581 \\
1632 \\
893 \\
902 \\
1049 \\
903 \\
716 \\
167 \\
119 \\
527 \\
882\end{array}$ & $\begin{array}{r}112 \\
87 \\
162 \\
149 \\
221 \\
312 \\
221 \\
138 \\
58 \\
62 \\
124 \\
132\end{array}$ & $\begin{array}{r}175 \\
78 \\
301 \\
175 \\
228 \\
308 \\
190 \\
123 \\
36 \\
26 \\
94 \\
168\end{array}$ & $\begin{array}{r}1.56 \\
.90 \\
1.86 \\
1.17 \\
1.03 \\
.99 \\
.86 \\
.89 \\
.62 \\
.42 \\
.76 \\
1.27\end{array}$ & $\begin{array}{r}6.3 \\
4.9 \\
9.1 \\
8.4 \\
12.4 \\
17.6 \\
12.4 \\
7.8 \\
3.3 \\
3.5 \\
7.0 \\
7.4\end{array}$ \\
\hline NNUAL & 48 & 477 & 148 & 102 & .69 & 100 \\
\hline
\end{tabular}

MAGNITUDE AND PROBABILITY OF ANNUAL LOW FLOW BASED ON PERIOD OF RECORD 1930-85

\begin{tabular}{|c|c|c|c|c|c|c|}
\hline \multirow{2}{*}{$\begin{array}{l}\text { PERIOD } \\
\text { (CON- } \\
\text { SECU- } \\
\text { TIVE } \\
\text { DAYS) }\end{array}$} & \multicolumn{6}{|c|}{$\begin{array}{l}\text { DISCHARGE, IN } \text { FT }^{3} / \mathrm{S} \text {, FOR INDICATED RECURRENCE } \\
\text { INTERVAL, IN YEARS, AND ANNUAL NON- } \\
\text { EXCEEDANCE PROBABILITY, IN PERCENT }\end{array}$} \\
\hline & $\begin{array}{c}2 \\
508\end{array}$ & $\begin{array}{c}5 \\
20 \%\end{array}$ & $\begin{array}{l}10 \\
108\end{array}$ & $\begin{array}{l}20 \\
58\end{array}$ & $\begin{array}{l}50 \\
28\end{array}$ & $\begin{array}{r}100 \\
18\end{array}$ \\
\hline $\begin{array}{r}1 \\
3 \\
7 \\
14 \\
30 \\
60 \\
90 \\
120 \\
183\end{array}$ & $\begin{array}{l}26 \\
27 \\
29 \\
31 \\
35 \\
43 \\
49 \\
55 \\
67\end{array}$ & $\begin{array}{l}21 \\
21 \\
22 \\
24 \\
27 \\
33 \\
39 \\
44 \\
52\end{array}$ & $\begin{array}{l}18 \\
19 \\
20 \\
21 \\
24 \\
30 \\
35 \\
38 \\
47\end{array}$ & $\begin{array}{l}17 \\
17 \\
18 \\
19 \\
22 \\
27 \\
31 \\
35 \\
43\end{array}$ & $\begin{array}{l}15 \\
15 \\
16 \\
17 \\
20 \\
24 \\
28 \\
31 \\
41\end{array}$ & $\begin{array}{l}14 \\
15 \\
15 \\
16 \\
19 \\
22 \\
26 \\
28 \\
40\end{array}$ \\
\hline
\end{tabular}

MAGNITUDE AND PROBABILITY OF ANNUAL HIGH FLOW BASED ON PERIOD OF RECORD $1929-85$

\begin{tabular}{|c|c|c|c|c|c|c|}
\hline \multirow{2}{*}{$\begin{array}{l}\text { PERIOD } \\
\text { (CON- } \\
\text { SECU- } \\
\text { TIVE } \\
\text { DAYS) }\end{array}$} & \multicolumn{6}{|c|}{$\begin{array}{l}\text { DISCHARGE, IN FT } 3 / \mathrm{S}, \text { FOR INDICATED RECURRENCE } \\
\text { INTERVAL, IN YEARS, AND ANNUAL } \\
\text { EXCEEDANCE PROBABILITY, IN PERCENT }\end{array}$} \\
\hline & $\begin{array}{c}2 \\
508\end{array}$ & $\begin{array}{c}5 \\
208\end{array}$ & $\begin{array}{l}10 \\
108\end{array}$ & $\begin{array}{l}25 \\
48\end{array}$ & $\begin{array}{l}50 \\
28\end{array}$ & $\begin{array}{r}100 \\
18\end{array}$ \\
\hline $\begin{array}{r}1 \\
3 \\
7 \\
15 \\
30 \\
60 \\
90\end{array}$ & $\begin{array}{r}1140 \\
883 \\
672 \\
495 \\
366 \\
263 \\
219\end{array}$ & $\begin{array}{r}3340 \\
2330 \\
1570 \\
1050 \\
723 \\
512 \\
435\end{array}$ & $\begin{array}{r}6190 \\
4040 \\
2490 \\
1560 \\
1040 \\
739 \\
640\end{array}$ & $\begin{array}{r}12400 \\
7480 \\
4140 \\
2420 \\
1540 \\
1110 \\
984\end{array}$ & $\begin{array}{r}20000 \\
11300 \\
5810 \\
3230 \\
1990 \\
1450 \\
1310\end{array}$ & $\begin{array}{r}31100 \\
16700 \\
7910 \\
4190 \\
2520 \\
1860 \\
1720\end{array}$ \\
\hline
\end{tabular}

DURATION TABLE OF DAILY MEAN FLOW FOR PERIOD OF RECORD 1929-85

DISCHARGE, IN FT ${ }^{3} / \mathrm{S}$, THAT WAS EQUALED OR EXCEEDED FOR INDICATED PERCENT OF TIME

\begin{tabular}{|c|c|c|c|c|c|c|c|c|c|c|c|c|c|c|}
\hline 58 & 108 & 158 & 208 & 258 & 308 & 408 & 508 & 608 & 708 & 758 & 808 & 858 & 908 & 958 \\
\hline 486 & 293 & 204 & 157 & 127 & 108 & 84 & 72 & 64 & 58 & 55 & 51 & 46 & 40 & 31 \\
\hline
\end{tabular}


09430600 MOGOLLON CREER NEAR CLIFF, NM (Hydrologic bench-mark station)

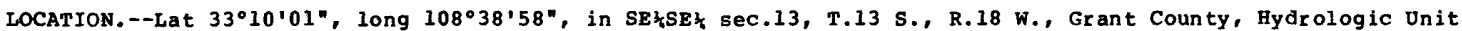
15040001 , on $r i g h t$ bank $0.3 \mathrm{mi}$ downstream from Rain Creek, $0.8 \mathrm{mi}$ downstream from Gila wilderness Boundary, 12 mi upstream from mouth, and $14 \mathrm{mi}$ north of Cliff.

DRAINAGE AREA. $--69 \mathrm{mi}^{2}$.

PERIOD OF RECORD.--March 1967 to current year.

GAGE.--Water-stage recorder. Elevation of gage is 5,440 ft above National Geodetic Vertical Datum of 1929 , from topographic map.

REMARKS. --No diversion above station.

AVERAGE DISCHARGE.--18 years (water years 1968-85), 32.0 ft $3 / \mathrm{s}, 23,180$ acre-ft/yr.

EXTREMES FOR PERIOD OF RECORD.--Maximum discharge, 10,800 ft $3 / \mathrm{s}$, Aug. 12, 1967, gage height, 13.7 ft, from

floodmarks, from rating curve extended above $220 \mathrm{ft} / \mathrm{s}$ on basis of slope-area measurement of peak flow; no flow at times.

STATISTICAL SUMMARIES

MEAN MONTHLY AND MEAN ANNUAL DIScharges 1968-85

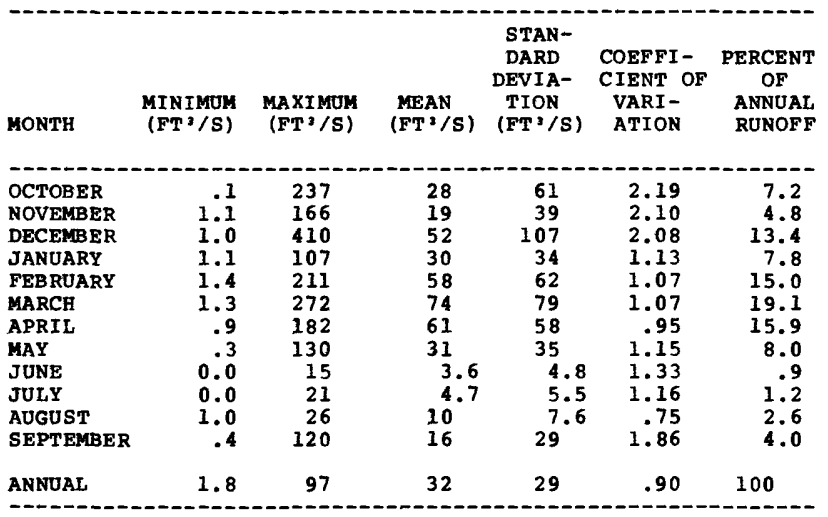

MAGNITUDE AND PROBABILITY OF ANNUAL LOW FLOW BASED ON PERIOD OF RECORD 1969-85

\begin{tabular}{|c|c|c|c|c|c|c|}
\hline \multirow{2}{*}{$\begin{array}{l}\text { PERIOD } \\
\text { (CON- } \\
\text { SECU- } \\
\text { TIVE } \\
\text { DAYS) }\end{array}$} & \multicolumn{6}{|c|}{$\begin{array}{l}\text { DISCHARGE, IN FT' }{ }^{3 / S} \text {, FOR INDICATED RECURRENCE } \\
\text { INTERVAL, IN YEARS, AND ANNUAL NON- } \\
\text { EXCEEDANCE PROBABILITY, IN PERCENT }\end{array}$} \\
\hline & $\begin{array}{c}2 \\
508\end{array}$ & $\begin{array}{c}5 \\
208\end{array}$ & $\begin{array}{l}10 \\
108\end{array}$ & $\begin{array}{l}20 \\
58\end{array}$ & $\begin{array}{l}50 \\
28\end{array}$ & 10 \\
\hline $\begin{array}{r}1 \\
3 \\
7 \\
14 \\
30 \\
60 \\
90 \\
120 \\
183\end{array}$ & $\begin{array}{r}0.0 \\
0.0 \\
0.0 \\
0.0 \\
.1 \\
.8 \\
1.5 \\
1.9 \\
4.0\end{array}$ & $\begin{array}{r}0.0 \\
0.0 \\
0.0 \\
0.0 \\
0.0 \\
.4 \\
.6 \\
.9 \\
.9 \\
2.1\end{array}$ & $\begin{array}{r}0.0 \\
0.0 \\
0.0 \\
0.0 \\
0.0 \\
0.0 \\
.3 \\
.6 \\
1.5\end{array}$ & $\begin{array}{r}0.0 \\
0.0 \\
0.0 \\
0.0 \\
0.0 \\
0.0 \\
.2 \\
.5 \\
1.2\end{array}$ & $\begin{array}{l}-- \\
-- \\
-- \\
-- \\
-- \\
--\end{array}$ & \\
\hline
\end{tabular}

MAGNITUDE AND PROBABILITY OF ANNUAL HIGH FLOW BASED ON PERIOD OF RECORD 1968-85

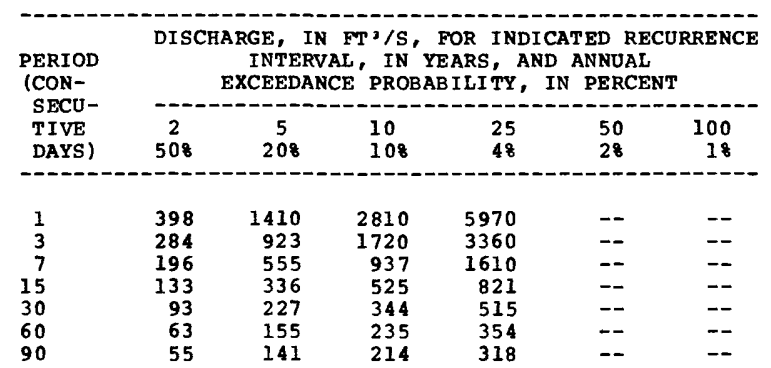

DURATION TABLE OF DAILY MEAN FLOW FOR PERIOD OF RECORD 1968-85 DISCHARGE, IN FT ${ }^{3 / S}$, THAT WAS EQUALED OR EXCEEDED FOR INDICATED PERCENT OF TIME

\begin{tabular}{|c|c|c|c|c|c|c|c|c|c|c|c|c|c|c|}
\hline 58 & 108 & 158 & 208 & 258 & 308 & 408 & 508 & 608 & 708 & 758 & 808 & 858 & 908 & 958 \\
\hline 143 & 89 & 59 & 37 & 25 & 19 & 11 & 6.2 & 3.3 & 2.0 & 1. & 1.3 & .9 & .4 & 0 \\
\hline
\end{tabular}


LOCATION,--Lat $32^{\circ} 43^{\prime} 37^{\prime \prime}$, long $108^{\circ} 40^{\prime} 30^{\prime \prime}$, in w sec.23, T.18 S., R.18 w., Grant County, Hydrologic Unit 15040002, on left bank $0.2 \mathrm{mi}$ downstream from Copper Canyon, $0.2 \mathrm{mi}$ upstream from Iower end of box canyon, $4.7 \mathrm{mi}$ northeast of Redrock, $14 \mathrm{mi}$ downstream from Mangas Creek, and at mile 539.2.

DRAINAGE AREA. $--2,829 \mathrm{mi}^{2}$.

PERIOD OF RECORD.--September 1904 to February 1905 (gage heights only). May 1905 to December 1906, January to December 1907 and July to October 1908 (gage heights only). November 1908 to December 1910 , January 1911 to January 1912 and May to June 1912 (gage heights only). August 1912 to September 1955 , October 1962 to current year. Monthly or annual discharge only for some periods, published in wsP I313. Published as "near Cliff"
1904-7.

REVISED RECORDS.--WSP 1213: 1906, 1911-15, 1931, 1936-37, 1939, 1941, 1944, 1945(P), 1946(M), 1947. WSP 1283 : Drainage area. WSP 1926: 1955. WDR NM-78-1: 1977.

GAGE. - Water-stage recorder. Elevation of gage is 4,090 ft above National Geodetic vertical Datum of 1929 , from plane table survey. Prior to Dec. 31, 1907, nonrecording gage at site 13.5 mi upstream at different datum. May 14, 1908, to July 16, 1909, nonrecording gage at site $0.2 \mathrm{mi}$ downstream at different datum.

REMARKS.--Diversions for irrigation of about 5,000 acres upstream from station. Gage height and rain gage satelite telemeter at gage.

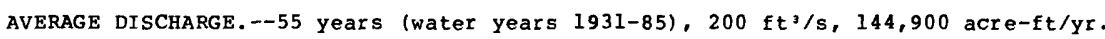

EXTREMES FOR PERIOD OF RECORD.--Maximum discharge, 48,800 $\mathrm{ft}^{3} / \mathrm{s}$, Dec. 19, 1978, gage height, $29.8 \mathrm{ft}$, in gage well, 34.1, ft from floodmarks, from rating curve extended above $9,500 \mathrm{ft} / \mathrm{s}$ on basis of slope-area measurement of peak flow; minimum, $2.2 \mathrm{ft} / \mathrm{s}$, Aug. 5,1947 .

STATISTICAL SUMMARIES

MEAN MONTHLY AND MEAN ANNUAL DISCHARGES 1931-85

\begin{tabular}{|c|c|c|c|c|c|c|}
\hline MONTH & $\begin{array}{c}\text { MINIMUM } \\
\left(\mathrm{FT}^{3} / \mathrm{S}\right)\end{array}$ & $\begin{array}{c}\text { MAXIMUM } \\
\left(\mathrm{FT}^{3} / \mathrm{S}\right)\end{array}$ & $\begin{array}{l}\text { MEAN } \\
\left(\mathrm{FT}^{3} / \mathrm{S}\right)\end{array}$ & $\begin{array}{l}\text { STAN- } \\
\text { DARD } \\
\text { DEVIA- } \\
\text { TION } \\
\left(\mathrm{FT}^{3} / \mathrm{S}\right)\end{array}$ & $\begin{array}{l}\text { COEFFI- } \\
\text { CIENT OF } \\
\text { VARI- } \\
\text { ATION }\end{array}$ & $\begin{array}{c}\text { PERCENT } \\
\text { OF } \\
\text { ANNUAI } \\
\text { RUNOFF }\end{array}$ \\
\hline $\begin{array}{l}\text { OCTOBER } \\
\text { NOVEMBER } \\
\text { DECEMBER } \\
\text { JANUARY } \\
\text { FEBRUARY } \\
\text { MARCH } \\
\text { APRIL } \\
\text { MAY } \\
\text { JUNE } \\
\text { JULY } \\
\text { AUGUST } \\
\text { SEPTEMBER }\end{array}$ & $\begin{array}{l}16 \\
48 \\
60 \\
64 \\
54 \\
40 \\
34 \\
27 \\
12 \\
11 \\
21 \\
9.8\end{array}$ & $\begin{array}{r}1768 \\
674 \\
2200 \\
1362 \\
1231 \\
1438 \\
1155 \\
996 \\
174 \\
239 \\
632 \\
1355\end{array}$ & $\begin{array}{l}166 \\
111 \\
242 \\
222 \\
329 \\
424 \\
269 \\
163 \\
56 \\
76 \\
166 \\
186\end{array}$ & $\begin{array}{r}319 \\
103 \\
447 \\
286 \\
350 \\
432 \\
262 \\
186 \\
44 \\
46 \\
126 \\
268\end{array}$ & $\begin{array}{r}1.92 \\
.93 \\
1.85 \\
1.29 \\
1.06 \\
1.02 \\
.97 \\
1.14 \\
.79 \\
.60 \\
.76 \\
1.44\end{array}$ & $\begin{array}{r}6.9 \\
4.6 \\
10.0 \\
9.2 \\
13.7 \\
17.6 \\
11.2 \\
6.8 \\
2.3 \\
3.1 \\
6.9 \\
7.7\end{array}$ \\
\hline ANNUAL & 48 & 629 & 200 & 154 & .77 & 100 \\
\hline
\end{tabular}

MAGNITUDE AND PROBABILITY OF ANNUAL LOW FLOW BASED ON PERIOD OF RECORD $1932-85$

\begin{tabular}{|c|c|c|c|c|c|c|}
\hline \multirow{2}{*}{$\begin{array}{l}\text { PERIOD } \\
\text { (CON- } \\
\text { SECU- } \\
\text { TIVE } \\
\text { DAYS) }\end{array}$} & \multicolumn{6}{|c|}{$\begin{array}{l}\text { DISCHARGE, IN FT'/S, FOR INDICATED RECURRENCE } \\
\text { INTERVAL, IN YEARS, AND ANNUAL NON- } \\
\text { EXCEEDANCE PROBABILITY, IN PERCENT }\end{array}$} \\
\hline & $\begin{array}{c}2 \\
508\end{array}$ & $\begin{array}{c}5 \\
208\end{array}$ & $\begin{array}{l}10 \\
108\end{array}$ & $\begin{array}{l}20 \\
58\end{array}$ & $\begin{array}{l}50 \\
28\end{array}$ & $\begin{array}{r}100 \\
18\end{array}$ \\
\hline $\begin{array}{r}1 \\
3 \\
7 \\
14 \\
30 \\
60 \\
90 \\
120 \\
183\end{array}$ & $\begin{array}{l}12 \\
14 \\
16 \\
19 \\
26 \\
37 \\
48 \\
60 \\
80\end{array}$ & $\begin{array}{r}6.8 \\
7.6 \\
9.1 \\
11 \\
15 \\
23 \\
32 \\
40 \\
55\end{array}$ & $\begin{array}{r}4.9 \\
5.5 \\
6.5 \\
8.0 \\
12 \\
18 \\
26 \\
31 \\
47\end{array}$ & $\begin{array}{r}3.7 \\
4.1 \\
4.9 \\
6.2 \\
9.3 \\
14 \\
21 \\
25 \\
42\end{array}$ & $\begin{array}{r}2.7 \\
3.0 \\
3.5 \\
4.5 \\
7.2 \\
11 \\
17 \\
19 \\
37\end{array}$ & $\begin{array}{l}15 \\
16 \\
34\end{array}$ \\
\hline
\end{tabular}

MAGNITUDE AND PROBABILITY OF ANNUAL HIGH FLOW BASED ON PERIOD OF RECORD 1931-85

\begin{tabular}{|c|c|c|c|c|c|c|}
\hline $\begin{array}{l}\text { PERIOD } \\
\text { (CON- }\end{array}$ & DIS & $\begin{array}{l}\text { RGE, } \\
\text { INTEF } \\
\text { XCEED? }\end{array}$ & $\begin{array}{l}\mathrm{FT}{ }^{3} / \mathrm{S} \\
\mathrm{IL}, \mathrm{IN} \\
\mathrm{E} \text { PRO }\end{array}$ & $\begin{array}{l}\text { OR IN } \\
\text { ARS, } \\
\text { ILITY }\end{array}$ & $\begin{array}{l}\text { CATED } \\
\text { D ANNU } \\
\text { IN PER }\end{array}$ & $\begin{array}{l}\text { CURRENCE } \\
\text { NT }\end{array}$ \\
\hline $\begin{array}{l}\text { TIVE } \\
\text { DAYS ) }\end{array}$ & $\begin{array}{c}2 \\
508\end{array}$ & $\begin{array}{c}5 \\
208\end{array}$ & $\begin{array}{l}10 \\
108\end{array}$ & $\begin{array}{l}25 \\
48\end{array}$ & $\begin{array}{l}50 \\
28\end{array}$ & $\begin{array}{r}100 \\
18\end{array}$ \\
\hline $\begin{array}{r}1 \\
3 \\
7 \\
15 \\
30 \\
60 \\
90\end{array}$ & $\begin{array}{r}1960 \\
1390 \\
1030 \\
725 \\
524 \\
364 \\
299\end{array}$ & $\begin{array}{r}6080 \\
3910 \\
2480 \\
1560 \\
1050 \\
731 \\
616\end{array}$ & $\begin{array}{r}11500 \\
6910 \\
3980 \\
2360 \\
1520 \\
1070 \\
923\end{array}$ & $\begin{array}{r}23400 \\
13000 \\
6660 \\
3700 \\
2280 \\
1640 \\
1450\end{array}$ & $\begin{array}{r}37700 \\
19800 \\
9330 \\
4980 \\
2970 \\
2180 \\
1960\end{array}$ & $\begin{array}{r}58700 \\
29300 \\
12700 \\
6510 \\
3780 \\
2820 \\
2600\end{array}$ \\
\hline
\end{tabular}

DURATION TABLE OF DAILY MEAN FLOW FOR PERIOD OF RECORD 1931-85

DISCHARGE, IN FT ${ }^{3} / S$, THAT WAS EQUALED OR EXCEEDED FOR INDICATED PERCENT OF TIME

\begin{tabular}{|c|c|c|c|c|c|c|c|c|c|c|c|c|c|c|}
\hline 58 & 108 & 158 & 208 & 258 & 308 & 408 & 508 & 608 & 708 & 758 & 808 & 858 & 908 & 958 \\
\hline 705 & 415 & 289 & 221 & 172 & 137 & 106 & 91 & 76 & 63 & 56 & 48 & 39 & 30 & 20 \\
\hline
\end{tabular}


GILA RIVER BASIN

09432000 GILA RIVER BELOW BLUE CREEK, NEAR VIRDEN, NM

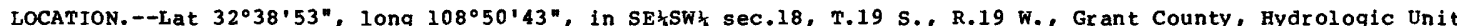
15040002 , on $r i g h t$ bank at head of canyon, $1.4 \mathrm{mi}$ downstream from Blue Creek, $10 \mathrm{mi}$ east of virden, $16 \mathrm{mi}$ upstream from New Mexico-Arizona state line, and at mile 523.6.

DRAINAGE AREA.--3.203 $\mathrm{mi}^{2}$, excluding Animas River Basin.

PERIOD OF RECORD.--May to November 1914, March to September 1915, July 1927 to cur rent year. July 1927 to May 1931 monthly discharge only, published in WSP 1313, computed as sum of flow at virden Bridge, 9 mi downstream, and in Sunset Canal. Published as Gila River near Duncan, AZ, 1914-15 and as Gila River at Fuller's Ranch, near Duncan, Az, 1931-38.

REVISED RECORDS.--WSP 1283: Drainage area. WSP 1313: 1929, 1931-32(M).

GAGE.--Water-stage recorder. Altitude of gage is 3,875 ft, from river-profile map. May 11 , 1914 to Sept, 30, 1915, at site $6 \mathrm{mi}$ downstream, 1,000 $\mathrm{ft}$ upstream from intake of sunset Canal. June 1 to July 7 , 1931, nonrecording gage at present site and datum. Since April 18, 1980, supplementary gage on left bank $800 \mathrm{ft}$ downstream at same datum., Since June 1980, crest-stage gage at supplementary gage site.

REMARKS.--Station is above all Duncan Valley diversions. Diversions for irrigation of about 6,200 acres above station.

AVERAGE DISCHARGE. --57 years (water years 1929-85), $188 \mathrm{ft}^{3} / \mathrm{s}, 136,200$ acre-ft/yx.

EXTREMES FOR PERIOD OF RECORD.--Maximum discharge, 58,700 ft3/s Dec. 19, 1978, gage height, 29.00 ft from rating curve extended above $38,000 \mathrm{ft}^{3} / \mathrm{s}$ on basis of slope-area measurement of peak fiow; minimum, $1 \mathrm{ft} / \mathrm{f}^{3} \mathrm{July} 14$, 1934 .

STATISTICAL SUMMARIES

MEAN MONTHLY AND MEAN ANNUAL DISCHARGES 1929-85

\begin{tabular}{|c|c|c|c|c|c|c|}
\hline MONTH & $\begin{array}{l}\text { MINIMUM } \\
\left(\mathrm{FT}^{3} / \mathrm{S}\right)\end{array}$ & $\begin{array}{c}\text { MAXIMUM } \\
\left(\mathrm{FT}^{*}{ }^{3} / \mathrm{S}\right)\end{array}$ & $\begin{array}{l}\text { MEAN } \\
\left(\mathrm{FT}^{3} / \mathrm{S}\right)\end{array}$ & $\begin{array}{l}\text { STAN- } \\
\text { DARD } \\
\text { DEVIA- } \\
\text { TION } \\
\left(\text { FT }^{3} / \mathrm{S}\right)\end{array}$ & $\begin{array}{l}\text { COEFFI - } \\
\text { CIENT OF } \\
\text { VARI - } \\
\text { ATION }\end{array}$ & $\begin{array}{c}\text { PERCENT } \\
\text { OF } \\
\text { ANNUAL } \\
\text { RUNOFF }\end{array}$ \\
\hline $\begin{array}{l}\text { OCTOBER } \\
\text { NOVEMBER } \\
\text { DECEMBER } \\
\text { JANUARY } \\
\text { FEBRUARY } \\
\text { MARCH } \\
\text { APRIL } \\
\text { MAY } \\
\text { JUNE } \\
\text { JULY } \\
\text { AUGUST } \\
\text { SEPTEMBER }\end{array}$ & $\begin{array}{l}5.4 \\
35 \\
48 \\
64 \\
61 \\
45 \\
28 \\
14 \\
4.4 \\
4.9 \\
9.4 \\
4.9\end{array}$ & $\begin{array}{r}1667 \\
707 \\
2485 \\
1387 \\
1277 \\
1464 \\
1138 \\
907 \\
183 \\
217 \\
966 \\
1507\end{array}$ & $\begin{array}{r}150 \\
111 \\
226 \\
233 \\
319 \\
395 \\
258 \\
142 \\
46 \\
75 \\
200 \\
183\end{array}$ & $\begin{array}{r}273 \\
105 \\
416 \\
298 \\
347 \\
435 \\
264 \\
168 \\
43 \\
53 \\
193 \\
270\end{array}$ & $\begin{array}{r}1.82 \\
.95 \\
1.84 \\
1.28 \\
1.09 \\
1.10 \\
1.02 \\
1.18 \\
.95 \\
.70 \\
.97 \\
1.48\end{array}$ & $\begin{array}{r}6.4 \\
4.7 \\
9.7 \\
10.0 \\
13.6 \\
16.9 \\
11.0 \\
6.1 \\
1.9 \\
3.2 \\
8.6 \\
7.8\end{array}$ \\
\hline NNUAL & 43 & 640 & 188 & 139 & .74 & 100 \\
\hline
\end{tabular}

MAGNITUDE AND PROBABILITY OF ANNUAL LOW FLOW BASED ON PERIOD OF RECORD 1930-85

\begin{tabular}{|c|c|c|c|c|c|c|}
\hline \multirow{2}{*}{$\begin{array}{l}\text { PERIOD } \\
\text { (CON- } \\
\text { SECU- } \\
\text { TIVE } \\
\text { DAYS) }\end{array}$} & \multicolumn{6}{|c|}{$\begin{array}{l}\text { DISCHARGE, IN FT'/S, FOR INDICATED RECURRENCE } \\
\text { INTERVAL, IN YEARS, AND ANNUAL NON- } \\
\text { EXCEEDANCE PROBABILITY, IN PERCENT }\end{array}$} \\
\hline & $\begin{array}{c}2 \\
508\end{array}$ & $\begin{array}{c}5 \\
208\end{array}$ & $\begin{array}{l}10 \\
108\end{array}$ & $\begin{array}{l}20 \\
58\end{array}$ & $\begin{array}{l}50 \\
28\end{array}$ & $\begin{array}{r}100 \\
18\end{array}$ \\
\hline $\begin{array}{r}1 \\
3 \\
7 \\
14 \\
30 \\
60 \\
90 \\
120 \\
183\end{array}$ & $\begin{array}{r}5.9 \\
6.3 \\
7.2 \\
9.5 \\
15 \\
29 \\
41 \\
58 \\
87\end{array}$ & $\begin{array}{r}3 . \\
3 . \\
3 . \\
4 . \\
6 . \\
15 \\
23 \\
33 \\
52\end{array}$ & $\begin{array}{r}2 . \\
2 . \\
3 . \\
4 . \\
9 . \\
16 \\
23 \\
38\end{array}$ & $\begin{array}{r}1.7 \\
1.8 \\
2.0 \\
2.2 \\
3.2 \\
7.0 \\
12 \\
16 \\
29\end{array}$ & $\begin{array}{r}1 . \\
1 . \\
1 . \\
1 . \\
2 . \\
4 . \\
8 . \\
11 \\
21\end{array}$ & $\begin{array}{r}3.5 \\
6.4 \\
7.8 \\
17\end{array}$ \\
\hline
\end{tabular}

MAGNITUDE AND PROBABILITY OF ANNUAL HIGH FLOW BASED ON PERIOD OF RECORD 1929-85

\begin{tabular}{|c|c|c|c|c|c|c|}
\hline \multirow{2}{*}{$\begin{array}{l}\text { PERIOD } \\
\text { (CON- } \\
\text { SECU- } \\
\text { TIVE } \\
\text { DAYS) }\end{array}$} & \multicolumn{6}{|c|}{$\begin{array}{l}\text { DISCHARGE, IN FT' }{ }^{3 / S} \text {, FOR INDICATED RECURRENCE } \\
\text { INTERVAL, IN YEARS, AND ANNUAL } \\
\text { EXCEEDANCE PROBABILI TY, IN PERCENT }\end{array}$} \\
\hline & $\begin{array}{c}2 \\
508\end{array}$ & $\begin{array}{c}5 \\
208\end{array}$ & $\begin{array}{l}10 \\
108\end{array}$ & $\begin{array}{l}25 \\
48\end{array}$ & $\begin{array}{l}50 \\
28\end{array}$ & $\begin{array}{r}100 \\
18\end{array}$ \\
\hline $\begin{array}{r}1 \\
3 \\
7 \\
15 \\
30 \\
60 \\
90\end{array}$ & $\begin{array}{r}1940 \\
1390 \\
982 \\
714 \\
526 \\
361 \\
291\end{array}$ & $\begin{array}{r}4910 \\
3340 \\
2150 \\
1450 \\
1010 \\
705 \\
582\end{array}$ & $\begin{array}{r}8170 \\
5380 \\
3260 \\
2090 \\
1410 \\
1000 \\
850\end{array}$ & $\begin{array}{r}14300 \\
9040 \\
5110 \\
3080 \\
1990 \\
1470 \\
1290\end{array}$ & $\begin{array}{r}20700 \\
12700 \\
6840 \\
3940 \\
2460 \\
1880 \\
1700\end{array}$ & $\begin{array}{r}29100 \\
17400 \\
8920 \\
4920 \\
2980 \\
2350 \\
2200\end{array}$ \\
\hline
\end{tabular}

DURATION TABLE OF DAILY MEAN FLOW FOR PERIOD OF RECORD 1929-85 DISCHARGE, IN FT'3/S, THAT WAS EOUALED OR EXCEEDED FOR INDICATEDD PERCENT OF TIME

\begin{tabular}{|c|c|c|c|c|c|c|c|c|c|c|c|c|c|c|}
\hline 58 & 108 & 158 & 208 & 258 & 308 & 408 & 508 & 608 & 708 & 758 & 808 & 858 & 908 & 958 \\
\hline 692 & 404 & 280 & 209 & 165 & 138 & 106 & 88 & 74 & 59 & 51 & 42 & 31 & 20 & 9.1 \\
\hline
\end{tabular}




\section{GILA RIVER BASIN}

09442680 SAN FRANCISCO RIVER NEAR RESERVE, NM

LOCATION, - Lat $33^{\circ} 44^{\prime} 12^{\prime \prime}$, long $108^{\circ} 46^{\prime} 14^{n}$, in NEłNWhSE sec. 35, T. 6 S, R. 19 w., Catron County, Hydrologic Unit 15040004 , on left bank $1,300 \mathrm{ft}$ downstream from Rainbow Bridge Canyon, 1.7 mi northwest of Reserve, and at mile 563.1 .

DRAINAGE AREA. $--350 \mathrm{mi}^{2}$, approximately.

PERIOD OF RECORD.--March 1959 to current year.

REVISED RECORDS.--WDR NM-78-1: 1977. WRD Nm-84-1: 1973, 1979-80.

GAGE.--Water-stage recorder. Elevation of gage is 5,820 ft above National Geodetic vertical Datum of 1929 , from topographic map. Prior to Dec. 15, 1972 at site 1,800 ft upstream at different datum.

REMARKS,--Possible minor regulation by Luna Lake, $27 \mathrm{mi}$ upstream. Diversions for irrigation of about 280 acres upstream from station.

AVERAGE DISCHARGE.--26 years (water years 1960-85), 28.3 $\mathrm{ft}^{3} / \mathrm{s}, 20,500$ acre-ft/yr.

EXTREMES FOR PERIOD OF RECORD.--Maximum discharge, 9,830 ft $3 / \mathrm{s}$, Oct. 1, 1983, gage height, $11.71 \mathrm{ft}$ recorded, 11.3 $\mathrm{ft}$, from outside floodmarks, from rating curve extended above $1,400 \mathrm{ft} / \mathrm{s}$ on basis of slope-area measurement of peak flow; minimum, $1.0 \mathrm{ft} 3 / \mathrm{s}$, Mar. $16,1959$.

EXTREMES OUTSIDE PERIOD OF RECORD.--Maximum stage, about $15 \mathrm{ft}$, as determined in $1962 \mathrm{from}$ old floodmarks. Major floods of Nov. 26, 1905 and Dec. 3, 1906, exceeded 20,000 ft3/s at Alma (downstream). See wSP 1313 .

STATISTICAL SUMMARIES

MEAN MONTHLY AND MEAN ANNUAL DISCHARGES 1960-85

\begin{tabular}{|c|c|c|c|c|c|c|}
\hline MONTH & $\begin{array}{c}\text { MINI MUM } \\
\left(\mathrm{FT}^{3} / \mathrm{S}\right)\end{array}$ & $\begin{array}{c}\text { MAXIMUM } \\
\left(\mathrm{FT}^{3} / \mathrm{S}\right)\end{array}$ & $\begin{array}{l}\text { MEAN } \\
\left(\mathrm{FT}^{3} / \mathrm{S}\right)\end{array}$ & $\begin{array}{c}\text { STAN- } \\
\text { DARD } \\
\text { DEVIA- } \\
\text { TION } \\
\left(\mathrm{FT}^{3} / \mathrm{S}\right)\end{array}$ & $\begin{array}{l}\text { COEFFI- } \\
\text { CIENT OF } \\
\text { VARI- } \\
\text { ATION }\end{array}$ & $\begin{array}{c}\text { PERCENT } \\
\text { OF } \\
\text { ANNUAL } \\
\text { RUNOFF }\end{array}$ \\
\hline $\begin{array}{l}\text { OCTOBER } \\
\text { NOVEMBER } \\
\text { DECEMBER } \\
\text { JANUARY } \\
\text { FEB RUARY } \\
\text { MARCH } \\
\text { APRIL } \\
\text { MAY } \\
\text { JUNE } \\
\text { JULY } \\
\text { AUGUST } \\
\text { SEPTEMBER }\end{array}$ & $\begin{array}{l}3.3 \\
5.2 \\
5.1 \\
5.7 \\
5.1 \\
4.2 \\
3.4 \\
2.8 \\
2.0 \\
1.8 \\
4.6 \\
5.1\end{array}$ & $\begin{array}{r}430 \\
211 \\
159 \\
78 \\
178 \\
336 \\
398 \\
162 \\
22 \\
28 \\
79 \\
172\end{array}$ & $\begin{array}{l}38 \\
18 \\
24 \\
19 \\
36 \\
78 \\
57 \\
18 \\
5.5 \\
8.8 \\
16 \\
21\end{array}$ & $\begin{array}{l}97 \\
40 \\
40 \\
20 \\
46 \\
97 \\
86 \\
32 \\
4.4 \\
6.4 \\
15 \\
33\end{array}$ & $\begin{array}{r}2.56 \\
2.17 \\
1.65 \\
1.04 \\
1.28 \\
1.23 \\
1.50 \\
1.77 \\
.80 \\
.72 \\
.94 \\
1.57\end{array}$ & $\begin{array}{r}11.1 \\
5.4 \\
7.1 \\
5.6 \\
10.5 \\
23.0 \\
16.9 \\
5.3 \\
1.6 \\
2.6 \\
4.7 \\
6.1\end{array}$ \\
\hline NNUAL & 6.1 & 101 & 28 & 27 & .94 & 100 \\
\hline
\end{tabular}

MAGNITUDE AND PROBABILITY OF ANNUAL LOW FLOW BASED ON PERIOD OF RECORD 1961-85

\begin{tabular}{|c|c|c|c|c|c|c|}
\hline \multirow{2}{*}{$\begin{array}{l}\text { PERIOD } \\
\text { (CON- } \\
\text { SECU- } \\
\text { TIVE } \\
\text { DAYS) }\end{array}$} & \multicolumn{6}{|c|}{$\begin{array}{l}\text { DISCHARGE, IN FT }{ }^{3} / \mathrm{S} \text {, FOR INDICATED RECURRENCE } \\
\text { INTERVAL, IN YEARS, AND ANNUAL NON- } \\
\text { EXCEEDANCE, PROBABILITY, IN PERCENT }\end{array}$} \\
\hline & $\begin{array}{c}2 \\
508\end{array}$ & $\begin{array}{c}5 \\
208\end{array}$ & $\begin{array}{l}10 \\
108\end{array}$ & $\begin{array}{l}20 \\
58\end{array}$ & $\begin{array}{l}50 \\
28\end{array}$ & $\begin{array}{r}100 \\
18\end{array}$ \\
\hline $\begin{array}{r}1 \\
3 \\
7 \\
14 \\
30 \\
60 \\
90 \\
120 \\
183\end{array}$ & $\begin{array}{l}2.3 \\
2.5 \\
2.8 \\
3.0 \\
3.5 \\
4.1 \\
4.7 \\
5.7 \\
7.2\end{array}$ & $\begin{array}{l}1.6 \\
1.7 \\
1.9 \\
2.1 \\
2.4 \\
2.8 \\
3.3 \\
4.2 \\
5.2\end{array}$ & $\begin{array}{l}1.4 \\
1.5 \\
1.6 \\
1.7 \\
1.9 \\
2.3 \\
2.7 \\
3.5 \\
4.8\end{array}$ & $\begin{array}{l}1.2 \\
1.3 \\
1.4 \\
1.5 \\
1.7 \\
1.9 \\
2.3 \\
3.0 \\
4.5\end{array}$ & $\begin{array}{l}1.1 \\
1.1 \\
1.2 \\
1.3 \\
1.4 \\
1.6 \\
2.0 \\
2.5 \\
4.3\end{array}$ & $\begin{array}{l}-- \\
-- \\
-- \\
-- \\
-- \\
-- \\
-- \\
--\end{array}$ \\
\hline
\end{tabular}

MAGNITUDE AND PROBABILITY OF ANNUAL HIGH FLOW BASED ON PERIOD OF RECORD 1960-85

\begin{tabular}{|c|c|c|c|c|c|c|}
\hline \multirow{2}{*}{$\begin{array}{l}\text { PERIOD } \\
\text { (CON- } \\
\text { SECU- } \\
\text { TIVE } \\
\text { DAYS) }\end{array}$} & \multicolumn{6}{|c|}{$\begin{array}{l}\text { DISCHARGE, IN FT' }{ }^{3} \text { S, FOR INDICATED RECURRENCE } \\
\text { INTERVAL, IN YEARS, AND ANNUAL } \\
\text { EXCEEDANCE PROBABILITY, IN PERCENT }\end{array}$} \\
\hline & 208 & 208 & 10 & 25 & $\begin{array}{r}50 \\
28\end{array}$ & 100 \\
\hline $\begin{array}{r}1 \\
3 \\
7 \\
15 \\
30 \\
60 \\
90\end{array}$ & $\begin{array}{r}261 \\
181 \\
133 \\
97 \\
72 \\
51 \\
41\end{array}$ & $\begin{array}{r}1010 \\
652 \\
421 \\
276 \\
197 \\
134 \\
108\end{array}$ & $\begin{array}{r}2250 \\
1320 \\
777 \\
477 \\
331 \\
222 \\
178\end{array}$ & $\begin{array}{r}5640 \\
2890 \\
1510 \\
853 \\
574 \\
381 \\
306\end{array}$ & $\begin{array}{r}10600 \\
4870 \\
2320 \\
1240 \\
818 \\
541 \\
434\end{array}$ & $\begin{array}{l}-- \\
-- \\
-- \\
-- \\
-- \\
--\end{array}$ \\
\hline
\end{tabular}

DURATION TABLE OF DAILY MEAN FLOW FOR PERIOD OF RECORD 1960-85 DISCHARGE, IN FT'3/S, THAT WAS EQUALED OR EXCEEDED FOR INDICATED PERCENT OF TIME 


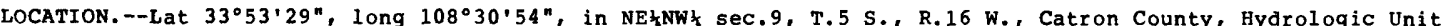
15040004 , on right bank $0.4 \mathrm{mi}$ upstream from first diversion, $1.4 \mathrm{mi}$ northeast of Aragon, and $8 \mathrm{mi}$ upstream from Apache Creek.

DRAINAGE AREA, --94 $\mathrm{mi}$.

PERIOD OF RECORD.--July 1966 to current year. 1955 to 1965 at site 0.6 mi upstream (drainage area, $89 \mathrm{mi} 2$ ), annual maximum only.

REVISED RECORD.--WDR NM-78-1: 1977.

GAGE.--Water-stage recorder and concrete control. Elevation of gage is $6,750 \mathrm{ft}$ above National Geodetic vertical Datum of 1929, from topographic map.

REMARKS.- -No diversion above station.

AVERAGE DISCHARGE.--19 years (water years 1967-85), 3.47 $\mathrm{ft}^{3} / \mathrm{s}, 2,510$ acre-ft/yr.

EXTREMES FOR PERIOD OF RECORD.--Maximum discharge, $660 \mathrm{ft} 3 / \mathrm{s}$ Oct. 2, 1983, gage height, $3.90 \mathrm{ft}$ in gage well, 4.23 ft from floodmarks, from rating curve extended above $80 \mathrm{ft}^{3} / \mathrm{s}$ on basis of slope-area measurements at gage heights $3.13 \mathrm{ft}$ and $3.90 \mathrm{ft}$ minimum, $1.1 \mathrm{ft} / \mathrm{s}$ July $22,1969$.

STATISTICAL SUMMARIES

MEAN MONTHLY AND MEAN ANNUAL DISCHARGES 1967-85

MAGNITUDE AND PROBABILITY OF ANNUAL LOW FLOW BASED ON PERIOD OF RECORD 1968-85

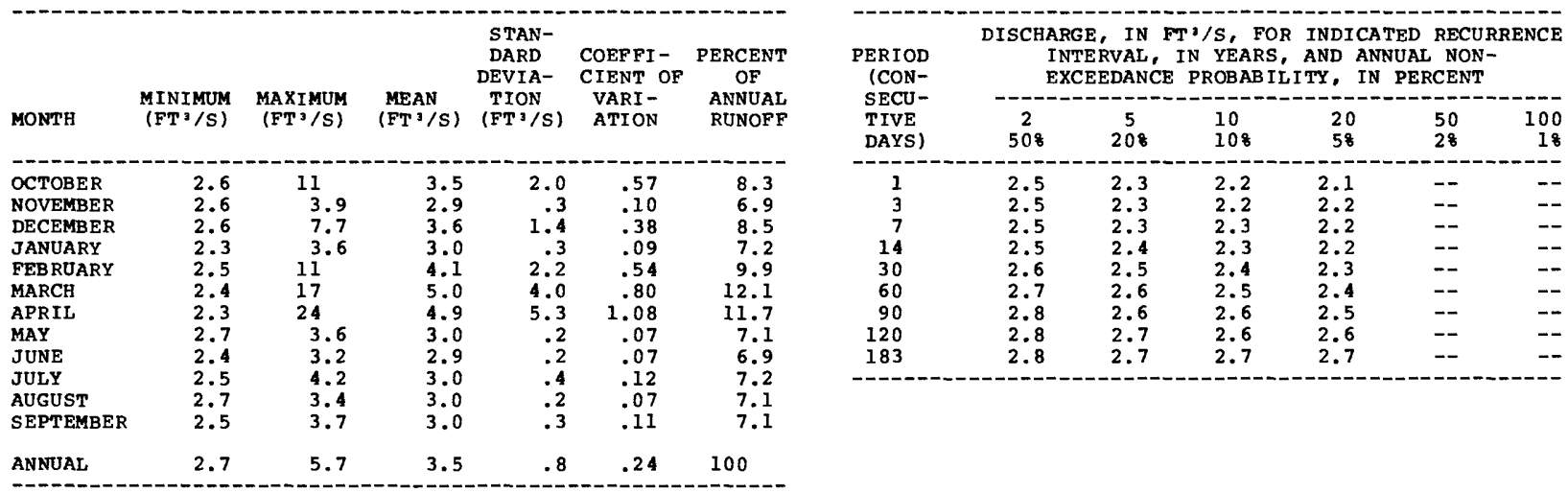

MAGNITUDE AND PROBABILITY OF ANNUAL HIGH FLOW BASED ON PERIOD OF RECORD 1967-85

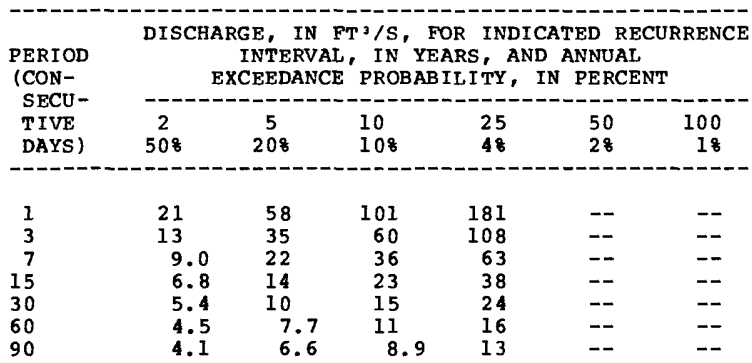

DURATION TABLE OF DAILY MEAN FLOW FOR PERIOD OF RECORD 1967-85 DISCHARGE, IN FT $3 / S$, THAT WAS EQUALED OR EXCEEDED FOR INDICATED PERCENT OF TIME

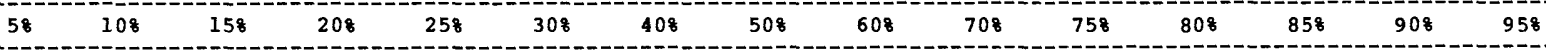

$\begin{array}{lllllllllllllll}4.3 & 3.5 & 3.3 & 3.2 & 3.2 & 3.1 & 3.0 & 3.0 & 2.9 & 2.8 & 2.7 & 2.7 & 2.6 & 2.6 & 2.5\end{array}$


GILA RIVER BASIN

09443000 SAN FRANCISCO RIVER NEAR ALMA, NM

LOCATION.--Lat $33^{\circ} 22^{\prime} 05^{\prime \prime}$, long $108^{\circ} 54^{\prime} 35^{\prime \prime}$, in SWłSEł sec.4, T.11 S., R. 20 w., Catron County, Hydrologic Unit 15040004 , on right bank $1.2 \mathrm{mi}$ downstream from Alma, $4 \mathrm{mi}$ northwest of Glenwood, $6 \mathrm{mi}$ upstream from whitewater Creek, and at mile 523.5 .

DRAINAGE AREA. $--1,546 \mathrm{mi}^{2}$.

PERIOD OF RECORD.--September 1904 to January 1914, fragmentary (see wSP 1313), January 1964 to current year. Prior to october 1911, published as "at Alma".

REVISED RECORD.--WDR NM-78-1: 1977.

GAGE.--Water-stage recorder. Elevation of gage is 4,840 ft above National Geodetic Vertical Datum of 1929 , from topographic map. Prior to Aug. 11, 1912, nonrecording gages at various sites, within 500 ft of each other, 0.8 mi upstream, at different datums. Aug. 11, 1912, to Feb. 2, 1914, nonrecording gage at approximately present site and datum. Jan. 10, 1964 to Nov.1, 1972, at datum $3.00 \mathrm{ft}$ higher.

REMARRS.--Diversions for irrigation of about 1,600 acres upstream from station.

AVERAGE DISCHARGE.--21 years (water years 1965-85), 88.4 ft $3 / \mathrm{s}, 64,050$ acre-ft/yr.

EXTREMES FOR PERIOD OF RECORD,--Maximum discharge, 56,600 ft ${ }^{3} / \mathrm{s}$, Oct. 2, 1983, gage height, $21.44 \mathrm{ft}$, present datum, from floodmarks in well, from rating curve extended above $9,000 \mathrm{ft} / \mathrm{s}$ on basis of slope-area measurements at gage heights $18.16 \mathrm{ft}$ and $21.44 \mathrm{ft}$; no flow many days.

EXTREMES OUTSIDE PERIOD OF RECORD.--Major floods probably occurred Jan. 19 and oct. 14, 1916, when discharges of $60,000 \mathrm{ft}^{3} / \mathrm{s}$ were computed at Clifton, $\mathrm{Az}$

STATISTICAL SUMMARIES

MEAN MONTHLY AND MEAN ANNUAL DISCHARGES 1965-85

\begin{tabular}{|c|c|c|c|c|c|c|}
\hline MONTH & $\begin{array}{l}\text { MINIMUM } \\
\left(\mathrm{FT}^{3} / \mathrm{S}\right)\end{array}$ & $\begin{array}{c}\text { MAXIMUM } \\
\left(\mathrm{FT}^{3} / \mathrm{S}\right)\end{array}$ & $\begin{array}{l}\text { MEAN } \\
\left(\mathrm{FT}^{3} / \mathrm{S}\right)\end{array}$ & $\begin{array}{c}\text { STAN- } \\
\text { DARD } \\
\text { DEVIA- } \\
\text { TION } \\
\left(\mathrm{FT}^{3} / \mathrm{S}\right)\end{array}$ & $\begin{array}{l}\text { COEFFI- } \\
\text { CIENT OF } \\
\text { VARI- } \\
\text { ATION }\end{array}$ & $\begin{array}{c}\text { PERCENT } \\
\text { OF } \\
\text { ANNUAL } \\
\text { RUNOFF }\end{array}$ \\
\hline OCTOBER & .1 & 1792 & 155 & 431 & 2.78 & 14.5 \\
\hline NOVEMBER & .2 & 502 & 42 & 109 & 2.60 & 3.9 \\
\hline DECEMBER & 6.3 & 1009 & 141 & 292 & 2.07 & 13.2 \\
\hline JANUARY & 6.9 & 420 & 71 & 108 & 1.53 & 6.6 \\
\hline FEBRUARY & 2.5 & 586 & 130 & 185 & 1.42 & 12.2 \\
\hline MARCH & 0.0 & 870 & 225 & 277 & 1.23 & 21.1 \\
\hline APRIL & 0.0 & 855 & 140 & 213 & 1.52 & 13.1 \\
\hline MAY & 0.0 & 427 & 47 & 96 & 2.04 & 4.4 \\
\hline JUNE & 0.0 & 42 & 6.2 & 11 & 1.70 & .6 \\
\hline JULY & 1.0 & 40 & 15 & 12 & .83 & 1.4 \\
\hline AUGUST & 4.1 & 251 & 48 & 55 & 1.14 & 4.5 \\
\hline SEPTEMBER & .3 & 301 & 49 & 82 & 1.68 & 4.6 \\
\hline ANNUAL & 6.3 & 283 & 89 & 94 & 1.05 & 100 \\
\hline
\end{tabular}

MAGNITUDE AND PROBABILITY OF ANNUAL LOW FLOW BASED ON PERIOD OF RECORD 1966-85

\begin{tabular}{|c|c|c|c|c|c|c|}
\hline \multirow{2}{*}{$\begin{array}{l}\text { PERIOD } \\
\text { (CON- } \\
\text { SECU- } \\
\text { TIVE } \\
\text { DAYS) }\end{array}$} & \multicolumn{6}{|c|}{$\begin{array}{l}\text { DISCHARGE, IN FT } 3 / S \text {, FOR INDICATED RECURRENCE } \\
\text { INTERVAL, IN YEARS, AND ANNUAL, NON- } \\
\text { EXCEEDANCE PROBAB ILITY, IN PERCENT }\end{array}$} \\
\hline & $\begin{array}{c}2 \\
508\end{array}$ & $\begin{array}{c}5 \\
208\end{array}$ & $\begin{array}{l}10 \\
108\end{array}$ & $\begin{array}{l}20 \\
58\end{array}$ & $\begin{array}{l}50 \\
28\end{array}$ & $\begin{array}{r}100 \\
18\end{array}$ \\
\hline $\begin{array}{r}1 \\
3 \\
7 \\
14 \\
30 \\
60 \\
90 \\
120 \\
183\end{array}$ & $\begin{array}{r}0.0 \\
0.0 \\
0.0 \\
0.0 \\
.5 \\
.9 \\
2.8 \\
7.2 \\
11\end{array}$ & $\begin{array}{r}0.0 \\
0.0 \\
0.0 \\
0.0 \\
0.0 \\
.1 \\
.5 \\
2.0 \\
6.2\end{array}$ & $\begin{array}{l}0.0 \\
0.0 \\
0.0 \\
0.0 \\
0.0 \\
0.0 \\
0.0 \\
.8 \\
5.0\end{array}$ & $\begin{array}{r}0.0 \\
0.0 \\
0.0 \\
0.0 \\
0.0 \\
0.0 \\
0.0 \\
.3 \\
4.4\end{array}$ & $\begin{array}{l}-- \\
-- \\
-- \\
-- \\
-- \\
-- \\
-- \\
--\end{array}$ & 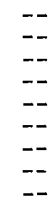 \\
\hline
\end{tabular}

MAGNITUDE AND PROBABILITY OF ANNUAL HIGH FLOW BASED ON PERIOD OF RECORD $1965-85$

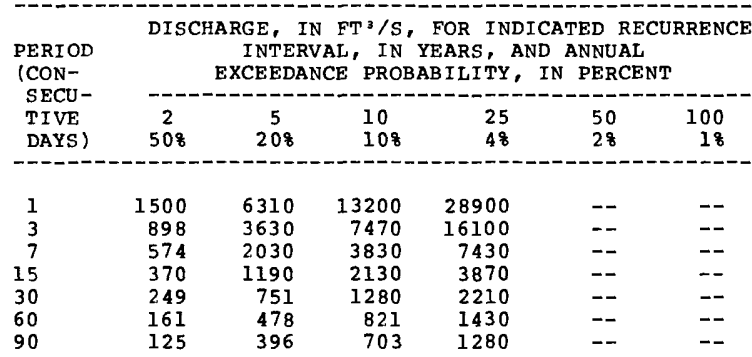

DURATION TABLE OF DAILY MEAN FLOW FOR PERIOD OF RECORD 1965-85

DISCHARGE, IN FT3/S, THAT WAS EQUALED OR EXCEEDED FOR INDICATED PERCENT OF TIME

\begin{tabular}{|c|c|c|c|c|c|c|c|c|c|c|c|c|c|c|}
\hline 58 & 108 & 158 & 208 & 258 & 308 & 408 & 508 & 608 & 708 & 758 & 80 옹 & 858 & 908 & 958 \\
\hline 404 & 184 & 106 & 65 & 43 & 31 & 18 & 12 & 7.6 & 3.7 & 2.2 & 1.2 & .5 & 0.0 & 0.0 \\
\hline
\end{tabular}


GILA RIVER BASIN

09444000 SAN FRANCISCO RIVER NEAR GLENWOOD, NM

LOCATION.--Lat $33^{\circ} 14^{\prime} 48^{\prime \prime}$, long $108^{\circ} 52.47^{\prime \prime}$, in NEłNWł sec.23, T.12 S., R. 20 w., Catron County, Hydrologic Unit 15040004 , on left bank $0.2 \mathrm{mi}$ upstream from hot springs, $5 \mathrm{mi}$ south of Glenwood, $6 \mathrm{mi}$ downstream from whitewater Creek, and at mile 511.5 .

DRA INAGE AREA. $--1,653 \mathrm{mi}^{2}$.

PERIOD OF RECORD.--October 1927 to current year. Monthly discharge only for some periods, published in wSP 1313.

REVISED RECORDS.--WSP 1213: 1931, 1934, 1936-37, 1940-42, 1943-44(M), 1945-47. WSP 1283: Drainage area. WDR NM-78-1: 1977. WDR NM-79-1: 1973, 1975-77'(P).

GAGE.--Water-stage recorder. Elevation of gage is 4,560 ft above National Geodetic vertical Datum of 1929 , from topographic map. Prior to Feb. 15, 1934, at site $4.5 \mathrm{mi}$ upstream at datum $98.82 \mathrm{ft}$ higher.

REMARKS.--Diversions for irrigation of about 2,000 acres upstream from station. Gage height and rain gage satellite telemeter at station.

AVERAGE DISCHARGE. --58 years (water years 1928-85), $82.9 \mathrm{ft} / \mathrm{s}, 60,060$ acre-ft/yr.

EXTREMES FOR PERIOD OF RECORD.--Maximum discharge, 37,100 ft $3 / \mathrm{s}$, oct. 2, 1983 , gage height, $18,15 \mathrm{ft}$ recorded, $20.80 \mathrm{ft}$ from outside floodmarks, from rating curve extended above 4,200 $\mathrm{ft}^{3} / \mathrm{s}$ on basis of slope-area measurements at gage heights $10.74 \mathrm{ft}, 15.6 \mathrm{ft}$ and $20.8 \mathrm{ft} ; \mathrm{minimum}, 1.5 \mathrm{ft} / \mathrm{s} \mathrm{Aug}$. 6,1961 .

EXTREMES OUTSIDE PERIOD OF RECORD.--Major floods probably occurred Jan. 19 and Oct. 14 , 1916 when discharges of $60,000 \mathrm{ft}^{3} / \mathrm{s}$ or greater were computed for station at Clifton, Az. On Nov. 26, 1905, a peak of $25,000 \mathrm{ft} / \mathrm{s}$ was measured (by float-area method) at station at Alma (about $12 \mathrm{mi}$ upstream, drainage area, 1,560 mi2); a similar measurement of $21,000 \mathrm{ft}^{3} / \mathrm{s}$ was made at the Alma station for peak of Dec. 3, 1906.

STATISTICAL SUMMARIES

MEAN MONTHLY AND MEAN ANNUAL DISCHARGES 1928-85

\begin{tabular}{|c|c|c|c|c|c|c|}
\hline MONTH & $\begin{array}{l}\text { MINIMUM } \\
\left(\mathrm{FT}^{3} / \mathrm{S}\right)\end{array}$ & $\begin{array}{c}\text { MAXIMUM } \\
\left(\mathrm{FT}^{3} / \mathrm{S}\right)\end{array}$ & $\begin{array}{l}\mathrm{MEAN} \\
\left(\mathrm{FT}^{3} / \mathrm{S}\right)\end{array}$ & $\begin{array}{l}\text { STAN- } \\
\text { DARD } \\
\text { DEVIA- } \\
\text { TION } \\
\left(\mathrm{FT}^{3} / \mathrm{S}\right)\end{array}$ & $\begin{array}{l}\text { COEFFI- } \\
\text { CIENT OF } \\
\text { VARI- } \\
\text { ATION }\end{array}$ & $\begin{array}{c}\text { PERCENT } \\
\text { OF } \\
\text { ANNUAL } \\
\text { RUNOFF }\end{array}$ \\
\hline $\begin{array}{l}\text { OCTOBER } \\
\text { NOVEMBER } \\
\text { DECEMBER } \\
\text { JANUARY } \\
\text { FEBRUARY } \\
\text { MARCH } \\
\text { APRIL } \\
\text { MAY } \\
\text { JUNE } \\
\text { JULY } \\
\text { AUGUST } \\
\text { SEPTEMBER }\end{array}$ & $\begin{array}{l}9.8 \\
11 \\
13 \\
14 \\
15 \\
11 \\
10 \\
8.7 \\
5.7 \\
13 \\
14 \\
7.7\end{array}$ & $\begin{array}{r}2026 \\
520 \\
1068 \\
509 \\
602 \\
1036 \\
1049 \\
593 \\
93 \\
108 \\
392 \\
282\end{array}$ & $\begin{array}{r}92 \\
42 \\
86 \\
76 \\
111 \\
182 \\
138 \\
71 \\
27 \\
37 \\
76 \\
56\end{array}$ & $\begin{array}{r}297 \\
66 \\
188 \\
110 \\
158 \\
237 \\
190 \\
102 \\
18 \\
18 \\
67 \\
49\end{array}$ & $\begin{array}{l}3.24 \\
1.58 \\
2.19 \\
1.44 \\
1.42 \\
1.30 \\
1.38 \\
1.43 \\
.69 \\
.49 \\
.87 \\
.87\end{array}$ & $\begin{array}{r}9.2 \\
4.2 \\
8.6 \\
7.7 \\
11.2 \\
18.3 \\
13.9 \\
7.2 \\
2.7 \\
3.8 \\
7.7 \\
5.7\end{array}$ \\
\hline NNUAL & 14 & 351 & 83 & 79 & .95 & 100 \\
\hline
\end{tabular}

MAGNITUDE AND PROBABILITY OF ANNUAL LOW FLOW BASED ON PERIOD OF RECORD 1929-85

\begin{tabular}{ccccccc} 
& DISCHARGE, IN FT /S, FOR INDICATED RECURRENCE \\
PERIOD & \multicolumn{5}{c}{ INTERVAL, IN YEARS, AND ANNUAL NON- } \\
(CON- & \multicolumn{3}{c}{ EXCEEDANCE } & PROBABIL ITY, IN PERCENT \\
SECU- & -2 & 5 & 10 & 20 & 50 & 100 \\
TIVE & 2 & 208 & 108 & 58 & 28 & 18 \\
DAYS) & 508 & 208 & 98 \\
\hline 1 & 9.9 & 6.2 & 4.7 & 3.8 & 2.9 & 2.4 \\
3 & 11 & 7.1 & 5.6 & 4.6 & 3.6 & 3.1 \\
7 & 12 & 8.3 & 6.8 & 5.7 & 4.7 & 4.2 \\
14 & 13 & 9.5 & 7.9 & 6.7 & 5.6 & 5.0 \\
30 & 15 & 11 & 9.6 & 8.5 & 7.4 & 6.8 \\
60 & 18 & 13 & 11 & 10 & 9.1 & 8.4 \\
90 & 20 & 15 & 13 & 11 & 9.9 & 9.1 \\
120 & 23 & 17 & 15 & 14 & 12 & 11 \\
183 & 29 & 22 & 19 & 18 & 17 & 16 \\
\hline
\end{tabular}

MAGNITUDE AND PROBABILITY OF ANNUAL HIGH FLOW BASED ON PERIOD OF RECORD $1928-85$

\begin{tabular}{|c|c|c|c|c|c|c|}
\hline \multirow{2}{*}{$\begin{array}{l}\text { PERIOD } \\
\text { (CON- } \\
\text { SECU- } \\
\text { TIVE } \\
\text { DAYS) }\end{array}$} & \multicolumn{6}{|c|}{$\begin{array}{l}\text { DISCHARGE, IN FT }{ }^{3} / \mathrm{S} \text {, FOR INDICATED RECURRENC } \\
\text { INTERVAL, IN YEARS, AND ANNUAL } \\
\text { EXCEEDANCE PROBABILITY, IN PERCENT }\end{array}$} \\
\hline & $\begin{array}{c}2 \\
508\end{array}$ & $\begin{array}{c}5 \\
208\end{array}$ & $\begin{array}{l}10 \\
108\end{array}$ & $\begin{array}{l}25 \\
48\end{array}$ & $\begin{array}{l}50 \\
28\end{array}$ & $\begin{array}{r}100 \\
18\end{array}$ \\
\hline $\begin{array}{r}1 \\
3 \\
7 \\
15 \\
30 \\
60 \\
90\end{array}$ & $\begin{array}{l}854 \\
542 \\
363 \\
257 \\
191 \\
139 \\
112\end{array}$ & $\begin{array}{r}2730 \\
1680 \\
1020 \\
659 \\
455 \\
326 \\
267\end{array}$ & $\begin{array}{r}5330 \\
3220 \\
1820 \\
1120 \\
737 \\
525 \\
438\end{array}$ & $\begin{array}{r}11400 \\
6750 \\
3540 \\
2020 \\
1260 \\
892 \\
765\end{array}$ & $\begin{array}{r}19200 \\
11200 \\
5550 \\
3020 \\
1800 \\
1270 \\
1110\end{array}$ & $\begin{array}{r}31300 \\
17800 \\
8440 \\
4370 \\
2500 \\
1760 \\
1580\end{array}$ \\
\hline
\end{tabular}

DURATION TABLE OF DAILY MEAN FLOW FOR PERIOD OF RECORD 1928-85

DISCHARGE, IN FT ${ }^{3} / S$, THAT WAS EQUALED OR EXCEEDED FOR INDICATED PERCENT OF TIME

\begin{tabular}{|c|c|c|c|c|c|c|c|c|c|c|c|c|c|c|}
\hline 58 & 108 & 158 & 208 & 258 & 308 & 408 & 508 & 608 & 708 & 758 & 808 & 858 & 908 & 958 \\
\hline 303 & 155 & 100 & 73 & 57 & 47 & 36 & 31 & 26 & 23 & 21 & 19 & 17 & 15 & 12 \\
\hline
\end{tabular}

